$\beta \& B$ A $1,015,394$

540

857

D 7

BIBLTOTHECA

SORIPTOROM GRAECORUM

\title{
BT ROMANORUM
}

830

80 (N) 0085

TEUBNERLANA
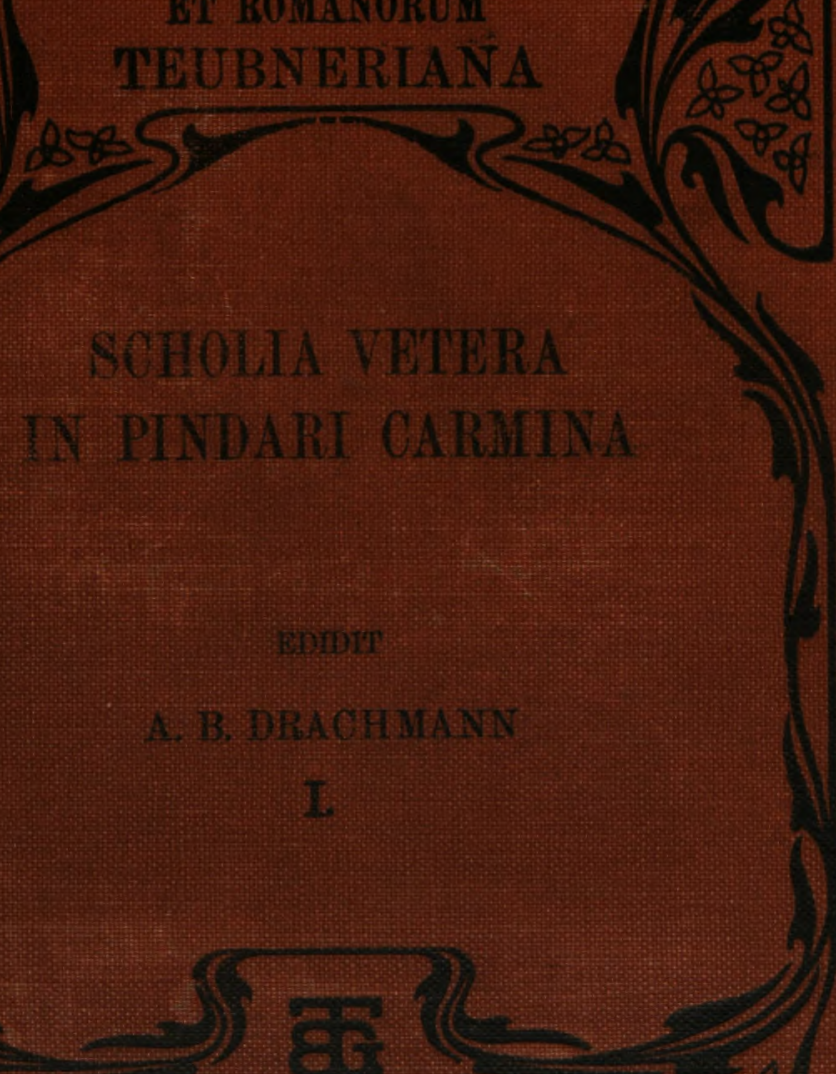

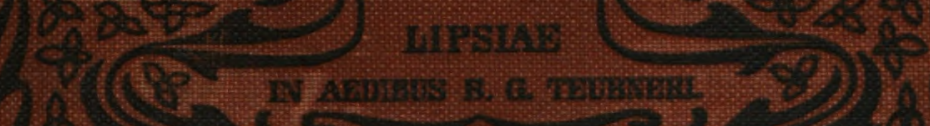




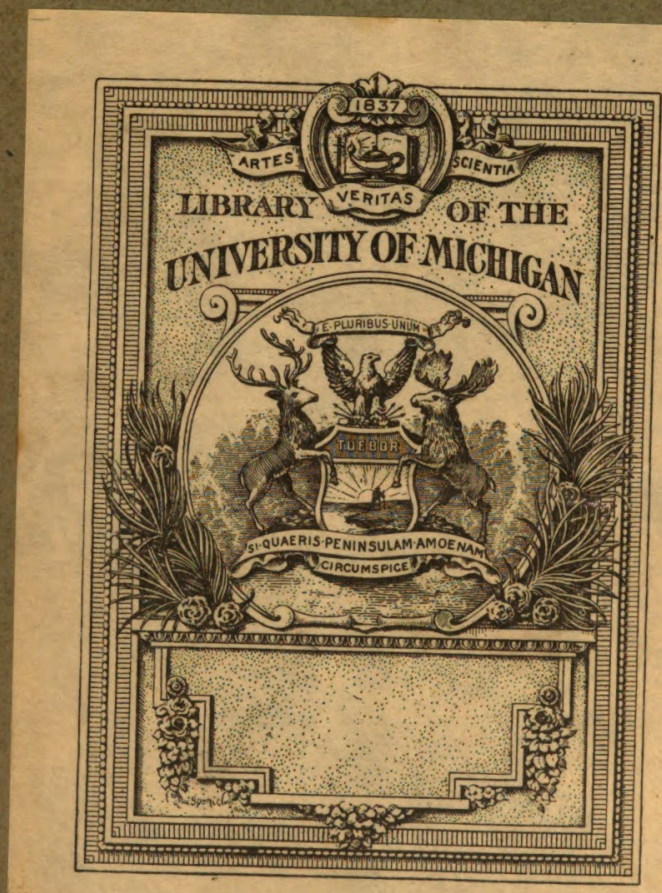

- 
888

$\mathrm{PHO}_{\mathrm{O}}$

537

DD 76 



\title{
SCHOLIA VETERA IN PINDARI CARMINA
}

\author{
RECENSUIT
}

A. B. DRACHMANT, ed.

VOL. I

SCHOLIA IN OLYMPIONICAS

ADIECTA EST TABULA PHOTOTYPICA.

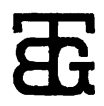

LIPSIAE

IN AEDIBUS B. G. TEUBNERI

MCMIII 
I

IIPSIAE: TYPIS B. G. TEUBNERI

Digitized by GOOgle 


\section{IOANNI LVDOVICO HEIBERG}

\section{PHILOLOGO}

D. 
Digitizad by Go ogle. 


\section{Praefatio.}

In scholiis veteribus Pindari Olympionicarum recensendis praecipue usus sum codicibus sex, qui iam a Mommseno in textu carminum constituendo adhibiti et siglis ABCDEQ notati sunt*). Sunt autem hi:

Ambrosianus C 222 inf. bombycinus**) saec. XIII. A Descripsit Keil Rh. Mus. VI (1848) p. 108-10; cfr. Studemund Anecd. p. 212-47; 0. Schroeder Philol. LIV (1899) p. 277. Continet praeter multa alia Pindari Ol. I-XII. Correctus est passim manu si non eadem quae textum et scholia scripsit, at certe aequali; quam a prima manu, quae raro correxit nec fere nisi in scribendo, non distinxi. Uno aut altero loco manus recens apparet. Imago phototypica huic volumini adfixa est huius codicis fol. $195^{\text {v }}$. Apographum scholiorum ab Eduardo Schwartz confectum. mihique liberalissime traditum primum ipse, iterum Otto Schroeder cum codice contulimus. Glossas ipse exscripsi, in quibusdam amica Heibergii opera adiutus. - Codicis Ambrosiani apographum, quod ad scholia attinet, est cod. Vratislaviensis Redigerani 40 prima pars; de quo codice Bed. tripartito cfr. Gerhardus ap. Boeckhium vol. II p. II sq.; Schroeder 1. 1. p. 286; Catal. codd. Graec. qui in Bibl. Urbica Vratislaviensi adservantur (Vratisl. 1889) p. 49 sqq. Boeckhius codicem, quem primus adhibuit, Vrat(islaviensem)

*) Codices Pindari Florentinos, Romanos, Mediolanenses, Venetos, Parisinos, Vindobonenses breviter descripsit Jul. Resler Philol. vol. IV (1849) p. 510-532; ne hoc in unoquoque codice commemorando repetam.

*) Hoc nomine olim vulgato codices in charta orientali scriptos hodie quoque designare commodum visum est. 
A vocavit; ego Red. insignivi, sed raro eius mentionem feci; notitiam eius lectionum debeo praeter Boeckhium Ottoni Schroeder.

B Vaticanus 1332 bomb. saec. XII. Descripsit Schroeder 1. 1. p. 278-80; cfr. Resler Ult. Pind. Isthmiae Scholia (diss. Vratislav. [1847]) p. 3 sq.; id. Philol. IV (1849) p. 525; Mommsen Rh. Mus. VI (1848) p. 435-36. Continuit olim omnia Pindari epinicia cum scholiis; nune foliis amissis lacunae effectae sunt haud paucae, in scholiis 01 . hae: I init. - 29; 69 med. (p. 34, 14 huius ed.) - 106; 171 - fin.; V 16 med. (p. 143, 13) - $48 \operatorname{med}$ (p. 151, 8); fortasse etiam IX, 46-59 (cfr. p. 278, 13*). - Ceterum aliis quoque modis codex pessime habitus est; quippe charta mollis et parum resistens multis locis vel humore prope dissoluta vel ipso atramento exesa est, ut pro litteris maculae lacunaeve informes existerent; tum totus codex ne ultra pessum daretur pelle quadam obductus est, quae si olim perlucida fuit, hodie certe in fuscum colorem obscurata efficit ut multis locis scriptura divinanda potius quam legenda sit, haud paucis legi omnino non possit**). Codicem Fulvii Ursinii olim fuisse constat; qui in exem-

*) Cum sch. 44 desinit f. $63, a$ sch. 60 b inçipit f. 64 . Fol. 63 autem olim a suo loco aberat; nam et ceteris minus est marginibus abscissis, et textum eius (vs. 40-59 vulg.) in imo f. $62^{\mathrm{r}}$ et summo f. $64^{\mathrm{r}}$ adscripsit manus recentior. Sed dubitationem movet, quod neque textus ulla pars excidit, et scholia vulgata 46-59 sine texta non folium, sed tantum paginam codicis $B$ expletura erant.

*) Hanc medelam, morbo sane peiorem, abhinc non plus triginta annis codici adhibitam esse mihi dixit $P$. Ehrle, egregius bibliothecae Vaticanae praefectus; cum eius testimonio optime convenit quod neque Resler illius rei mentionem facit et Tycho Mommsen, qui codicem anno 1847 tractavit, se eum obductum esse meminisse mihi quaerenti negavit. Resleri autem verbae haec sunt (Philol. IV, 625): „Difficilis lectu est quod passim situ et aetate margines eius sunt adesi, passim chartae panni adsuti ordoque foliorum perversus est". Feliciter sane accidit quod Mommsen omnia scholia ante contulit quam illad malum codici illatum est. 
plari suo editionis Romanae lectiones haud paucas, sed quae omnes ad textum pertinerent, enotavit*); ad scholia nihil aliud quam nomina quaedam fabularia, ut argumenta indicaret, apposuit. - Glossae partim prima, partim recentiore manu (Georgii Platanitae, cfr. Schroeder Philol. LIV p. 279) scriptae sunt; hanc, quae in textu scholiorum raro apparet, signavi $\mathbf{B}^{\mathbf{2}}$. - Scholia ipse contuli, glossas descripsi; postea Tycho Mommsen v. cl. et de his studiis optime meritus collationem suam abhine plus $\mathrm{L}$ annos, cum codicis condicio melior esset, factam insigni liberalitate mihi concessit. Hac cum mea diligenter comparata omnes locos, de quorum lectione dubitari posse visum est, benevola Schroederi, Heibergii, Hudii opera cum codice denuo conferendos curavi. - Ex B scholia O. XIV (non O. XI quoque, ut Abel Wien. Studd. IV p. 236 opinari videtur) descripsit librarius codicis Vaticanae 1333, quo $\beta$ adhibito de scriptura codicis $B$ in hac quidem parte certius affirmare licet. Hoc apographum siglo $\beta$ notavi.

Parisinus 2774- bomb. saeculi XIV. Descripsit Schroeder C 1. 1. p. 280. Continet praeter alia Pindari Olympia et partem Pythiorum (usque ad V 67 vulg.). - Multa correxit librarius ipse in scribendo, panca manus fere aequafis $\left(\mathbf{C}^{2}\right)$; deinde manus recens (saec. $\mathrm{XV}-\mathrm{XVI}, \mathbf{C}^{3}$ ) lemmata subduxit, glossas et varias lectiones addidit; cuius additamenta fere non curavi. - Scholia ipse contuli, glossarum nonnullas descripsi.

Laurentianus 32, 52 bomb. saec. XIV. Descripsit D Schroeder 1. 1. p. 281; cfr. Mommsen parerg. Pind. p. 4-6. Continet Pindari carmina omnia; Pindaro alligatus est alius codex, qui Theocriti carmina continet. Primi quaternionis primum folium periit; ceterorum margines magnam

-) Locos ubi codex et editio discreparent fere duobus punctis (:) supra vel infra versum positis et in codice et in libro impresso significabat, in hoc saepius addito v. c., h. $\theta$. vetus codex; quae puncta Schroeder Philol. LIV p. 279, quia exemplar Ursinianum inspicere non potuerat, Georgio illi qui codicem retractavit tribuit. 
partem exesi et charta recentiole refecti sunt, in qua manus recentissima ex alio codice ( $Q$, Laur. 32, 35) quae interierunt scholiorum partes supplere conata est. Ea additamenta signavi [D]; sicubi lectionem communi librariorum $D$ et [D] opera effectam afferre necesse fuit, quae [D]

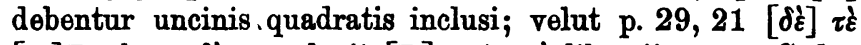
[D]D, h. $\theta . \delta \varepsilon$ supplevit [D] ante $\tau \dot{\varepsilon}$ librarii D. - Codex in hac quidem parte (neque enim eadem manus totum exaravit) litteris minutissimis, crebris compendiis, calamo et crasso et festinanti satis neglegenter scriptus ad legendum haud facilis, ad conferendum valde lubricus est. Contuli scholia, glossas fere neglexi.

E Laurentianus 32, 37 bomb. saec: XIV. Descripsit Schroeder p. $281 \mathrm{sq}$. Continet Theocriti carmina (cfr. Ziegler Theocr. ${ }^{3}$ p. V); Pindari Olympia et Pythia; tum Libanii epistulas alia manu scriptas. Ultimum Pindari folium (131) chartaceum est; continet P. XII 45-56 vulg.

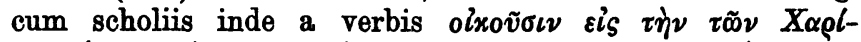

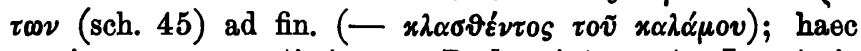
omnia manu recentissima ex D descripta sunt. In ceteris scholiis manus recens $\left(\mathrm{F}^{2}\right)$ raro apparet. - Codicem Euangelistae Capiferrei poetae Romani (Nolhac, Bibliothèque de Fulvio Orsini p. 257) olim fuisse ex inscriptioni-

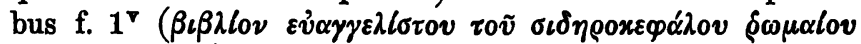
$x \alpha i \tau \tilde{\omega} \nu \varphi(\lambda \omega \nu)$ et $197^{\nabla}$ apparet. - Scholia contuli, glossas omnes perlegi et si quae notatu dignae videbantur descripsi. a Laurentianus 32, 35 bomb. saec. XIV. Continet Pindari Olympia et Pythia cum prolegomenis (p. 4,9-11, 11 h. ed.), scholiis, glossis (f. $1-89^{\mathrm{r}}$ ); tum f. $89^{\mathrm{v}}-91^{\mathrm{v}}$ alia manus $\left(Q^{b}\right)$ alteram vitam Pindari et metrica quaedam addidit*). In imo f. $91^{\mathbf{v}}$ eadem manus primum Iliadis versum cum scholiis scripsit. - Qui Pindarum scripsit, in scholiis varias lectiones vel correctiones supra versus addidit; saepe se ipse in scribendo ita correxit, ut quod

*) Huic nomen fuisse Malachiam ex subscriptione f. $8^{\text {r }}$

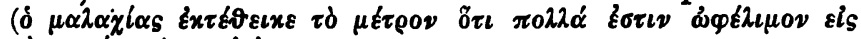

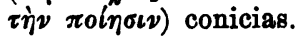


scriptum erat pumice detergeret et in litura meliora rescriberet. - Ex hoc codice vel simillimo eius descripta sunt scholia Vratislaviensis D (h. e. tertiae partis codicis Vrat. ${ }^{\circ}$ Redigerani tripartiti), quem adhibuit Boeckhius. Signavi Vrat. d, sed raro attuli. - Codicis Q scholia contuli, glossas fere non curavi.

Praeter hos libros a me inspecti et partim collati sunt:

Vaticanus 41 bomb. saec. XIV. Continet praeter vitam H Pindari recentiore manu scriptam Olympia et Pythia cum scholiis, deinde initium Batrachomachiae. - Contuli vitam, scholia $0 . I$ et partem 0 . II, alia passim per omnes Olympionicas, maxime ubi deficit $B$; nam fuit cum crederem ex hoc codice Calliergum scholia cod. B supplevisse.

Laur. 32, 33 bomb. saec. XIV. Continet praeter multa F alia Olympia et Pythia cum scholiis et glossis. Scholia O. I perlegi et passim contuli, alia pauca, maxime quae ad temporum rationes pertinent, notavi.

Gottingensis philol. 29 bomb. saec. XIII. Descripsit G Heyne $I^{3}$ praef. p. LI; Boeckhius I p. XVII. Adhibuit in scholiis iam Heyne (cfr. praef. p. XV sq.), postea Boeckhius, in primis saltem Olympiis (cfr. praef. vol. II, 1 p.V). Continet Olympia (praeter O. I, quod manu rec. additum est) et Pythia (usque ad XII, 38 vulg.; quae restant et praeterea N. I-III alius recensionis sunt); tum Nicandri Theriaca et Alexipharmaca. - Codicem non totum contuli (quod nunc paenitet); sed tantum numerum locorum aliquam ob causam memorabilium inspexi, ut omnibus scholiis collatis non ita magnum fructum proventurum esse sperem.

Vaticanus 42 bomb. saec. XIV. Continet Olympia K (praeter IV, $17-\mathrm{V}, 6$ vulg.) et Pythiorum quattuor prima (usque ad IV, 495 vulg.) cum prolegomenis, scholiis, glossis. Quantum operae in libro nullius pretii impenderim, referre trodet.

Ambrosianus E 103 sup. bomb. saec. XIII-XIV. Con- N tinet Olympia cum scholiis et post haec scholia metrica (quibus singula carmina carent) $\mathrm{O}$. I-XI cum prolego- 
menis. Contuli pauca scholia O. I et XIV et omnia O. XII.

(P Parisinus 2403 (V Mommseni) saec. XIII-XIV. Descripsit Schroeder p. 283. Continet Olympia, Pythia, partem Nemeorum. Scholia Germani veteribus intermixta habet, et omnino cum Caesareo Vindobonensi (U Mommseni) artissime coniunctus est (cfr. Mommsen Sch. Germ. p. XXIV). Contuli prolegomena Ol., multa scholia O. I, partem O. VI, omnia 0. XII, metrica pleraque. Invitus a siglo Mommseni discessi, cum $\mathbf{P}$ signarem; sed siglo $\mathbf{V}$ aliter utendum erat.

Praeterea obiter inspexi: Vat. 902 (L; saec. XIV, non XII), turpissimam chartam; Leid. Q 4 (0); Vat. Urb. 144 (R); Angelicum C, 1, 1; Vat. 121 (T); Marc. 475 (Y). Vindobonensem 130 (U) a Mommseno totum collatum habeo, sed rarissime adhibui; eiusdem ex collationibus aliquam notitiam habeo codicum 0 et T. Denique Perusini B 43 (M) particulas meum in usum contulit Heiberg.

De horum codicum cognatione et auctoritate ita hoc loco disseram, ut omissa omni disputatione quid sentiam breviter exponam, alibi de iis rebus fusius explicaturus. Igitur in 0 . II-XII duo diversa scholiorum genera exstant, unum solo codice A eiusque apographo Redigerano servatum, quod ob id ipsum Ambrosianum vocatur, alterum ceterorum omnium qui noti sunt codicum commune, quod ab antiquissimo codice B Vaticanum appellavit Mommsen. In O. XIII et XIV, ubi A deficit, solis scholiis Vaticanis utimur; neque aliter res habet in 0. I. Nam etsi scholia eius carminis etiam codice A continentur, tamen hac quidem in parte is est consensus eius cum ceteris codicibus, ut non duo genera diversa, sed duas recensiones unius generis traditas esse appareat. Ex his una codicibus ACFKN, altera (B)DEHQ (cum PR Ang.) continetur; certum eius rei documentum est ordo scholiorum 0 . I, 1, cuius conspectum hoc loco subicio. 


\begin{tabular}{|c|c|c|c|c|c|c|c|}
\hline A & $\mathbf{C}$ & F K & $\mathbf{E} \mathbf{R}$ & H & $\mathbf{Q}$ & D & ed. Rom. \\
\hline inscr. a & & $\begin{array}{l}\text { inscr. a } \\
\text { inscr. b }\end{array}$ & \multirow{10}{*}{$\begin{array}{c}\text { inscr. a } \\
\text { inscr. b } \\
1^{\mathrm{a}} \\
1^{\mathrm{b}} \\
1^{\mathrm{c}} \\
4^{\mathrm{a}} \\
9^{\mathrm{a}-0} \\
14^{\mathrm{c}} \\
5 \\
16^{\mathrm{b}} \\
19^{\mathrm{b}} \\
1^{\mathrm{a}} \\
(\mathrm{b}) \\
18^{\mathrm{b}}\end{array}$} & $\begin{array}{c}1^{\circ} \\
\text { inscr. } \\
-\end{array}$ & $\begin{array}{c}1^{0} \\
\text { inscr. a } \\
1^{\mathrm{d}}\end{array}$ & \multirow{3}{*}{$\begin{array}{c}1^{1^{0}} \\
\mathrm{XX}^{2} \text { lyr. } \\
\text { inscr. a } \\
\text { inscr. b }\end{array}$} & $\underset{10}{\text { inscr. } b+a}$ \\
\hline & $1^{a}$ & & & inscr. $b$ & inscr.b & & 14 \\
\hline & $1^{\mathrm{d}}$ & & & $1^{2}$ & $1^{a}$ & & $1^{b}$ \\
\hline & $1^{b}$ & & & $1^{b}$ & 1 b & $1^{2}$ & $1^{c}$ \\
\hline & $1^{\mathrm{c}}$ & & & & $1^{\circ}$ & $1^{b}$ & $1^{d}$ \\
\hline & $1^{\bullet}$ & & & $1^{\mathrm{d}}$ & $1^{\mathrm{d}}$ & $1^{\mathrm{c}}$ & $1^{t}$ \\
\hline & 4 & & & $\begin{array}{l}1^{8} \\
18\end{array}$ & $\begin{array}{l}1^{8} \\
18\end{array}$ & $1^{8}$ & $1^{8}$ \\
\hline & & & & & & & \\
\hline & & & & & & & \\
\hline & & & & & & & \\
\hline
\end{tabular}

Tamen FK versus finem sch. 159 mutati alteram recensionem sequuntur*). Contra $\mathrm{D}$, qui initio $\mathrm{O}$. I cum $\mathrm{HQ}$ consentit, a sch. fere $97 \mathrm{~b}$ ad $\mathrm{AC}$ transit ibique usque ad finem carminis permanet. - Ex his duabus recensionibus, ut hoc statim absolvam, neutram per omnia sequi licet; immo ex utraque pro natura loci quae lectio melior videtur eligenda est.

Inter codices scholiorum Vaticanorum B tam multa habet propria - nam et in rebus minutioribus saepe discrepat et multo pauciora scholia habet quam ceteri -, ut unam quasi classem efficiat, ceteri CDEHQ alteram. Ex his rursus E propius quam ceteri a B abest. C (cum $\mathrm{N}$, quantum eum novi) recensionem interpolatam et licenter mutatam praebet; quae in solo O. XIV a B propius quam ceterorum abest. HQ, quibus etiam $G$ sine dubio adnumerandus est, unam classem efficiunt, quae plura scholia habet quam ceteri. Ex his pleraque recentiora sunt; haud pauca ex Ambrosianis transscripta, sed in transscribendo interpolata sunt; pauca et vetera et egregia reperiuntur,

*) 159 a. e. f. FK cum $C$ faciunt ( $A$ iam in sch. 157 deticit); ab 159d (quod in CFK post $159 \mathrm{f}$ legitur) $E$ sequuntur 
ex quibus maxime insigne est sch. O. I 33. Denique D permulta scholia omisit, alia contraxit; in rebus minutioribus cum C saepe consentit, sed interpolationis gravioris vitio caret. - De reliquis codicibus quos quidem notos habeam pauca sufficient. $F$ et $K$ extra $O$. I cum $\mathbf{E}$ fere consentiunt; item P (i. e. V Mommseni) RTU, quorum PU Germani scholia veteribus inserta habent. $O$ cum $C N$ aliqua affinitate coniunctus videtur, sed passim cum B mirum in modum consentit. $M$ et $Y$, quorum uterque in occidente scriptus est, recensionem interpolatam, sed ab C diversam praebent.

In iis scholiis Ambrosianis quae solo codice A tradita sunt recensio facilis, omnis difficultas in emendatione est; nam A cum ab interpolatione liber sit, singula verba tam foede corrupta saepe exhibet, ut de vero restituendo despexandum videatur. Itaque correctis quae certo iudicio sanari posse visa sunt, cetera fere intacta reliqui, sed ut corruptelas indicarem et coniecturas dubias cum aliorum tum meas sub textu adhiberem. Ubi A cum Vaticanis non modo in summa rerum, sed etiam in verbis consentit, illum ducem ita secutus sum, ut nisi aperte corruptus esset, eius lectiones etiam omnium Vaticanorum consensui praeferrem, consensum autem cuiusvis codicis Vaticani cum A certissimum veritatis indicem haberem. Sed ubi solis Vaticanis utimur, difficilior est recensio. Neque enim codice. B, quamquam ceteris antiquior est et haud paucis locis solus verum servavit, tamquam certo duce uti licet; qui et in scholiis disponendis lemmatisque particulisve velut $\alpha \lambda \lambda \omega s$, ov้v, $\varphi \eta \sigma i$ similibus addendis omittendisve liberius versatus est et licentis mutationis atque interpolationis passim convincitur*). Accedit quod in scholiis

*) Duo huius rei exempla afferam. Sch. 0. IX 133 a et b duae glossae sunt, quae cum lemmate carerent,. in archetypo codicum CDEQ post $134 \mathrm{~d}$ falso insertae erant. In $\mathrm{B}$ recto quidem loco, post 132, leguntur; sed dum transponuntur, et lem-

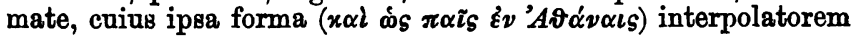


Vaticanorum et Ambrosiani communibus A non semper cum B, sed saepe cum ceteris facit. Itaque in scholiis Vaticanis recensendis eam legem tenui, ut consensum CDEQ pro fundamento haberem nec lectiones singulares codicis B nisi ubi aperte meliora praeberet reciperem, sin autem ad $B$ alius codex, in primis $\mathrm{E}$, accederet, reliquos posthaberem. In lemmatis quidem describendis et similibus rebus minoribus administrandis certas leges inveniri posse omnino desperavi. Ceterum in scholiis describendis non eandem diligentiam et verecundiam observatam esse, quam in tertu boni quidem librarii praestare solent, nemini ignotum est. Itaque et quid antiquitus fuerit permultis locis dignosci omnino nequit, et in eligendo, reiciendo, textu constituendo sibi constare difficile est; qua in re nec mihi satisfeci nec aliis me satisfecisse credo; a peritis veniam aliquam impetraturum spero.

Scholiorum veterum recensio praeter codices quibus ipsa tradita sunt etiam commentatores recentioris aetatis, qui is scholiis usi sunt, respicere debet. Thomas enim Magister et Triclinius in verbis Pindari interpretandis nostris scholiis passim ita usi sunt, ut ea ad verbum fere repeterent. Sed cum neuter eorum melioribus codicibus quam quos hodie habemus usus sit, ad textum constituendum parum adiumenti afferunt. Item Is. Tzetzes, dum scholia metrica in versus redigit, codice usus est nostri $\mathbf{Q}$ simillimo. Eorundem scholiorum metricorum Triclinius novam dedit recensionem et quaedam e suo ingenio recte emendavit. - Praeter Byzantinos Pindari commentatores etiam Jo. Tzetzes in variis scriptis suis nostris scholiis usus est; cuius locos plerosque debeo humanitati Scheorii, qui ex copiis suis quae in rem

arguit, locupletatae et $\grave{\eta}$ in $\nu \iota x \eta_{\sigma} \alpha_{S}$ mutato ad similitudinem iusti scholii 132 b redactae sunt. - Sch. O. XIII 116 e (p. 380,

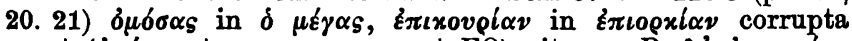

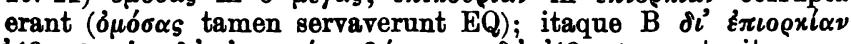

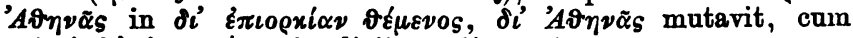
ceteri $\delta i$ i $\pi \iota$ lo $x i \alpha \nu$ simpliciter retinerent. 
erant liberalissime mecum communicavit. Tzetzem uberiora quam nos scholia Pindarica habuisse ex uno aut altero loco (0. VII 36) efficere voluit Scheerius; equidem dum eam rem in medio relinquo, eiusmodi locos inter textum et apparatum integros expressi, ceteros ibidem nominare satis habui. Denique aliorum quoque scriptorum locos cum scholiis Pindaricis aliqua affinitate coniunctos ut potui conquisivi et eandem rationem secutus, si qui ex commentariis Pindaricis a nostris diversis petiti esse visi erant, descripsi, reliquos dumtaxat nominavi. . Neque tamen occultissima et remotissima quaeque investigare aut propositum fuit aut, si fuisset, tempus sivisset; itaque aliis qui maioribus doctrinae subsidiis instructi in hoc genere elaborare volent, sat multa relicta esse certum habeo. Quamquam ad verba scholiorum emendanda exiguus fructus ex tali labore peti posse videtur.

Editionem meam ita institui, ut ex sex illis codicibus ABCDEQ scholia quidem omnia reciperem, glossas autem, -postquam in singulis codicibus plane diversas esse vidi, fere neglegerem. Ex solo codice B glossas prima manu scriptes, quas re vera ad vetera scholia pertinere apertum est, omnes recepi; item ex $\mathrm{E}$ varias lectiones inter versus adscriptas. Ex codicibus quorum partes tantum collatas habui, $H$ saepius adhibui, ne in quibusdam scholiis sola $Q$ auctoritate confiderem; reliquos ubi e re visum est in apparatu nominavi, sed eorum lectiones etiam sicubi enotatas habui omnes apponere minime volui. Scholia a codicibus aliena et ex aliis auctoribus adsumpta inter textum et apparatum ferri iam supra demonstravi.

Scholia Ambrosiana ot Vaticana, ut par est, separata tuli, exceptis iis locis (sunt autem pauci), ubi A cum Vaticanis ita consentit, ut ex utrisque unus textus commode effici possit. Ad singula scholia sigla codicum ex quibus desumpta sunt in margine apposui. Scholia diversi

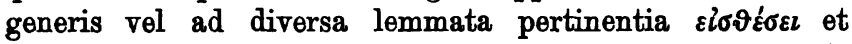
numero versus addito, scholia eiusdem generis et lemmatis 
spatio relicto distinxi*). Praeterea quae ad eundem versum pertinent litteris velut $\mathrm{a} b \mathrm{c}$ additis numeravi. Qua in re id quod his quidem in scholiis fieri necesse erat, magis commodo eorum qui hac editione usuri sunt quam ipsi rei et veritati consului; neque enim fieri potuit ut scholia diversae originis ubique secernerentur aut quae ab initio unum fuerunt non aliquando dirimerentur. Cum illis litteris spatiisque ita coniuncta sunt sigla codicum in margine adposita, ut ubicunque ponuntur, ad initium novi scholii spatio et littera signati referantur, ubi omittuntur, ex proximo superiore loco repetenda sint. Quodsi quando codex aliquis partem tantum scholii servavit, ad lineam transversam ( 1 ) in textu interpositam sigla marginis referuntur; eademque linea usus sum, si in uno textus versu plura spatia. occurrunt, ut ad cuius scholii initium sigla referrentur indicarem. Ceterum universa haec ratio, qua quibus ex codicibus unumquodque scholium parsve scholii edatur extemplo perspicuum fit, ut ad usum facilis et commoda est, ita solis verbis paulo difficilius explicatur. Scholia in eodem codice repetita esse siglis velut $Q^{\text {ab }}, Q^{\text {bis }}$ indicavi. Siglo uncinis incluso, velut (C), significatur, discrepantias eius codicis solito graviores (ut in $\mathrm{C}$ violentas mutationes, in D absurdas omissiones) in apparatu omissas esse; quod praeter CD raro vel nusquam feci**).

Propter eam codicum naturam quam supra (p. XIII) demonstravi, apparatum criticum tota lectionum farragine onerare editoris non est; immo. singulorum codicum naturs et quae inter omnes intercedat ratione perspecta,

*) In codicibus plerisque singula scholia, quae quidem lemmata habeant, tribus quatuorve punctis $(\because$ vel $\because)$ distinguuntur; post lemmata duplex fere punctum (:) ponitur. Sed propter universam scholiorum nostrorum naturam, quam infra demonstrabo, tota haec distinguendi ratio adeo nullius momenti est, ut eam in editione mea sciens praetermiserim.

- In $B$ eadem ratione uno alterove loco (velut p. 342,18 ) usus sum, ut scholium in ceteris codicibus separatum ferri, in B alio loco textri insertum esse significarem; sed lectiones codicis $B$ in apparatu nusquam sciens omisi. 
quidquid re vera traditum est, religiose adhibere, cetera abicere debet. Itaque in universum eas mihi leges proposui, ut omnes quisquilias, velut accentuum spirituumve rationes a vulgata abhorrentes, Ny paragogicum additum omissumve, similia praeterirem; deinde etiam singulos singulorum codicum errores vel interpolationes, abi de vera lectione ex ceteris constaret, omitterem. A priore lege raro nec fere nisi in $\mathbf{A}$ et $\mathbf{B}$ discessi; alteram ita observavı, ut cautior quam audacior videri mallem. Itaque primum ubicunque unius vel duorum codicum fide textus constat, eorum no apertissima quidem vitia praetermisi. Deinde codicis A omnes lectiones attuli, etiam in 0 . I, nam in ceteris carminibus id facere res ipsa coëgit. Item codicis B omnes lectiones adhibui, quod et omnium antiquissimus est et multa habet singularia. In ceteris Vaticanis legem severius tenui; tamen et ex E praeter itacismos et similia omnia fere attuli, quod is codex in rebus quibusdam, ut in ordine scholiorum, fideliorem quam ceteri archetypi imaginem praebet; et ubicumque duo pluresve codices' in eadem lectione ita consentiunt, ut ex eo consensu non modo ad textum constituendum, sed ad affinitates codicum diudicandas aliquid effici posse videatur, talia omnia diligenter adnotare volui. Quo factum est ut in $0 . I$, ubi codices in tres quatuorve classes omnes ad eundem archetypum redeuntes discedunt, apparatus paulo uberior quam in ceteris carminibus evaderet. Item ubicumque $B$ abest, ex codicibus DEQ nihil fere quod ullius momenti esse videretur praetermisi. Contra codicis interpolati C lectiones singulares, utpote quae ad textum constituendum plerumque nihil omnino valeant, ne apparatum ultra omnem modum inutiliter oneràrem, permultas omisi; de hoc igitur codice ex silentio meo concludere non licet. Denique etiam animi causa nonnulla quae ad somnolentiam vel licentiam librariorum illustrandam pertinerent me addidisse fateor. Ut autem ratio mea ad diudicandum facilis et aperta esset, in uno carmine 0 . XII omnes lectiones quas in apparatu praeterii in fine huius prae- 
fationis exposui. - De singulis codicibus hoc addo, D neglegentissime soriptum esse et in omittendo saepe incredibilem in modum peccare; $H$ vitiis minutis et futtilibus abundare; Q plerumque diligenter scriptum esse, sed passim multa vitia in breve spatium quasi congerere, ut arehetypum eius ad legendum varium fuisse conicias.

In apparatu $\mathrm{O}$. II-XII consensum codicem Vaticanorum, ubi Ambrosiano opponuntur, siglo V significavi; idem V in 0 . I facere nolui, ne ad consensum $\mathrm{AC}(\mathrm{DFK})$ significandum novum siglum introducerem. Coterum illo siglo V leotio archety $\mathrm{i}$ Vaticanorum, singulis singulorum codicum vitiis praetermissis, notatur. - Siglo uncis incluso, velut (E), significatur, eum codicem in ea quidem lectione qua de agitur cum ceteris consentire, sed aliquo vitio in apparatu omisso laborare. Ut ad pag. 142, 13 notatum est: $\pi \varepsilon \mu-$

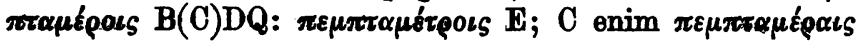
habet, quod non fuit indicandum. Similem in modum verba partesve verborum in apparatu uncinis inclusi, ubi duo pluresve codices in minutiis discrepant, in summa re consentiunt. Ut 0 . XIV metr. epod. $\gamma^{\prime}$ (p. 389, 4. 5), ubi 'Adoxuoúóvecov habet $\mathrm{D}$, 'AAxpocóviov $\mathrm{E}$, in apparatu est: 'Adxucubo (E)เov DE. Denique iisdem uncinis usus sum ad terminationes vocabulorum in codicibus (maxime $A B$ ) per compendium omissas significandas; velut , $\lambda \eta \eta_{\gamma}(\omega v)$ A" significat, in A $\lambda \eta^{\prime}$ vel simile quid, non $\lambda \eta^{\prime} \gamma \omega \nu$ aut $\lambda \widetilde{\eta \gamma^{\prime}}$ scriptum esse. Eiusmodi autem compendia ibi tantum notavi ubi de vera lectione aliquam ob causam dubitari posse videtur, quam ob rem etiam interrogationis siglum saepius addidi, ut 145, $17 x \alpha \tau \alpha \sigma x \varepsilon v \alpha \sigma \tau(\eta \nu$ ?) $\mathrm{DH}$, quod compendium utriusque codicis et in $x \alpha \tau \alpha \sigma x \varepsilon v \alpha \sigma \tau \delta \nu$ solvi potest. Ubi plures codices in eadem lectione consentiunt, sed unus eorum eam compendio obscuravit, siglo velut $\cdot \mathbf{E}^{\text {cp }}$ usus

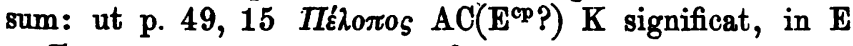

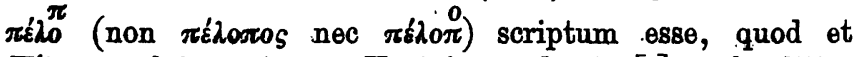
Iltharce solvi possit. - Uncinis quadratis [] verba litterasve quae hodie legi nequeunt inclusi; litteras dubiae

8chor. m Proparux ed. Drachmann. 
lectionis punctis infra positis notavi. Haec signa in B fere solo occurrunt; neque tamen ubicunque in B scriptura obscurata est, in apparatu dubitationis signa posui, sed ibi tantum ubi de vera lectione aliquam ob causam dubitari posse videtur. - Signum interrogationis ad siglum codicis appositum ad eum solum codicem pertinet cuius siglo appositum est; ut BDE? significat, de lectione codicum BD constare, de $\mathbf{E}$ non item. Hoc autem sigla eo consilio usus sum, ut quid in codice scriptum esset certo distingui non posse indicarem; dubitationes meas de collationis fidelitate, ut suboriri fere necesse fuit, ita lectoribus obtrudi nulla ratio est.

Ordinem scholiorum qui in codicibus est, si a mea editione discrepat, diligenter notavi, post singula scholia numero scholii quod in codice sequitur litteris minutis expresso. Et hoc quidem ideo necessario faciendum erat, quod si ordo scholiorum ignoratur, multae discrepantiae in lemmatis positae recte intellegi non possunt; sed nescio an etiam dispositionem cuiusque codicis tabella composita illustrare debuerim. In disponendis scholiis magnopere differunt codices. Scilicet archetypi codicum nostrorum eiusdem generis fuerunt cuius etiamnunc sunt codices Aristophanis et Homeri Pindaricis pluribus saeculis vetustiores, qui scholia alia in margine exteriore scripta, alia in margine superiore vel inferiore addita, glossas autem inter ipsos versus insertas habent. At in iis codicibus Pindaricis quibus nos utimur haec omnia in unum corpus scholiorum marginalium conflata sunt (nam glossae interlineares omnes fere praeter codicis $B$ recentiores sunt). Ut in Olympionicarum prima A post scholia cuiusque paginae archetypi glossas eiusdem iusto ordine collocavit, in ceteris carminibus glossas scholiis fere intermixtas habet. Inter Vaticanos praeter ceteros $\mathrm{E}$ vestigia antiquae formae servavit, reliqui diversae originis scholia plus minusve in ordinem redegerunt. Itaque ordine scholiorum et lemmatum ratione diligenter observatis vetera scholia marginalia, quae lemmate antiquitus tradito plerumque insignia sunt, 
a paraphrasibus et historiis, quae lemmate fere carebant, itemque a glossis brevibusque adnotationibus interlinearibus vel intramarginalibus distinguere saepe licet; denique scholia recentiora et quae ex recensione Ambrosiana in quosdam codices Vaticanos translata sunt saepe ipso ordine arguuntur. Haec. omnia typis exprimere, in ea praesertim editione in qua ordo scholiorum numeros versuum et ordinem lemmatum necessario sequatur, infinitae dubitationis opus fuisset; sed tamen ubicunque ordo in codieibus turbatus certa antiquae formae signa praebebat et disponendi aliquam libertatem relinquebat (nam alioquin codicum ordo cum ratione huius editionis consentiens sequendus erat), primo loco vetera quae visa erant scholia (glossis illa quidem saepe interspersa), cetera additamenta deinceps quantum fieri potuit collocavi.

Edita sunt haec scholia primum Romae a. 1515 a Zacharia Calliergo Cretensi (r). Is in scholiis pro funda- $r$ mento editionis habuisse videtur codicem $B$, sed ut etiam ex aliis codicibus (non ex solo $H$, ut putabat Mommsen. praef. ed. mai. p. XV not. 16 et suppl. p. 96) tota scholia et lectiones a B discrepantes reciperet. Eius textum secuti sunt editores posteriores usque ad Boeckhium; quorum consensum littera $\nabla$ signavi. Paucas novas lectiones e.v codice nescio quo petitas praebet margo editionis Brubachianae (Francofurti 1542); etiam pauciora correxerunt editores Oxonienses (1697). Primus D. Beckius. in editione Lipsiae a. 1792 emissa et editionem principem diligenter excussit et haud pauca ex ingenio suo correxit. In scholiis metricis emendandis Beckio praeierat Pauwius Notis in Pindarum (Trajecti ad Rh. 1747). Beckii editio scholiorum fundamentum fuit Heynianae (h) alteri maiori h (a. 1798), in quam primum scholia recepit; sed praeterea Heynius codicem Gottingensem passim inspexit*). Postremo Boeckhius codicibus Gottingensi et Vratislaviensibus usus

2) Usus sum editione Heyniana Lipsiensi a. 1817, quae repetitio est ed. Gottingensis alterius (1798). 
novam prorsus formam his scholiis induit et admirabili quae in illo fuit doctrina adiutus plurimos locos emendavit. Sed neque codicum satis plenam notitiam habuit nec scholia diversi generis ubique separavit, augendi quam distinguendi studiosior. - Lectiones vulgatas ubi a mea discrepant in apparatu plerumque commemoravi, exceptis tamen lemmatis similibusque rebus, in quibus primus editor satis libere versatus est; neque vero si qua seholia vel apud Boeckhium vel in mea editione vulgatis nova accesserunt, notandum duxi. In recentioribus editionibus si qua a vulgatis abhorrentia inveni, ad primum auctorem relata diligenter adnotari; sed ex Boeckhianis multa omittenda erant, quod is Ambrosiana cum Vaticanis haud raro ita coniunxit, ut novum textum efficeret. Quae ad scholia nostra emendanda praeter editores contulerunt viri docti, ut potui conquisivi; sed pauci ipsa scholia dedita opera tractaverunt, in quibus praeter Lehrsium (Die Pindarscholien. Lipsiae 1873) h: l. nominabo Horn (De Aristarchi studíis Pindaricis. Diss. Gryphiswald. 1883); Feine (De Aristarcho Pindari interprete. Diss. Jenens. 1883); Sybel (De scholiis vett. in Pindari carmina. Diss. Marburg. 1872). Ad scholia Ambrosiana O. I et II emendanda nonnihil contulit C. E. Ch. Schneider (Apparatus Pindarici supplementum. Vratislaviae 1844). Cetera per editiones Pindari, annales philologicos, miscellanea veterum virarum doctorum adeo dispersa leguntur, ut multa me fugisse certus sim. Coniecturas ad verba Pindari pertinentes, quas viri docti ex scholiis petiverunt, silentio praetermisi, ut verum fateor, deterritus exemplo Abelii.

Restat ut gratias agam iis viris doctis qui studia mea variis modis adiuverant. Quantum debeam Bchroedero, ex ipse hac editione parum apparet; qui non solum plagularum corrigendarum taedio suscepto ad textum et apparatum emendandum plurima contulit, sed etiam per multos annos et consilio et labore ita mihi adfuit, ut et in universa re et in singulis administrandis vix quicquam eo nesciente novaverim. Apographum codicis Ambrosiani 
Eduardo Schwartz, collationes cum B tum aliorum codicum Tychoni Mommsen accepta me referre iam supra dixi; hunc quidem interim mortem obiisse eo magis doleo, quod et doni tanti liberalitate homini sibi ignoto traditi et operis tot annis ante a se incohati, iam vero ab alio ex parte perfecti rationem nemo meliore iure reposcere potuisset. Scheerii benevolentiae me auctarium testimoniorum debere iam supra demonstravi. Praeter Heibergium, Sehroederam, Hudium etiam Carolus. Hölk nonnullos locos codicum Laurentianorum, de quibus dubitatio inciderat, meum in usum inspicere voluit. Denique: institutis Carlsbergico, quod otium ad haec studia necessarium liberalitate sua mihi paravit, et Hielmstierne-Rosencroniano, cuius opibus sublevatus codicum conferendi causa. peregrinationes feci, praefectisque bibliothecarum Vaticanae Ehrle, Laurentianae Biagi, Ambrosianae Ceriani, Parisinae 0 mont v. cll., qui eadem omnes humanitate codiees conferemdi laborem quantum in iis fuit faciliorem mihi reddere voluerunt, gratum animum libenter profiteor.

Scribebam Hauniae mense Januario a. MDCCCCIII.
A. B. Drachmann. 


\section{- Supplementum apparatus O. XII.}

Hoc supplemento continentur omnes lectiones codicum CDEQ, quae in apparatum non receptae sunt.

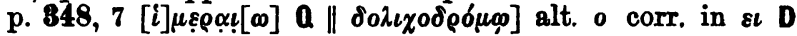

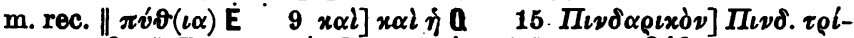

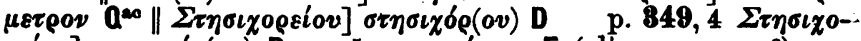

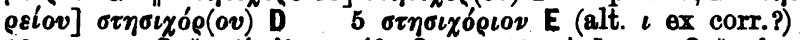

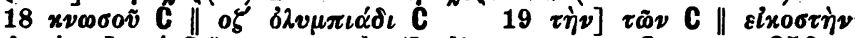

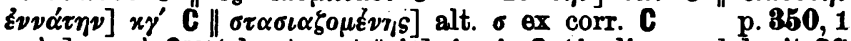

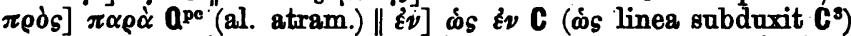

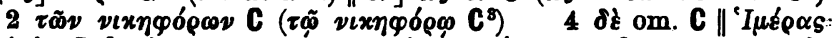
${ }_{x \alpha l}$ in D legi nequeunt 10 Elevveplov om. C 10.11 El-

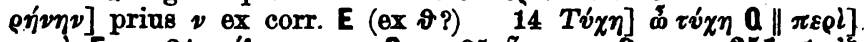

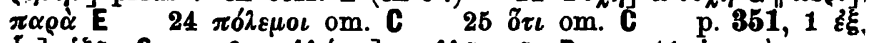

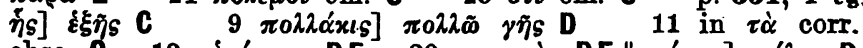

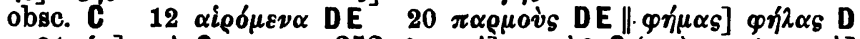

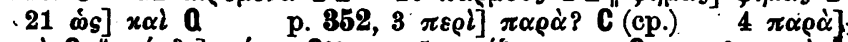

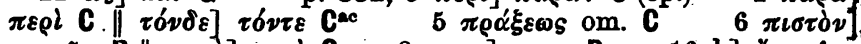
$\pi \iota \sigma \tau \tilde{\omega} \nu$ D $\| \pi \alpha \rho \alpha] \pi \varepsilon \rho l$ C $\quad 8 \varphi \eta \sigma l] \varphi \alpha \sigma \iota$ D 16 l.] $\xi_{\mu \pi \alpha \lambda \iota \nu}$

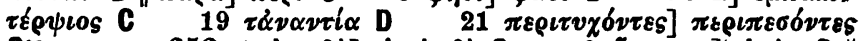

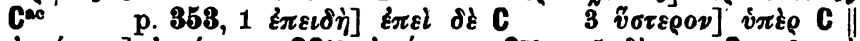

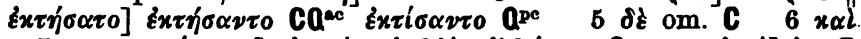

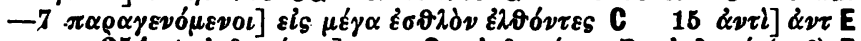

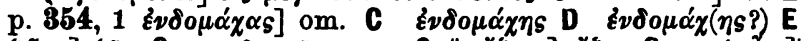

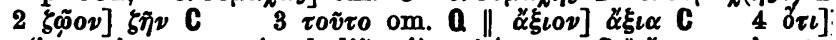
+ (i. e. signum novi scholii) viz

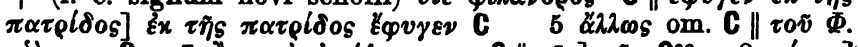

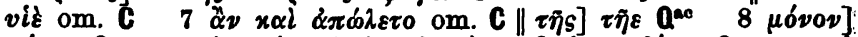

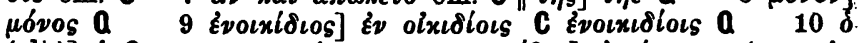

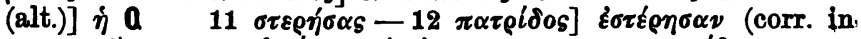

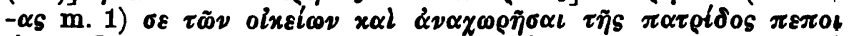

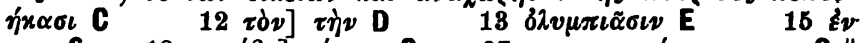

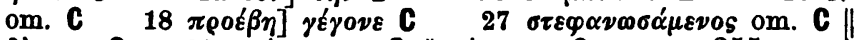

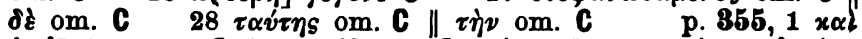

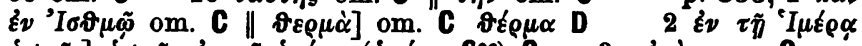

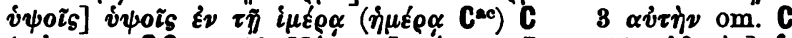

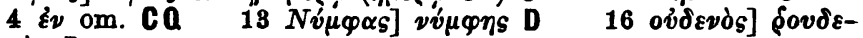
vòs D 


\section{Addenda et corrigenda.}

Praeter locos scholiorum recentiorum in apparatu nominatos afferre debueram etiam hos:

p. 246, 21-247, 5 cfr. Thom. 292, 3.

p. 248, 19 sq. = Germ. 294, 11.

p. 276, 1-3 cfr. Thom. 317, 20.

p. 276, $22-26$ cfr. Thom. 319, 5.

Dum ad scholia metrica recentiora sero attendo, correctiones nonnullas a Triclinio factas vel mihi vel sliis tribui. Sunt autem hae:

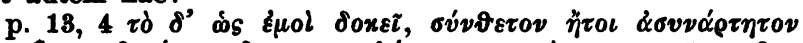

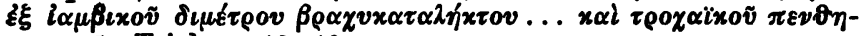

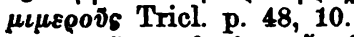

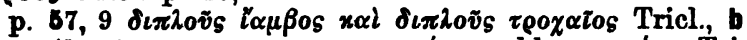

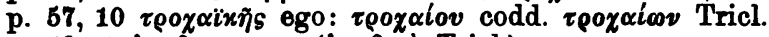

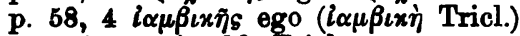

p. 105, 1 xal add. Tricl.

p. $105,10 \dot{\alpha} \pi \delta]$ हो $x$ Tricl. ft. recto

p. 128, 12 \& $\nu \alpha \pi \alpha i \sigma \tau \omega \nu$ Tricl.

p. 128, 15 suppl. et Tricl.

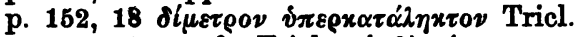

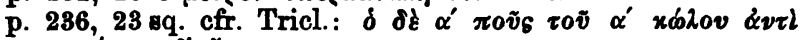

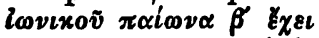

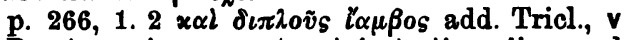

Praeterea in apparata et in testimoniis suppleri et corrigi possunt haec:

p. 38, 3 iे $\sigma \tau^{\prime}$ A (non AQ): $\eta^{\prime} \sigma \vartheta^{\prime}$ H Q r

p. 43, $\left.17 \mu \eta^{\prime} v\right]$ dé CD (delend. signum interrogationis)

p. 60, 5 cfr. Bekkeri anecd. I, 203, 12

p. 66, 4 a cum DEH, non cum C facit

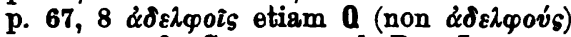

p. 68, 16 cfr. Cram. anecd. Par. I, 286, 27

p. $69,1-20$ cfr. id. ibid. $15-27$

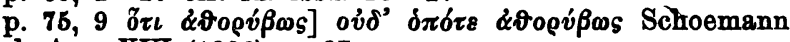

Philol. Anz. XVI (1886), p. 87

p. 99, 18-20 cfr. Cram. anecd. Par. I, 264, 25

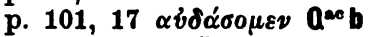

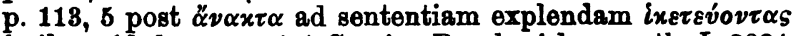
vel simile quid deesse putat Crusius Roscheri lex. myth. I, 2824 


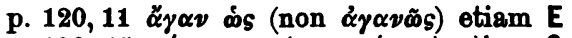

p. $120,15 \mu \varepsilon v \tau o \iota ~ \gamma \varepsilon$ (non $\mu \varepsilon v \tau o \iota$ ) etiam $\mathbf{Q}$; itaque $\mu \varepsilon v \tau o \iota \gamma \varepsilon$ in textu ponere debui

p. 144, $4 \Gamma \varepsilon \lambda \omega \omega v$ iam Horn de Aristarchi stadd. p. 30

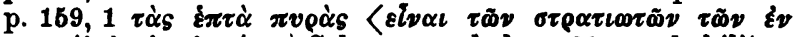

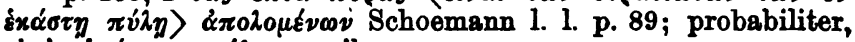

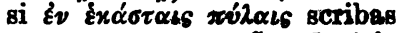

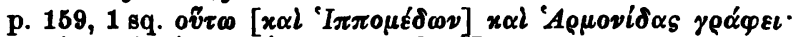

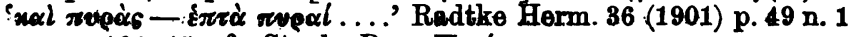

p. 164, 15 cfr. Steph. Byz. Iıєás

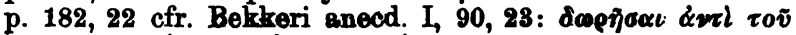

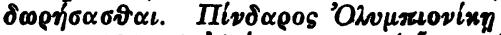

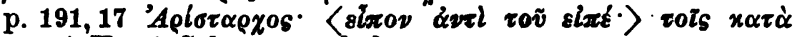
(cetera ut Horn) Schoemann 1. 1. p. 90

p. 289,16 cfr. EM 426,30

p. 845,17 pro $C$ scribend. $C^{8}$. 


\section{Index siglorum.}

$A=$ Ambrosianus C 222 inf.

$A^{\text {ac }}=\mathrm{A}$ ante correctionem

$A^{p e}=A$ post correctionem

$A^{\text {corr }}$ vel $A^{\text {ecorr }}=A$ post correctionem (ubi quid ante correctionem fuerit dinosci nequit)

$\mathbf{A}^{\mathbf{1}}=\mathbf{A}$ infra (in ipso texta)

$A^{*}=A$ supra versum

$A^{c p}=A$ compendio (si compendium in omissa terminatione consistit; cfr. p. XVII)

$A^{81}=$ glossa interlinearis codicis A

$\mathbf{A}^{\text {mg }}=$ scholium marginale (extra ordinem scholioram scriptum) codicis A

$A^{2}, A^{b}=A$ priore, $A$ posteriore loco ( $\mathrm{nbi}$ idem scholium bis scriptum est)

$B=$ Vaticanus 1332

C = Parisinus 2774

D = Laurentianus 32,62

[D] = eiusdem supplementa ex Laur. 32, 35 (Q) petita; cfr. pag. VIII

$\mathrm{E}=$ Laurentianus 32, 37

F = Laurentianus 32,33

6 = Gottingensis philol. 29

H = Vaticanus 41

$K=$ Vaticanus 42

$\mathbf{L}=$ Vaticanus 902

$M=$ Perusinus B 43

$N=$ Ambrosianus $\mathrm{E} 103$ sup.

$0=$ Leidensis Q 4

$P=$ Parisinus 2403 (V Mommseni)

$a=$ Laurentianus 32,35

$a^{b}=Q$ posteriore loco (cfr. pag. VIII)

$R=$ Vaticanus Urbinas 144

$\mathbf{T}=$ Vaticanus 121

$U=$ Vindobonensis 130

$Y=$ Marcianus 475

$V$ =codicum Vaticanorum consensus (cfr. pag. XVII) Ang. = codex Angelicus C, 1, 1 
Red. = Vratislaviensis Redigerani 40 prima pars (cfr. pag. V sq.)

Vrat $^{\mathrm{d}}$ = eiusdem tertia pars (cfr. pag. IX)

$\boldsymbol{\theta}=$ Ambr. C 22 sup. (Thomanus)

b $=$ Boeckh (editio Boeckhiana)

h = Heyne (ed. Heyniana Lipsiensis a. 1817)

$r=$ editio Romana a. 1515

$v=$ textus vulgatus (a Romana usque ad Heynianam vel Boeckhianam)

Ab. = Abel (editiones Abelianae scholiorum veterum in Nemea et Isthmia a. 1884, recentiorum in Olympia et Pythia a. 1891)

Beck = editio Beckiana (altera non mutata, Lipsiae 1811)

Bothe $=$ Pindar von F. H. Bothe, Berlin 1808

Brub. = ed. Brubachiana (Francofurti 1542)

Gedike = Pindari carmina selecta ed. Fr. Gedike, Berol. 1786 Oxon. = ed. Oxoniensis (1697)

Stephanus = ed. Pauli Stephani, 1599

n. 1 . = non liquet

s. s. = supra scripto vel scriptis

1. = lemma (lemmatis, lemmati etc.)

( ) includuntur: 1) terminationes compendio omissae; 2) sigla codicum quoram lectiones omittuntur (cfr. pag. XV. XVII); 3) partes verboram in quibus codices discrepant, ubi ea discrepantia non notatur (cfr. pag. XVII)

[] includuntur: 1) verba partesve verboram quae in codicibus olim fuerunt, sed hodie legi nequeunt; 2) supplementa librarii [D]

I ad sigla codicum in margine apposita pertinet (cfr. pag. XV) Punctis infra litteras positis litterae dubiae lectionis notantur.

Pindari carmina et fragmenta ad editionem Schroederianam, Hesiodi loci ad editionem Rzachianam minorem, Comicorum Atticorum fragmenta ad Kockianam citantur. 


\section{$B \operatorname{los} \Pi \iota \nu \delta \alpha \rho o v$.}

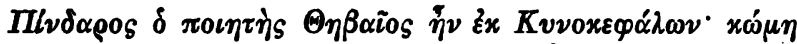

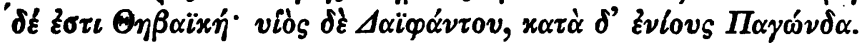

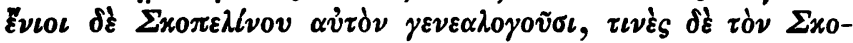

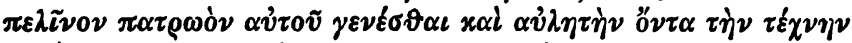

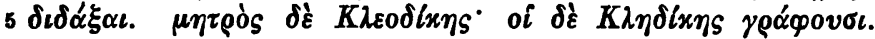

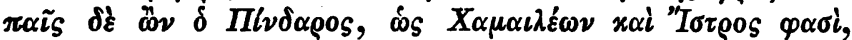

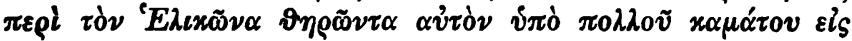

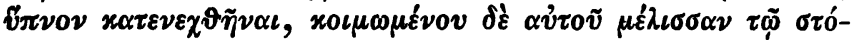

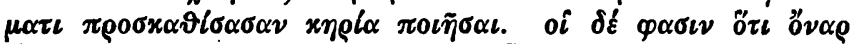

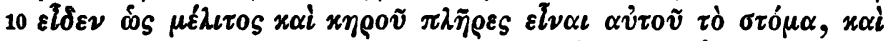

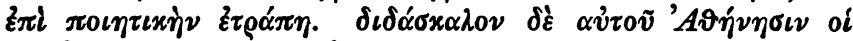

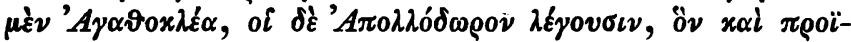

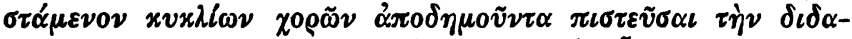

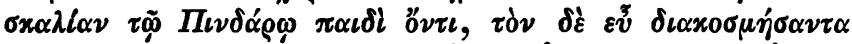

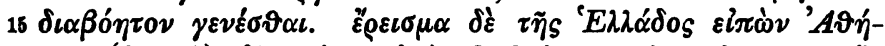

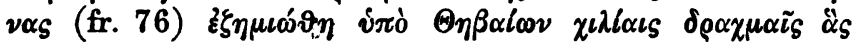

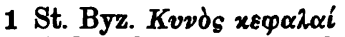

6 Paus. 9, 23, 2. Philostr. im. 2, 12. Ael. v. h. 12, 45. Anth. Plan. 30515 Isocr. $\pi \varepsilon \rho l \alpha \nu \tau \iota \delta$. 166. [Aesch.] ep. 4, 2 sq. Paus. 1, 8, 4. Tzetzes ad Hes. Op. 412 (p. 224 Gsfd.)

Vitam Ambr. primus edidit J. G. Schneider ante Nicandri Theriaca e cod. Vratisl.; repetiit post Boeckhium C. E. Chr. Schneider app. Pind. suppl. (Vratisl. 1844) p. 441 Kvvooxe-

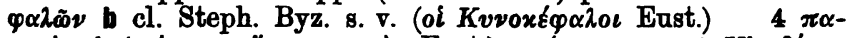

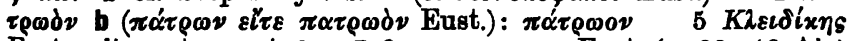

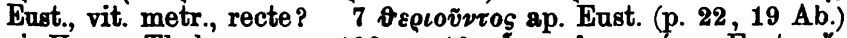

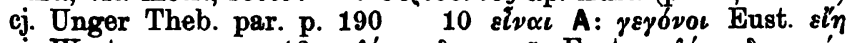

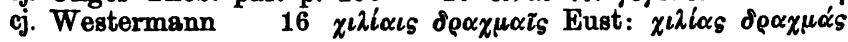




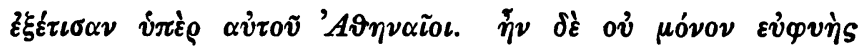

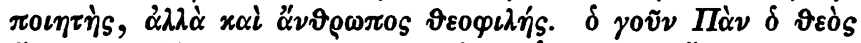

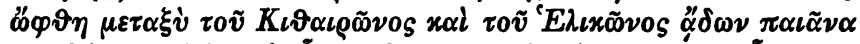

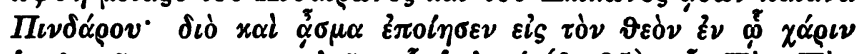

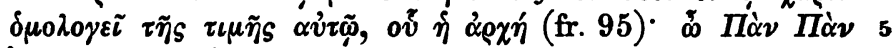

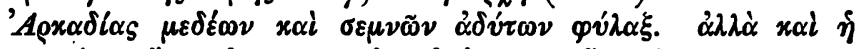

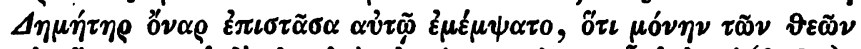

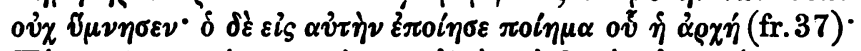

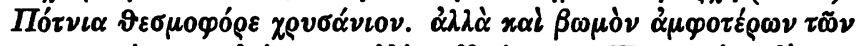

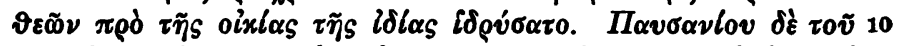

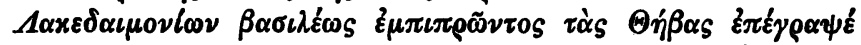

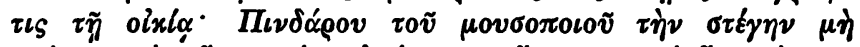

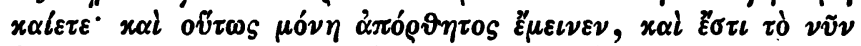

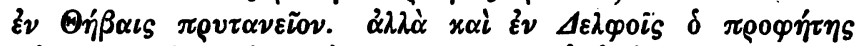

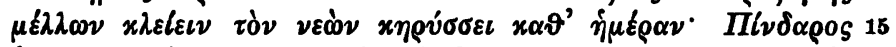

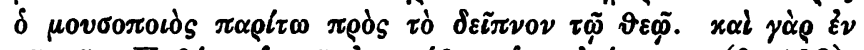

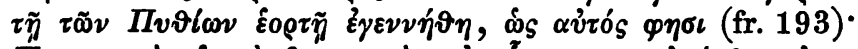

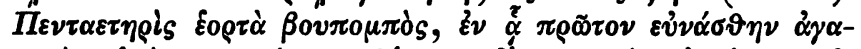

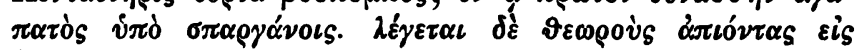

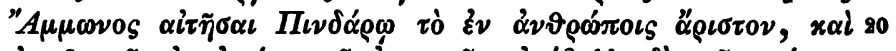

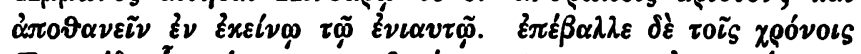

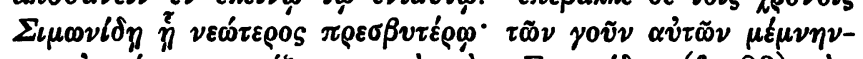

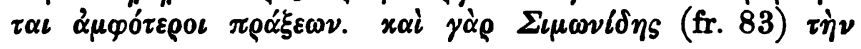

2 sch. P. 3, 139. Philostr. im. 2,12 6 Paus. 9, 23, 3 9 sch. P. 3, 137. Paus. 9, 25, 3 18 Plut. qu. conv. 8, 1, 6 19 Plut. cons. ad Apoll. 14 (p. 109 a)

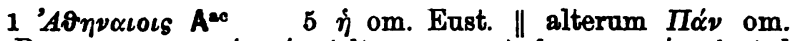

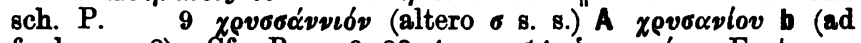
fr. hymn. 8). Cfr. Paus. 9, 23, 4 14 $\delta$ vecoxópos Eust.

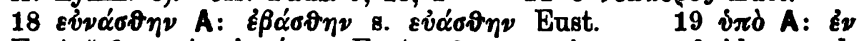

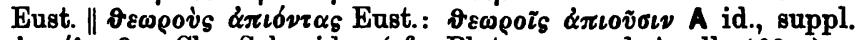
$\xi \nu \tau \varepsilon i 2 \alpha \sigma \theta \alpha \iota \mathrm{Chr}$. Schneider (cfr. Plat. cons. ad Apoll. 109 a) $20{ }^{\prime} A \pi t \delta 2 \lambda \omega \nu \alpha$ v. Leatsch Philol. 11 p. 17 (cl. eod. ibid.) \| $\Pi_{\iota \nu-}$

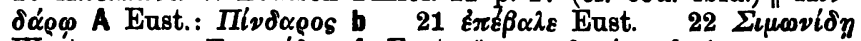

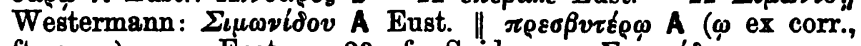
ft. ex $\alpha$ ): - 


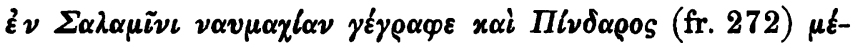

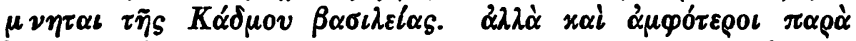
'IÉ

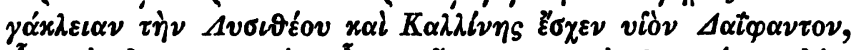

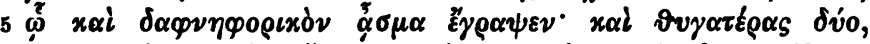

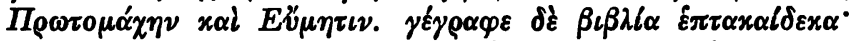

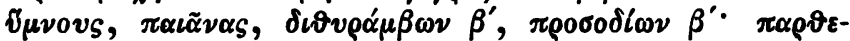

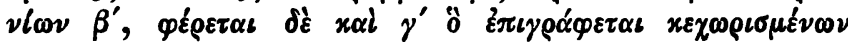

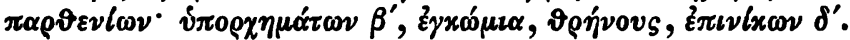

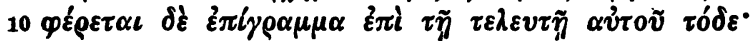

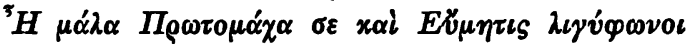

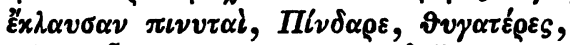

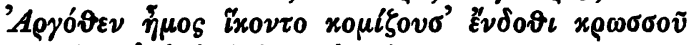

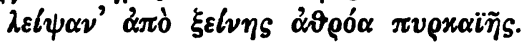

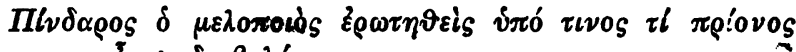

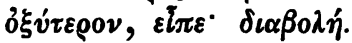

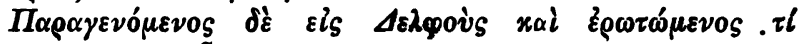
$\pi \alpha^{\prime} \rho \varepsilon \sigma \tau \iota ~ \vartheta v ́ \sigma \omega \nu, \varepsilon i \pi \varepsilon^{*} \pi \alpha \iota \tilde{\alpha} \nu \alpha$.

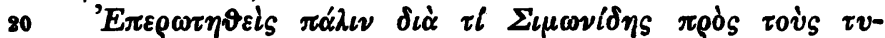

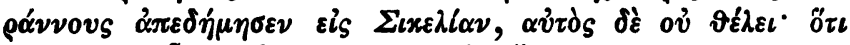

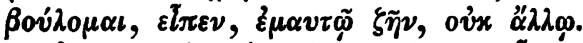

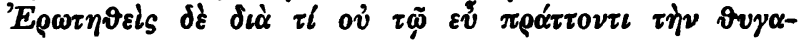

6 sch. P. 3, $139 \quad 18$ Paus 10, 24, 5

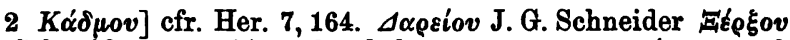

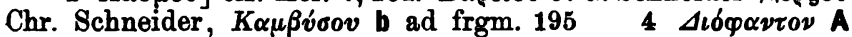

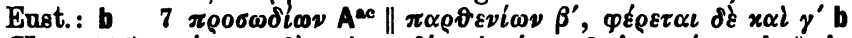

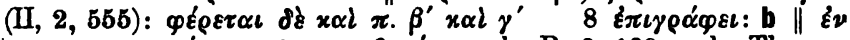

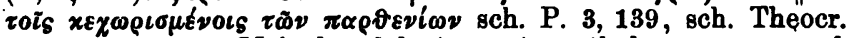
2, $10 \quad 11$ os Meineke delect. poet. anthol. p. 239: $\tau \varepsilon A$

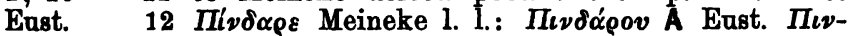

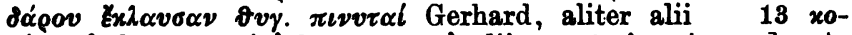

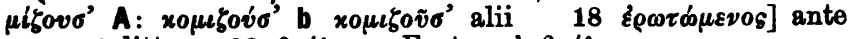

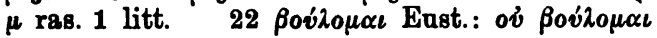




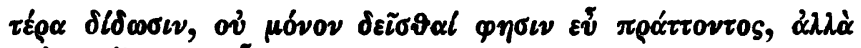

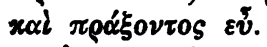

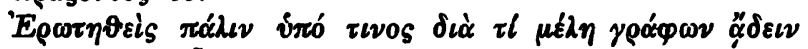

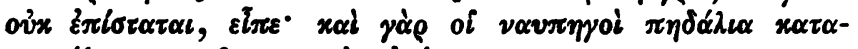

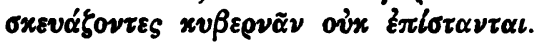

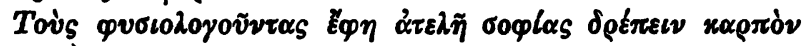
(fr. 209).

EHKQP' $\theta$

$\Pi \iota v \delta \alpha \dot{\rho} \circ v$ vยvos.

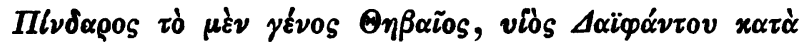

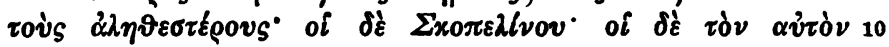

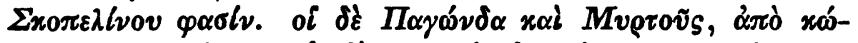

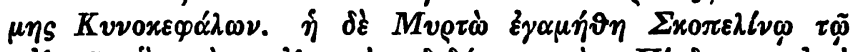

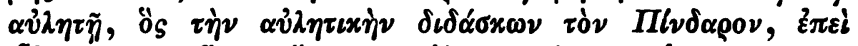

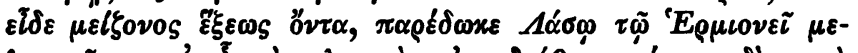

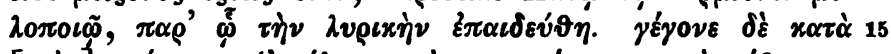

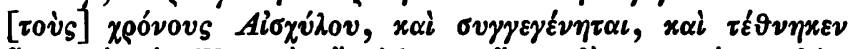

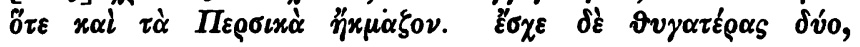

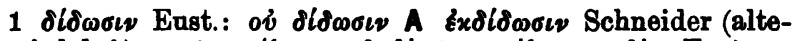

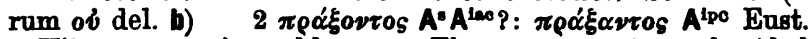
8 Vitam quae in codd. recc. Thomana vocatur ed. Abel 3, p. 31-39 ex codd. recc., qui cum v fere conspirant $\| \Pi \iota \nu \delta \alpha ́ \rho o v$

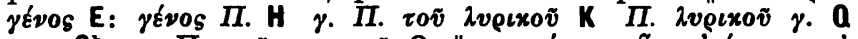

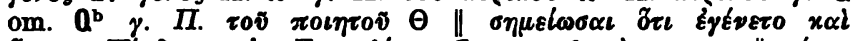

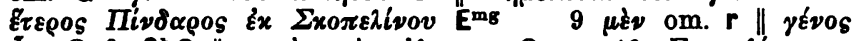

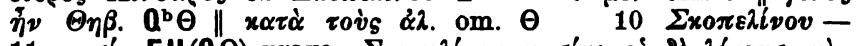

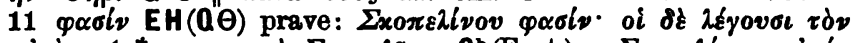

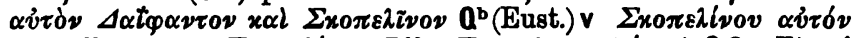

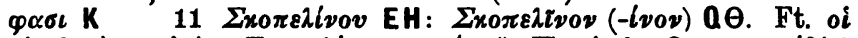

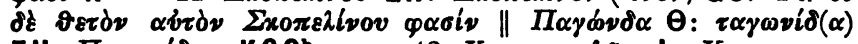

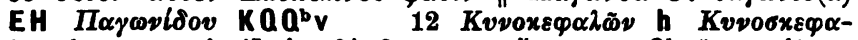

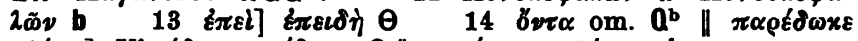

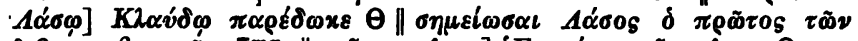

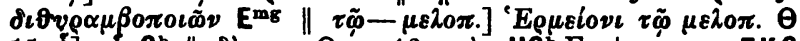

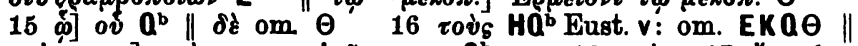

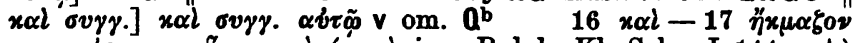

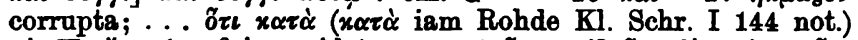

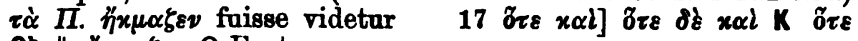
$a^{b} \| \eta_{x \mu \alpha \xi \varepsilon v} \theta$ Eust. $v$ 


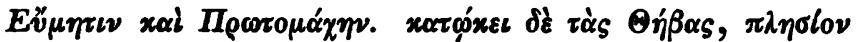

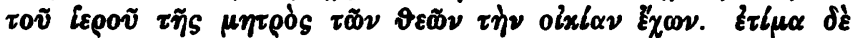

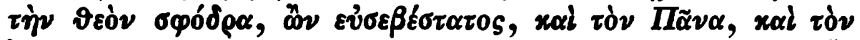

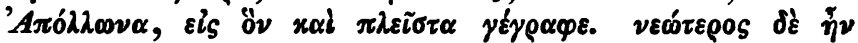

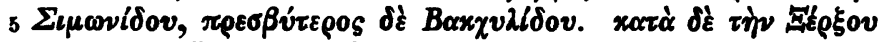

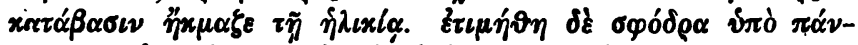

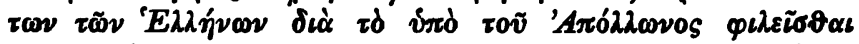

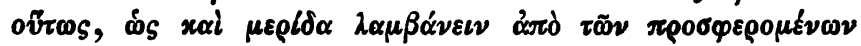

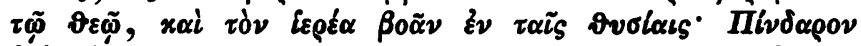

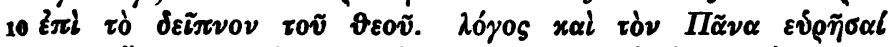

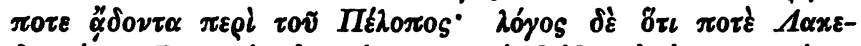

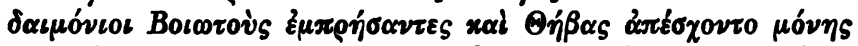

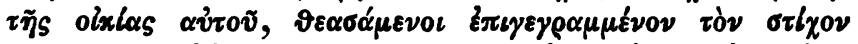

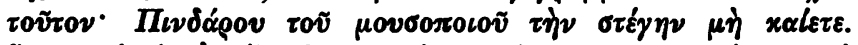

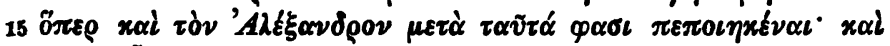

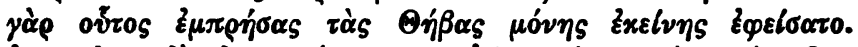

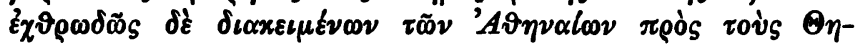

8 Paus. 9. 23, 3. Plut. de sera num. vind. 13. 15 Arr. exped. Al. 1, 9, 10. Dio Chr. de regno 2 p. 27 Ddf. Tzetzes Chil. 7, $\mathbf{4 1 0}$

1 Eซ̋r

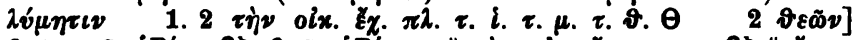

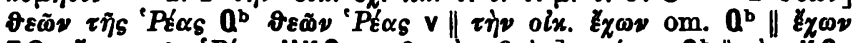

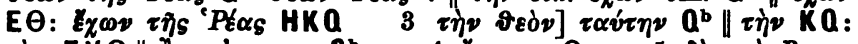

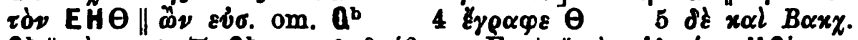

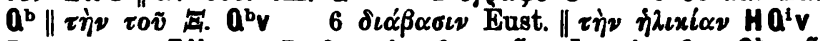

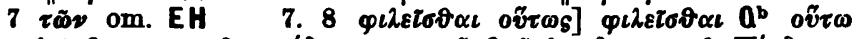

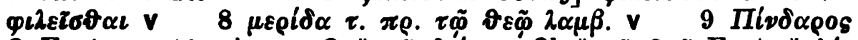

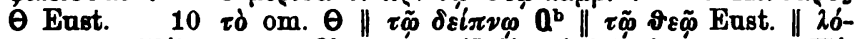

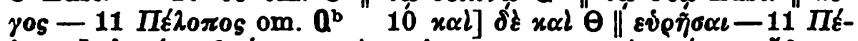

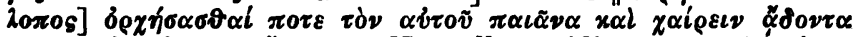

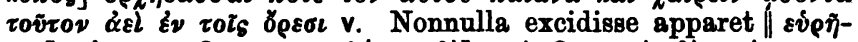

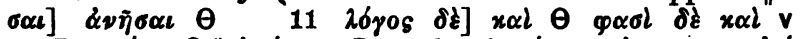

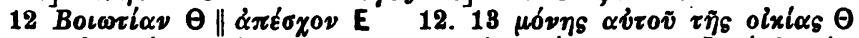

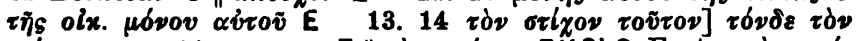

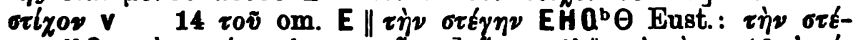

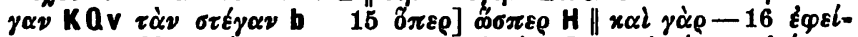

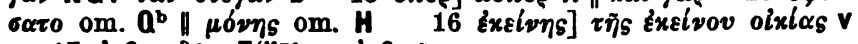

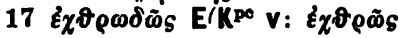




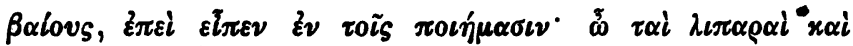

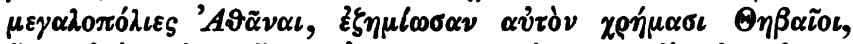

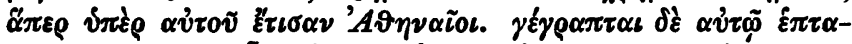

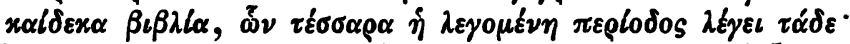

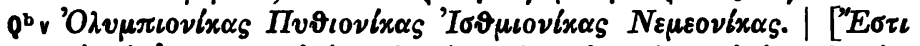

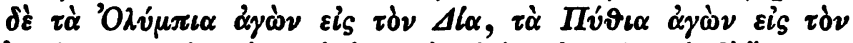

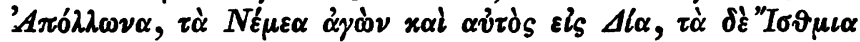

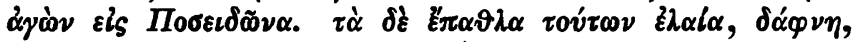

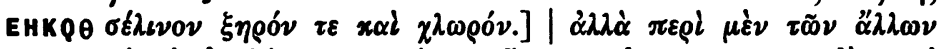

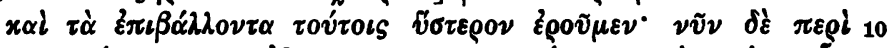

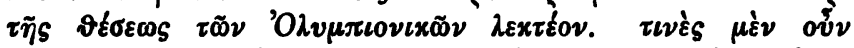

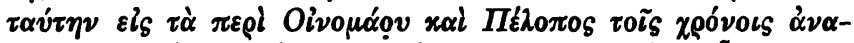

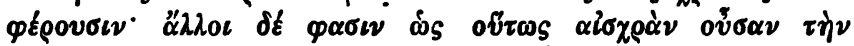

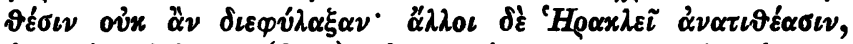

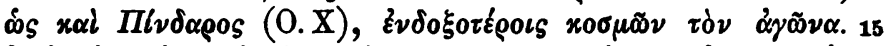

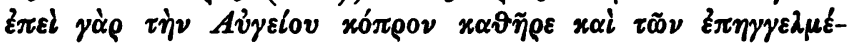

\section{8 sch. 0. 3, 2716 Tzetzes ad Lyc. 41}

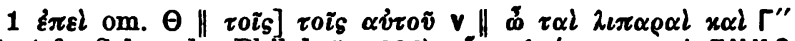

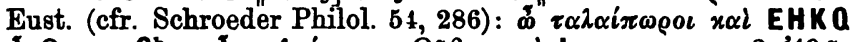

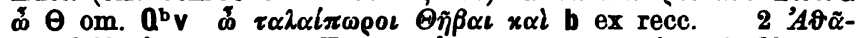

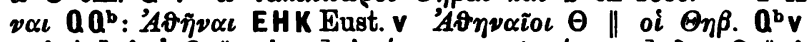

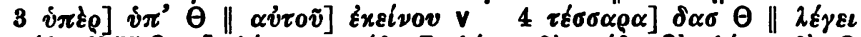

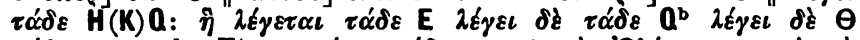

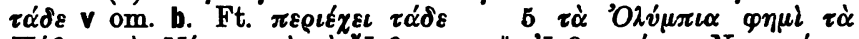

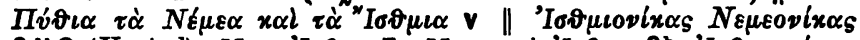

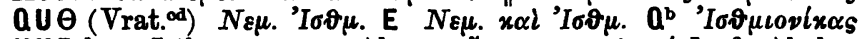
H KP hyp. Isthm. p. 349, $5 \mathrm{Ab} .5^{\text {" }} E \sigma \tau \iota-9$ $\left.\chi^{\lambda \omega \rho \sigma \delta}\right]$ cfr. Abel 3,

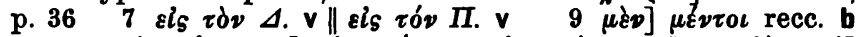

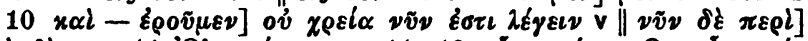

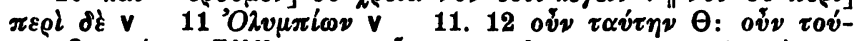
$\tau \omega \nu$ Q $\tau o v i \omega \nu$ EHK om. $v$ oṽ $\tau \alpha v \tau \tau \alpha$ b ex recc. $12 x \alpha i$ om.

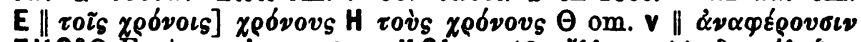

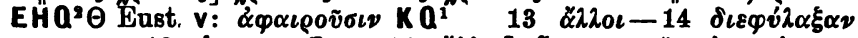

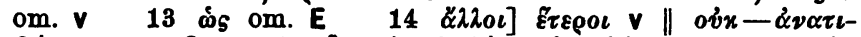

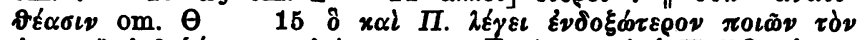

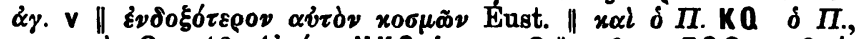

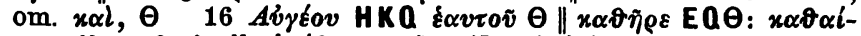

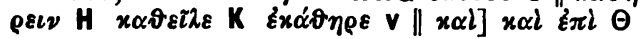




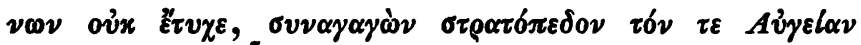

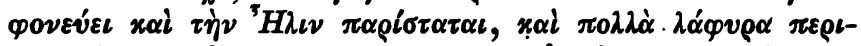

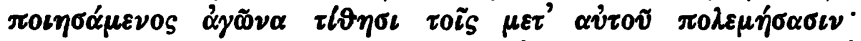

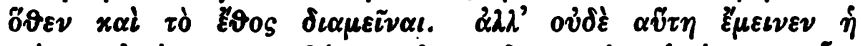

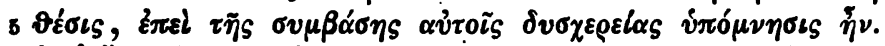

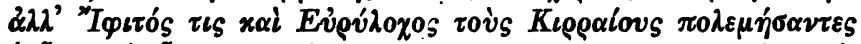

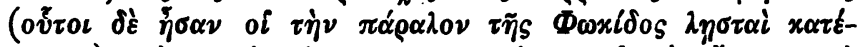

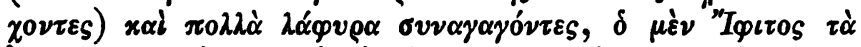

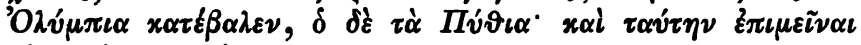

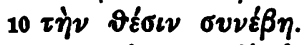

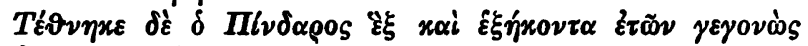

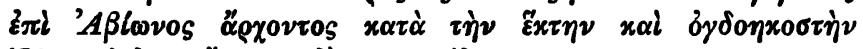

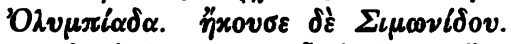

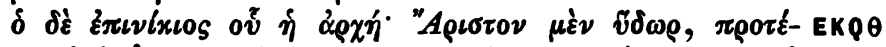

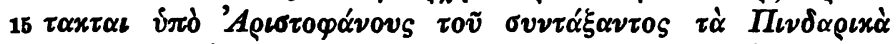

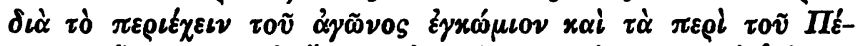

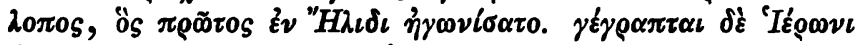

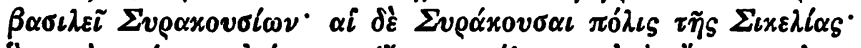

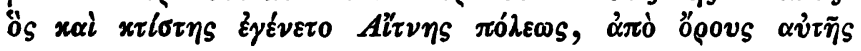

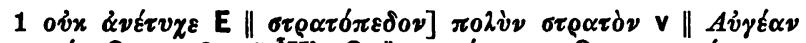

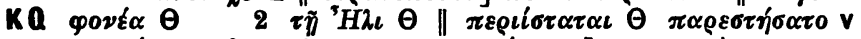

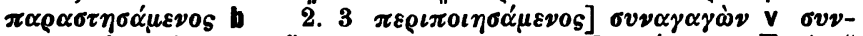

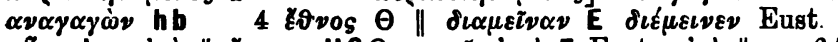

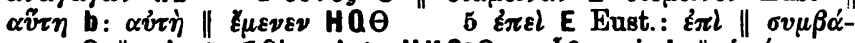

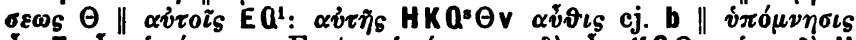

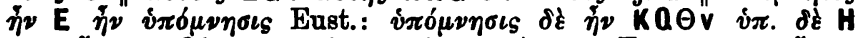

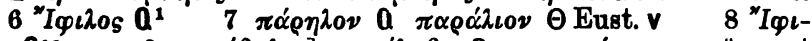

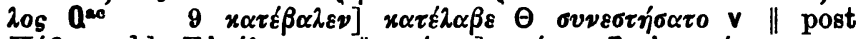

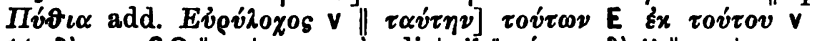

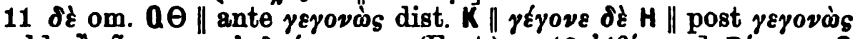

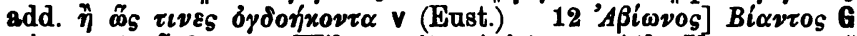

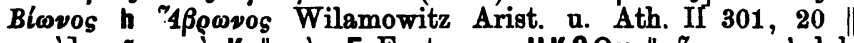

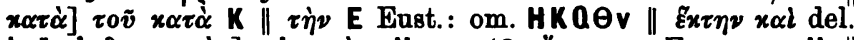

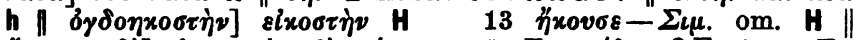

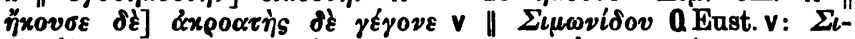

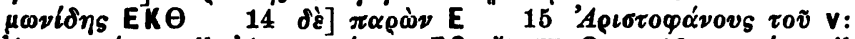

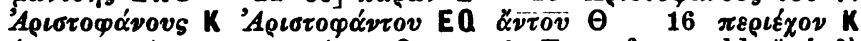
(o ex corr.) $17 \pi \rho \omega \tau \omega_{g} a \quad 18 \Sigma v \rho \rho$. fere codd. $\| \dot{\eta} \delta \dot{\varepsilon}$

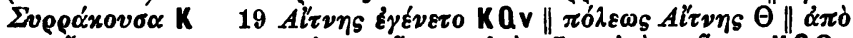

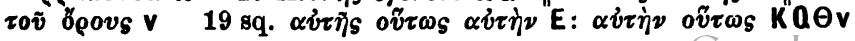




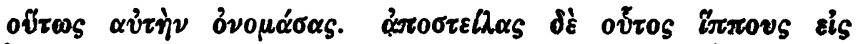

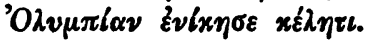

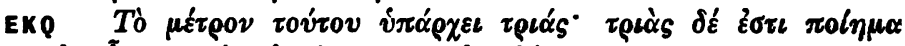

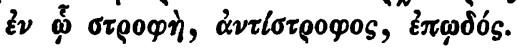

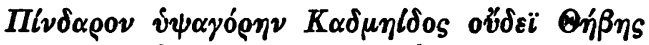

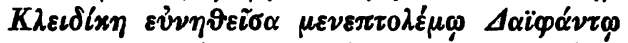

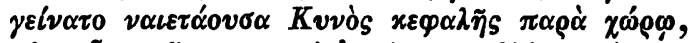

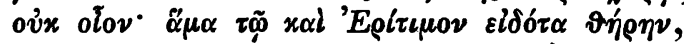

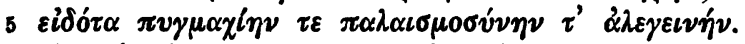

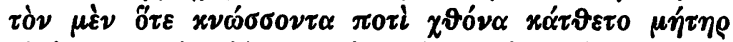

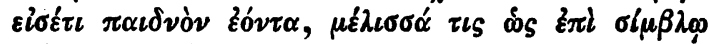
$\chi \varepsilon i \lambda \varepsilon \sigma \iota \nu \eta \eta \iota \alpha^{\prime} \chi 0 \iota \sigma \iota \quad \tau \iota \vartheta \alpha \iota \beta \omega ́ \sigma \sigma o v \sigma \alpha$ $\pi 0 \tau \tilde{\alpha} \tau 0$.

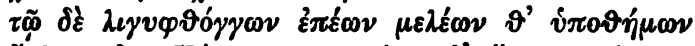

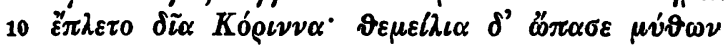

3 sch. Ar. av. 1731. sch. Germ. ed. Momms. p. 2. Eust. p. 30 Ab. 11 Plut. glor. Ath. 4

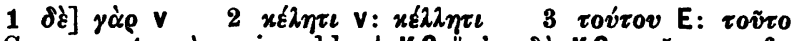

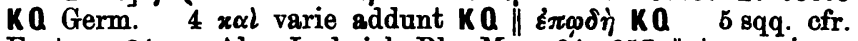
Eust. p. 24 sq. Ab. Ludwich Rh. Mus. 34, 357 || tres primos

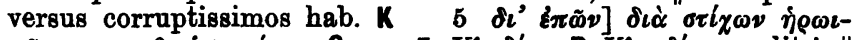

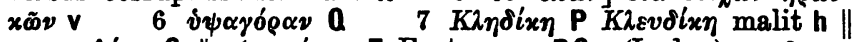
$\mu \varepsilon \nu \varepsilon \pi 0 \lambda \varepsilon \mu \omega 0$ Q $\Delta \alpha \iota \varphi \alpha^{\prime} \nu \tau \omega$ E Eust.: - $\tau \eta$ PQV (Ludw.) $8 x \varepsilon-$

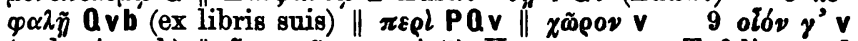

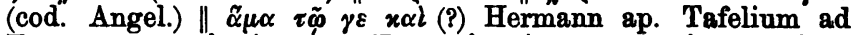

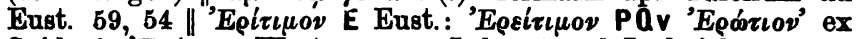

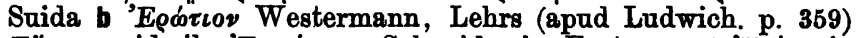

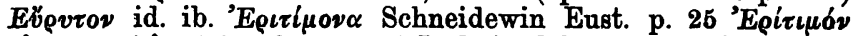

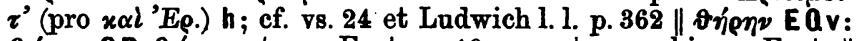

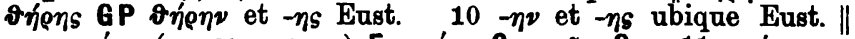

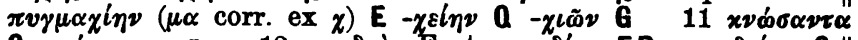

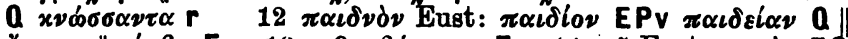

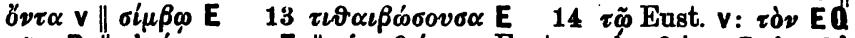

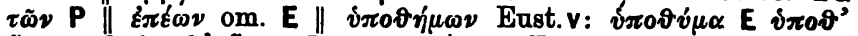
$\tilde{v} \mu \nu \omega \nu$ Q $\delta \pi \sigma \vartheta \vartheta^{\prime} \tilde{v} \omega \nu$ P 15 día EP Eust. 


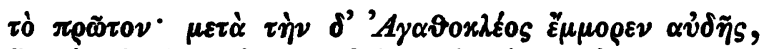

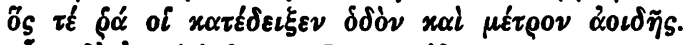

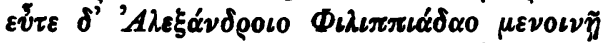

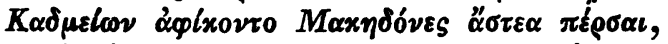

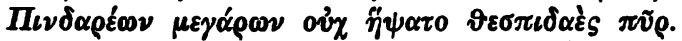

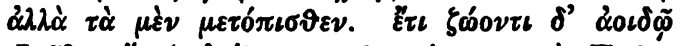

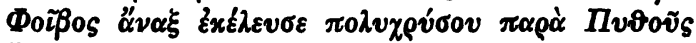

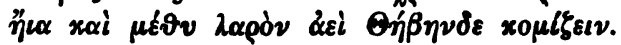

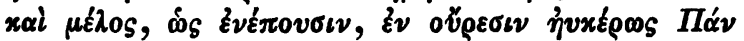

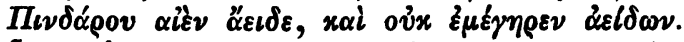

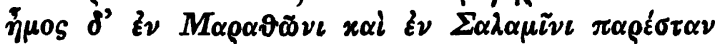

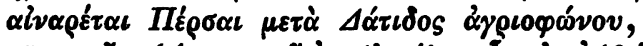

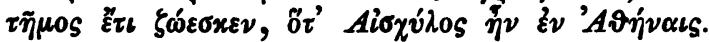

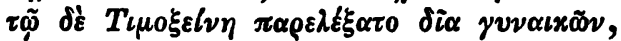

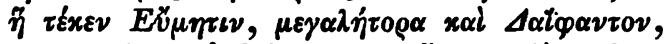

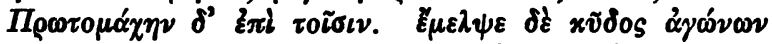

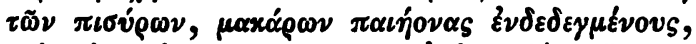

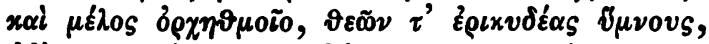

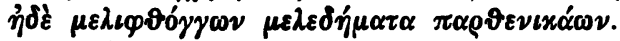

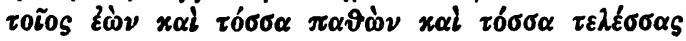
80

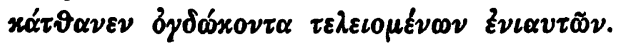

1 'A $\alpha \propto \vartheta 0 x \lambda \varepsilon_{\varepsilon}$ cod. Angel. (teste Mommseno sch. Germ.

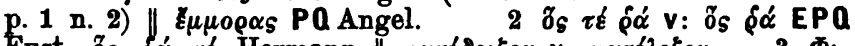

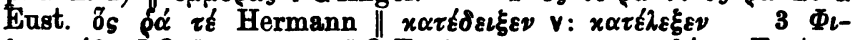

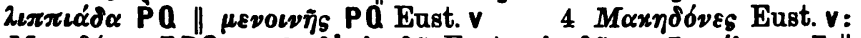

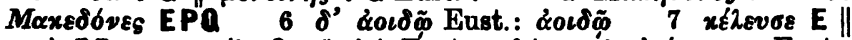

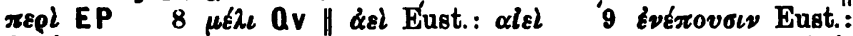

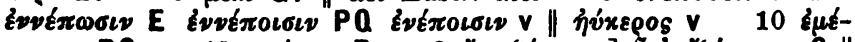
yeL

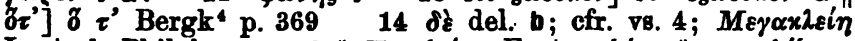

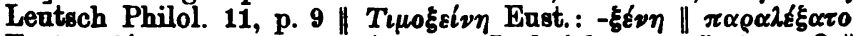

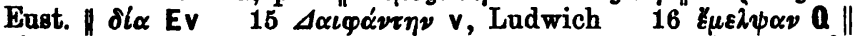

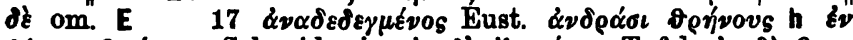

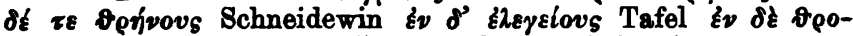
visuov́s Bergk" p. 370. Dithyrambos omitti mirum est

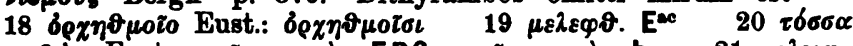

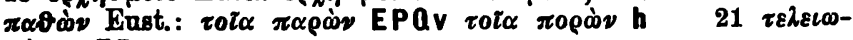
$\mu \in \dot{v} \omega \boldsymbol{\nu}$ EP 


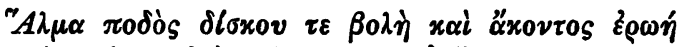

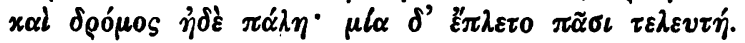

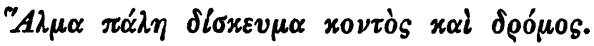

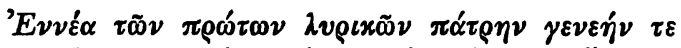

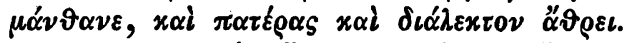

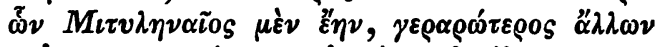

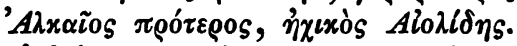

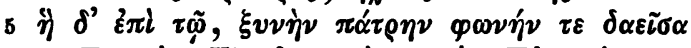

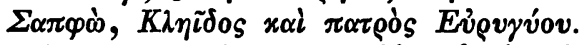

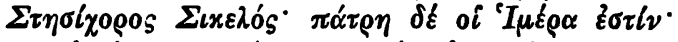

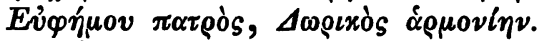
'I

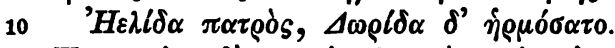

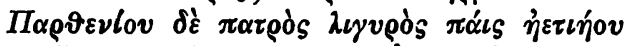

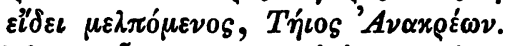

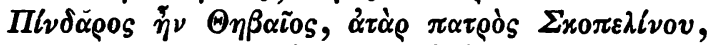

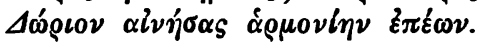

1-4 sch. Isthm. 1, 35. Eust. p. 29 Ab. id. ad Il. 1320, 18. sch. Germ. ed. Mo. p. 1

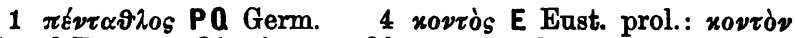

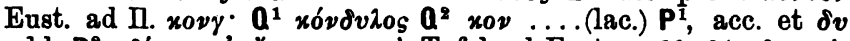

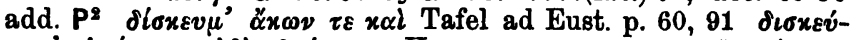

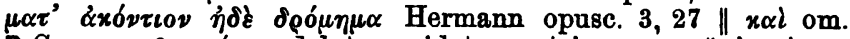

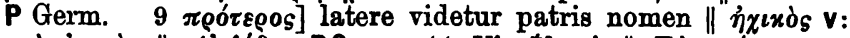

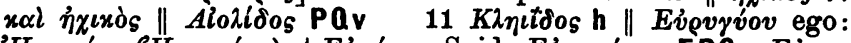

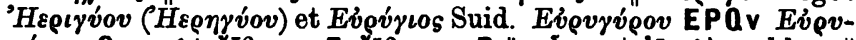

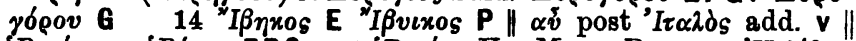

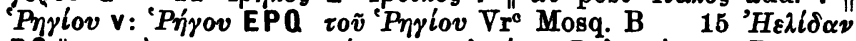

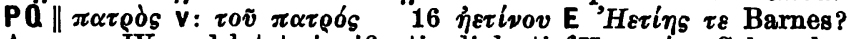
Anacr.p.IV; sed latet significatio dialecti (Hoıovei $\omega$ ? Schroeder

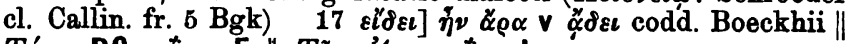

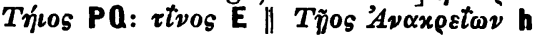




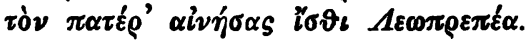

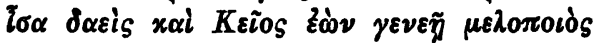

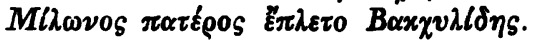

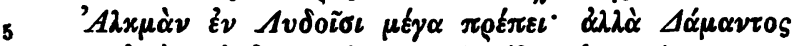

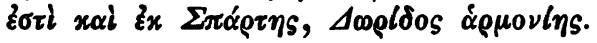

80

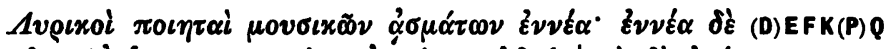

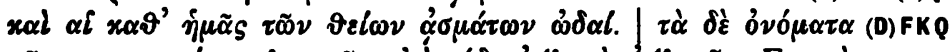

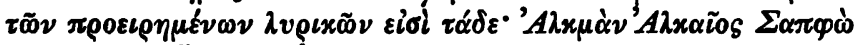

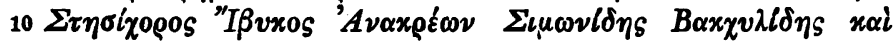
IIlvdapos.

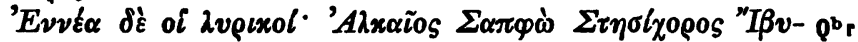

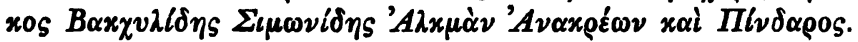

9-11 cfr. laterculum Coislinianum (Usener Dion. de imit. p. 130)

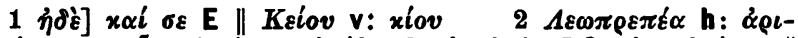

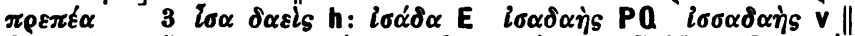

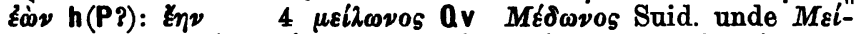

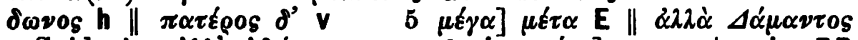

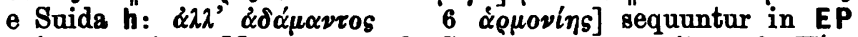
epigrammata a Mommseno sch. Germ. p. 2 recensita (Els $\Pi_{i}^{\prime} \nu$ $\delta \alpha \rho o v$ om. E) $7 \mathrm{sq}$. P non contali; in D pleraque h. sch. exesa \| cfr. Sitzungsber. d. bayer. Ak. 1870, II, 79 sqq. $7 \dot{\alpha} \sigma \mu$.

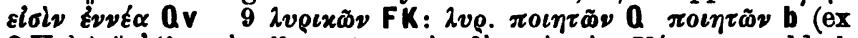

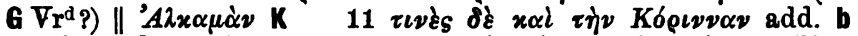

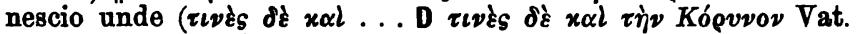

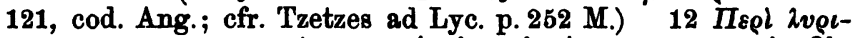

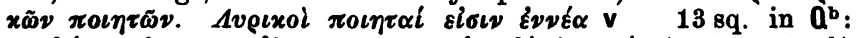

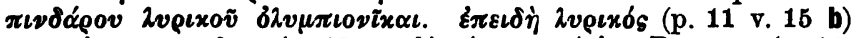

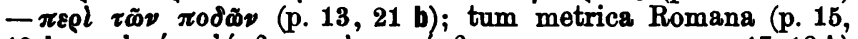

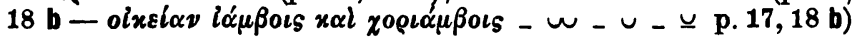




\section{Scholia in 0lympionicarum carmen $I$.}

CF $Q$

CFO

AE

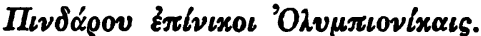

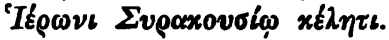

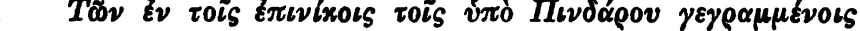

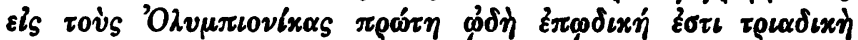

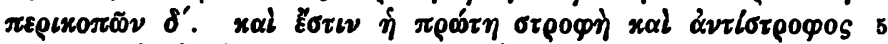

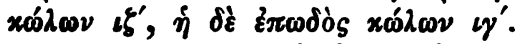

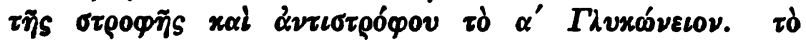

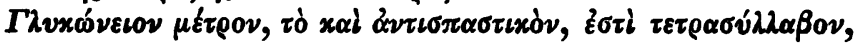

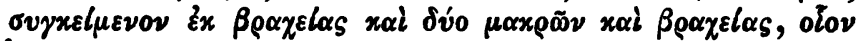

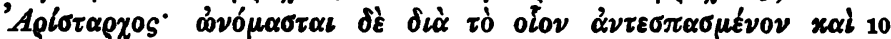

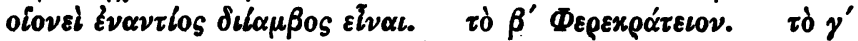

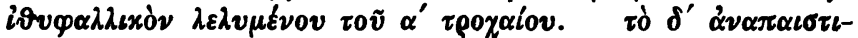

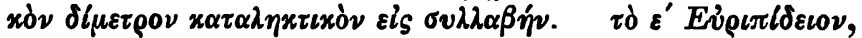

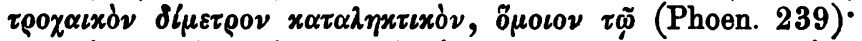

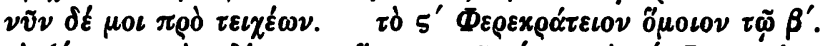

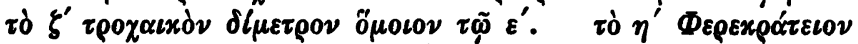

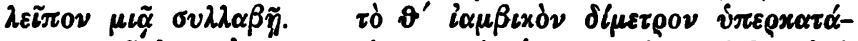

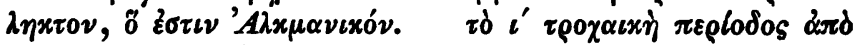

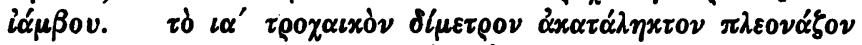

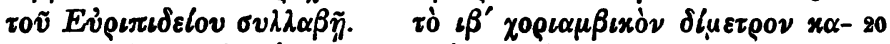

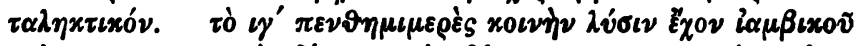

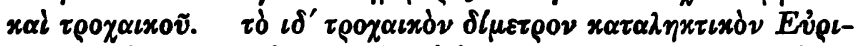

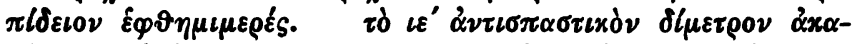

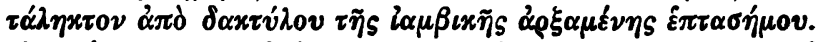

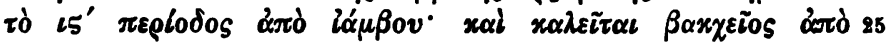

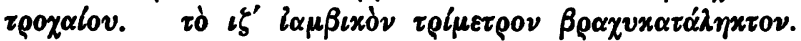

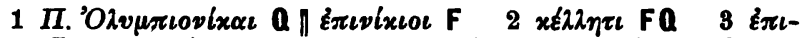

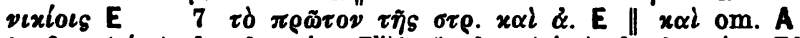

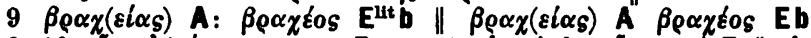

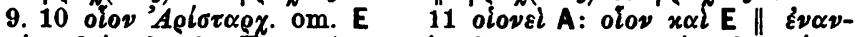

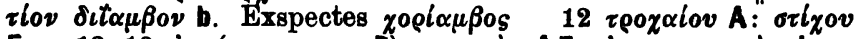
E $12.13 \dot{\alpha} \alpha \alpha(\alpha$ ex corr. E) $\pi \alpha \iota \sigma \tau \iota x \dot{\nu} \nu$ A E: $\alpha \nu \tau \iota \sigma \pi \alpha \sigma \tau \iota x \dot{\nu} \nu$ b

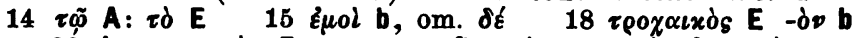

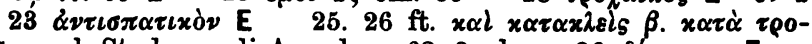

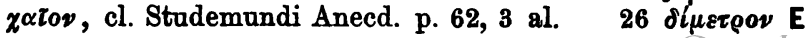




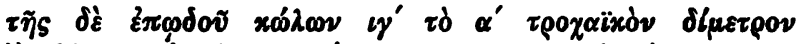

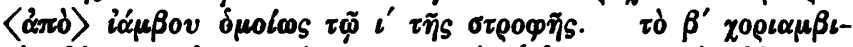

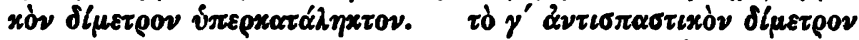

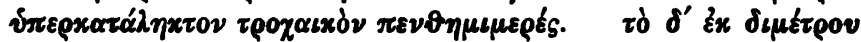

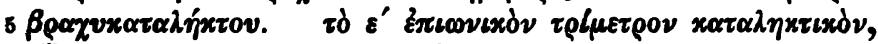

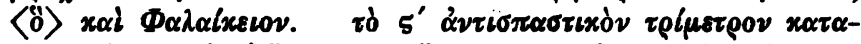

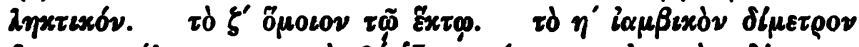

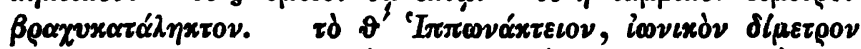

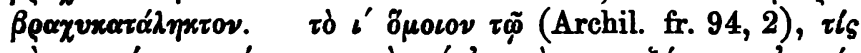

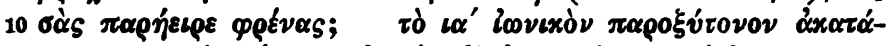

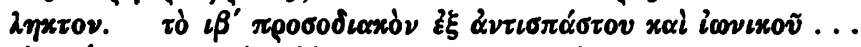

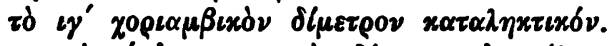

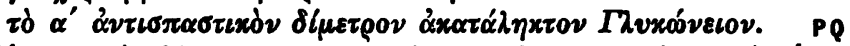

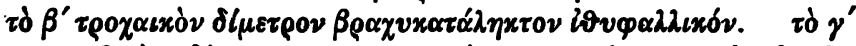

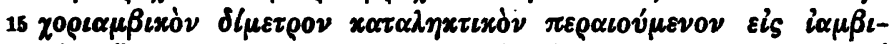

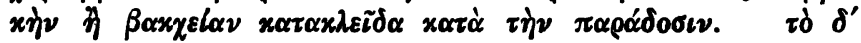

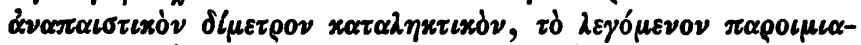

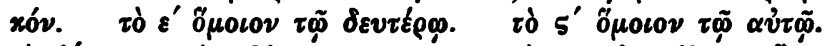

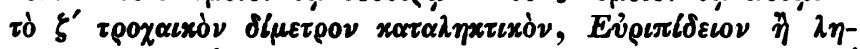

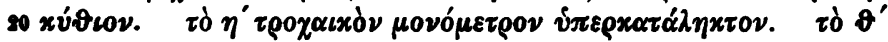

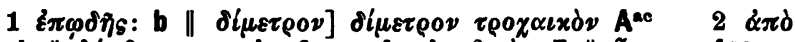

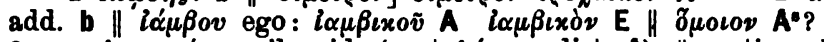

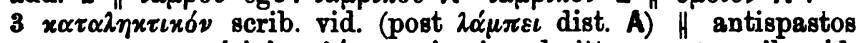

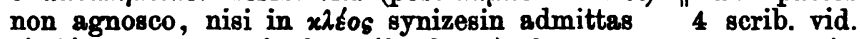

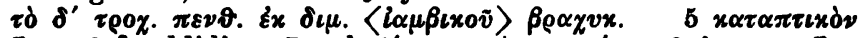

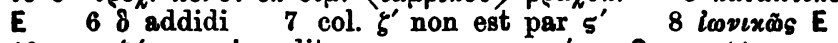

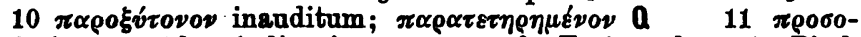
dıxòv: ego || lac. indicsvi 13 sqq. cfr. Tzetzes de metr. Pind. Trichas de metris p. 52 sqq. (cum $P$ fere consentit) 13 \& $x \alpha i$

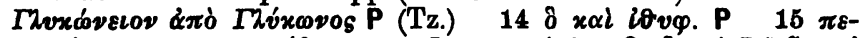

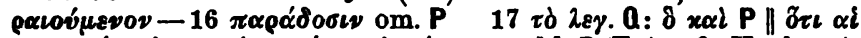

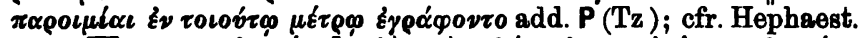

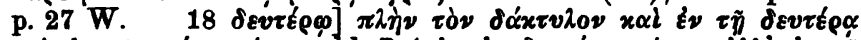

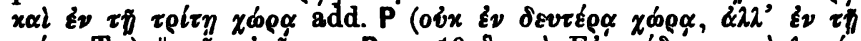

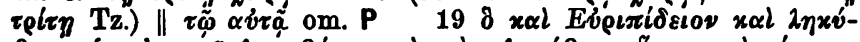

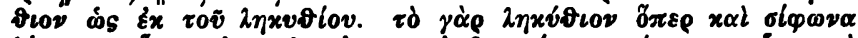

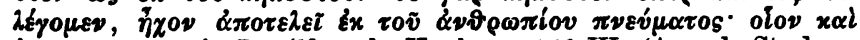

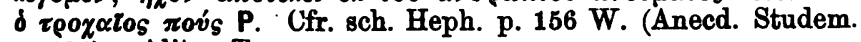
p. 134). Aliter Tz. 


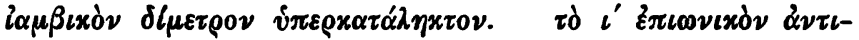

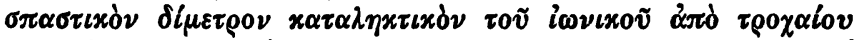

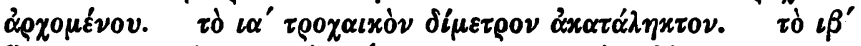

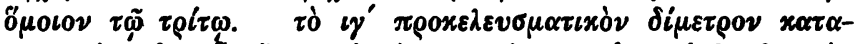

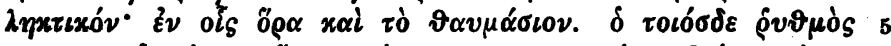

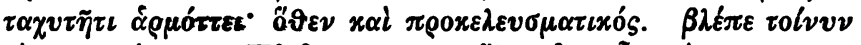

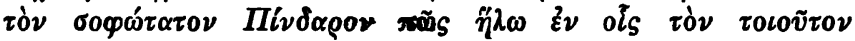

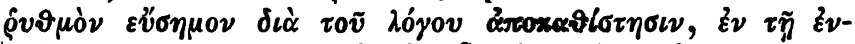

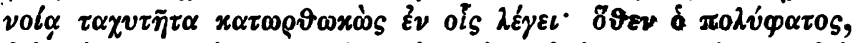

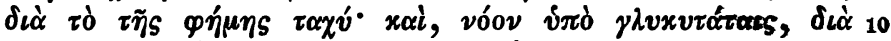

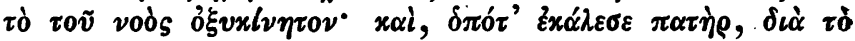

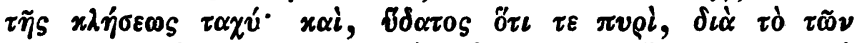

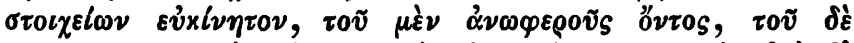

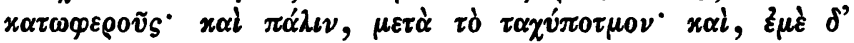

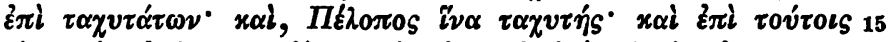

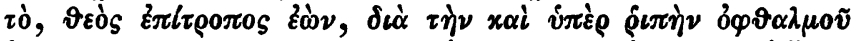

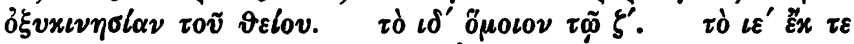

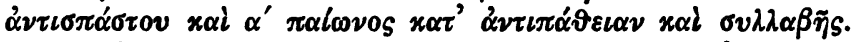

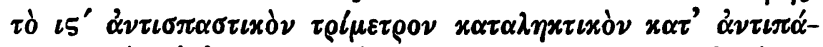

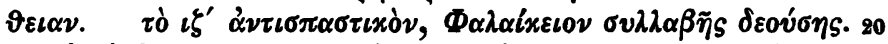

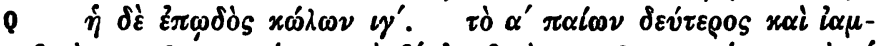

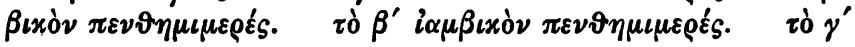

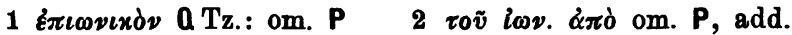

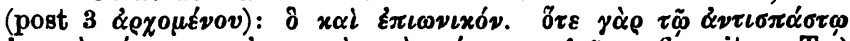
i

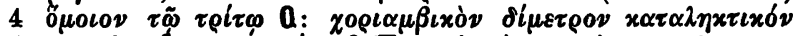

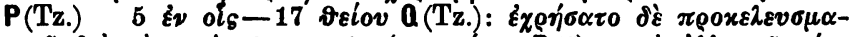

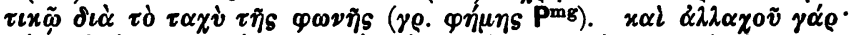

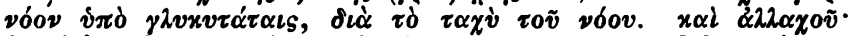

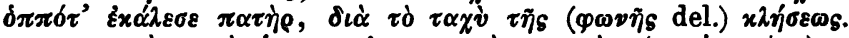

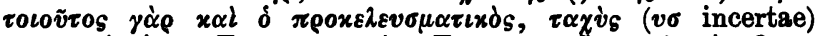

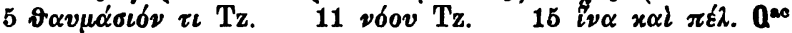

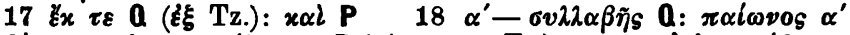

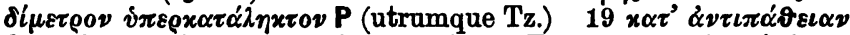

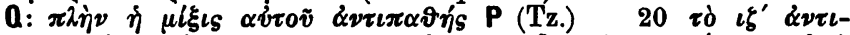

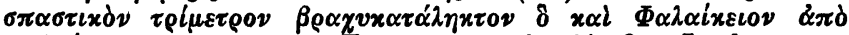
$\Phi \alpha \lambda \alpha i x o v$ P (utrumque Tz.) $21 \dot{\varepsilon} \pi \oplus \delta \dot{\eta}$ a. In hoc voc. des. metr. P 


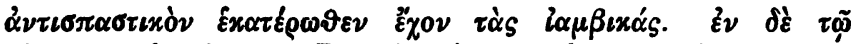

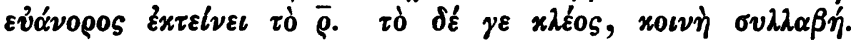

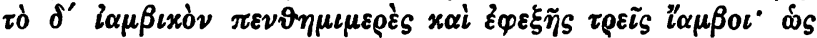

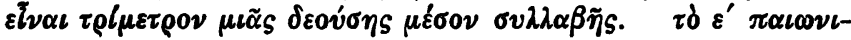

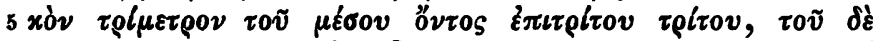

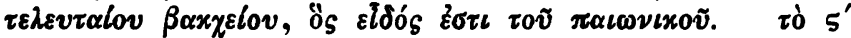

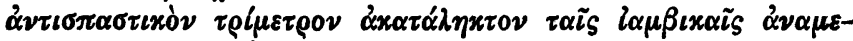

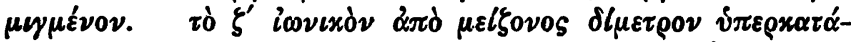

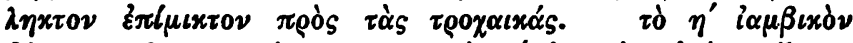

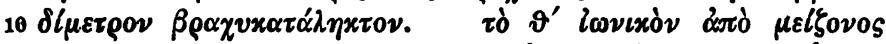

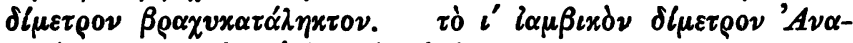

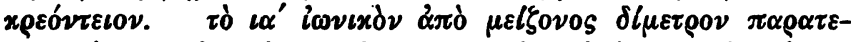

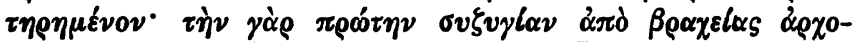

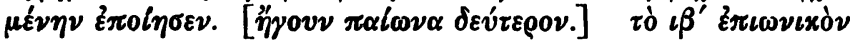

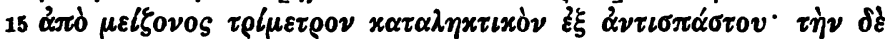

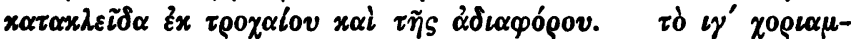

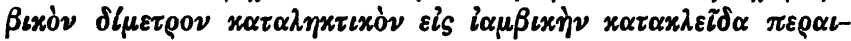

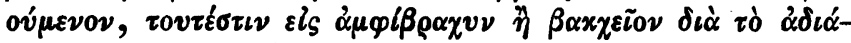

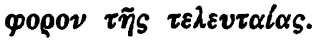

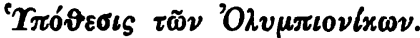

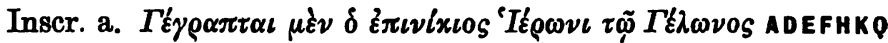

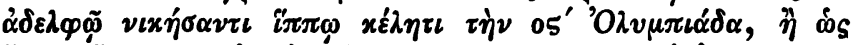

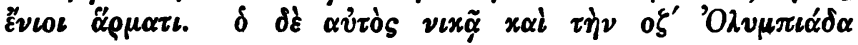

13 Hephaest. p. 37 W. 14 sch. Hephaest. p. 193, 7 W.

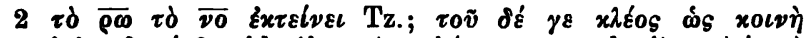

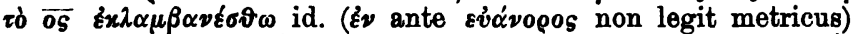

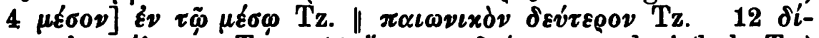

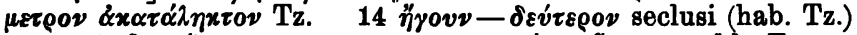

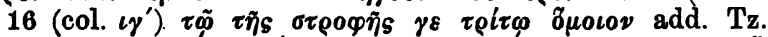

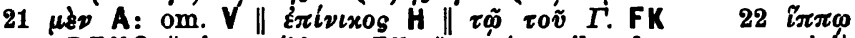

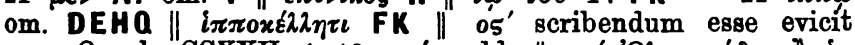

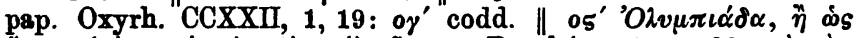

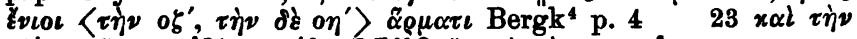

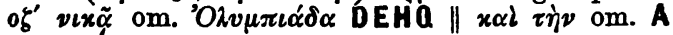




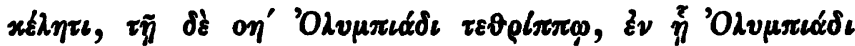

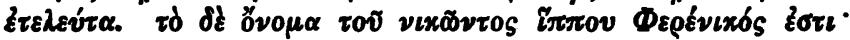

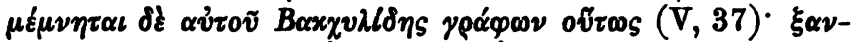
จór

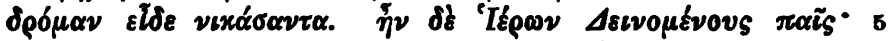

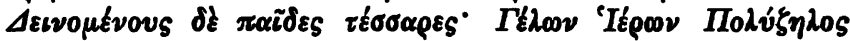

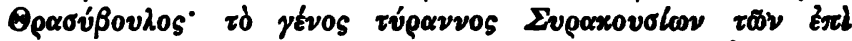

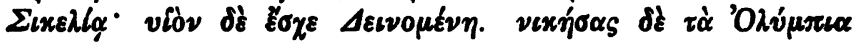

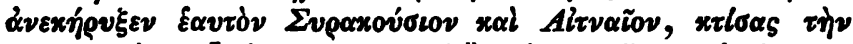

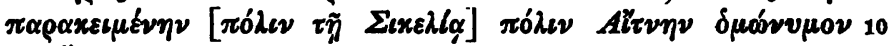

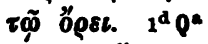

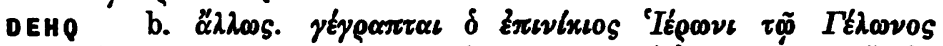

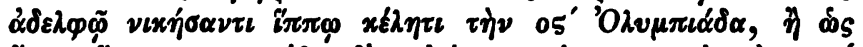

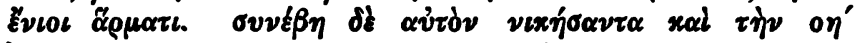

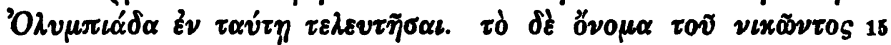

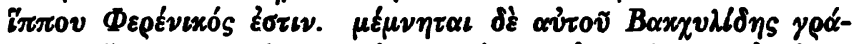

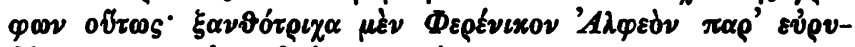

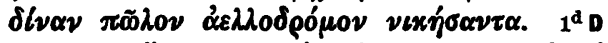

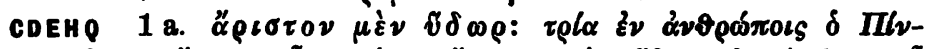

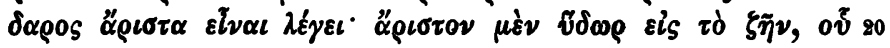

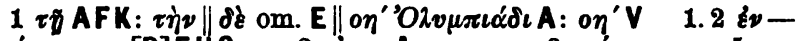

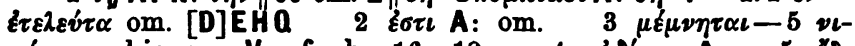

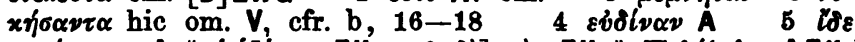

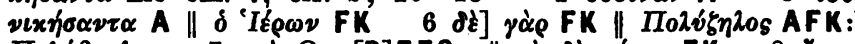

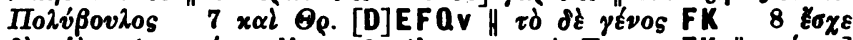

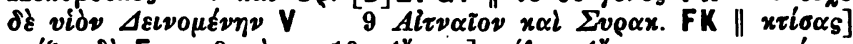

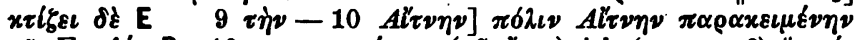

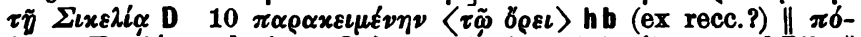

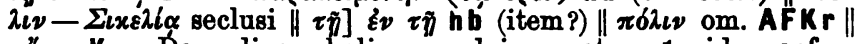

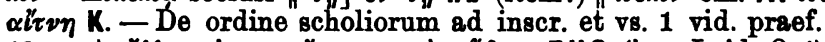

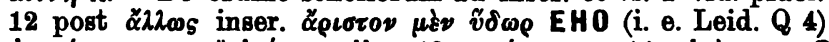
छ̇ $\left.05^{\prime}\right] ~ o \gamma^{\prime}$ codd., cfr. ad p. 15, $\left.22 \| \hat{\eta} \omega_{s}\right] \dot{\omega s} \delta \xi \mathrm{E} 14 x \alpha \downarrow \tau \eta \dot{\eta} \nu$

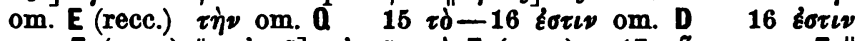

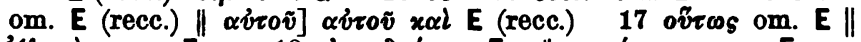

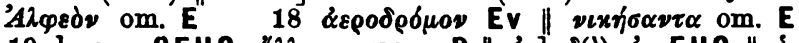

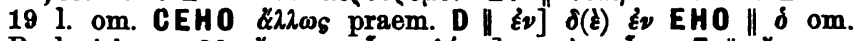

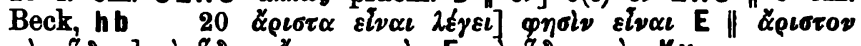

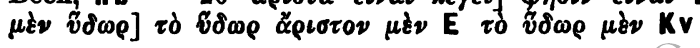




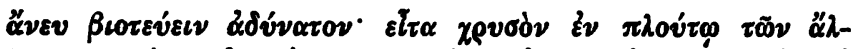

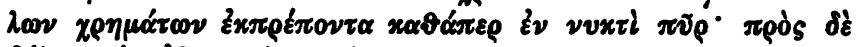

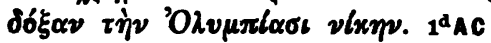

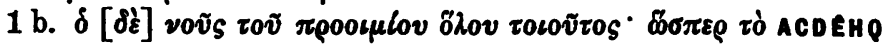

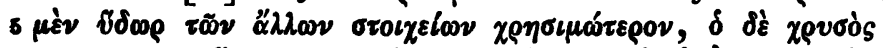

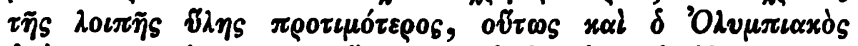

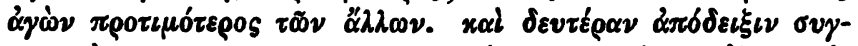

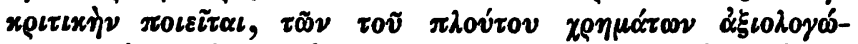

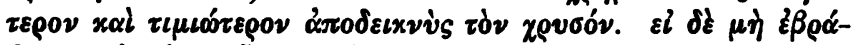

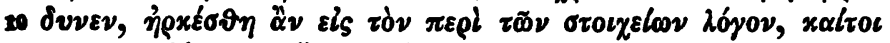

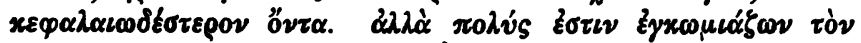

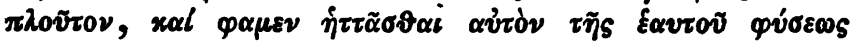

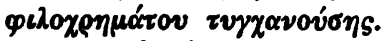

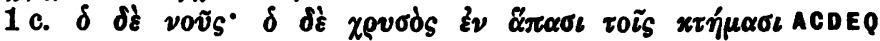

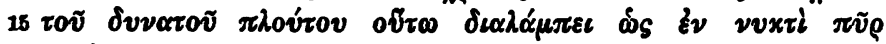
xณĹ́์

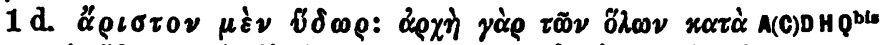

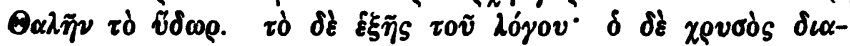

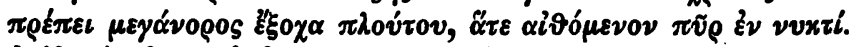

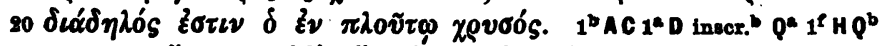

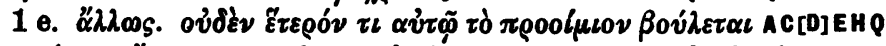

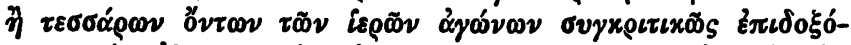

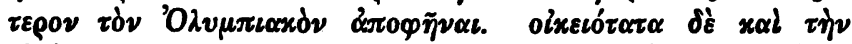

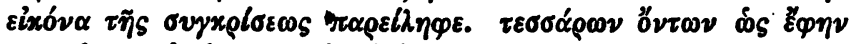

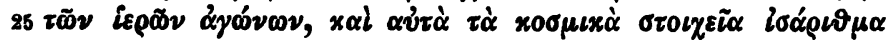

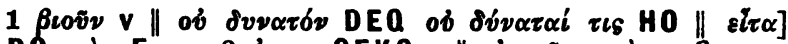

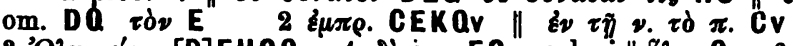

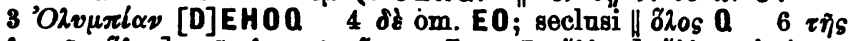

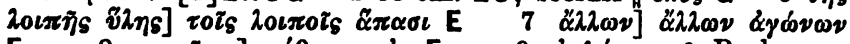

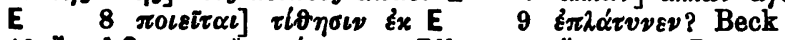

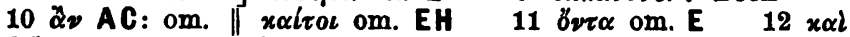

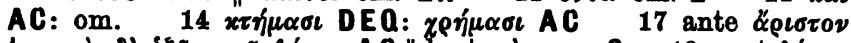

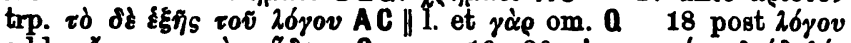

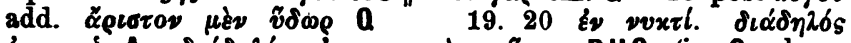

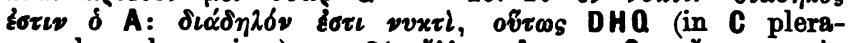

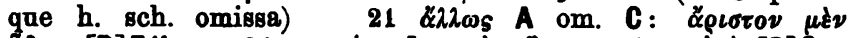

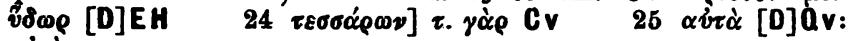
$\alpha \dot{v i d s}$ 


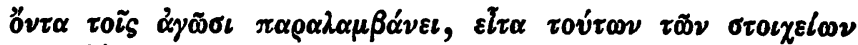

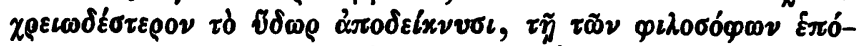

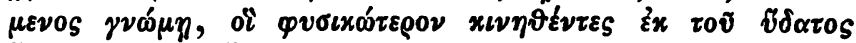

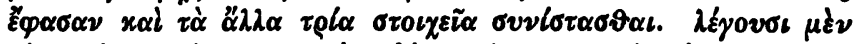

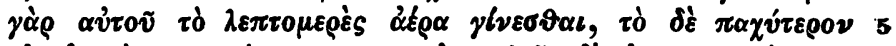

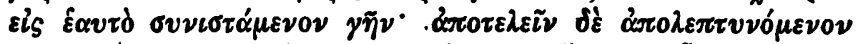

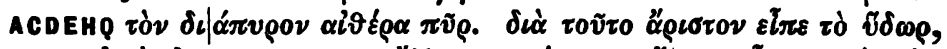

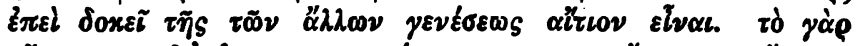

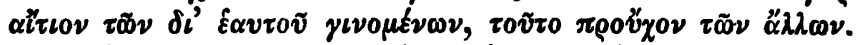

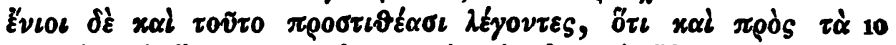

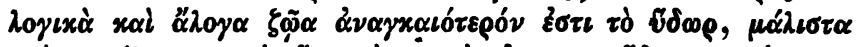

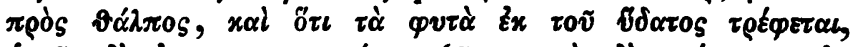

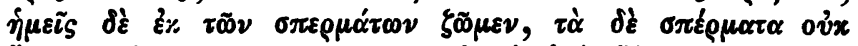

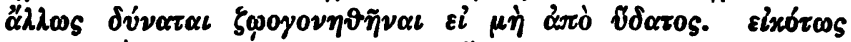

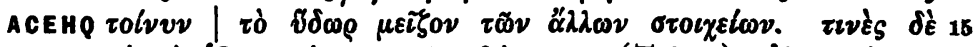

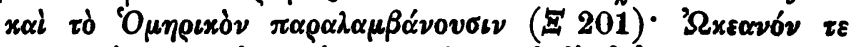

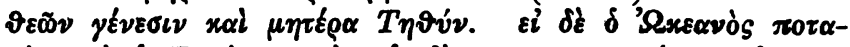

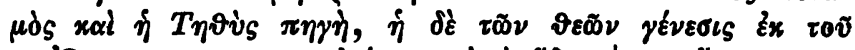

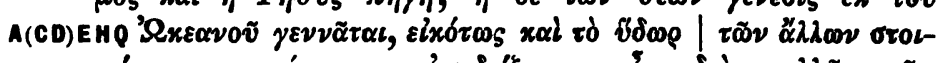

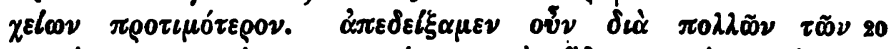

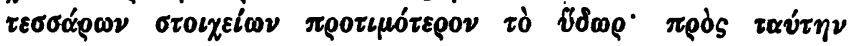

10 Aristot. met. 1, 3. Dorogr. graec. p. 91.170

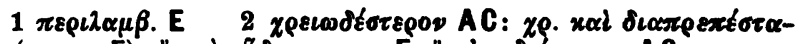

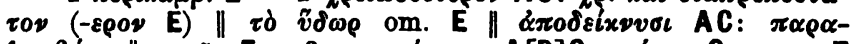

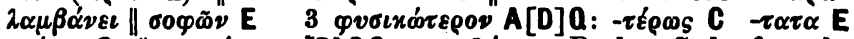

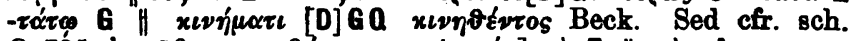

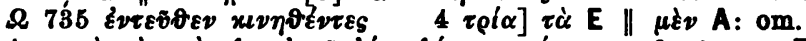

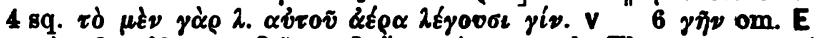

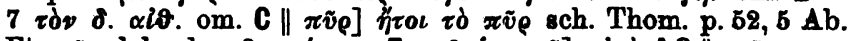

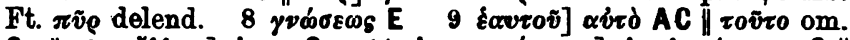

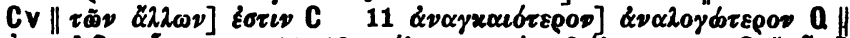

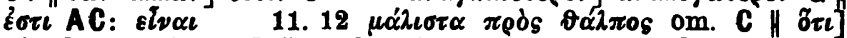

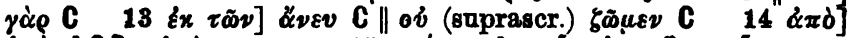

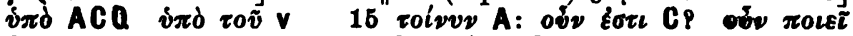

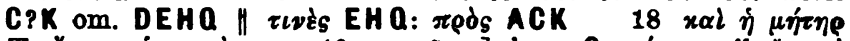

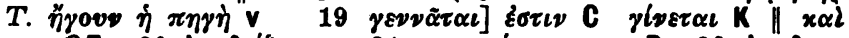

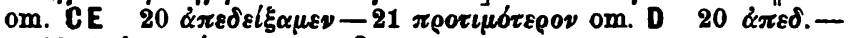

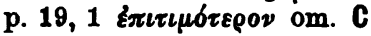




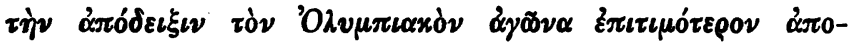

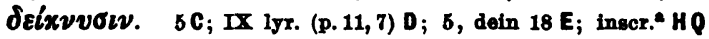

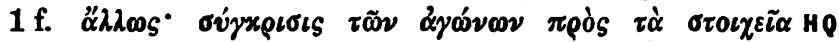

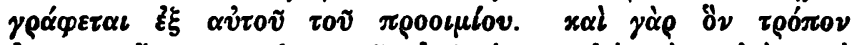

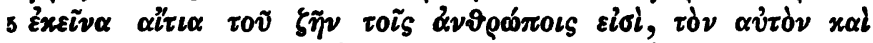

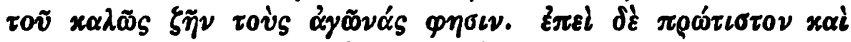

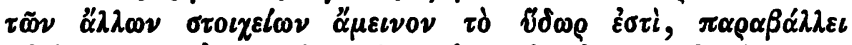

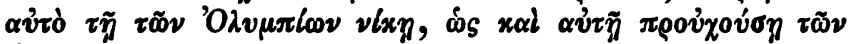

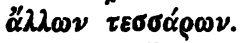

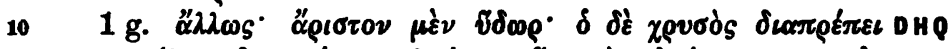

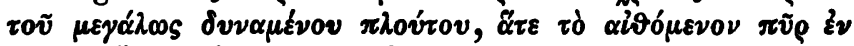

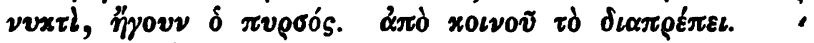

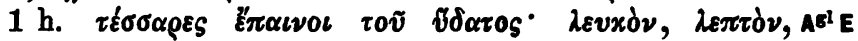

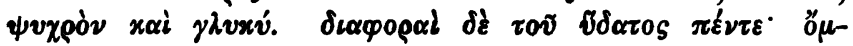

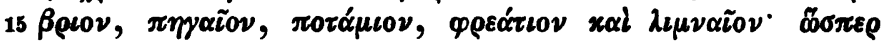

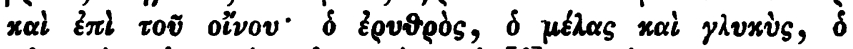

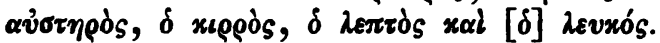

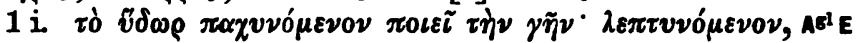

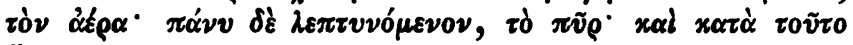

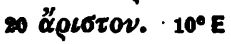

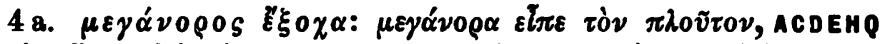

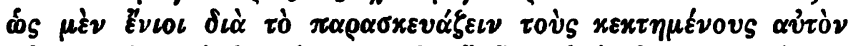

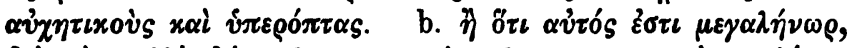

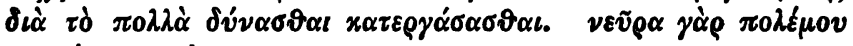
25 Xovoós. $9 \mathrm{E} 13^{\mathrm{b}} \mathrm{C}$.

13 sch. Aesch. Prom. 14416 Ath. I, 26 c

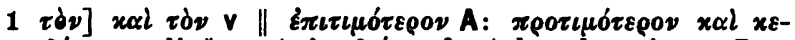

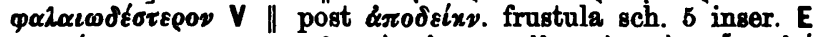

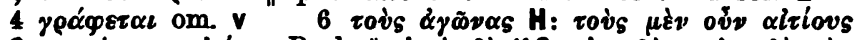

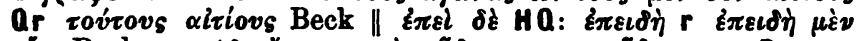

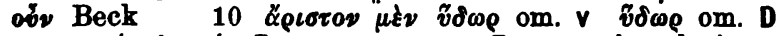

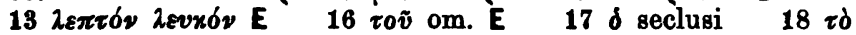

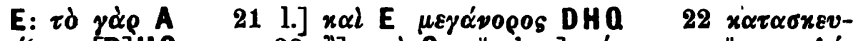

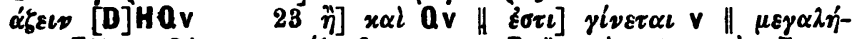

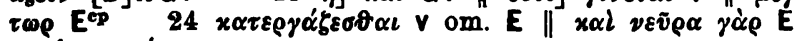
$25 \delta$ xevobs $v$ 


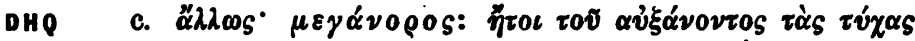

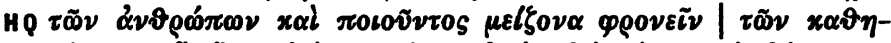

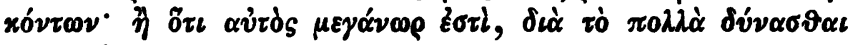

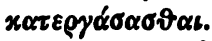

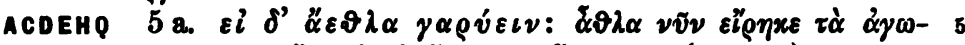

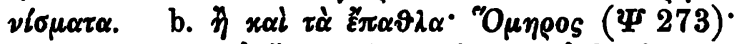

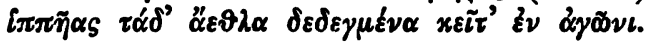

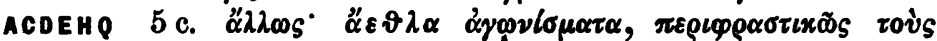

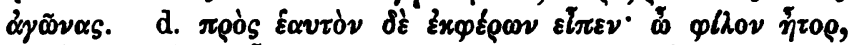

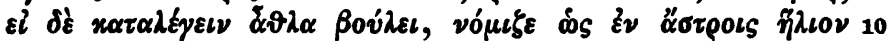

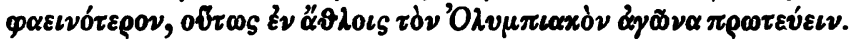

DнР

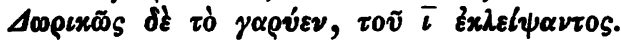

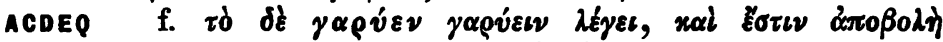

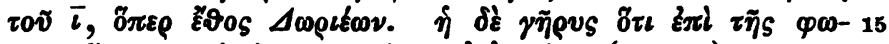

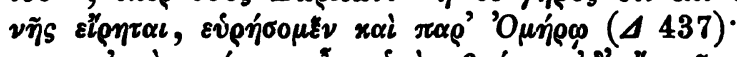

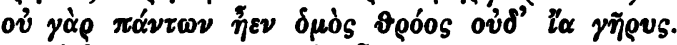

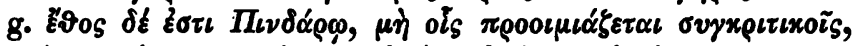

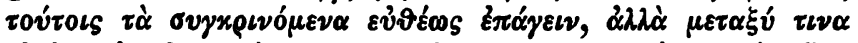

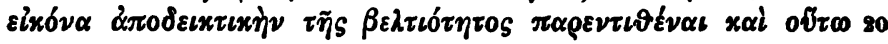

sch. 4 d. Suid., Phot., lex. Bachm. 296, 5: $\mu \varepsilon \gamma \alpha \dot{\alpha} 0 \rho 0$ s

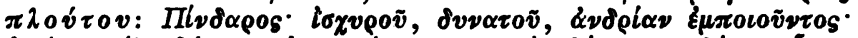

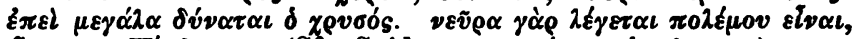

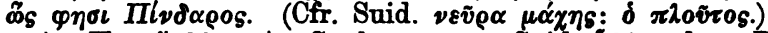

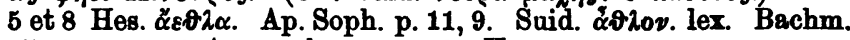
37, 6. 615 Ap. soph. p. 54, 29. Hes. $\gamma \tilde{\partial} \rho v s$

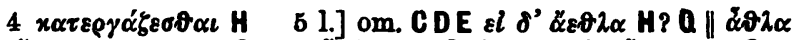

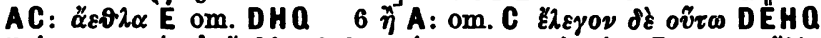

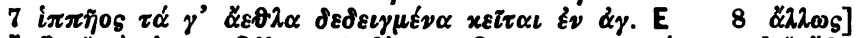

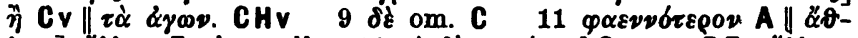

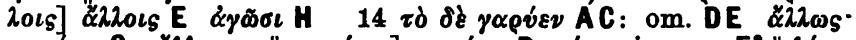

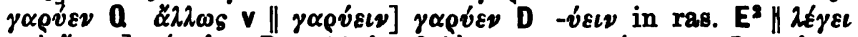

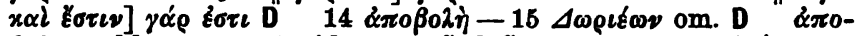

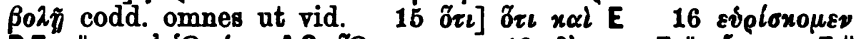

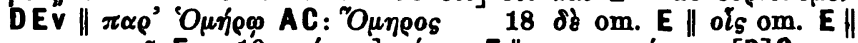

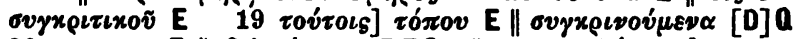

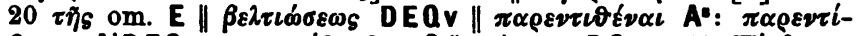

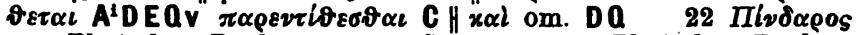
om. Phot. lex. Bachm. $24 \tilde{\omega}_{S} \varphi$. II. om. Phot. lex. Bachm. 


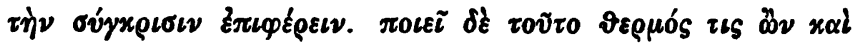

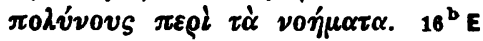

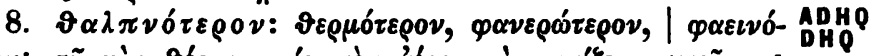

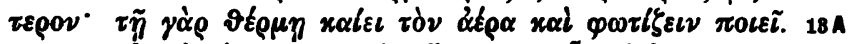

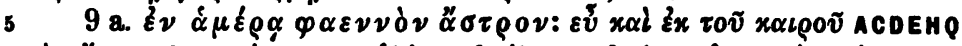

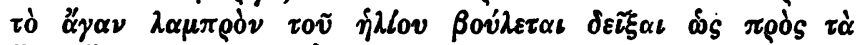

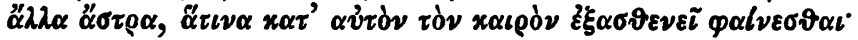

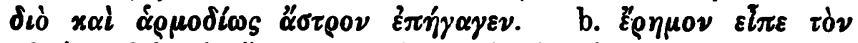

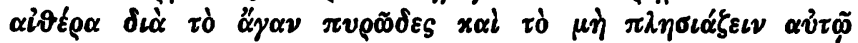

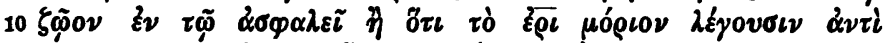

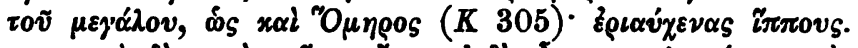

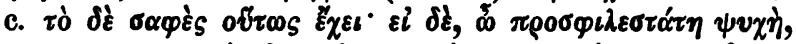

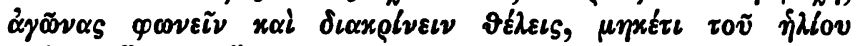

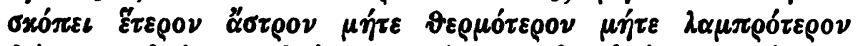

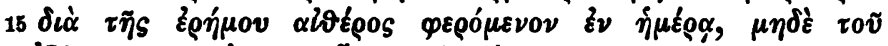

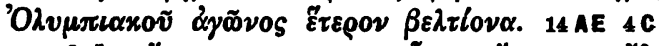

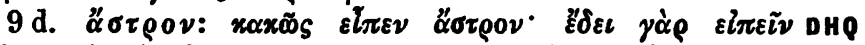

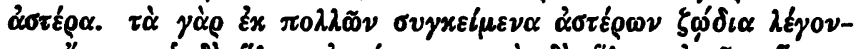

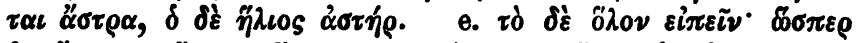

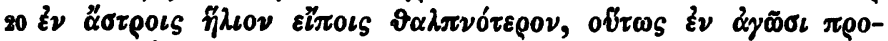

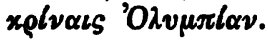

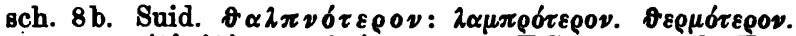

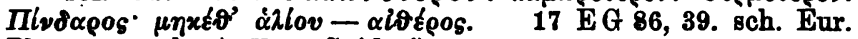
Phoen. 1. sch. A E 5. Suid. äorpov

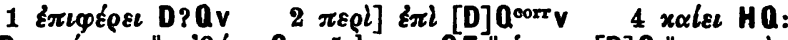

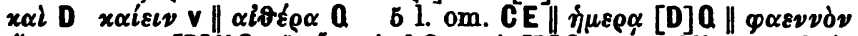

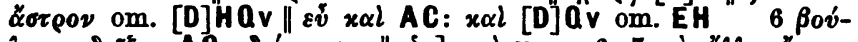

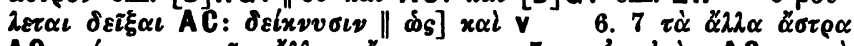

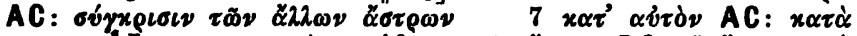

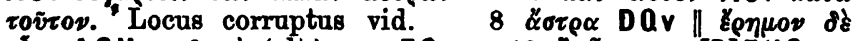

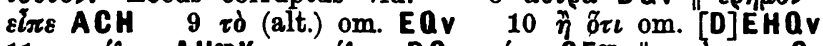

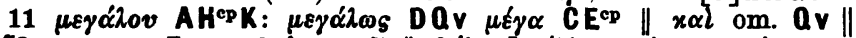

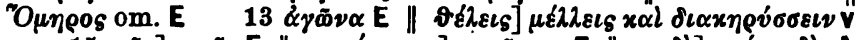

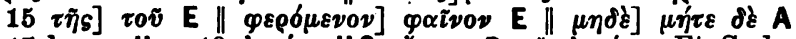

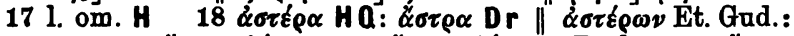

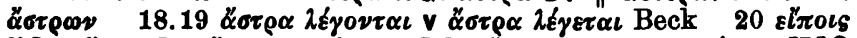

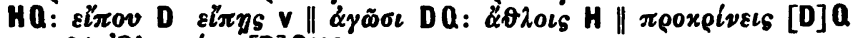
$21{ }^{\prime} O$ L $\mu \pi i \alpha s[D] Q^{\infty}$ ? 


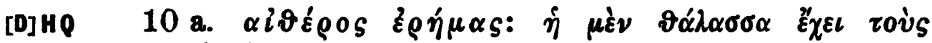

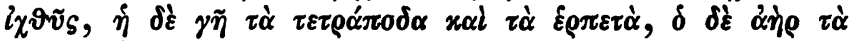

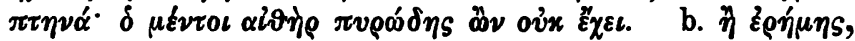

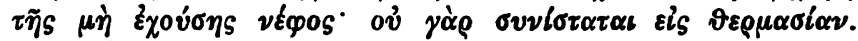

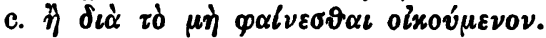

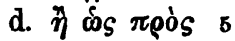

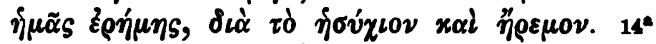

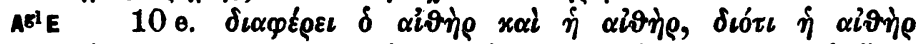

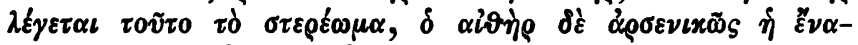

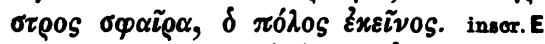

[D]н 13 а. ठ̆

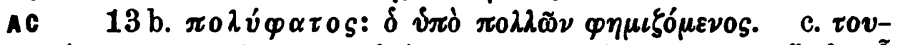

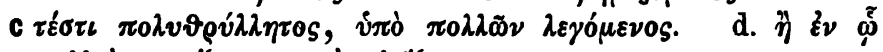

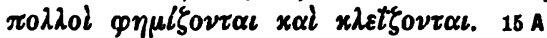

[D]но 13 е. $\pi$ о

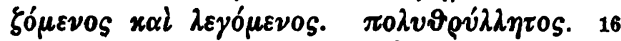

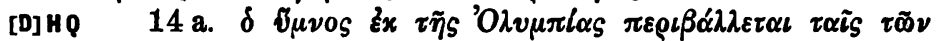

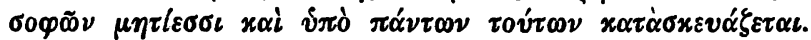

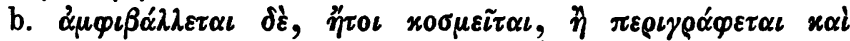

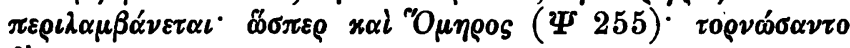
$\delta \tilde{\varepsilon}^{\prime} \sigma \tilde{\eta} \mu \alpha$.

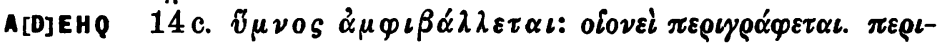

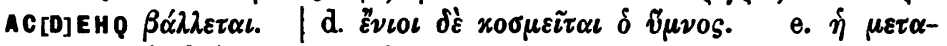

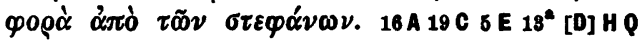

A 15. $x \varepsilon \lambda \alpha \delta \varepsilon i \nu: ~ \delta \mu \nu \varepsilon \tilde{\nu} .17$

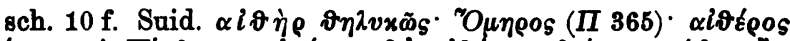

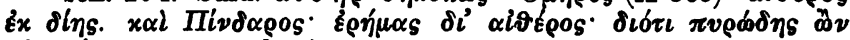

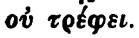
18 sch. A $\Psi 255$

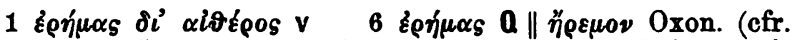

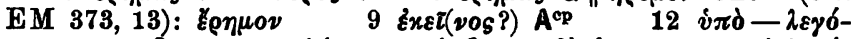

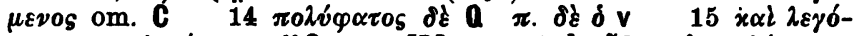

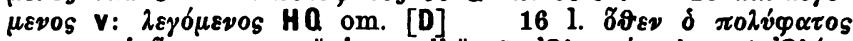

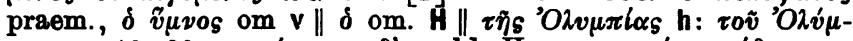

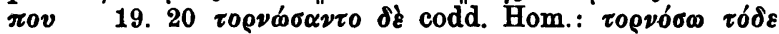

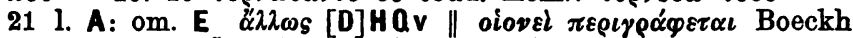

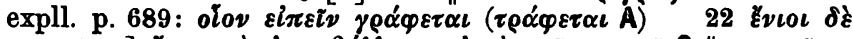

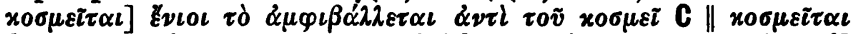

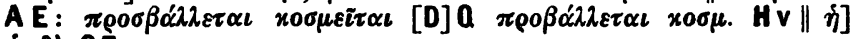
$\dot{\eta}$ de CE 


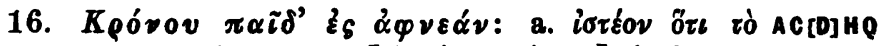

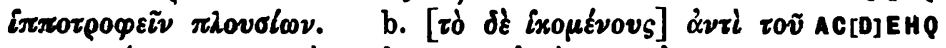

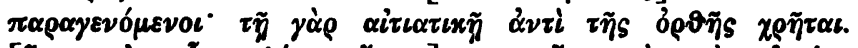

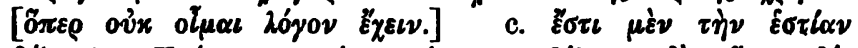

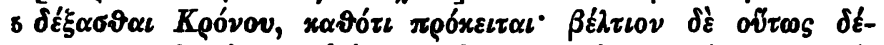

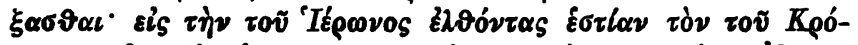

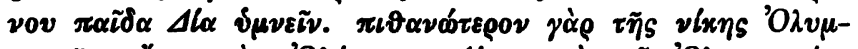

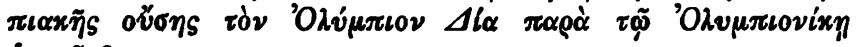
$\hat{v} \mu \nu \varepsilon \tilde{u} \sigma \vartheta \alpha$. 19 AE $99 \mathrm{C}$

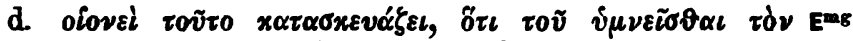

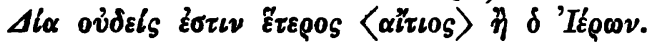

17. $\mu \alpha \dot{\alpha} x \alpha \iota \rho \alpha \nu: \varepsilon v j \delta \alpha l \mu o \nu \alpha$. $\pi \lambda 0 v \sigma i \alpha v$.

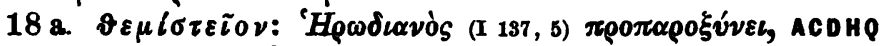

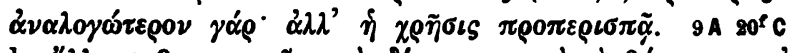

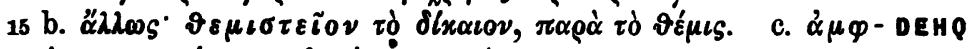

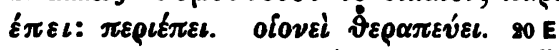

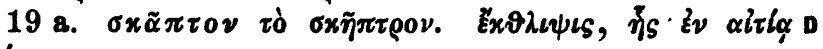
$\pi \alpha \rho \varepsilon ́ \mu \pi \tau \tau \omega \sigma \iota \varsigma$.

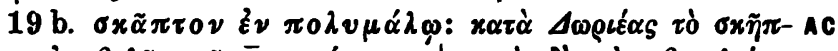

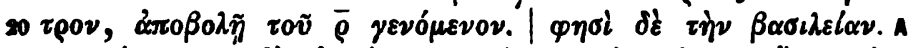

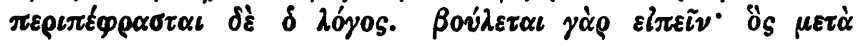

17 EM 101, 23. Eust. I. 104, 21. 1158, $33 \quad 19$ Eust. Il. 104,22

1 l. sic A: Kৎóvov $\pi \alpha \tau \delta \alpha$ C[D]HQ om. E \| Ordo: b. c. a.

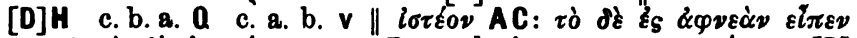

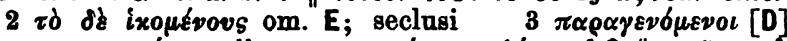

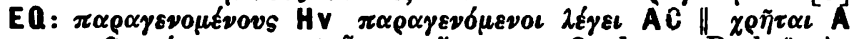

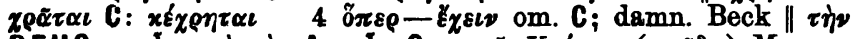

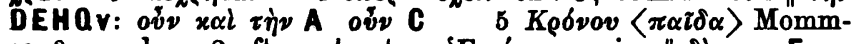

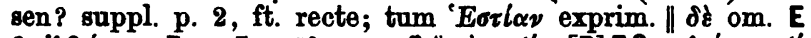

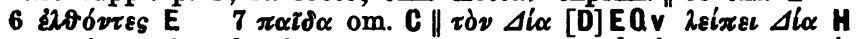

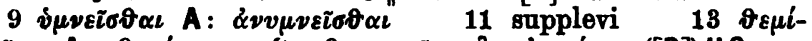

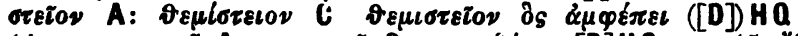

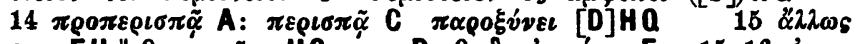

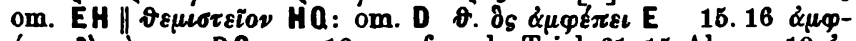
$\varepsilon \pi \varepsilon \iota$ dो $\tau \delta$ $\pi \varepsilon \rho$. DQv 19 sq. cfr. sch. Tricl. 61, $15 \mathrm{Ab}$. 19 है

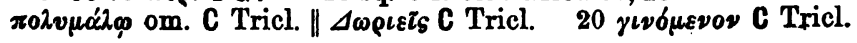




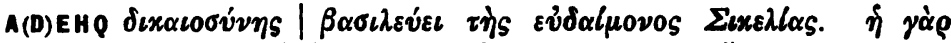

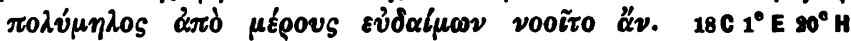

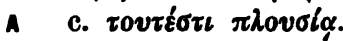

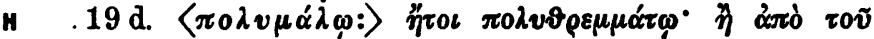

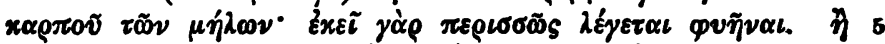

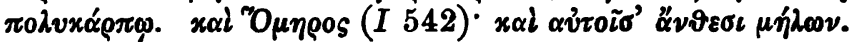

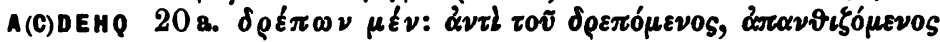

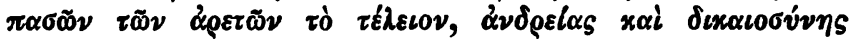

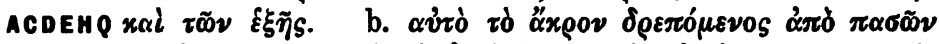

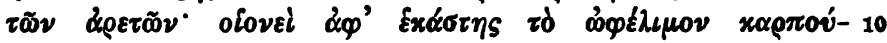

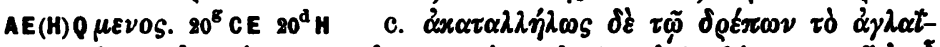

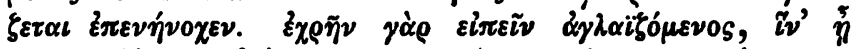

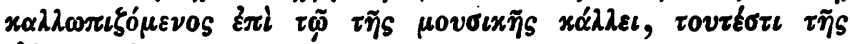

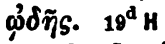

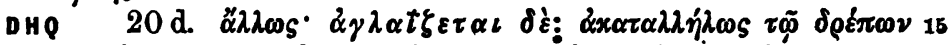

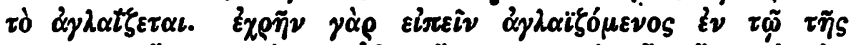

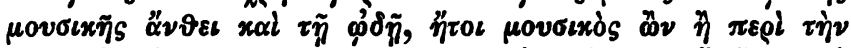

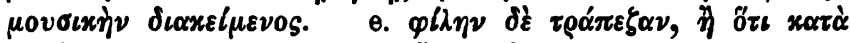

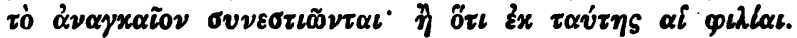

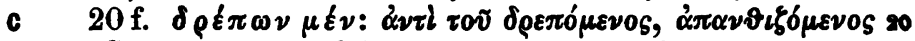

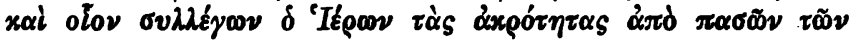

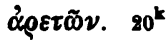

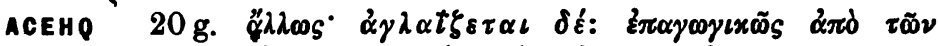

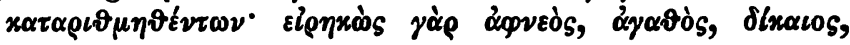

4 Eust. I. 301, 23. Suid. $\pi 0 \lambda v \mu \eta^{\prime} \lambda$ 5 sch. AB I 542

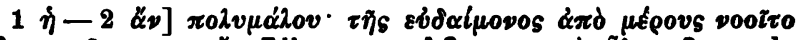

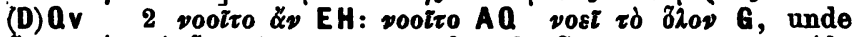

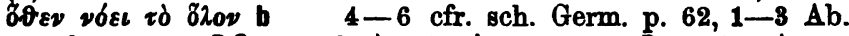

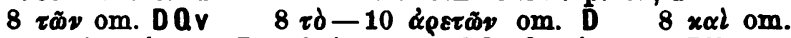

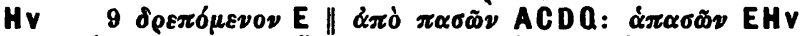

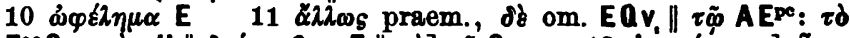

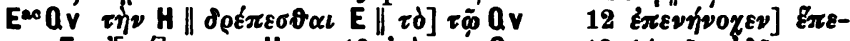

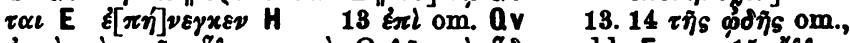

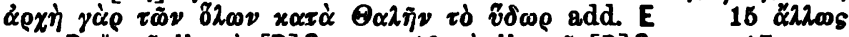

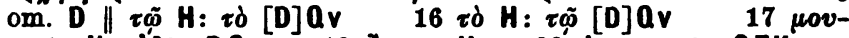

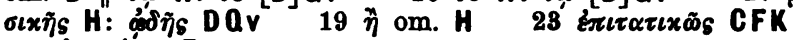

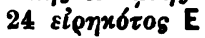




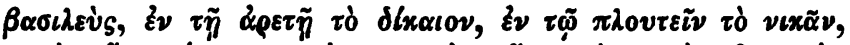

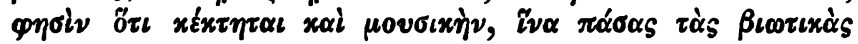

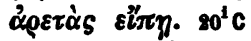

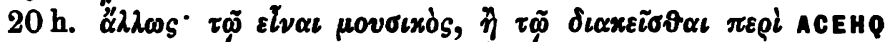

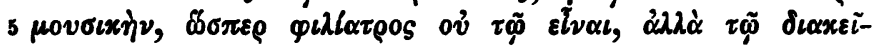
$\sigma \vartheta \alpha t .20^{\circ} \mathrm{C}$

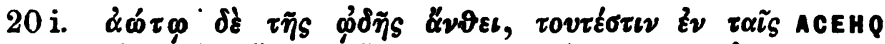

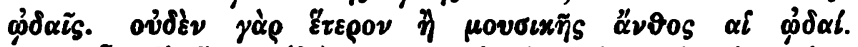

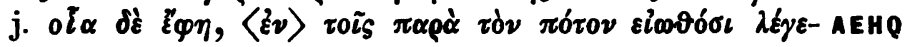
$10 \sigma \vartheta \alpha \varepsilon_{\text {. }}$ 24:AC

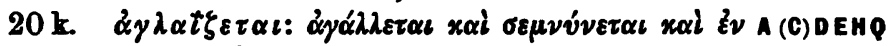

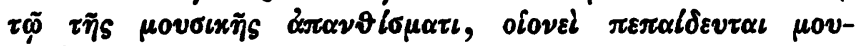

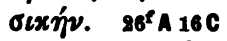

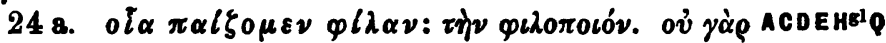

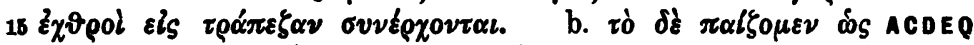

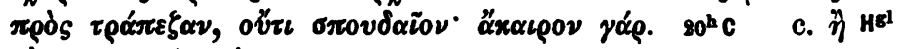

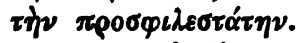

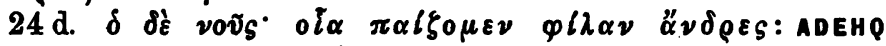

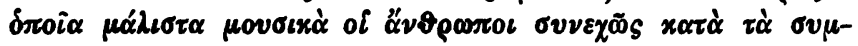

14 EM 794, 12. Eust. I. 1118, 59

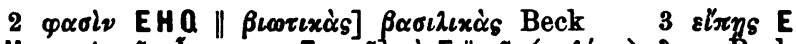

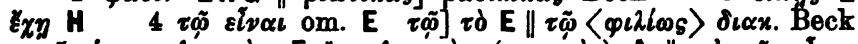
5 is

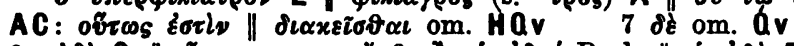

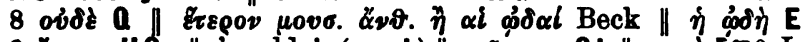

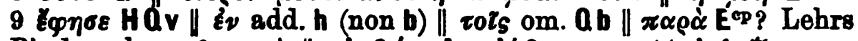

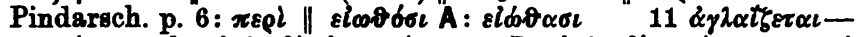

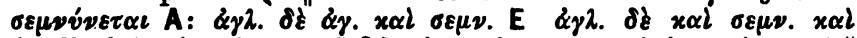

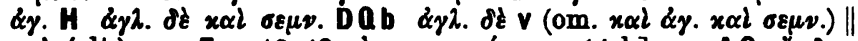

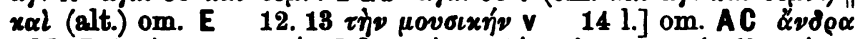

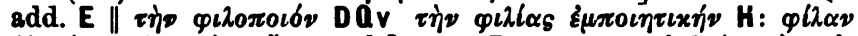

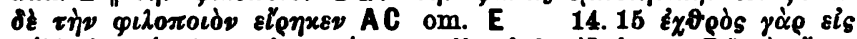

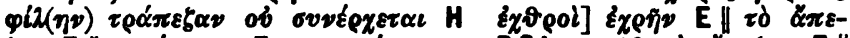

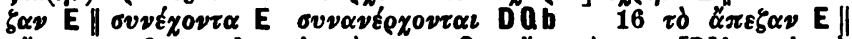

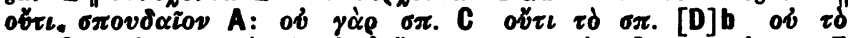

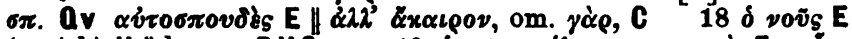

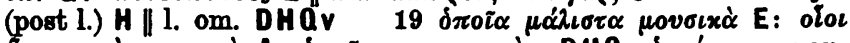

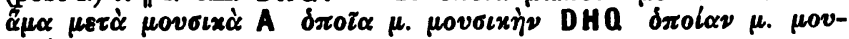
oเxทे V 


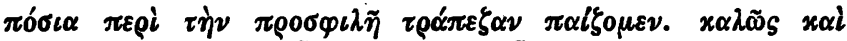

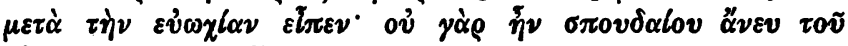
$\varepsilon v ่ \omega \chi \varepsilon \tilde{\imath} \sigma \vartheta \alpha \iota \quad x \iota \vartheta \alpha \rho l \zeta \varepsilon \iota \nu$.

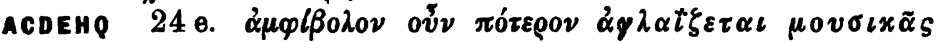

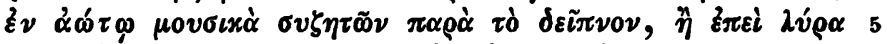

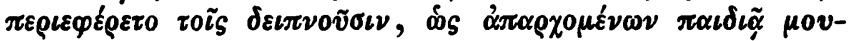

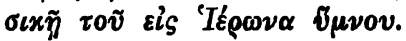

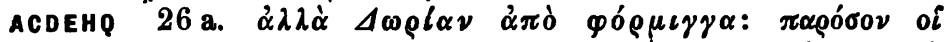

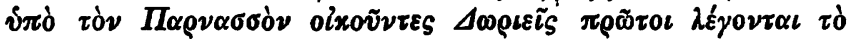

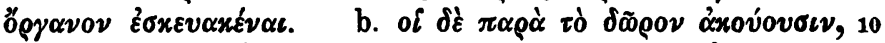

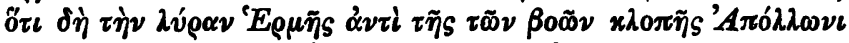

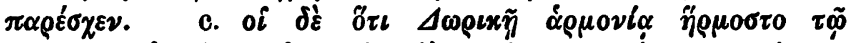

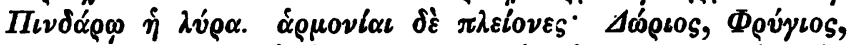

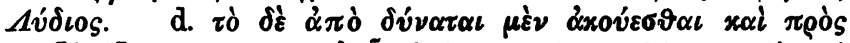

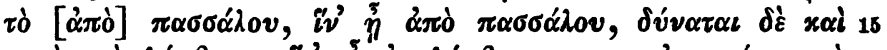

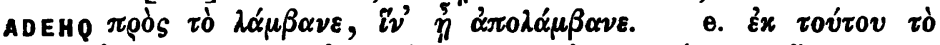

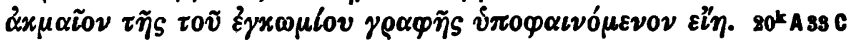

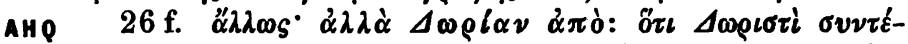

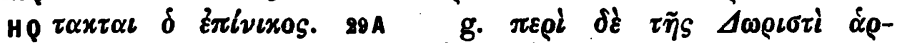

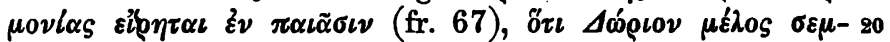

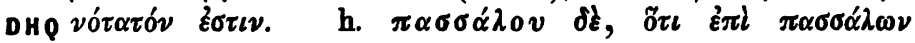

21 Eust. ad Od. 1428, 58. 1586, 9

$1 \pi \rho 0 \sigma \varphi \iota \lambda \tilde{\eta}] \pi \rho \circ \varepsilon \varepsilon \mu \varepsilon \varepsilon v \eta v \mathrm{E} \| \pi \alpha i \xi o \mu \varepsilon \nu$ om. A $\| x \alpha \lambda \tilde{\omega} s]$

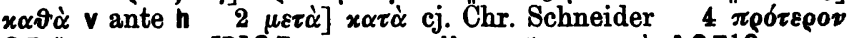

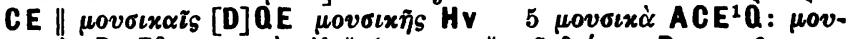

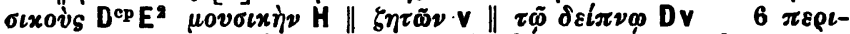

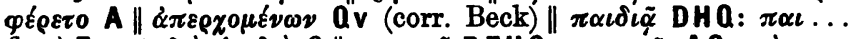

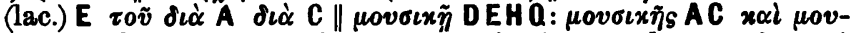

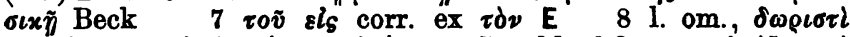

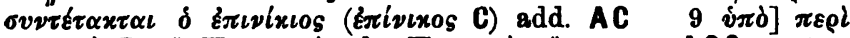

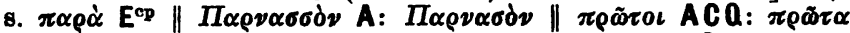

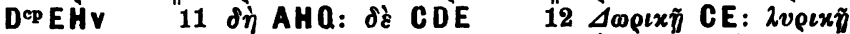

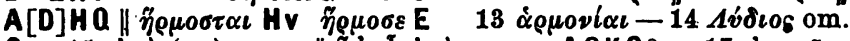

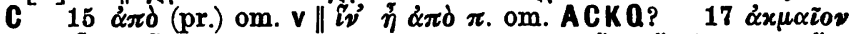

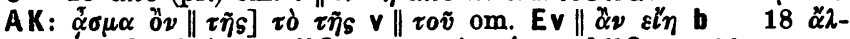

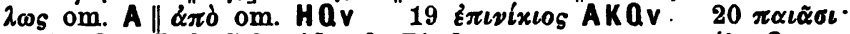

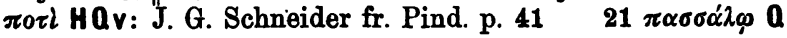




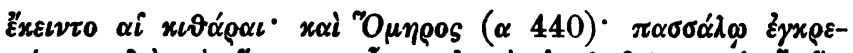

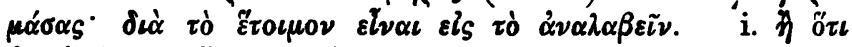

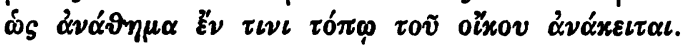

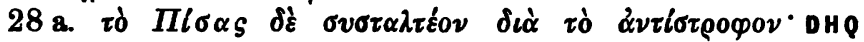

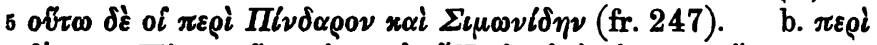

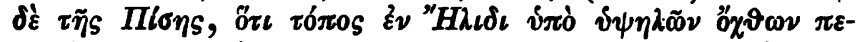

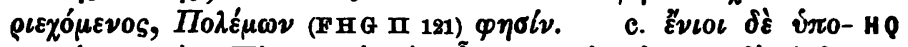

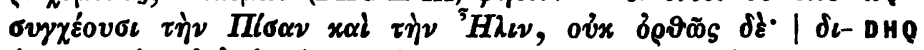

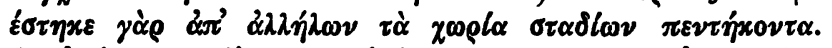

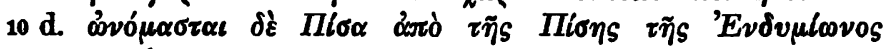
จvyazৎós.

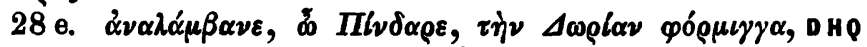

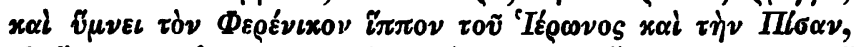

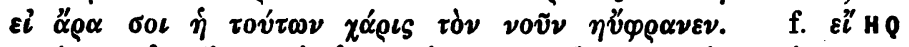

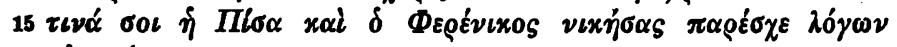

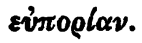

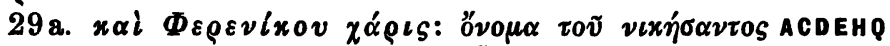

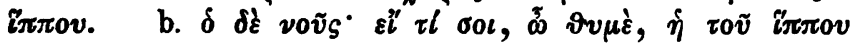

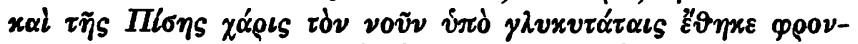

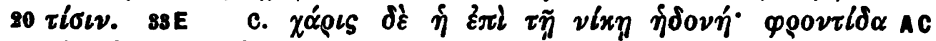

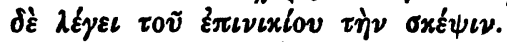

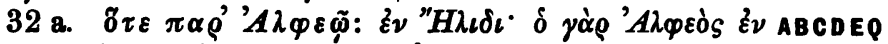

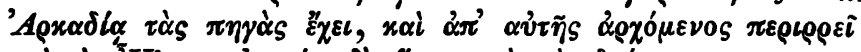

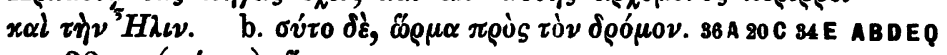

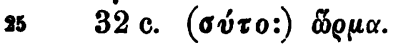
$B$ BI

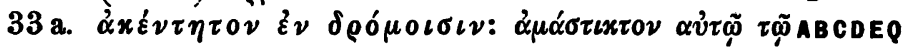

9 cfr. sch. 0. 10,51 a. 55b 10 sch. Theocr. 4, 29

1 debuit afferre $\vartheta 67.105 \| \varepsilon^{2} \gamma x \rho \varepsilon \mu \alpha^{\prime} \sigma \alpha \sigma \alpha$ et $\alpha y x \rho \varepsilon \mu$. codd.

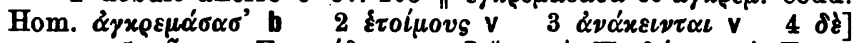

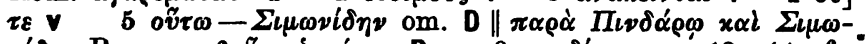

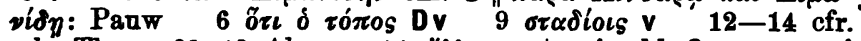
sch. Thom. 63, $18 \mathrm{Ab}$. $14 \% \alpha \lambda \lambda_{\text {s. }}$ ante $\varepsilon l$ add. Qv $15 \delta$

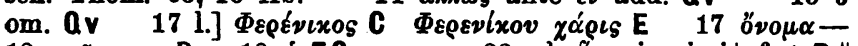
18 voṽ om. D 18 i EQv: om. 22 ab $\delta \tau \varepsilon$ incipit f. 1 B\| 1. om. C $C^{1} \| \tilde{o} \tau \varepsilon$ om. AC $23 \alpha \dot{v} \tau \eta_{S}$ C: $\alpha \dot{v} \tau \tilde{\omega} \nu(-\delta \nu$ E)

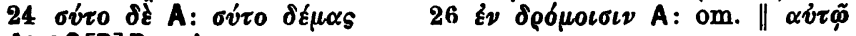
$A^{\text {eP }}$ ? $[D] P: \alpha \dot{v} \tau \tilde{v}$ 


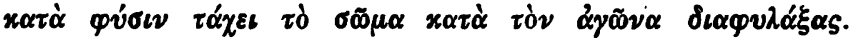

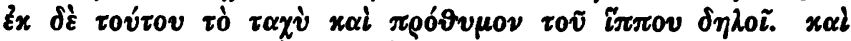

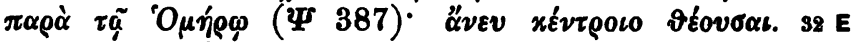

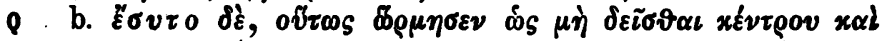

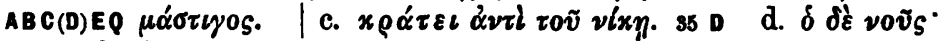
5

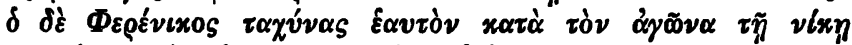

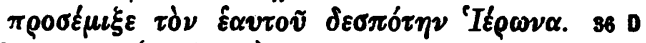

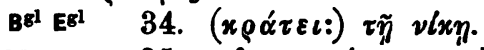

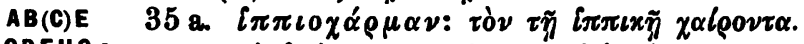

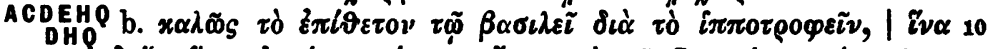

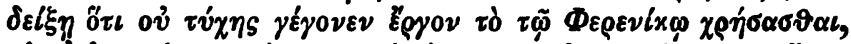

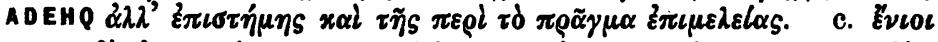

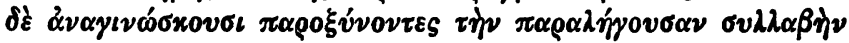

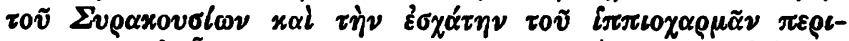

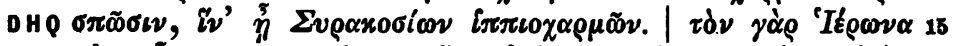

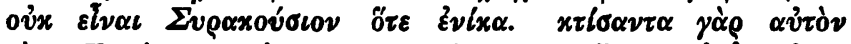

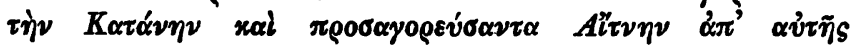

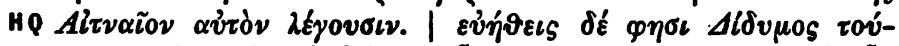

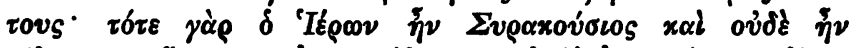

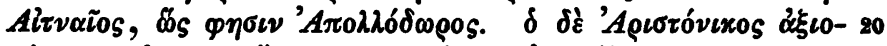

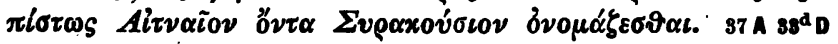

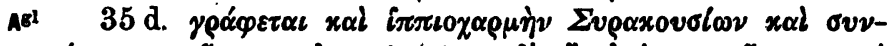

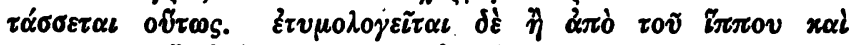

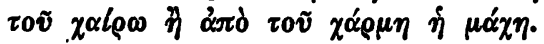

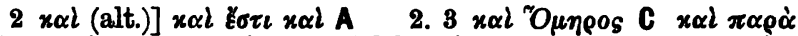

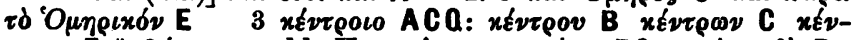

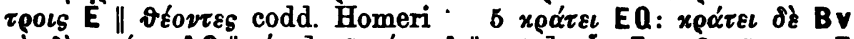

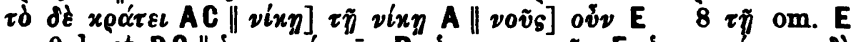

9 1. et DQ $\| i \pi \pi\left\llcorner 0 \chi \alpha \alpha_{\rho} \mu \bar{\alpha} \nu\right.$ B $i \pi \pi\llcorner 0 \chi \alpha \rho \mu \tilde{\alpha} \nu \mathrm{E} i \pi \pi\llcorner 0 \chi \alpha \rho \mu \eta \nu$ d'

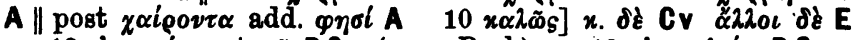

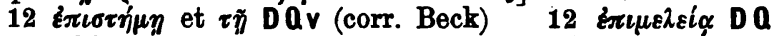

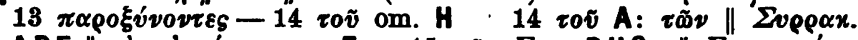

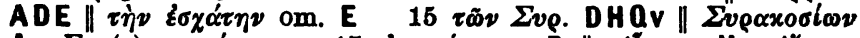

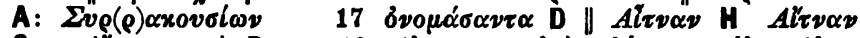

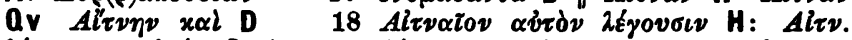

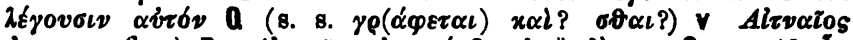

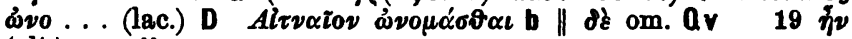
(alt.) om. H 


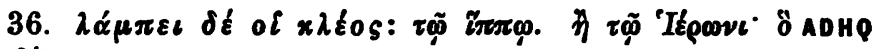
xal $\beta \varepsilon \lambda \tau \iota 0 v . ~ 38 \mathrm{~A}$

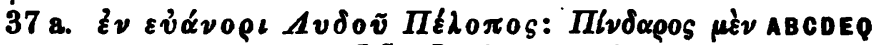

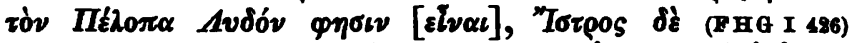

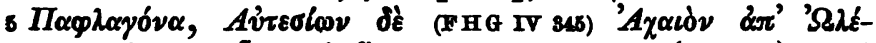

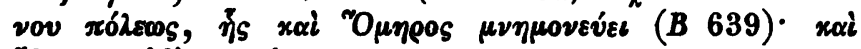

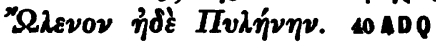

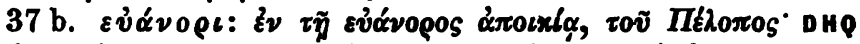

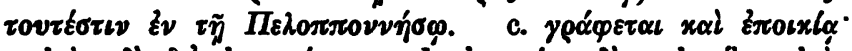

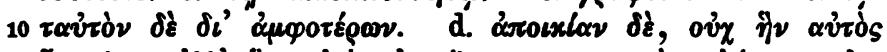

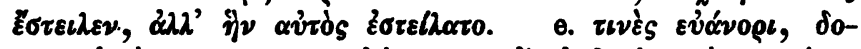

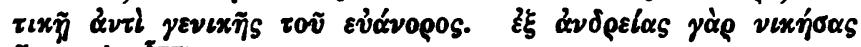

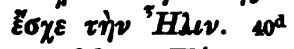

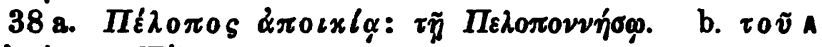

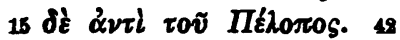

38 c. $(\alpha \pi 0\llcorner x l \alpha:) x \alpha \tau o u x l \alpha$.

$B$ st

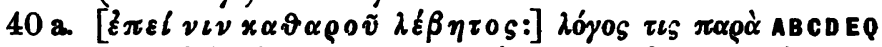

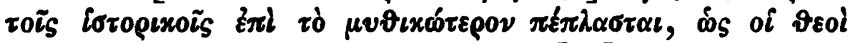

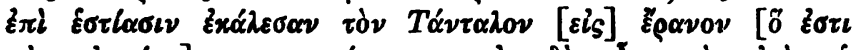

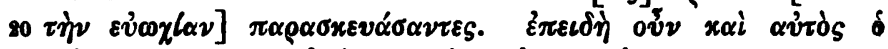

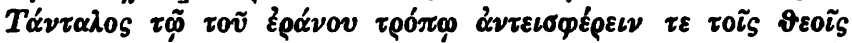

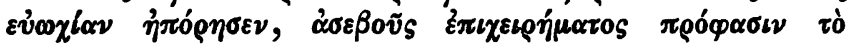

3 Diod. 4, 74. Tzetzes ad Lyc. 15019 Apoll. epit. 2, 3

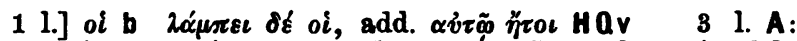

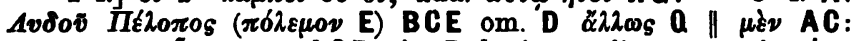
om. 4 sival om. ACE; in B legi nequit 5 Aivedicov

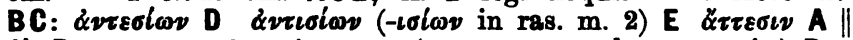

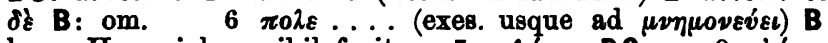
locus Homeri huc nihil facit $7 \pi \varepsilon \lambda \eta \eta \eta \nu$ DQr 8 \&vávopl]

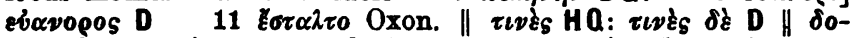

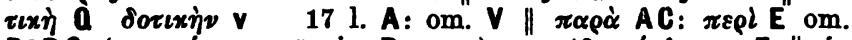

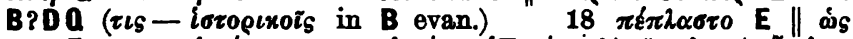

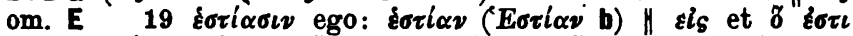

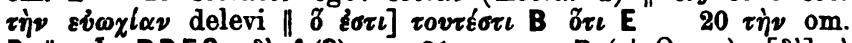
D ${ }_{0} \nu \nu$ BDEQ: $\delta \xi$ A (C) $21 \tau \varepsilon$ om. B (et Oxon.) [ $\left.\delta \dot{\varepsilon}\right] \tau \dot{\varepsilon}$

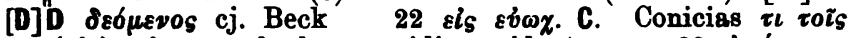
$\theta$. $\left\langle\varepsilon l_{s}\right\rangle \varepsilon \dot{v} \omega x$; sed plura excidisse videntur $22 \eta \eta^{\prime} \delta \rho \eta \sigma \alpha \nu$

E \| $\pi \rho \delta \varphi \alpha \sigma i \nu$ om. B 


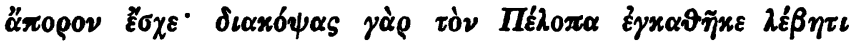

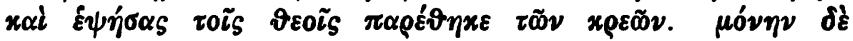

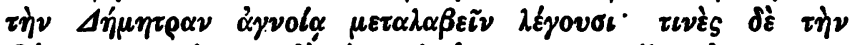

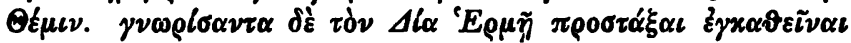

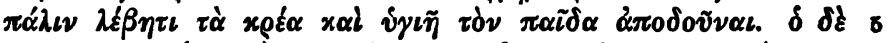

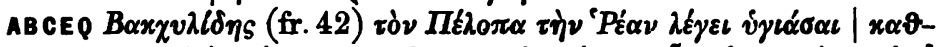

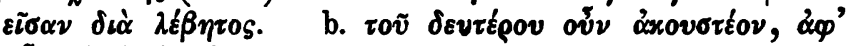

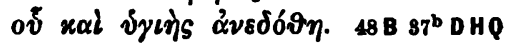

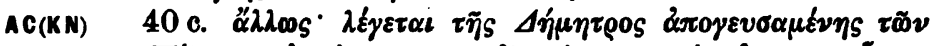

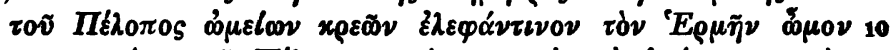

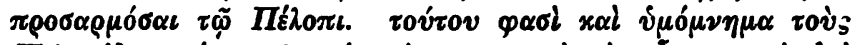

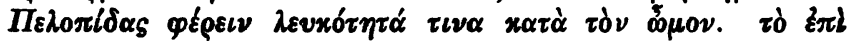

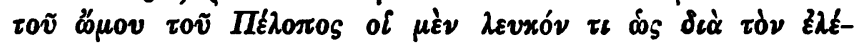

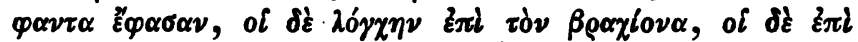

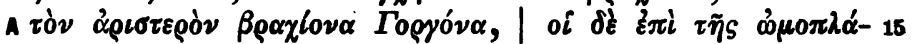

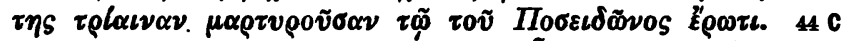

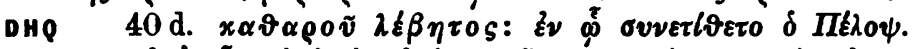

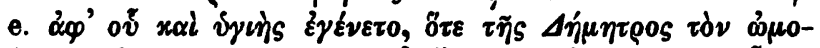

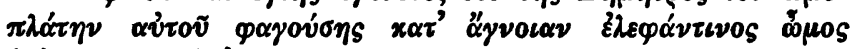

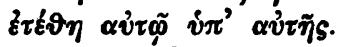

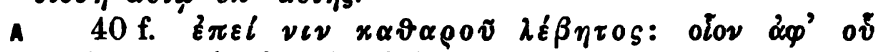

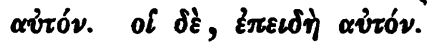

11 Tzetzes ad Lyc. 152 (p. 411 M.)

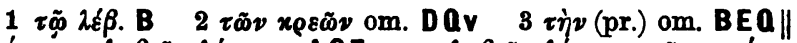

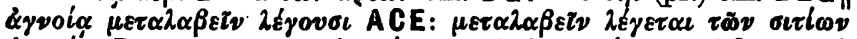

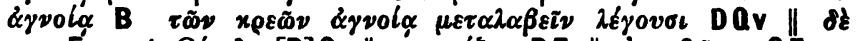

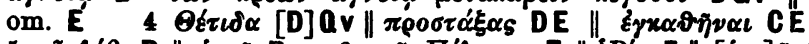

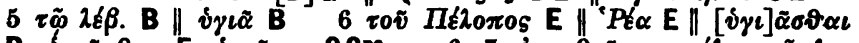

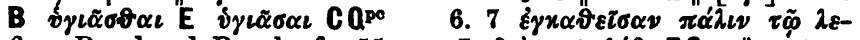

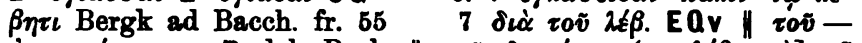

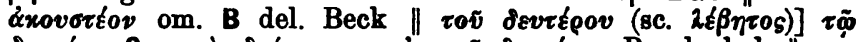

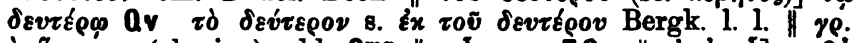

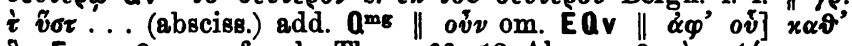

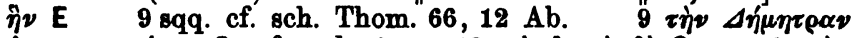

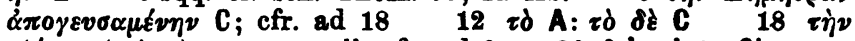

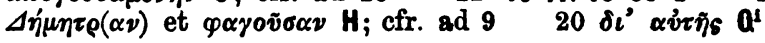




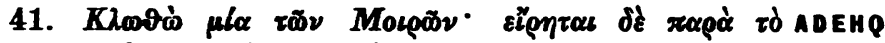

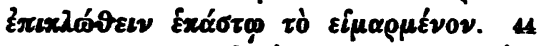

42 a. $x \varepsilon x \alpha \delta \mu \varepsilon \dot{v} \nu \nu: x \varepsilon x 0 \sigma \mu \eta \mu t v o v$. ss A

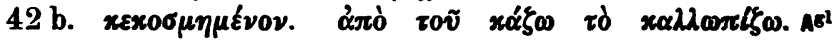

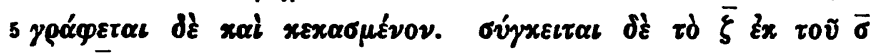

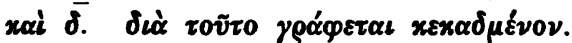

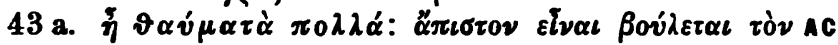

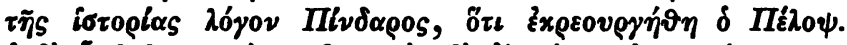

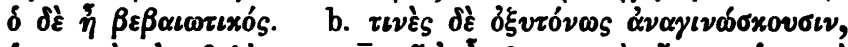

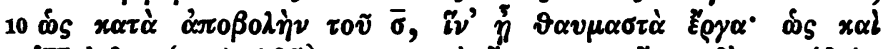

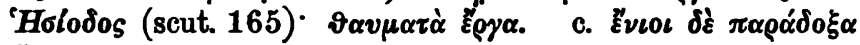
$\eta \eta \hat{x}$

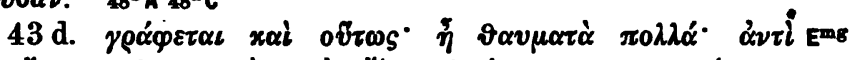

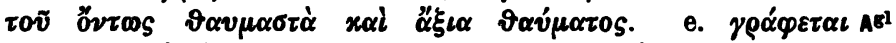

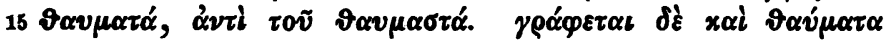
$\pi 0 \lambda \lambda \alpha ́$.

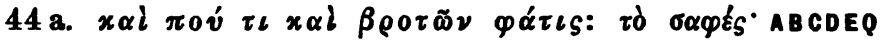

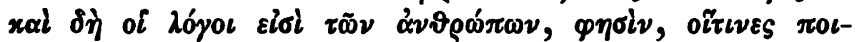

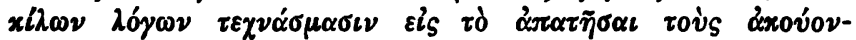

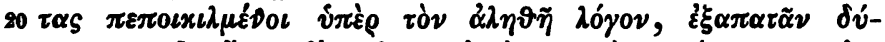

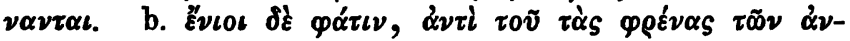

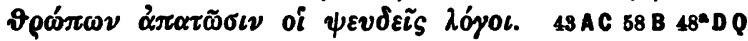

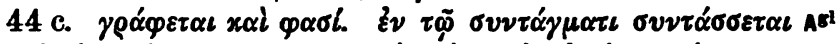

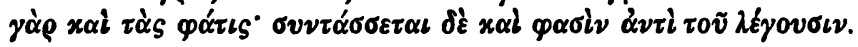

1 sch. Hes. th. 2119 sch. Hes. scut. 154

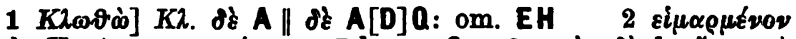

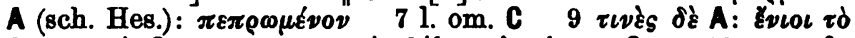

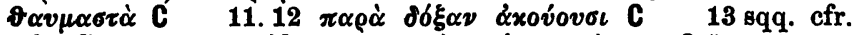

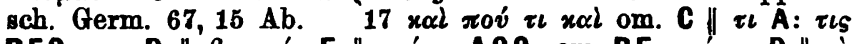

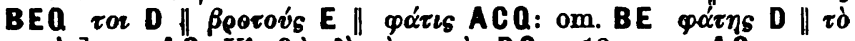

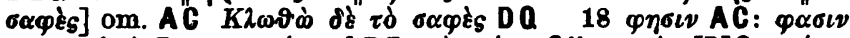

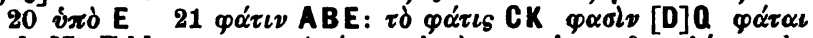

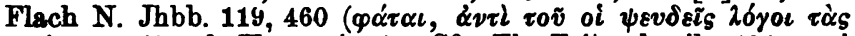

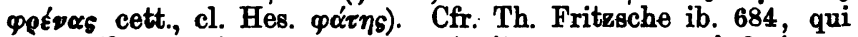

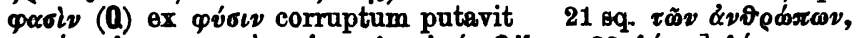

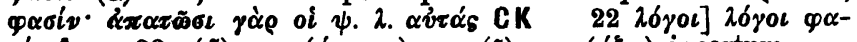
oiv A $23 \tau(\tilde{\omega}) \sigma v \nu \tau(\alpha \gamma \mu \alpha \tau \iota)$ an $\tau(\tilde{\eta}) \sigma v \nu \tau\left(\alpha^{\prime} \xi \varepsilon \iota\right)$ incertum 


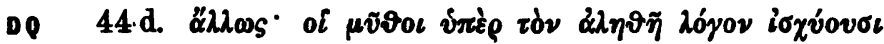

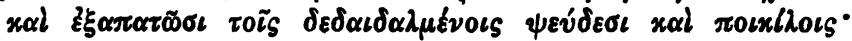

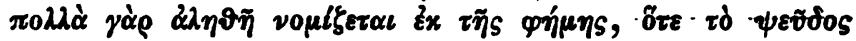
$\pi \rho 0 \sigma \lambda \alpha^{\prime} \beta \eta$. $\quad 8^{\mathrm{b}}$

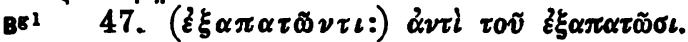

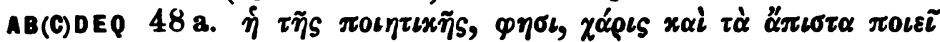
$\pi L \sigma \tau \alpha^{\prime} . \quad 50 \wedge 4^{\mathrm{d} D Q}$

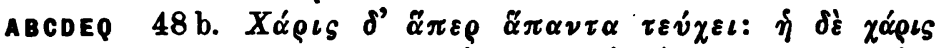

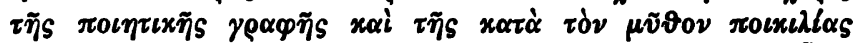

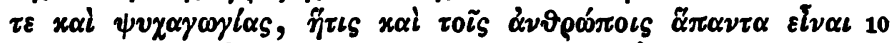

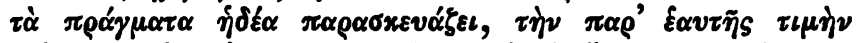

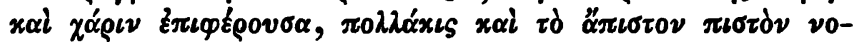

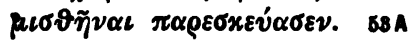

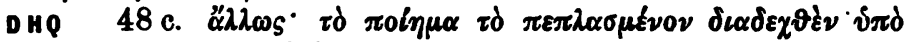

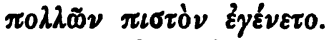

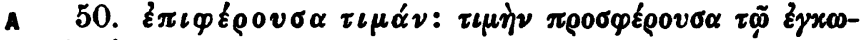
$\mu\llcorner\alpha \xi 0 \mu \varepsilon \dot{v} \nu$. $\quad$ ธ9

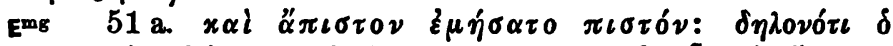

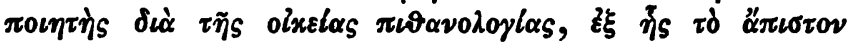

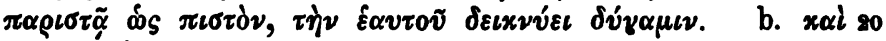

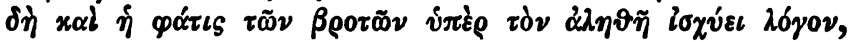

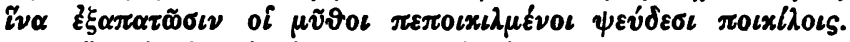

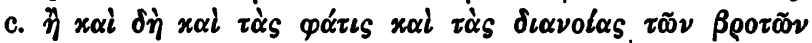

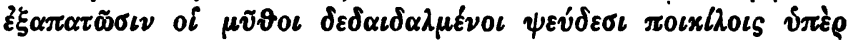

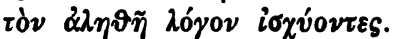

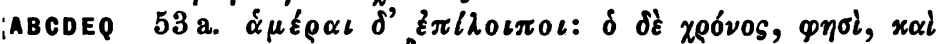

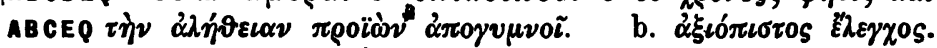

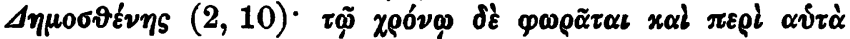

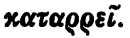

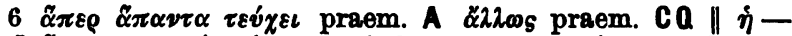

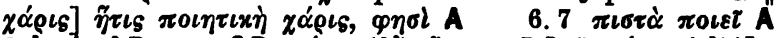

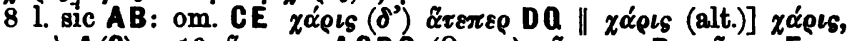
$\varphi \eta \sigma i \mathrm{~A}(\mathrm{C}) \quad 10 \tilde{\alpha} \pi \alpha \nu \tau \alpha$ ACDQ (0xon.): $\tilde{\alpha} \pi \alpha \sigma \nu$ BV $\tilde{\alpha} \pi \varepsilon \rho \mathrm{E}$

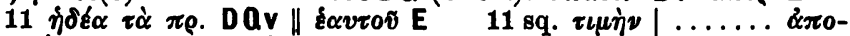

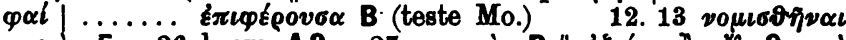

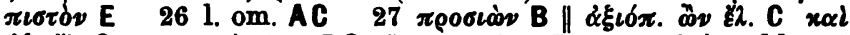

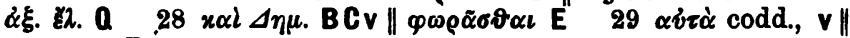

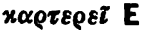




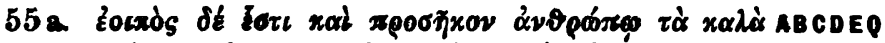

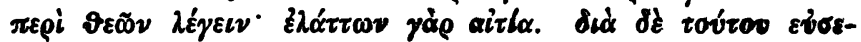

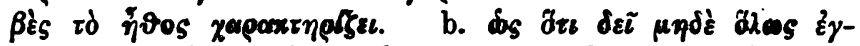

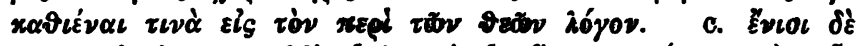

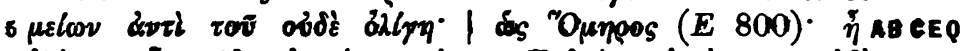

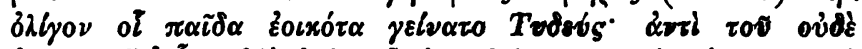

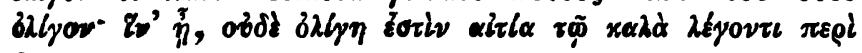
จะฒข. แล

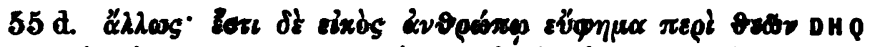

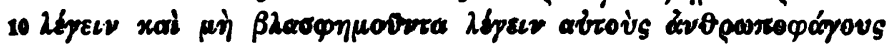

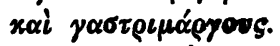

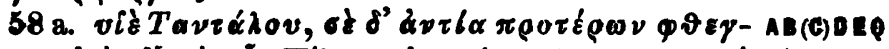

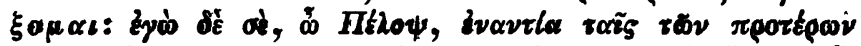

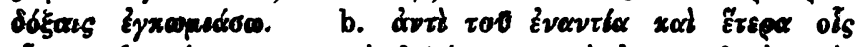

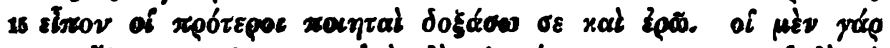

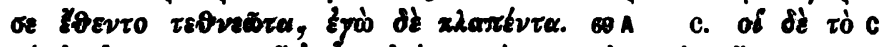

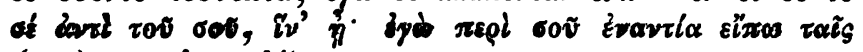

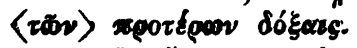

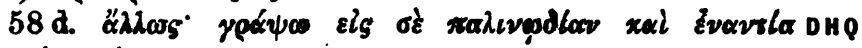

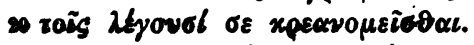

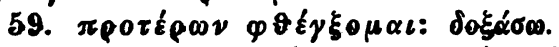

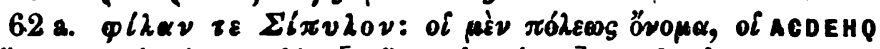

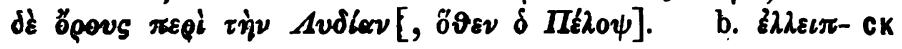

\section{5 sch. A E 800. Eust. 1. 610, 6. Suid. bilyov}

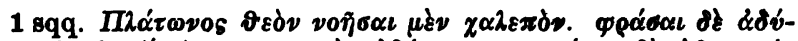

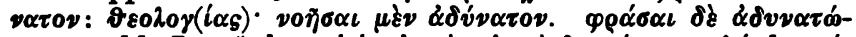

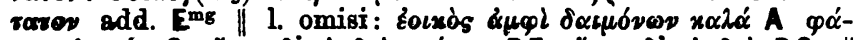

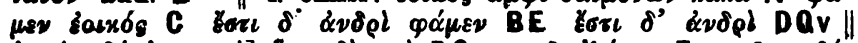

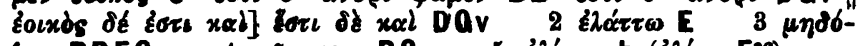
Laog BDEQ 4 ชต̃ข om. BQ 5 bilyou h (bilyo Em)

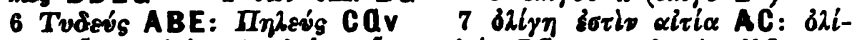

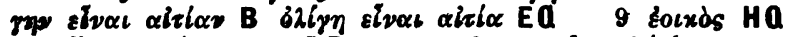

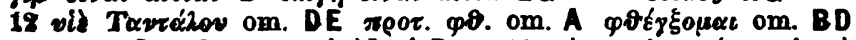

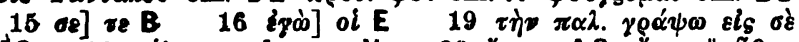

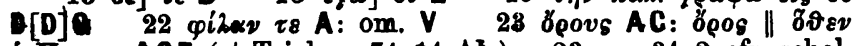
\& II. om. ACE (et Tricl. p. 74, 14 Ab.) 23-p. 34, 2 cfr. ochol. Tricl. p. 72, 18 Ab. Lehrs Pindarsch. p. 41

Sogoz. ns Proparum ed. Drachmann 


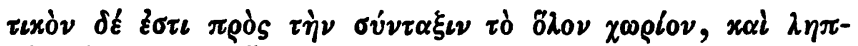

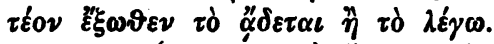

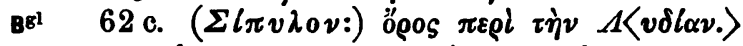

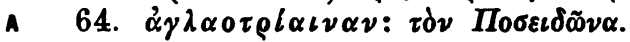

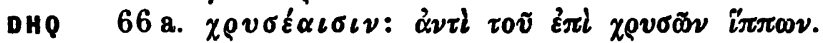

Bв1D HO b. $\mu \varepsilon \tau \alpha \beta \iota \beta \alpha^{\prime} \sigma \alpha \iota, \mu \varepsilon \tau \alpha \gamma \alpha \gamma \varepsilon \tilde{\nu} \nu$.

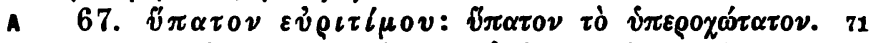

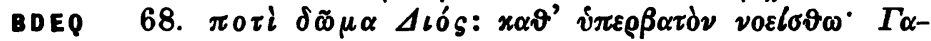

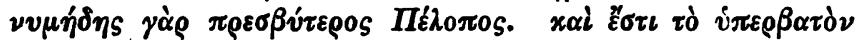

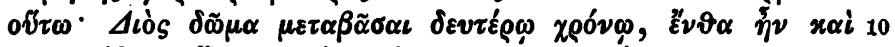

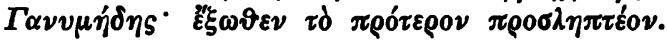

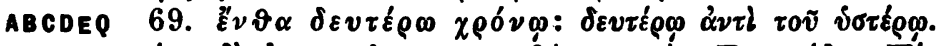

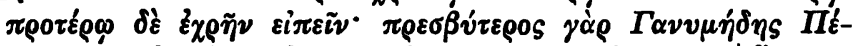

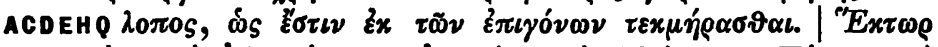

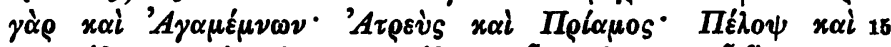

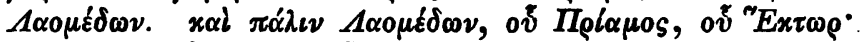

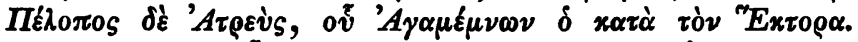

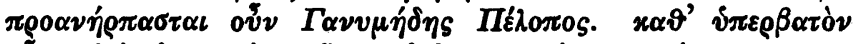

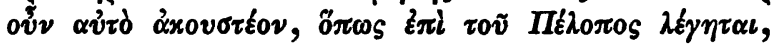

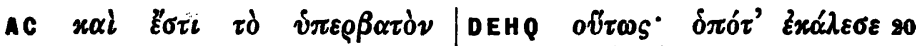

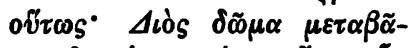

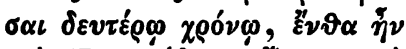

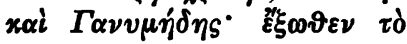

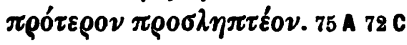

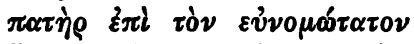

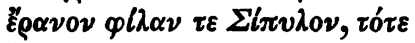
$\dot{\alpha} \gamma \lambda \alpha 0 \tau \rho l \alpha \iota v \alpha \nu \mu \varepsilon \tau \alpha \beta \tilde{\alpha} \sigma \alpha \iota \delta \varepsilon v-$

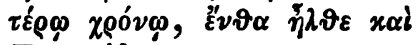
$\Gamma \alpha \nu v \mu \eta \dot{\delta} \delta \xi .720$

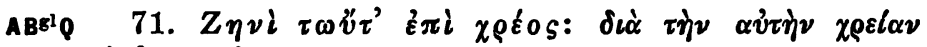

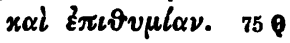

$6 \mu \varepsilon \tau \alpha \beta \iota \beta \alpha^{\prime} \sigma \alpha \iota$ B : $\mu \varepsilon \tau \alpha \beta \iota \beta \alpha^{\prime} \sigma \alpha S$ DQ $-\sigma \varepsilon \iota \nu$ H $\| \mu \varepsilon \tau \alpha\langle\gamma \alpha \gamma \varepsilon i v ?\rangle$

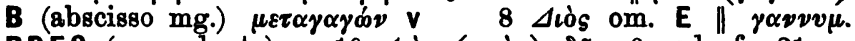

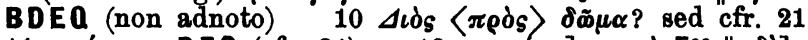

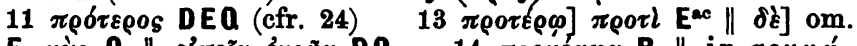
E yò Q

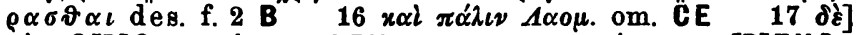

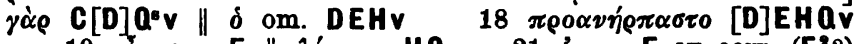
19 ovv om. E \| $\lambda \varepsilon \varepsilon \varepsilon \tau \alpha \iota$ HQ 21 Q $\alpha \nu \mu$. E ex corr. (E2?) $24 \pi \rho \delta \tau \varepsilon \rho \circ$ et $-o \nu$ A (cfr. 11) $\| \eta^{2} \lambda v \varepsilon$ om. E $26 \varepsilon \pi l$

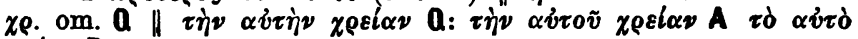
$x \rho \operatorname{cog}_{\mathrm{g}} \mathrm{B}$ 


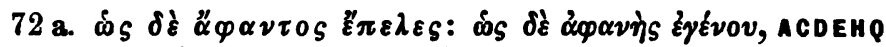

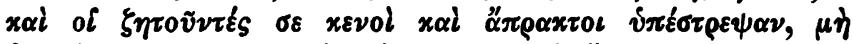

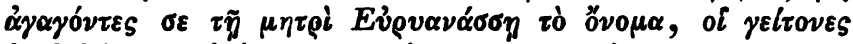

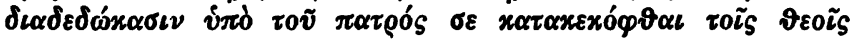

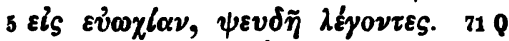

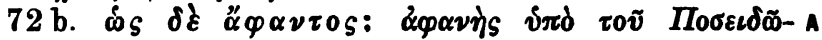
vos. 77

75 a. 

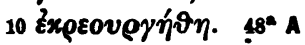

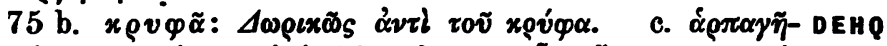

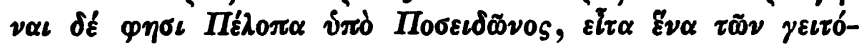

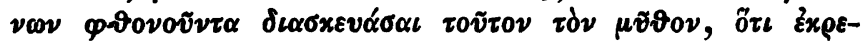

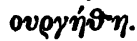

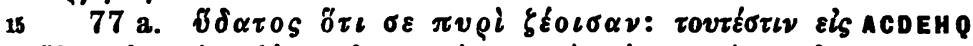

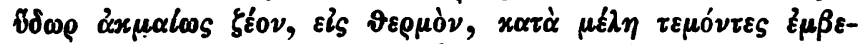

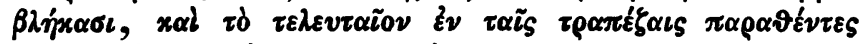

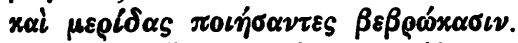

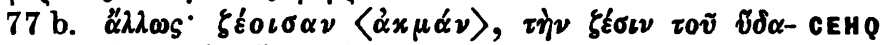

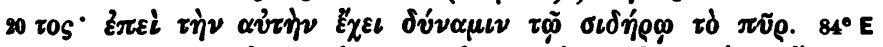

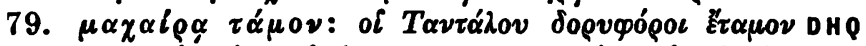

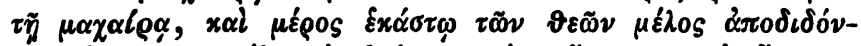

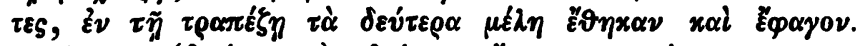

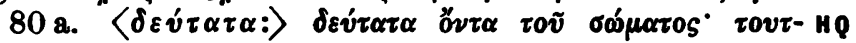

11 sch. P. 3, 65. Herodian. I, 489, 17

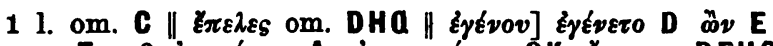

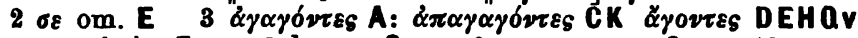

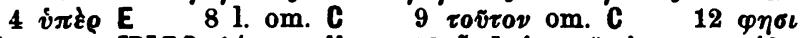

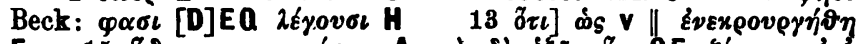

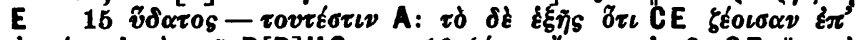

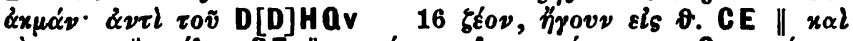

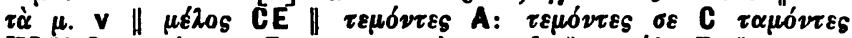
[D] (H)Q $\tau \varepsilon \mu \omega_{0} \nu$ of E $18 x \alpha i$ om. A $\|\mu \varepsilon \rho i \delta \alpha E\| x \alpha \tau \alpha-$

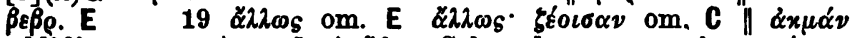

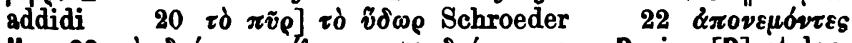

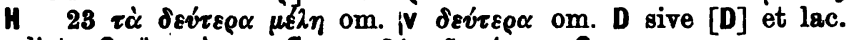

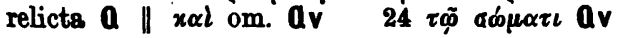




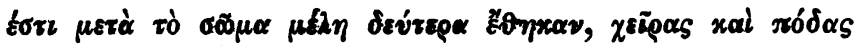

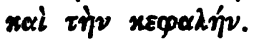

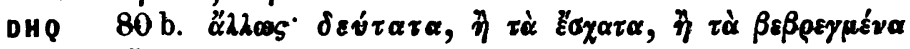

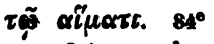

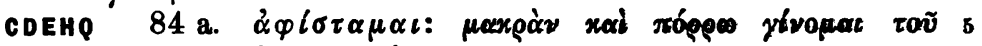

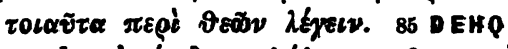

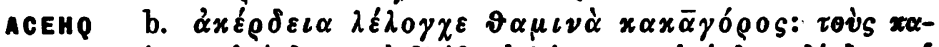

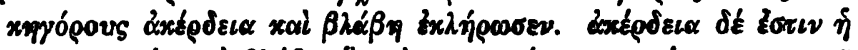
ACDEH

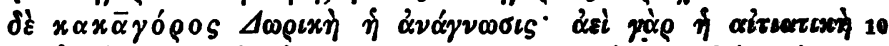

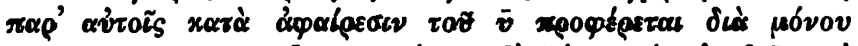

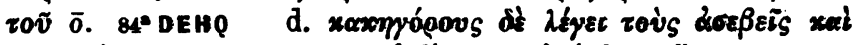

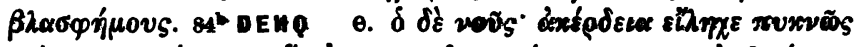

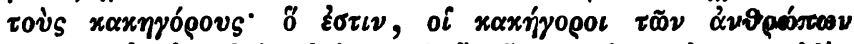

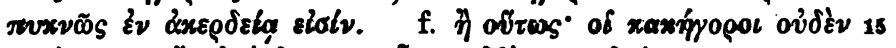

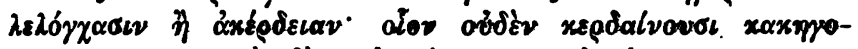

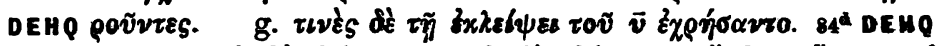

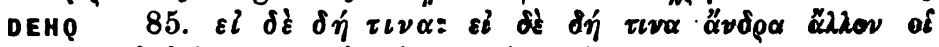

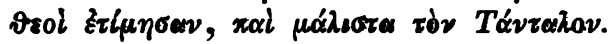

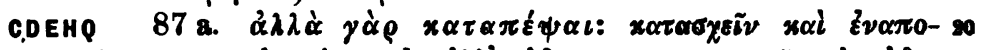

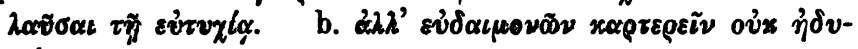

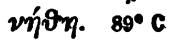

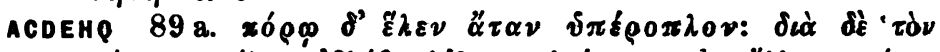

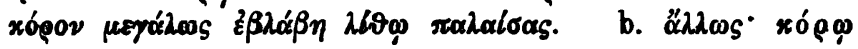

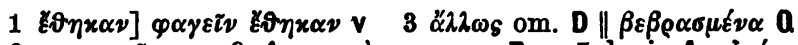

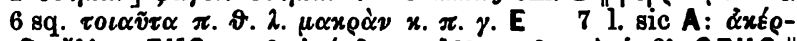

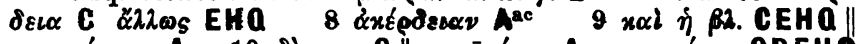

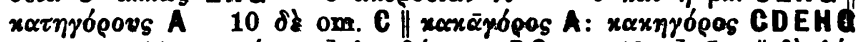

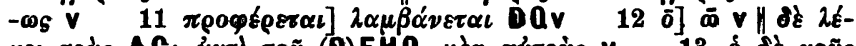

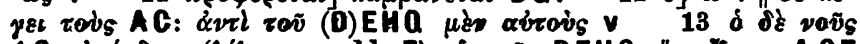

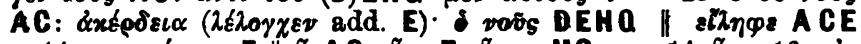

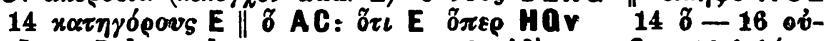

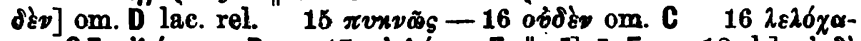

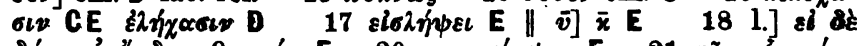

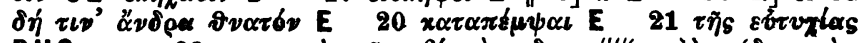

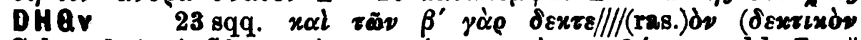

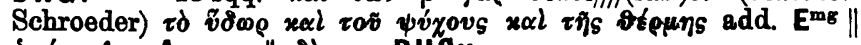
viźporiov A: om. \# de om. DHQV 


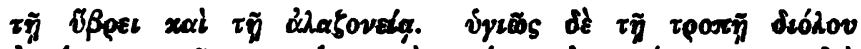

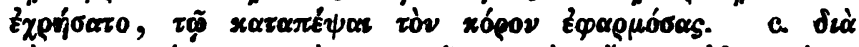

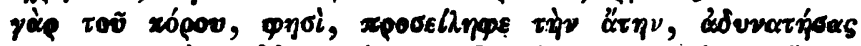

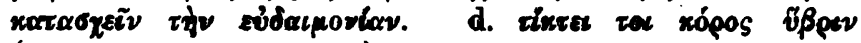
5 (paroem. I, 308. II, 218). $91 \mathrm{C}$

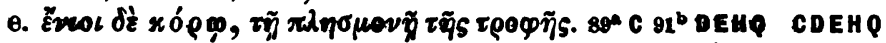

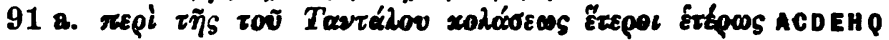

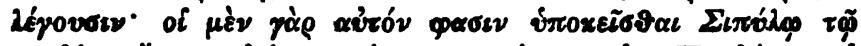

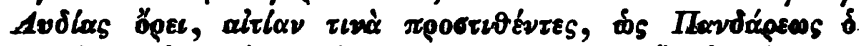

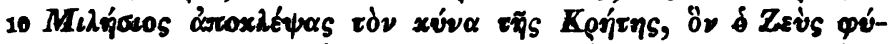

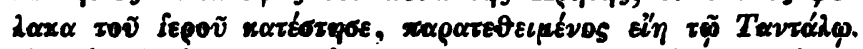

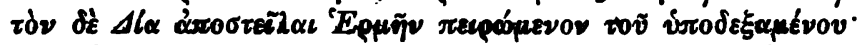

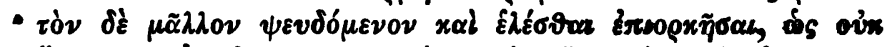

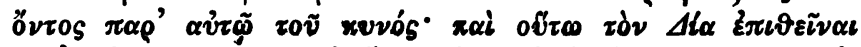

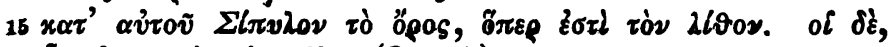

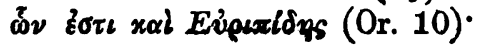

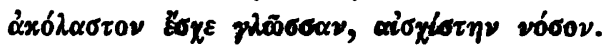

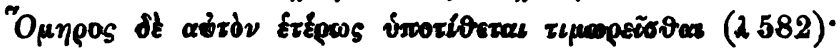

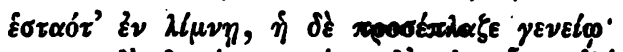

э

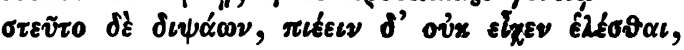

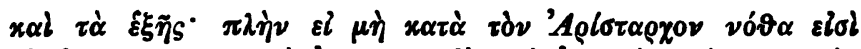

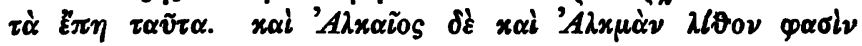

8 sch. $\tau$ 518. $v$ 66. Anton. Lib. $36 \quad 15$ sq. Tzetzes Chil. 5,47021 sch. $\lambda 568$.

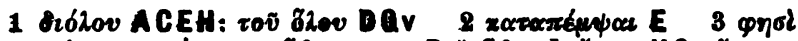

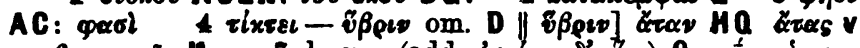

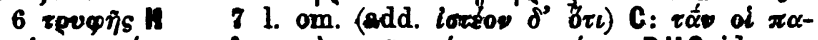

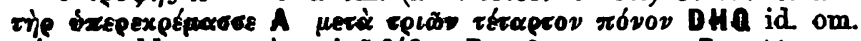

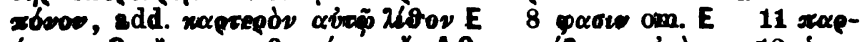

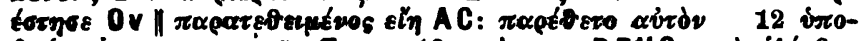

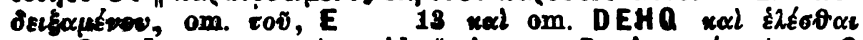

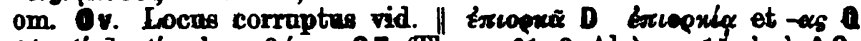

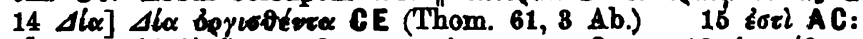

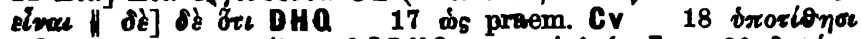

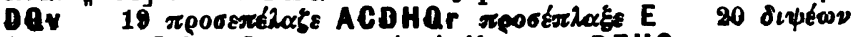

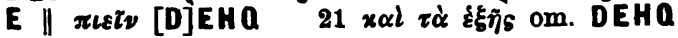




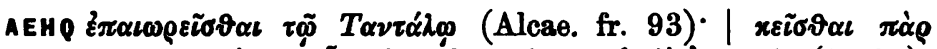

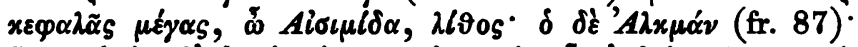

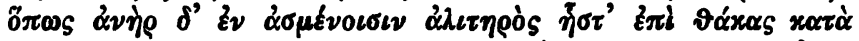

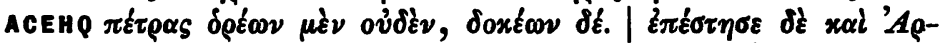

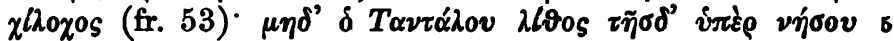

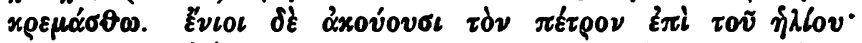

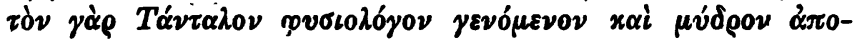
ต

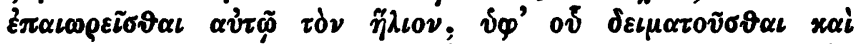

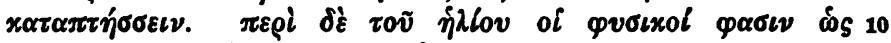

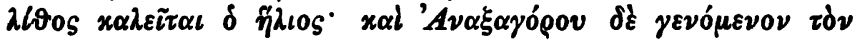

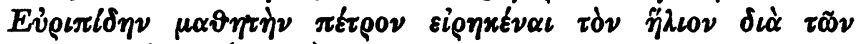

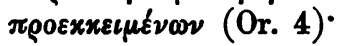

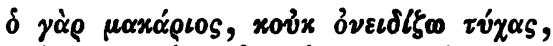

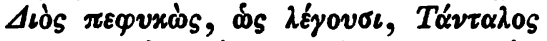

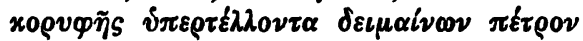

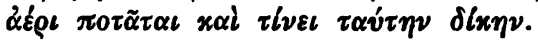

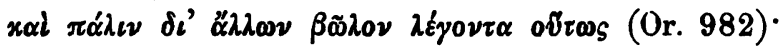

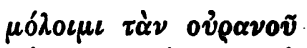

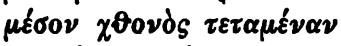

80

$\alpha i \omega \rho \eta \eta_{\mu \alpha \sigma \iota} \pi \dot{\varepsilon} \tau \rho \alpha \nu$

3 Eust. ad Od. 1701, 236 sch. Eur. Or. 582. Tzetzes Chil. 6, 458-62. 478-82. Eust. ad Od. 1700, 60

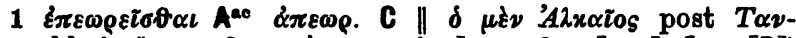

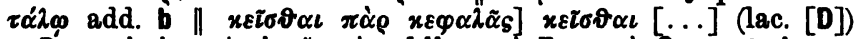
$\tilde{\sigma} \mathrm{D}^{\prime}$, omissis ceteris \| $\pi \dot{\alpha} \rho$ AH: $\pi \varepsilon \rho l$ E $\pi \alpha \rho \dot{\alpha} \alpha$

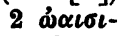

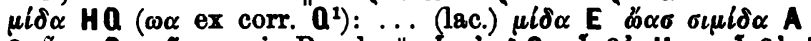
$3 \delta \pi \eta$ Q

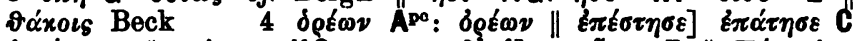

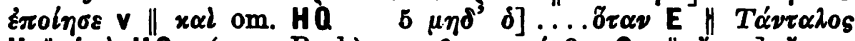

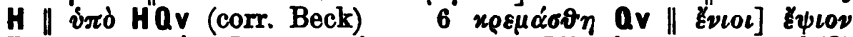

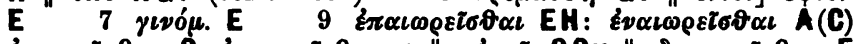

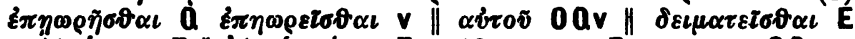

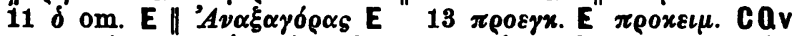

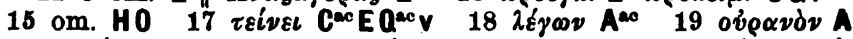

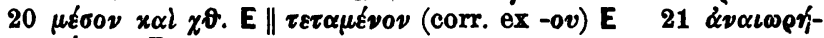
$\mu \alpha \sigma \iota \pi \dot{r} \rho \circ \nu \mathrm{E}$ 


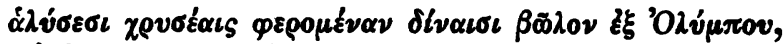

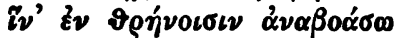

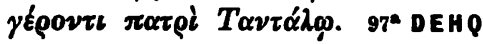

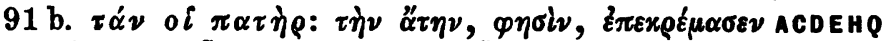

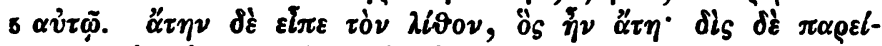

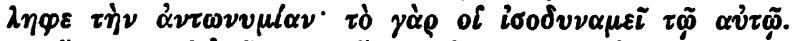

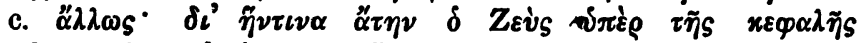

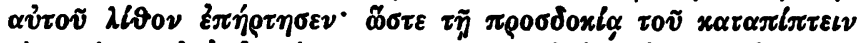

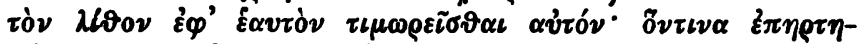

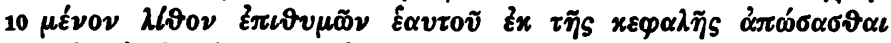

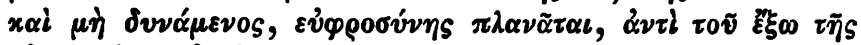

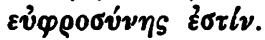

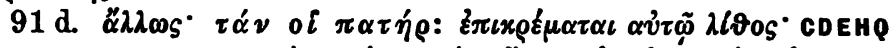

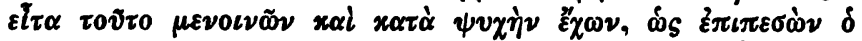

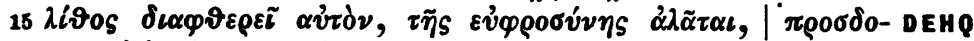

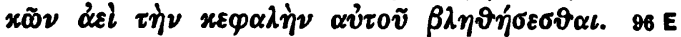

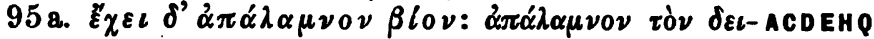

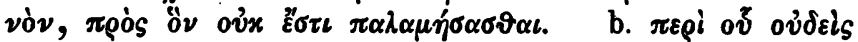

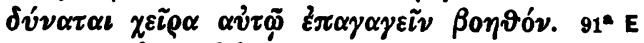

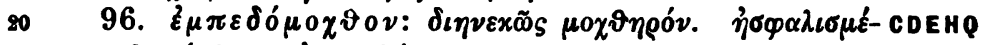

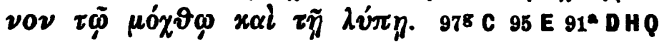

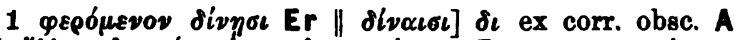

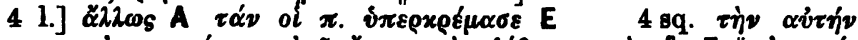

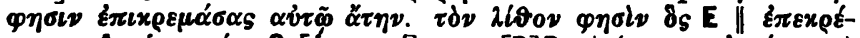

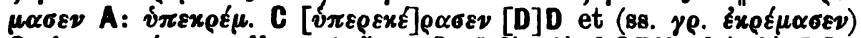
Q

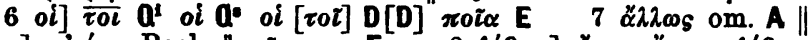

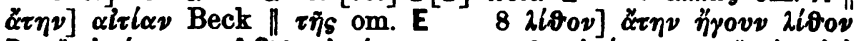

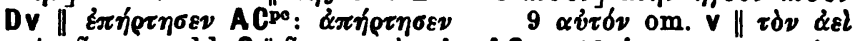

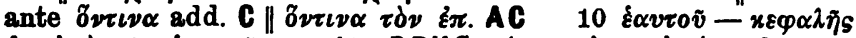

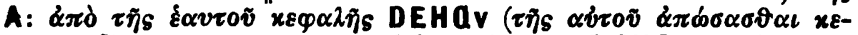

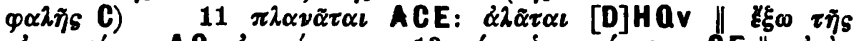

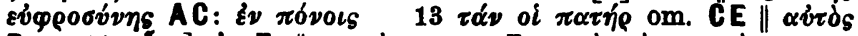

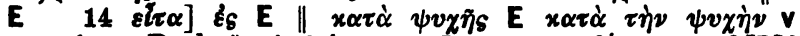

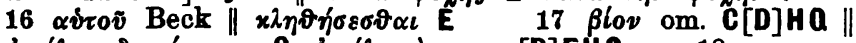

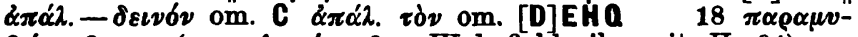

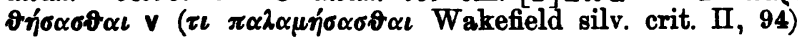

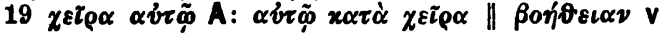




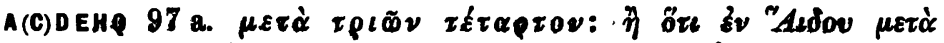

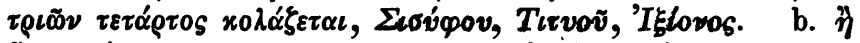

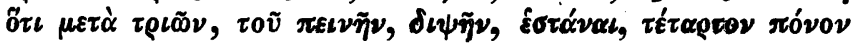

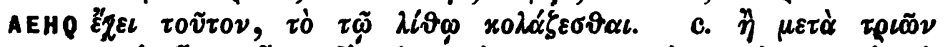

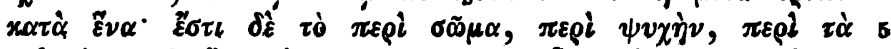

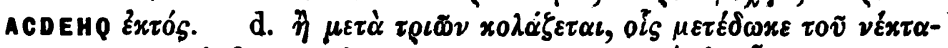

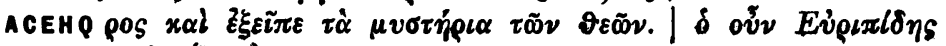
$\varphi \eta \sigma l \nu$ (1. c.)

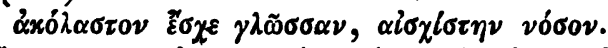

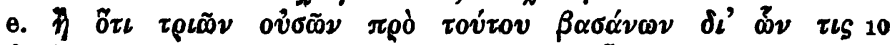

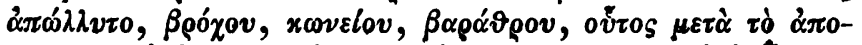

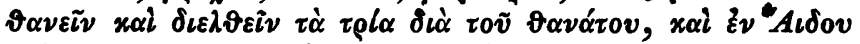

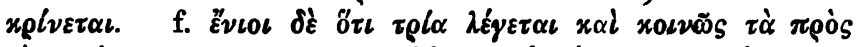

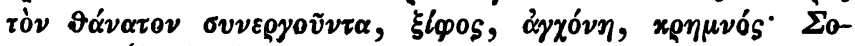

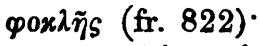

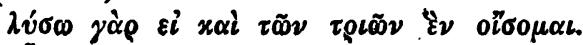

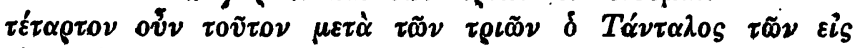

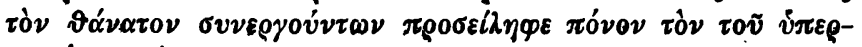

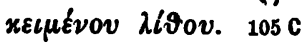

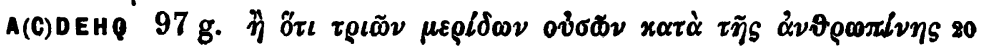

10 et 13 Zenob. 6,11. Apostol.16,20. Diogenian. (paroem. II)

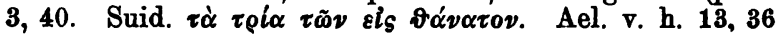

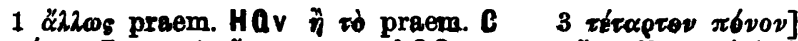

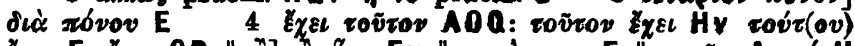

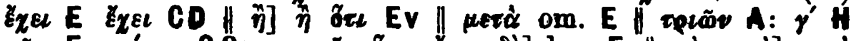

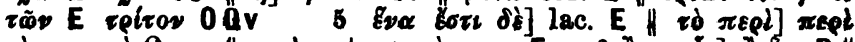

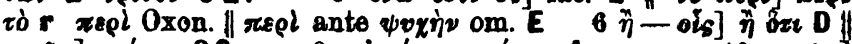

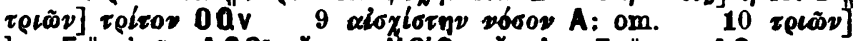

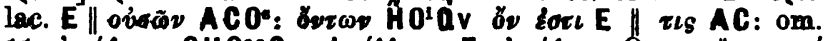

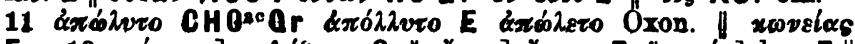

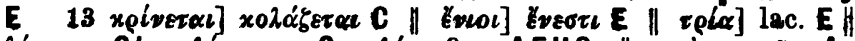

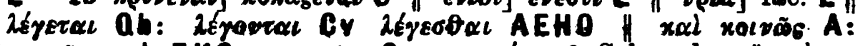

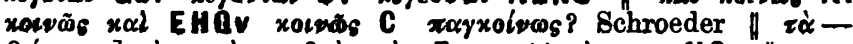

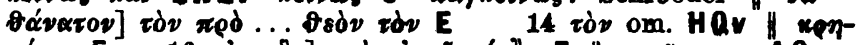

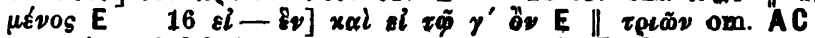

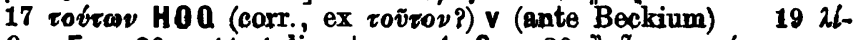

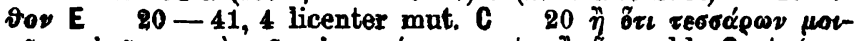

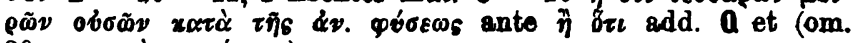
20 sq. $x \alpha \tau \dot{\alpha}-\varphi v ́ \sigma \varepsilon \omega \varsigma) v$ 


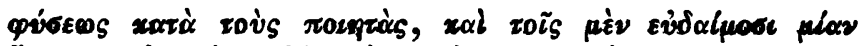

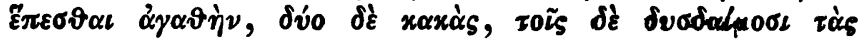

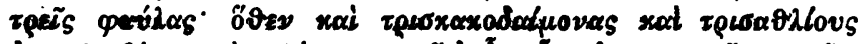

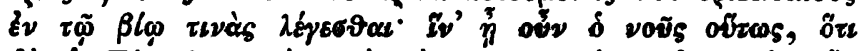

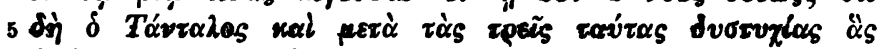

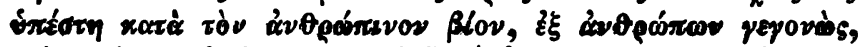

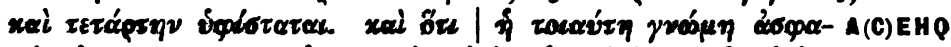

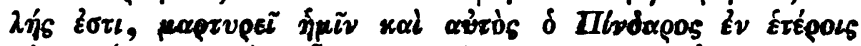

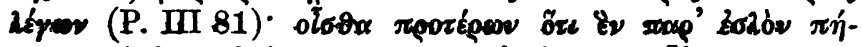

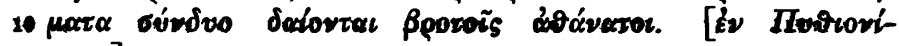

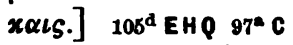

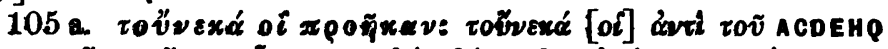

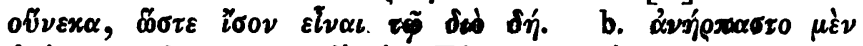

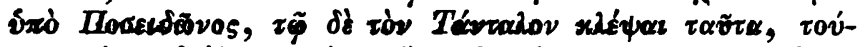

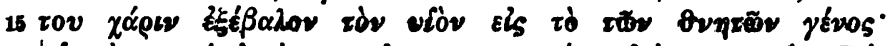

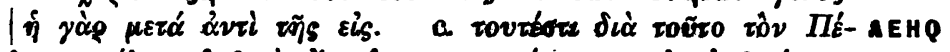

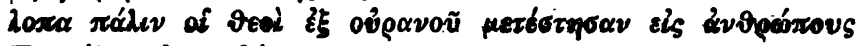

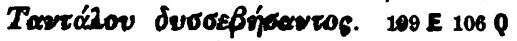

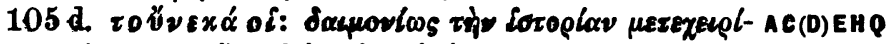

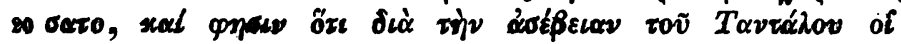

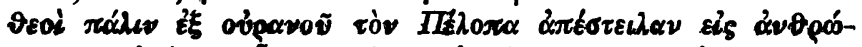

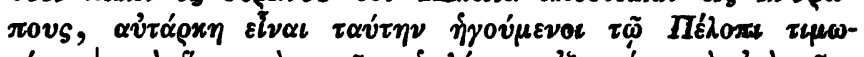

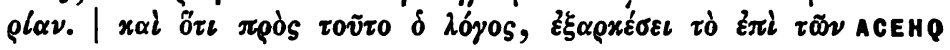

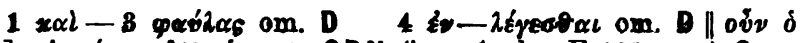
vovic] ơ movov Ace o voṽs CDN (i. e. Ambr. E 108 sup.) 0

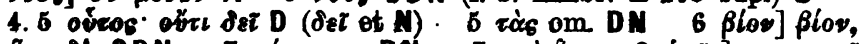

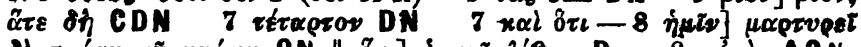

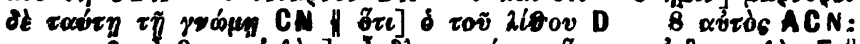

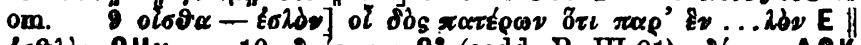

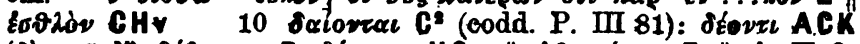

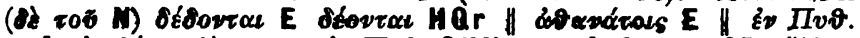

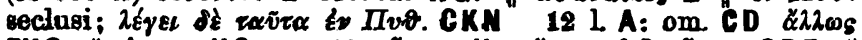

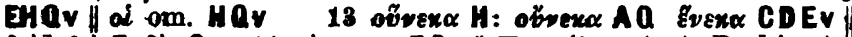

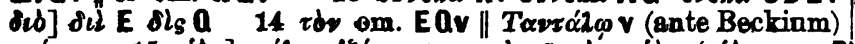

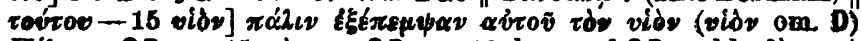
$\Pi \xi \lambda_{0 \pi \alpha} \mathrm{CD} \quad 16$ tb om. CD $19 \mathrm{l}$. om. ACD, add. $\delta \xi$ post

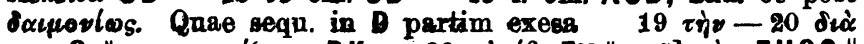

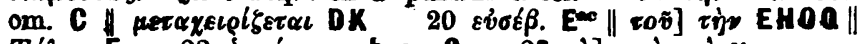
Tádiov E 22 ท่ 


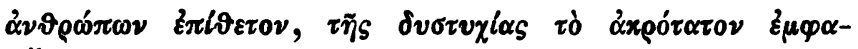

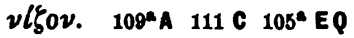

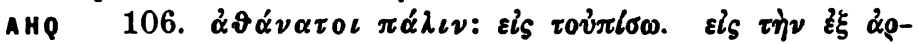

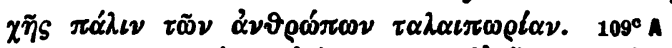

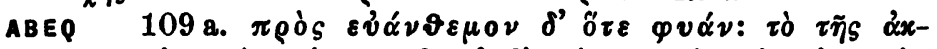
6

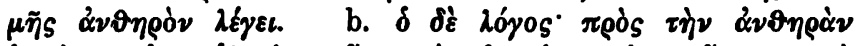

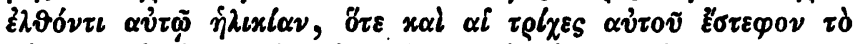

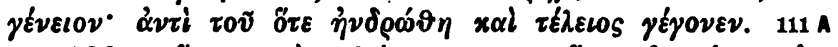

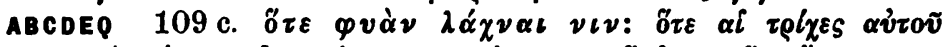

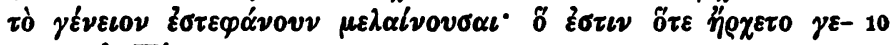
$\nu \varepsilon\left\llcorner\tilde{\alpha} \nu \delta \Pi \hbar \lambda_{0 \psi} \psi 112\right.$ A $113 \mathrm{C}$

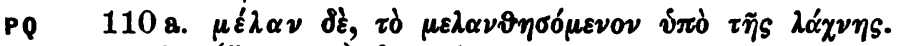

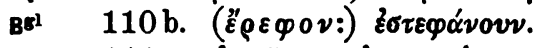

ABCDEQ 111 a. É $\tau$ ¿

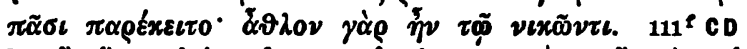

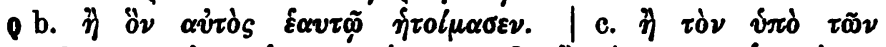

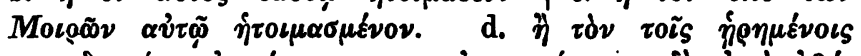

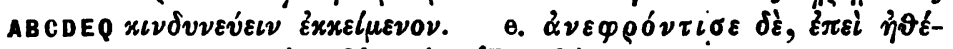

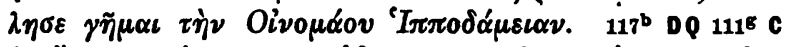

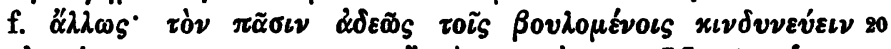

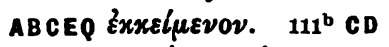

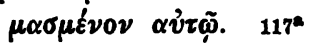

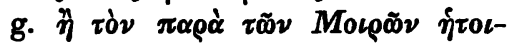

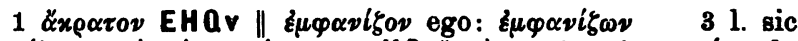

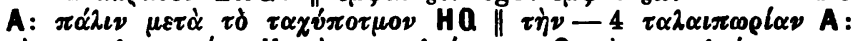

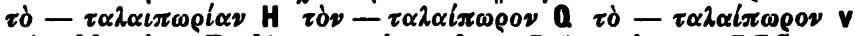
(et add. yḱvos Beck) 5 inc. f. 11 B $\| \varphi v \alpha^{\prime} \nu$ om. BEd

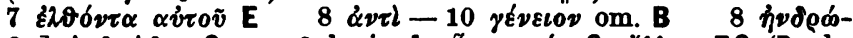

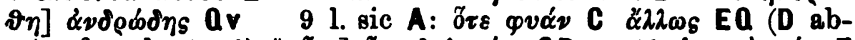

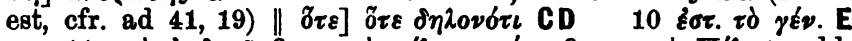

$11 x \alpha i<v \delta \rho \circ \tilde{\sigma} \sigma \theta \alpha \iota x \alpha i$ z

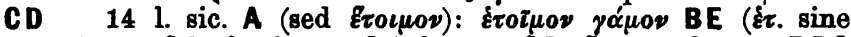

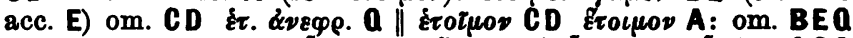

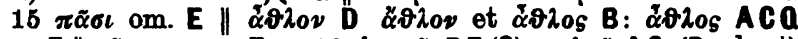

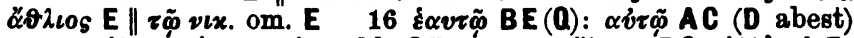

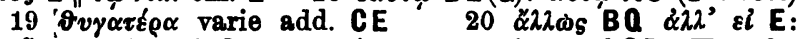

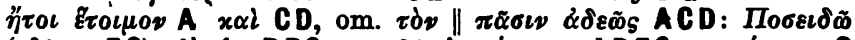

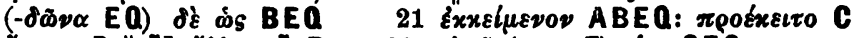

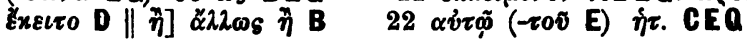




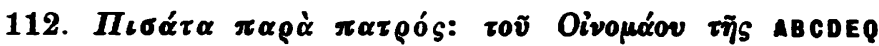

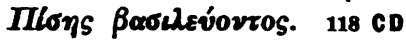

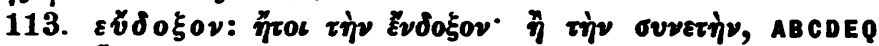

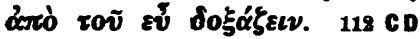

114. द̇

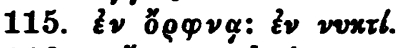

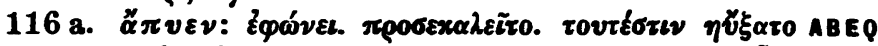

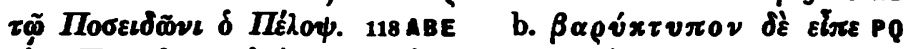

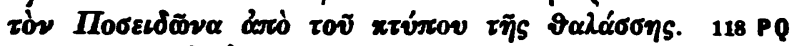

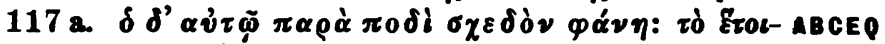

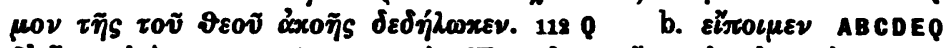

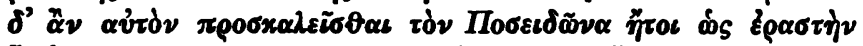

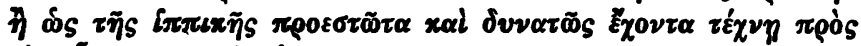

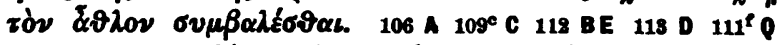

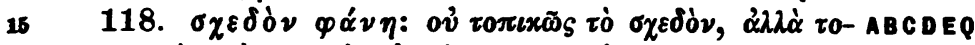

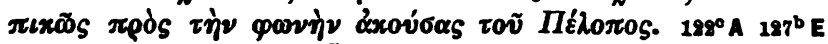

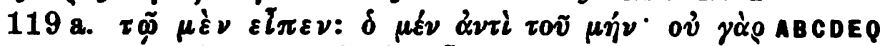

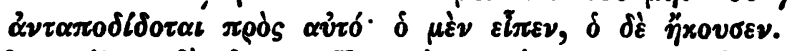

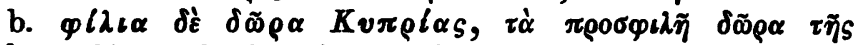

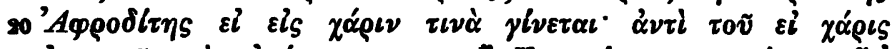

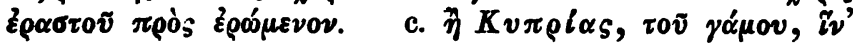

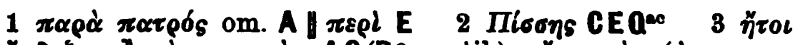

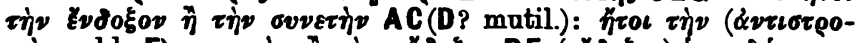

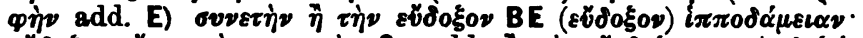

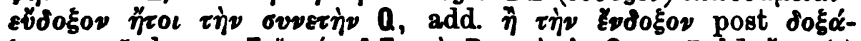

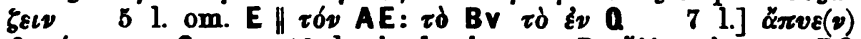

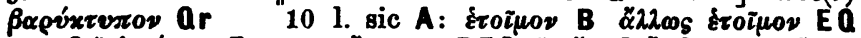

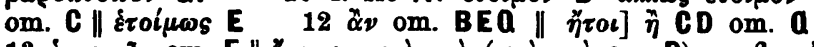

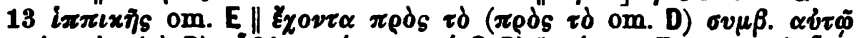

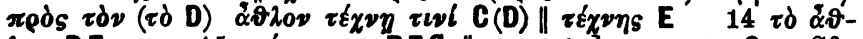

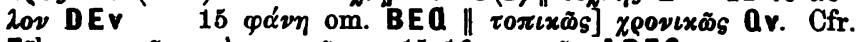

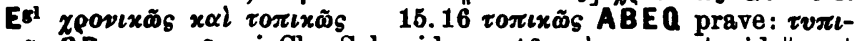

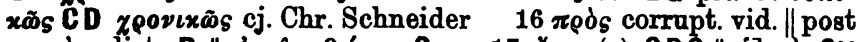

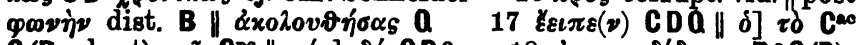
Q (D abest) $\left.\tau \tilde{\varphi} C^{p c} \| \mu \eta^{\prime}\right]$ ] $\delta \xi$ CD ? $18 \alpha v \tau \alpha \pi 0 \delta l \delta o \tau \alpha \iota$ B ? (D):

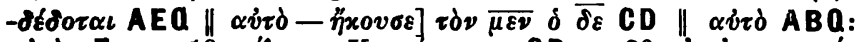

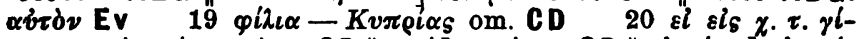

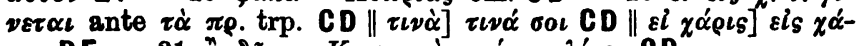

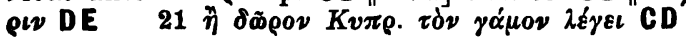




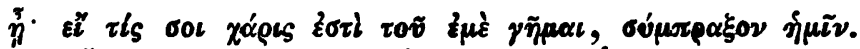

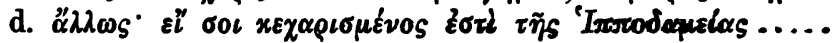

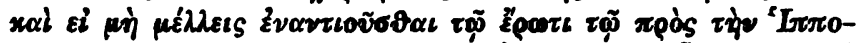

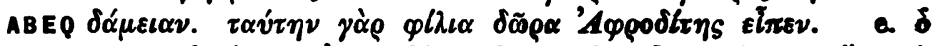

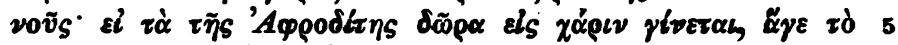

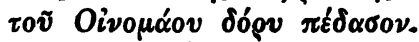

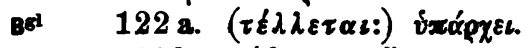

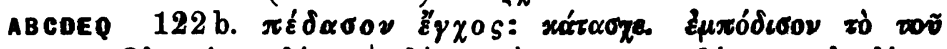

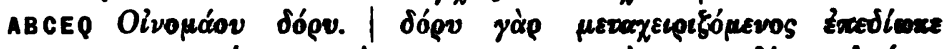

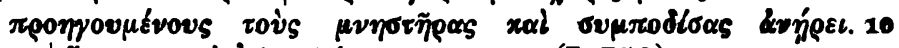

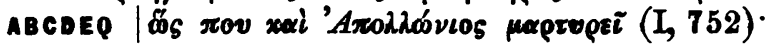

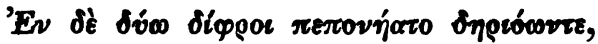

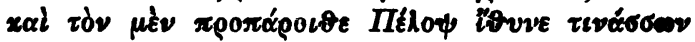

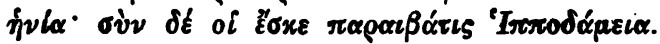

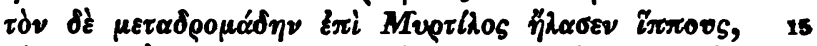

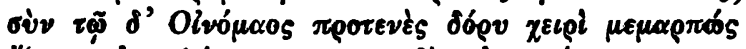

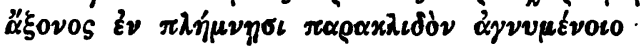

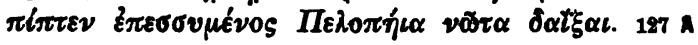

11-18 Tzetzes ad Lyc. 156

1 el $\tau \iota s$ ABDQ: $\varepsilon i \tau \imath$ Cv $\tilde{y} \tau \iota$ E

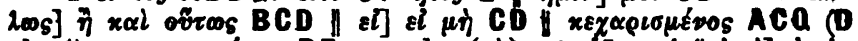

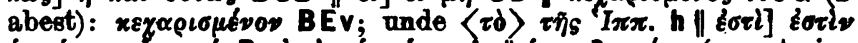

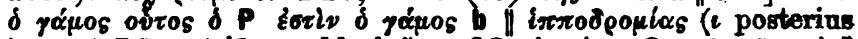

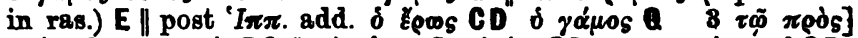

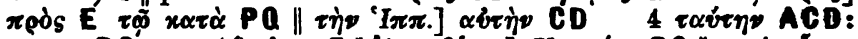

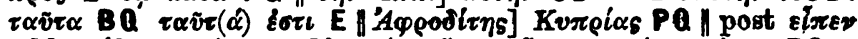

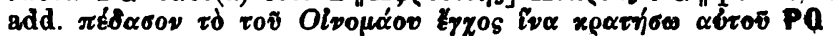

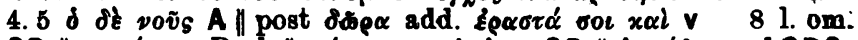

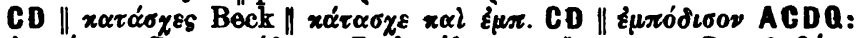

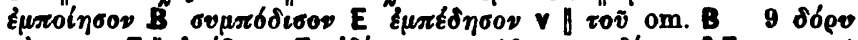

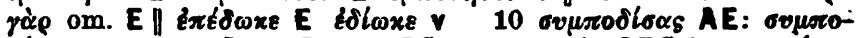
$\partial l \xi \omega \nu 11 \pi 0 v] \pi \alpha$ D $\pi \varepsilon \rho$ EQV 12 dv́o CEQ \| $\pi \varepsilon \pi 0 v \varepsilon i \alpha \tau 0$

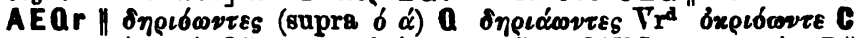

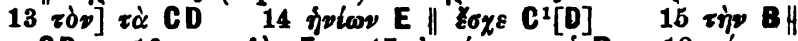

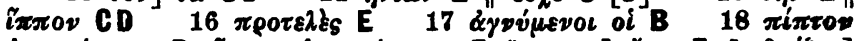

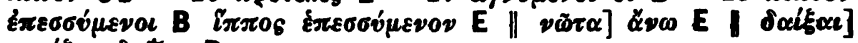
$\tau \iota \nu \alpha{ }^{\prime} \xi \iota \delta \alpha \operatorname{t\zeta } \varepsilon \iota$ B 


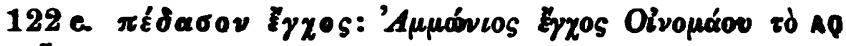
\&ppax קpoovev. 119 A

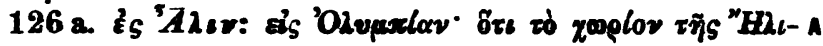
dog. 155

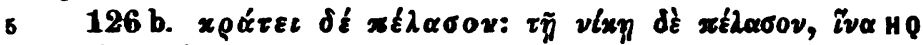

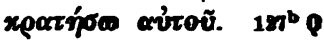

127 a.

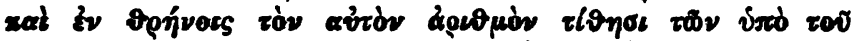

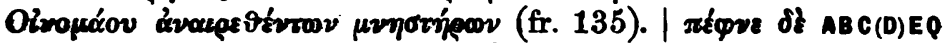

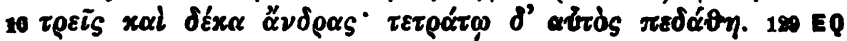

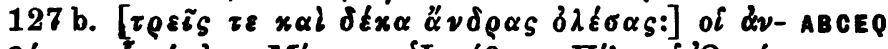

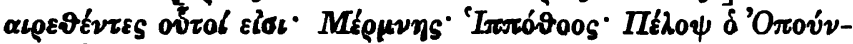

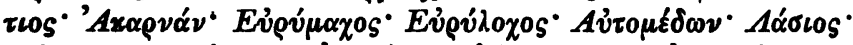

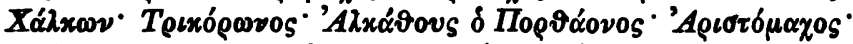

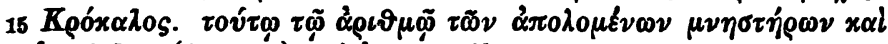

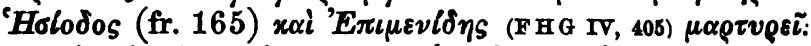

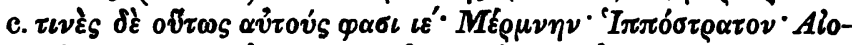

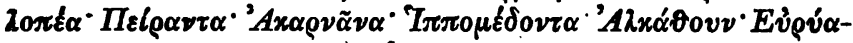

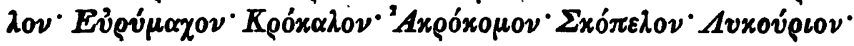

11-p. 46, 6 Paus. 6, 21, 10. 11 (Hes. fr. 165)

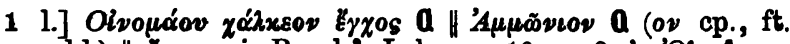
postea add.) \|| Evvos cj. Bergk ${ }^{2}$, Lehrs p. $10 \quad 3 \varepsilon_{6}{ }^{\prime} O \lambda_{1}$. A

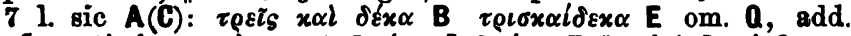

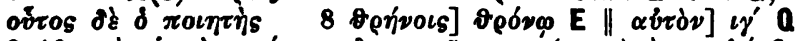

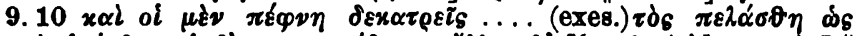

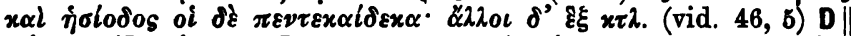

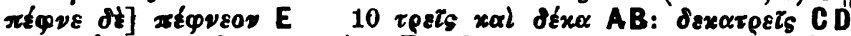

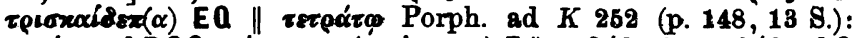

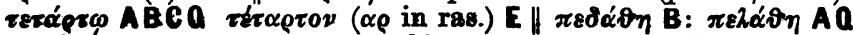

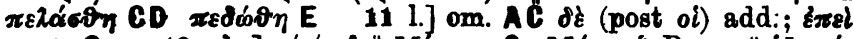

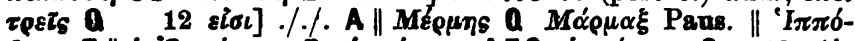

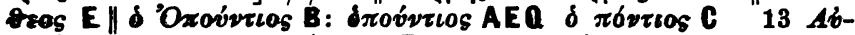

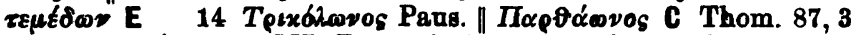

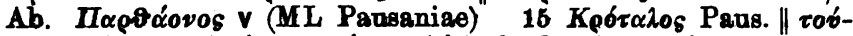

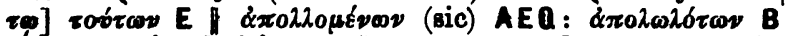

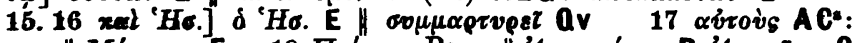

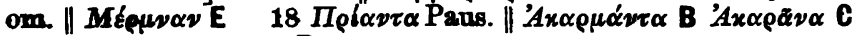
19 Avxoṽejor Paus. 


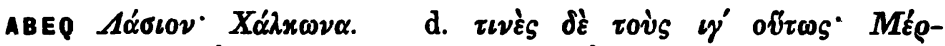

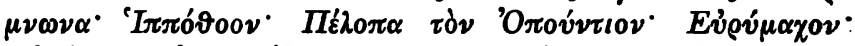

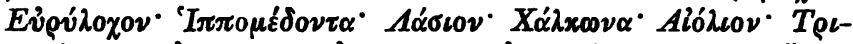
А В (D) Е

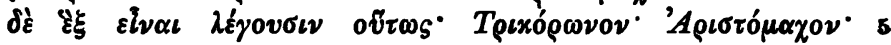

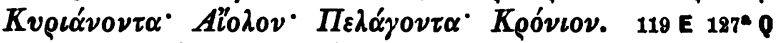

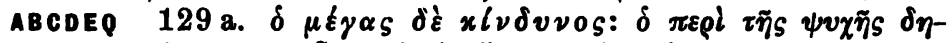

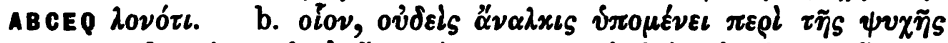

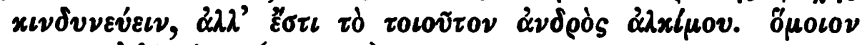

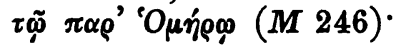

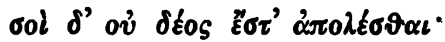

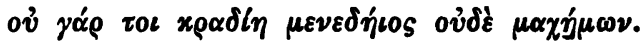

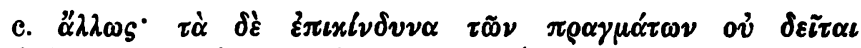

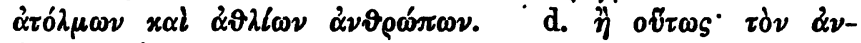

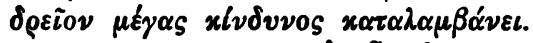

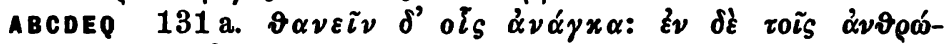

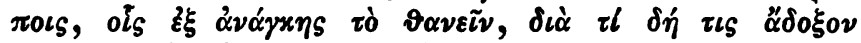

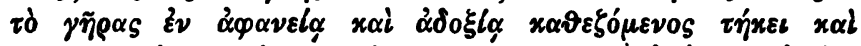

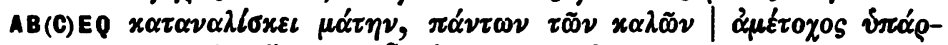

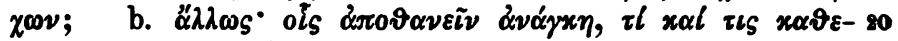

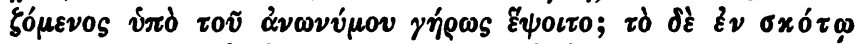

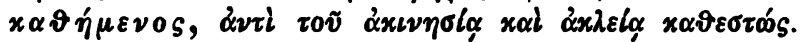

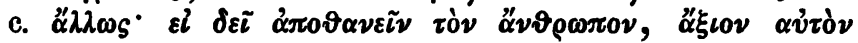

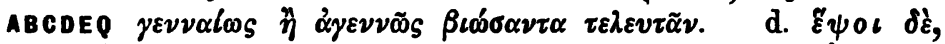

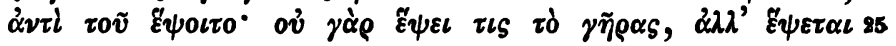

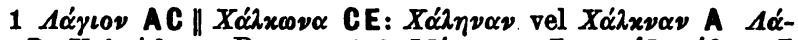

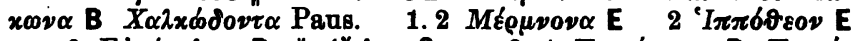

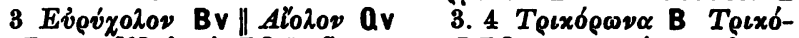

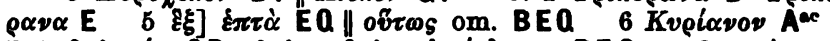

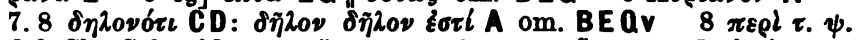

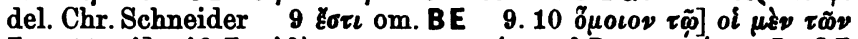

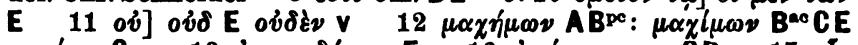

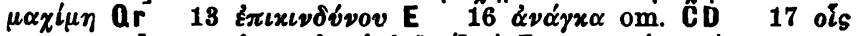

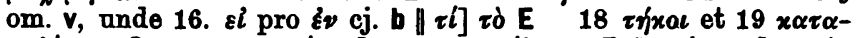

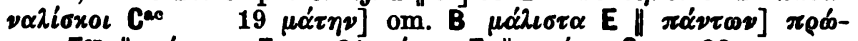

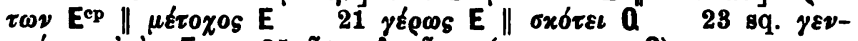

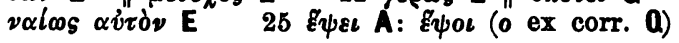




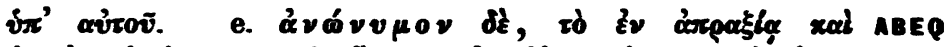

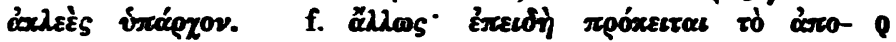

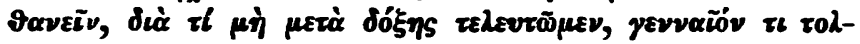

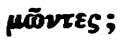

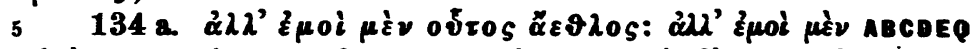

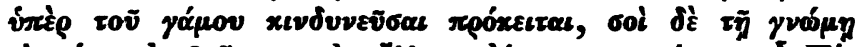

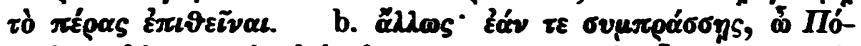

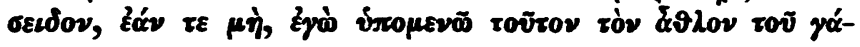
Hov. 137 A

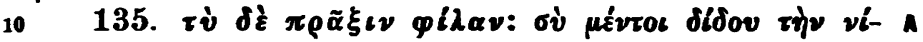

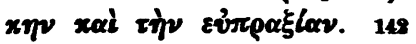

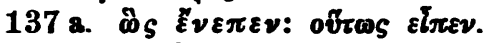

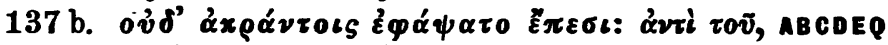

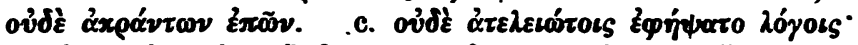

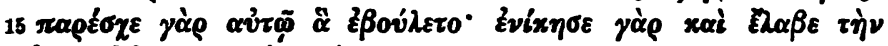

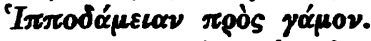

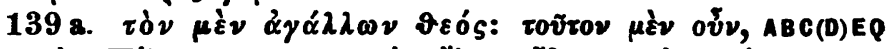
$\varphi \eta \sigma \zeta$, тòv $\Pi \varepsilon z^{2}$

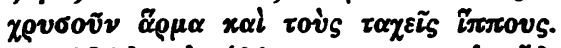

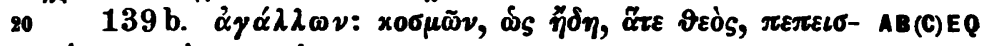

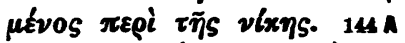

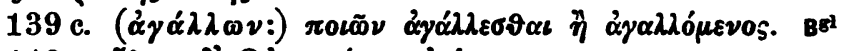

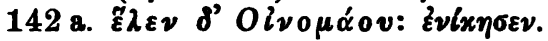

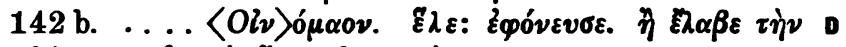

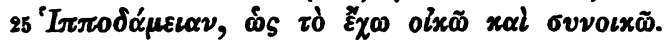

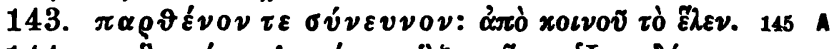

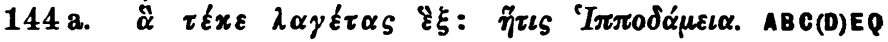

5 1. om. Q $\| \alpha \lambda \lambda^{\prime}$ (alt.) om. BEv $5.6 \varphi \eta \sigma \iota$ varie add. CD $6 \tau \tilde{\eta}$

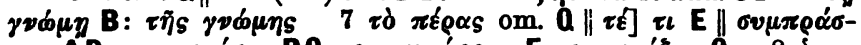

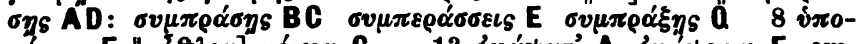

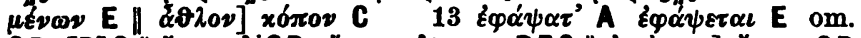

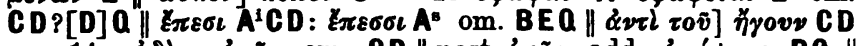

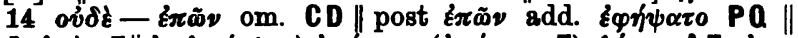

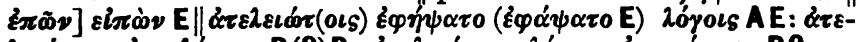

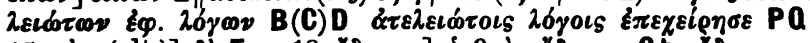

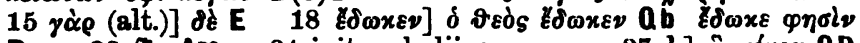
Bv 23 Eq Apc 24 init. scholii exesum 27 l.] $\hat{\alpha} \tau \xi x \in v$ CD

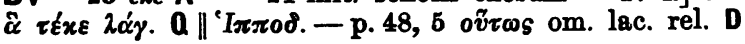




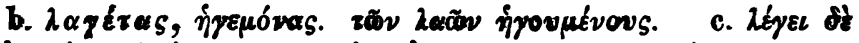

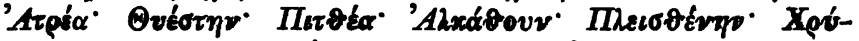

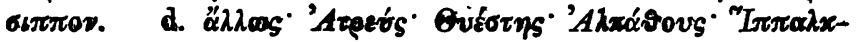

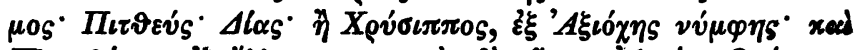

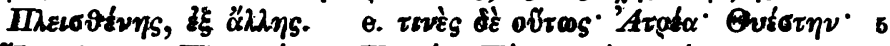
"Inscodixpov' II

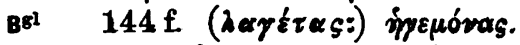

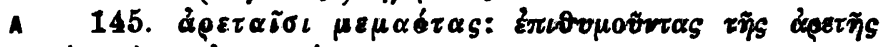

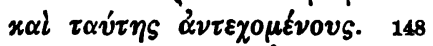

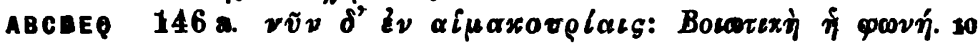

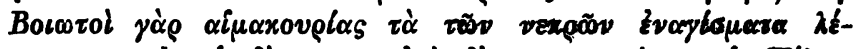

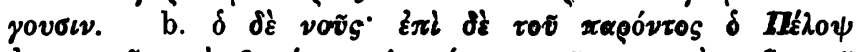

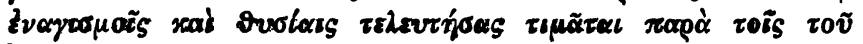

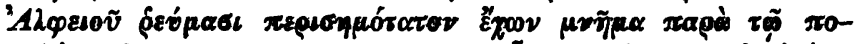

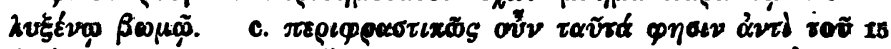

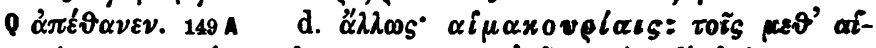

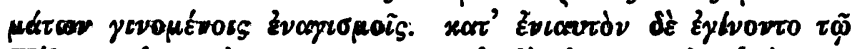

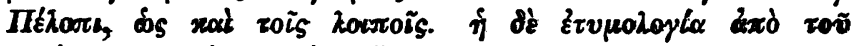

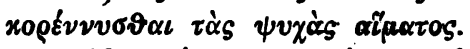

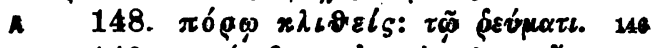

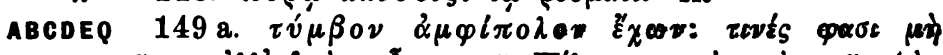

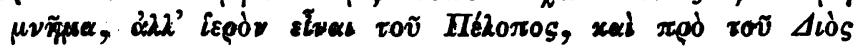

1-6 seh. Eur. Or. 5. Tzetzes exeg. Il. p. 68 sq- mant.

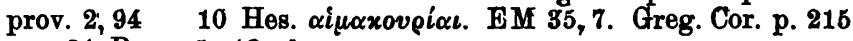
21 Paus. 5, 13, 1 sq.

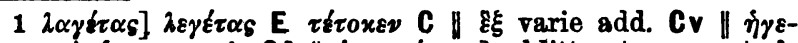

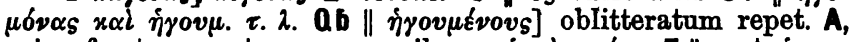
prius frustra conatus supra scribere; $\dot{\eta} \gamma \dot{\alpha} \rho, \mu \sigma v \eta \mathrm{E} \|$ post $\eta_{\gamma o v-.}$

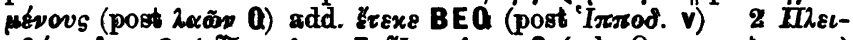

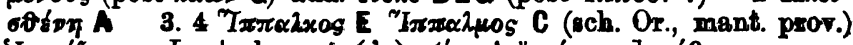

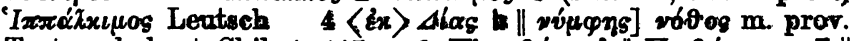

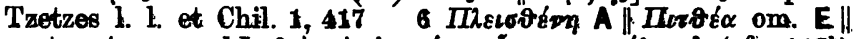

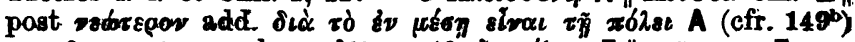

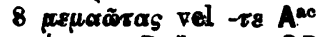

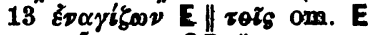

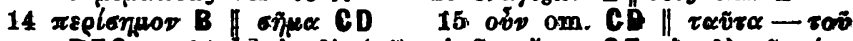

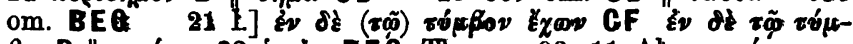

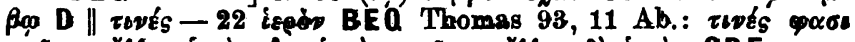

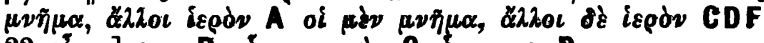

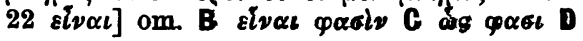




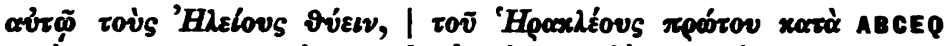

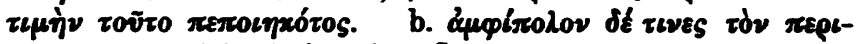

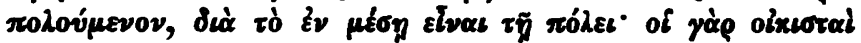

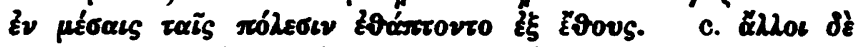

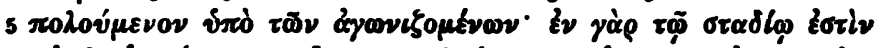

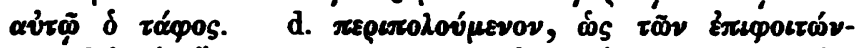

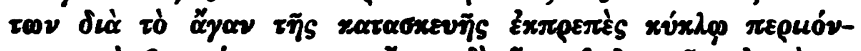

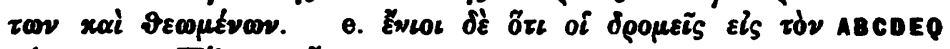

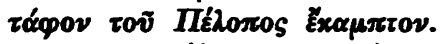

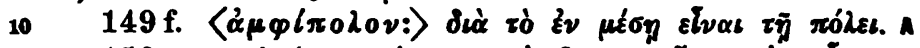

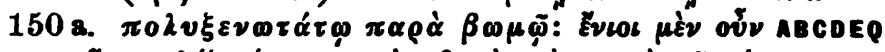

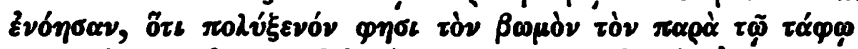

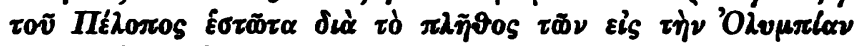

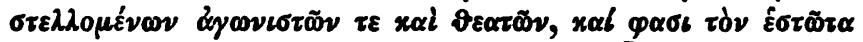

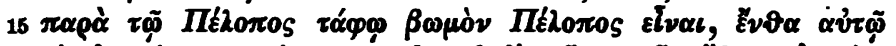

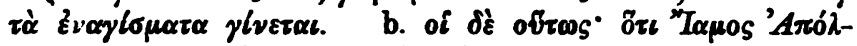

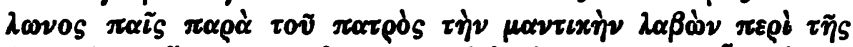

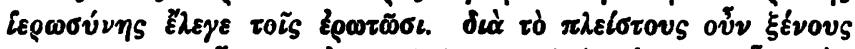

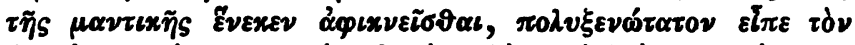

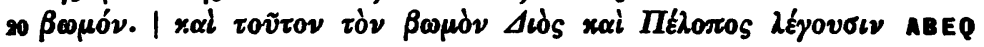

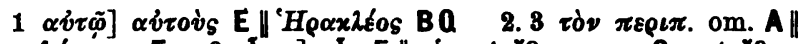

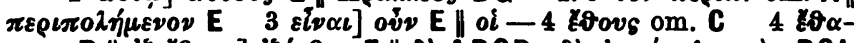

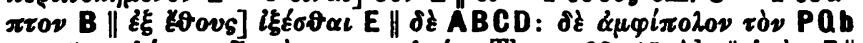

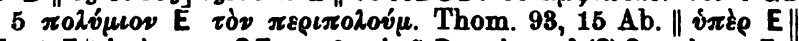

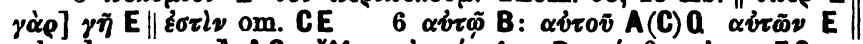

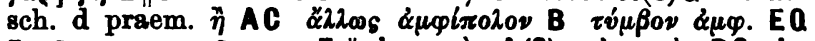

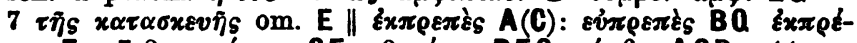

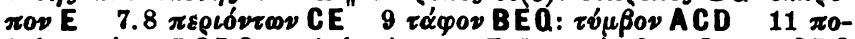

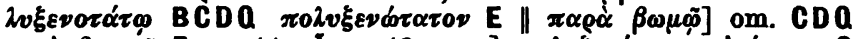

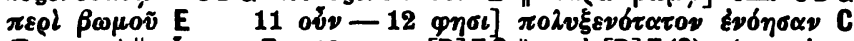

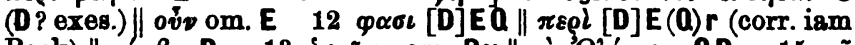

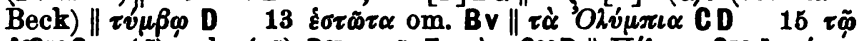

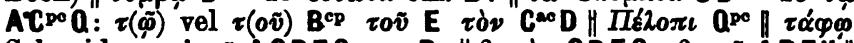
Schneider: $\alpha \dot{v} \tau \sigma \tilde{v}$ ACDEQ om. BV $\| \beta \omega \mu \delta \nu$ CDEQ: $\beta \omega \mu \tilde{\omega}$ ABFK\|. $\Pi \dot{2} 20 \pi 0 s$ AC(E

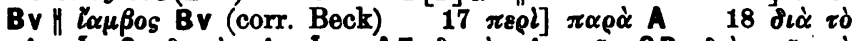

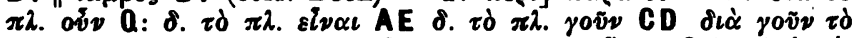

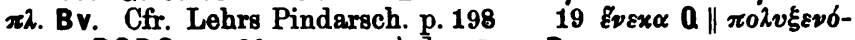

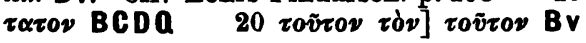

Bchoz. ix Pridarux ed. Drachmann. 


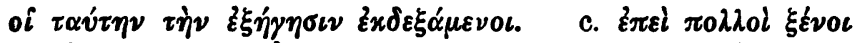

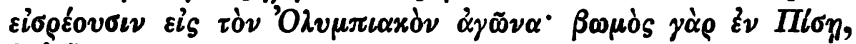

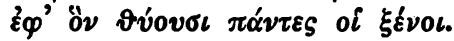

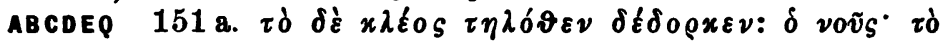

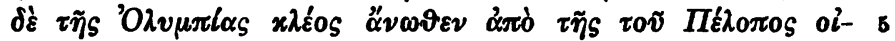

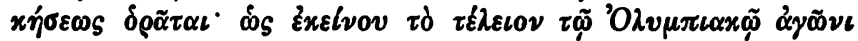

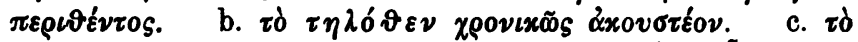

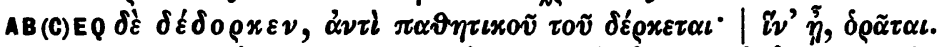

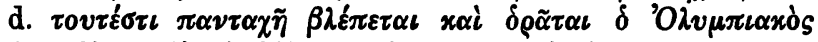

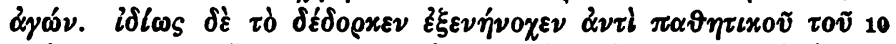

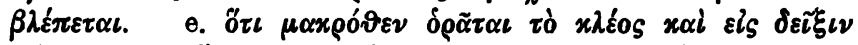

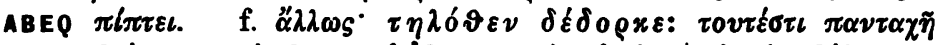

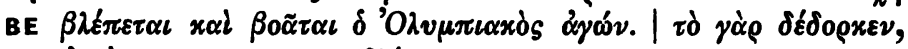

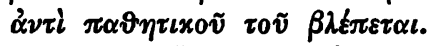

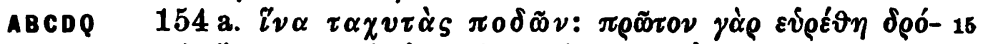

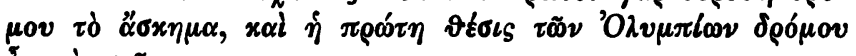

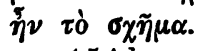

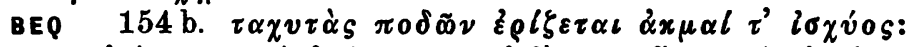

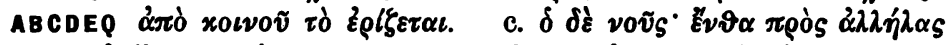

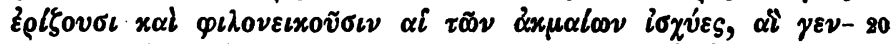

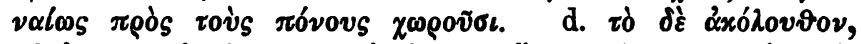

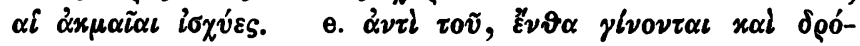

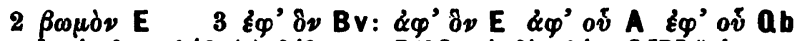

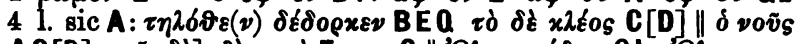

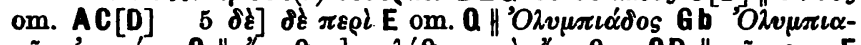

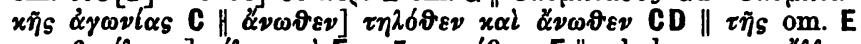

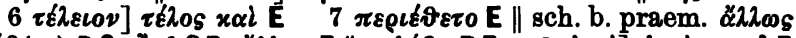

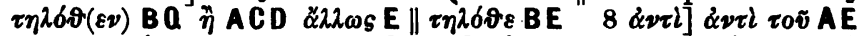

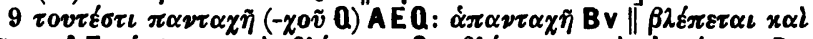

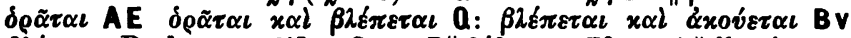

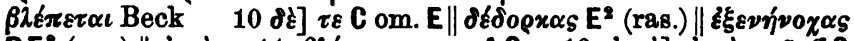

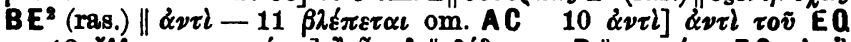

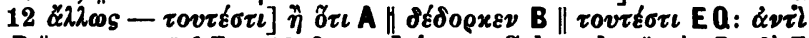

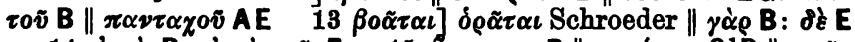

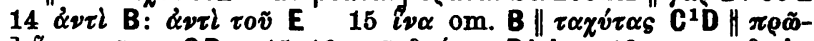

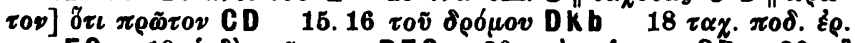

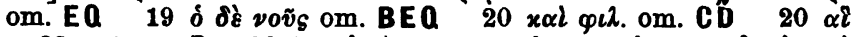

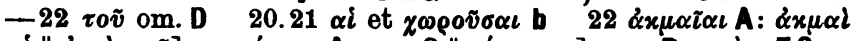

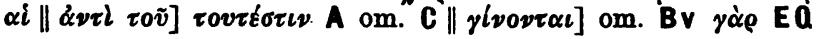




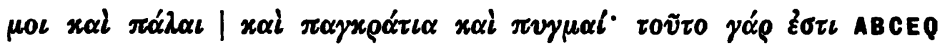

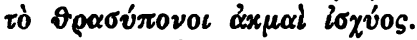

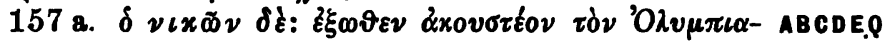

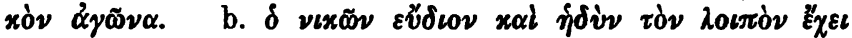

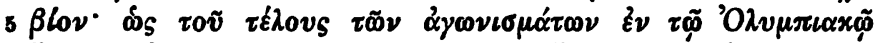

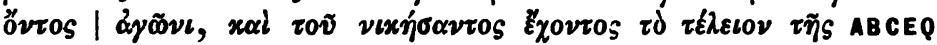

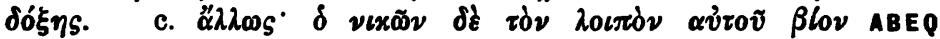

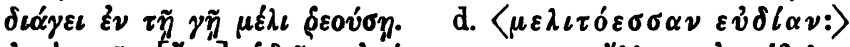

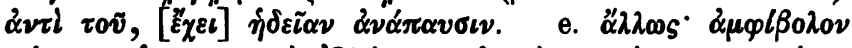

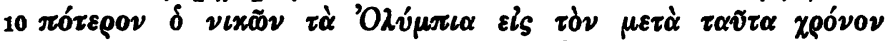

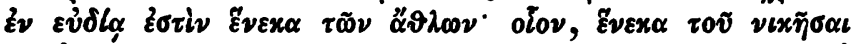

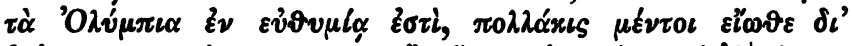

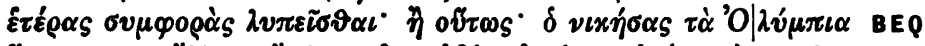

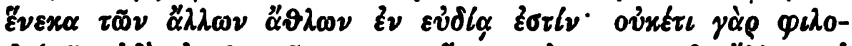

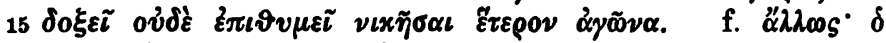

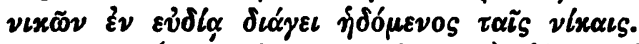

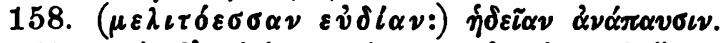
Bel

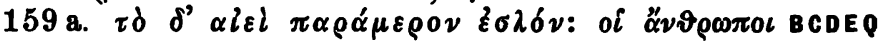

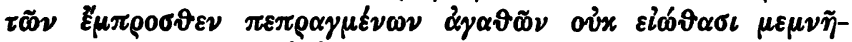

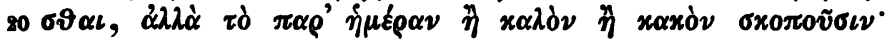

8 Exod. 3, 8.

$\left.1 \pi \alpha^{\prime} \lambda \alpha \iota\right] \pi \alpha \lambda \alpha^{\prime} \tau(\iota \alpha)$ E \| $x \alpha l \quad \pi \alpha \gamma x \rho . \quad x \alpha \ell \quad \pi v \gamma \mu \alpha i$ om. C \|

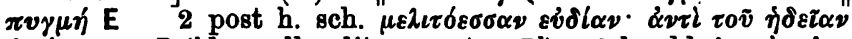

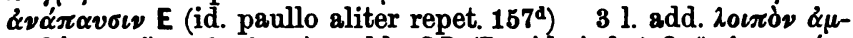

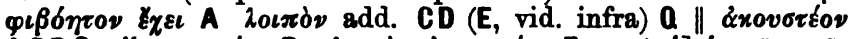

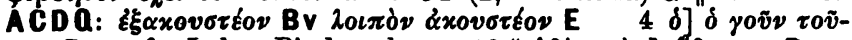

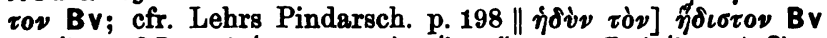

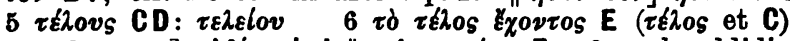

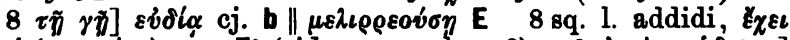
delevi (om. et v) cum E* (vid. supra ad v8. 3) 9 \& $\alpha \nu i-j \delta \varepsilon i \alpha \nu]$

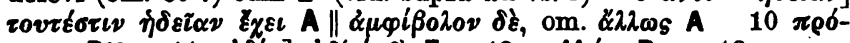

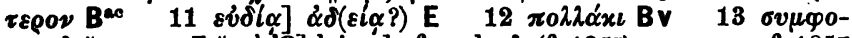

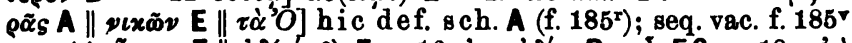

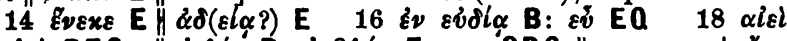

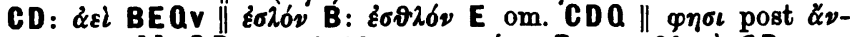

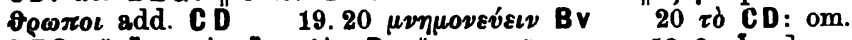

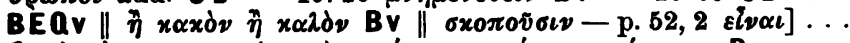

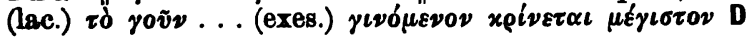




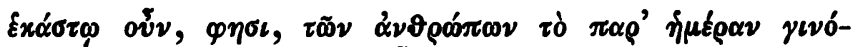

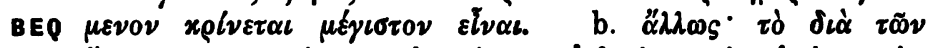

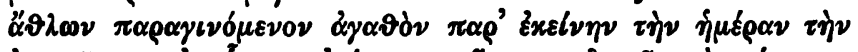

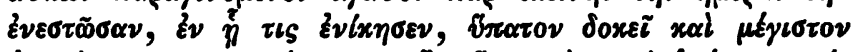

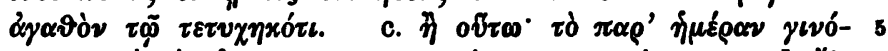

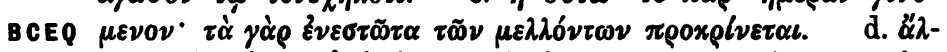

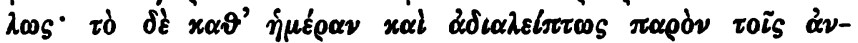

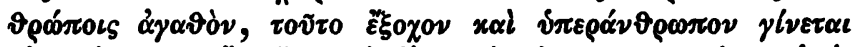

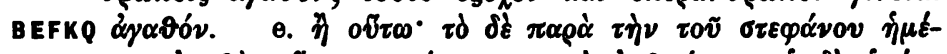

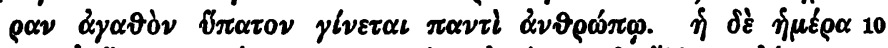

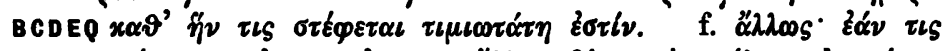

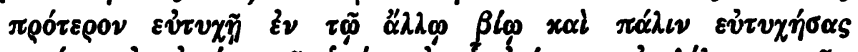

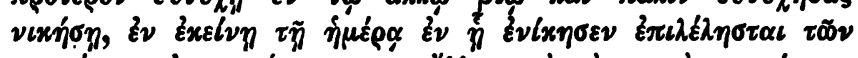

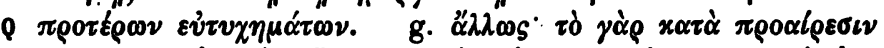

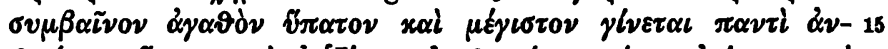

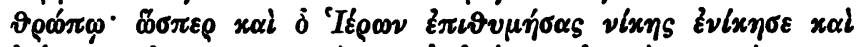

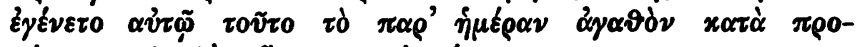

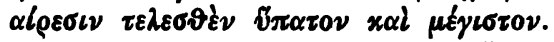

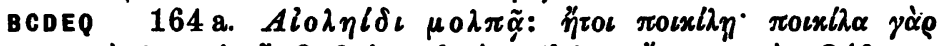

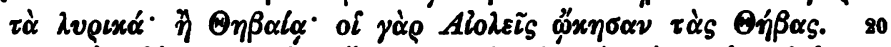

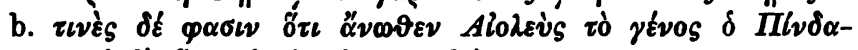

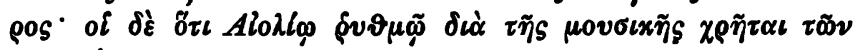

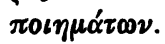

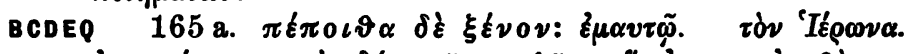

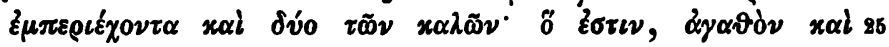

$1 \tau \dot{\alpha}$ et $\gamma \iota \nu \delta \mu \varepsilon \nu \alpha$ E $2 \delta \iota \dot{\alpha}$ scripsi: $\delta \dot{\varepsilon} 3 \pi \varepsilon \rho \iota \gamma \iota \nu . ~ E$

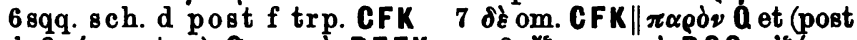

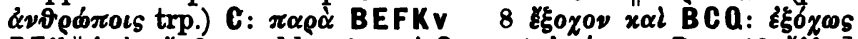

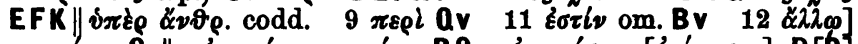

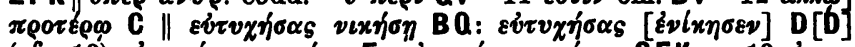

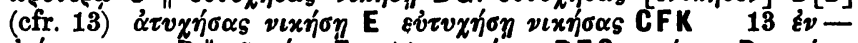

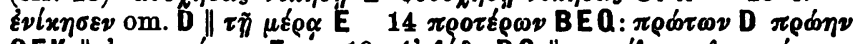

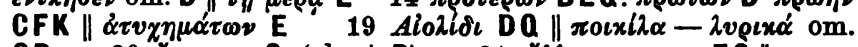

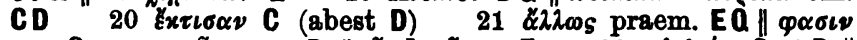

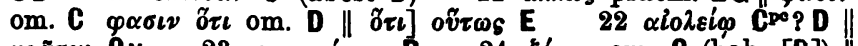

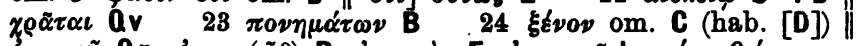

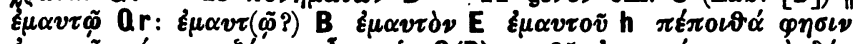

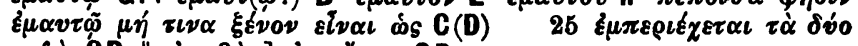

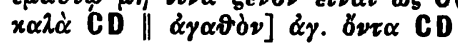




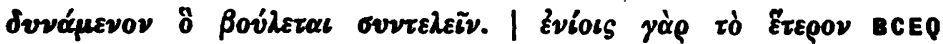

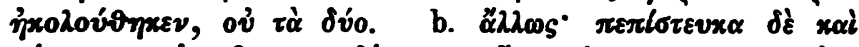

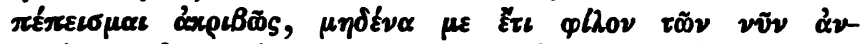

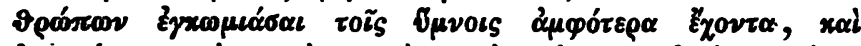

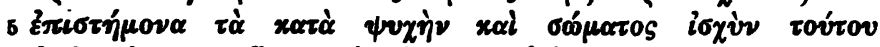

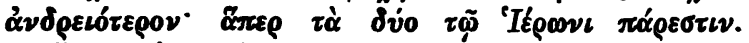

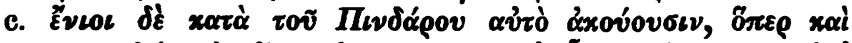

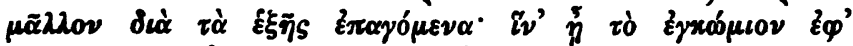

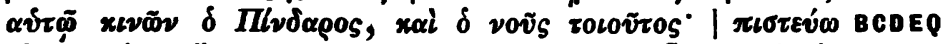

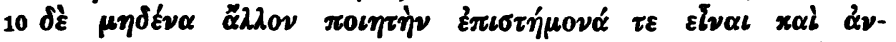

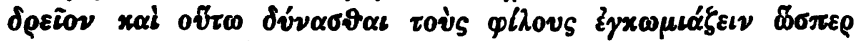

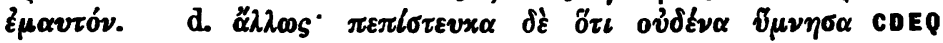

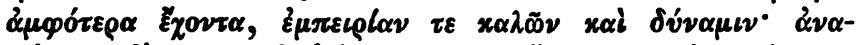

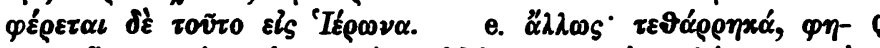

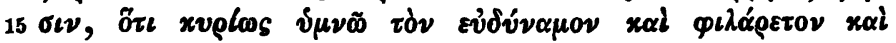

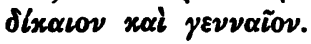

169 a. $\delta \alpha \iota \delta \alpha \lambda \omega \sigma \varepsilon \dot{\varepsilon} \varepsilon \nu v \tilde{y} \nu \omega \nu \pi \tau v \chi \alpha \tilde{\iota}_{S}: x 0 \sigma \mu \eta \dot{\sigma} \sigma \varepsilon \nu$. BCDEQ

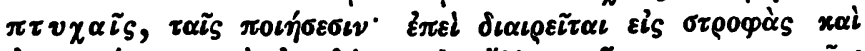

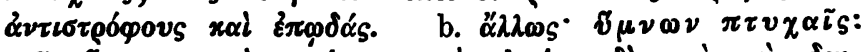

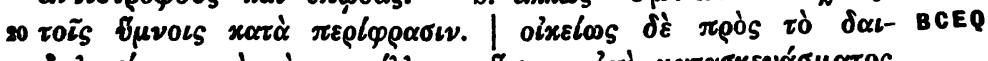

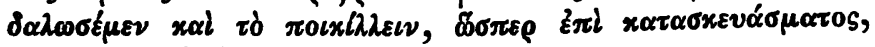

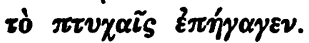

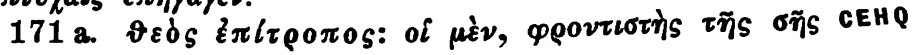

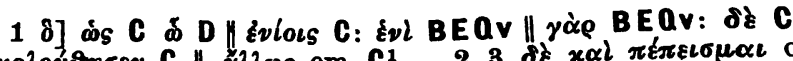
$2 \eta x$ x

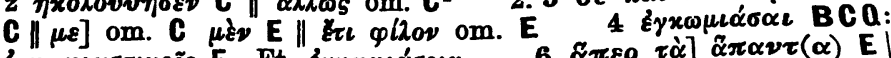

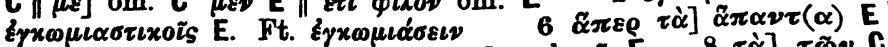

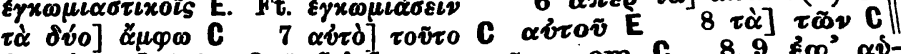

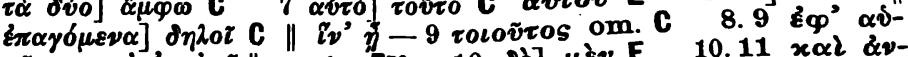

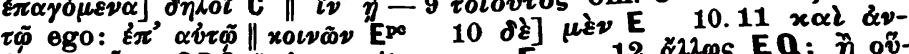

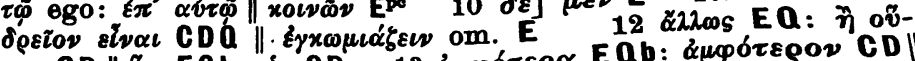

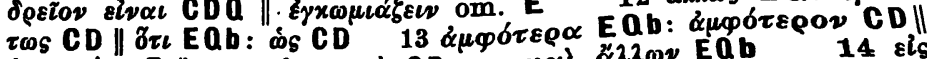

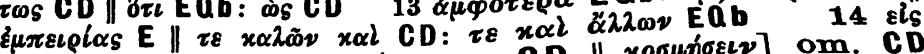

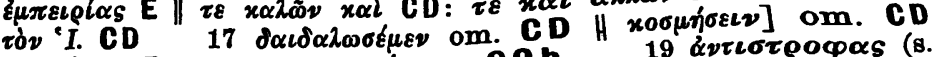

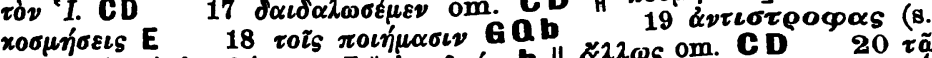

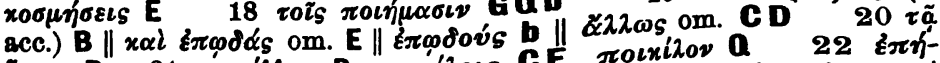

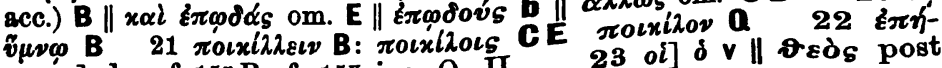

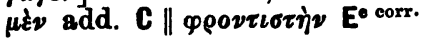




\section{4}

Scholia

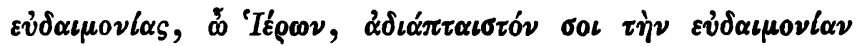

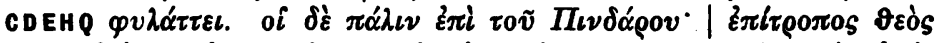

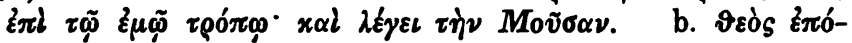

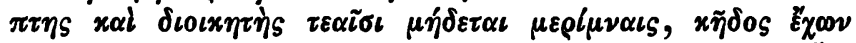

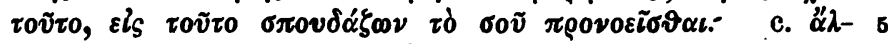

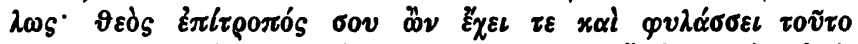

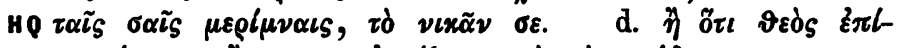

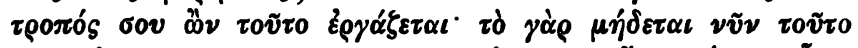

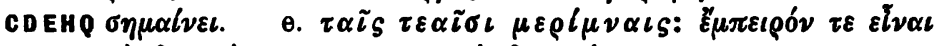

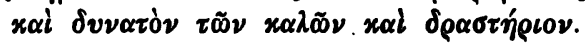

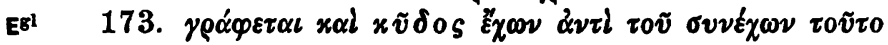
$x \tilde{v} \delta 0 s$.

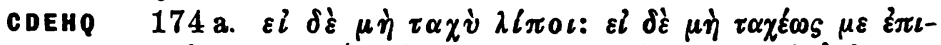

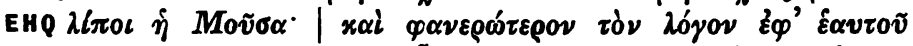

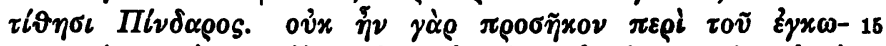

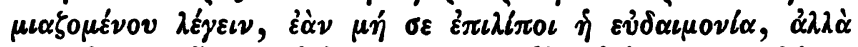

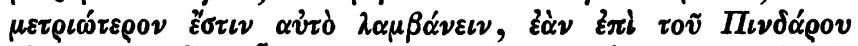

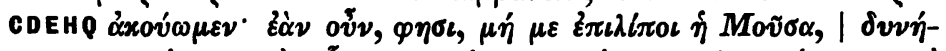

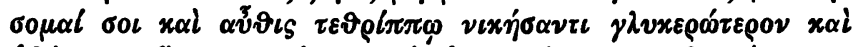

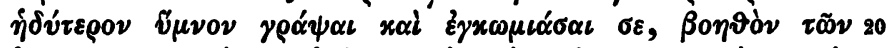

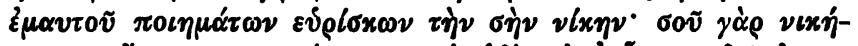

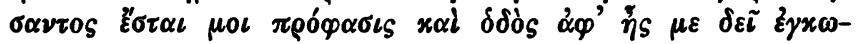
$\mu \iota \alpha ́ \sigma \alpha \iota$.

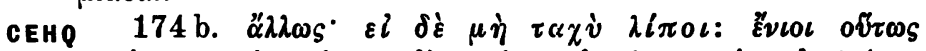

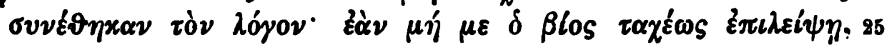

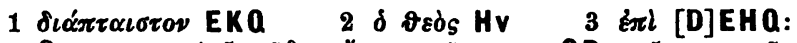

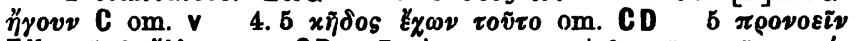

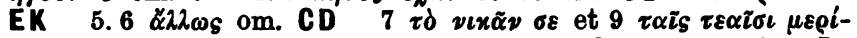
$\mu \nu \alpha \iota s$ om. Ev (haplogr.) $\| \nu \iota x \tilde{\alpha} \nu \sigma \varepsilon] \nu \iota x \tilde{\eta} \sigma \alpha \iota Q^{20}$ ? $\nu \iota x \tilde{\eta}(\sigma \alpha \iota) \sigma \varepsilon \mathrm{D}$ ?

8 vข̃v om. H 9 sq. ad 165 pertinere vid. $9 \tau \alpha t_{5}-$ $\mu \varepsilon \rho i \mu \nu \alpha \iota s$ HQ: om. E (vid. supra) $\grave{\eta}$ C $\grave{\eta} \tau \dot{\partial}$ D $10 \delta v \nu \alpha \tau \dot{\nu}$ ]

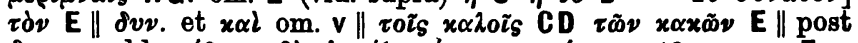

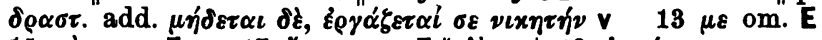

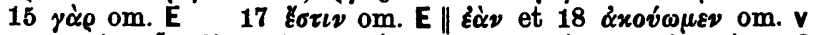

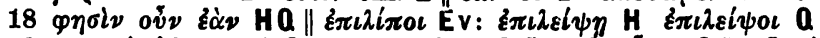

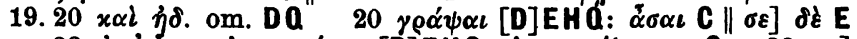

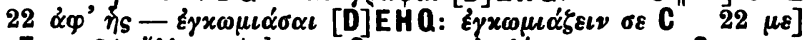

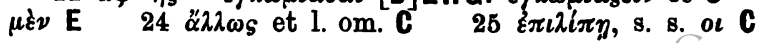




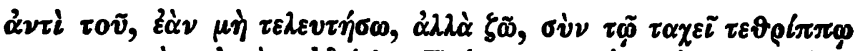

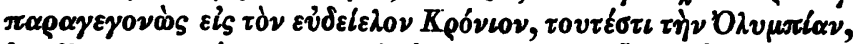

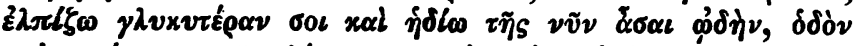

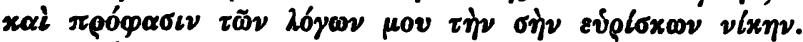

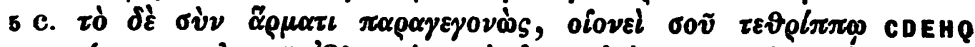

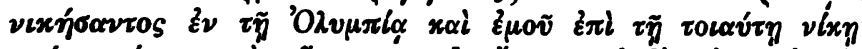

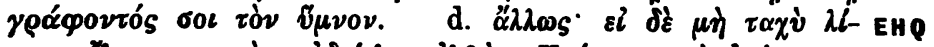

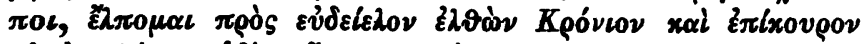

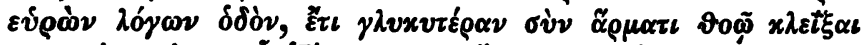

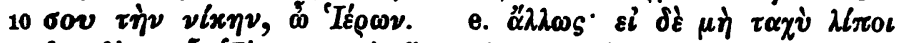

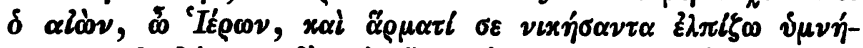

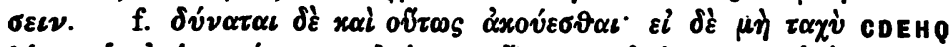

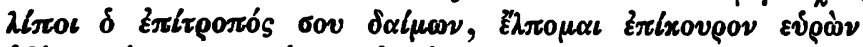

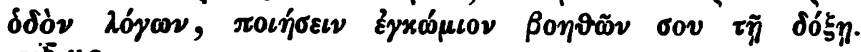
$15179^{-5} \mathrm{HQ}$

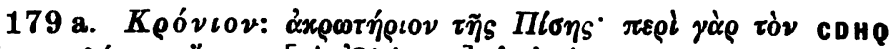

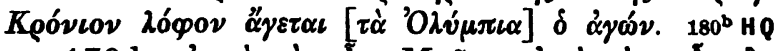

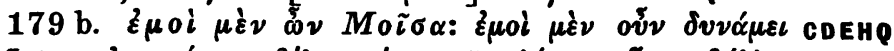

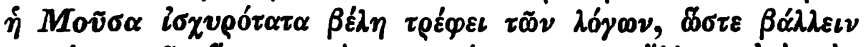

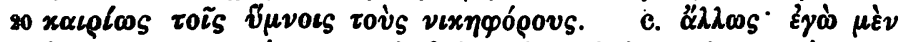

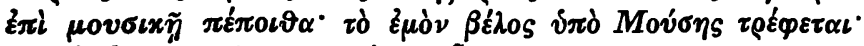

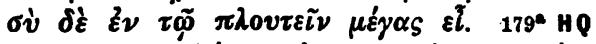

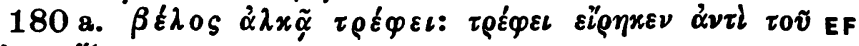
$\dot{\alpha} \lambda x \grave{\eta} \nu \alpha \tilde{v} \xi \varepsilon$.

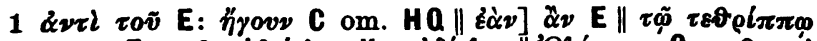

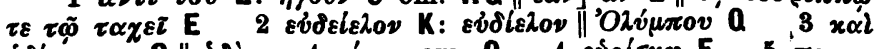

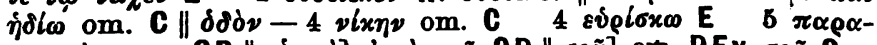

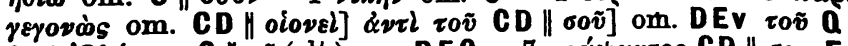

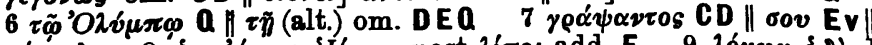

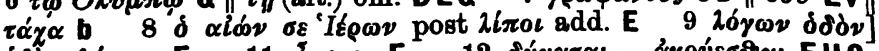

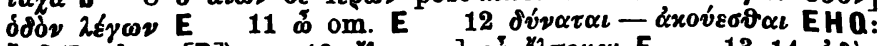

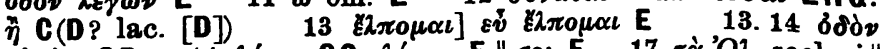

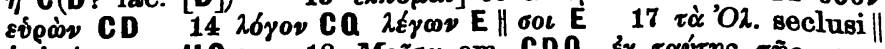

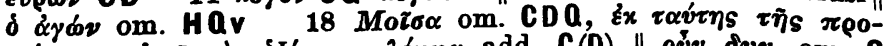

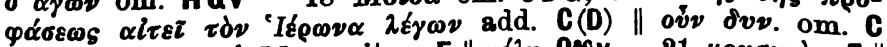

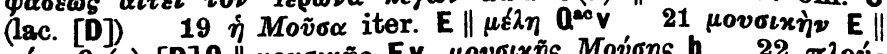

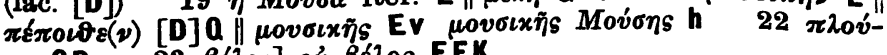

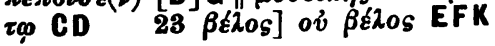




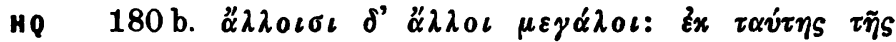

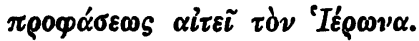

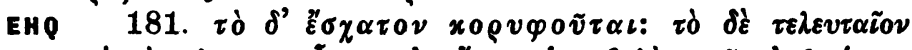

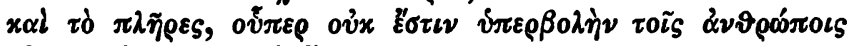

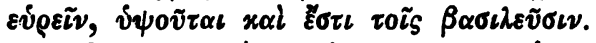

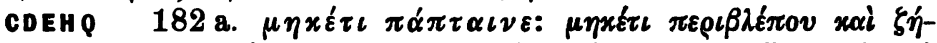

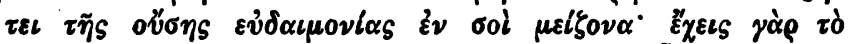

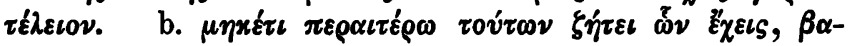
$\sigma \iota \lambda \varepsilon \dot{s} s$ yevó

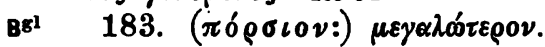

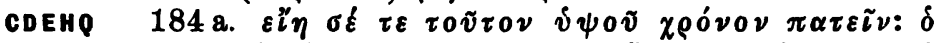

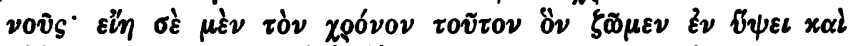

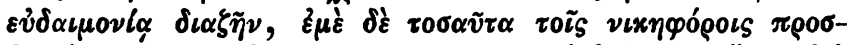

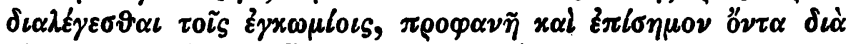

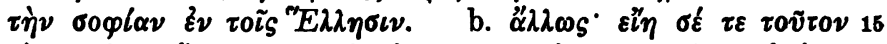

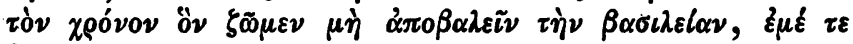

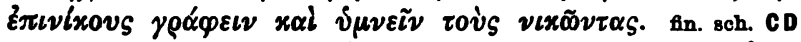

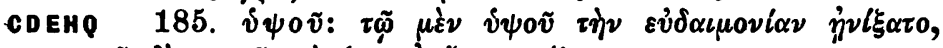

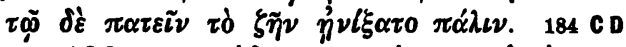

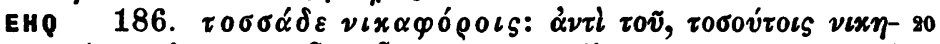

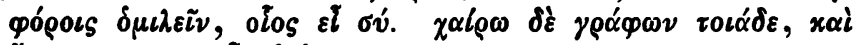

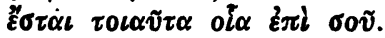

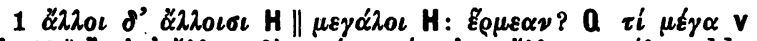

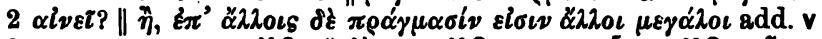

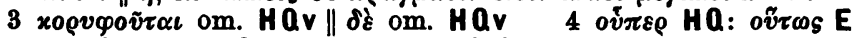

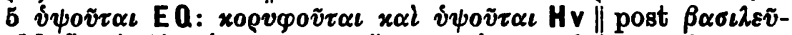

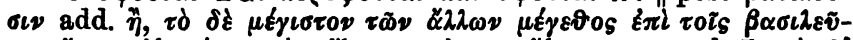

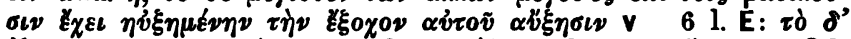

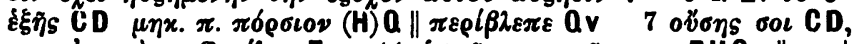

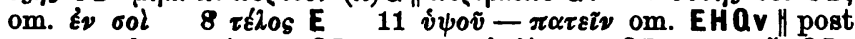

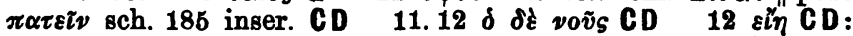

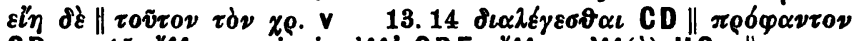

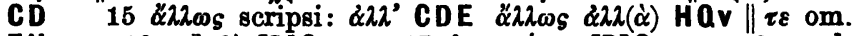

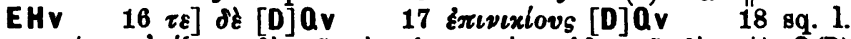

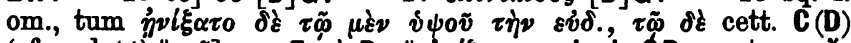

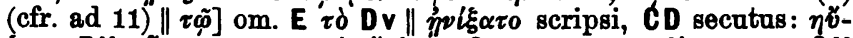

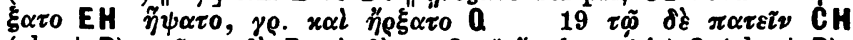
(abest D): $\tau \tilde{\omega} \pi$. $\delta \dot{E} \mathrm{E} \tau \dot{\delta} \delta \xi \pi$. Qv $\| \not \nu^{2} \xi \alpha \tau 0$ (sic) C (abest D):

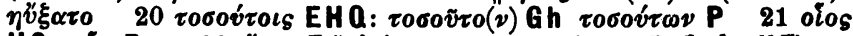

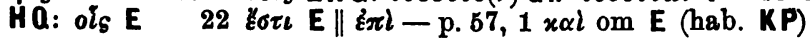




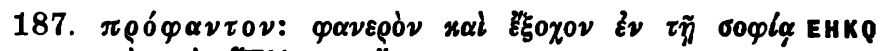

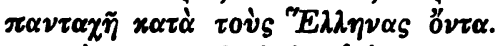

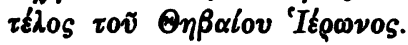

EFKP

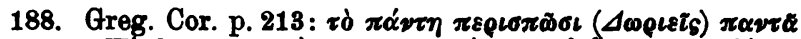

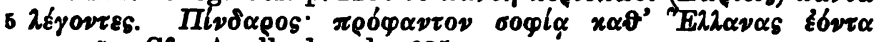
$\pi \alpha v \tau \tilde{\alpha}$. Cfr. Apoll. de adv. 625.

\section{Scholia in Olympionicarum carmen II.}

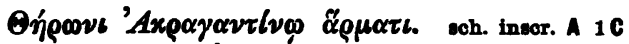

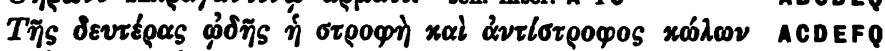

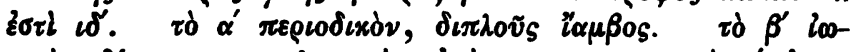

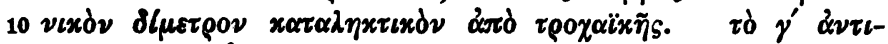

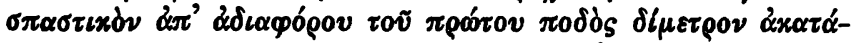

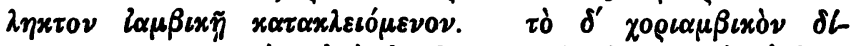

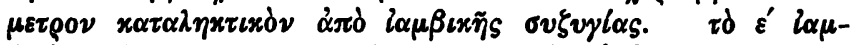

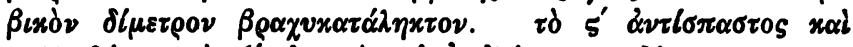

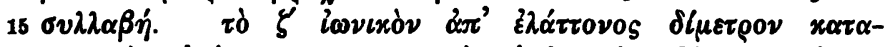

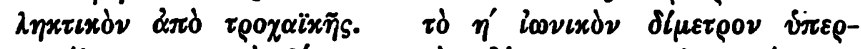

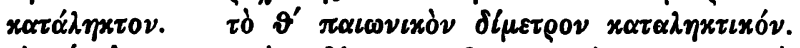

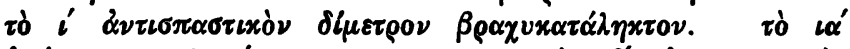

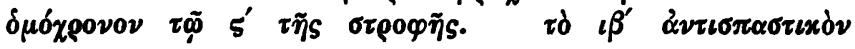

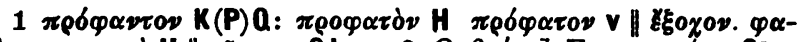

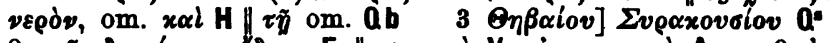

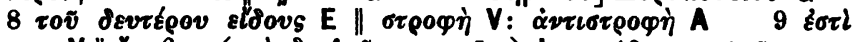

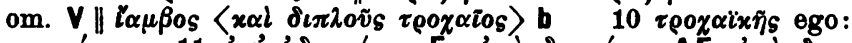

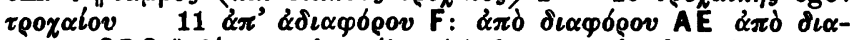

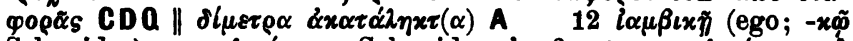

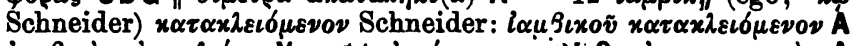

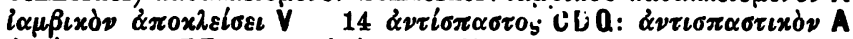

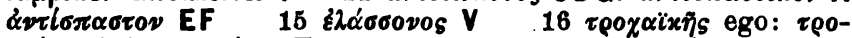

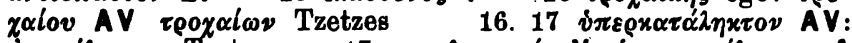

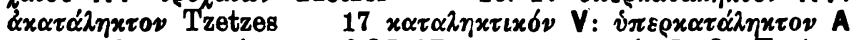

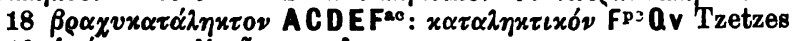

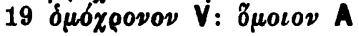




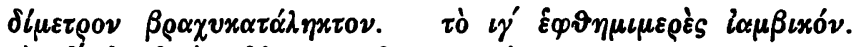

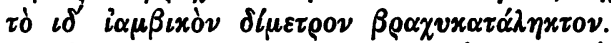

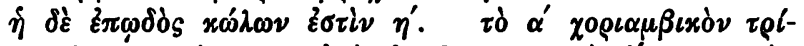

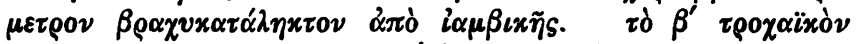

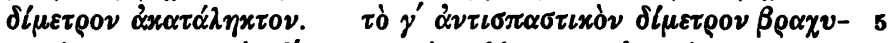

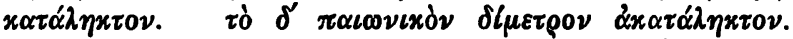

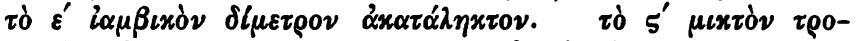

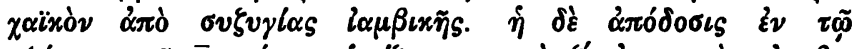

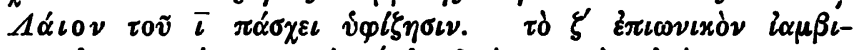

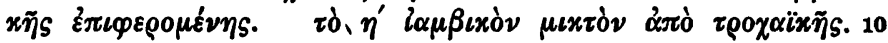
$15 \mathrm{~A} 23 \mathrm{C} 1 \mathrm{E}$

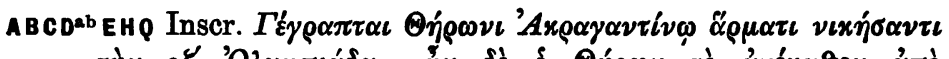

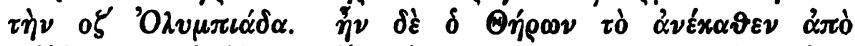

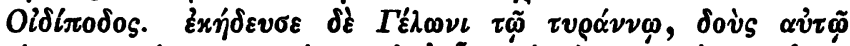

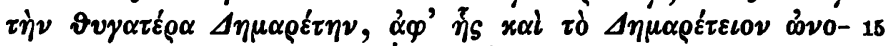

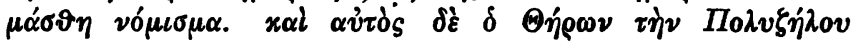

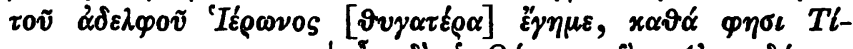

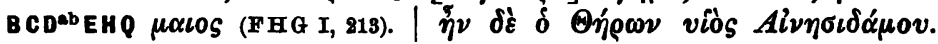
8 BD $^{\mathrm{A} E H Q ~} 11 \mathrm{CD}^{\mathrm{b}}$

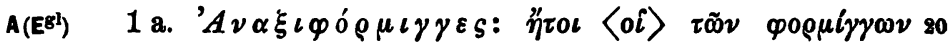

$3 \varepsilon \pi \omega \delta \dot{\eta}$ E $\| \varepsilon \sigma \tau i \nu$ om. V $\| \alpha^{\prime}$ om. A $3.4 \delta i \mu \varepsilon \tau \rho o \nu$ E

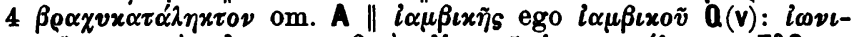

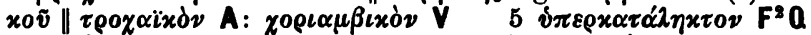

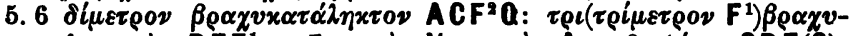

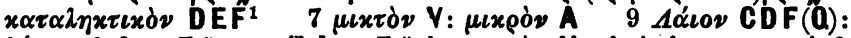

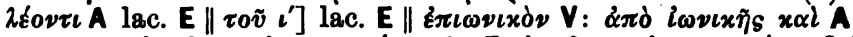

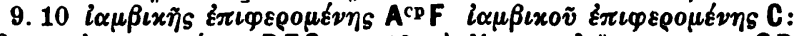

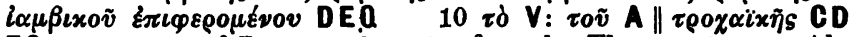

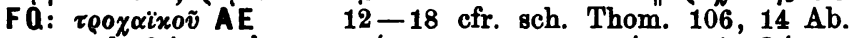

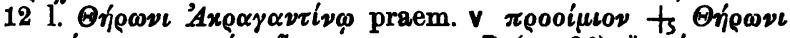

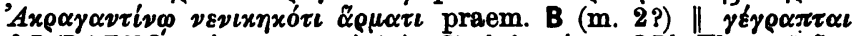

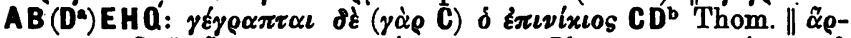

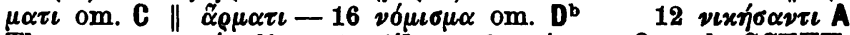

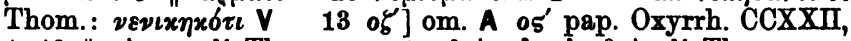

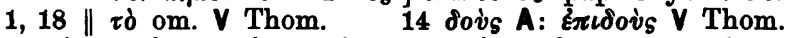

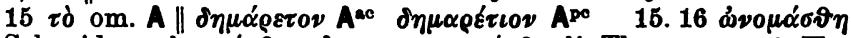

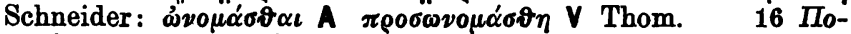

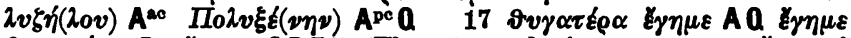
จv

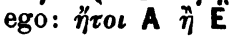




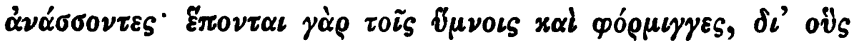

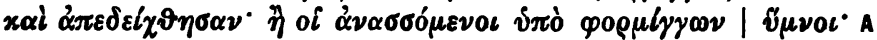
$\pi \rho o ́ \tau \varepsilon \rho \nu \nu$ jò̀

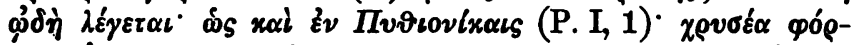

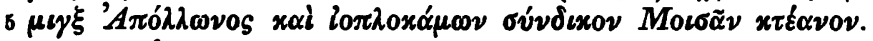

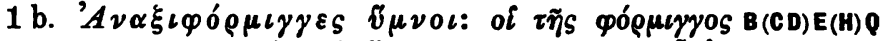

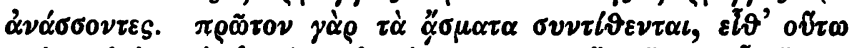

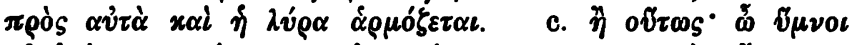

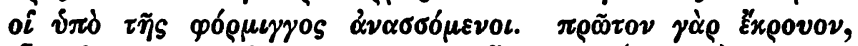

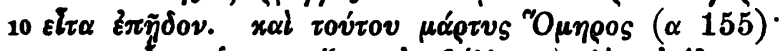

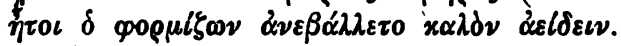

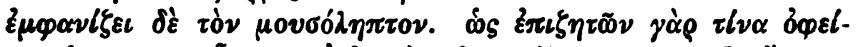

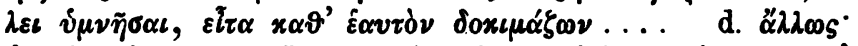

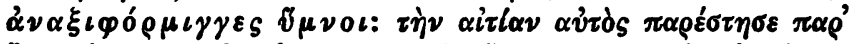

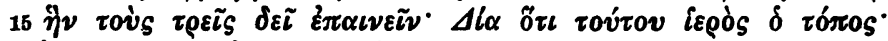

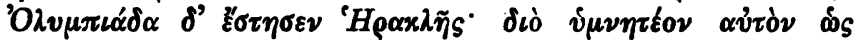

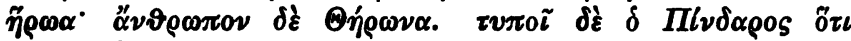

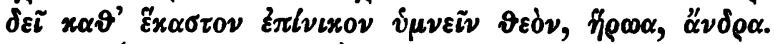

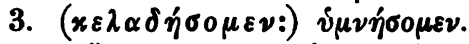

Be1

80

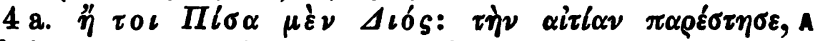

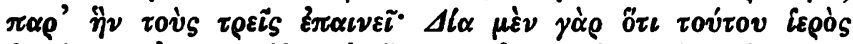

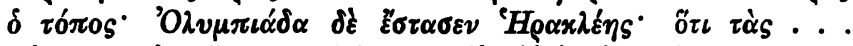

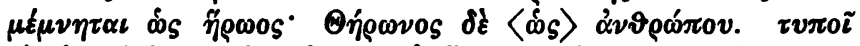

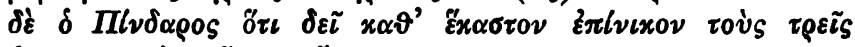

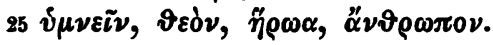

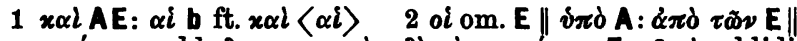

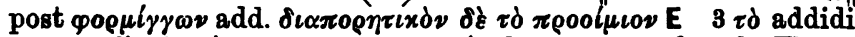

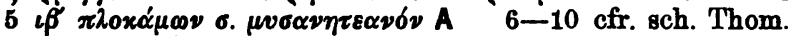

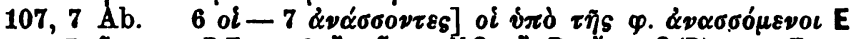

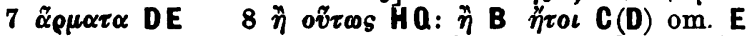

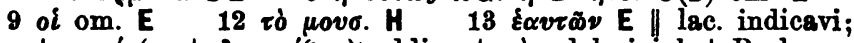

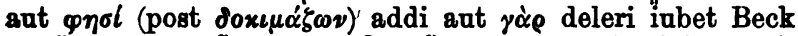

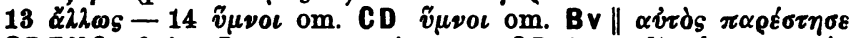

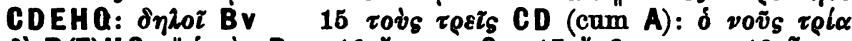

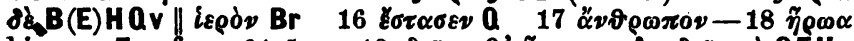
hic om. E; cfr. p. 61, $518 \delta \varepsilon \tau x \alpha \theta^{\prime}$ Ex $18 \sigma 0 \nu$ A: $\delta \varepsilon \tau x \alpha \downarrow$ CEHV

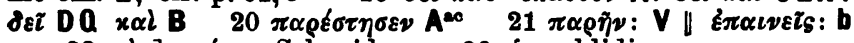

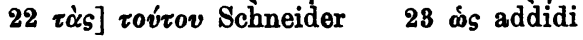




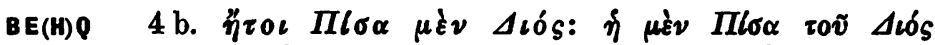

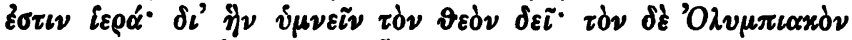

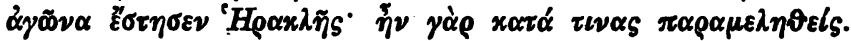

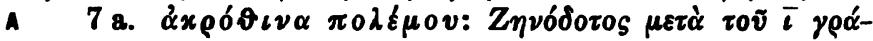

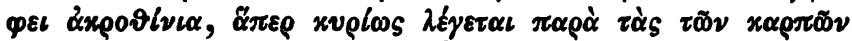

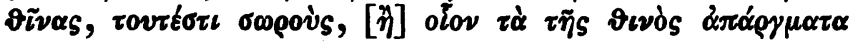

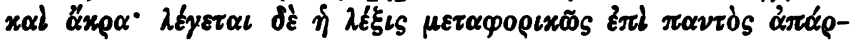

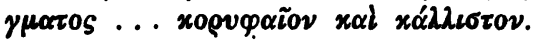

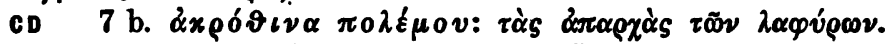

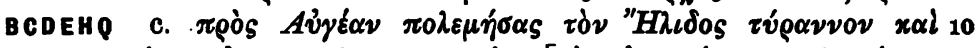

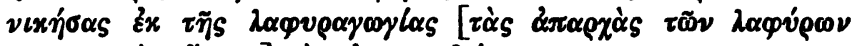

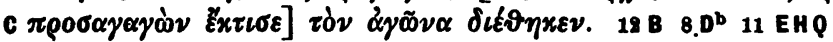

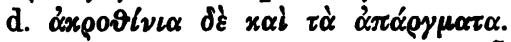

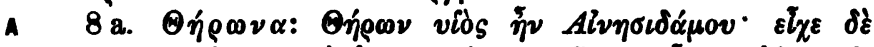

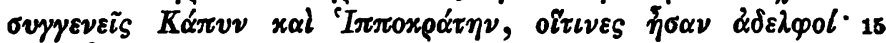

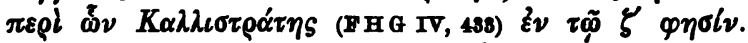

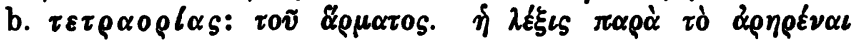

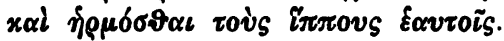

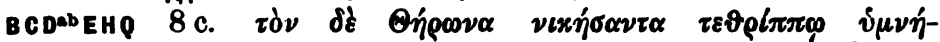

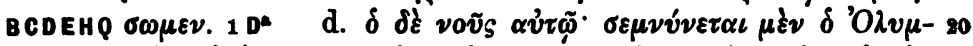

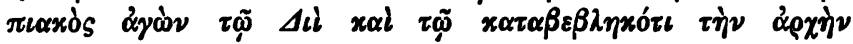

5 EM 53, 10. EG 29, 40. Hes. Suid. s. v. lex. Bach. 56, 14. sch. Eur. Phoen. 203. Eust. Od. 1862, 2910 cfr. p. 7, 1 sqq.

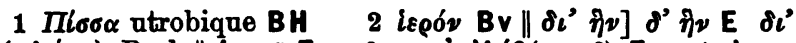

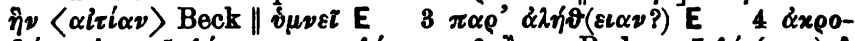

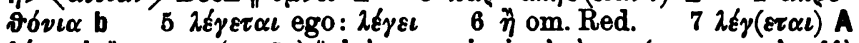
$\lambda \xi \xi \varepsilon \iota$ b $\|\mu \varepsilon \tau \alpha \varphi o(\rho \iota x \tilde{\omega} s)\| \varepsilon \pi l \pi$. scripsi: $\alpha \pi \delta$

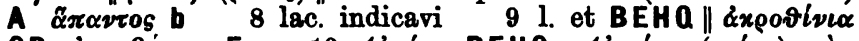

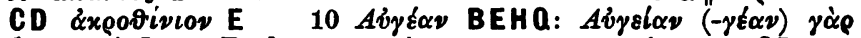
CD \| $\tau \dot{\partial} \nu] \tau \eta_{\zeta}$ Beck $11 \tau \dot{\alpha} s-12 \pi \rho 0 \sigma \alpha \gamma \alpha \gamma \dot{\omega}$ om. CD

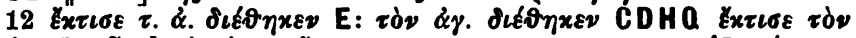

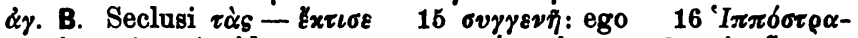

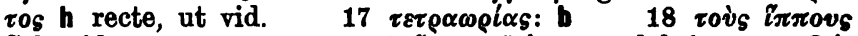

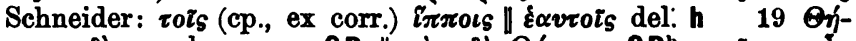

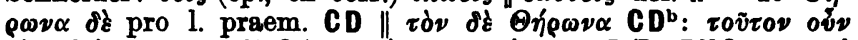

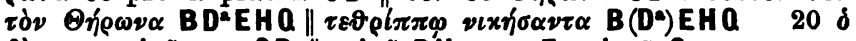

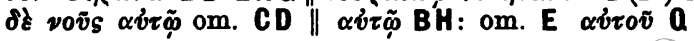




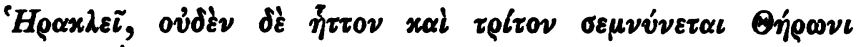

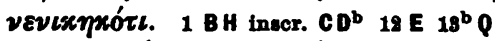

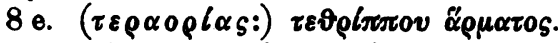

Bgi

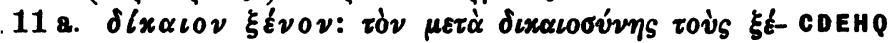

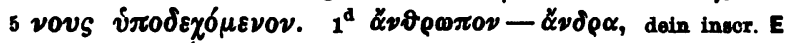

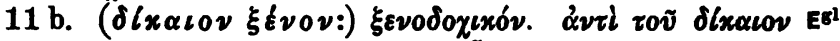

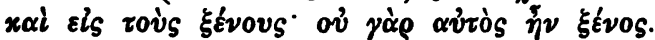

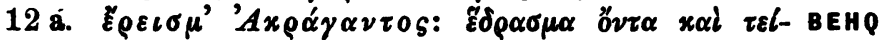

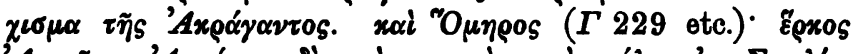

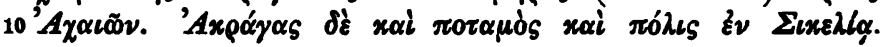
$13^{\circ} \mathrm{HQ}$

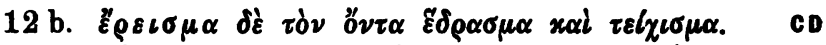

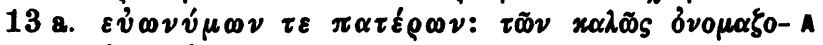

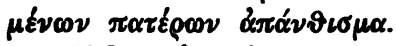

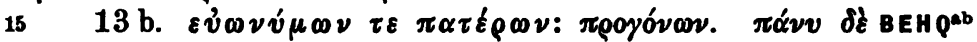

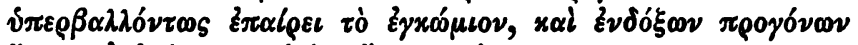

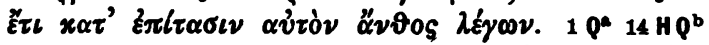

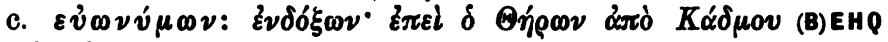

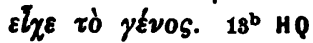

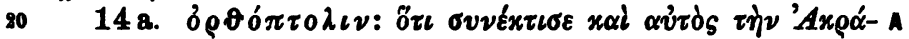

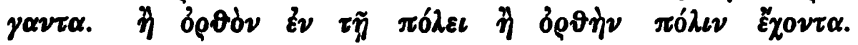
motr. A

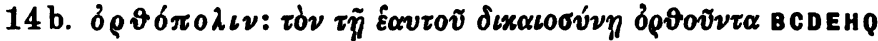

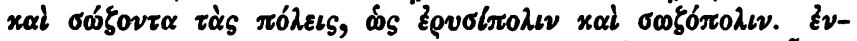

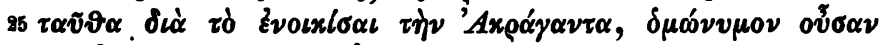

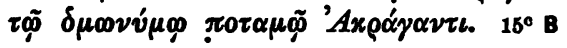

18 Eust. vit. Pind. 12, 19 Ab.

$6 \xi \xi \varepsilon v 0 \delta \circ \chi\left\llcorner x 6 \nu\right.$ scripsi: $\xi \varepsilon \nu 0 \delta 6 \chi(0 \nu) \tau \delta \nu E^{\mathrm{op}}, 8 \varepsilon \delta \rho \alpha \sigma \mu \alpha$

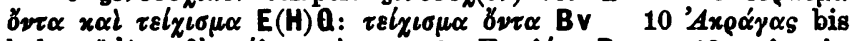

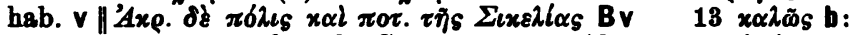

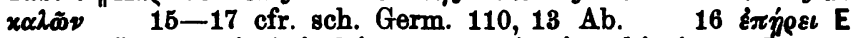

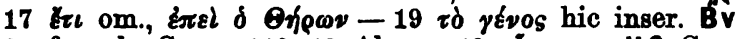
18. 19 cfr. sch. Germ. 110, 12 Ab. 19 sixe om. HQ Germ.

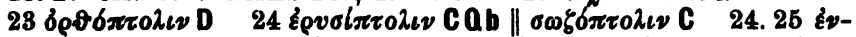

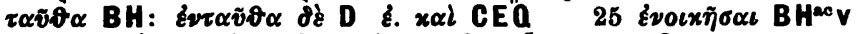

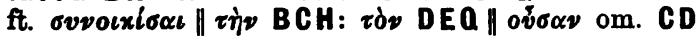




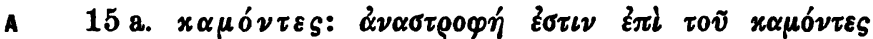

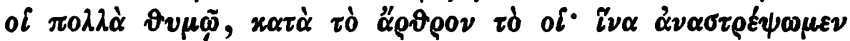

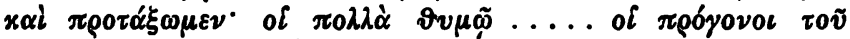

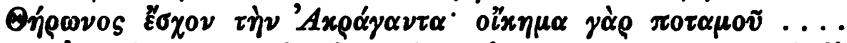

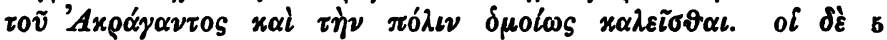

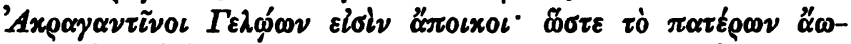

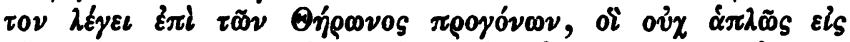

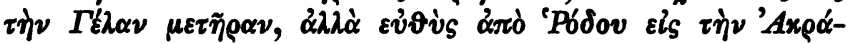

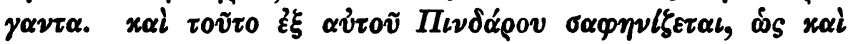

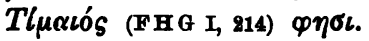

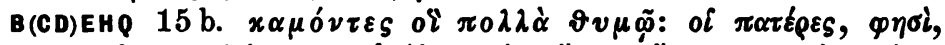

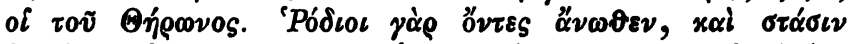

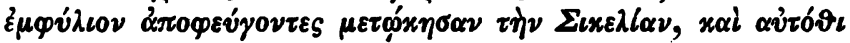

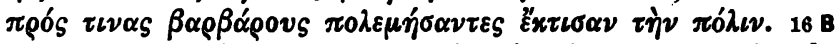

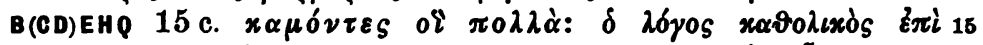

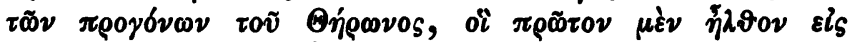

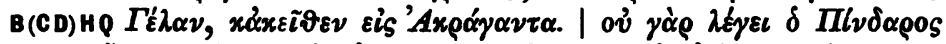

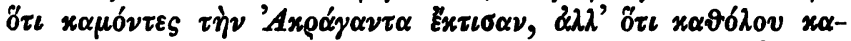

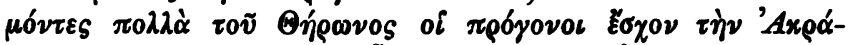

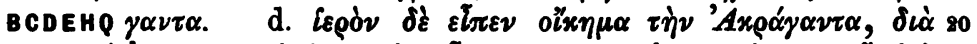

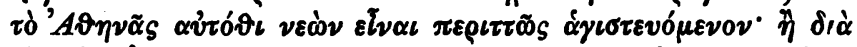

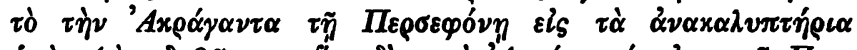

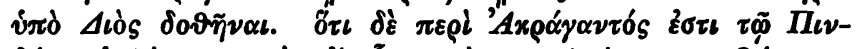

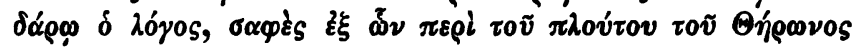

1 Eust. vit. Pind. 10, 7 Ab. 22 sch. N. 1, 17. Plut. Tim. 8,4

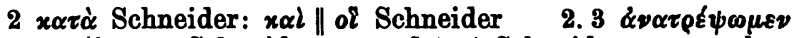
$x \alpha i \pi \rho 0 \sigma \tau \alpha ́ \xi \omega \mu \varepsilon v$ : Schneider 3 oi (pr.) Schneider 3 sq. lac.

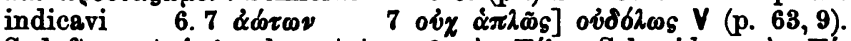
Sed ft. post $\dot{\alpha} \pi \lambda \tilde{\omega} s$ lac. stat. $8 \tau \dot{\eta} \nu \Gamma \xi \lambda \alpha \nu$ Schneider: $\tau \delta \nu \Gamma \xi-$

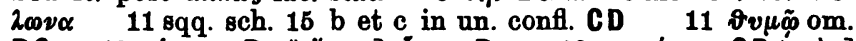

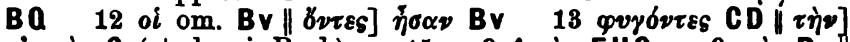

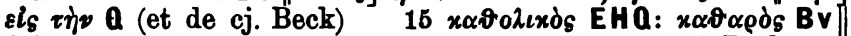

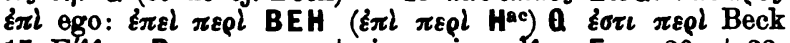
$17 \Gamma \varepsilon \lambda \lambda \alpha \nu$ B semper, ceteri saepius; $\eta \lambda \alpha \nu$ E 20 et $22 \tau \dot{\eta} \nu]$

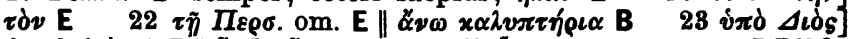

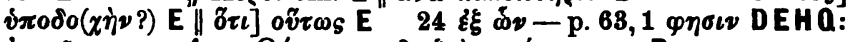

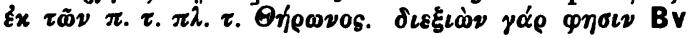




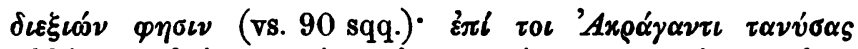

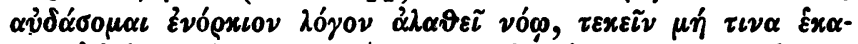

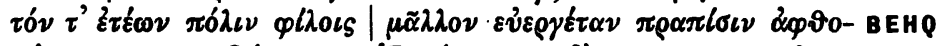

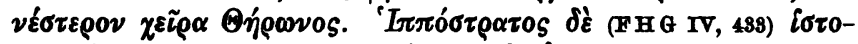

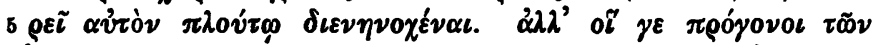

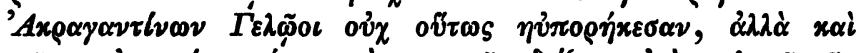

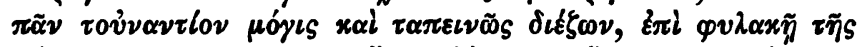

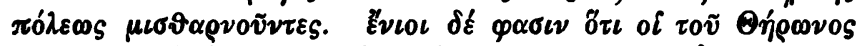

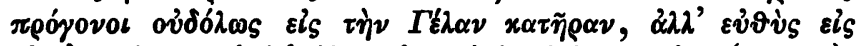

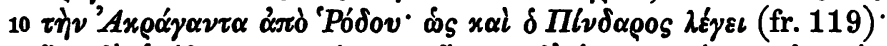

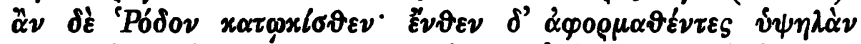

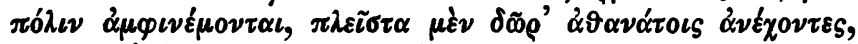

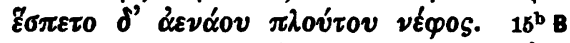

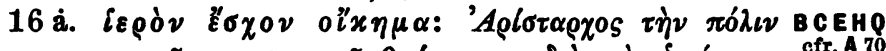

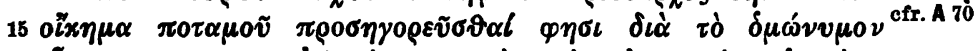

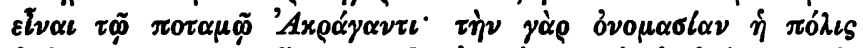

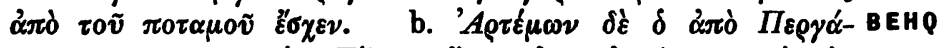

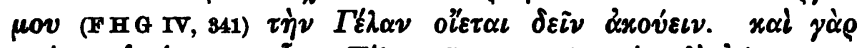

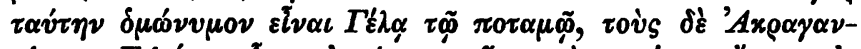

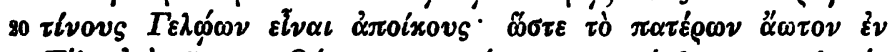

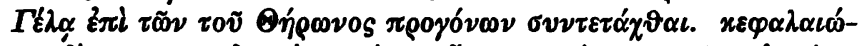

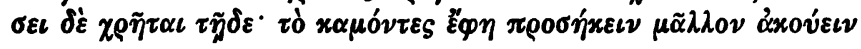

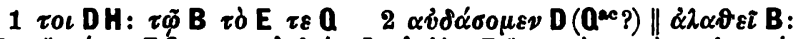

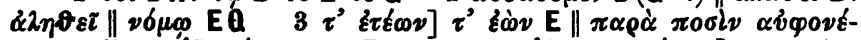

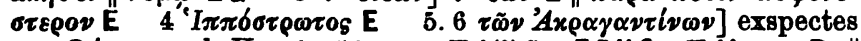

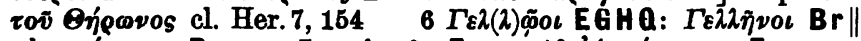

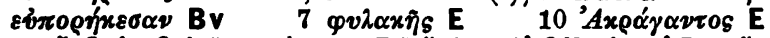

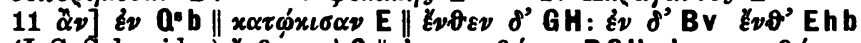

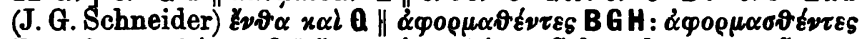

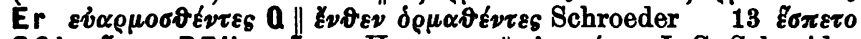

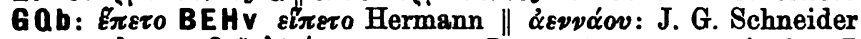

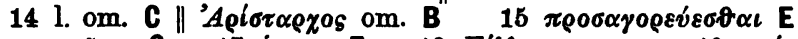

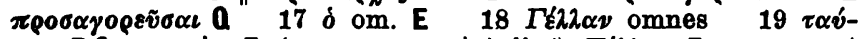
$\tau \eta \nu$ B Q: $\tau \alpha v \tau \dot{\nu} \nu \mathrm{E}$ (corr. ex $\tau \alpha \nu \tau \dot{\nu} \nu) \mathrm{H} \| \Gamma \dot{\varepsilon} 2 \lambda \alpha \nu \mathrm{E}$; mox post

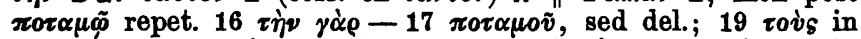

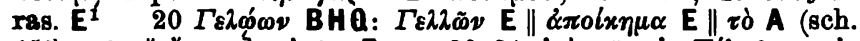

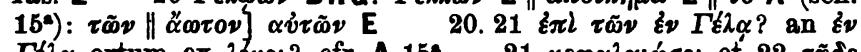

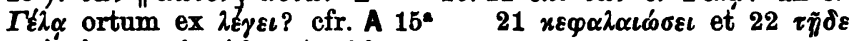
scripsi : $x \varepsilon \varphi \alpha \lambda \alpha \iota \omega \delta \varepsilon \varepsilon$ et $\tau \tilde{\omega} \delta \varepsilon$ 


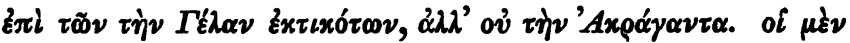

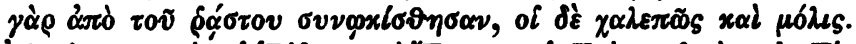

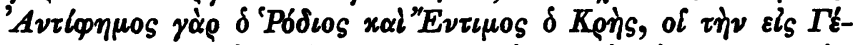

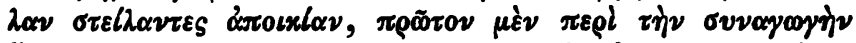

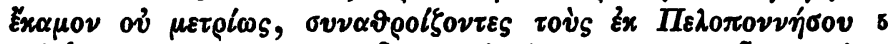

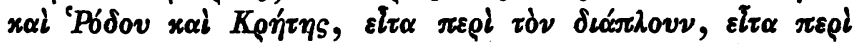

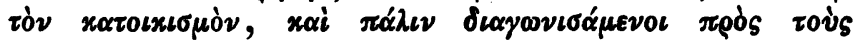

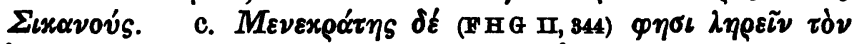

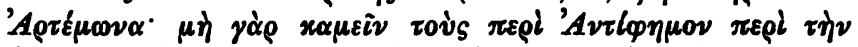

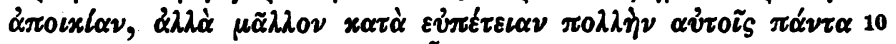

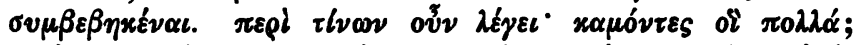

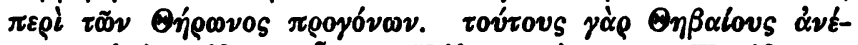

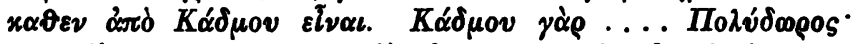

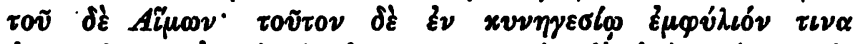

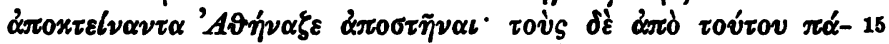

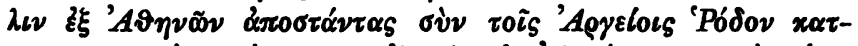

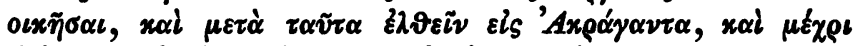

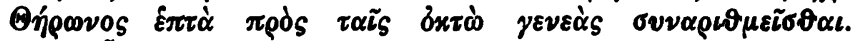

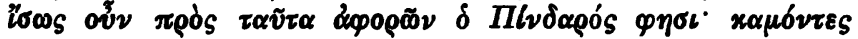

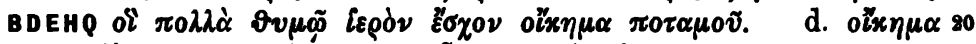

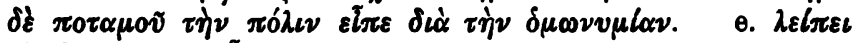

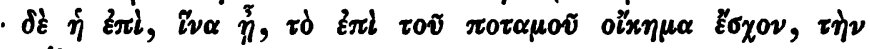
$\pi \delta \alpha_{\iota v}$.

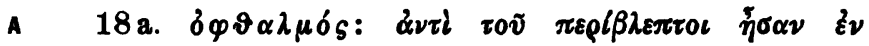

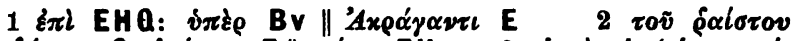

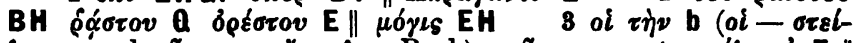

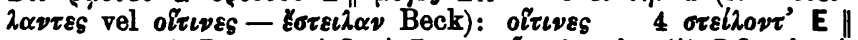

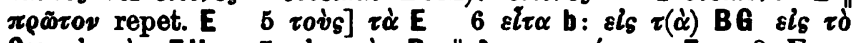
Qv $\varepsilon l_{s} \tau \dot{\delta} \nu$ EH 7 oix

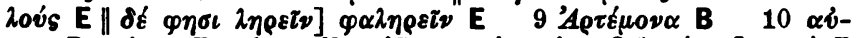

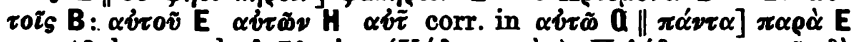

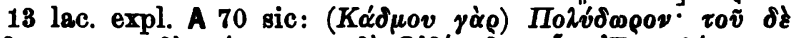

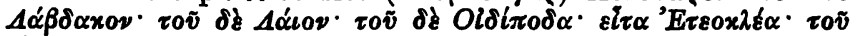

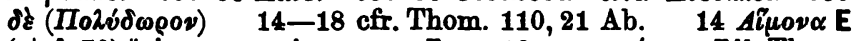

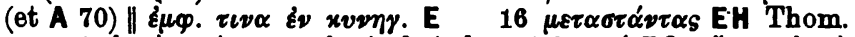

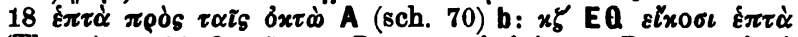

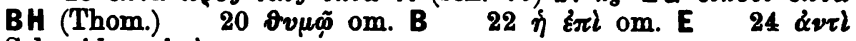
Schneider: $\& \pi \dot{\delta}$ 


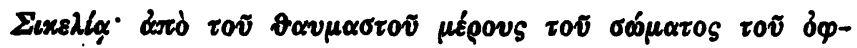

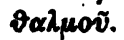

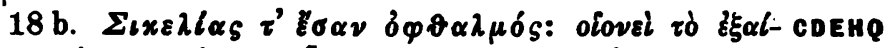

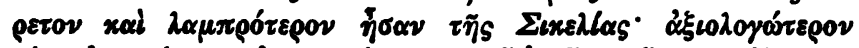

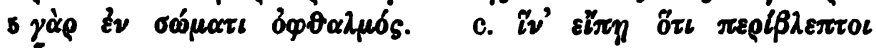

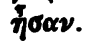

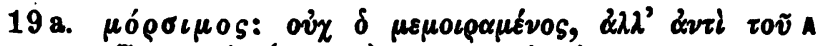

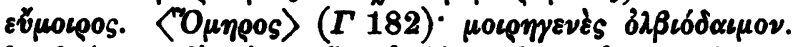

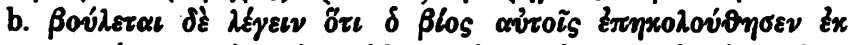

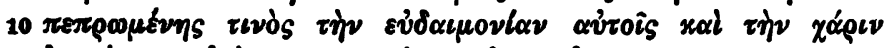

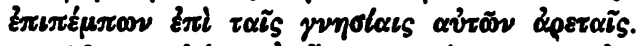

$19 \mathrm{c.} \mathrm{\alpha i \omega ́ \nu} \tau$ '

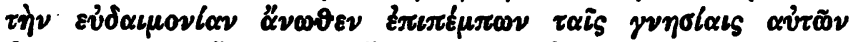

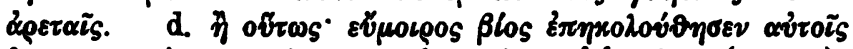

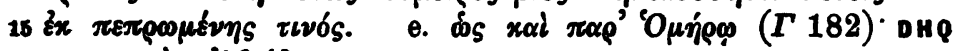

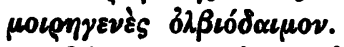

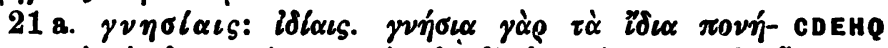

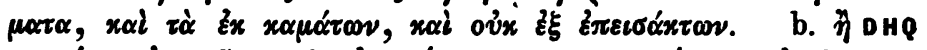

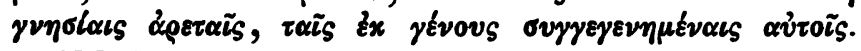
20 220 CDEHQ

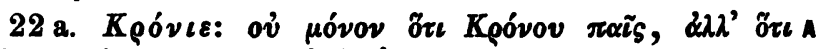

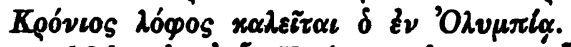

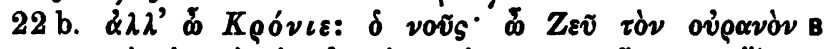

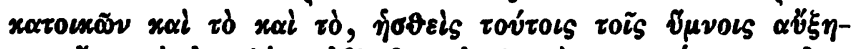

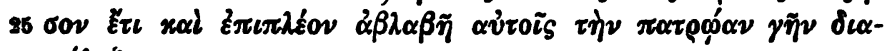

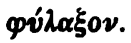

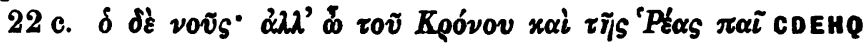

7 Eust. Il. 402, 9

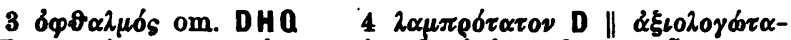

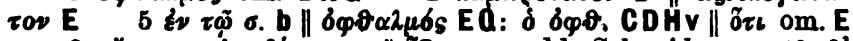

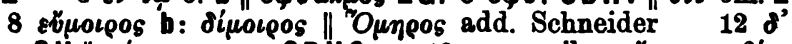

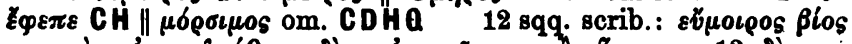

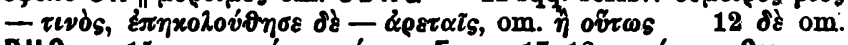

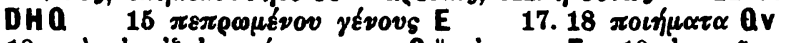

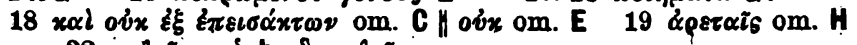
$22 x \alpha \lambda \varepsilon i \tau \alpha \iota \delta$ b: $\delta$ x $\alpha \lambda \varepsilon i \tau \alpha \iota$

Bchox. ma Pardaryx ed. Drachmann. 


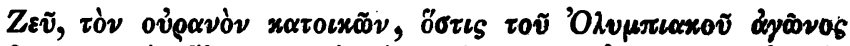

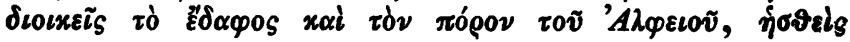

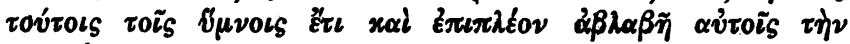

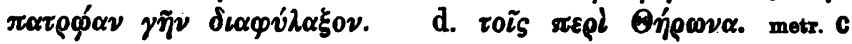
27 DEHQ

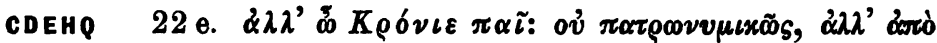

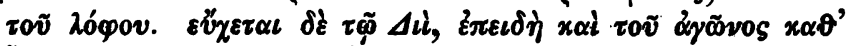

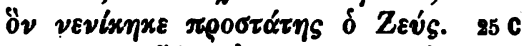

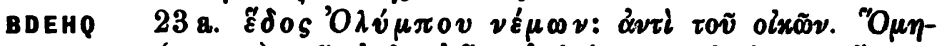

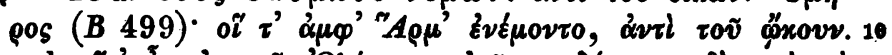

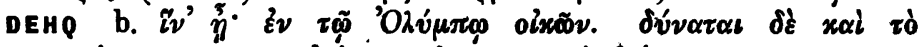

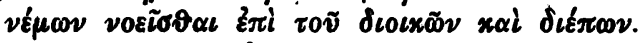

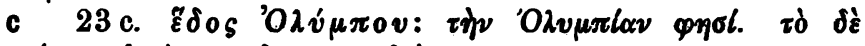

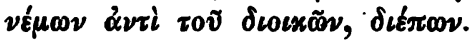

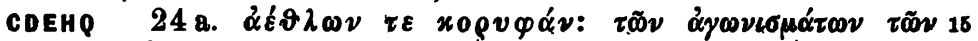

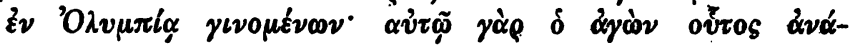
$x \varepsilon \iota \tau \alpha \iota . \quad 27 \mathrm{C}$

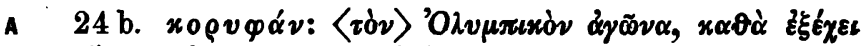

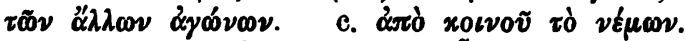

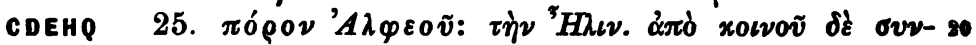

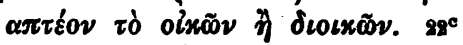

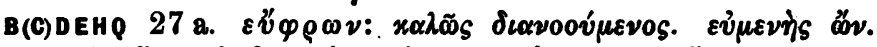

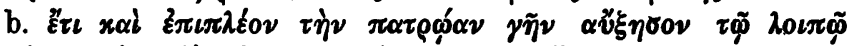

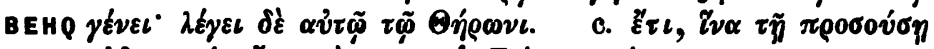

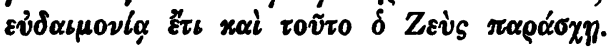

9 Eust. Il. 636, 18. Od. 1554, 21

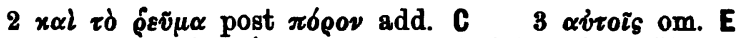

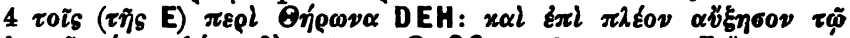

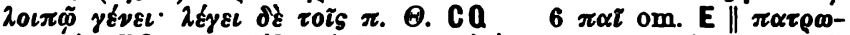

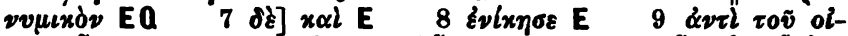

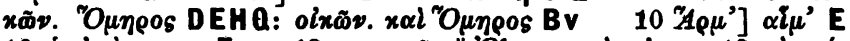

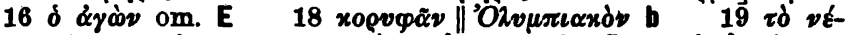

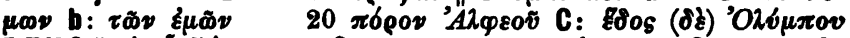

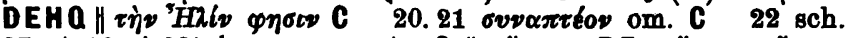

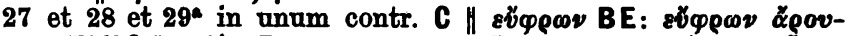

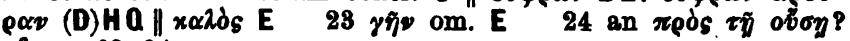
cfr. p. 69,24 


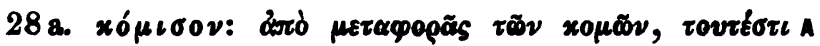

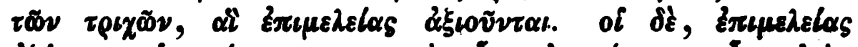

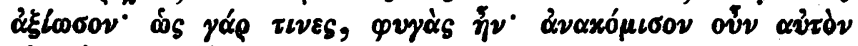
sis $\pi \dot{\nu} \nu \pi \alpha \tau \rho l \delta \alpha$.

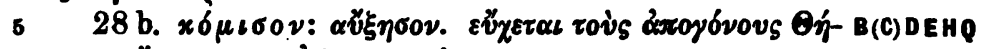

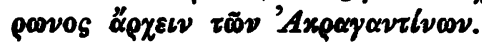

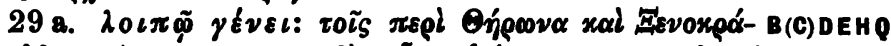

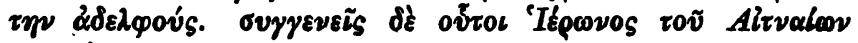
Boointeos. $81 \mathrm{C}$

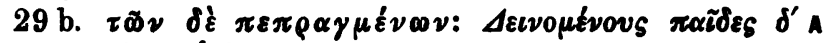

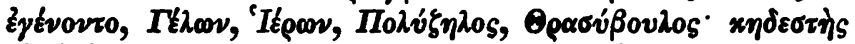

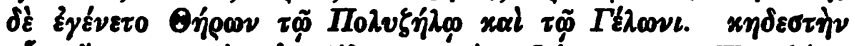

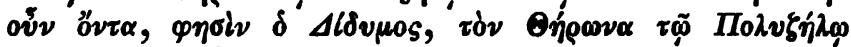

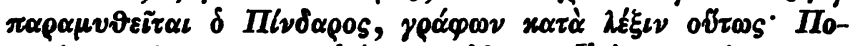

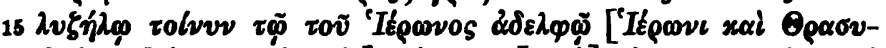

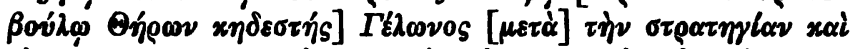

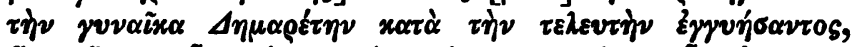

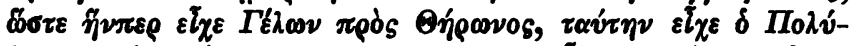

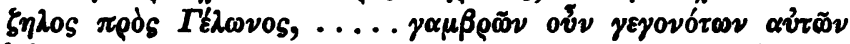

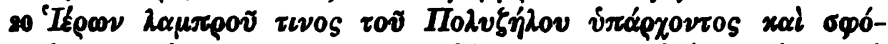

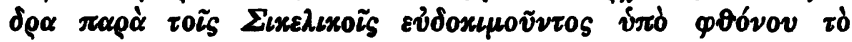

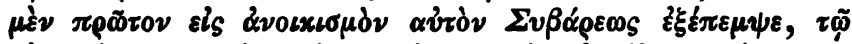

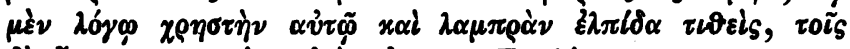

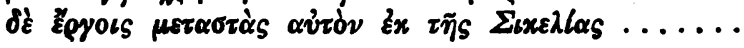

1 sch. VL E 359. EM 526, 44. Hes. Suid. $x 6 \mu \iota \xi \varepsilon$. Zon. 1245

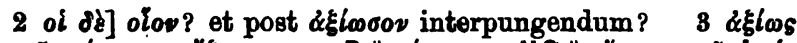

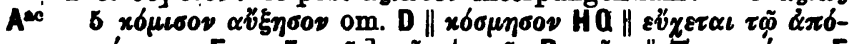

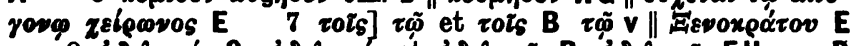

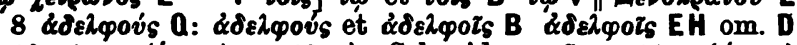

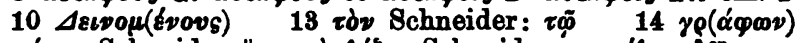

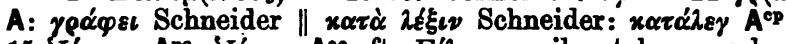

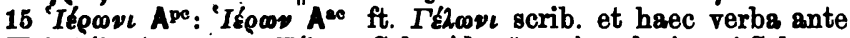

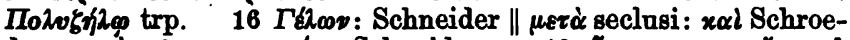

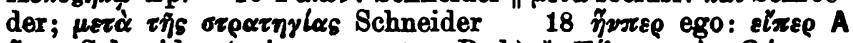

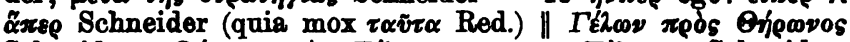

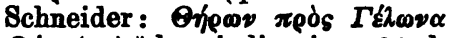
$19 \Gamma$ Éloovos Schneider:

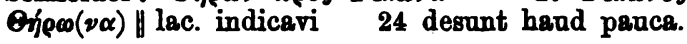




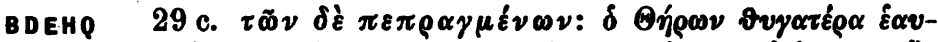

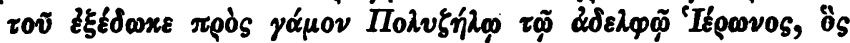

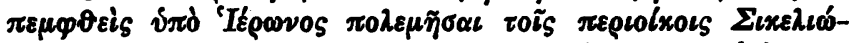

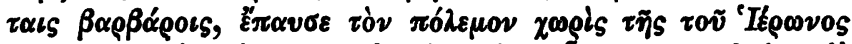

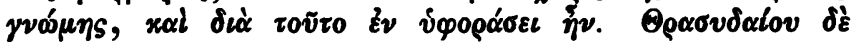

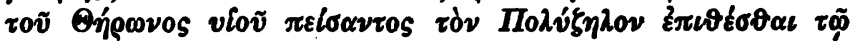

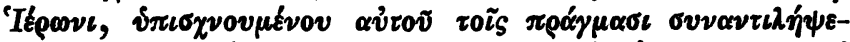

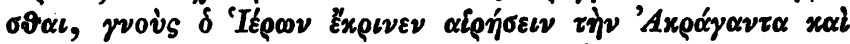

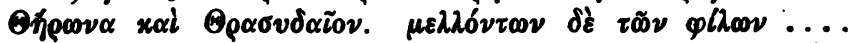

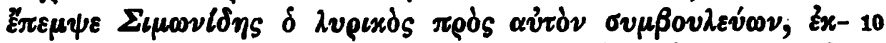

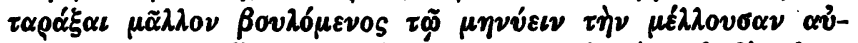

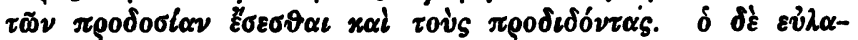

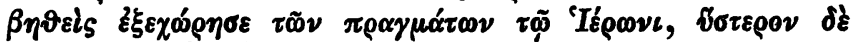

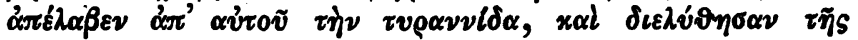

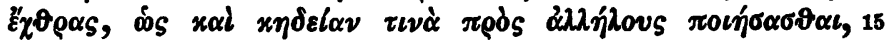

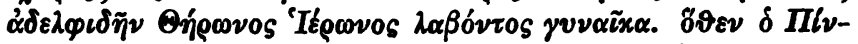

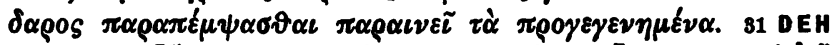

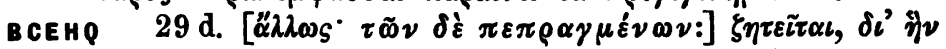

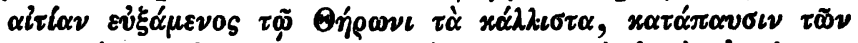

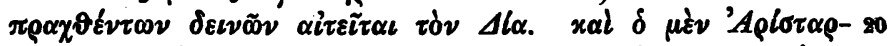

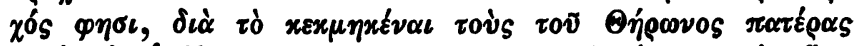

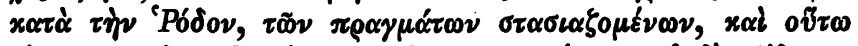

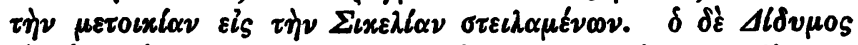

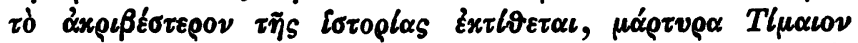

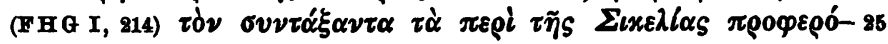

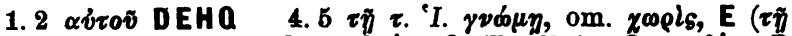

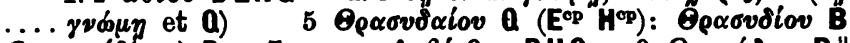

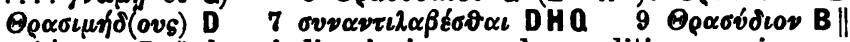
$\mu \varepsilon \lambda 6 \nu \tau \omega \nu$ B || lac. indicavi, in qua de proditione amicoram

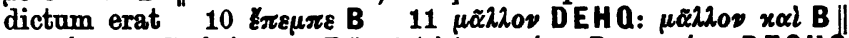

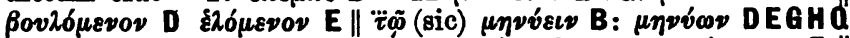

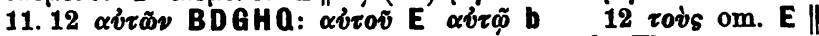

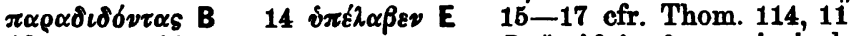
Ab. $16 \alpha \delta \varepsilon \lambda \varphi \iota \delta \tilde{\eta} v-\gamma v v \alpha \tau x \alpha$ om. D \| $\alpha \delta \varepsilon \lambda \varphi \iota \delta \eta \tilde{v}$ scripsi cl.

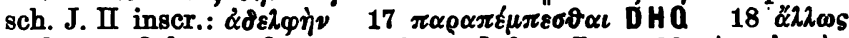

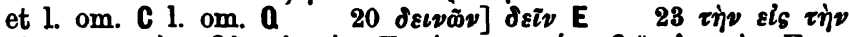

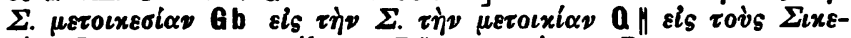
$\lambda l_{\alpha \nu}$ B $25 \sigma v \mu \pi \rho \alpha \xi \alpha \nu \tau \alpha$ E $\| \pi \rho \circ \varphi \varepsilon \rho \sigma \mu \varepsilon \nu \alpha$ E 


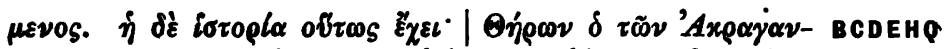

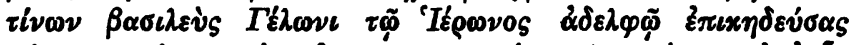

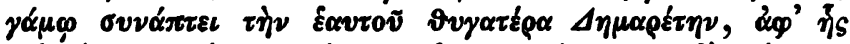

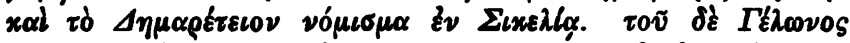

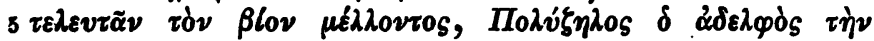

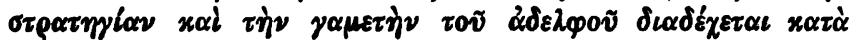

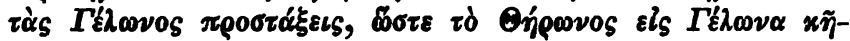

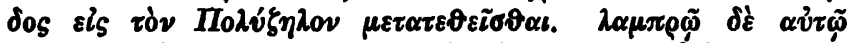

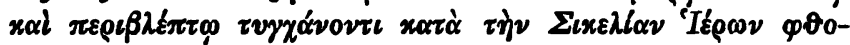

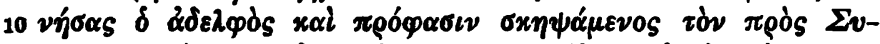

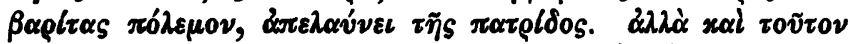

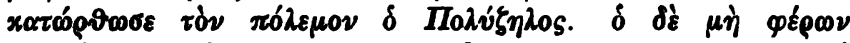

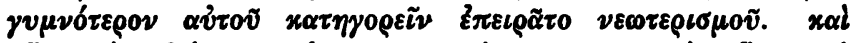

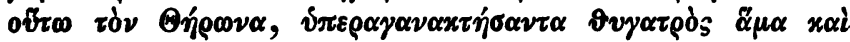

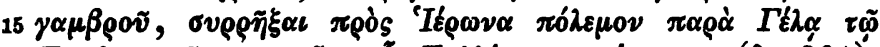

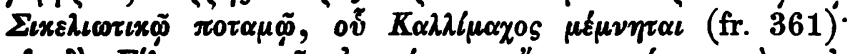

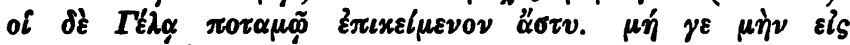

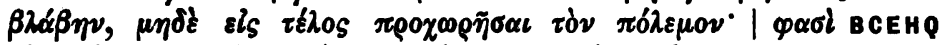

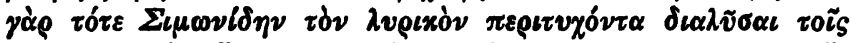

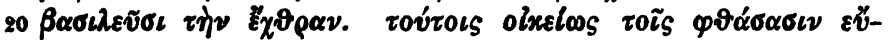

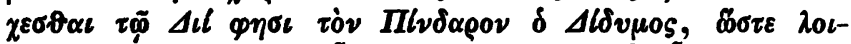

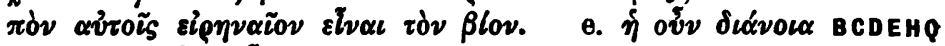

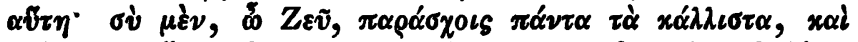

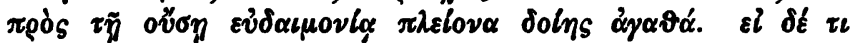

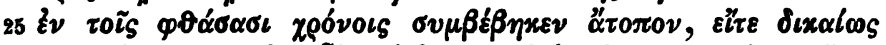

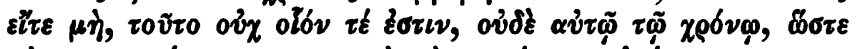

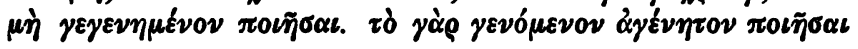

1-16 cfr. Thom. 113, 22 Ab.

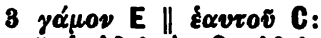

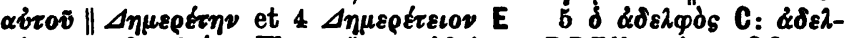

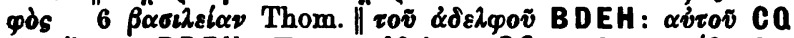

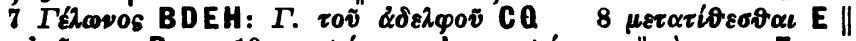

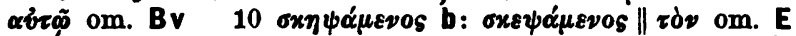

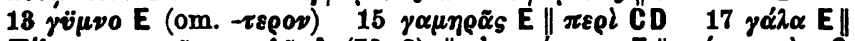

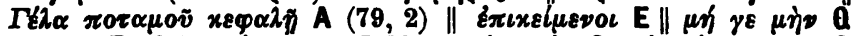

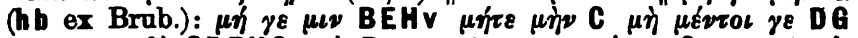
$18 \mu \eta \delta \xi$ CDEHQ: $\mu \eta े$ B $19 \pi \alpha \rho \alpha \tau v \delta \nu \tau \alpha$ C 20 ol-

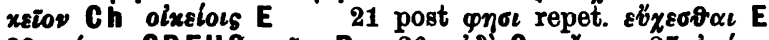

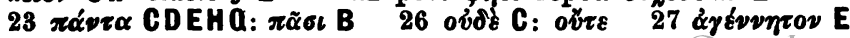




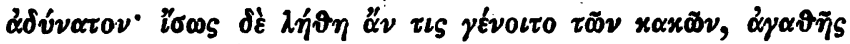
$\mu 0 l \rho \alpha S \delta\llcorner\alpha \delta \varepsilon \xi \alpha \mu t \nu \eta$ S. 34 CDEHQ

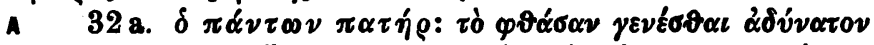

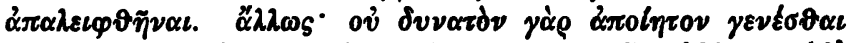

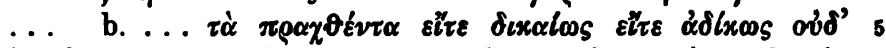

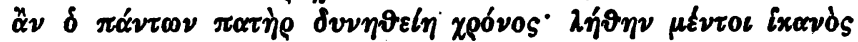

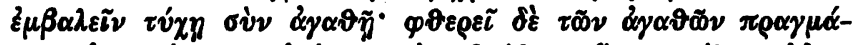

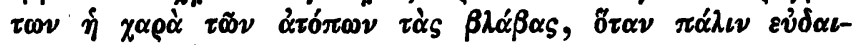
$\mu 0 \nu l \alpha \pi \alpha \rho \alpha y \dot{\varepsilon} \nu \eta \tau \alpha$.

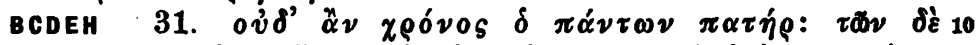
$\pi \varepsilon \pi \rho \alpha \gamma \mu \varepsilon \nu \omega \nu$ हैอ

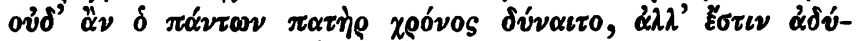

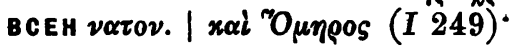

\section{ov่dé $\tau \iota \mu \tilde{\eta} \chi 0 S$}

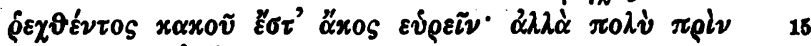

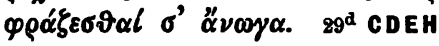

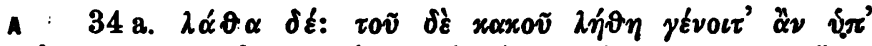

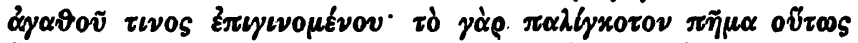

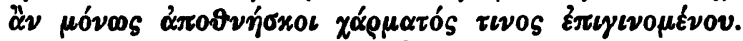

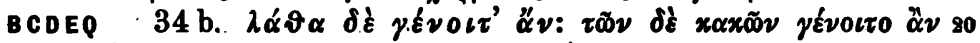

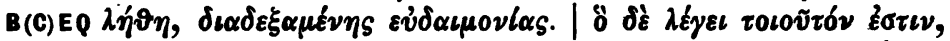

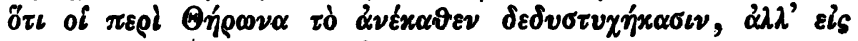

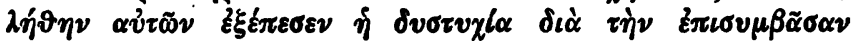

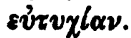

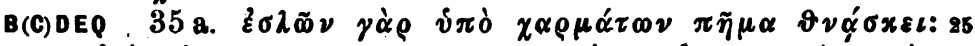

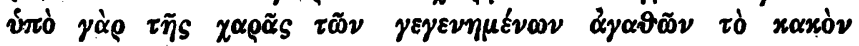

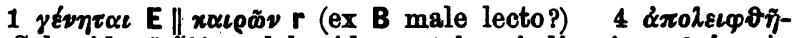

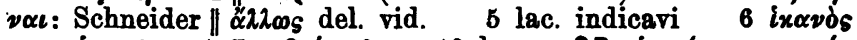

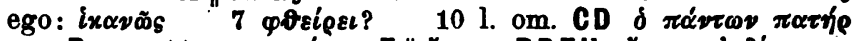
om. BV $11 \pi \varepsilon \pi \rho \omega \mu \varepsilon v \omega \nu$ E Hै हैo

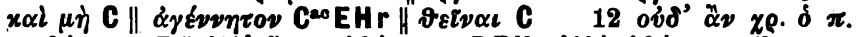

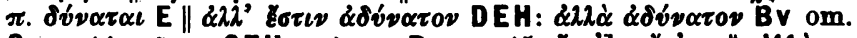

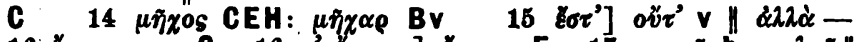

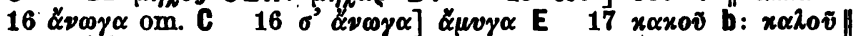

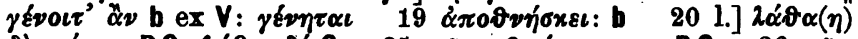

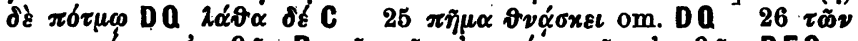

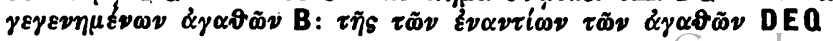




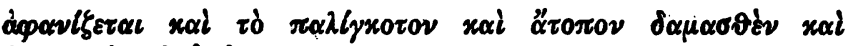

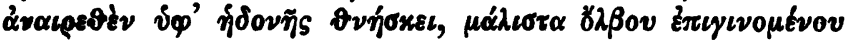

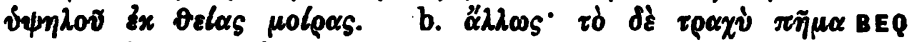

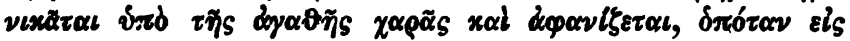

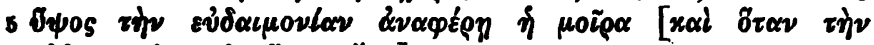

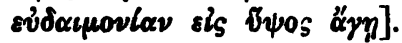

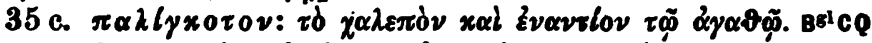

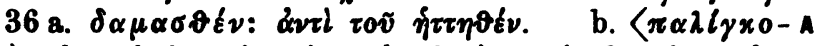

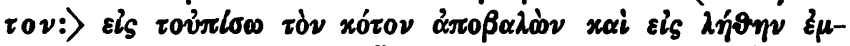

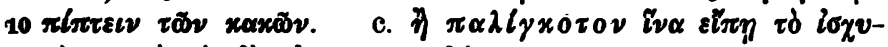

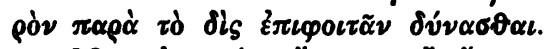

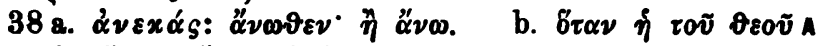

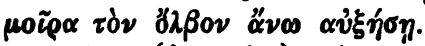

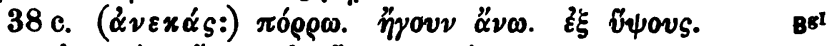

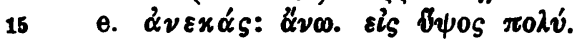

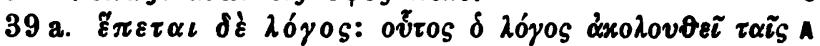

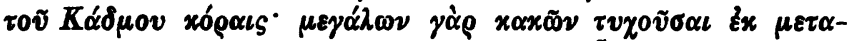

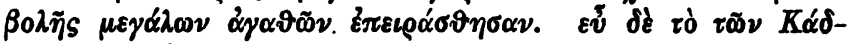

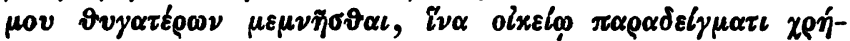

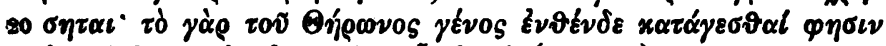

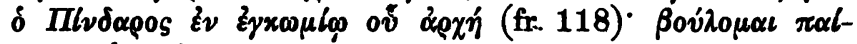

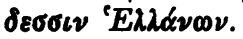

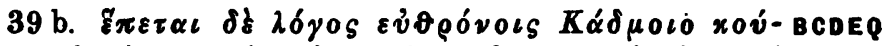

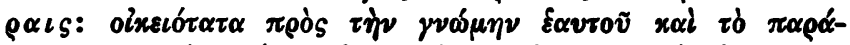

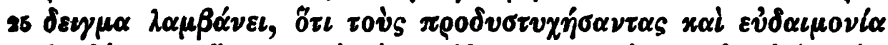

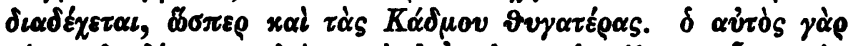

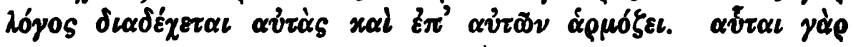

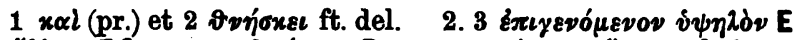

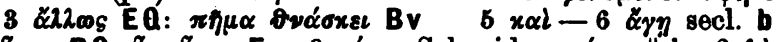

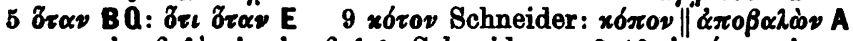

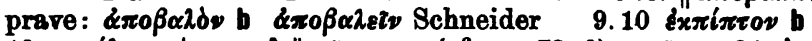

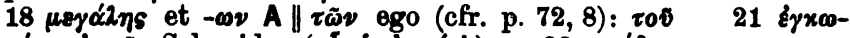

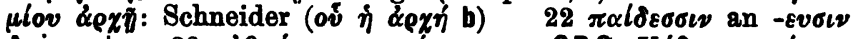

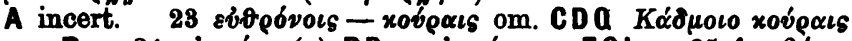

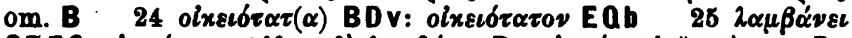

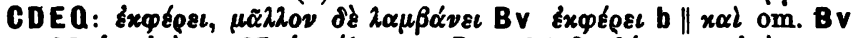

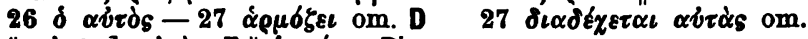
Bv $\| \alpha \dot{v} \dot{\omega} \nu] \alpha \dot{v} \tau \dot{\eta} \nu \mathrm{E} \| \dot{\alpha} \rho \mu \delta \sigma \varepsilon \iota \mathrm{B}^{\mathrm{i} v}$ 


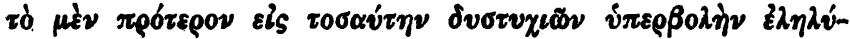

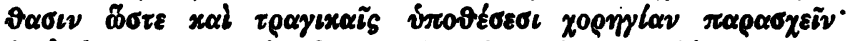

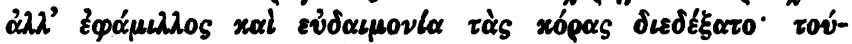

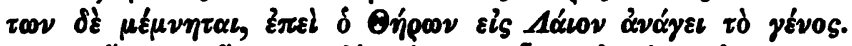

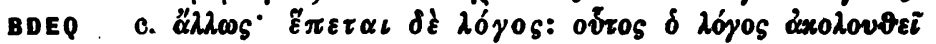

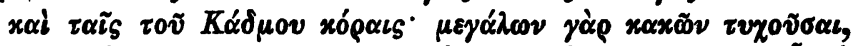

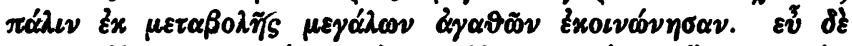

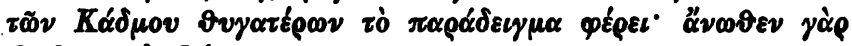

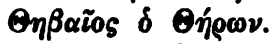

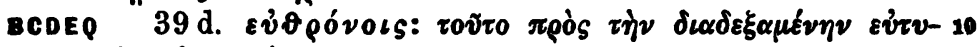
$x^{i \alpha \nu} \dot{\alpha} x o v \sigma \tau \hat{\varepsilon} \sigma \nu$.

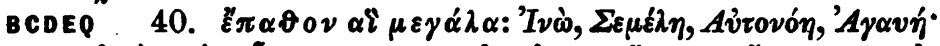

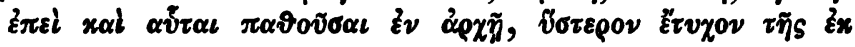
$\vartheta \varepsilon \tilde{\omega} \nu \tau \iota \mu \tilde{\eta} s$.

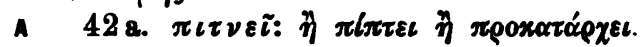

BCDEQ 42 b. $\pi l \tau \nu \varepsilon \tilde{i}: x \alpha \tau \alpha \pi \tau \pi \tau \varepsilon \iota$.

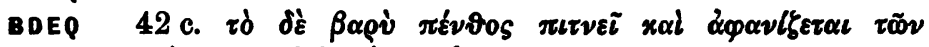

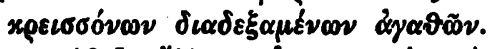

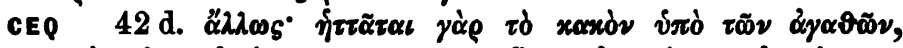

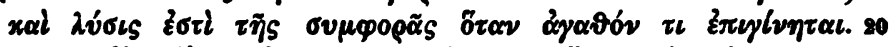

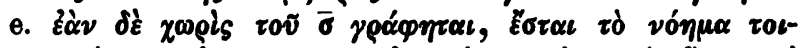

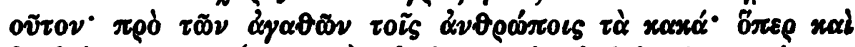

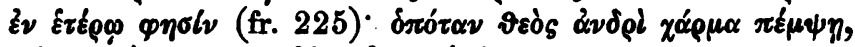

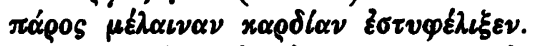

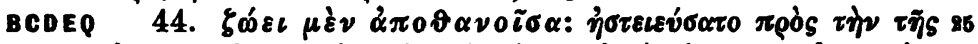

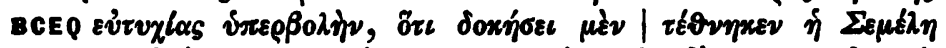

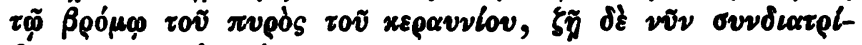

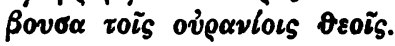

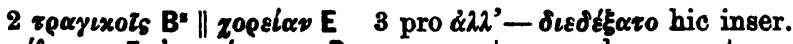

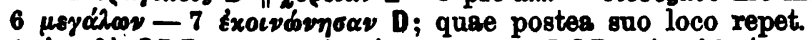

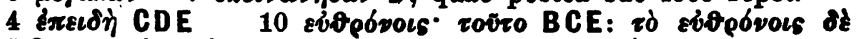

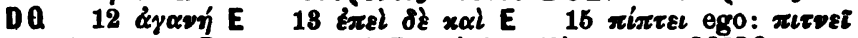

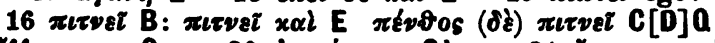

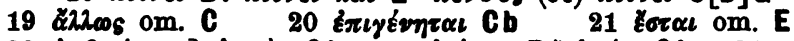

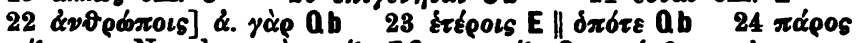

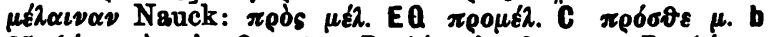

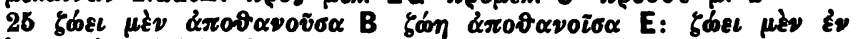

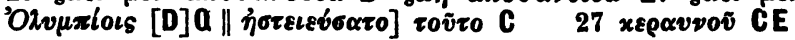




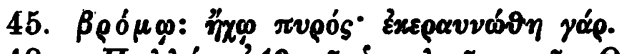

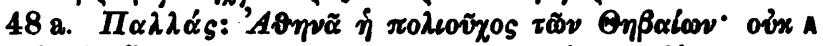

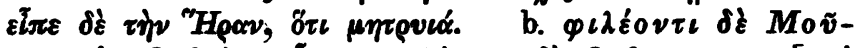

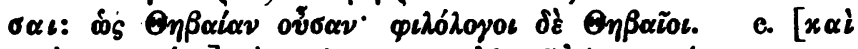

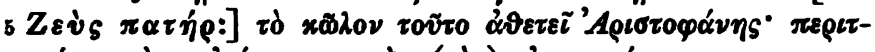

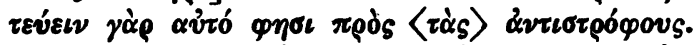

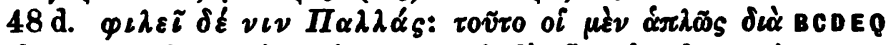

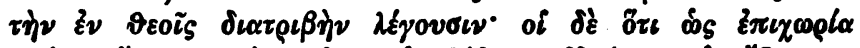

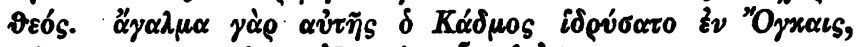

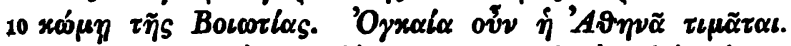

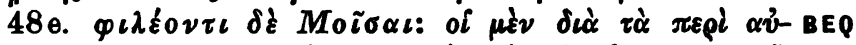

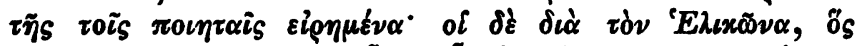

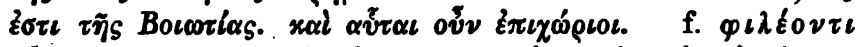

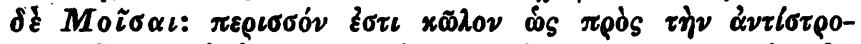

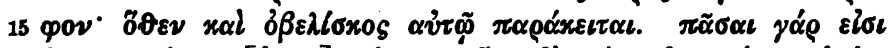

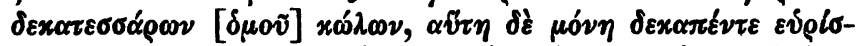

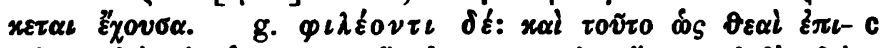

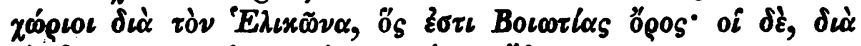

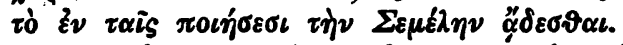

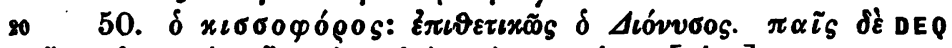

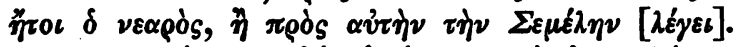

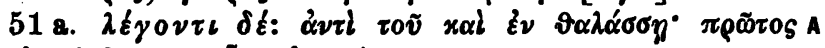
yà

8 sch. Aesch. Sept. 149. Hes. 'O Lyc. 122522 Eust. vit. Pind. 10, 8 Ab.

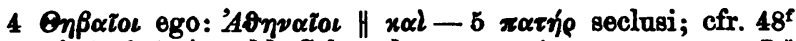

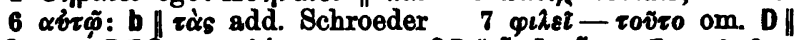

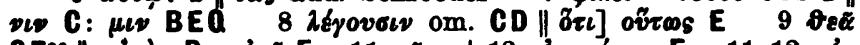

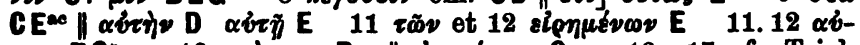

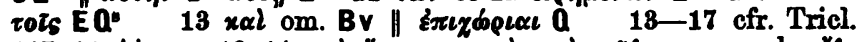

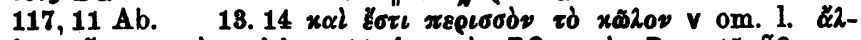

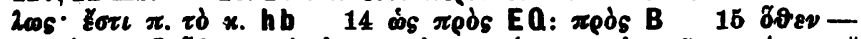

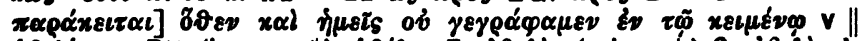

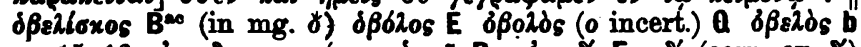

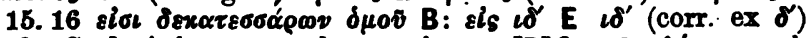

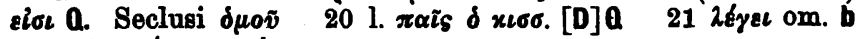

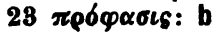




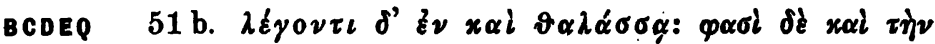

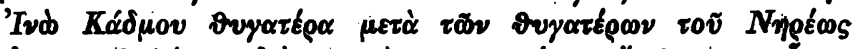

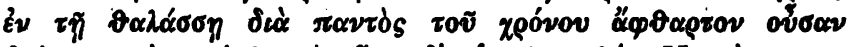

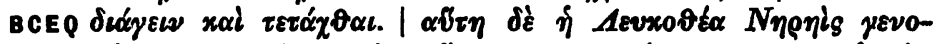

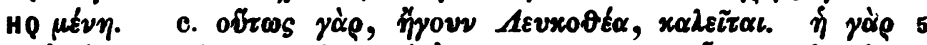

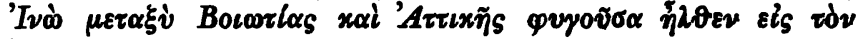

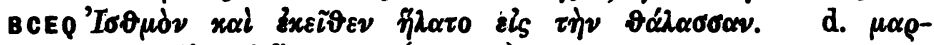

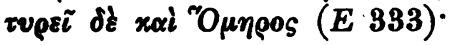

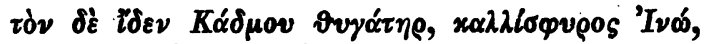

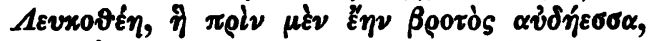

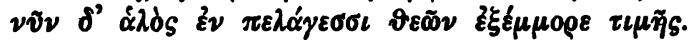

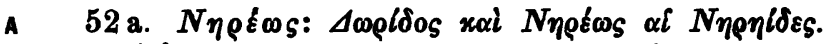

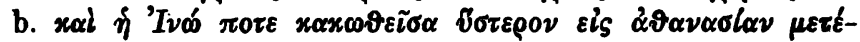

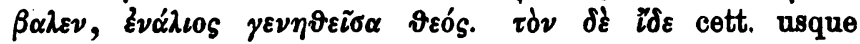
ad $\tau \iota \mu \tilde{\eta} s$.

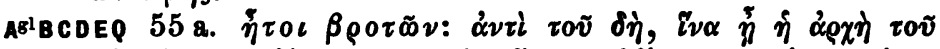

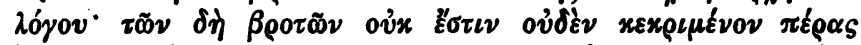

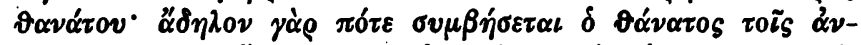

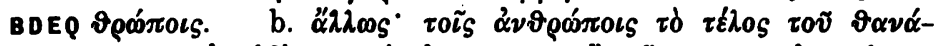

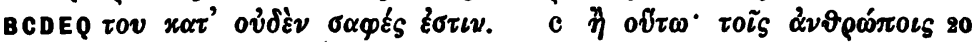

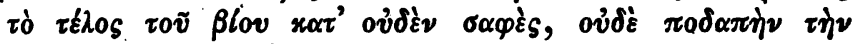

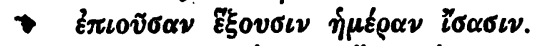

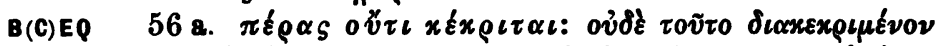

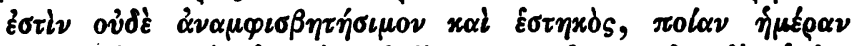

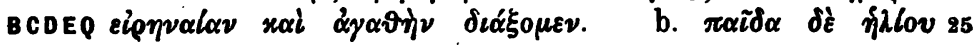

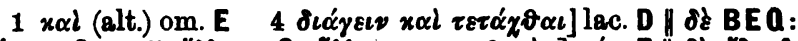

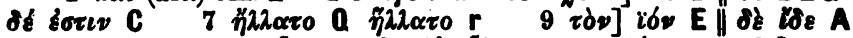

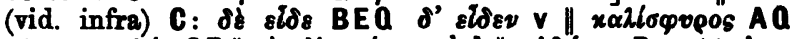

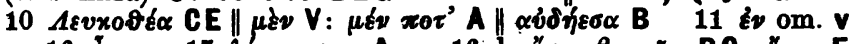

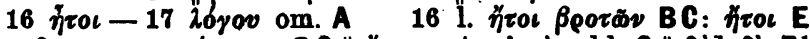

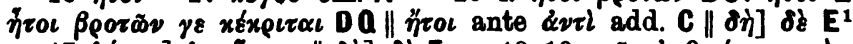

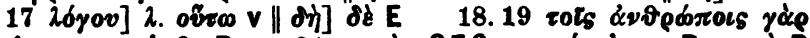

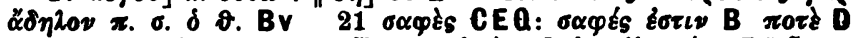

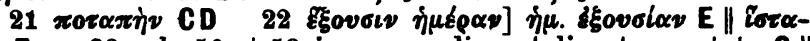
ouv E 23 sch. 56 et 58 inverso ordine et licenter mutata C \|

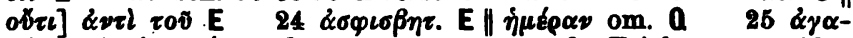

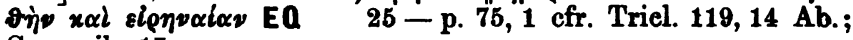
Germ. ib. 17 


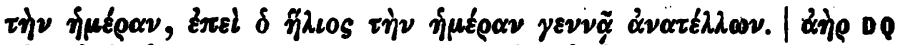

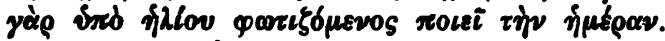

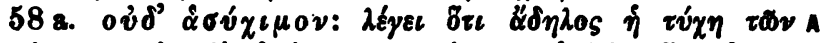

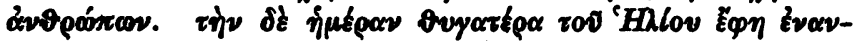

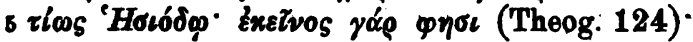

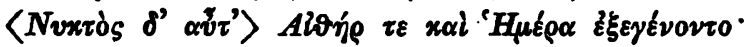

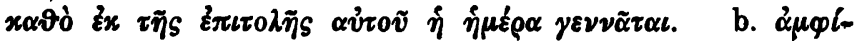

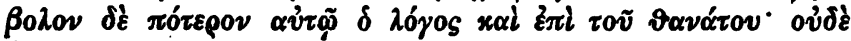

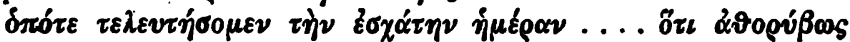

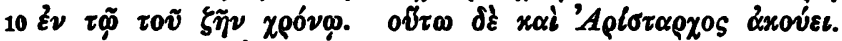

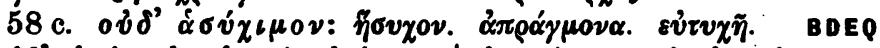

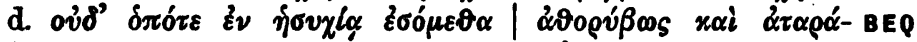

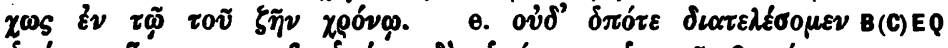

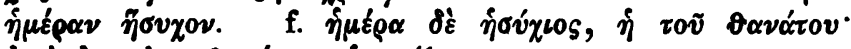

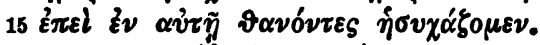

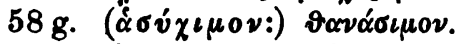

Bs!

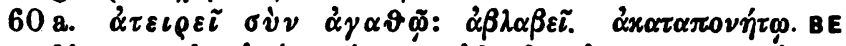

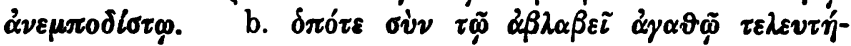

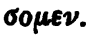

20

61. ( $\left(\varepsilon \varepsilon \lambda \varepsilon v \tau \alpha^{\prime} \sigma o \mu \varepsilon \nu:\right) \pi \lambda \eta \varrho \omega ́ \sigma o \mu \varepsilon \nu$.

$B$ Bl

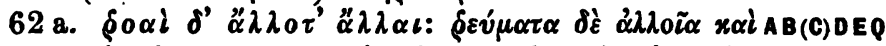

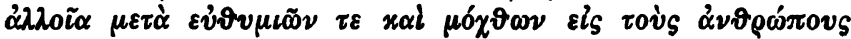

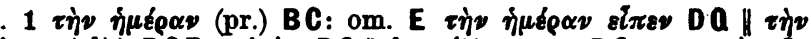

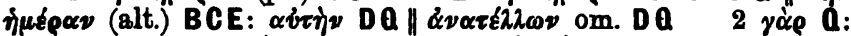

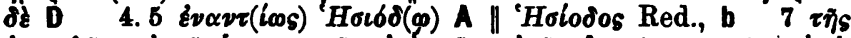

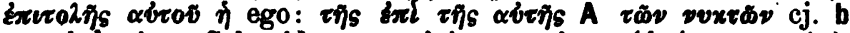

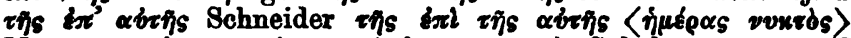

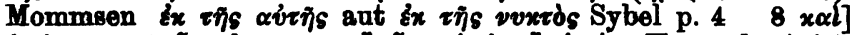

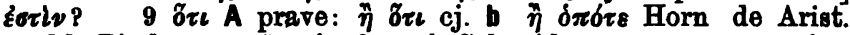
stadd. Pind. p. 15 ||

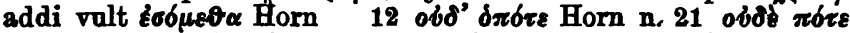

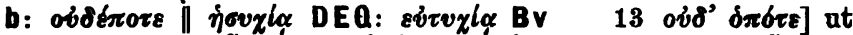

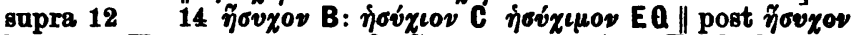
lac. stat. Horn 14-16 efr. Germ. 119, 11 Ab.; Tricl. ib. 13

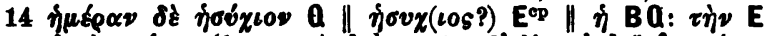

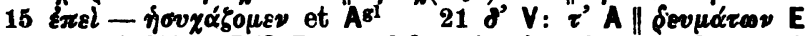

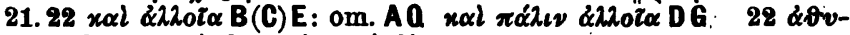
$\mu \varepsilon \tilde{\omega} \nu$ A $\| \tau \varepsilon x \alpha \iota$ A: $x \alpha \iota \mu \varepsilon \tau \dot{\alpha}$ V 


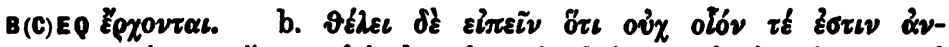

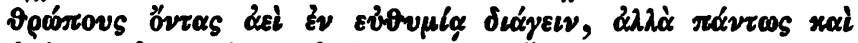

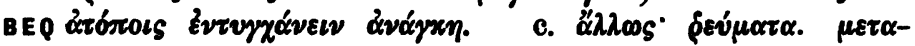

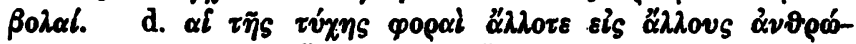

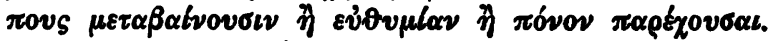

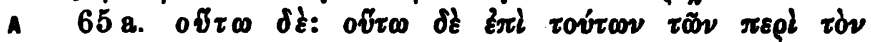

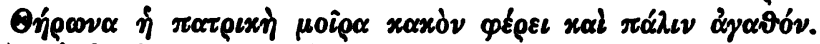

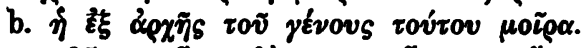

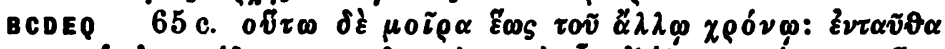

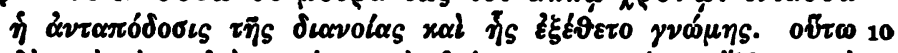

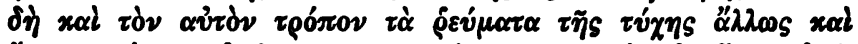

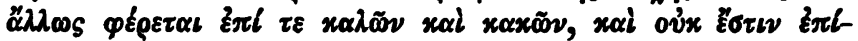

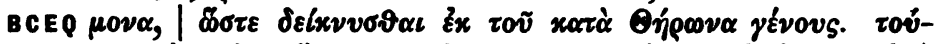

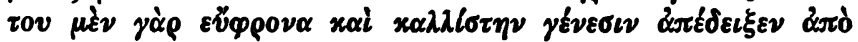

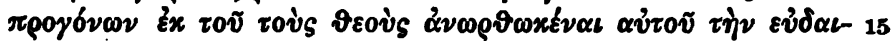

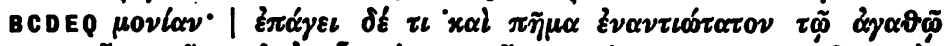

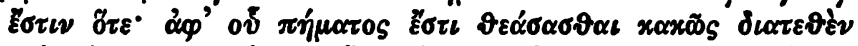

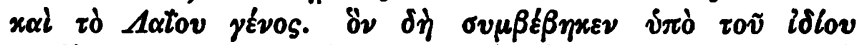

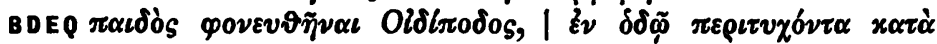

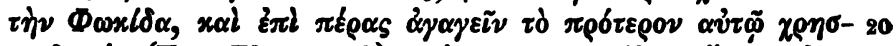

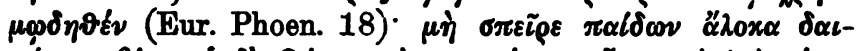

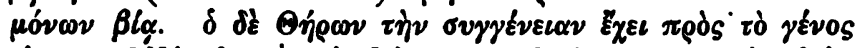

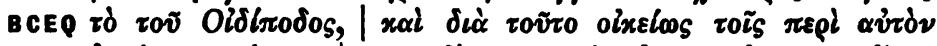

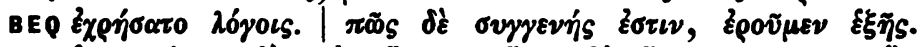

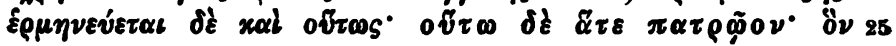

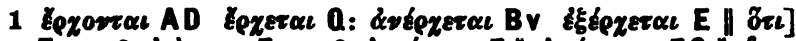

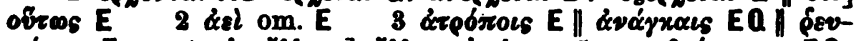

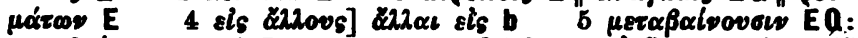

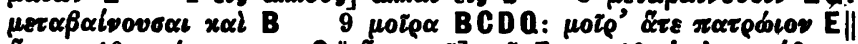

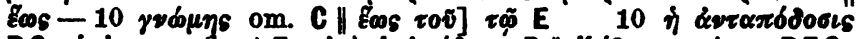

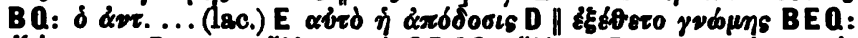

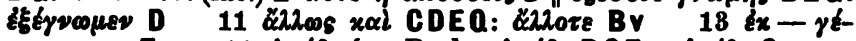

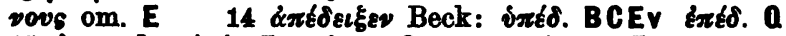

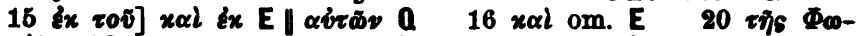

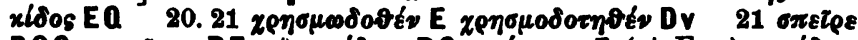

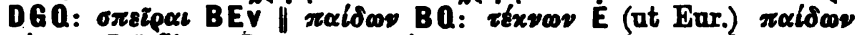

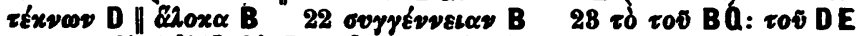

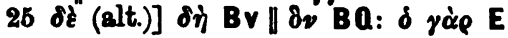




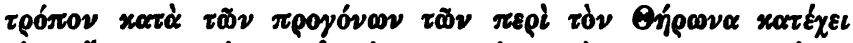

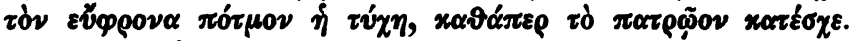

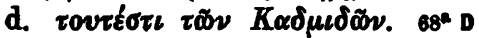

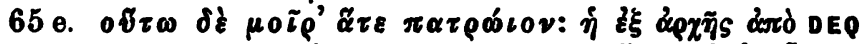

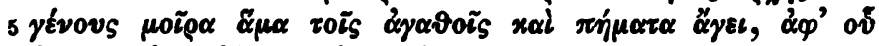

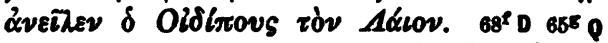

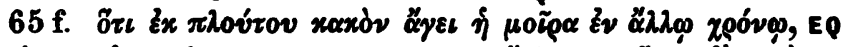

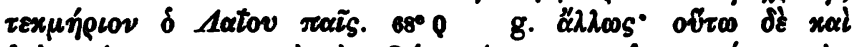

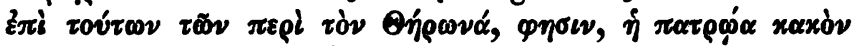
10 ă

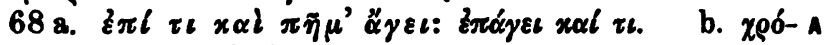

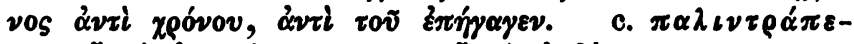

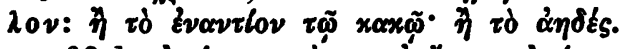

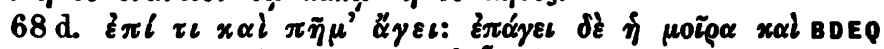

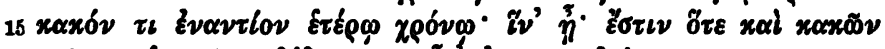

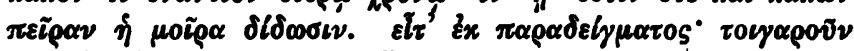

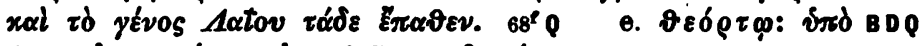

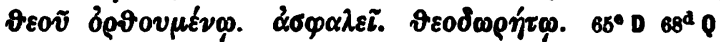

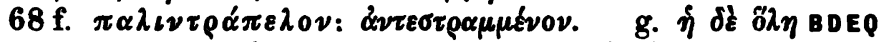

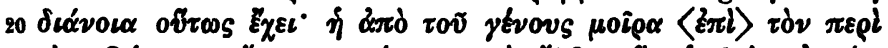

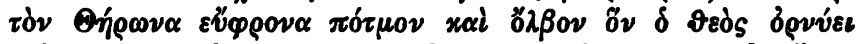

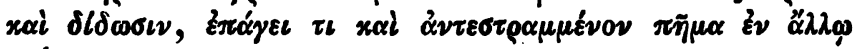

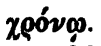

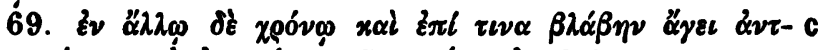

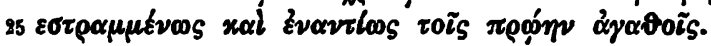

19 Suid. Phot. lex Bachm. 326, 30

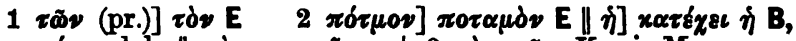

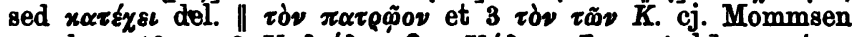

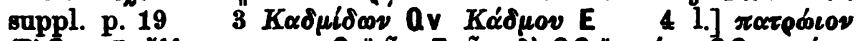

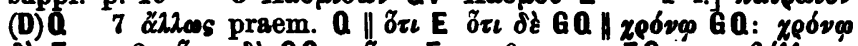

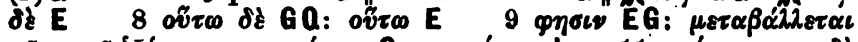

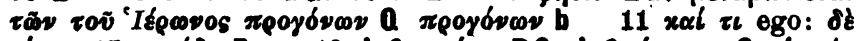

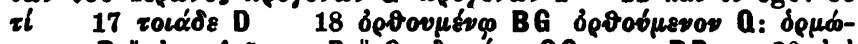

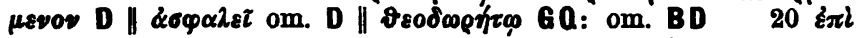

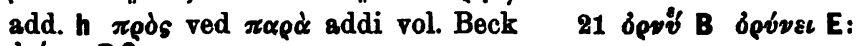
beivec Dav 


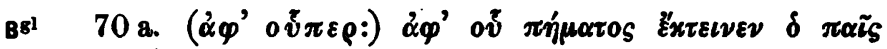
$\tau \grave{\nu} \nu \pi \alpha \tau \varepsilon \dot{\rho} \rho \alpha$.

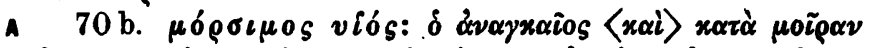

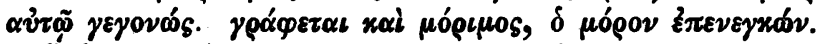

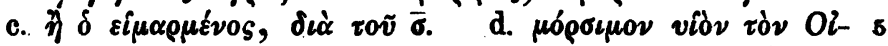

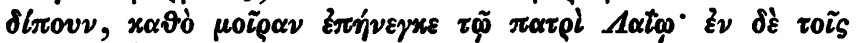

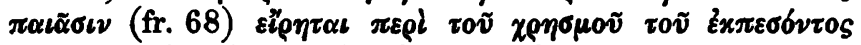

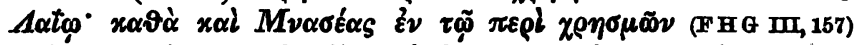

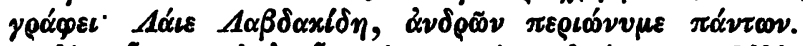

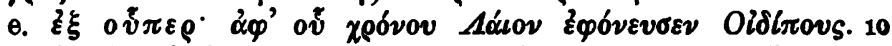

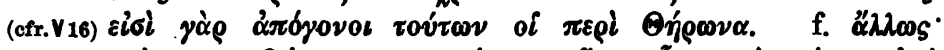

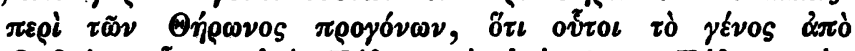

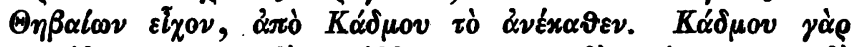

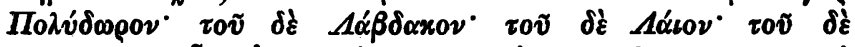

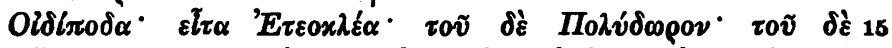

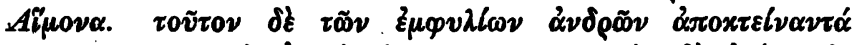

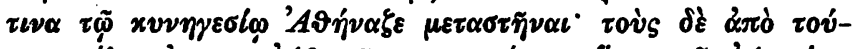

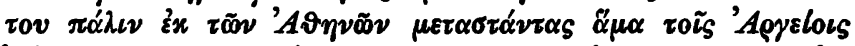

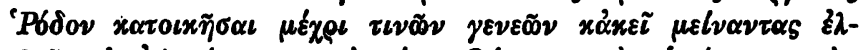

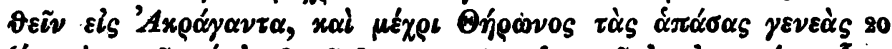

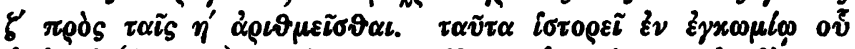

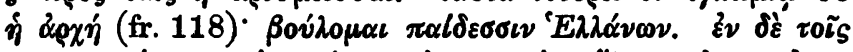

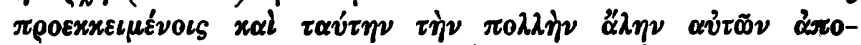

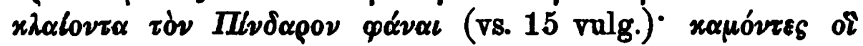

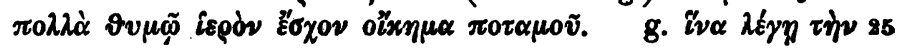

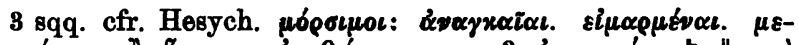

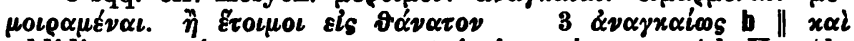

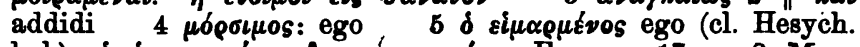

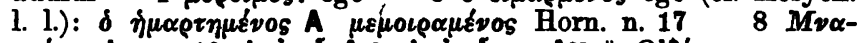

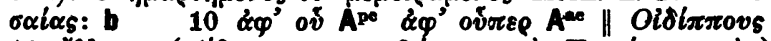

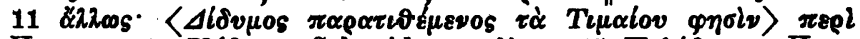
Horn 13 Kóduov: Schneider ex V 15 Modudopos: Horn

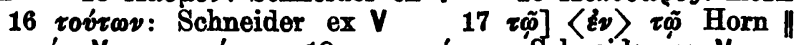

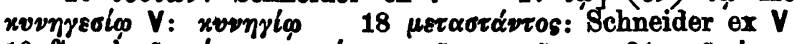

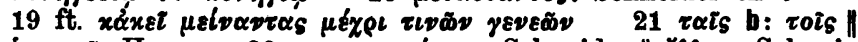

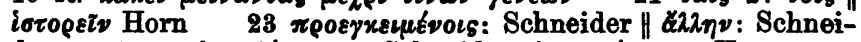

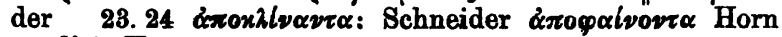
25 dist. Horn 


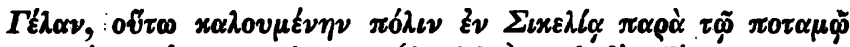

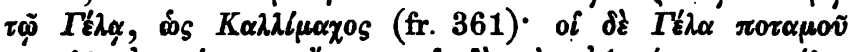

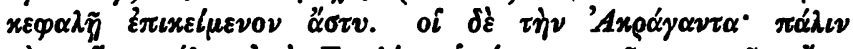

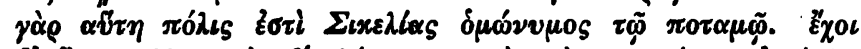

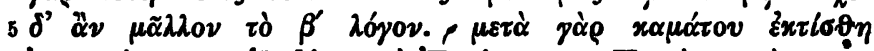

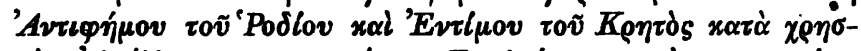

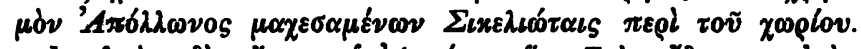

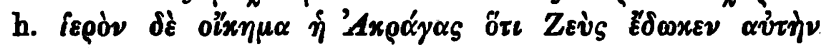

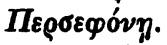

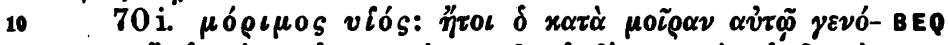

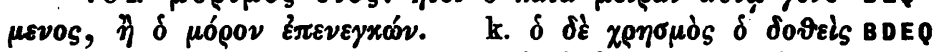

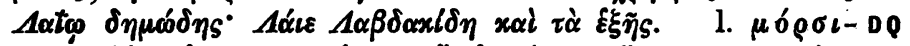

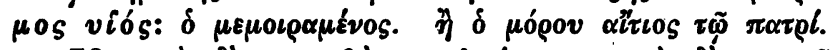

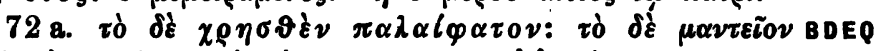

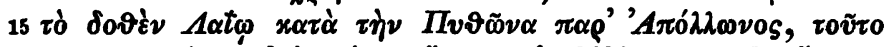

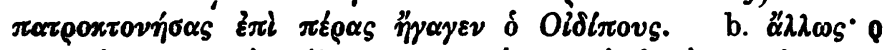

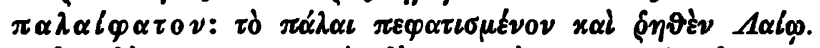

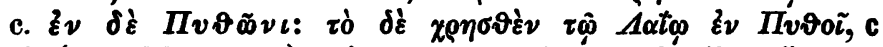

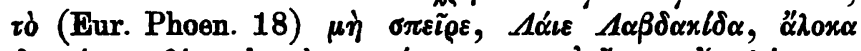

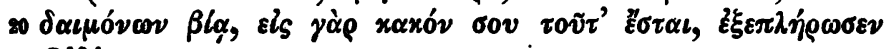
Oldiscovs.

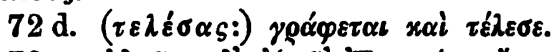

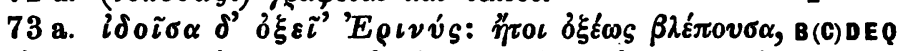

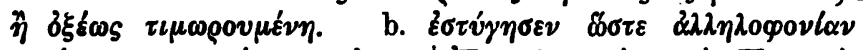

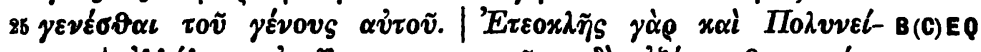

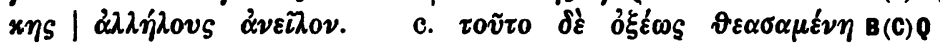

11 anth. Pal. 14, 67. hyp. Soph. O. T. et Eur. Phoen.

$2 \delta \xi \Gamma \lambda_{\alpha} V: \delta^{\prime} d y \in \lambda_{\alpha}$

5 ft. $\boldsymbol{\tau} \dot{\alpha} \alpha^{\prime}$, cl. V

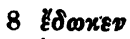

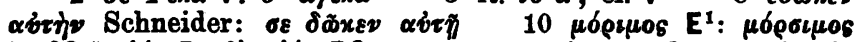

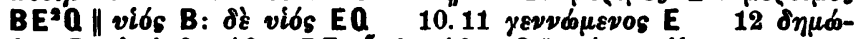

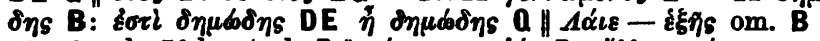

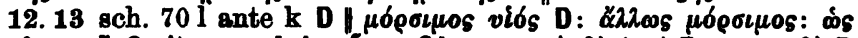

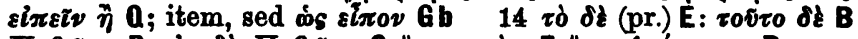

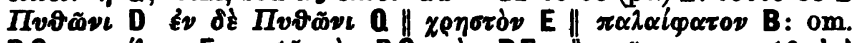

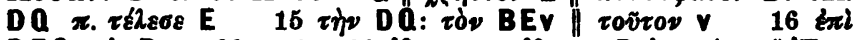

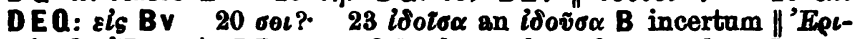
ขv́s C: 'Epเvขv́s BE om. DQ || sch. 73 frustula quaedam C 


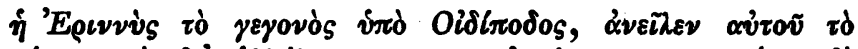

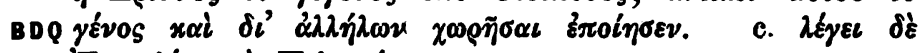

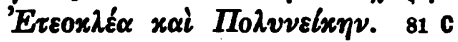

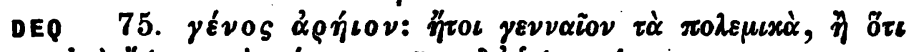

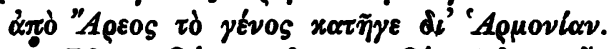

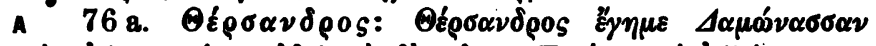

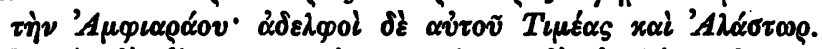

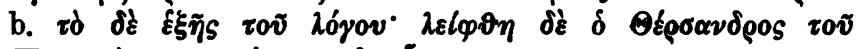

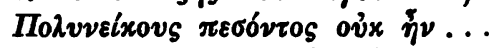

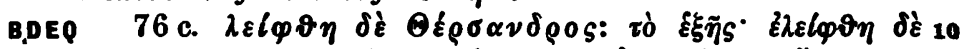

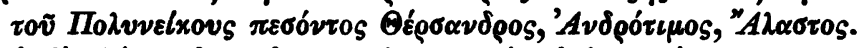

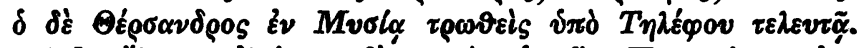

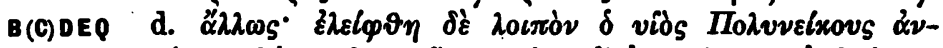

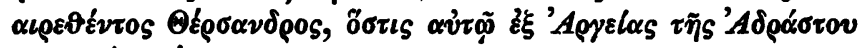

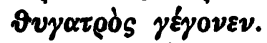

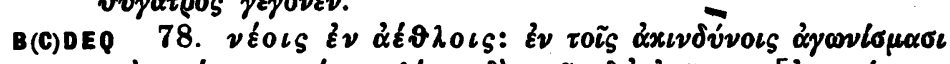

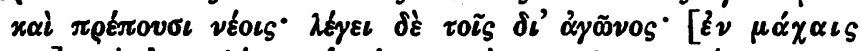

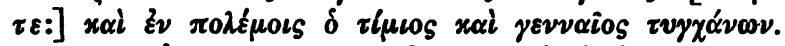

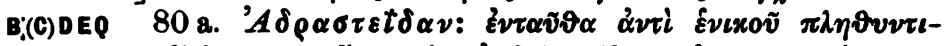

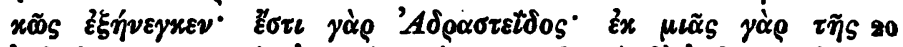

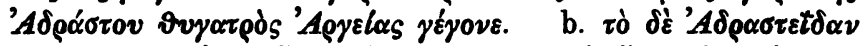

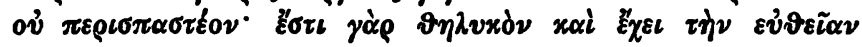

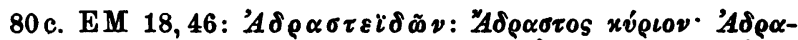

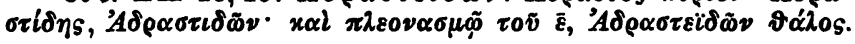

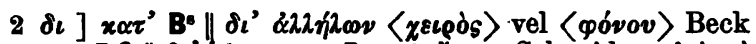

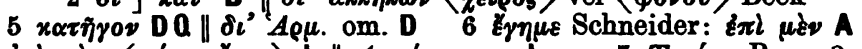

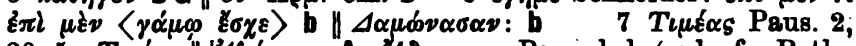

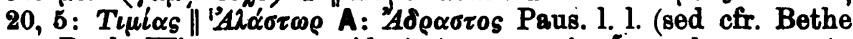
ap. Pauly-Wissowa 8. v. Alastor) 9 oix $\eta_{\nu}$ verba corrupta

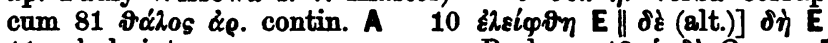
11 schol. integrum non esse susp. Beck $12 \delta \delta \xi \theta$. om. D\|

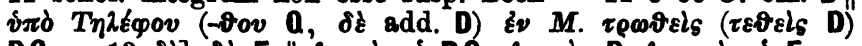

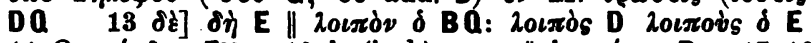

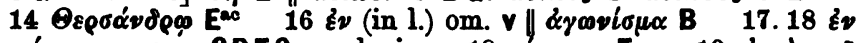

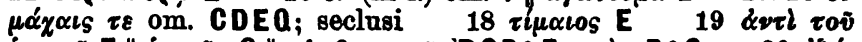

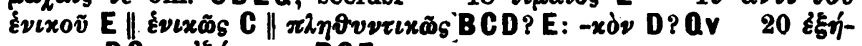

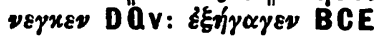




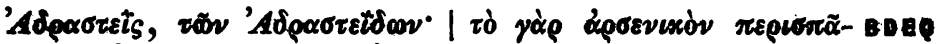

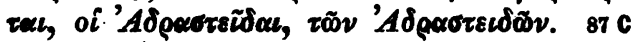

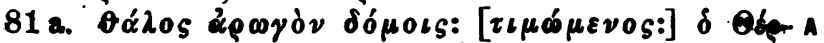

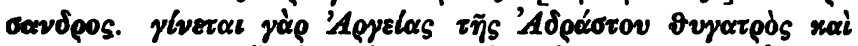

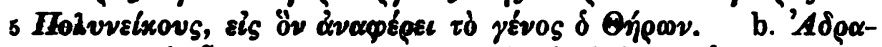

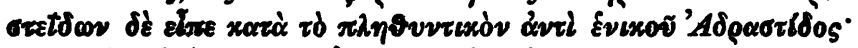

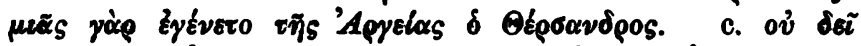

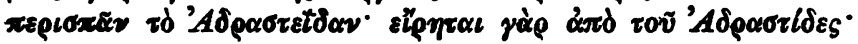
Lavodies.

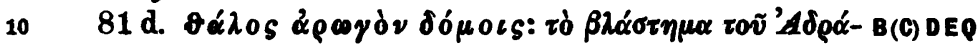

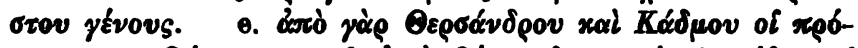

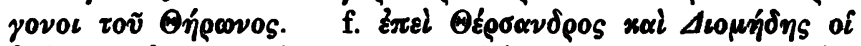

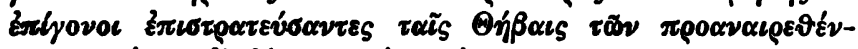

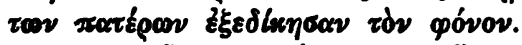

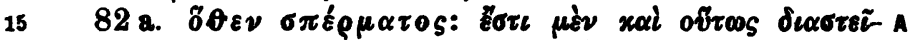

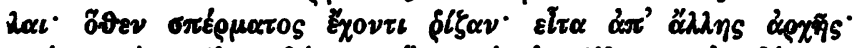

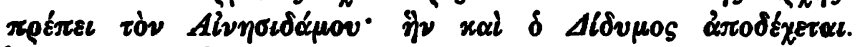

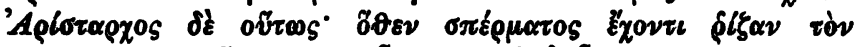

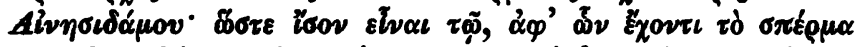

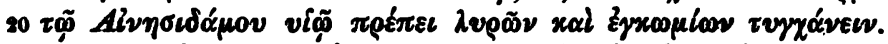

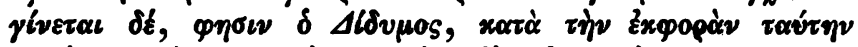

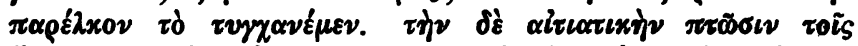

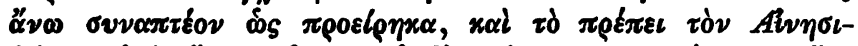

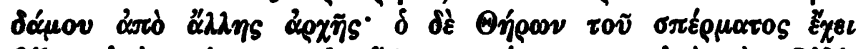

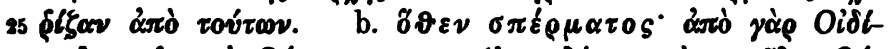

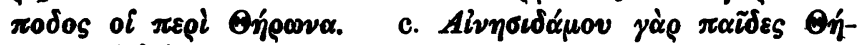

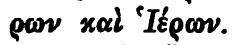

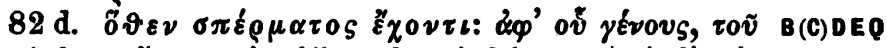

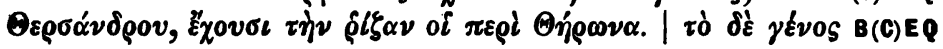

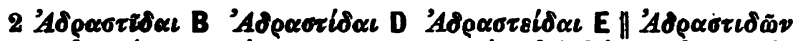

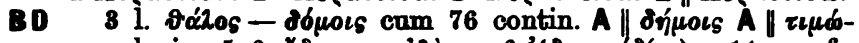

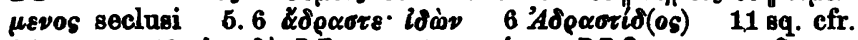

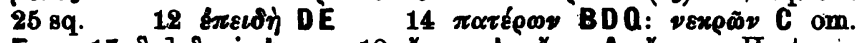

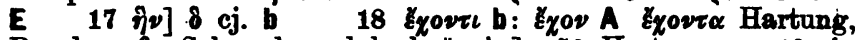

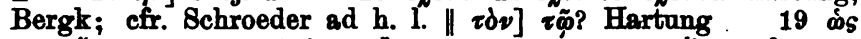

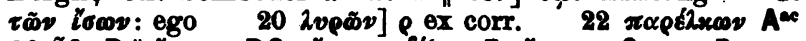

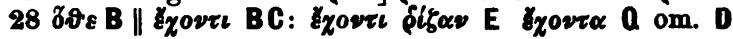

Schoz. ix Promaruic ed. Drachmann. 


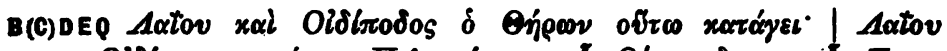

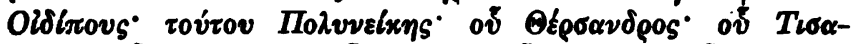

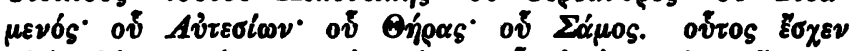

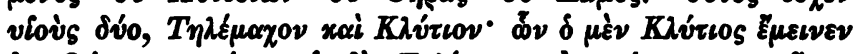

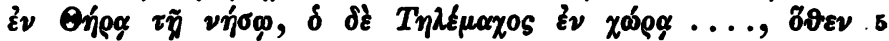

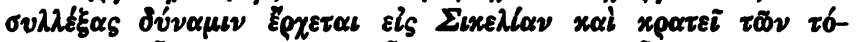

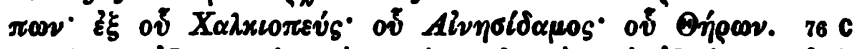

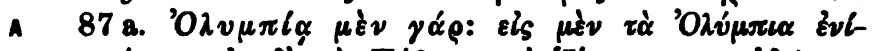

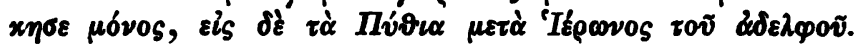

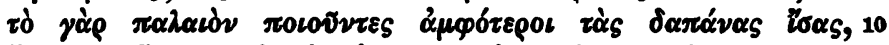

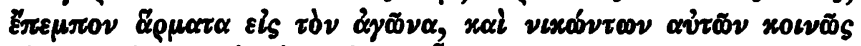

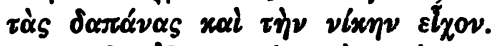

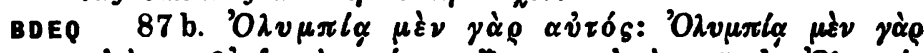

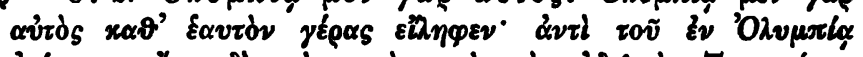

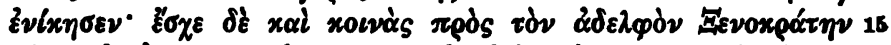

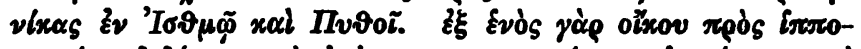

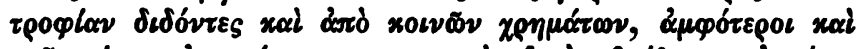

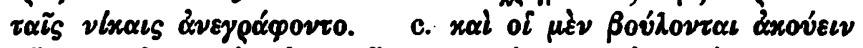

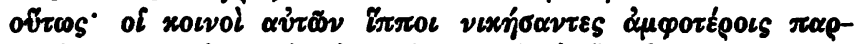

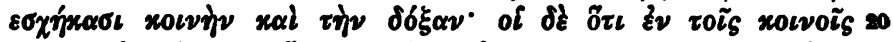

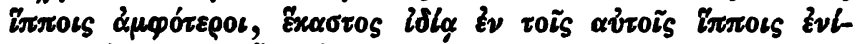

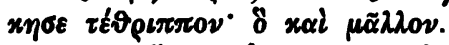

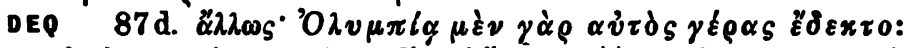

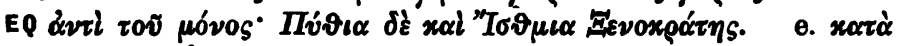

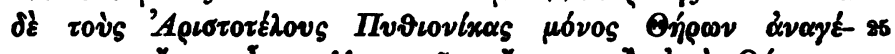

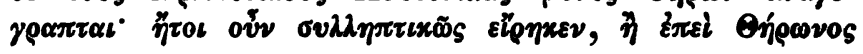

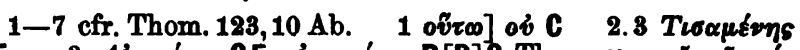

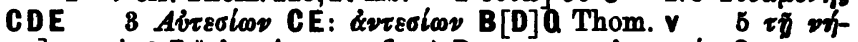

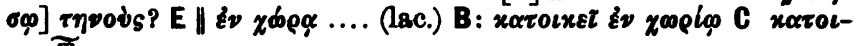

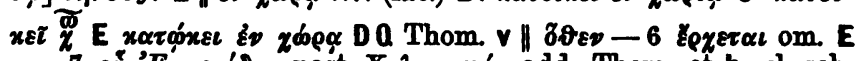

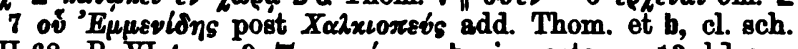
0. III 68. P. VI 49 Esvoxpórovs b vix recte 13 l.] om.

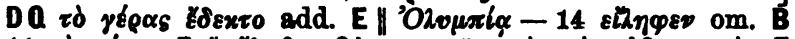

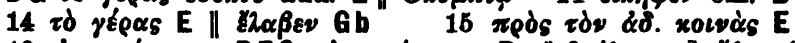

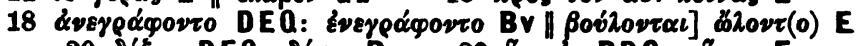

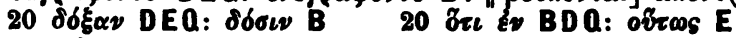

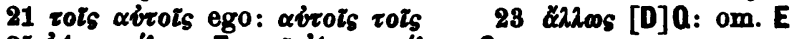

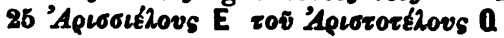




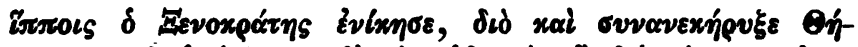

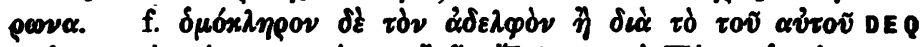

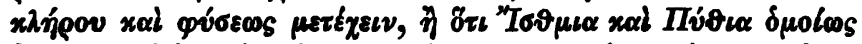

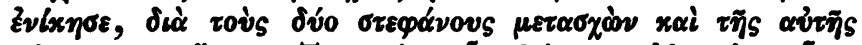

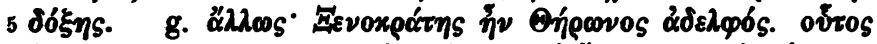

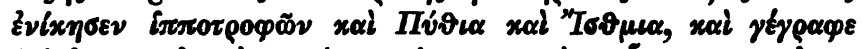

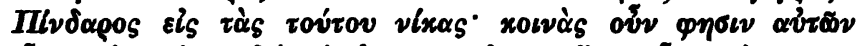

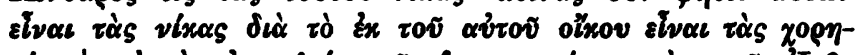

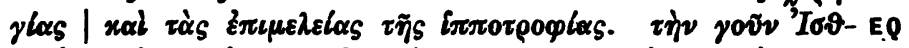

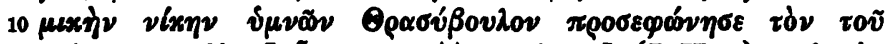

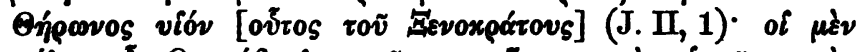

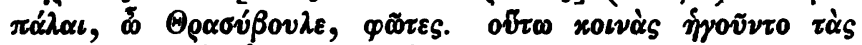

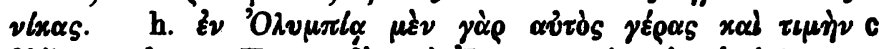

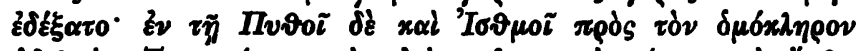

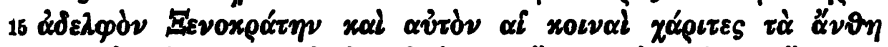

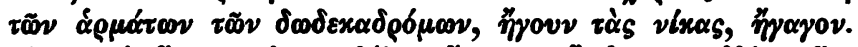
of xocvoi i

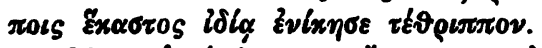

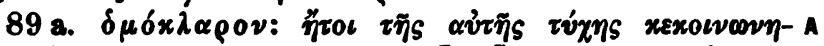

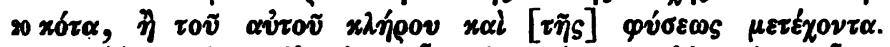

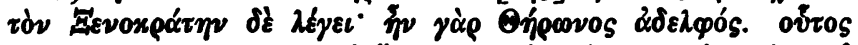

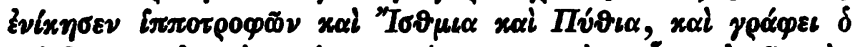

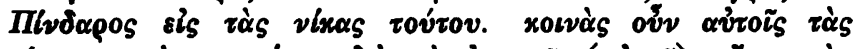

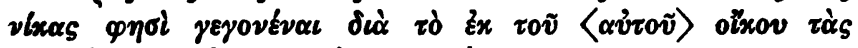

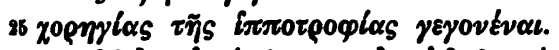

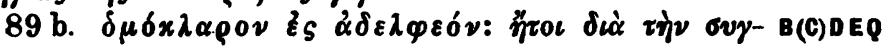

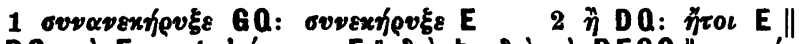

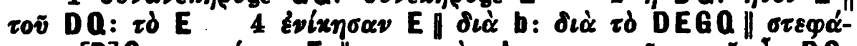

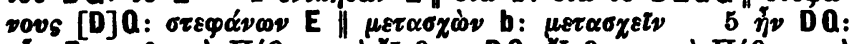

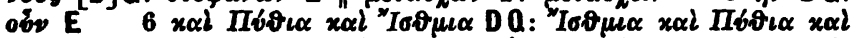

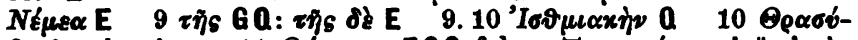

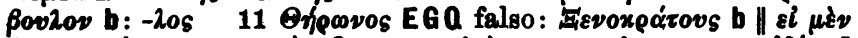

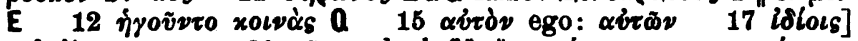

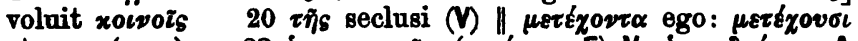

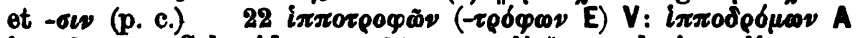

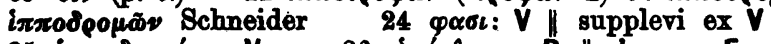

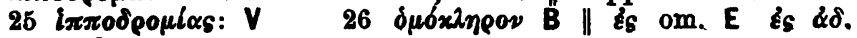
om. DQ 
YéveLóv

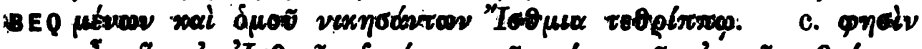

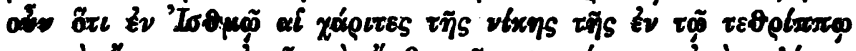

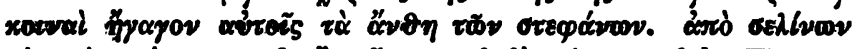

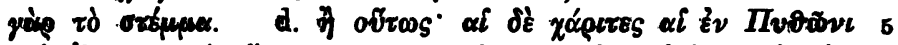

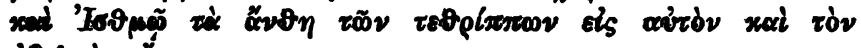

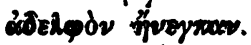

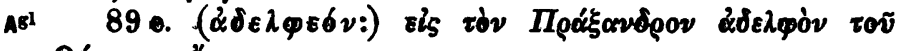
Oxpowes ővra.

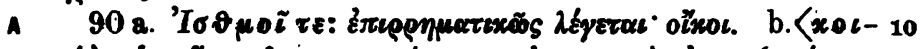

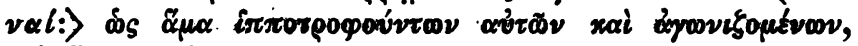
mod \&́pa vaxcóveov.

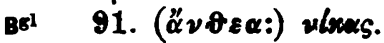

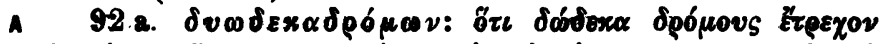

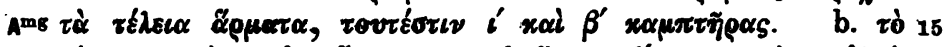

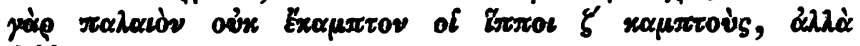
Sowdexo.

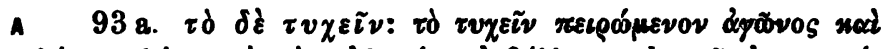

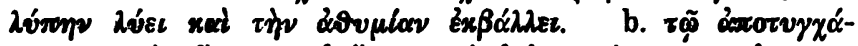

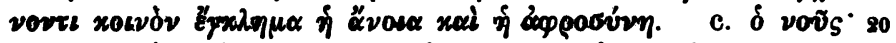

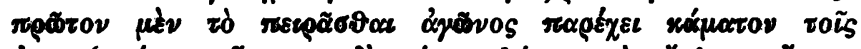

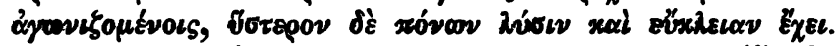

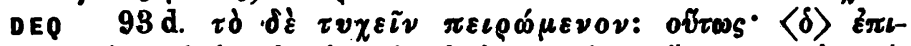

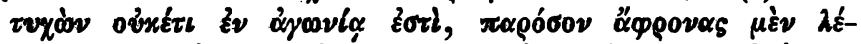

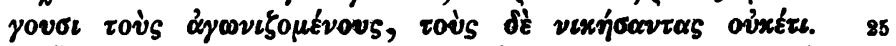

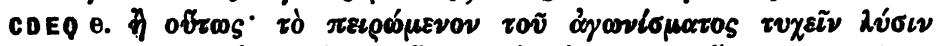

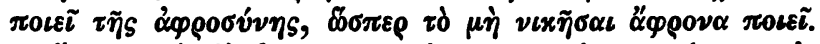

BEQ f. "ैג

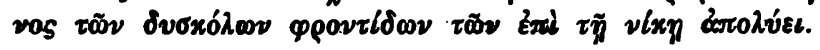

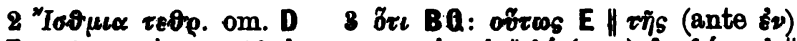

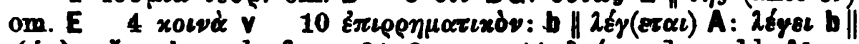

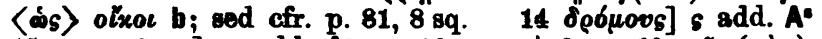

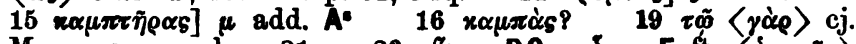

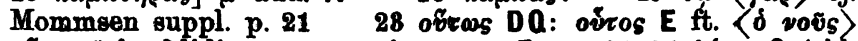

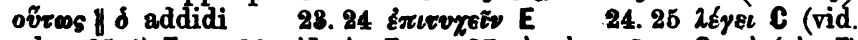

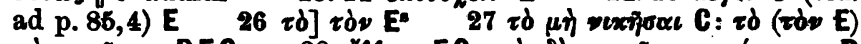

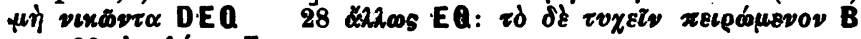

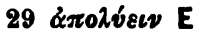




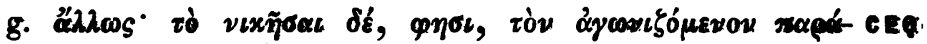

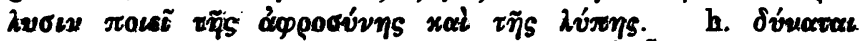

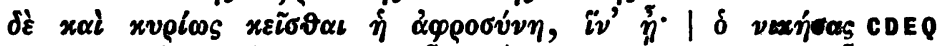

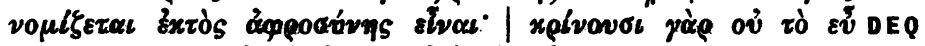

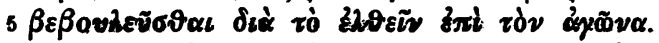

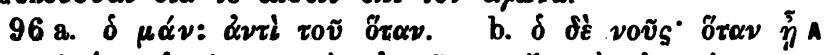

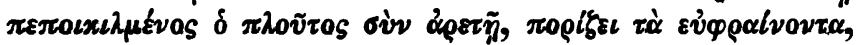

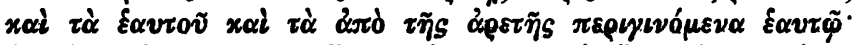

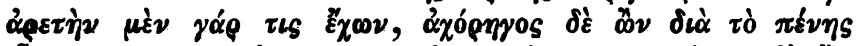

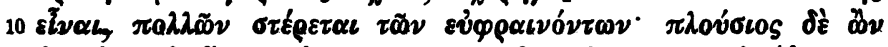

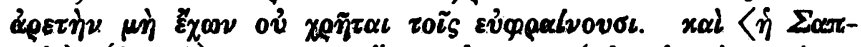

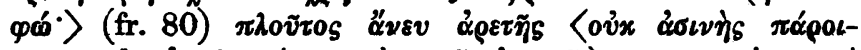

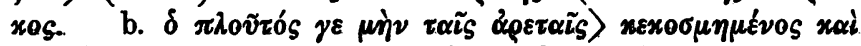

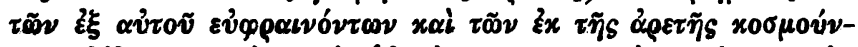

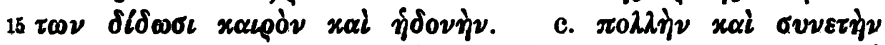

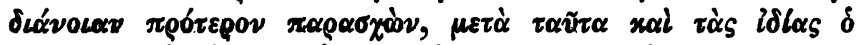

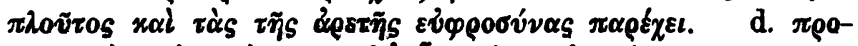

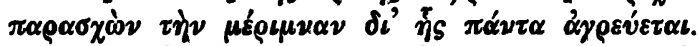

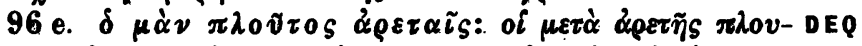

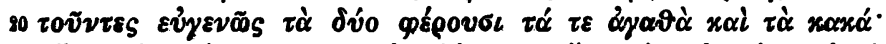

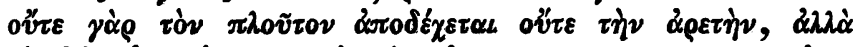

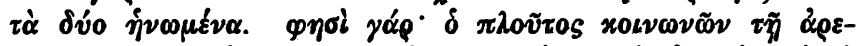

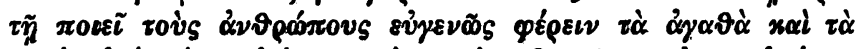

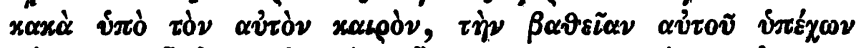

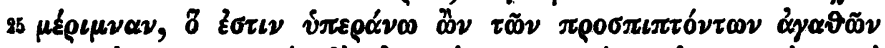

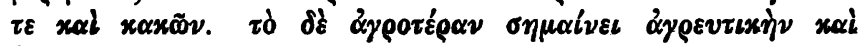

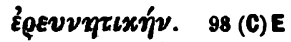

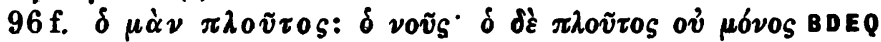

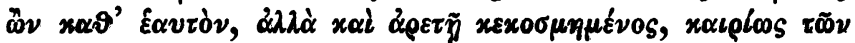

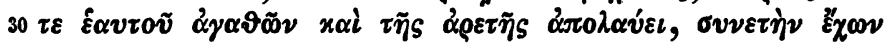

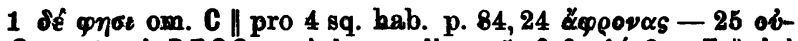

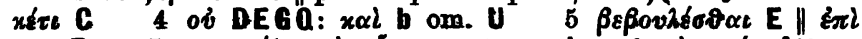

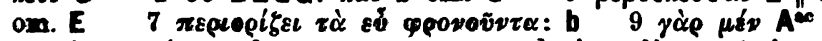
10 Expponoúverov $A^{\text {ac }} 11.12 .13$ supplevi ex $V 19$ descaĩs

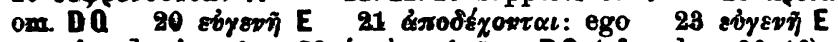

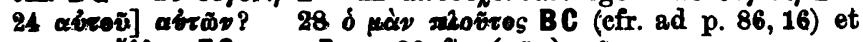

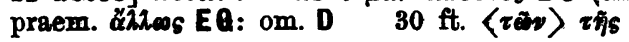




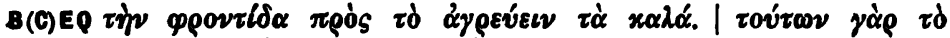

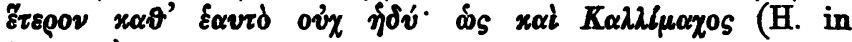
Jov. 95)

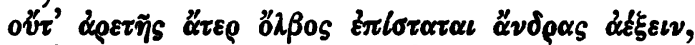

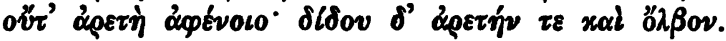

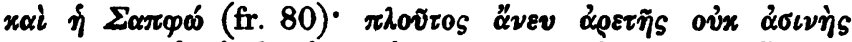

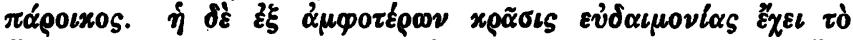

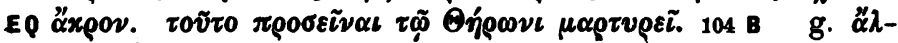

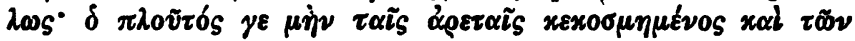

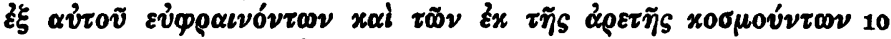

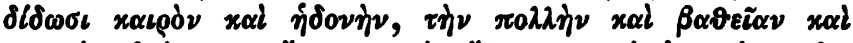

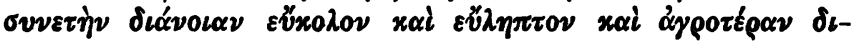

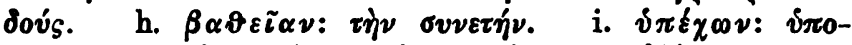

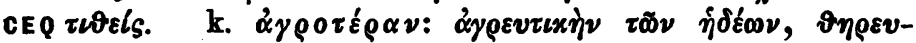

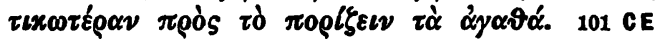

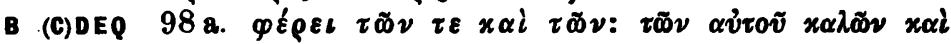

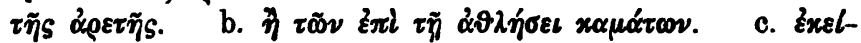

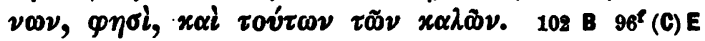

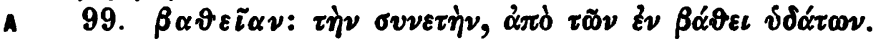

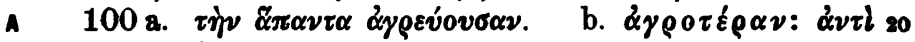

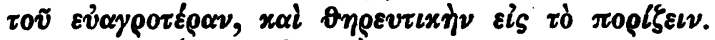

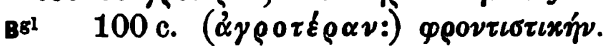

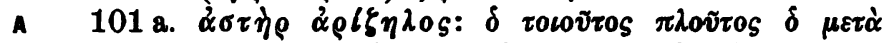

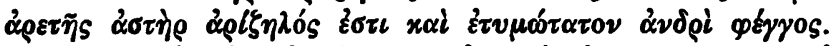

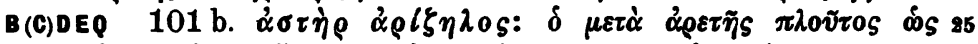

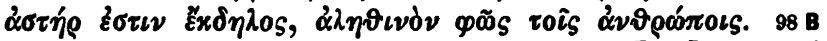

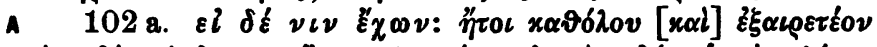

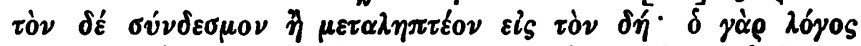

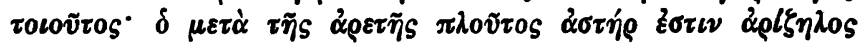

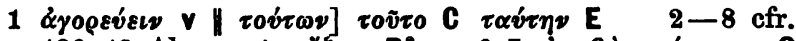

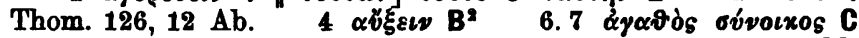

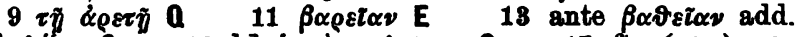

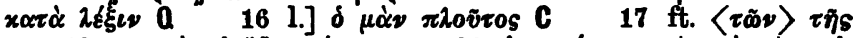

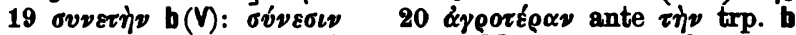

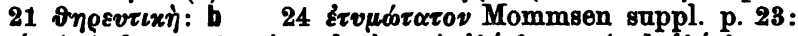

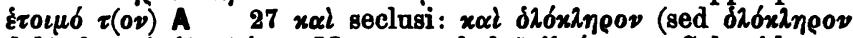

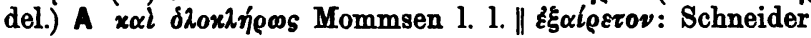




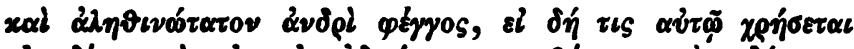

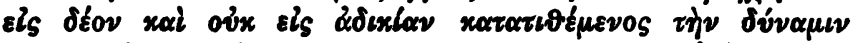

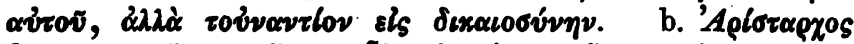

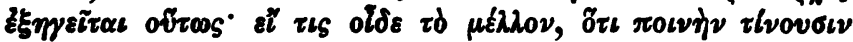

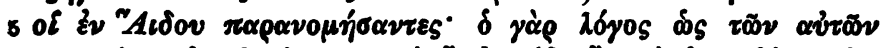

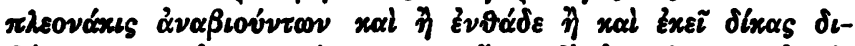

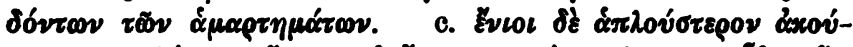

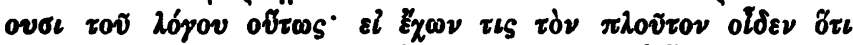

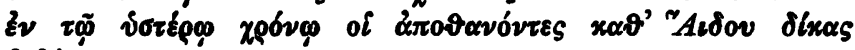

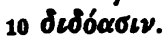

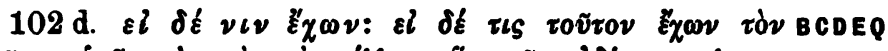

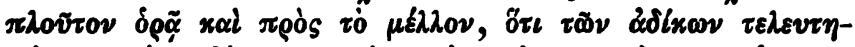

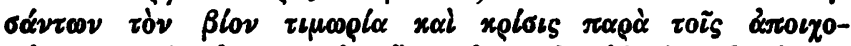

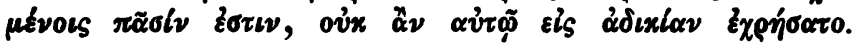

$15106 \mathrm{~B} 105 \mathrm{C}$

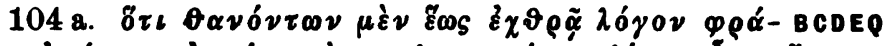

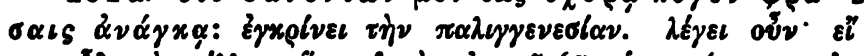

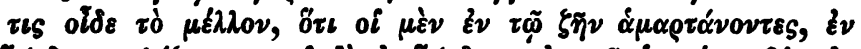

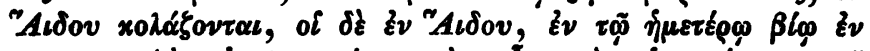

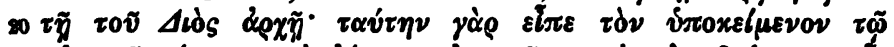

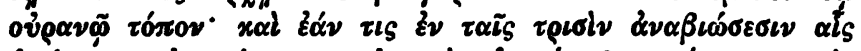

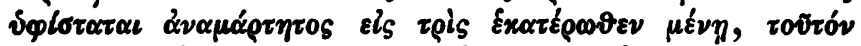

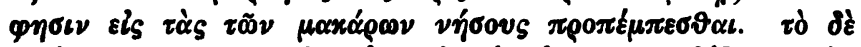

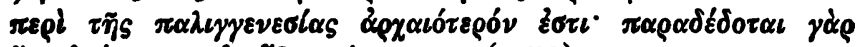

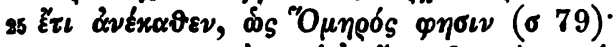

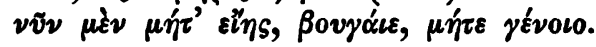

23 Porphyr. ad o 79. Eust. ad Od. 1838, 40

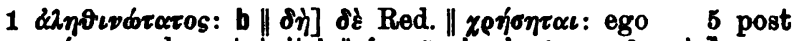

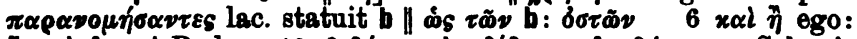

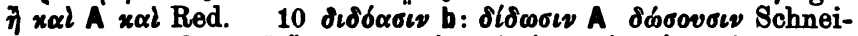

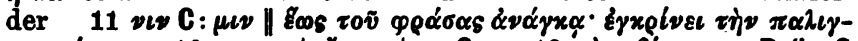
yeveolov ex $16 \mathrm{sq}$. post Exwo trp. C 13 idv plov om. B (in $\mathbf{a}$

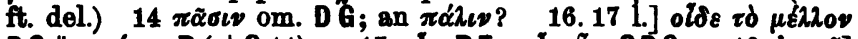
DQ

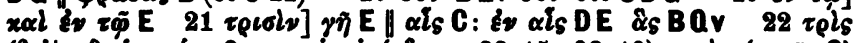

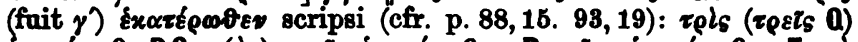

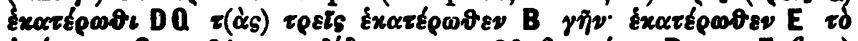

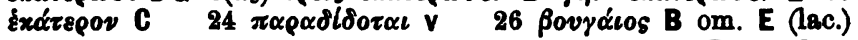




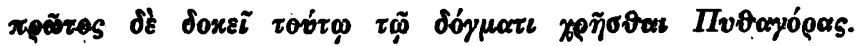
$113 \mathrm{C}$

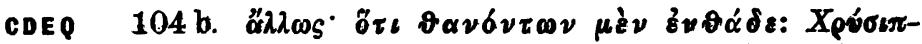

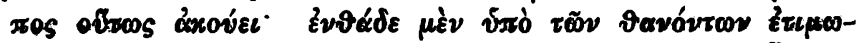

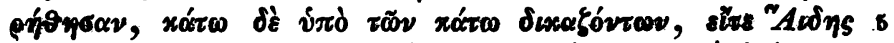

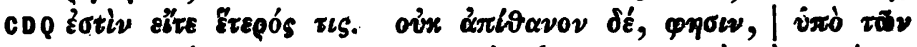

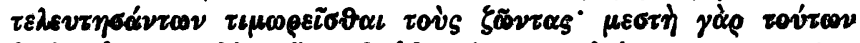

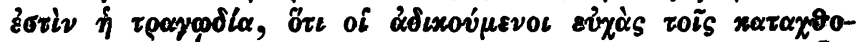

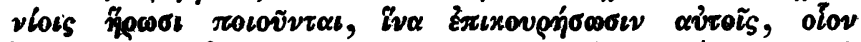

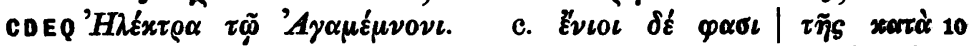

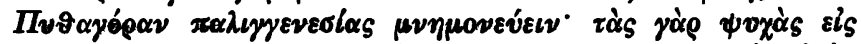

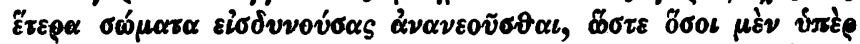

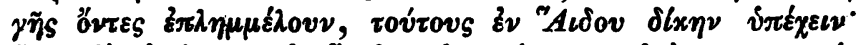

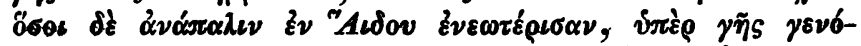

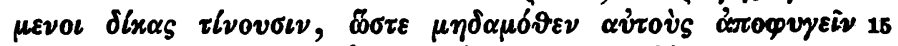

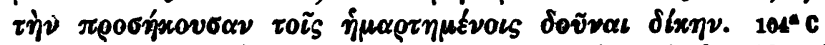

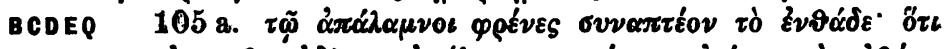

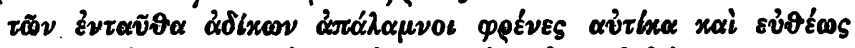

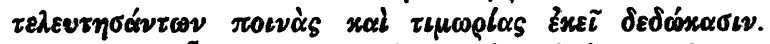

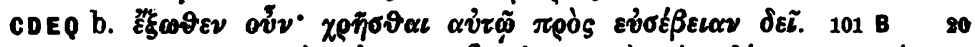

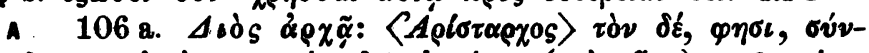

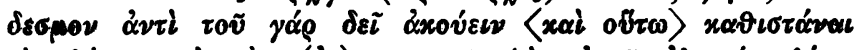

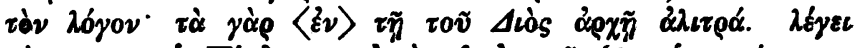

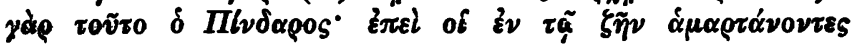

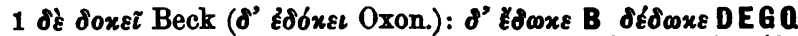

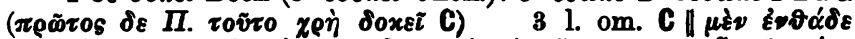

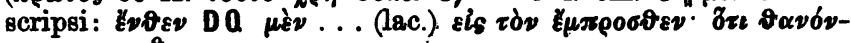

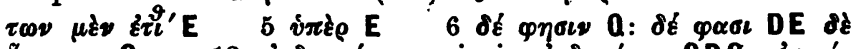

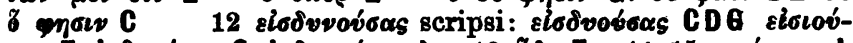

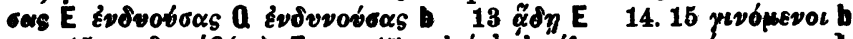

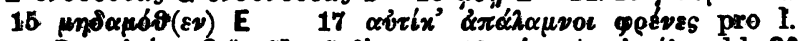

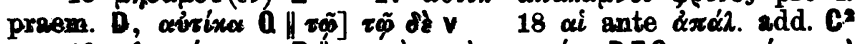

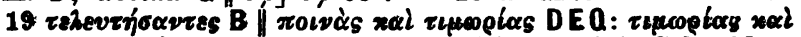
rocò̀es B upwolos C 21 sqq. Aristarchi esse vidit Schneider; appl. JLHeiberg vòv dé

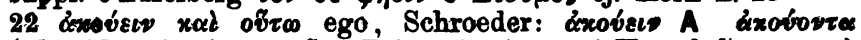

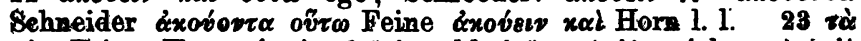

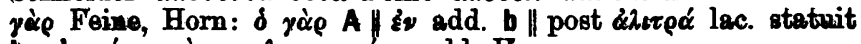

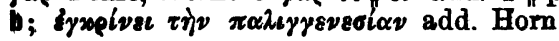




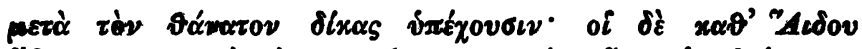

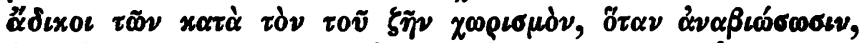

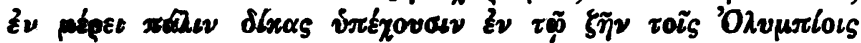

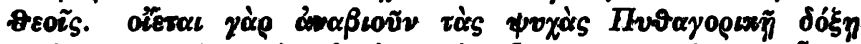

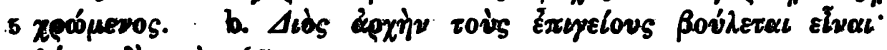
$\lambda \dot{e}$ -

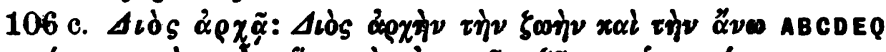

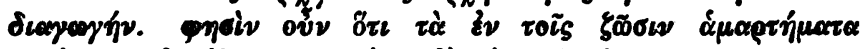

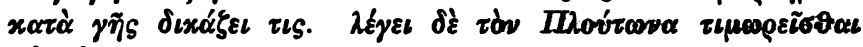
20 ariveds. 108 BDEQ

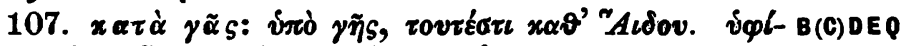

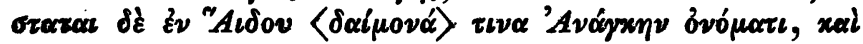

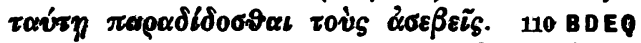

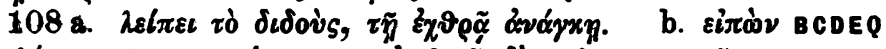

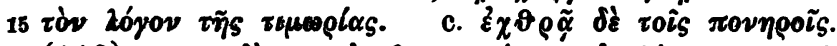

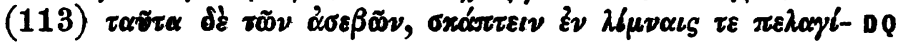

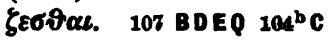

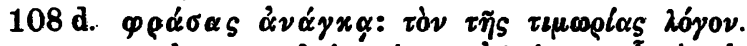

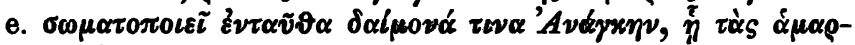

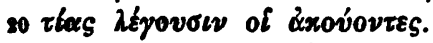

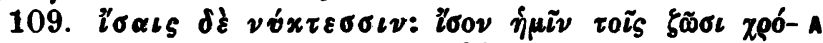

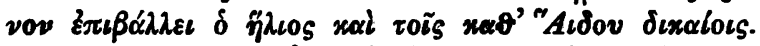

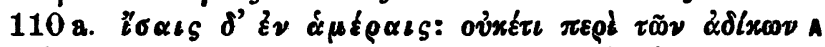

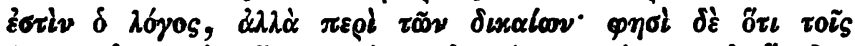

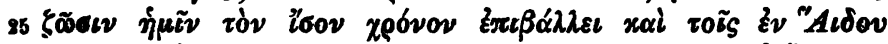

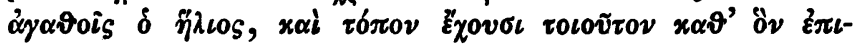

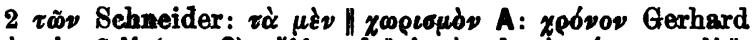

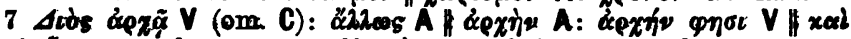

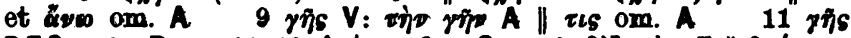

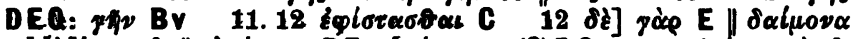

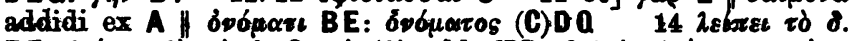

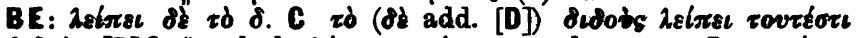

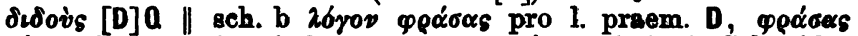
26 yov Q ${ }_{15} \varepsilon_{2} \rho \dot{\alpha}$ CD 18 post 26jov distinxit Schneider

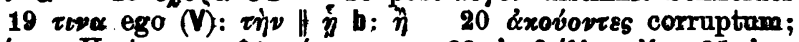

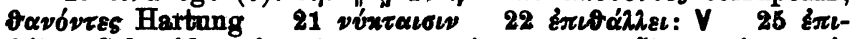

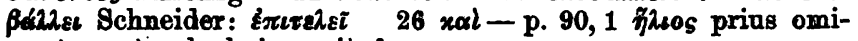
serat, postea h. l. inseruit A 


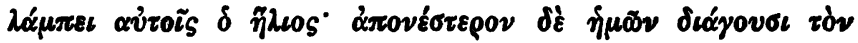
xpóvov.

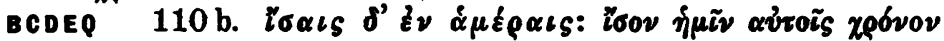

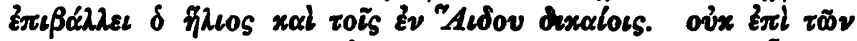

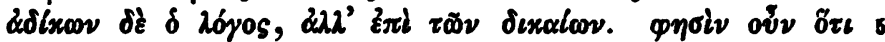

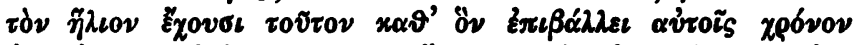

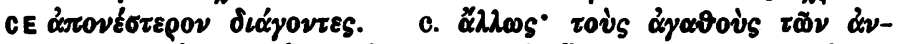

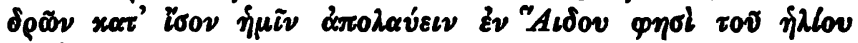

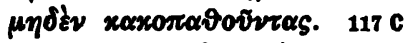

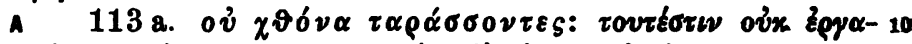

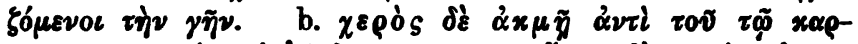

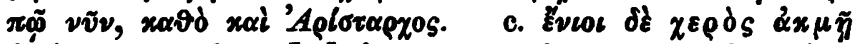

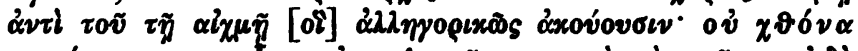

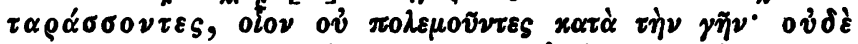

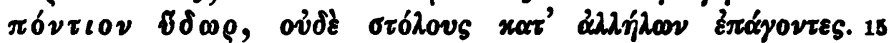

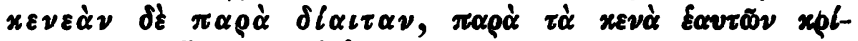

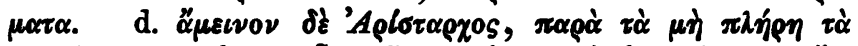

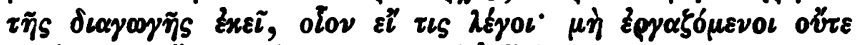

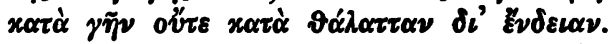

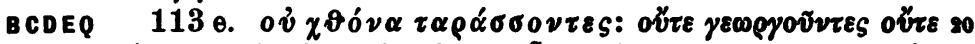

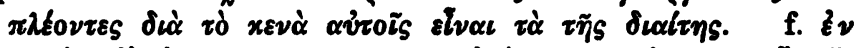

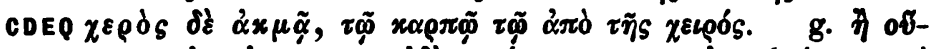

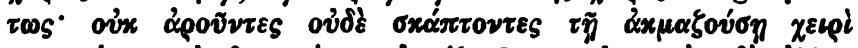

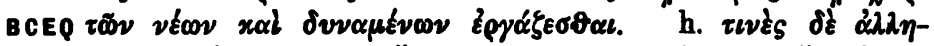

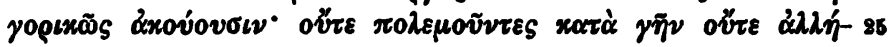

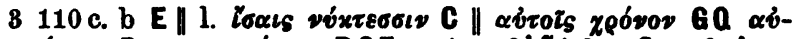

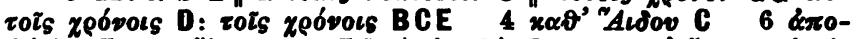

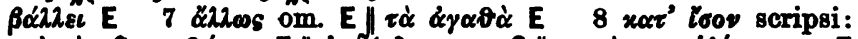

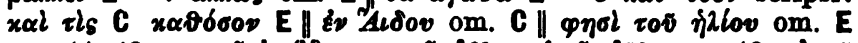
11. $12 x \alpha \rho \pi \tilde{\omega}$ b $(V): x \alpha \rho x \tilde{\omega} A^{20} \chi \alpha \lambda_{x \tilde{\varphi}} A^{p 0} \quad 13 \alpha l_{x \mu \tilde{y}}$

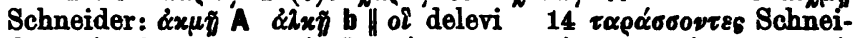

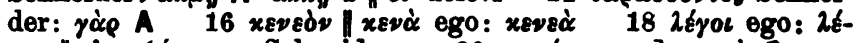

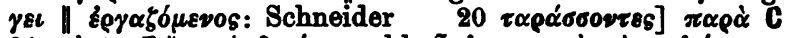

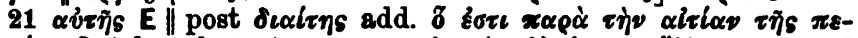

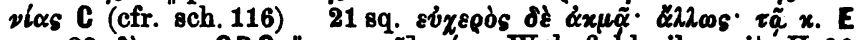

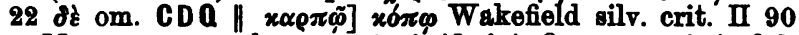

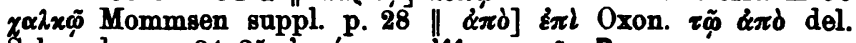

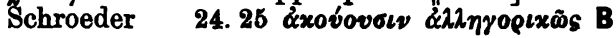




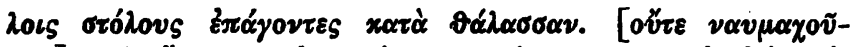

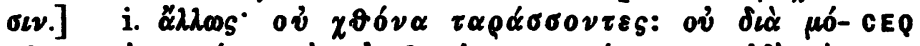

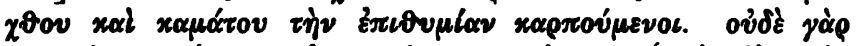

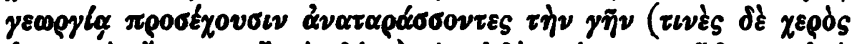

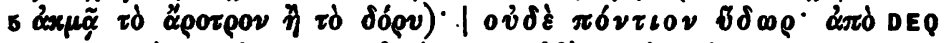

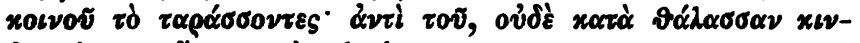

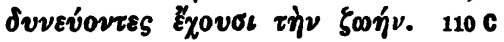

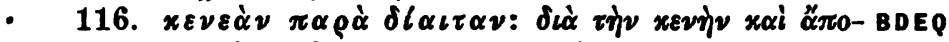

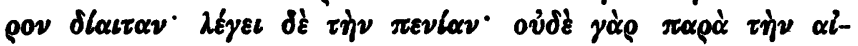

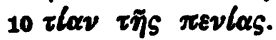

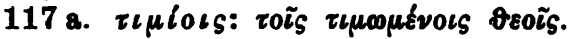

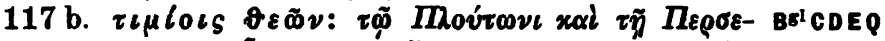

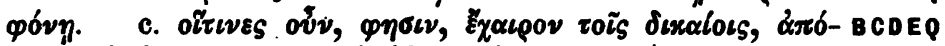

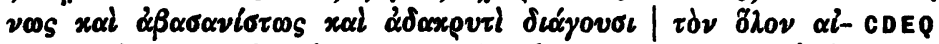

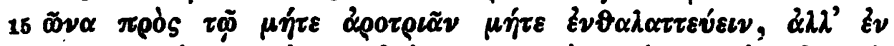

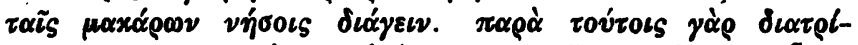

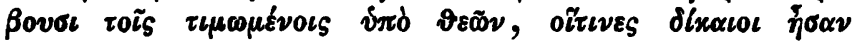

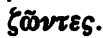

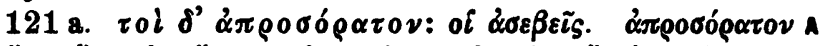

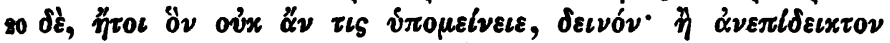

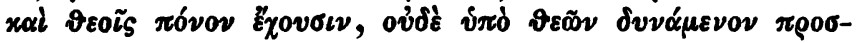

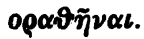

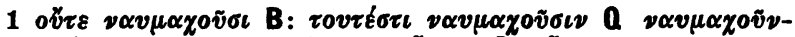

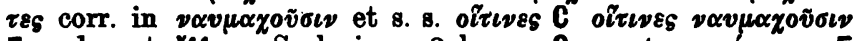

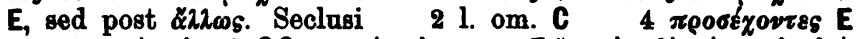

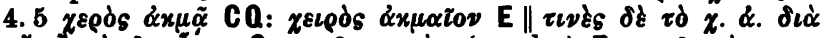

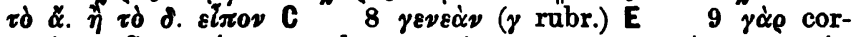

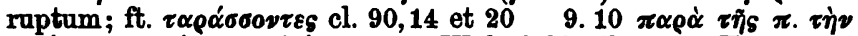
$\alpha i \tau i \alpha \nu \mathrm{E} \pi \varepsilon \rho i \tau^{2} \nu \alpha \alpha i \tau i \alpha \nu$ rĩs $\pi$. Wakefield silv. crit. II, $90 \pi \varepsilon \rho$

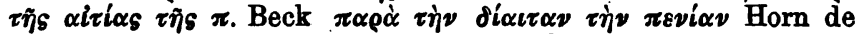
Ar. studd. Pind. n. $27 \quad 16 \tau \alpha \tau_{S}$ DEQ: $\tau \alpha \tau_{S} \tau \tilde{\omega} \nu$ C $\pi \alpha \rho \dot{\alpha}$

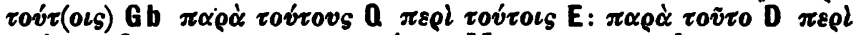
$\tau \alpha \delta \tau \alpha \iota s$ C $17 \tau o \tau_{S} \tau \iota \mu \omega \mu \xi \nu o \iota s$ Mommsen suppl. p. 29: $\tau \alpha \tilde{t}_{S}$

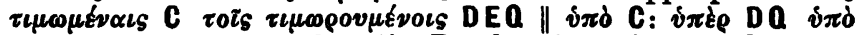

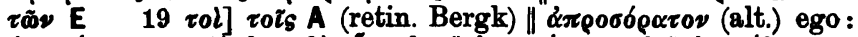

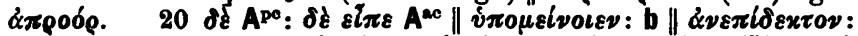

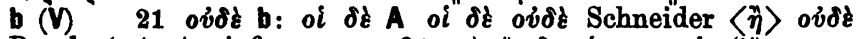
Bergk (retento infra $\pi \rho 00 \rho \alpha \vartheta \tilde{\eta} v \alpha \iota) \| \delta v v \alpha \dot{\mu} \mu \varepsilon v 0 \iota:$ b (V) 21. $22 \pi \rho 00 \rho \alpha \vartheta \tilde{\eta} \nu \alpha \iota: V$ 


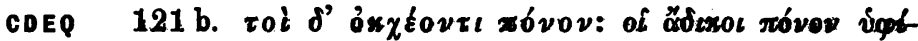

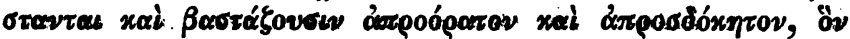

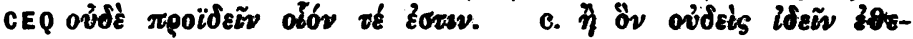

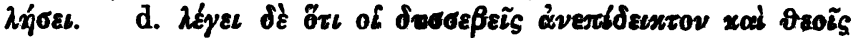

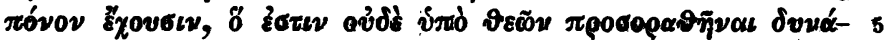

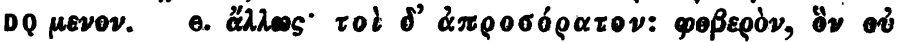
$\tau 0 \lambda \mu \tilde{\alpha}$ $\tau \iota s \pi \rho 0 \sigma \beta \lambda \hat{\psi} \psi \alpha \iota$.

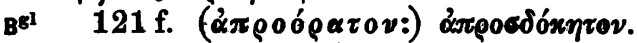

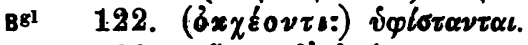

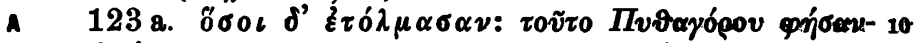

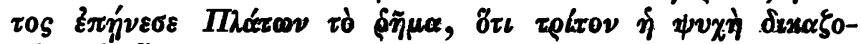

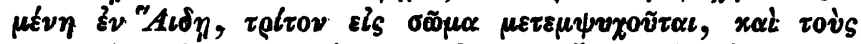

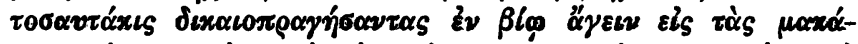

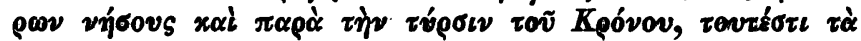

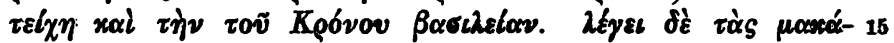

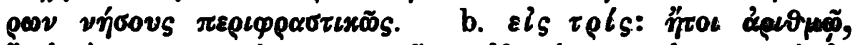

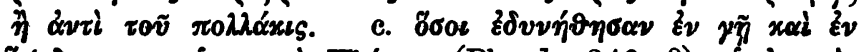

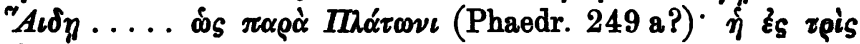

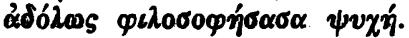

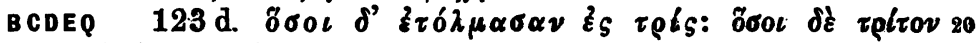

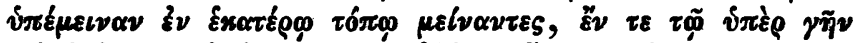

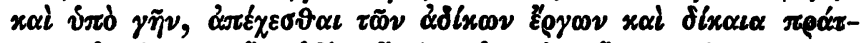

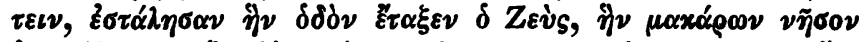

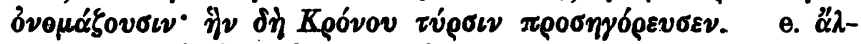

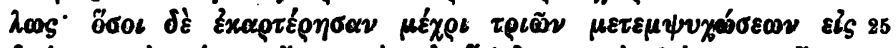

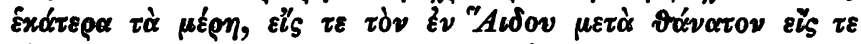

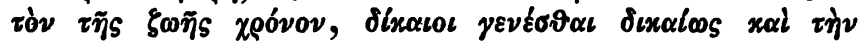

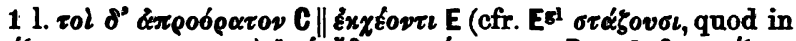

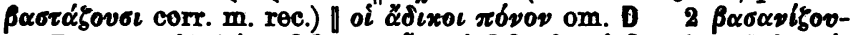

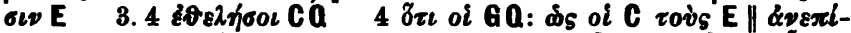

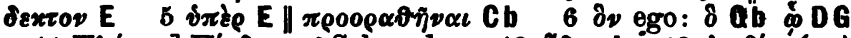

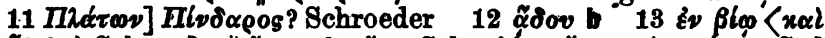

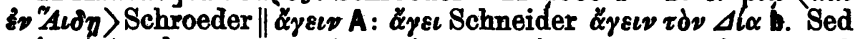

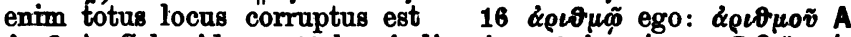

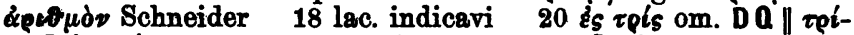

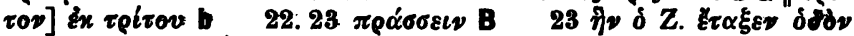

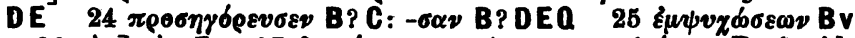

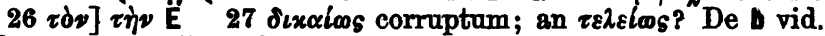
ad p. 93, $1 \| x \alpha i] \tau \varepsilon$ C 


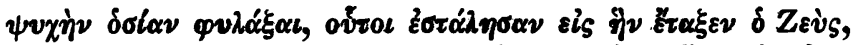

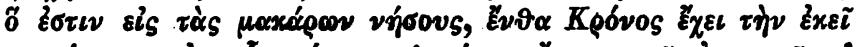

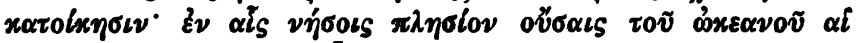

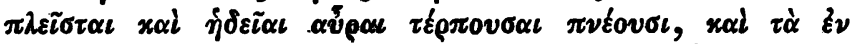

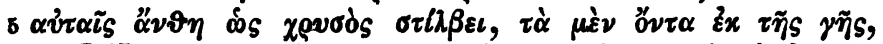

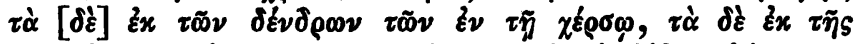

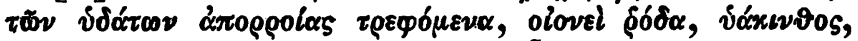

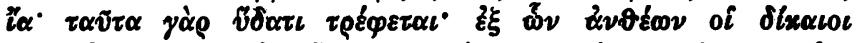

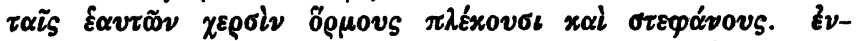

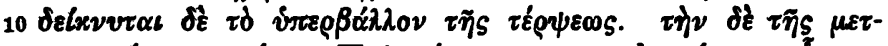

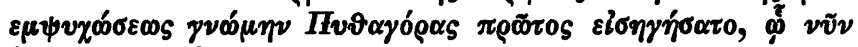

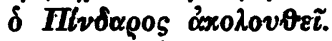

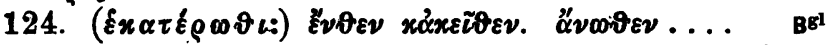

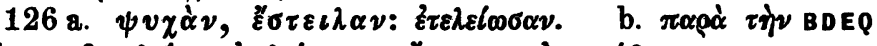

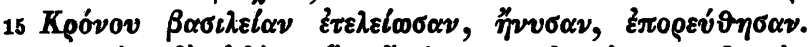

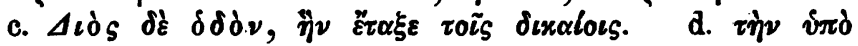

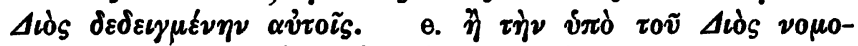

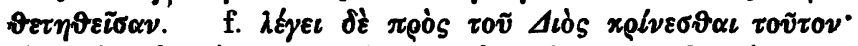

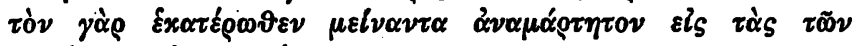

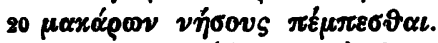

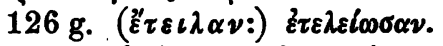

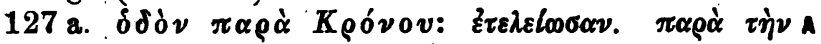

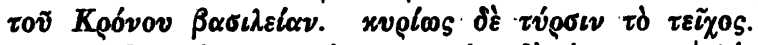

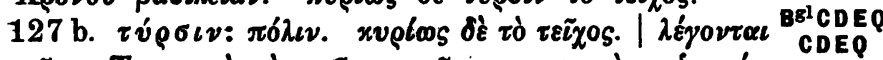

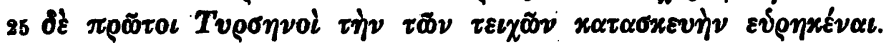

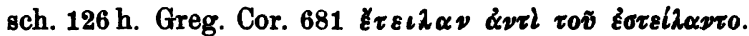
24 sch. Lycophr. 717

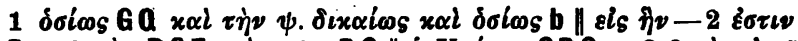

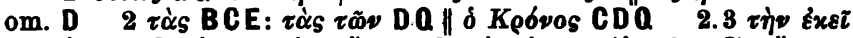

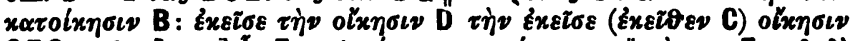

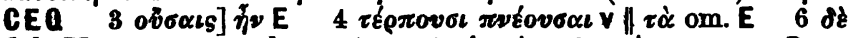
del. Mommsen suppl. p. 317 oiovel-8 rpéqera om. D

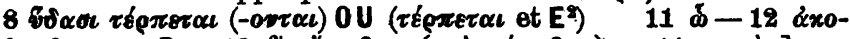

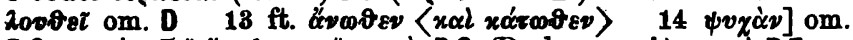

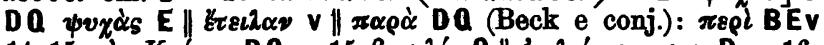

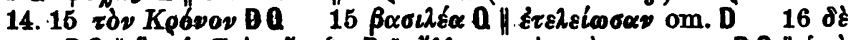

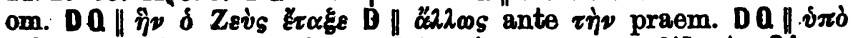

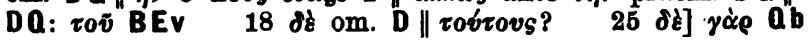




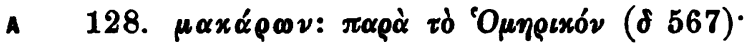

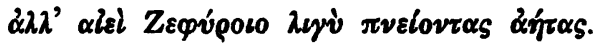

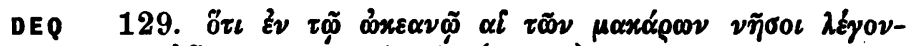

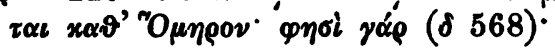

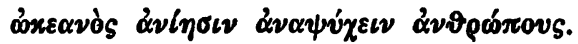

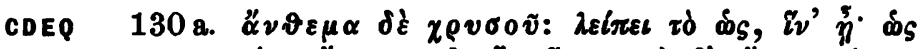

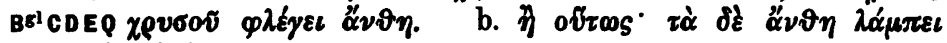

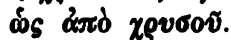

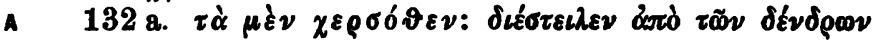

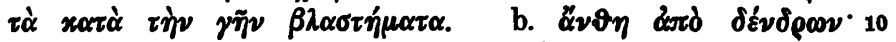

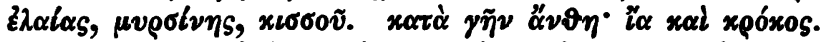

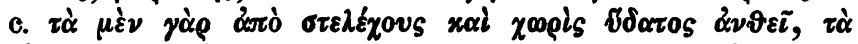

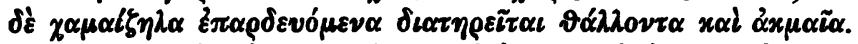

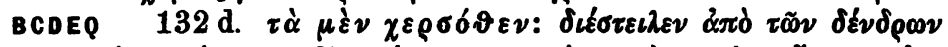

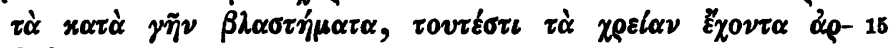

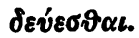

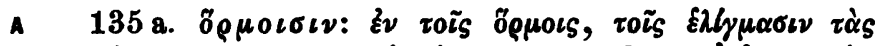

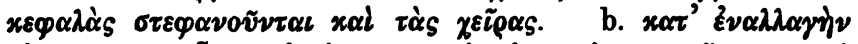

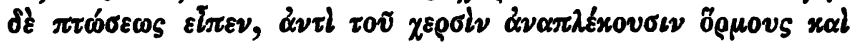

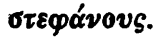

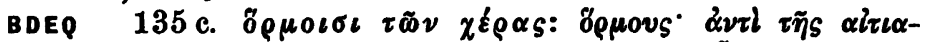

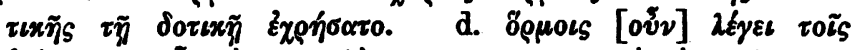

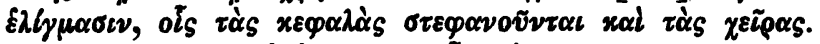

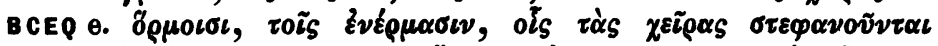

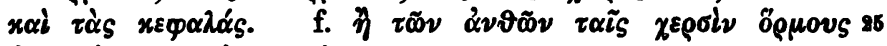

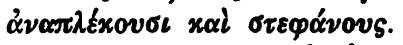

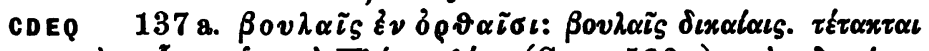

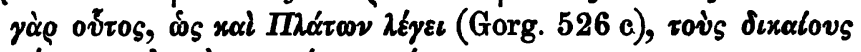

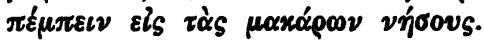

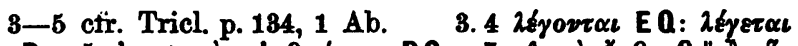

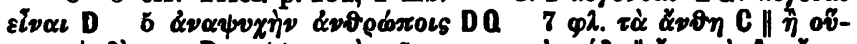

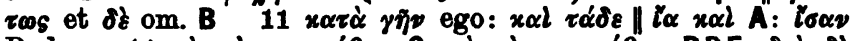

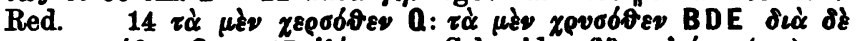

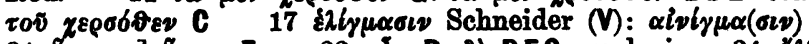

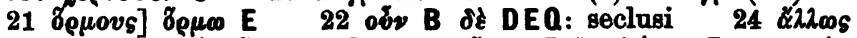

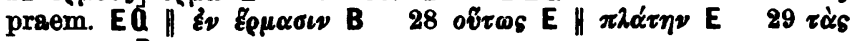
$\tau \tilde{\omega} \nu \mu \alpha x$. D 


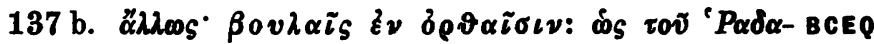

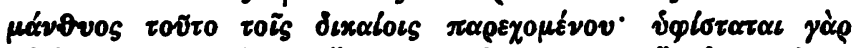

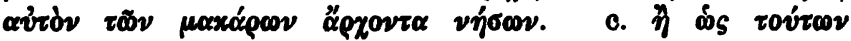

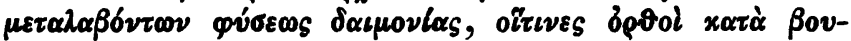

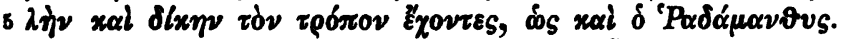

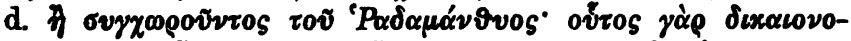

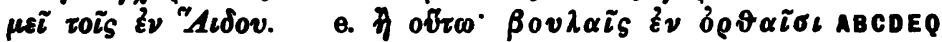

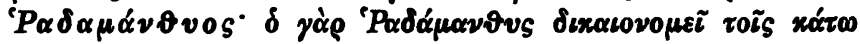

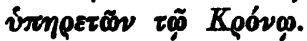

10138 a.

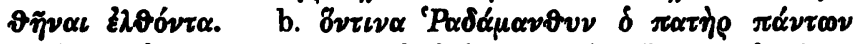

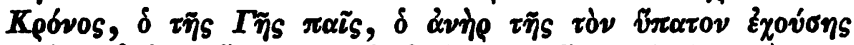

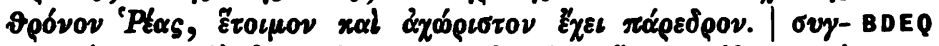

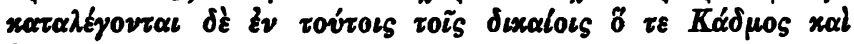
$15 \delta \Pi \eta \lambda \varepsilon v$ s.

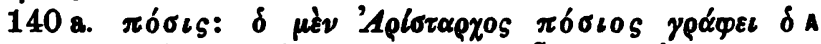

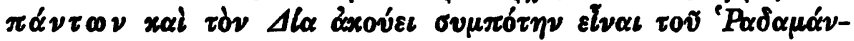

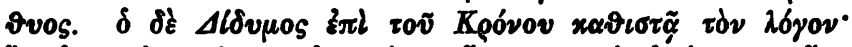

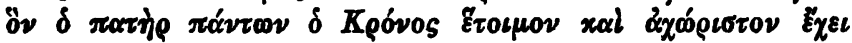

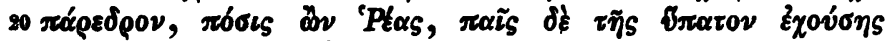

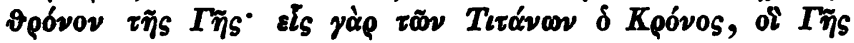

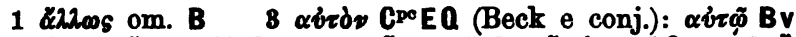

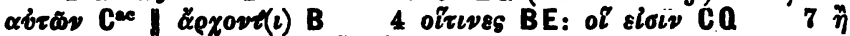

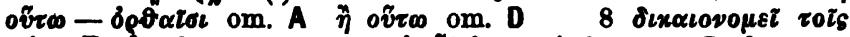

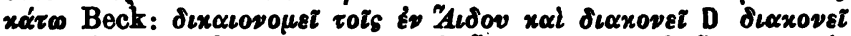

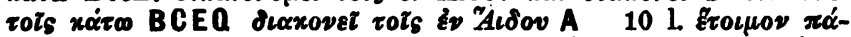

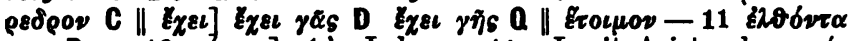
om. D $16 \pi 0 \sigma \nu$ s] $\Delta l o s$ Lehrs p. 11. „Legit Aristarchus $\pi \delta-$

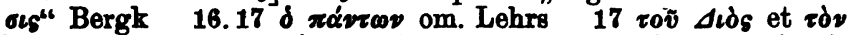

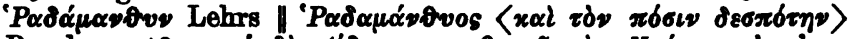

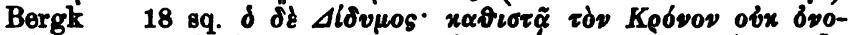

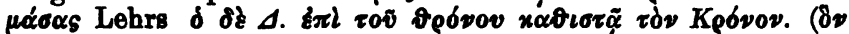

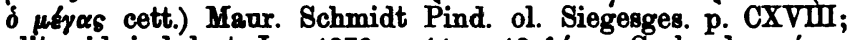
aliter id. ind. lect. Jen. 1879, p. 1118 26yov Gerhard: xp6vov

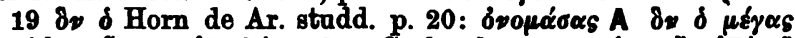

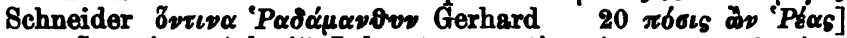

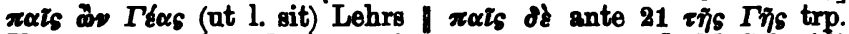

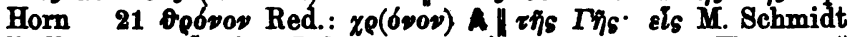
11. 11. ( $\Gamma \eta_{s:}$ zis Kó́vos M. Schmidt: $\chi \rho(6 \nu 0 s)$ A opderos Red. 


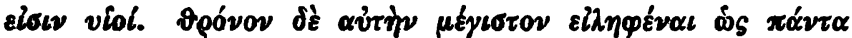

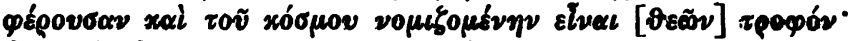

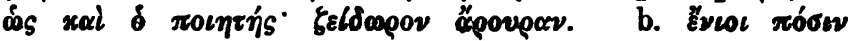

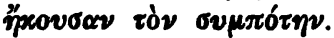

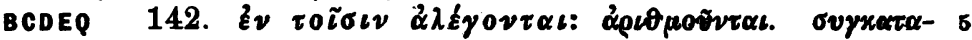

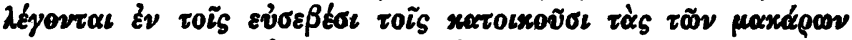

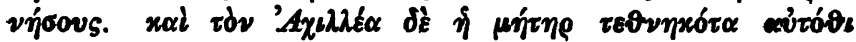
गु

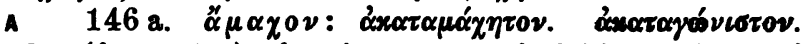

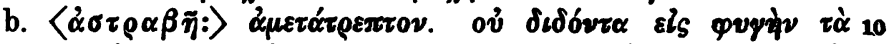

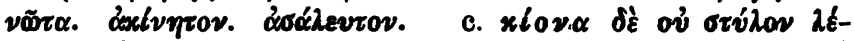

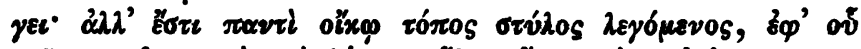

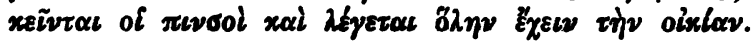

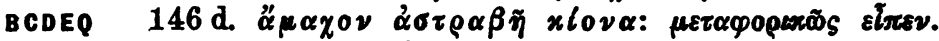

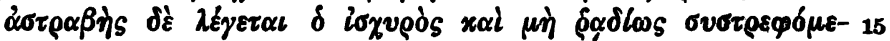

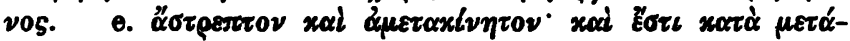

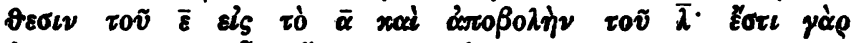

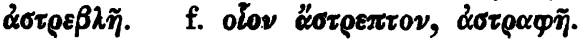

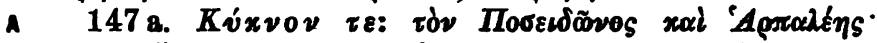

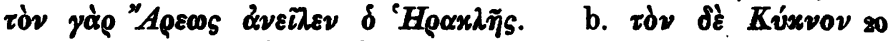

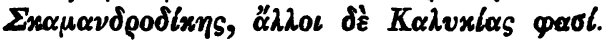

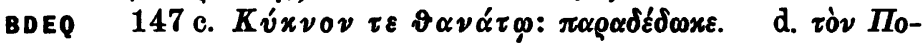

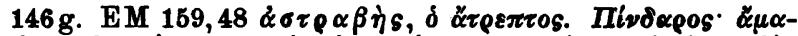

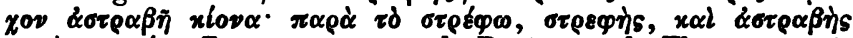

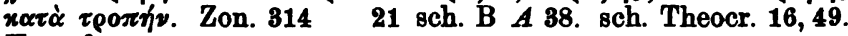
Hyg. f. 157

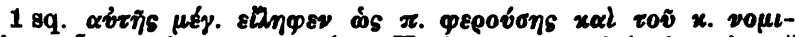

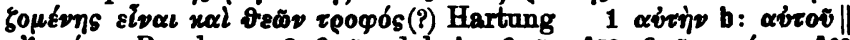

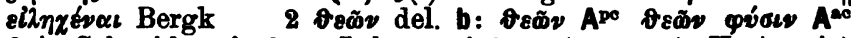

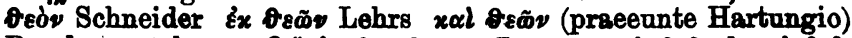

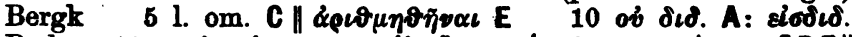

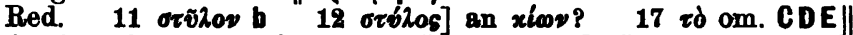

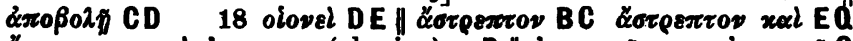

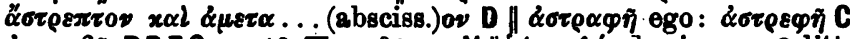

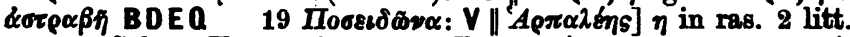

21 Calyce Hyg., sch. Th. et II.

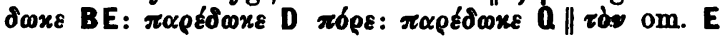




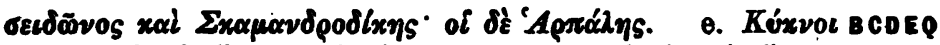

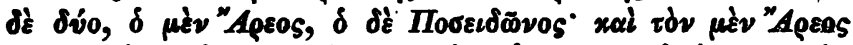

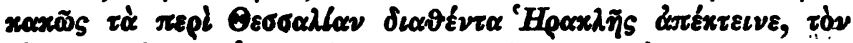

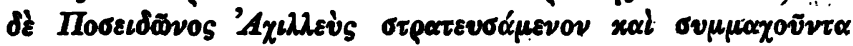

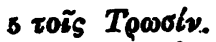

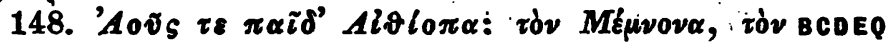

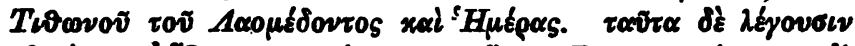

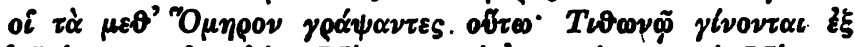

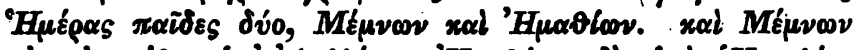

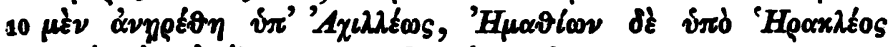

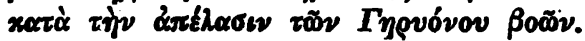

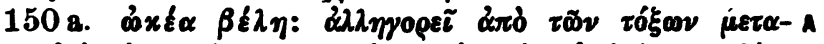

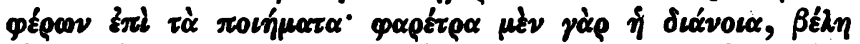

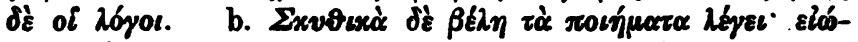

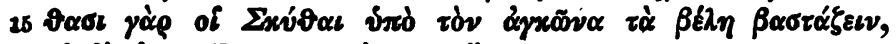

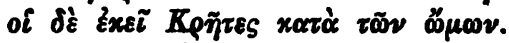

150 c. $\operatorname{\omega x} \varepsilon \alpha \beta \beta \varepsilon \lambda \eta$ :

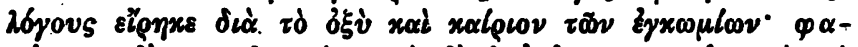

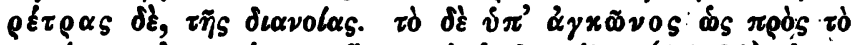

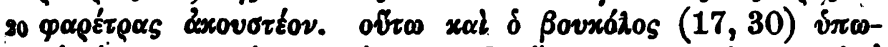

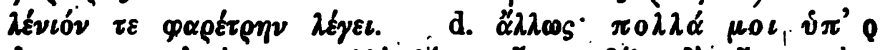

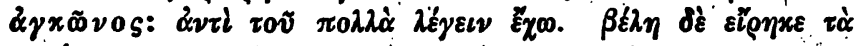

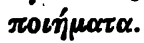

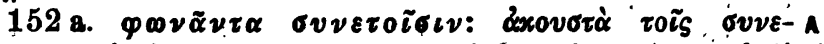

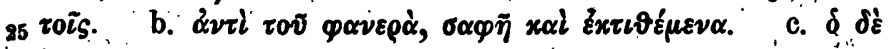

1-5 sch. Ar. Ran. 963. - Thom. 136, 18: Lv́o Kúxivol

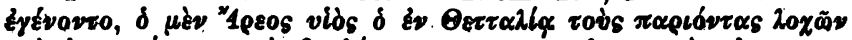

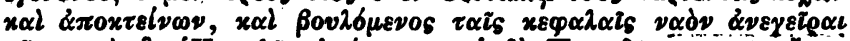

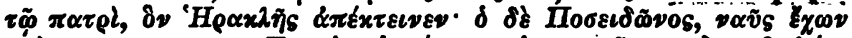

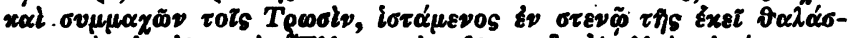

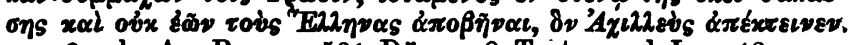

6 sch. Ar. Ran. p. 631 Dü. $\quad 8$ Tzetzes ad Lyc. 18

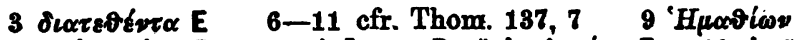

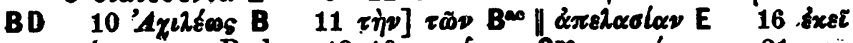

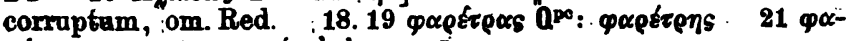

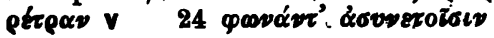

Sohor. wr Pardarux ed. Drachmann. 


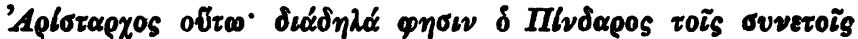

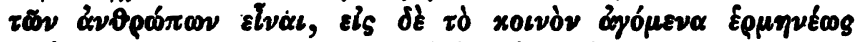

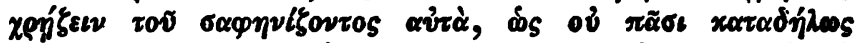

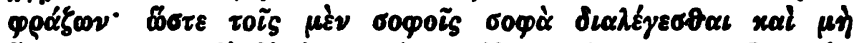

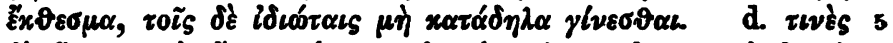

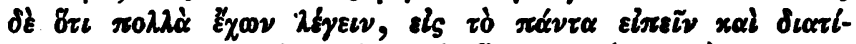

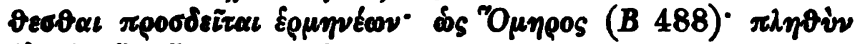

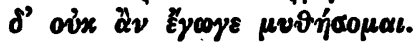

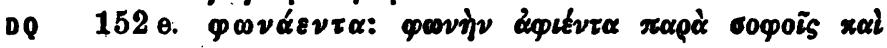

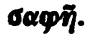

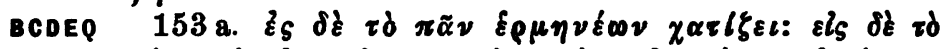

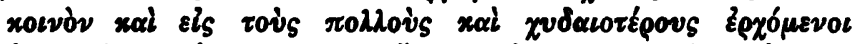

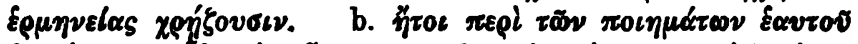

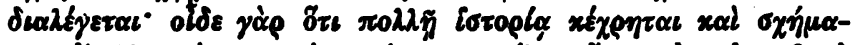

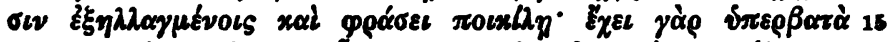

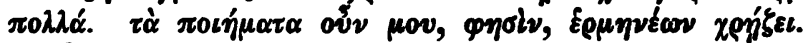

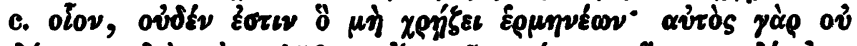

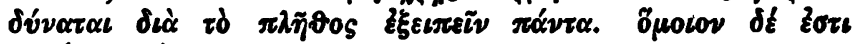
$\tau \tilde{\text { }}$ (B 488)

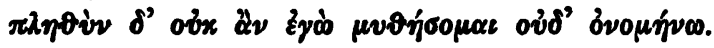

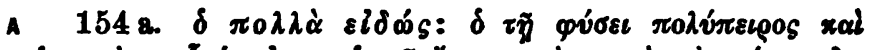

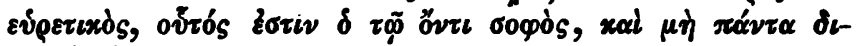

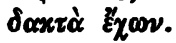

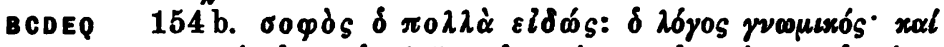

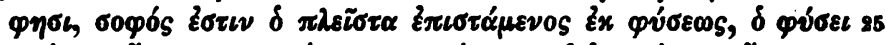

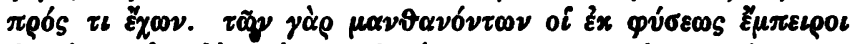

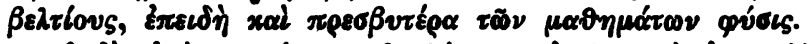

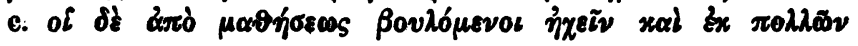

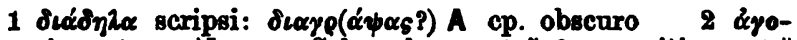

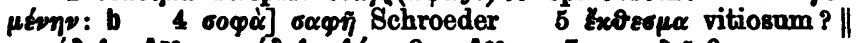

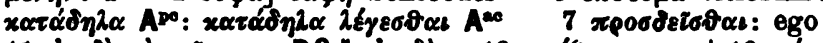

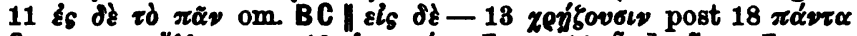

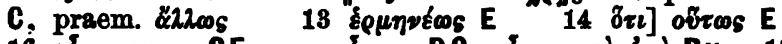

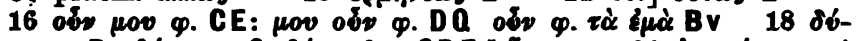

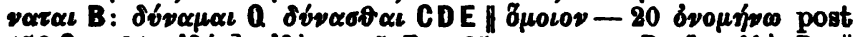

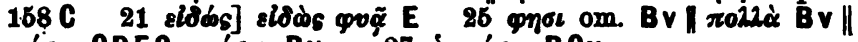

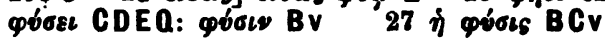




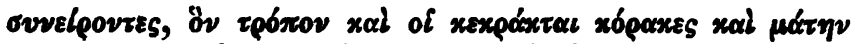

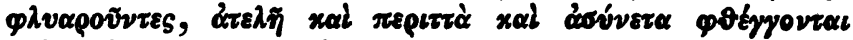

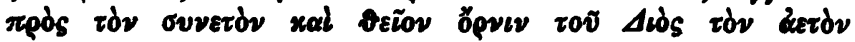

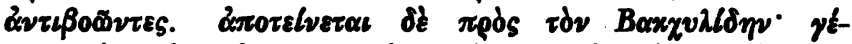

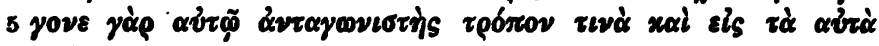

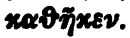

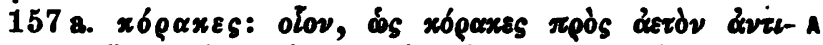

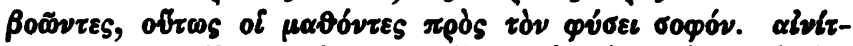

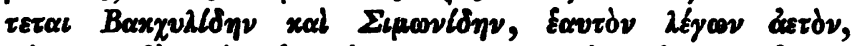

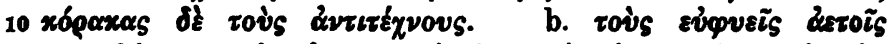

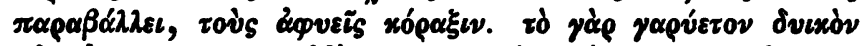

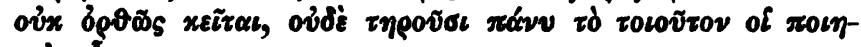
rai ovirow 164

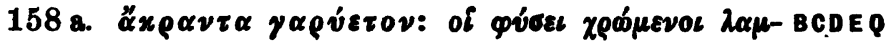

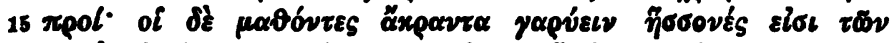

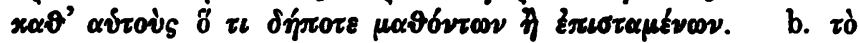

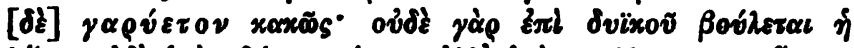

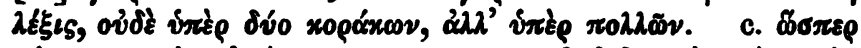

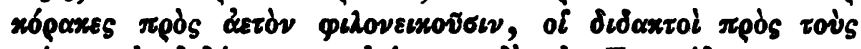

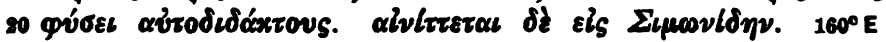

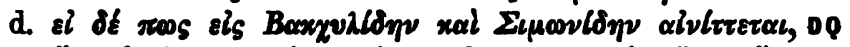

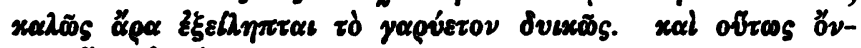
$\tau \cos z_{\chi \varepsilon t} \delta$ hójos.

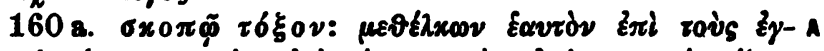

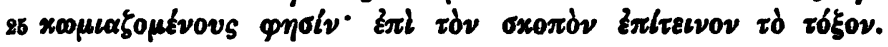

11 Gregor. Cor. p. $218 \mathrm{~S}$.

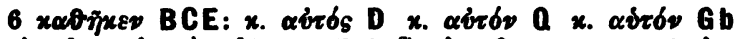

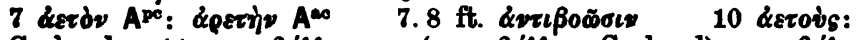

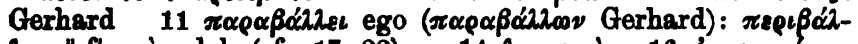

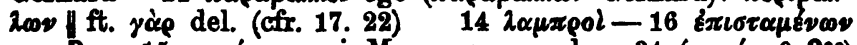

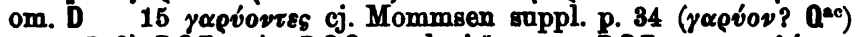

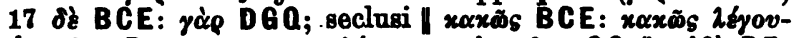

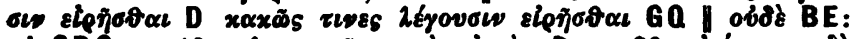

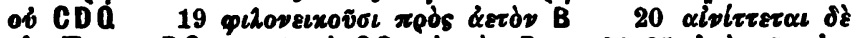

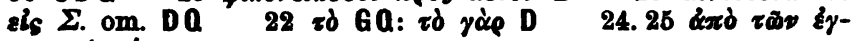

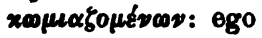




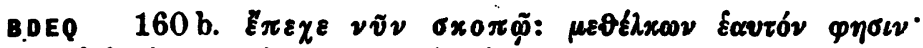

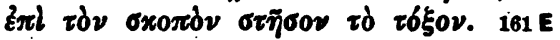

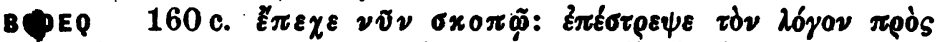

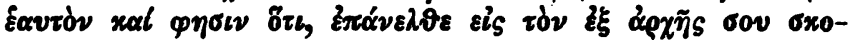

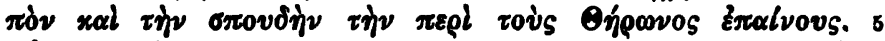

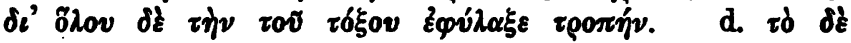

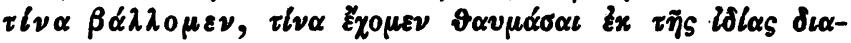
volos. $160^{\mathrm{b} E}$

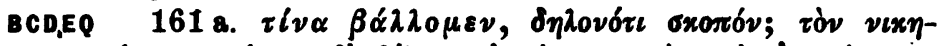

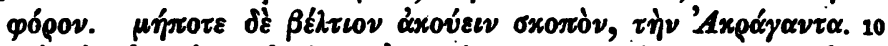

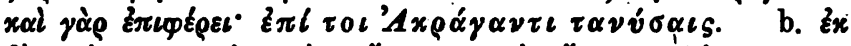

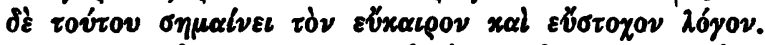

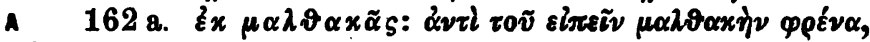

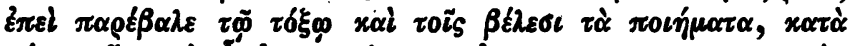

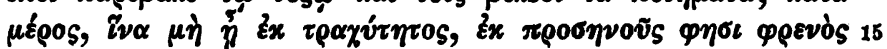

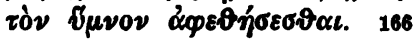

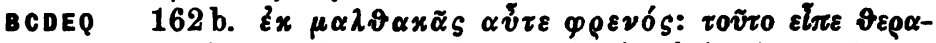

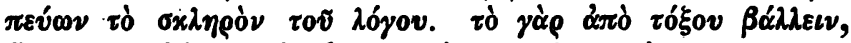
§ోs

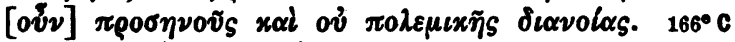

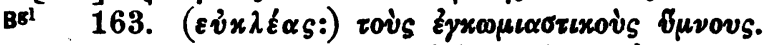

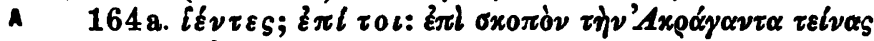

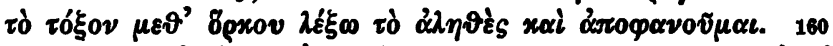

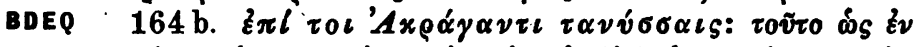

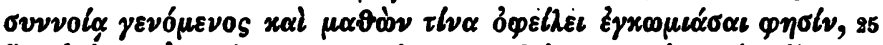

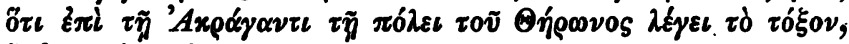

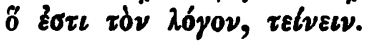

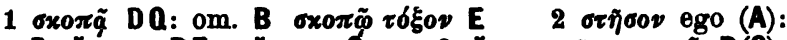
$\sigma \tau \eta \tilde{\sigma \alpha}$ D $\sigma \tau \eta \eta \alpha$ BEv

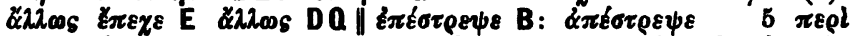

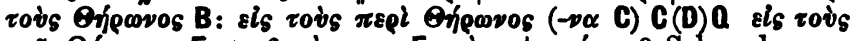

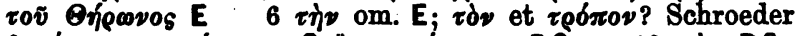

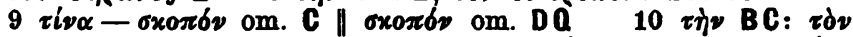

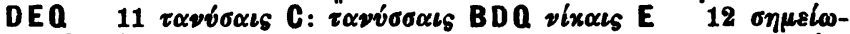

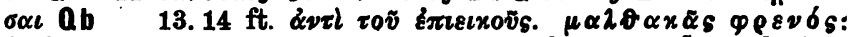

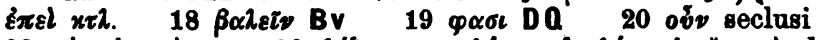

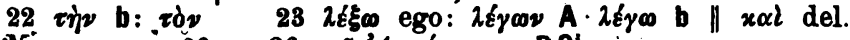

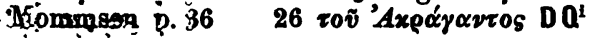




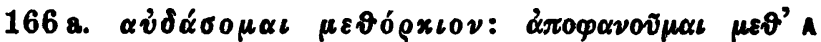

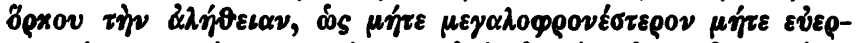

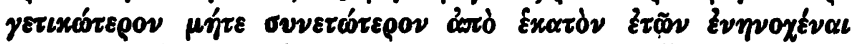

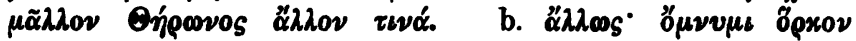

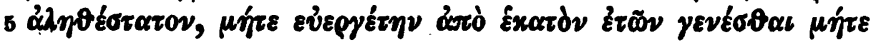

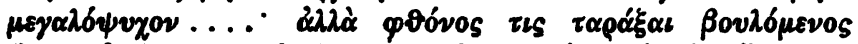

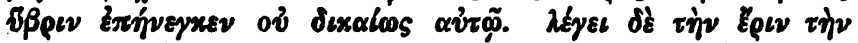

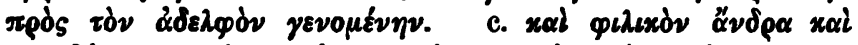

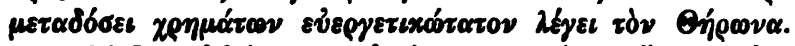

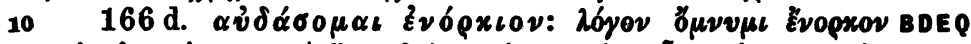

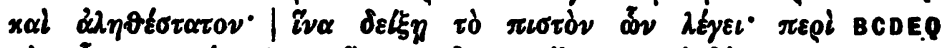

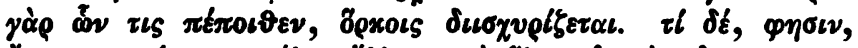

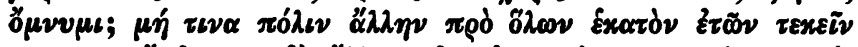

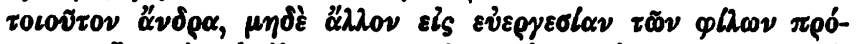

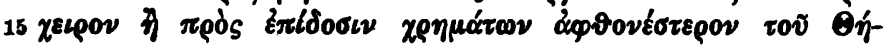
pwovos. $178^{1} C 173^{1} E Q$

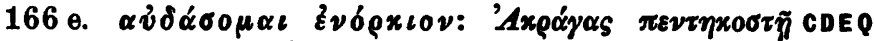

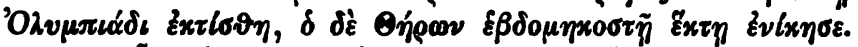

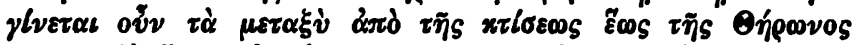

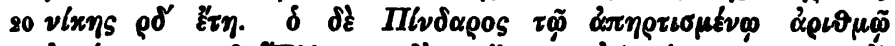

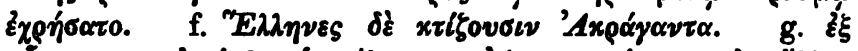

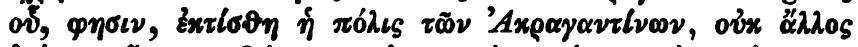

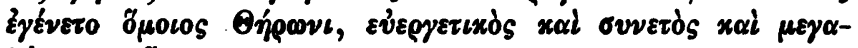

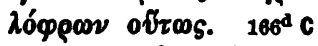

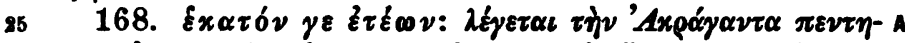

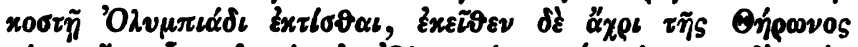

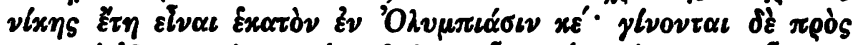

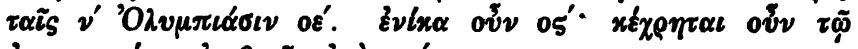

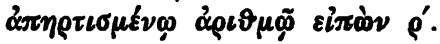

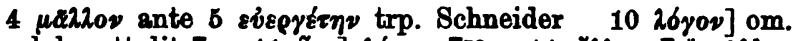

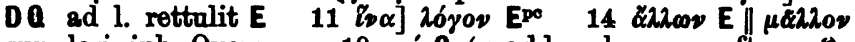
suppleri iub. Oxonn. 18 os' Q (5 add. rubr., corr. ft. ex '); cfr. pap. Oxyrrh. CCXXII, 1, 18: $0^{\prime}$ CEP of D os iे of b

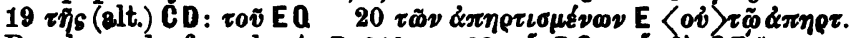
Bergk; sed cfr. sch. A B 64922 ov DQ: ov dह CE $\|$ Q

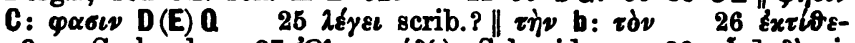

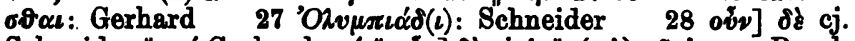

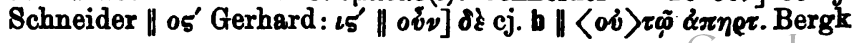




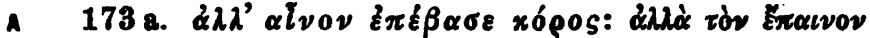

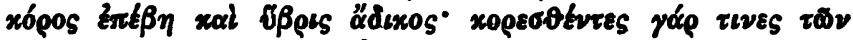

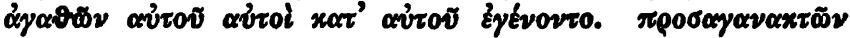

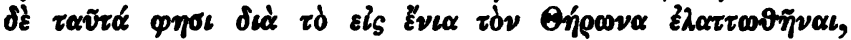

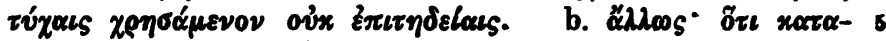

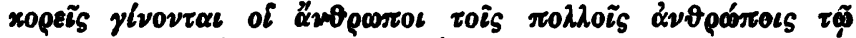

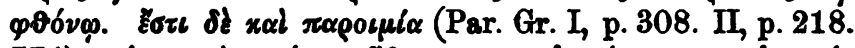

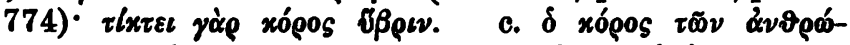

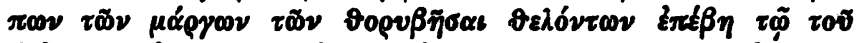

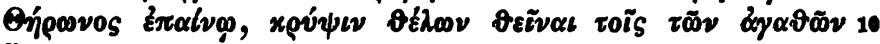
(1) E้pyoss.

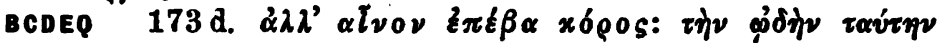

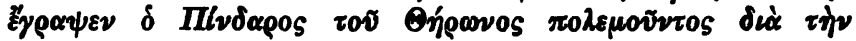

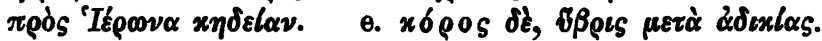

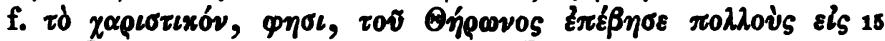

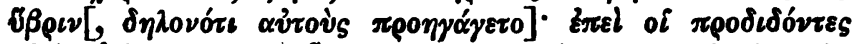

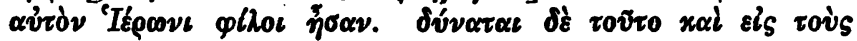

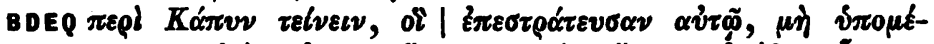

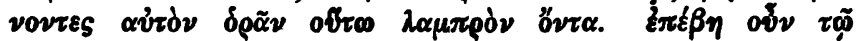

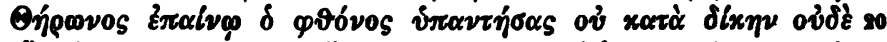

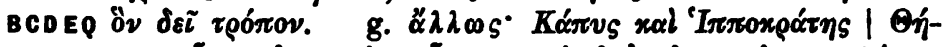

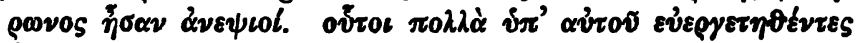

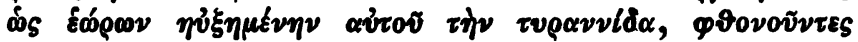

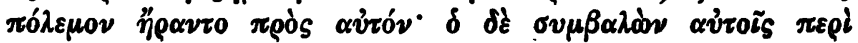

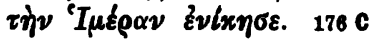

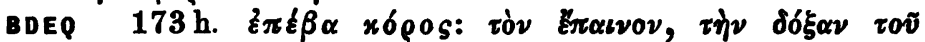

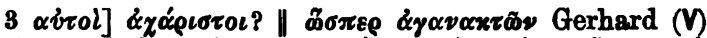

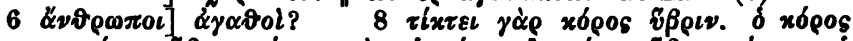

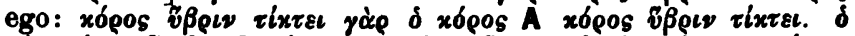

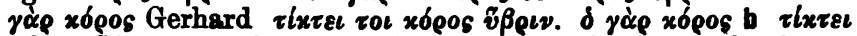

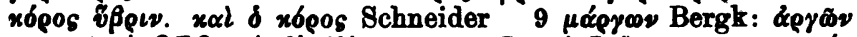

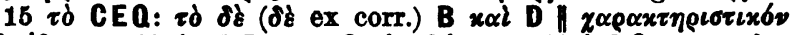

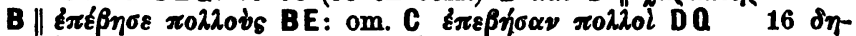

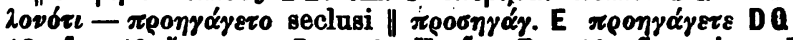

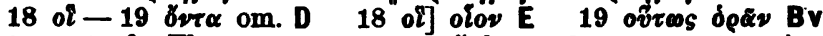

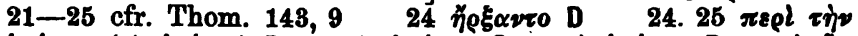

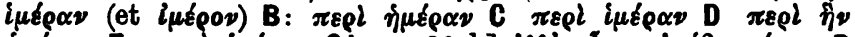

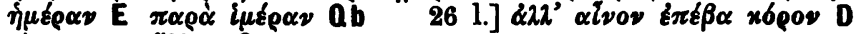
et praem. canos Q 


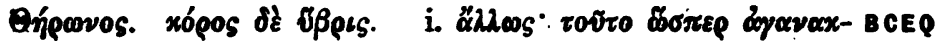

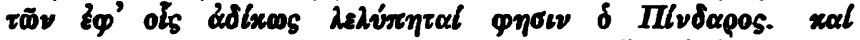

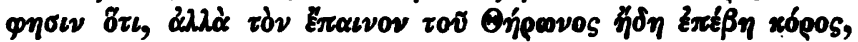

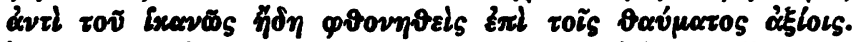

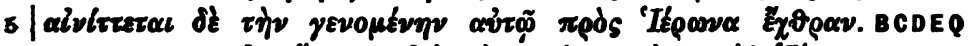

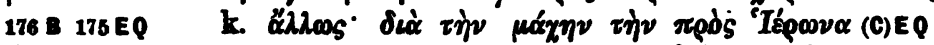

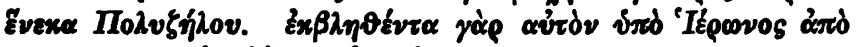

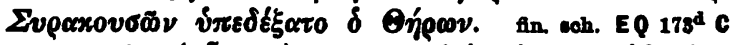

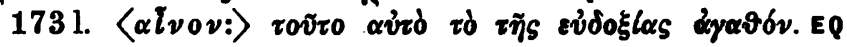
$10166^{\mathrm{b}} \mathrm{EQ}$

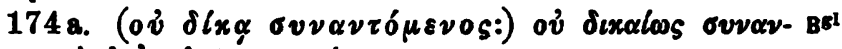

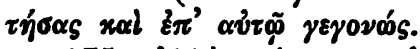

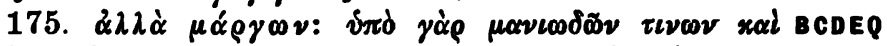

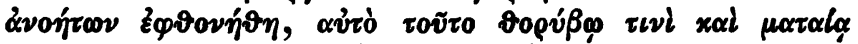

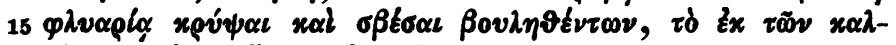

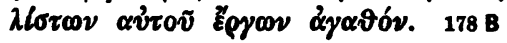

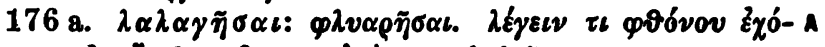
$\mu \varepsilon v 0 \nu . \quad$ b. $\hat{\eta े ~}$

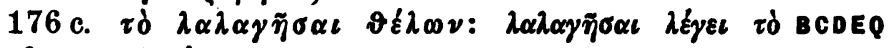

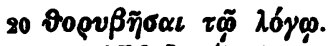

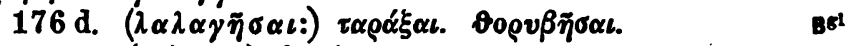

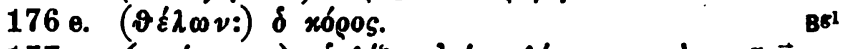

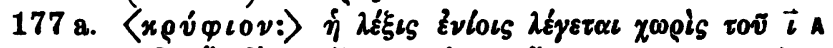

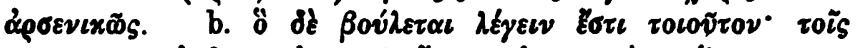

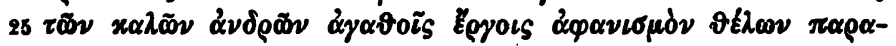

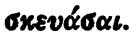

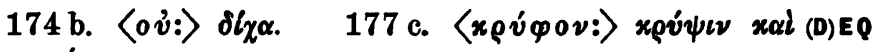

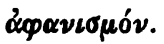

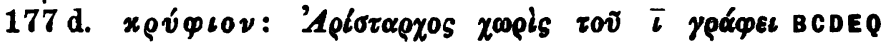

1 if $\tilde{v} \beta \rho c_{5} \mathrm{DQ} \quad 2$ post $\Pi l \nu \delta \alpha \rho \circ g$ sch. 175 inser. C

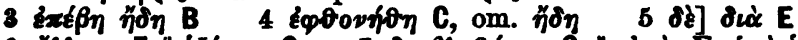

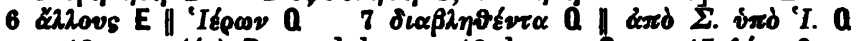

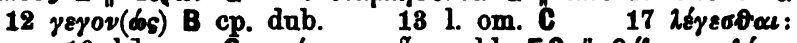

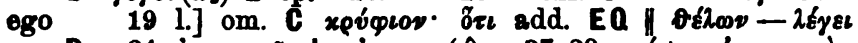

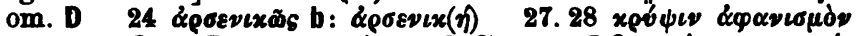

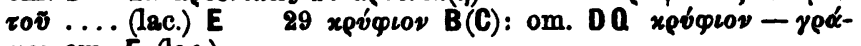
甲ei om. E (lac.) 


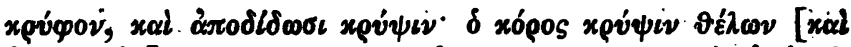

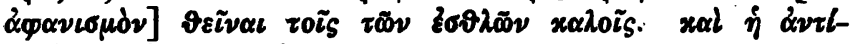

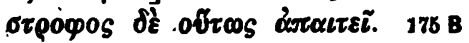

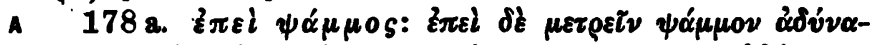

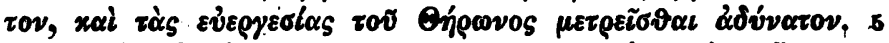

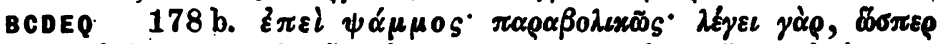

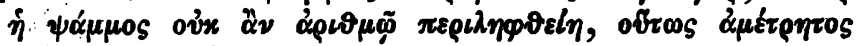

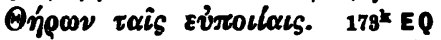

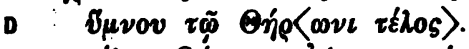

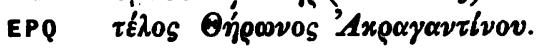

\section{Scholia in Olympionicarum carmen III.}

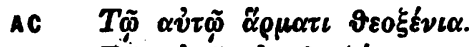

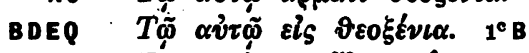

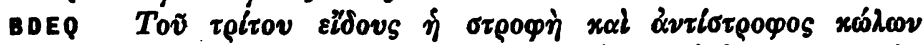

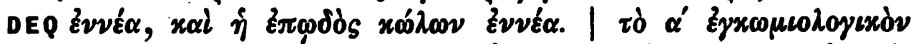

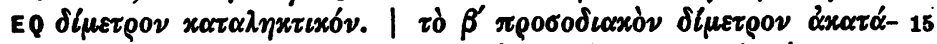

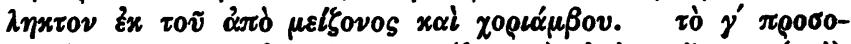

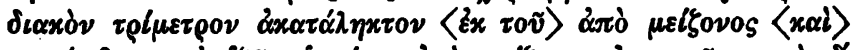

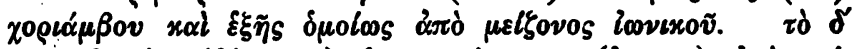

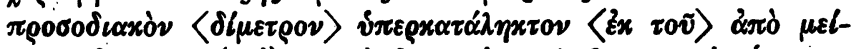

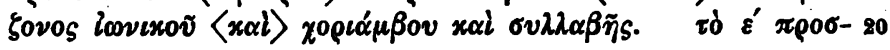

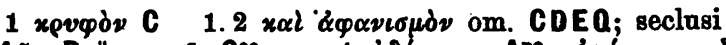

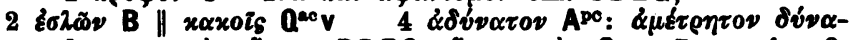

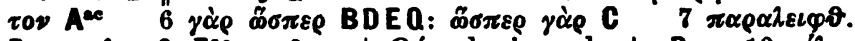

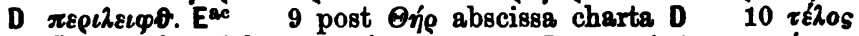

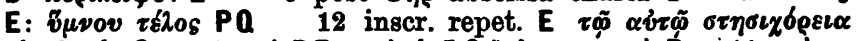

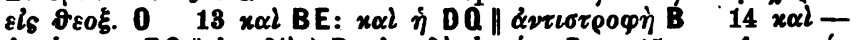

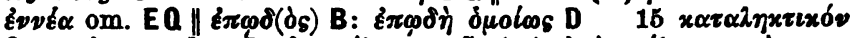

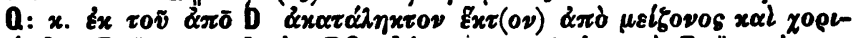

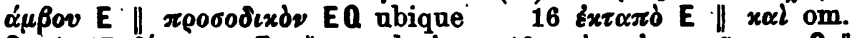

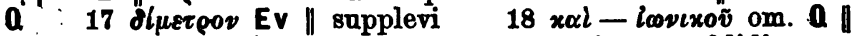

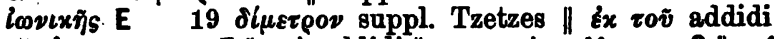

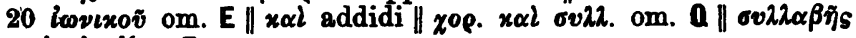
scripsi: $\varepsilon \xi \tilde{\eta} \tilde{s} \mathrm{E}$ 


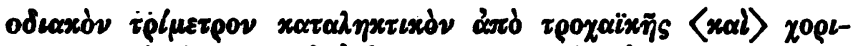

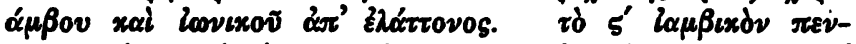

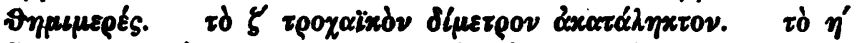
ซ̋

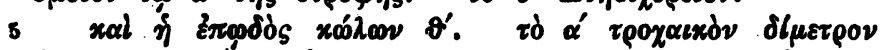

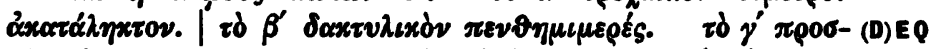

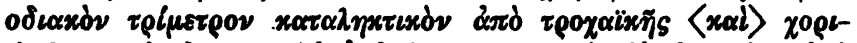

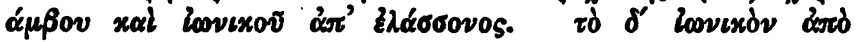

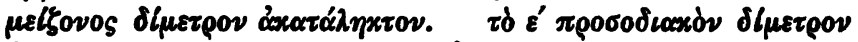

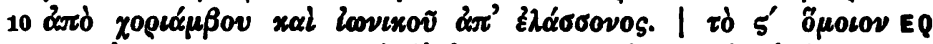

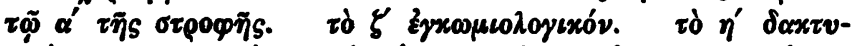

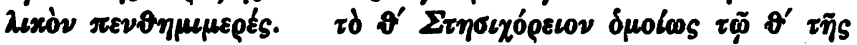

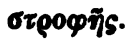

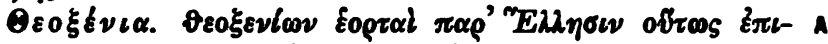

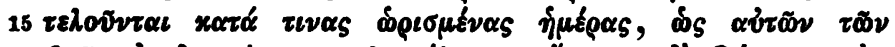

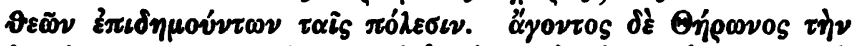

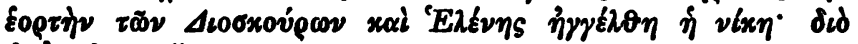

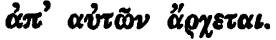

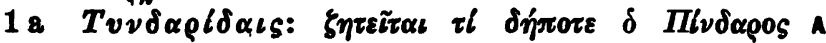

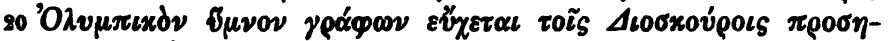

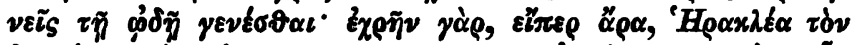

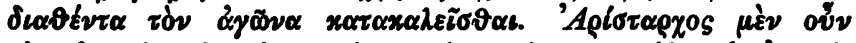

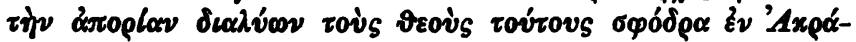

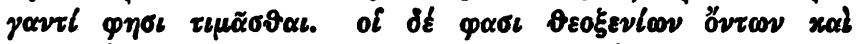

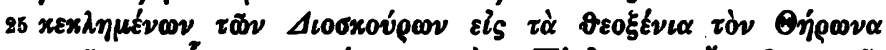

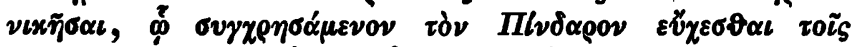

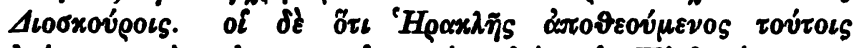

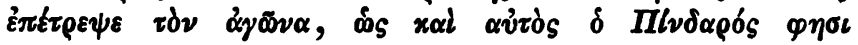

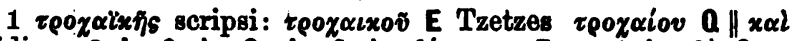

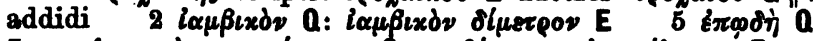

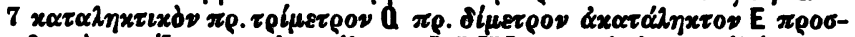

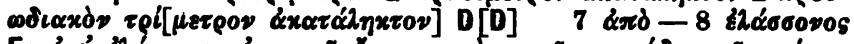

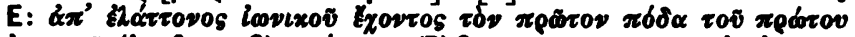

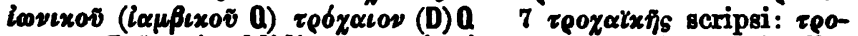

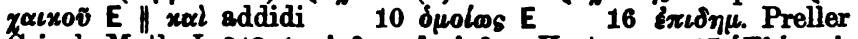

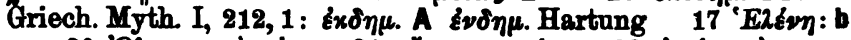

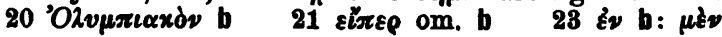




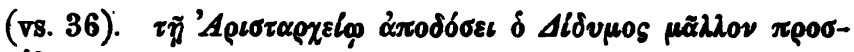
$\tau$ toseral.

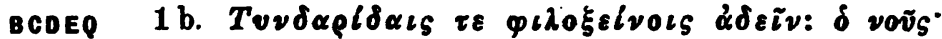

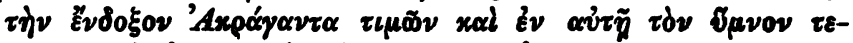

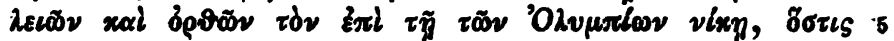

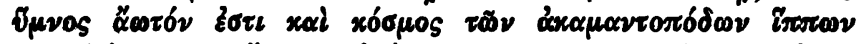

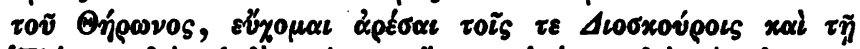

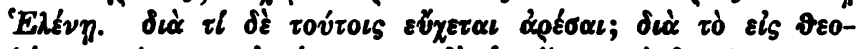

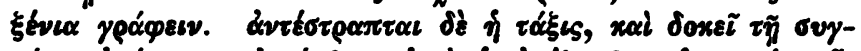

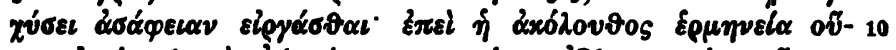

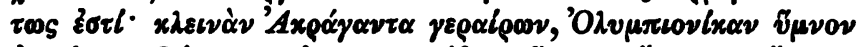

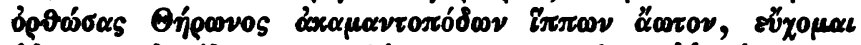

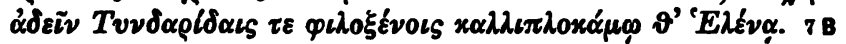

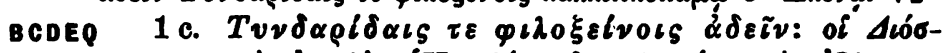

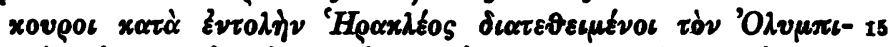

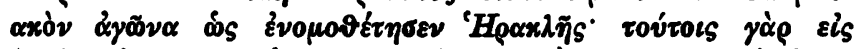

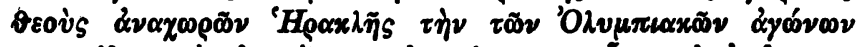

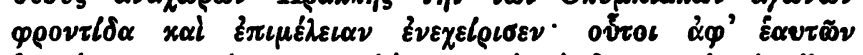

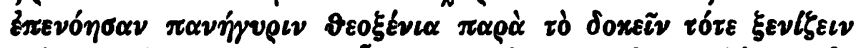

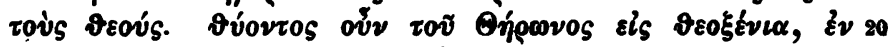

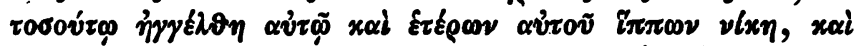

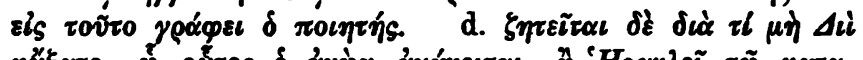

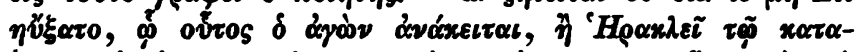

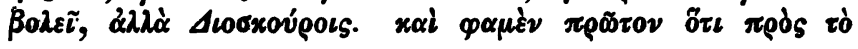

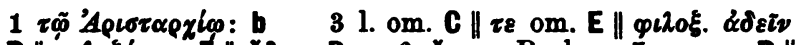

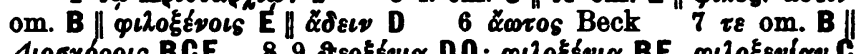

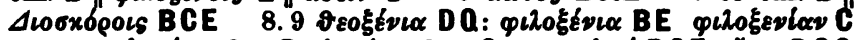

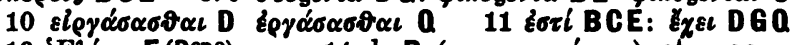

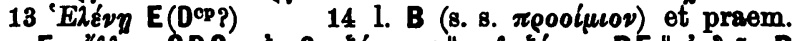

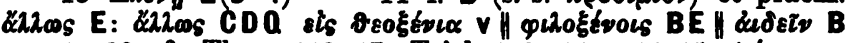

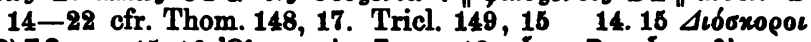

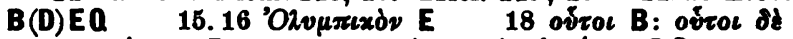

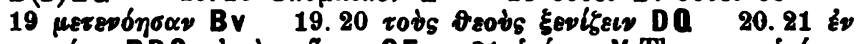

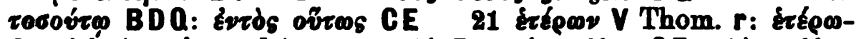

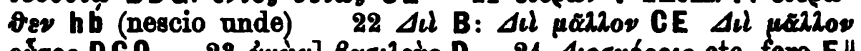

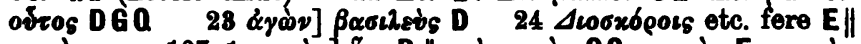

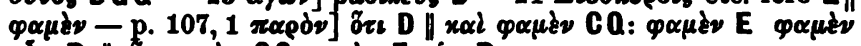

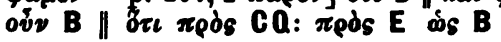




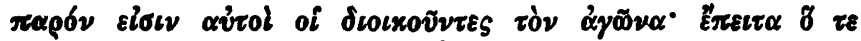

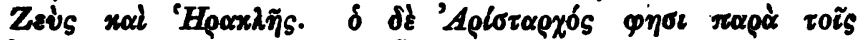

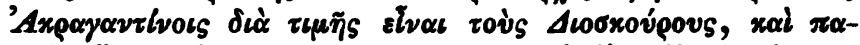

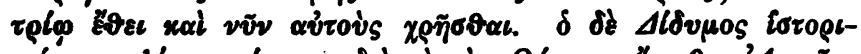

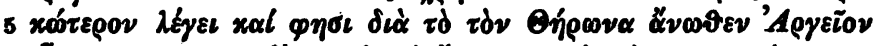

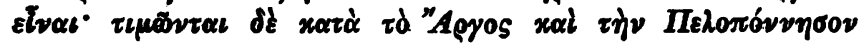

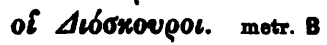

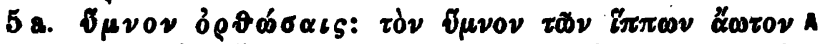

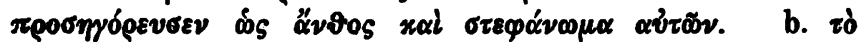

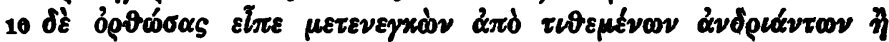

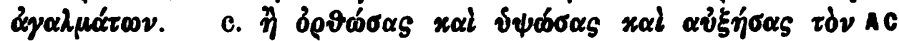

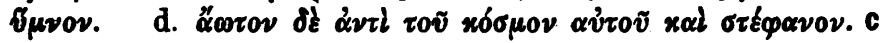

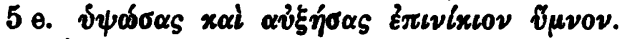
Bg1

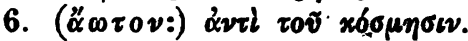

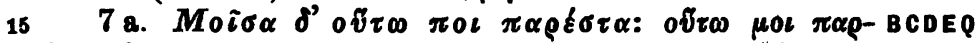

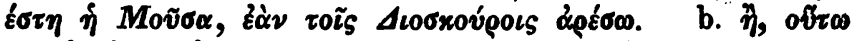

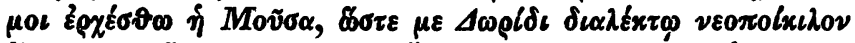

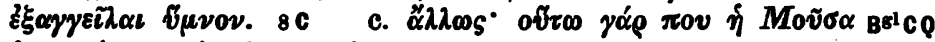

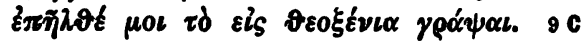

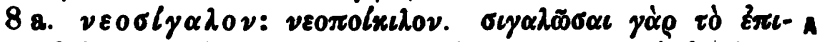

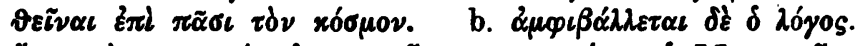

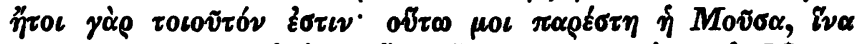

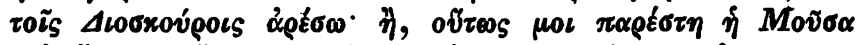

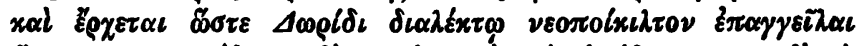

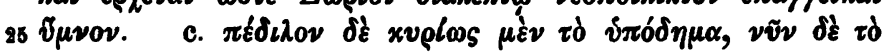

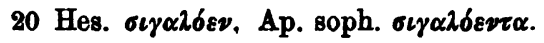

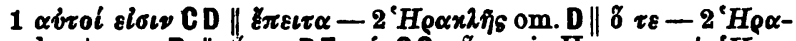

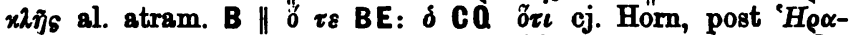
$x \lambda \eta_{s}$ lac. ind. Locus nondum exped. vid. 3 Alooxópovs BCE

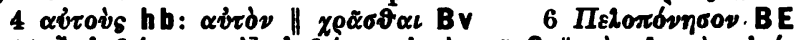

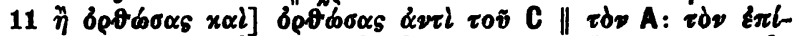

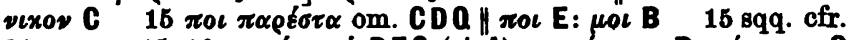
21 sqq. $15.16 \pi \alpha \rho \varepsilon \sigma \tau \eta \eta \dot{\eta}$ DEQ (et A): $\pi \alpha \rho \varepsilon \sigma \tau \eta x \varepsilon$ B $\pi \alpha \alpha_{\rho} \varepsilon \sigma \tau \iota \nu$ C

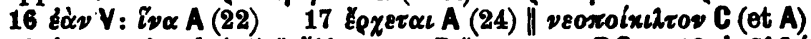

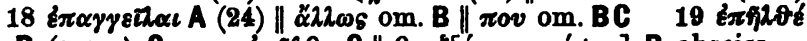

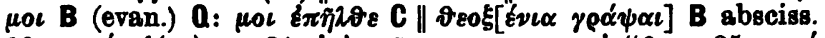

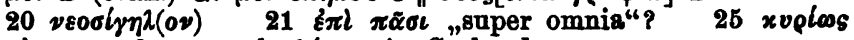

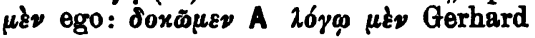




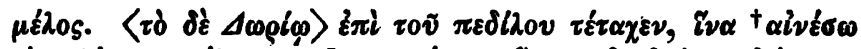

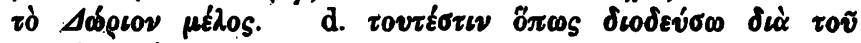

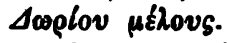

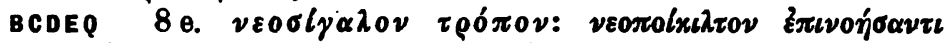

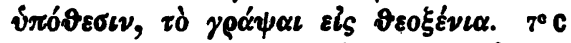

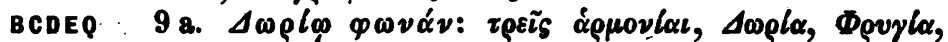

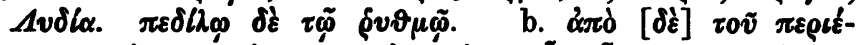

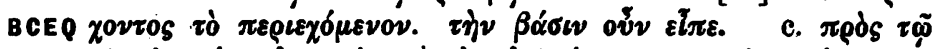

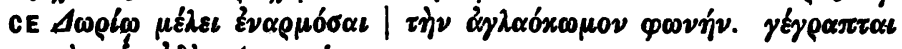

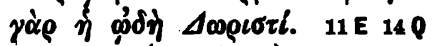

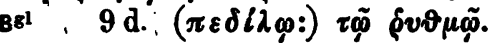

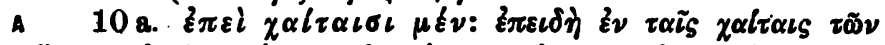

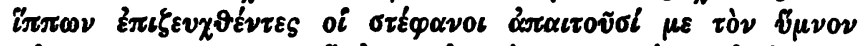

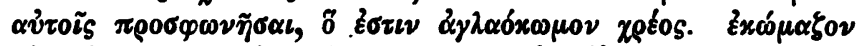

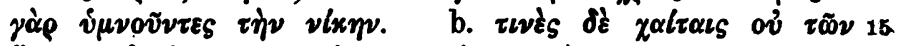

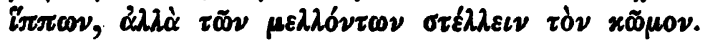

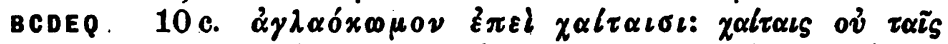

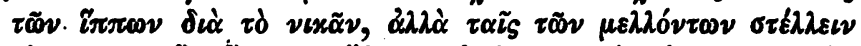

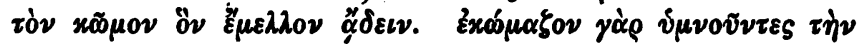

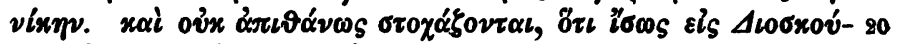

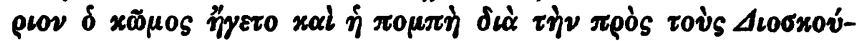

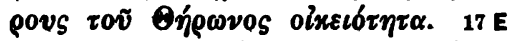

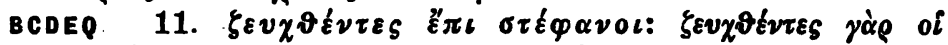

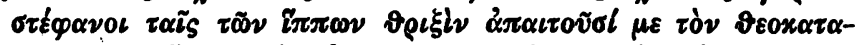

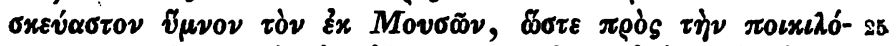

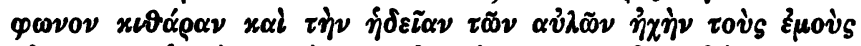

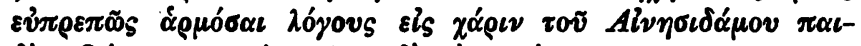

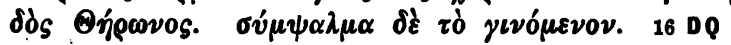

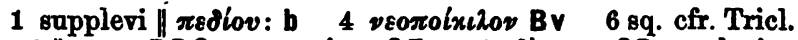

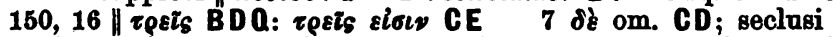

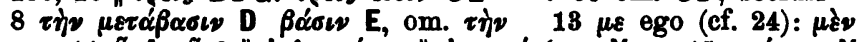

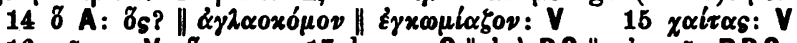

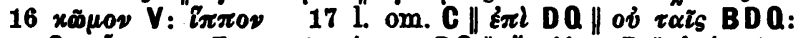

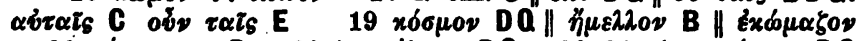

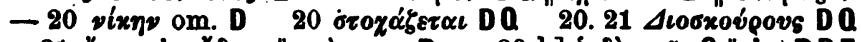

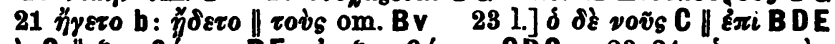

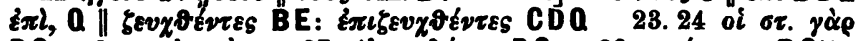

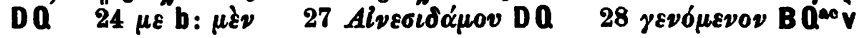




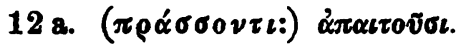

Bs1

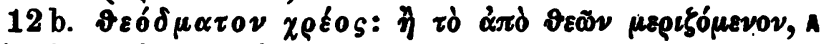

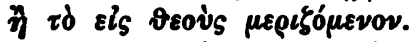

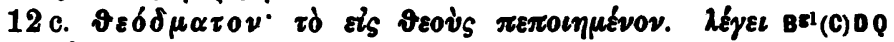

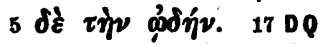

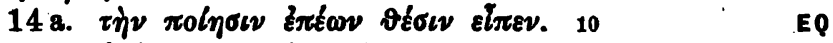

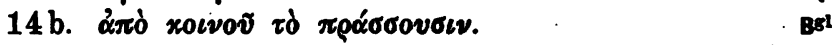

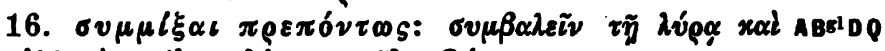

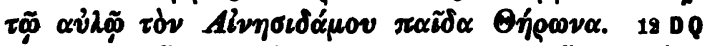

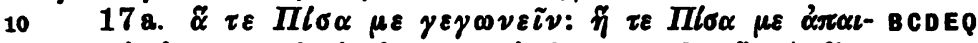

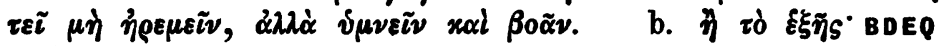

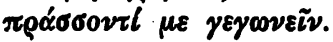

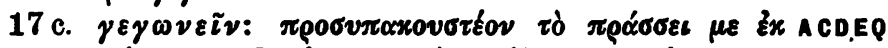

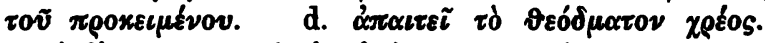

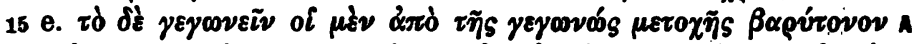

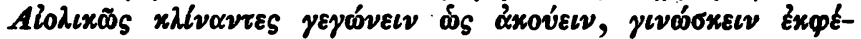

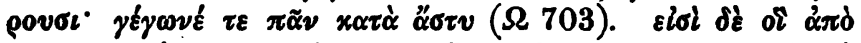

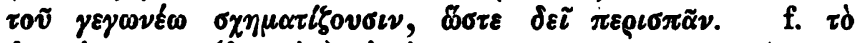

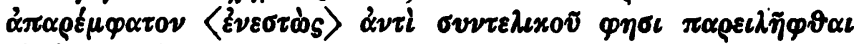

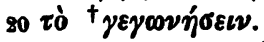

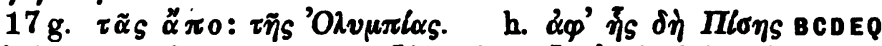

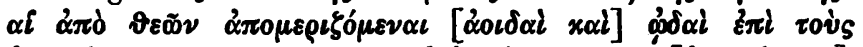

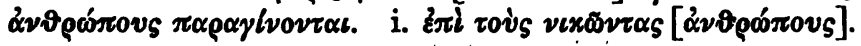

\section{EM 391, 27 15 Herodian. II 83, 8}

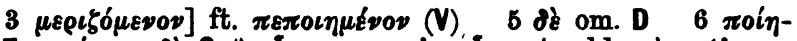

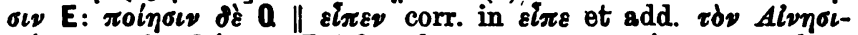

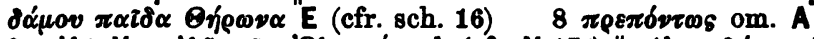

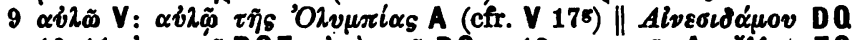

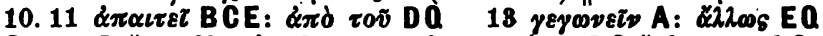

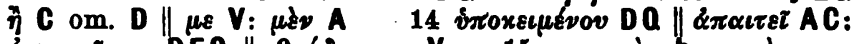

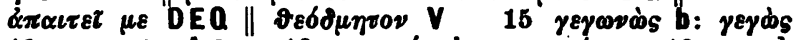

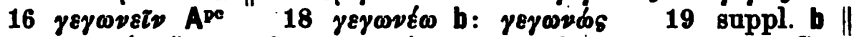

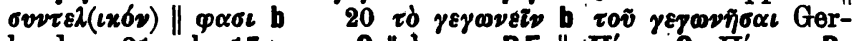

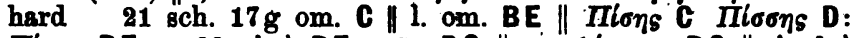

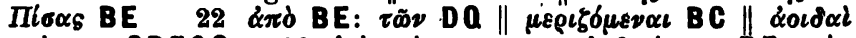

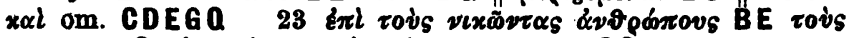

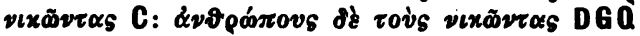




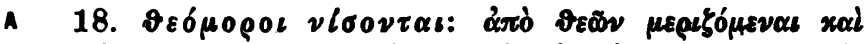

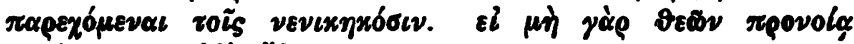

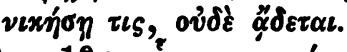

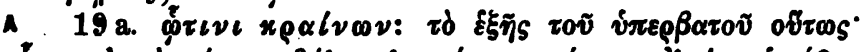

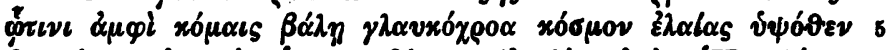

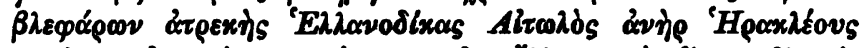

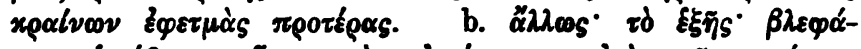

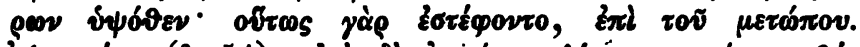

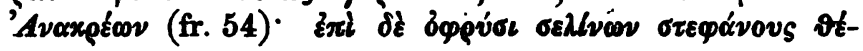
revol.

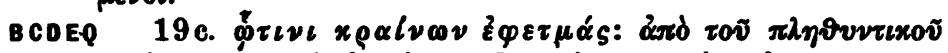

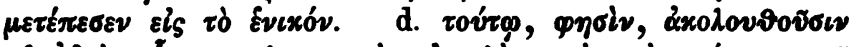

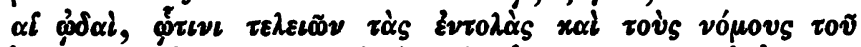

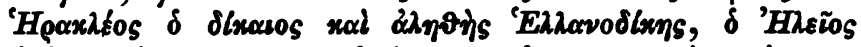

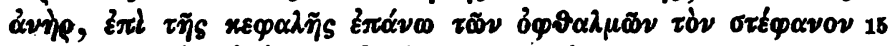

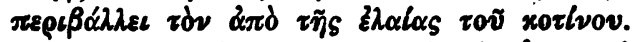

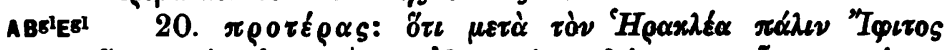

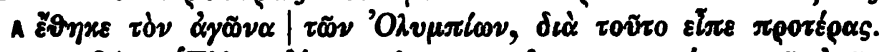

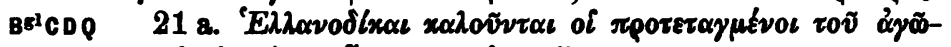

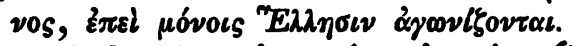

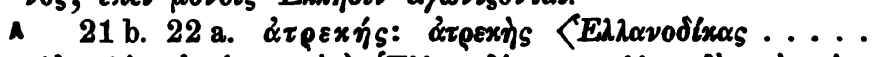

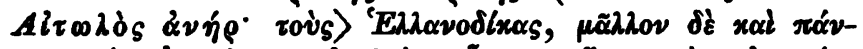

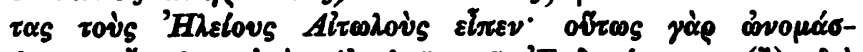

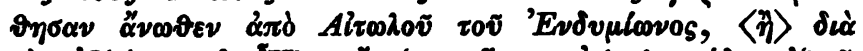

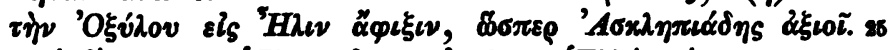

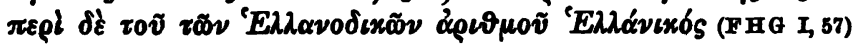

8 Athen. 15, 674 e. 19 EM 831, 20. Bekk. anecd. 248, 32

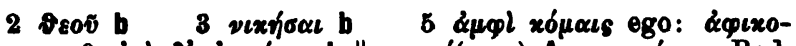

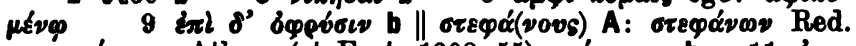

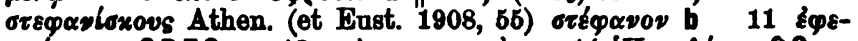

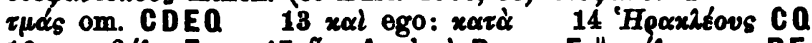

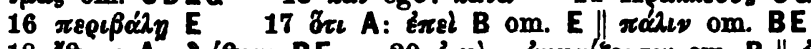

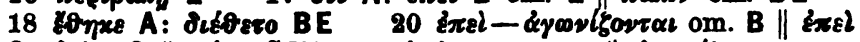

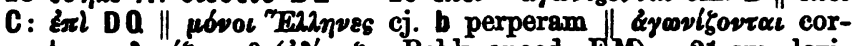
ruptum: dixásovot? (żlxakov Bekk. snecd., EM) 21 supplevi; cf. ad p. 111, 324 suppl. b 


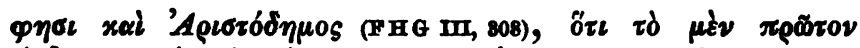

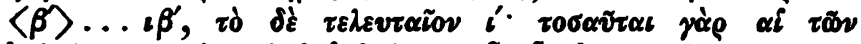

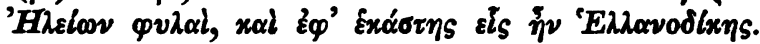

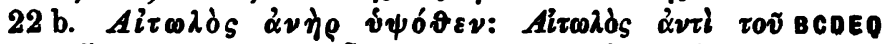

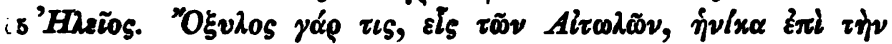

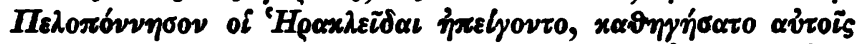

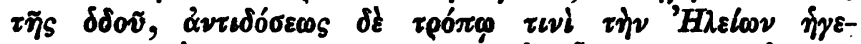

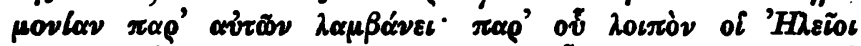

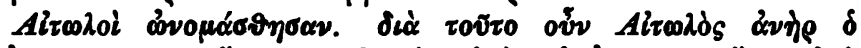

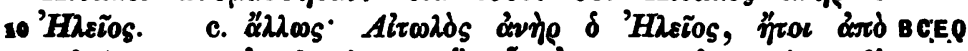

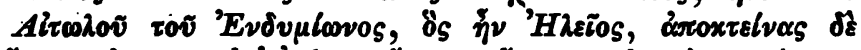

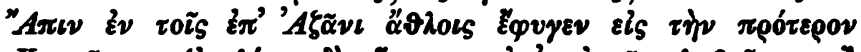

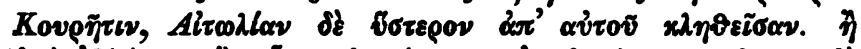

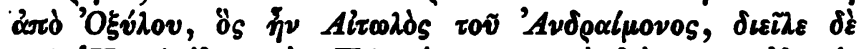

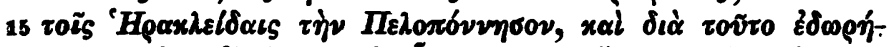

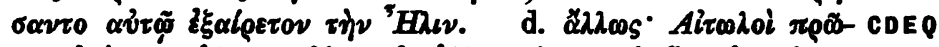

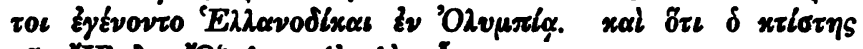

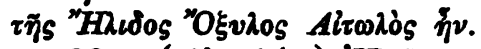

22 e. (Aircolós:) 'Hheíos.

$\mathbf{B e}^{\mathbf{I}}$

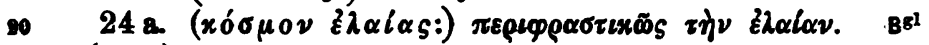

b. $(\tau \dot{\alpha} \nu$ :) $\tau \dot{\eta} \nu \dot{\varepsilon} \boldsymbol{\varepsilon} \alpha \dot{\alpha} \boldsymbol{\alpha} \nu$.

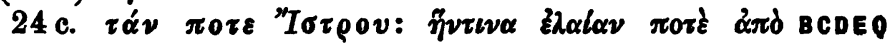

10 Pans. 5, 1, 8. Harpocr. Suid. EM 331, 2416 Tzetzes Chil. 12, 870

2 supplevi cl. Paus. V 9, $4 \|<\beta] \beta$ b 3 post $h$. sch. add.

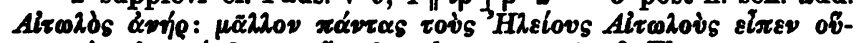

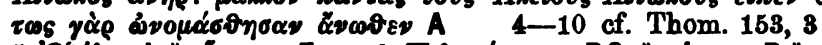

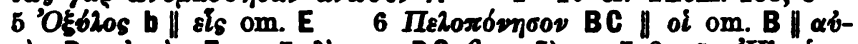

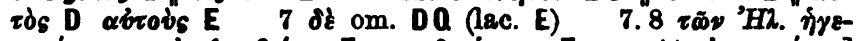

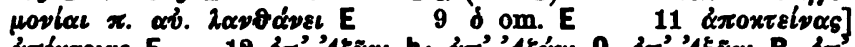

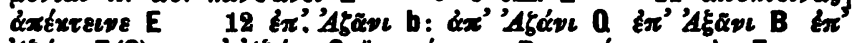

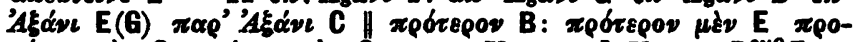

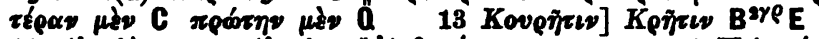

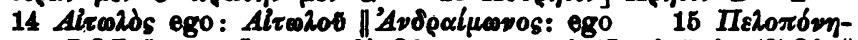

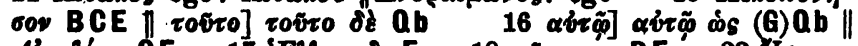

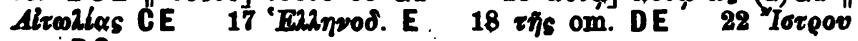
om. DQ. 


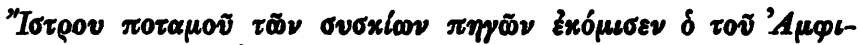

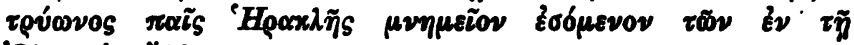

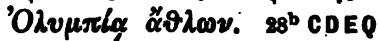

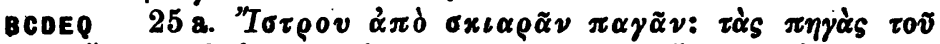

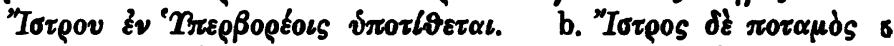

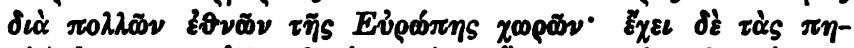

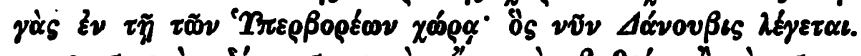

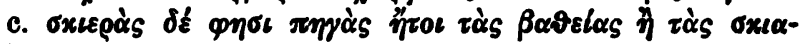

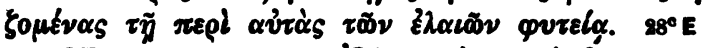

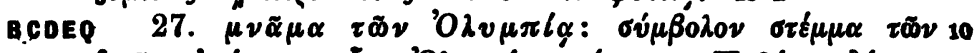

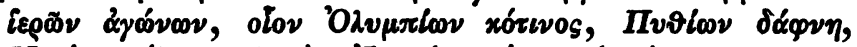

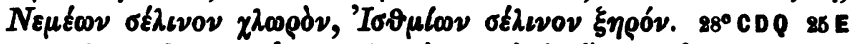

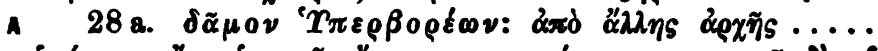

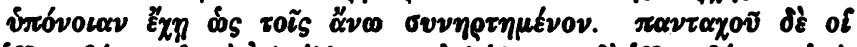

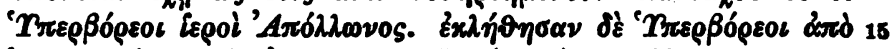

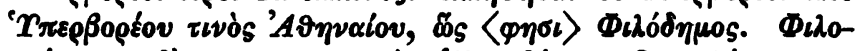

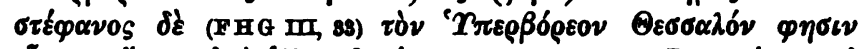

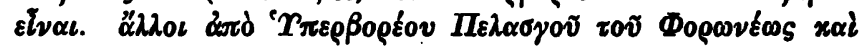

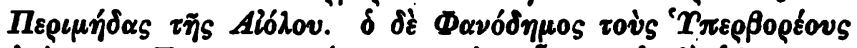

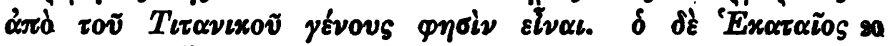

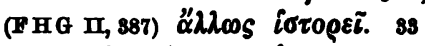

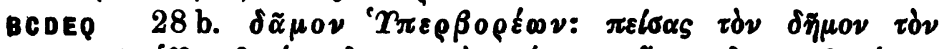

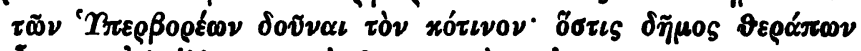

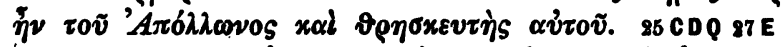

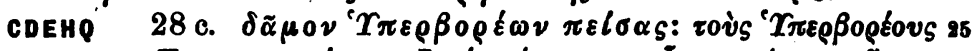

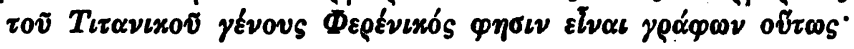

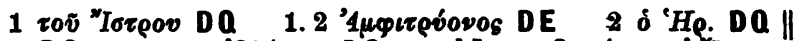

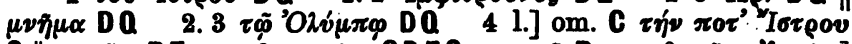

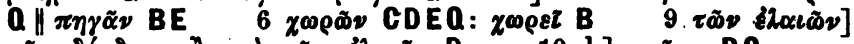

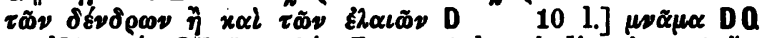

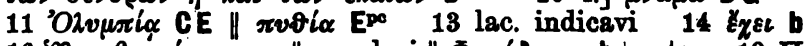

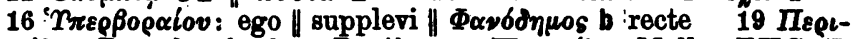

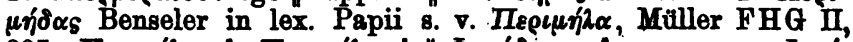

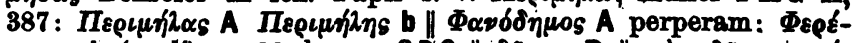

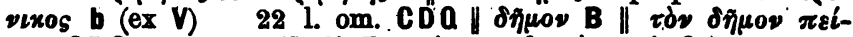

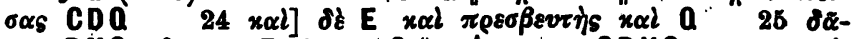

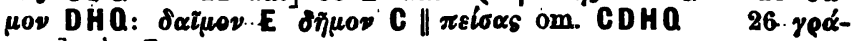
gov] rò̀ $\mathrm{E}$ 


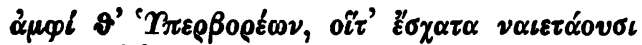

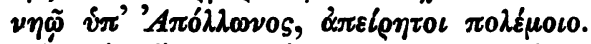

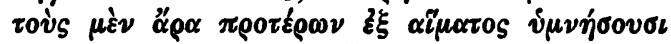

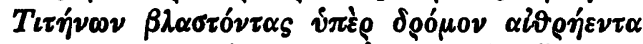

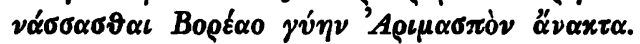

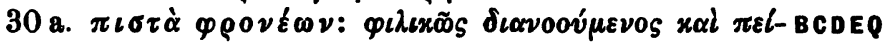

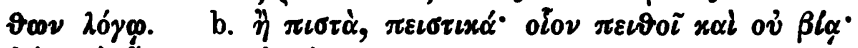

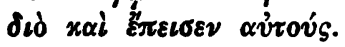

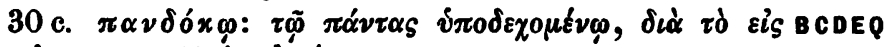

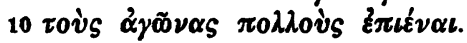

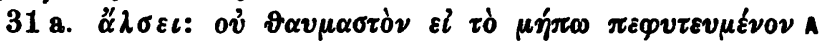

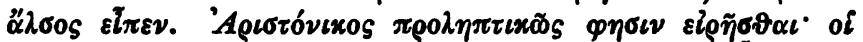

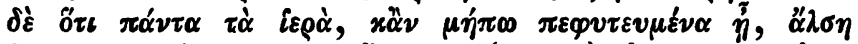

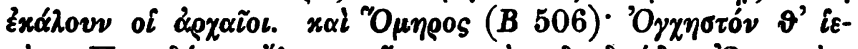

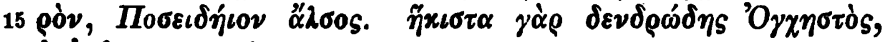

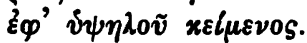

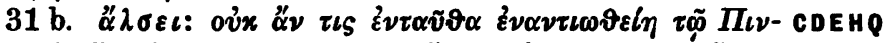

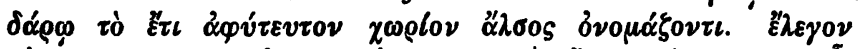

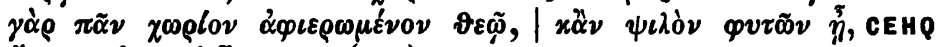

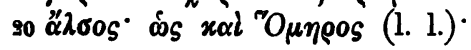

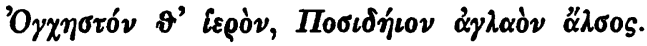

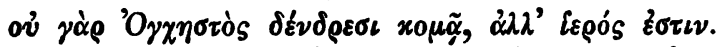

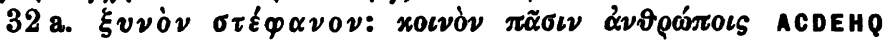

1-5 Tzetzes Chil. 7, $680 \quad 11$ Strabo 9, 412

1 ¿a $\mu \varphi i \delta^{\prime}$ Hermann obss. crit. in quosdam locos Aesch. et Eur. p. $36 \quad 2$ $2 \eta \tilde{\omega}$ Tzetzes: $\nu \alpha \tilde{\omega}$ DEGH $\alpha \tilde{\omega}$ C $\nu \alpha \dot{\nu} \nu$ a

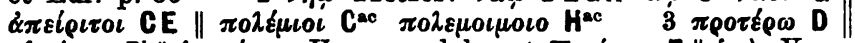

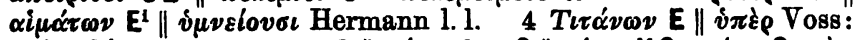

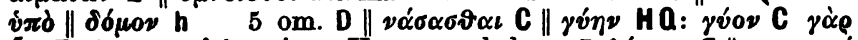

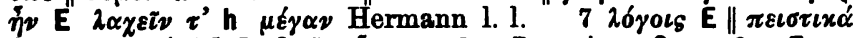

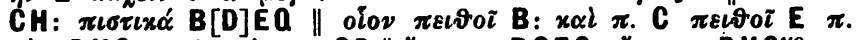

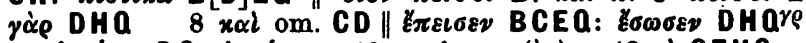

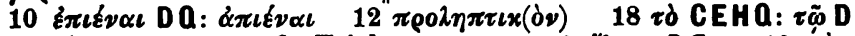

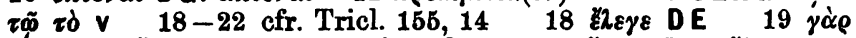

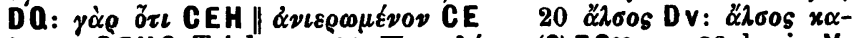

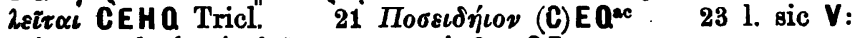

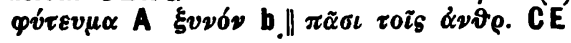

Schox. uv Pindarux ed. Drachmann. 
CDEHQ

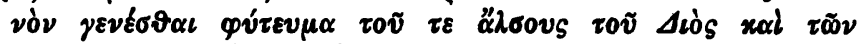

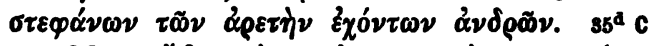

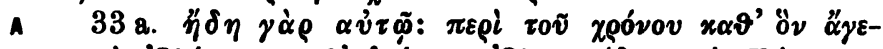

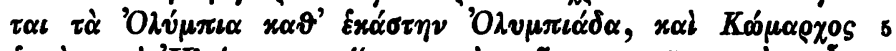

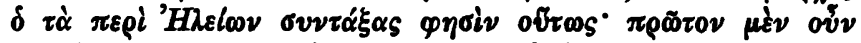

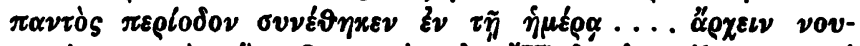

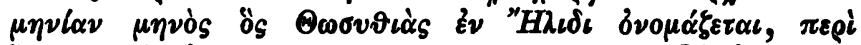

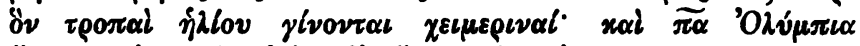

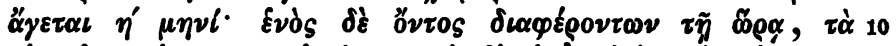

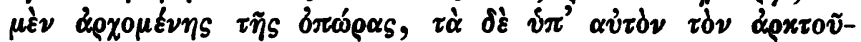

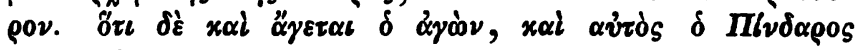

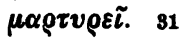

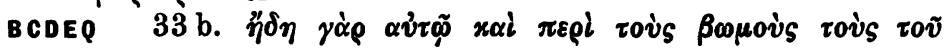

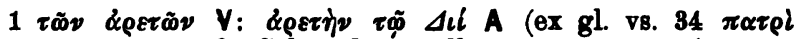

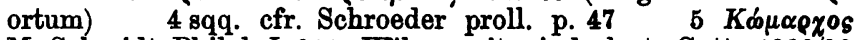
M. Schmidt Philol. I 644 Wilamowitz ind. lect. Gott. 1889/90 p. 10: $\widetilde{x \omega}$ A По $\varepsilon_{\varepsilon \omega \nu}$ Sybel Herm. V 203 'A

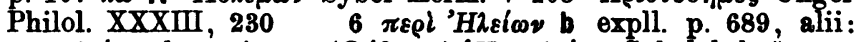

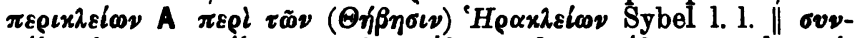

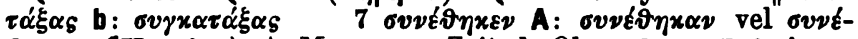
$\vartheta_{\eta} \alpha \varepsilon v\left\langle H_{\rho} \alpha x \lambda \eta \tilde{s}\right\rangle$ A. Mommsen Zeit d. Ol. p. 8 7.8 $\varepsilon^{2} \tau \tilde{\eta}$

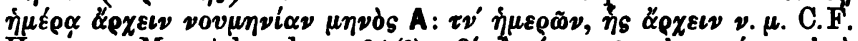

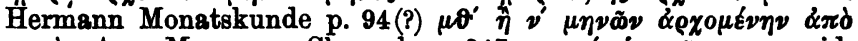

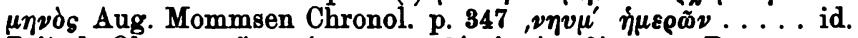

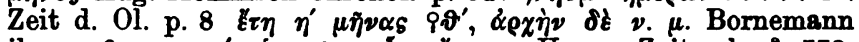

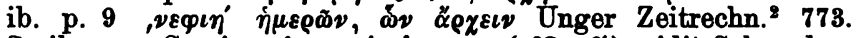
Scrib. cum Gemino $\varepsilon \tau \tilde{\omega} \nu \nu \eta^{\prime}, \eta_{\mu \varepsilon \rho \tilde{\omega} \nu}\left\langle, \beta \lambda^{\prime} \times \beta^{\prime}\right\rangle$ vidit Schroeder;

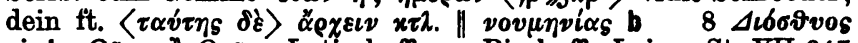
cj. b Ovos $\hat{\eta}$ @vtos Latischeff ap. Bischoff. Leipz. St. VII 347

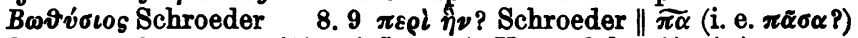

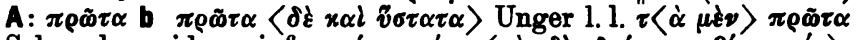
Schroeder; idem infra $\eta^{\prime} \mu \eta \nu l,\left\langle\tau \dot{\alpha} \delta \varepsilon \dot{\delta} \delta \varepsilon v i \tau \varepsilon \rho \alpha \theta^{\prime} \mu \eta \nu l^{\circ}\right\rangle$.

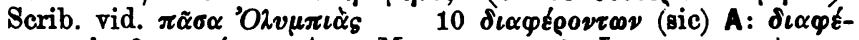

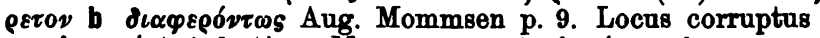

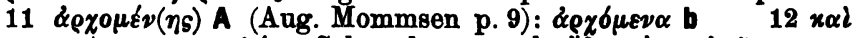

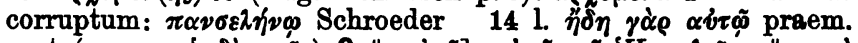

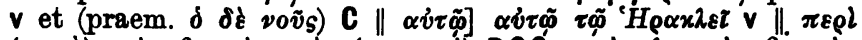

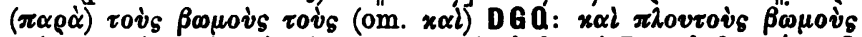

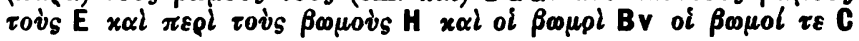




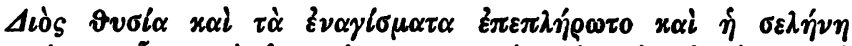

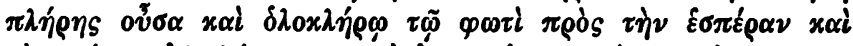

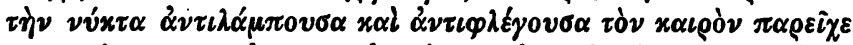

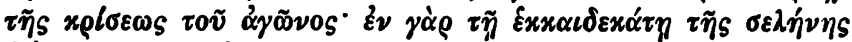

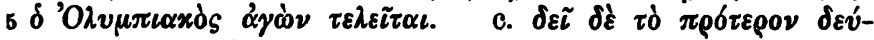

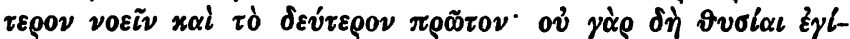

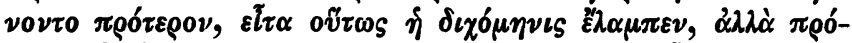

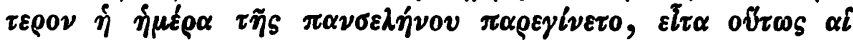

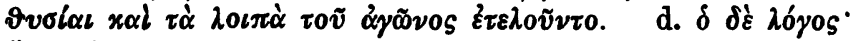

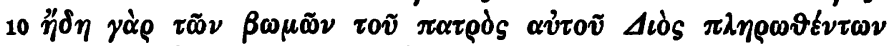

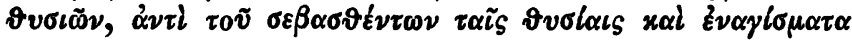
$\lambda \alpha \beta o ́ v \tau \omega \nu . \quad 35^{\circ} \mathrm{CE}$

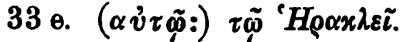

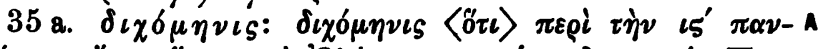

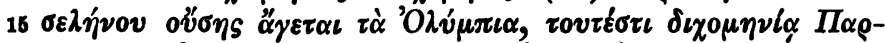

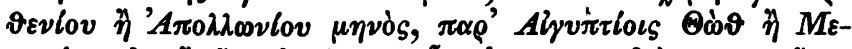

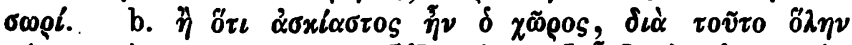

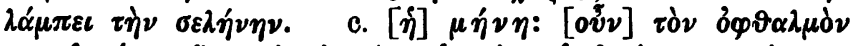

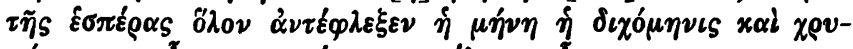

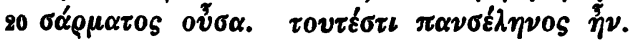

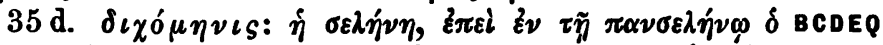

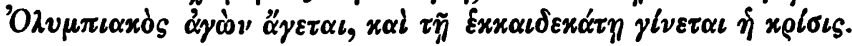

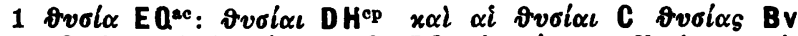

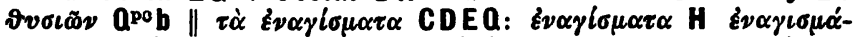

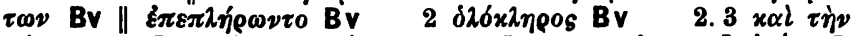

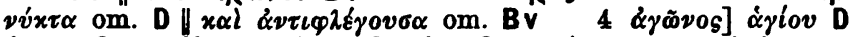

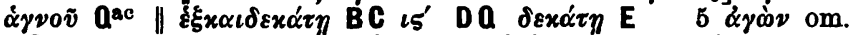

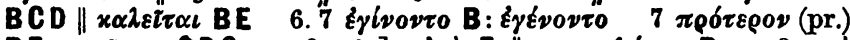

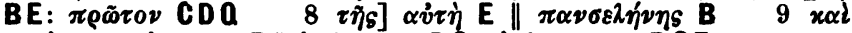

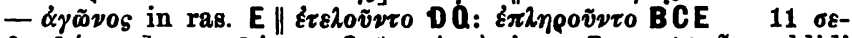

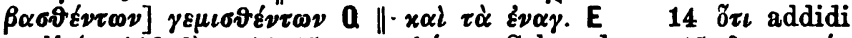

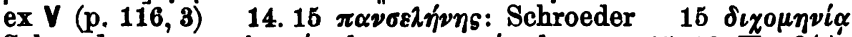
Schroeder, ego: oi $\mu \eta v i \alpha A^{\text {ac }} \nu o v \mu \eta v i \alpha A^{\text {po }} 15.16 \Pi_{\alpha \rho \vartheta}(\varepsilon)-$

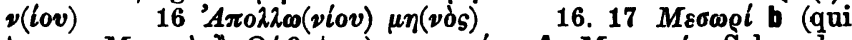

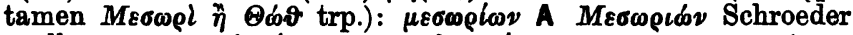

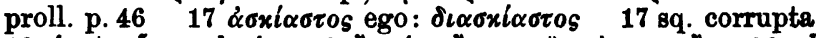

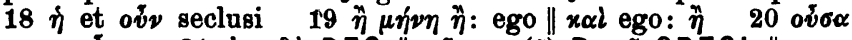

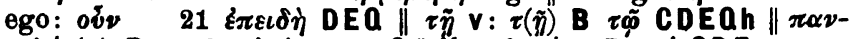
$\sigma \varepsilon \lambda \eta \dot{\nu} \nu(\omega)$ B $22 \dot{\alpha} \gamma \dot{\omega} \nu$ om. Q $\| \varepsilon \xi \xi \alpha \iota \delta \varepsilon x \alpha \tau \eta$ B $15^{\prime}$ CDE 


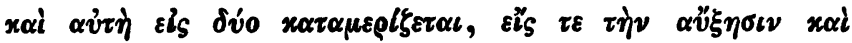

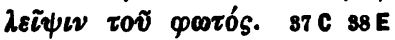

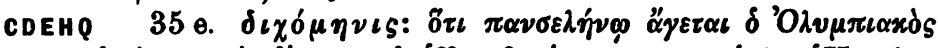

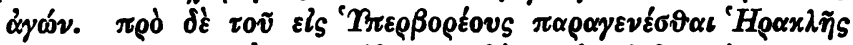

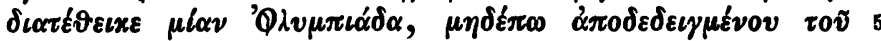

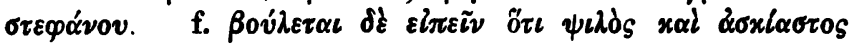

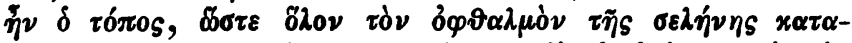

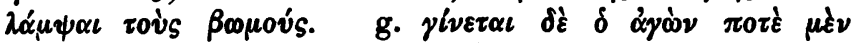

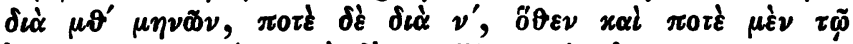

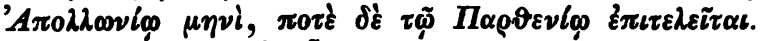

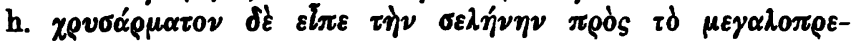

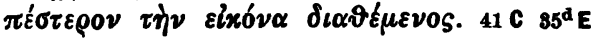

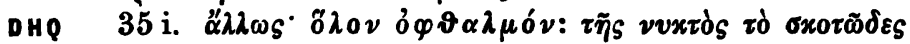

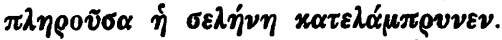

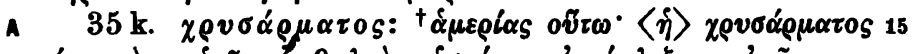

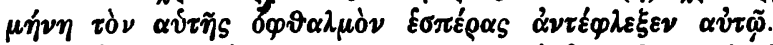

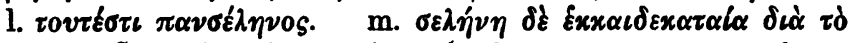

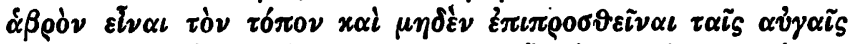

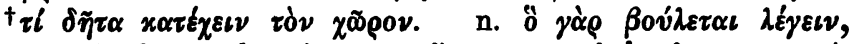

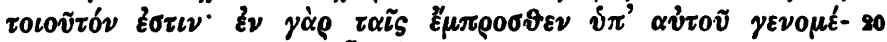

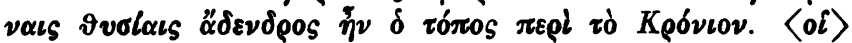

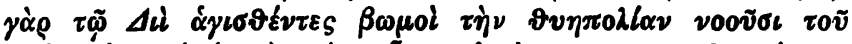

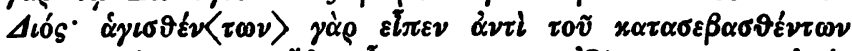

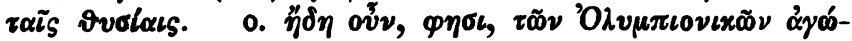

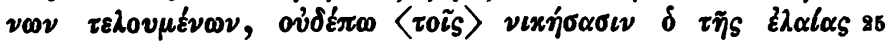

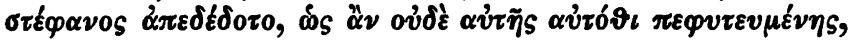

\section{8-9 Tzetzes ad Lyc. 41. id. Chil. I 581}

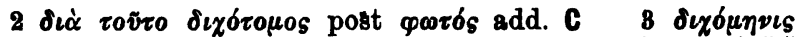

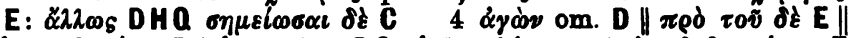

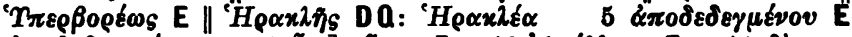

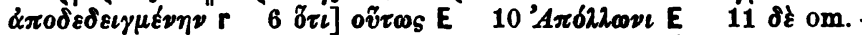

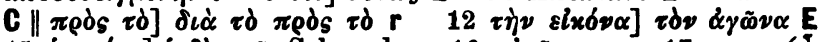

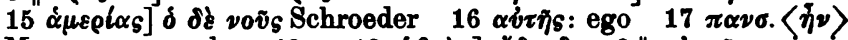

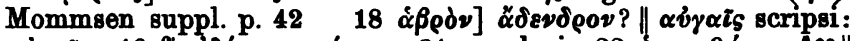

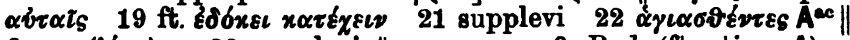

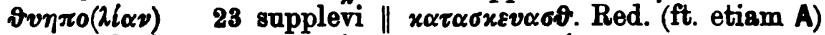

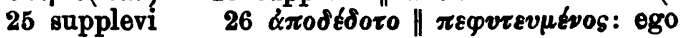




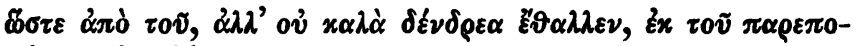

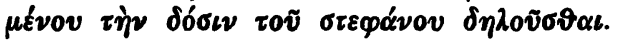

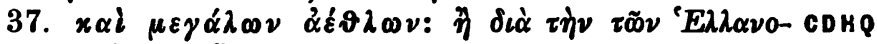

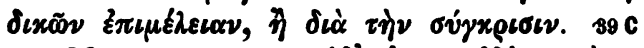

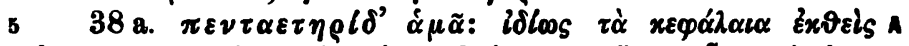

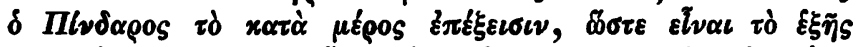

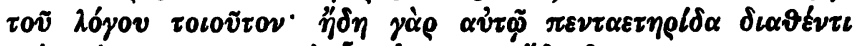

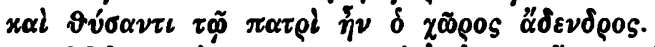

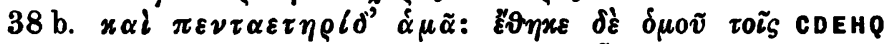

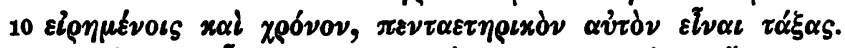

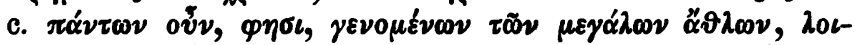

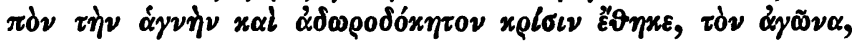

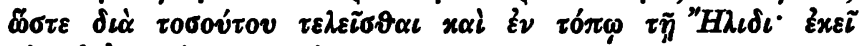

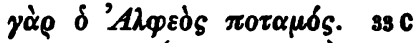

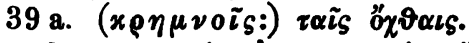

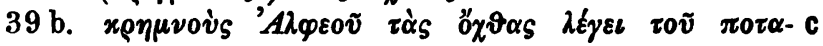
น०ṽ. $38 \mathrm{C}$

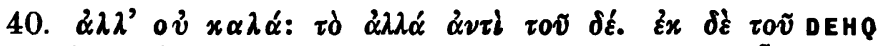

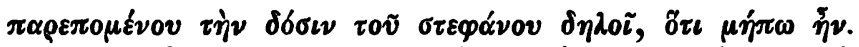

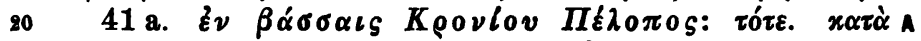

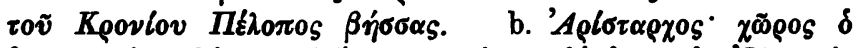

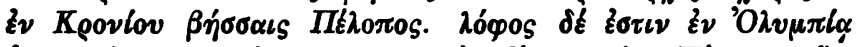

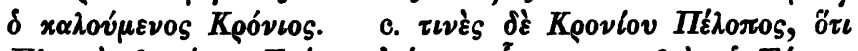

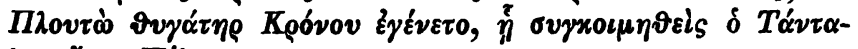

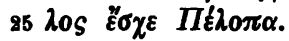

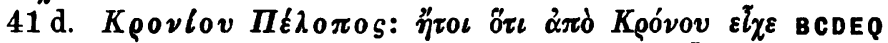

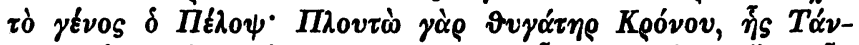

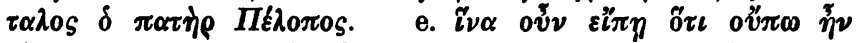

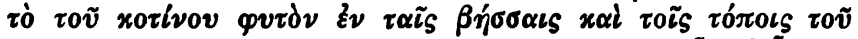

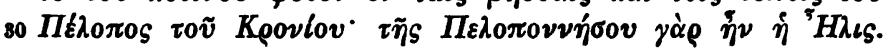

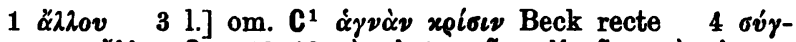

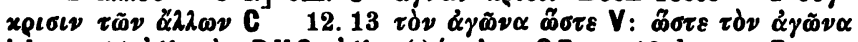

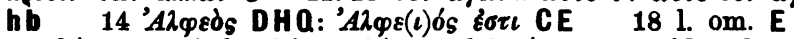

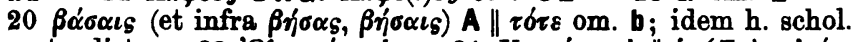

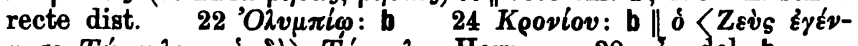

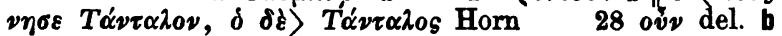

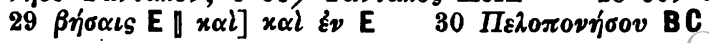




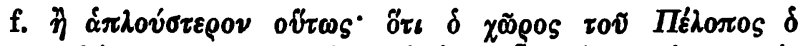

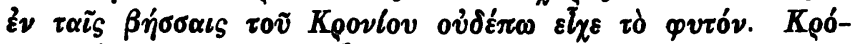

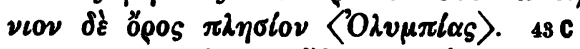

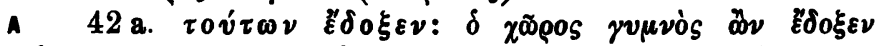

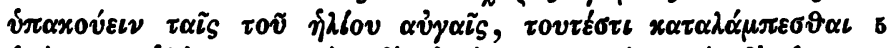

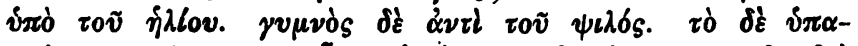

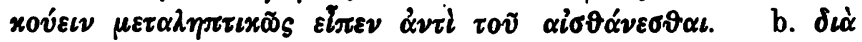

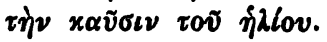

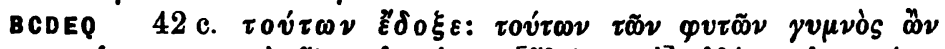

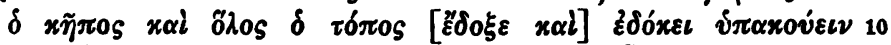

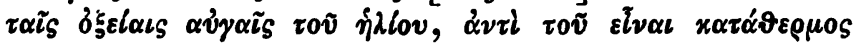

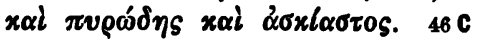

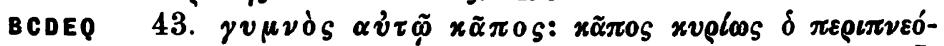

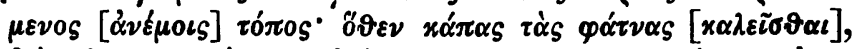

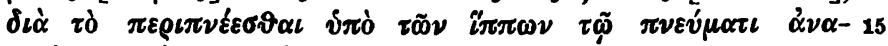

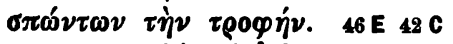

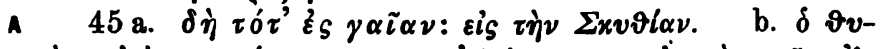

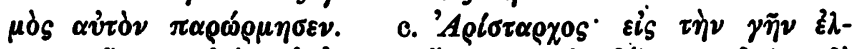

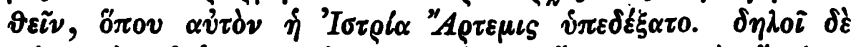

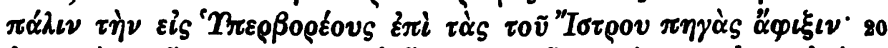

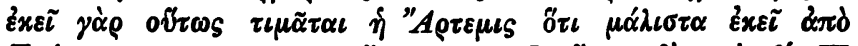

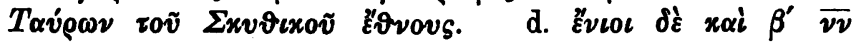

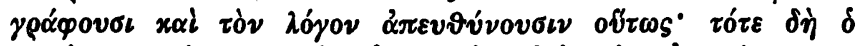

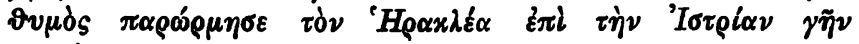

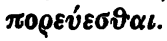

13 EM 489, 40. 511, 6. Zon. 1203. Or. 79, 19. Eust. I. 595, 23. Od. 1546, 4014 Hes. xár $10 \mathrm{~s}$

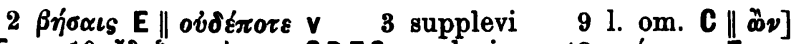

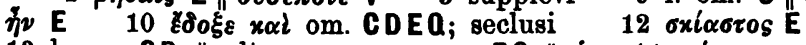

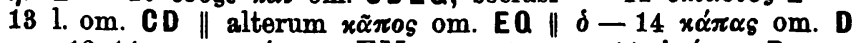

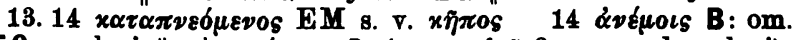

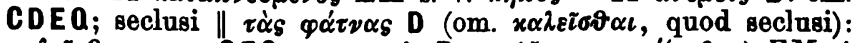

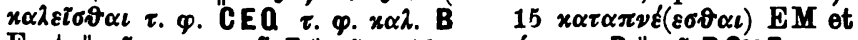

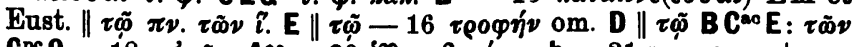

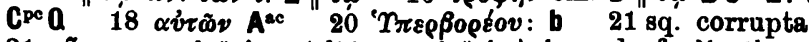

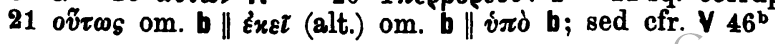




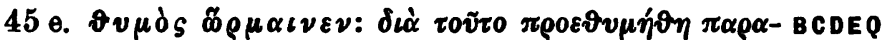

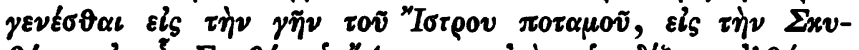

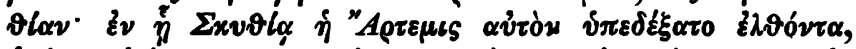

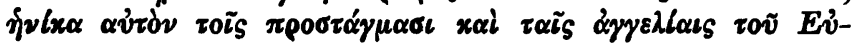

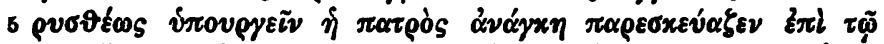

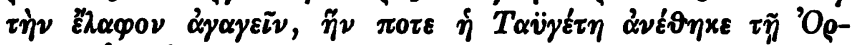

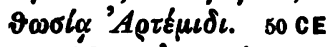

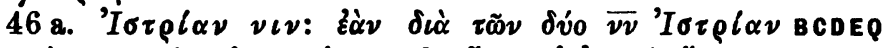

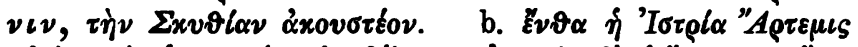

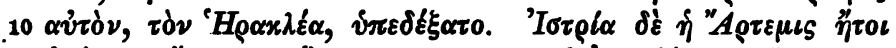

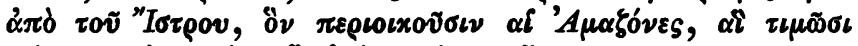

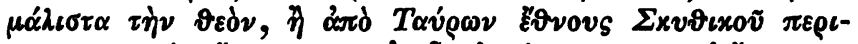

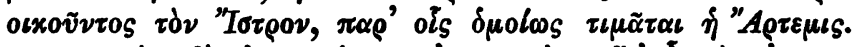

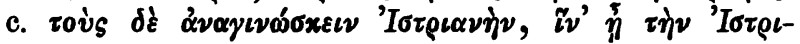

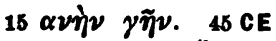

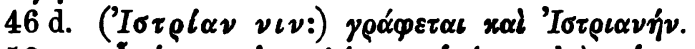
881

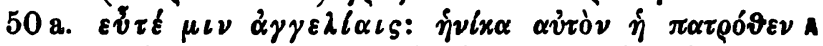

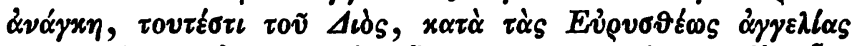

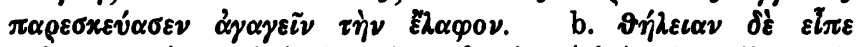

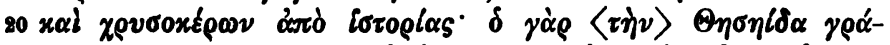

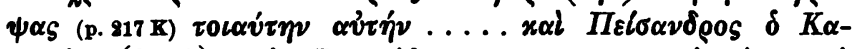

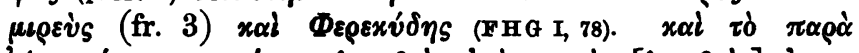

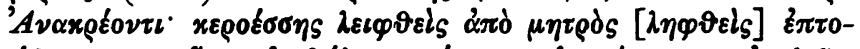

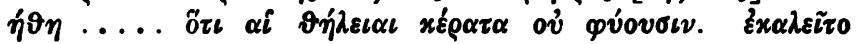

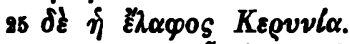

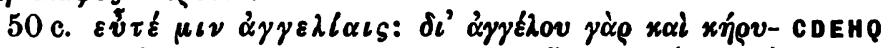

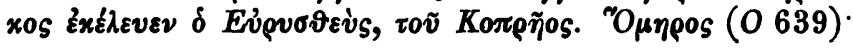

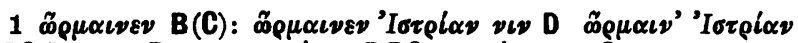

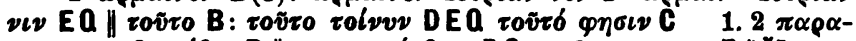

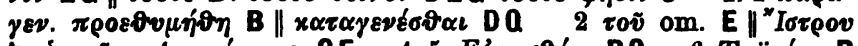

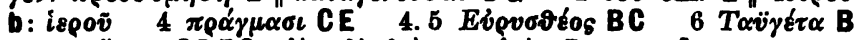

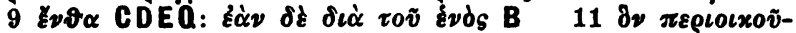

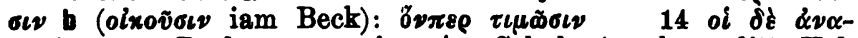

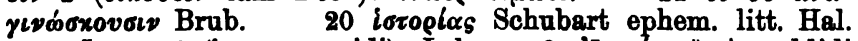

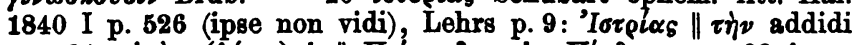

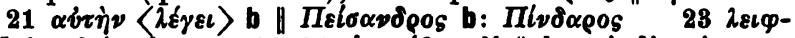

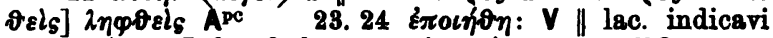

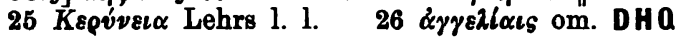




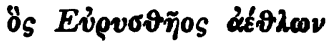

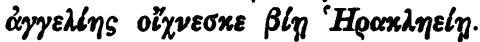

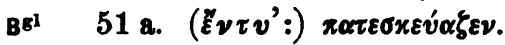

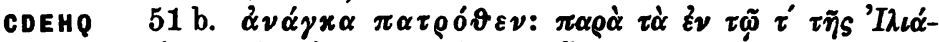

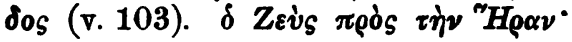

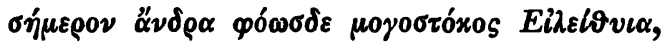

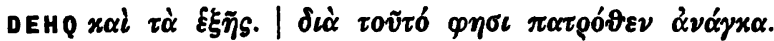

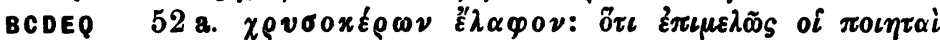

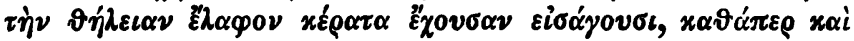

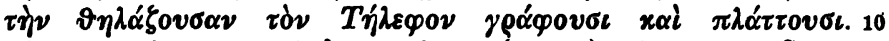

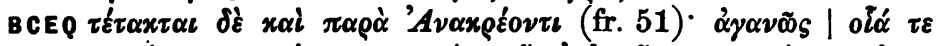

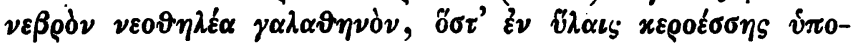

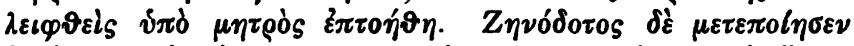

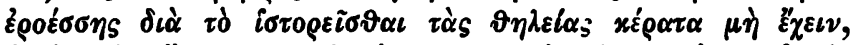

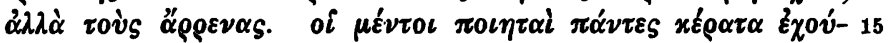

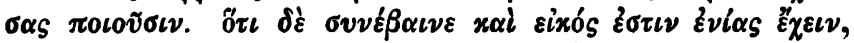

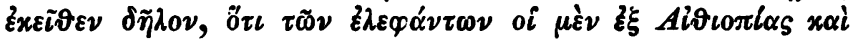

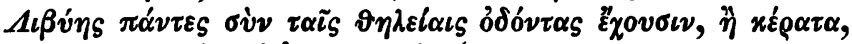

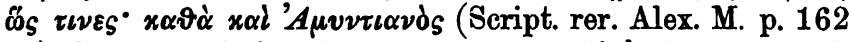

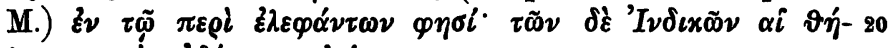

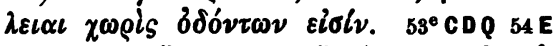

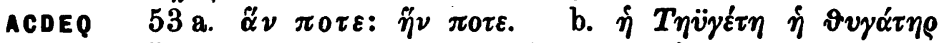

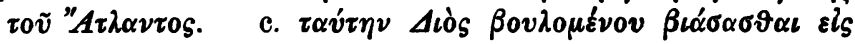

8 Ael.h. an. 7, 3917 Tzetzes sch. Chil. IV 92 (p. 109 Pr.)

$2 l_{\chi \nu \varepsilon \sigma x \varepsilon}$ CEr \| 'H $\left.{ }^{\prime} \alpha x \lambda \eta \varepsilon i \eta\right]$ om. Har lac. D $4 \tau \tilde{\varphi}$

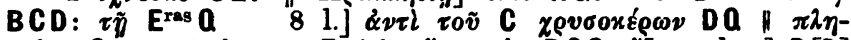

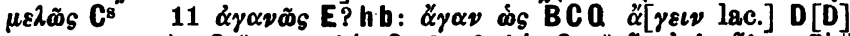

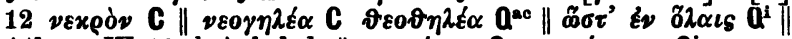

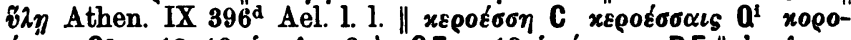

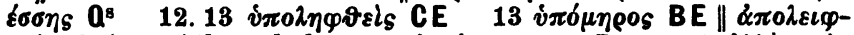

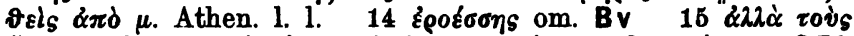

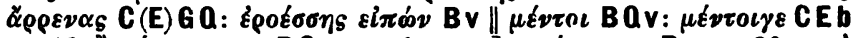

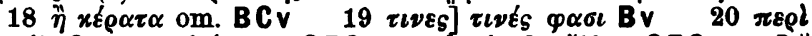

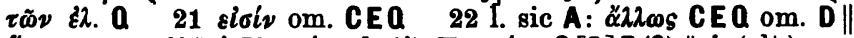

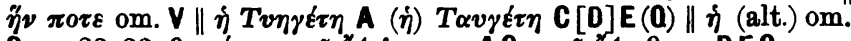

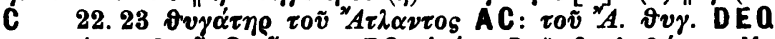

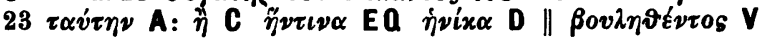




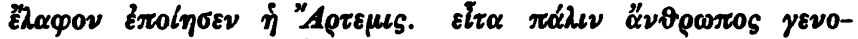

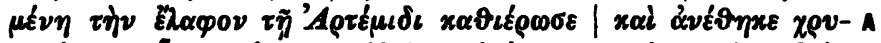

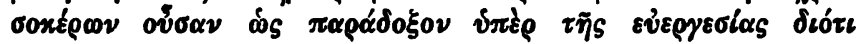

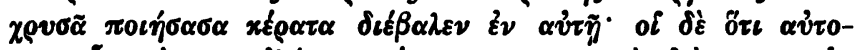

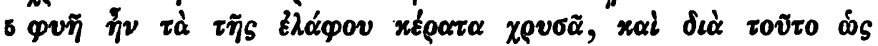

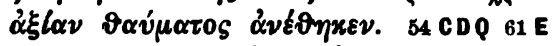

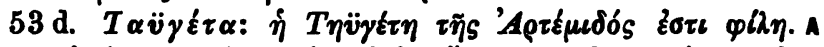

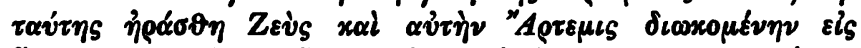

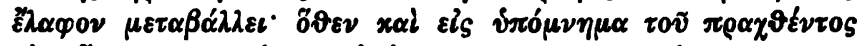

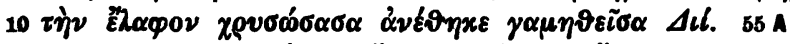

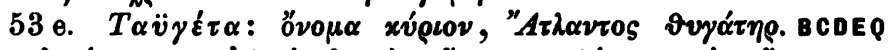

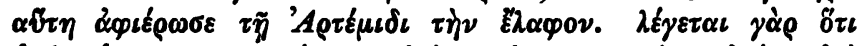

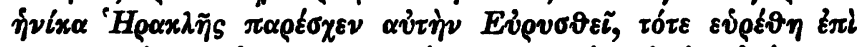

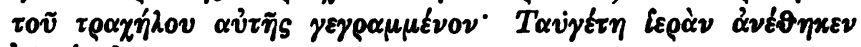
15 'A

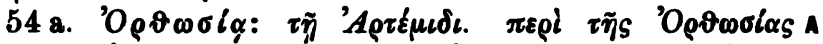

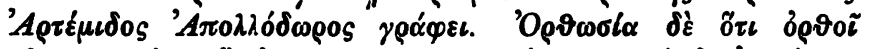

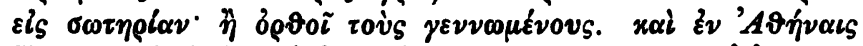

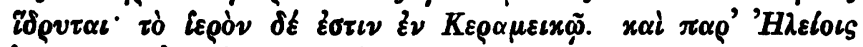

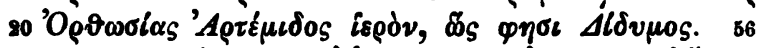

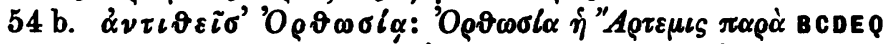

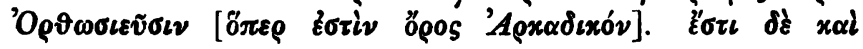

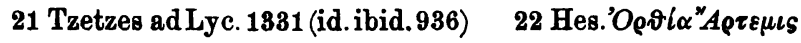

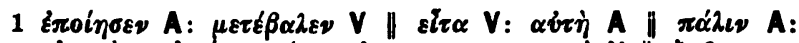

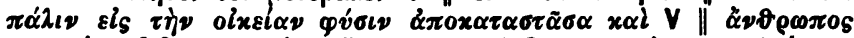

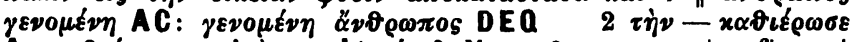

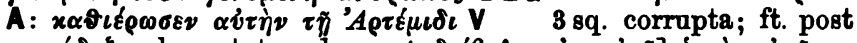

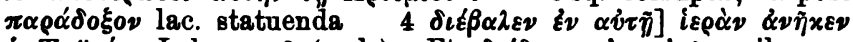

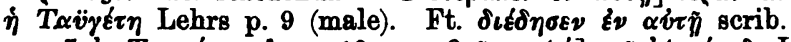

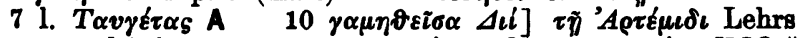

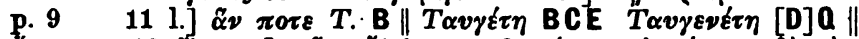

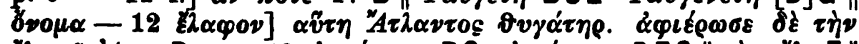
Zn. $\tau \tilde{y}{ }^{\prime} A \rho \tau$. Bv 12 dq

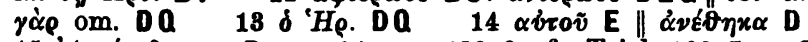

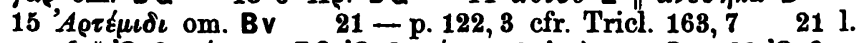

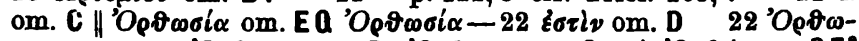

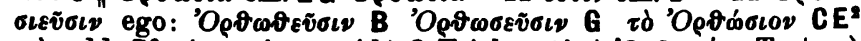

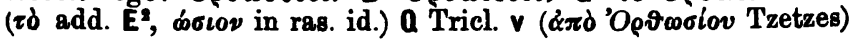


'A

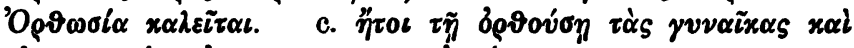

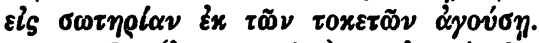

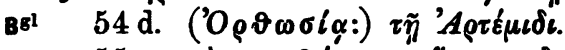

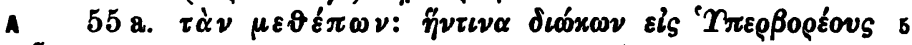
$\eta \tilde{\eta} 29 \varepsilon v .51$

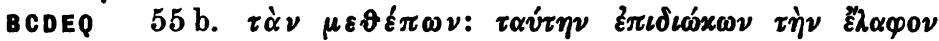

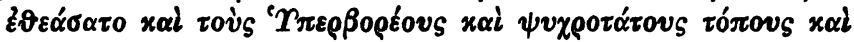

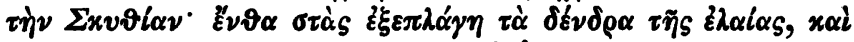

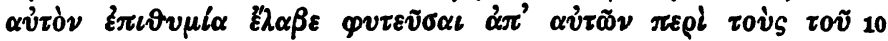

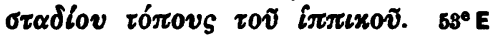

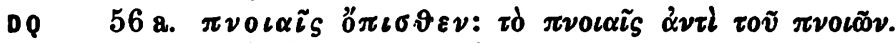

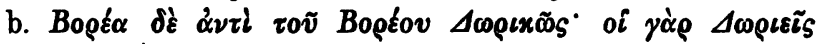

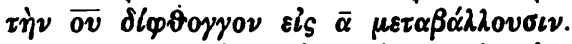

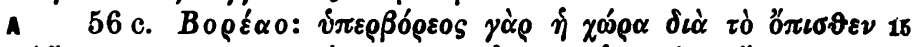

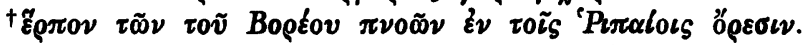

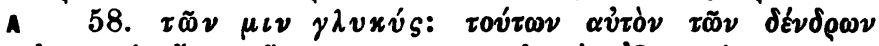

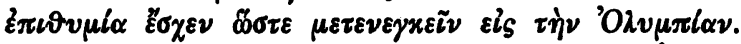

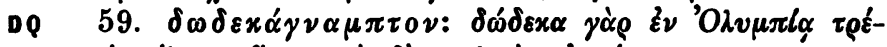

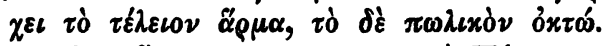

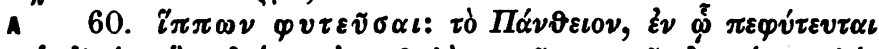

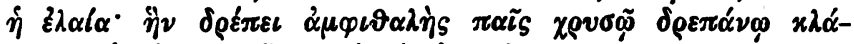

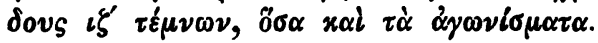

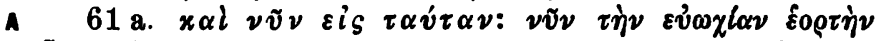

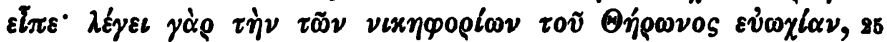

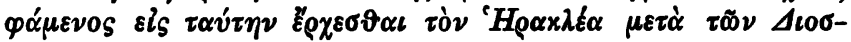

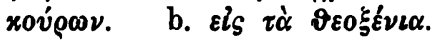

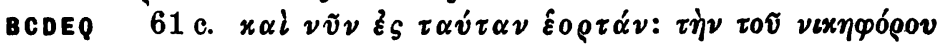

21-22 Aristot. mirab. 51. sch. Ar. Plut. 586 (= Suid. xo-

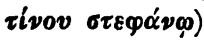

(ad p. 121) 22 ö $\pi \varepsilon \rho-' A \rho x$. delevi (hab. Tzetzes Tricl.) \|'A $\rho x \alpha-$

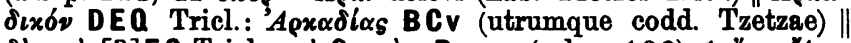

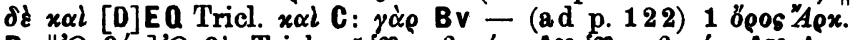

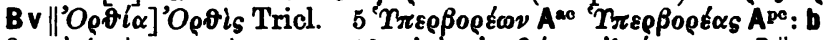

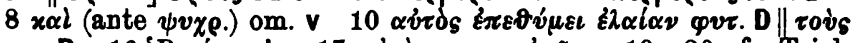

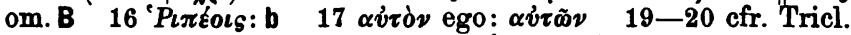

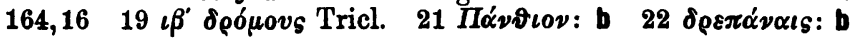




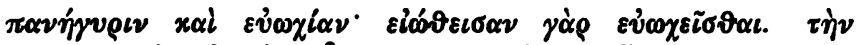

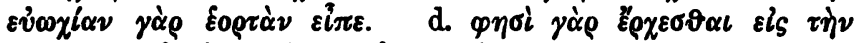

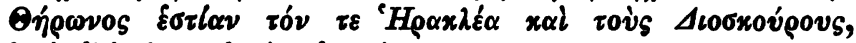

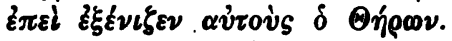

5 62 a. $\alpha \nu \tau \iota \vartheta \varepsilon \varepsilon$

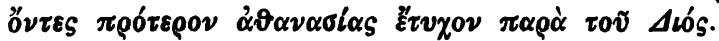

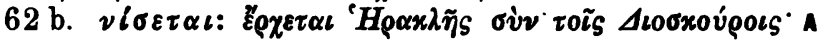

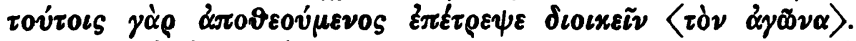

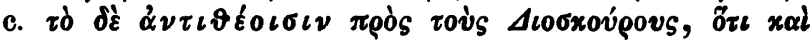

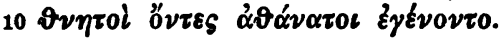

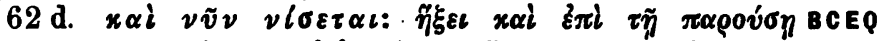

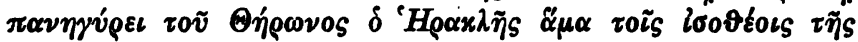

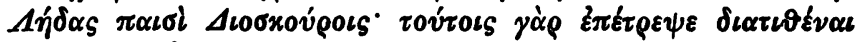

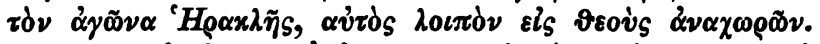

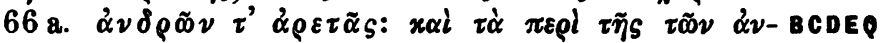

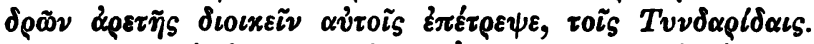

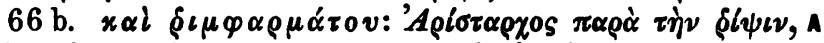

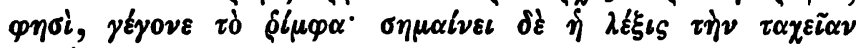

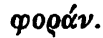

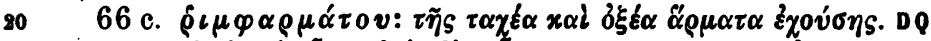

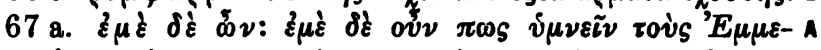

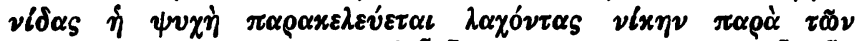

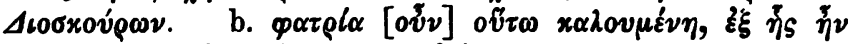

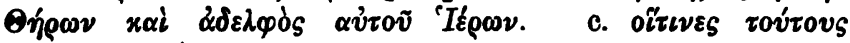

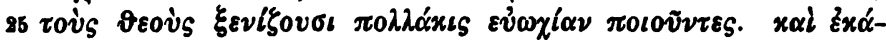

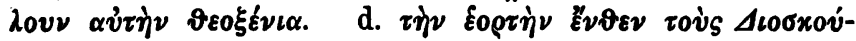

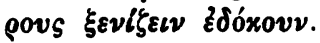

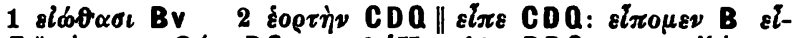

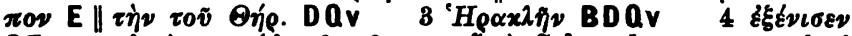

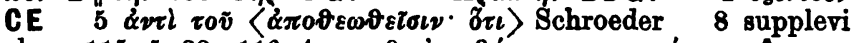

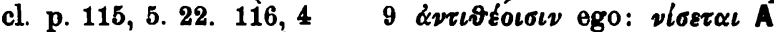

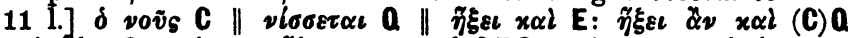

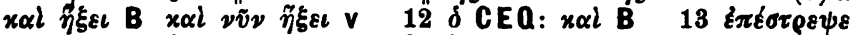

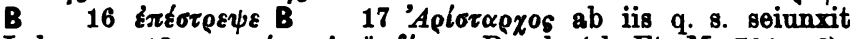

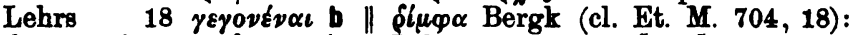

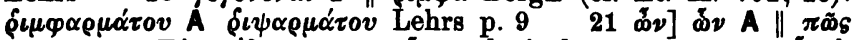

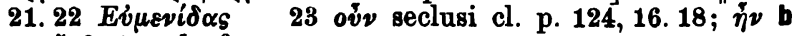
26 हैข $\nu$ ? sed cfr. p. 87, 22 


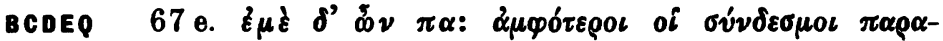

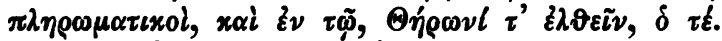

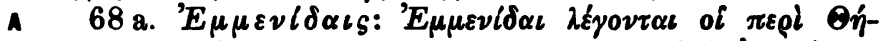

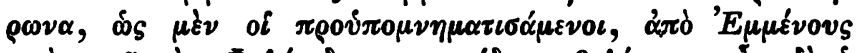

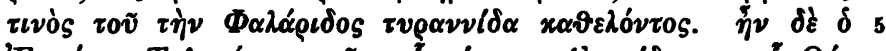

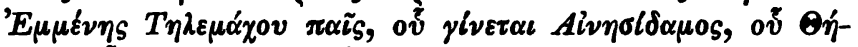

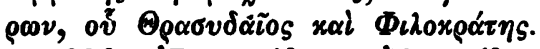

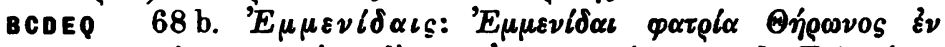

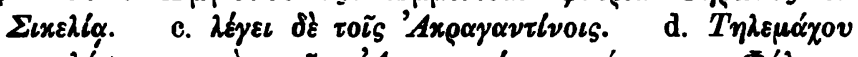

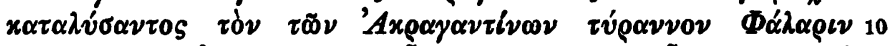

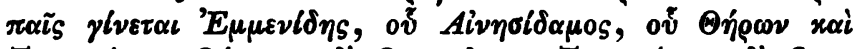

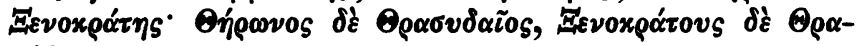
бúßovios.

Bgl 71. ( $(\pi \lambda \varepsilon l \sigma \tau \alpha \iota \sigma \iota:) \pi \varepsilon \pi \lambda \eta \rho \omega \mu \varepsilon \dot{\nu} \alpha \iota s$.

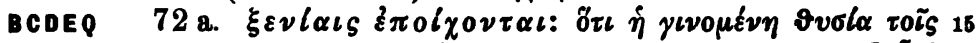

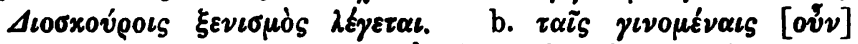

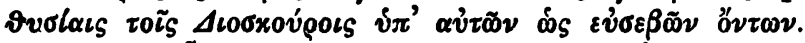

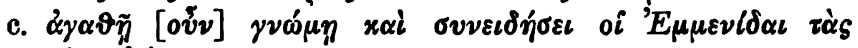

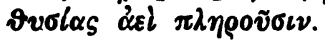

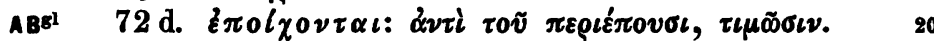

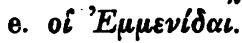

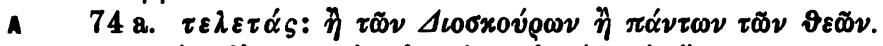

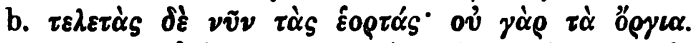

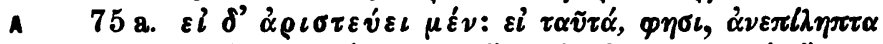

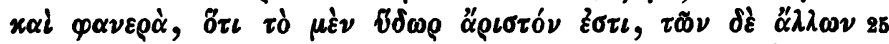

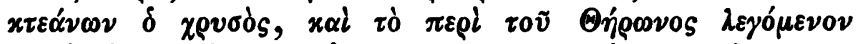

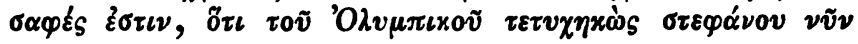

$1 \pi \tilde{\alpha}$ DQ: $\pi \alpha \tilde{\imath}$ BE om. C $\|$ oi $\sigma \dot{\nu} \nu \delta \varepsilon \sigma \mu o \iota$ CDEQ: $\sigma \dot{\nu} \nu \delta \varepsilon \sigma \mu o \iota$

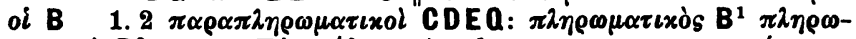

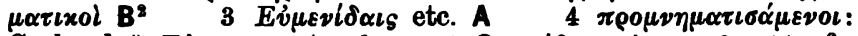

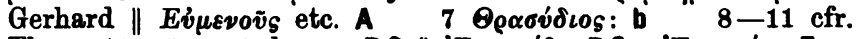

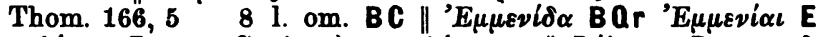

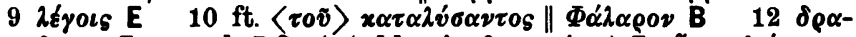

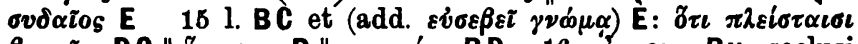

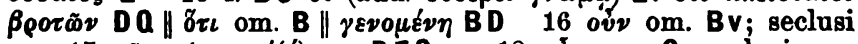

$17 \tau \tilde{\omega} \nu \Delta \iota 0 \sigma \times 0 \hat{v}(6) \rho \omega \nu$ DEQ 18 ờv om. C; seclusi

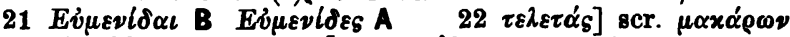

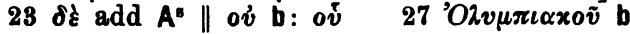




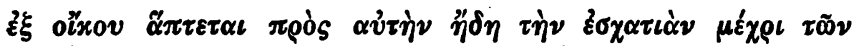

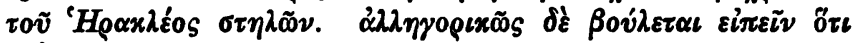

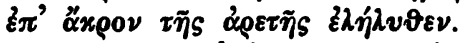

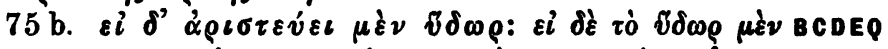

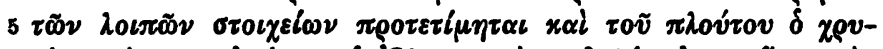

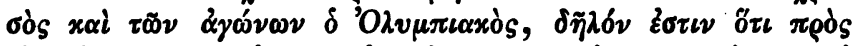

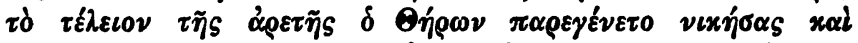

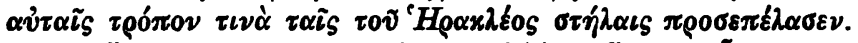

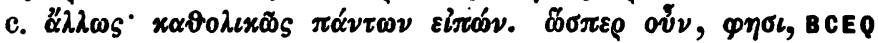

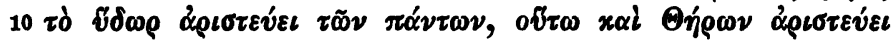

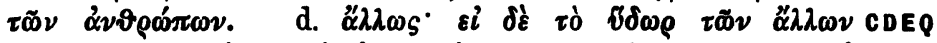

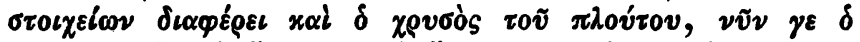

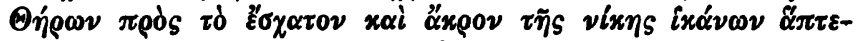

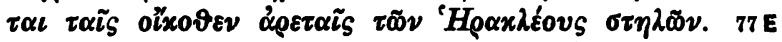

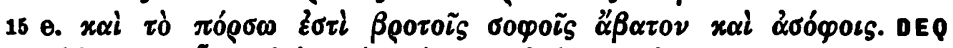

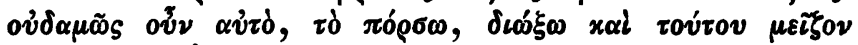

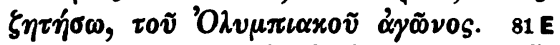

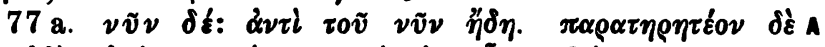

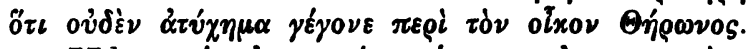

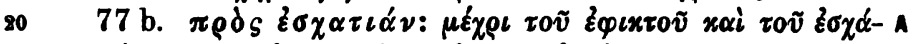

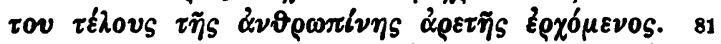

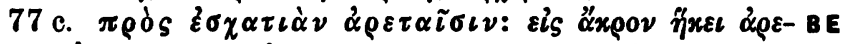

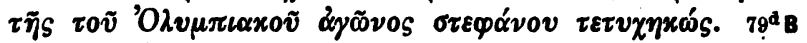

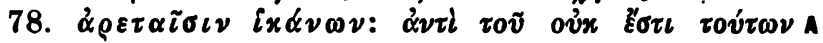

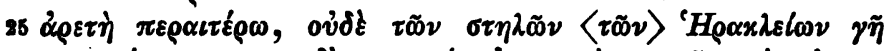

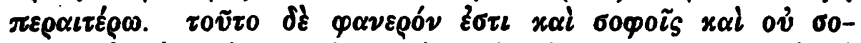

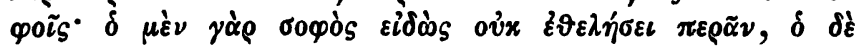

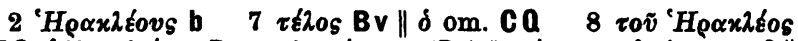

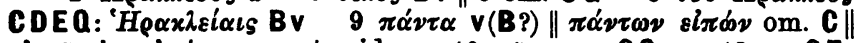
$\varepsilon i \pi \varepsilon \tilde{\imath} \nu \mathrm{h}$; $\varepsilon i \pi \dot{\omega} \nu$ corrupt. vid. $10 \tau \tilde{\omega} \nu$ om. CQ $12 \gamma \varepsilon$ CE:

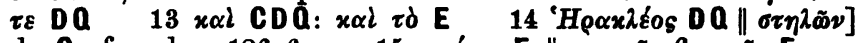

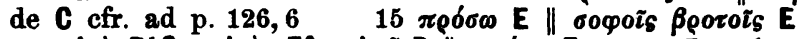

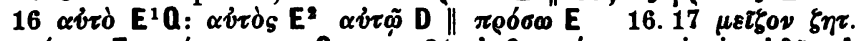

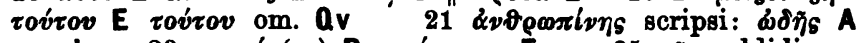

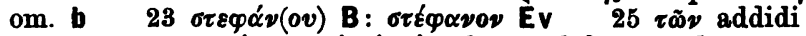
25. $26 \gamma \tilde{\eta} \pi \varepsilon \rho \alpha \iota \tau \varepsilon \rho \omega$ scripsi: in A cp. dub., quod $\pi \varepsilon \rho \alpha \iota \tau \varepsilon \rho \omega \omega$ solvit Schroeder; sed ab $\gamma \eta$ certe incipit 


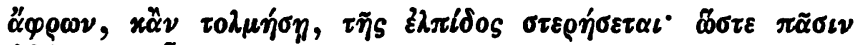

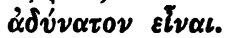

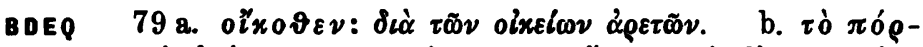

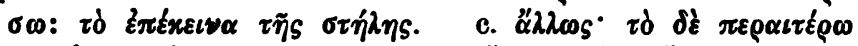

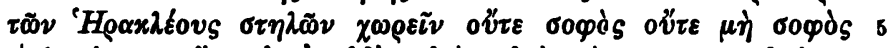

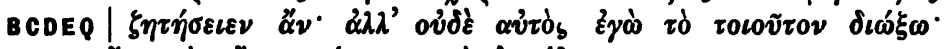

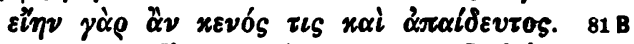

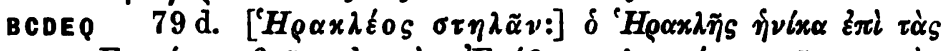

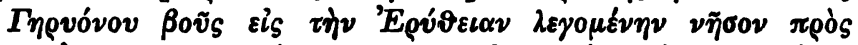

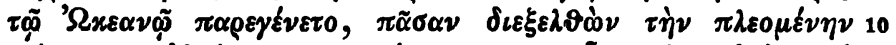

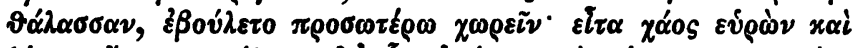

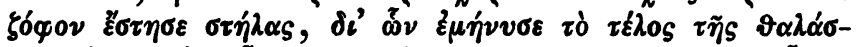

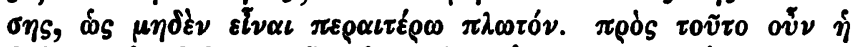

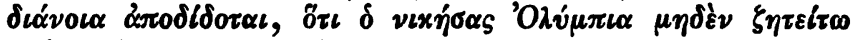

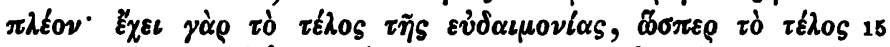

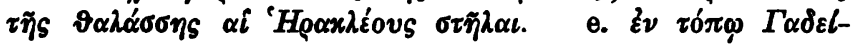
pols. $79^{\circ} B \quad 75^{\circ} \mathrm{E}$

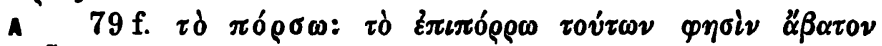

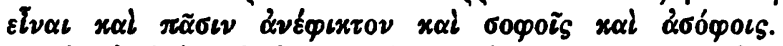

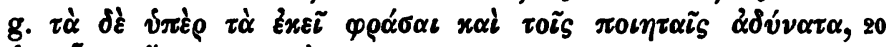

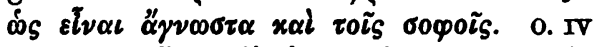

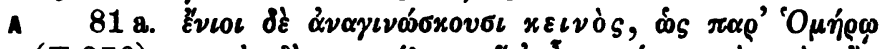

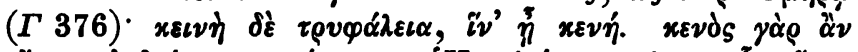

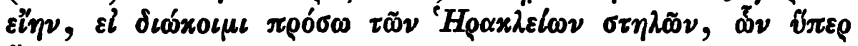
$\alpha$ ' $\gamma \alpha \nu .78$

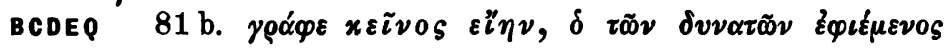

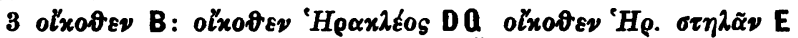

3. $4 \pi \rho \sigma \sigma \omega \omega$ BE $4 \tau \tilde{\omega} \nu \sigma \tau \eta \lambda \tilde{\omega} \nu \mathrm{BV} \| \ddot{\alpha} \lambda \lambda_{0}$ om. BV $\| \delta \dot{\mathrm{DE} Q}$ :

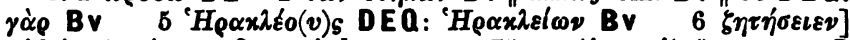

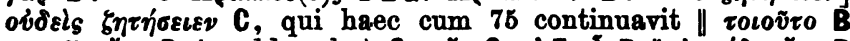

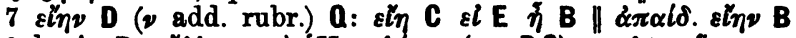

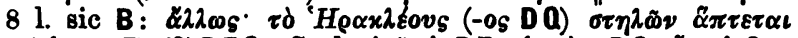

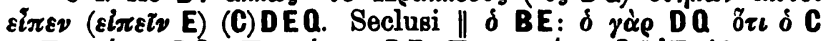

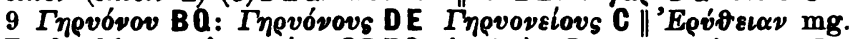

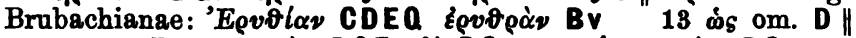

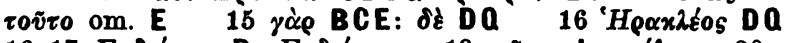

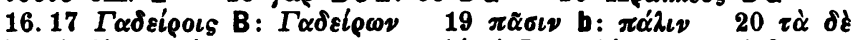
b: $\tau \dot{\partial} \delta^{\prime} \varepsilon \| \varphi \vartheta \alpha^{\prime} \sigma \alpha \iota: \operatorname{ego} 26 \gamma \rho\left(\alpha^{\prime} \varphi \varepsilon\right)$ B: $\gamma \rho\left(\alpha^{\prime} \varphi \varepsilon \tau \alpha \iota\right) x \alpha \iota$ C om.

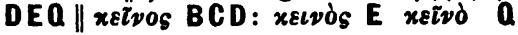




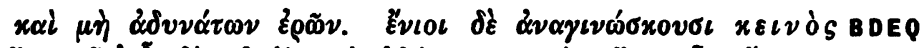

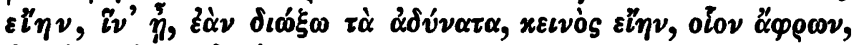

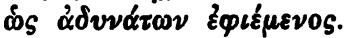

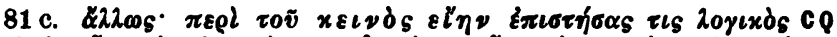

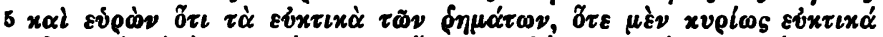

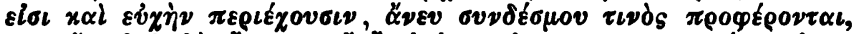

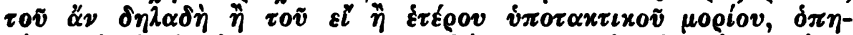

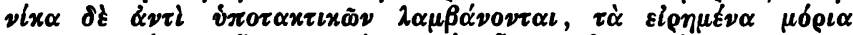

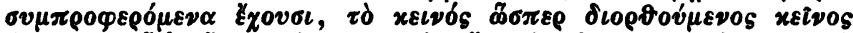

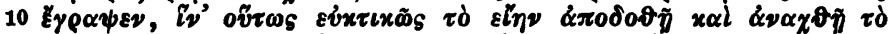

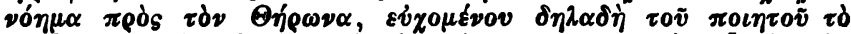

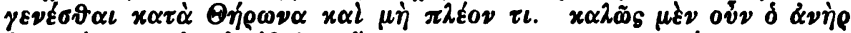

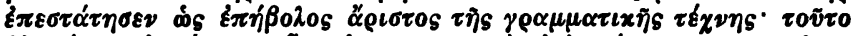

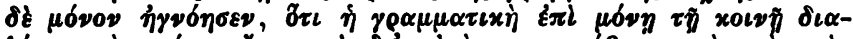

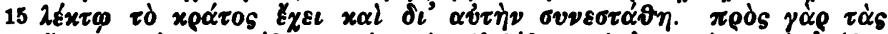

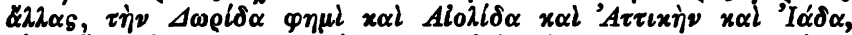

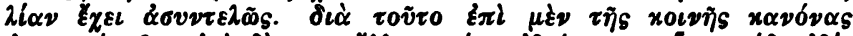

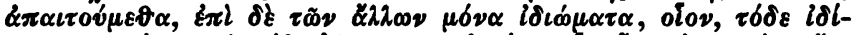

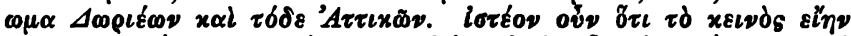

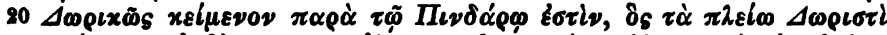

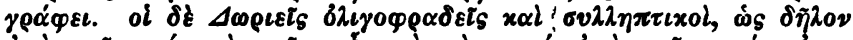

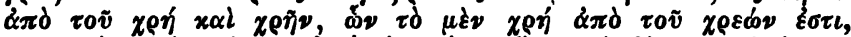

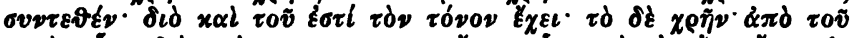

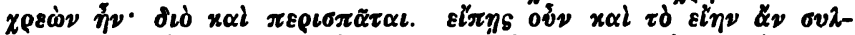

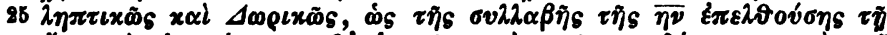

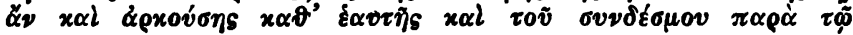
$\Delta \omega \rho \iota \varepsilon$.

$\tau \varepsilon \hat{\lambda o s} \theta \varepsilon \circ \xi \varepsilon \nu i \omega \nu$.

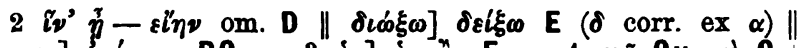

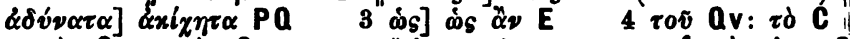

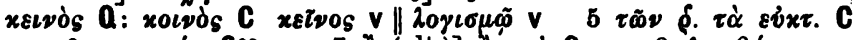

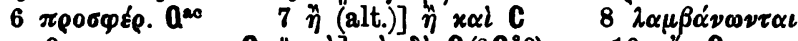

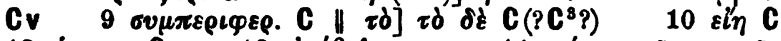

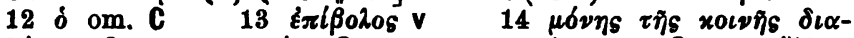

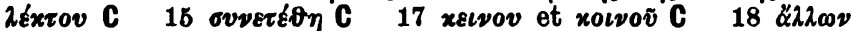

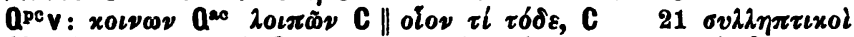

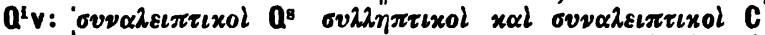

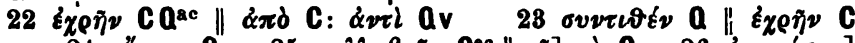

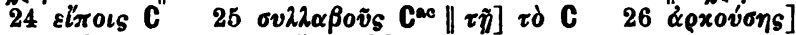

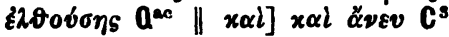




\section{Scholia in 0lympionicarum carmen IV.}

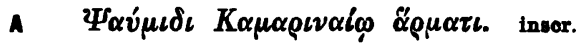

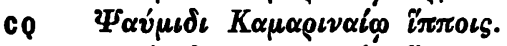

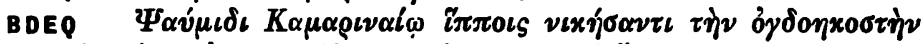

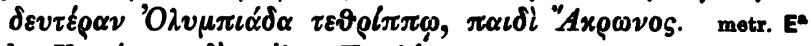

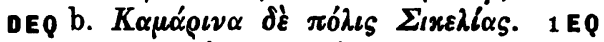

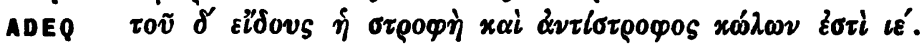

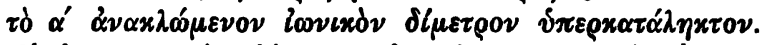

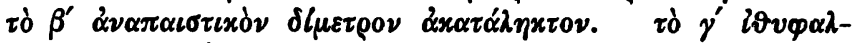

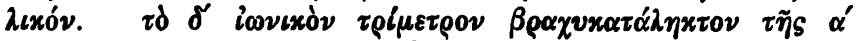

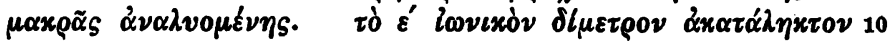

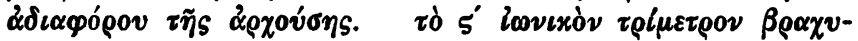

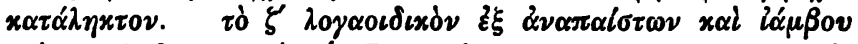

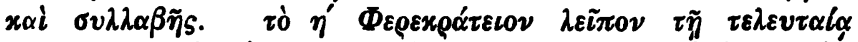

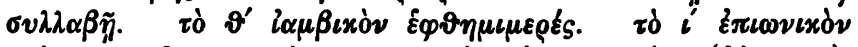

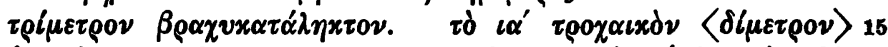

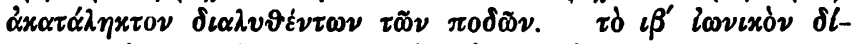

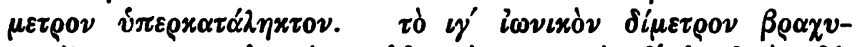

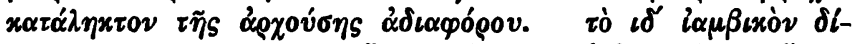

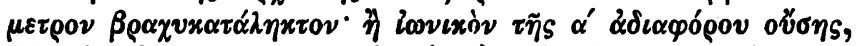

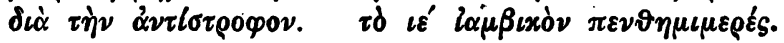

$1 \psi \alpha \dot{\mu} \mu \iota \delta \iota 2$ sqq. inscr. post metr. hab. DQ ibid. repet.

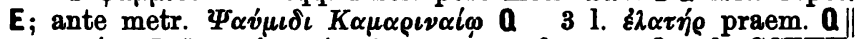

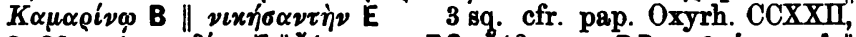

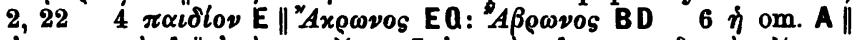

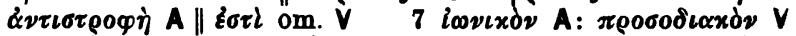

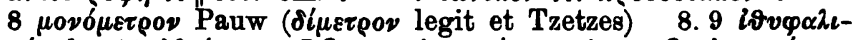

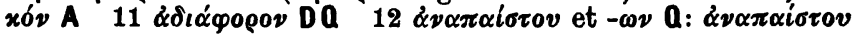
ADE. Corr. iam Pauw $13 x \alpha \downarrow \sigma v \lambda \lambda \alpha \beta \tilde{\eta} \mathrm{S}$ EQ Tzetzes: $\delta \iota \sigma v \lambda-$

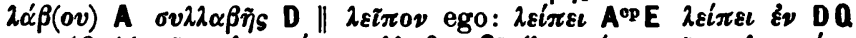

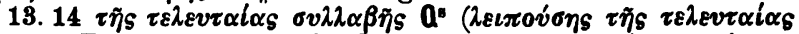

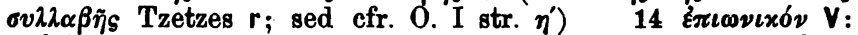

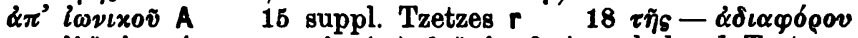

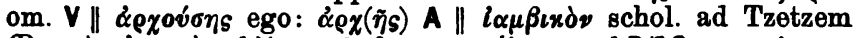

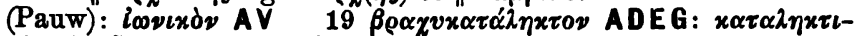

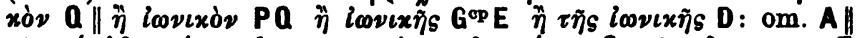

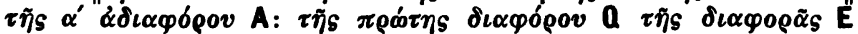

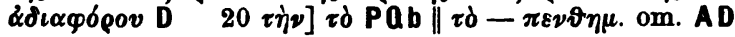




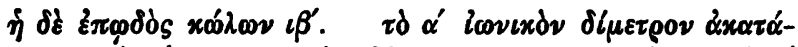

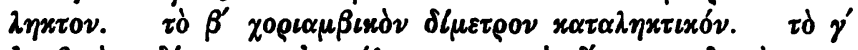

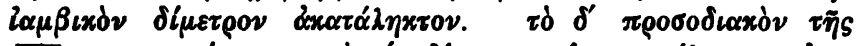

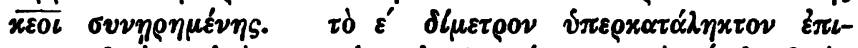

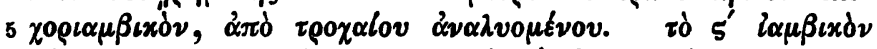

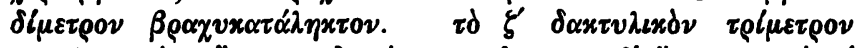

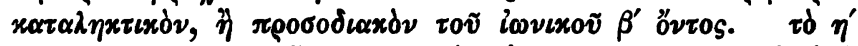

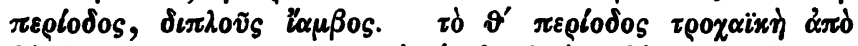

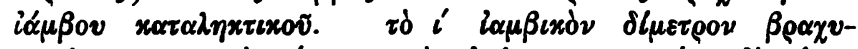

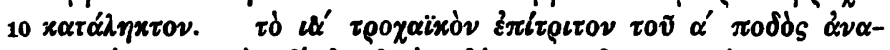

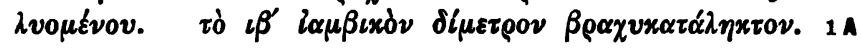
inscr. DEQ

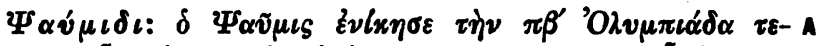

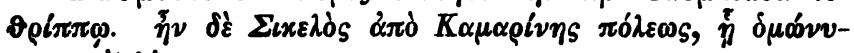
$15 \mu 0 S$ xai $\lambda l \mu \nu \eta$. metr.

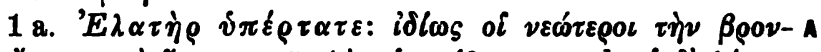

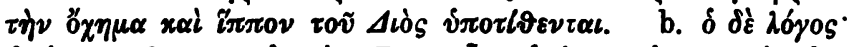

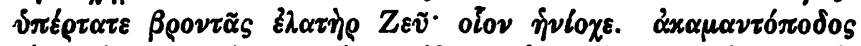

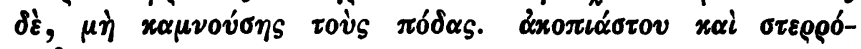
20 rodos.

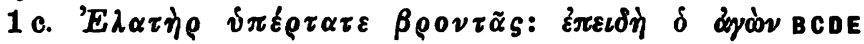

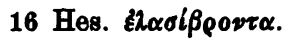

$1 \hat{\eta}-\imath \beta^{\prime}$ om. D $\| x \omega \lambda_{\omega \nu}$ EQ: $\varepsilon l_{S}$ A $\left.\| \alpha^{\prime}\right] \alpha^{\prime} \tau \tilde{\eta} s \varepsilon \pi \omega \delta \tilde{\eta}_{S} \mathrm{D}$

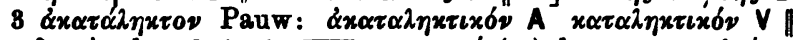

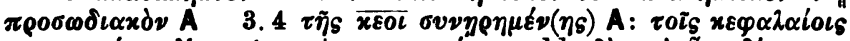

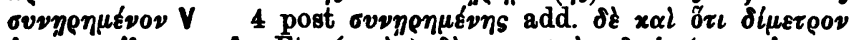

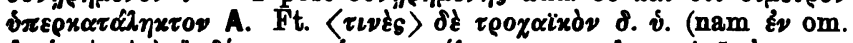

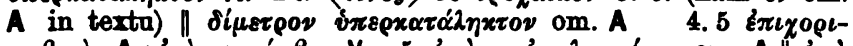

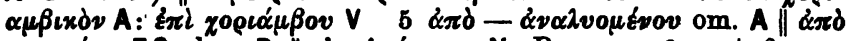

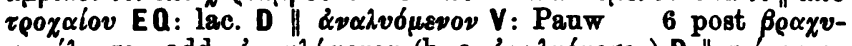

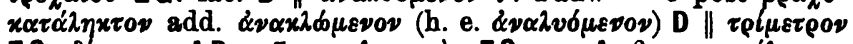

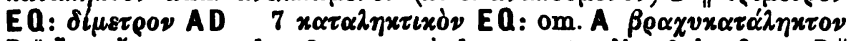
D $\| \hat{\eta}-b v \tau$ -

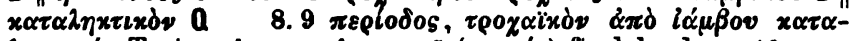
$\lambda \eta x \tau\llcorner x \sigma \nu$ Tzetzes $b$; $x \alpha \tau \alpha \lambda \eta x \tau\llcorner x \circ \tilde{v}$ (s. -xbv) ft. delend. $10 \tau \rho \circ-$

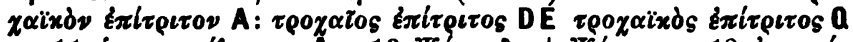

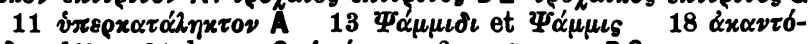

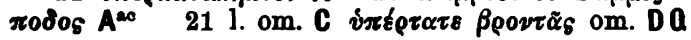

Sohor. m Predsex ed. Drachmann. 


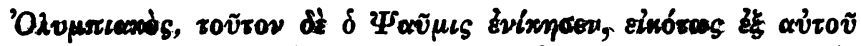

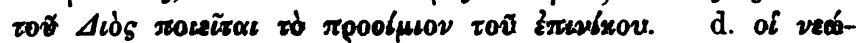

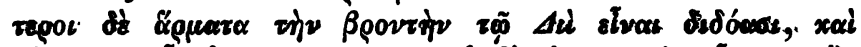

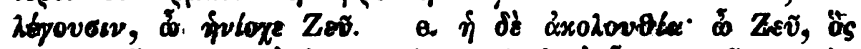

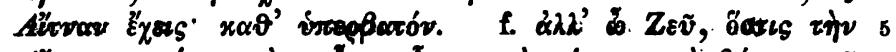

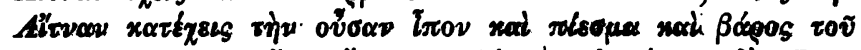

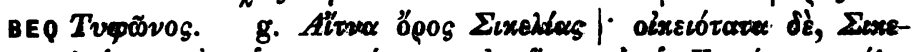

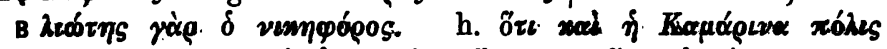

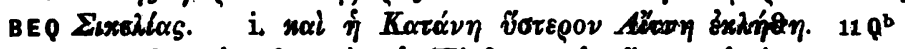

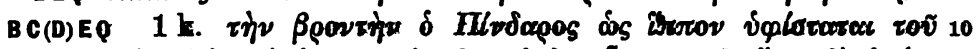

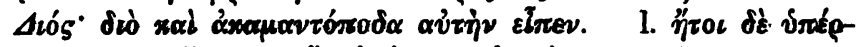

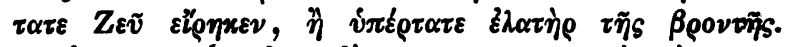

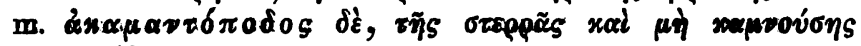
rovis $\pi \delta_{\delta} \alpha$ g.

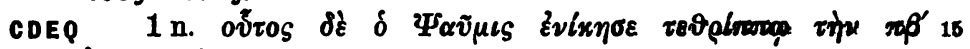
'Qגopscsésd $\alpha$.

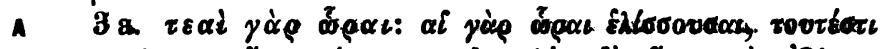

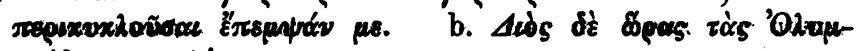

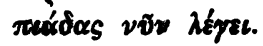

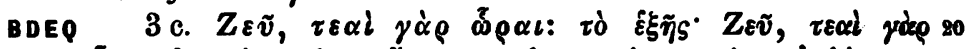

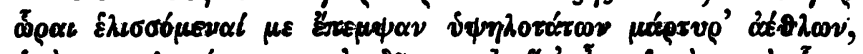

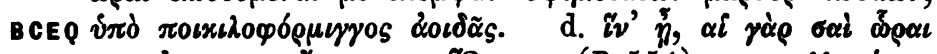

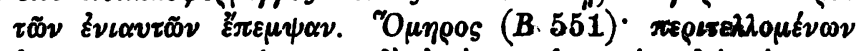

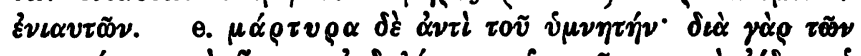

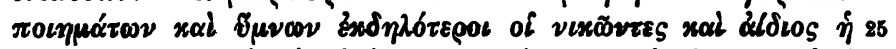

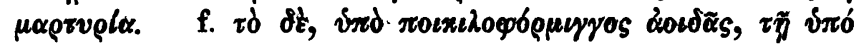

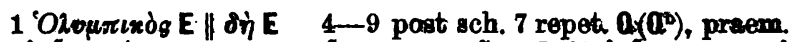

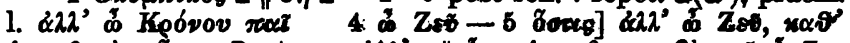

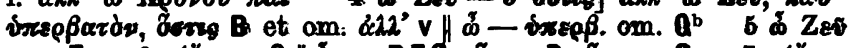

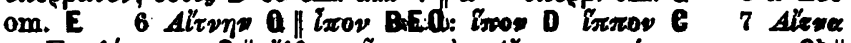

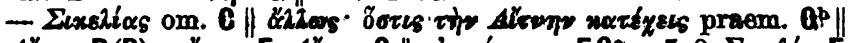

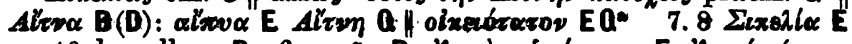

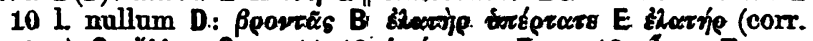

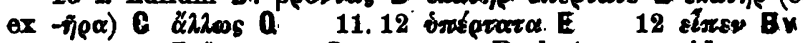

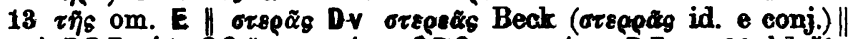

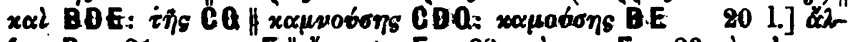

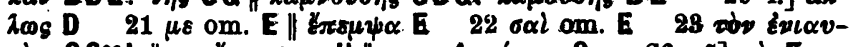

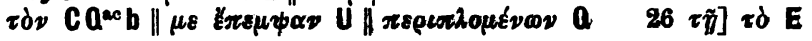




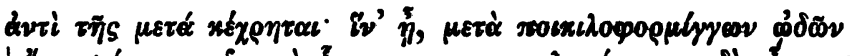

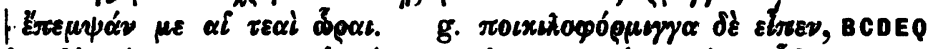

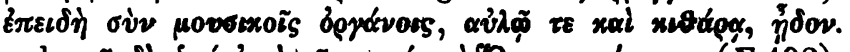

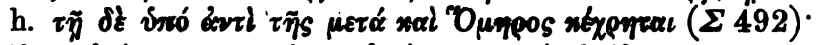

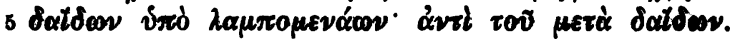

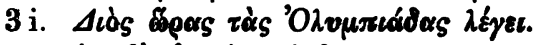

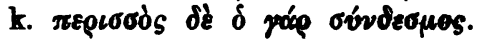

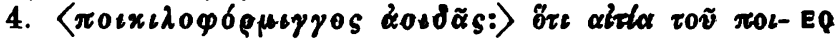

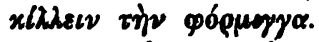

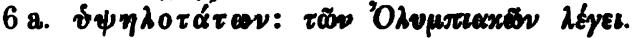

b. róxp-A

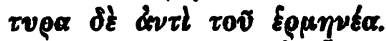

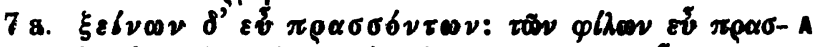

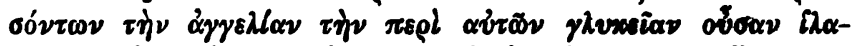

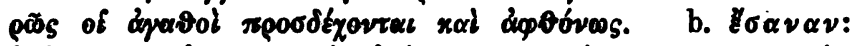

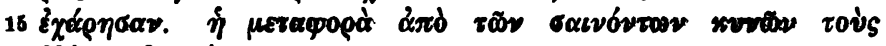
islovs decrótros.

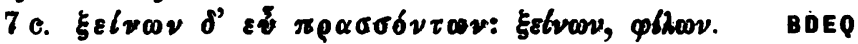

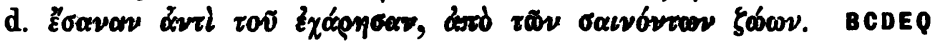

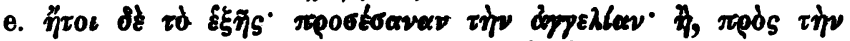

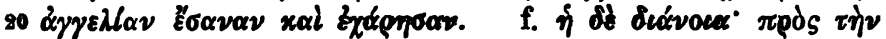

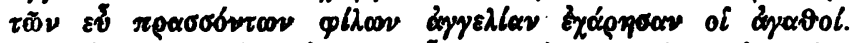

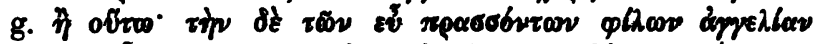

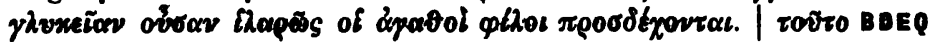

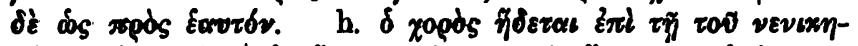

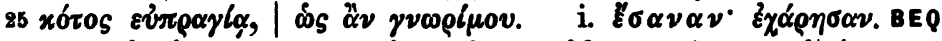

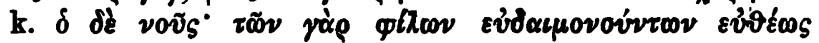

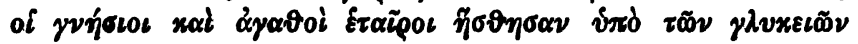

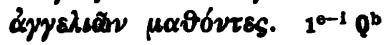

4 sch. A $\Sigma 492$

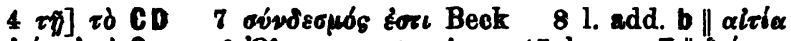

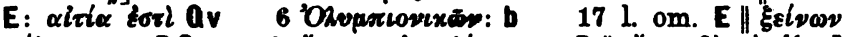

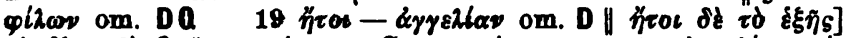

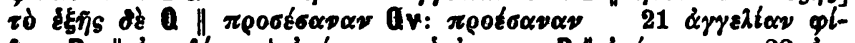

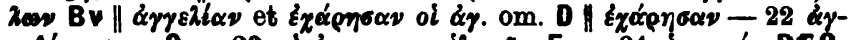

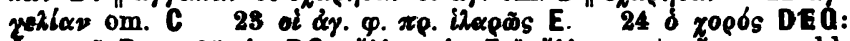

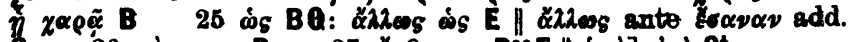

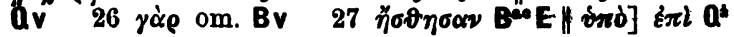




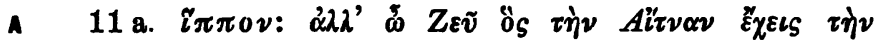

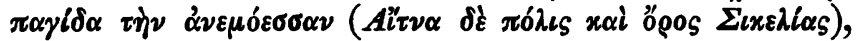

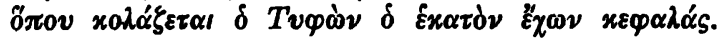

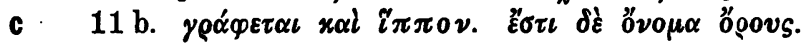

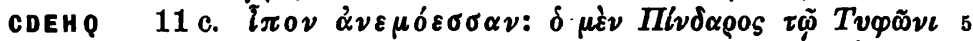

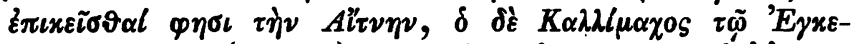

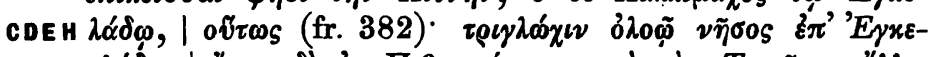

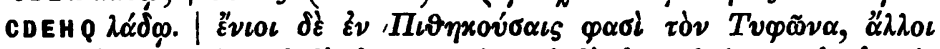

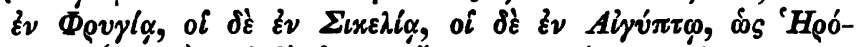

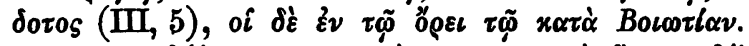

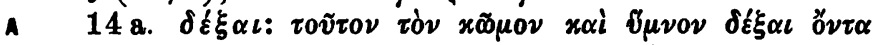

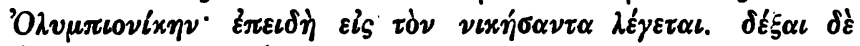

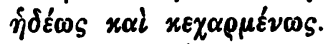

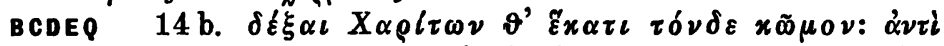

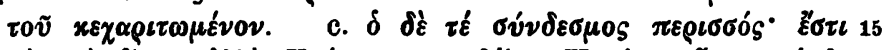

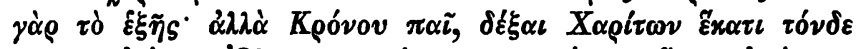

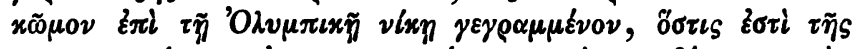

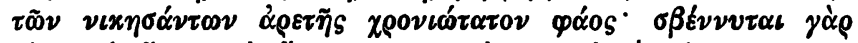

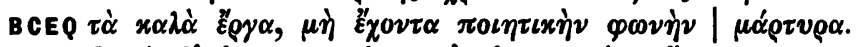

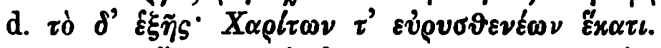

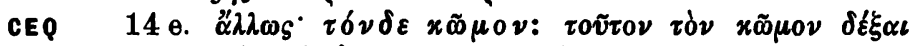

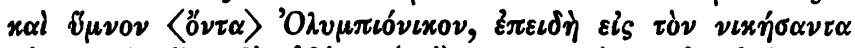

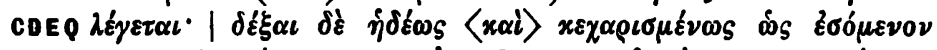

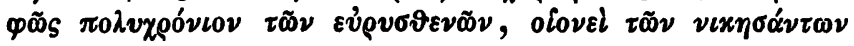

8 Tzetzes ad. Lyc. 177. sch. P. 1, 31

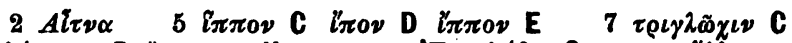

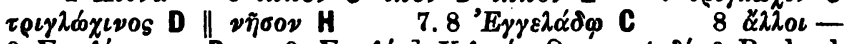

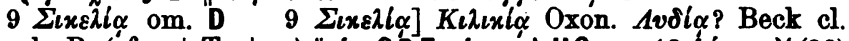

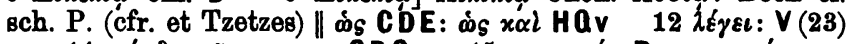

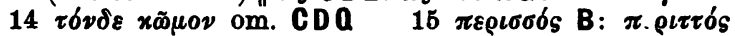

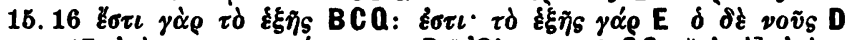

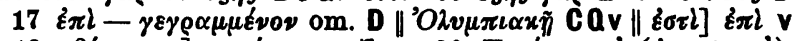

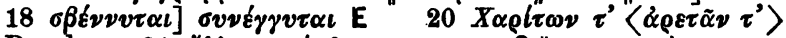

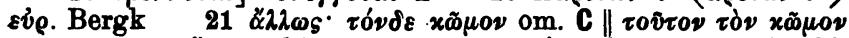
om. E 22 bै $\nu \tau \alpha$ add. b ex A $23 \quad \lambda \varepsilon \gamma \varepsilon \iota$ Q (ut A) $\| x \alpha i$ add.

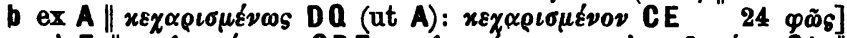

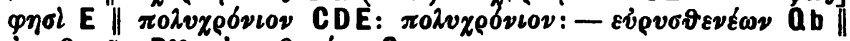

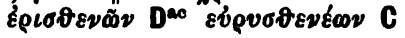




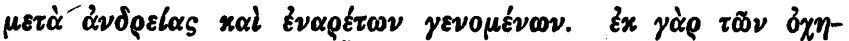

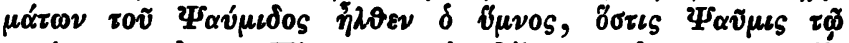

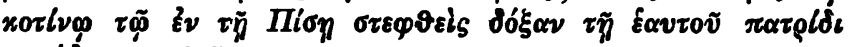

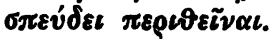

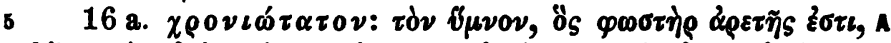

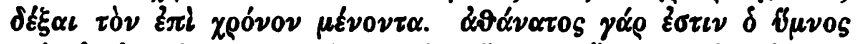

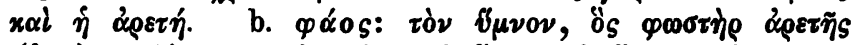

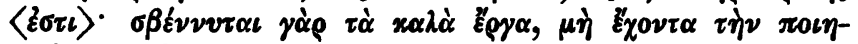

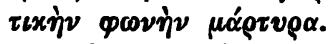

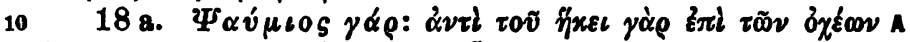

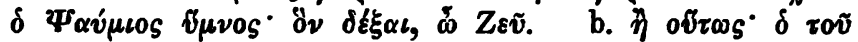

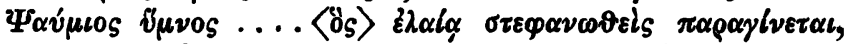

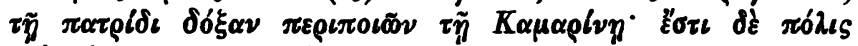
$x \alpha \ell \lambda l \mu \nu \eta \tau \tilde{\eta} s \quad \Sigma i x \varepsilon \lambda l \alpha s$.

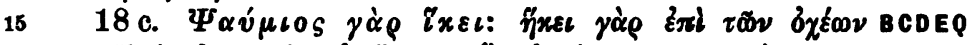

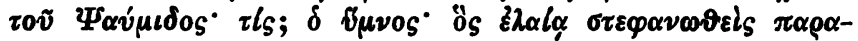

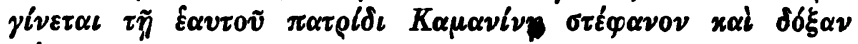
pépoov. $24 \mathrm{E}$

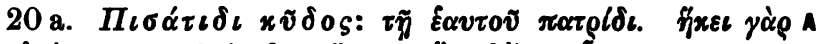

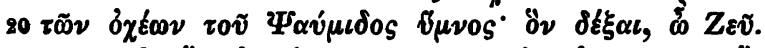

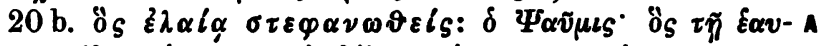

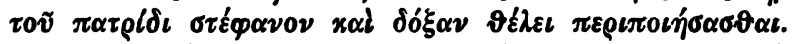

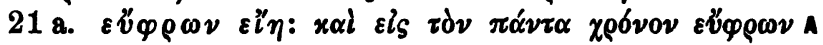

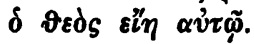

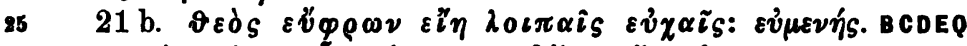

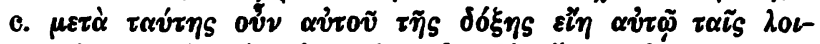

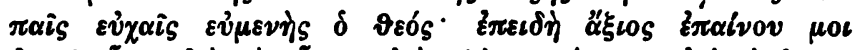

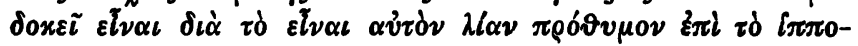

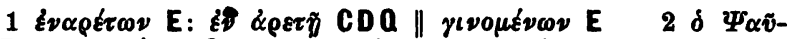

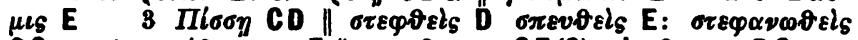

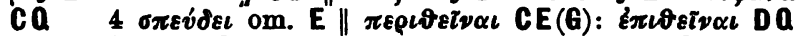

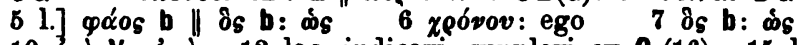
$10 \varepsilon \pi l \quad V:$ : $\alpha \delta \delta \quad 12$ lac. indicavi, supplevi ex C (16) 16 l. B:

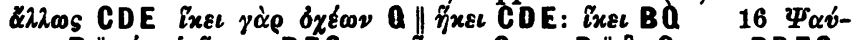

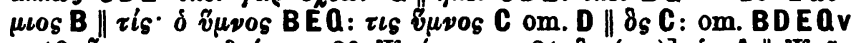

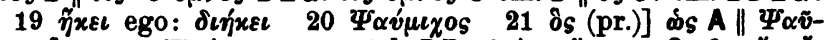

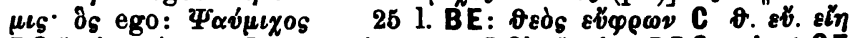

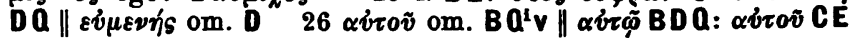




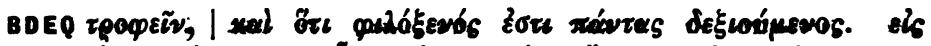

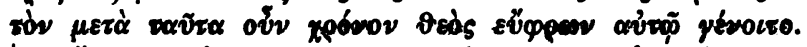

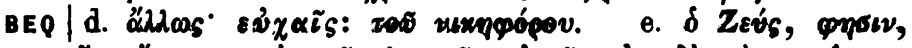

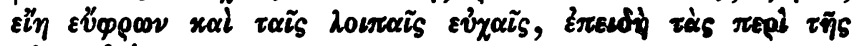
uhñ Ėtélacev. ME

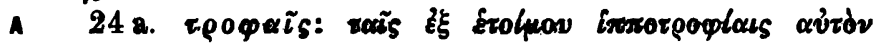

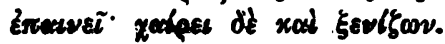

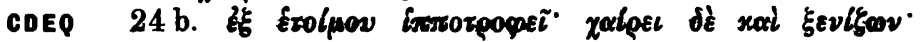
$\delta \iota \dot{~} \varepsilon \pi \alpha \iota v \tilde{~ \alpha u ̛ ́ r o ́ v . ~} 21 \mathrm{E}$

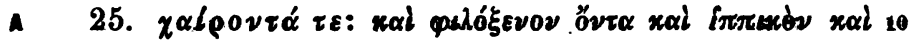

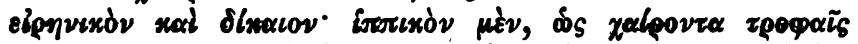

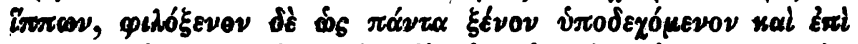

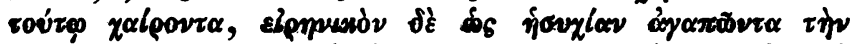

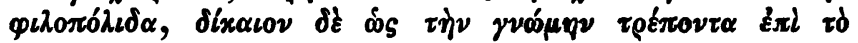
nocoupótrarov sai \&ub́deviov.

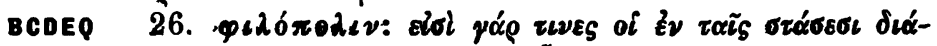

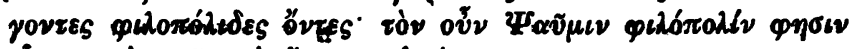

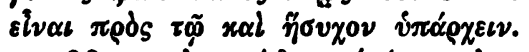

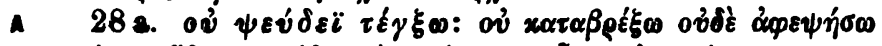

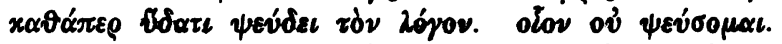

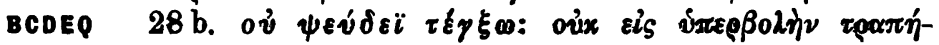

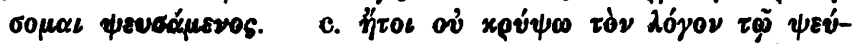

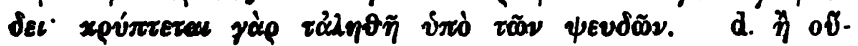

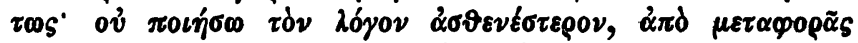

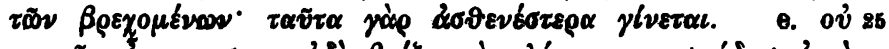

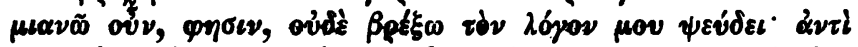

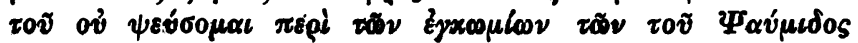
$\lambda$ troov.

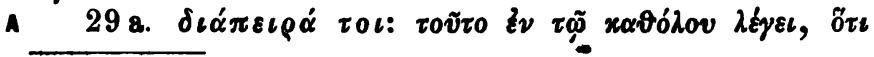

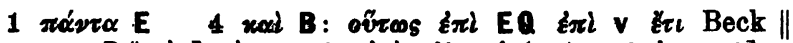

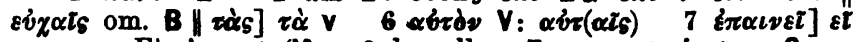

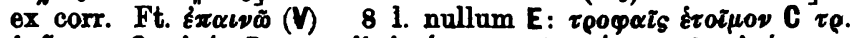

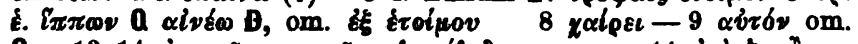

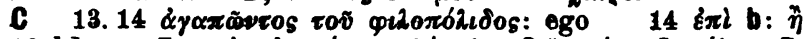

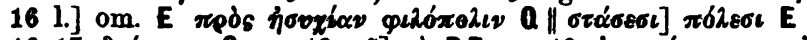

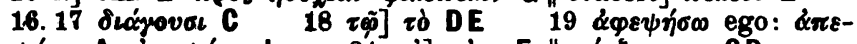

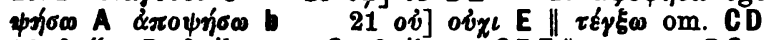

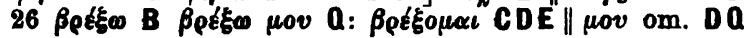




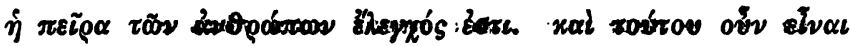

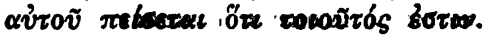

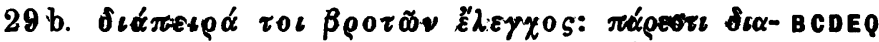

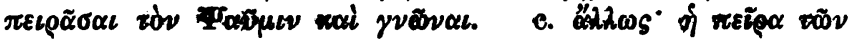

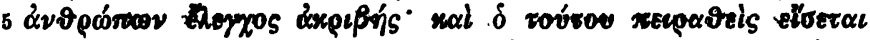

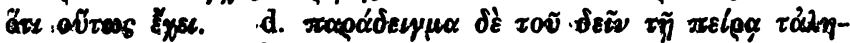

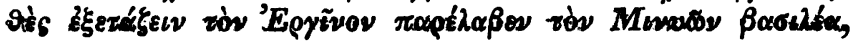

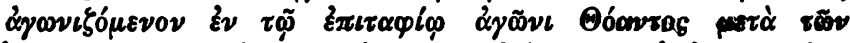

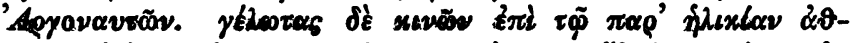

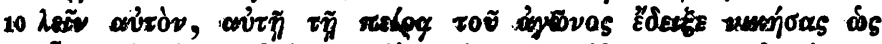

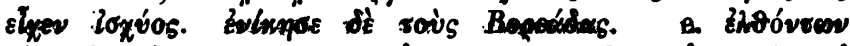

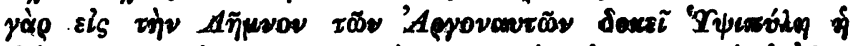

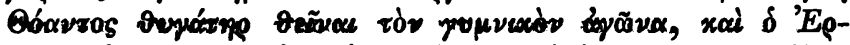

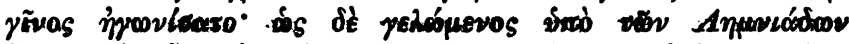

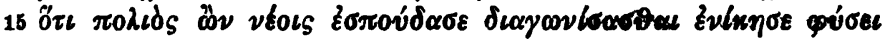

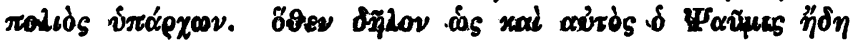

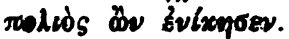

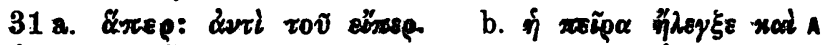

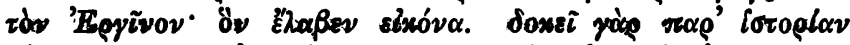

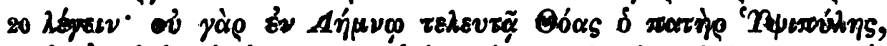

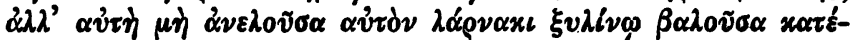

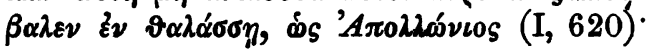

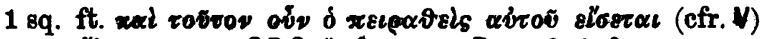

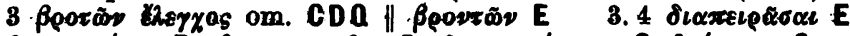

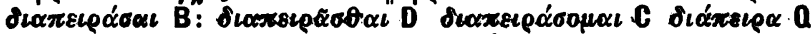

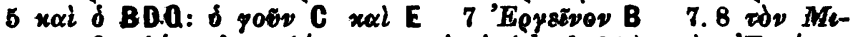

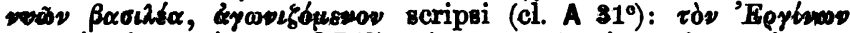

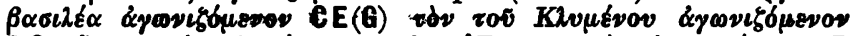

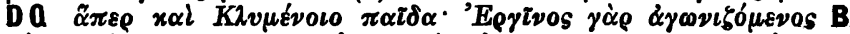

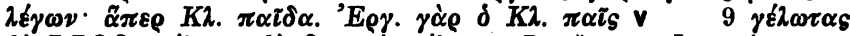

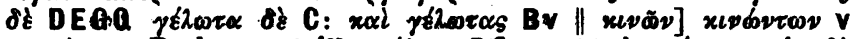

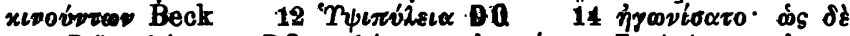

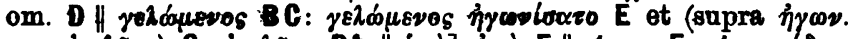

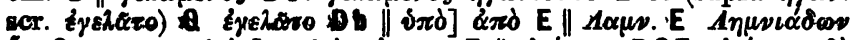

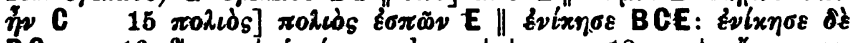

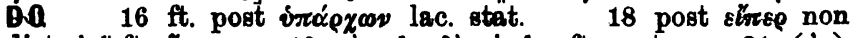
dist. b || ft. $\tilde{\eta} \pi \varepsilon \rho \quad 19 . \gamma \alpha \dot{\rho} \cdot A$ : $\delta \varepsilon$ cj. b, ft. recte $21\langle\varepsilon v\rangle$ $\lambda \alpha \dot{\rho} \nu \alpha x i$ b 


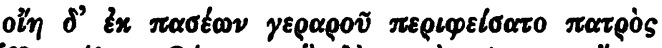

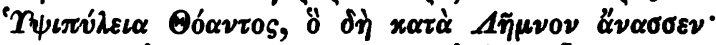

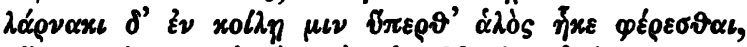

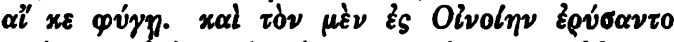

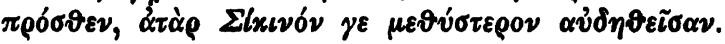

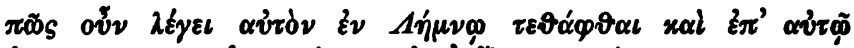

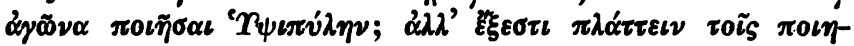

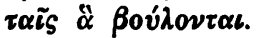

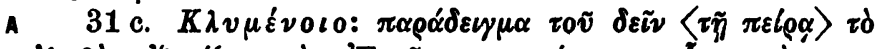

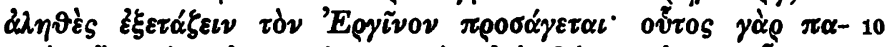

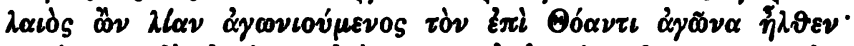

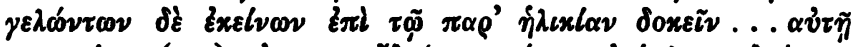

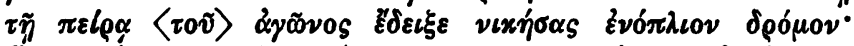

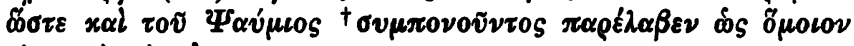

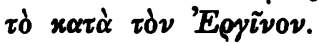

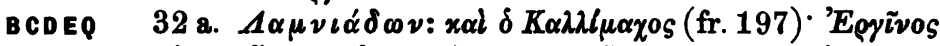

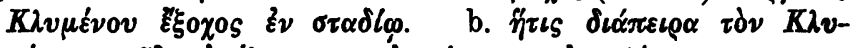

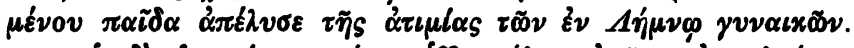

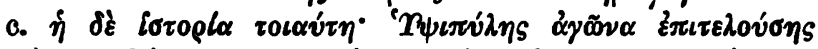

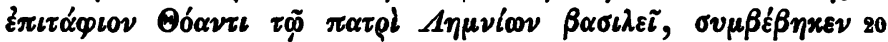

32 d. Apostol. 7, 95 (= sch. Thom. p. 179, 1 Ab.): 'E

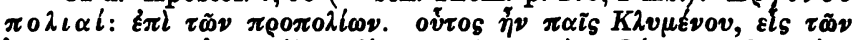

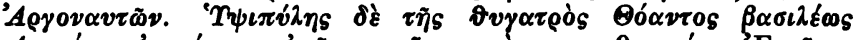

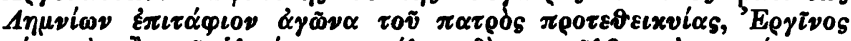

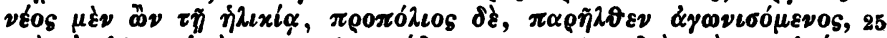

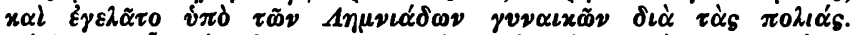

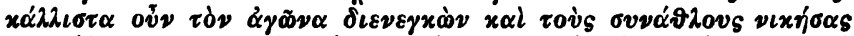

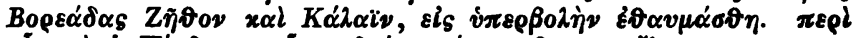

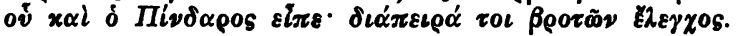

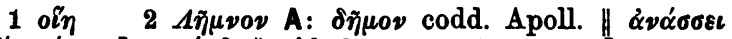

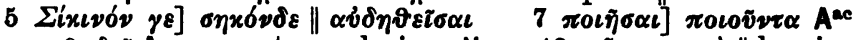

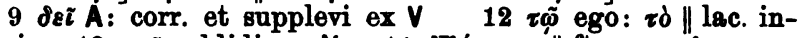

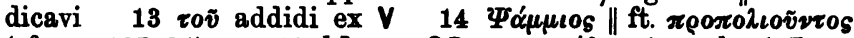
(cfr. p. 137, 24) 161.$]$ om. CD $\Lambda \alpha \mu \nu \iota \alpha ́ \delta$.. (cp. obsc.) E $A$.

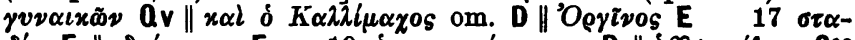

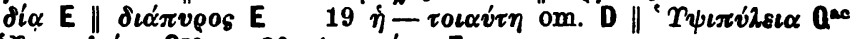

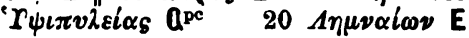




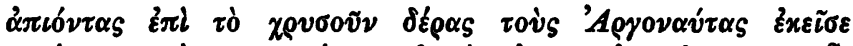

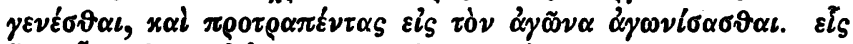

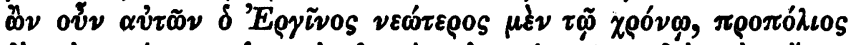

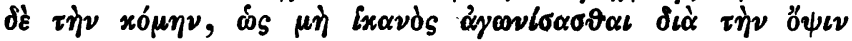

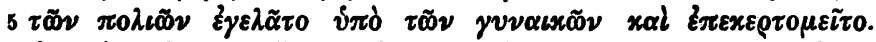

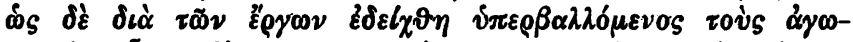

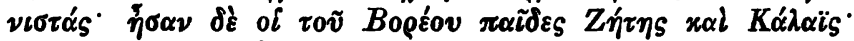

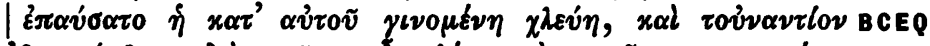

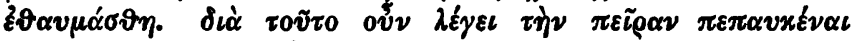

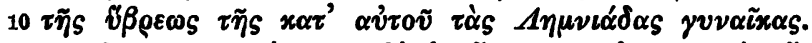

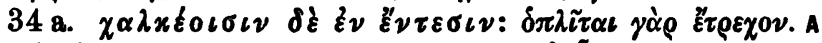

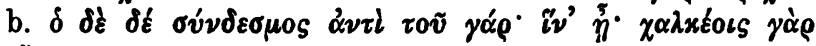

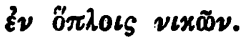

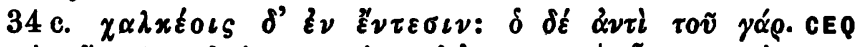

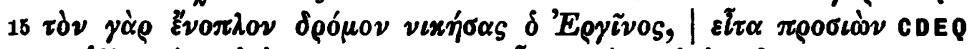

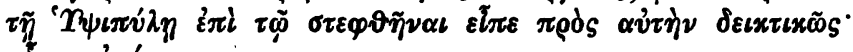

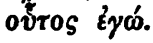

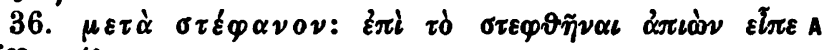

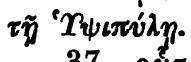

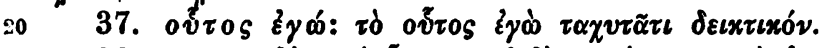

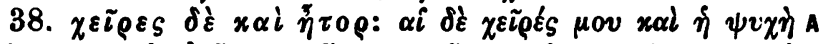

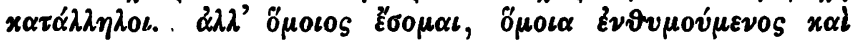
$\pi \rho \alpha ́$ $\tau \tau \omega \nu$.

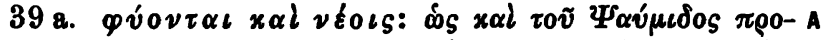

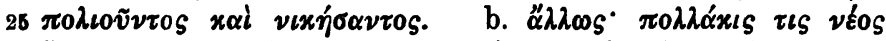

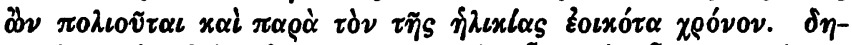

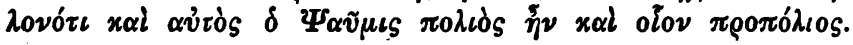

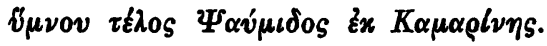

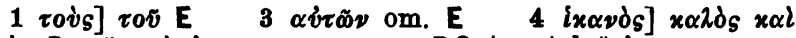

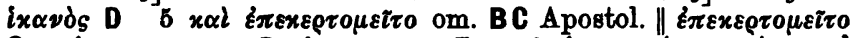

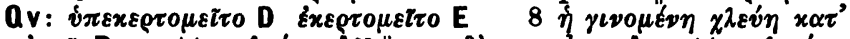

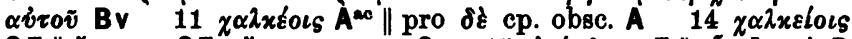

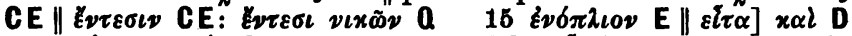

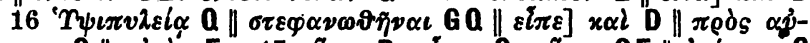

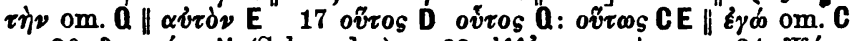

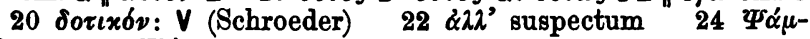

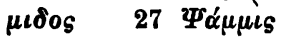




\section{Scholia in Olympionicarum carmen V.}

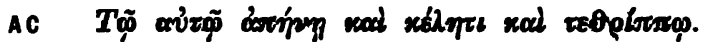

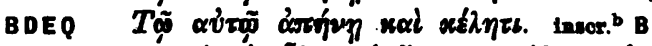

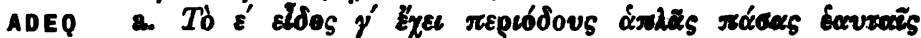

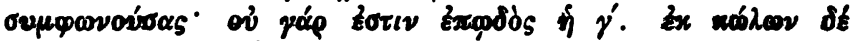

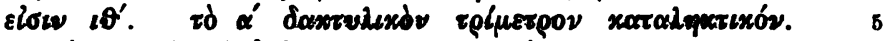

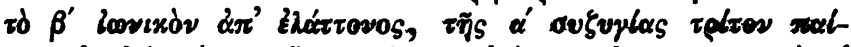

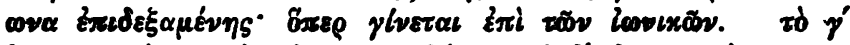

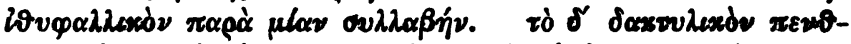

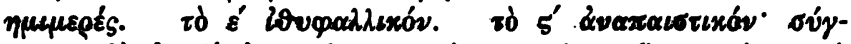

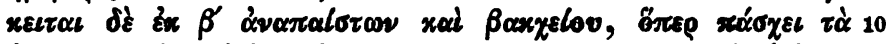

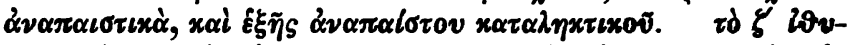

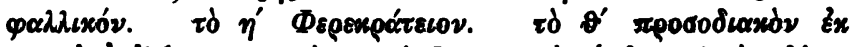

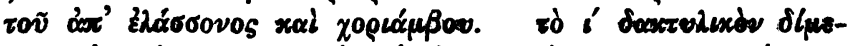

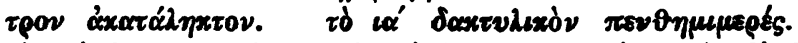

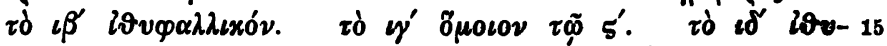

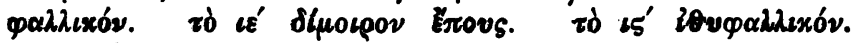

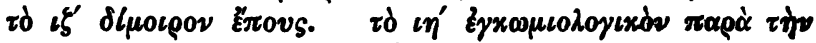

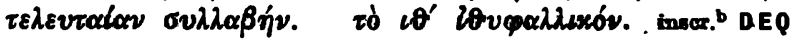

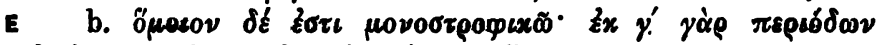

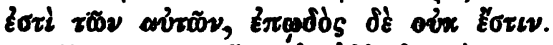

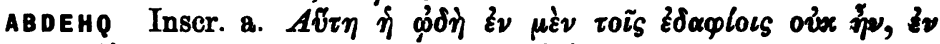

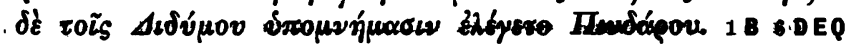

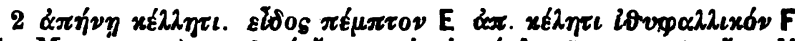

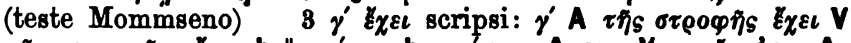

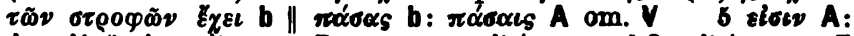

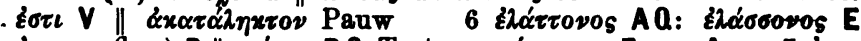

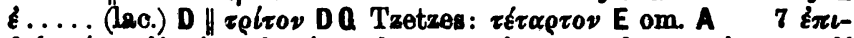

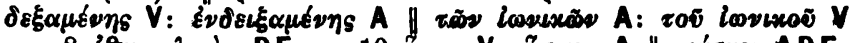

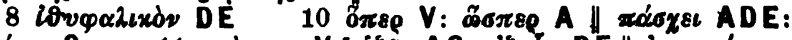

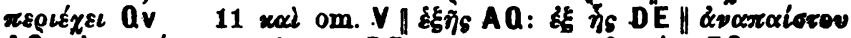

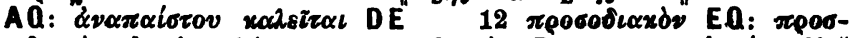

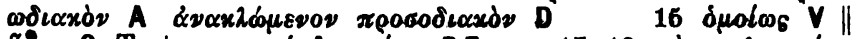

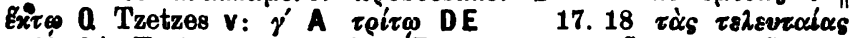

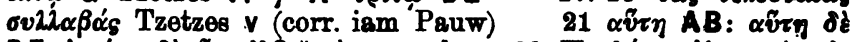
DE $\tau \tilde{\eta}_{S} \tau \alpha \dot{\delta} \delta \varepsilon \mathrm{A}$ 
Inscr. b. ГÉ

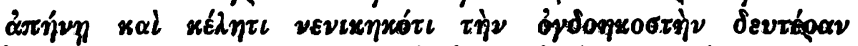

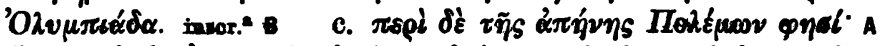
मैy

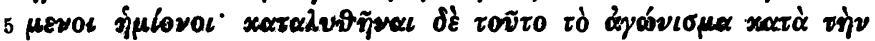

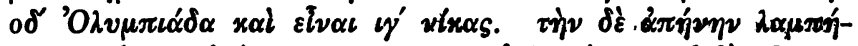

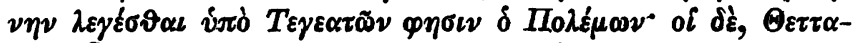

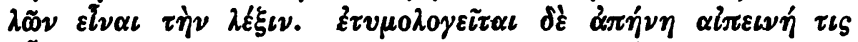

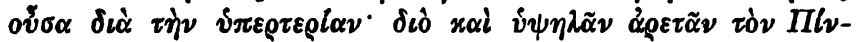

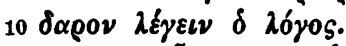

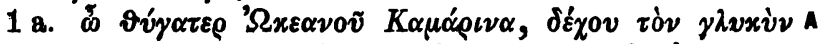

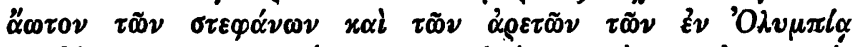

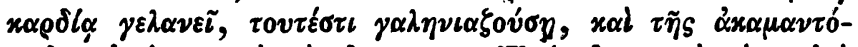

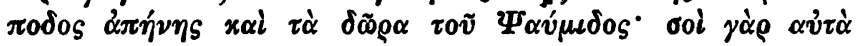
$15 \pi \alpha \rho \alpha x 0 \mu l \zeta \varepsilon \varepsilon$.

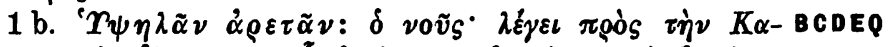

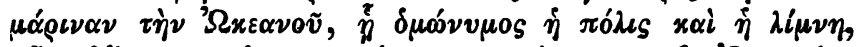

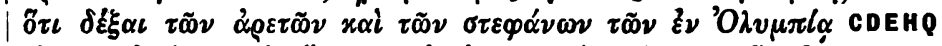

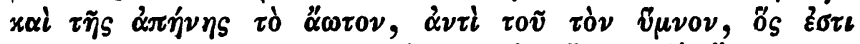

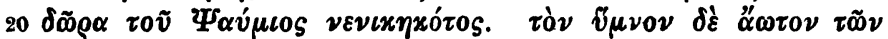

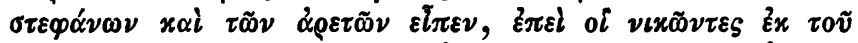

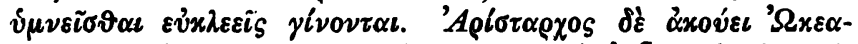

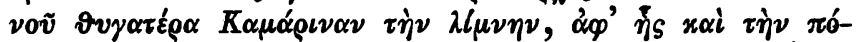

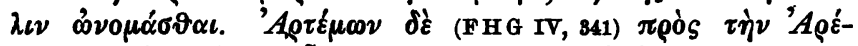

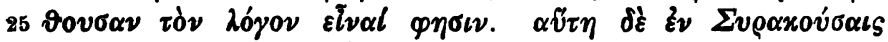

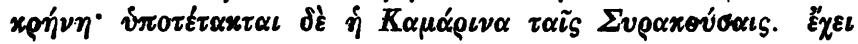

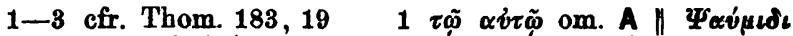

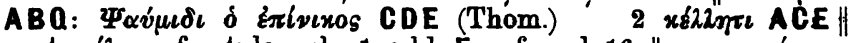

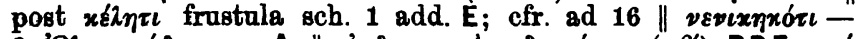

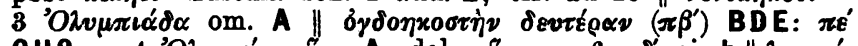

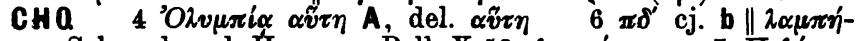

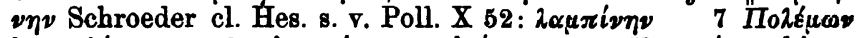

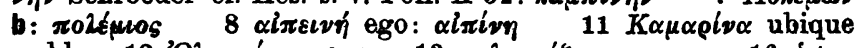

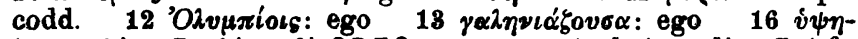

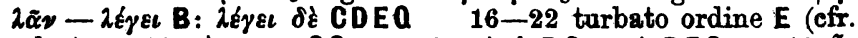
ad 2) $16 \tau \dot{\eta} v$ om. CQ $17 x \alpha i \dot{\eta}$ BC: xal DEQ 19 ôs

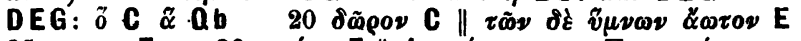

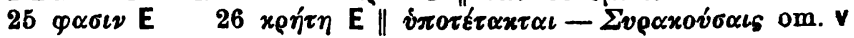




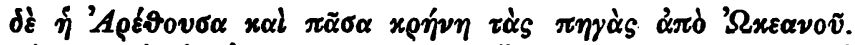
CEн $\beta$ Q

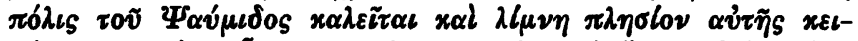

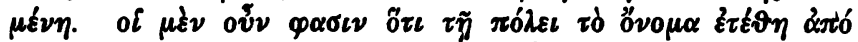

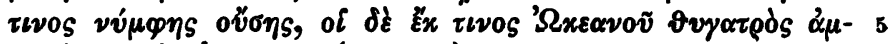
porépaıs, ios 'Holodos (th. 364)'

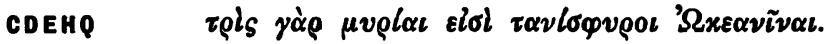

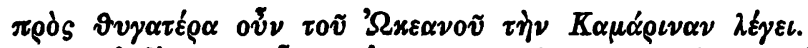

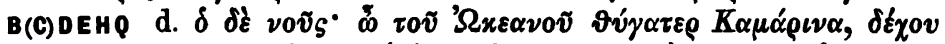

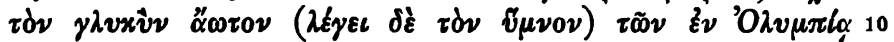

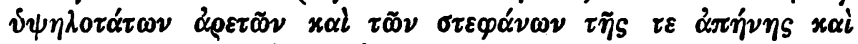

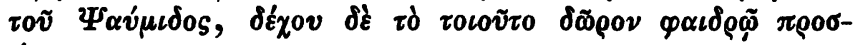
மóc⿻. insor." $D Q$ metr. ${ }^{b} E$

C 5. $x \alpha \rho \delta l \alpha \quad \gamma \varepsilon \lambda \alpha \nu \varepsilon \tilde{\imath}: \gamma \alpha \lambda \eta \nu i \omega ́ \sigma \eta x \alpha \ell$ $\varphi \alpha \iota \delta \rho \tilde{\alpha}$.

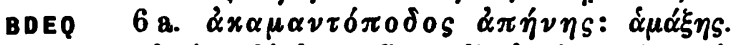

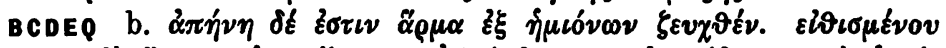

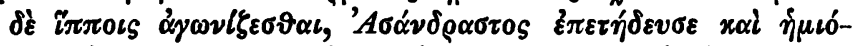

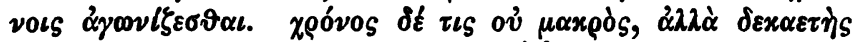

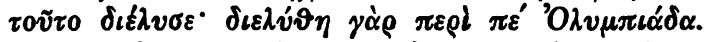

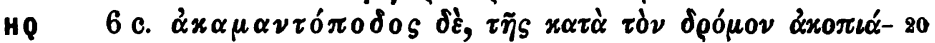

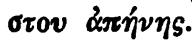

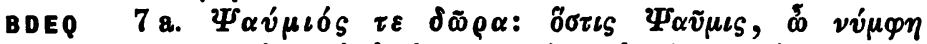

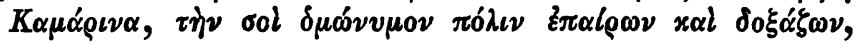

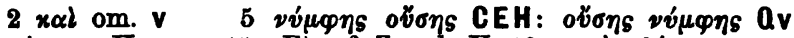

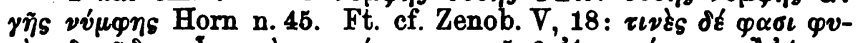

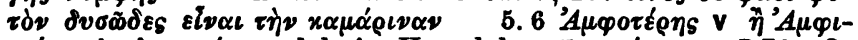

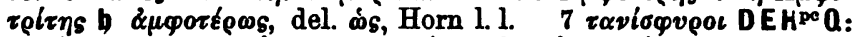

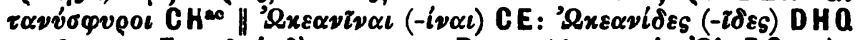

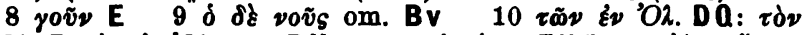

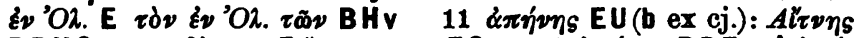

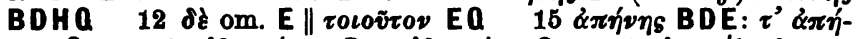

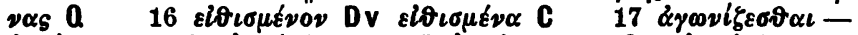

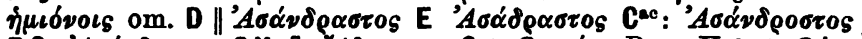

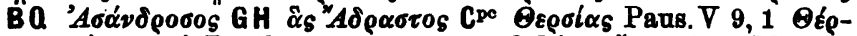

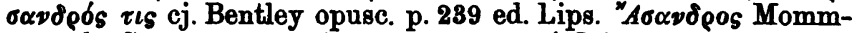

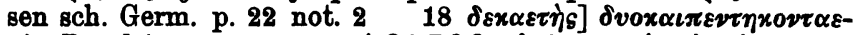

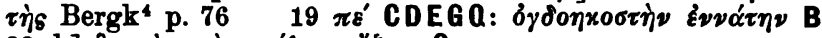

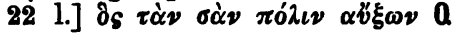




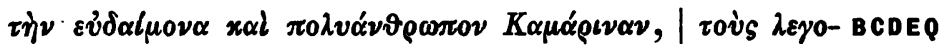

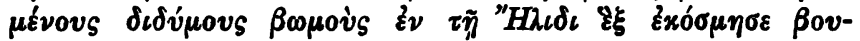

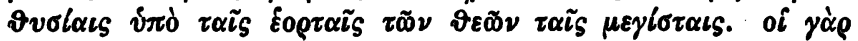

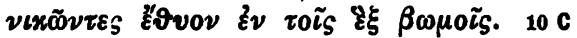

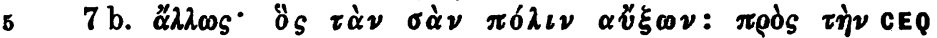

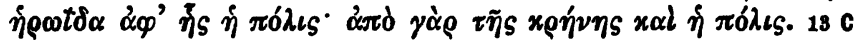

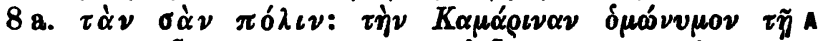

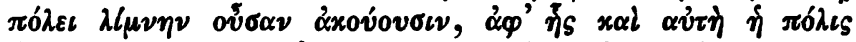

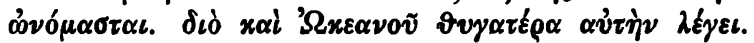

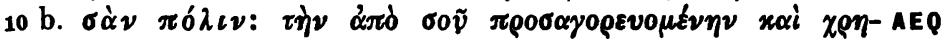

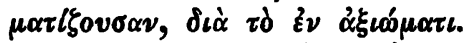

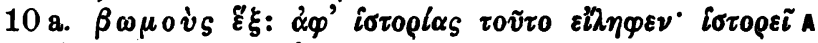

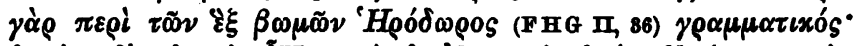

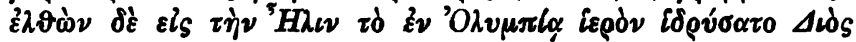

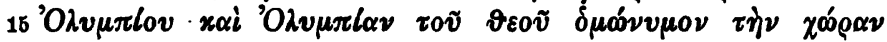

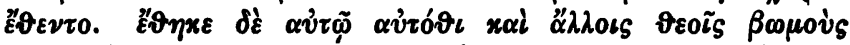

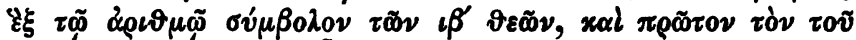

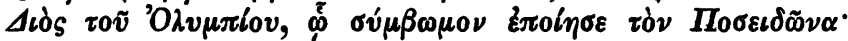

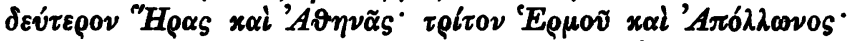

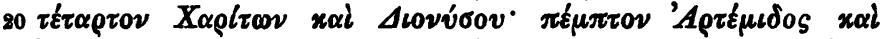

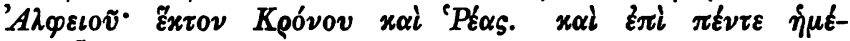

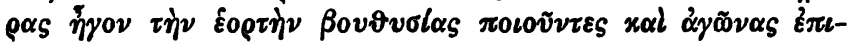
$\tau \varepsilon \lambda \circ \tilde{\nu} \nu \tau \varepsilon$.

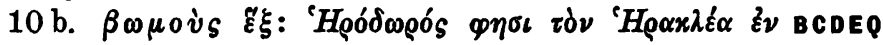

$1 \beta \omega \mu \nu v_{\xi} \xi \xi$ pro 1. praem. C $\left.2 \xi \xi\right] x \alpha i$ E 3 iso BCE:

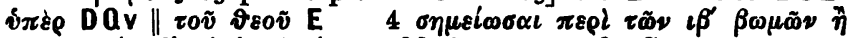
$\mu \tilde{\alpha} \lambda \lambda o \nu 5^{\prime}, \iota \beta^{\prime} \delta \dot{\varepsilon} \alpha \gamma \alpha \lambda \mu \alpha^{\prime} \tau \omega \nu$ add. D 5-6 efr. Germ. 185, 12

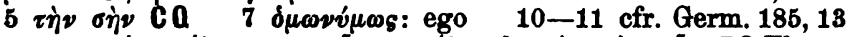

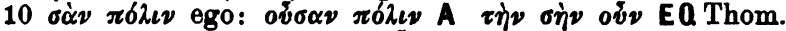

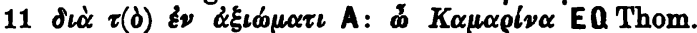

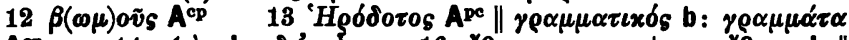

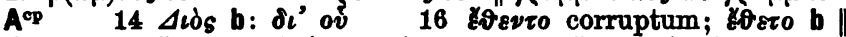

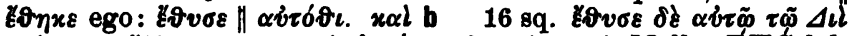

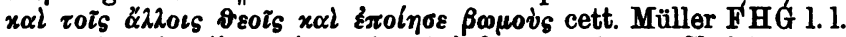

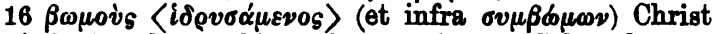

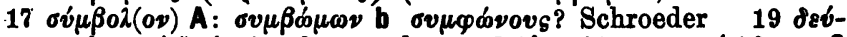

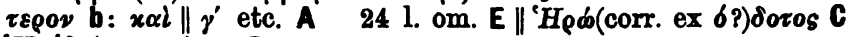

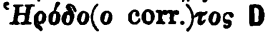




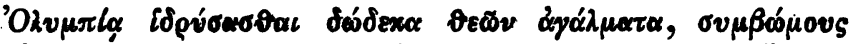

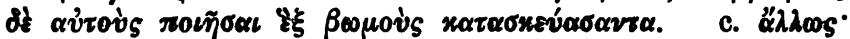

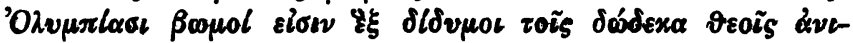

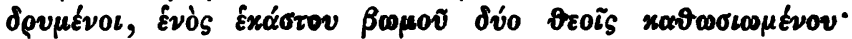

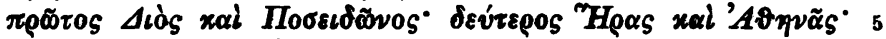

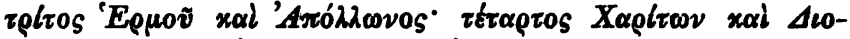

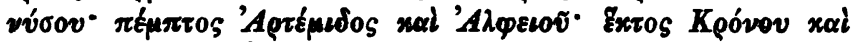

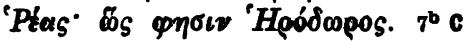

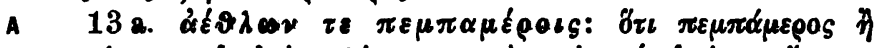

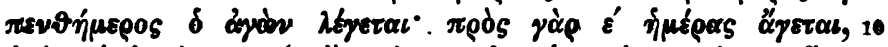

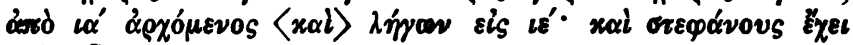

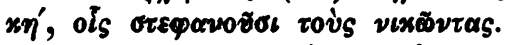

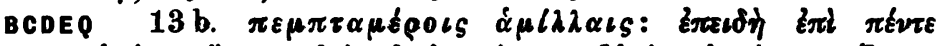

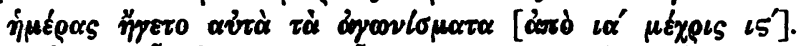

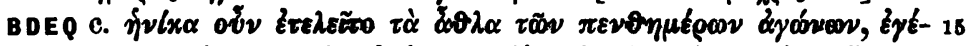

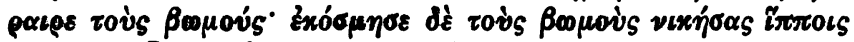

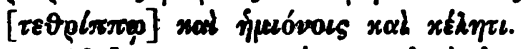

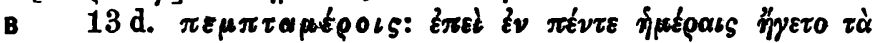

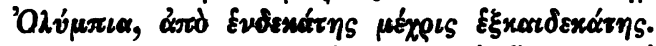

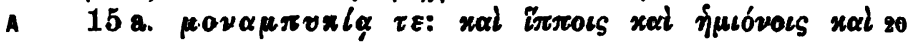

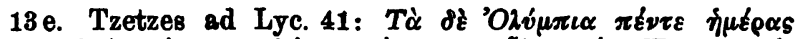

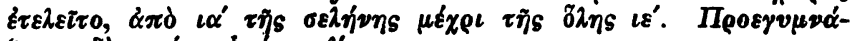
Gove de ropiny inuepas $\lambda^{\prime}$.

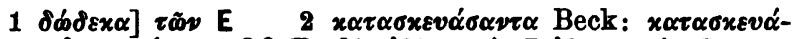

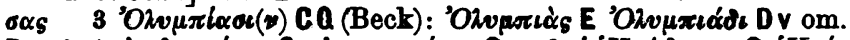

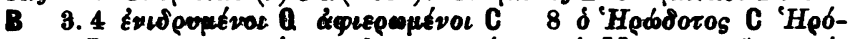

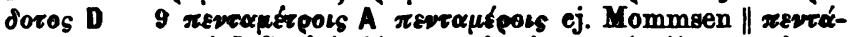

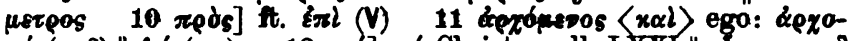

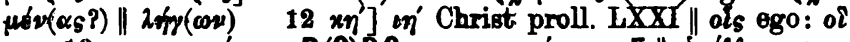

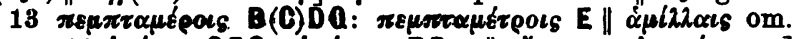

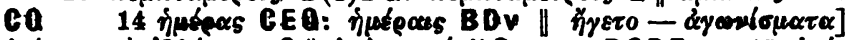

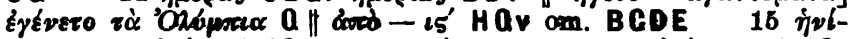

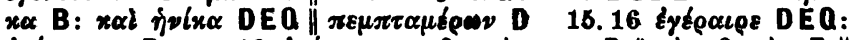

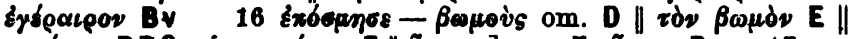

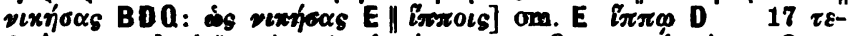

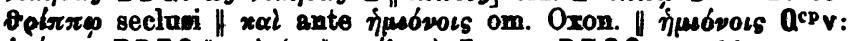

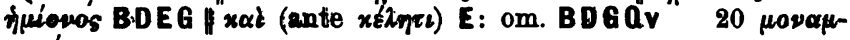
zvxís $\gamma \varepsilon$ 


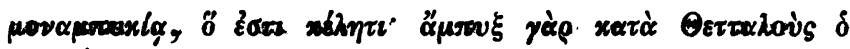
xecicxóg.

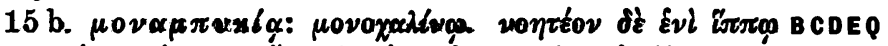

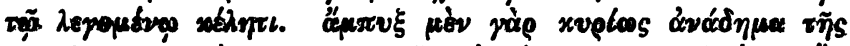

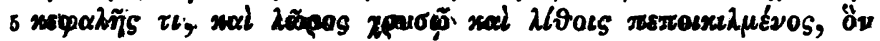

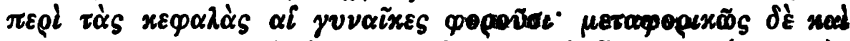

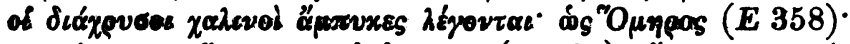

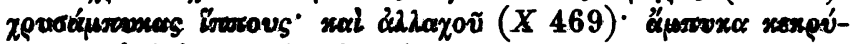

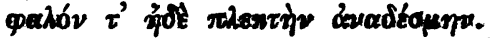

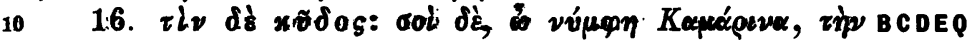

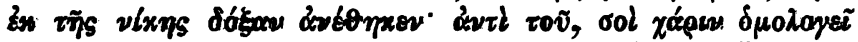

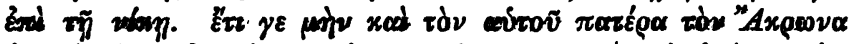

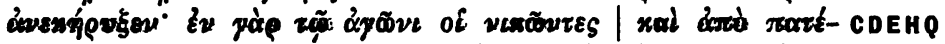

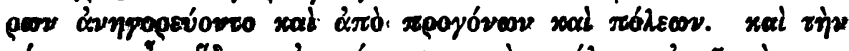

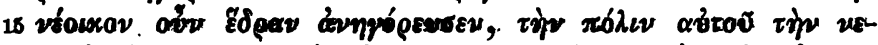

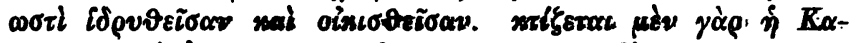

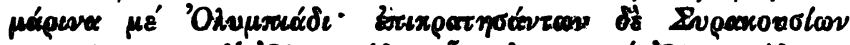

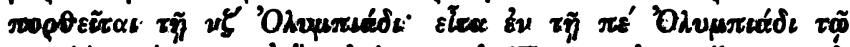

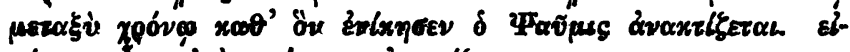

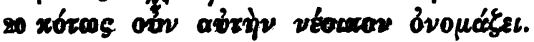

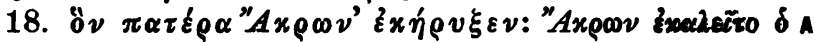

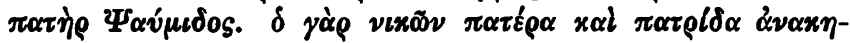
péroes.

4 sch. T E 358. Hes., Suid., EM 86;20. Ap. soph., Hes.,

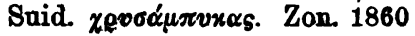

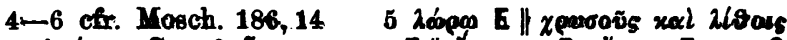

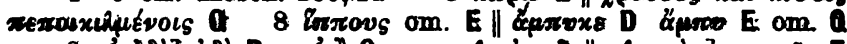

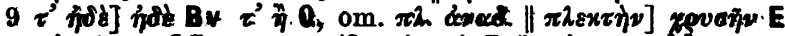

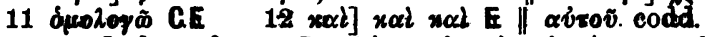

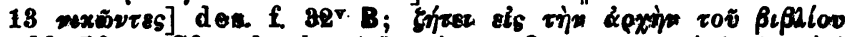

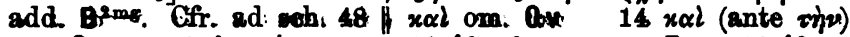

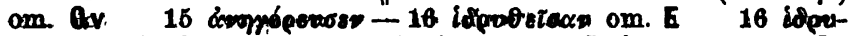

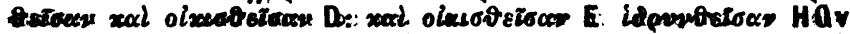

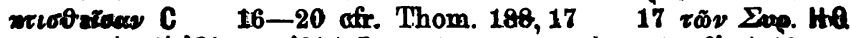

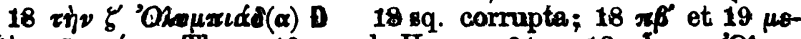

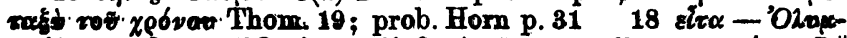

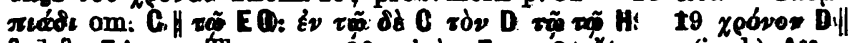

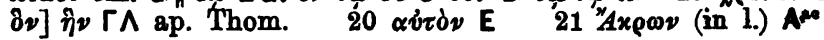




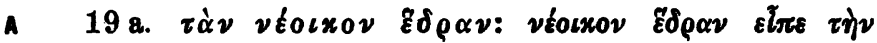

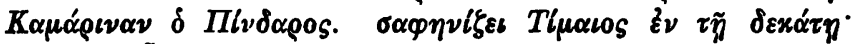

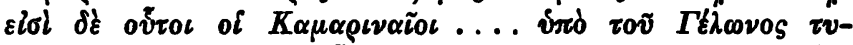

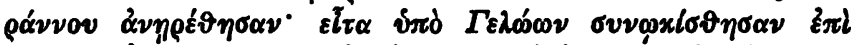

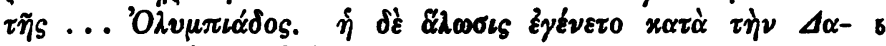

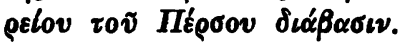

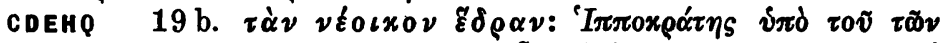

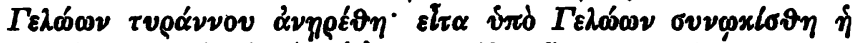

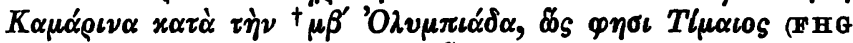

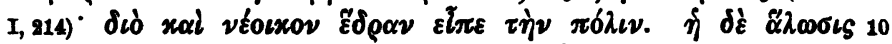

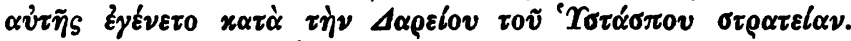

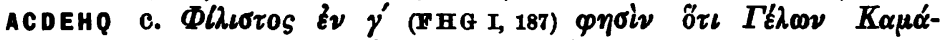

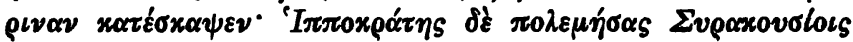

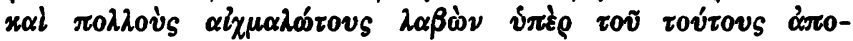

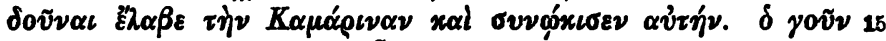

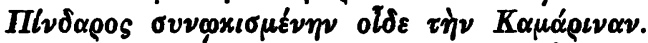

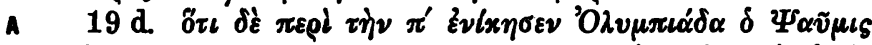

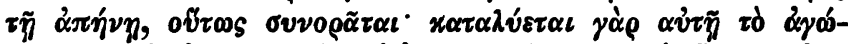

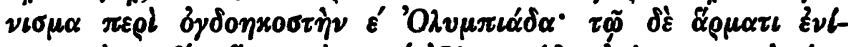

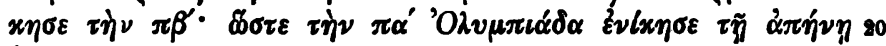

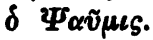

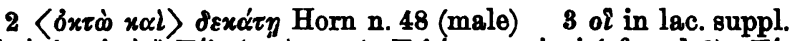
b \|

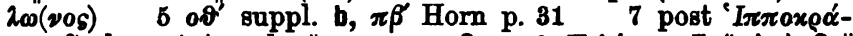

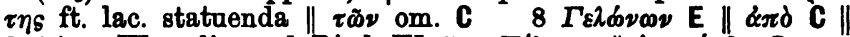

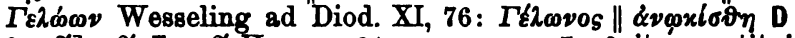
$\left.9 \mu \beta^{\prime}\right] \times \beta^{\prime}$ E $\pi \beta^{\prime}$ Horn $p .31$ ex recc. 7-9 ita constitui vo-

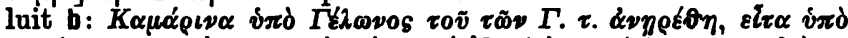

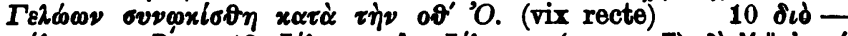

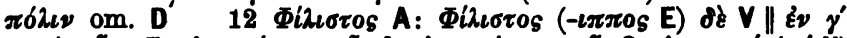

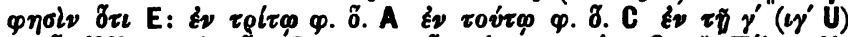

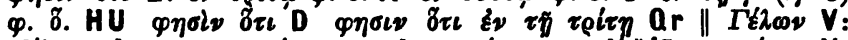

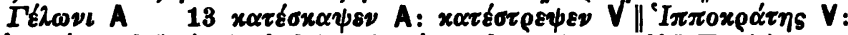

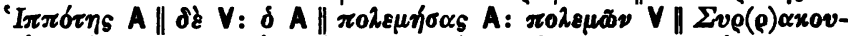

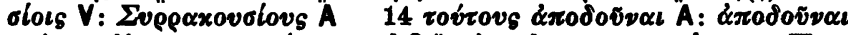

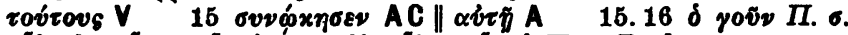

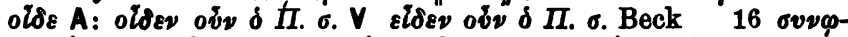

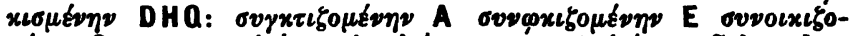

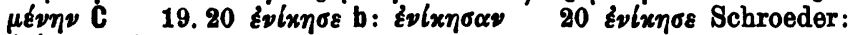

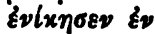




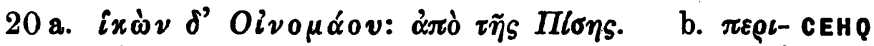

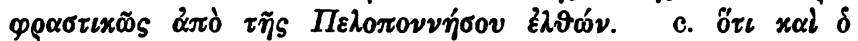

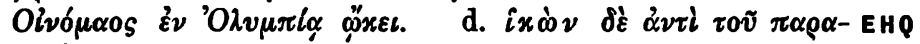
yEvó

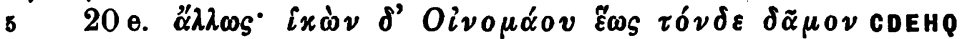

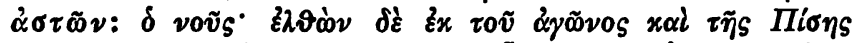

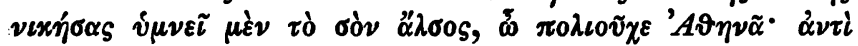

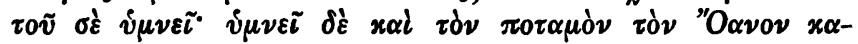

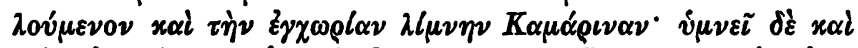

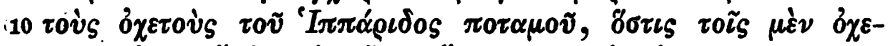

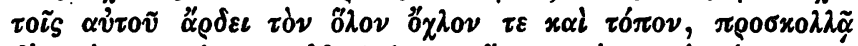

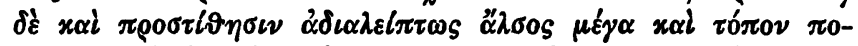

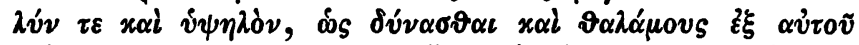

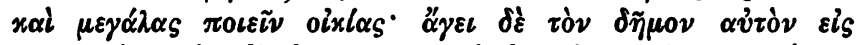

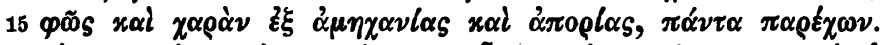

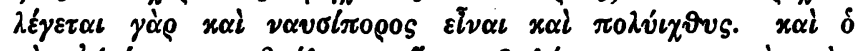

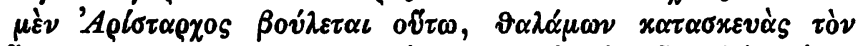

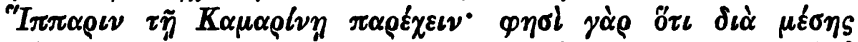

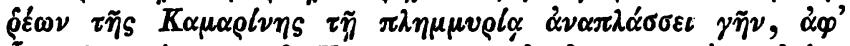

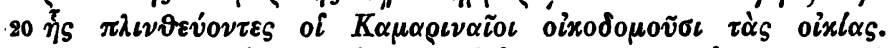

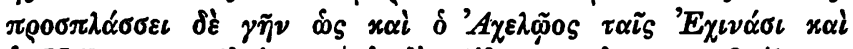

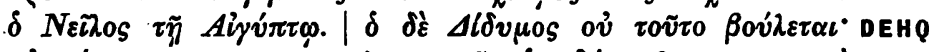

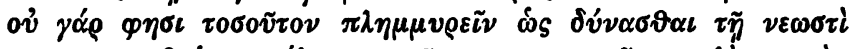

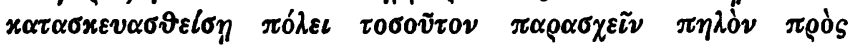

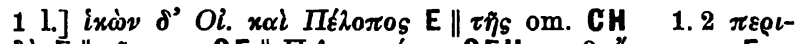

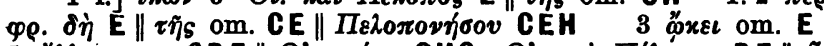

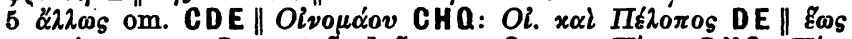

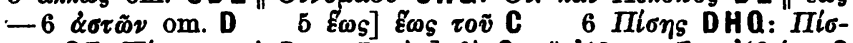

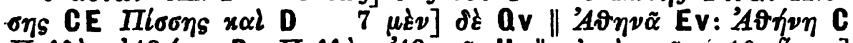

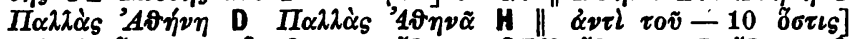

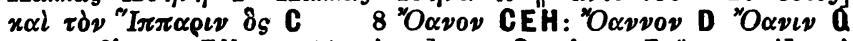

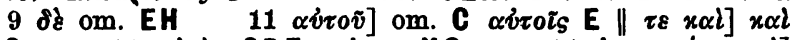

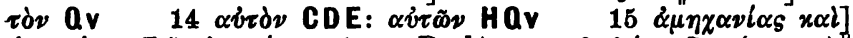

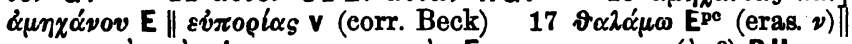

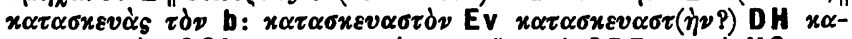

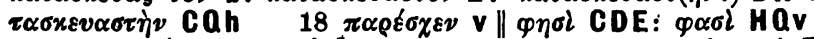

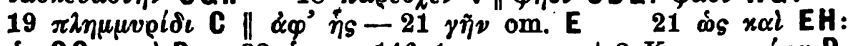

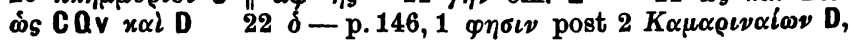

Schox. no Pexdarux ed. Drachmann. 


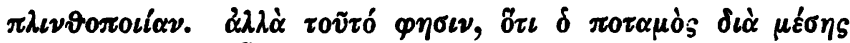

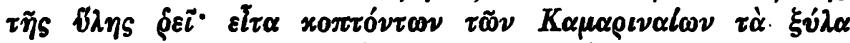

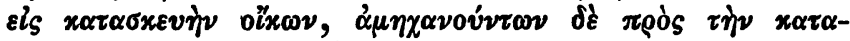

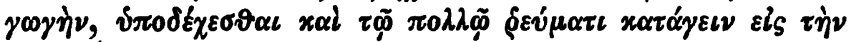
Ен

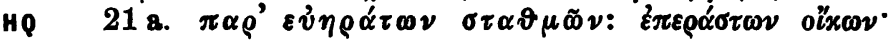

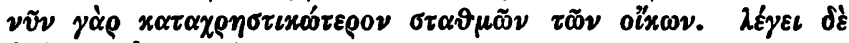

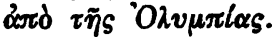

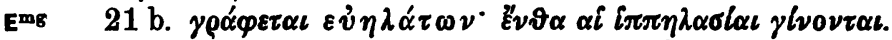

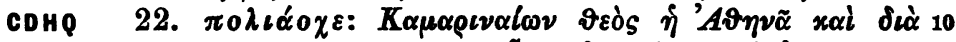

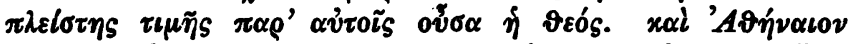

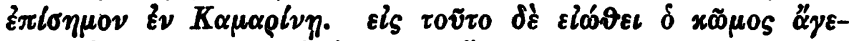

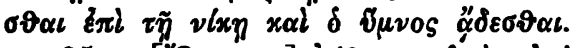

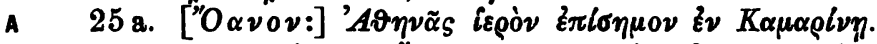

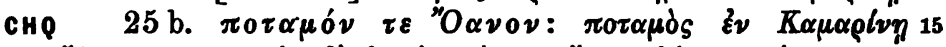

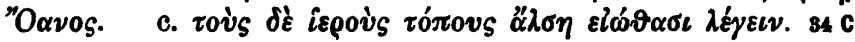

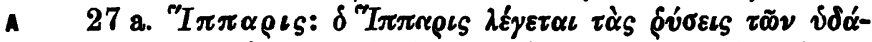

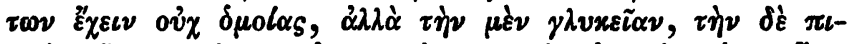

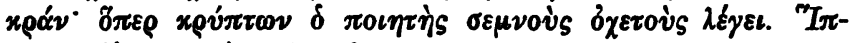

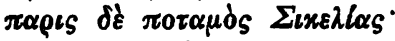

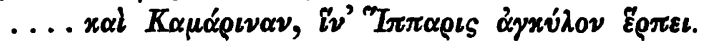

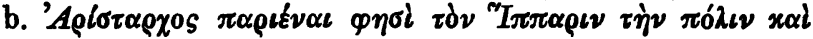

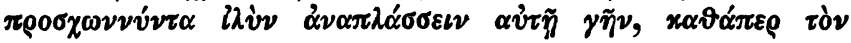

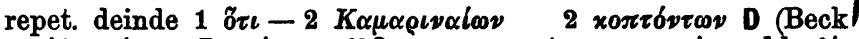

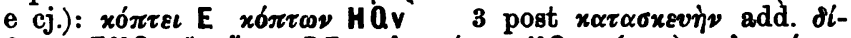

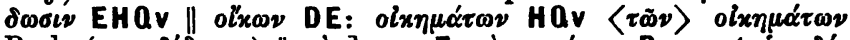

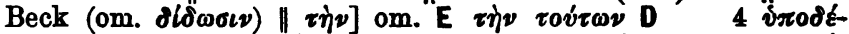

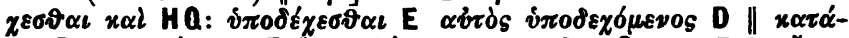

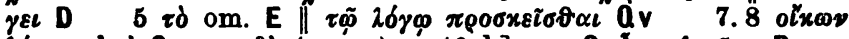

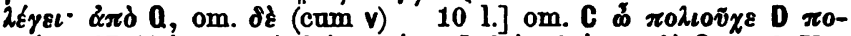

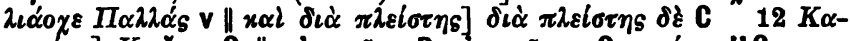

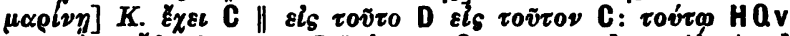

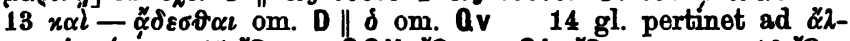

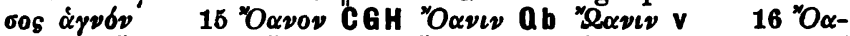

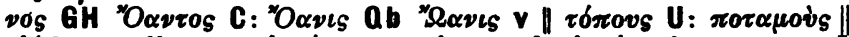

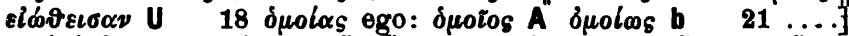

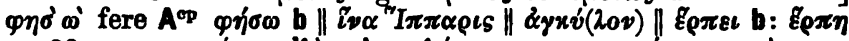

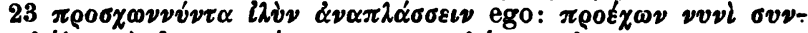

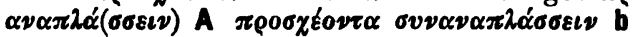




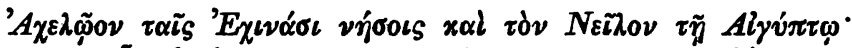

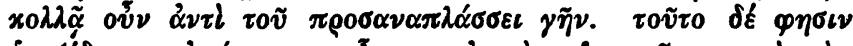

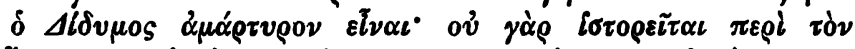

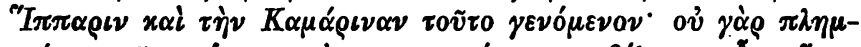

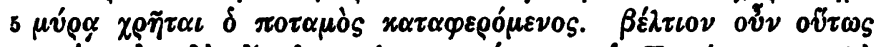

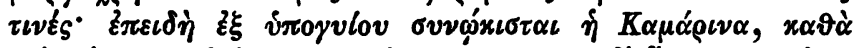

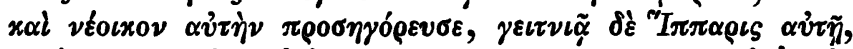

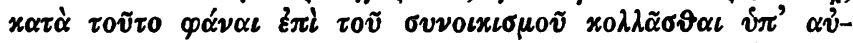

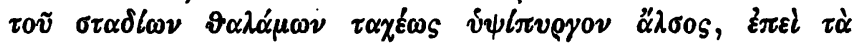

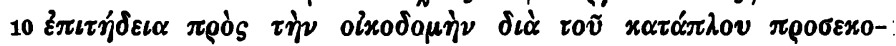

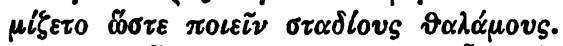

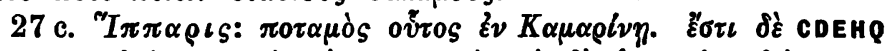

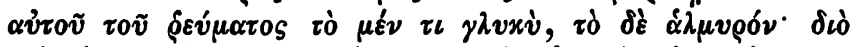

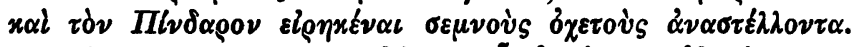
15

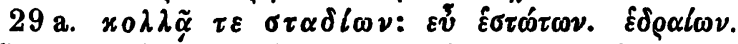
CDEHQ

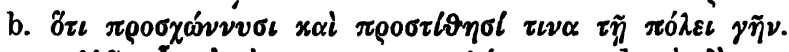

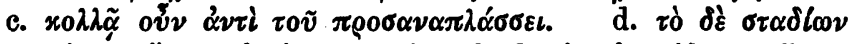

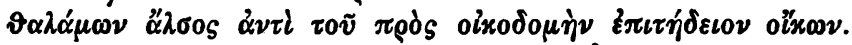

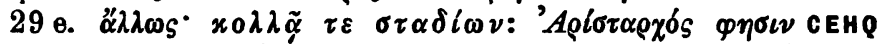

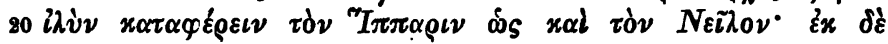

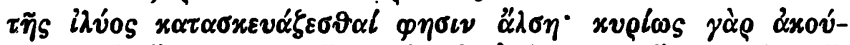

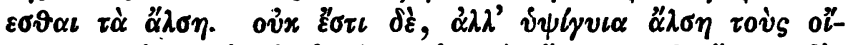

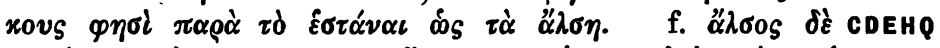

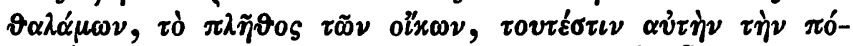

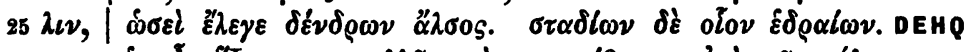

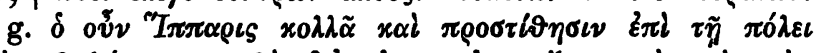

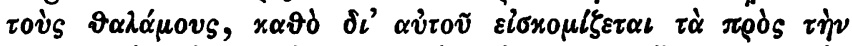

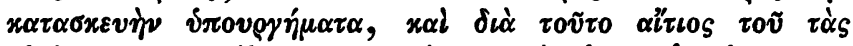

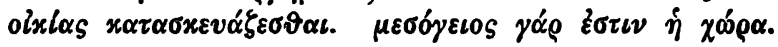

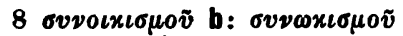

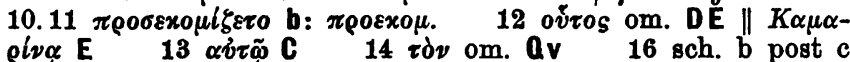
9 vifigviov cj. b

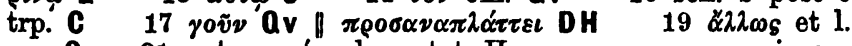
om. C 21 ante xv९lios lac. stat. Horn, quam $\theta$ recc. sic ex-

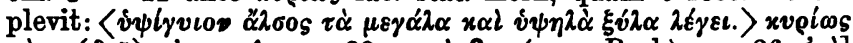

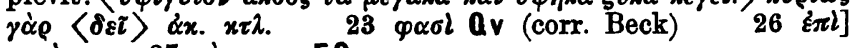
$\pi \varepsilon \rho l v 27$ v 


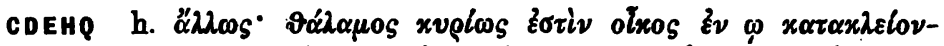

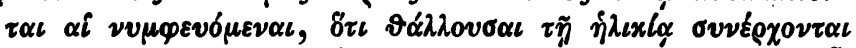

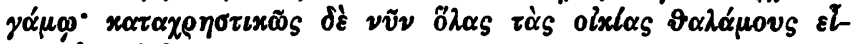
$\pi \varepsilon \nu \delta \Pi l \nu \delta \alpha \rho o s$. $22 \mathrm{C} 37 \mathrm{D}$

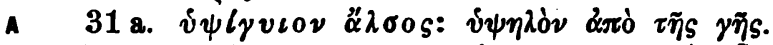

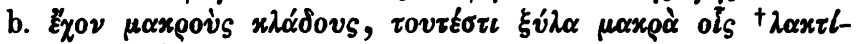

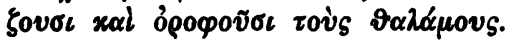

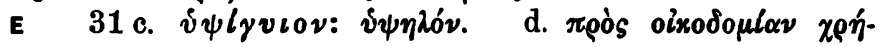

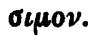

E 32. $\delta \pi^{\prime} \dot{\alpha} \mu \eta \chi \alpha \nu l \alpha s: \delta \pi^{\prime} \dot{\alpha} \pi 0 \rho l \alpha s$.

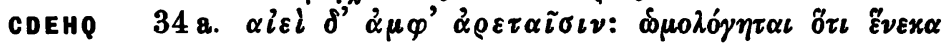

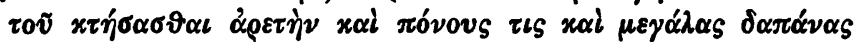

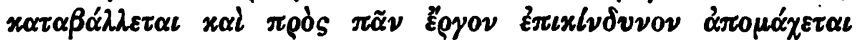

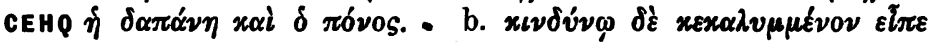

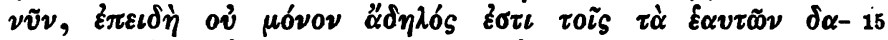

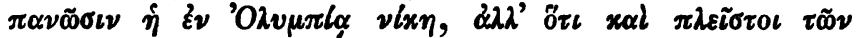

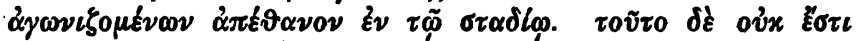

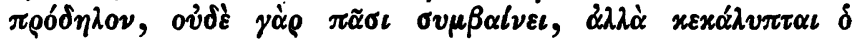

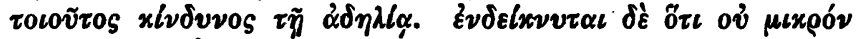

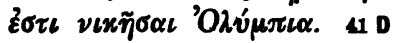

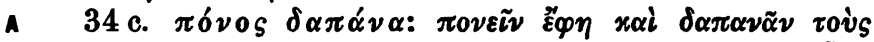

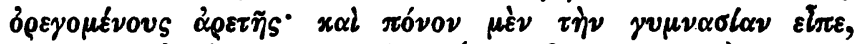

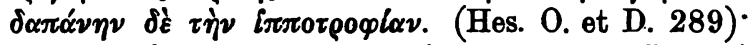

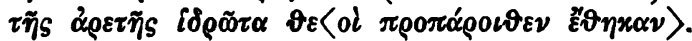

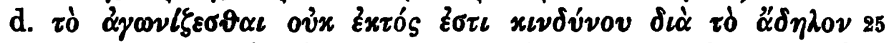

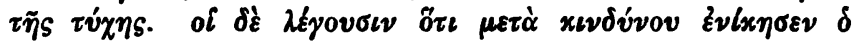

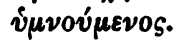

A $36 \mathrm{a} . x \iota \nu \delta v ́ v \omega x \varepsilon x \alpha \lambda v \mu \mu \varepsilon v 0 \nu$ : $\pi \rho \dot{s} s \dot{\eta} \nu v l x \eta \nu^{\circ} \alpha_{\alpha} \delta \eta$

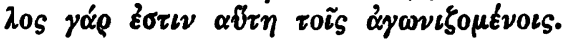

1 EM 441, 13. Hes. $\vartheta \alpha^{\prime} \lambda \alpha \mu \circ$

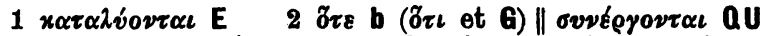

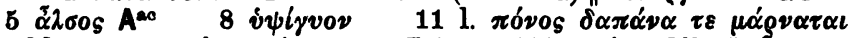

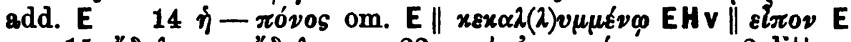

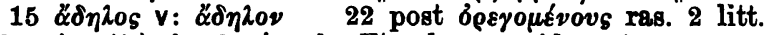
$24 \vartheta \varepsilon\langle o l$ cett. $>$ b: $\vartheta$ $\lambda v \mu \mu \varepsilon v$ os $A$, bis scr. 


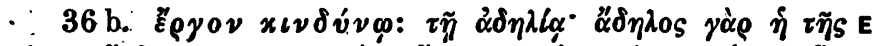

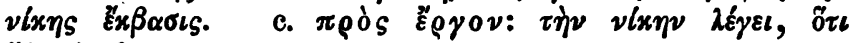

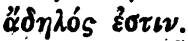

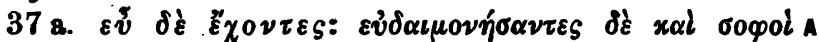

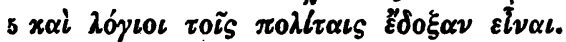

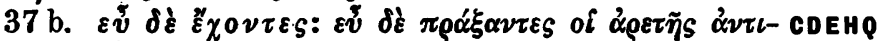
$\pi 0 \iota 0$ ำ

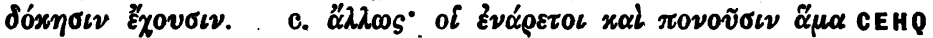

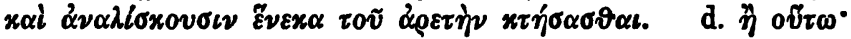

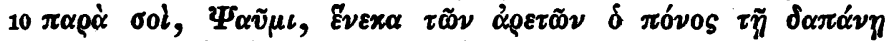

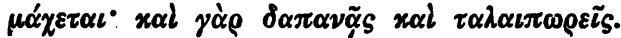

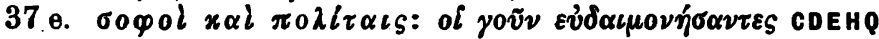

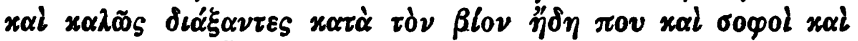

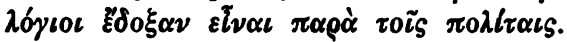

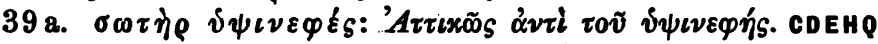

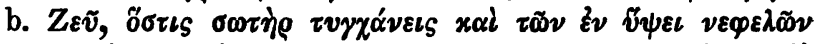

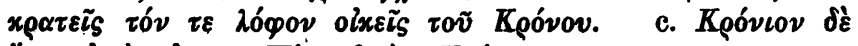

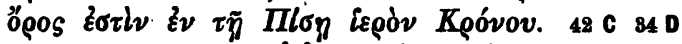

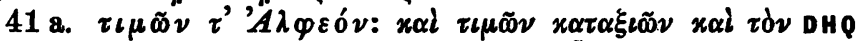

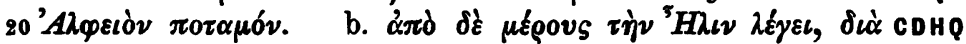

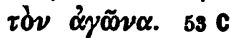

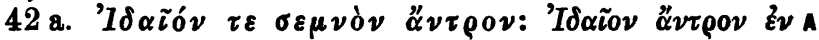

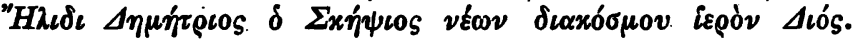

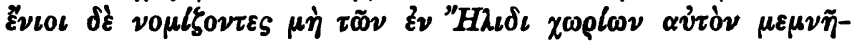

(15) Herodian. II, 695, 22

$6 \delta^{\prime}$ Exovzes CD 12 1. (H) Q: om. C rogoi D rogol $\pi 0-$

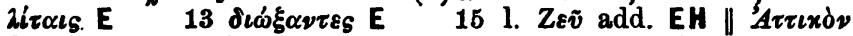

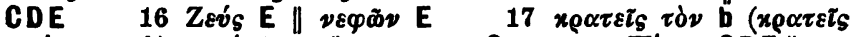

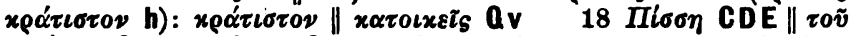

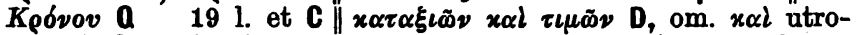
que. Delevi virgulam post $\tau \iota \mu \tilde{\omega} \nu 20 \delta \xi$ et $\lambda \dot{\varepsilon} \gamma \varepsilon \iota$ om. C

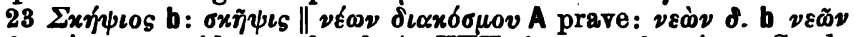

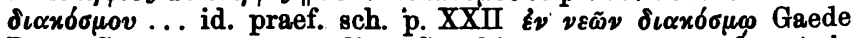
Dem. Sc. quae supers. (diss. Gryphisw. 1880) n. 74 (fr. 54) $\varepsilon^{2} v$

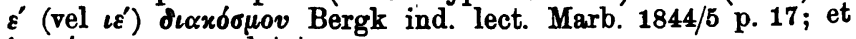
in $\nu \varepsilon \hat{\omega} \omega \nu$ numerus latet 


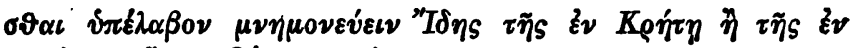

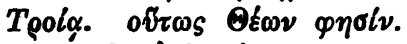

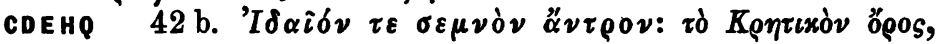

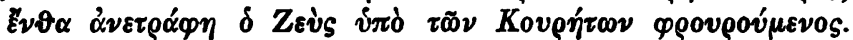

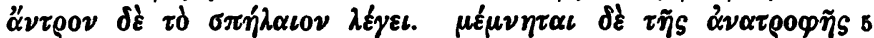

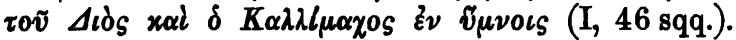

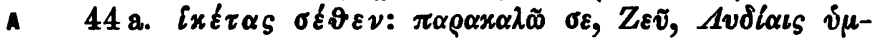

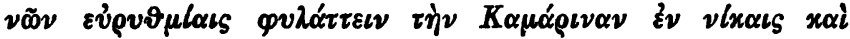

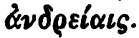

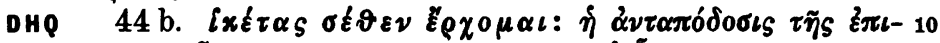

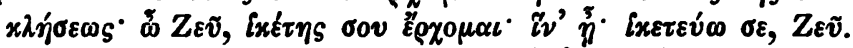

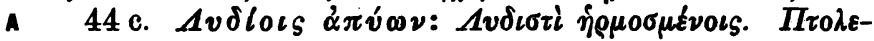

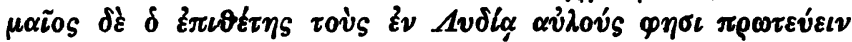

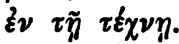

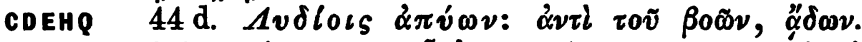
15

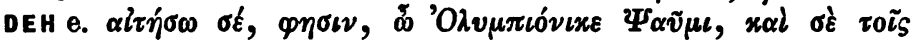

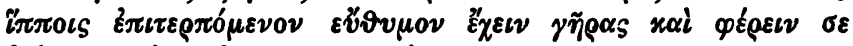

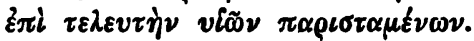

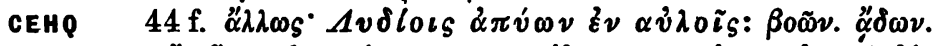

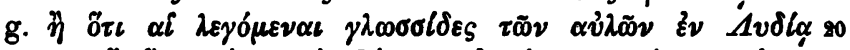

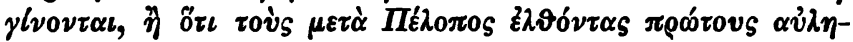

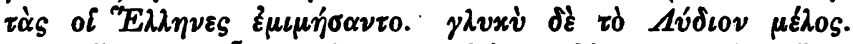

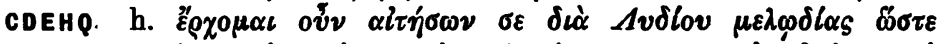

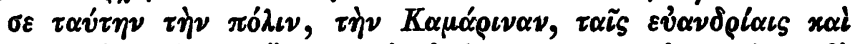

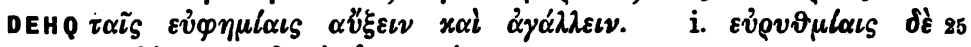

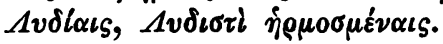

21 Plut. de mus. 15. Paus. 9, 5, 4(?)

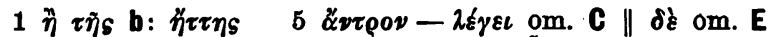
10 l. om. D \| E̊

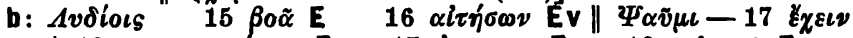

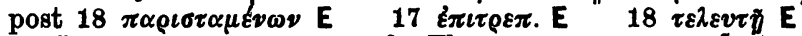

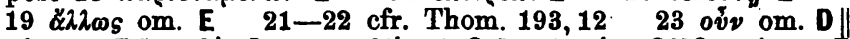

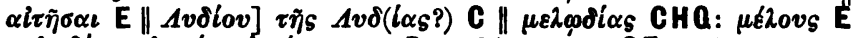

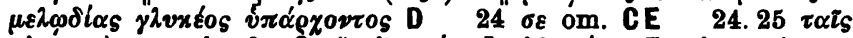

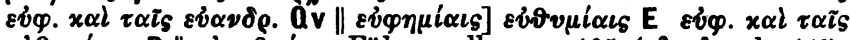

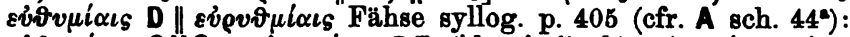

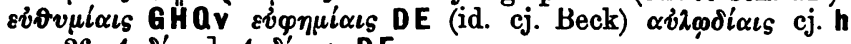

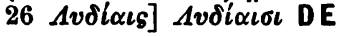




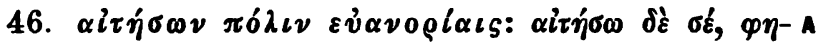

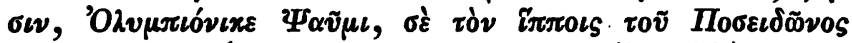

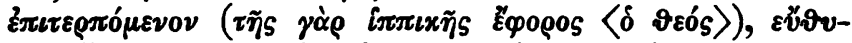

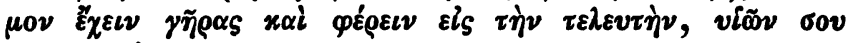
$5 \pi \alpha \rho \iota \sigma \tau \alpha \mu \varepsilon \dot{\nu} \omega \nu$.

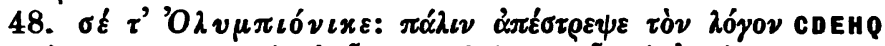

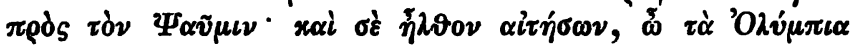

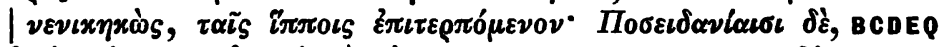

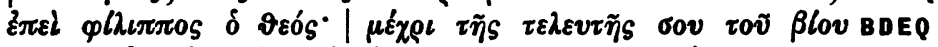

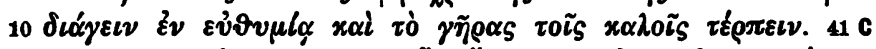

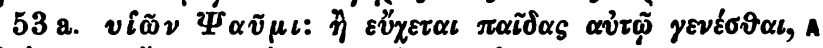

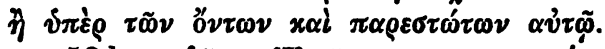

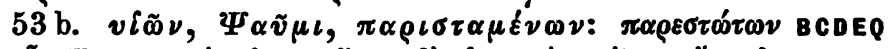

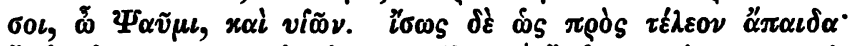

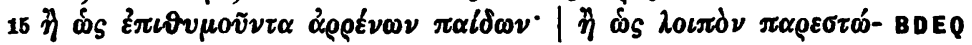
$\tau \omega \nu \alpha v i \tilde{\varphi} x \alpha i \pi \alpha \ell \delta \omega \nu$.

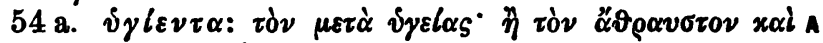
vंyı

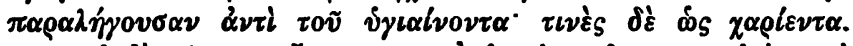

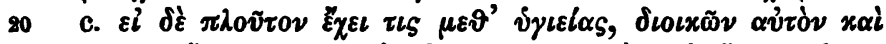

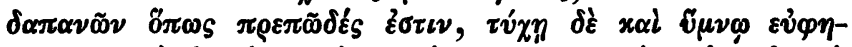

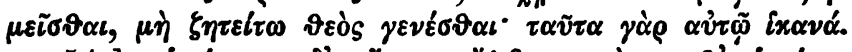

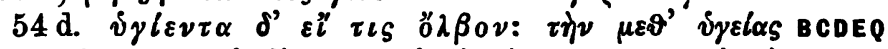

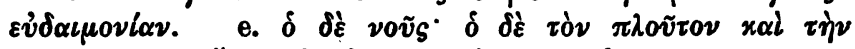

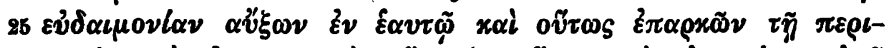

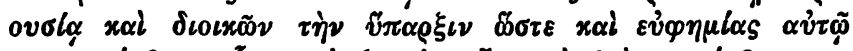

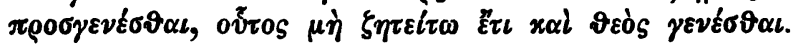

54 f. Choerobosc. in Theodos. can. p. 204, 22 G. (209, 4 Hilg.):

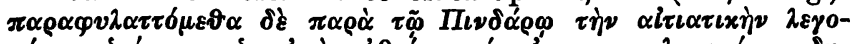

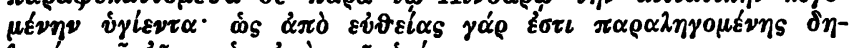

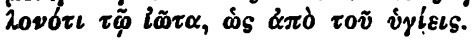

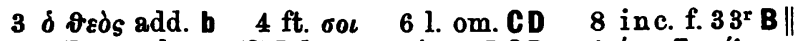

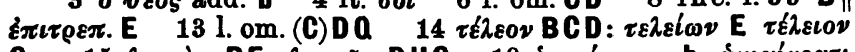

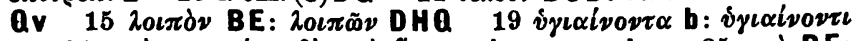

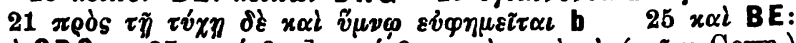

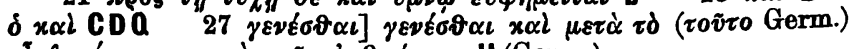

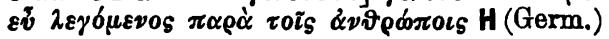




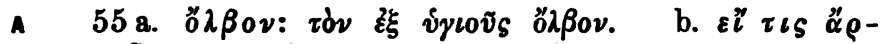

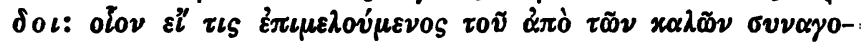

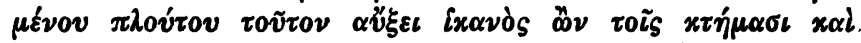

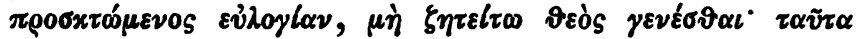

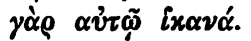

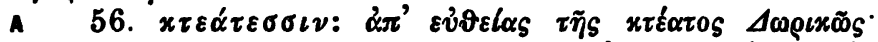

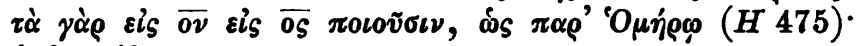

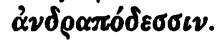

DQ $\tau \hat{\text { żlos. }}$

\section{Scholia in Olympionicarum carmen VI.}

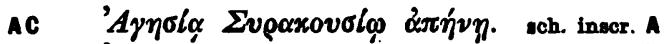

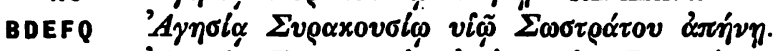

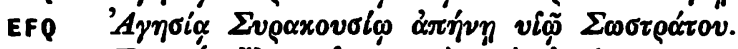

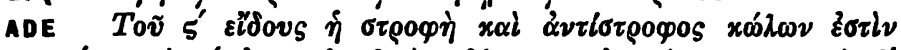

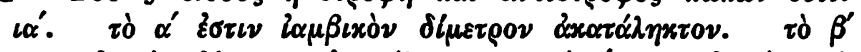

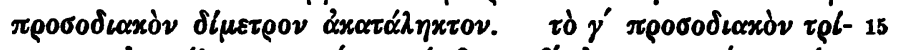

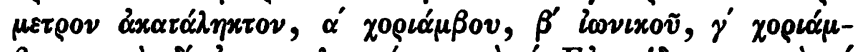

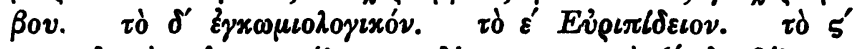

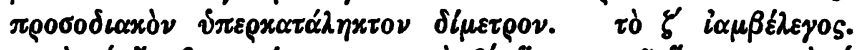

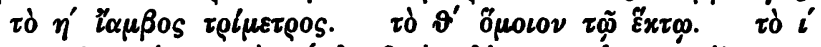

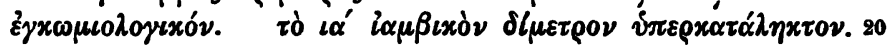

6 Herodian. I $391(=$ II 772, $35=$ II 936, 21). EM 542, 8

$3 \alpha \tilde{v} \xi \varepsilon \iota$ ego: $\alpha \tilde{v} \xi \varepsilon \nu 7 \quad 7 \varepsilon l_{S}$ (alt.) A: $\xi \xi$ b (e Red. male lecto)

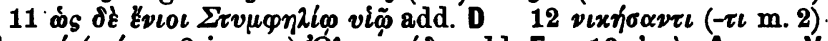

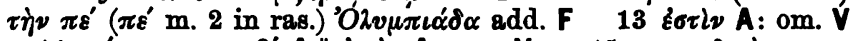

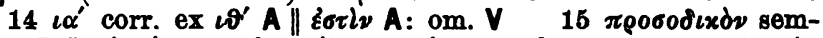
per $\mathrm{E} \| \tau \dot{\partial} \gamma^{\prime}-16 \dot{\alpha} \alpha \alpha \tau \alpha^{\prime} \lambda \eta x \tau 0 \nu$ in mg. A $15 x \rho 0 \sigma 0 \delta\llcorner\alpha x \dot{\partial} \nu$

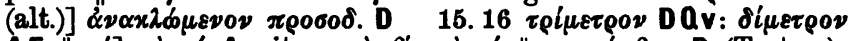

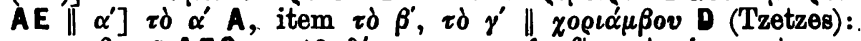

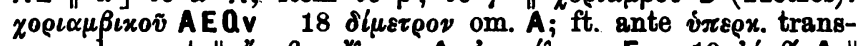

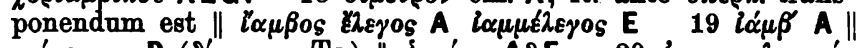

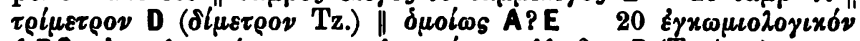

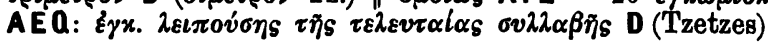




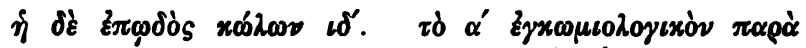

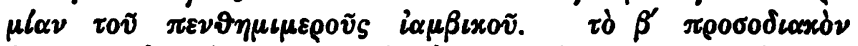

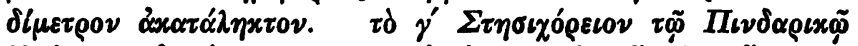

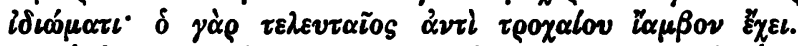

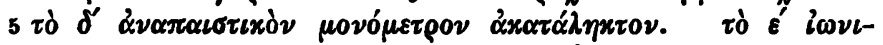

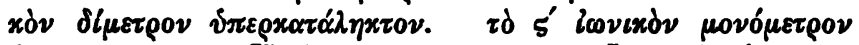

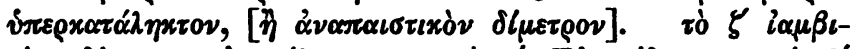

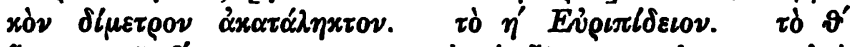

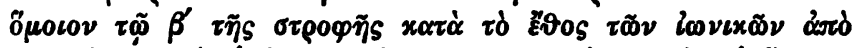

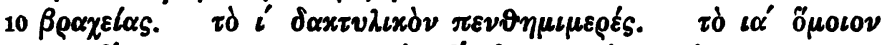

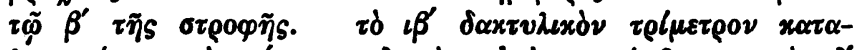

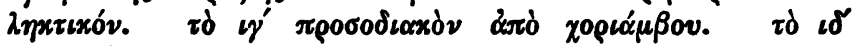

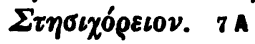

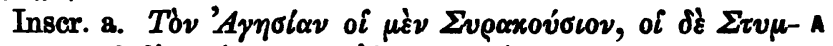

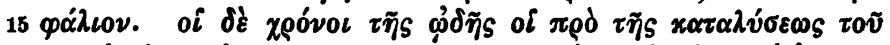

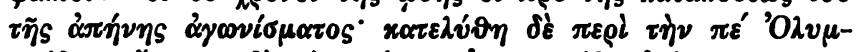

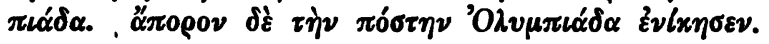

Inscr. b. 'A

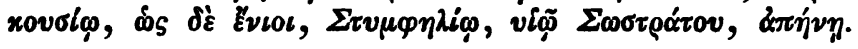

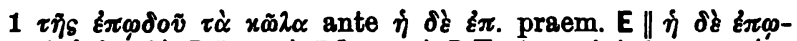

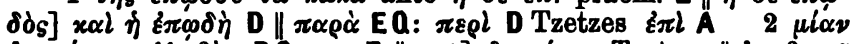

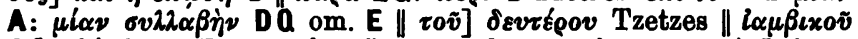

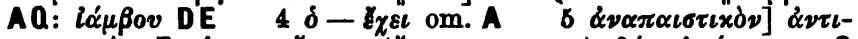

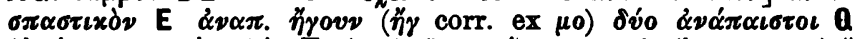

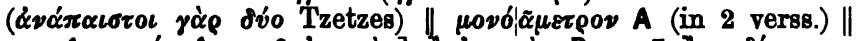

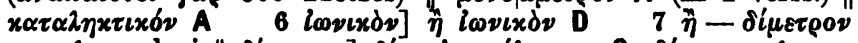

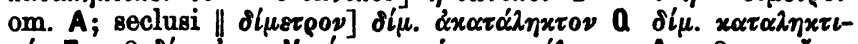

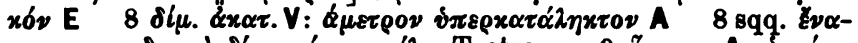

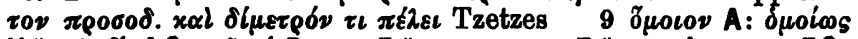

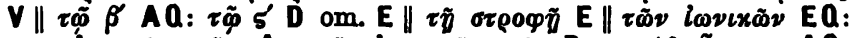

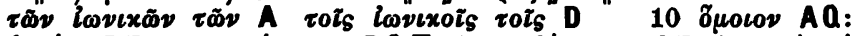

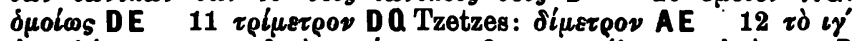

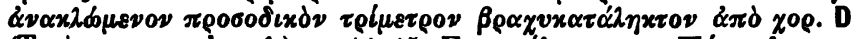

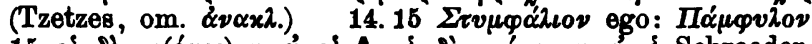

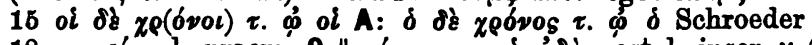

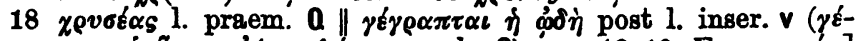

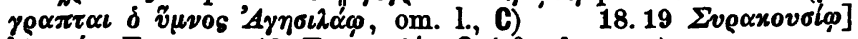

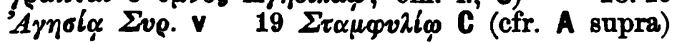




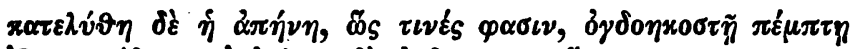

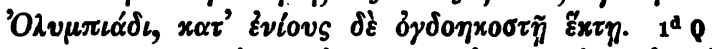

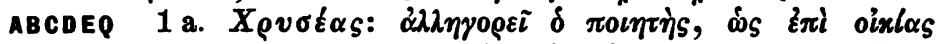

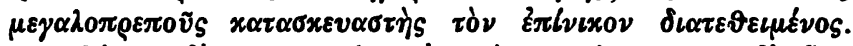

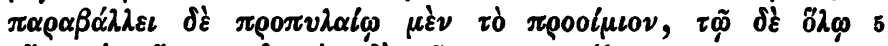

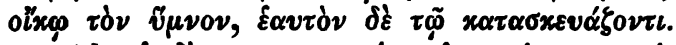

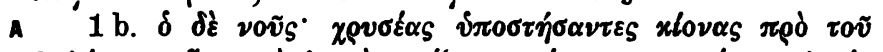

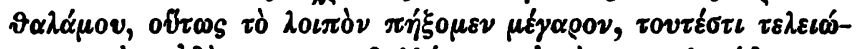

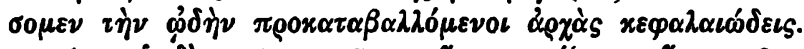

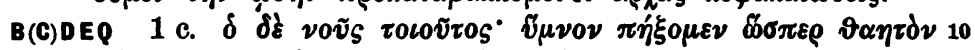

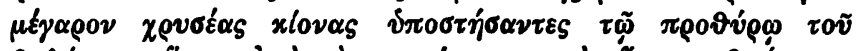

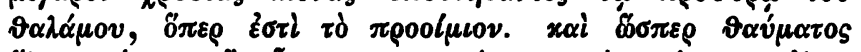

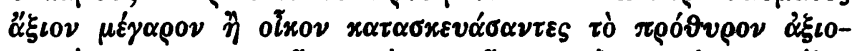

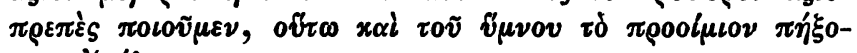

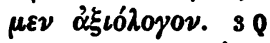

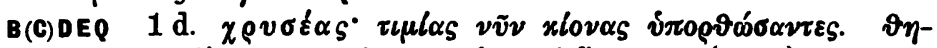

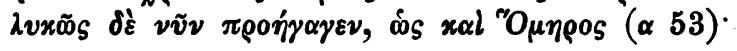

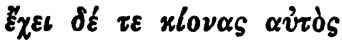

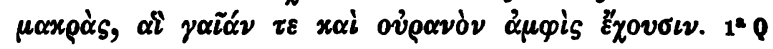

BCEQ 3. ஸ́

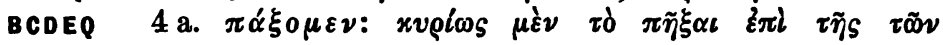

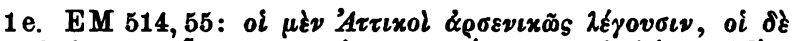

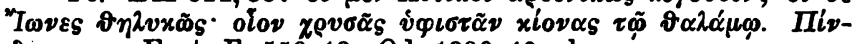

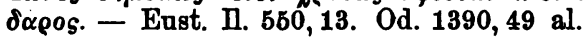

$1 \delta \gamma \delta . \pi \xi \mu \pi \tau \eta] \bar{\pi} \bar{\pi} / \| /$ (ras.) E $2 \varepsilon x \tau \eta] \quad \delta \gamma \delta \delta \eta$ C $3 \not \rho v-$

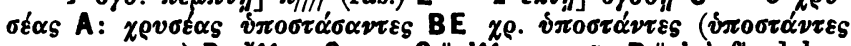

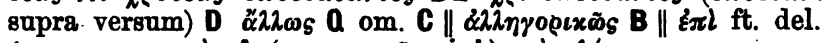

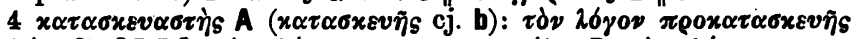

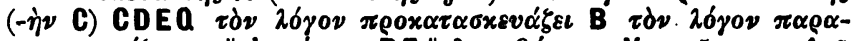

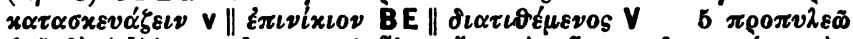

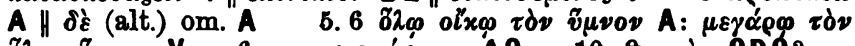

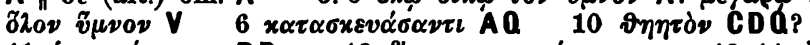

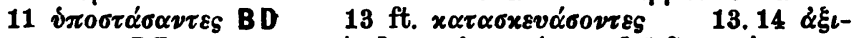

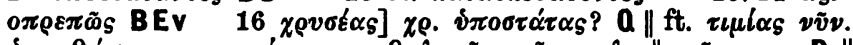

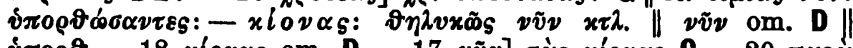

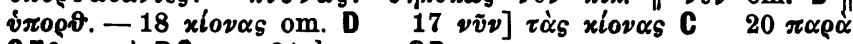
CE?: $\pi \varepsilon \rho l$ B Qv 21 l. om. CD 


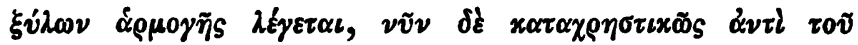

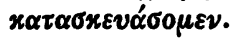

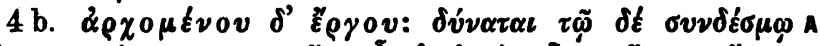

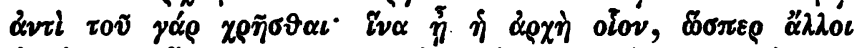

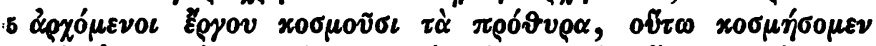

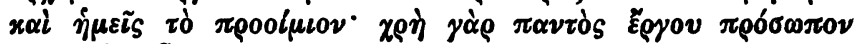

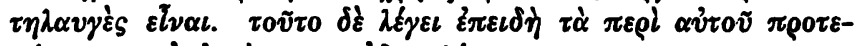

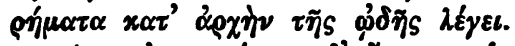

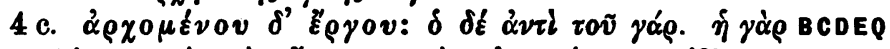
$10 \alpha^{2} x \alpha_{0}$

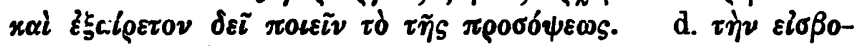

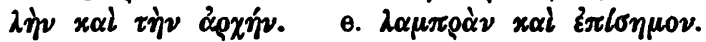

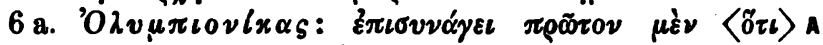

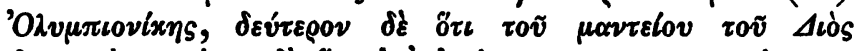

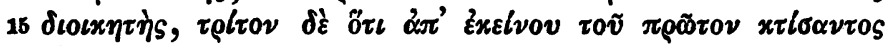

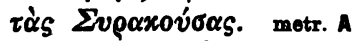

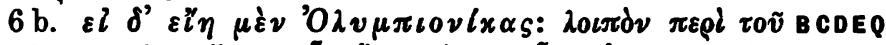

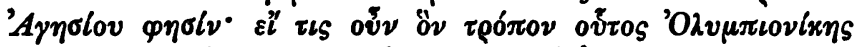

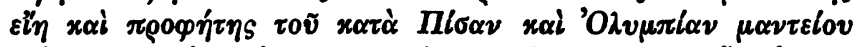

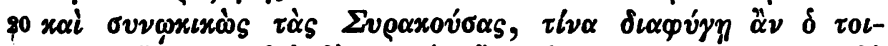

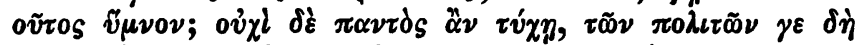

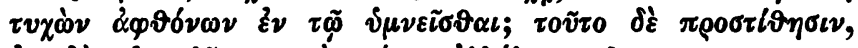

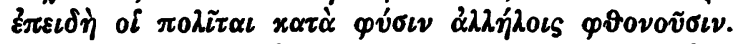

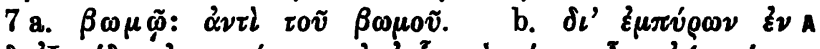

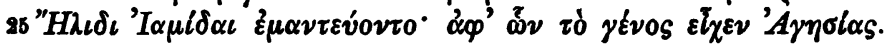

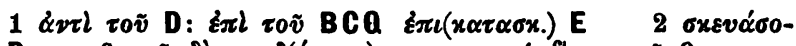

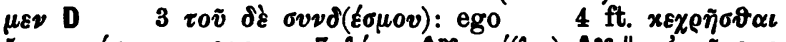

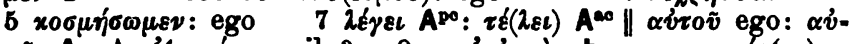

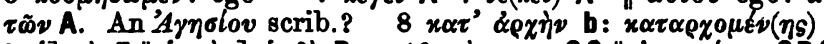

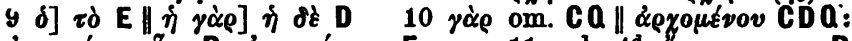

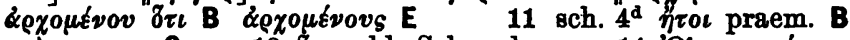

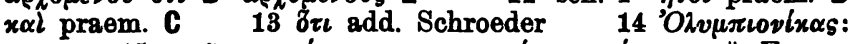

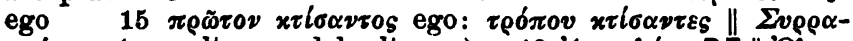

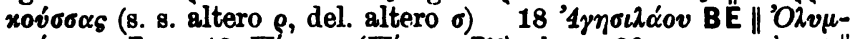

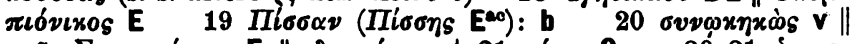

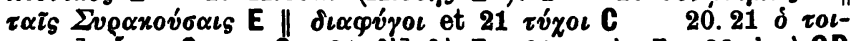

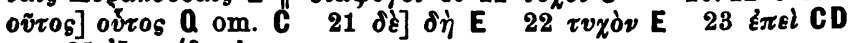
25 'I $\alpha \mu \mu \ell \delta \iota: b$ 


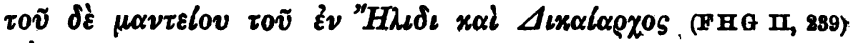
$\mu \varepsilon \dot{\mu} \mu \nu \eta \tau \alpha \iota$.

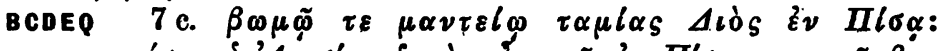

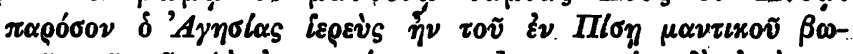

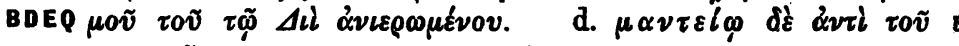
$\mu \alpha \nu \tau \iota x \tilde{\omega}$.

Bg1 7 e. (

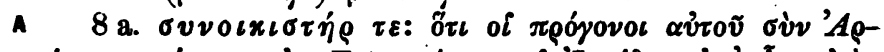

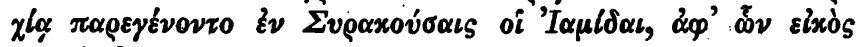

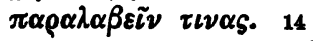

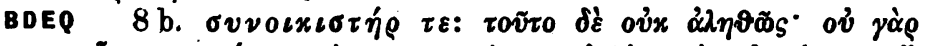

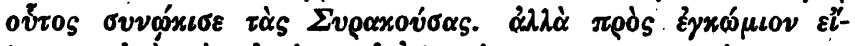

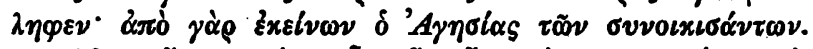

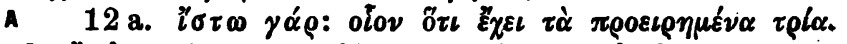

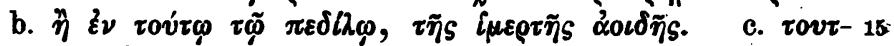

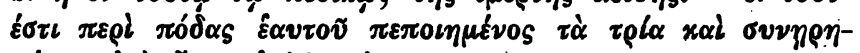

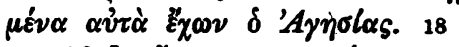

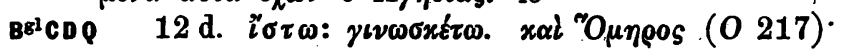

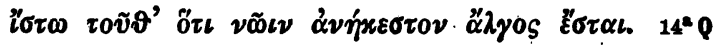

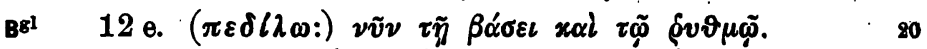

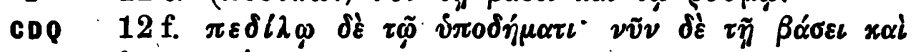

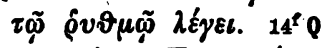

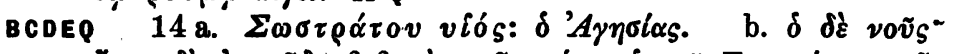

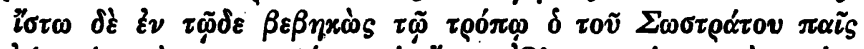

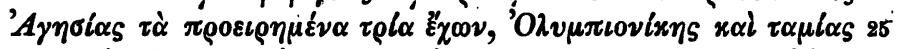

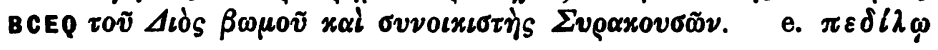

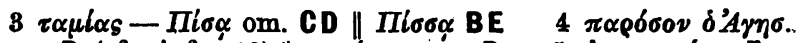
hic om. B (cfr. infra 13) $\| \pi \alpha \rho \sigma ́ \sigma o \nu$ om. D 5 \& $2 \nu \varepsilon \rho \omega \mu \varepsilon \varepsilon_{\omega} \mathrm{E}$

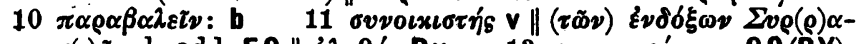

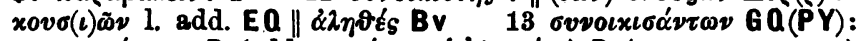

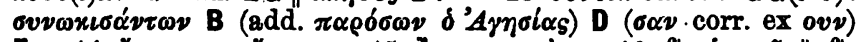

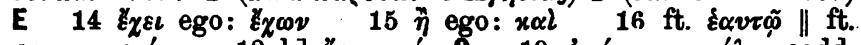

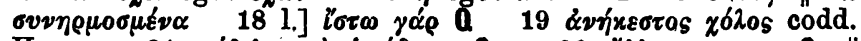

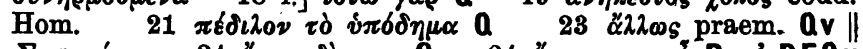

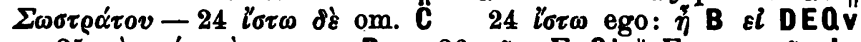

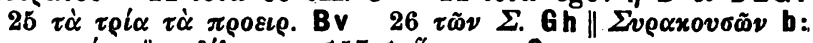

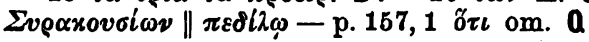




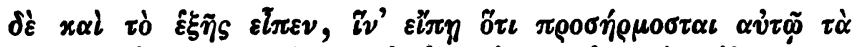

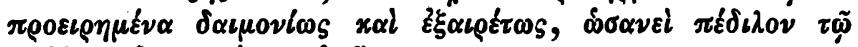

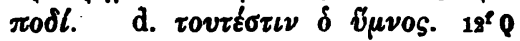

BEQ

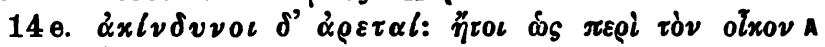

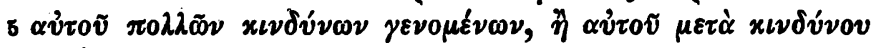

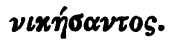

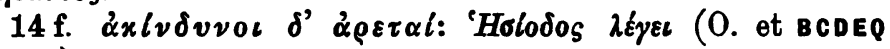
D. 289)

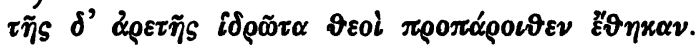

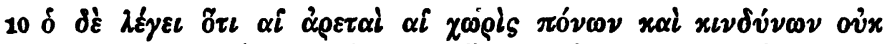

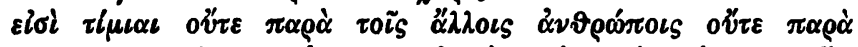

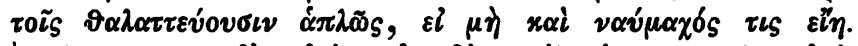

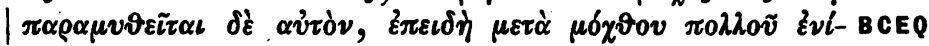
$x \eta \sigma \varepsilon \nu$.

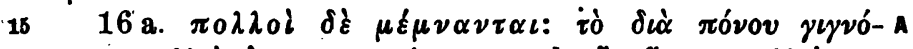

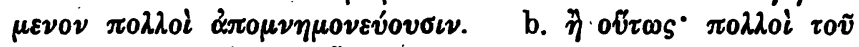

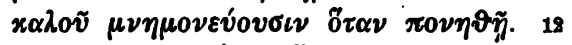

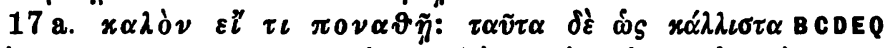

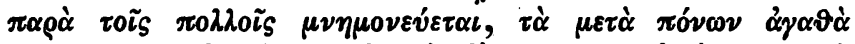

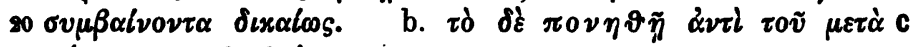

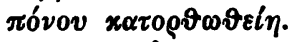

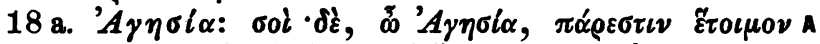

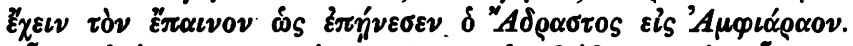

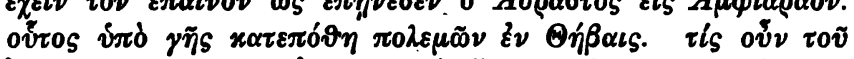

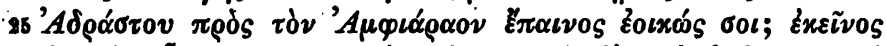

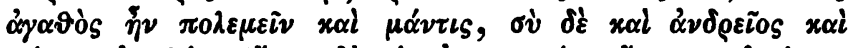

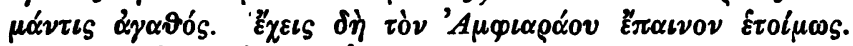

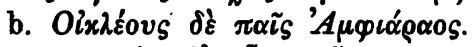

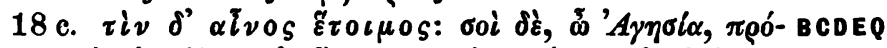

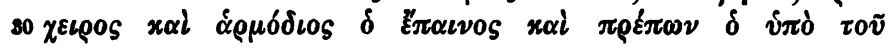

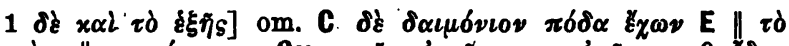

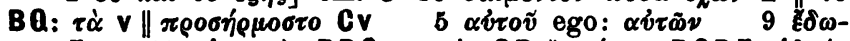

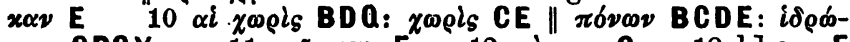
$\tau \omega \nu$ GPQYv 11 roĩs om. E $12 \mu \eta \dot{~ o m . ~ C ~} 18$ l.] om. E

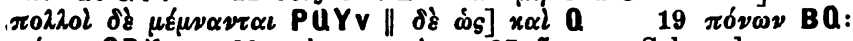

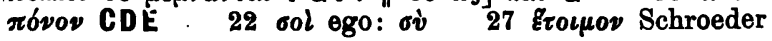




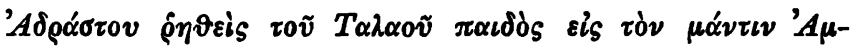

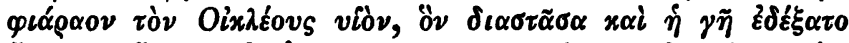

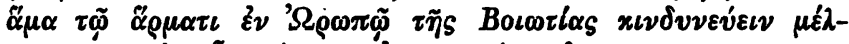

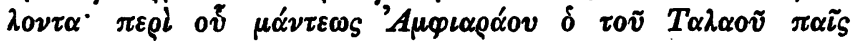

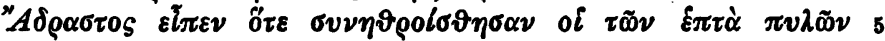

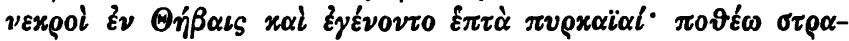

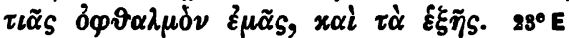

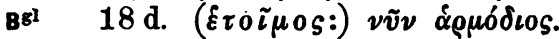

Bg1 21 a. (ż $\pi \varepsilon l$ :) $\alpha \varphi \varphi^{\prime}$ ov.

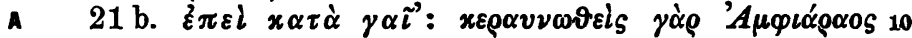

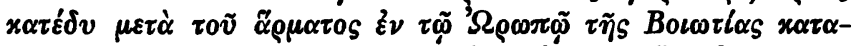

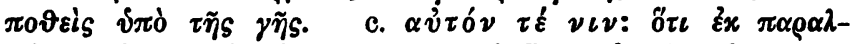

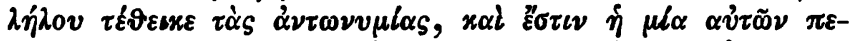

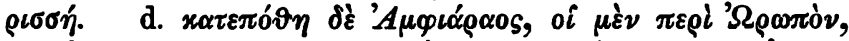

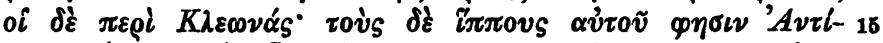

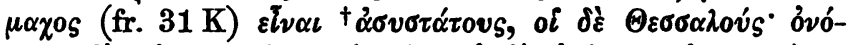

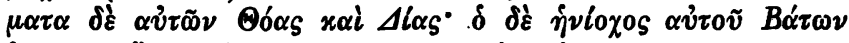

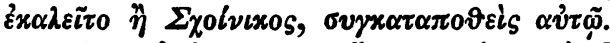

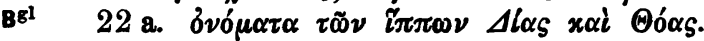

Bgl 22 b. ( $\left.\varepsilon^{\prime \prime} \mu \alpha \rho \psi \varepsilon \nu:\right) i \pi \varepsilon \delta \varepsilon ́ \xi \alpha \tau 0$.

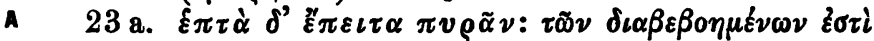

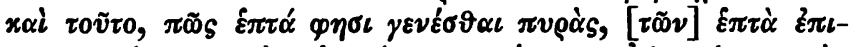

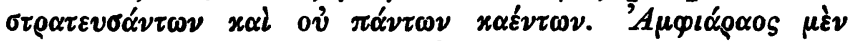

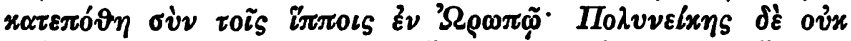

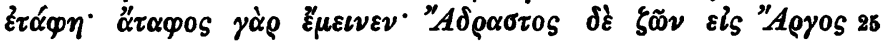
$\dot{\alpha} \pi \tilde{\eta} \lambda \vartheta \varepsilon v . \quad x \alpha \tau \alpha \lambda \varepsilon i \pi 0 \nu \tau \alpha \iota \delta^{\gamma}, T v \delta \varepsilon \dot{s} s, K \alpha \pi \alpha \nu \varepsilon \dot{s} s, \Pi \alpha \rho \vartheta \varepsilon v 0-$

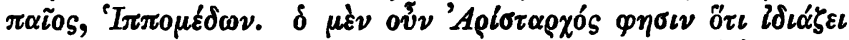

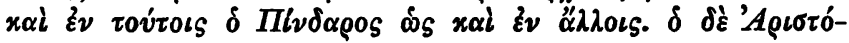

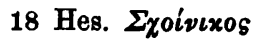

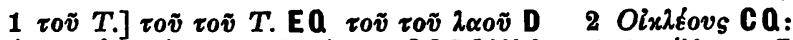

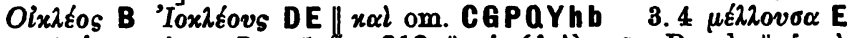

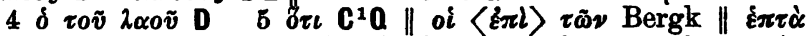

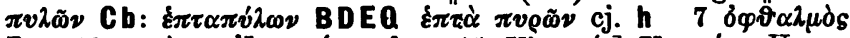

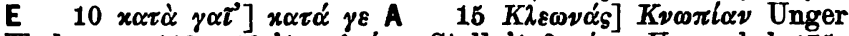

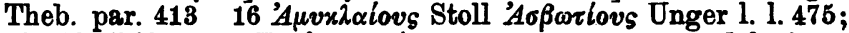

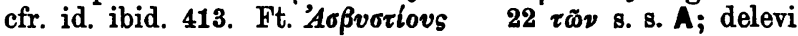




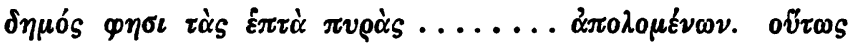

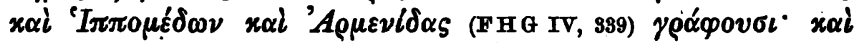

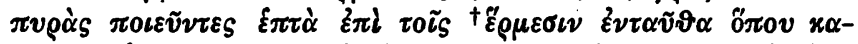

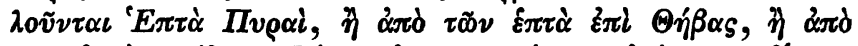

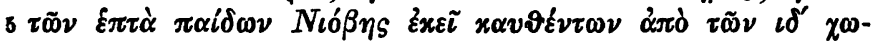

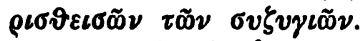

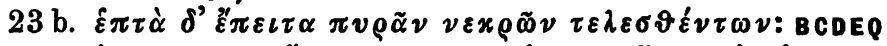

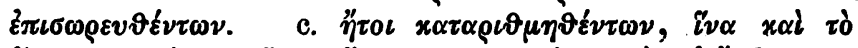

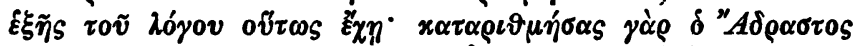

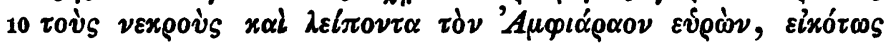

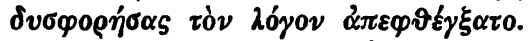

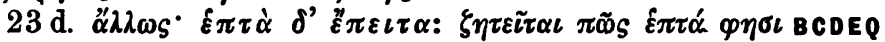

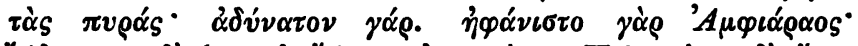

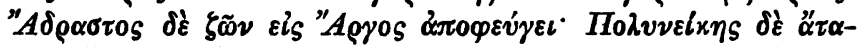

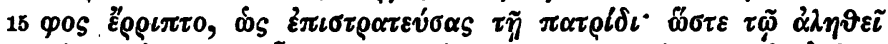

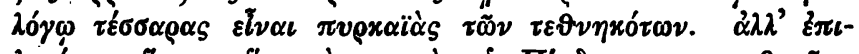

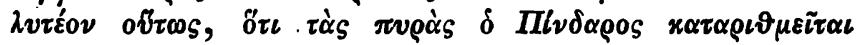

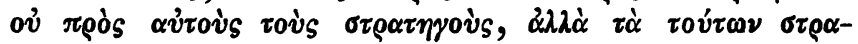

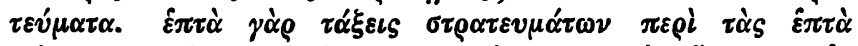

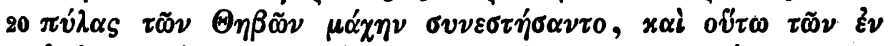

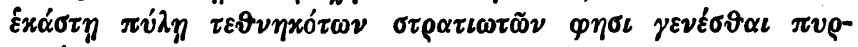
roïós. $27 \mathrm{E}$

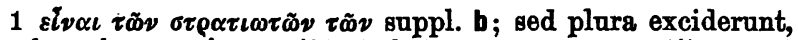
cfr. sch. $23^{\mathrm{a}} \quad 2$ 'I $I \pi \pi^{2} \mu \varepsilon^{\prime} \delta(\omega \nu)$ A (ex p. 158, 27 repetitum, ut

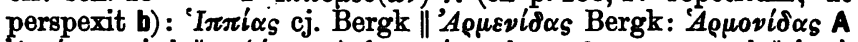

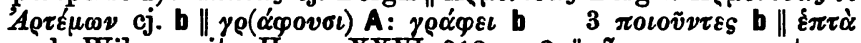

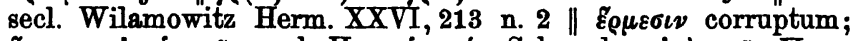

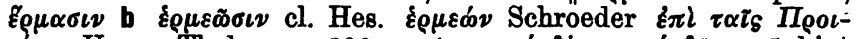

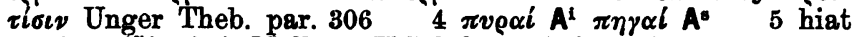

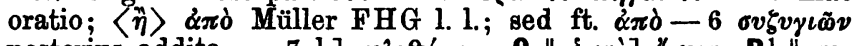

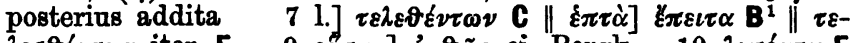

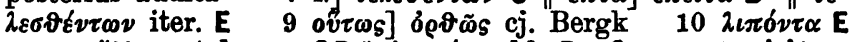

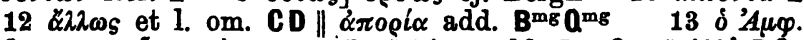

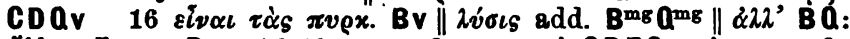

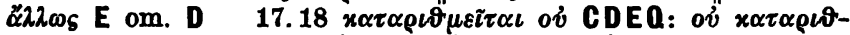

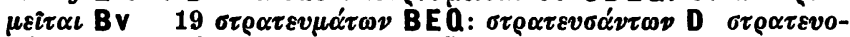

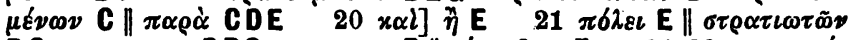

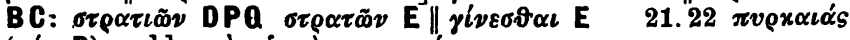

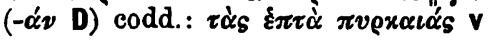




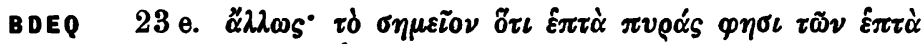

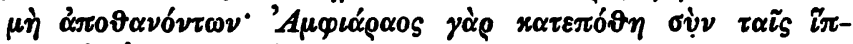

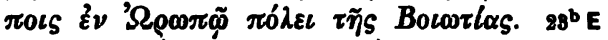

Bgl $23 \mathrm{f}$. $(\tau \varepsilon \lambda \varepsilon \sigma \vartheta \varepsilon v \tau \omega \nu:) \sigma v \nu \alpha \chi \vartheta \dot{\tau} \nu \tau \omega \nu$.

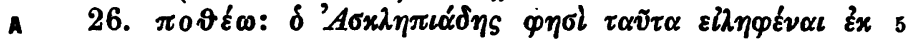

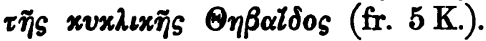

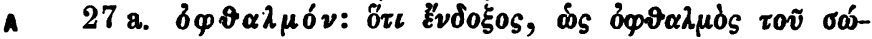

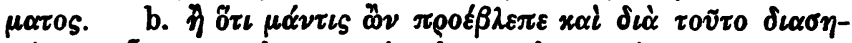

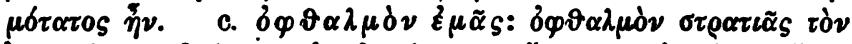

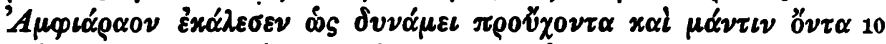

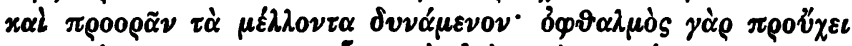

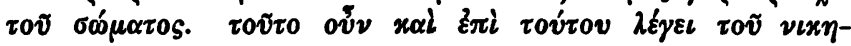
фópov.

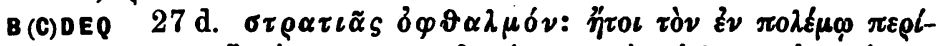

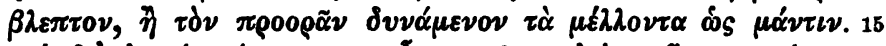

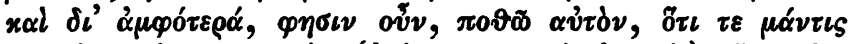

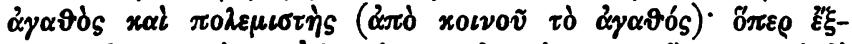

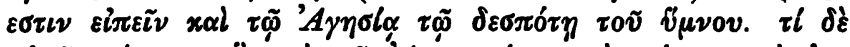

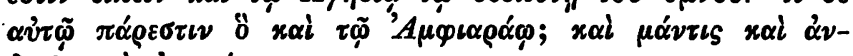

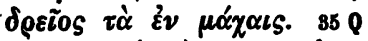

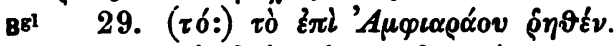

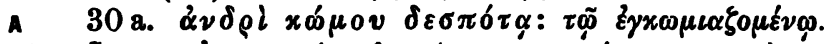

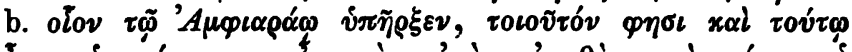

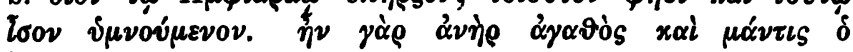
'A

14-17 Tzetzes Chil. 10, 739 sq.

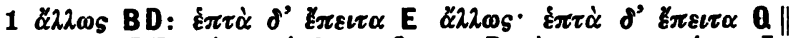

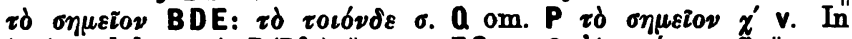
textu ad h. v. + B(B's) \|

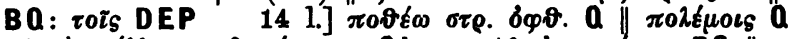

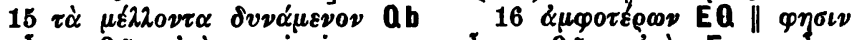

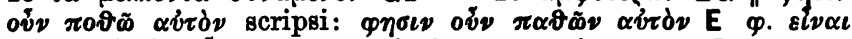

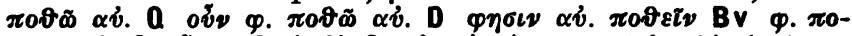

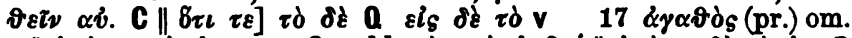

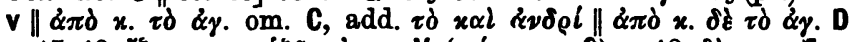

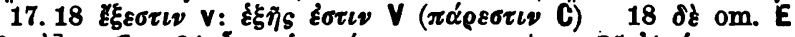

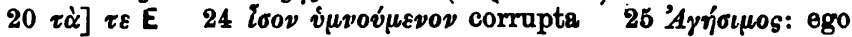




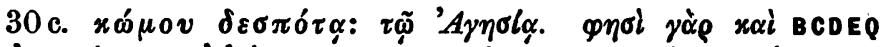

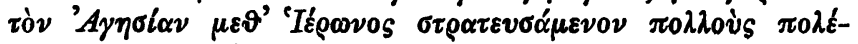

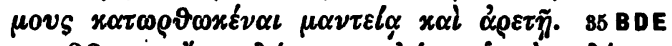

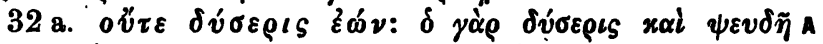

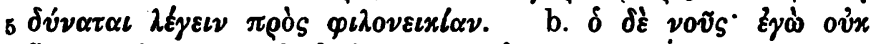

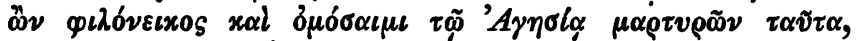

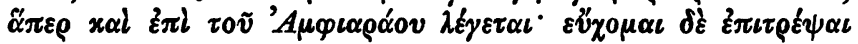

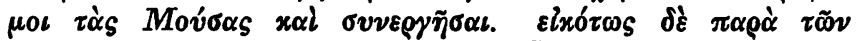

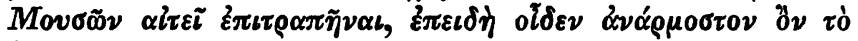

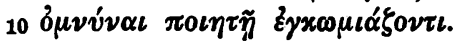

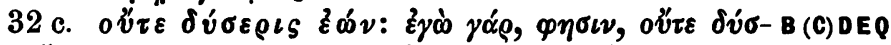

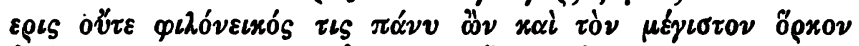

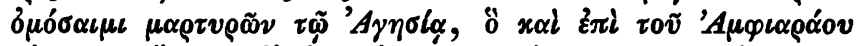

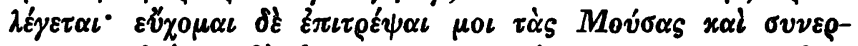

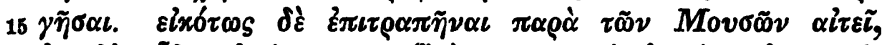

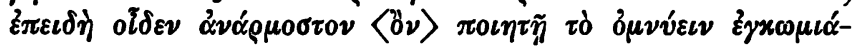

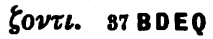

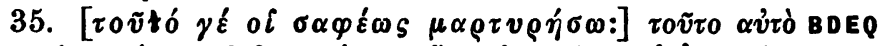

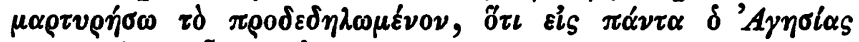

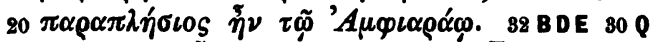

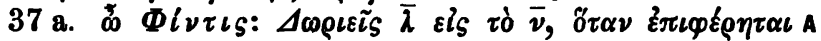

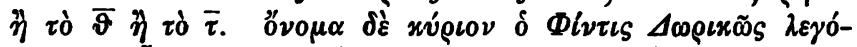

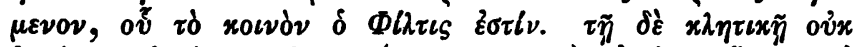

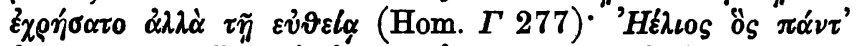

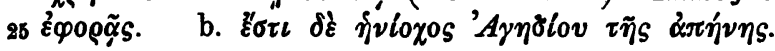

21 Eust. II. 918, 28. sch. Theocr. 1, $77 \quad 23$ sch. A $\Gamma 277$

1 q $\alpha \sigma \iota$ CG?Pb \| $x \alpha \imath$ om. BCv $2 \mu \varepsilon \tau \dot{\alpha}$ CD $2.3 \pi 0 \lambda \varepsilon-$

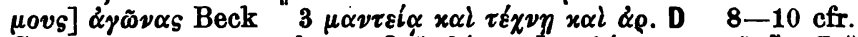

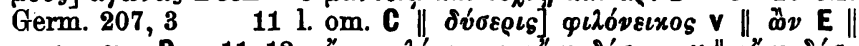

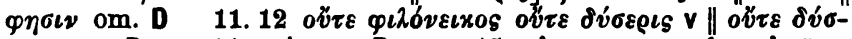

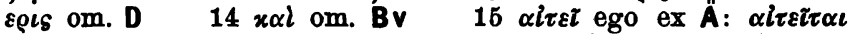

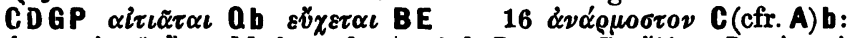
$\dot{\alpha} \rho \mu 0 \sigma \tau \delta \nu \|$ oै $v$ add. b ex A 18 l. B: om. E $\alpha \lambda \lambda \omega s$ D $\tau \dot{\alpha} x \alpha l$

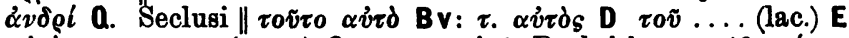

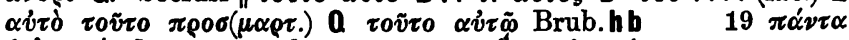

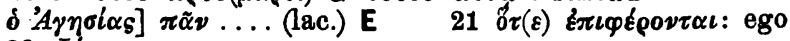

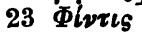

Sohor. ix Prodaux ed. Drachmann. 


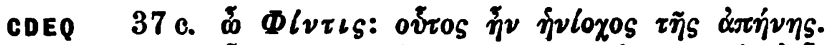

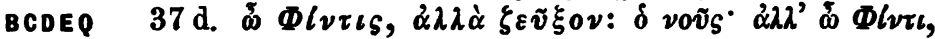

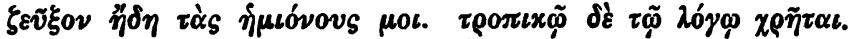

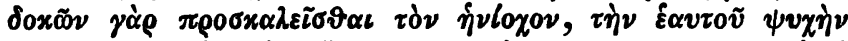

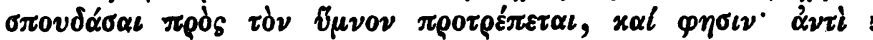

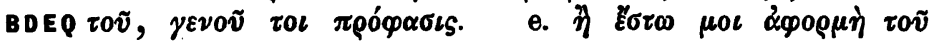

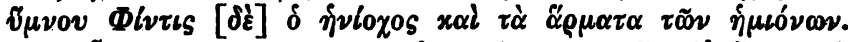

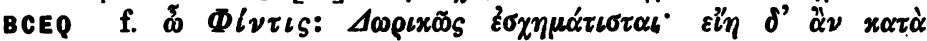

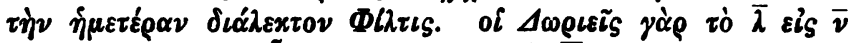

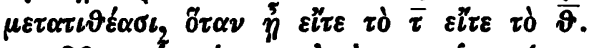

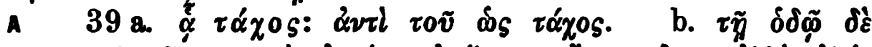

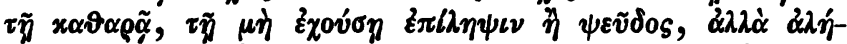

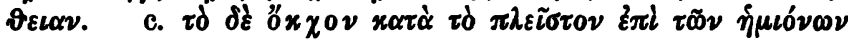
$\tau \iota \boldsymbol{\varepsilon} \alpha \sigma \iota \nu$.

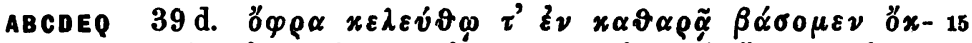

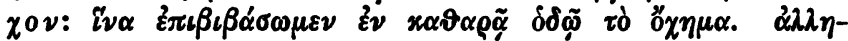

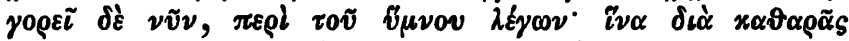

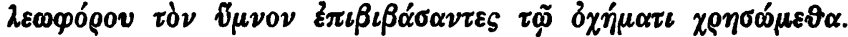

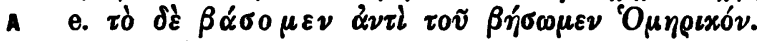

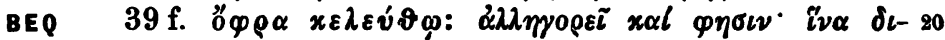

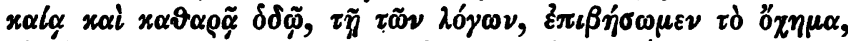

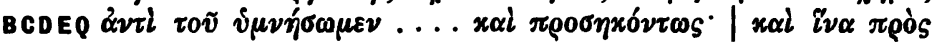

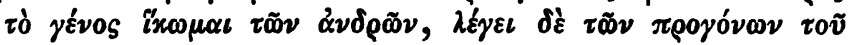

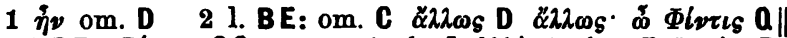

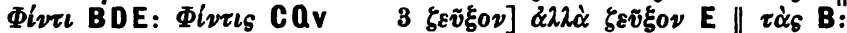

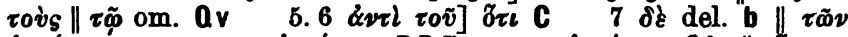

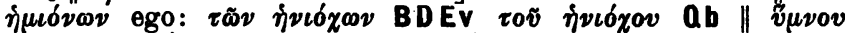

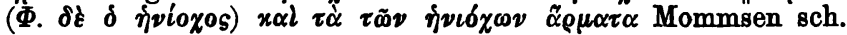

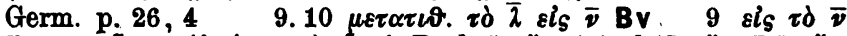

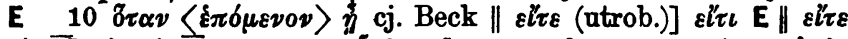

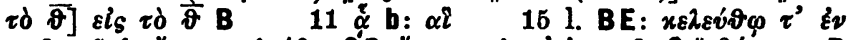

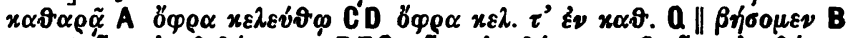

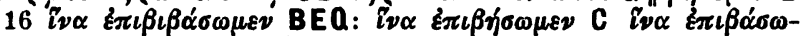

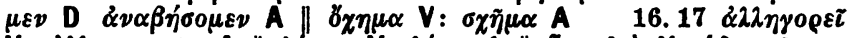

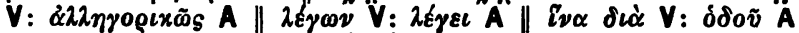

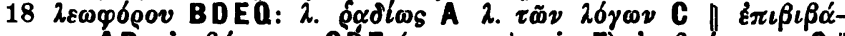

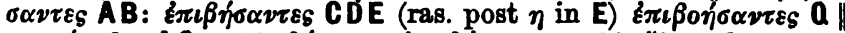
$\chi \rho \eta \sigma \delta \mu \varepsilon \vartheta \alpha$ A Q $19 \beta \hat{\eta} \sigma \omega \mu \varepsilon \nu$ b: $\beta \dot{\eta} \sigma 0 \mu \varepsilon \nu \quad 20 \not 2 \lambda \lambda \omega s$ 1. praem.

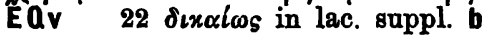




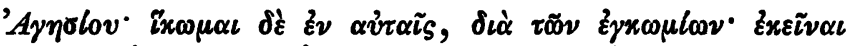

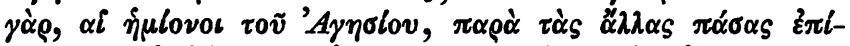

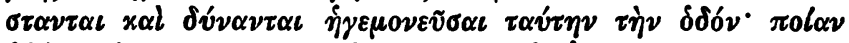

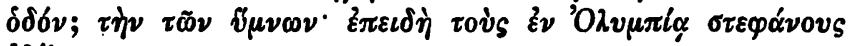

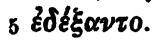

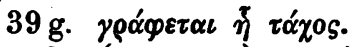

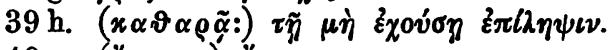

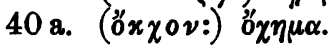

Bmg Egl

Bg1

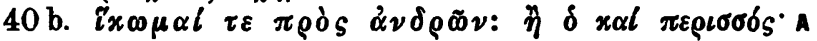

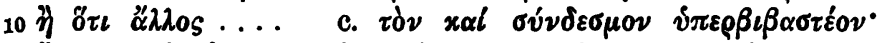

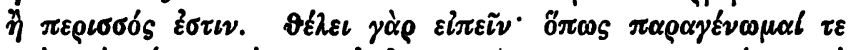

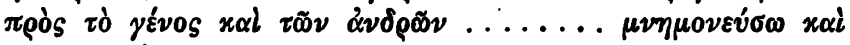
$\tau \tilde{\omega} \nu \pi \rho \circ \delta \sigma \omega \omega \nu$.

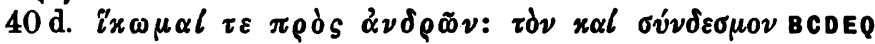

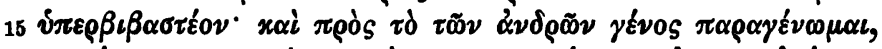

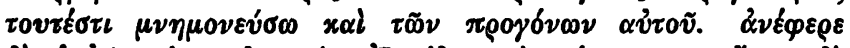

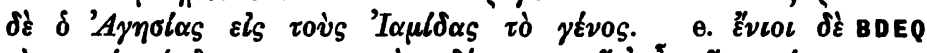

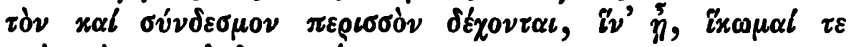

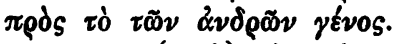

2041 a. $(x \alpha l:) \tau \dot{\delta} x \alpha l \quad \pi \varepsilon \rho l \sigma \sigma o \dot{\nu} x \alpha \tau \dot{\alpha} . \ldots$.

Bg1

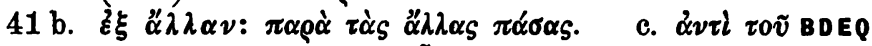

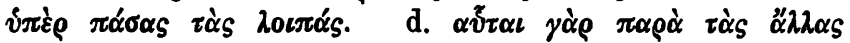

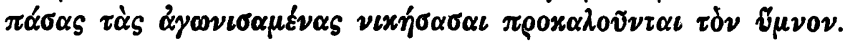

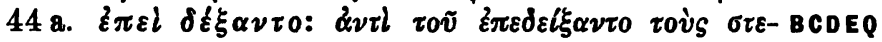

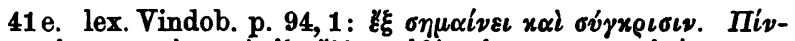

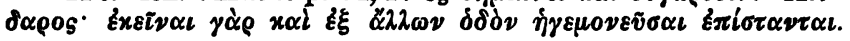

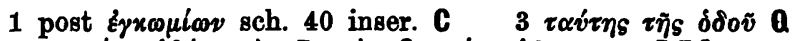

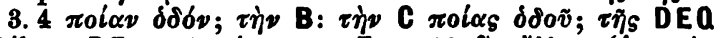

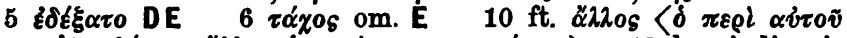

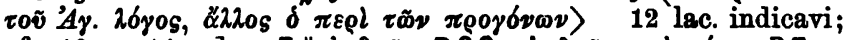

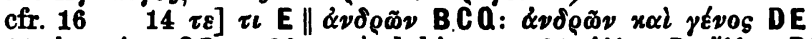
$16 \alpha v \alpha \varphi t e \varepsilon \varepsilon$ CD $20 x \alpha \tau \dot{\alpha}$ dubium $21 \alpha \lambda \lambda \tilde{\alpha} \nu$ D $\alpha \lambda \lambda \tilde{\alpha} \nu$ PQ

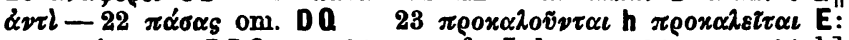
$\pi \rho 0 \sigma x \alpha \lambda_{0} v \tau \tau \alpha \iota$ B DQv 24 sqq. cfr. Lehrs p. 194 sq. 24 1.]

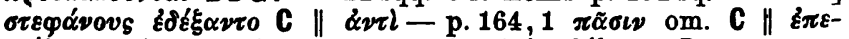
$\delta \varepsilon l \xi \alpha \nu \tau o$ et $\pi \tilde{\alpha} \sigma \nu \nu($ p. 164, 1) om. BV $\| \dot{\alpha} \pi \varepsilon \delta \delta \xi \xi \nu \tau \tau$ D 


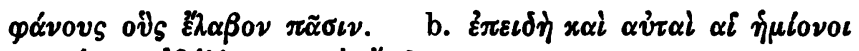

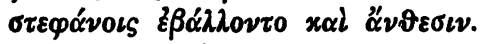

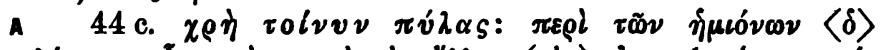

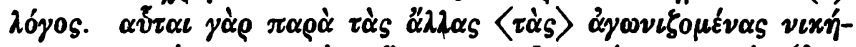

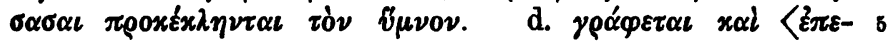

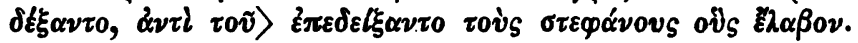

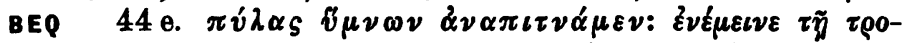

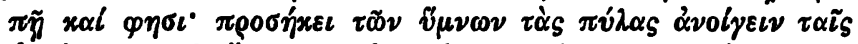

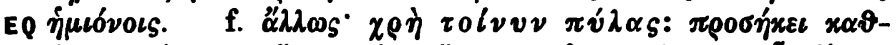

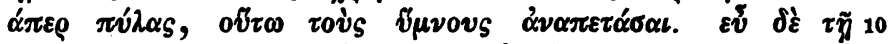

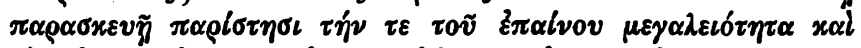

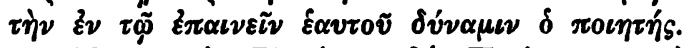

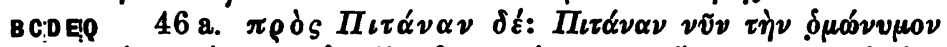

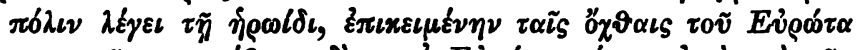

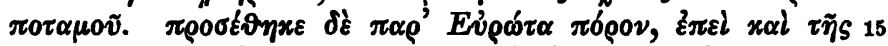

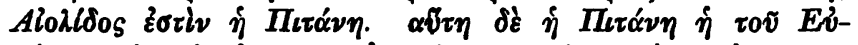

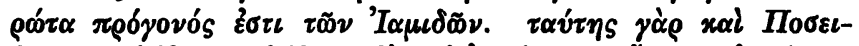

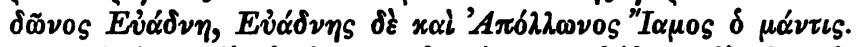

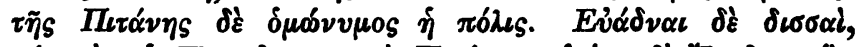

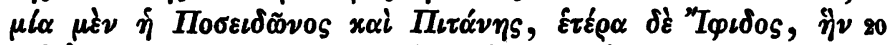

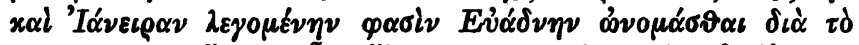

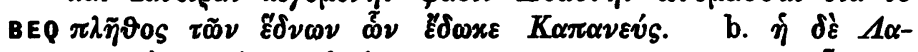

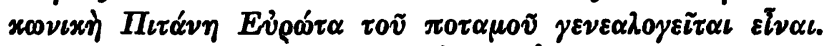

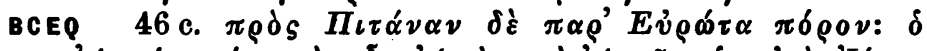

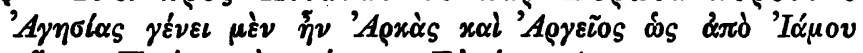

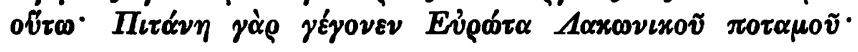

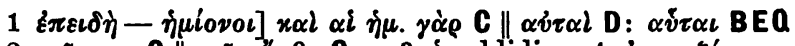

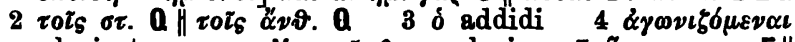

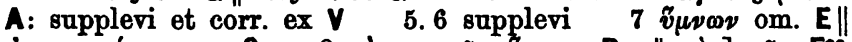

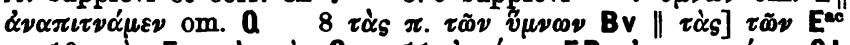

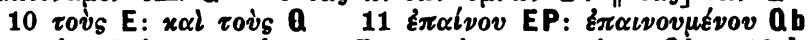

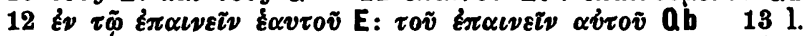

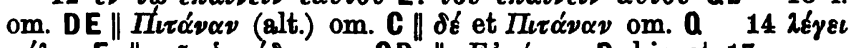

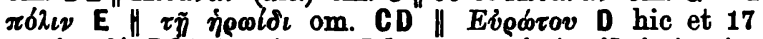

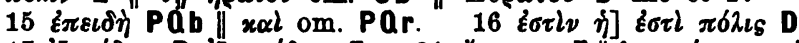

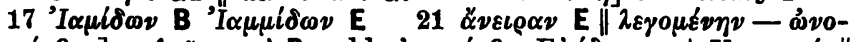

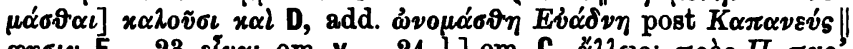

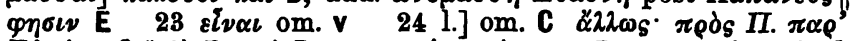

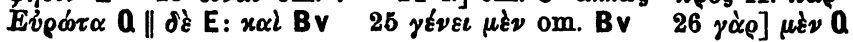




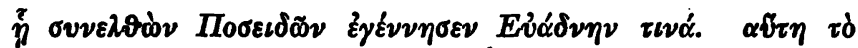


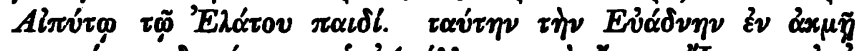

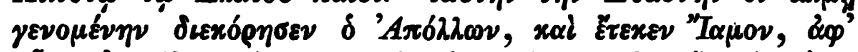

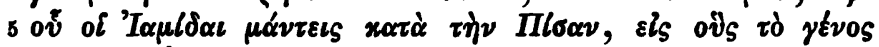

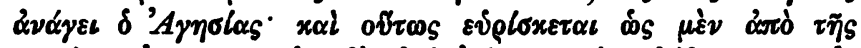

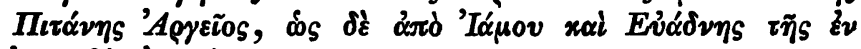

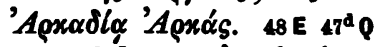

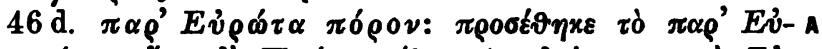

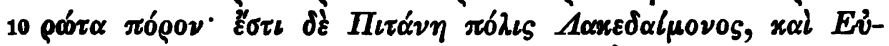

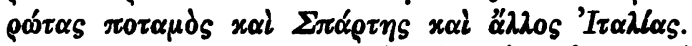

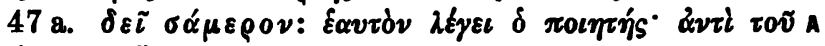

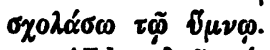

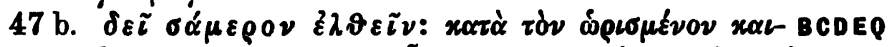

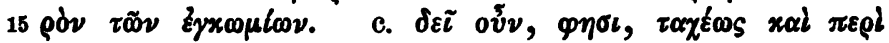

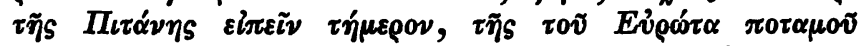

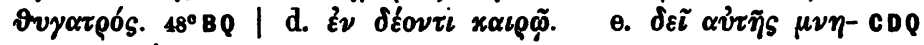

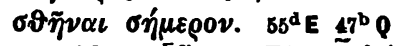

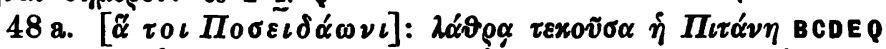

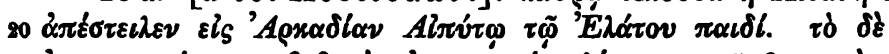

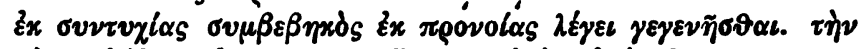

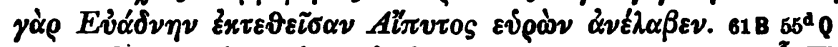

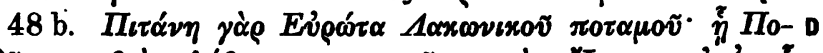

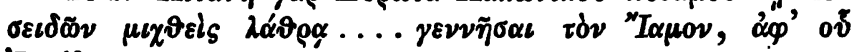
25 'I $\alpha \mu l \delta \alpha \iota$.

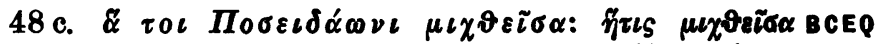

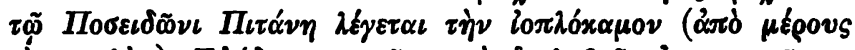

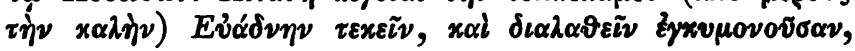

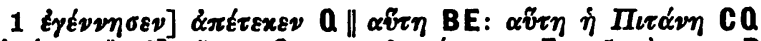

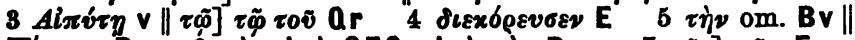
$\Pi i \sigma \sigma \alpha \nu$ B $6 \mu \xi \nu \& \pi \delta$ CEQ: $\alpha \pi \delta \mu \xi \nu$ BV $\left.7 \tau \eta_{\xi}\right] \tau \tilde{\omega} \nu \mathrm{E}$

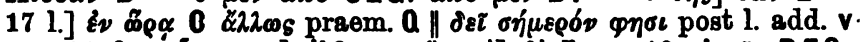

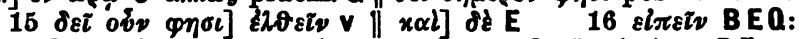

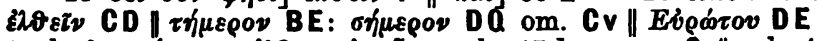

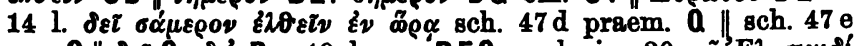
om. C $\| \delta \varepsilon \tau$ Q: $\delta \iota^{\prime}$ D 19 l. om. 'BEQ; seclusi $20 \tau \tilde{\omega}^{\prime} E \lambda$. $\pi \alpha \iota \delta \imath$

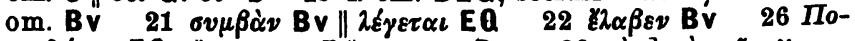

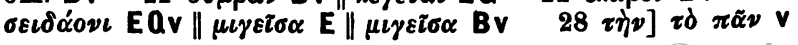




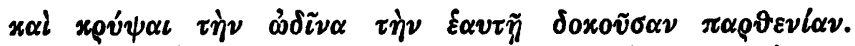

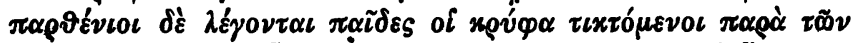

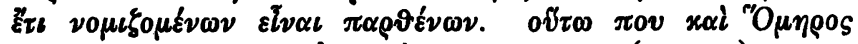

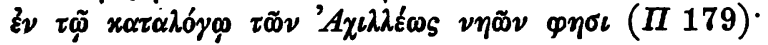

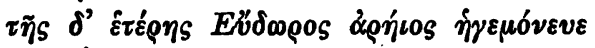
$\pi \alpha \rho \vartheta t v L o s$.

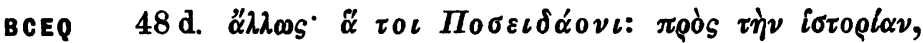

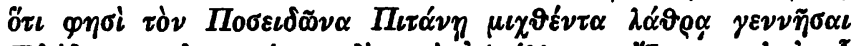

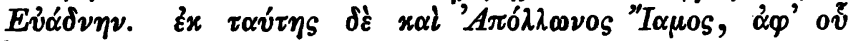
'I $\alpha \mu l \delta \alpha \iota$.

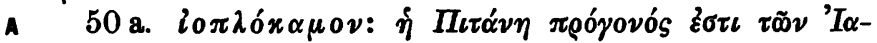

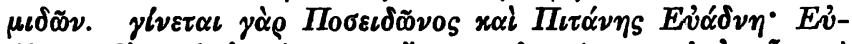

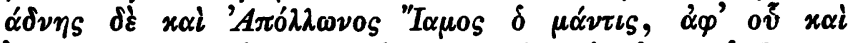

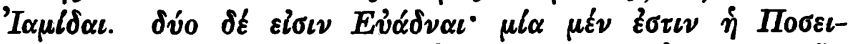

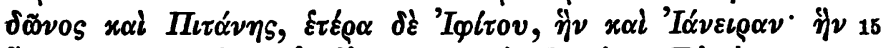

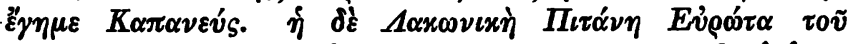

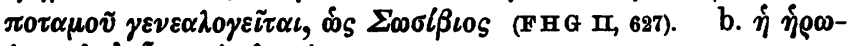

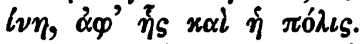

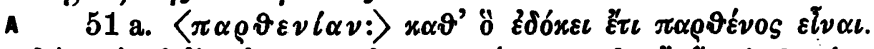

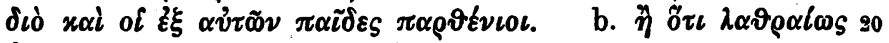

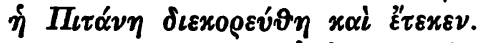
tóxov.

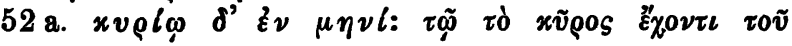

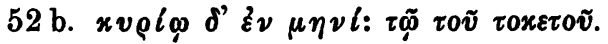

c. $\delta$ ขoṽs

2. 3 sch. A $\Delta$ 499. sch. ABT $\Pi$ 180. EG 410, 32. EM 236, 27. Suid. Phot. 20 sch. B $\Pi 180$

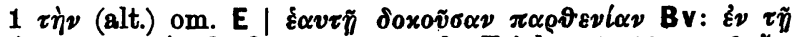

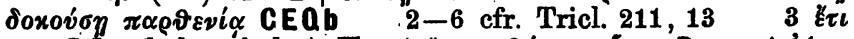

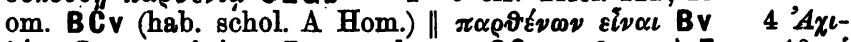

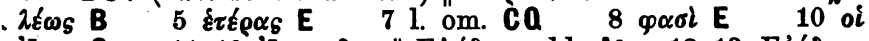

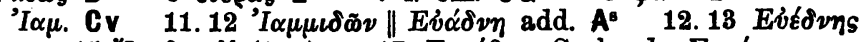
15 "I

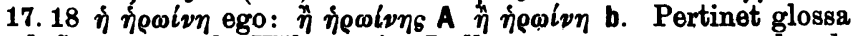

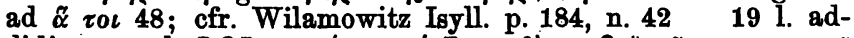

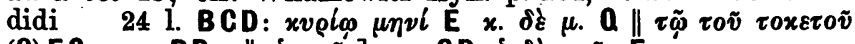

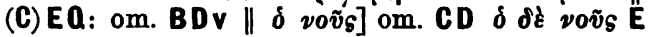




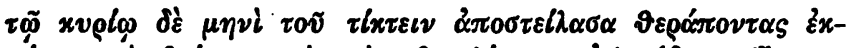

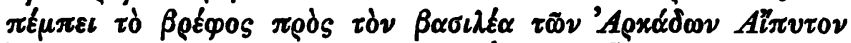

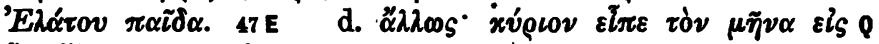

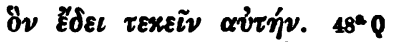

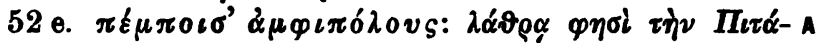

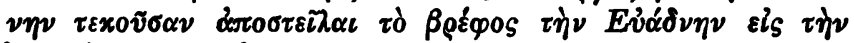
'A

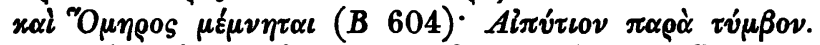

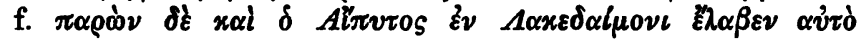

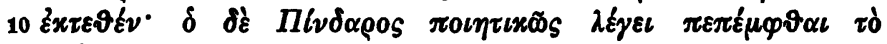
Béśos.

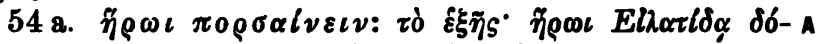

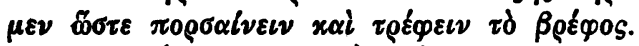

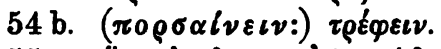

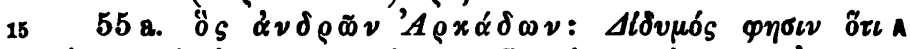

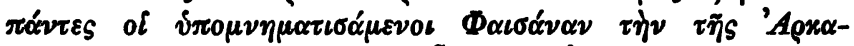

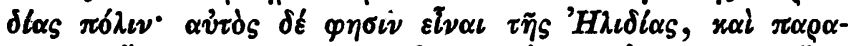

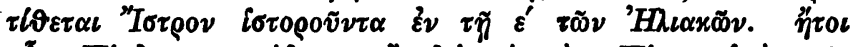

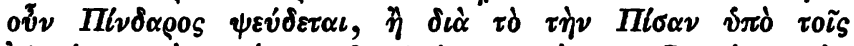

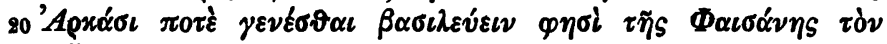
Aliturov. 57

$55 \mathrm{~b}$.

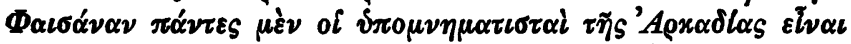

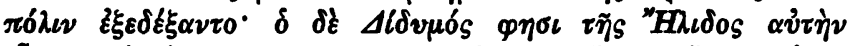

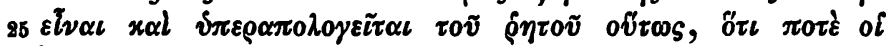

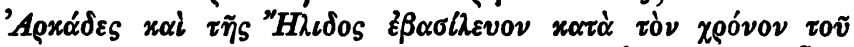

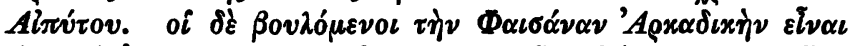

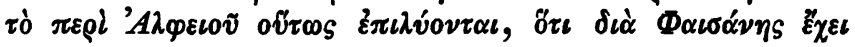

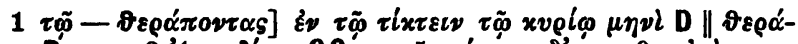

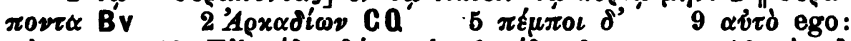

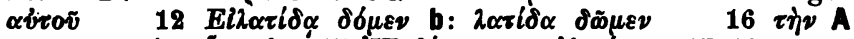

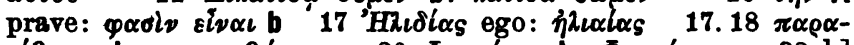

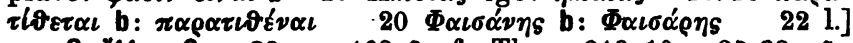

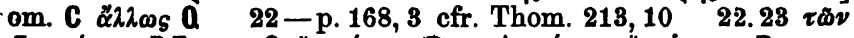

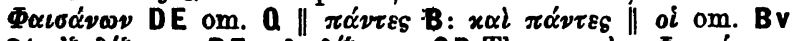

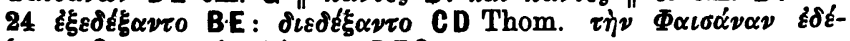

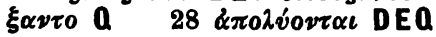




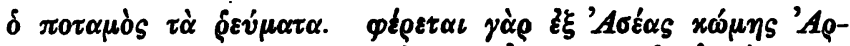

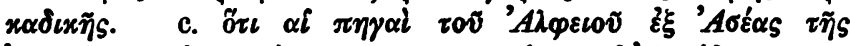
'A

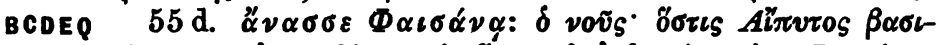

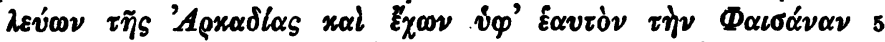

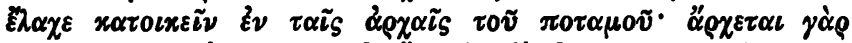

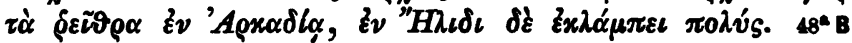
$55^{\mathrm{b}} \mathrm{EQ}$

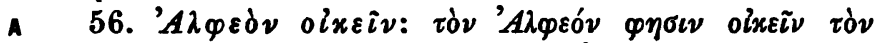

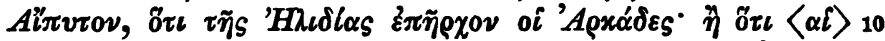

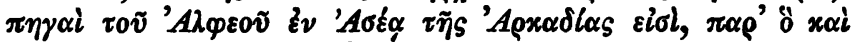

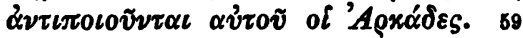

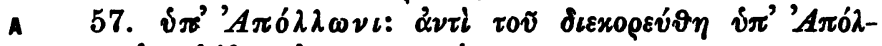

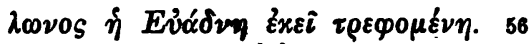

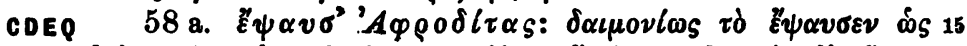

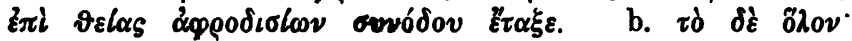

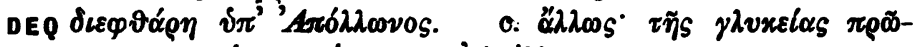

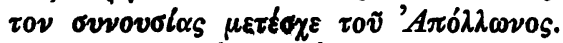

A 59 a o ởं'

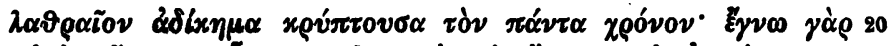

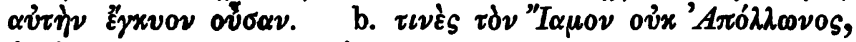

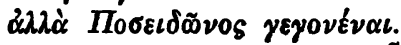

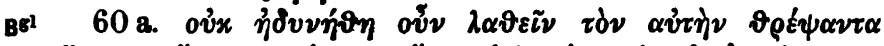

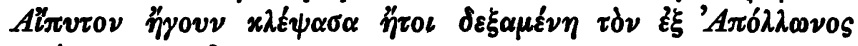

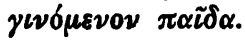

1 St. B. 'Aotó

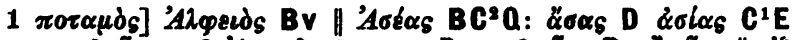

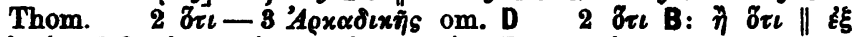

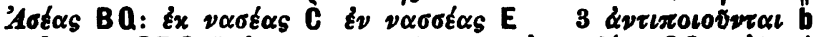
4 l. om. CDQ $\| \delta$ voṽs om. E 5 'Apxadlas CQv: 'Apxádos

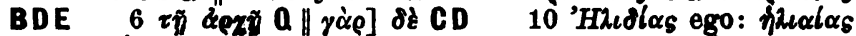

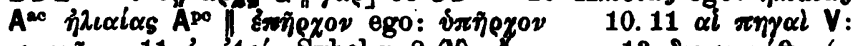

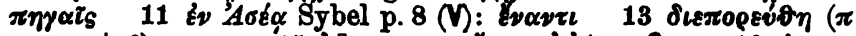

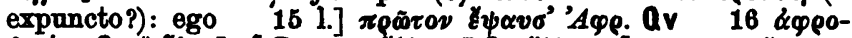

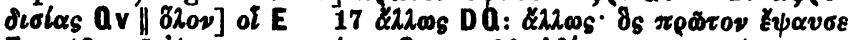

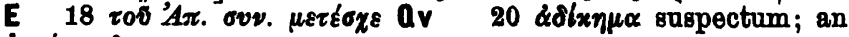

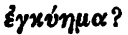




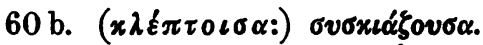

B'1

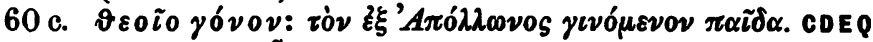

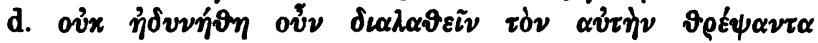

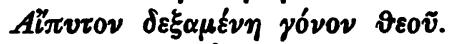

5

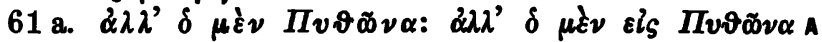

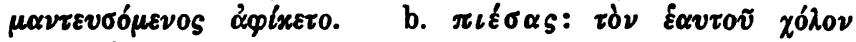

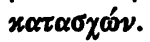

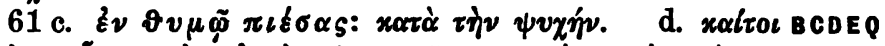

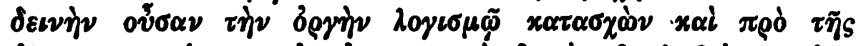

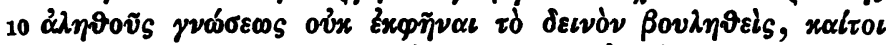

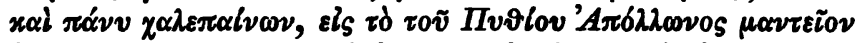

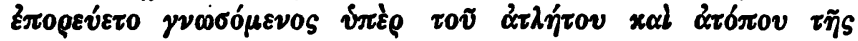

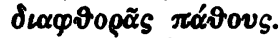

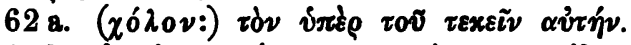
Bgl

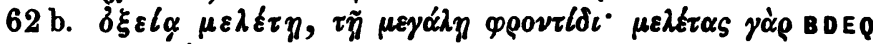

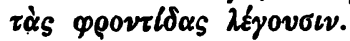

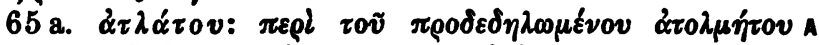

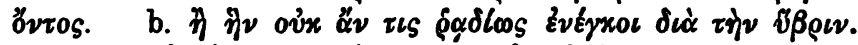

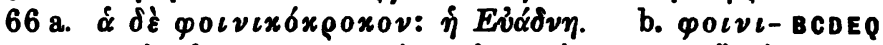

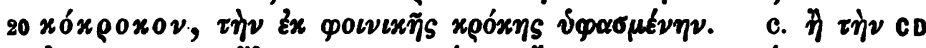

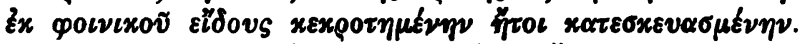

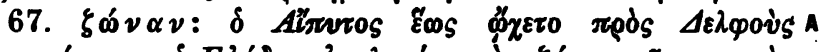

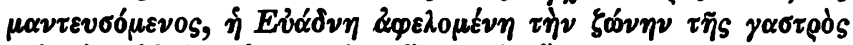

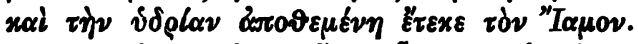

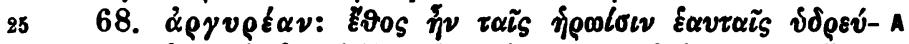

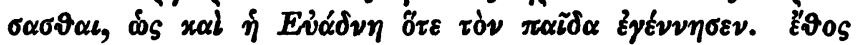

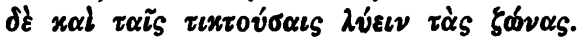

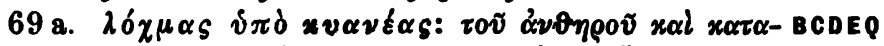

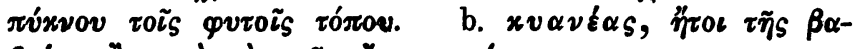

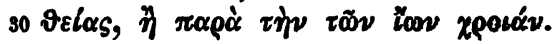

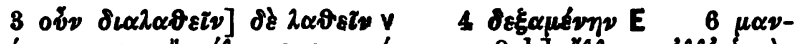

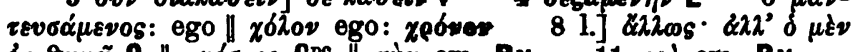

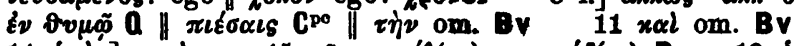

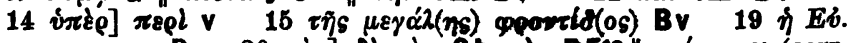

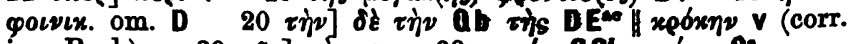

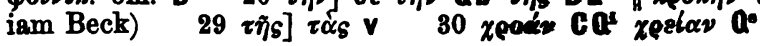




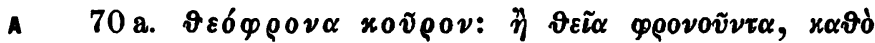

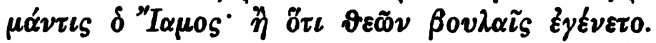

BCDEQ 70 b. $\tau l x \tau \varepsilon \vartheta \varepsilon \delta ́ \varphi \rho O \nu \alpha$ x० v๊

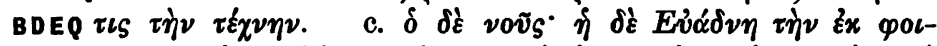

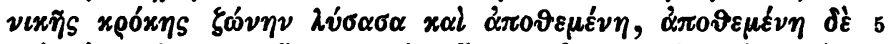

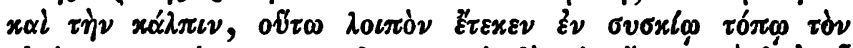

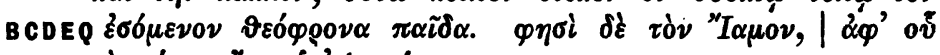

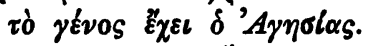

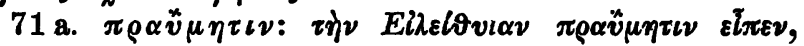

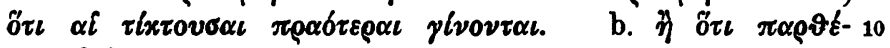

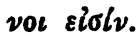

BDE 71 b. $\pi \rho \alpha \tilde{v} \mu \eta \tau l v \tau^{\prime} E i \lambda \varepsilon l \vartheta v \iota \alpha \nu: \pi \rho \alpha \varepsilon i \alpha \nu . \quad \varepsilon \dot{v} \mu \varepsilon v \eta \tilde{\eta}$.

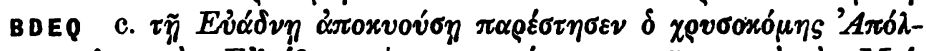

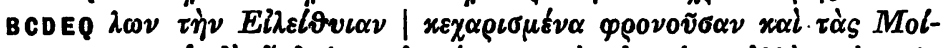

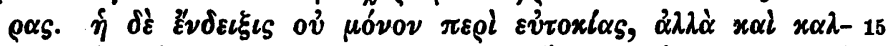

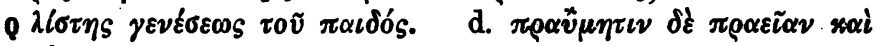
$\varepsilon \dot{v} \mu \varepsilon v \tilde{\eta}$.

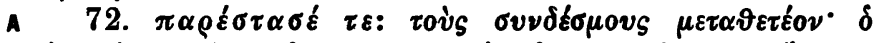

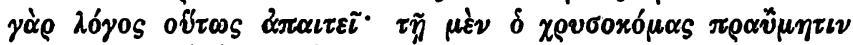

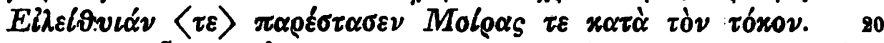

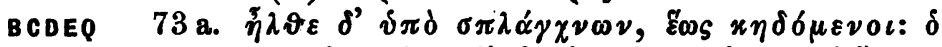

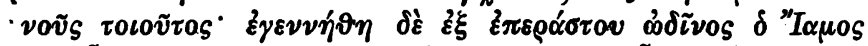

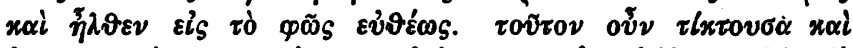

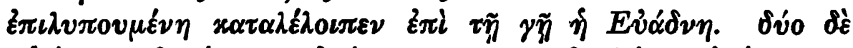

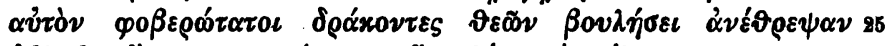

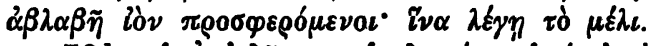

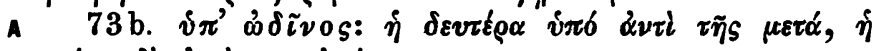

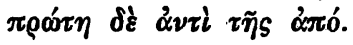

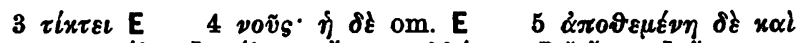

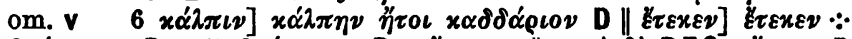

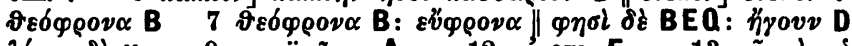

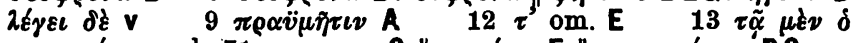

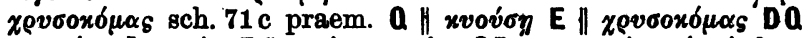

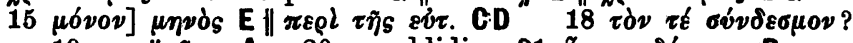

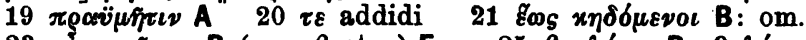

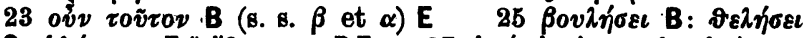

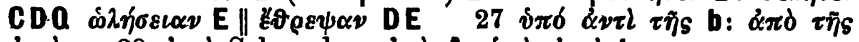

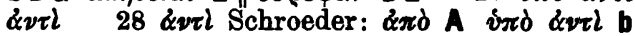




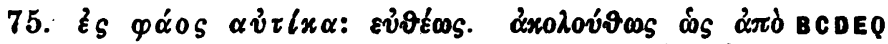

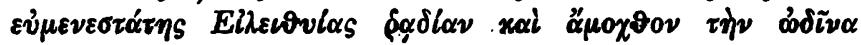

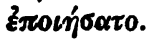

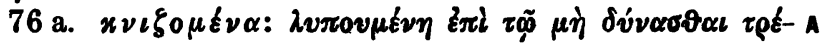

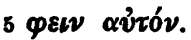

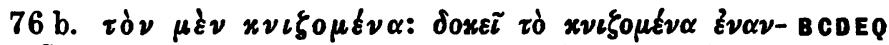

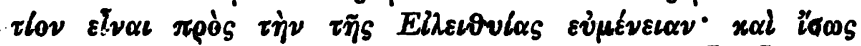

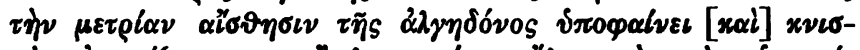

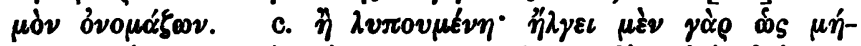

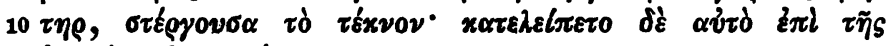

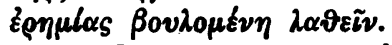

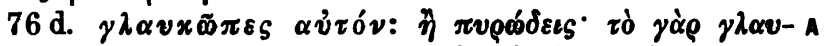

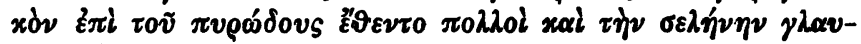

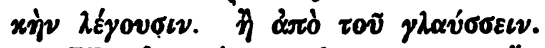

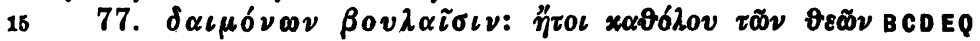

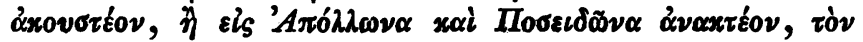

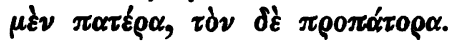

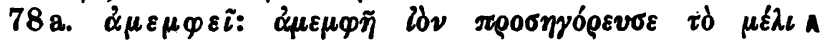

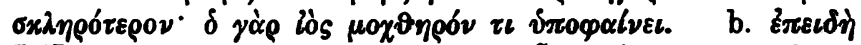

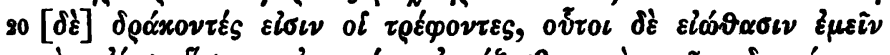

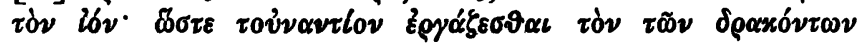

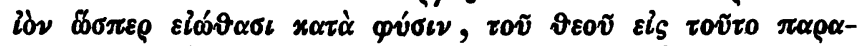

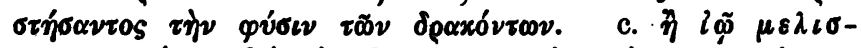

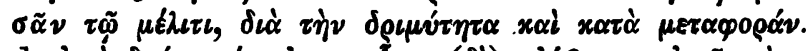

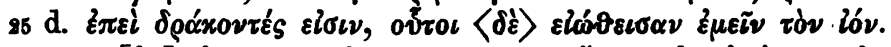

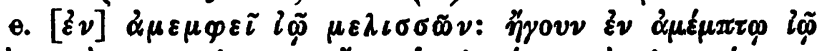

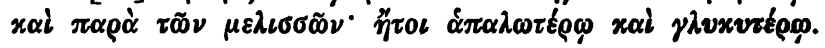

12 EM 233, $21 \quad 14$ ib. 36

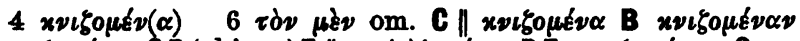

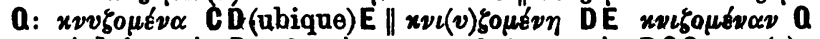

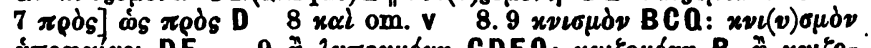

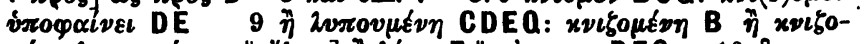

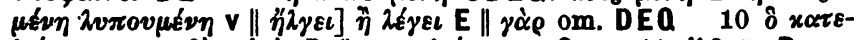

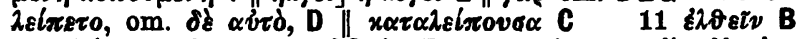

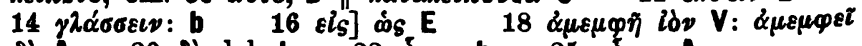

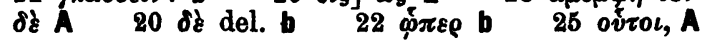




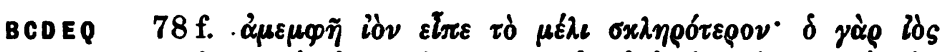

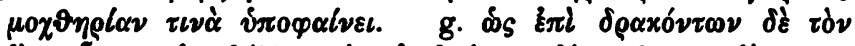

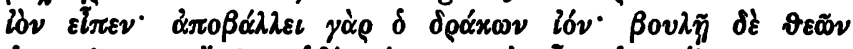

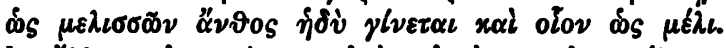

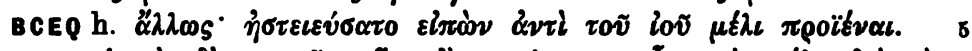

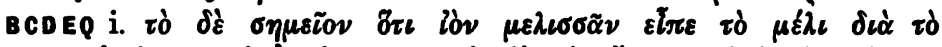

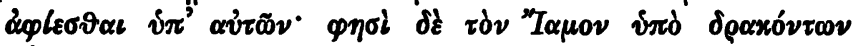

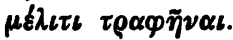

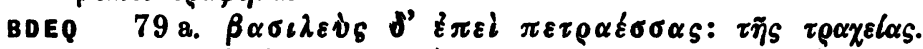

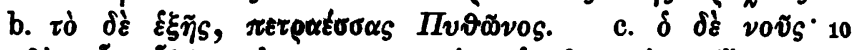

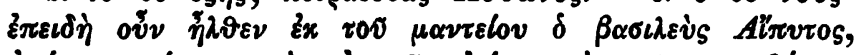

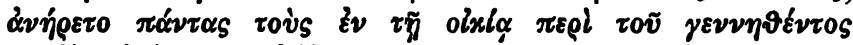

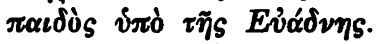

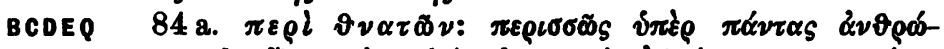

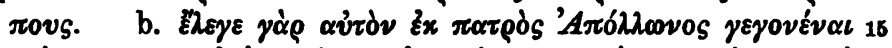

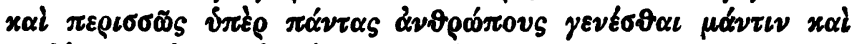

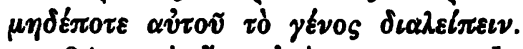

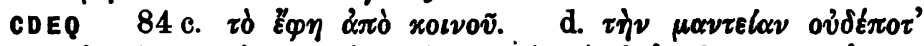

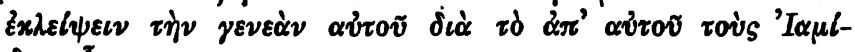

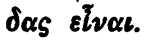

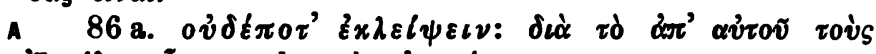

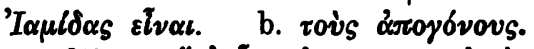

A 87 a.

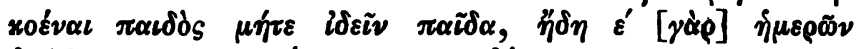

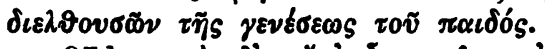

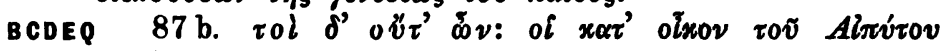

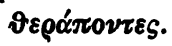

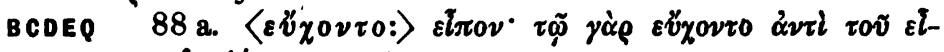

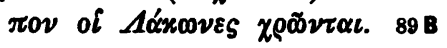

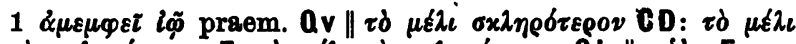

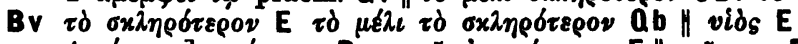

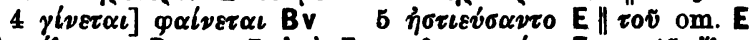

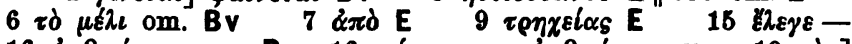

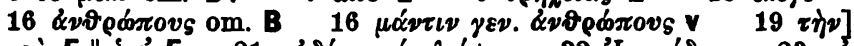

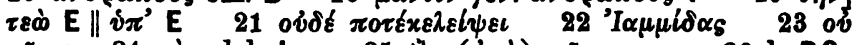

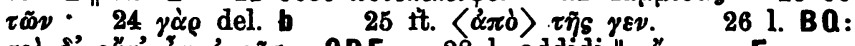

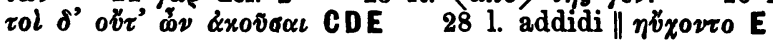




\section{Olymp. V1 78-91}

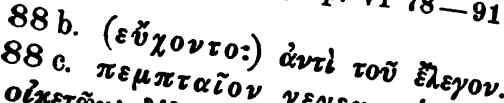

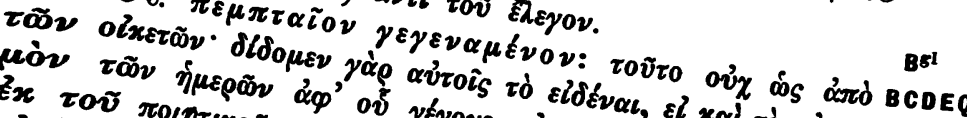

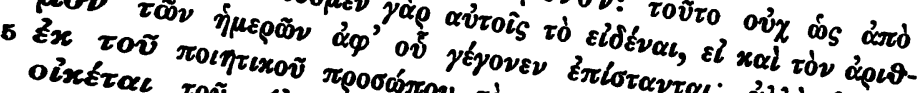

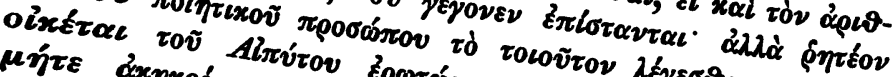

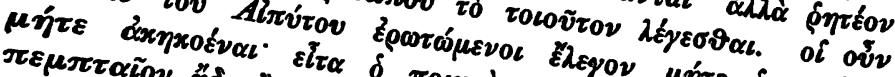

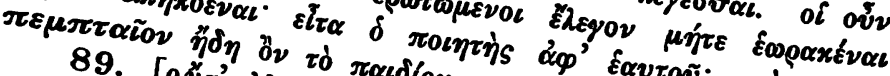

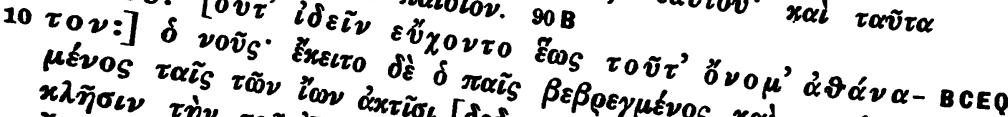

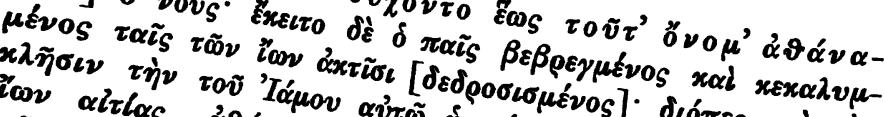

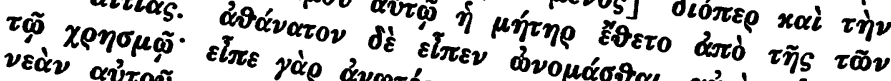

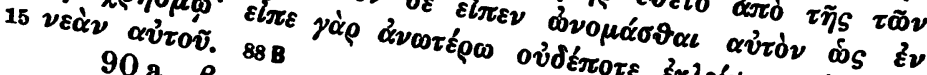

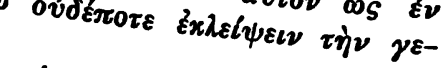

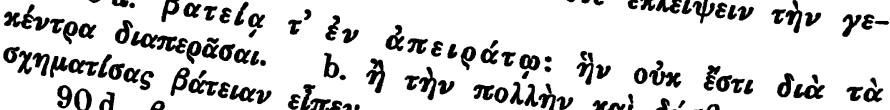

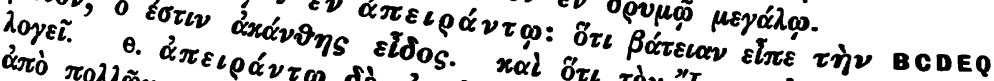

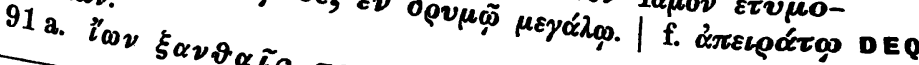

20 Hes. Bó $\tau_{0}$.

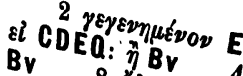

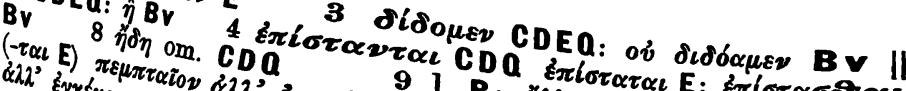

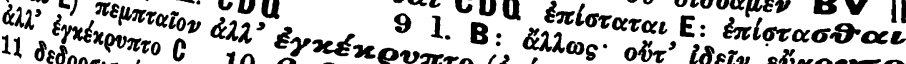

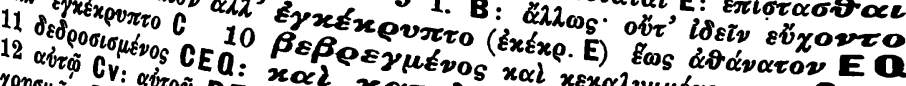

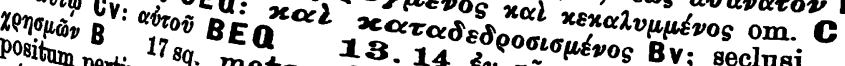

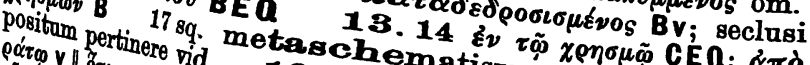

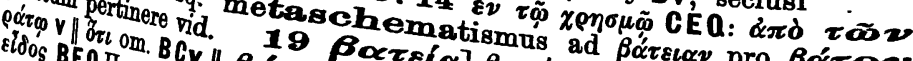

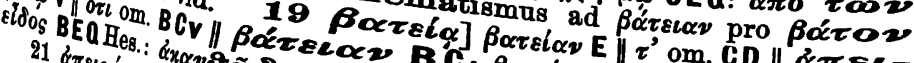

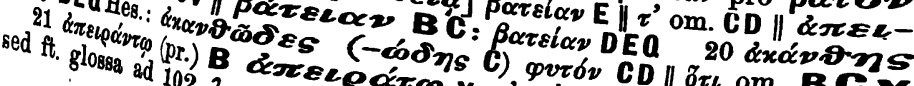

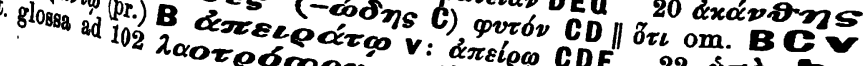
pertinet $23 \dot{\alpha} x \tau i \sigma_{\iota}$ Apo 


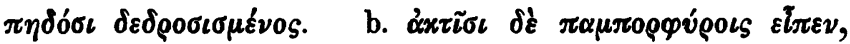

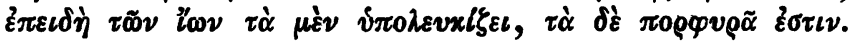

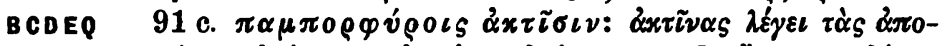

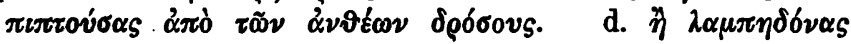

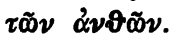

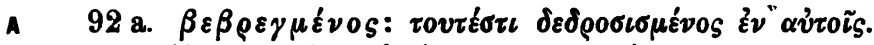

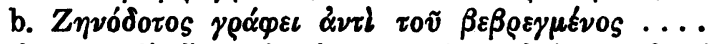

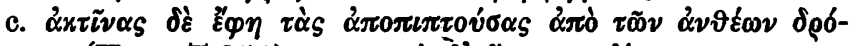

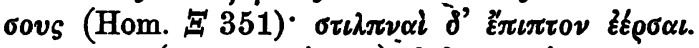

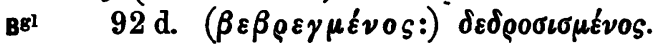

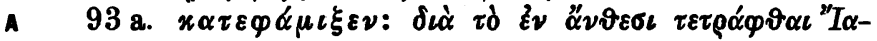

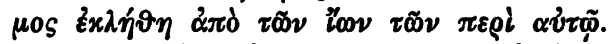

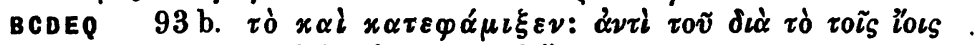

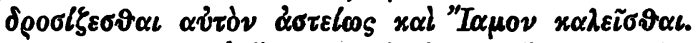

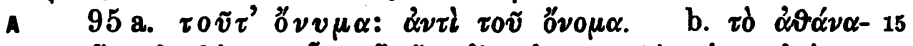

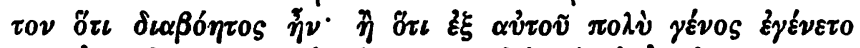

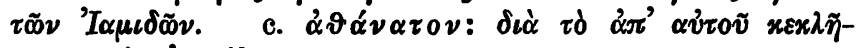

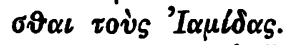

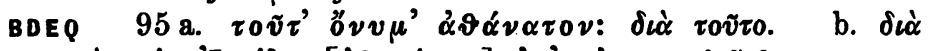

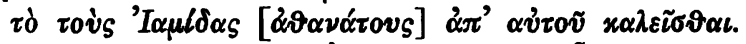

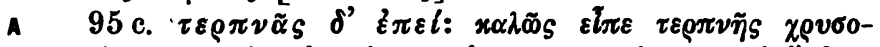

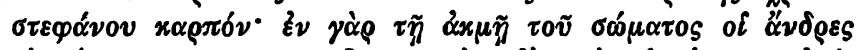

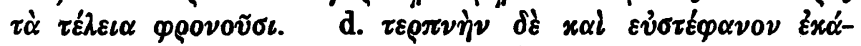

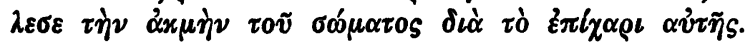

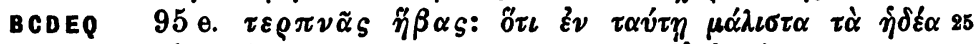

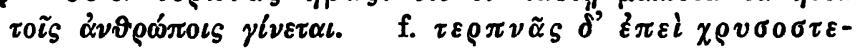

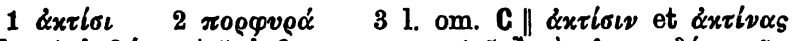
BDE $4 \dot{\alpha} \nu \vartheta \hat{\varepsilon} \omega \nu$ et $5 \dot{\alpha} \nu \vartheta \tilde{\omega} \nu \nu$ omnes $4.5 \hat{\eta} \tau \dot{\eta} \nu \lambda \alpha \mu \pi \tau \delta \dot{\alpha} \nu \alpha \tau \tilde{\omega} \nu$

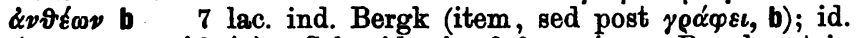
$\langle\pi \tilde{\alpha} \nu \sigma \tilde{\omega} \mu \alpha \alpha \dot{\alpha} \beta \varrho \sigma v\rangle$, Schneidewin $\beta \varepsilon \beta \rho \alpha \gamma \mu \varepsilon v 0$, Rauchenstein (N. Jhbb. 91, 656) $\pi \varepsilon \varphi \rho \alpha \gamma \mu \varepsilon \dot{v} 0$ suppl. 9 a $\pi \dot{\varepsilon} \pi i \pi \tau o \nu$ codd. Hom.

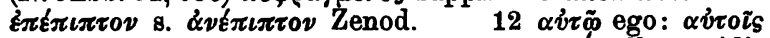

$13 x \alpha i$ om. BCV 14 post $x \alpha \lambda \varepsilon \tau \sigma \theta \alpha \iota$ nonnulla excidisse vid.

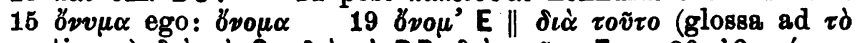

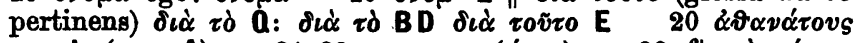

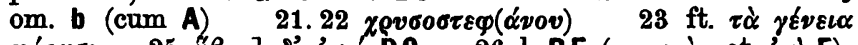

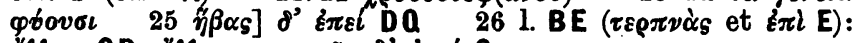

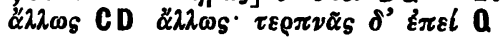




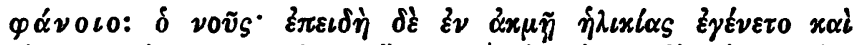

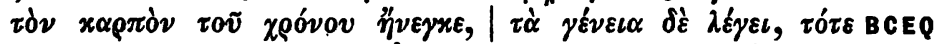

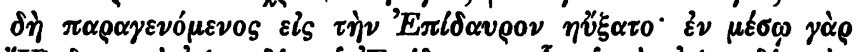

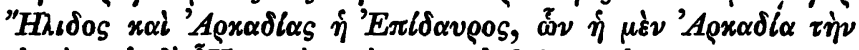

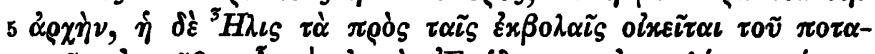

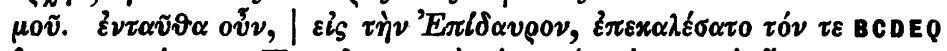

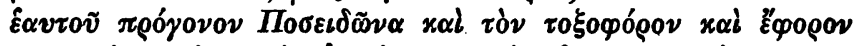

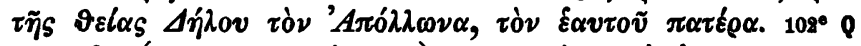

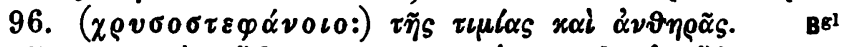

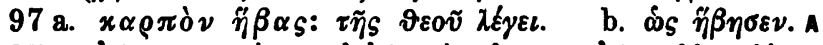

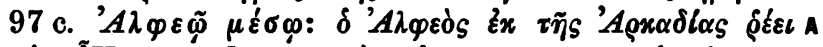

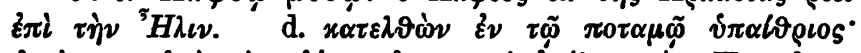

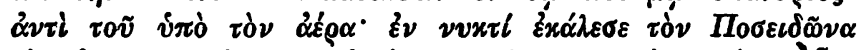

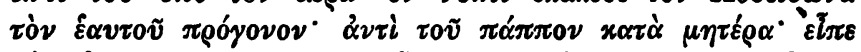

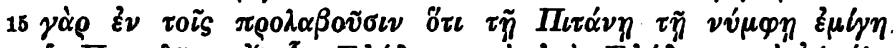

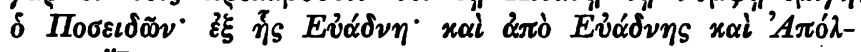

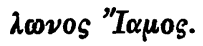

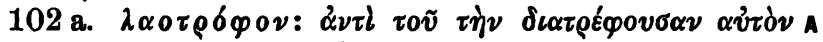

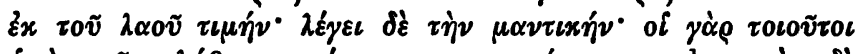

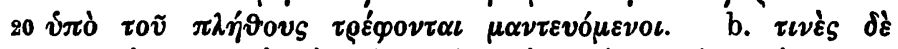

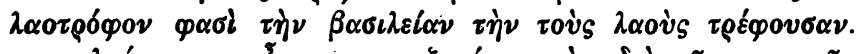

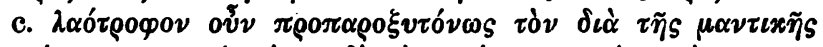

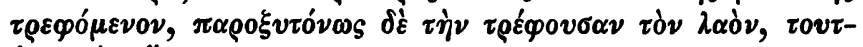

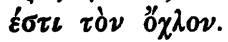

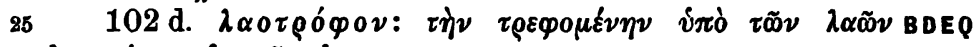

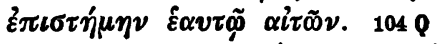

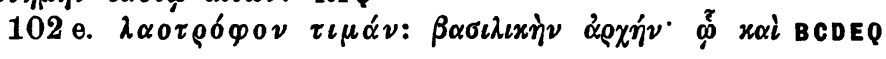

13 Hes. $v \pi \alpha$ io

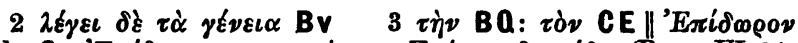

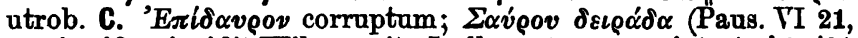

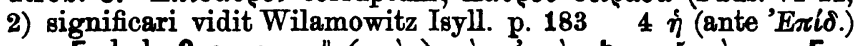

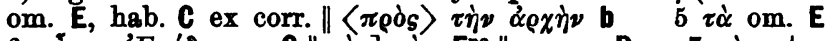

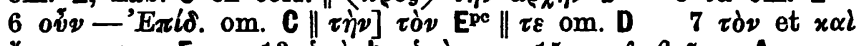

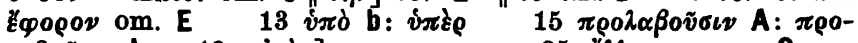

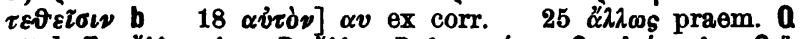

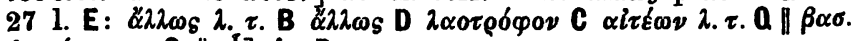

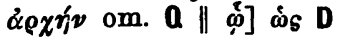




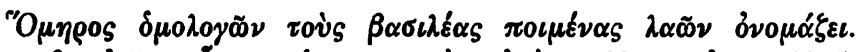

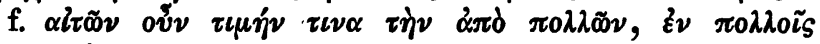

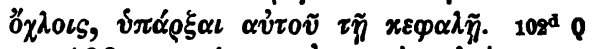

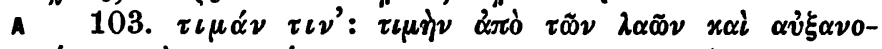

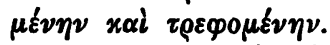

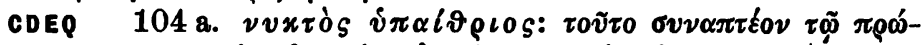

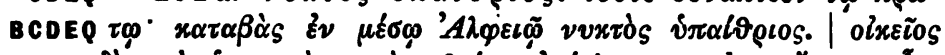

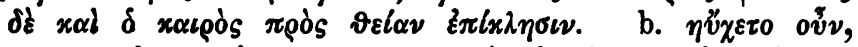

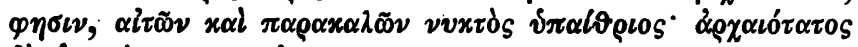

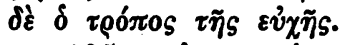

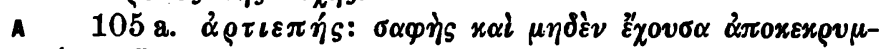

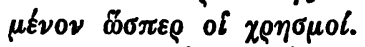

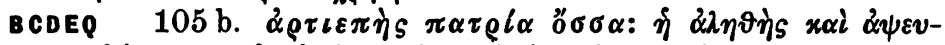

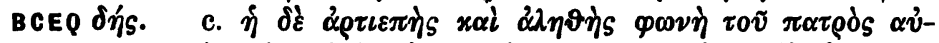

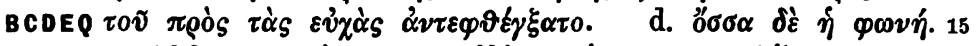

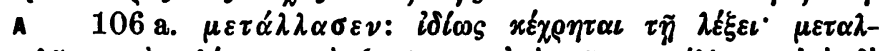

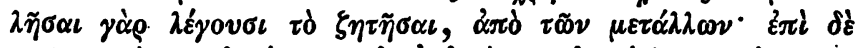

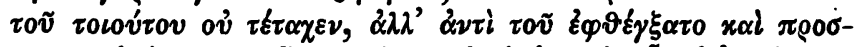

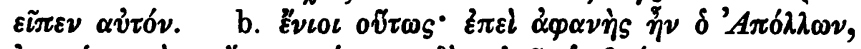

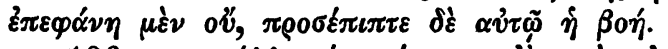

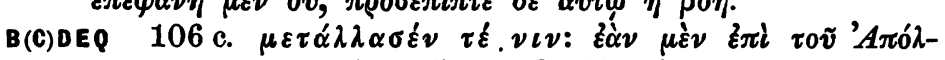

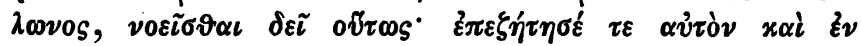

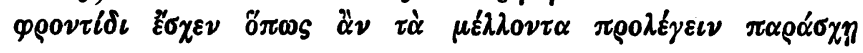

16 sch. $\alpha$ 231. EM 580, 53. Zon. 1348

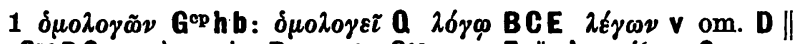

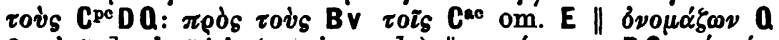

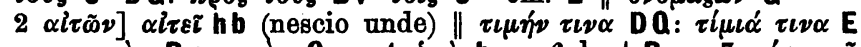
$\tau \iota \nu \alpha \tau u \mu \eta \eta \nu$ BV $\tau \iota \mu \eta \nu$ C 4 is b 6 l. et B $7 \mu \varepsilon \delta \varphi \tau \tilde{\varphi}$

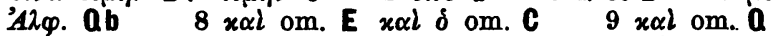

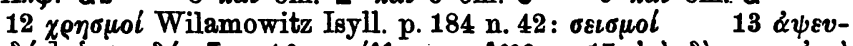

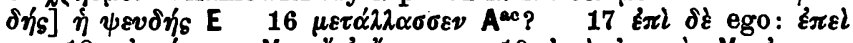

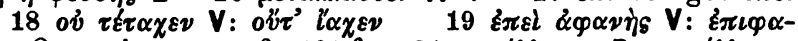
vìs. Q. s. vix sana; cfr. $106 \mathrm{f} 21 \mu \varepsilon \tau \alpha^{\prime} \lambda \lambda \alpha \sigma \varepsilon \nu$ D: $\mu \varepsilon \tau \alpha^{\prime} \lambda \lambda \alpha \sigma \sigma \varepsilon$ BE $\mu \varepsilon \tau \dot{\alpha} \lambda \lambda \alpha \sigma \varepsilon$ Q $\mu \varepsilon \tau \alpha \lambda \alpha^{\prime} \sigma \sigma \varepsilon$ C \| $\nu(\nu$ B: $\mu \iota \nu$ \| post $\mu \iota \nu$ lac.

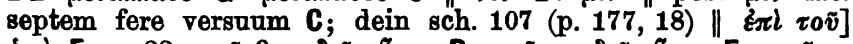

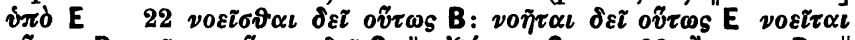

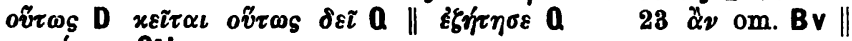
$\pi \alpha \rho \alpha^{\prime} \sigma \chi 0 \iota \mathbf{Q}^{\circ} \mathrm{b}$ 


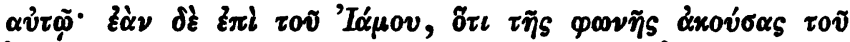

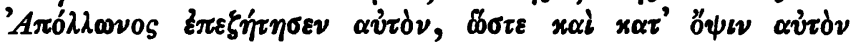

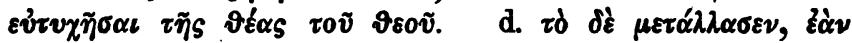

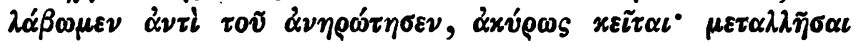
8 yó

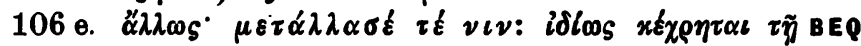

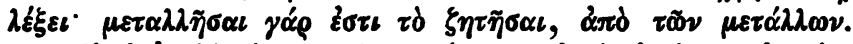

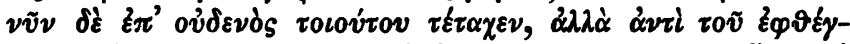

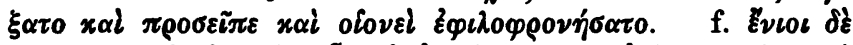

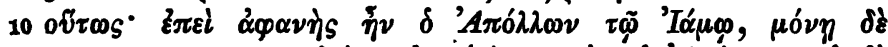

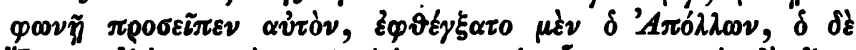

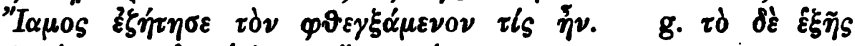

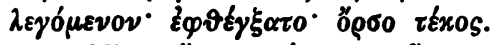

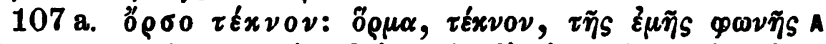

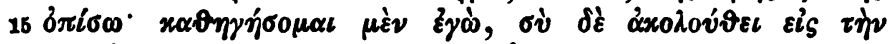

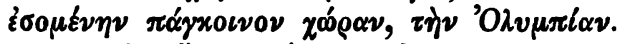

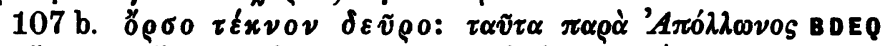

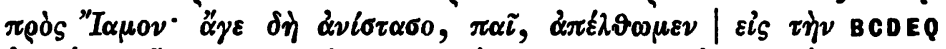

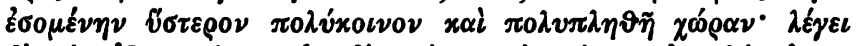

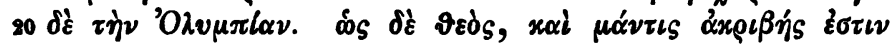

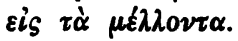

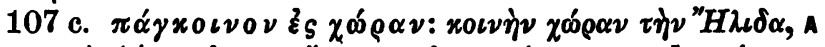

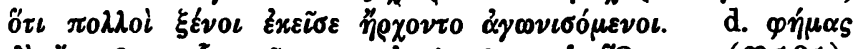

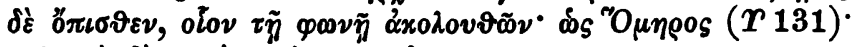

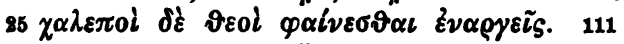

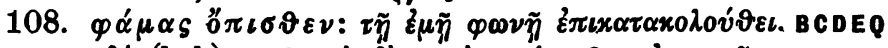

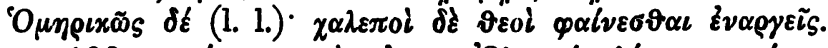

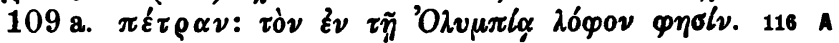

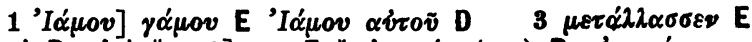

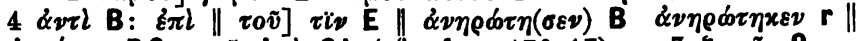

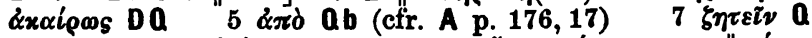

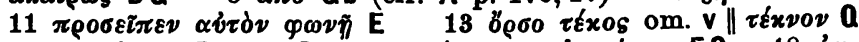

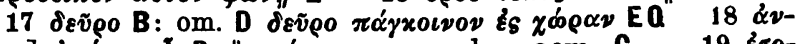

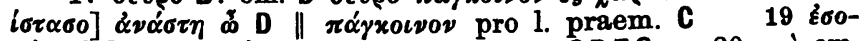

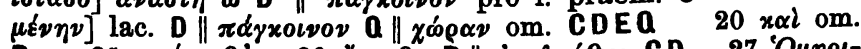

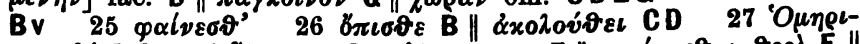

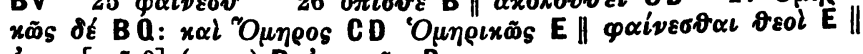

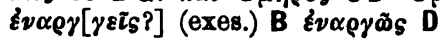

Schor. mi Pardagy ed. Drachmann. 


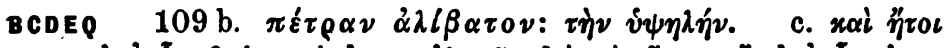

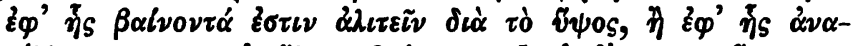

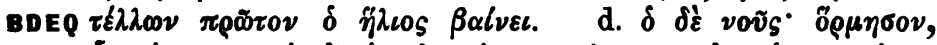

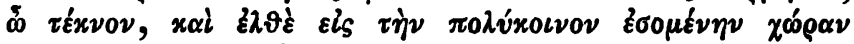

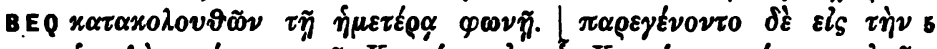

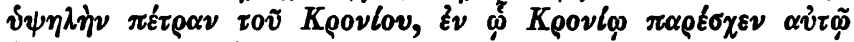

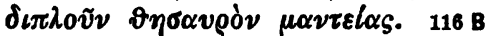

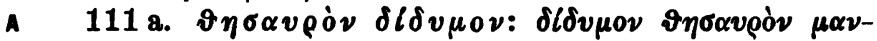

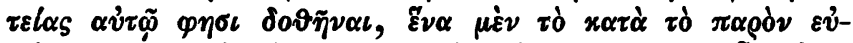

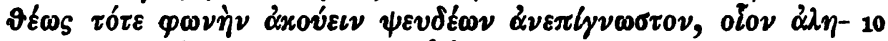

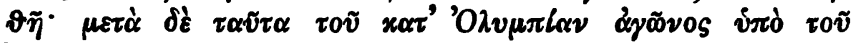

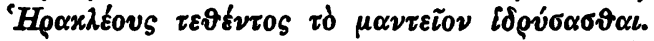

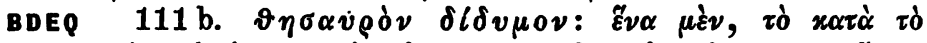

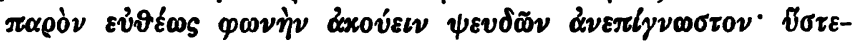

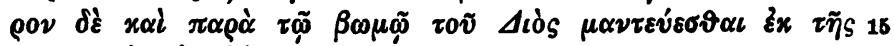

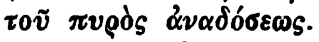

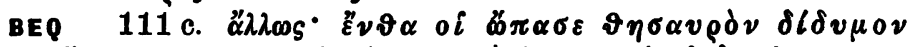

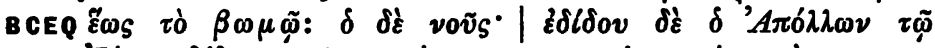

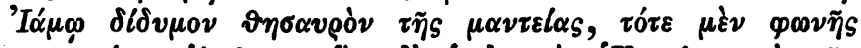

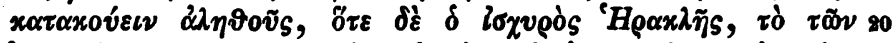

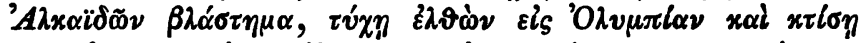

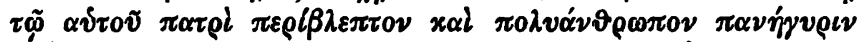

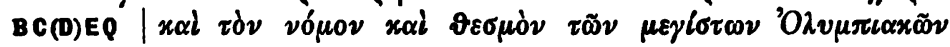

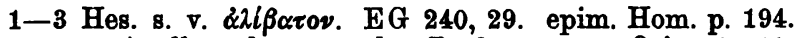
EM 427, 39. Apoll. soph. 83, 25. lex. Bachm. 250, 4. Orion 67, 10. Zonaras p. 986. (Sch. Eur. Hipp. 732 Schw.)

1 ทैं

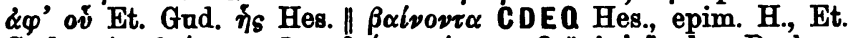

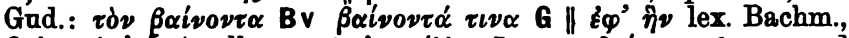

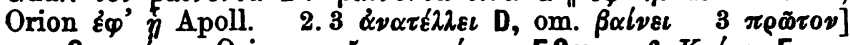

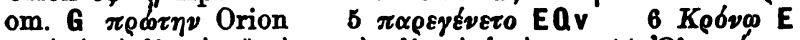

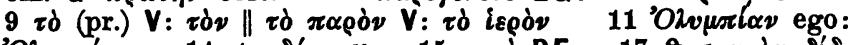

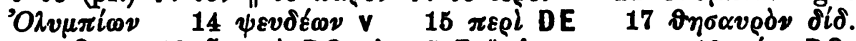

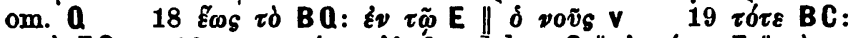

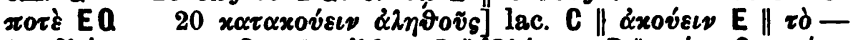

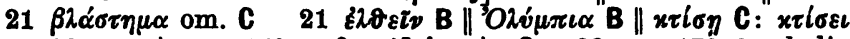

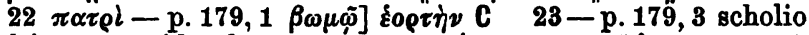
$111^{\mathrm{d}}$ inser. D; vid. ad 179,4 $23 \mu \varepsilon y i \sigma \tau \omega \nu$ om. D || OAv 


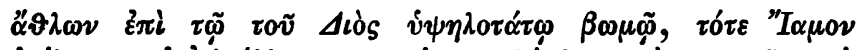

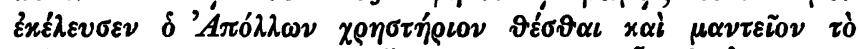

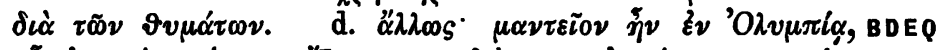

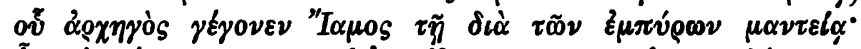

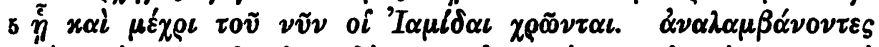

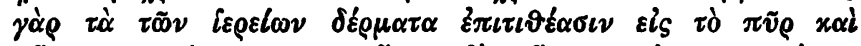

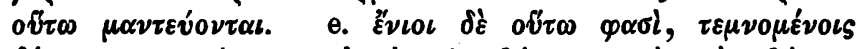

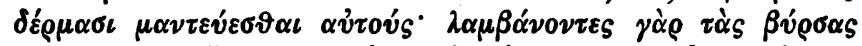

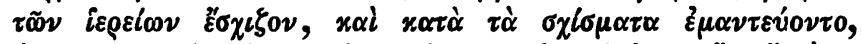

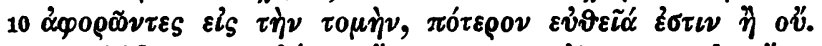

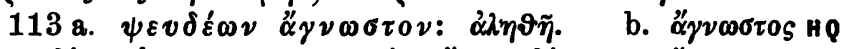

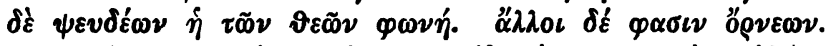

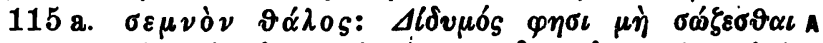

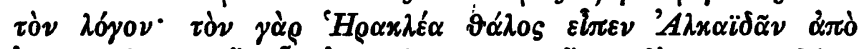

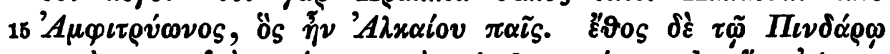

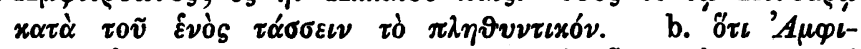

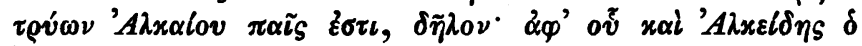

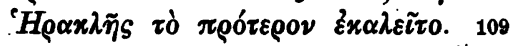

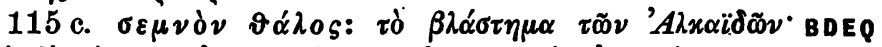

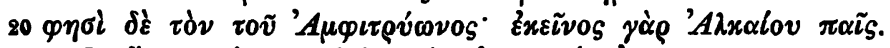

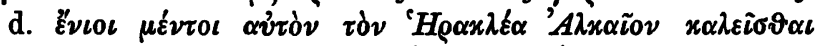

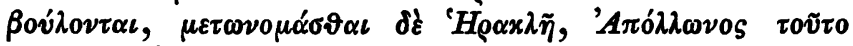
$\chi \varrho \eta \sigma \mu о \delta 0 \tau \eta \dot{\sigma} \sigma \nu \tau \sigma \mathrm{s} .120 \mathrm{~B}$

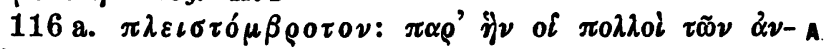

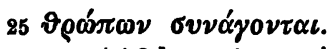

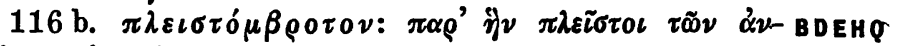

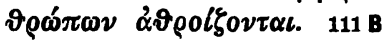

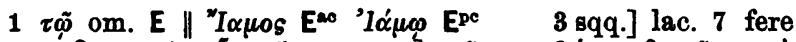

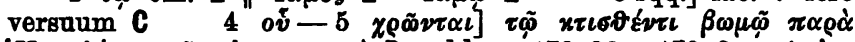

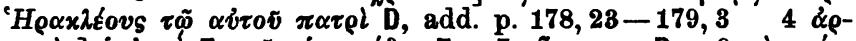

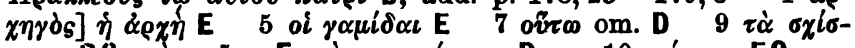

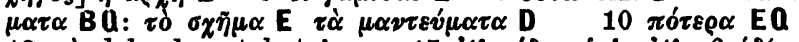

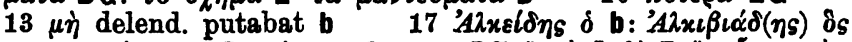

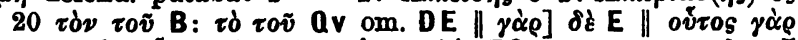

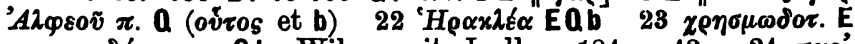

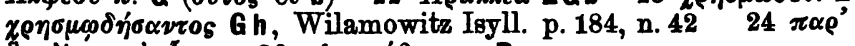

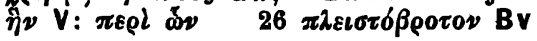




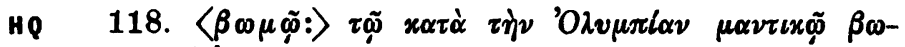

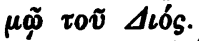

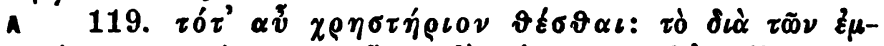

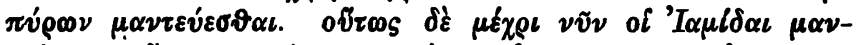

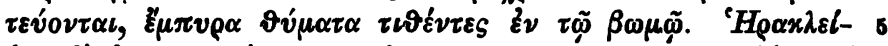

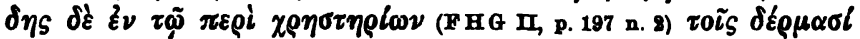

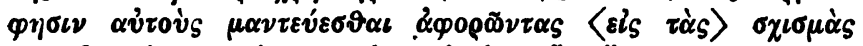

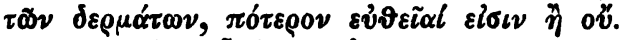

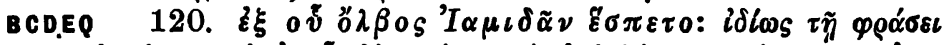

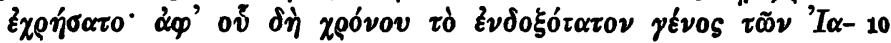

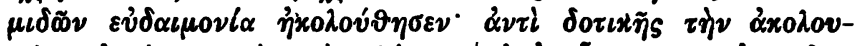

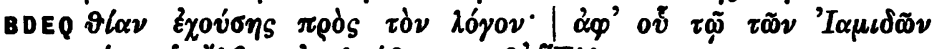

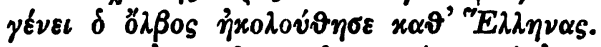

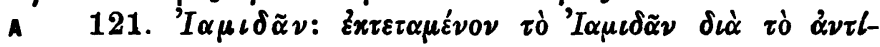

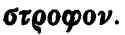

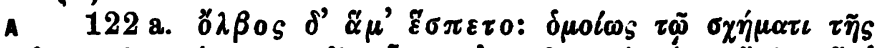

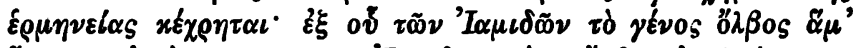

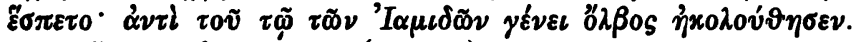

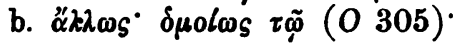

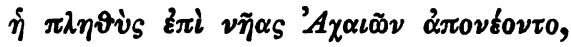

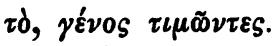

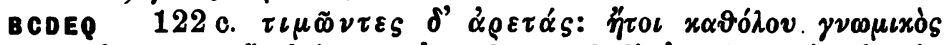

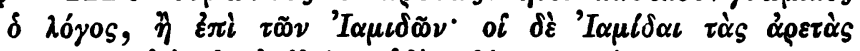

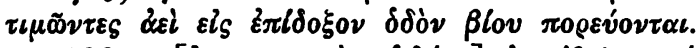

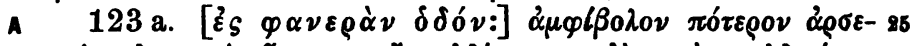

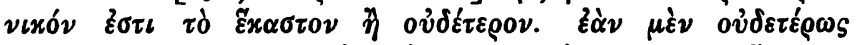

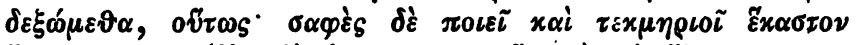
है

1 1.] Z Z

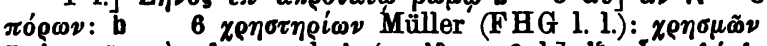

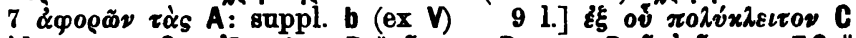

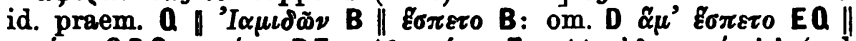

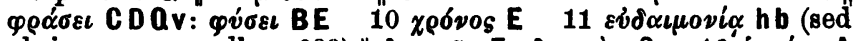

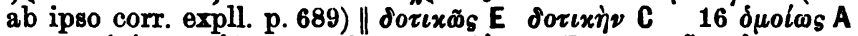

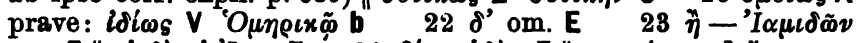

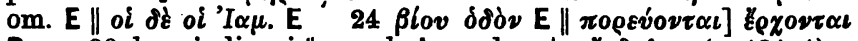

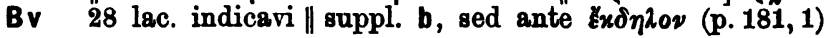




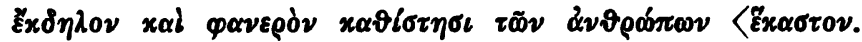

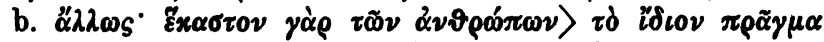

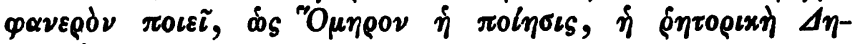
$\mu 0 \sigma \theta \varepsilon v \eta v$.

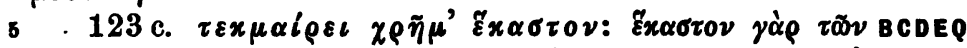

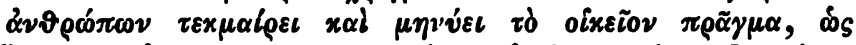

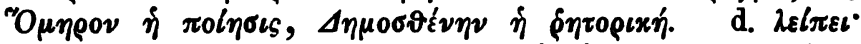

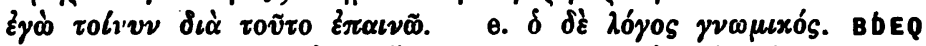

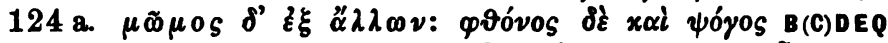

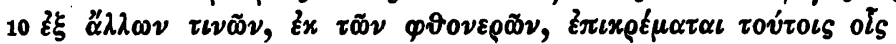

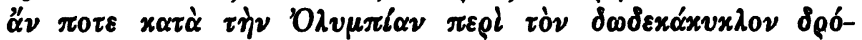

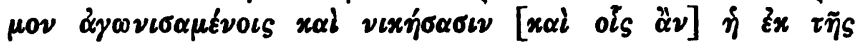

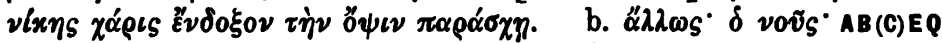

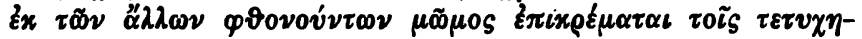

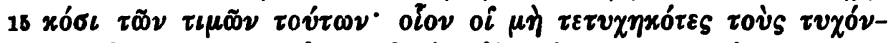

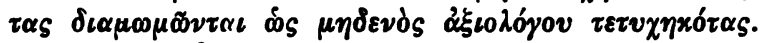

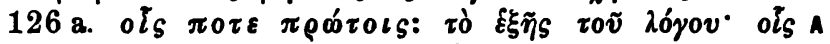

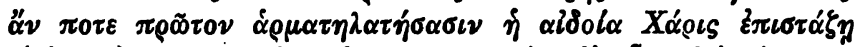

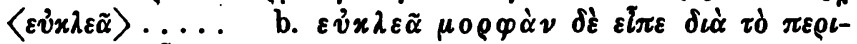

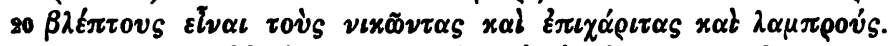

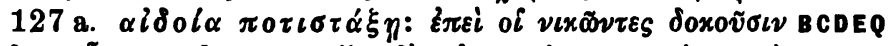

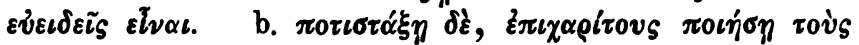
$\nu เ x \tilde{\omega} \nu \tau \alpha \varsigma$.

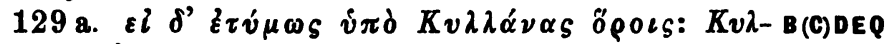

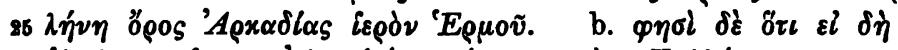

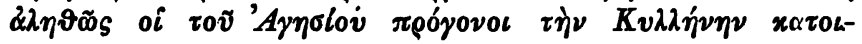

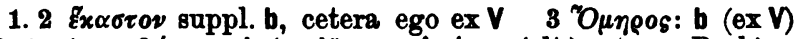

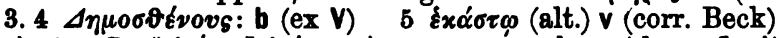
$7 \times \alpha \iota \Delta \eta \mu$. BV $\| \lambda \varepsilon i \pi \varepsilon l] \lambda \varepsilon i \pi \varepsilon \iota \tau \dot{v} \mathrm{~V} 9$ sqq. sch. a et b conflavit C

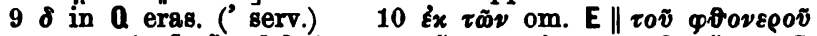

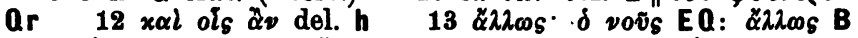

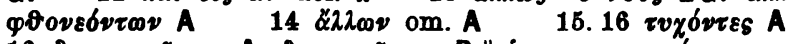

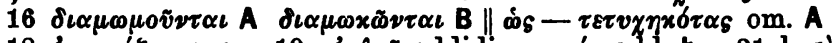

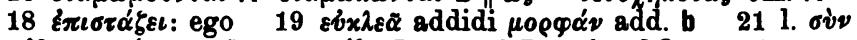

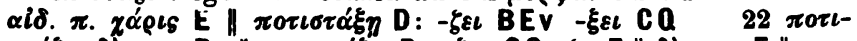

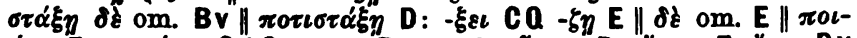

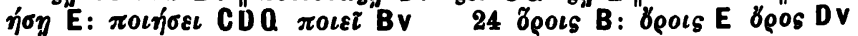
om. CQ 25 boos om. E 


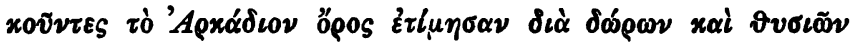

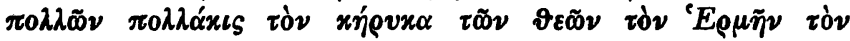

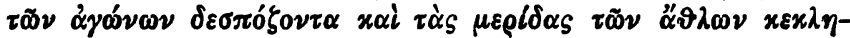

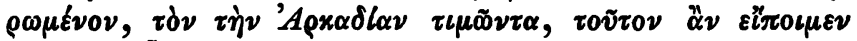

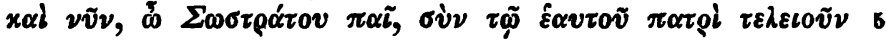

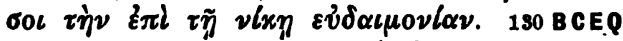

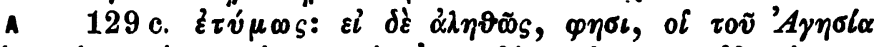

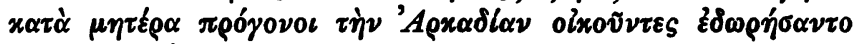
$\vartheta v \sigma l \alpha s \tau \tilde{\omega}{ }^{`} E \rho \mu \tilde{\eta}$.

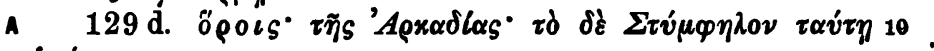

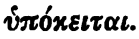

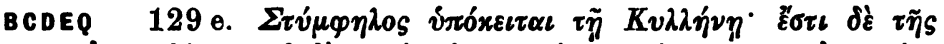

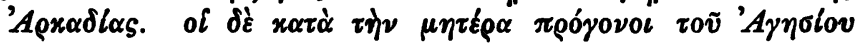

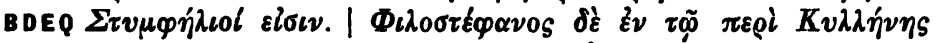

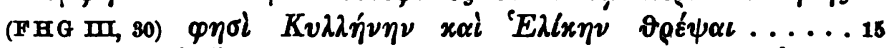

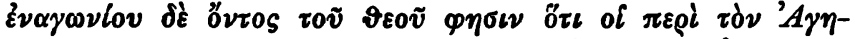

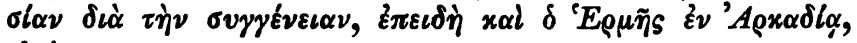

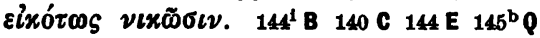

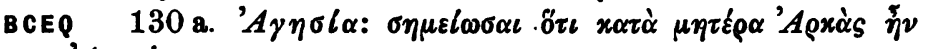
'A

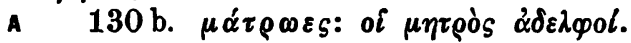

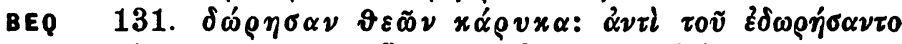

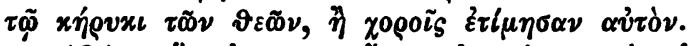

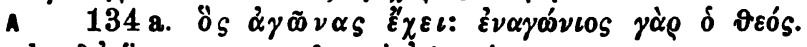

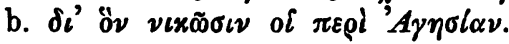

15 Serv. ad Aen. IV $252 \quad 21$ sch. B $\Pi 717$.

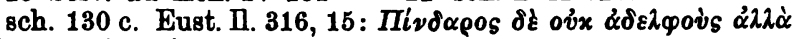

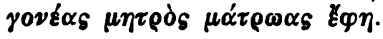

1 'A

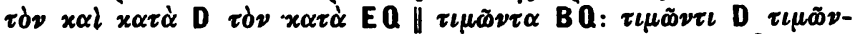

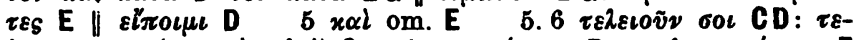

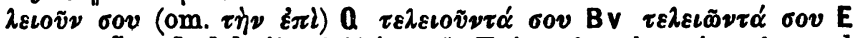

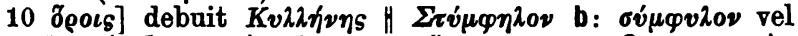

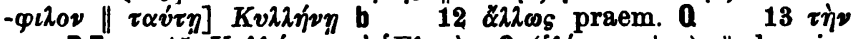

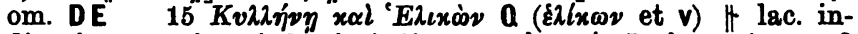

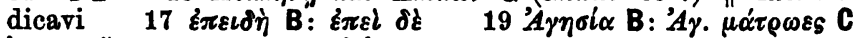

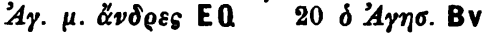




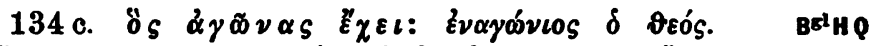

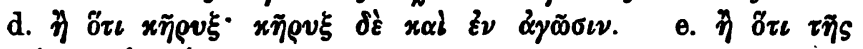

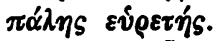

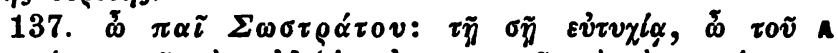

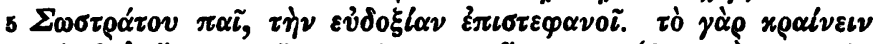

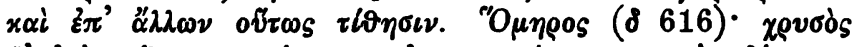

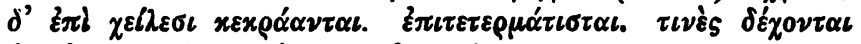

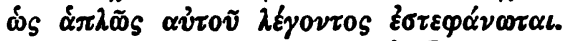

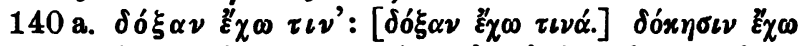

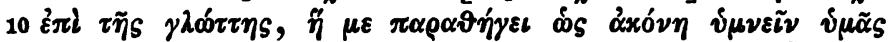

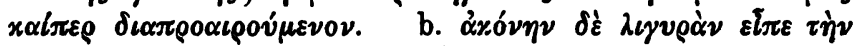

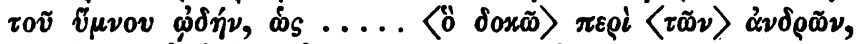

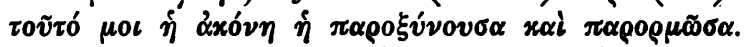

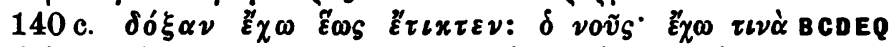

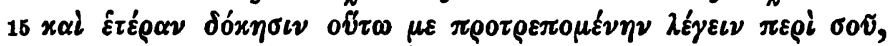

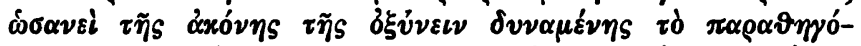

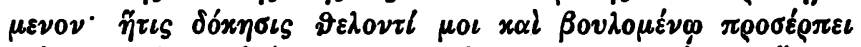

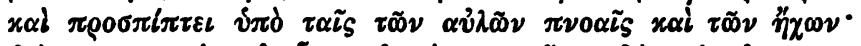

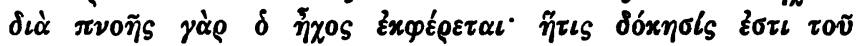

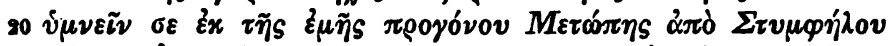

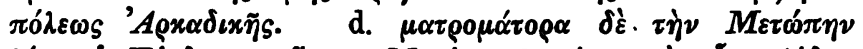

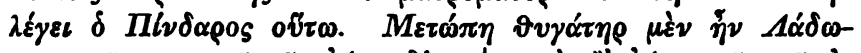

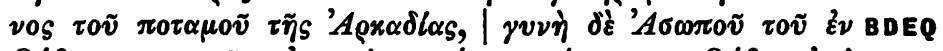

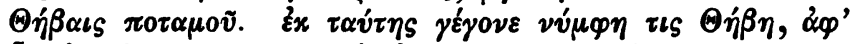

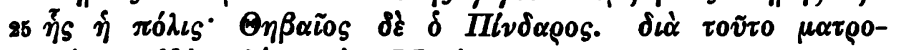

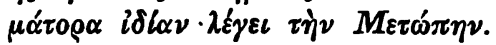

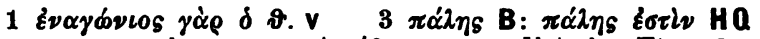

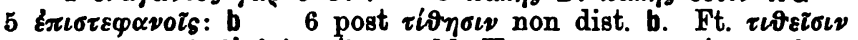

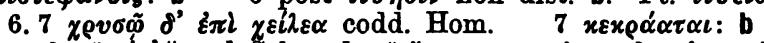

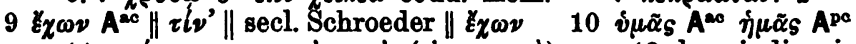

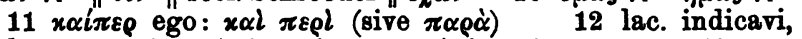

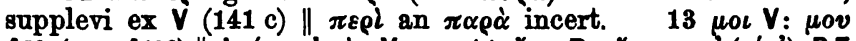

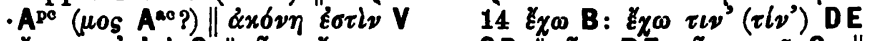

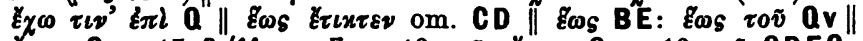

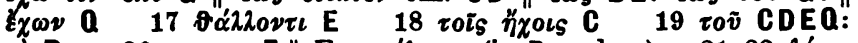

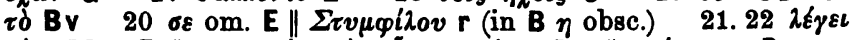

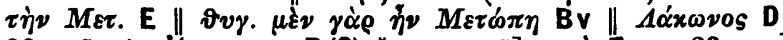

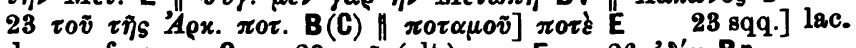
decem fere vr. C 23 rov (alt.) om. E 26 idía Br 


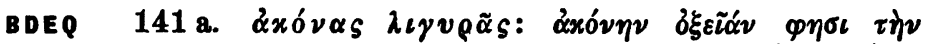

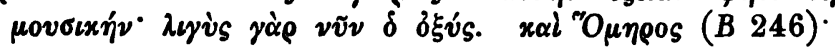

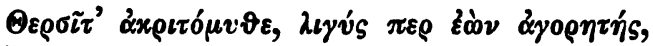
iб $x \varepsilon 0$.

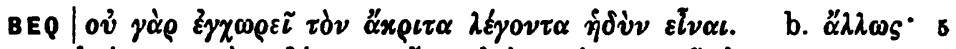

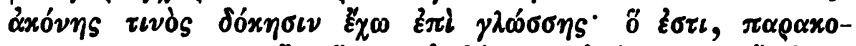

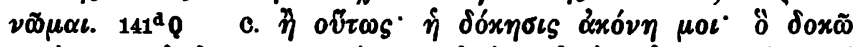

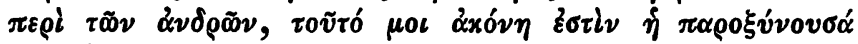

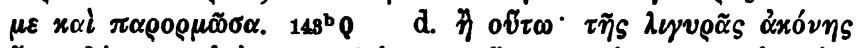

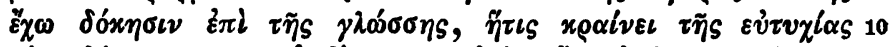

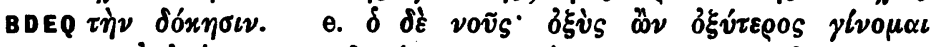

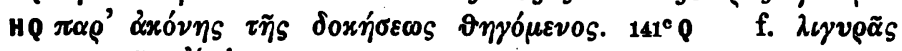
$\nu \tilde{v} \nu$ iñs $\partial \xi \varepsilon l \alpha \varsigma$.

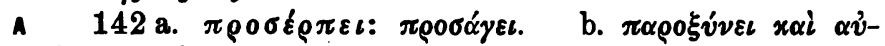

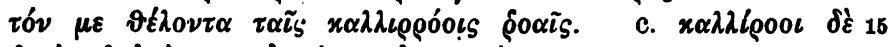

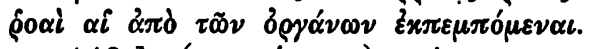

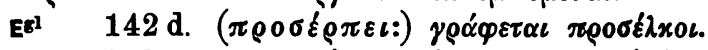

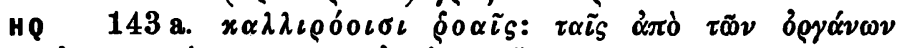

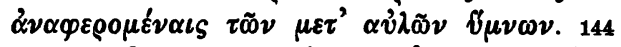

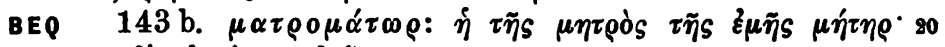

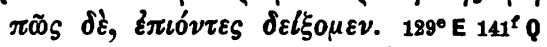

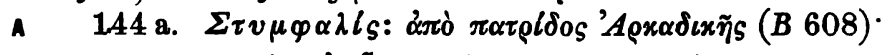

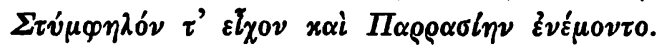

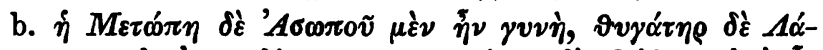

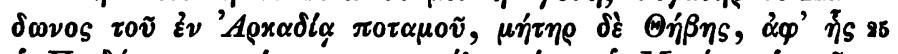

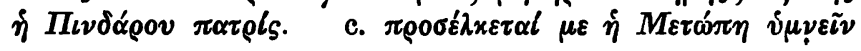

2 Eust. Il. 213, 35. Hes. EM 564, 55. EG 369, 54

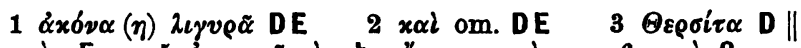

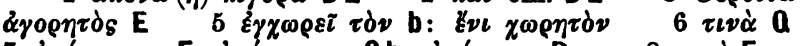

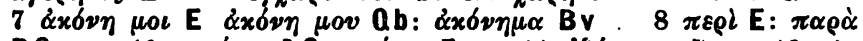

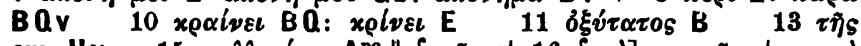

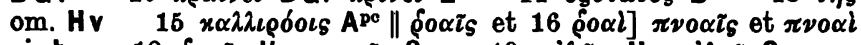

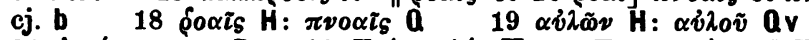

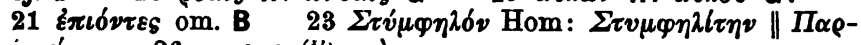

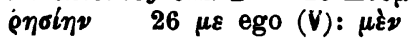




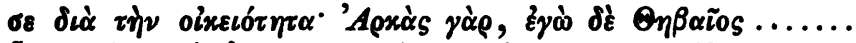

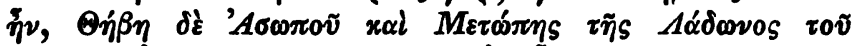

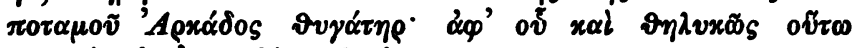

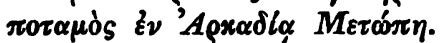

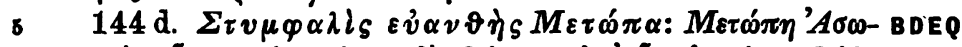

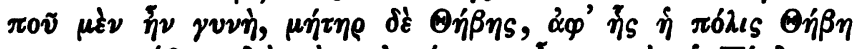

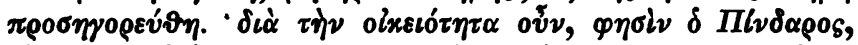

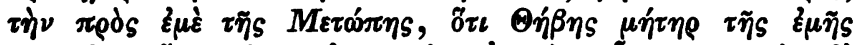

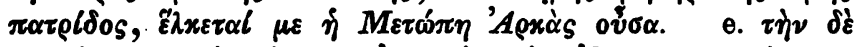

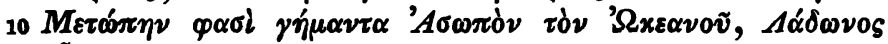

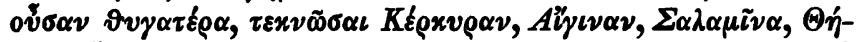

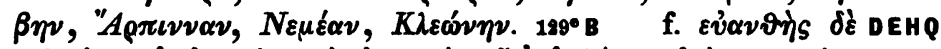

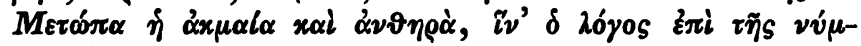
$\varphi \eta S \alpha^{2} x 0 v i \eta \alpha \iota$. $145^{\mathrm{b}} \mathrm{E} 129^{\circ} \mathrm{O}$

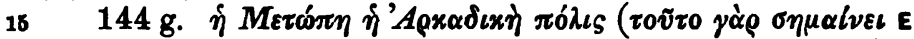

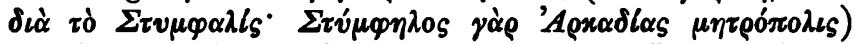

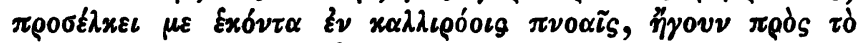

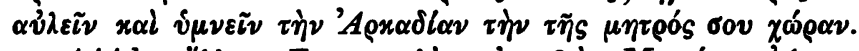

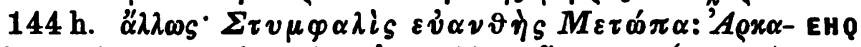

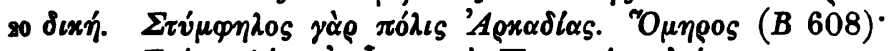

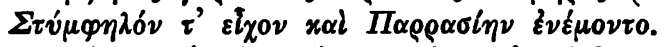

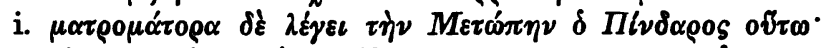

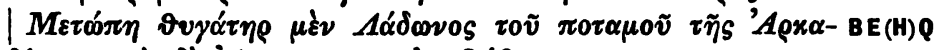

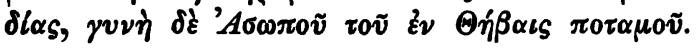

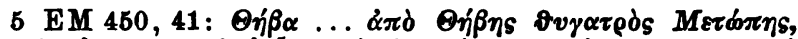

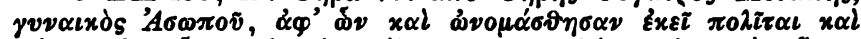

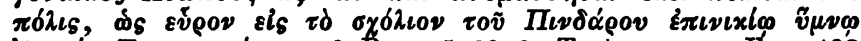
'Ay

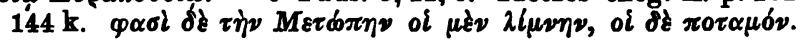
Thom. 232, 5

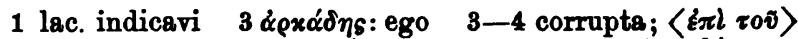

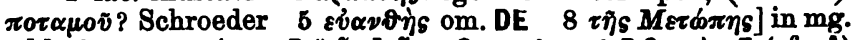

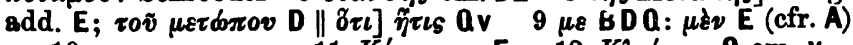

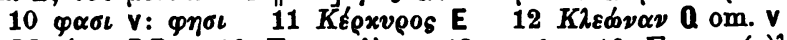

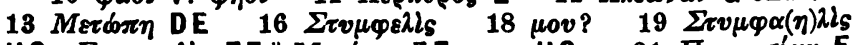

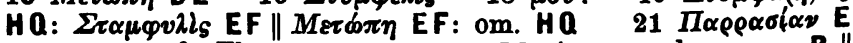

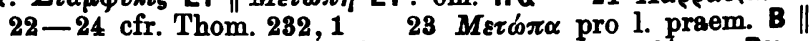

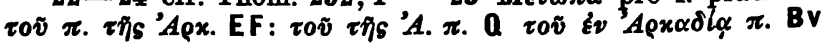




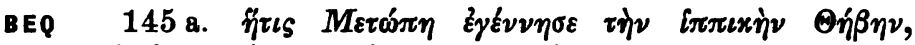

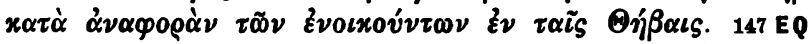

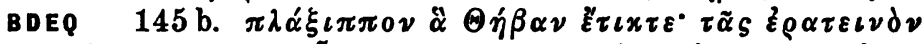

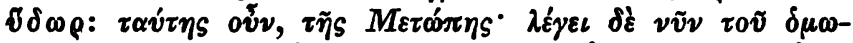

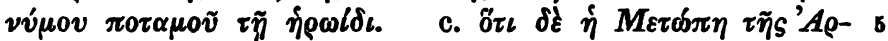

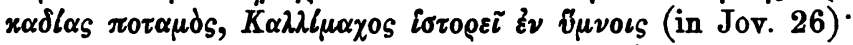

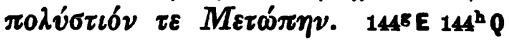

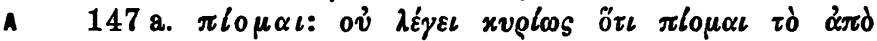

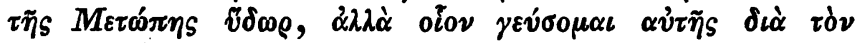

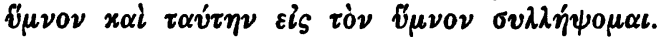

BDEQ $147 \mathrm{~b}$. $\pi l 0 \mu \alpha \iota \dot{\alpha} \nu \delta \rho \alpha ́ \sigma \iota \nu \alpha i \chi \mu \alpha \tau \alpha \tilde{\sigma} \sigma \iota \nu \tilde{\varepsilon} \omega \varsigma \tau 0 \tilde{v} \forall \mu-$

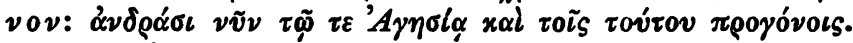

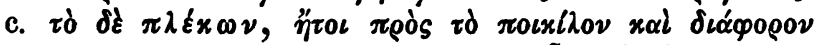

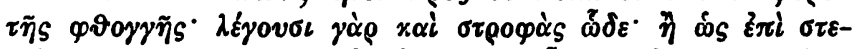

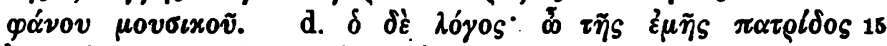

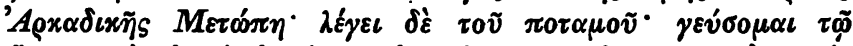

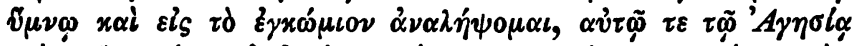

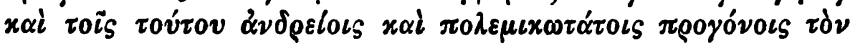

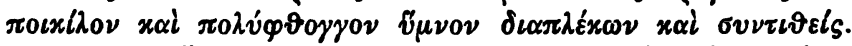
BCDEQ $148 \mathrm{a}$. ő

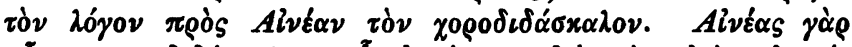

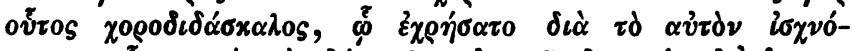

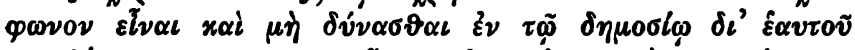

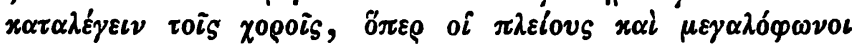

1 sq. cum is quae praecedunt conj. BE $2 \tau \alpha \tau_{S}$ om.

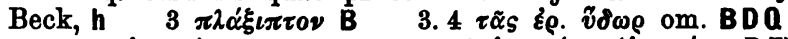

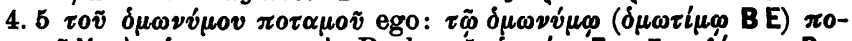

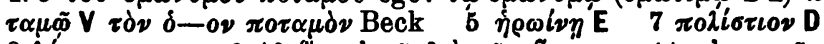

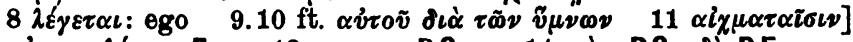

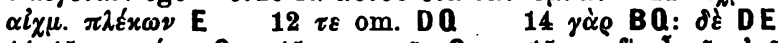

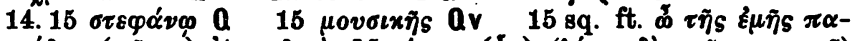

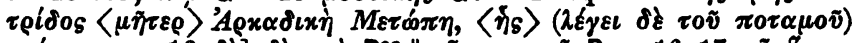

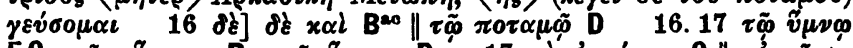

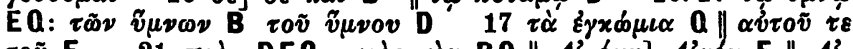

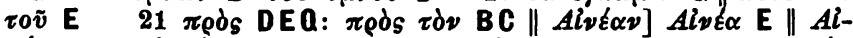

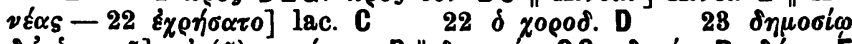

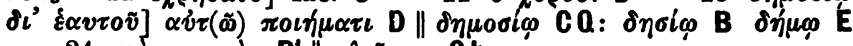

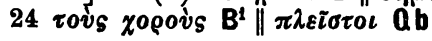




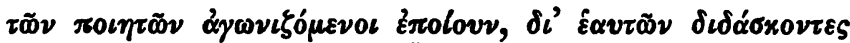

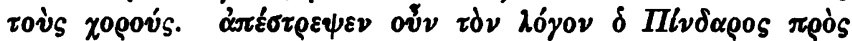

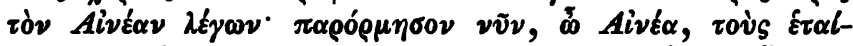

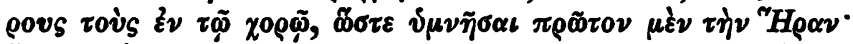

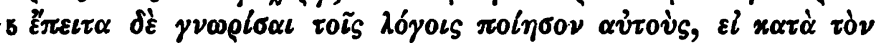

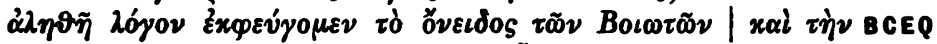

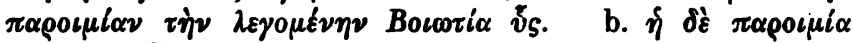

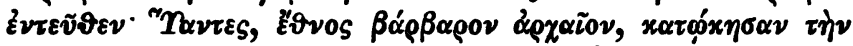

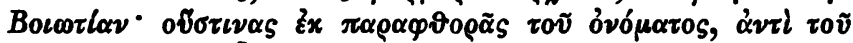

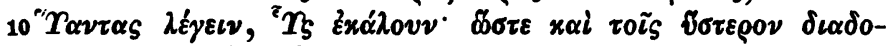

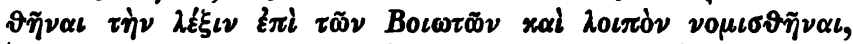

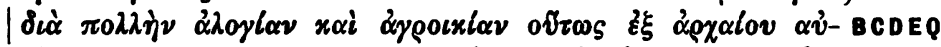

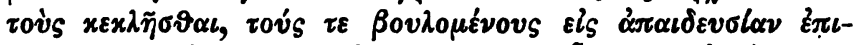

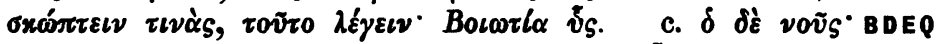

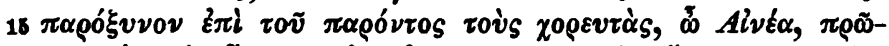

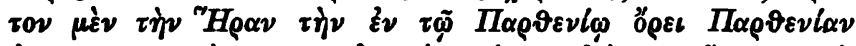

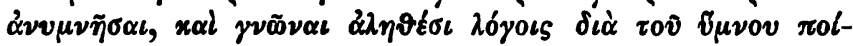

8 sch. Ap. Rh. 3, 1242. - Apost. 5, 11. Tzetzes ad Lyc. 433:

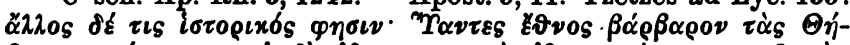

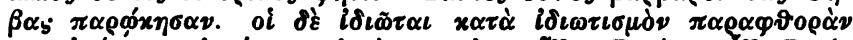

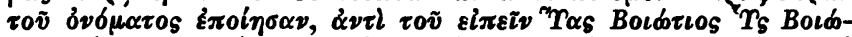

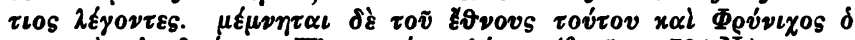

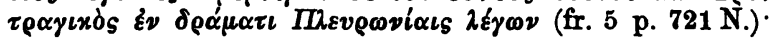

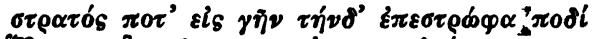

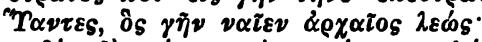

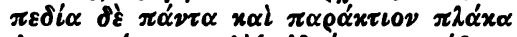

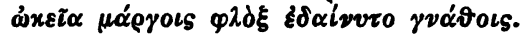

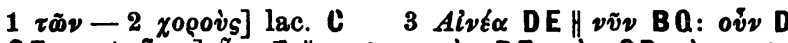
om. CE $4 \tilde{\omega} \sigma \tau \varepsilon] \tilde{o} \tau \varepsilon$ E $\| \pi \rho \tilde{\omega} \tau 0 \nu \mu \dot{\nu} \nu \mathrm{BE}: \mu \dot{\nu} \mathrm{CD} \mu \dot{\varepsilon} \nu \pi \rho \tilde{\omega}-$

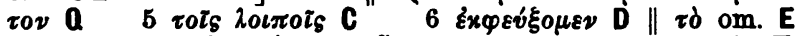

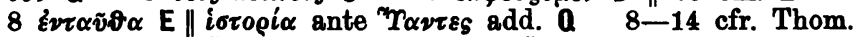

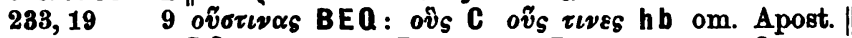
$\pi \alpha \rho \alpha \varphi \vartheta 0 \rho \tilde{\alpha} S$ BC: $\pi \alpha \rho \alpha \omega \rho \tilde{\alpha} S_{S} \mathrm{E}^{\alpha c}-\varphi \circ \rho \tilde{\alpha}_{S} \mathrm{E}^{\mathrm{pc}} \pi \varepsilon \rho \iota \varphi \circ \rho \tilde{\alpha}_{S}$ Q $\mu \varepsilon \tau \alpha-$

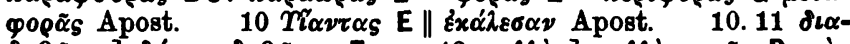

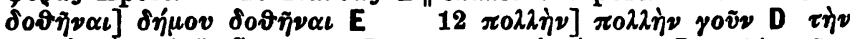

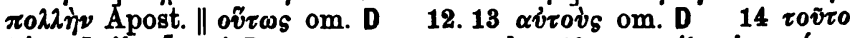

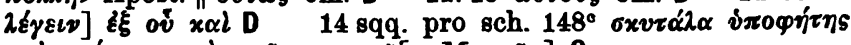

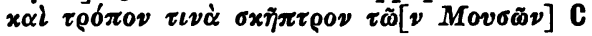




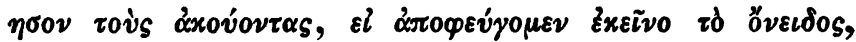

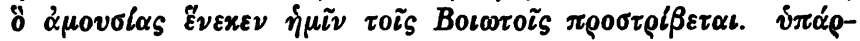

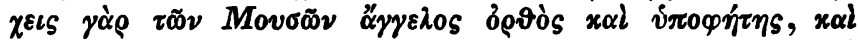

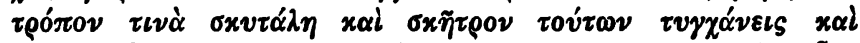

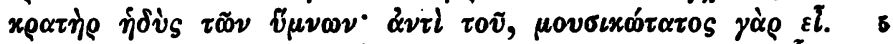

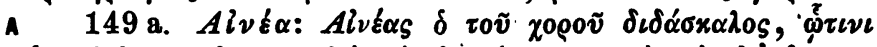

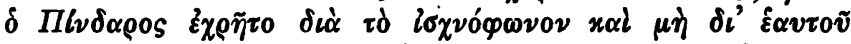

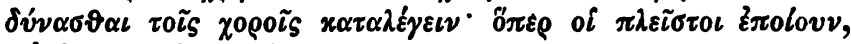

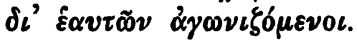

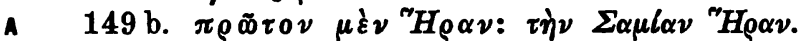

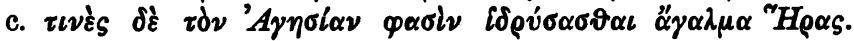

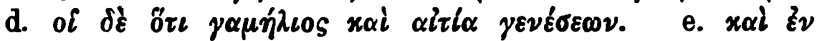

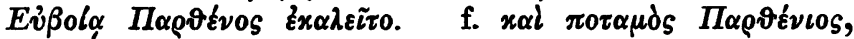

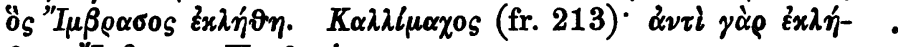
$\vartheta \eta s, " I \mu \beta \rho \alpha \sigma \varepsilon, \Pi \alpha \rho \vartheta \varepsilon \nu l o v$.

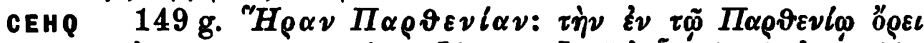

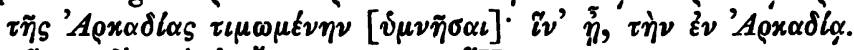

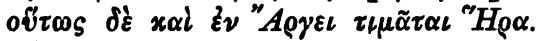

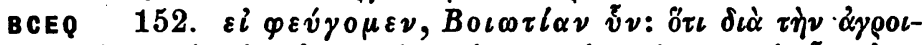

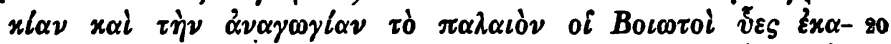

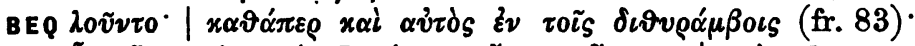

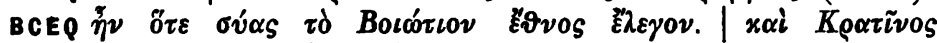

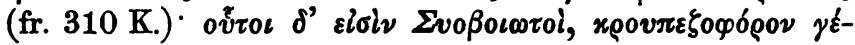

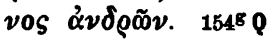

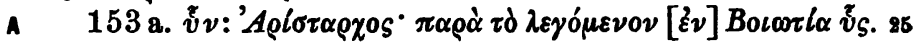

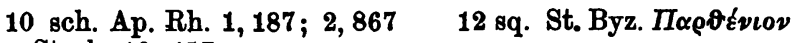
13 sq. Strab. 10, 457

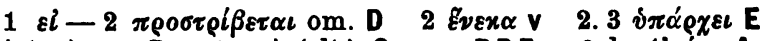
$3 x \alpha l$ (pr.) om. B $4 x \alpha l$ (alt.) Q: om. BDE 6 l. Aivés A 8 rov̀s xopoìs A; cfr. supra 186, $24 \quad 10 \sigma \widetilde{\alpha \mu l \alpha \nu}$ A $\sigma \alpha \lambda \alpha \mu i \alpha \nu$

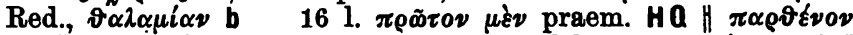

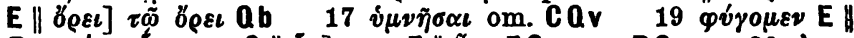

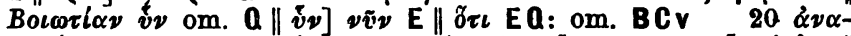

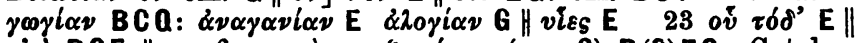

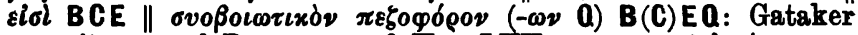

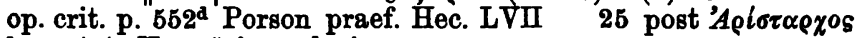
lac. stat. Horn $\|$ है $v$ seclusi 


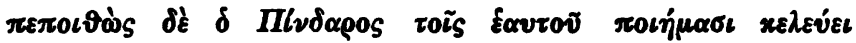

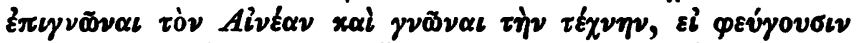

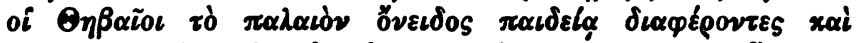

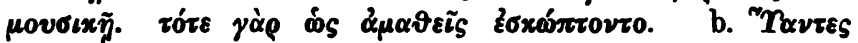

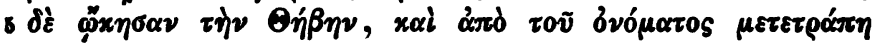

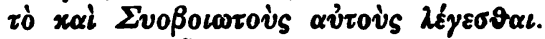

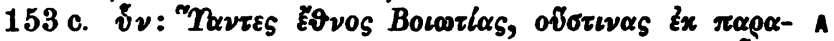

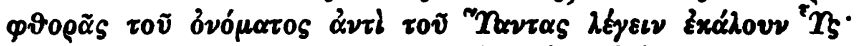

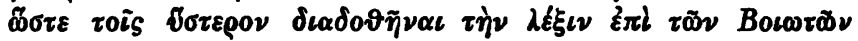

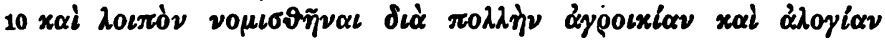

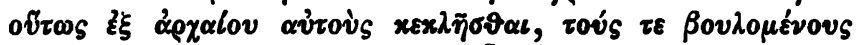

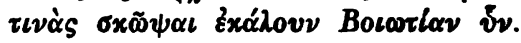

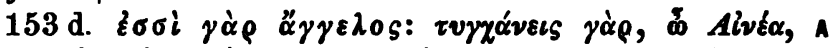

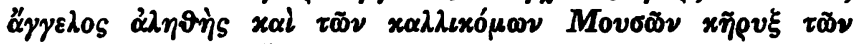

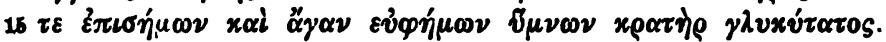

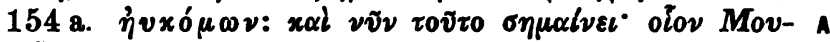

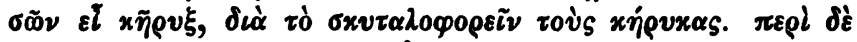

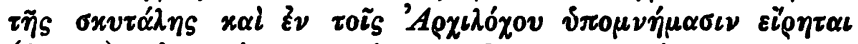

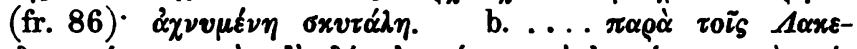

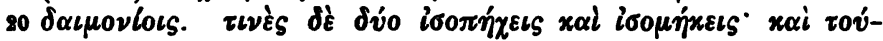

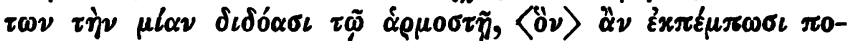

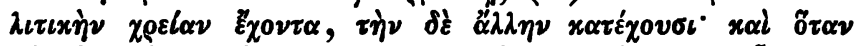

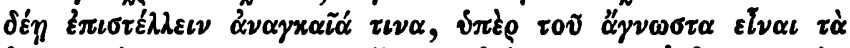

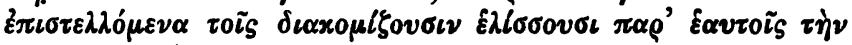

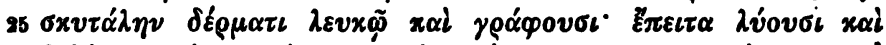

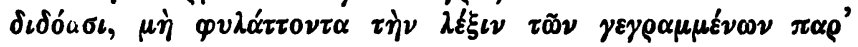

20-p. 190, 3 sch. Ar. av. 1283. Phot. et Suid. 8. v. $\sigma x v-$

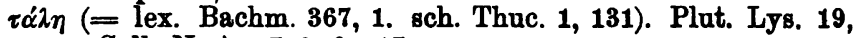
- 5-7. Gell. N. A. 17, 9, 6-17

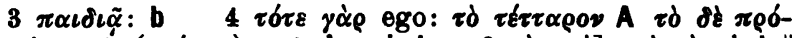

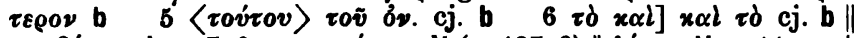

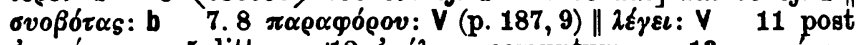

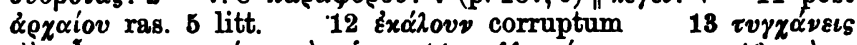

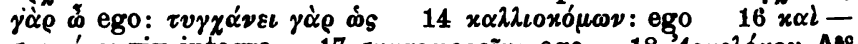

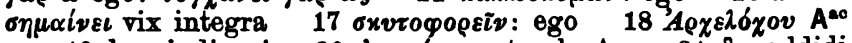

19 lac. indicavi 20 i $\sigma 0 \pi \eta x \varepsilon u_{S}$ et $8 \mathrm{ch}$. Ar. $21 \delta v$ addidi $26 \mathrm{ft} . \mu \grave{\eta} \varphi v \lambda \alpha \tau \tau \dot{\alpha} \nu \tau \omega \nu \tau \dot{\eta} \nu \tau \alpha \dot{\xi} \xi \nu$ 


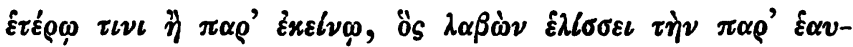

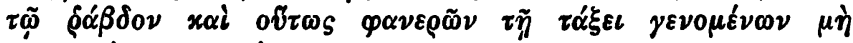

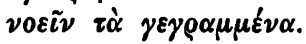

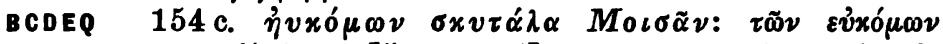

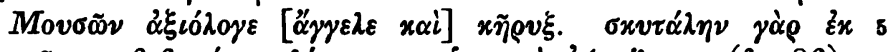

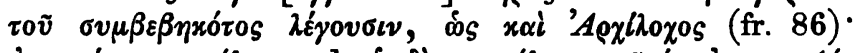

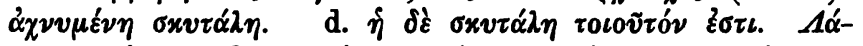

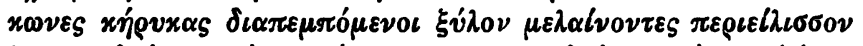

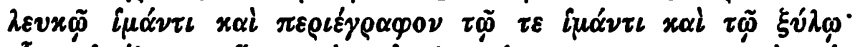

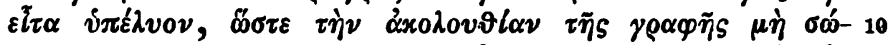
$\xi \varepsilon \sigma \vartheta \alpha \iota, \varepsilon l \mu \grave{\eta} \pi \rho 0 \sigma \tau \varepsilon \vartheta \varepsilon l \eta \eta$

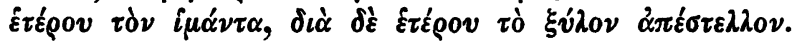

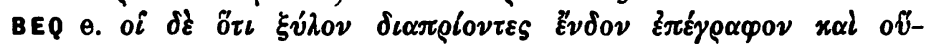

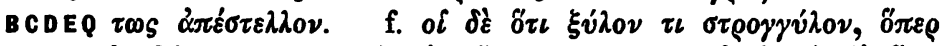

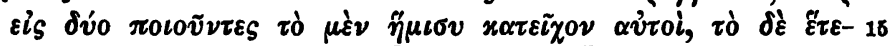

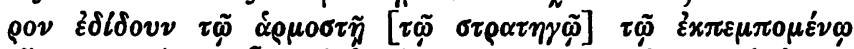

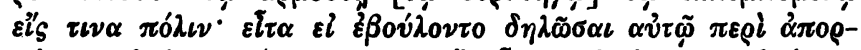

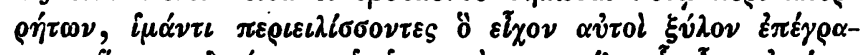

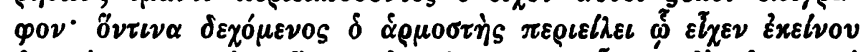

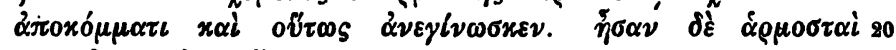

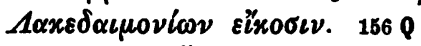

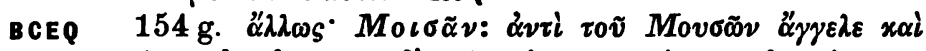

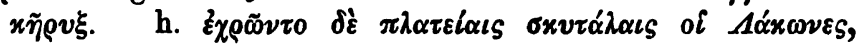

7-12 Tzetzes Chil. IX 135-55? 14-21 cfr. ad $154^{\circ}$

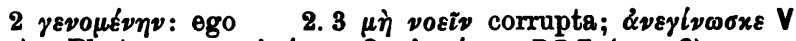

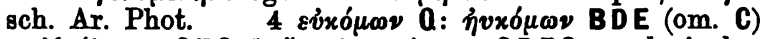

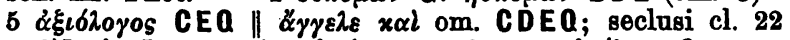

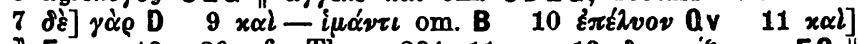

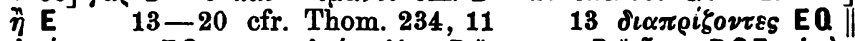

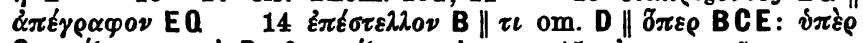

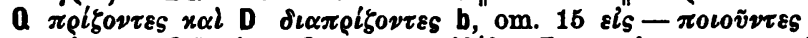

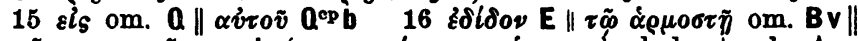

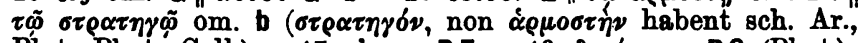

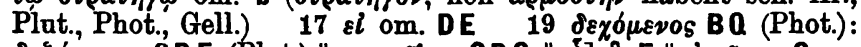

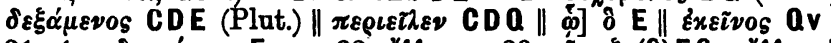

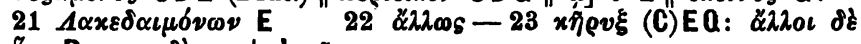

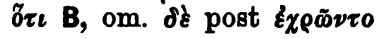




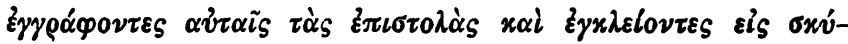

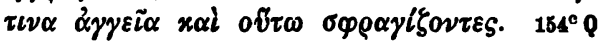

156 a. $\Sigma v \rho \alpha x 0 \sigma \sigma \tilde{\alpha} \nu \tau \varepsilon x \alpha i$ 'O $O \tau v \gamma l \alpha s:$ 'O

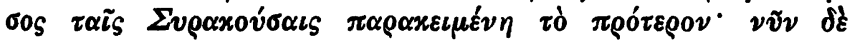

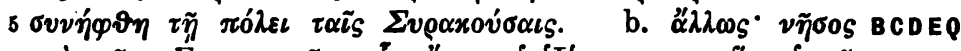

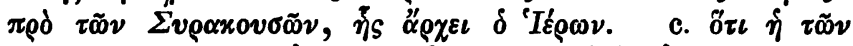

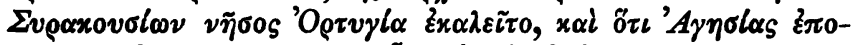

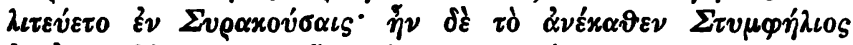

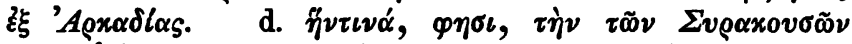

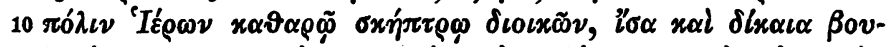

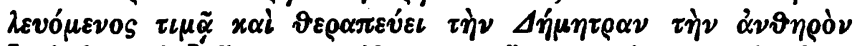

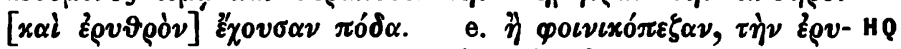

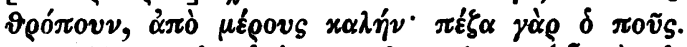

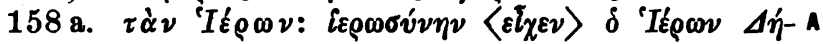

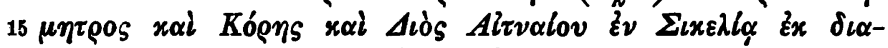

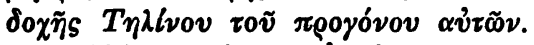

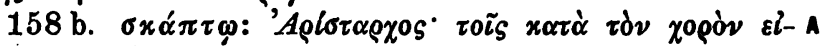

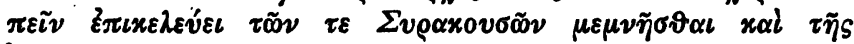

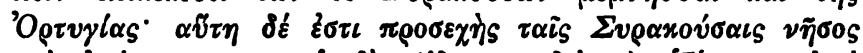

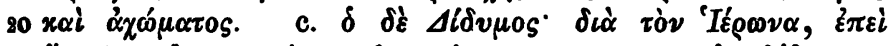

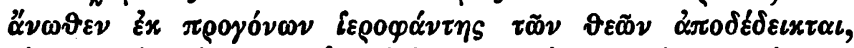

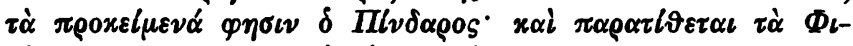

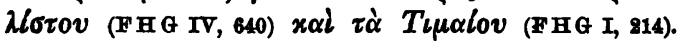

4 sq. sch. N. 1, 2 (p. 18, 10 Ab.). sch. P. 2, 1012 sch. Did.

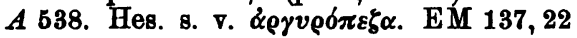

$1 \alpha \dot{\delta} \tau \alpha \tilde{\tau}_{S}$ C: $\alpha \dot{v} \tau 0 \tau_{S}$ BEv $\alpha \dot{v} \tau \tilde{\omega} \nu$ GQb 3 l. B: om. E $x \alpha l$

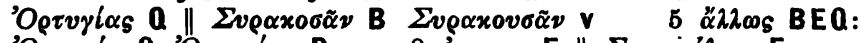

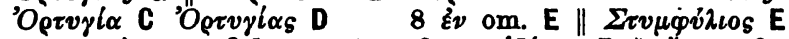

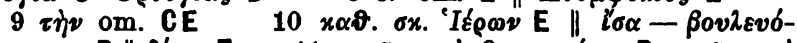

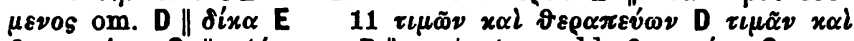

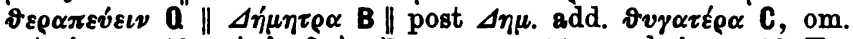

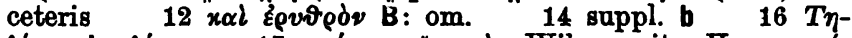
$\lambda i \nu 0 v$ b: $\lambda i$ i

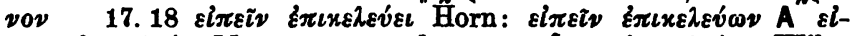

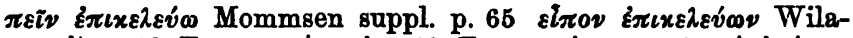

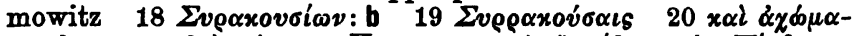

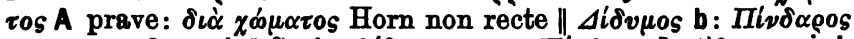

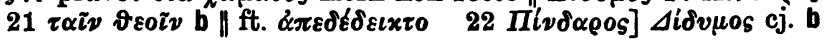




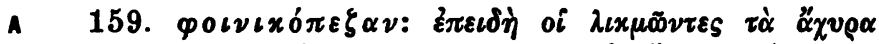

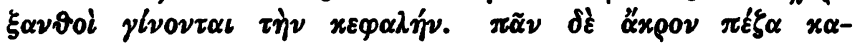
$\lambda \varepsilon \tilde{\tau} \tau \alpha \iota$.

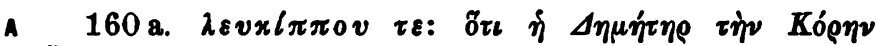

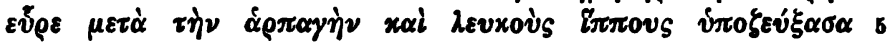

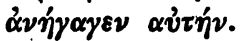

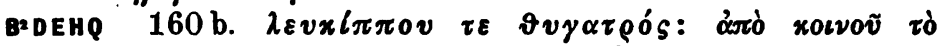

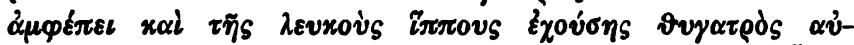

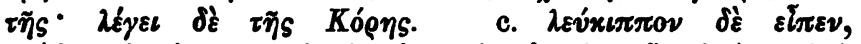

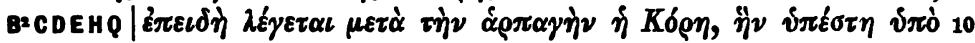

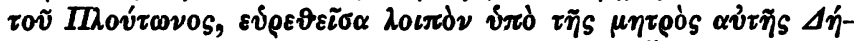

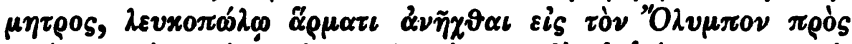

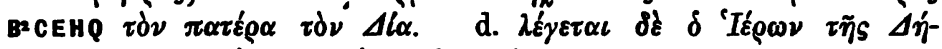

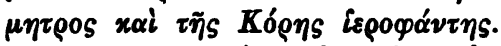

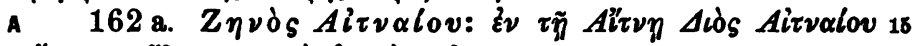

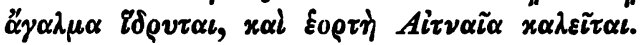

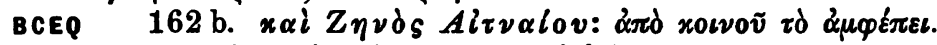

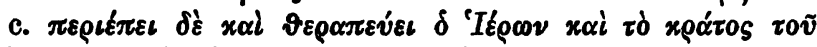

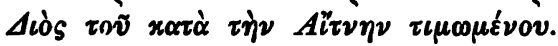

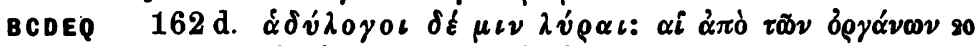

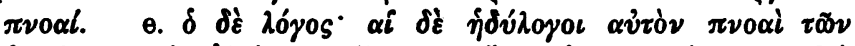

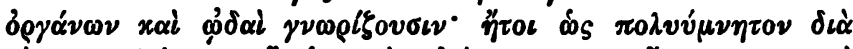

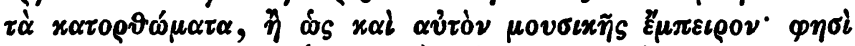

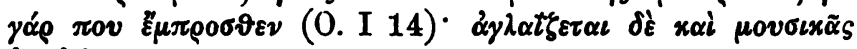

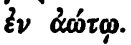

2 sq. sch. B $\Omega 272$

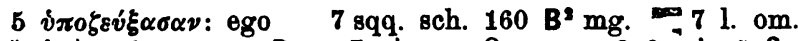

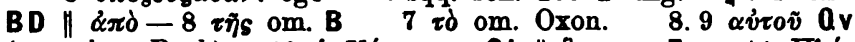

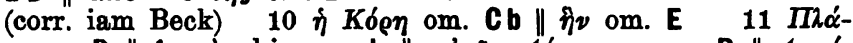

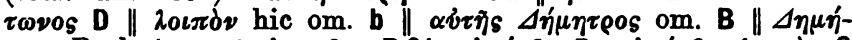

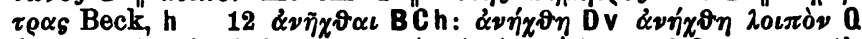

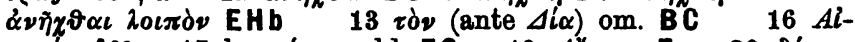

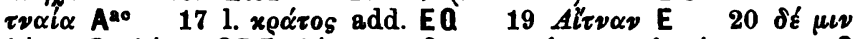

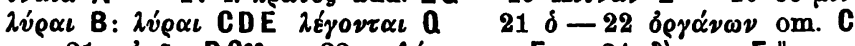

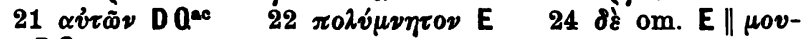
$\sigma \iota x \tilde{\eta}_{S} \mathrm{DQV}$ 


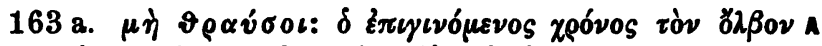

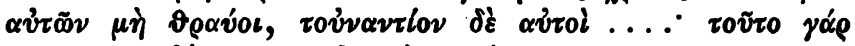

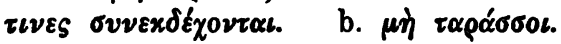

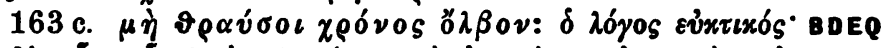

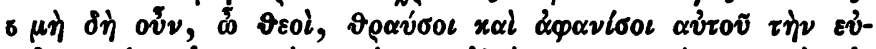

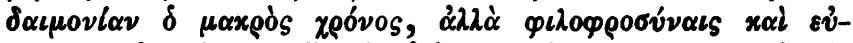

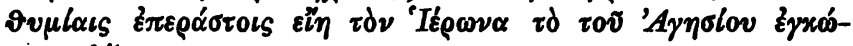
$\mu i O \nu \delta \hat{\varepsilon} \xi \alpha \sigma \vartheta \alpha \iota$.

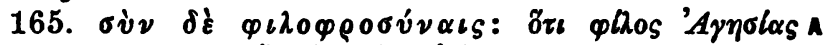
10 'IÉ

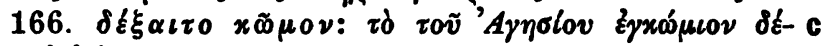
$\xi \alpha \iota \tau o \delta$ 'T $\varepsilon^{\prime} \rho \omega \nu$.

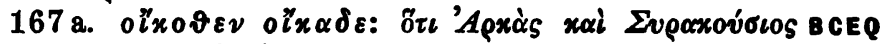

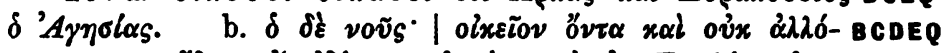

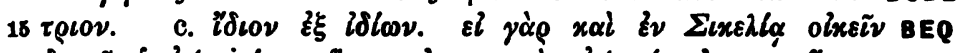

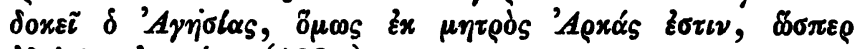

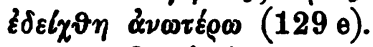

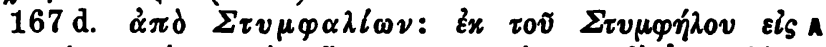

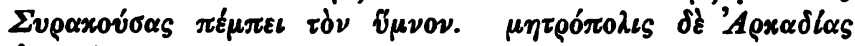
so रं $\Sigma \tau \dot{\nu} \mu \varphi \eta \lambda$ os.

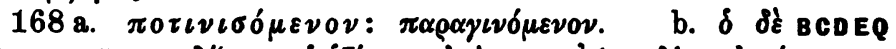

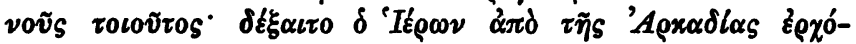

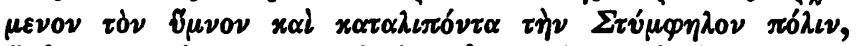

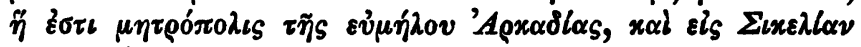
$25 \pi \alpha \rho \alpha y \iota v o ́ \mu \varepsilon v 0 \nu$.

170 a. $\dot{\alpha} \gamma \alpha \vartheta \alpha i \delta \xi \quad \pi \xi \lambda_{0} \nu \tau \alpha \iota: \alpha \dot{\alpha} \alpha \vartheta \alpha i \quad \delta \xi, \varphi \eta \sigma \iota ; x \alpha l_{A}$

19 et 24 cfr. sch. V $144 \mathrm{~g}$

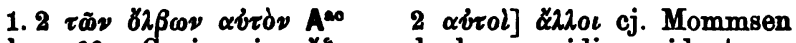
suppl. p. 66. Conieceris $\alpha$

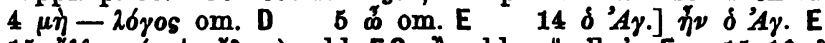

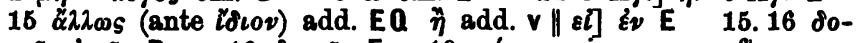

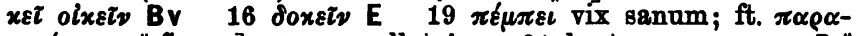

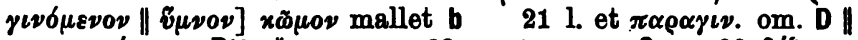

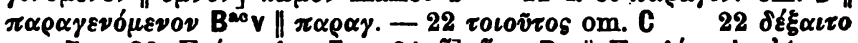

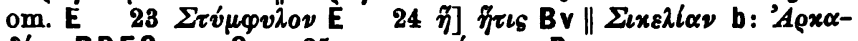

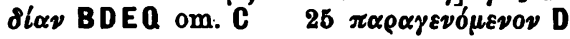

Soroc. na Padarex ed. Drachmann. 


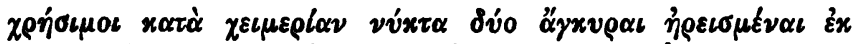

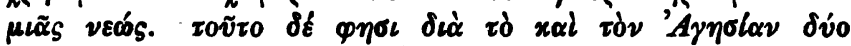

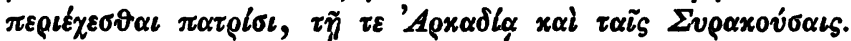

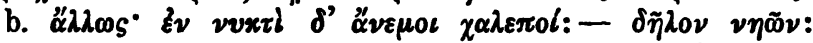

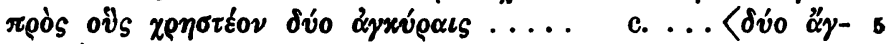

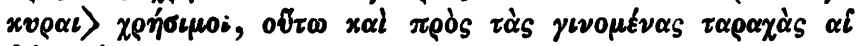

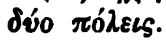

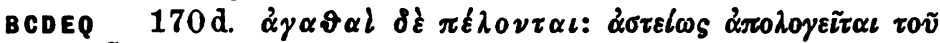

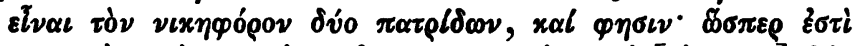

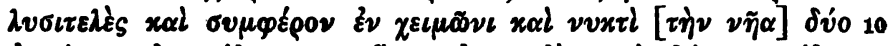

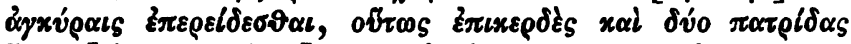

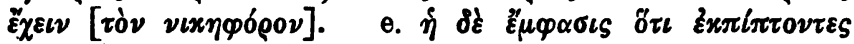

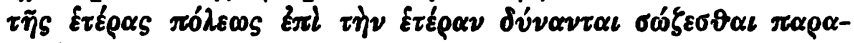
yเvó

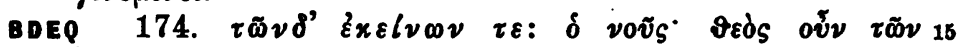
'A

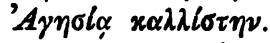

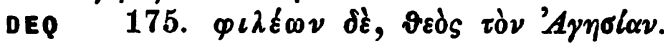

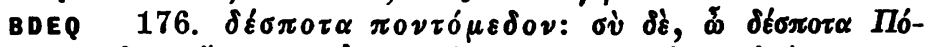

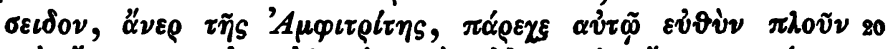

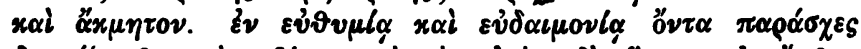

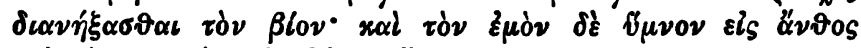

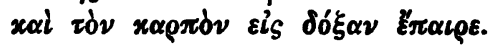

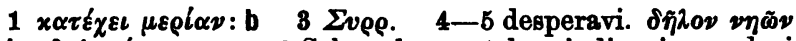

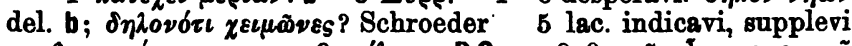

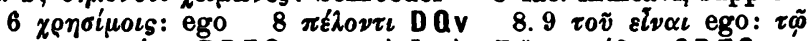

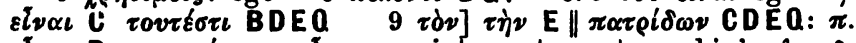

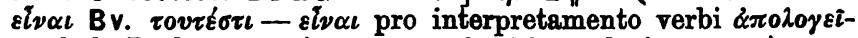
$\tau \alpha \iota$ hab. Beck $10 \tau \eta \dot{\nu} \nu \eta \hat{\alpha}$ om. CDEQ; seclusi $12 \tau \dot{\delta} \nu \nu \iota x$. om. CDEQ; seclusi || ö

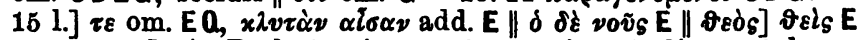

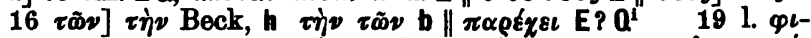

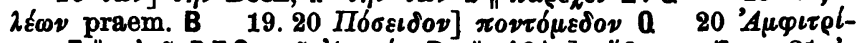

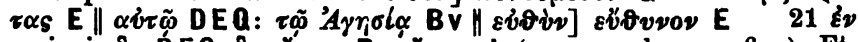
scripsi: $\delta \nu$ D́EQ $\delta v \ell_{v}^{\prime} \tau \varepsilon$ Bv $z_{v} \tau \varepsilon$ b (paragrapho praefixa). Ft.

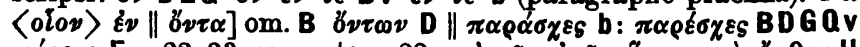

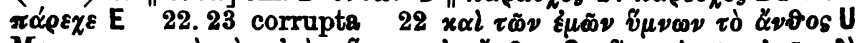

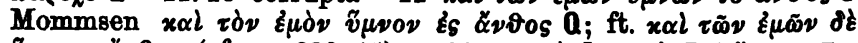

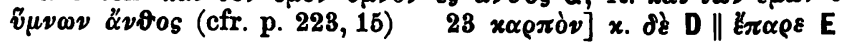




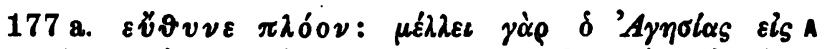

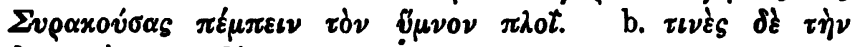

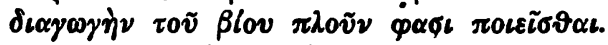

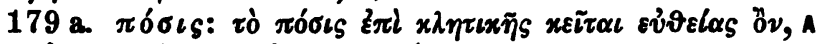

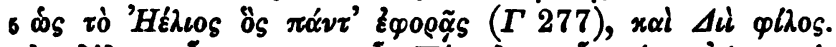

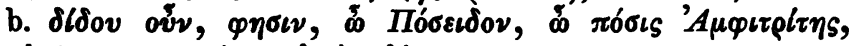

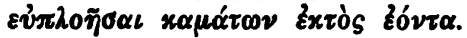

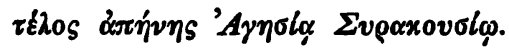

\section{Scholia in Olympionicaram carmen VII.}

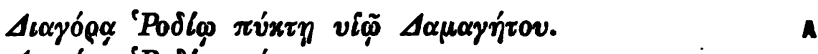

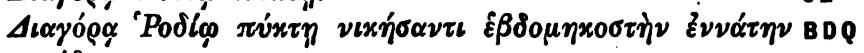

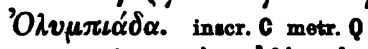

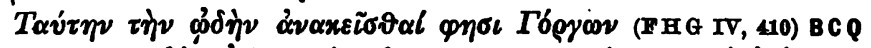

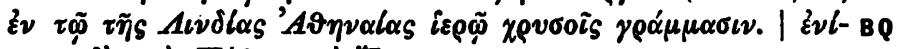

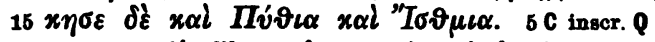

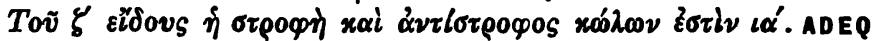

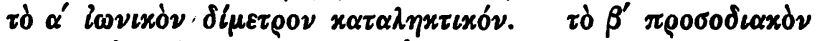

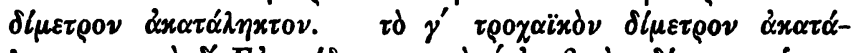

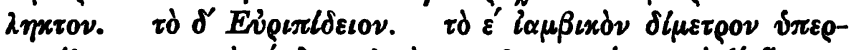

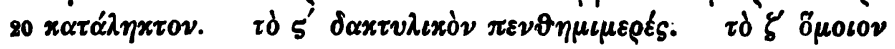

\section{Bch. A $\Gamma 277$}

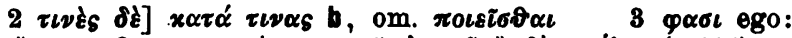

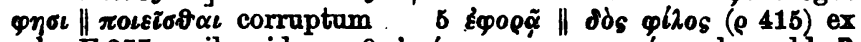

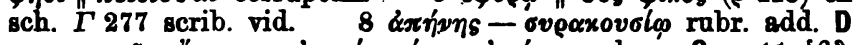

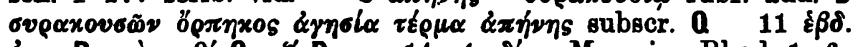

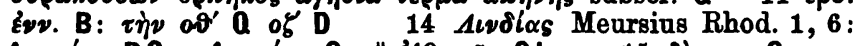

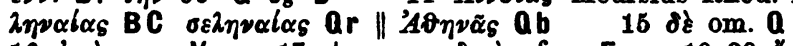

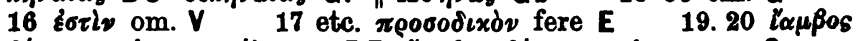

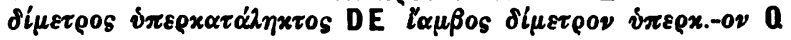




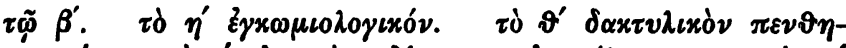

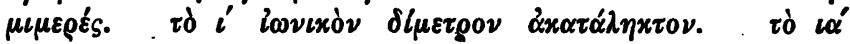

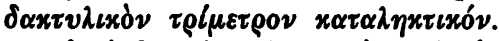

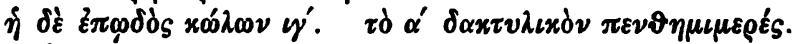

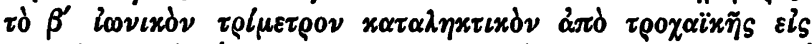

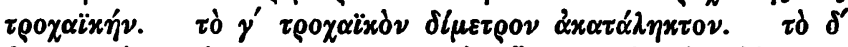

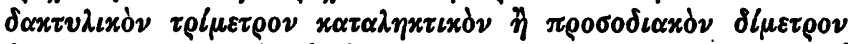

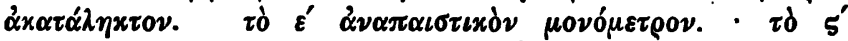

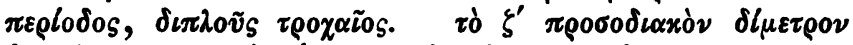

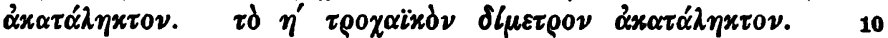

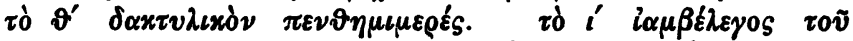

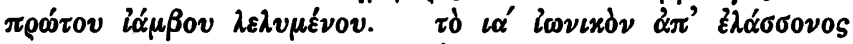

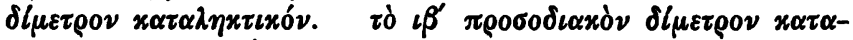
$\lambda \eta x \tau \iota x o ́ v . \quad \tau \dot{~} \iota \gamma^{\prime} \Sigma_{\tau} \eta \sigma \iota \chi \delta \rho \varepsilon \iota 0 \nu . \quad$ p. 195, 13-150

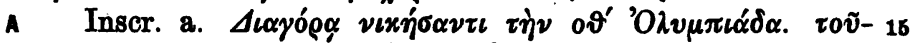

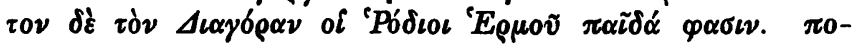

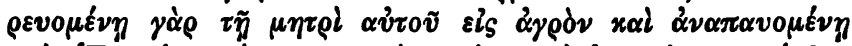

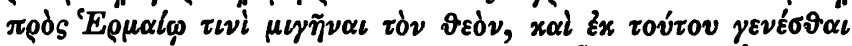

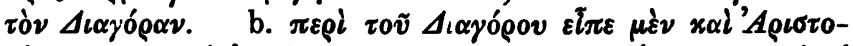

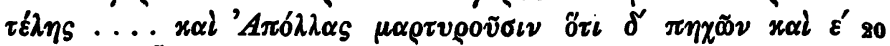

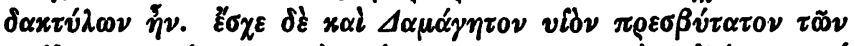

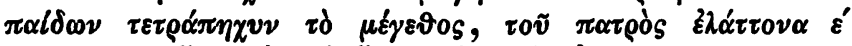

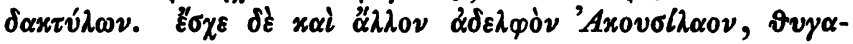

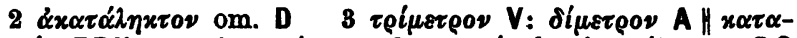

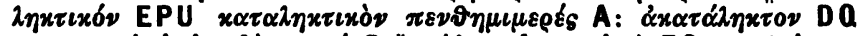

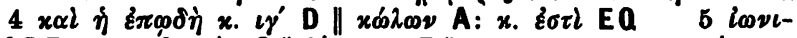

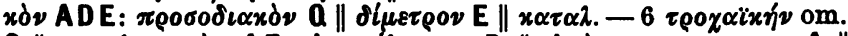

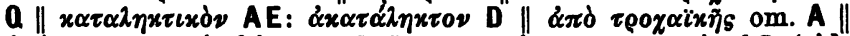

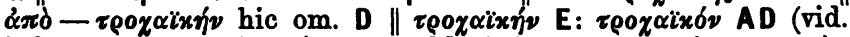

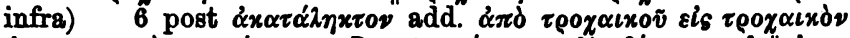

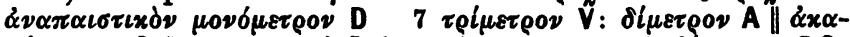
$\tau \alpha \dot{\lambda} \eta x \tau o \nu$ a

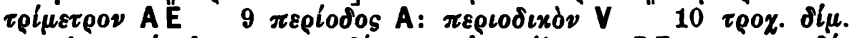

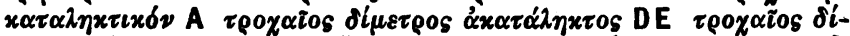

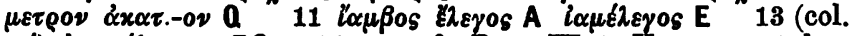
$\left.\iota \alpha^{\prime}\right) \alpha^{\prime} x \alpha \tau \alpha^{\prime} \lambda \eta \tau \tau o v$ EQ 15 sqq. cfr. Paus. VI 7; V 6,8. 20 lac.

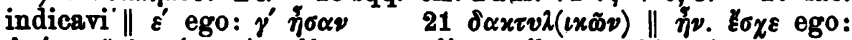

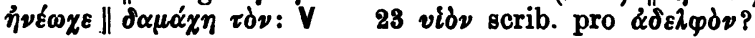




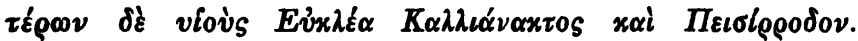

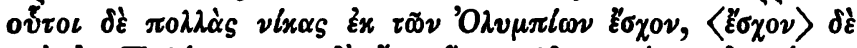

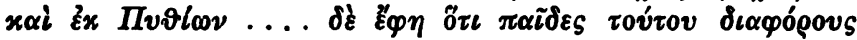

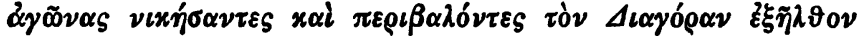

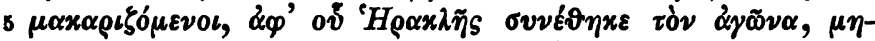

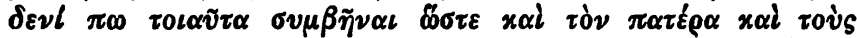

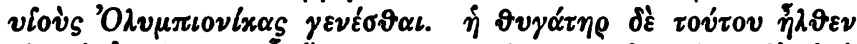

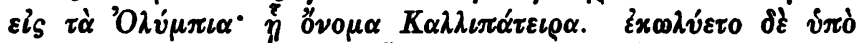

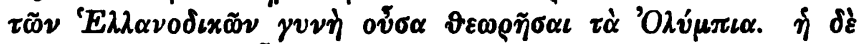

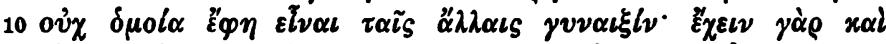

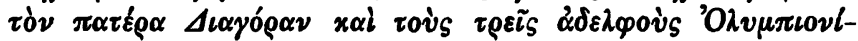

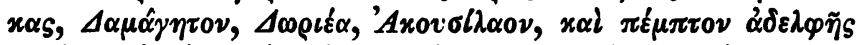

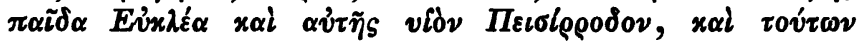

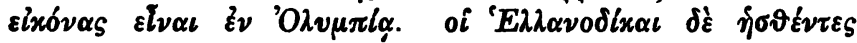

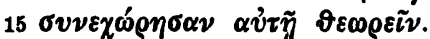

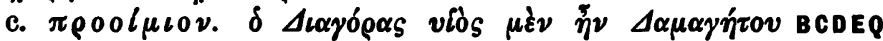

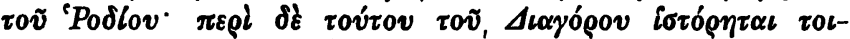

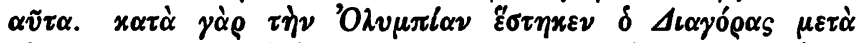

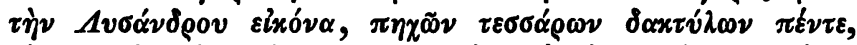

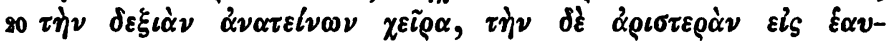

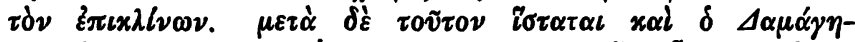

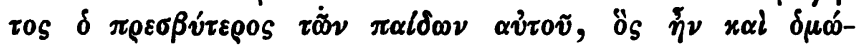

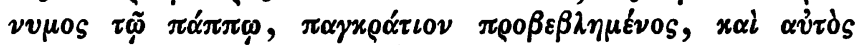

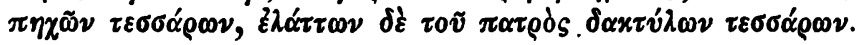

7-15 Paus. 5, 6, 7 sq. 17-p. 199, 1 Tzetzes Chil.I 694-618

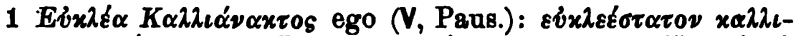

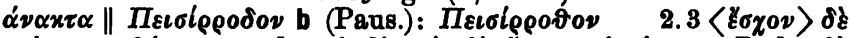
$x \alpha l$ ego: $\delta \varepsilon x \alpha \quad 3$ lac. indicavi, $\delta \xi \xi \xi \eta \eta$ scripsi cum Red.: $\delta \xi$

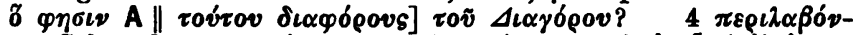

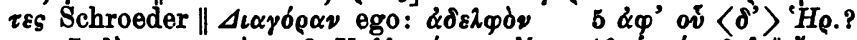

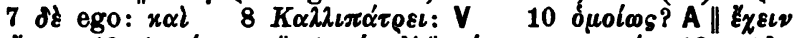

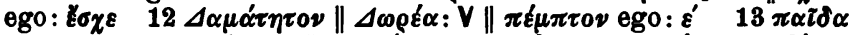

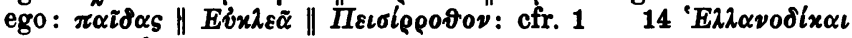

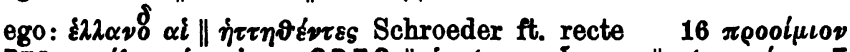
$B^{\text {mg: }}$ 甲

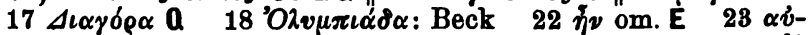

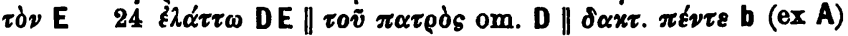




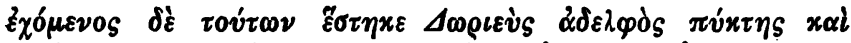

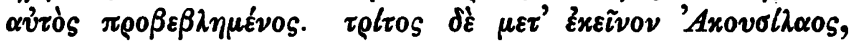

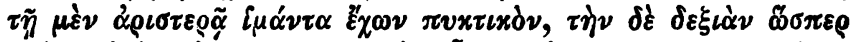

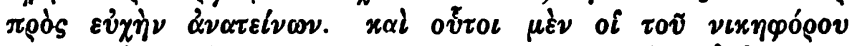

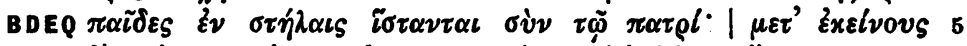

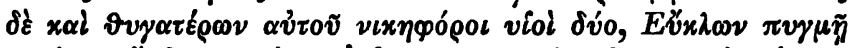

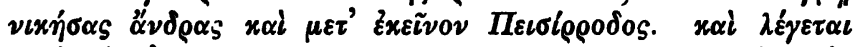

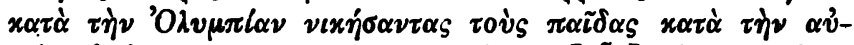

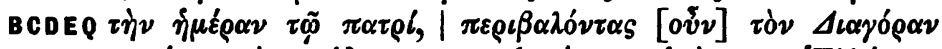

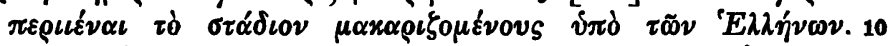

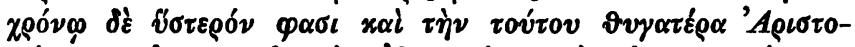

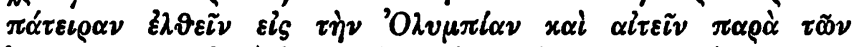

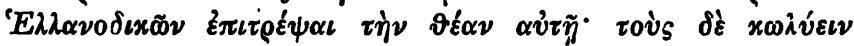

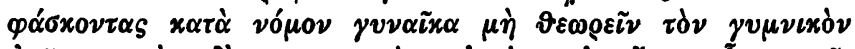

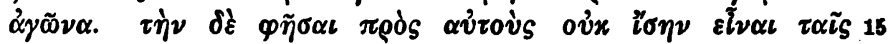

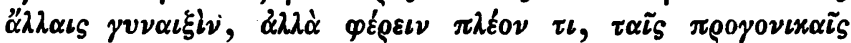

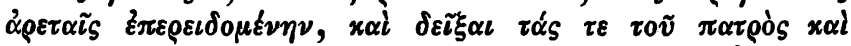

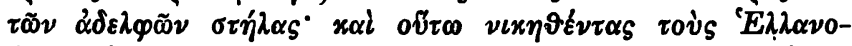

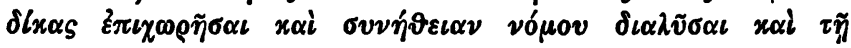

11-p. 199, 2 Aesch. epist. 4, 5. Ael. v. h. 10, 1. Val. Max. 8, 15 ext. 4. Gell. N. A. 3, 15

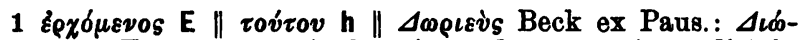

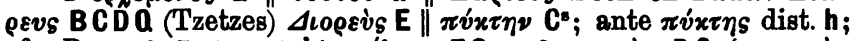

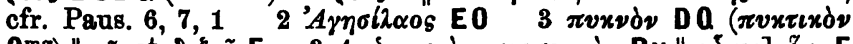

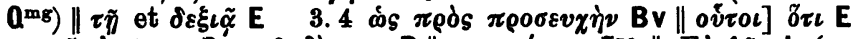

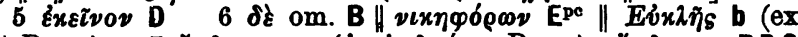

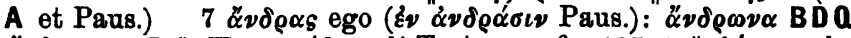

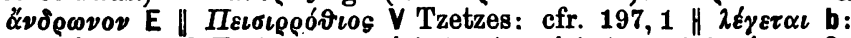

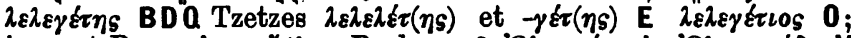

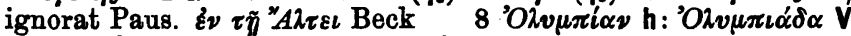

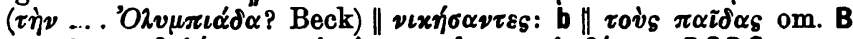

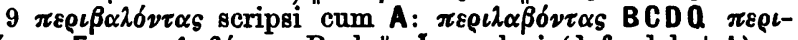
$\lambda \alpha \beta \sigma o v \tau \varepsilon$ E $\pi \alpha \rho \alpha \lambda \alpha \beta \sigma v \tau \alpha_{S}$ Beck $\|$ ov̀v seclusi (defendebat $b$ )

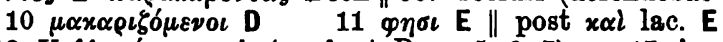

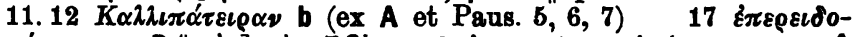

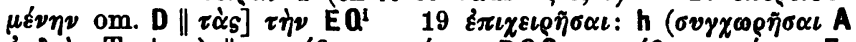

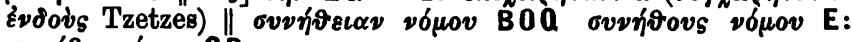
$\sigma v \nu \tilde{v} \vartheta \eta \nu \delta \mu \nu \nu$ CD 


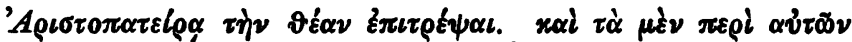

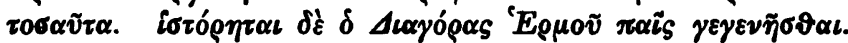

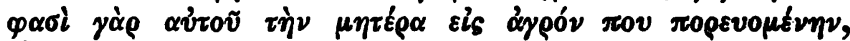

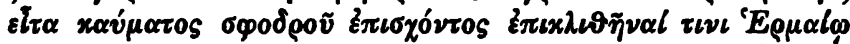

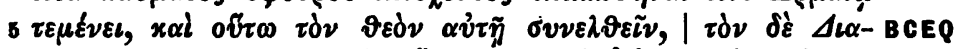

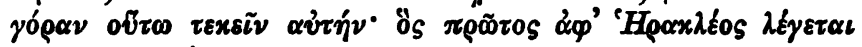

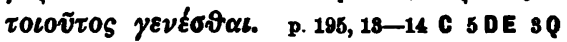

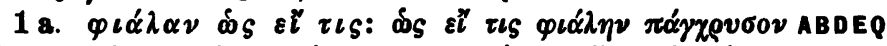

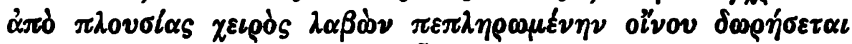

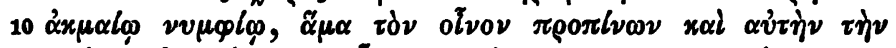

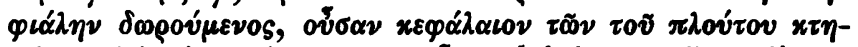

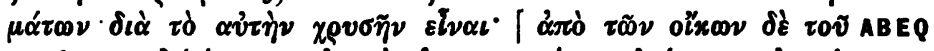

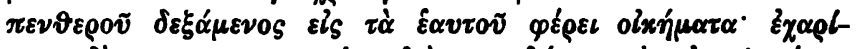

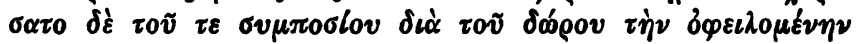

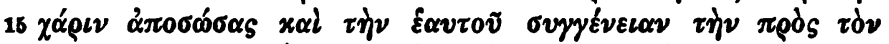

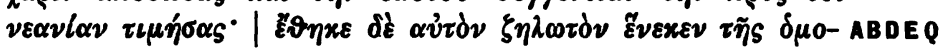

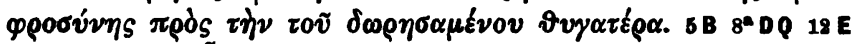

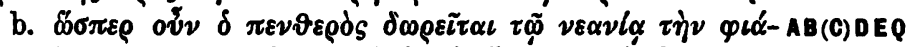

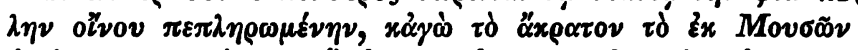

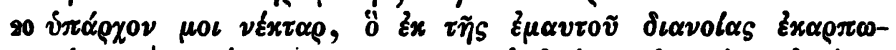

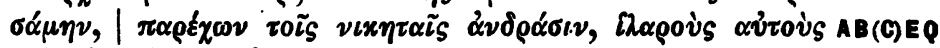

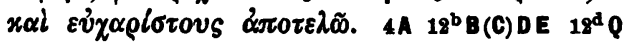

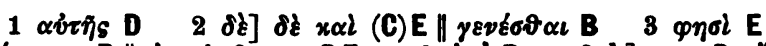

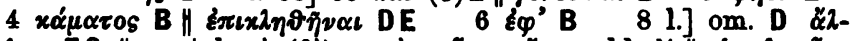

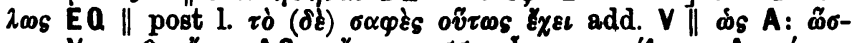

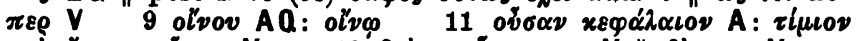

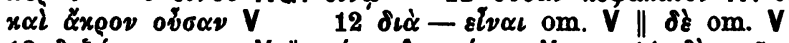

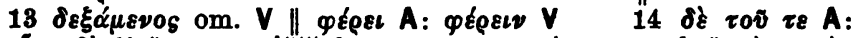

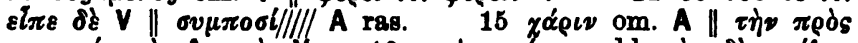

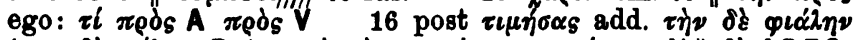

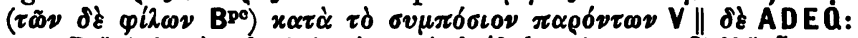

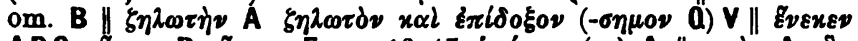

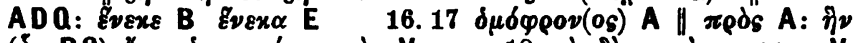

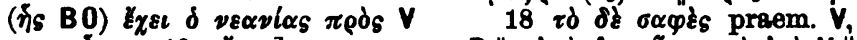

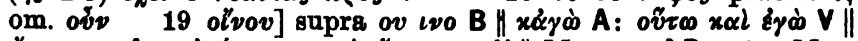

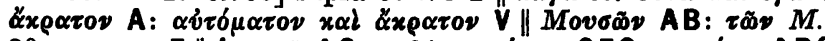
$20 \mu o \iota$ om. E\| $\varepsilon_{\alpha v \tau o v}$ AQ $21 \pi \alpha \rho \varepsilon z \omega \nu$ CEO: $\pi \alpha \rho \varepsilon z \omega$ AB Q $\|$

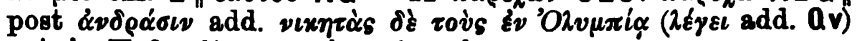

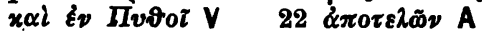




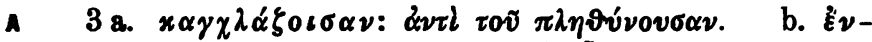

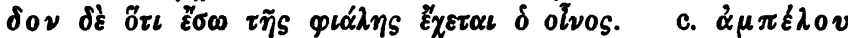

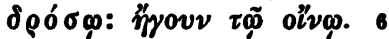

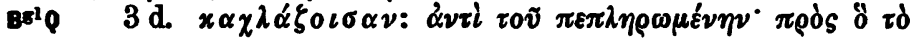

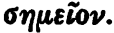

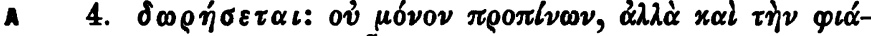

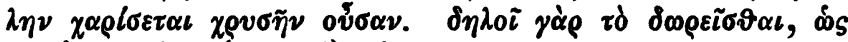

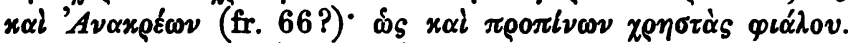

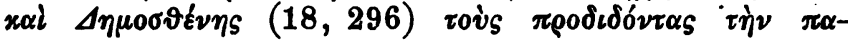
$\tau \rho l \delta \alpha \ldots 8$

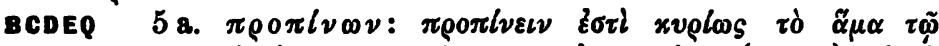

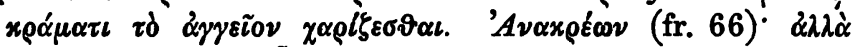

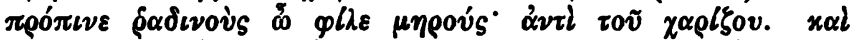

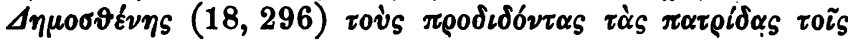

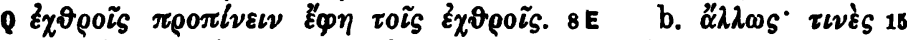

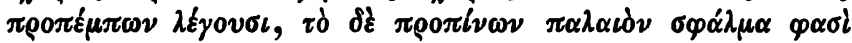

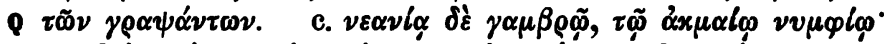

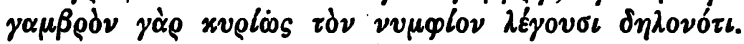

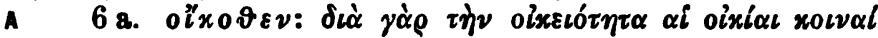

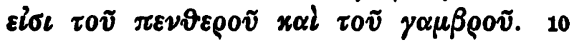

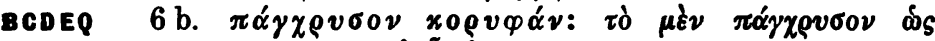

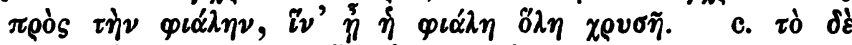

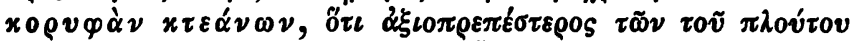

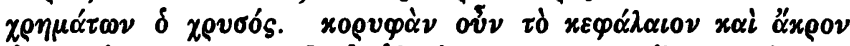

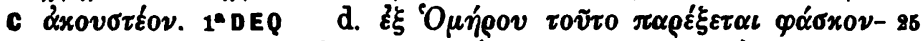

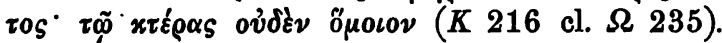

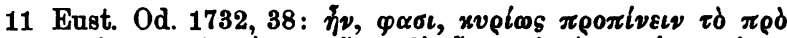

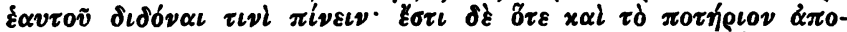

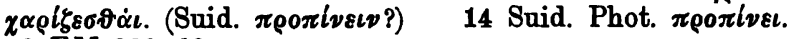

18 EM 220, 39

$4 \alpha \nu \tau i \tau o \tilde{v}$ et $\pi \rho \delta_{s}-5 \sigma \eta \mu \varepsilon i ̃ o \nu$ om. B $9.10 \pi \alpha \tau \rho i \alpha v:$ V.

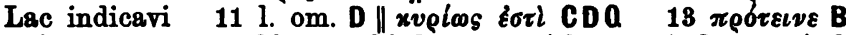

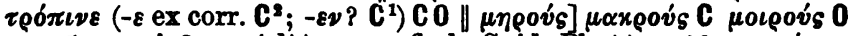

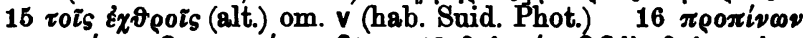

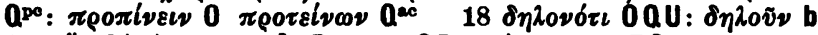

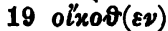

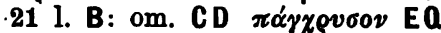

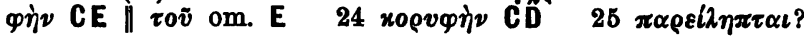




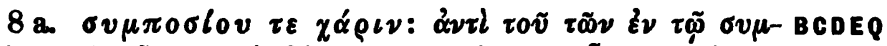

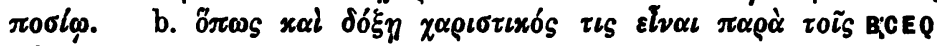
$\pi \ell \nu 0 v \sigma \iota \nu$.

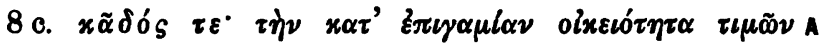

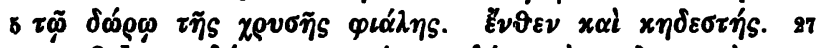

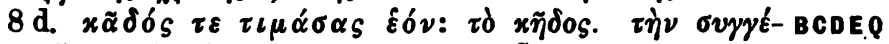

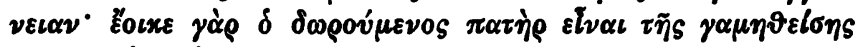

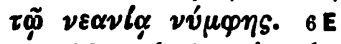

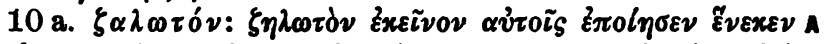

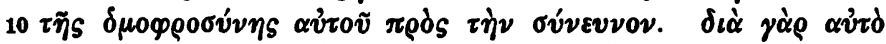

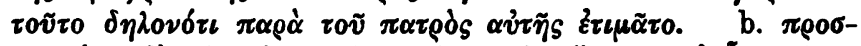

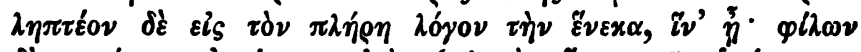

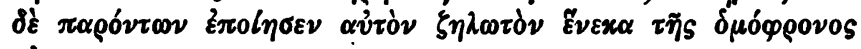

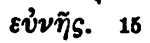

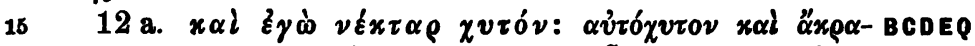

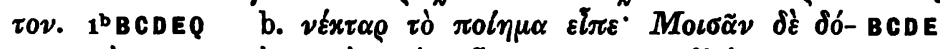

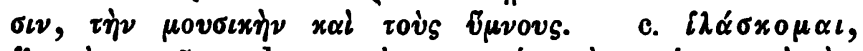

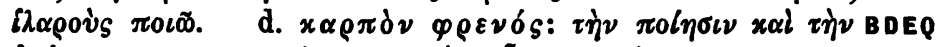

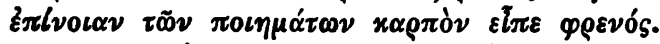

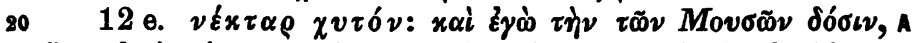

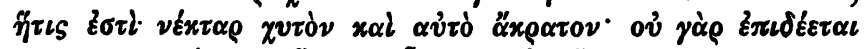

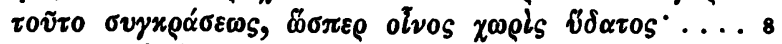

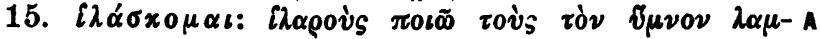

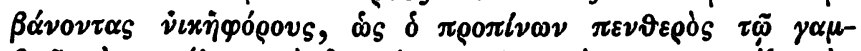

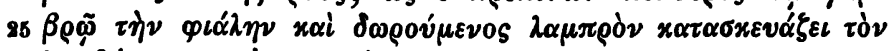

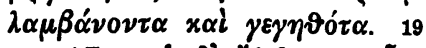

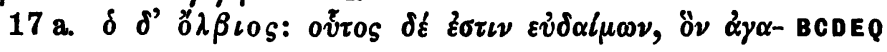

4 EM 509, 51

$1 \tau \tau \chi_{\alpha}^{\alpha} \rho \iota \nu$ B: om. $\| \tau \tilde{\omega} \nu$ om. BD $\| \tau \tilde{\omega}$ om. BCDEv (hab. GOQU) $1.2 \pi 0 \sigma i n$ E $2 x \alpha \iota \delta \delta \xi \eta$ CÉ

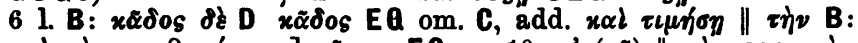

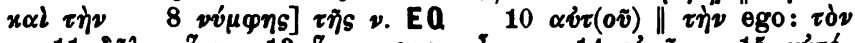

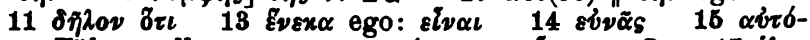

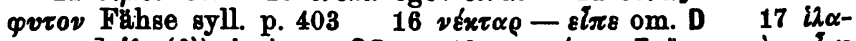

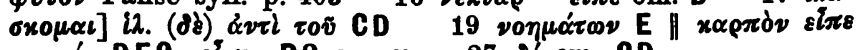

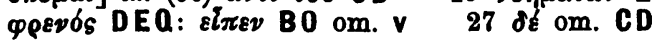




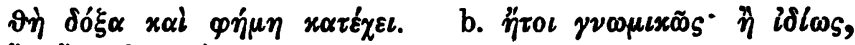

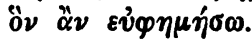

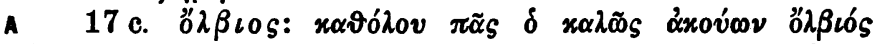

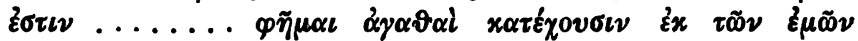

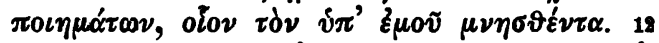

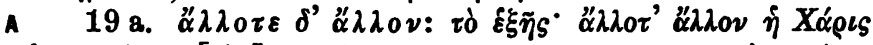

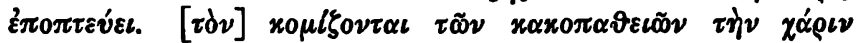

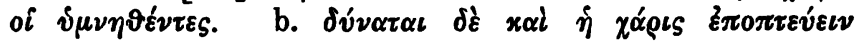

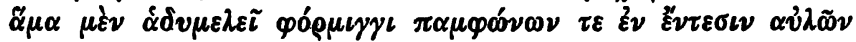

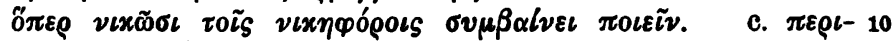

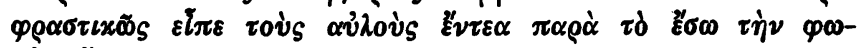

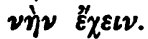

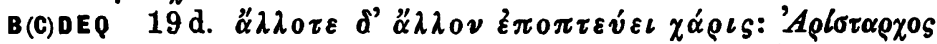

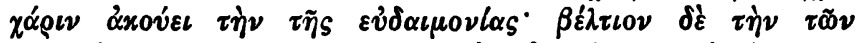

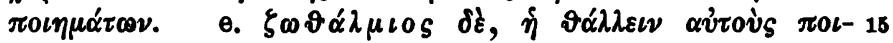

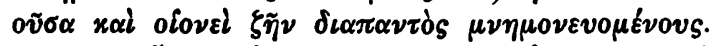

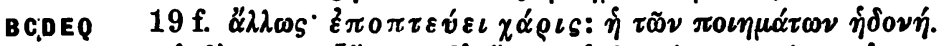

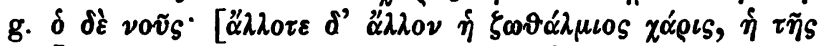

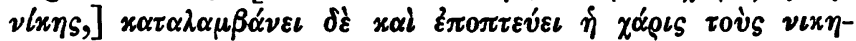

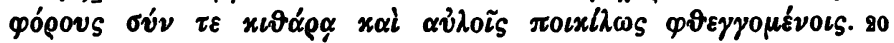

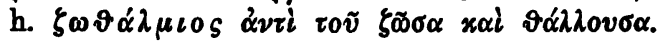

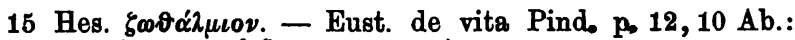

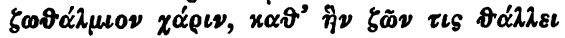

$3 \mathrm{ft} .\langle\hat{\eta}\rangle x \alpha \vartheta \hat{\vartheta} \lambda_{0 v} \quad 4\langle\hat{\eta}\rangle$ in lac. suppl. b $\| \varphi \eta \mu \alpha l$

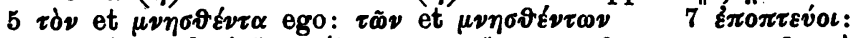
ego \| $\tau \dot{\partial} \nu$ seclusi $\| x o \mu l \zeta o \nu \tau \alpha$ : ego \| $x \alpha x \circ \pi \alpha \vartheta \tilde{\omega} \nu$ : ego $8 x \alpha \downarrow$

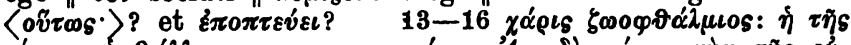

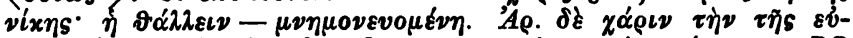

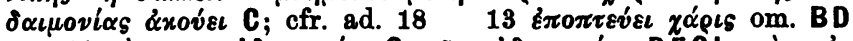

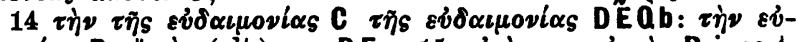

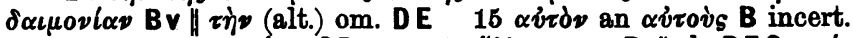

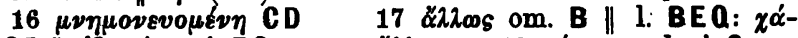

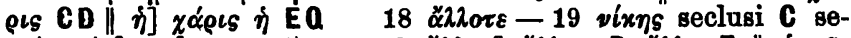

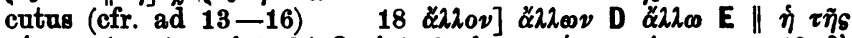

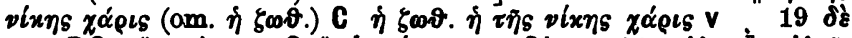

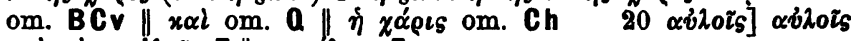

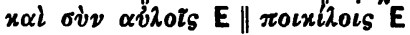




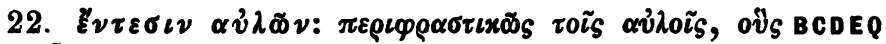

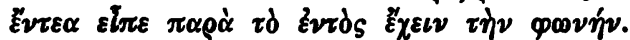

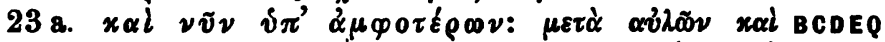

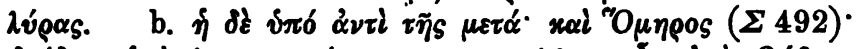

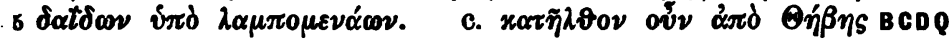

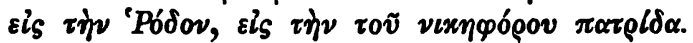

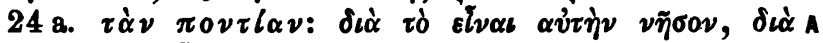

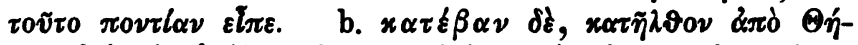

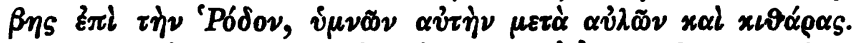

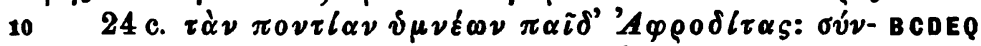

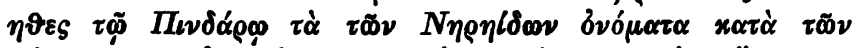

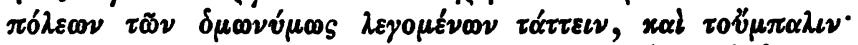

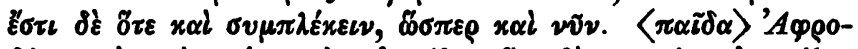

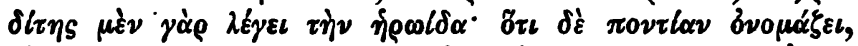

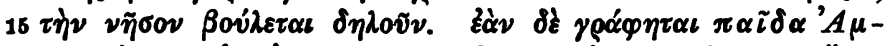

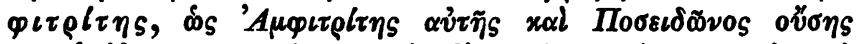

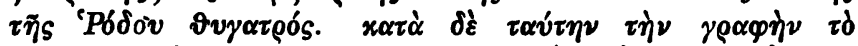

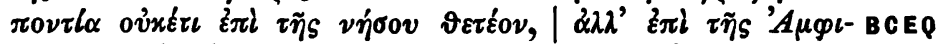

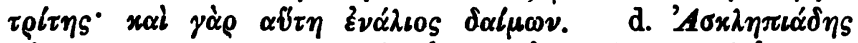

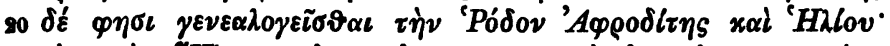

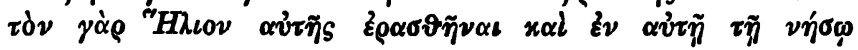
$\mu(\gamma \tilde{\eta} \nu \alpha \varepsilon$.

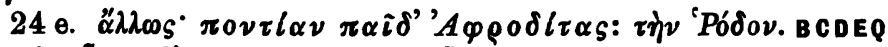

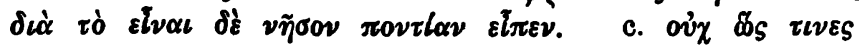

1 cf. EM 345, $17 \quad 4$ sch. A $\Sigma 492$

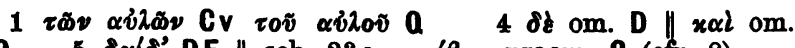

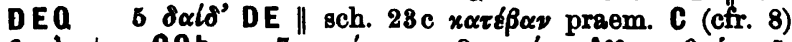

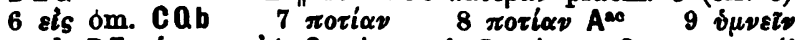
10 l. BE: $\delta \mu \nu . \pi$. 'A. C $\tau \dot{\alpha} \nu \pi$. $\delta$. D $\tau \dot{\alpha} \nu \pi$. a $12 \pi \dot{\alpha} \lambda \varepsilon \omega \nu]$

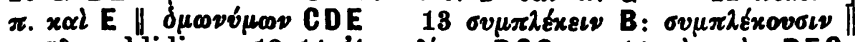

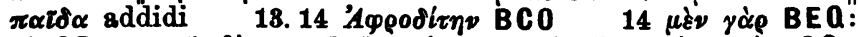

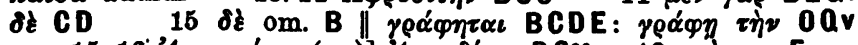

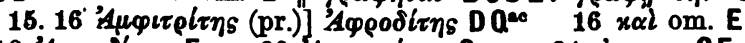

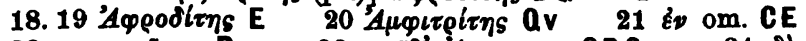

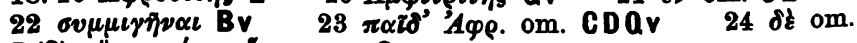
$B(C) \vee H \cdot \pi 0 \nu \tau l \alpha \nu$ elizev om. Cv 


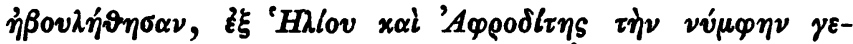

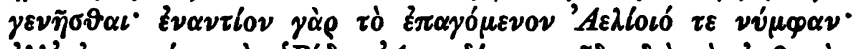

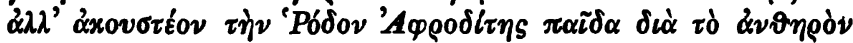

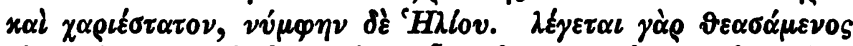

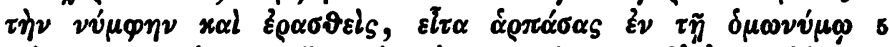

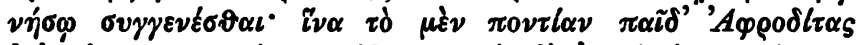

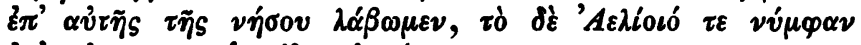

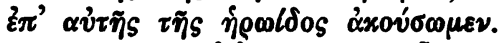

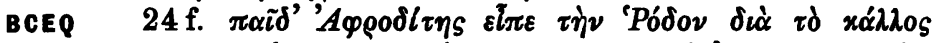

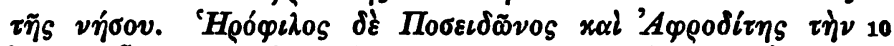

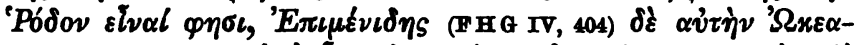

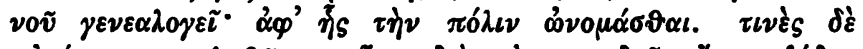

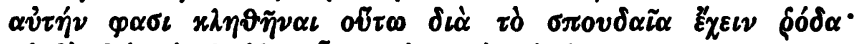

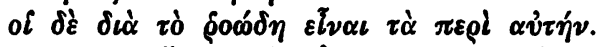

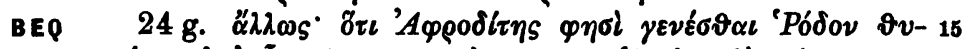

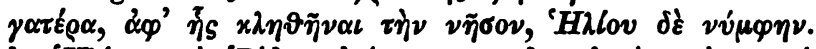

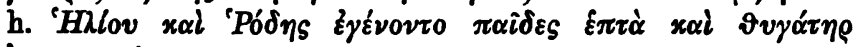
'Hภ̨

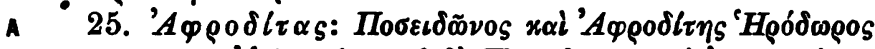

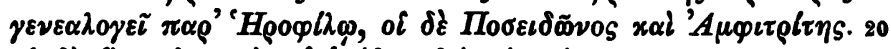

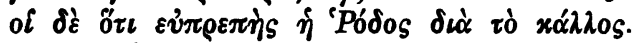

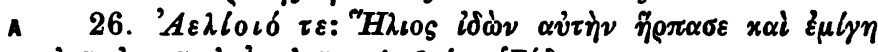

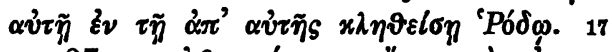

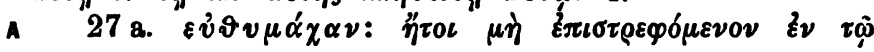

\section{EM 705, 620 Apollod. 1, 4,6}

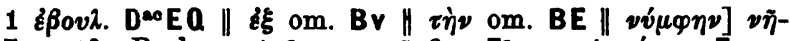

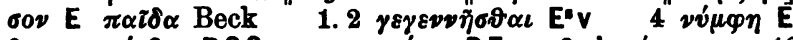

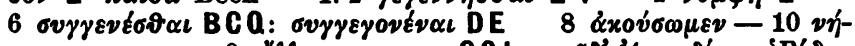

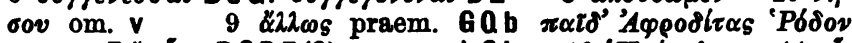

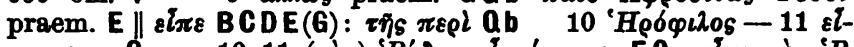

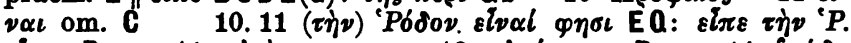

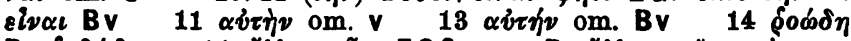

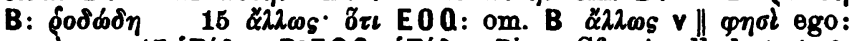

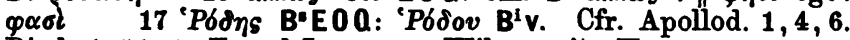
Diod. 5, 55, 5. Tz. ad Lyc. 923. Wilamowitz Herm. 14, 457 sqq.

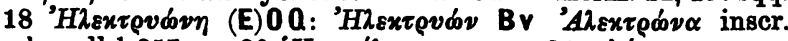

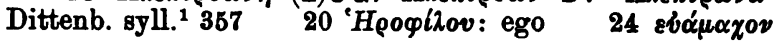




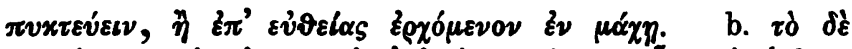
$\pi \varepsilon \lambda \omega \omega_{\rho}$

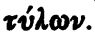

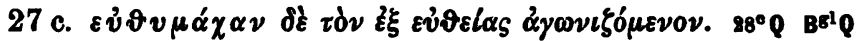

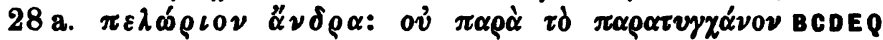

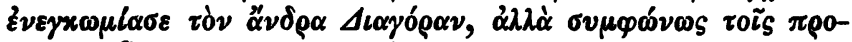

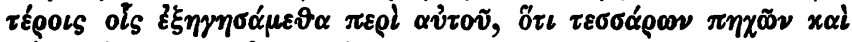

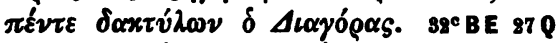

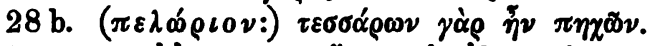

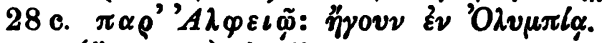

30. ( $\alpha \pi 0 เ v \alpha:) ~ \alpha \nu \tau l \delta \omega \rho \alpha$.

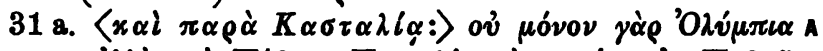

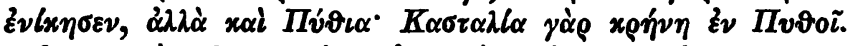
15 Acayópov.

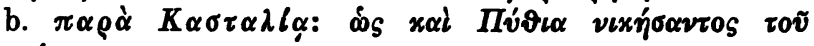

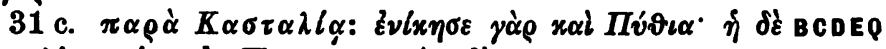

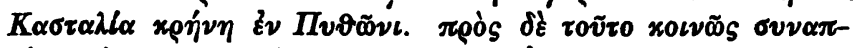

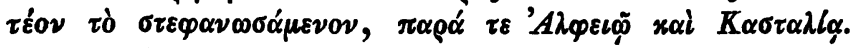
36 B $32^{\circ} \mathrm{CDQ}$ 34 E

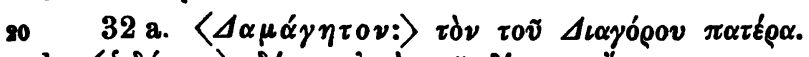

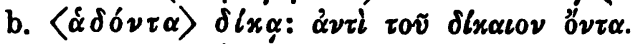

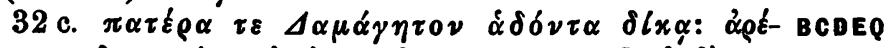

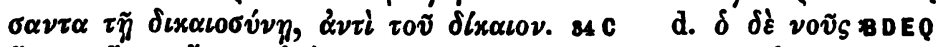

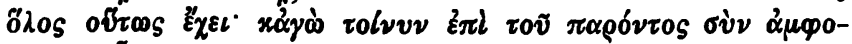

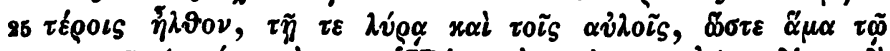

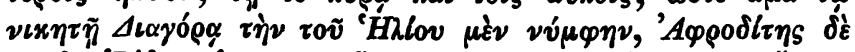

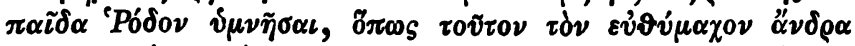

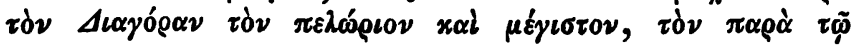

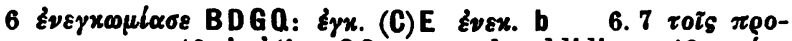

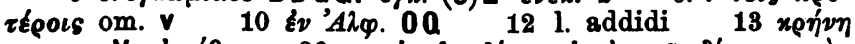

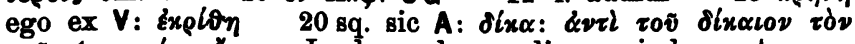

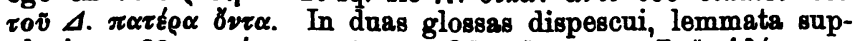

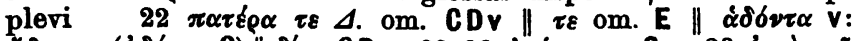

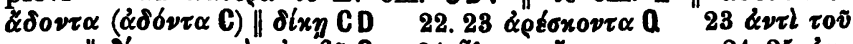

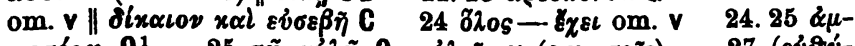

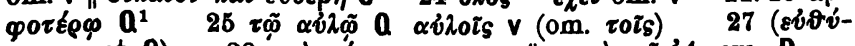

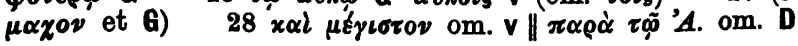




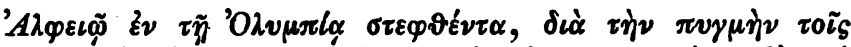

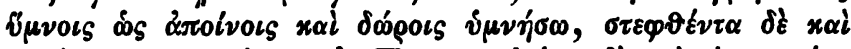

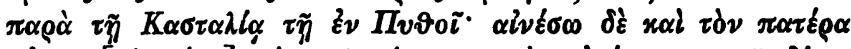

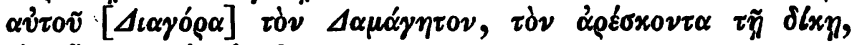

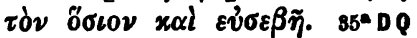

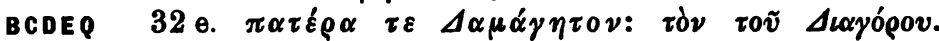

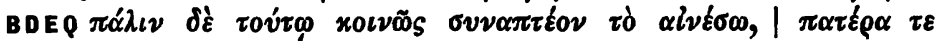

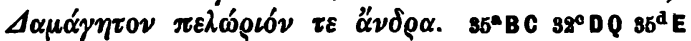

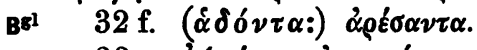

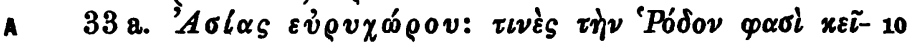

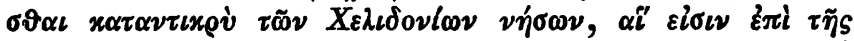

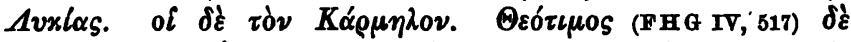

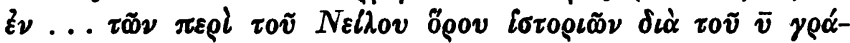

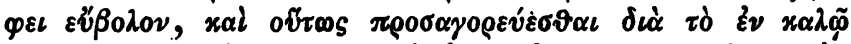

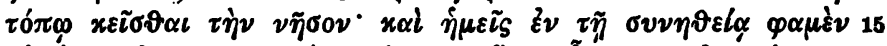

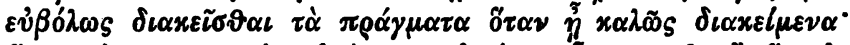

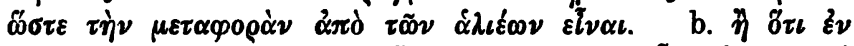

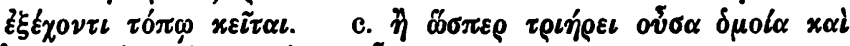

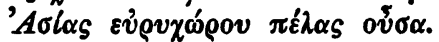

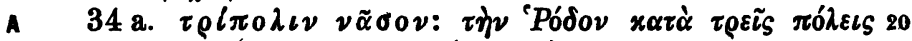

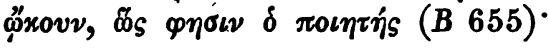

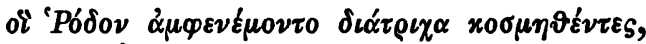

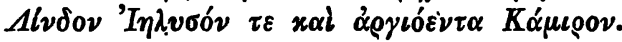

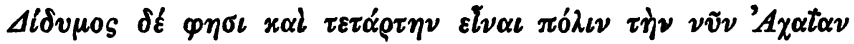
$x \alpha \lambda \circ v \mu \varepsilon \dot{v} \eta \nu$.

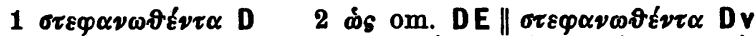

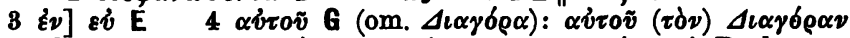

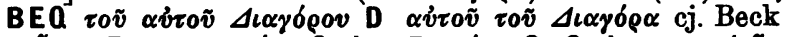

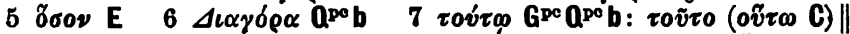

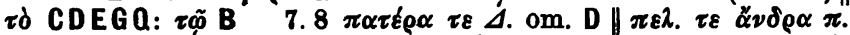

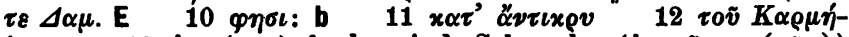
2ov? $13 \dot{\varepsilon} v \tau\left(\tilde{\omega} \nu\right.$ ?) A: lac. ind. Schroeder ( $\left.\xi_{\nu} \tau \tilde{\omega} \ldots\langle\tau \tilde{\omega} \nu\rangle\right)$ ह่ข $\tau \tilde{\omega}$ b $\tau \tilde{\omega} \nu$ Neilov $\tilde{0} \rho \omega \nu \| \bar{v} b: \bar{\mu}$ A $\bar{\nu}$ Mommsen suppl. p. 70, servato

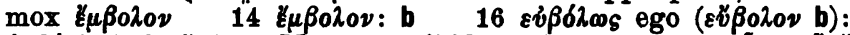

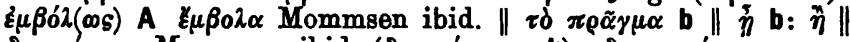

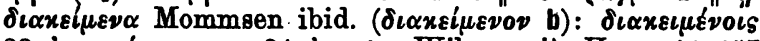

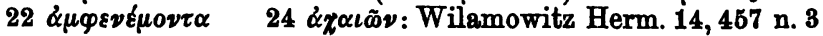




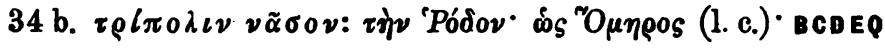

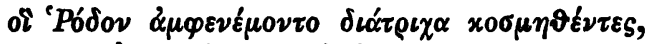

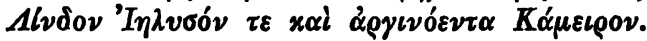

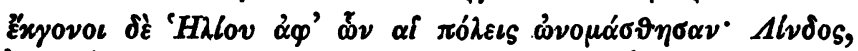

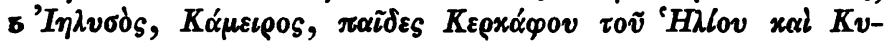

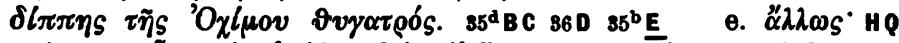

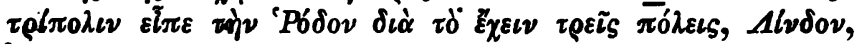

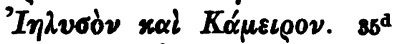

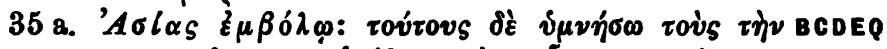

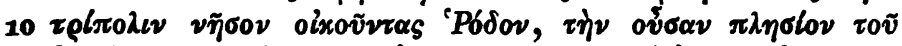

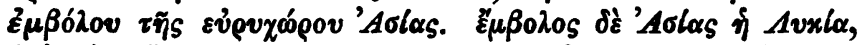

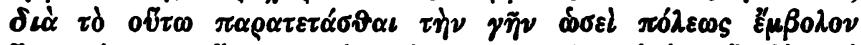

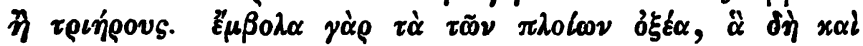

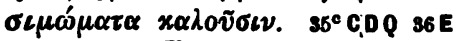

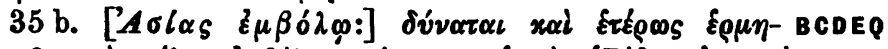

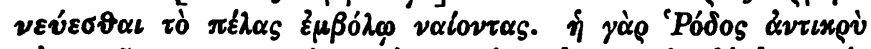

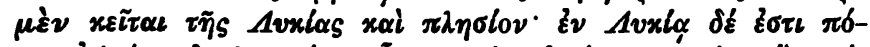

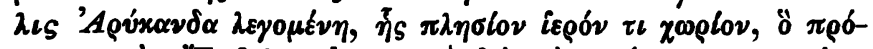

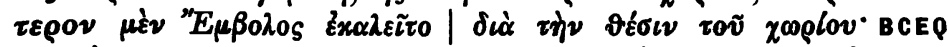

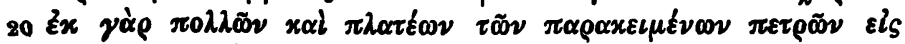

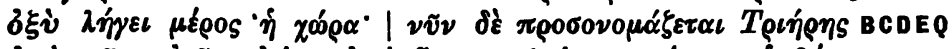

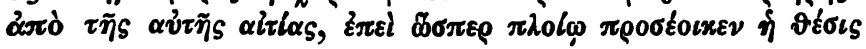

4 Strab. 14, 654. Diod. 5, 57. St. Byz. Aívoog. Káulpos. Eust. II. 315, 28. Tzetzes ad Lyc. 923. 11 Eust. Od. 1405, 20. 48 sq.

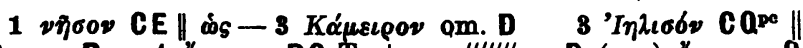

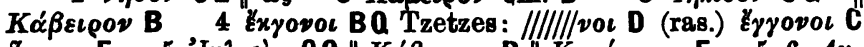

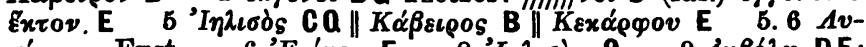

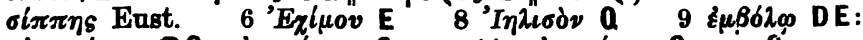

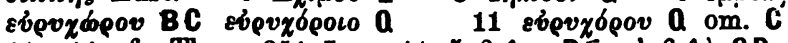

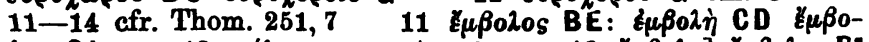

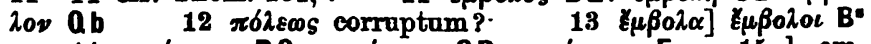

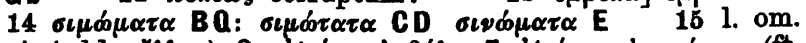

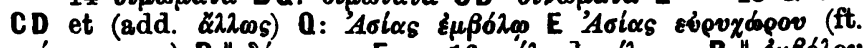

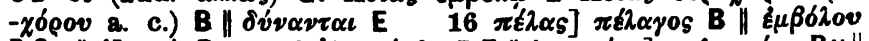

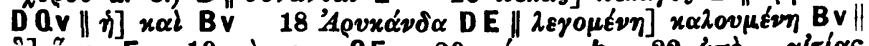

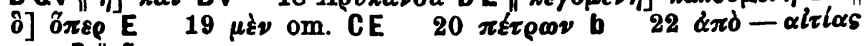

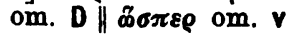




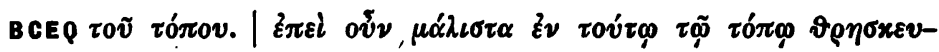

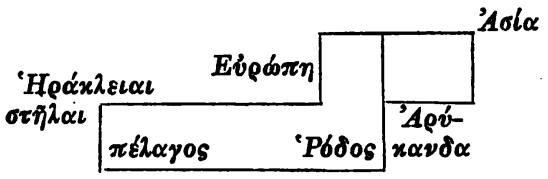

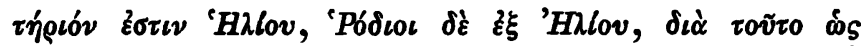

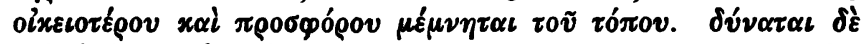

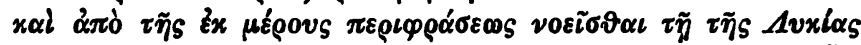

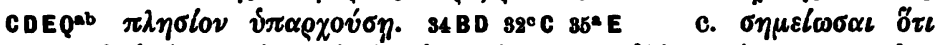

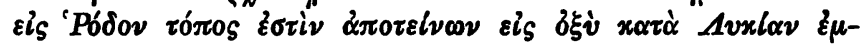
$\beta o ́ \lambda \omega \pi \alpha \rho \alpha \lambda \dot{\eta} \sigma \iota s$ s. $35 \mathrm{~b} \mathrm{CDQ} Q^{\mathrm{a}} 31 \mathrm{E} 34 \mathrm{Q}^{\mathrm{b}}$

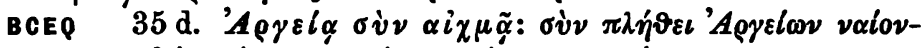

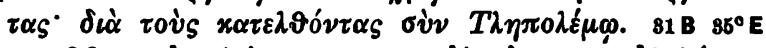

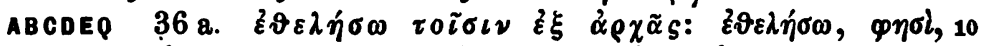

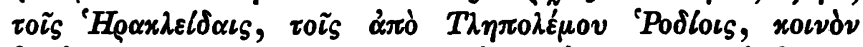

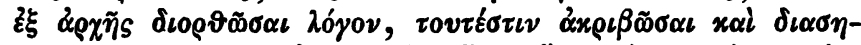

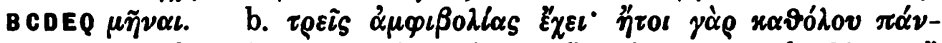

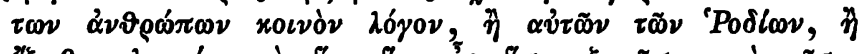

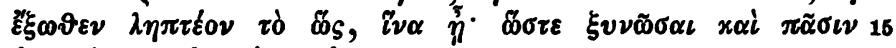

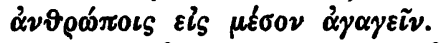

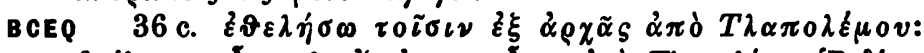

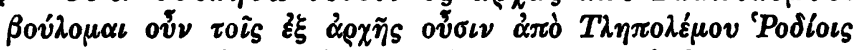

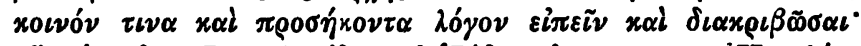

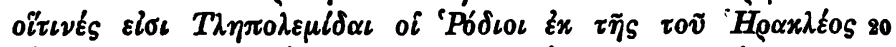

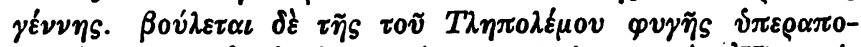

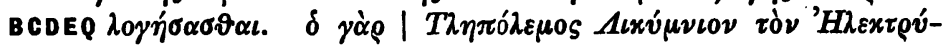

$1 \tau \delta \pi 0 v] \pi \delta \vartheta 0 v$ E \| figuram adpictam hab. BEG

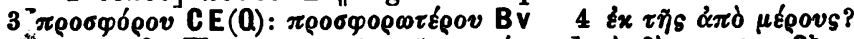

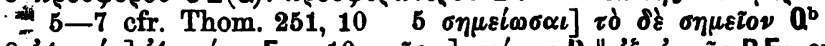
8 'A

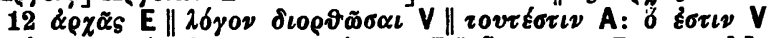

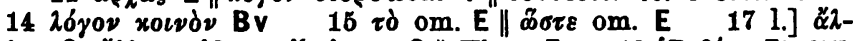

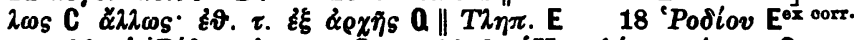

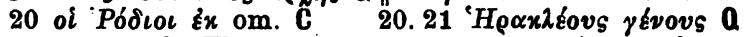

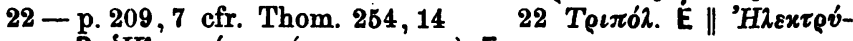
ovos D ' $H \lambda \varepsilon x \tau \rho v ́ \omega \nu \alpha$ (corr. ex -0s) E 


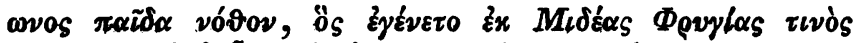

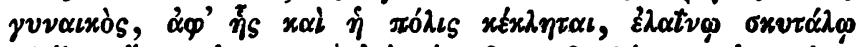

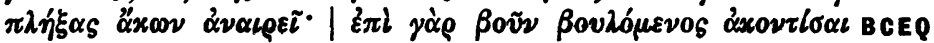

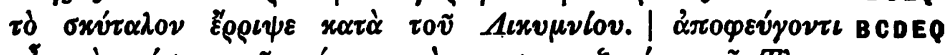

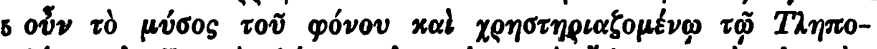

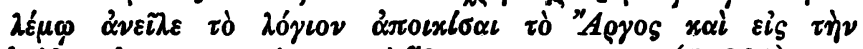

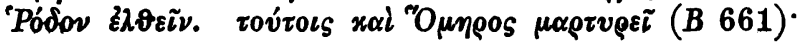

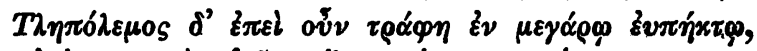

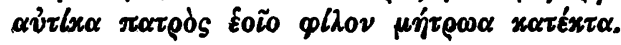

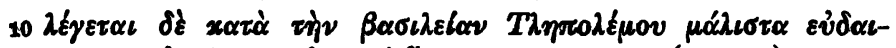

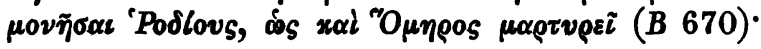

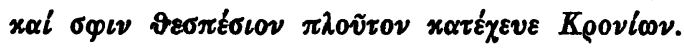

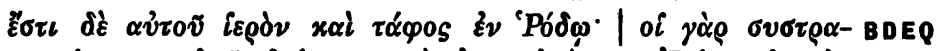

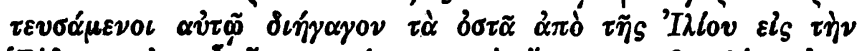

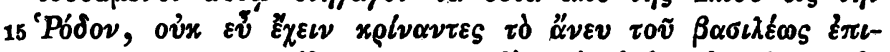

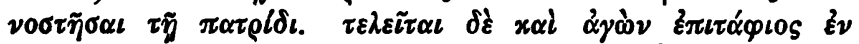

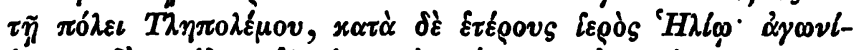

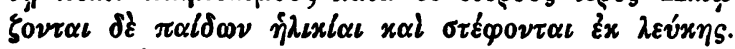

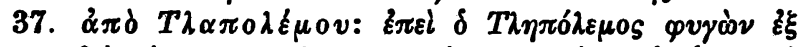

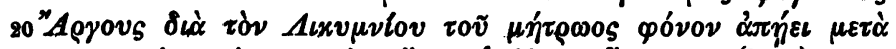

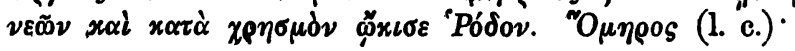

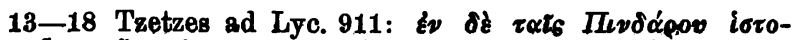

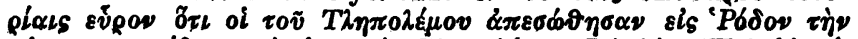

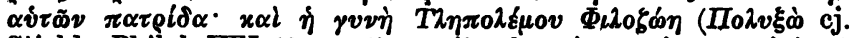

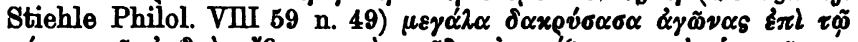

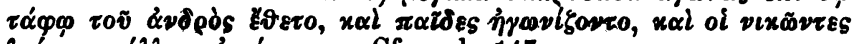

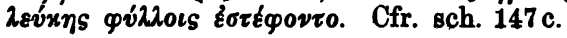

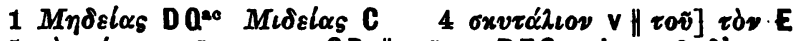

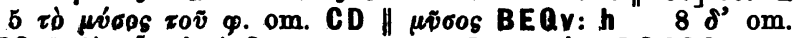

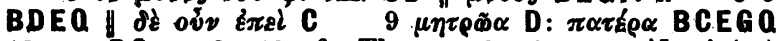
12 .opi. BQ 13-18 cfr. Thom. 272, 17 14 $\tau \dot{\alpha}] \alpha \dot{v} \tau(o \tilde{v}) \tau \dot{\alpha} \mathrm{D}$

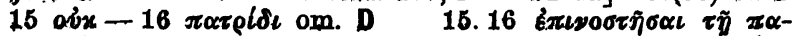

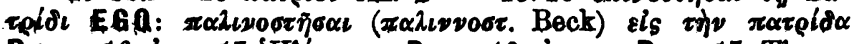

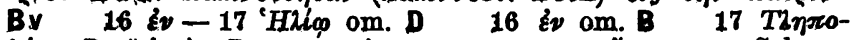

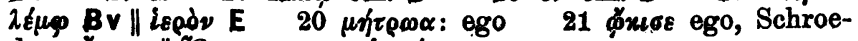

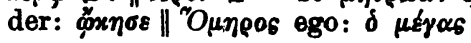

Bchol. nx Purdaruz ed. Drachmann. 


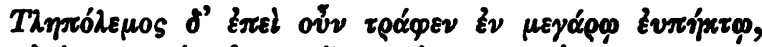

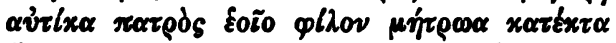

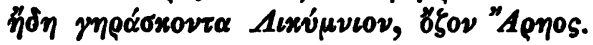

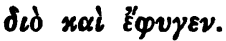

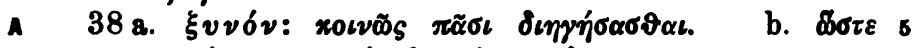

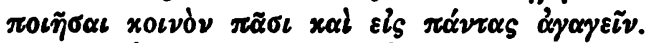

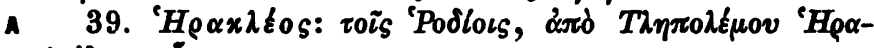

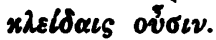

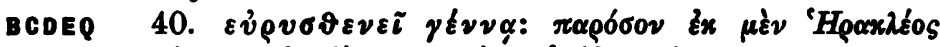

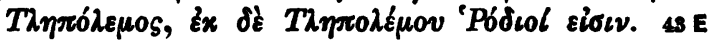

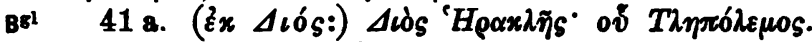

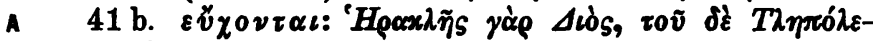

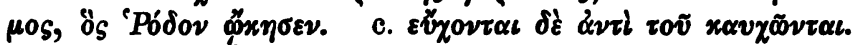

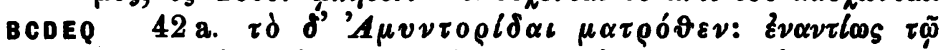

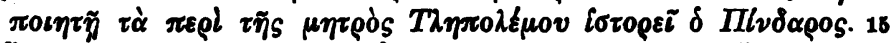

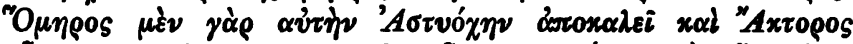

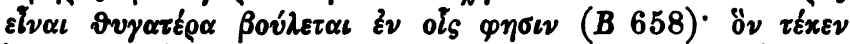

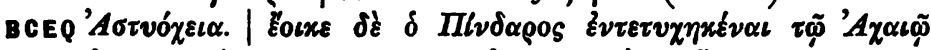

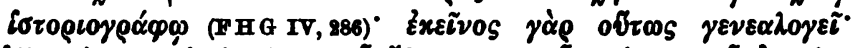

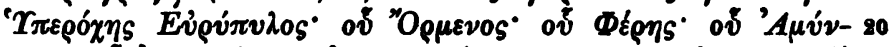

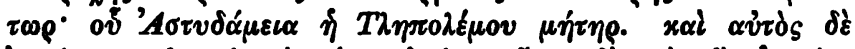

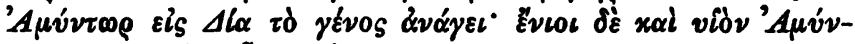

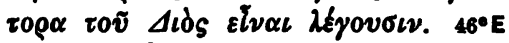

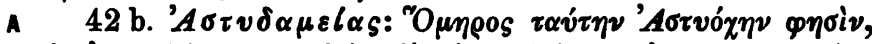

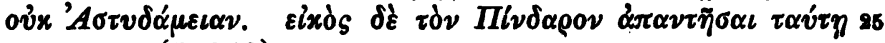
$\tau \tilde{\eta}$

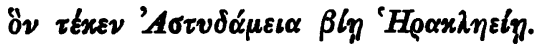

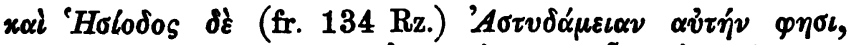

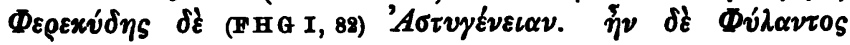

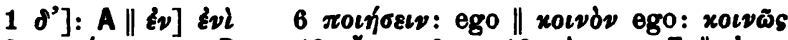

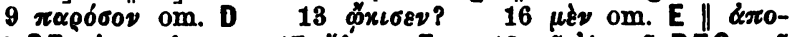

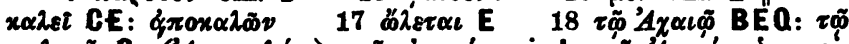

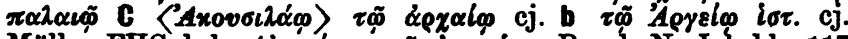

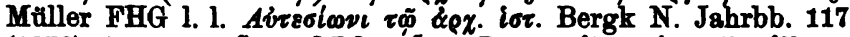

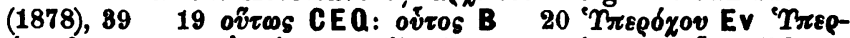

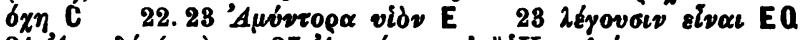

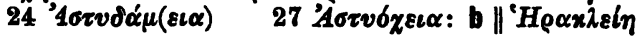




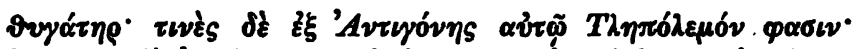

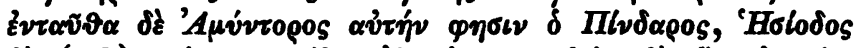

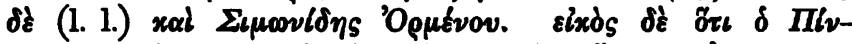

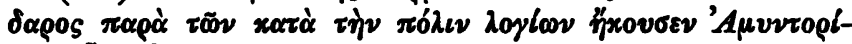

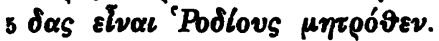

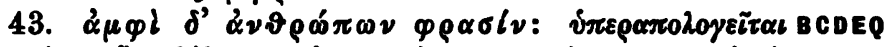

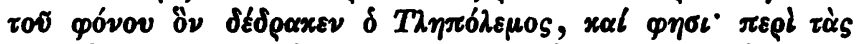

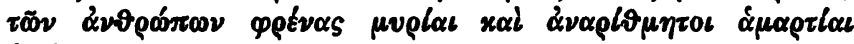

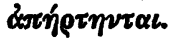

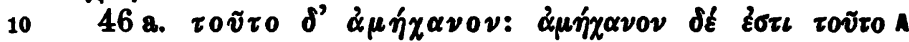

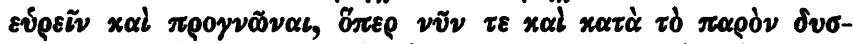

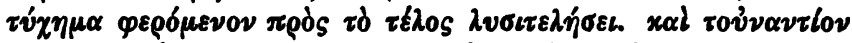

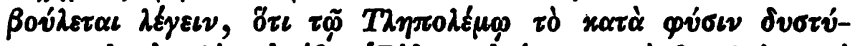

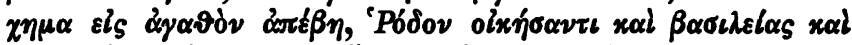

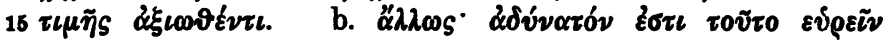

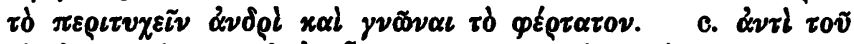

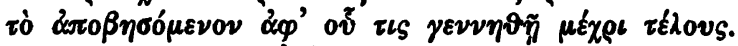

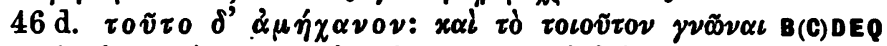

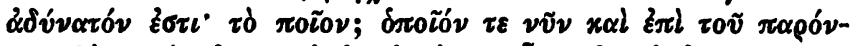

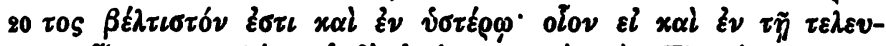

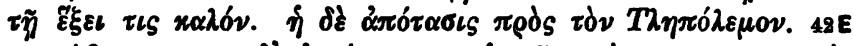

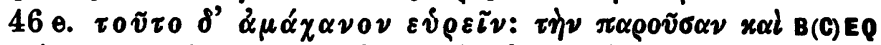

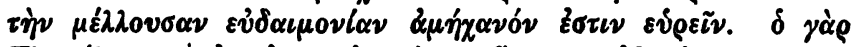

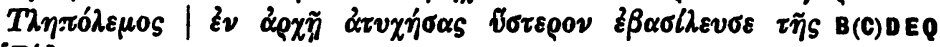
$25{ }^{\mathrm{C}} \mathrm{Pó} \delta$ ov.

48. ( $(v \chi \varepsilon \tilde{\imath} \nu:) \sigma v \mu \beta \tilde{\eta} \nu \alpha \iota$. Bel

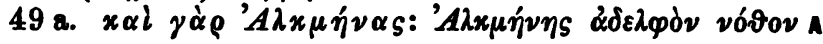

27-p. 212, 5 Apollod. 2, 4, 4. Tzetzes ad Lyc. 932

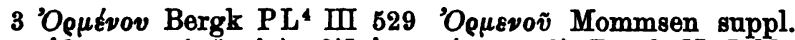

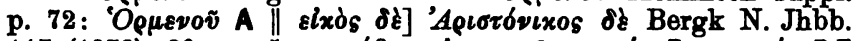

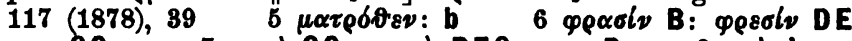

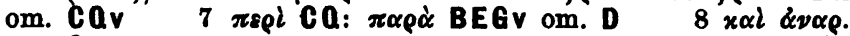

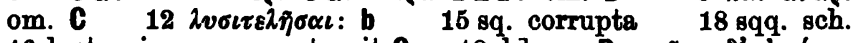
$46 \mathrm{~d}$ et $e$ in unum contraxit C 18 l.] om. D rovio $\delta^{\prime} \alpha \mu \alpha^{\prime} \chi^{\alpha-}$

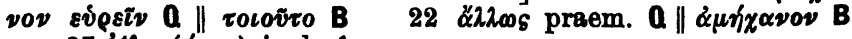
$27 ' A \lambda \mu \mu(\eta v \alpha s)$ in 1 . A 


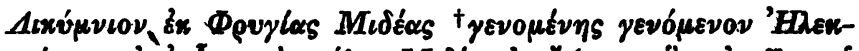

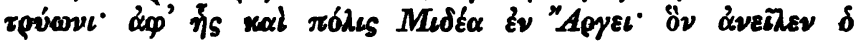

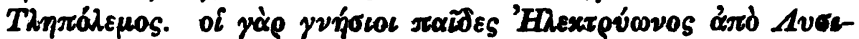

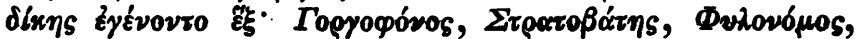

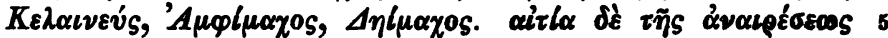

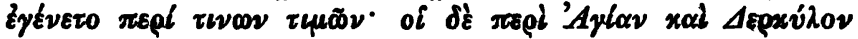
(FHG IV, 386)

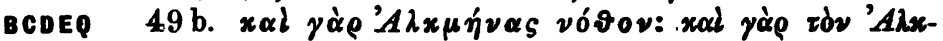

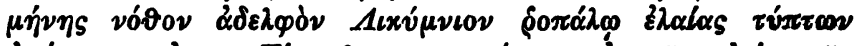

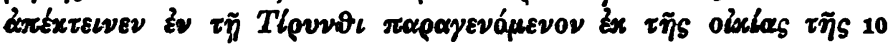

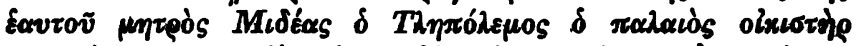

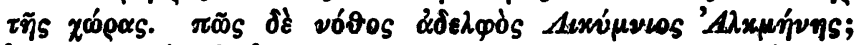

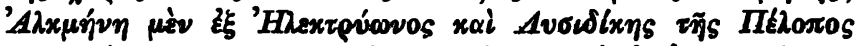

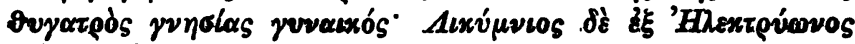

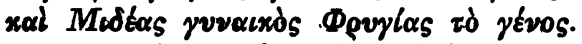

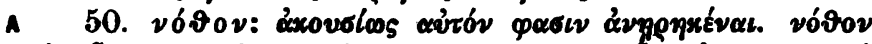

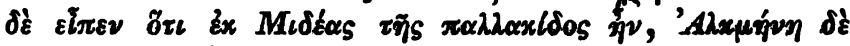

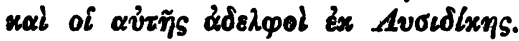

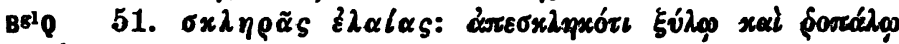
Elatup.

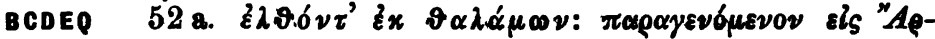

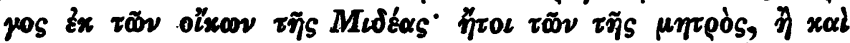

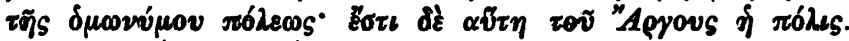

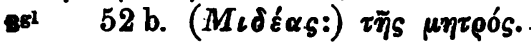

4 sch. T T 116

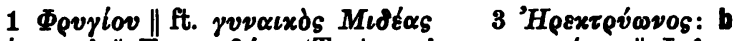

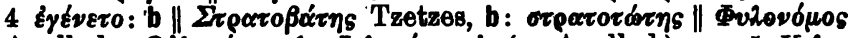

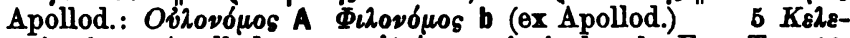
ขev́s: b ex Apollod. $6{ }^{\prime} A \gamma i \alpha \nu$ scripsi cl. sch. Eur. Tro. 16.

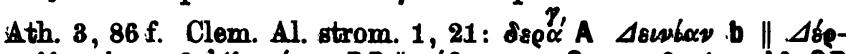

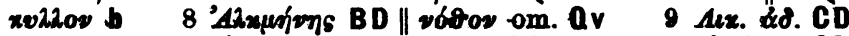

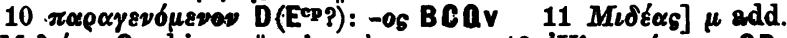

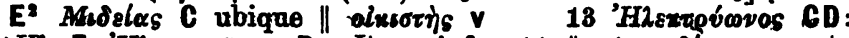

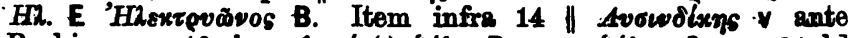

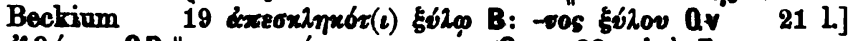

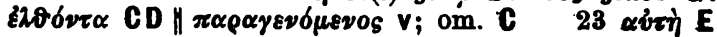




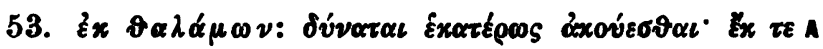

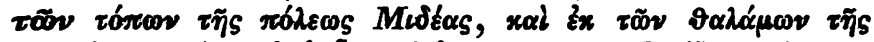

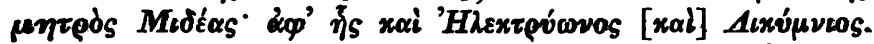

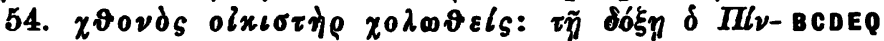

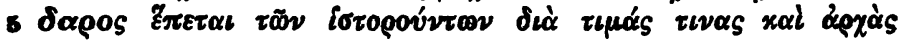

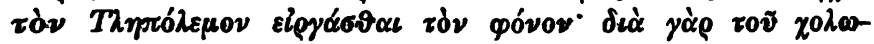

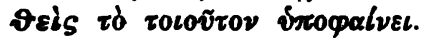

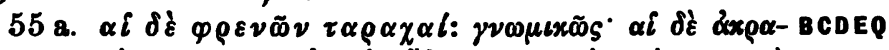

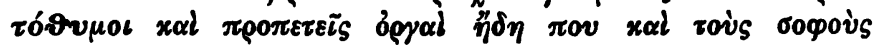

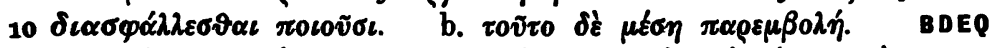

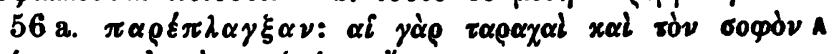

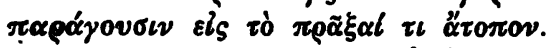

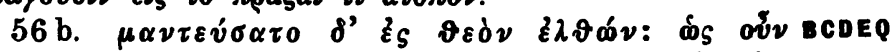

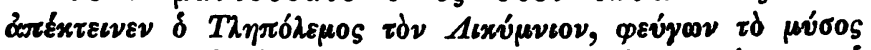

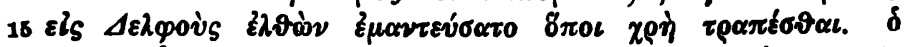

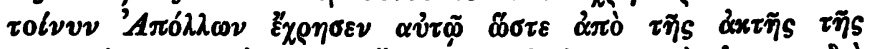

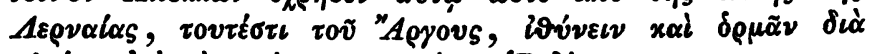

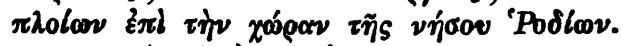

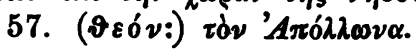

Bel

80

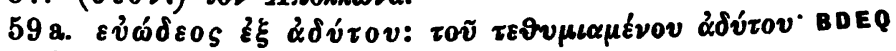

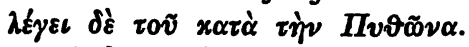

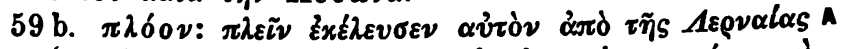

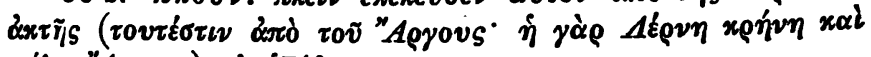

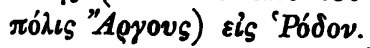

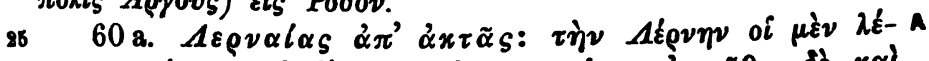

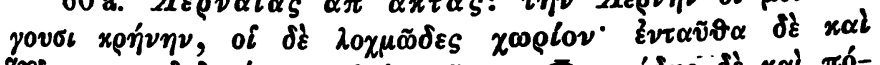

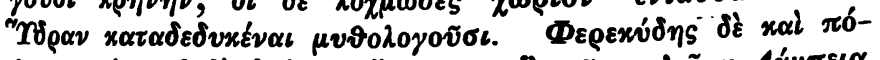

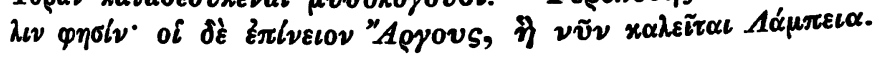

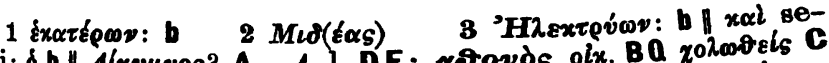

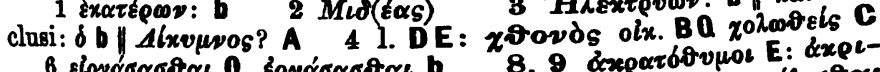

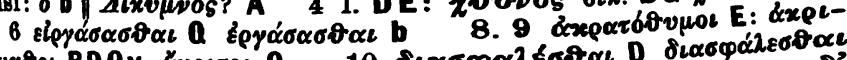

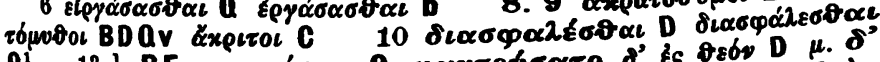

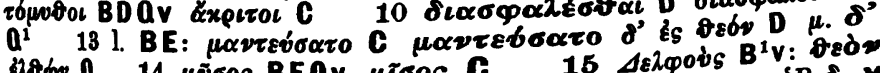

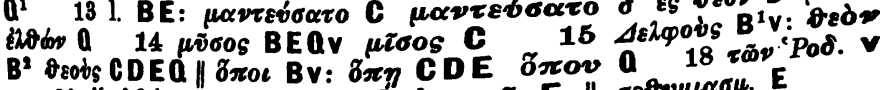

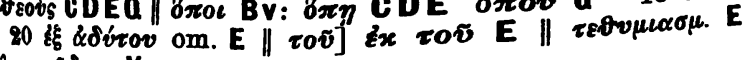

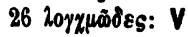




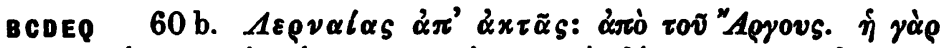

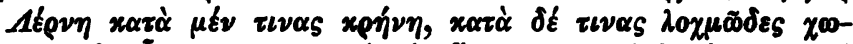

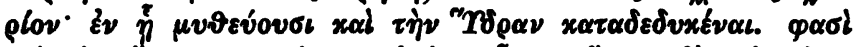

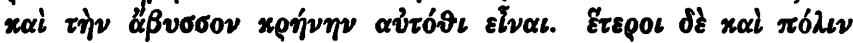

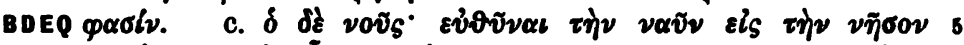

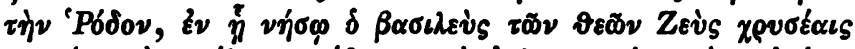

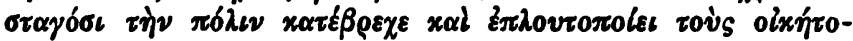

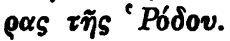

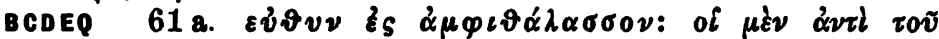

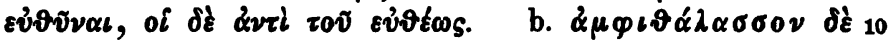

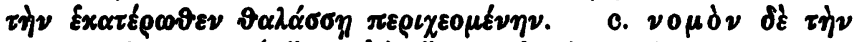

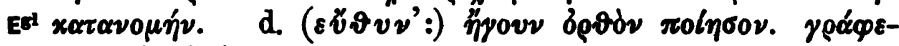

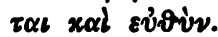

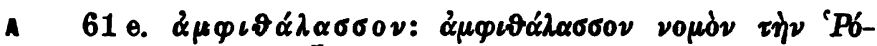

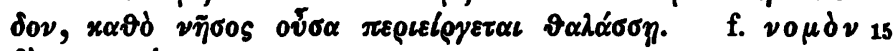
$\delta \xi, x \alpha \tau \alpha v \varepsilon \mu \eta \sigma \iota v$.

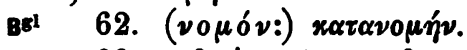

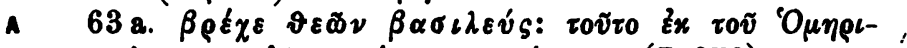

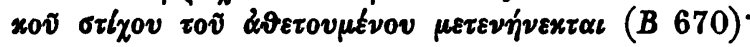

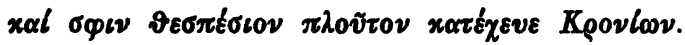

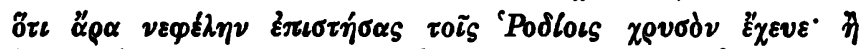

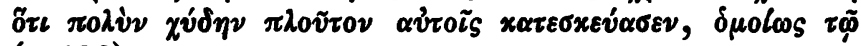
(o 426).

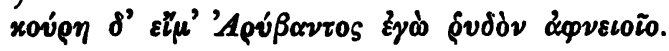

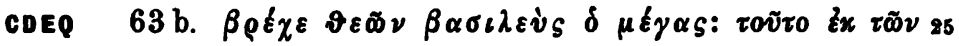

26 sch. A B 670. Strab. 14, 655

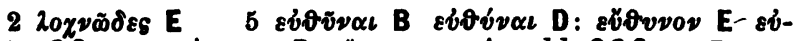

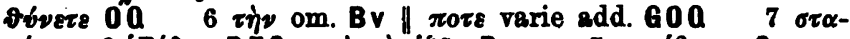

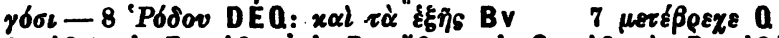

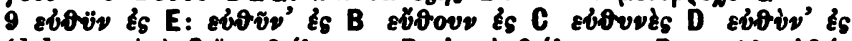

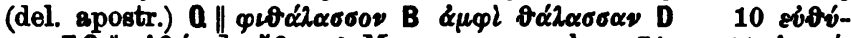

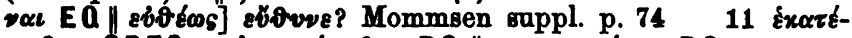

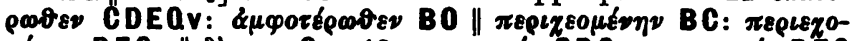

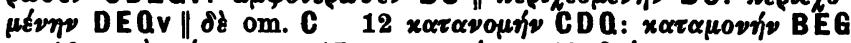

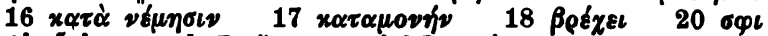

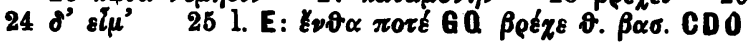




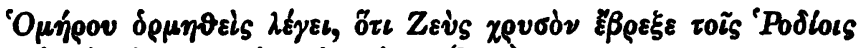

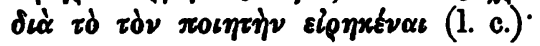

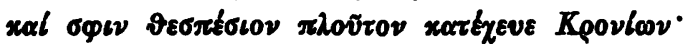

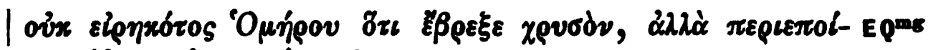

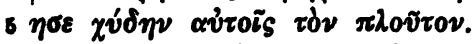

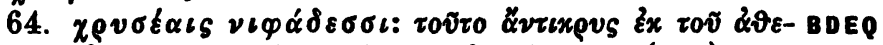

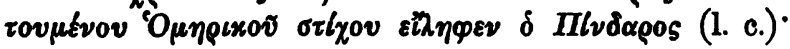

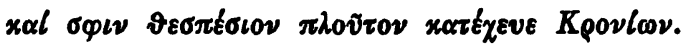

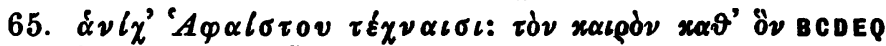

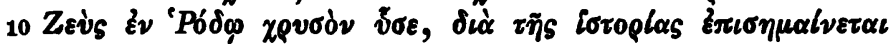

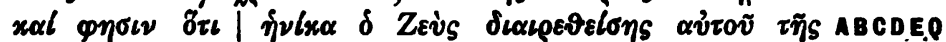

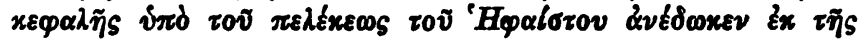

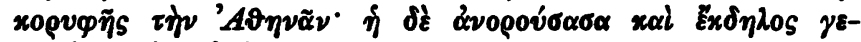
$\nu 0 \mu \varepsilon \nu \eta \mu \xi \gamma \alpha \varepsilon \beta \delta \eta \sigma \varepsilon v$.

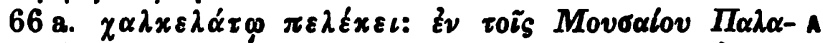

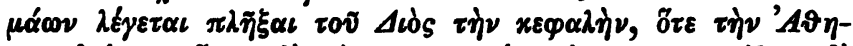

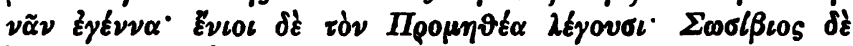

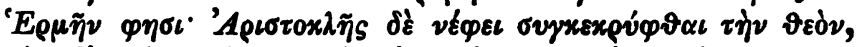

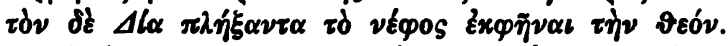

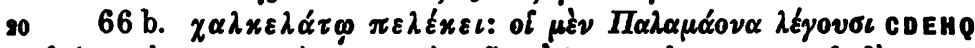

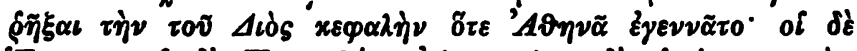

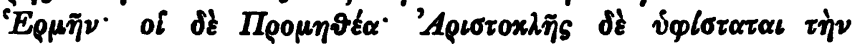

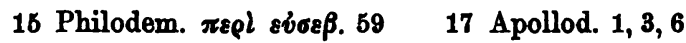

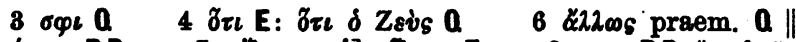

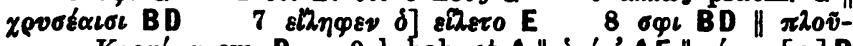

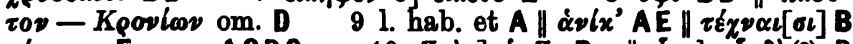

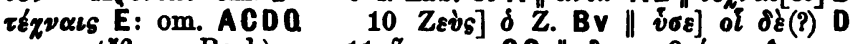

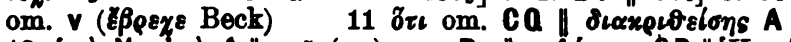

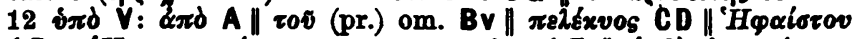

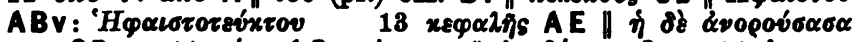

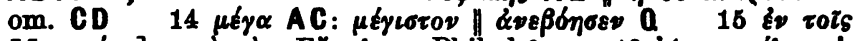

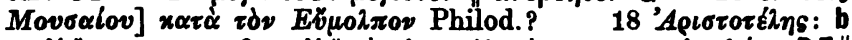

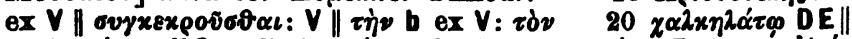

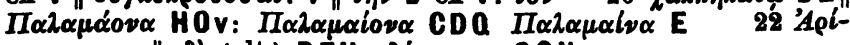

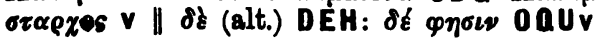




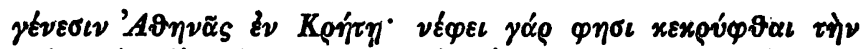

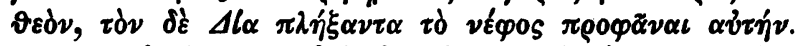

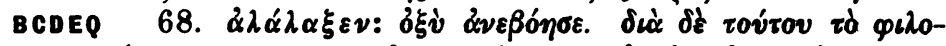

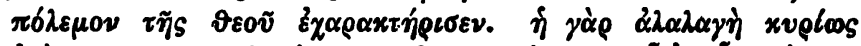

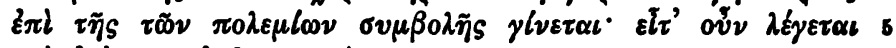

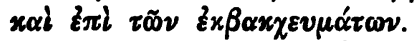

A 70 a. Ỏ̉ 

CGOQ 70 b. O

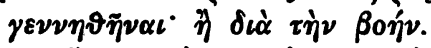

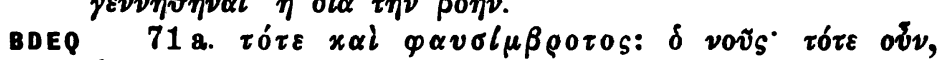

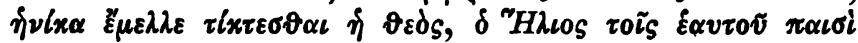

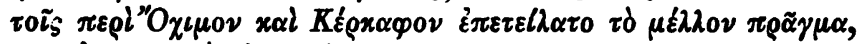

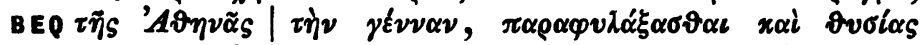

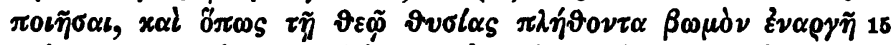

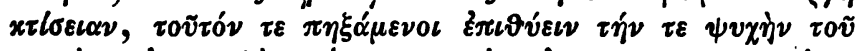

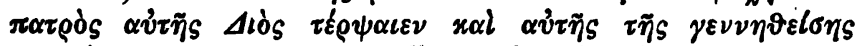

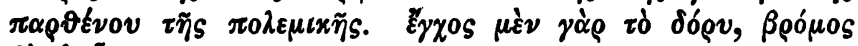
$\delta \dot{\varepsilon} \delta \dot{\eta} \chi 0 \mathrm{~s}$.

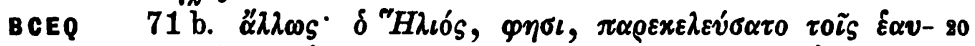

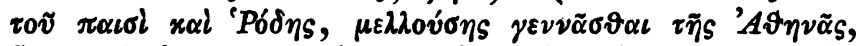

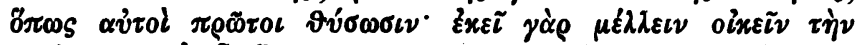

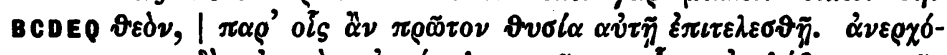

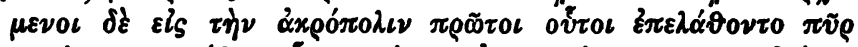

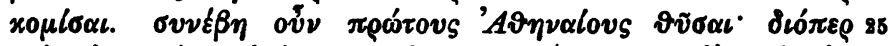

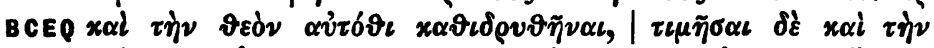

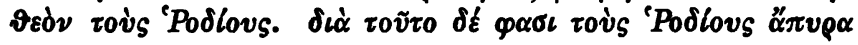

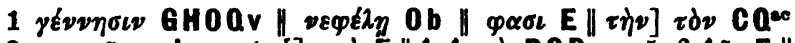

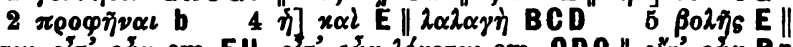

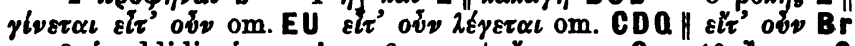
$8 \delta$ addidi; $\eta$ om. b $9 \nu v \nu$ et भैं

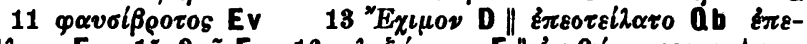

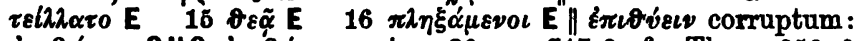

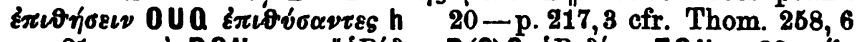

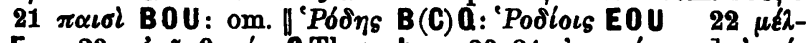

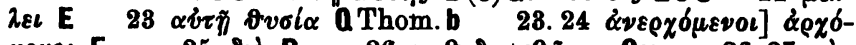

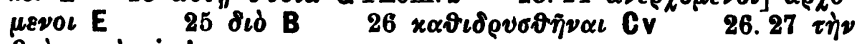

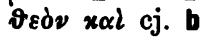




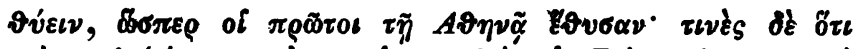

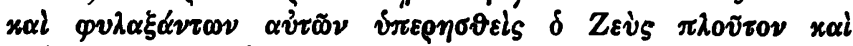

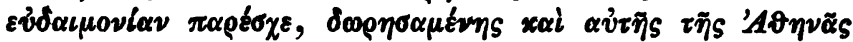

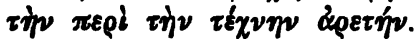

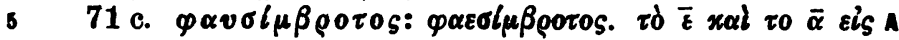

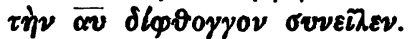

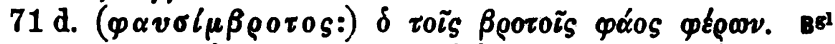

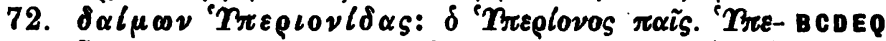

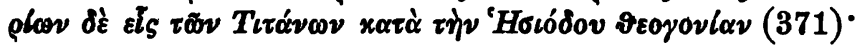

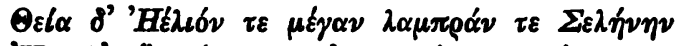

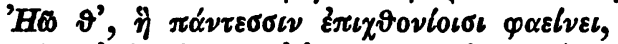

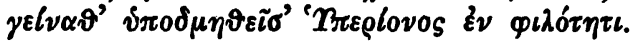

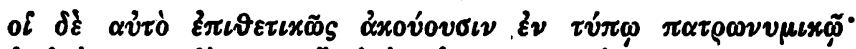

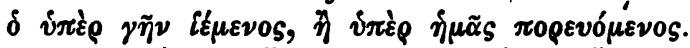

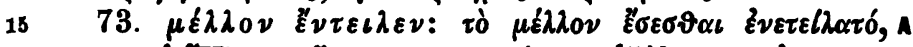

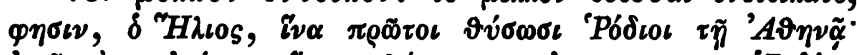

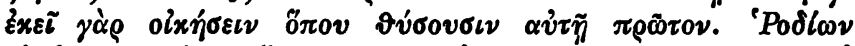

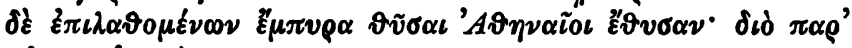

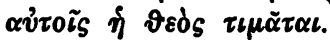

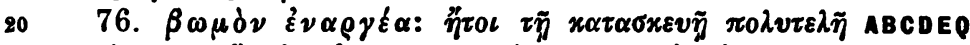

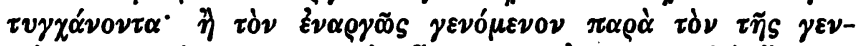

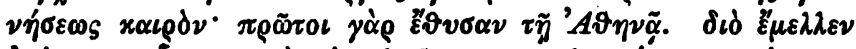

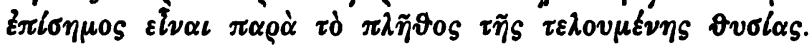

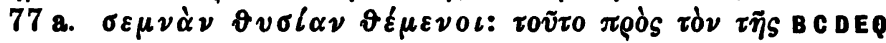

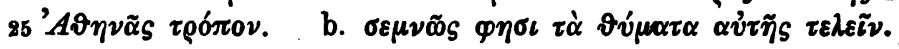

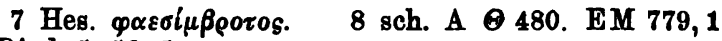
17 Diod. 5, 66, 5

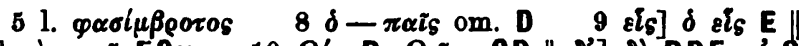

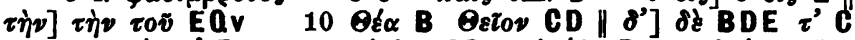

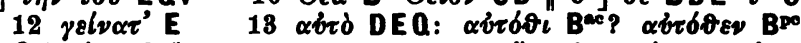

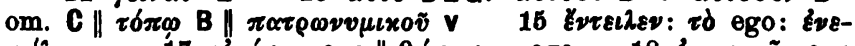

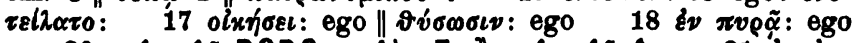

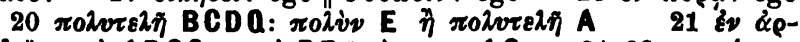

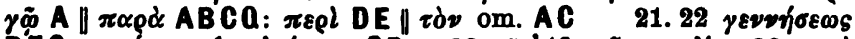

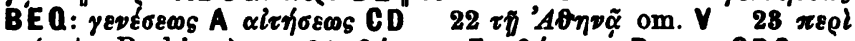
$v$ (ante Beckium) 24 ot $\mu \varepsilon v o i$ E: $\theta \varepsilon_{\mu \varepsilon \nu \alpha i}$ B om. CDQ

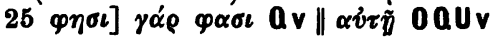




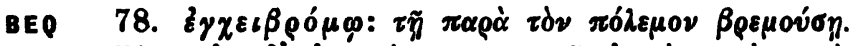

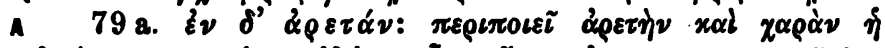

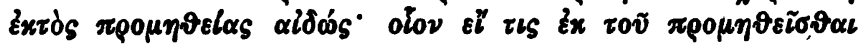

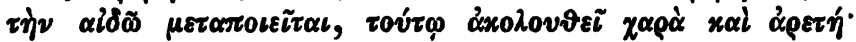

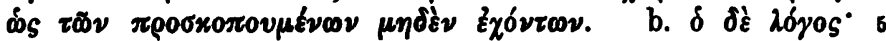

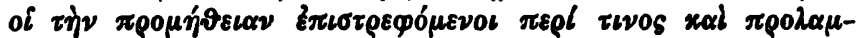

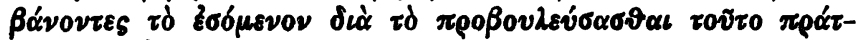

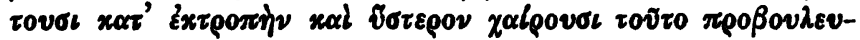

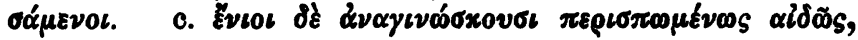

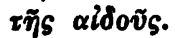

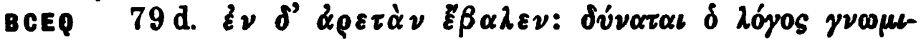

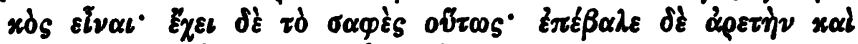

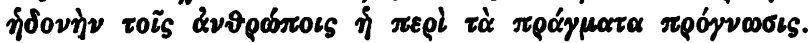

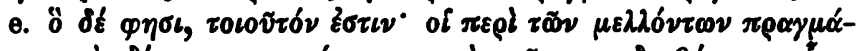

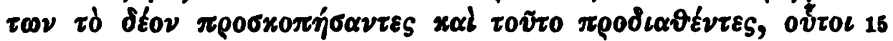

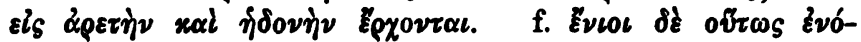

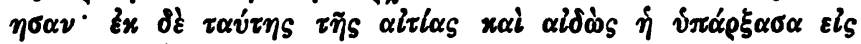

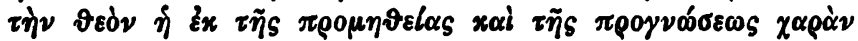

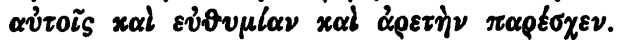

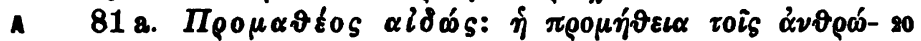

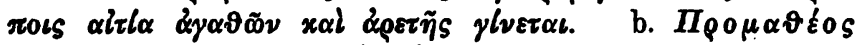

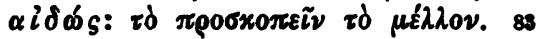

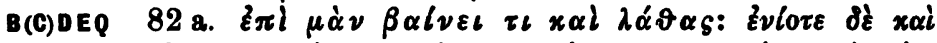

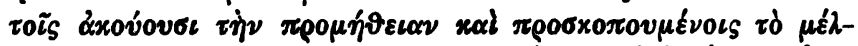

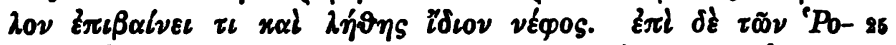

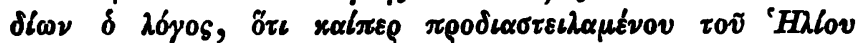

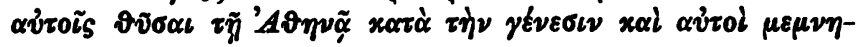

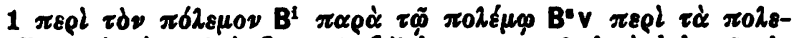

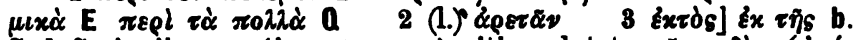

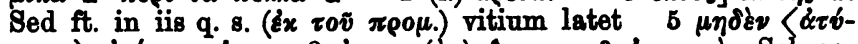

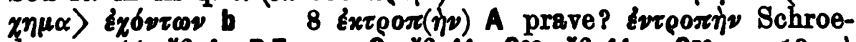

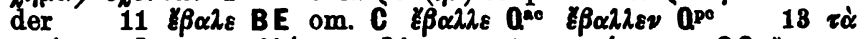

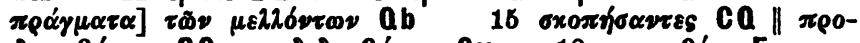

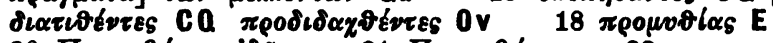

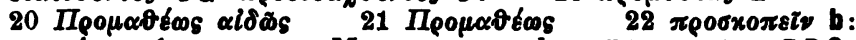

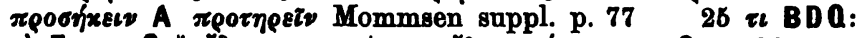

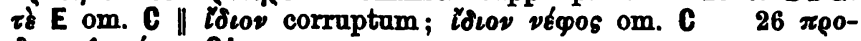

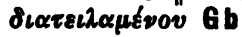




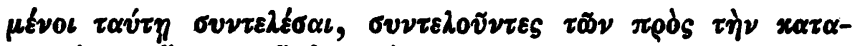

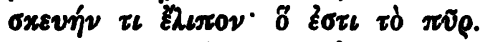

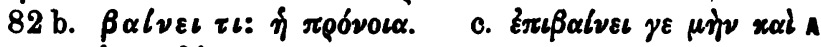

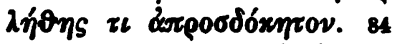

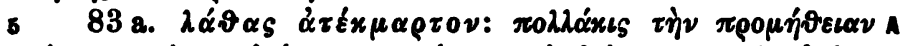

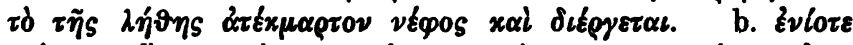

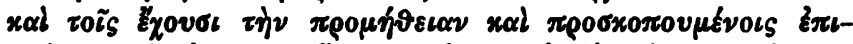

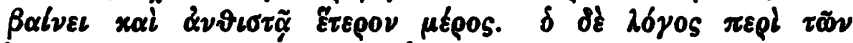

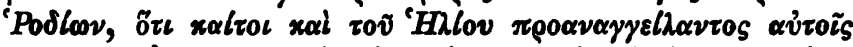

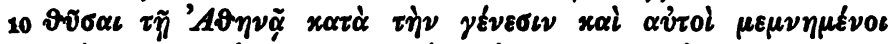

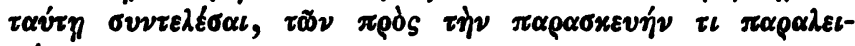

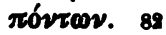

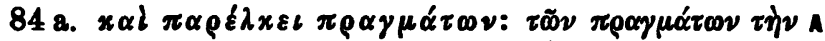

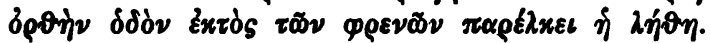

15

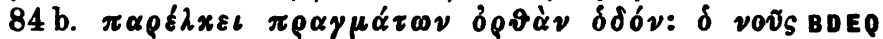

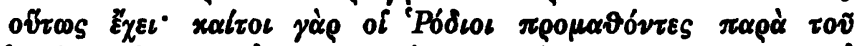

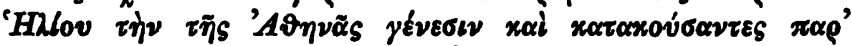

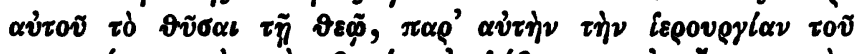

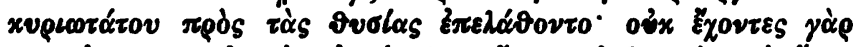

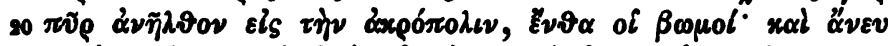

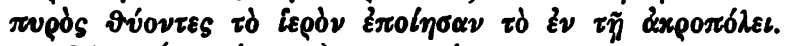

84 c. $(\pi \alpha \rho \varepsilon \lambda x \in \iota:) \pi \alpha \rho \alpha \tau \rho \varepsilon \pi \varepsilon \iota$.

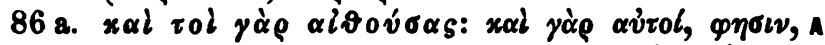

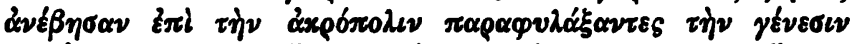

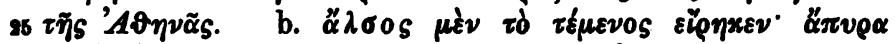

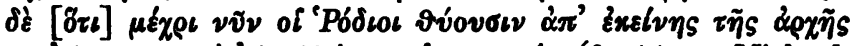

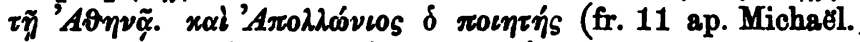

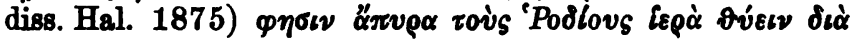

\section{6-27 Diod. 5, 66, 7}

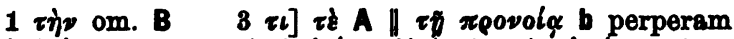

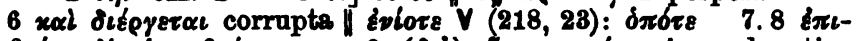

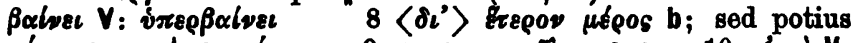

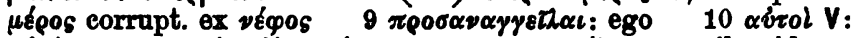

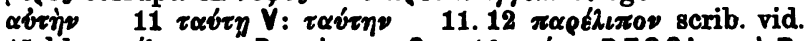

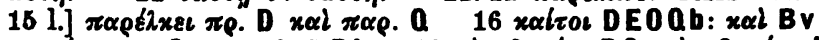

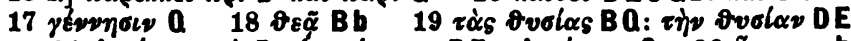

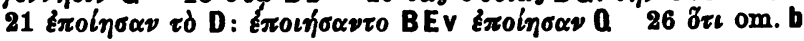




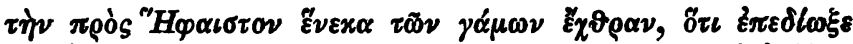

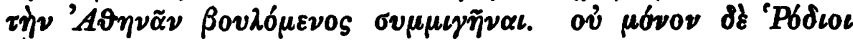

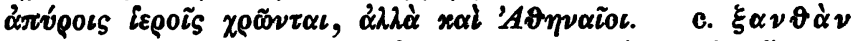

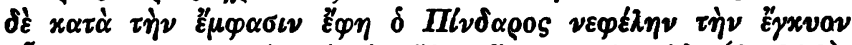

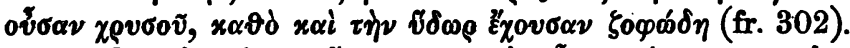

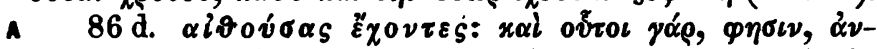

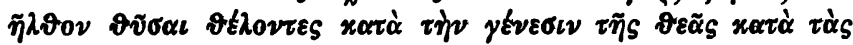

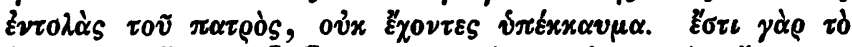

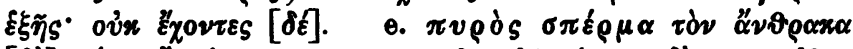

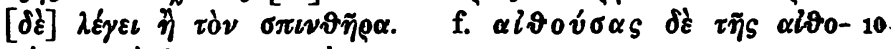

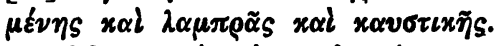

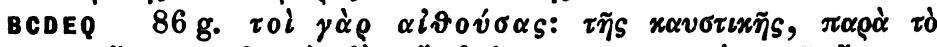

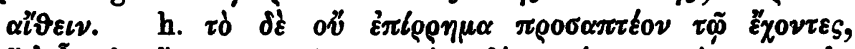

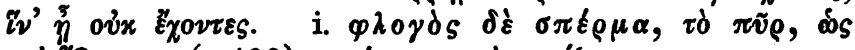

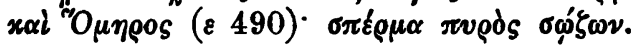

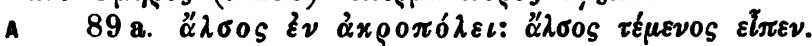

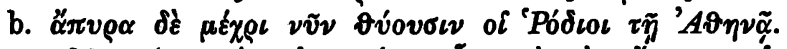

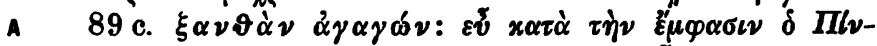

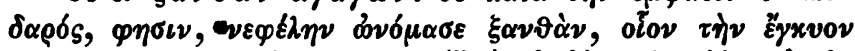

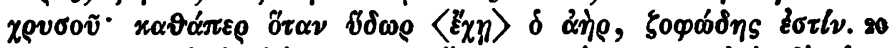

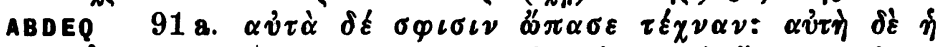

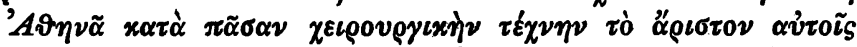

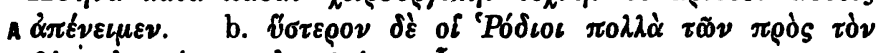

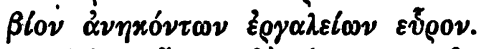

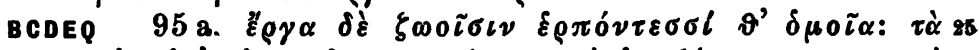

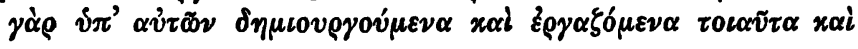

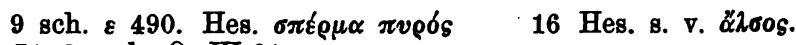
EM 71, 2. sch. 0. III 31

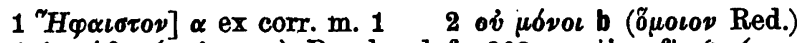

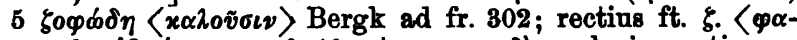

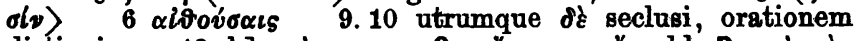

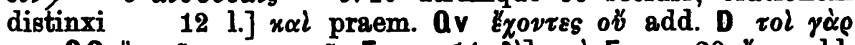

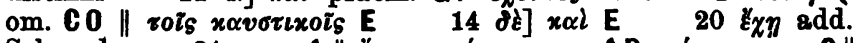

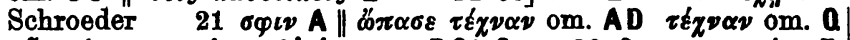

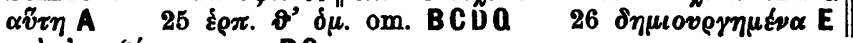

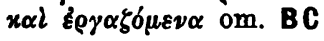




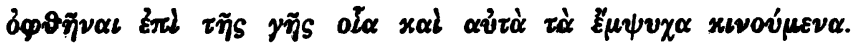

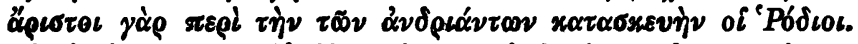

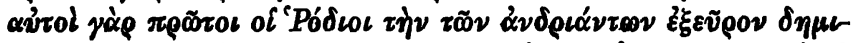

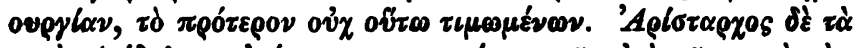

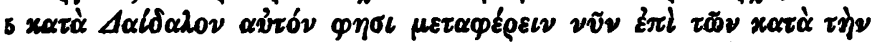

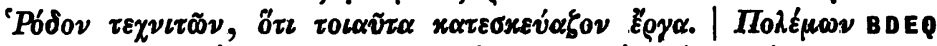

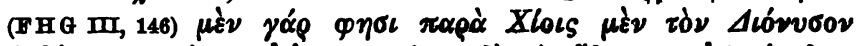

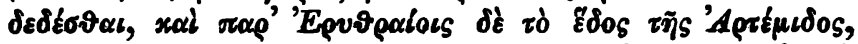

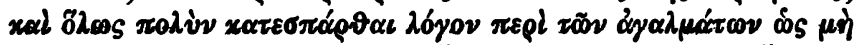

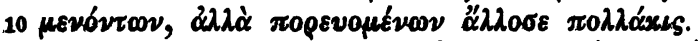

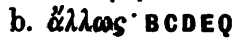

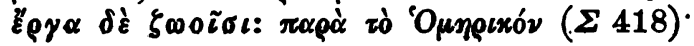

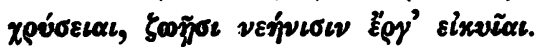

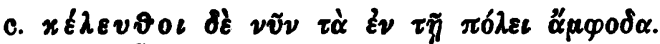

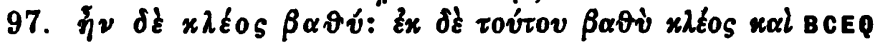

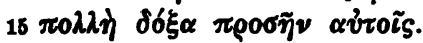

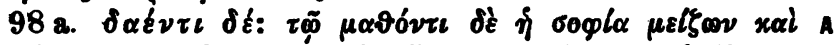

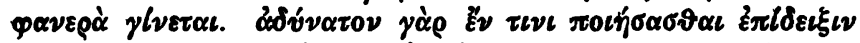

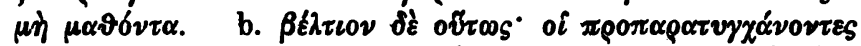

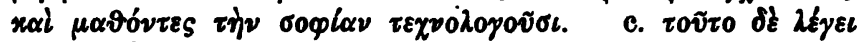

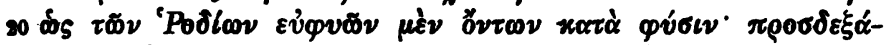

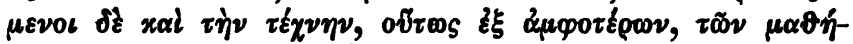

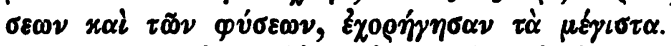

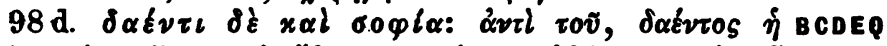

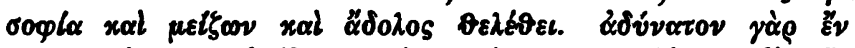

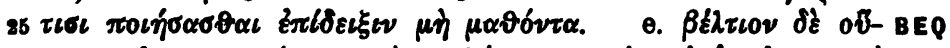

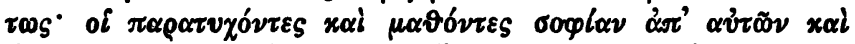

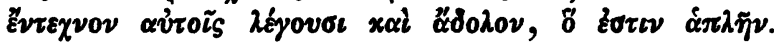

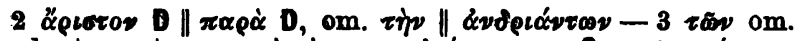

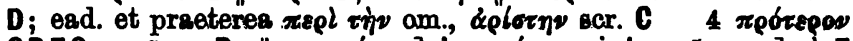

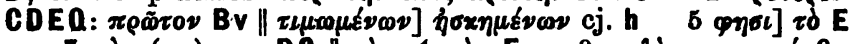

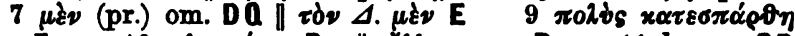

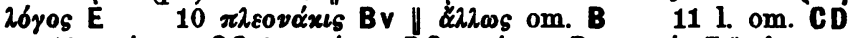

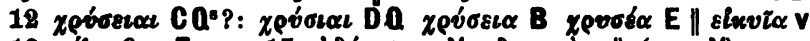

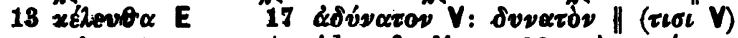

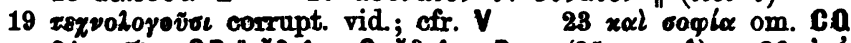

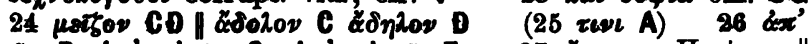

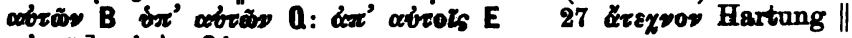

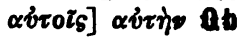




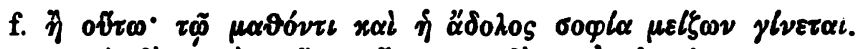

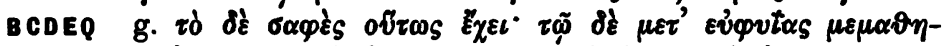

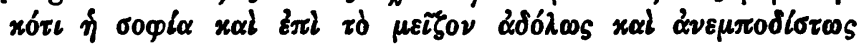

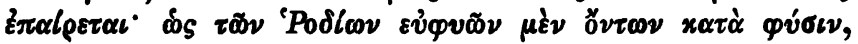

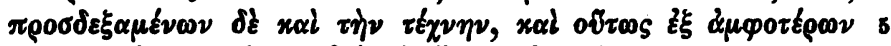

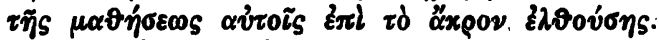

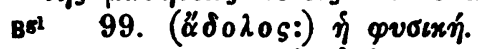

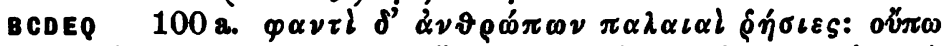

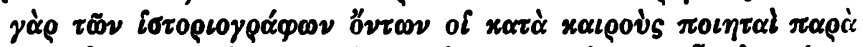

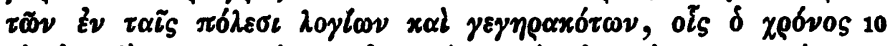

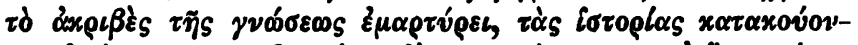

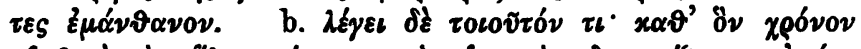

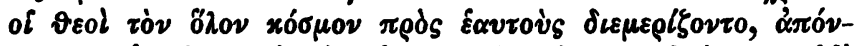

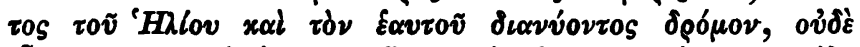

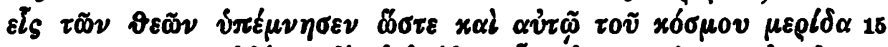

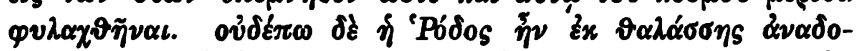

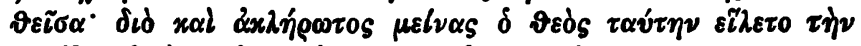

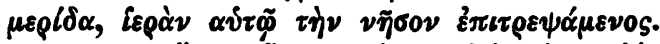

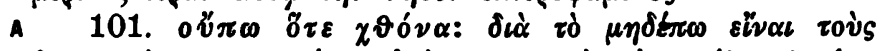

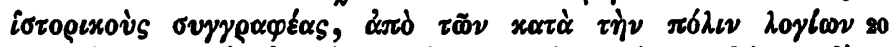

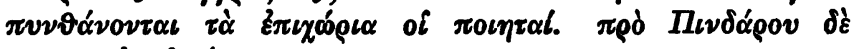

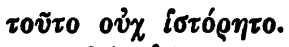

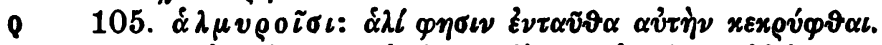

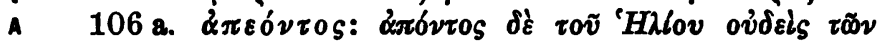

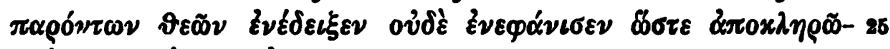

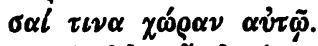

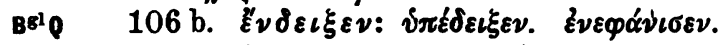

$B^{81} Q$ 107. $\lambda \alpha \dot{\alpha} \chi 0 \mathrm{~s}: x \lambda \tilde{\eta} \rho \circ \nu . \mu \varepsilon \rho l \delta \alpha$.

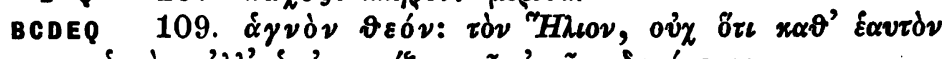

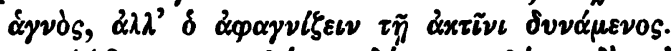

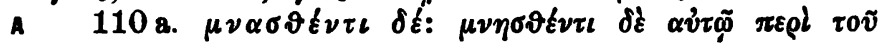

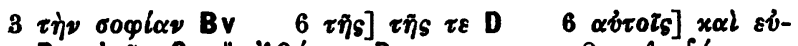

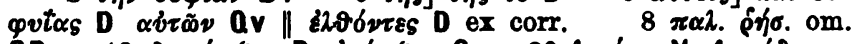

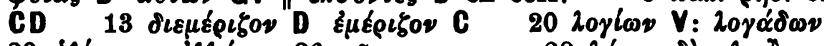

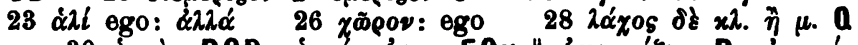

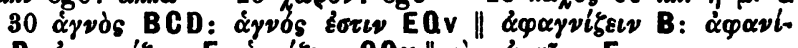

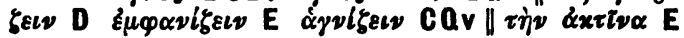




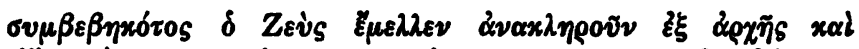

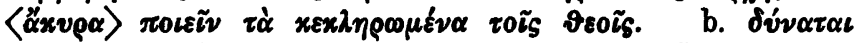

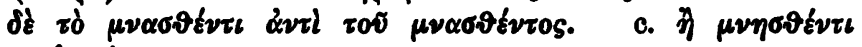

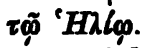

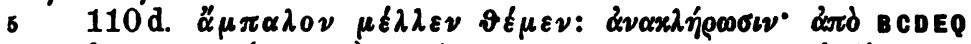

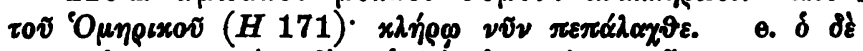

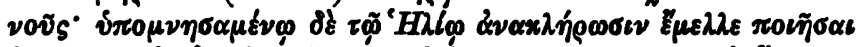

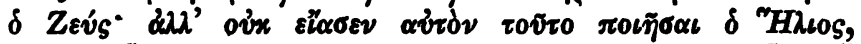

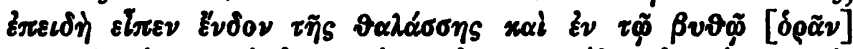

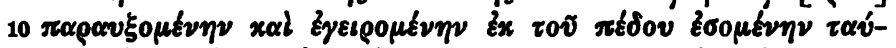

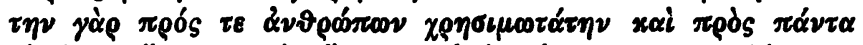

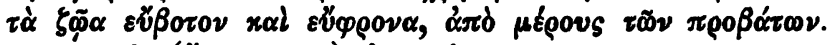

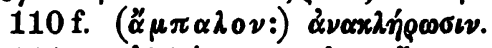

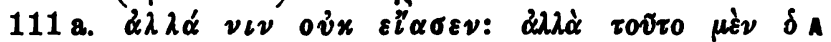

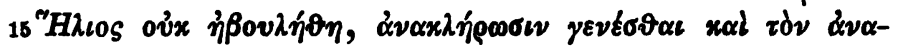

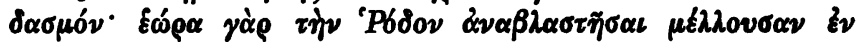

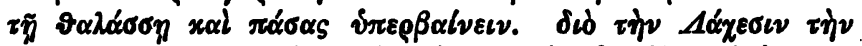

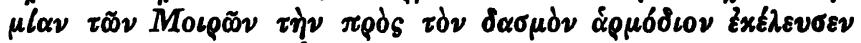

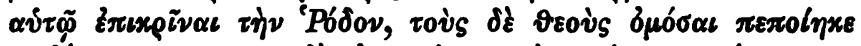

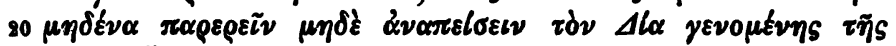

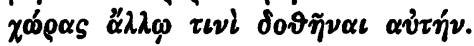

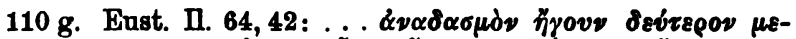

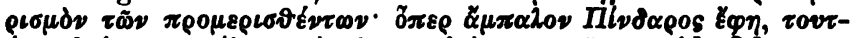

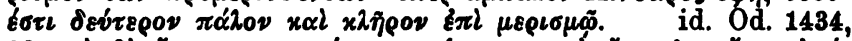

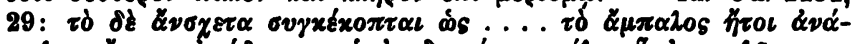

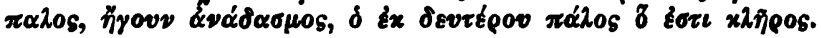

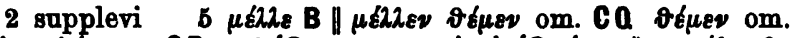

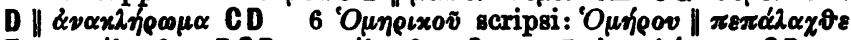

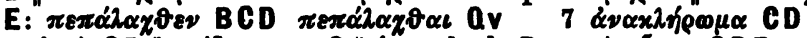

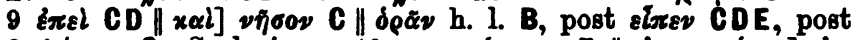

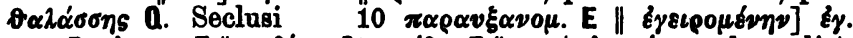

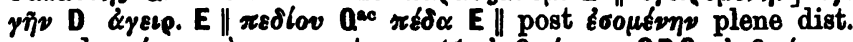

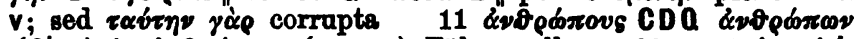

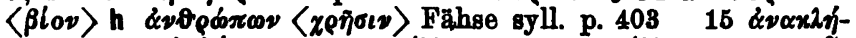

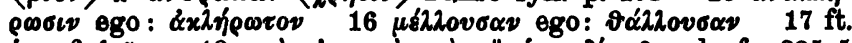

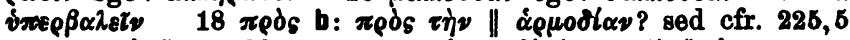

$19 \alpha \delta \tau \tilde{\omega} \quad 20 \pi \alpha \rho \alpha \iota \rho \varepsilon \tau$ : b ex V $(224,10) \| \& \nu \alpha \pi \varepsilon \tau \sigma \alpha \iota:$

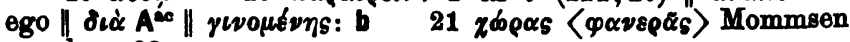
suppl. p. 83 


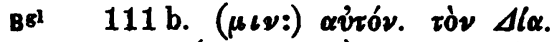

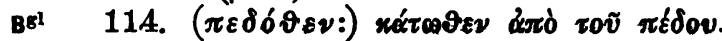

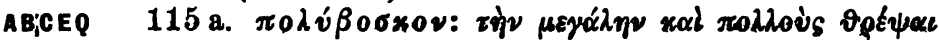

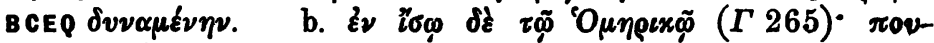

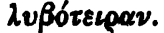

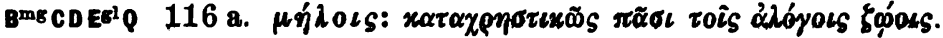

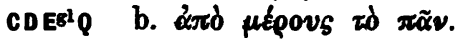

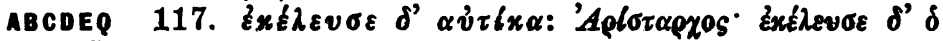

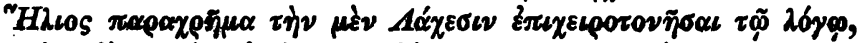

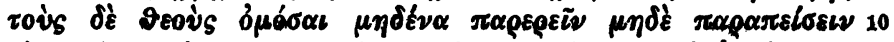

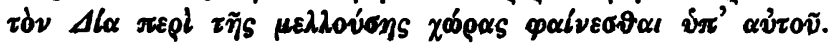

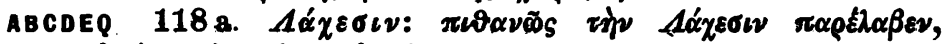

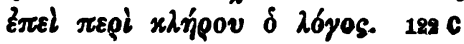

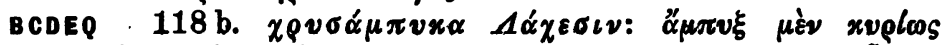

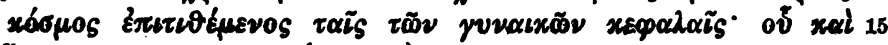

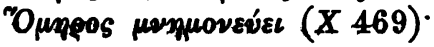

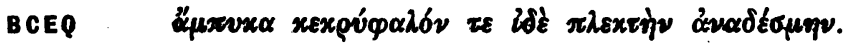

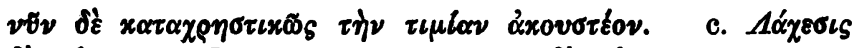

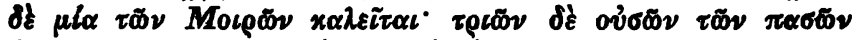

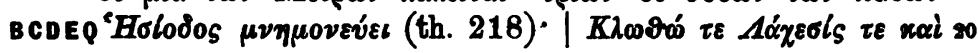

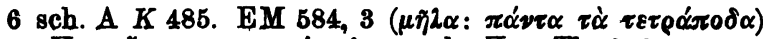

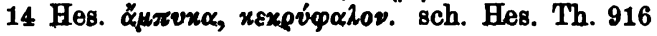

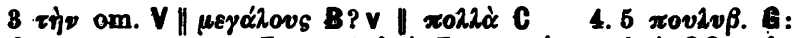

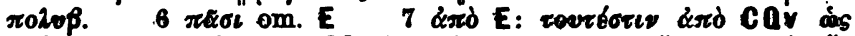

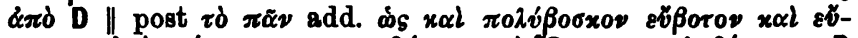

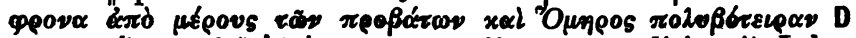

$8 x \leqslant$ xevor V \|'Aplorapyos om. V; a seqq. disiunrit Lehrs p. 8 ||

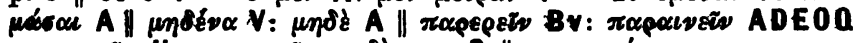

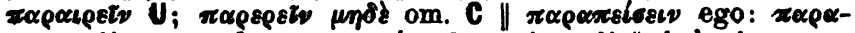

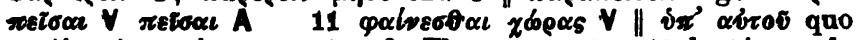
pertineat neseio $12-13 \mathrm{cfr}$. Thom. 268, 16121 . Á'xerev A: om. V, add. de post $\pi \omega \theta \alpha \nu \tilde{s} ;$ daloss V

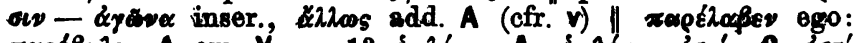

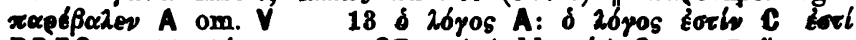

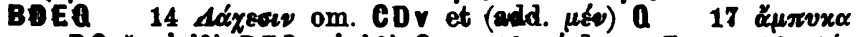

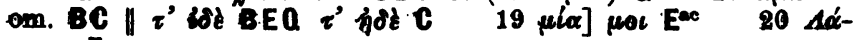

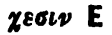




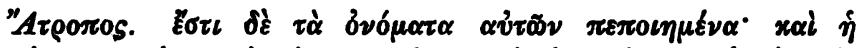

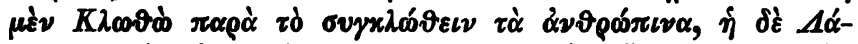

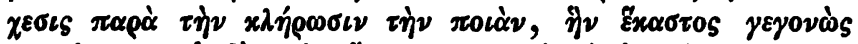

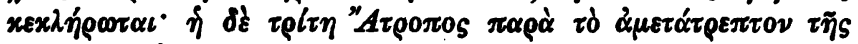

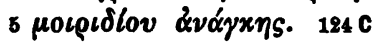

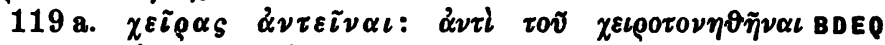
$\pi 0 \iota \tilde{\eta} \sigma \alpha \iota, x \alpha \tau \dot{\alpha} \mu \varepsilon \tau \omega \nu v \mu l \alpha \nu$.

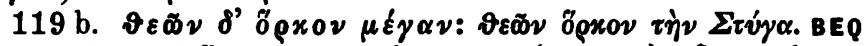

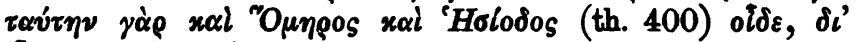

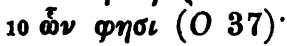

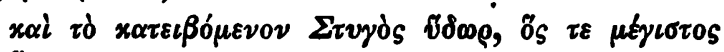
őpxos.

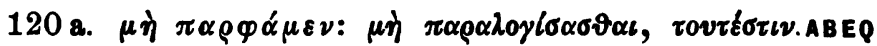

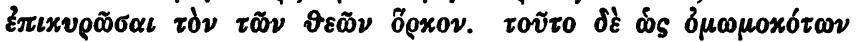

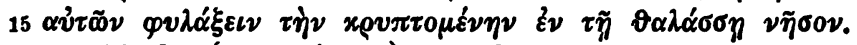

120 b. $\left(\pi \alpha \rho \varphi \alpha^{\prime} \mu \varepsilon \nu:\right) \pi \alpha \rho \alpha \beta \tilde{\eta} \nu \alpha \iota$. Bs1

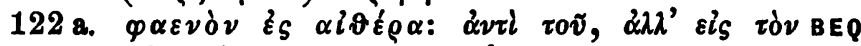

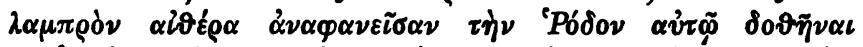

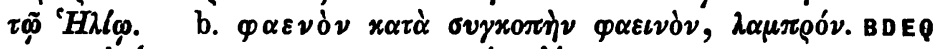

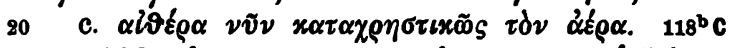

BCDEQ

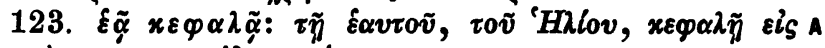

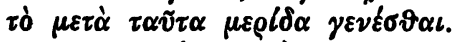

124 a. ( Bg1

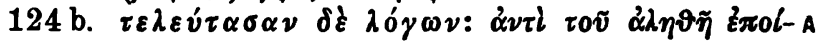
$25 \eta \sigma \alpha \nu \tau \dot{\alpha} \delta \delta^{\prime} \xi \alpha \nu \tau \alpha$.

1-5 sch. Hes. th. 211. Tzetzes ad Lyc. 144

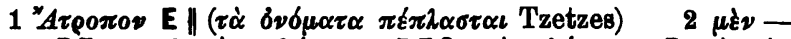

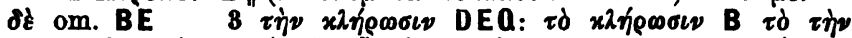

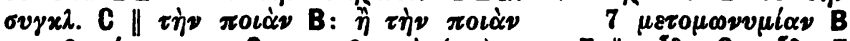

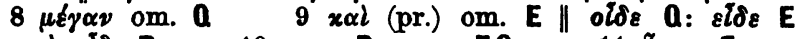

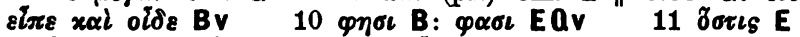

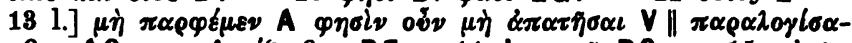

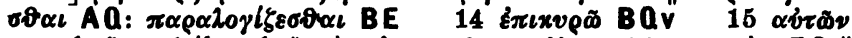

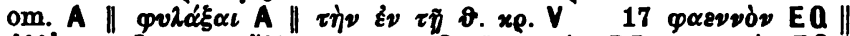

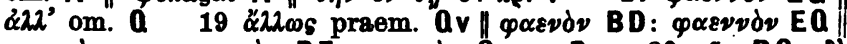

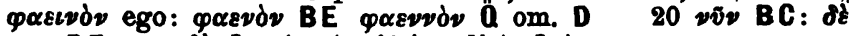

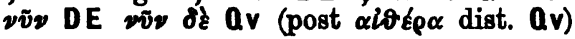

Bchor. ar Perparox od. Drachmann. 


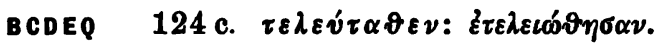

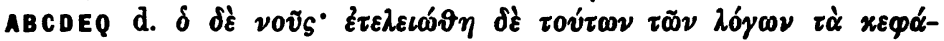

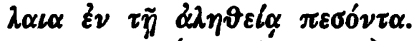

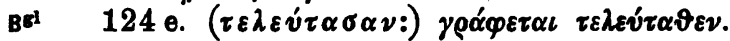

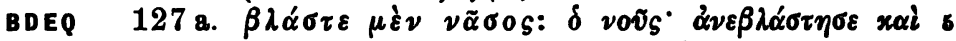

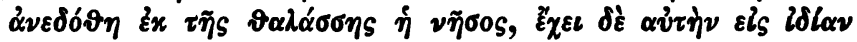

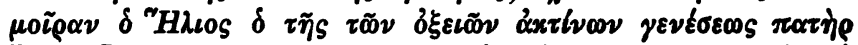

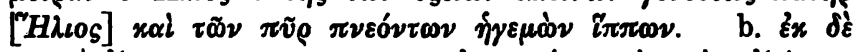

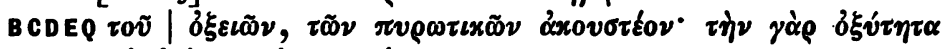

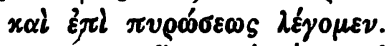

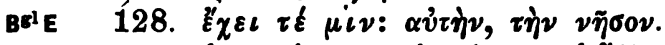

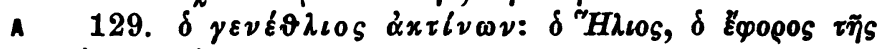

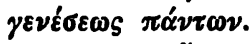

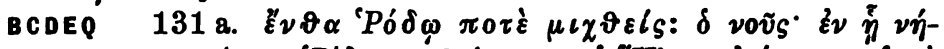

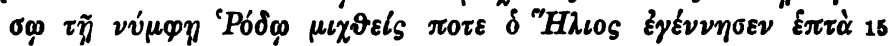

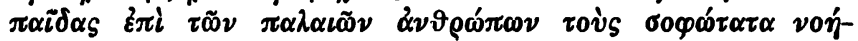

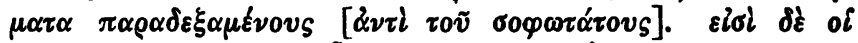

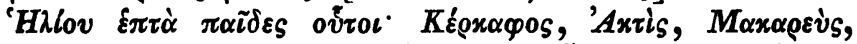

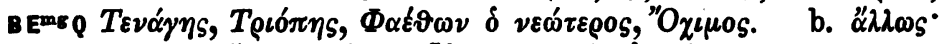

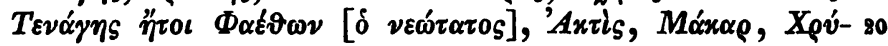

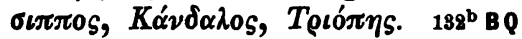

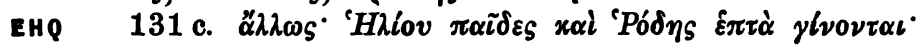

17 Diod. 5, 56. Tzetzes Chil. 4, 360

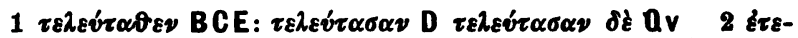

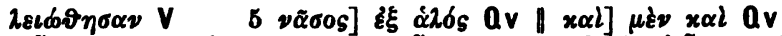

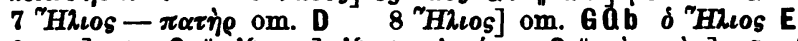

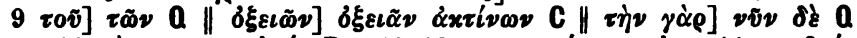

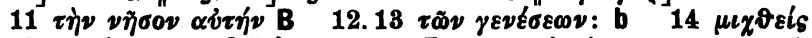

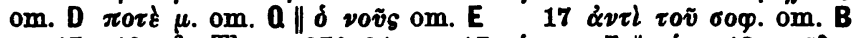
17-19 cfr. Thom. 270, $21 \quad 17$ oi om. E $\|$ oi $-18 \pi \alpha i \delta \varepsilon s$

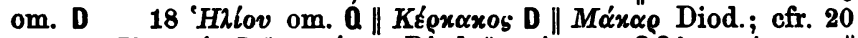

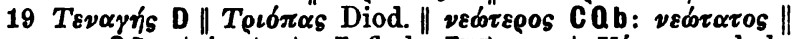

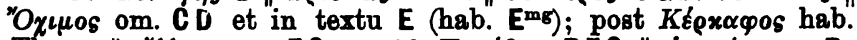

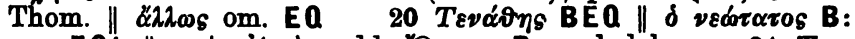

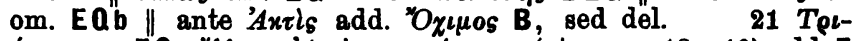

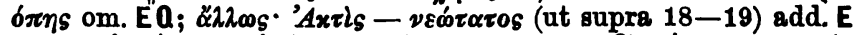

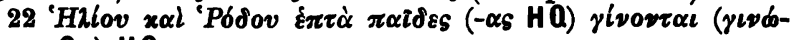

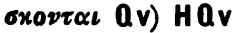




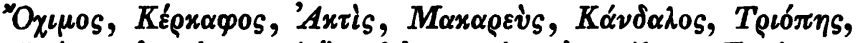

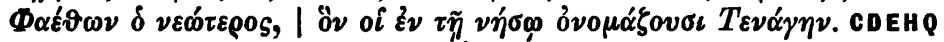

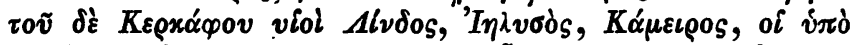

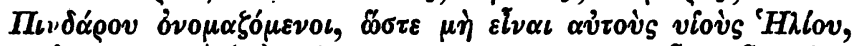

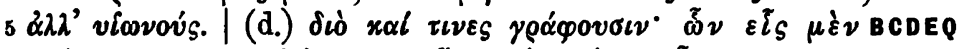

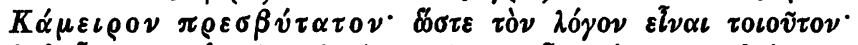

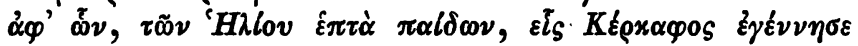

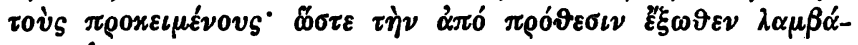

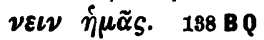

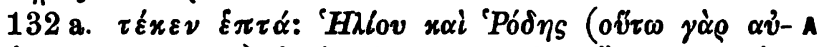

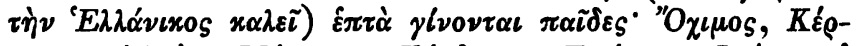

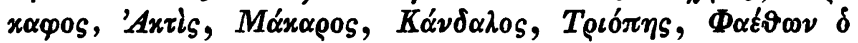

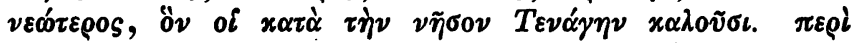

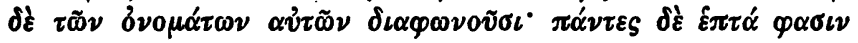

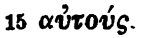

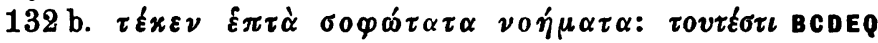

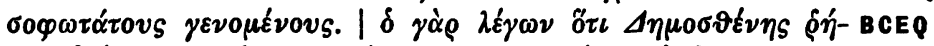

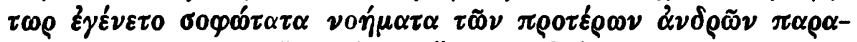

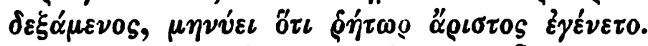

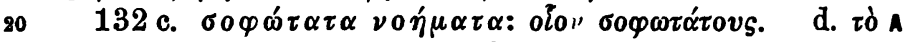

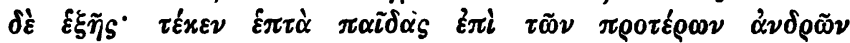

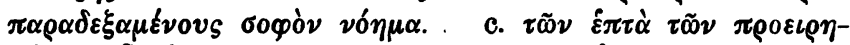

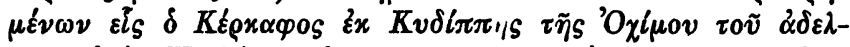

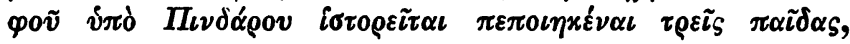

22 Diod. 5, 57. Strab. 14, 654. St. Byz. Alvdos.

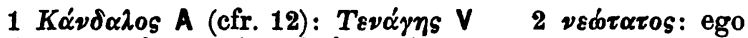

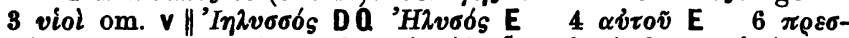

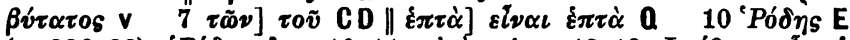

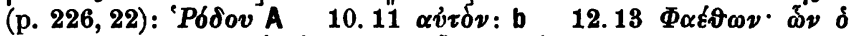

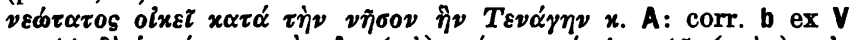

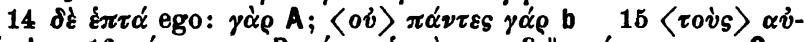

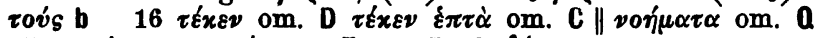

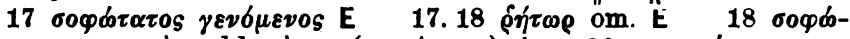

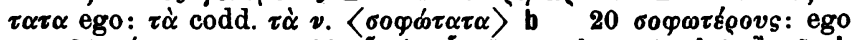

$21 \tau \varepsilon \dot{x}$ :

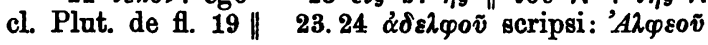




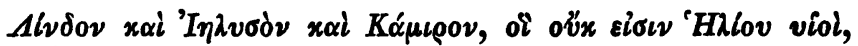

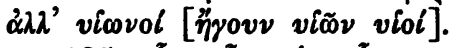

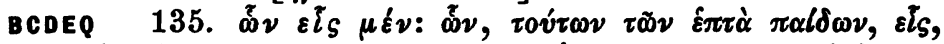

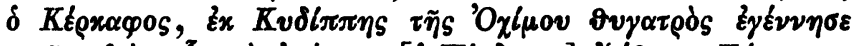

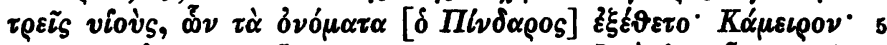

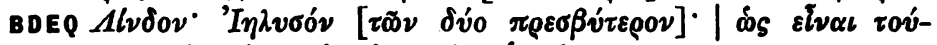

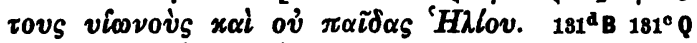

Bg1 138 a. ( $\left.\tau_{\rho} l_{\chi \alpha} \alpha\right) \tau \rho\llcorner\chi \tilde{\omega} s$.

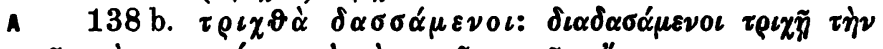

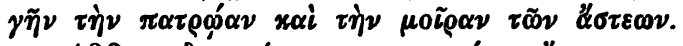

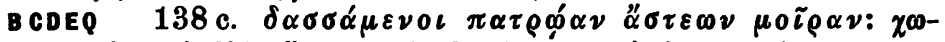

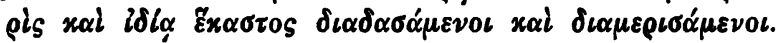

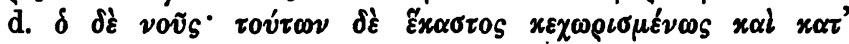

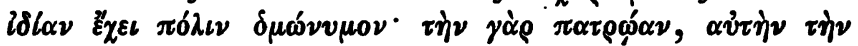

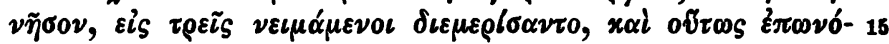

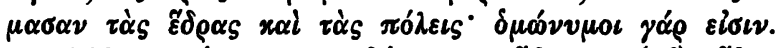

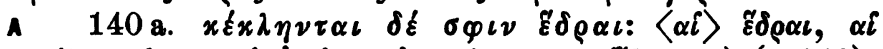

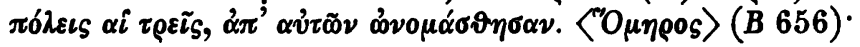

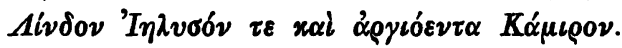

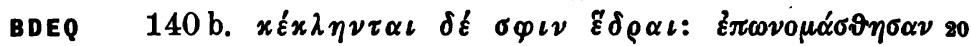

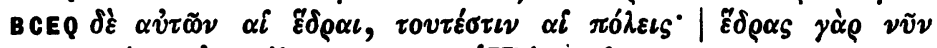

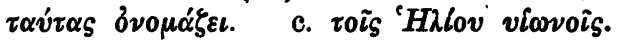

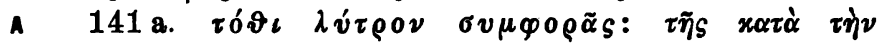

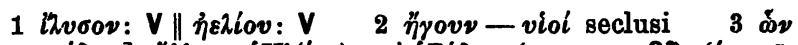

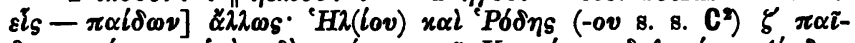

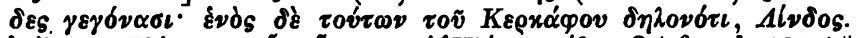

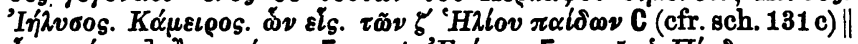

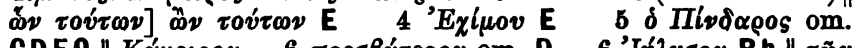

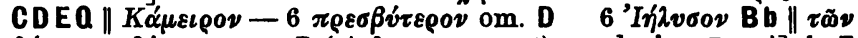

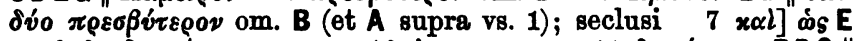

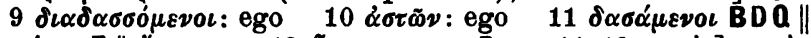

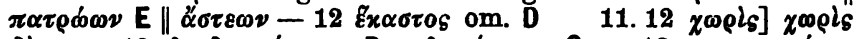

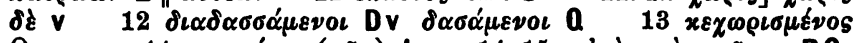

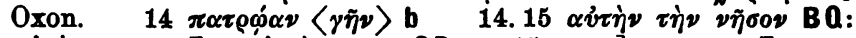

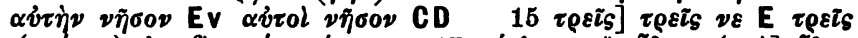

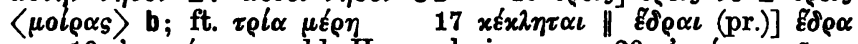

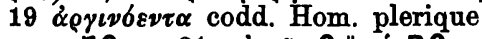

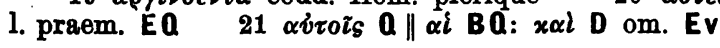




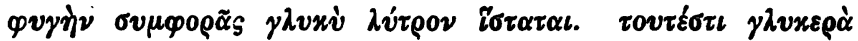

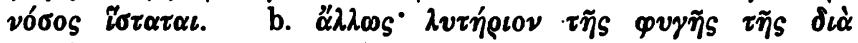
Aєxúpviov.

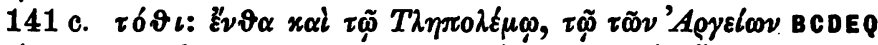

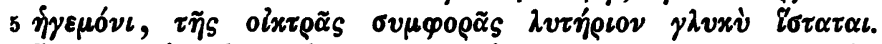

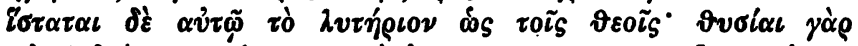

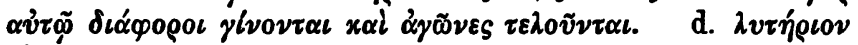

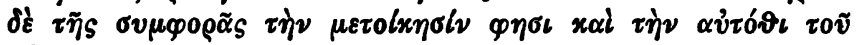

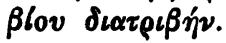

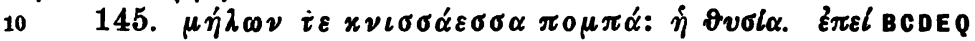

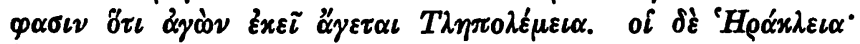

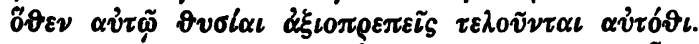

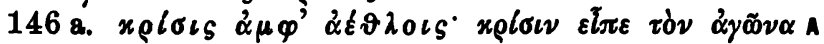

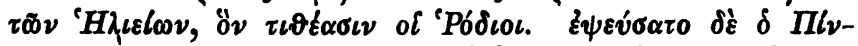

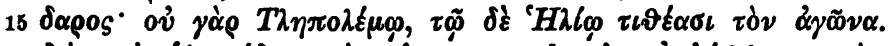

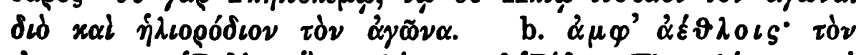

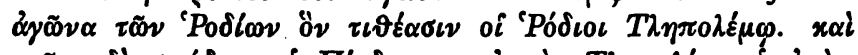

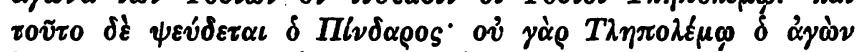

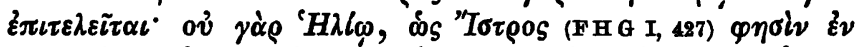

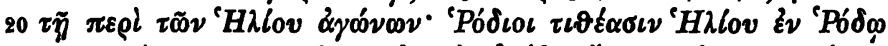

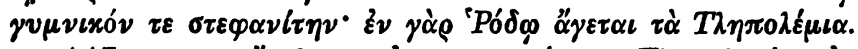

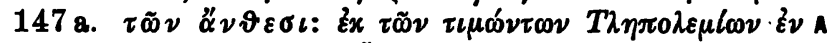

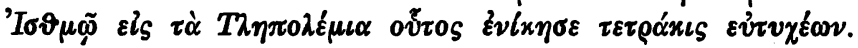

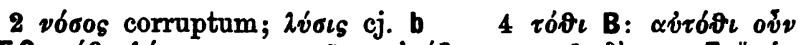

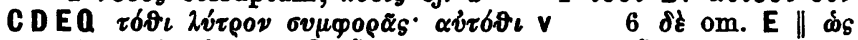

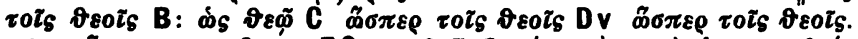

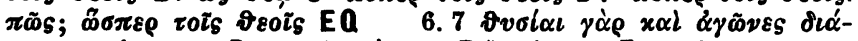

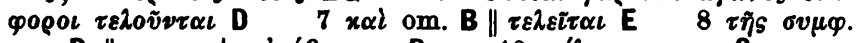

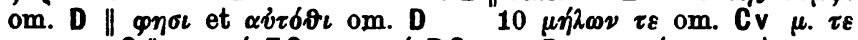

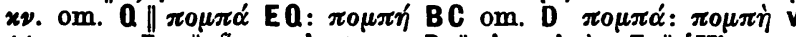

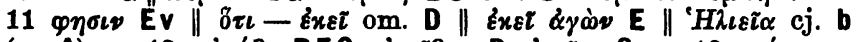

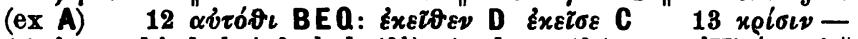

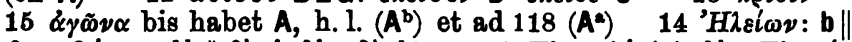

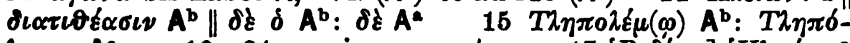

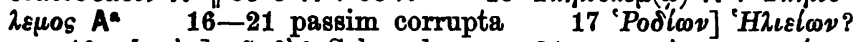
19 ov jò $\rho] \tau \tilde{\omega}$ dहे? Schroeder

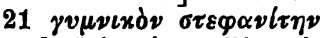
$\langle\dot{\alpha} \gamma \tilde{\omega} \nu \alpha\rangle$ b; sed potius post $\tau \varepsilon$ lac. statuenda $\| \varepsilon \nu$ jò $\rho-T \lambda \eta \pi 0 \lambda$. ft. a ceteris seiungenda 22.23 glossae cum lemmatis confusae et corruptae; pro $\varepsilon i_{S} \delta i_{S}$ cj. Schroeder 


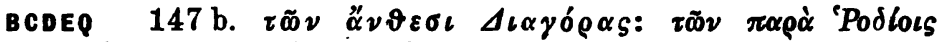

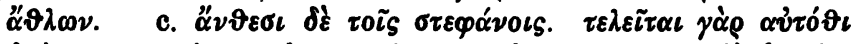

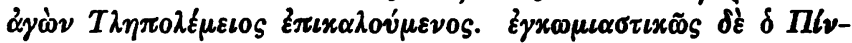

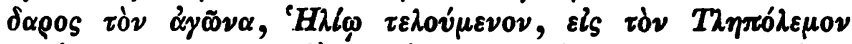

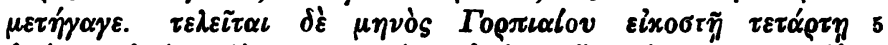

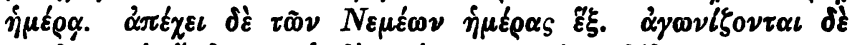

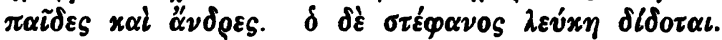

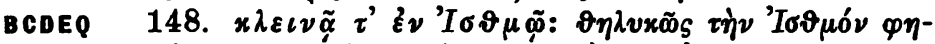

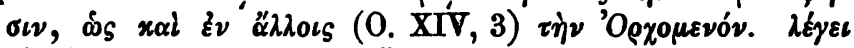

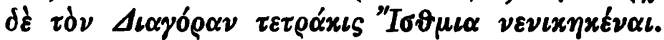

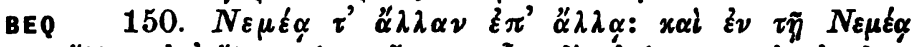

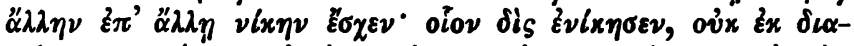

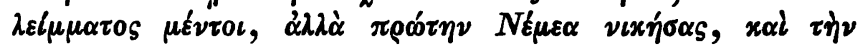

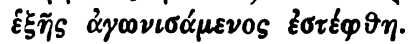

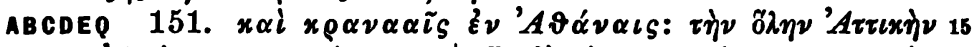

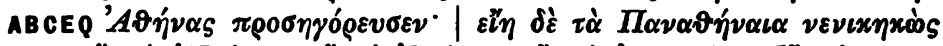

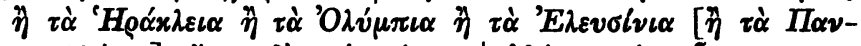

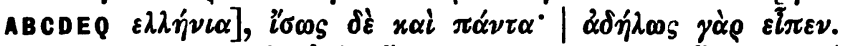

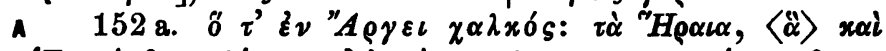

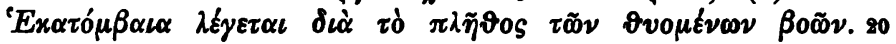

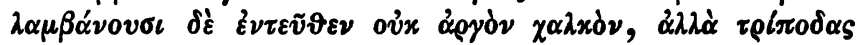

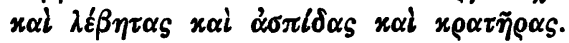

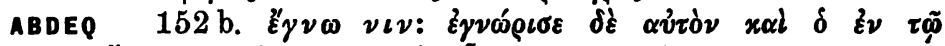

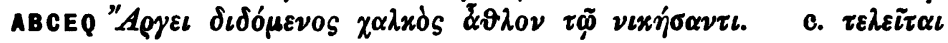

$2 \tau \varepsilon \lambda \varepsilon i \tau \alpha \iota-3 \varepsilon \pi \iota x \alpha \lambda$. om. C $3 \delta^{\prime} \varepsilon$ om. E $4{ }^{\circ} H \lambda l o v$ E

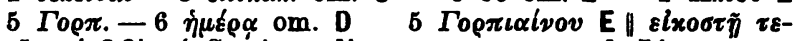
$\tau \alpha \rho \tau \eta] x \alpha^{\prime} \mathrm{CQ}^{1}{ }^{\prime}$ Corsinus diss. agon. p. 101 ed. Lips.

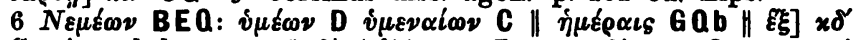
Corsinus l. l. p. $102 \| \delta \xi$ (alt.) om. E $7 \delta \xi$ om. C $9 x \alpha l$

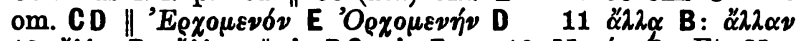

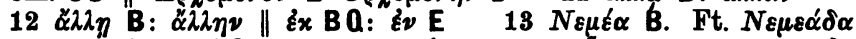

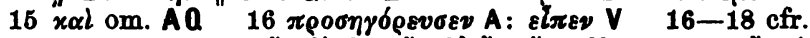
Thom. 273, $11 \quad 16 \varepsilon i \eta \eta \eta$

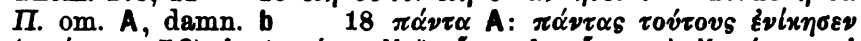

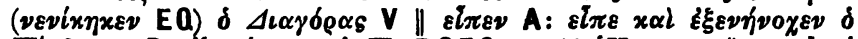

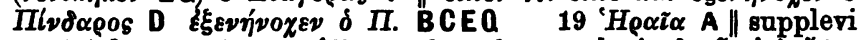

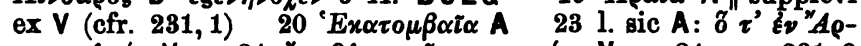

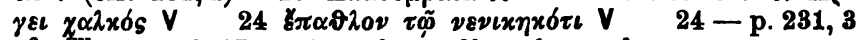
cfr. Thom. 273,17 $24 \tau \varepsilon \lambda \varepsilon i \tau \alpha \iota$ V: $x \alpha \lambda \varepsilon \tau \tau \alpha \iota A$ 


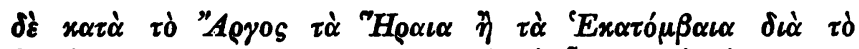

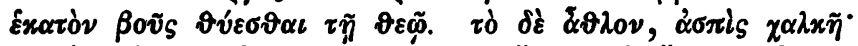

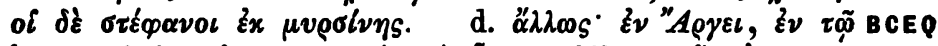

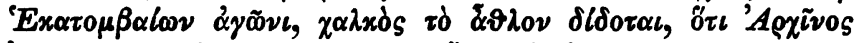

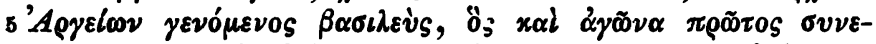

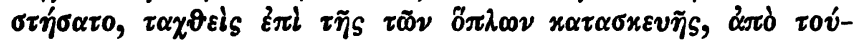

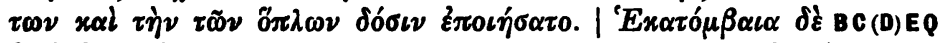

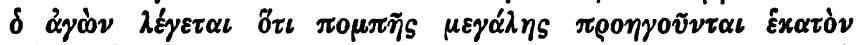

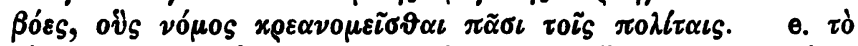

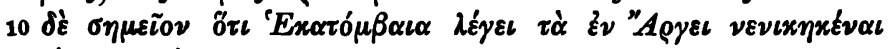

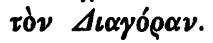

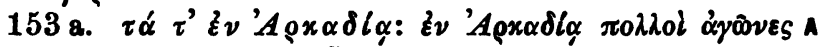

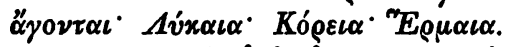

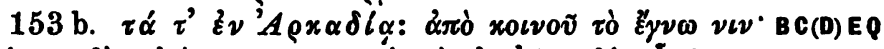

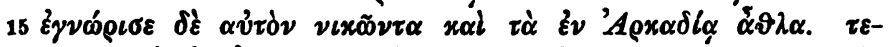

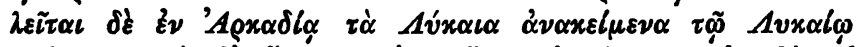

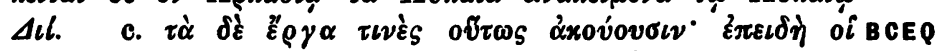
$\alpha$

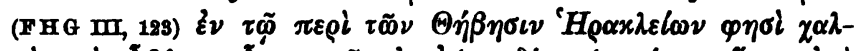

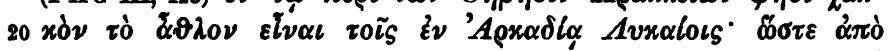
xoเvov $\tau \dot{\alpha}$ हैอ

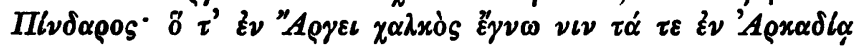

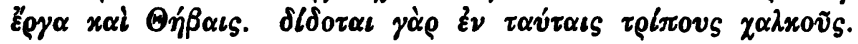

\section{Polyaen. 3, 8}

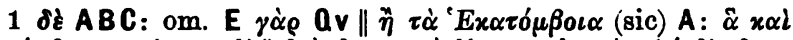

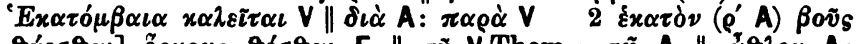

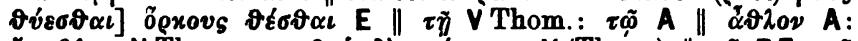

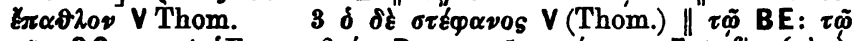

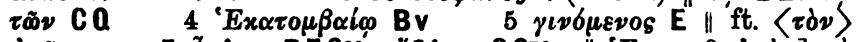

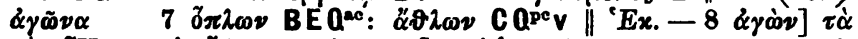

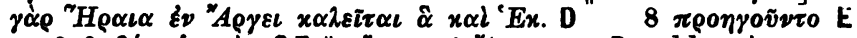

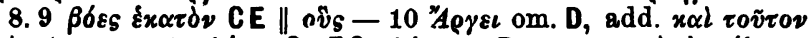

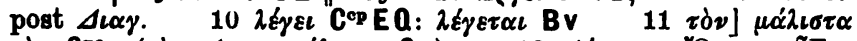

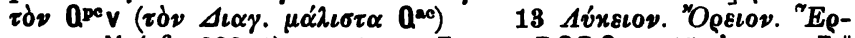

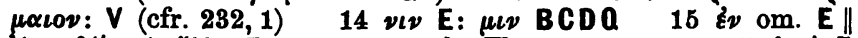

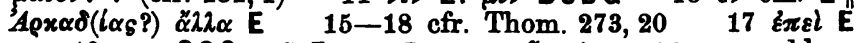
$19 \tau \tilde{\omega} \nu$ CGQ: $\tau \tilde{\varphi}$ E om. B 21 ő $h \quad 22 \mu \iota \nu$ codd., $v$ 23 Qท́pps B 


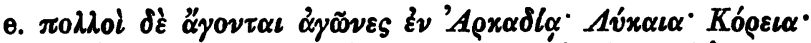

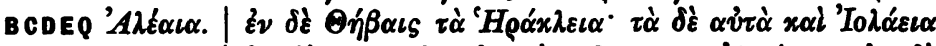

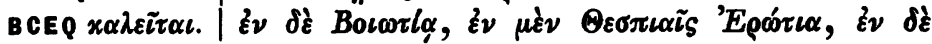

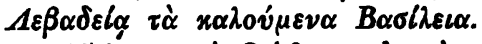

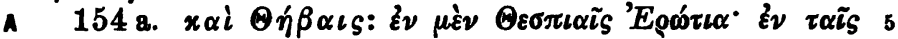

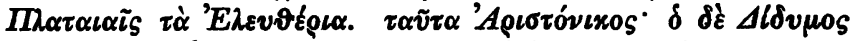

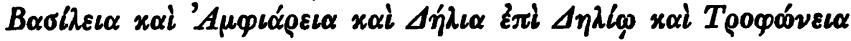
$\varepsilon \nu \Lambda \varepsilon \beta \alpha \delta \varepsilon l \alpha$.

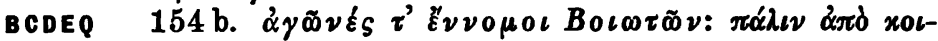

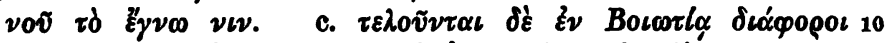

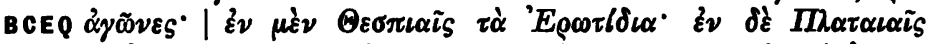

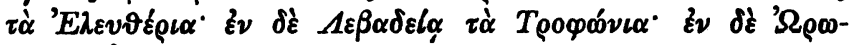
$\pi \tilde{\omega} \tau \dot{\alpha}$ 'A $A \varphi \iota \alpha \dot{\alpha} \alpha \iota \alpha$.

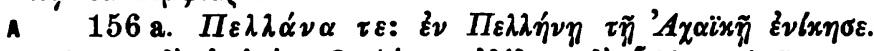

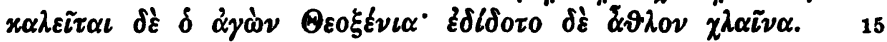

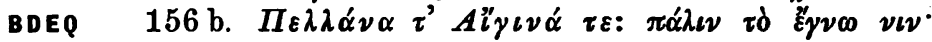

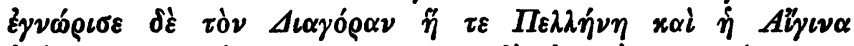

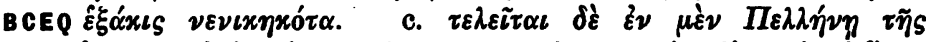

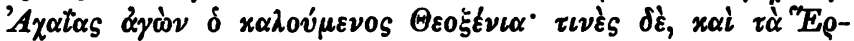

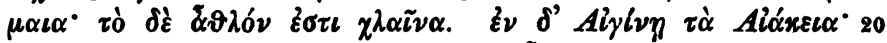

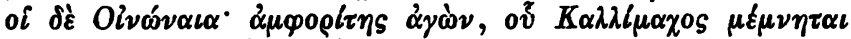

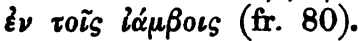

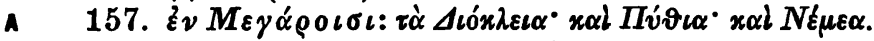

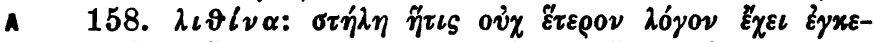

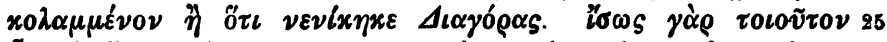

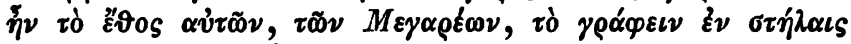

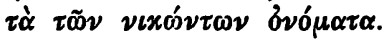

$2{ }^{\prime} I \sigma \lambda \alpha \iota \alpha$ B $3 \Theta \varepsilon \sigma \pi \varepsilon i \alpha \iota s$ B $\quad 5 \vartheta \varepsilon \sigma \pi \varepsilon \sigma L \alpha \tau_{S} \quad 6 \pi \lambda \alpha-$

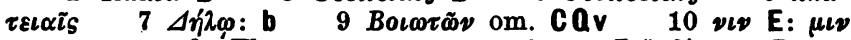

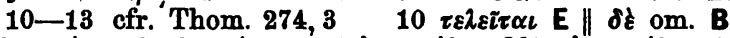

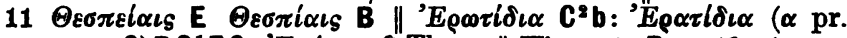

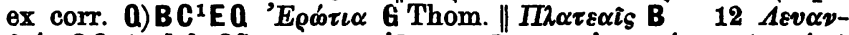

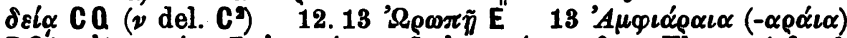

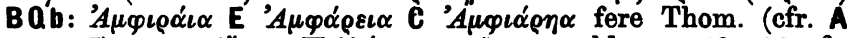

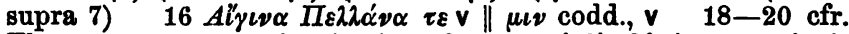

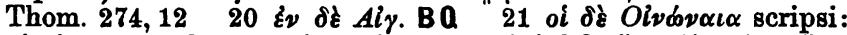
oi $\delta \grave{\varepsilon}$, o .... (lac.) B oi $\delta \xi \overline{~ o i ~ E ~}$

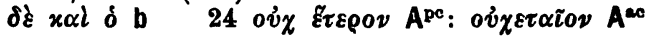




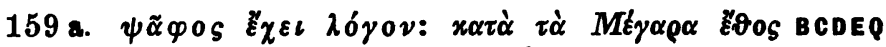

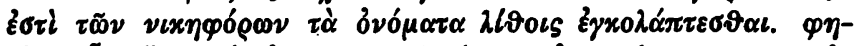

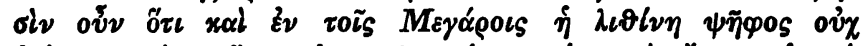

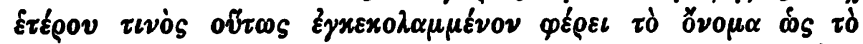

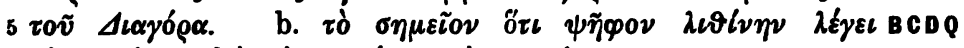

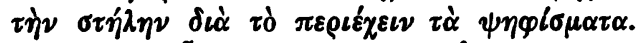

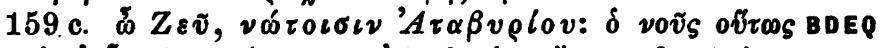

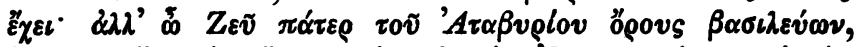

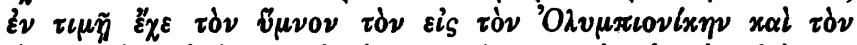

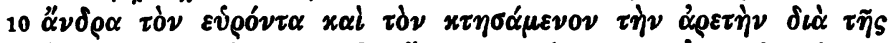

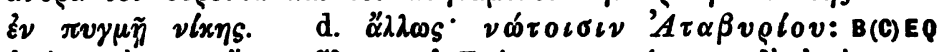

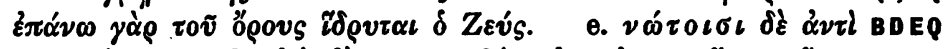

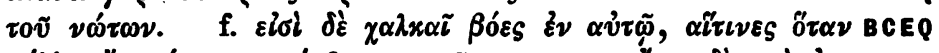

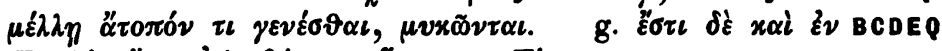

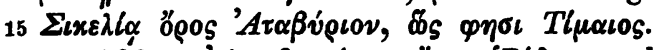

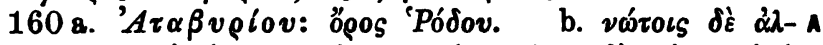

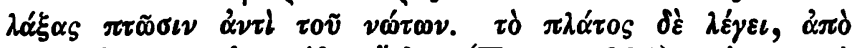

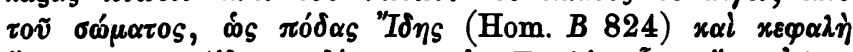

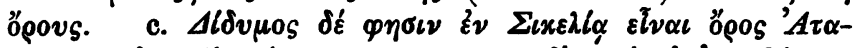

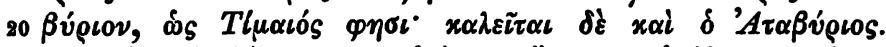

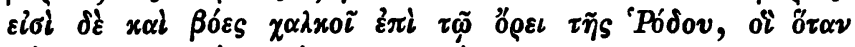

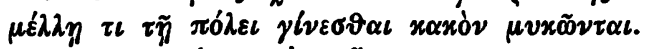

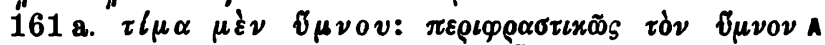

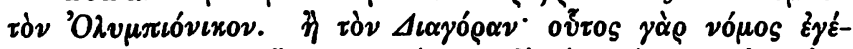

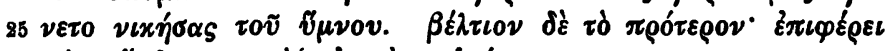

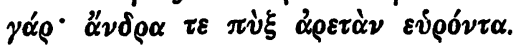

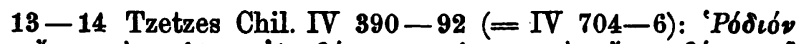

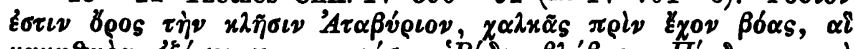

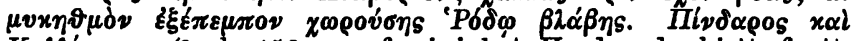

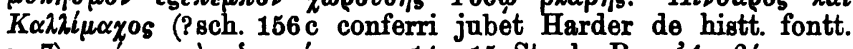

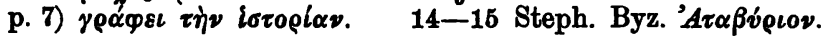

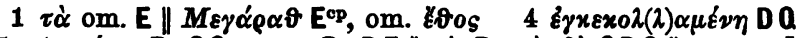

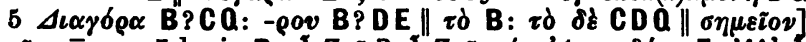

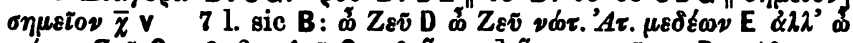

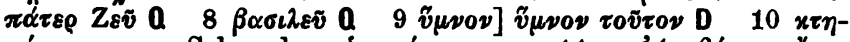

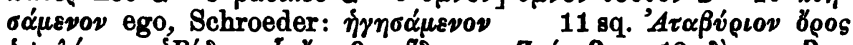

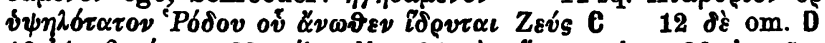

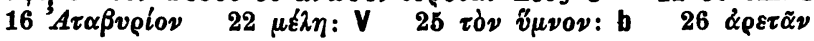




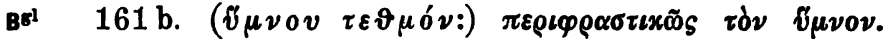

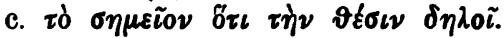

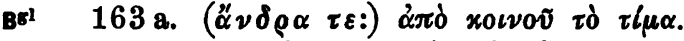

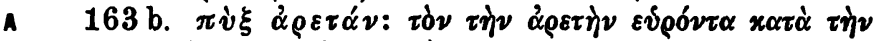

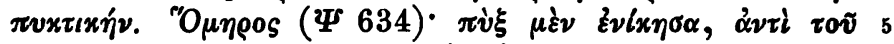

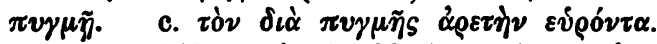

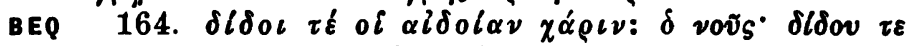

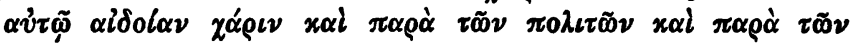

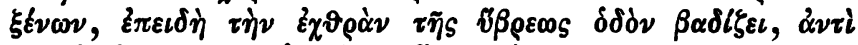

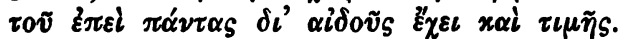

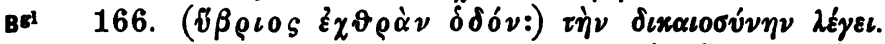

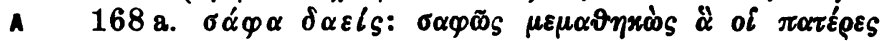

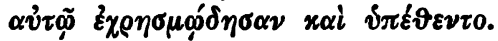

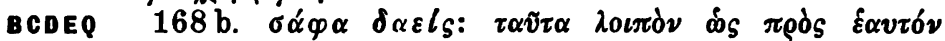

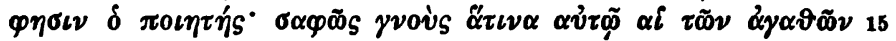

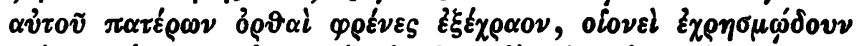

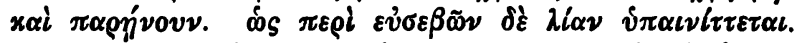

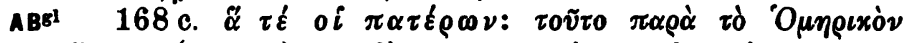

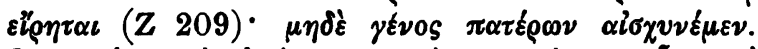

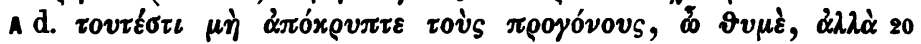

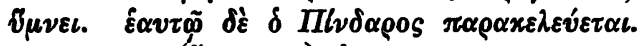

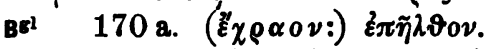

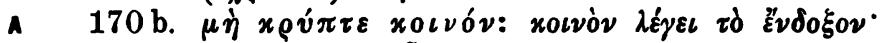

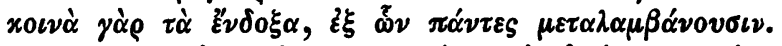

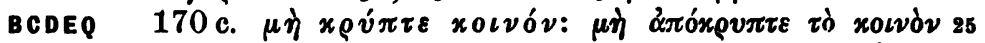

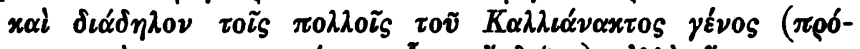
yovos jà

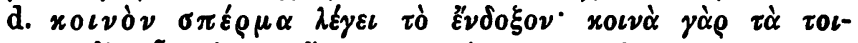

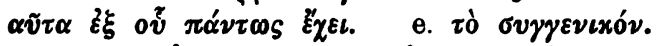

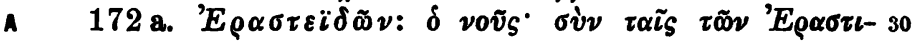

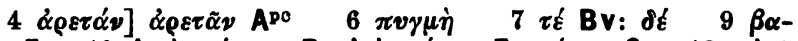

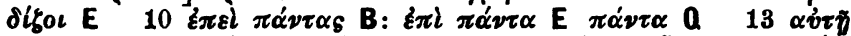

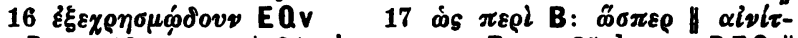

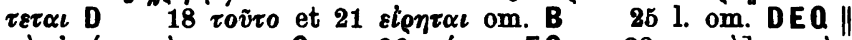

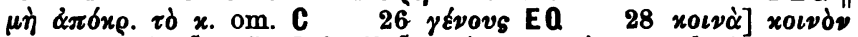

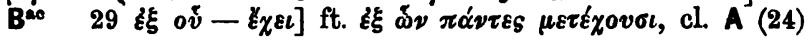




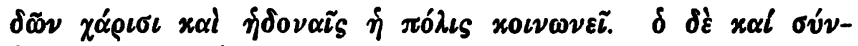

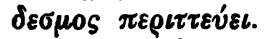

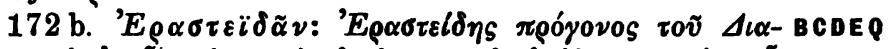

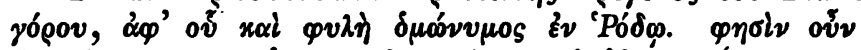

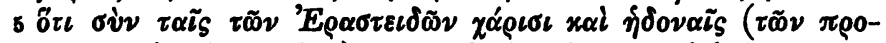

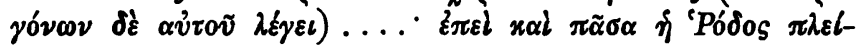

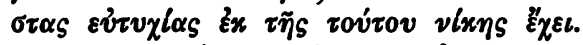

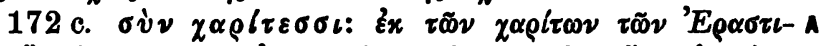

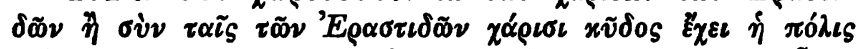

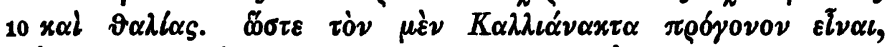

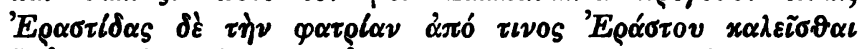

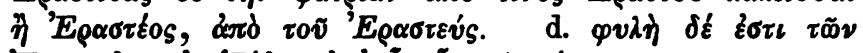
'E

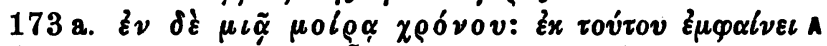

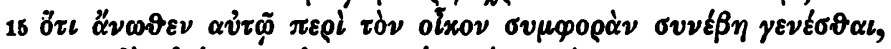

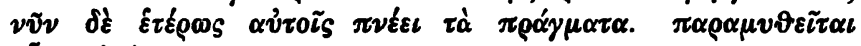

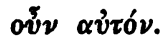

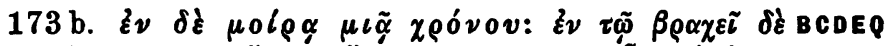

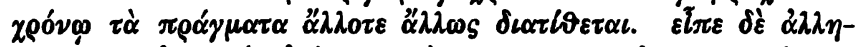

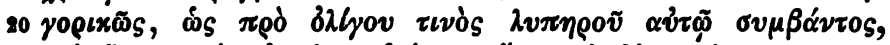

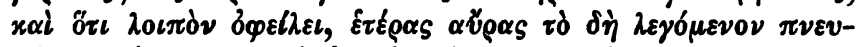

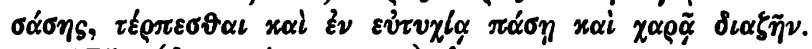

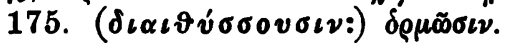

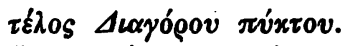

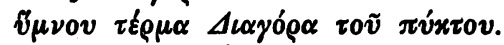

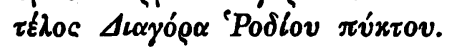

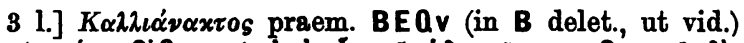

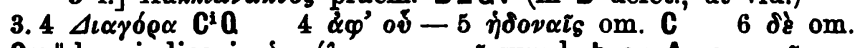

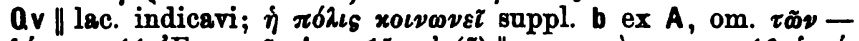

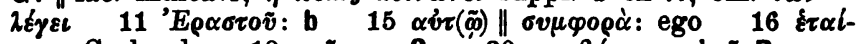

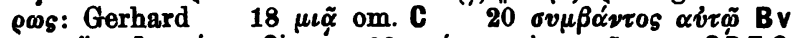

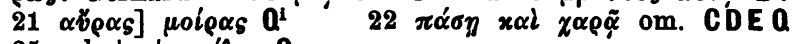
25 sub tertu $\tau$ énos 0 . 


\section{Scholia in Olympionicarum carmen VIII.}

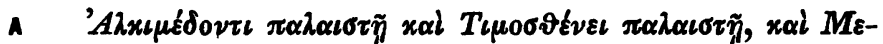

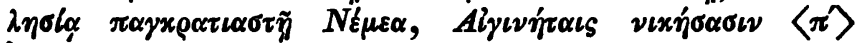

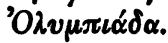

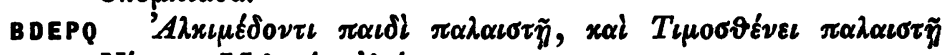

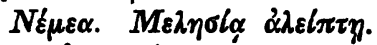

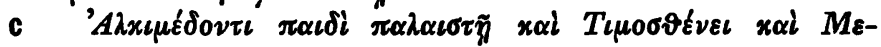
$\lambda \eta \sigma l \alpha, \pi \alpha \gamma x \rho \alpha \tau \iota \alpha \sigma \tau \tilde{\eta}$.

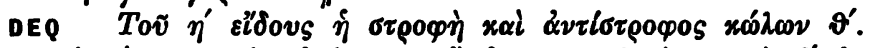

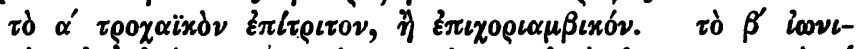

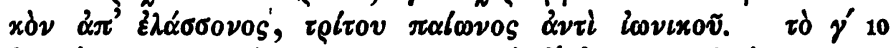

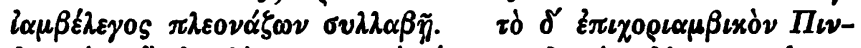

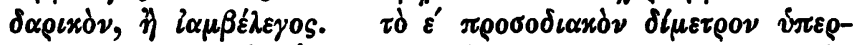

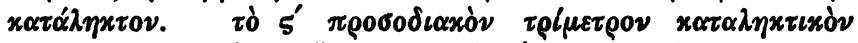

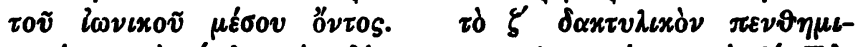

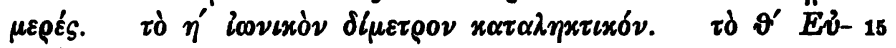
$\rho \iota \pi l \delta \varepsilon \iota 0 \nu$.

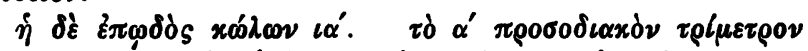

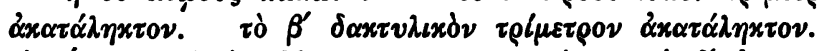

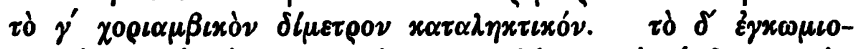

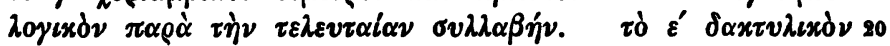

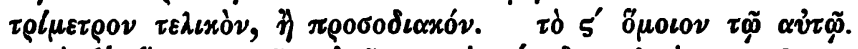

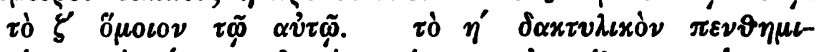

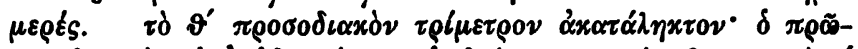

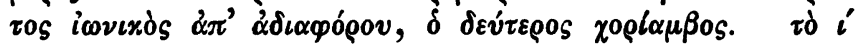

$2 \pi^{\prime}$ addidi ex V (inscr.) $4 \pi \alpha \lambda \alpha \iota \sigma \tau \tilde{\eta}$ (alt.)] $\pi \alpha \lambda \alpha \iota \sigma \tau \tilde{\eta} \tau \tilde{\omega}$

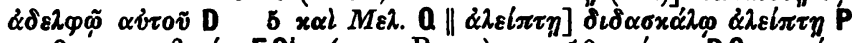

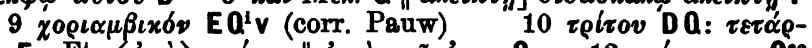

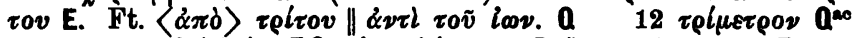

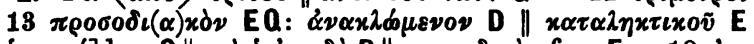

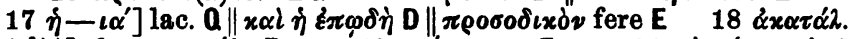

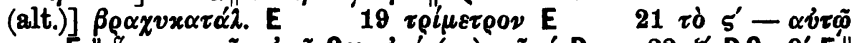

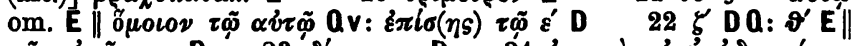

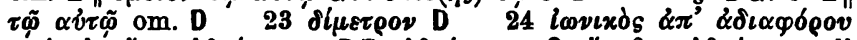

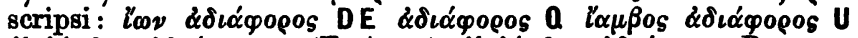

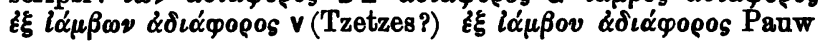




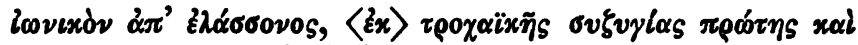

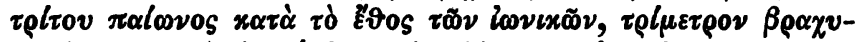

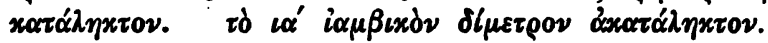

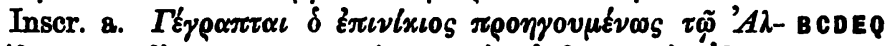

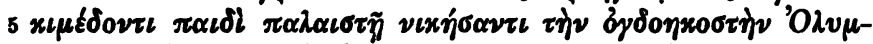

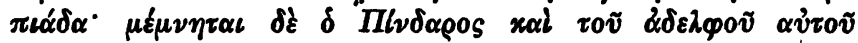

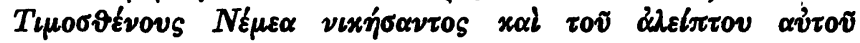

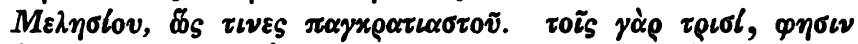

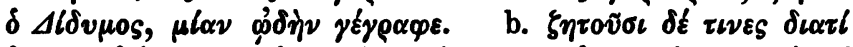

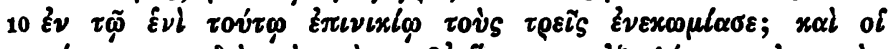

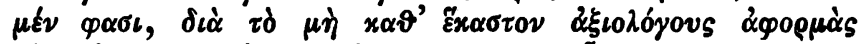

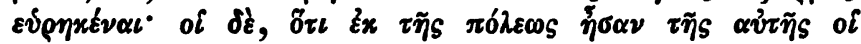

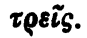

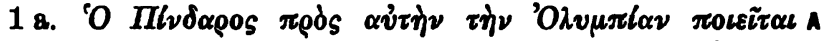

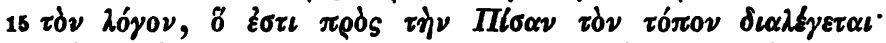

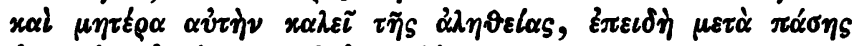

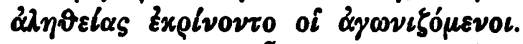

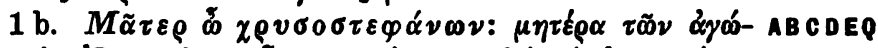

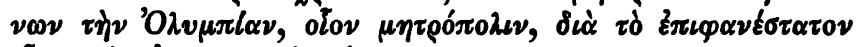

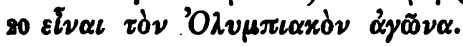

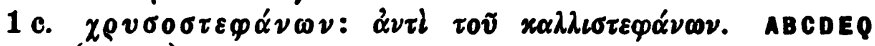

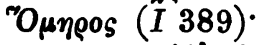

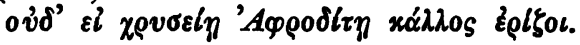

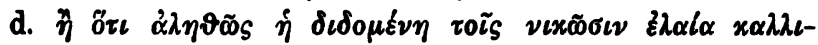

24 Aristot. mirab. 51. sch. Ar. Plut. 586. Paus. 5, 15, 3

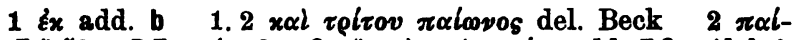

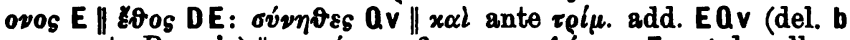

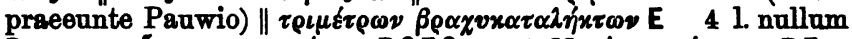

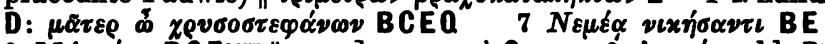
$8 \mathrm{M} \iota \lambda \eta \sigma i o v$ BCE

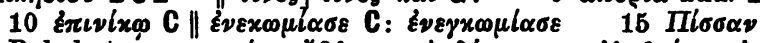

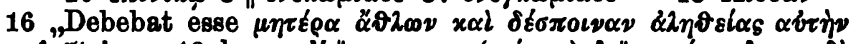
$x \alpha \lambda \varepsilon \tau^{6}$ b $18 \mathrm{l}$. om. V $\|\chi \rho v \sigma \varepsilon 0 \sigma \tau \varepsilon(\varphi \alpha \dot{\alpha} \nu \omega \nu) A\| \mu \eta \tau \varepsilon \rho \alpha A: \mu$. $\delta \varepsilon$

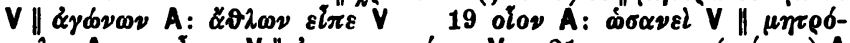

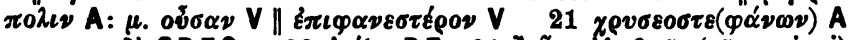

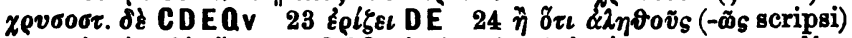

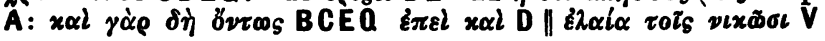




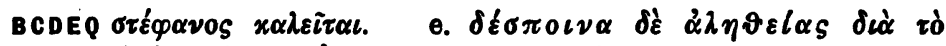

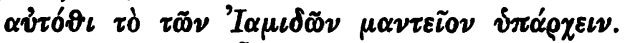

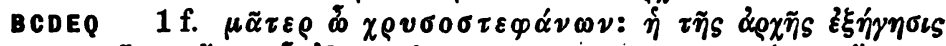

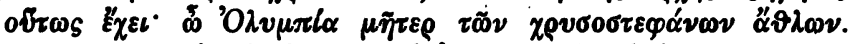

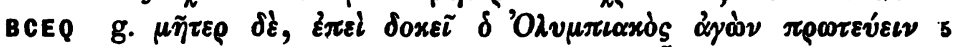

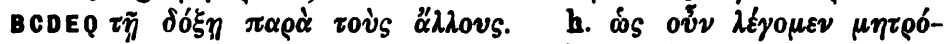

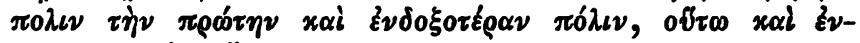

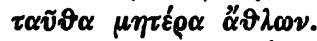

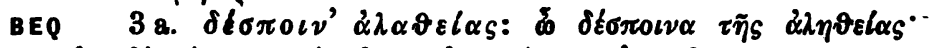

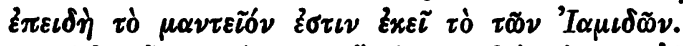

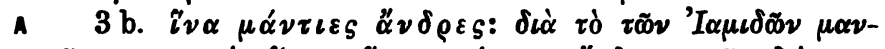

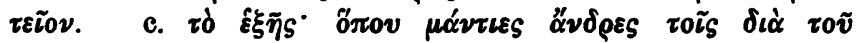

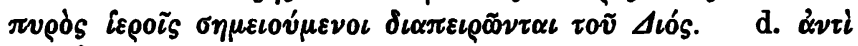

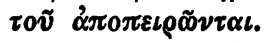

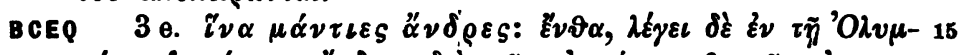

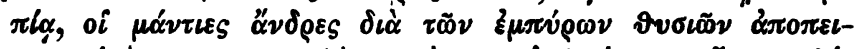

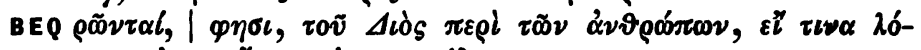

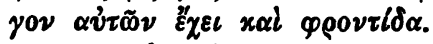

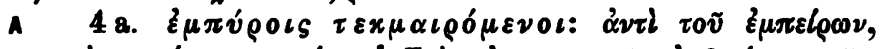

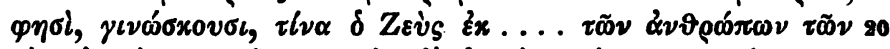

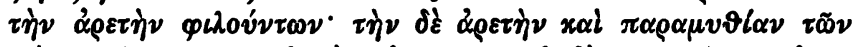

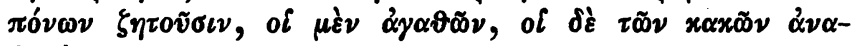
$\beta 0 \lambda \dot{v} v$.

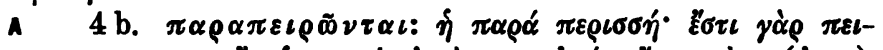

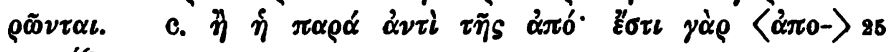
$\pi \varepsilon \iota \alpha^{\prime} \xi o v \sigma \iota \nu$.

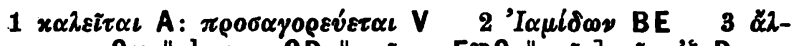

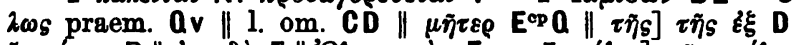

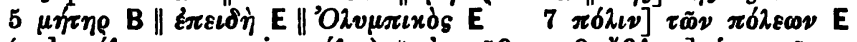

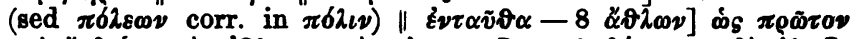

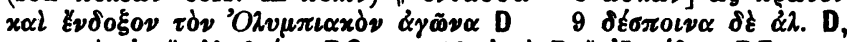
om. ceteris \| $\alpha \lambda \eta \vartheta \varepsilon i \alpha s$ B Q 10 ह $\pi \varepsilon l$ B \| 'I $\alpha \mu l \delta \omega \nu$ BE

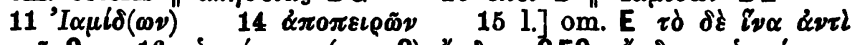

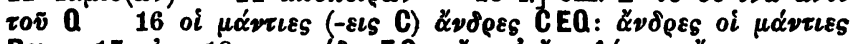

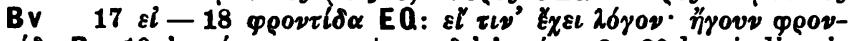

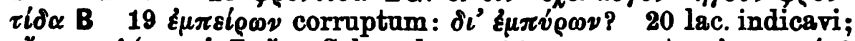

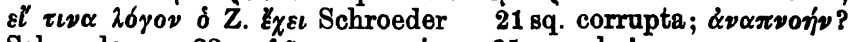
Schroeder $22 x \alpha \hat{\tilde{\omega}} \nu$ : correxi 25 suppl. b 


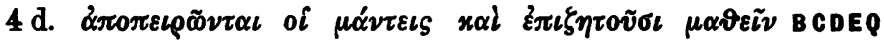

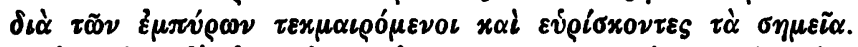

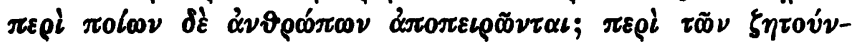

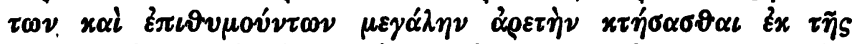

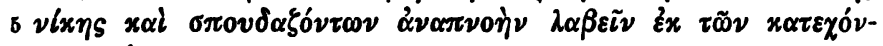
$\tau \operatorname{cov} \mu \hat{\sigma} \chi \partial 00 \nu$. ${ }^{\mathrm{m}} \mathrm{Q}$

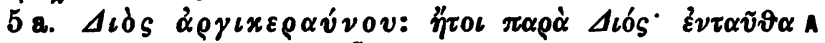

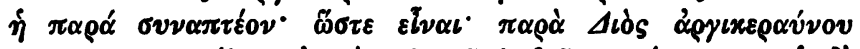

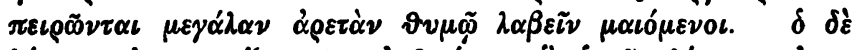

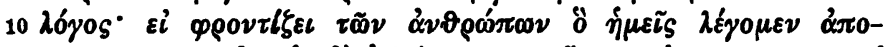

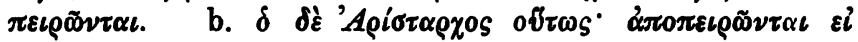

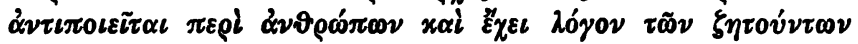

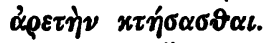

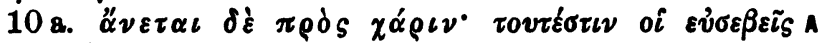

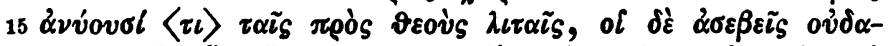
$\mu \tilde{\omega} s$. b. भु

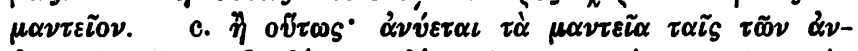

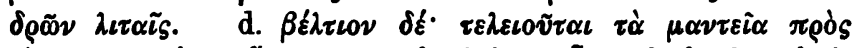

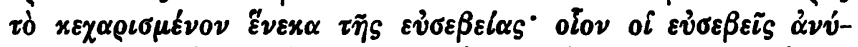

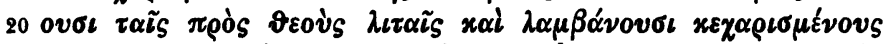

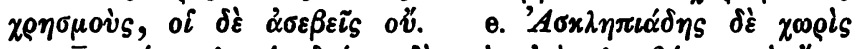

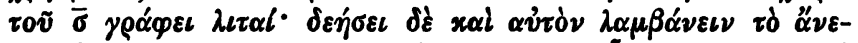

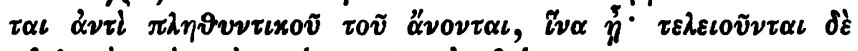

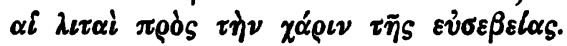

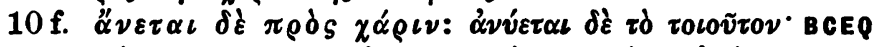

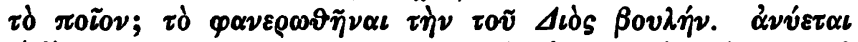

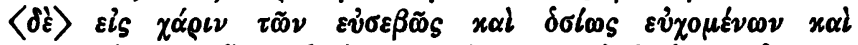

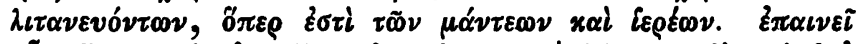

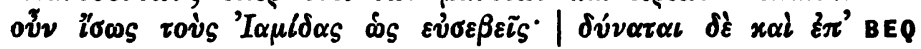

1 1. nullum CD: $\pi \alpha \rho \alpha \pi \varepsilon \iota \rho \tilde{\omega} \nu \tau \alpha \iota$ B $\alpha 2 \lambda \lambda_{0}$ EQv $9-11$ corrupta; $\mu \varepsilon \gamma \alpha \dot{\alpha} \alpha \nu-\lambda \alpha \beta \varepsilon i \nu$ l. novi sch. videntur $9 \beta \alpha \lambda \varepsilon \tau \nu A$ (defendit $b$ ut ex varia lectione ortum) $11 \alpha \pi 0 \pi \varepsilon \iota \rho \tilde{\omega} \nu \tau \alpha \iota$ (alt.)

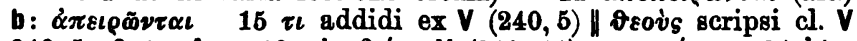

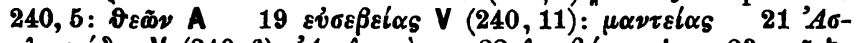

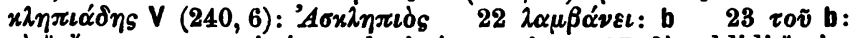

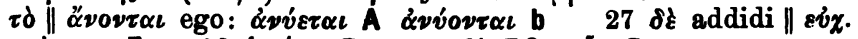

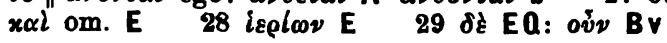




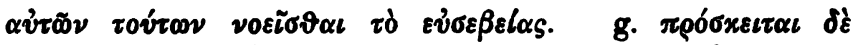

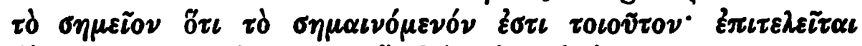

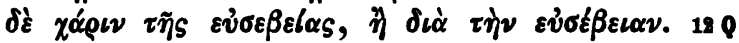

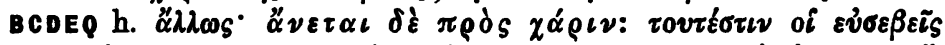

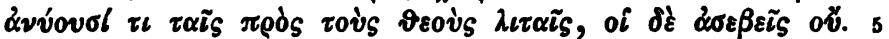

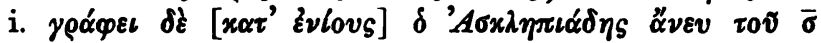

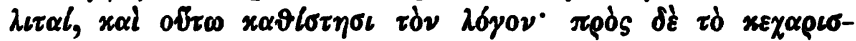

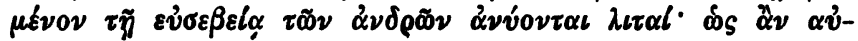

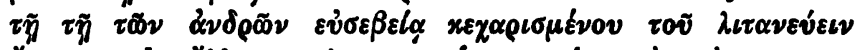

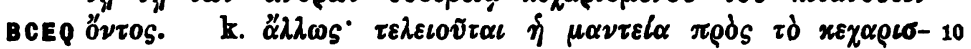

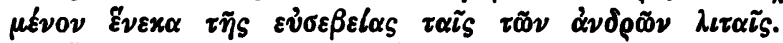

BCDEQ 1. भै

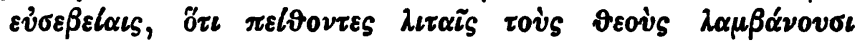

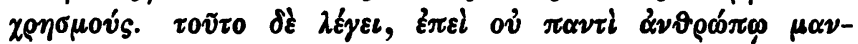

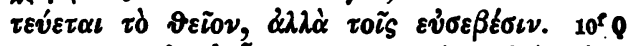

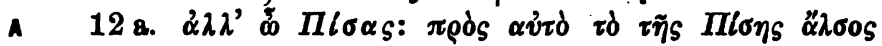

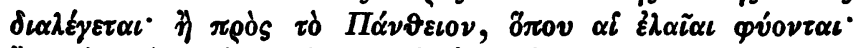

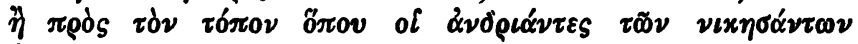

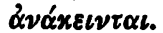

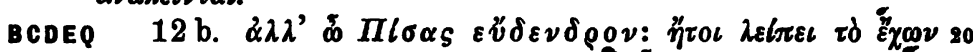

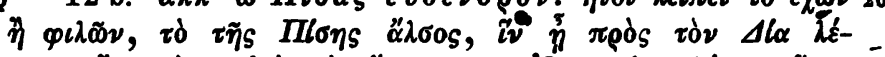

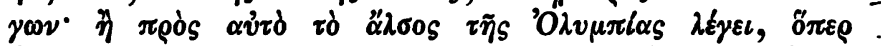

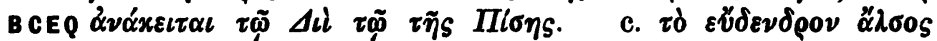

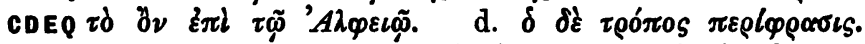

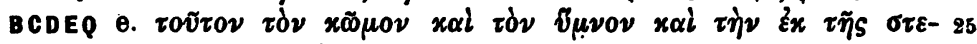
$\varphi \alpha \nu \eta \varphi 0 \varrho l \alpha \varsigma \pi \rho \sigma ́ \sigma \delta \varepsilon \xi \alpha \iota \nu l x \eta \nu$.

17 Aristot. mirab. 51. sch. Ar. Plat. 586. sch. 0. 3,60

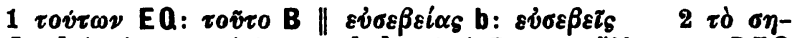

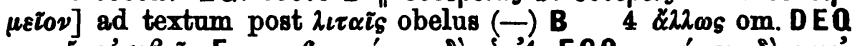

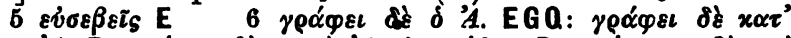

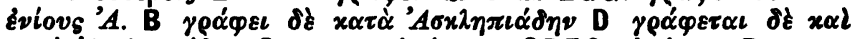

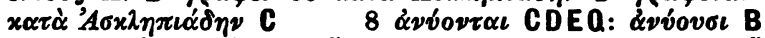

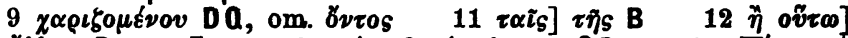

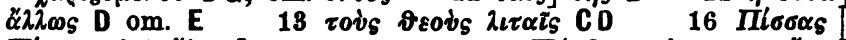

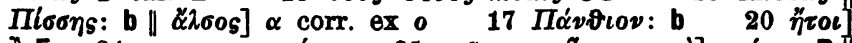

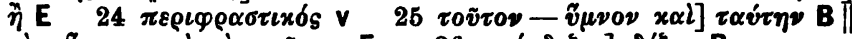

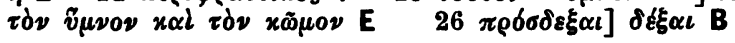




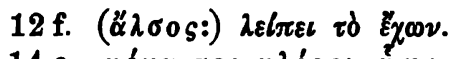

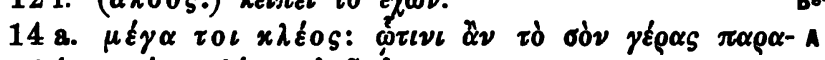

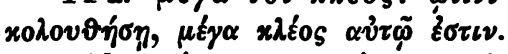

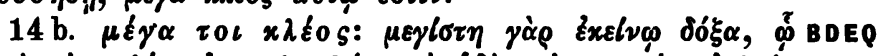

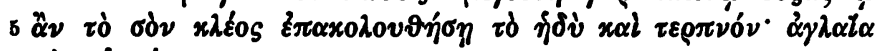

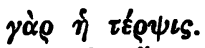

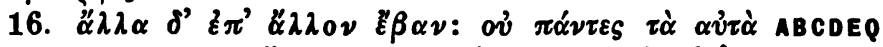

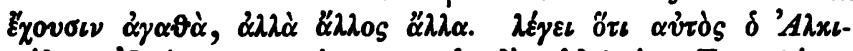

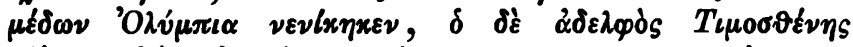

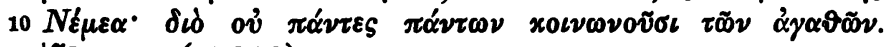

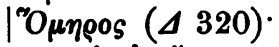

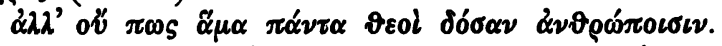

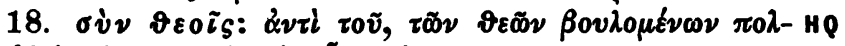

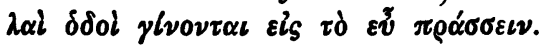

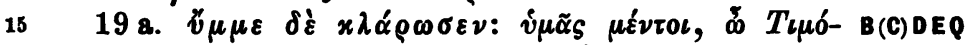

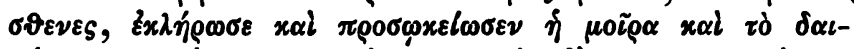

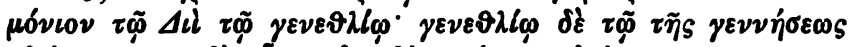

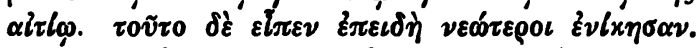

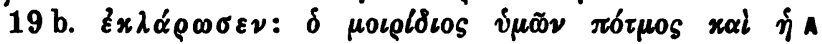

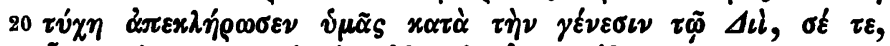

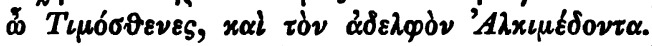

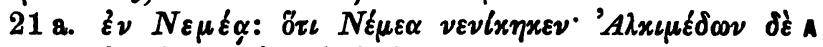

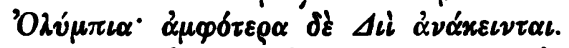

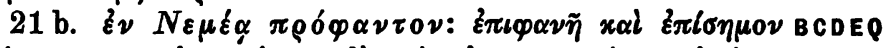

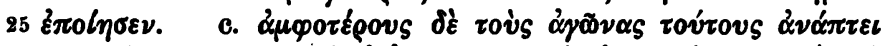

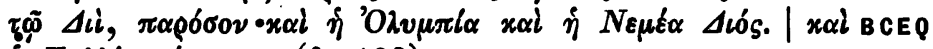
$\delta K \alpha \lambda \lambda l \mu \alpha \chi o ́ s ~ \varphi \eta \sigma \iota$ (fr. 193).

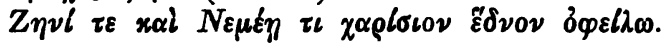

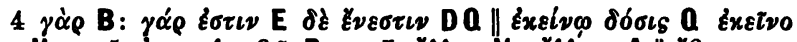

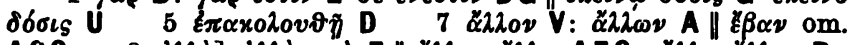

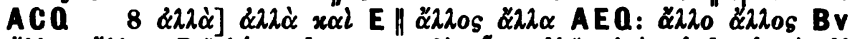

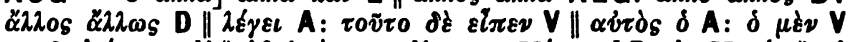

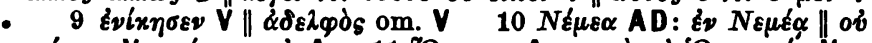

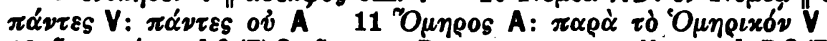
$12 \alpha \mu \alpha \pi \alpha v \tau \alpha$ AC(E) Q: $\alpha \pi \alpha \nu \tau \alpha$ B r $13 \tau 0 \tilde{v}$ om. H 15 l. BC(E):

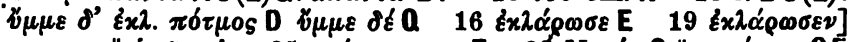

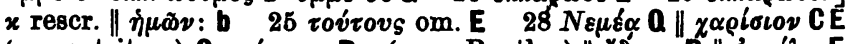

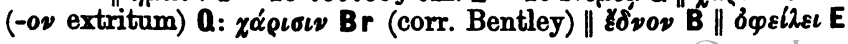

Sohox. In Perdarux ed. Drachmann. 


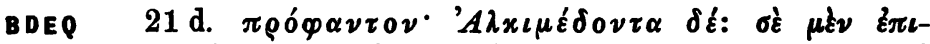

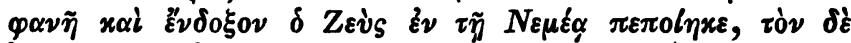

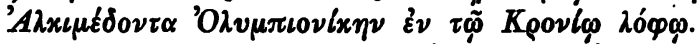

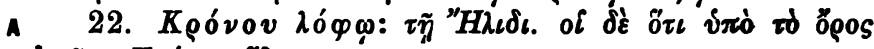

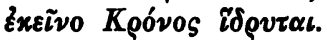

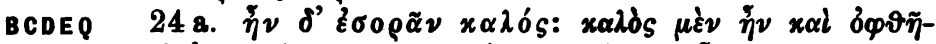

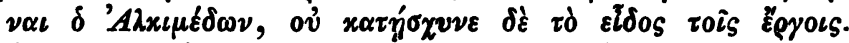

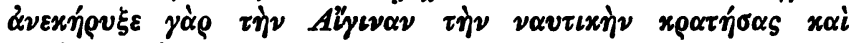
$\nu เ x \eta ́ \tilde{\sigma \alpha} \pi \alpha \dot{\lambda} \eta$.

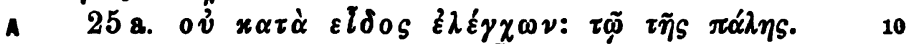

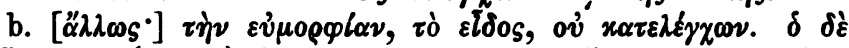

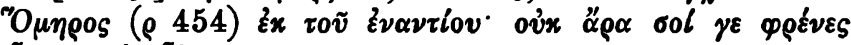

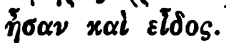

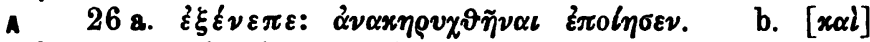

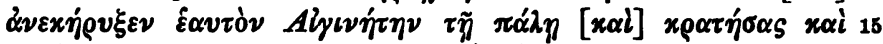

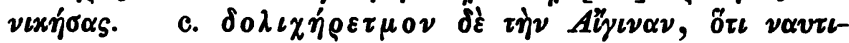

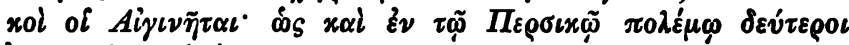

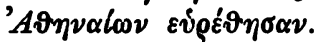

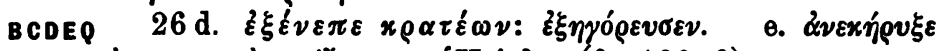

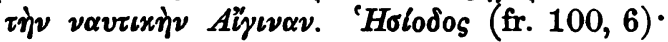

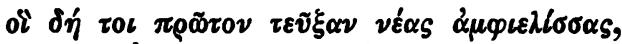

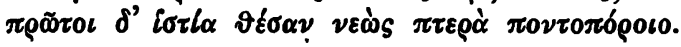

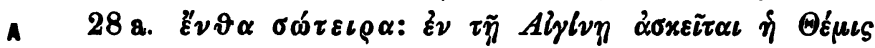

sch. 24 b. lex. Vind. 111, 16: $x \alpha \lambda \delta s \delta \& \gamma \alpha \vartheta \delta$ s. $x \alpha \downarrow x \alpha \lambda \delta s$

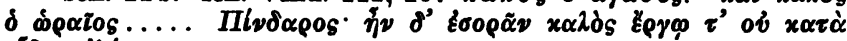

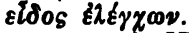

20 sch. Nem. 3, 21 (p. 79 Ab.). Tzetzes ad Lyc. 176

1 sqq. sch. $21 \mathrm{~d}$ praec. praem hb $32 \delta \varphi \varphi] \tau \tilde{\omega} \lambda \delta \varphi \varphi \mathrm{E}$

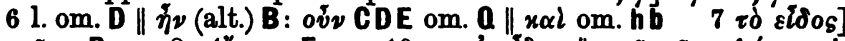

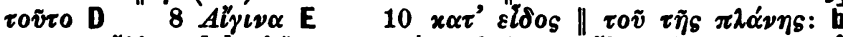

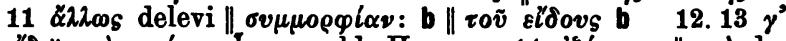

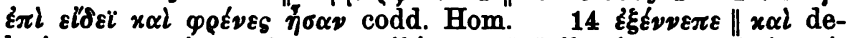

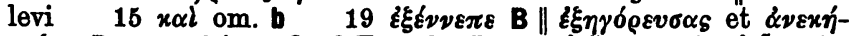

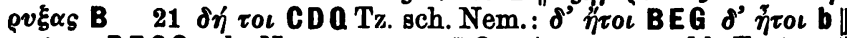

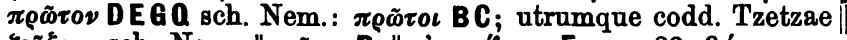

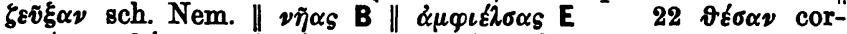
ruptum; $\theta \varepsilon \sigma \sigma \alpha \nu \mathbf{r} \| \nu \varepsilon \dot{s}$ omnes (etiam G) 


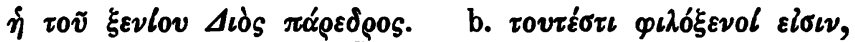

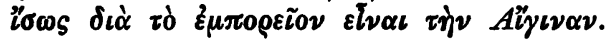

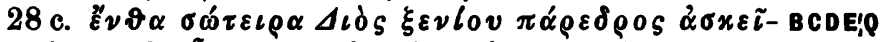

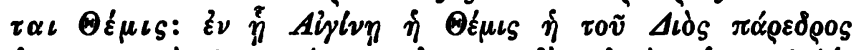

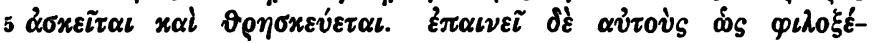

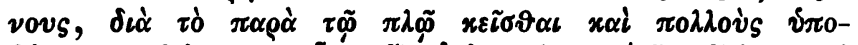

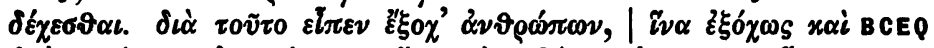

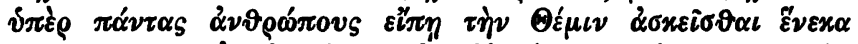

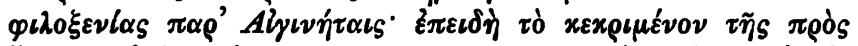

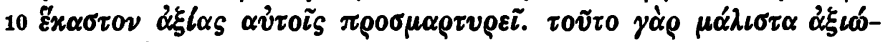

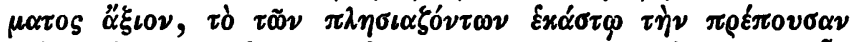

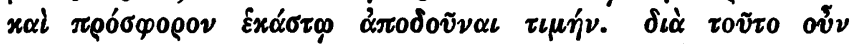

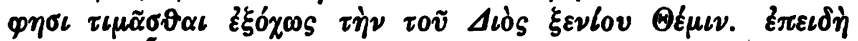

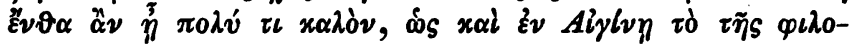

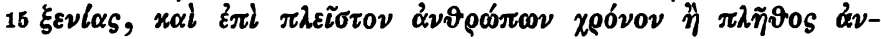

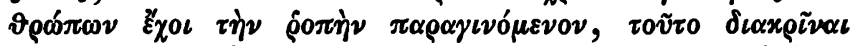

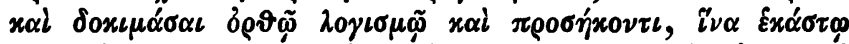

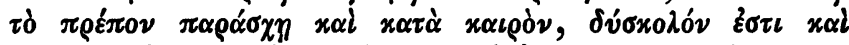

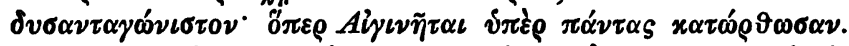

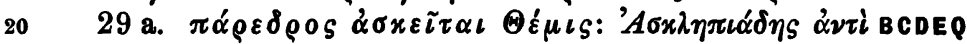

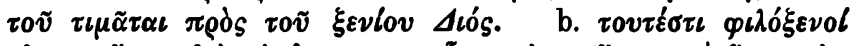

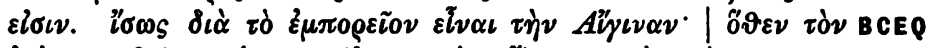

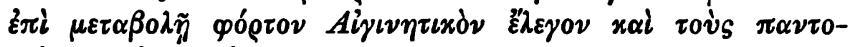

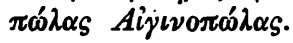

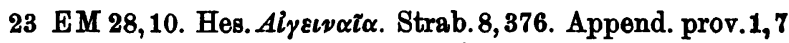

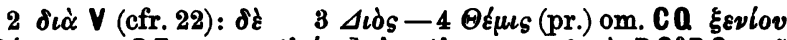

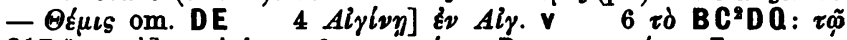

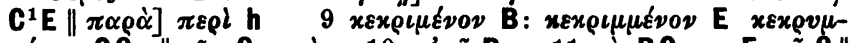

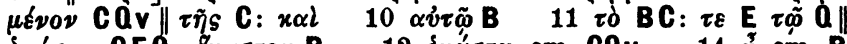

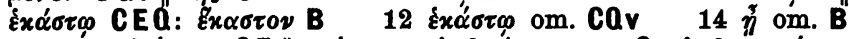

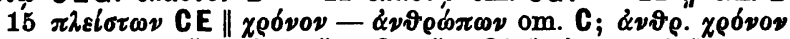

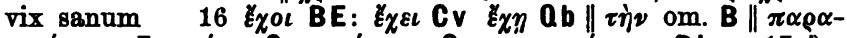

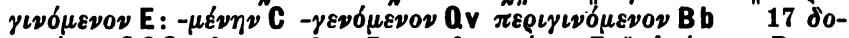

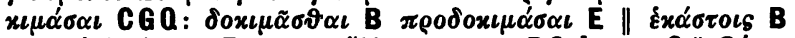

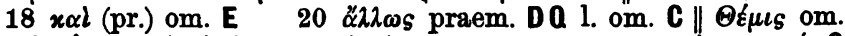

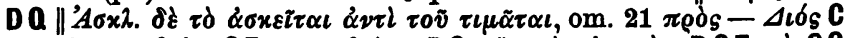

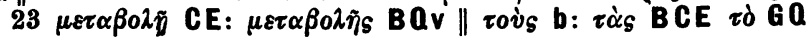




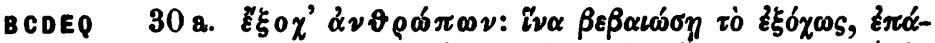

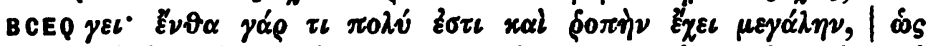

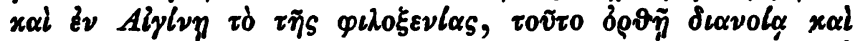

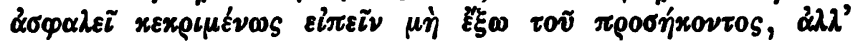

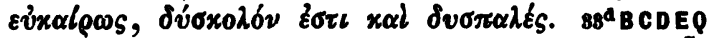

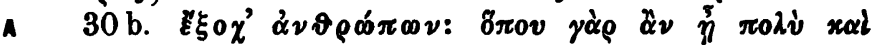

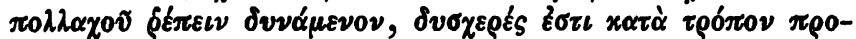

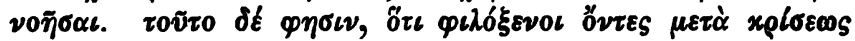

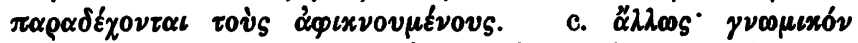

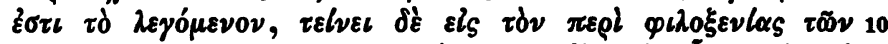

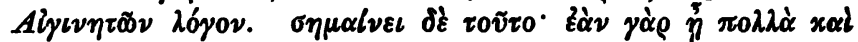

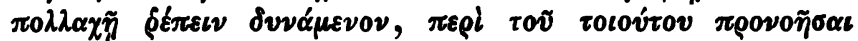

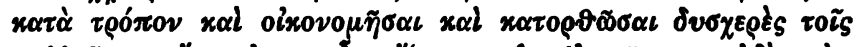

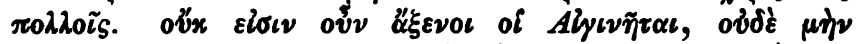

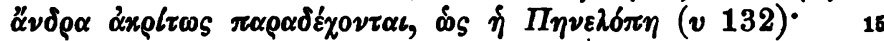

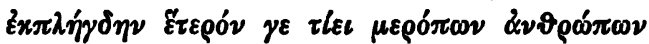

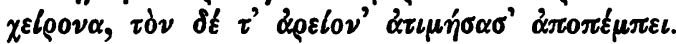

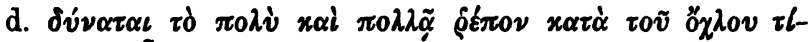

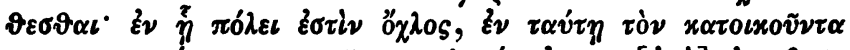

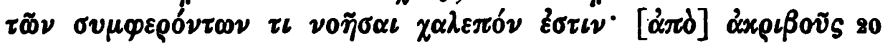

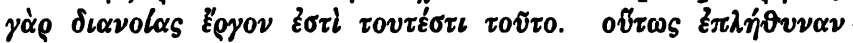

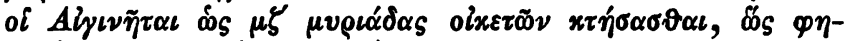

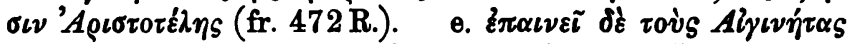

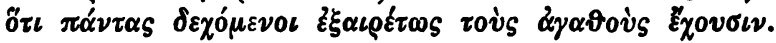

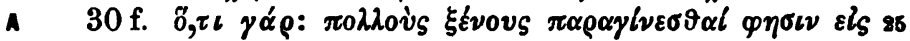

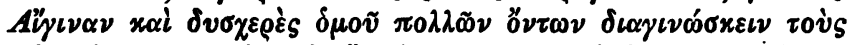

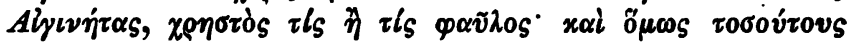

\section{Athen. 6, $272 \mathrm{~d}$}

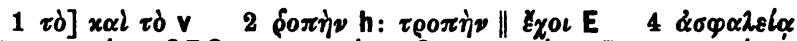

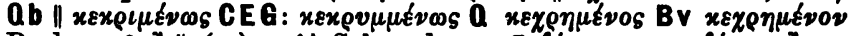
Beck $6 \hat{\eta} \|\langle\tau \iota\rangle \pi 0 \lambda$ Schroeder 7

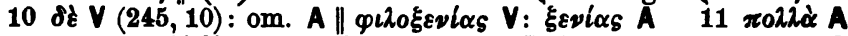

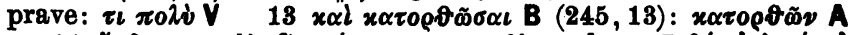

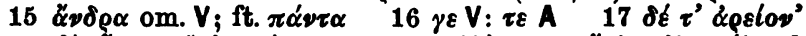

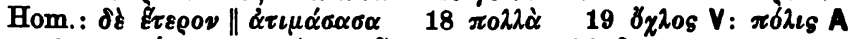

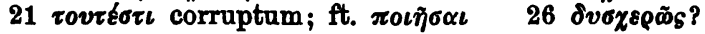




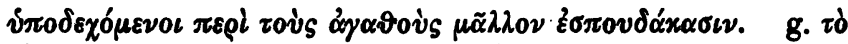

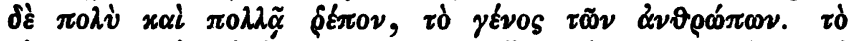

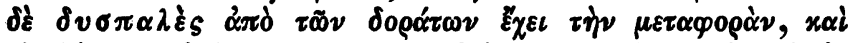

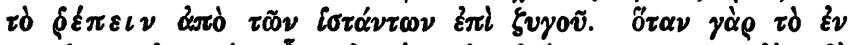

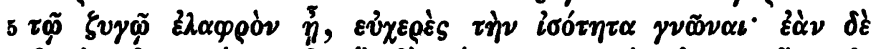

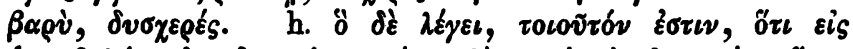

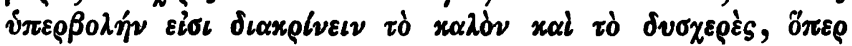

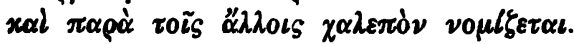

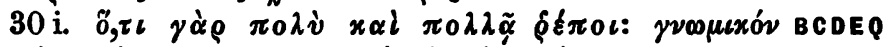

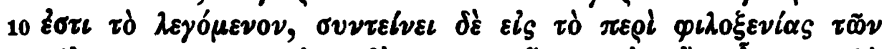

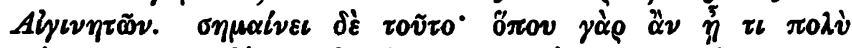

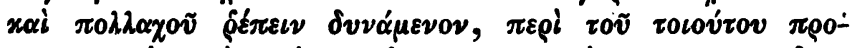

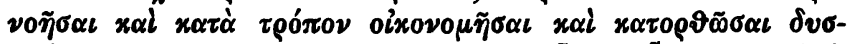

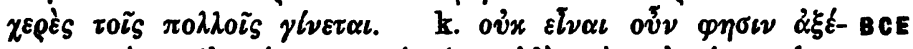

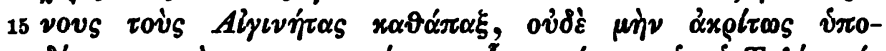

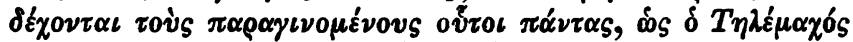

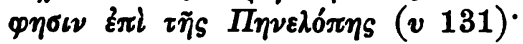

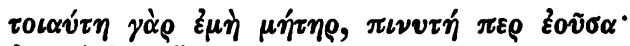

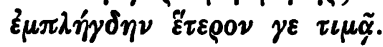

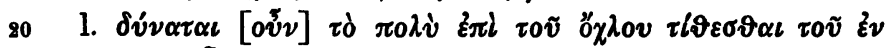

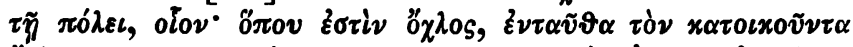

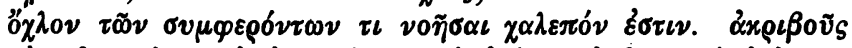

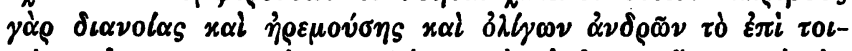

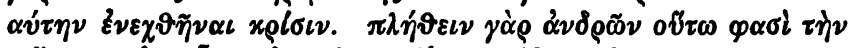

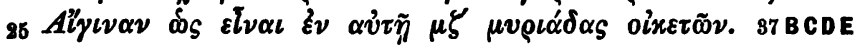

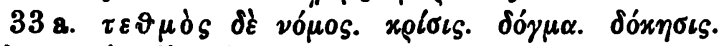

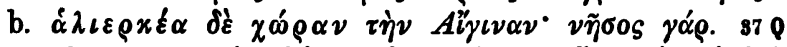

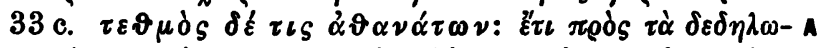

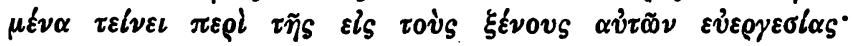

3 Eust. II. 674, 12(?)

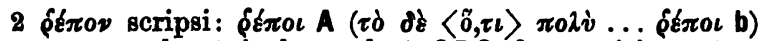
5 iे 9 sqq. sch. $30 \mathrm{i}-\mathrm{l}$ var. lect. CDQ fere omisi $13 \tau \rho \delta-$ $\pi 0 \nu] \nu \delta \mu \sigma \nu$ E || $x \alpha \tau 0 \rho \vartheta \tilde{\sigma} \sigma \alpha \iota$ B: $x \alpha \tau^{\prime} \delta \rho \vartheta \dot{0} \nu$ (C)DEQ $14 \tau 0 \tau_{S}$

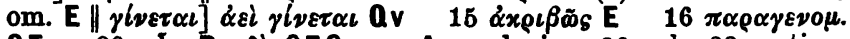
CE 20 ov̉ $\mathrm{B}$ : dè CEQ om. A; seclusi 26 sch. 33 a etiam in $U$, sed post $33 \mathrm{~d}$ 
$\varphi \eta \sigma i$ yà ö

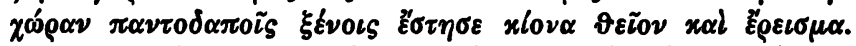

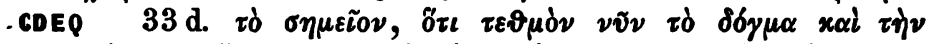

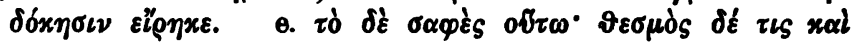

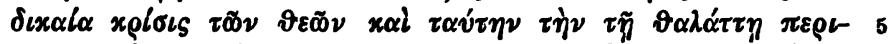

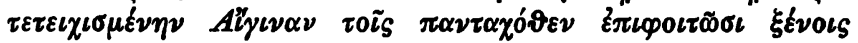

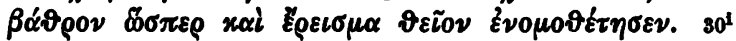

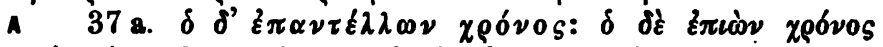

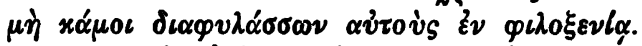

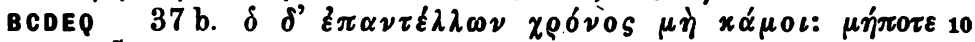

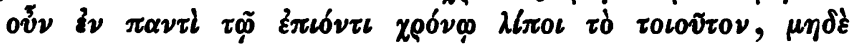

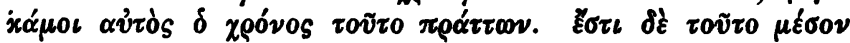

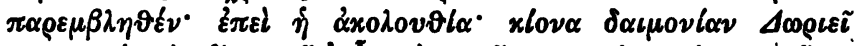

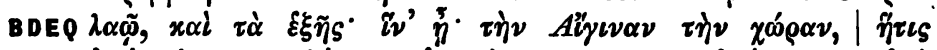

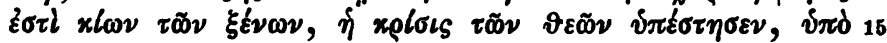

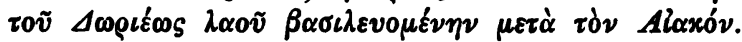

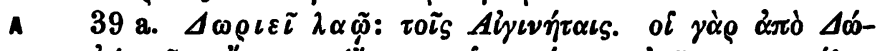

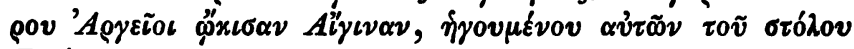

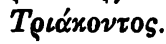

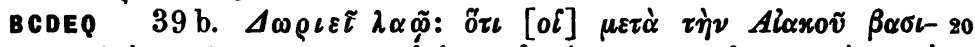

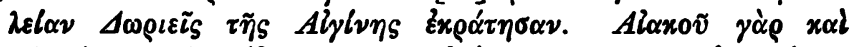

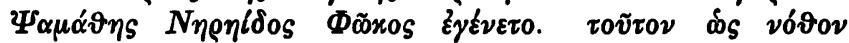

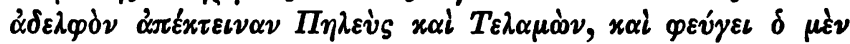

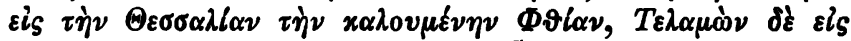

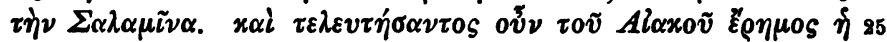

25-p. 247, 3 cfr. Tzetzes ad Lyc. 176 (p. 450 M.). id. Chil. VII 319

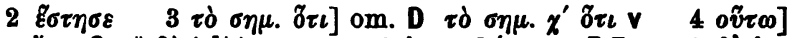

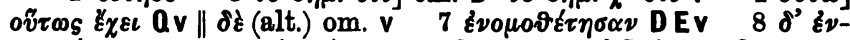

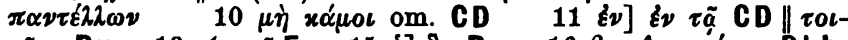

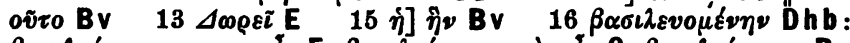

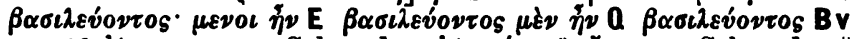

18 'A

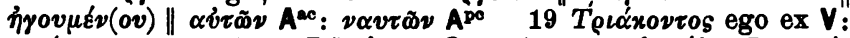

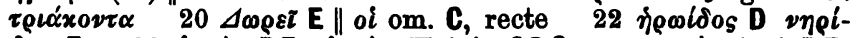

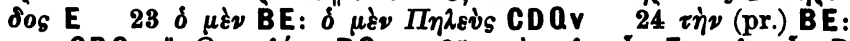

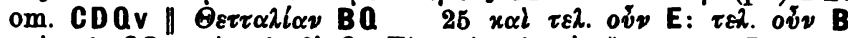

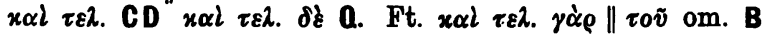




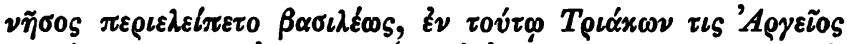

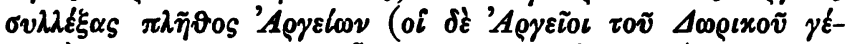

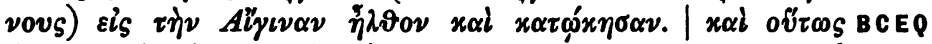

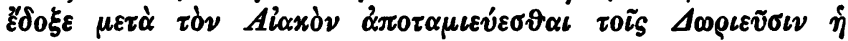

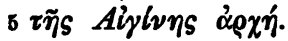

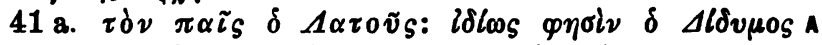

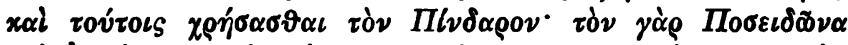

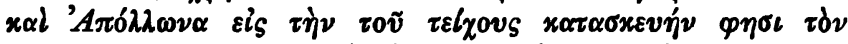

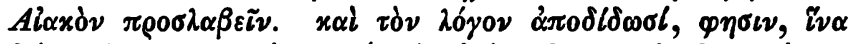

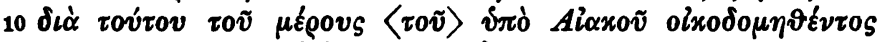

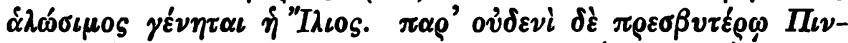

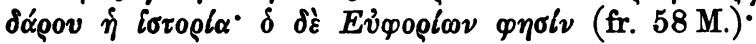

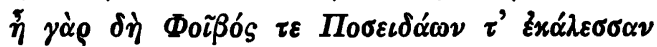

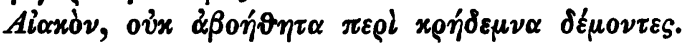

15

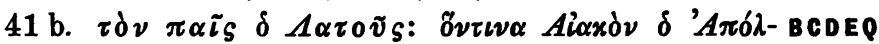

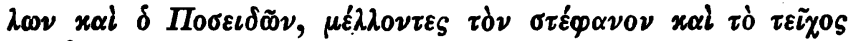

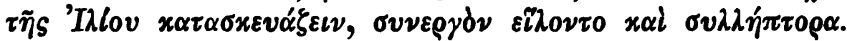

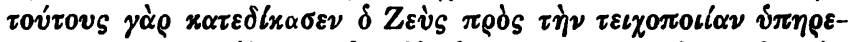

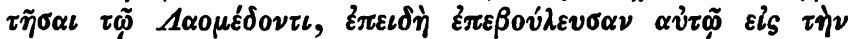

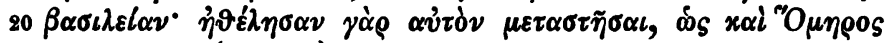
$\mu \nu \eta \mu 0 v \varepsilon v \varepsilon_{\varepsilon \iota}(A 399)^{\circ}$

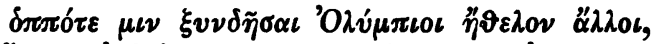

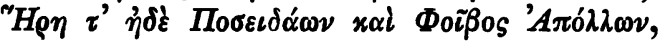

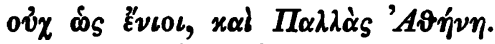

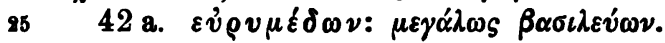

i8 sch. A $A$ 399. sch. BT $A$ 400. sch. T $\Phi 444$

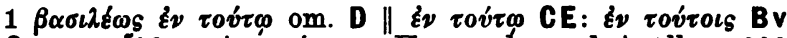

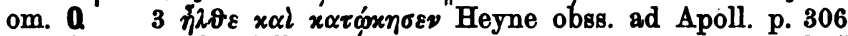

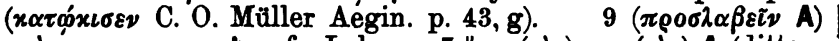
$x \alpha l-\varphi \eta \sigma \iota \nu$ om. b; cfr. Lehrs p. $7 \| \varphi \eta(\sigma i v)^{\cdot} \varphi \eta(\sigma i v)$ A (dittogr.)

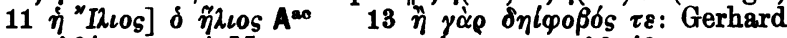

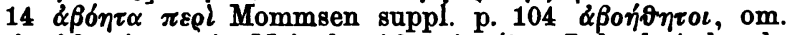

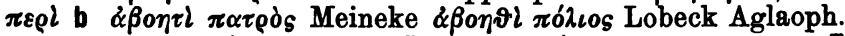

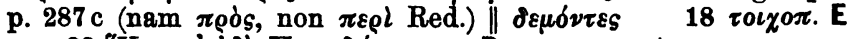

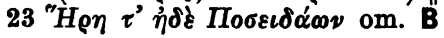




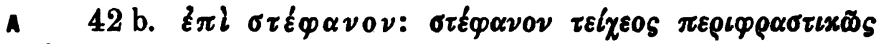

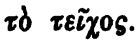

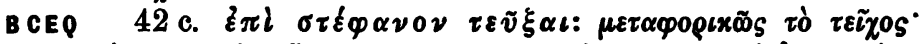

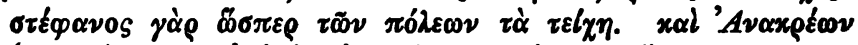

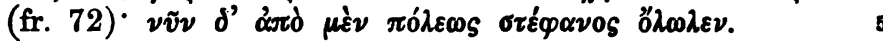

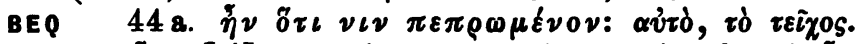

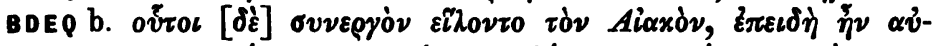

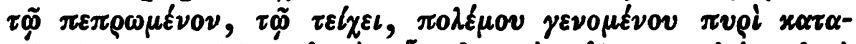

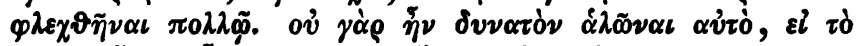

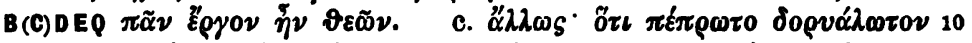


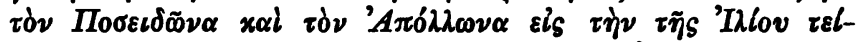

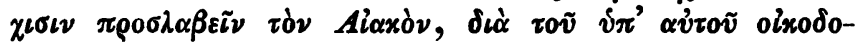

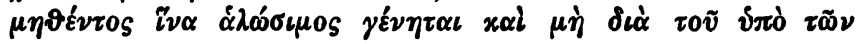
$\vartheta \varepsilon \tilde{\nu}$.

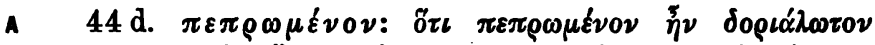

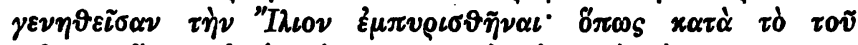

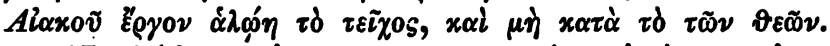

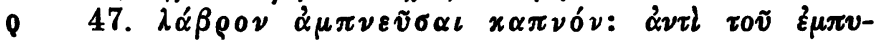

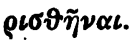

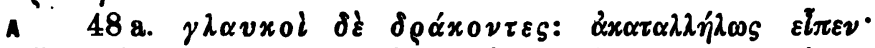
20

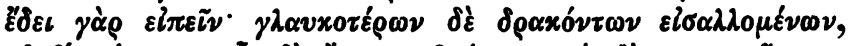

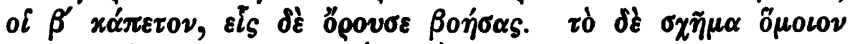

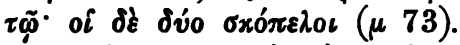

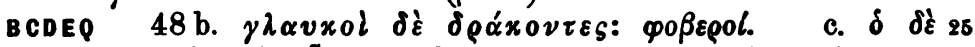

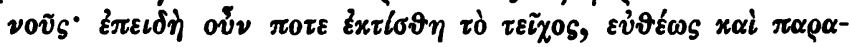

23 Eust. Od. 1713, 52

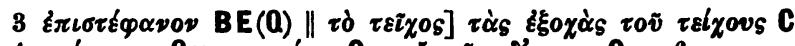

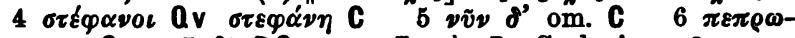

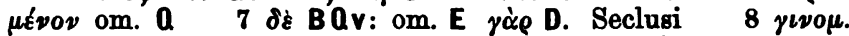

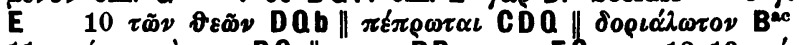
$11 \tau \alpha \dot{v} \tau \eta \nu \tau \dot{\eta} \nu \pi$. DQ \| $\varphi \eta \sigma \iota \mathrm{BD}: \varphi \alpha \sigma \iota$ Edv $12.13 \tau \varepsilon i\{\iota \sigma \iota v$

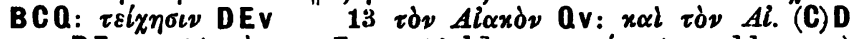
om. BE $14 \mu \grave{\eta}$ om. E 16 l.] $\pi \varepsilon \pi \rho \omega \mu \varepsilon \dot{\nu \eta}$ ( $\pi \varepsilon$ add. p. c.)

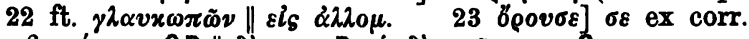

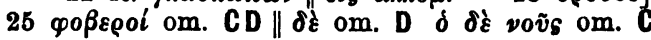




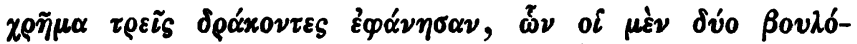

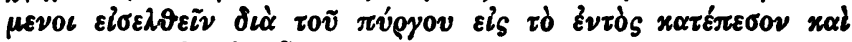

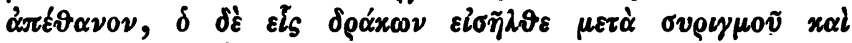

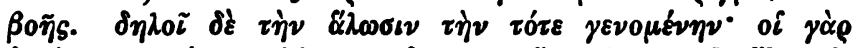

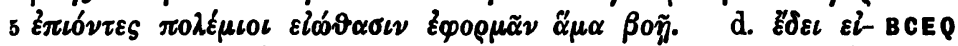

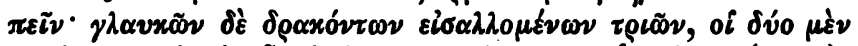

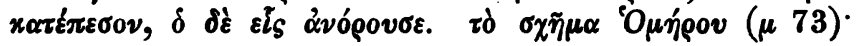

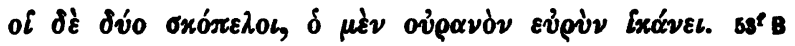

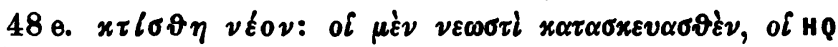

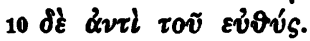

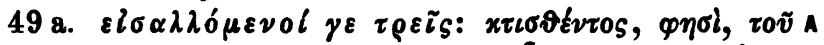

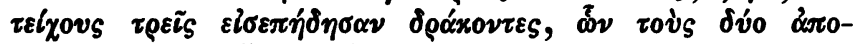

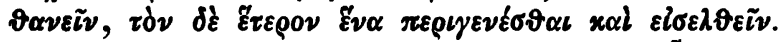

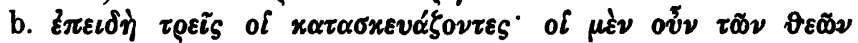

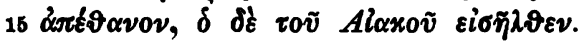

50. ( $x \alpha \dot{\pi} \pi \tau \tau 0 \nu:) x \alpha \tau k \pi \varepsilon \sigma o \nu$.

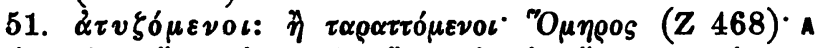

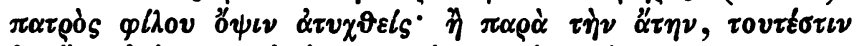

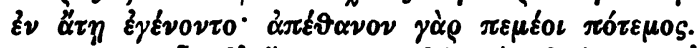

2052 \&. $\varepsilon l_{S} \delta^{\prime}$ '̆

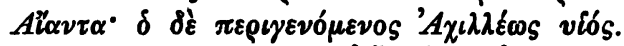

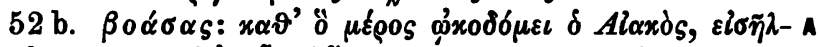

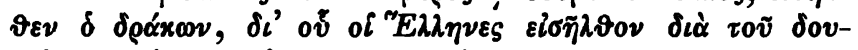

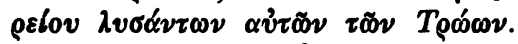

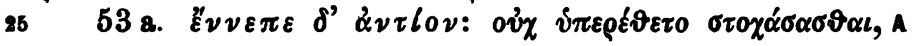

17 sch. BT Z 468. EM 168, 15. Hesych. Ap. soph. 46, 22 18 Orion. 11, 17. Eust. Il. 623, 35. 1007, 20. Hesych. s. v.

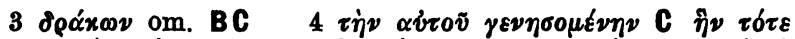

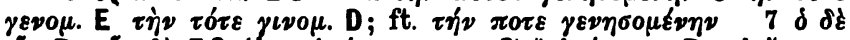

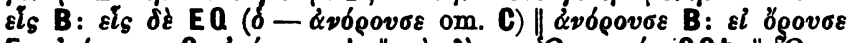

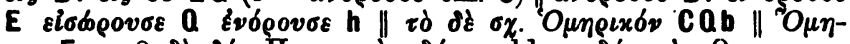

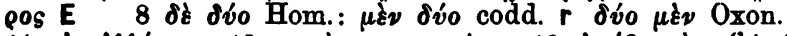

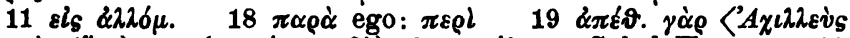

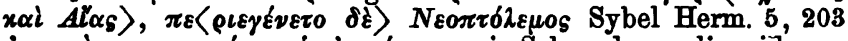

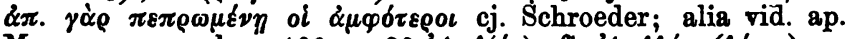

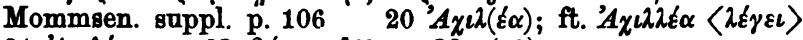

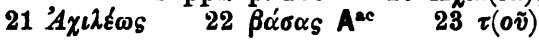




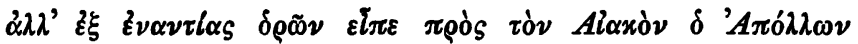

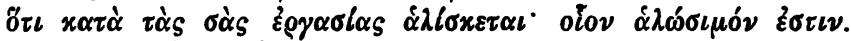

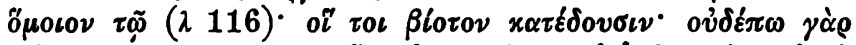

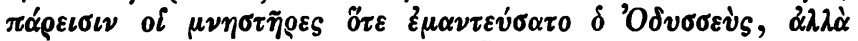

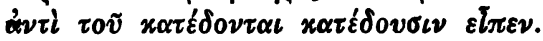

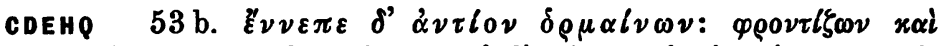

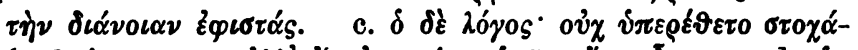

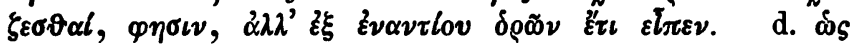

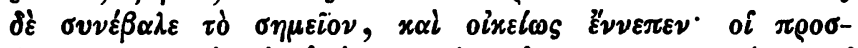

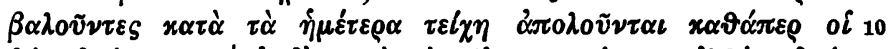

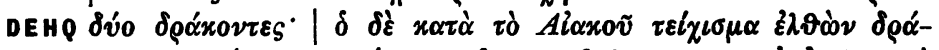

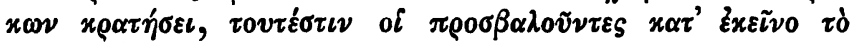

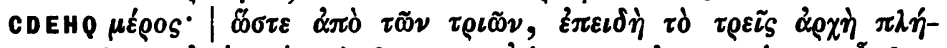

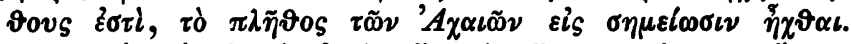

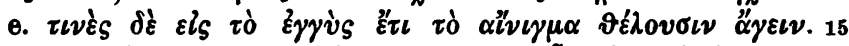

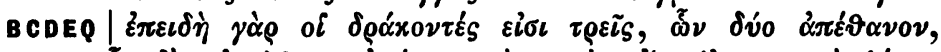

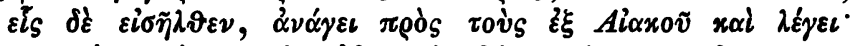

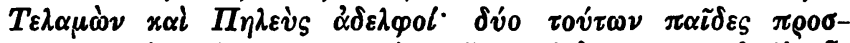

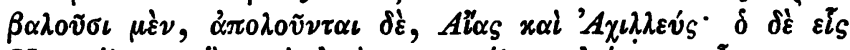

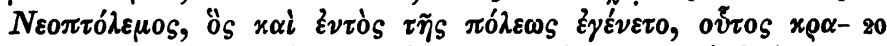

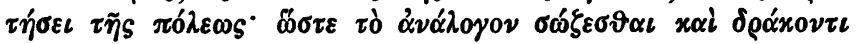

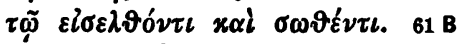

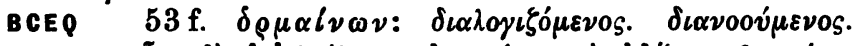

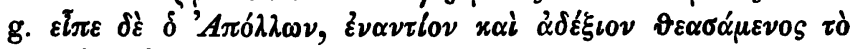

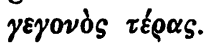

2 ante $\varepsilon$ eq $\alpha \sigma i \alpha \delta$ ras., $\sigma$ corr. (in scrib.); fuit ft. $\pi \varepsilon \rho \gamma \alpha \mu$

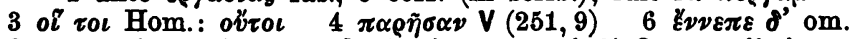

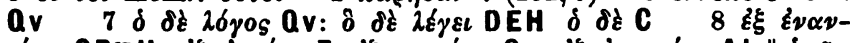

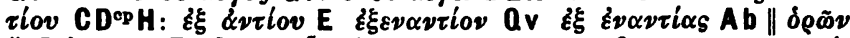
$\left.\varepsilon_{\tau \iota}\right] \delta \rho \mu \tilde{\omega} \nu$ ? Beck, b $\left.\| \varepsilon i \pi \varepsilon v\right]$ cetera seq. $55 \mathrm{c}$; cfr. 53 a $9 \sigma v \nu \varepsilon^{-}$

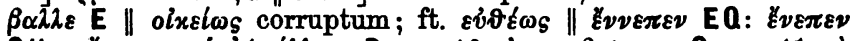

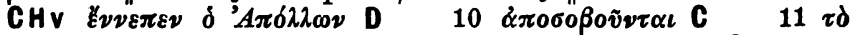

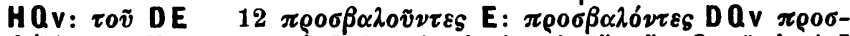

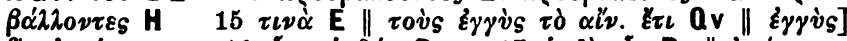

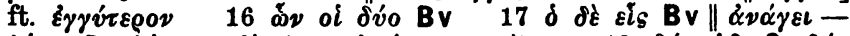

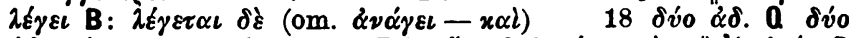

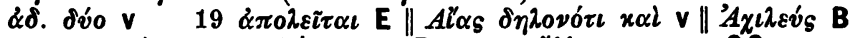
$21 x \alpha \iota-22 \sigma \omega \vartheta \hat{\varepsilon} \nu \tau \iota$ om. D $23 \alpha \alpha \lambda \omega \omega$ praem. CQv $24 \dot{\alpha} \delta \xi \xi$ Lov] $\alpha \dot{\delta} \circ \xi o \nu$ BV 


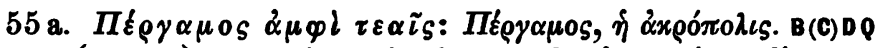

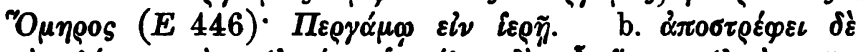

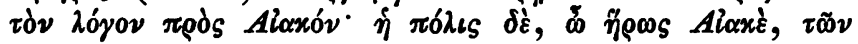

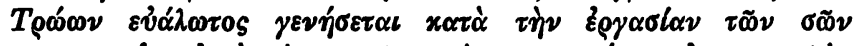

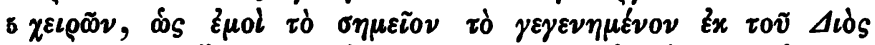

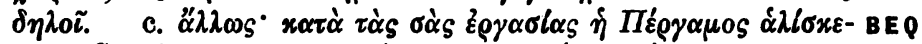

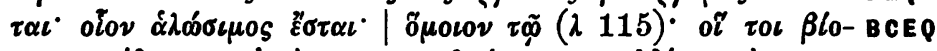

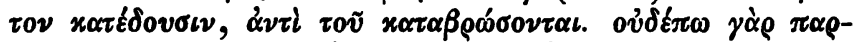

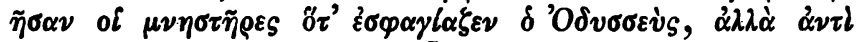

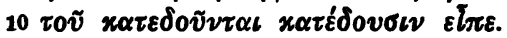

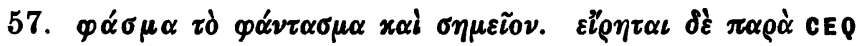

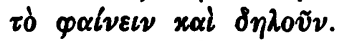

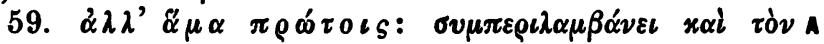

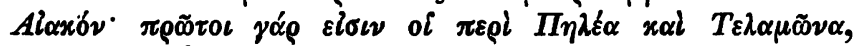

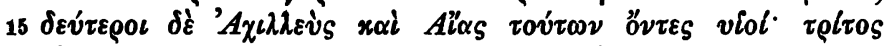

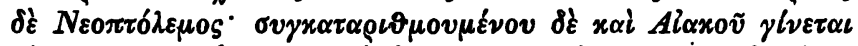

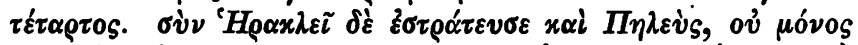

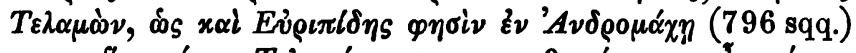

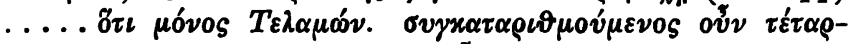

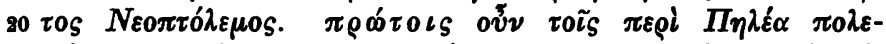

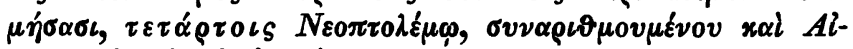

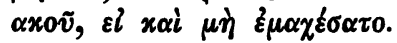

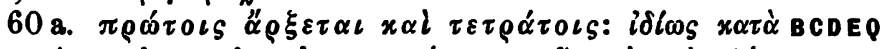

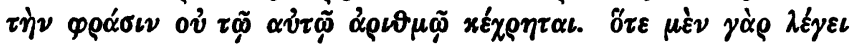

1 sch. $\Delta$ 508. Ap. soph. Hesych. 11 Hesych. 17 sch. Eur. Andr. 796

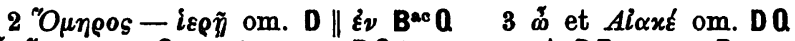

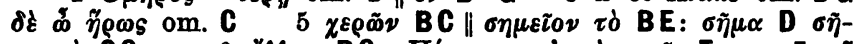

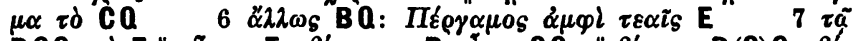

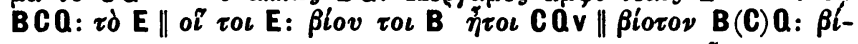

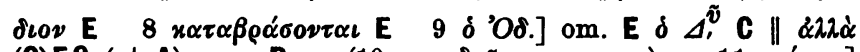

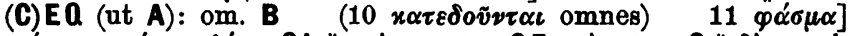

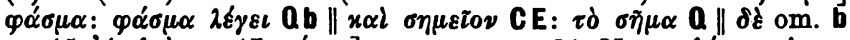

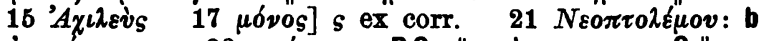

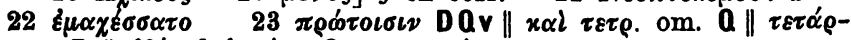

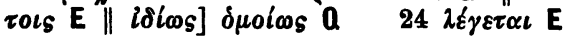




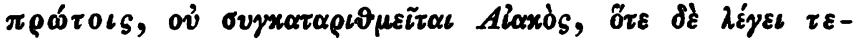

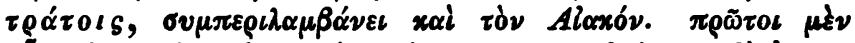

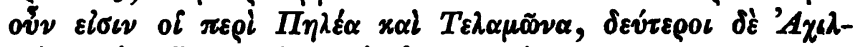

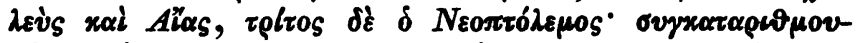

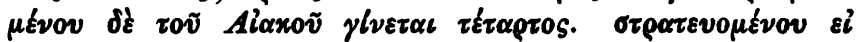

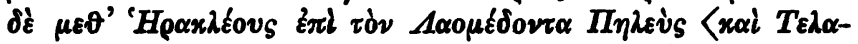

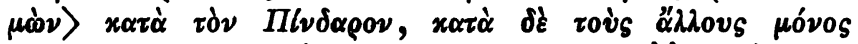

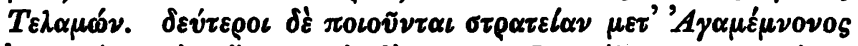

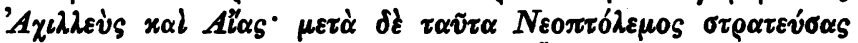

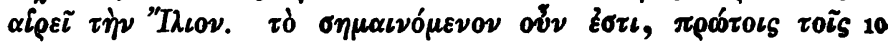

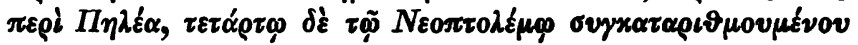

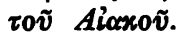

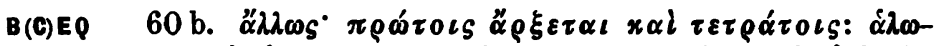

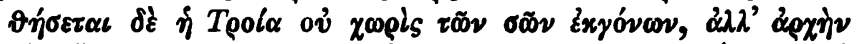

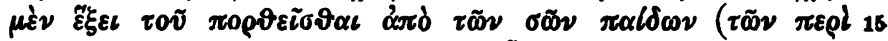

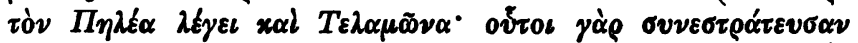

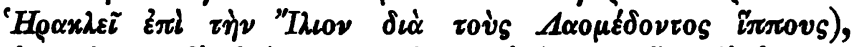

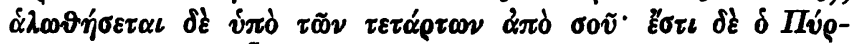

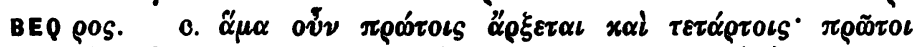

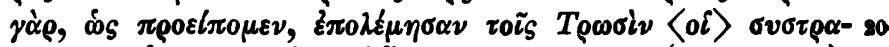

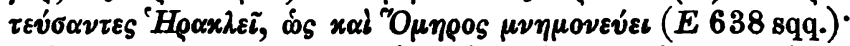

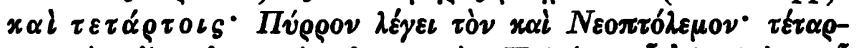

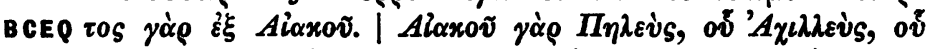

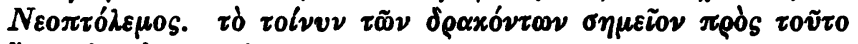

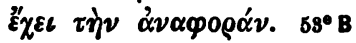

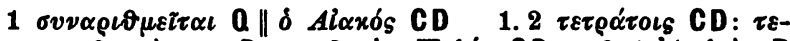

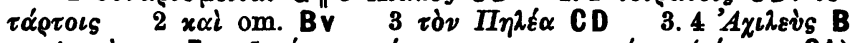

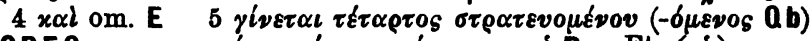

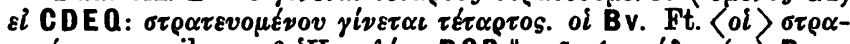

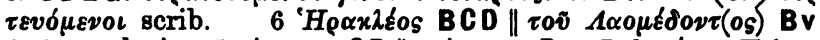

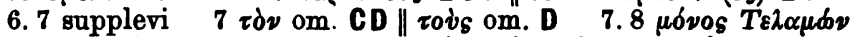

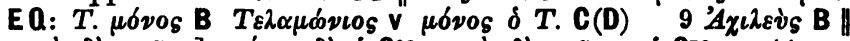

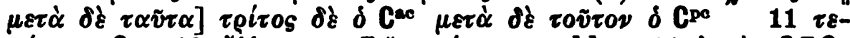

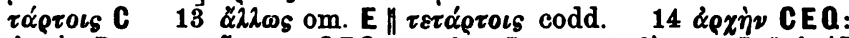

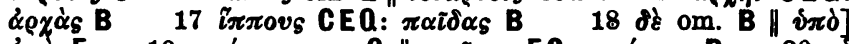
dist $E$

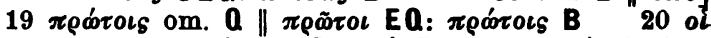

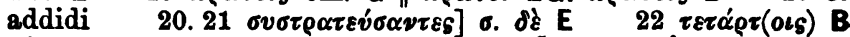

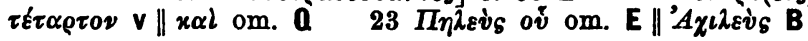




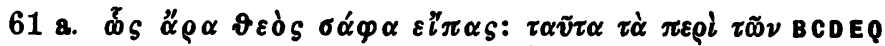

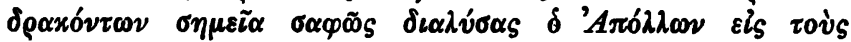

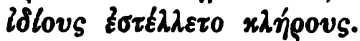

61 b. ( Bel

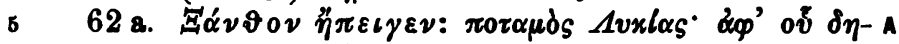

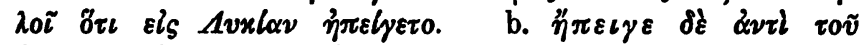

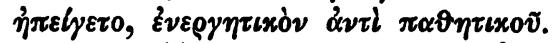

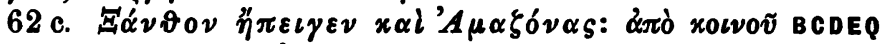

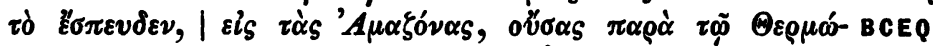

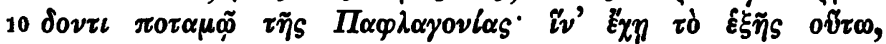

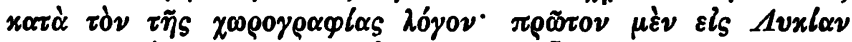

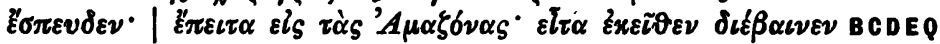

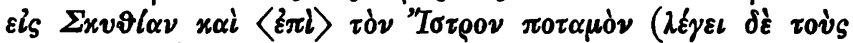

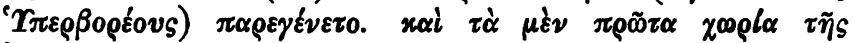

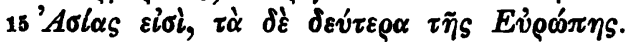

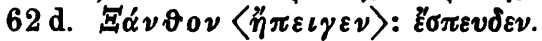

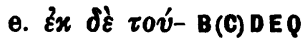

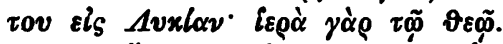

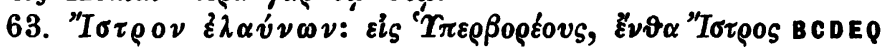

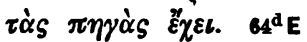

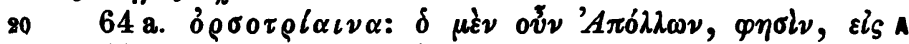

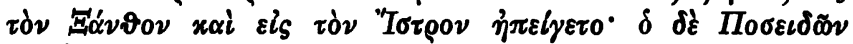

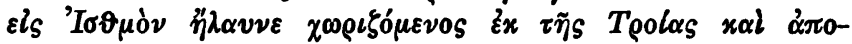

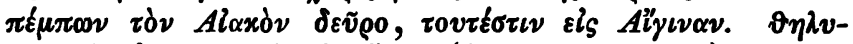

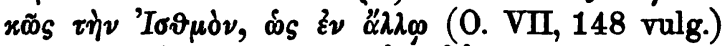

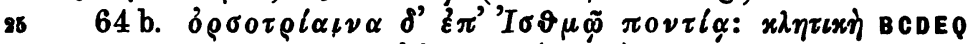

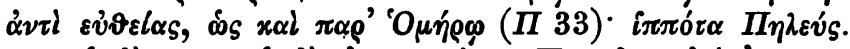

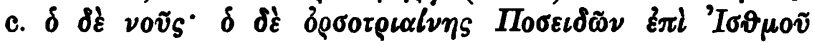

4 Gregor. Cor. p. 481 S. 25 sch. $A$ 175. EG 393, 32. sch. T $H$ 384. Eust. Il. 686, 25. Greg. Cor. p. 96. $603 \mathrm{~S}$.

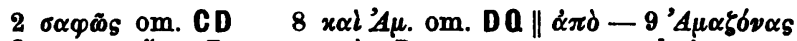
om. C 10 \& $\chi \circ \iota$ E $11 \mu \dot{1}$ B : om. 13 suppl. h

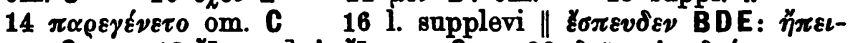

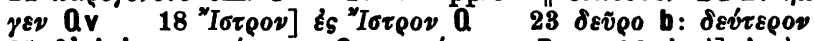

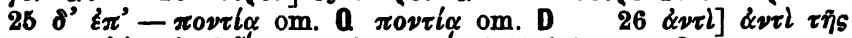

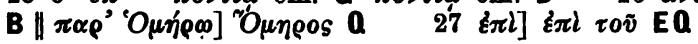




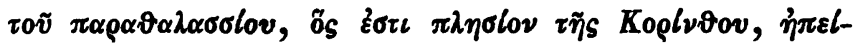

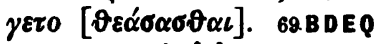

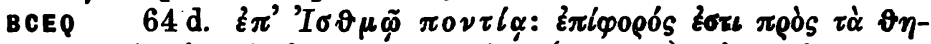

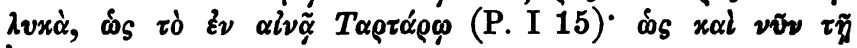
'I $\sigma \nabla \mu \tilde{\omega}$. ${ }^{\circ} \mathbf{4}^{\mathrm{D}} \mathrm{E}$

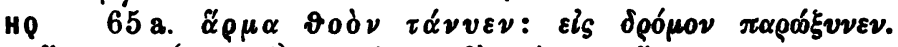

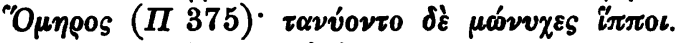

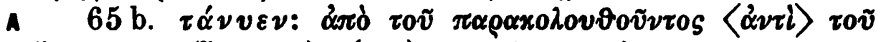

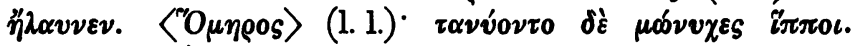

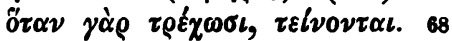

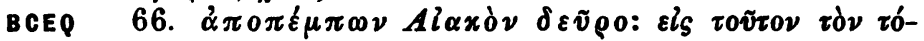

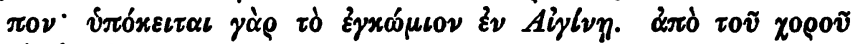

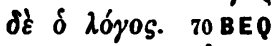

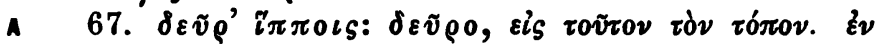
Alylvin. 69

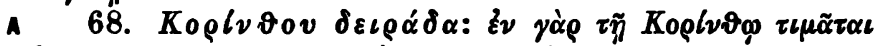

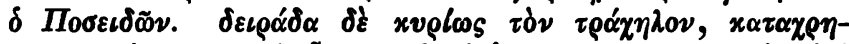

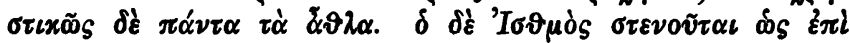

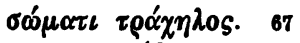

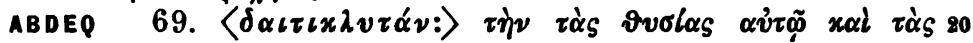

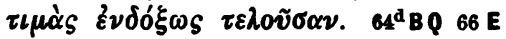

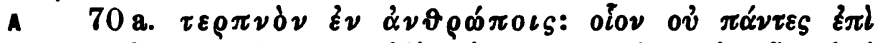

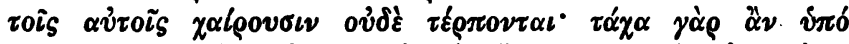

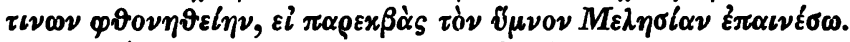

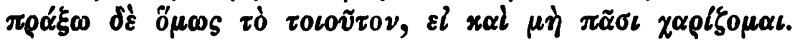

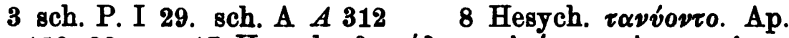

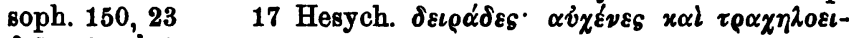

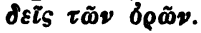

$1.2 \varepsilon \pi \varepsilon i \gamma \varepsilon \tau \alpha \iota$ EQ $2 \vartheta \varepsilon \alpha \dot{\sigma} \alpha \sigma \vartheta \alpha \iota$ (ex gl. ortum?) seclnsi.

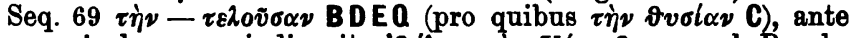

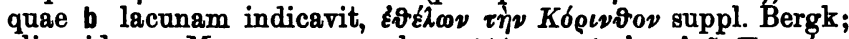

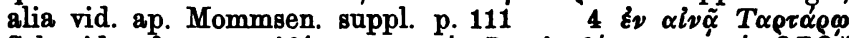

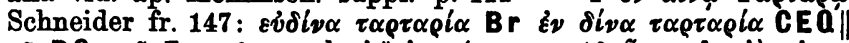

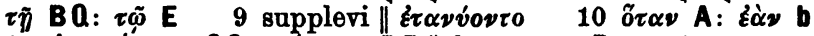

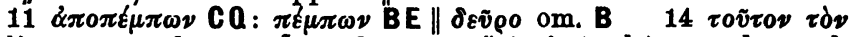

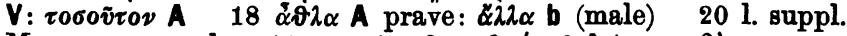

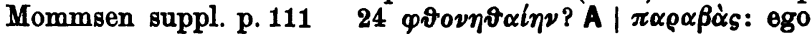




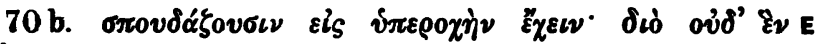

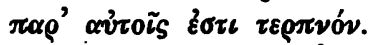

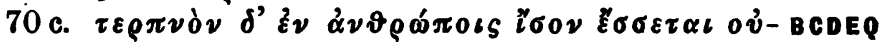

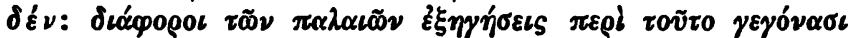

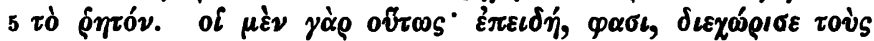

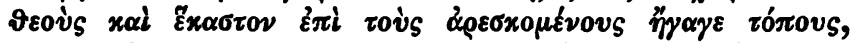

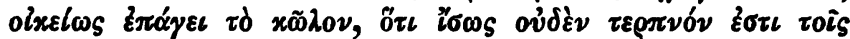

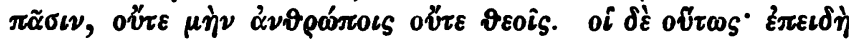

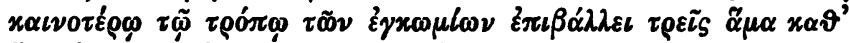

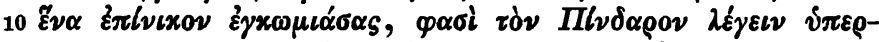

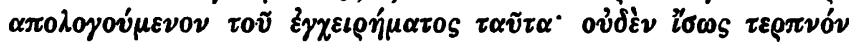

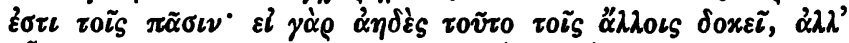

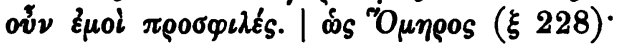

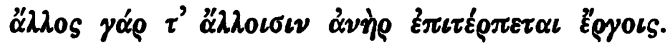

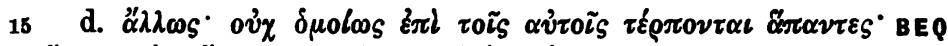

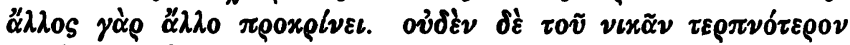

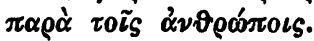

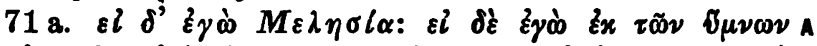

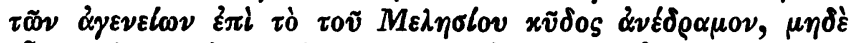

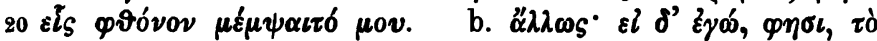

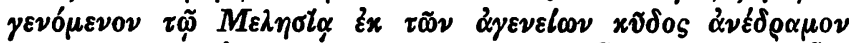

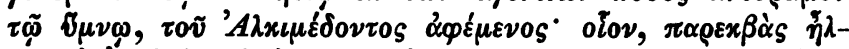

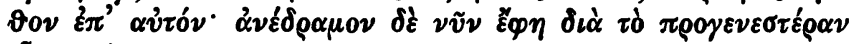

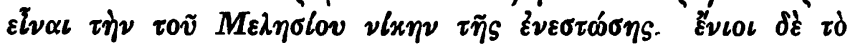

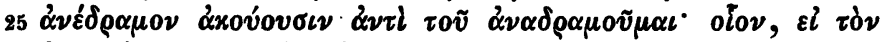

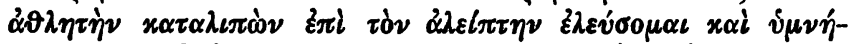

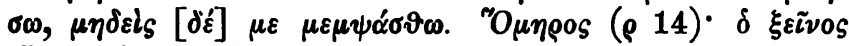

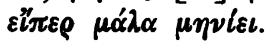

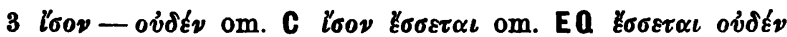

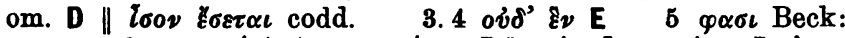

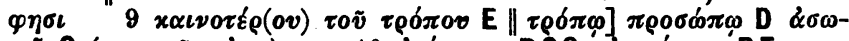

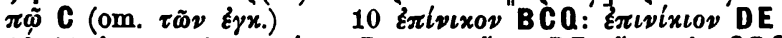

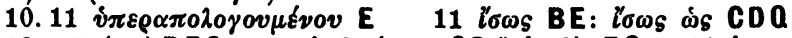

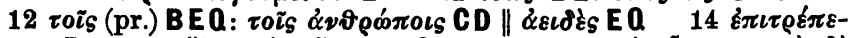

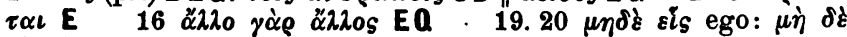
$\varepsilon i_{S}$ A $\mu \eta \delta \varepsilon l_{S} \varepsilon i_{S}$ Mommsen suppl. p. $11322 \pi \alpha \rho \alpha \beta \dot{\alpha} s:$ ego

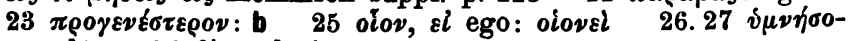

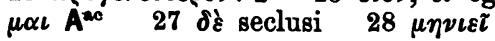




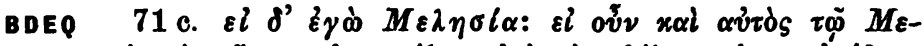

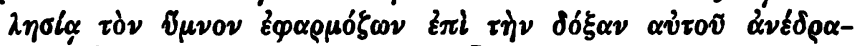

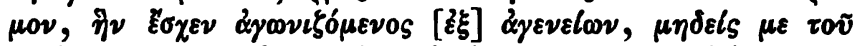

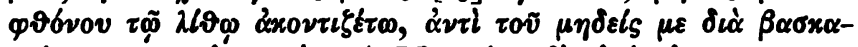

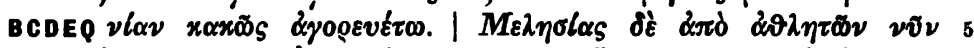

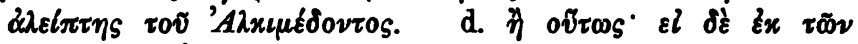

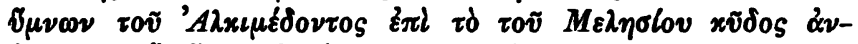

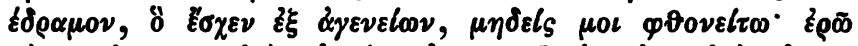

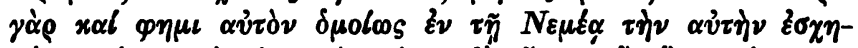

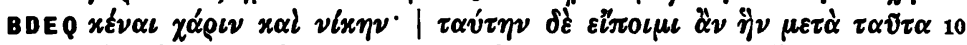

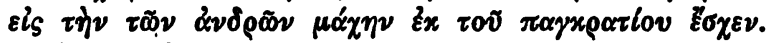

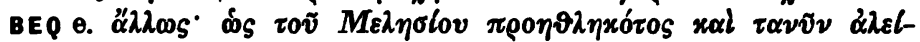

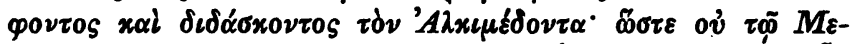

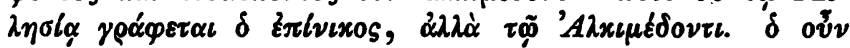

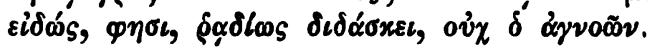

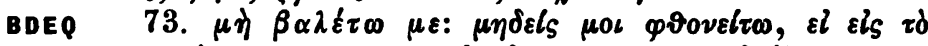

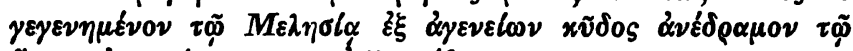

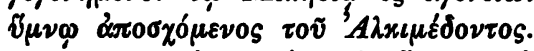

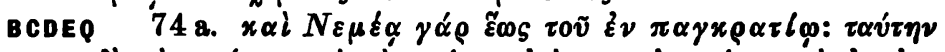

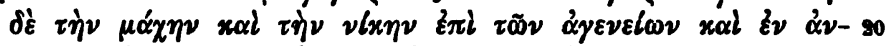

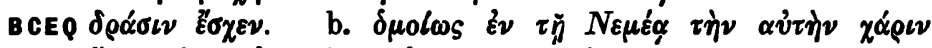

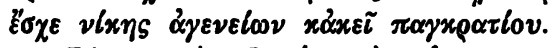

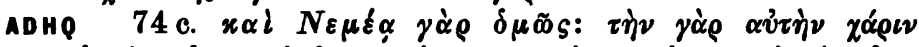

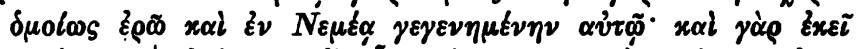

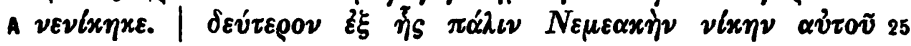

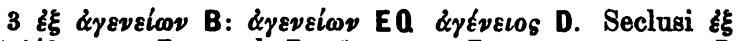

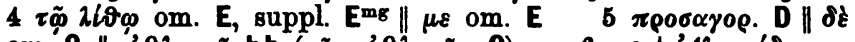

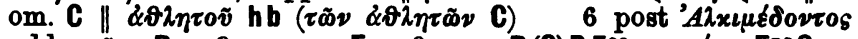
add. $x \varepsilon \tilde{i} \nu \alpha$ B $8 \mu 0 \iota$ om. E $9 \varphi \eta \mu \iota$ B (C)DE D

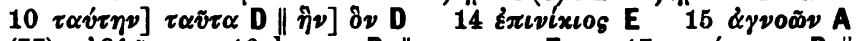

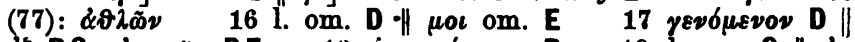

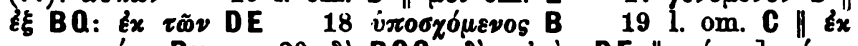

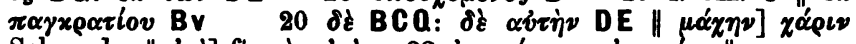

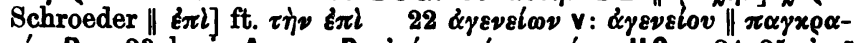

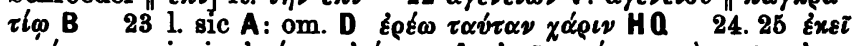

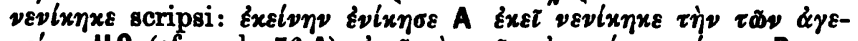

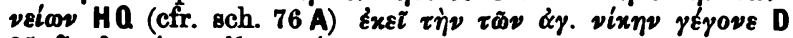

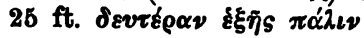




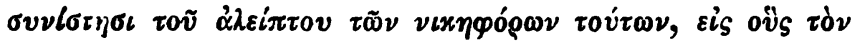

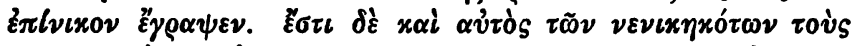

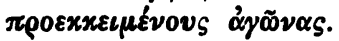

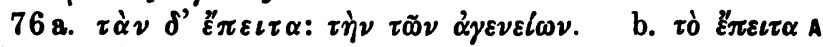

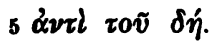

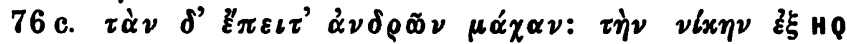

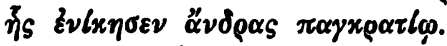

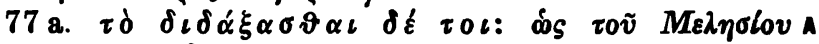

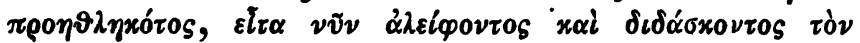

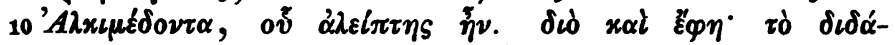

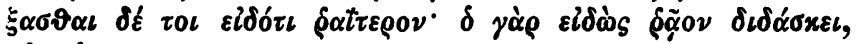

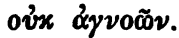

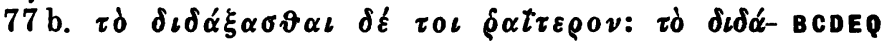

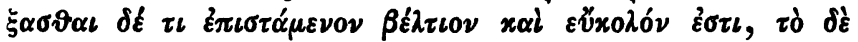

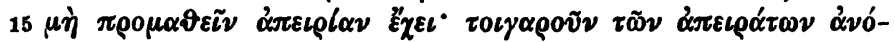

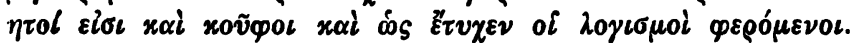

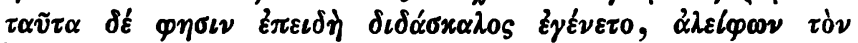

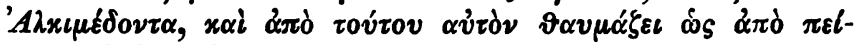

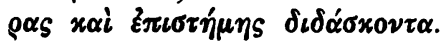

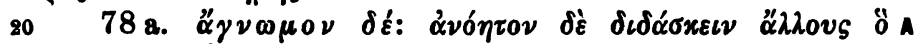

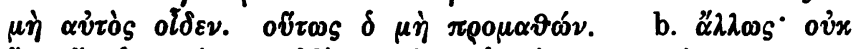

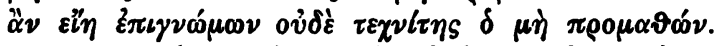

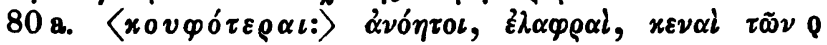
$\dot{\alpha} \mu \alpha \vartheta \tilde{\omega} \nu \alpha \tilde{i} \delta\llcorner\alpha \dot{\nu} \nu\llcorner\alpha \iota$.

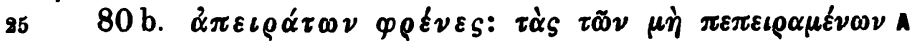

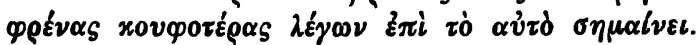

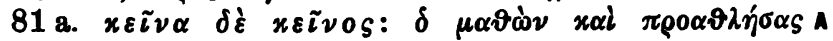

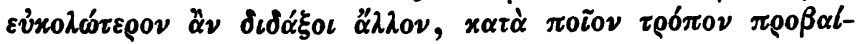

$6 \delta \xi \pi 0 \tau^{\prime} H \| \mu \alpha_{\alpha} \alpha \nu$ om. Q $6.7 \mathrm{ft} . \tau \dot{\eta} \nu \nu i x \eta \nu:-\varepsilon \xi \xi \tilde{\xi}$

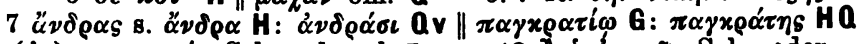

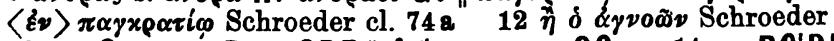

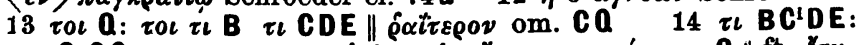

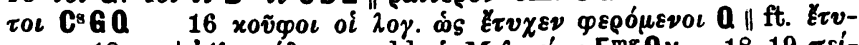

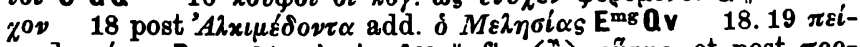

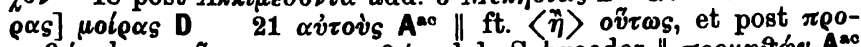

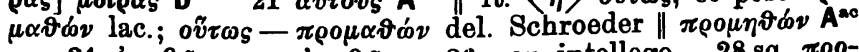
$24 \alpha \mu \alpha \vartheta \tilde{\omega} \nu$ ego: $\alpha \gamma \alpha \vartheta \tilde{\omega} \nu 26$ non intellego 28 sq. $\pi \rho \circ-$

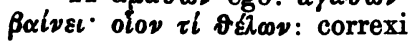

Schor. ax Pridarox ed. Drachmann. 


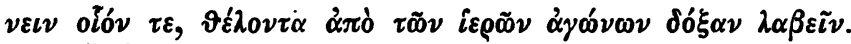

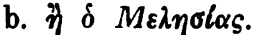

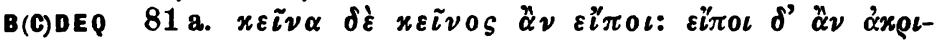

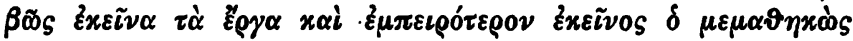

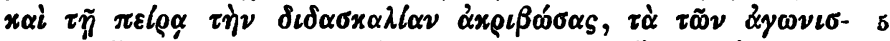

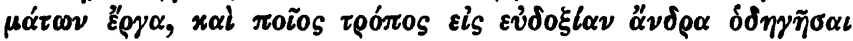

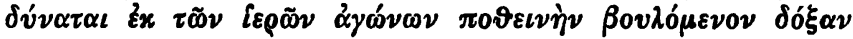

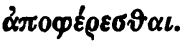

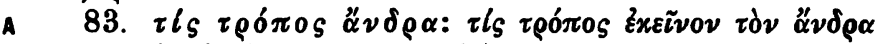

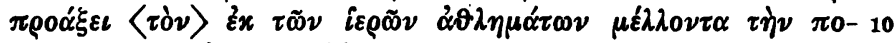

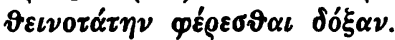

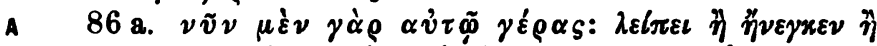

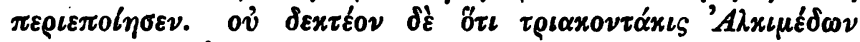

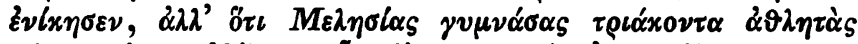

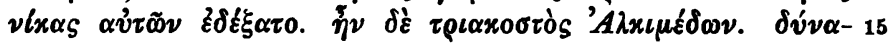

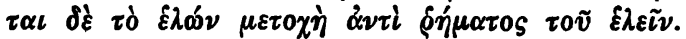

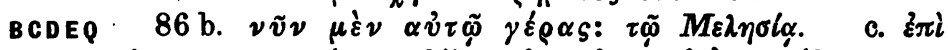

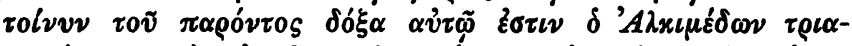

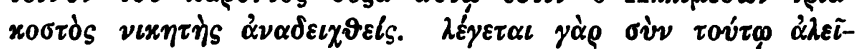

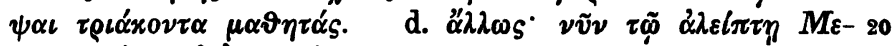

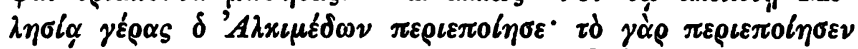

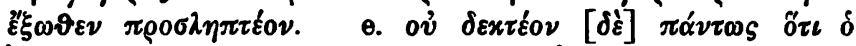

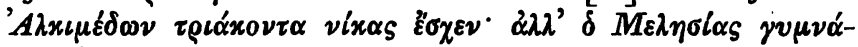

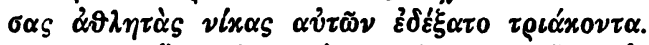

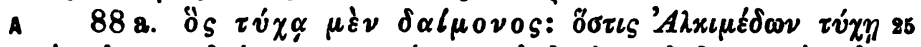

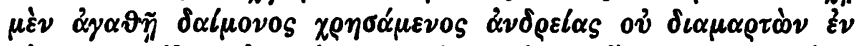

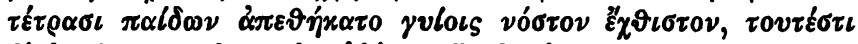

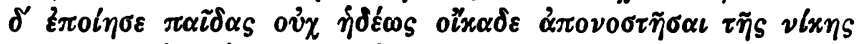

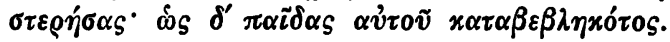

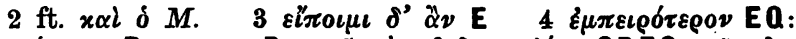

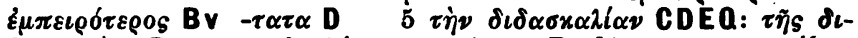

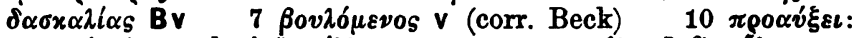

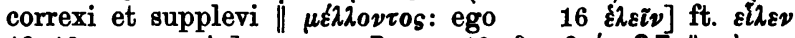

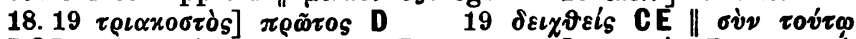

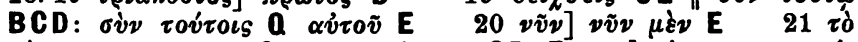
$\gamma \dot{\alpha} \rho \pi \varepsilon \rho \varepsilon \pi$. om. D Q $22 \delta \xi$ om. CD?E; seclusi $24 \tau \rho\llcorner\alpha \dot{-}-$

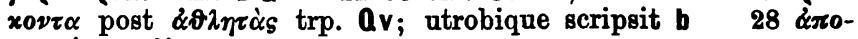

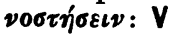




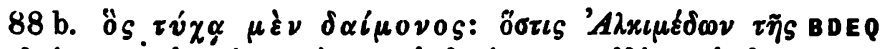

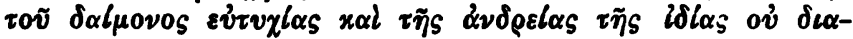

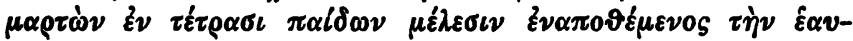

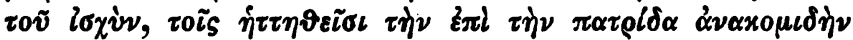

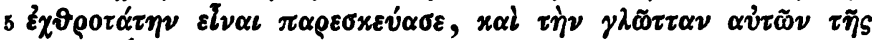

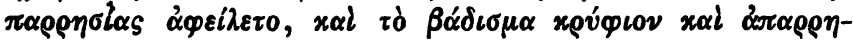

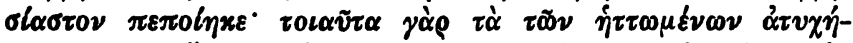

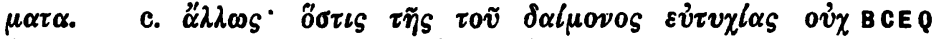

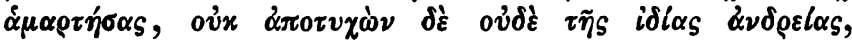
10 Ė

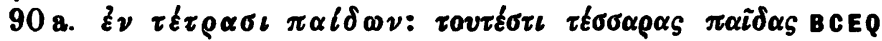

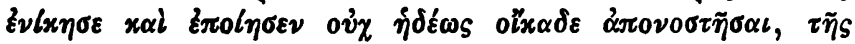

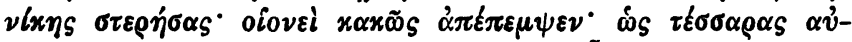

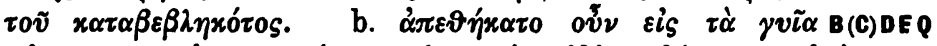

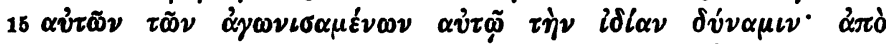

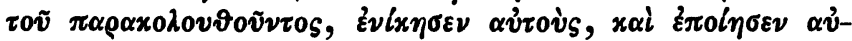

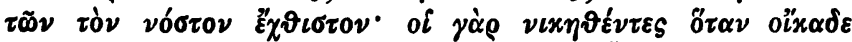

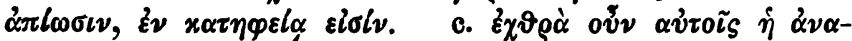

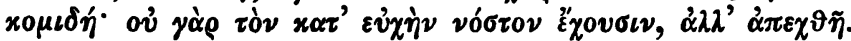

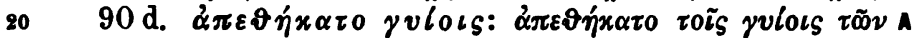

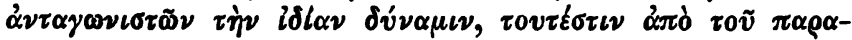

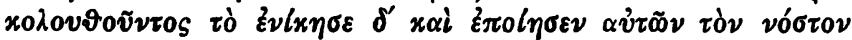

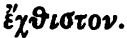

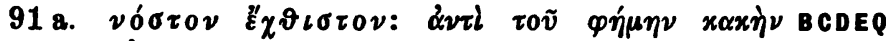

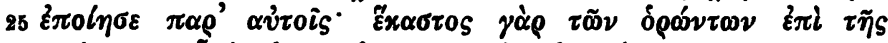

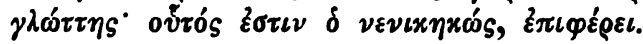

$3 \tau \varepsilon \varepsilon \alpha \rho \sigma \iota$ DEQ 4 iो̀े (ante $\pi \alpha \tau \rho$.) om. E 6 post

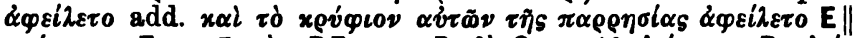

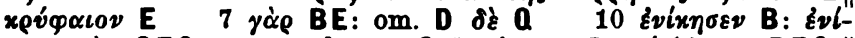

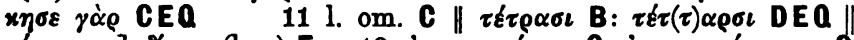

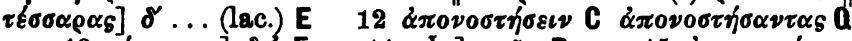

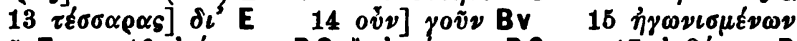

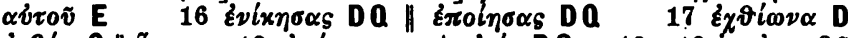

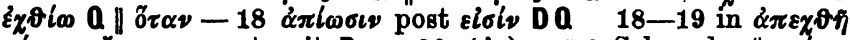

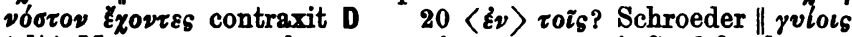
(alt.) Mommsen suppl. p. 116: viots 22 id ft. delend.

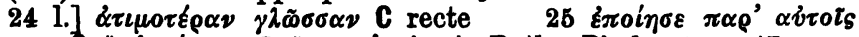

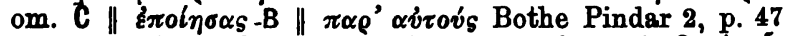

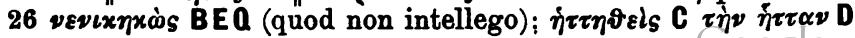




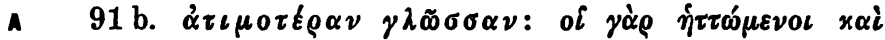

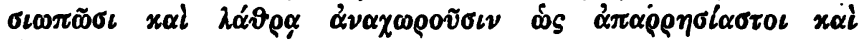

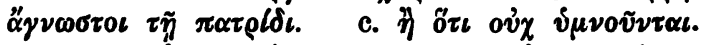

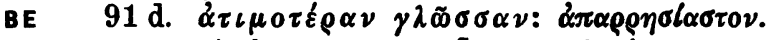

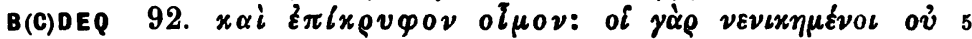

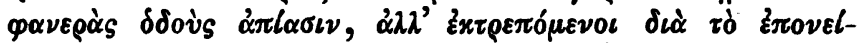

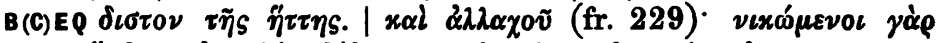

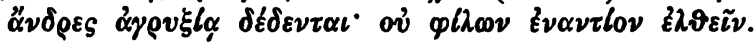

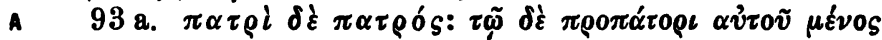

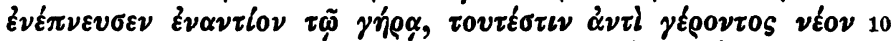

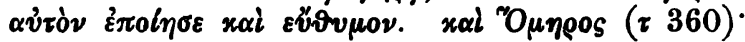

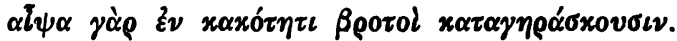

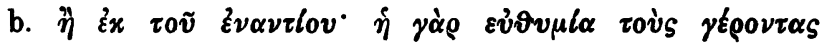

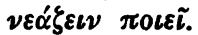

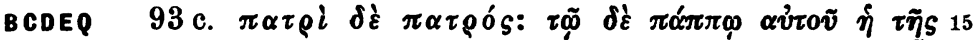

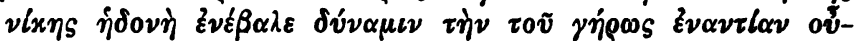

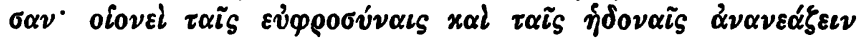

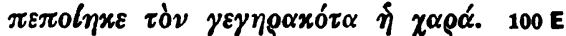

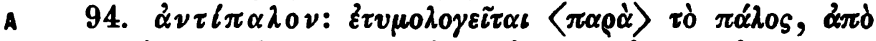

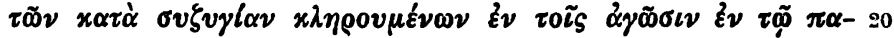
$\lambda \alpha \ell \varepsilon \iota \nu$ ऐे $\pi \alpha \gamma x \rho \alpha \tau \iota \alpha \dot{\xi} \xi \varepsilon \nu$.

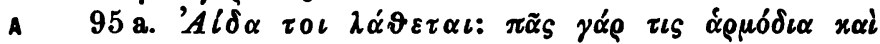

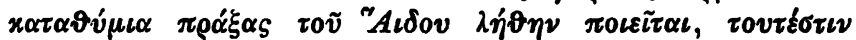

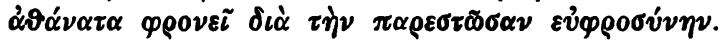

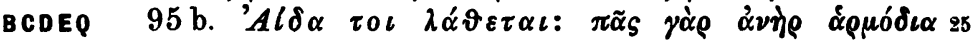

19 EM 111, 38. EG 61, 15. Eust. Il. 419, 12, al.

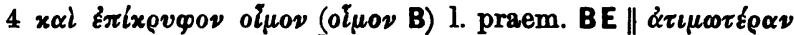

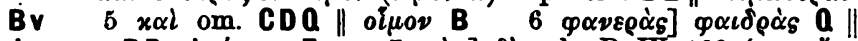

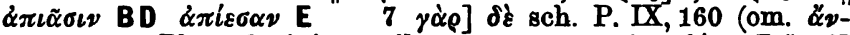

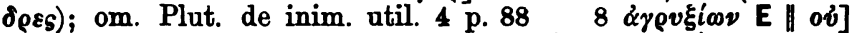

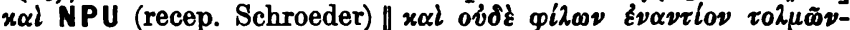

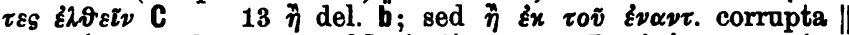

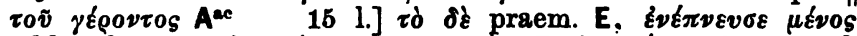

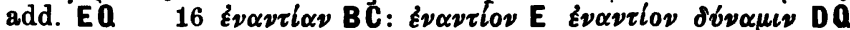

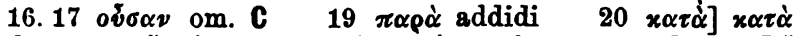

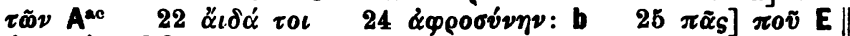
$\dot{\alpha} \rho \mu_{0} \delta i^{\prime} \omega s \mathrm{CQ}$ 


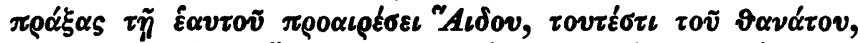

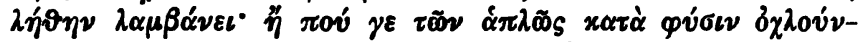

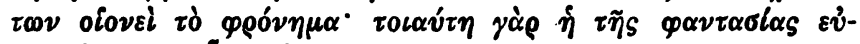

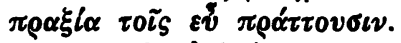

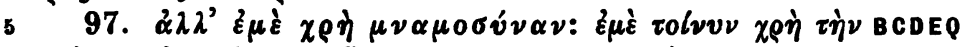

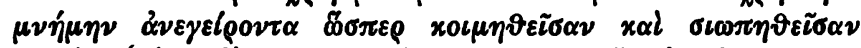

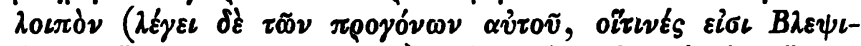

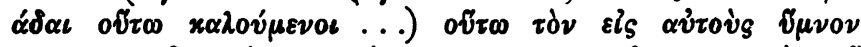

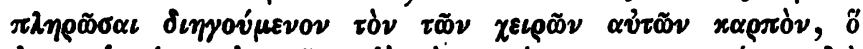

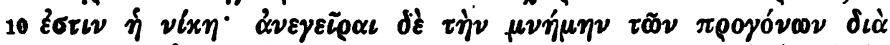

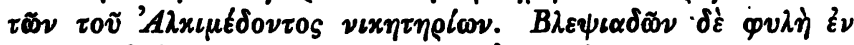

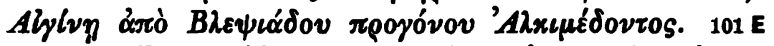

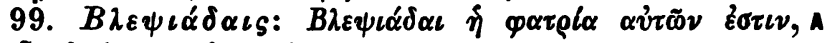

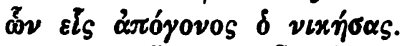

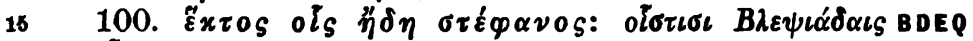

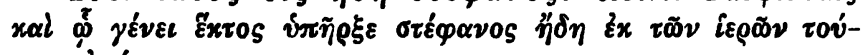

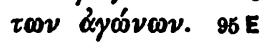

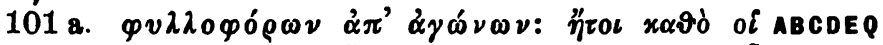

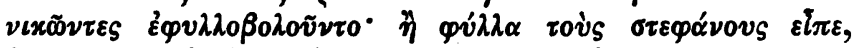

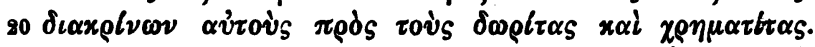

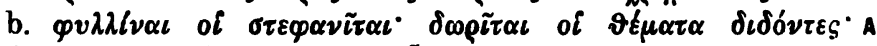

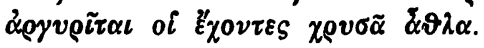

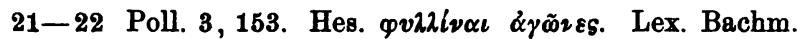
410, 9. EM 802, 38. - Plut. praec. ger. reip. 27 p. 820

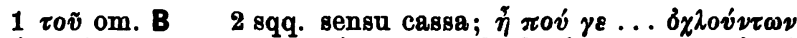

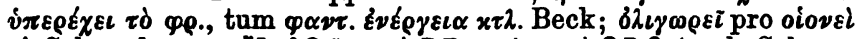

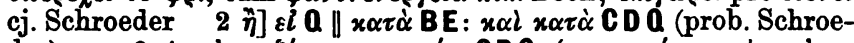

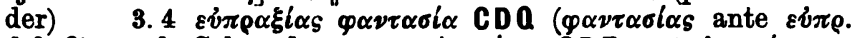

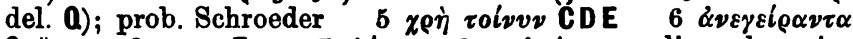

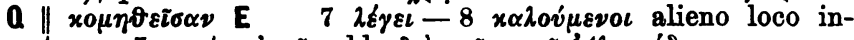

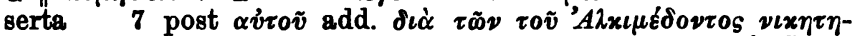

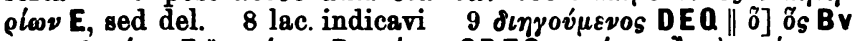

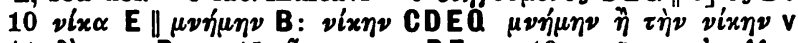
11 dzे om. D 15 Ex

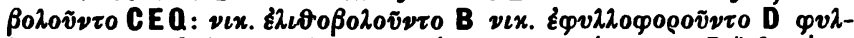

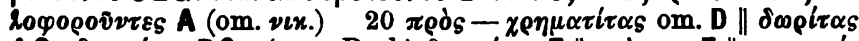

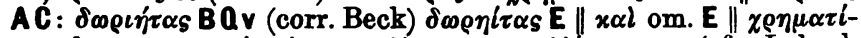

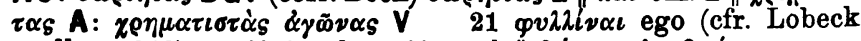

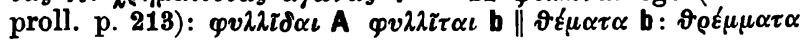




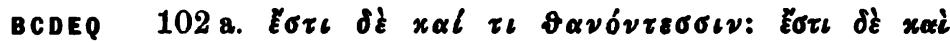

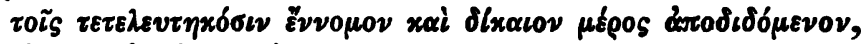

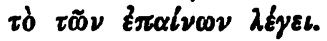

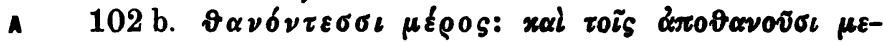

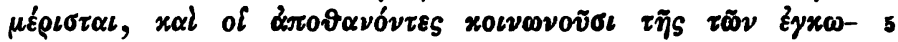

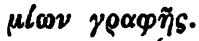

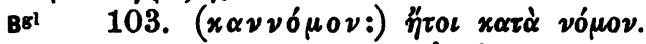

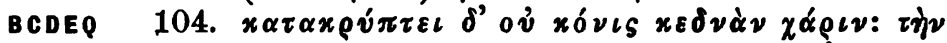

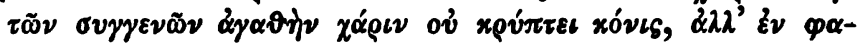

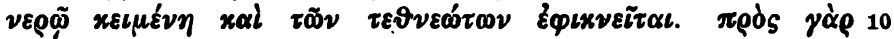

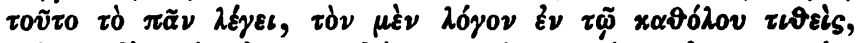

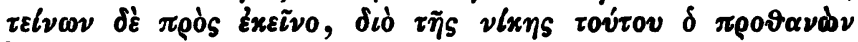

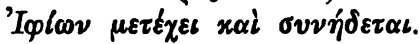

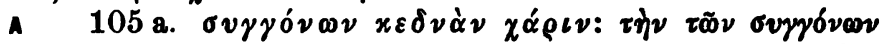

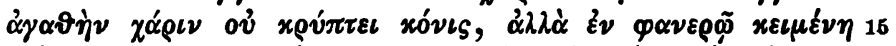

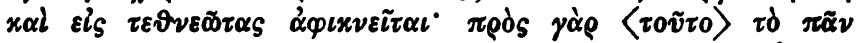

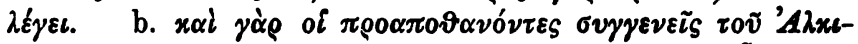

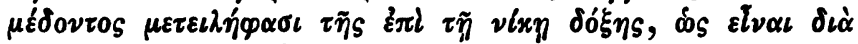

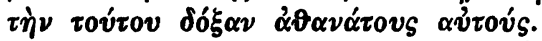

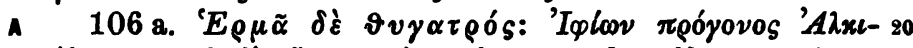

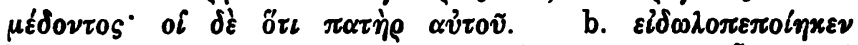

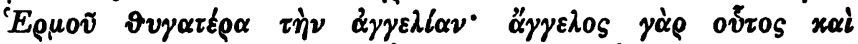

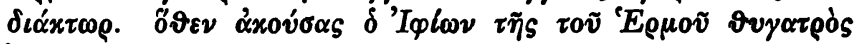

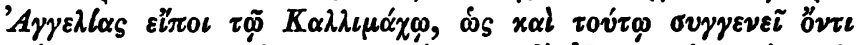

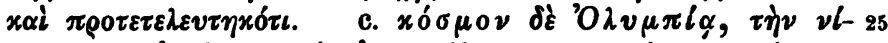

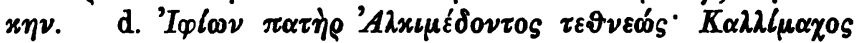

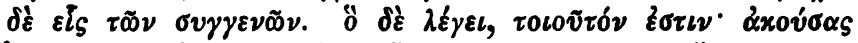

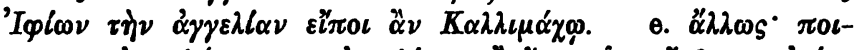

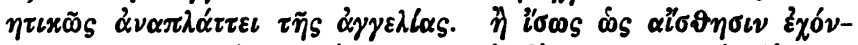

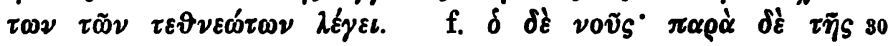

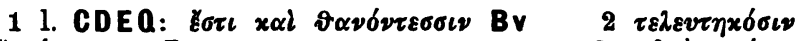

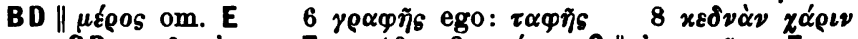

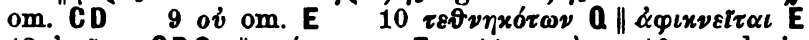

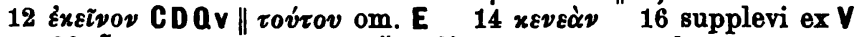

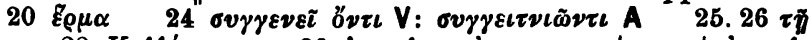
vixy $28 K \alpha \lambda \lambda i \mu \alpha \chi 0 s \quad 29 \& \nu \alpha \pi \lambda$. $\tau$. $\alpha \gamma \gamma$. corrupta; aut $\alpha \nu \alpha \pi \lambda$. $\tau \dot{\alpha} s \dot{\alpha} \gamma \gamma$. aut $\alpha \nu \alpha \pi \lambda$. . . . . (lac.) $\tau \tilde{\eta} s \dot{\alpha} \gamma \gamma$. cj. Schroeder 


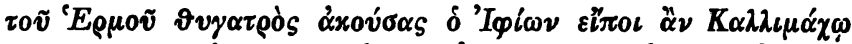

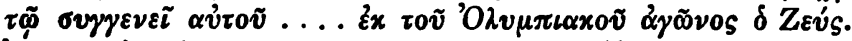

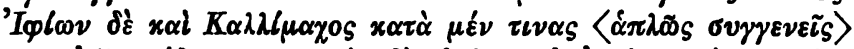

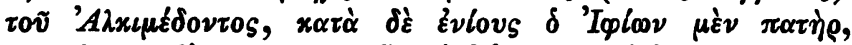

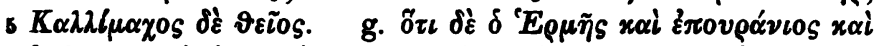

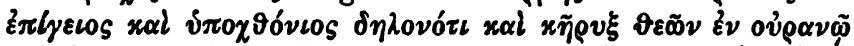

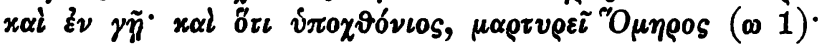

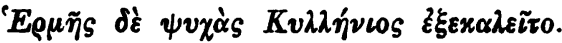

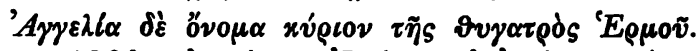

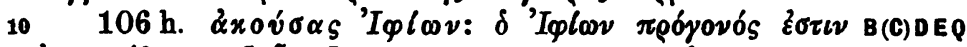

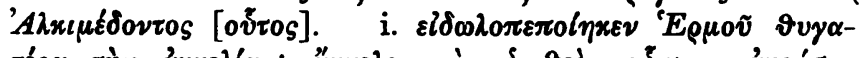

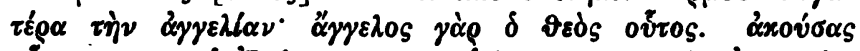

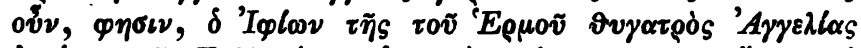

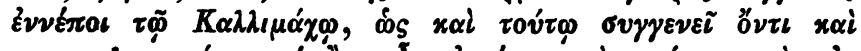

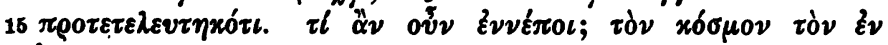

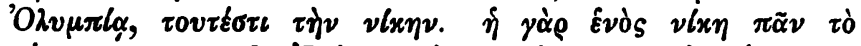

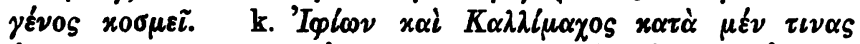

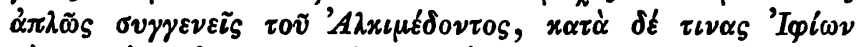

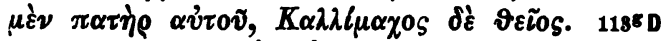

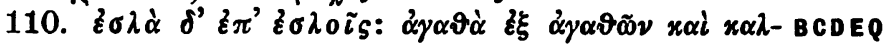

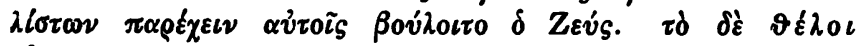

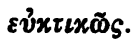

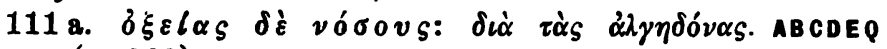
"Opпроs ( 1 269).

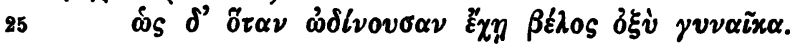

\section{Eust. II. 561, 34}

2 lac. indicari 3 supplevi ex $V \quad 4$ Évicos $A^{\text {ac }} \quad 5 K \alpha \lambda \lambda_{l}$ $\mu \alpha \chi(o v ?$ sine acc.) $\cdot \delta \delta \dot{\varepsilon} v i \sigma s: V \| \delta \dot{\varepsilon}$ (alt.) ft. delend. $9 \dot{\alpha} \gamma \gamma \varepsilon \hat{k}(\ell \alpha)$

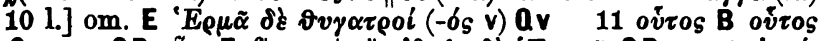

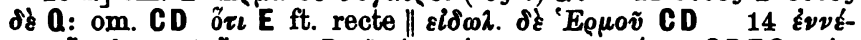

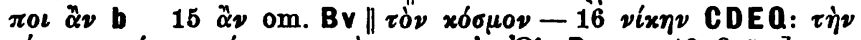

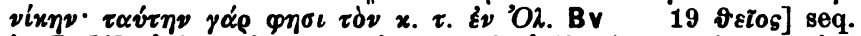

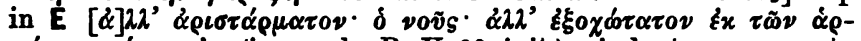

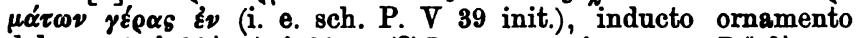

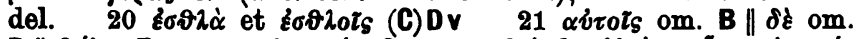

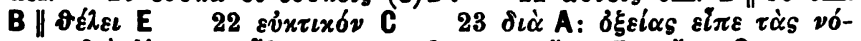

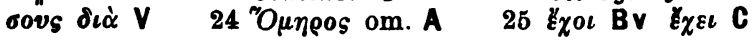




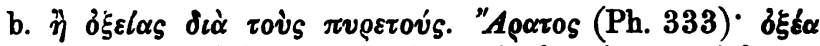

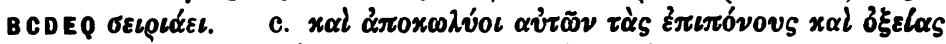

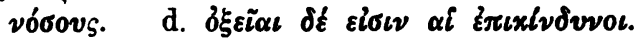

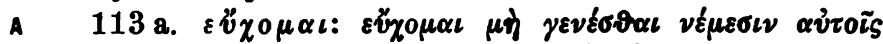

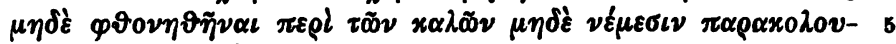

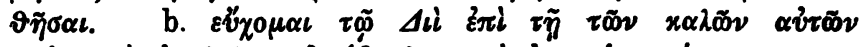

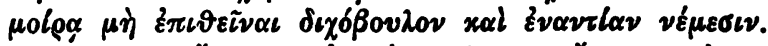

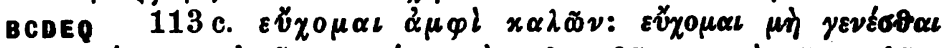

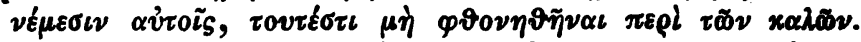

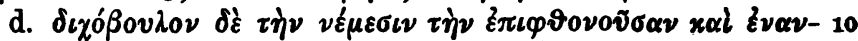

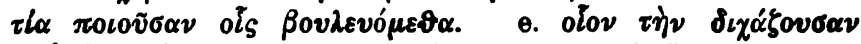

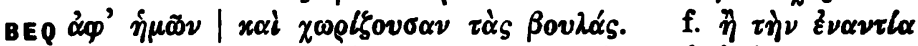

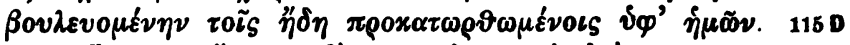

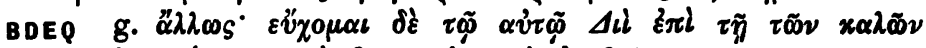

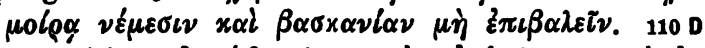

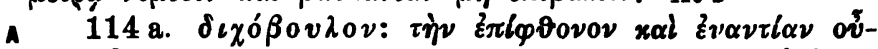

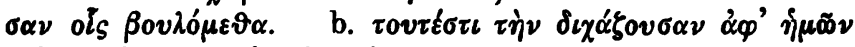

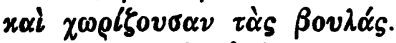

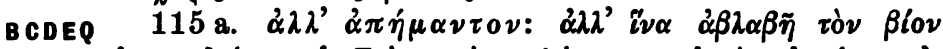

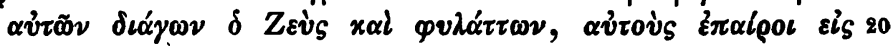

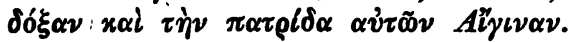

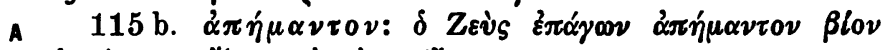

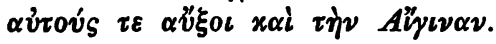

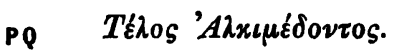

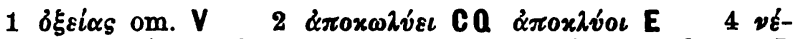

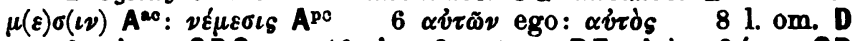

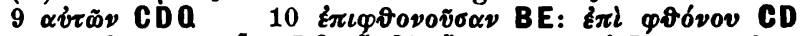

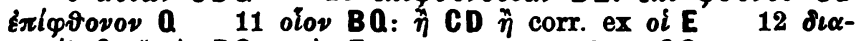

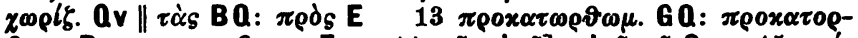

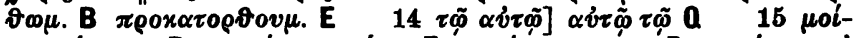

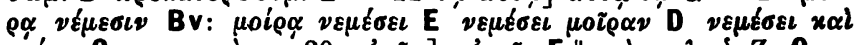

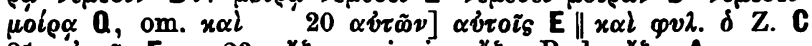

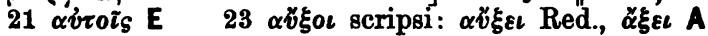




\section{Scholia in 0lympionicarum carmen IX.}

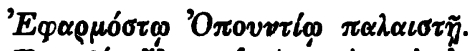

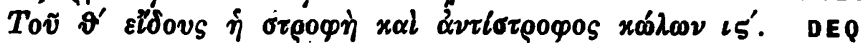

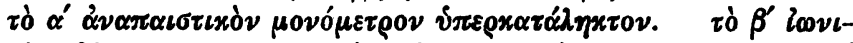

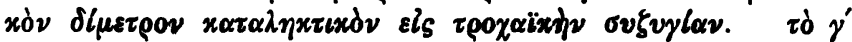

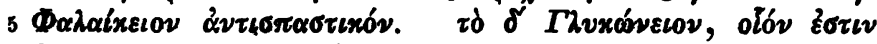

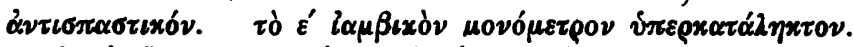

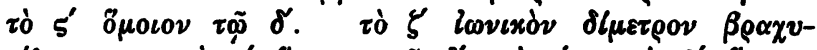

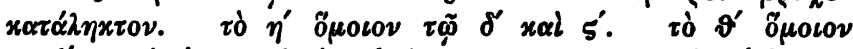

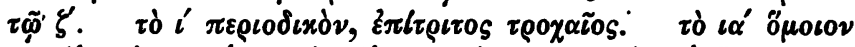

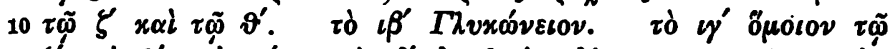

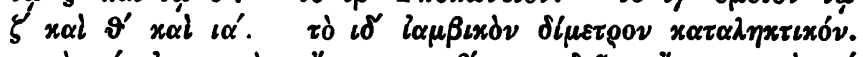

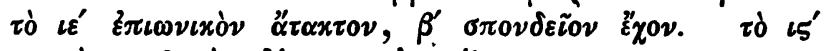

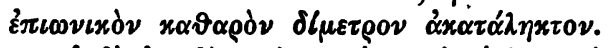

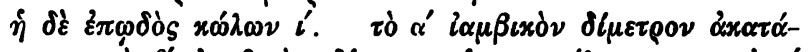

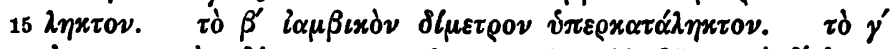

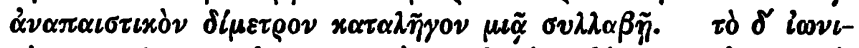

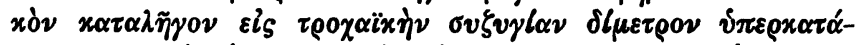

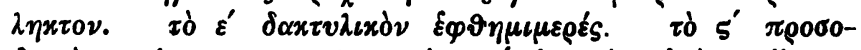

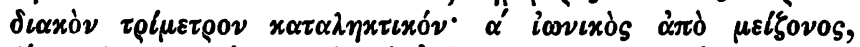

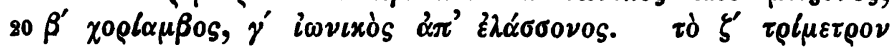

2 post $\iota 5^{\prime}$ add. $\dot{\eta} \delta \xi \xi \xi \pi \omega \delta \dot{\eta} x \omega \dot{\lambda} \omega \nu(\xi \sigma \tau l) \iota^{\prime} E Q$. $3 \dot{v} \pi \varepsilon \rho-$

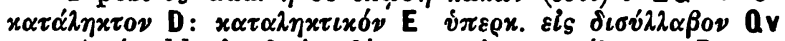

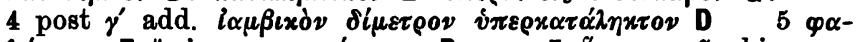

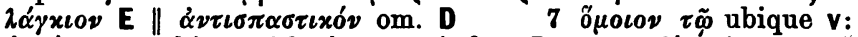

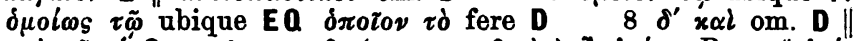

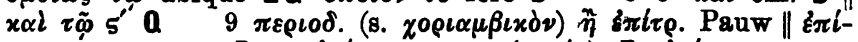

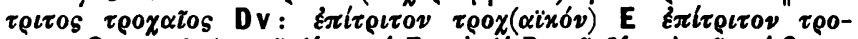

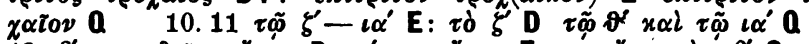

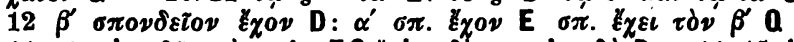

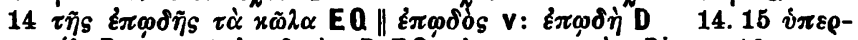

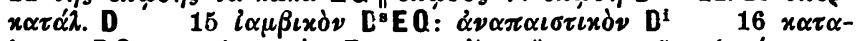

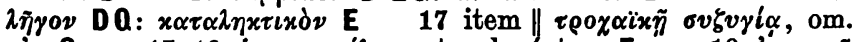

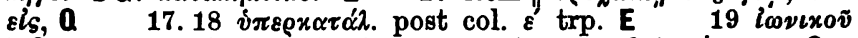

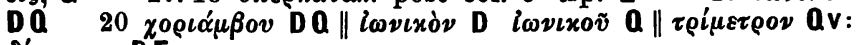

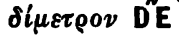




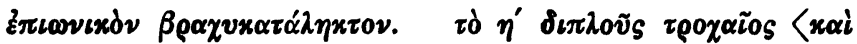

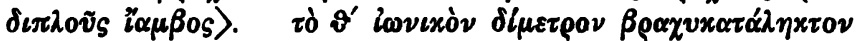

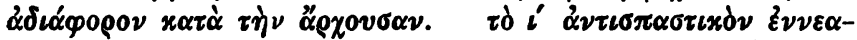

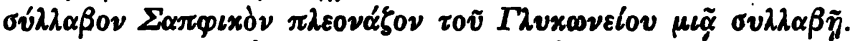

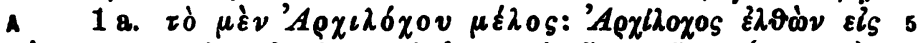

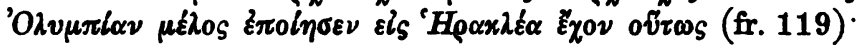

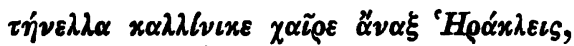

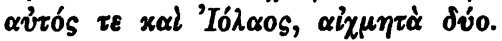

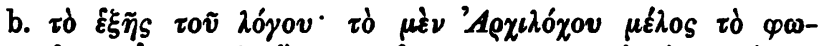

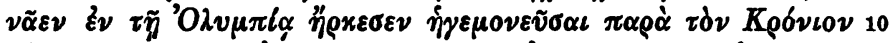

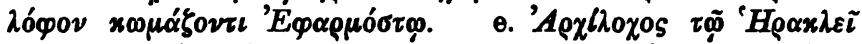

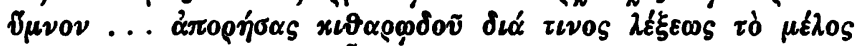

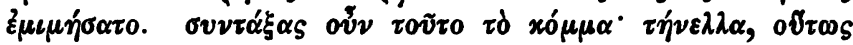

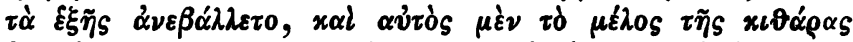

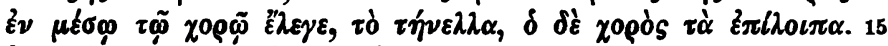

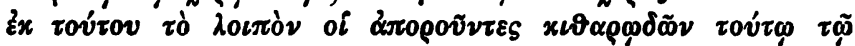

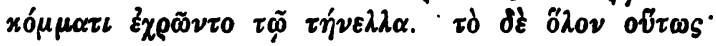

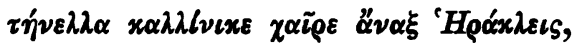

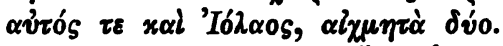

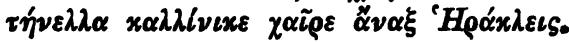

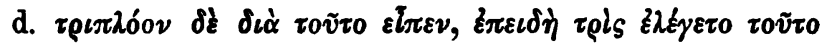

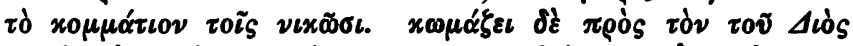

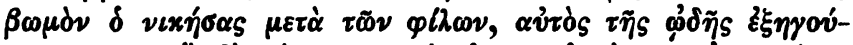

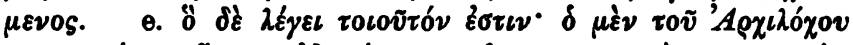

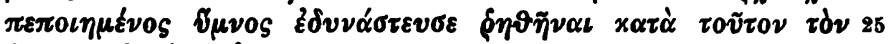

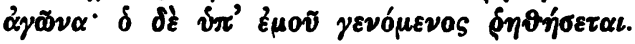

5 sch. Ar. 8v. 1764

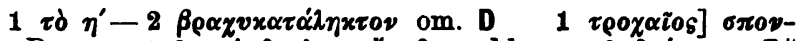

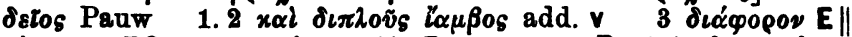

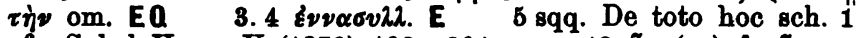

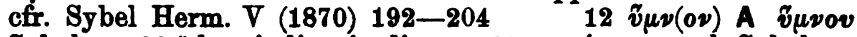

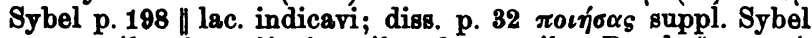

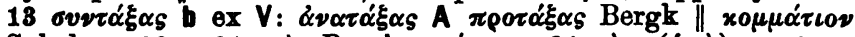

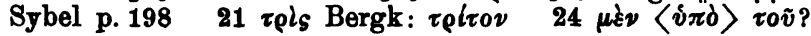




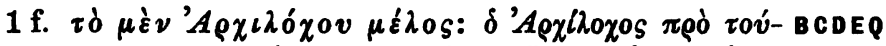

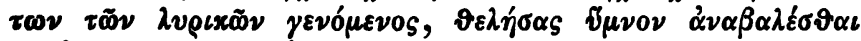

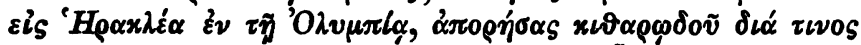

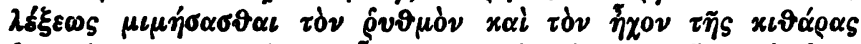

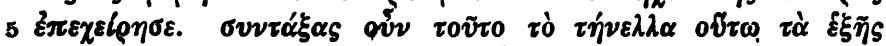

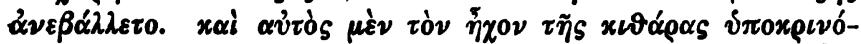

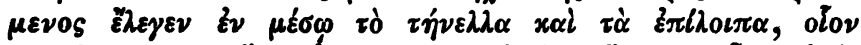

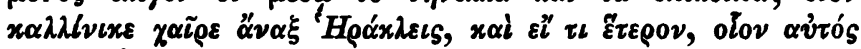

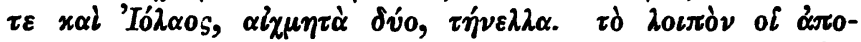

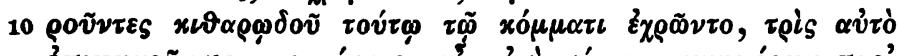

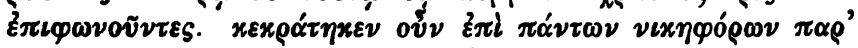

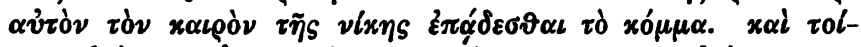

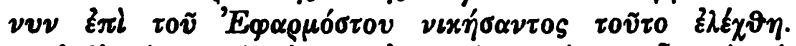

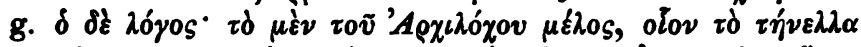

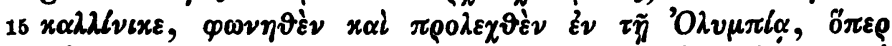

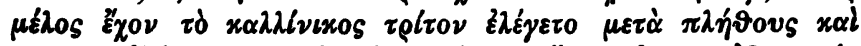

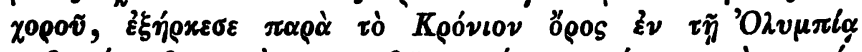

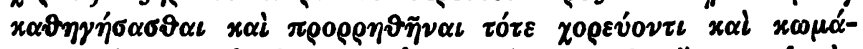

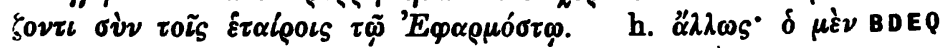

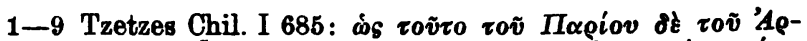

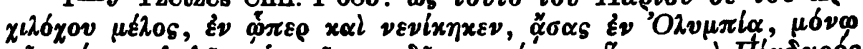

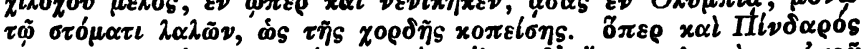

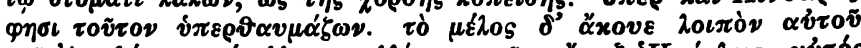

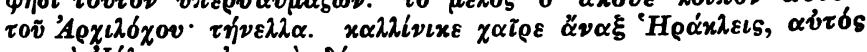

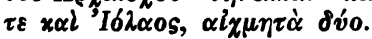

$2 \dot{\alpha} \nu \alpha \lambda \alpha \beta \varepsilon \dot{\varepsilon} \sigma \alpha \iota$ B $\alpha \nu \alpha \beta \dot{\alpha} \lambda \lambda \varepsilon \sigma \vartheta \alpha \iota$ D $3 \dot{\alpha} \pi 0 \rho \eta j \sigma \alpha$ Gerhard ex A: Évòs ov́x Evóves h. Quae seq. licenter mutavit Sybel

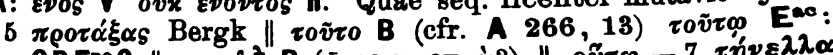

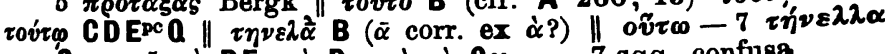

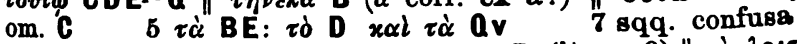

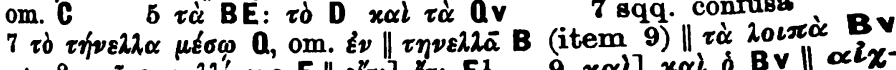

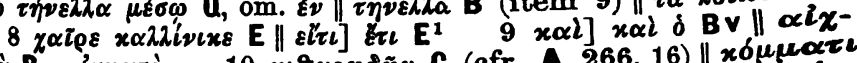
$\mu \eta \tau \dot{\alpha} \mathrm{B}$ : $\alpha i x \mu \alpha \tau \dot{\alpha} \quad 10 x \omega \sigma \alpha \rho \omega \delta \tilde{\omega} \nu$ C (cfr. A 266, 16) $\| x \delta \mu \mu \alpha \tau \iota$

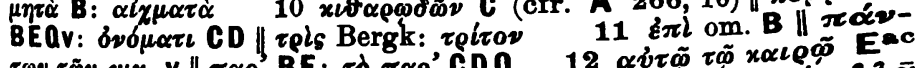

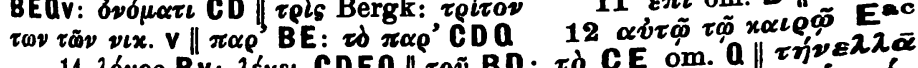

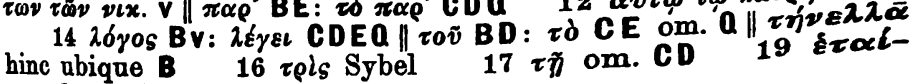

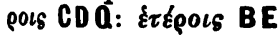




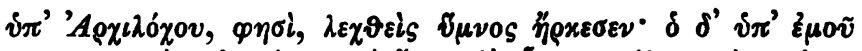

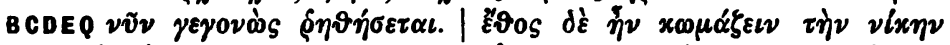

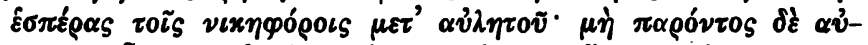

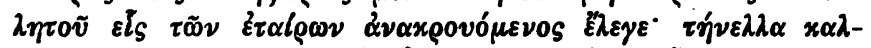

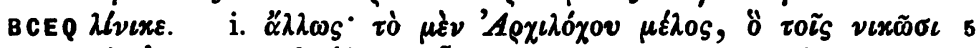

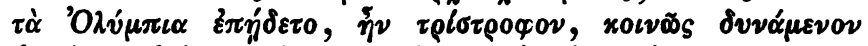

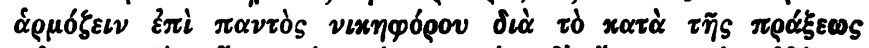

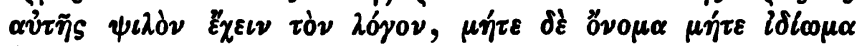

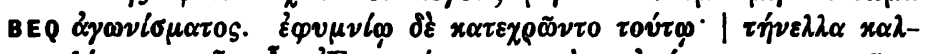

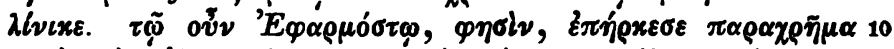

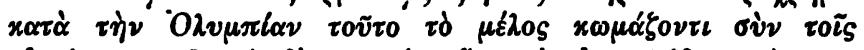

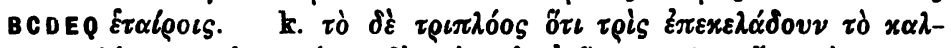

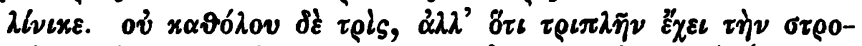

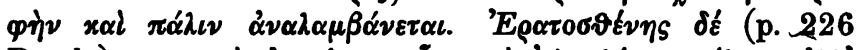

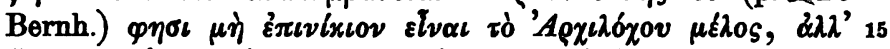

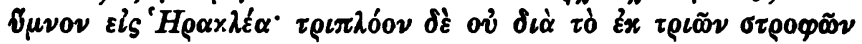

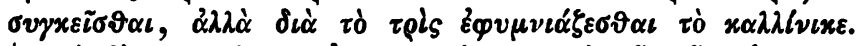

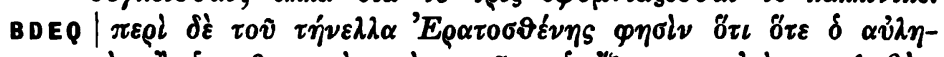

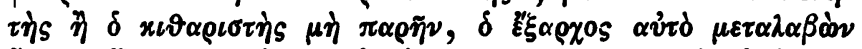

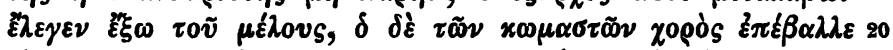

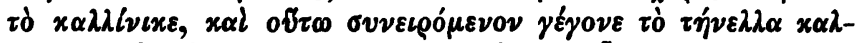

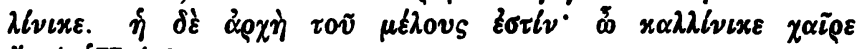

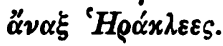

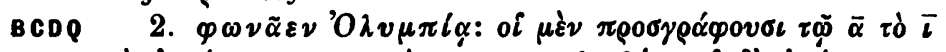

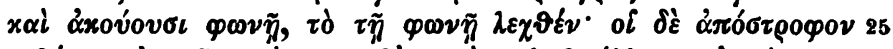

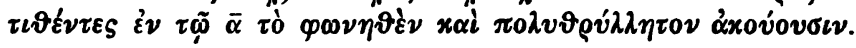

$1 \varphi \eta \sigma l$ om. D $3 \varepsilon_{\xi}^{\prime} \pi \hat{\varepsilon} \rho \alpha S$ V (corr. Pauw) $4 \pi \rho \sigma \alpha \nu \alpha-$

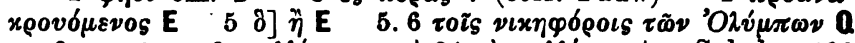

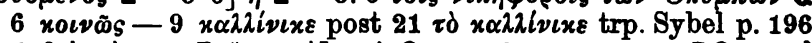

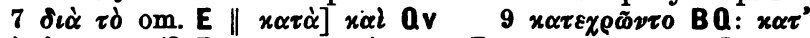

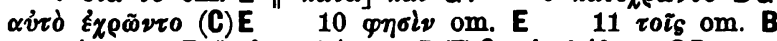

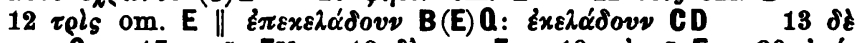

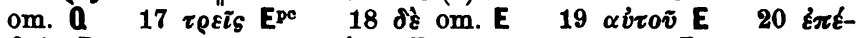

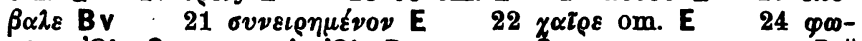
$\nu \tilde{\alpha} \varepsilon \nu{ }^{\prime} O \lambda$. Q: $\varphi \omega \nu \tilde{\alpha} \varepsilon^{\prime}{ }^{\prime} O \lambda$. B $\varphi \omega \nu \tilde{\alpha} \varepsilon \nu$ C $\varphi \omega \nu \tilde{\alpha}$ 8. $\varphi \omega \nu \alpha \tilde{\omega} \nu$ D \|

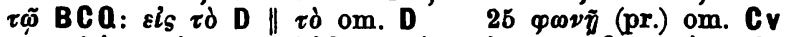

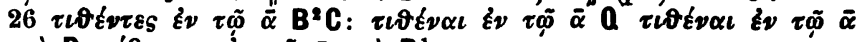

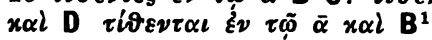




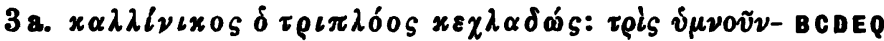

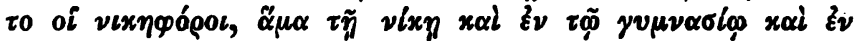

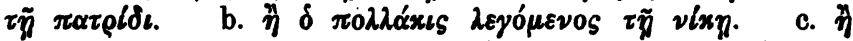

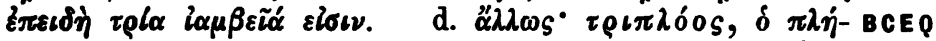

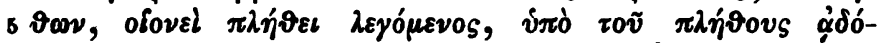

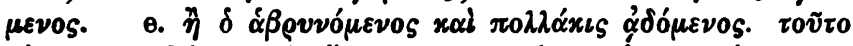

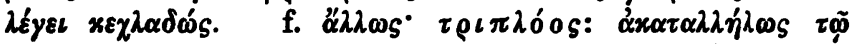

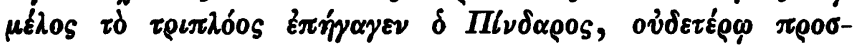

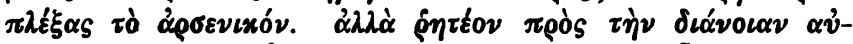

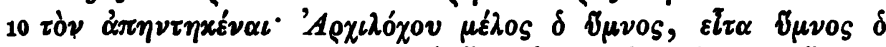

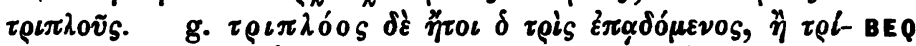

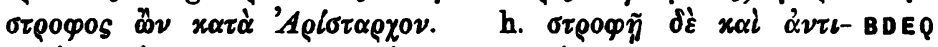

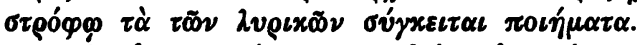

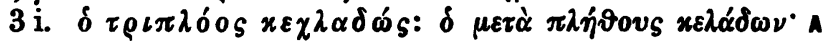

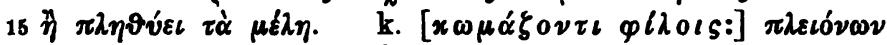

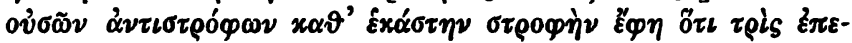

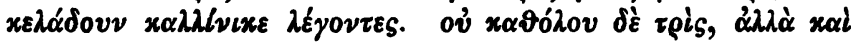

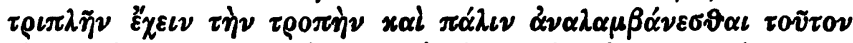

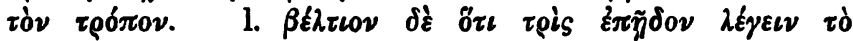

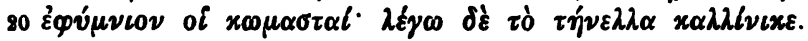

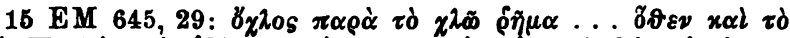

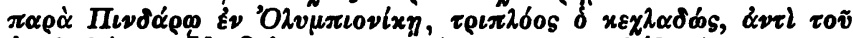
$\delta \pi \lambda \eta \vartheta \dot{v} \omega \nu$. Id. Orion. 122, $29\left(188,12:-\pi \lambda \eta^{\prime} \theta \omega v\right)$.

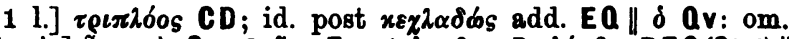

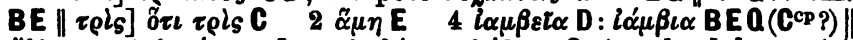

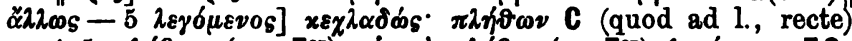

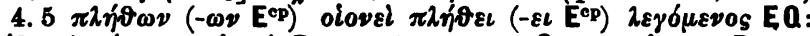

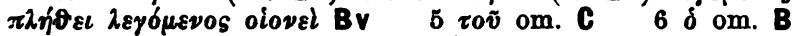

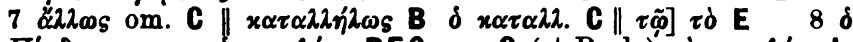

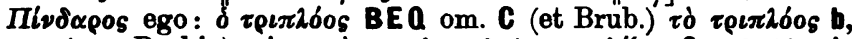

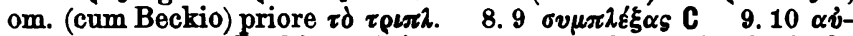

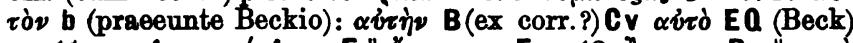

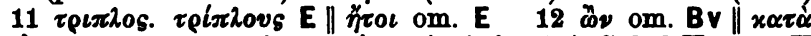

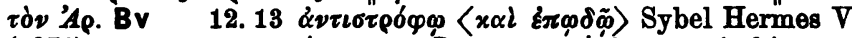

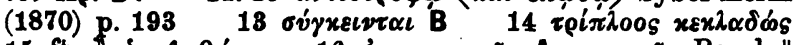

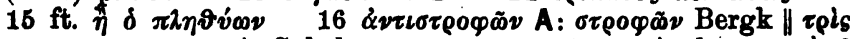

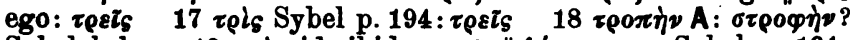
Sybel 1. 1. $19 \tau \rho i_{S}$ id. ibid.: $\tau \rho \varepsilon \tau_{s} \| \lambda \varepsilon \xi \varepsilon \nu$ om. Sybel p. 194:

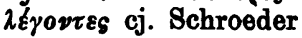




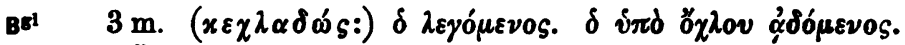
n. भै $x \varepsilon x \lambda \alpha \delta \dot{\omega} s, \delta x \varepsilon \lambda \alpha ́ \delta \omega \nu$.

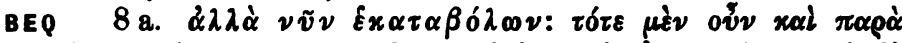

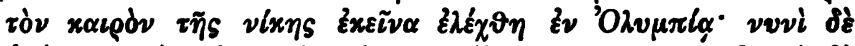

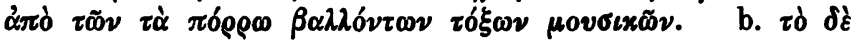

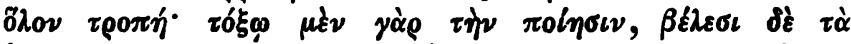

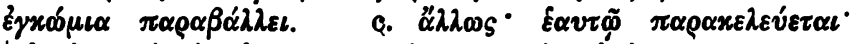

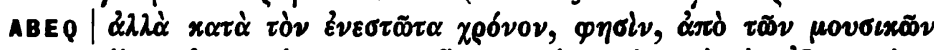

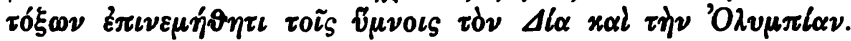

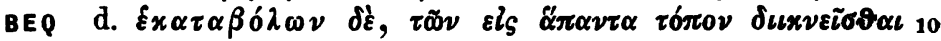

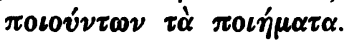

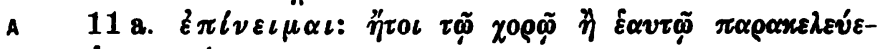

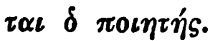

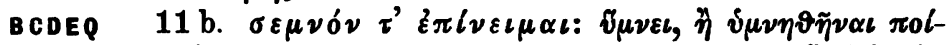

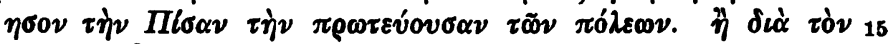

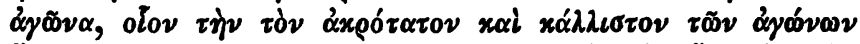

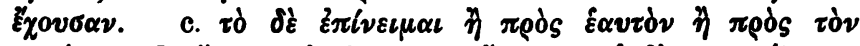

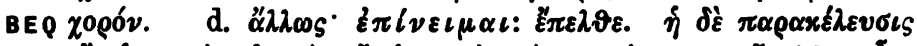

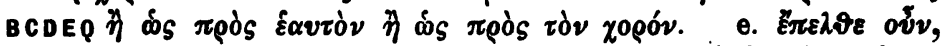

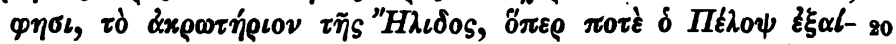

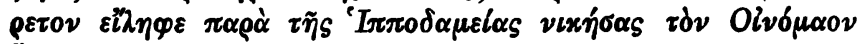
๕๊๊ขov.

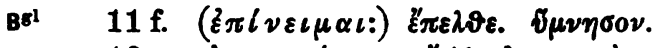

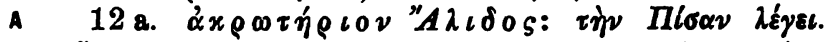

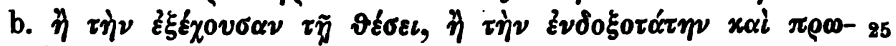
$\tau \varepsilon$ ข́ov $\alpha \nu$.

Bg1 12 b. (" $\left.A \lambda_{\iota} \delta_{0 \varsigma}:\right) \tau \tilde{\eta} s{ }^{\prime} O \lambda v \mu \pi l \alpha s$.

1 sq. glossa incertum an $B^{2} \quad 3$ l. et $A\|\varepsilon x \alpha \tau \eta \beta \delta \lambda \omega \nu A\| \pi \alpha \rho \dot{\alpha}$ EQ: $\pi \varepsilon \rho i \mathrm{~B} 4 \delta \xi \mathrm{BQ}$ : $\delta \xi x \alpha i \mathrm{E} \quad 5 \beta \alpha \lambda \sigma \nu \tau \omega \nu \mathrm{E} 6 \tau \delta \xi \varphi$

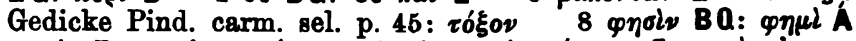

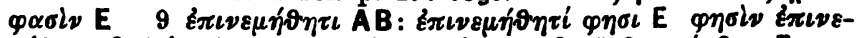

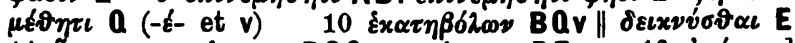

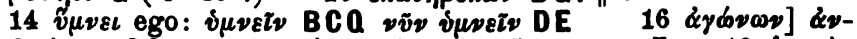

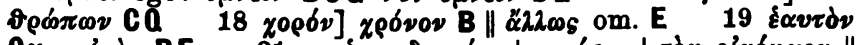

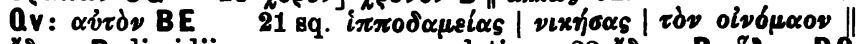

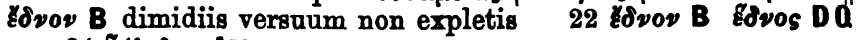
24 "Aledos Ape 


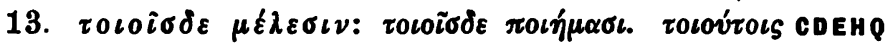

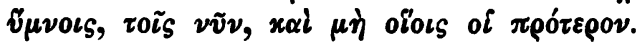

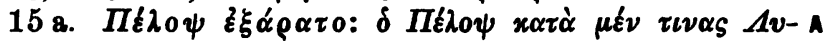

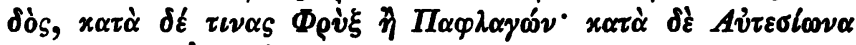
5 (FHG IV, 345) 'Axalós.

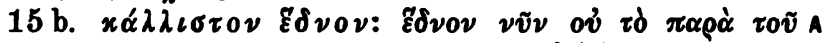

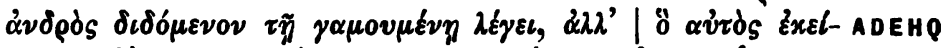

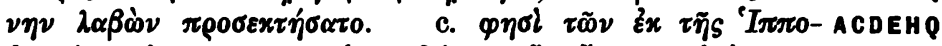

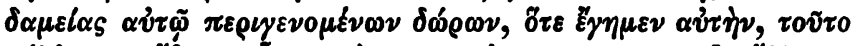

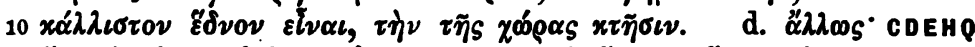

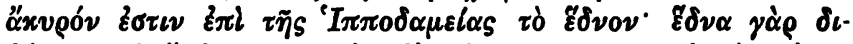

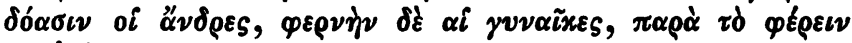
$\mu \varepsilon \vartheta^{\prime} \varepsilon \alpha v \tau \tilde{\nu} \nu$.

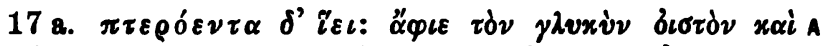

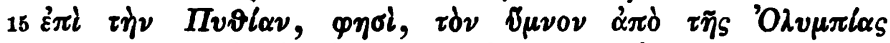

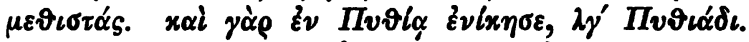

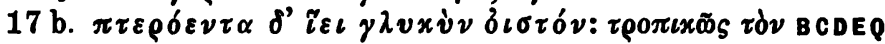

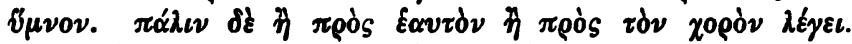

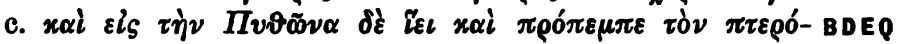

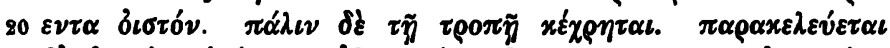

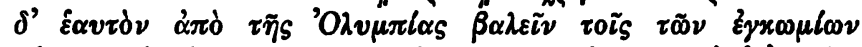

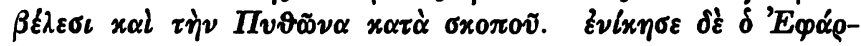

3 Tzetzes ad Lyc. 150. sch. O. I 37 6-8 et 9-13 sch. $\alpha$ 276. $\beta$ 53 12 EM 790, 43. 49. EG 651, 6. Orion. 160, 11

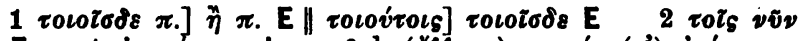

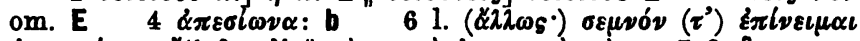

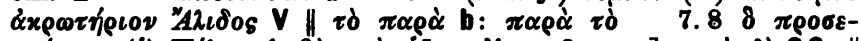

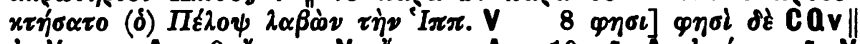
Ex V: om. A 9 घ

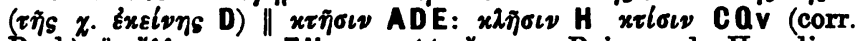

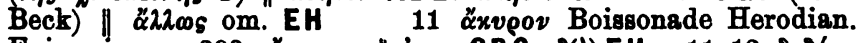

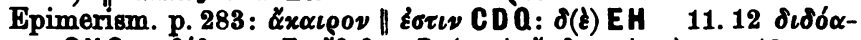

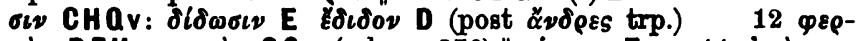

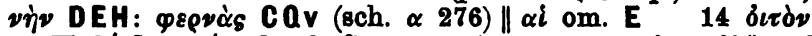

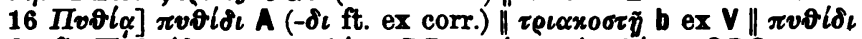

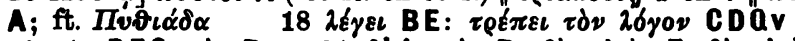

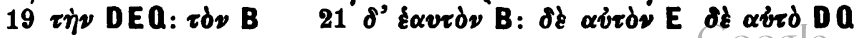




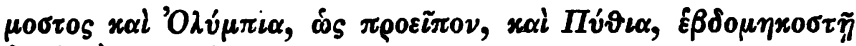

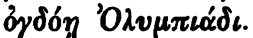

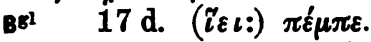

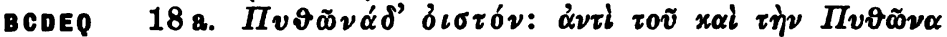

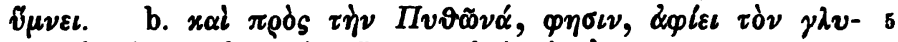

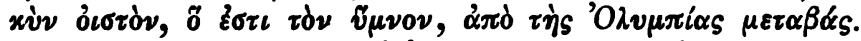

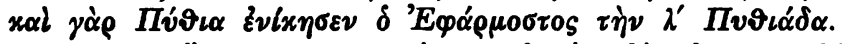

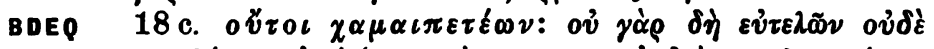
$\mu \iota x \rho \tilde{\nu} \nu$ hó

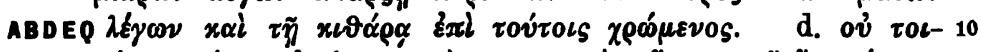

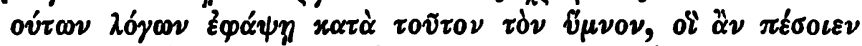

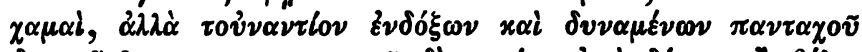

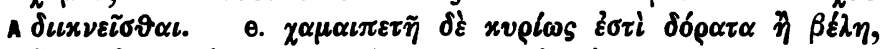

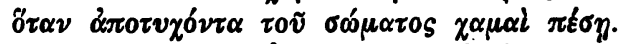

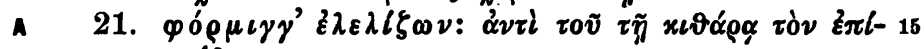

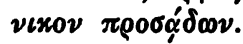

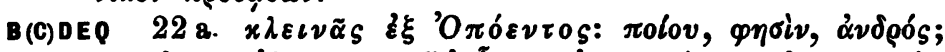

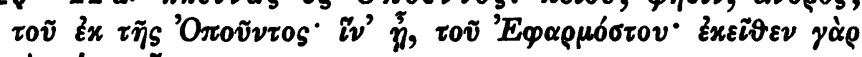

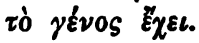

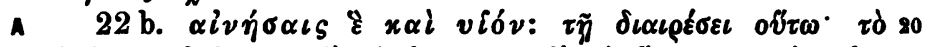

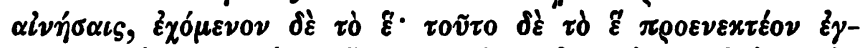

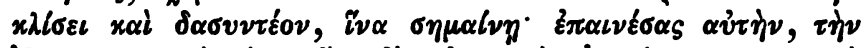

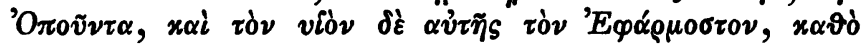

$1 \pi \rho \circ \varepsilon \pi 0 \nu$ BE: $\pi \rho \circ \varepsilon i \pi \varepsilon$ DQv. Sch. 0. II 70 d. 82 a conferri iubet Schroeder proll. p. 60 ¿ $\delta \gamma \delta 6 \eta \eta$ scripsi ex pap. Oxyrh. CCXXII, 1, 37 (cj. iam Hermann ap. h); $\tau \rho i \tau \eta$ codd. 4 l.] \&a-

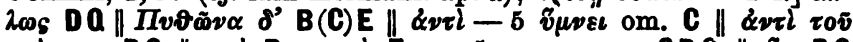

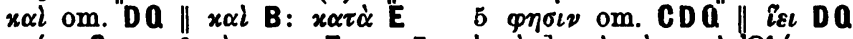

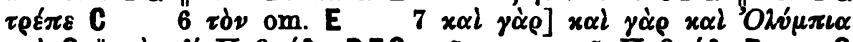

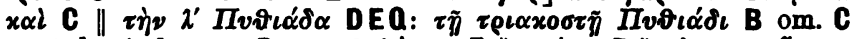

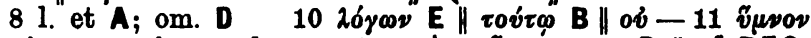

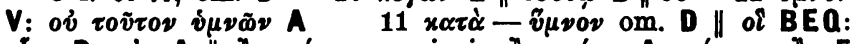

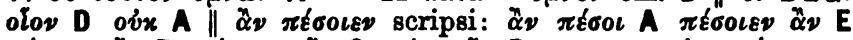

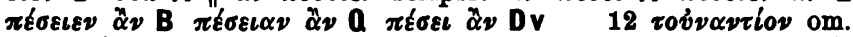

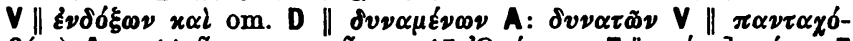

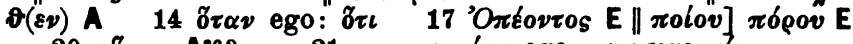

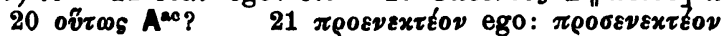

21. $22 \varepsilon^{\prime} \gamma \times \lambda l \sigma \varepsilon \iota$ Mommsen suppl. p. 120: $\varepsilon^{\prime} \gamma \times \lambda \eta^{\prime} \sigma \varepsilon \iota 22 \delta \alpha \sigma v \nu-$

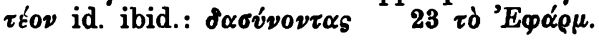




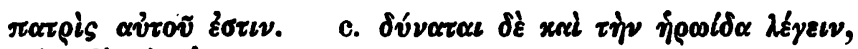

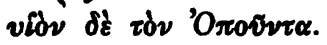

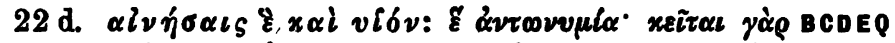

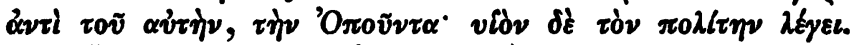

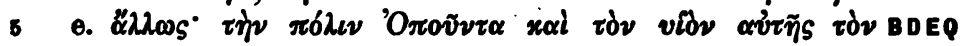

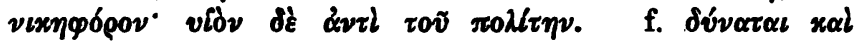

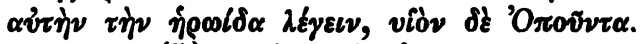

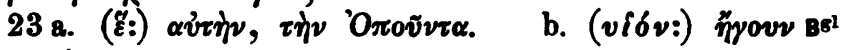

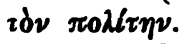

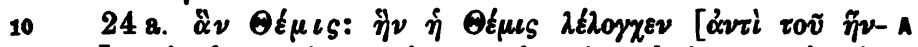

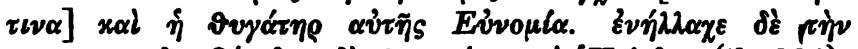

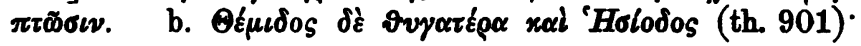

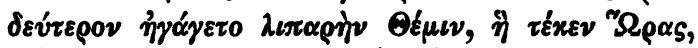

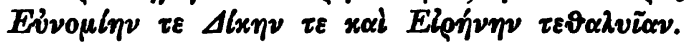

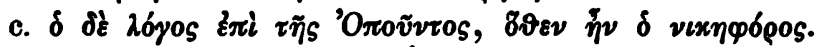

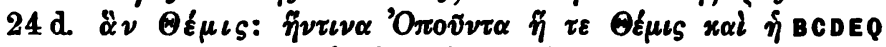

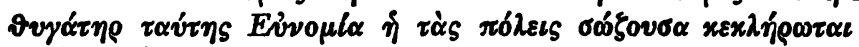

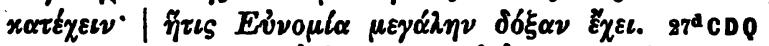
BDEQ

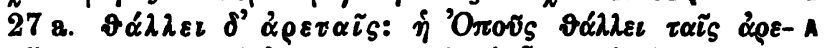

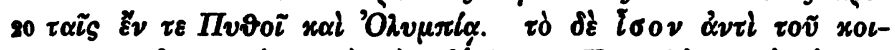

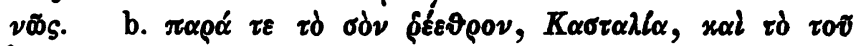

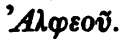

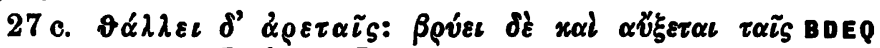

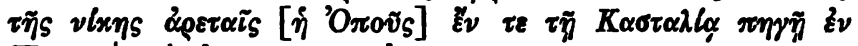

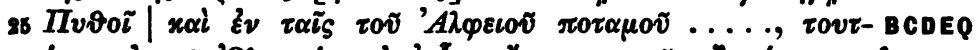

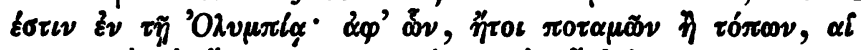

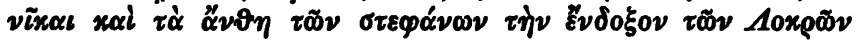

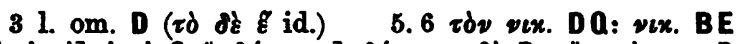

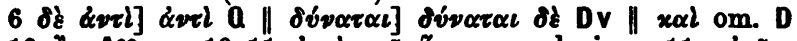

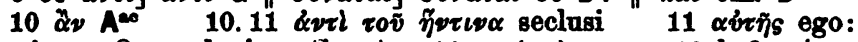

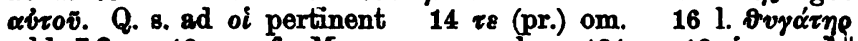
add. E a 19 sq. cfr. Mommsen suppl. p. 12119 iे ego:

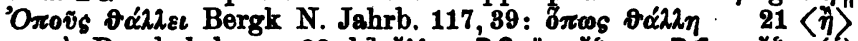

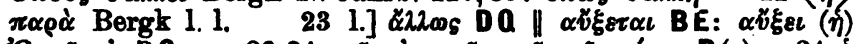

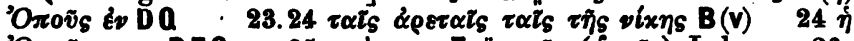

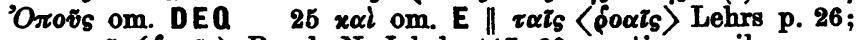
$\pi 0 \tau \alpha \mu 0 v$ 〈 $\mu \rho \tilde{v}\left\langle\pi \rho 0 x \operatorname{\alpha } \tau_{\boldsymbol{S}}\right\rangle$

Schox. In Prwdarex ed. Drachmann. 


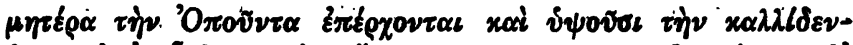

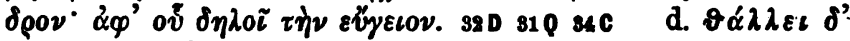

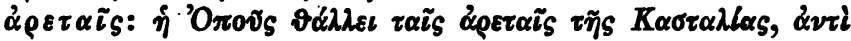

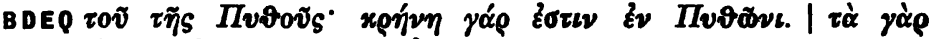

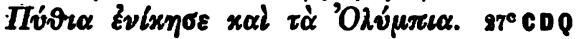

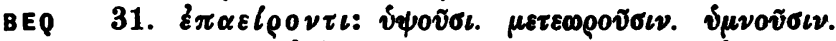

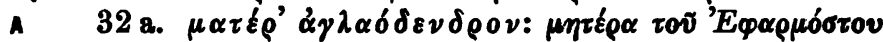

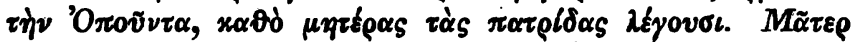

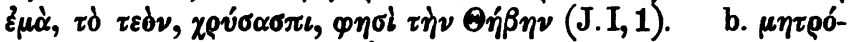

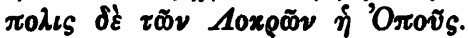

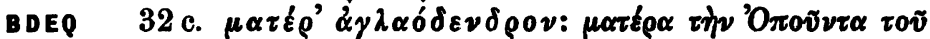

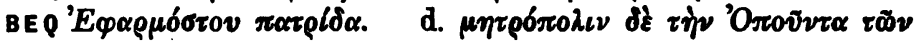

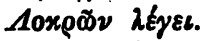

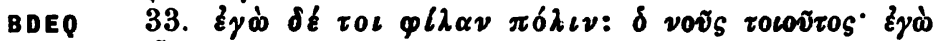

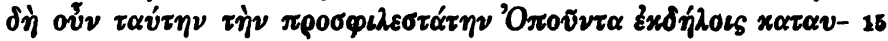

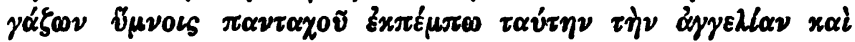

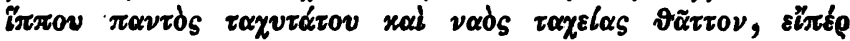

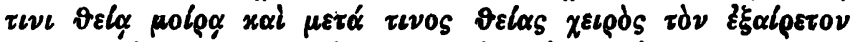

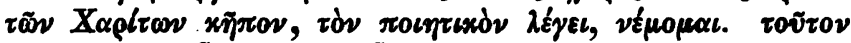

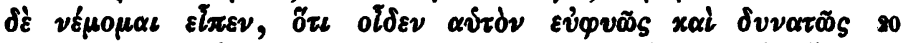

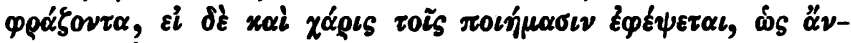

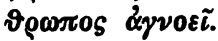

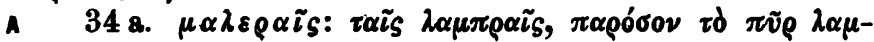

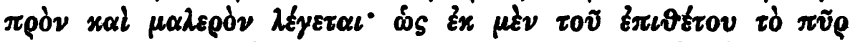

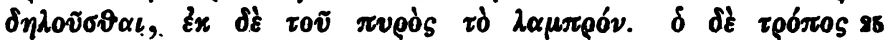

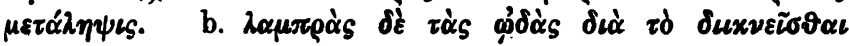

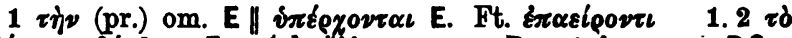

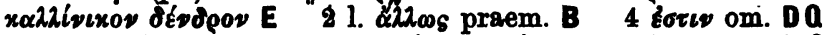

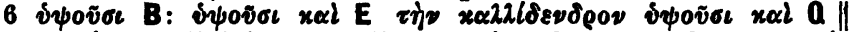

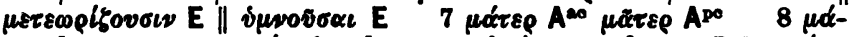

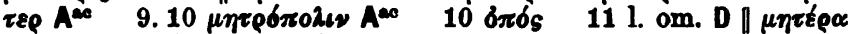

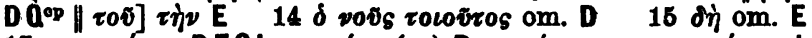

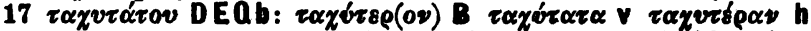

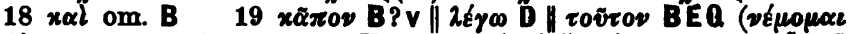

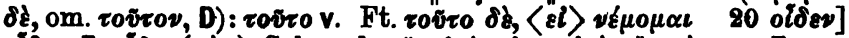

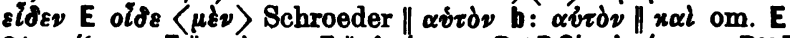

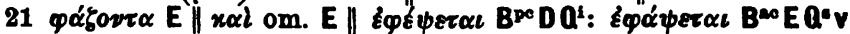

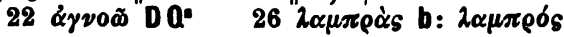




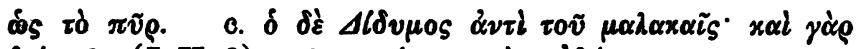

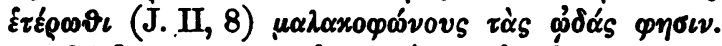

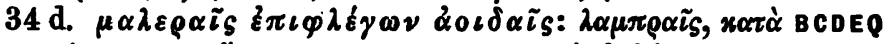

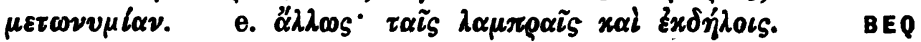

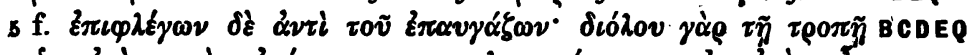

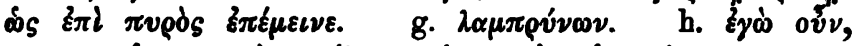

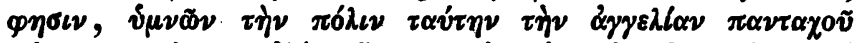

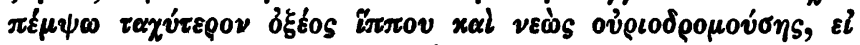

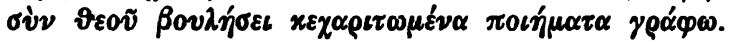

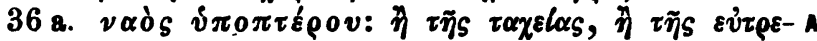

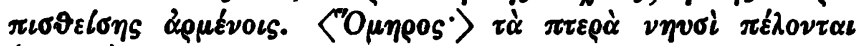
( $\lambda$ 124).

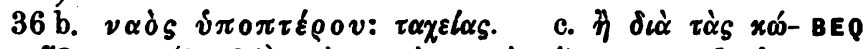

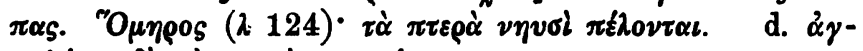

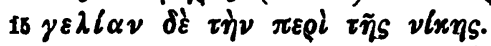

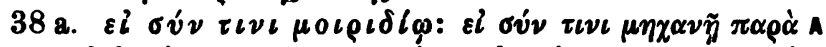

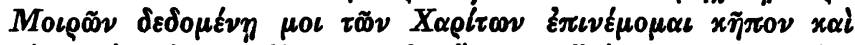

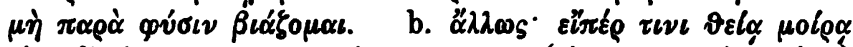

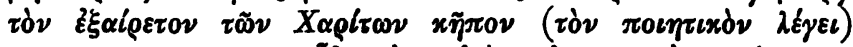

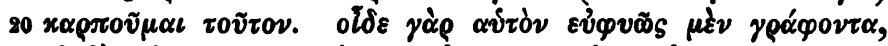

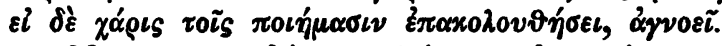
$\tau \varepsilon ́ \chi \nu \eta$.

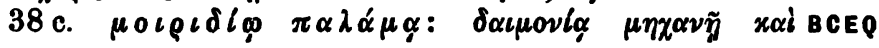

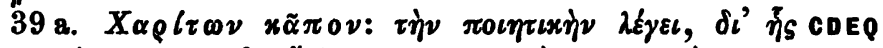

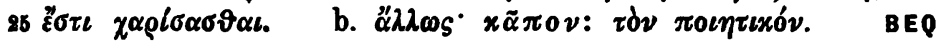

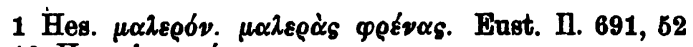

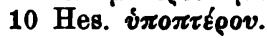

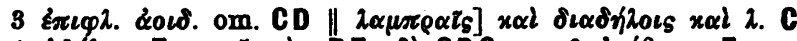

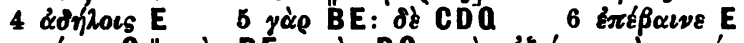

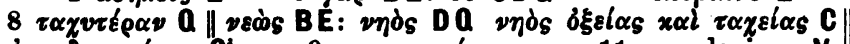

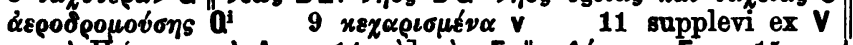

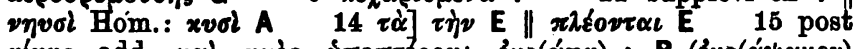

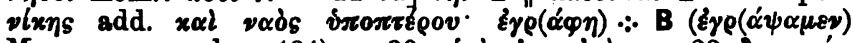

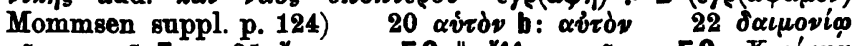

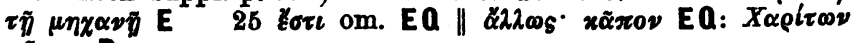
xथ̃ $\pi 0 \nu \mathrm{B}$ 


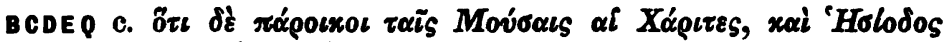

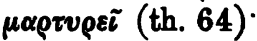

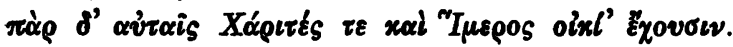

BDEQ 40. $x \varepsilon i \tilde{\nu} \alpha \iota$ jò

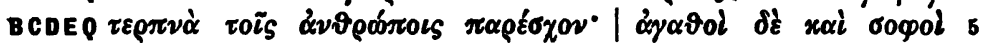

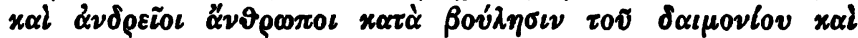

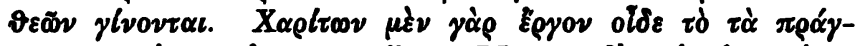

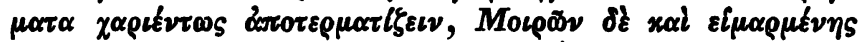

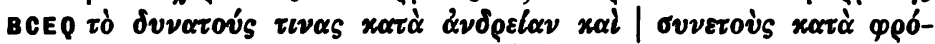

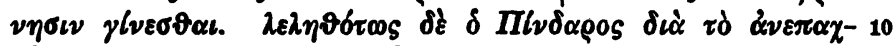

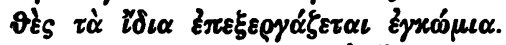

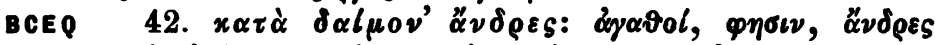

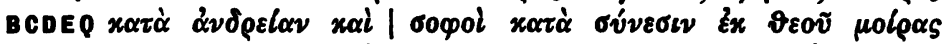

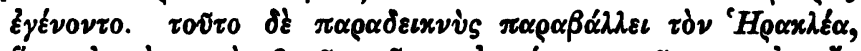

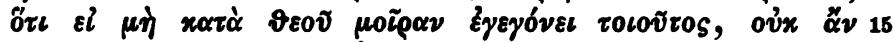

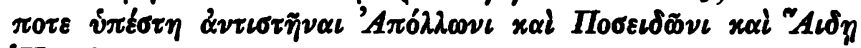

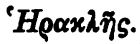

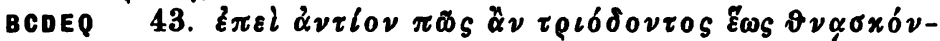

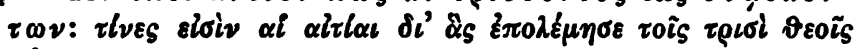
$\delta$ 'H

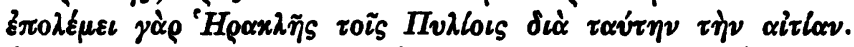

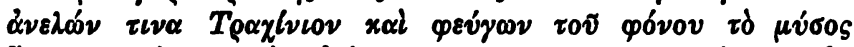
Eั

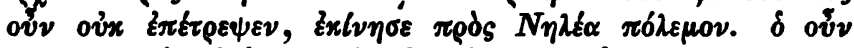

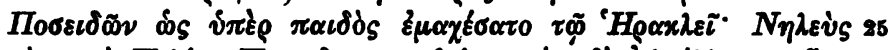

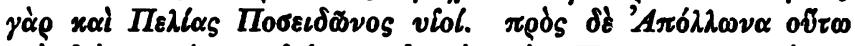

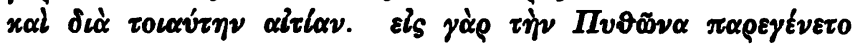

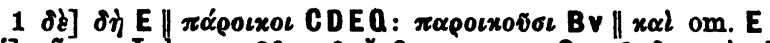

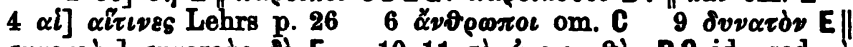

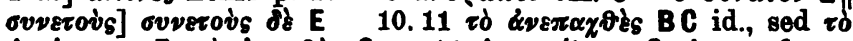

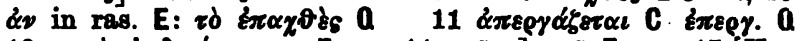

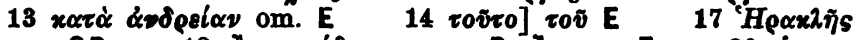

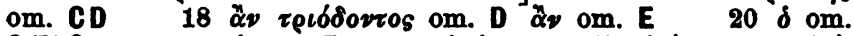

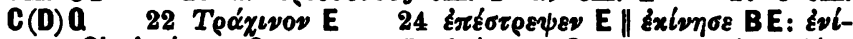

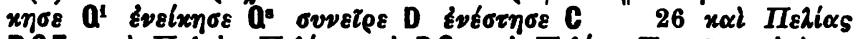

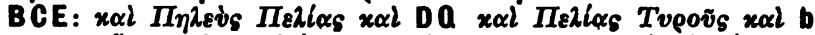

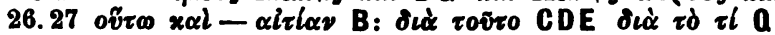

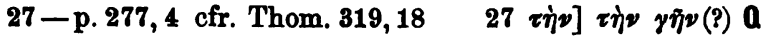




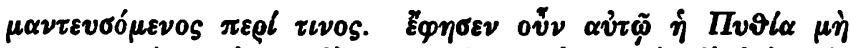

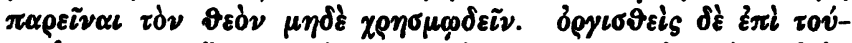

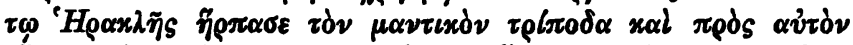

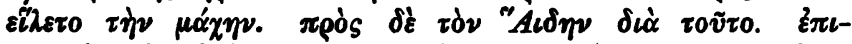

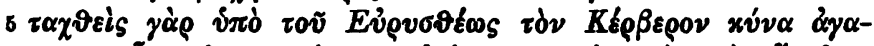

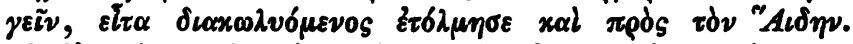

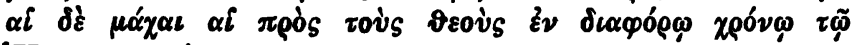

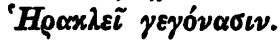

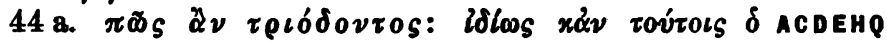

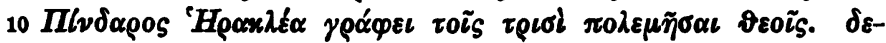

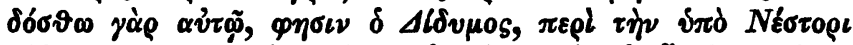

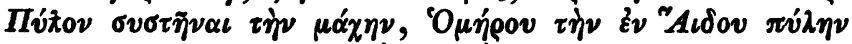

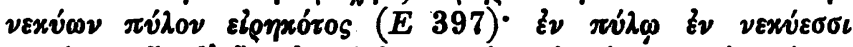

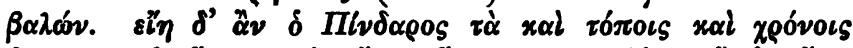

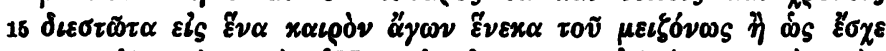

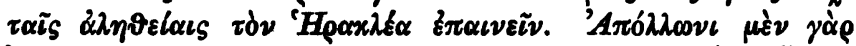

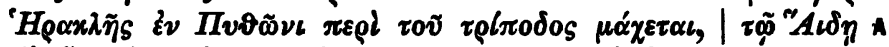

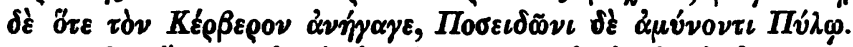

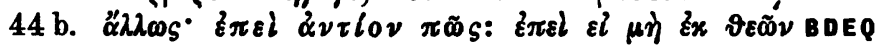

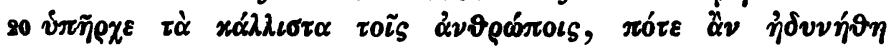

\section{4-6 sch. BT E $395 \quad 12$ sch. A E 397}

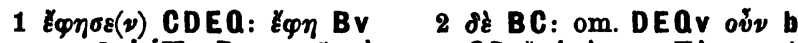

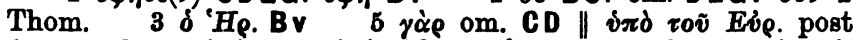

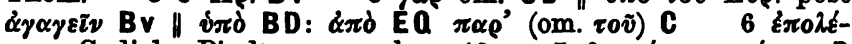

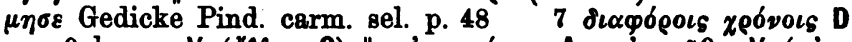

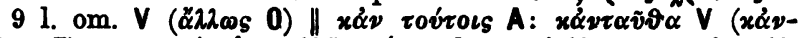
$\tau \varepsilon \tilde{\theta} \theta \varepsilon v$ E) $10 \tau \dot{\partial} \nu$ 'He. V \|

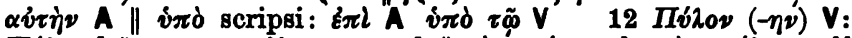

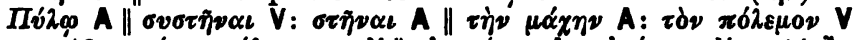

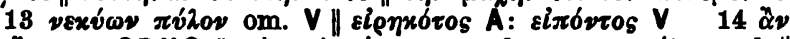

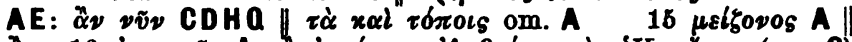

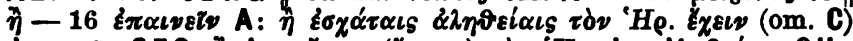

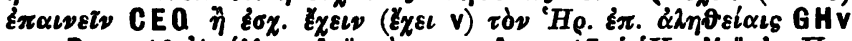

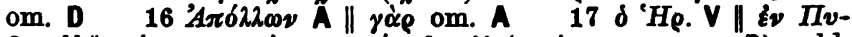

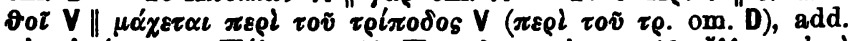

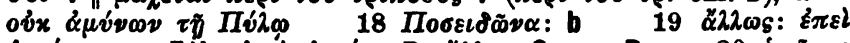

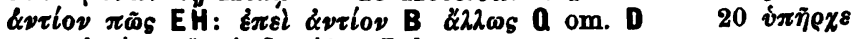
ego: $v \pi \alpha \dot{\alpha} \propto \chi \varepsilon \| \pi \delta \tau \varepsilon] \pi \delta \vartheta \varepsilon v$ Lehrs p. 26 


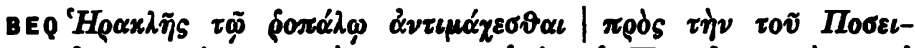

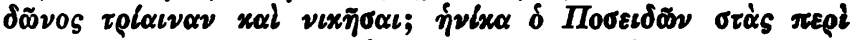

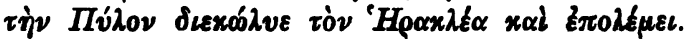

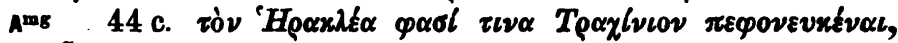

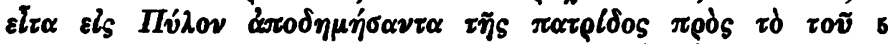

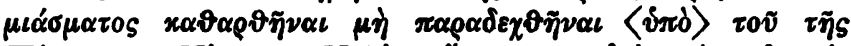

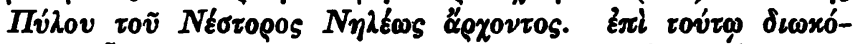

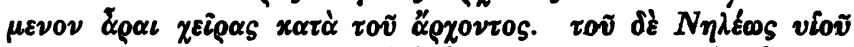

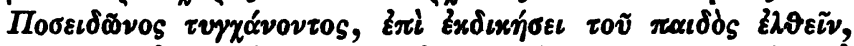

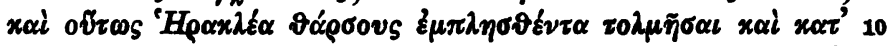

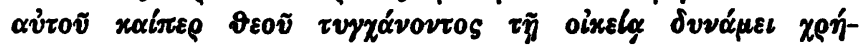
$\sigma \alpha \sigma \vartheta \alpha \iota$.

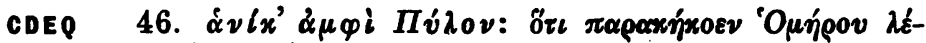

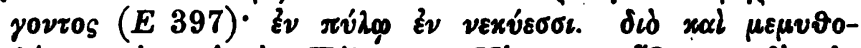

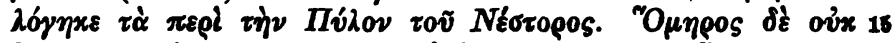

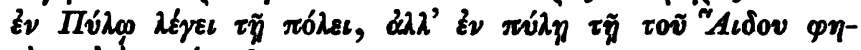

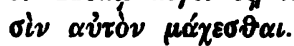

A 48. ทै

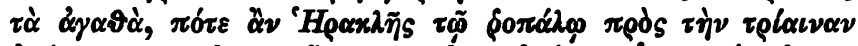

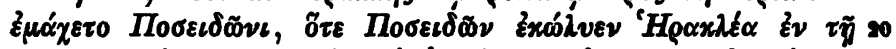

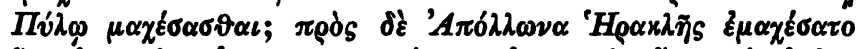

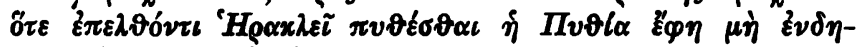

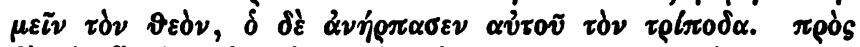

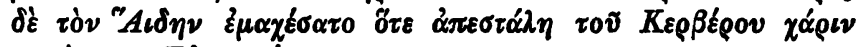

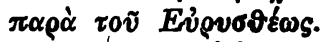

CDEP 50'a. O

13 sch. AT E 397

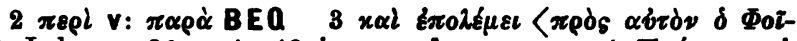

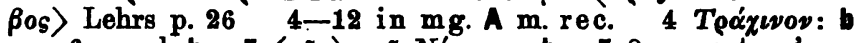

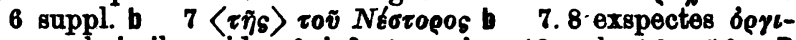

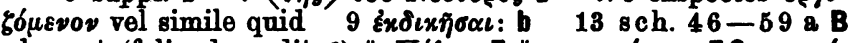

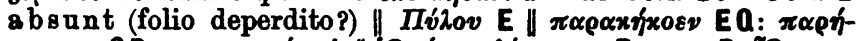

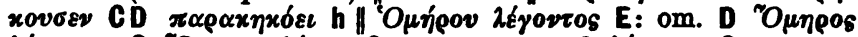

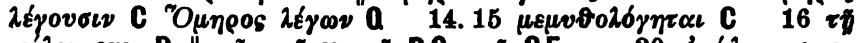

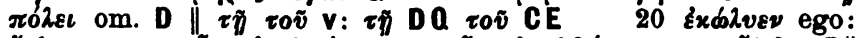

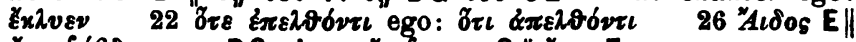

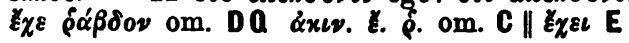




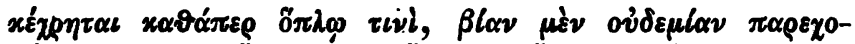

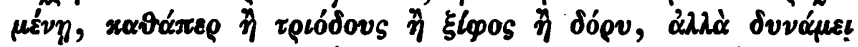

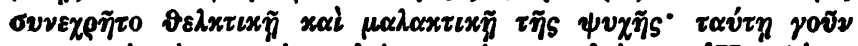

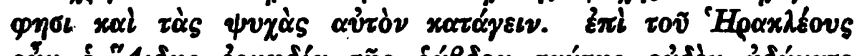

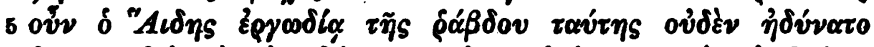

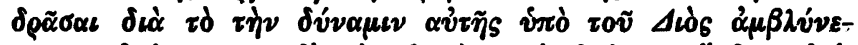

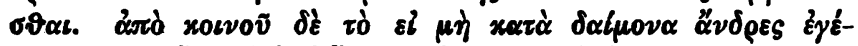

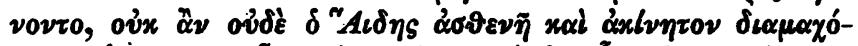

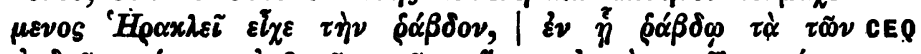

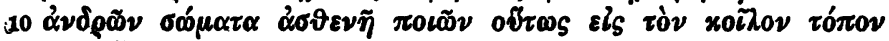

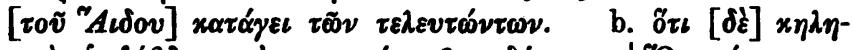

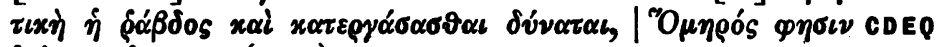

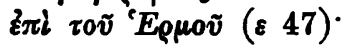

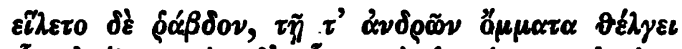

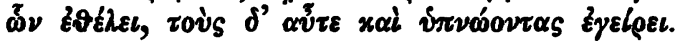

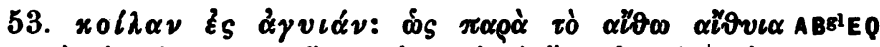

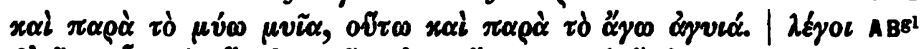

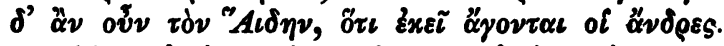

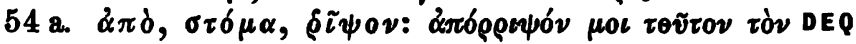

16 Herodian. I 281, 23. Steph. Byz. ¿yvió. EM 14, 21. EG 7, 39. Lex. Bachm. 17, 1. Orion. 22,17

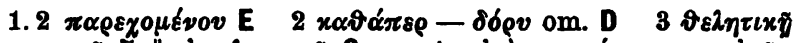

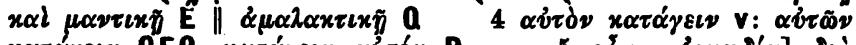

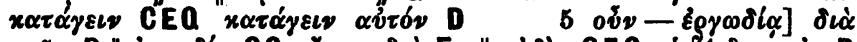

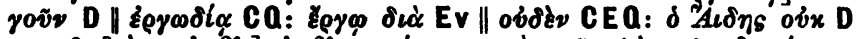

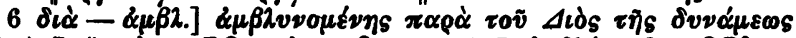

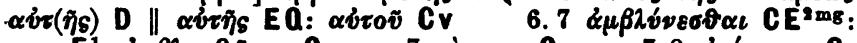

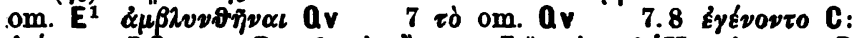

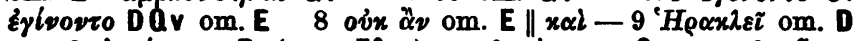

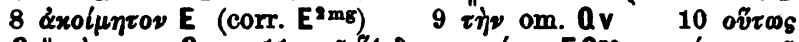

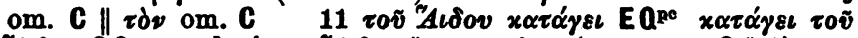

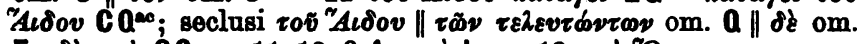

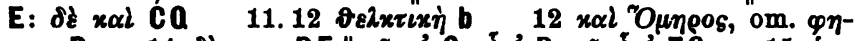

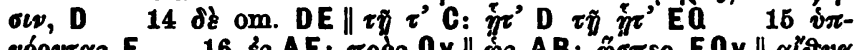

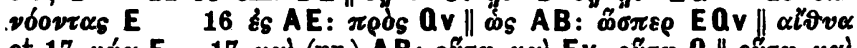

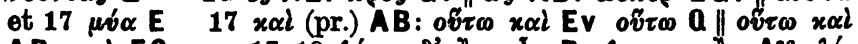

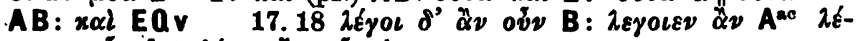

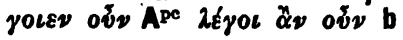




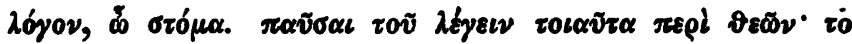

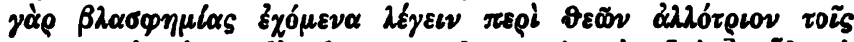

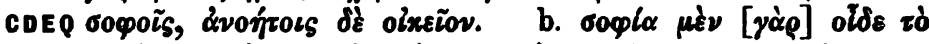

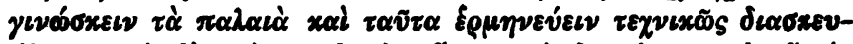

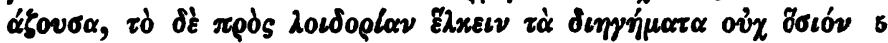

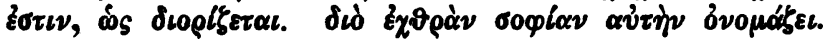

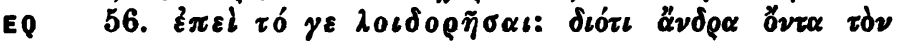

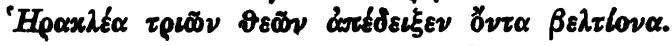

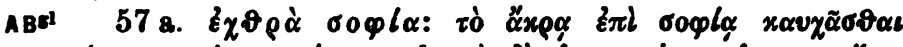

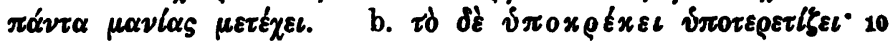

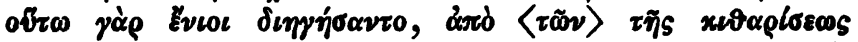

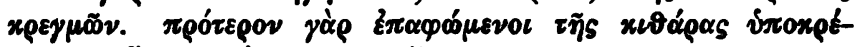

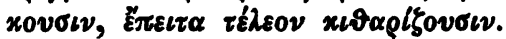

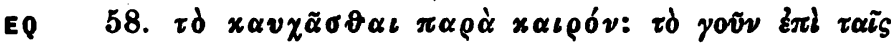

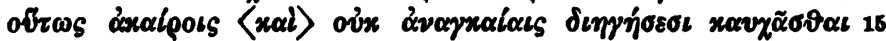

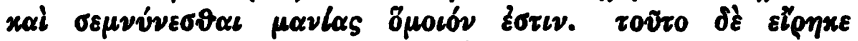

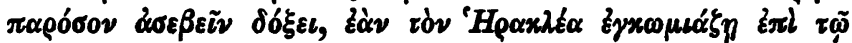

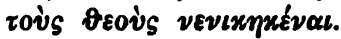

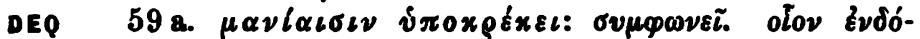

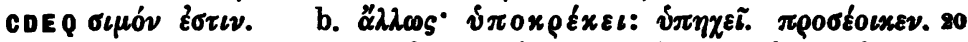

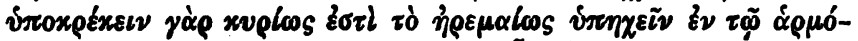

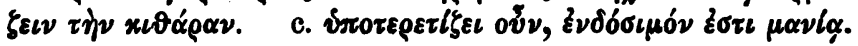

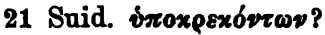

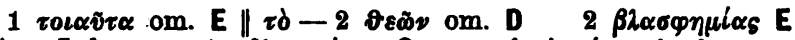

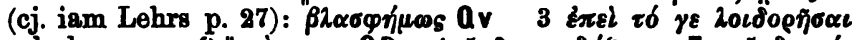

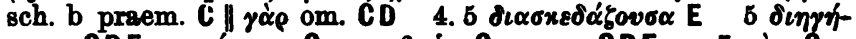

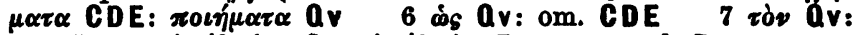

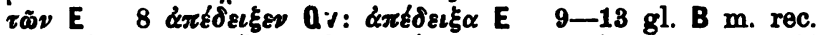

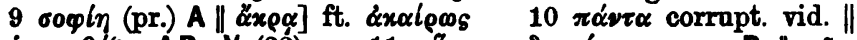

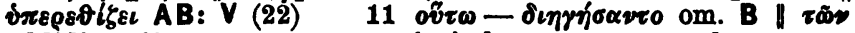

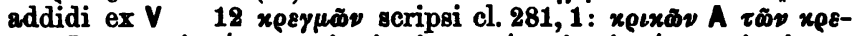

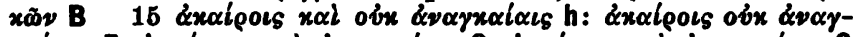

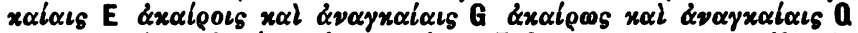

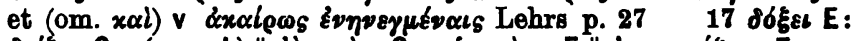

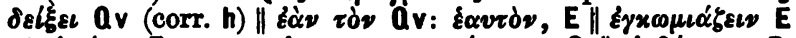

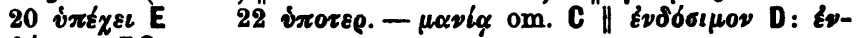

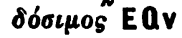




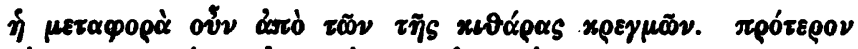

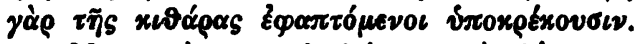

60 a. $\mu \eta \eta \nu \tilde{v} \nu \lambda \alpha \lambda \alpha \gamma \varepsilon \iota: \mu \eta\rangle$

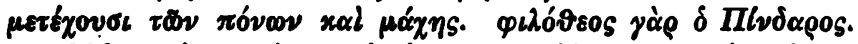

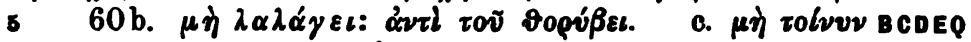

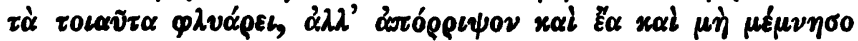

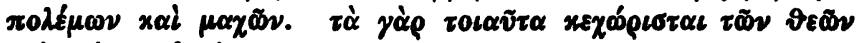

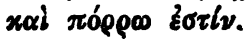

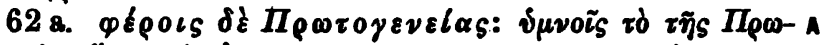

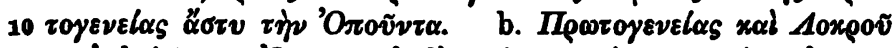

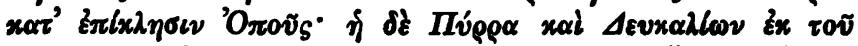

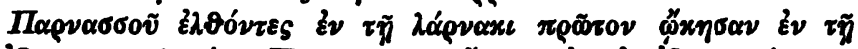

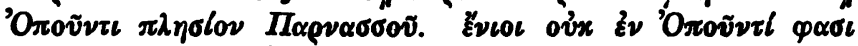

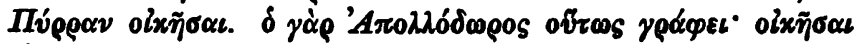

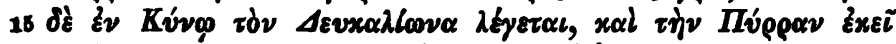

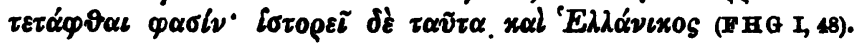

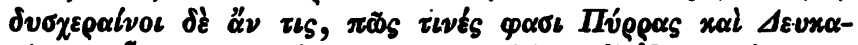

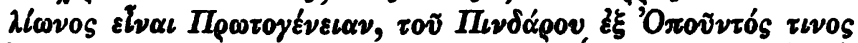

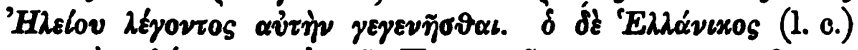

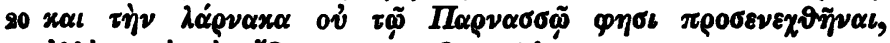

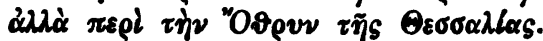

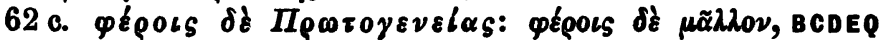

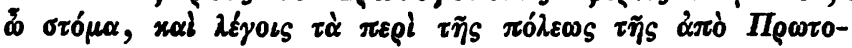

12 Strab. IX 425

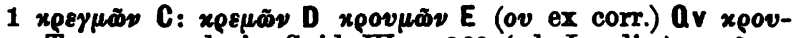

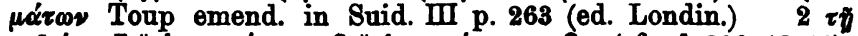

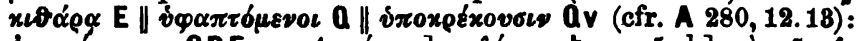

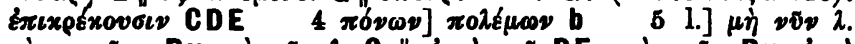

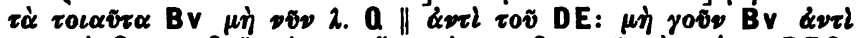

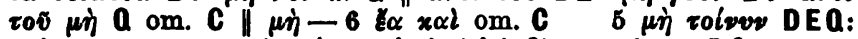

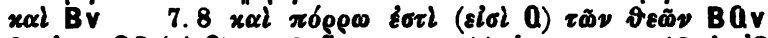

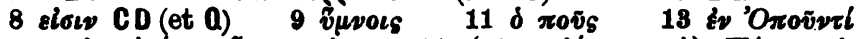

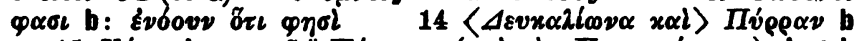

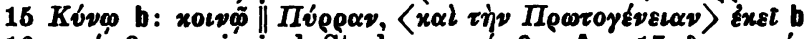

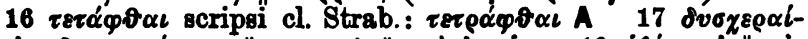

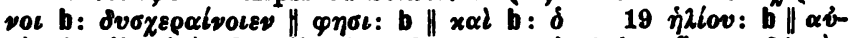

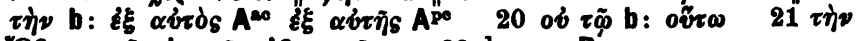

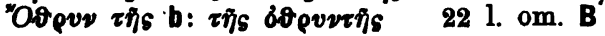




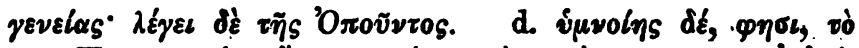

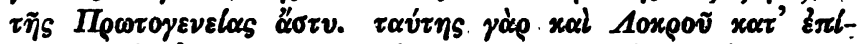

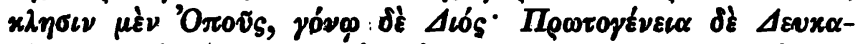

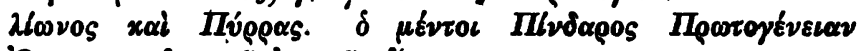

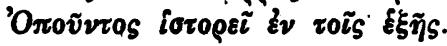

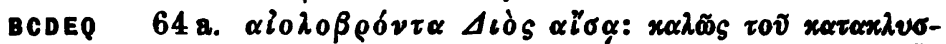

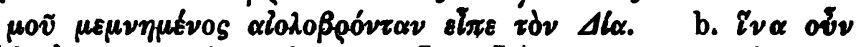

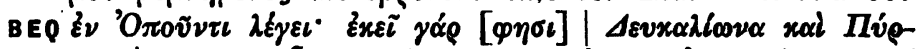

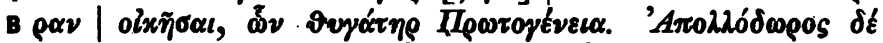

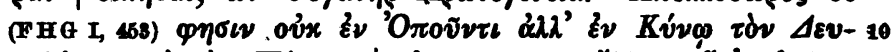

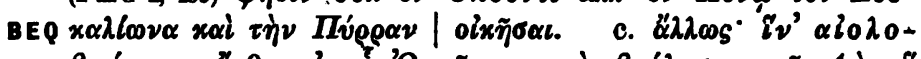

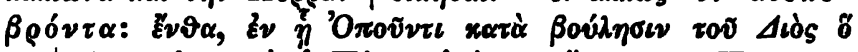

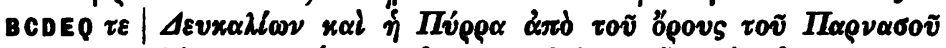

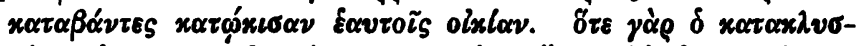

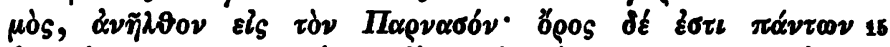

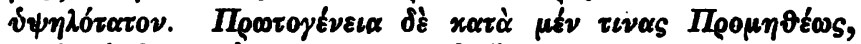

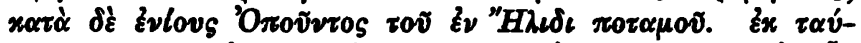

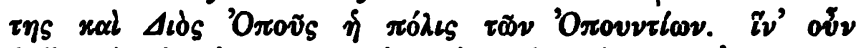

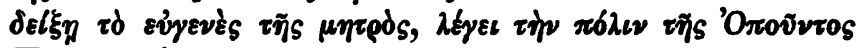

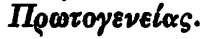

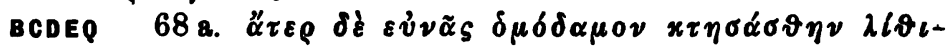

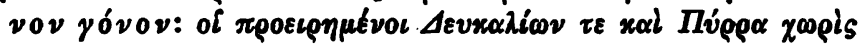

17 sch. Ap. Rh. IV 1780

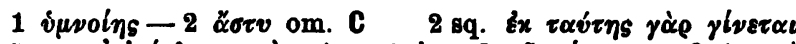

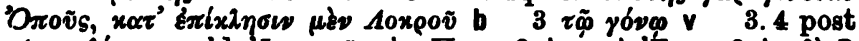

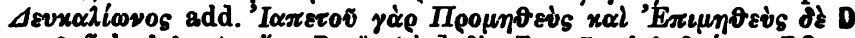

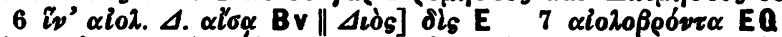

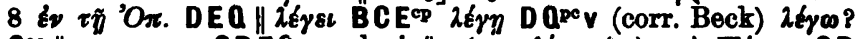

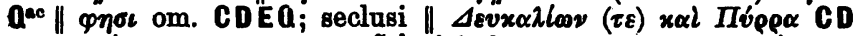

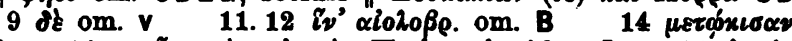

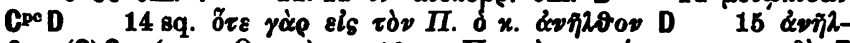

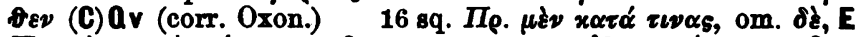

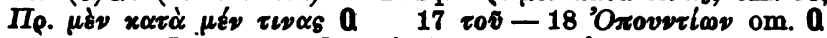

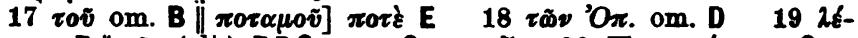

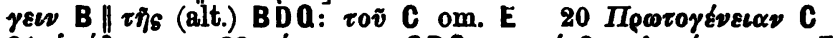

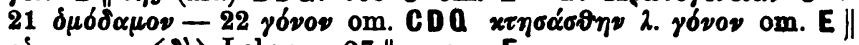

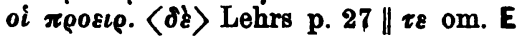




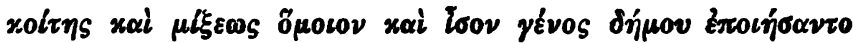

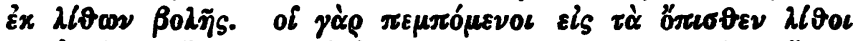

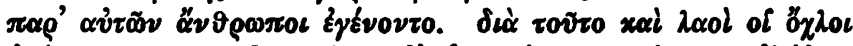

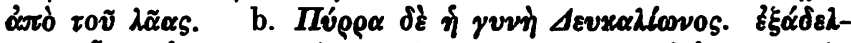

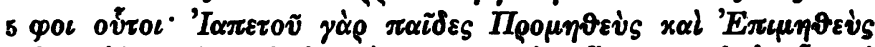

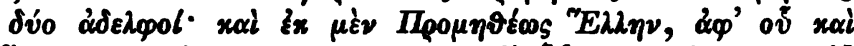

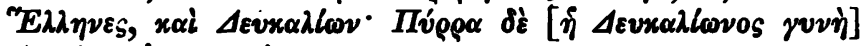

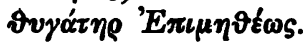

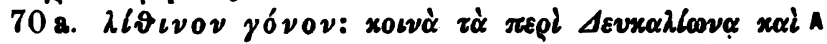

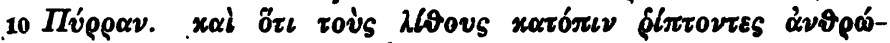

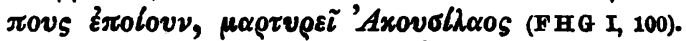

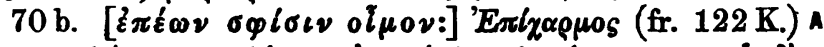

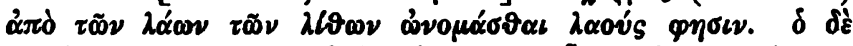

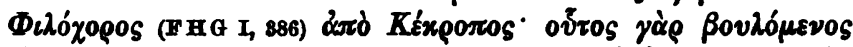

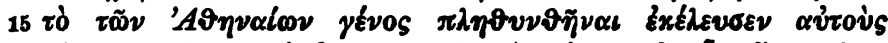

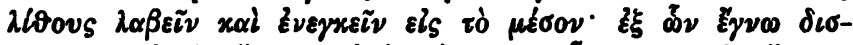

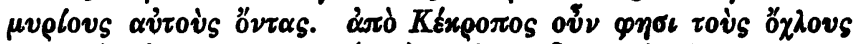

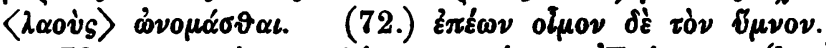

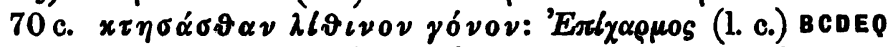

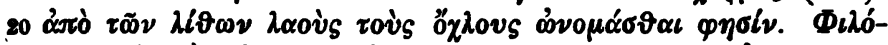

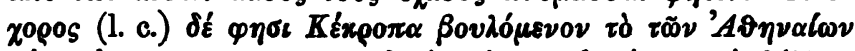

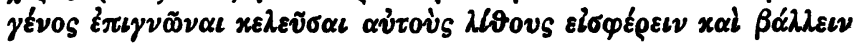

3 Apollod.1, 7,2,5. sch. A A126. Ap. soph. 107,1. EG 362,31. EM 553, 116 sch. Ap. Rh. III 108614 et 21 Eust. D. 23, 35

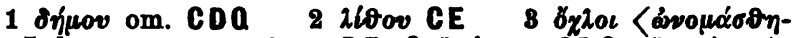

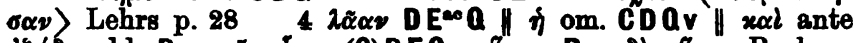

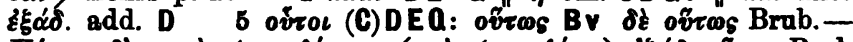

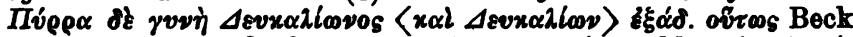

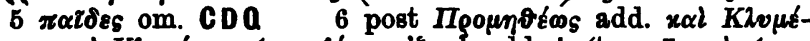

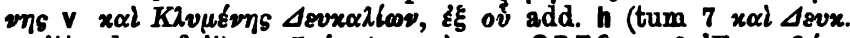

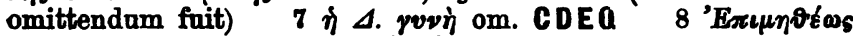

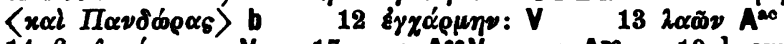

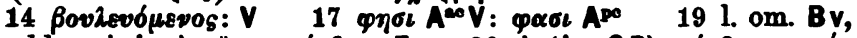

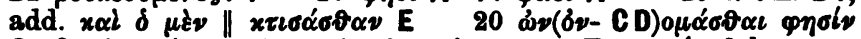

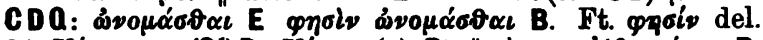

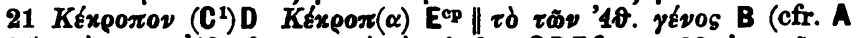

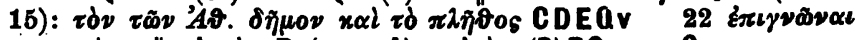
suspectum $\| \alpha \dot{v} \tau o \dot{s}$ B (cum A): $\alpha \dot{v} \tau \dot{\nu} \nu$ (D)EQ om. C 


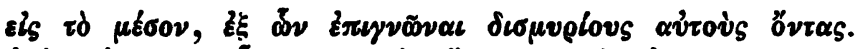

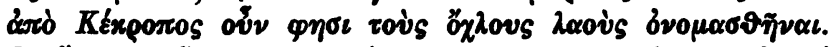

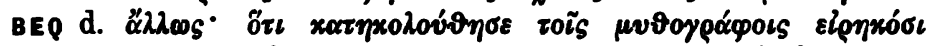

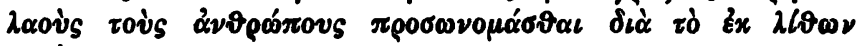
$\gamma \varepsilon \nu \in \sigma \sigma \theta \alpha \iota^{\circ}$

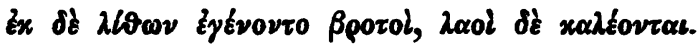

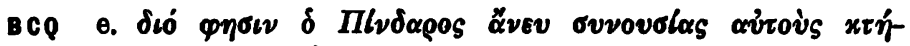
$\sigma \alpha \sigma \theta \alpha \iota$ גlo

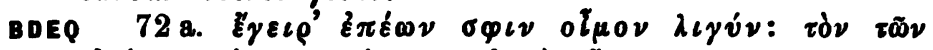

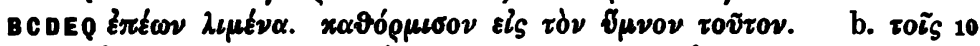

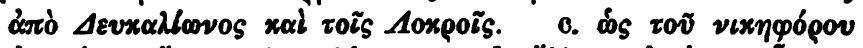

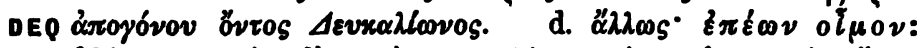

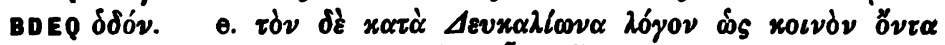

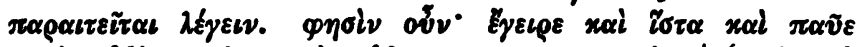

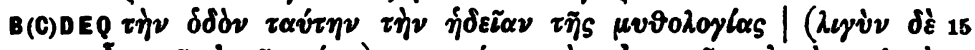

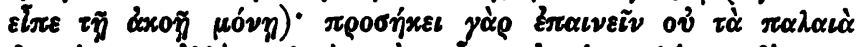

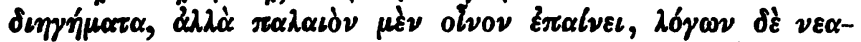

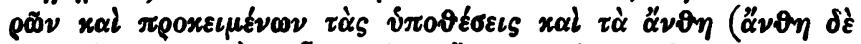

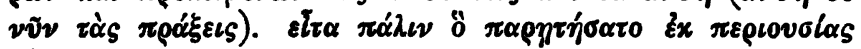
$\lambda$ Ėgel. 75 B

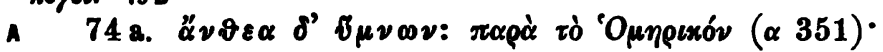

21 Eust. Od. 1421, 47

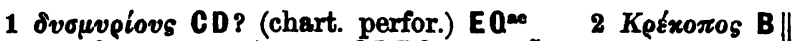
$\varphi \eta \sigma \iota$ BV (cfr. A 283, 17): om. CDEQ 3 б $\tau \iota$ om. Bv $\| x \alpha \tau \eta-$

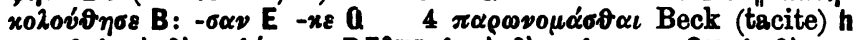

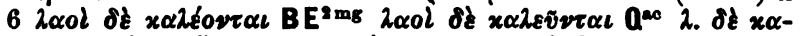

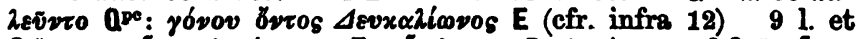

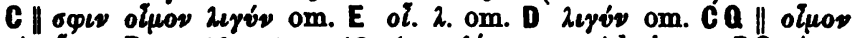

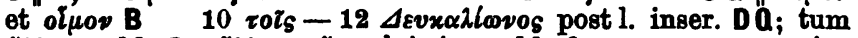

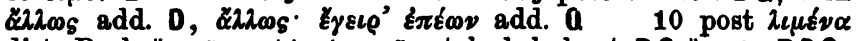

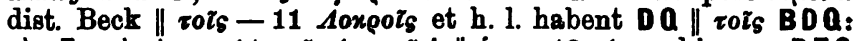

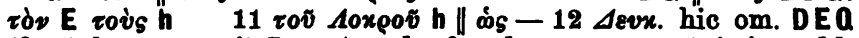
(frustalum servavit E post 70d, cfr. ad 6) 12 post $\varepsilon \pi \varepsilon^{\prime} \omega \nu$ add.

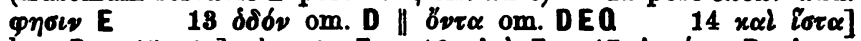

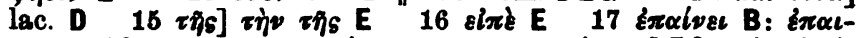

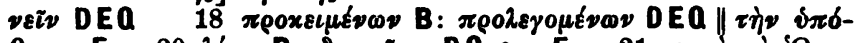

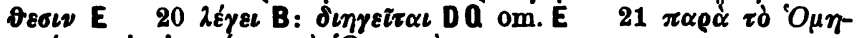

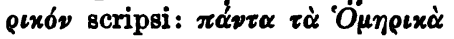




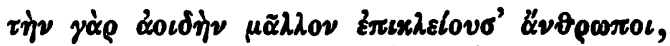

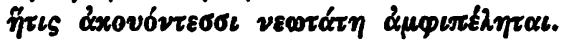

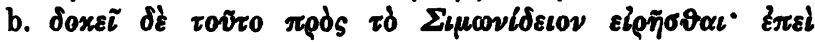

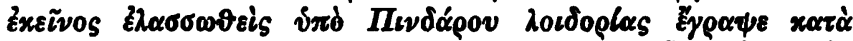

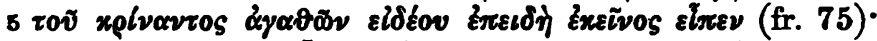

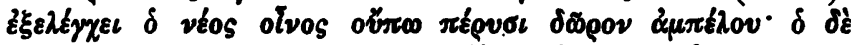

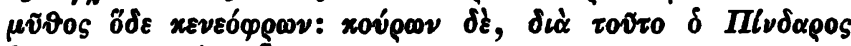

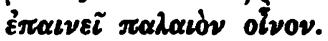

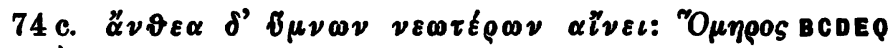
$10(\alpha 351)$.

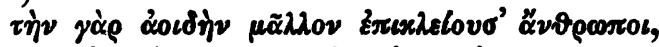

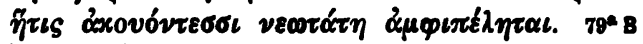

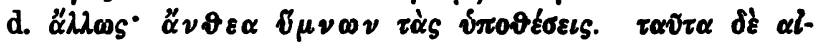

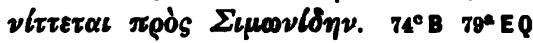

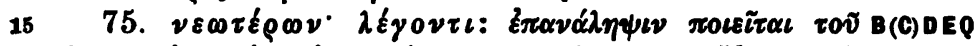

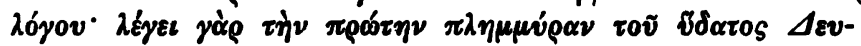
roגloovos. $74^{\mathrm{d}} \mathrm{B} 79^{\mathrm{a}} \mathrm{CD} 78^{\mathrm{C}} \mathrm{E} 79^{\mathrm{b}} \mathrm{Q}$

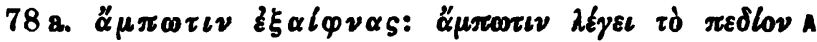

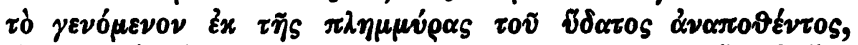

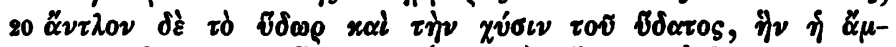

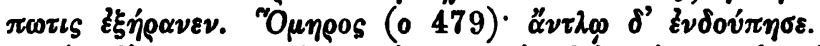

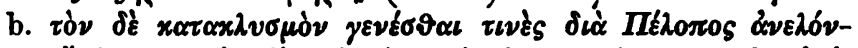

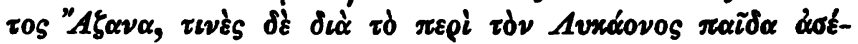

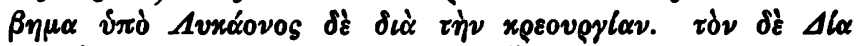

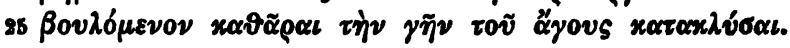

20 Hesych. ävนนันข.

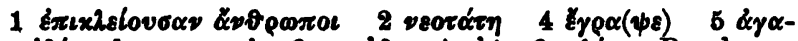

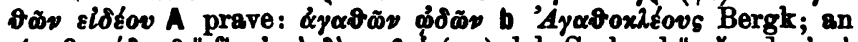

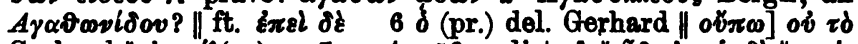

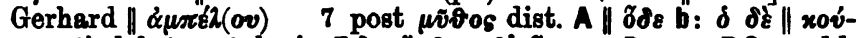

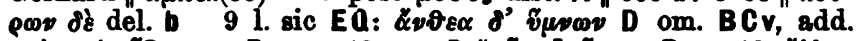

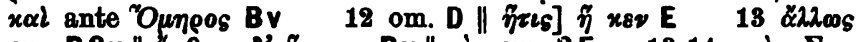

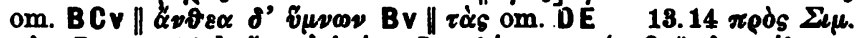

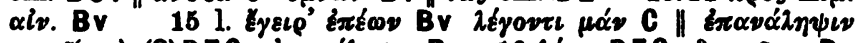

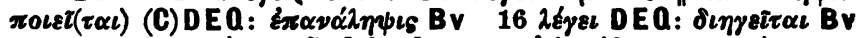

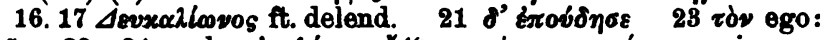

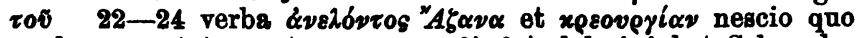
modo permutata sunt; porro 24 dz diò deleri iubet Schroeder 


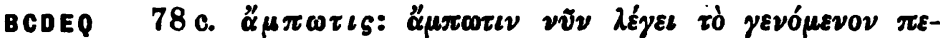

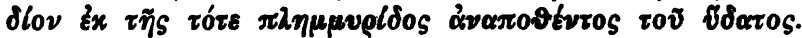

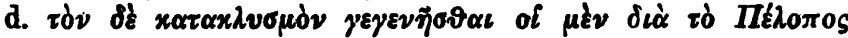

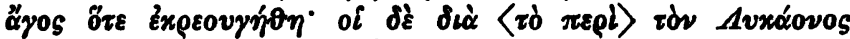

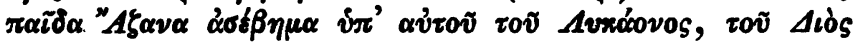

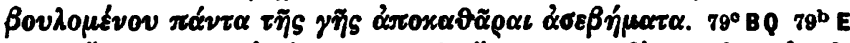

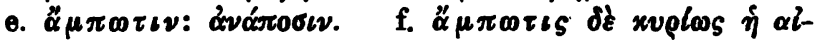

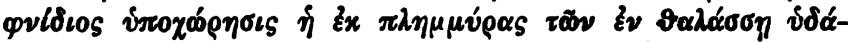

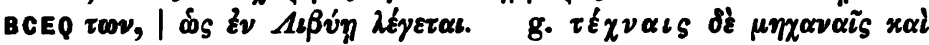

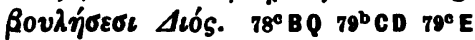

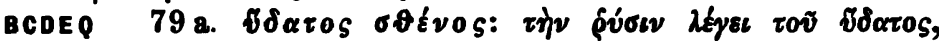

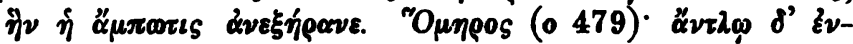

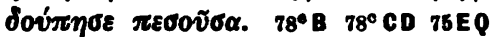

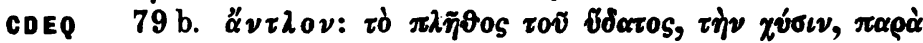

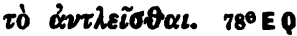

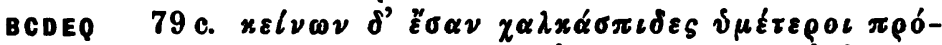

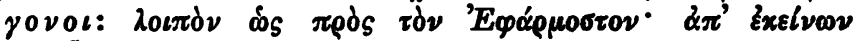

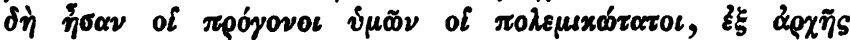

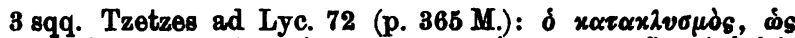

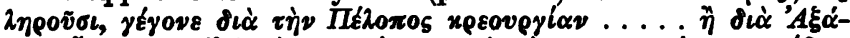

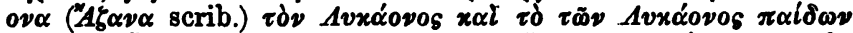

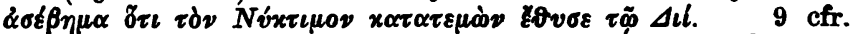
Diod. 3, 40, 9. Eust. ad Dion. Per. 198. EM 86, b0. EG 46, 22

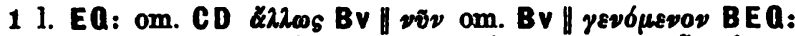

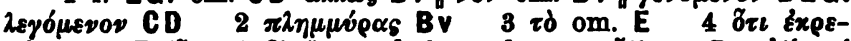

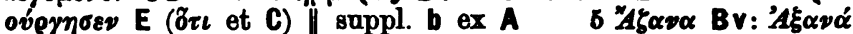

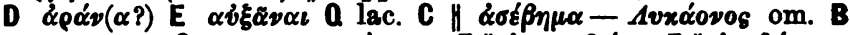

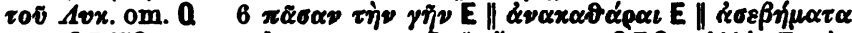

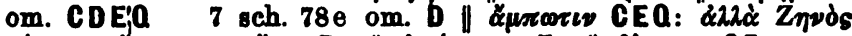

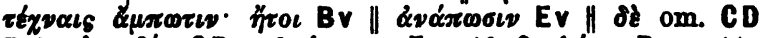

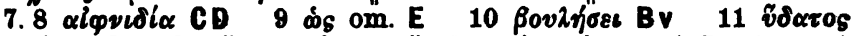

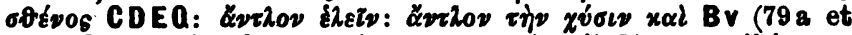

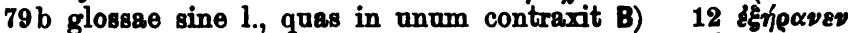

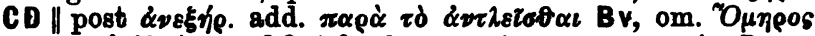

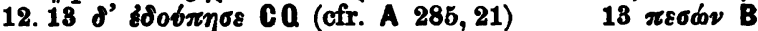

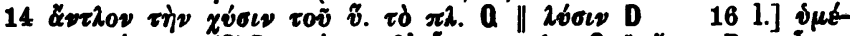

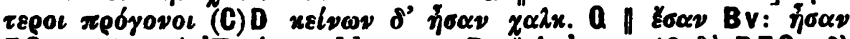

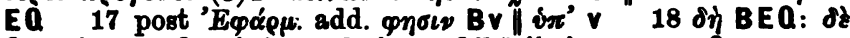

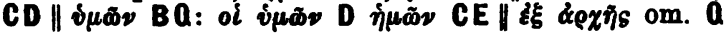




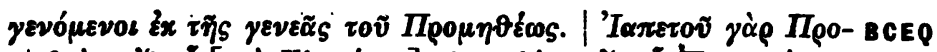

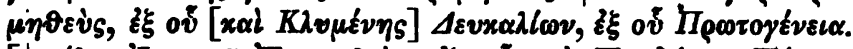

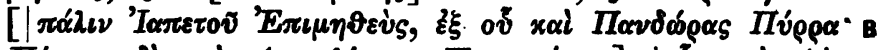

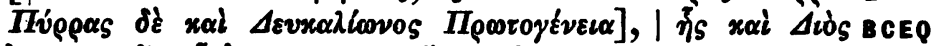

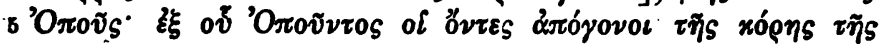

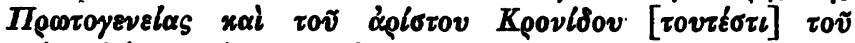

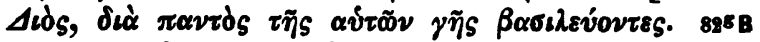

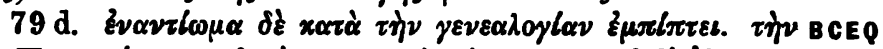

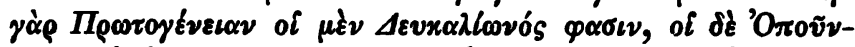

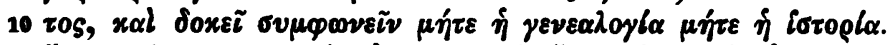

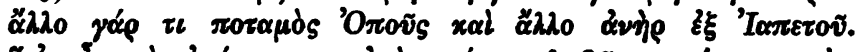

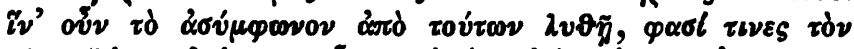

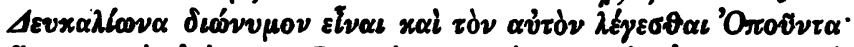

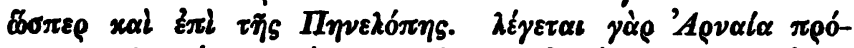

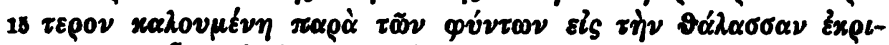

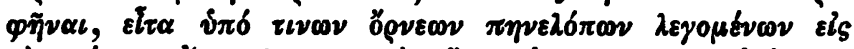

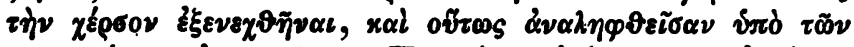

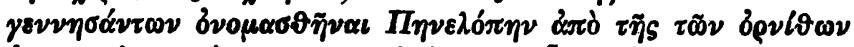

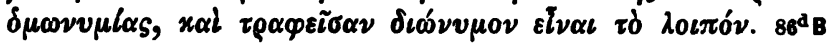

\section{4-19 Tzetzes ad Lyc. 792}

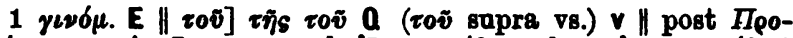

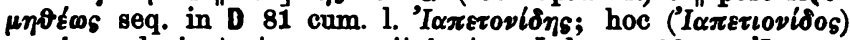
nescio unde in textum recepit b; tum Lehrs p. $28 \tau \tau_{5}$ 'I $\alpha \tau \varepsilon \tau L-$

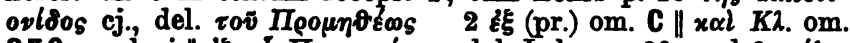

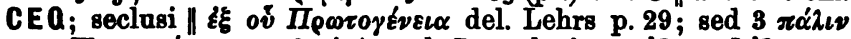

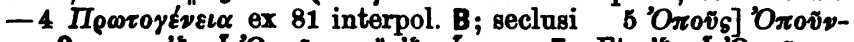

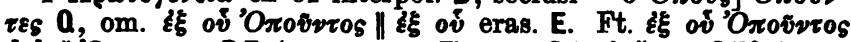

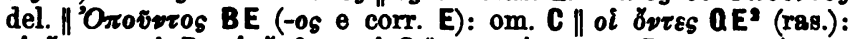
of 8vres $x \alpha 2$ B of zudpes of C

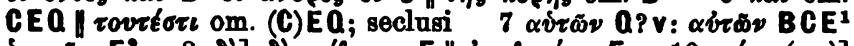

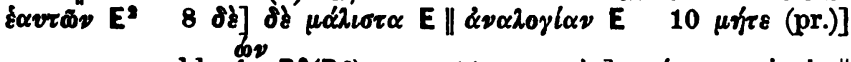

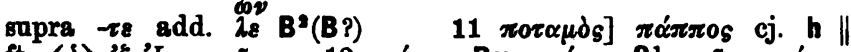

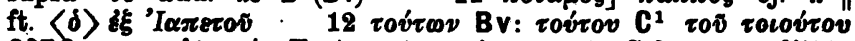

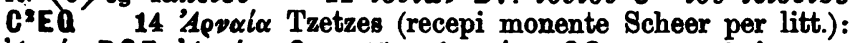

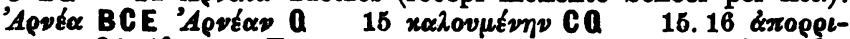

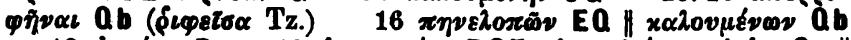

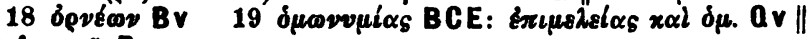
นอขี วดเสอขี B 


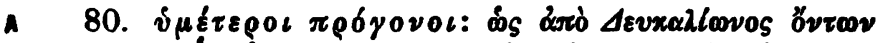

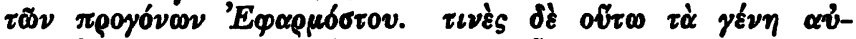

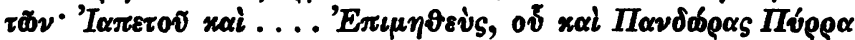

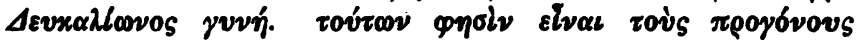

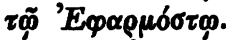

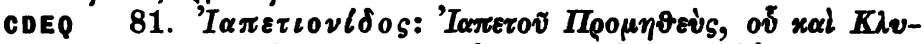

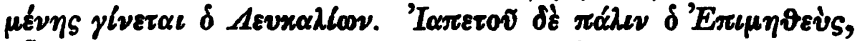

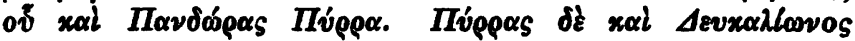

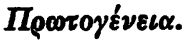

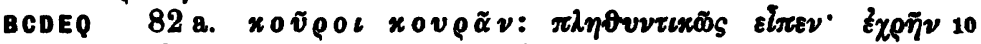

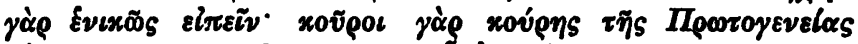

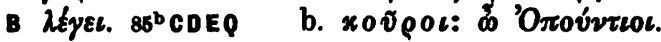

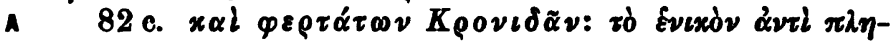

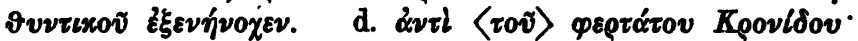
Aiòs yà

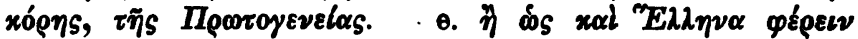

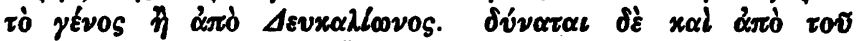

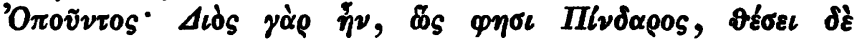
Aoxpoṽ.

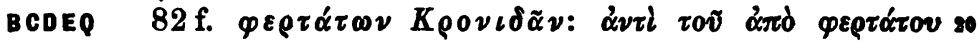

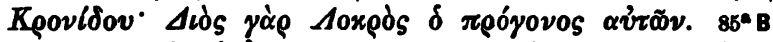

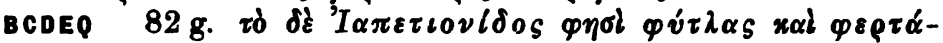

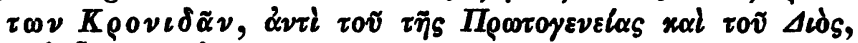

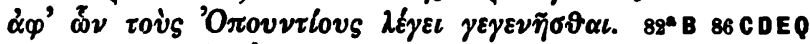

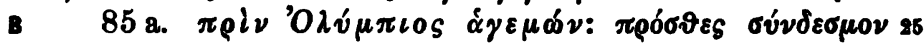

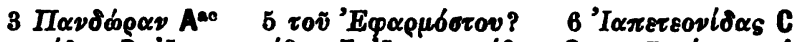

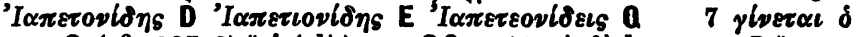
om. C (cfr. 287, 2) $\| \delta$ (alt.) om. CQ 10 rd $\delta \xi$ 1. praem. B $\|$ nov-

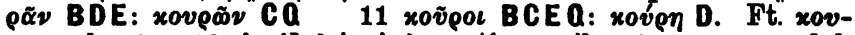

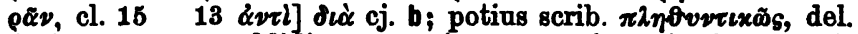

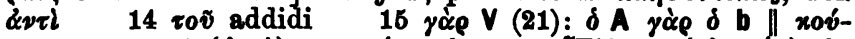

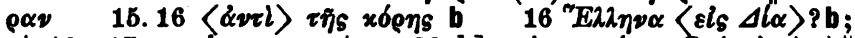

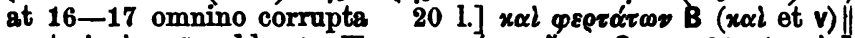

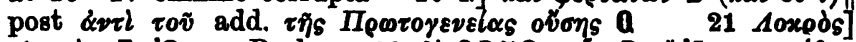

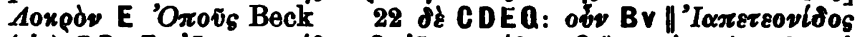

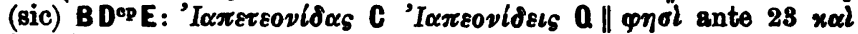

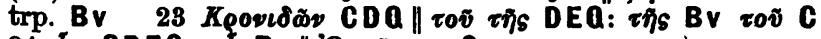

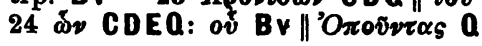




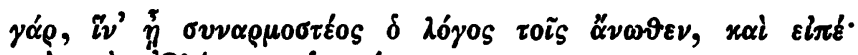
$\pi \rho i v$ yò 'O 'O

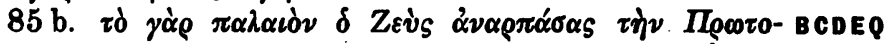

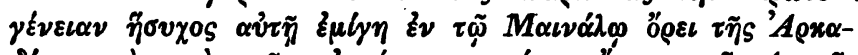

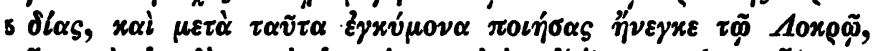

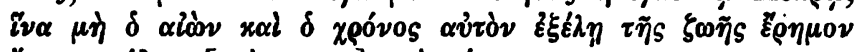

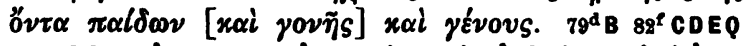

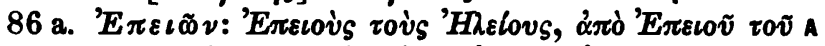

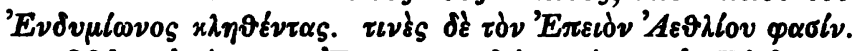

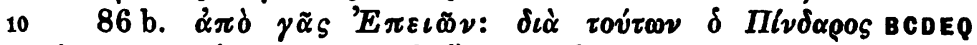

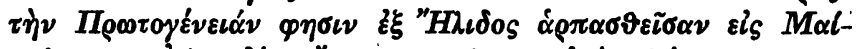

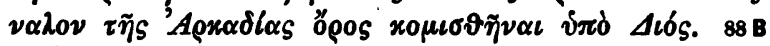

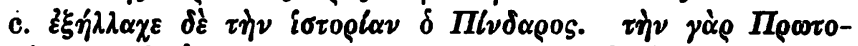

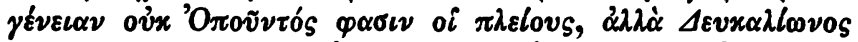

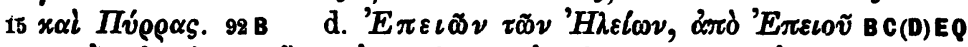

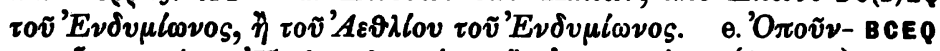

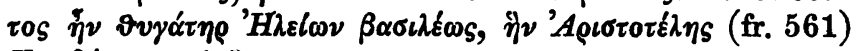

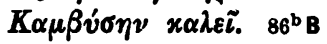

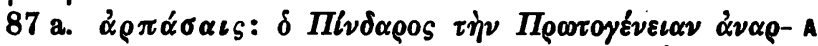

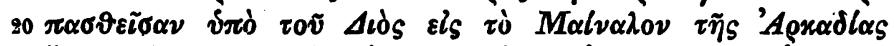

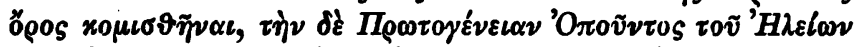

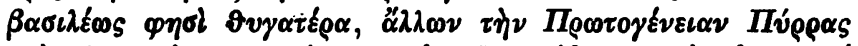

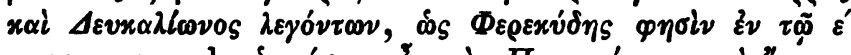

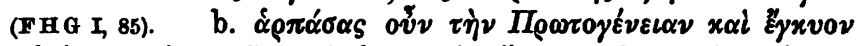

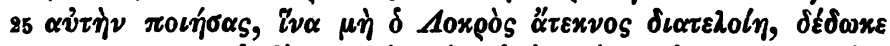

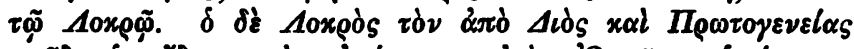

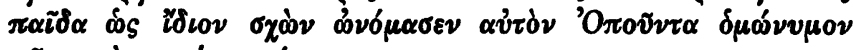

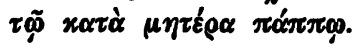

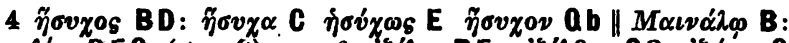

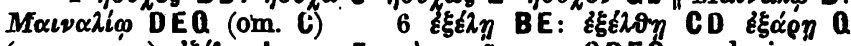

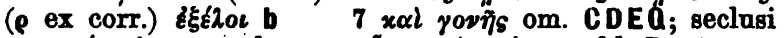

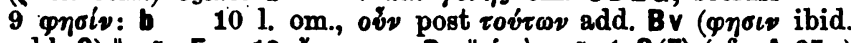

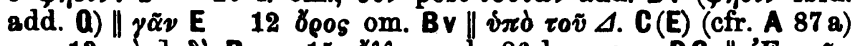

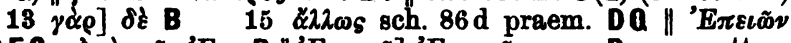

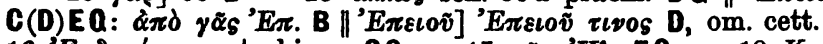

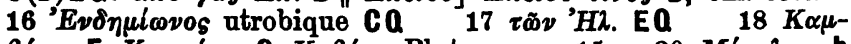

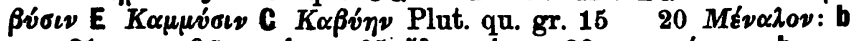

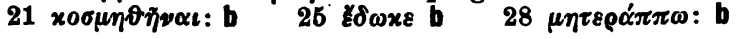

Sohol. m Prodarux ed. Drachmann. 


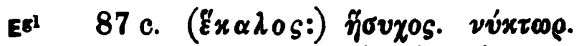

A 88 a. $\mu l_{\chi} \vartheta \eta: \mu \varepsilon \tau \dot{\alpha}$ jò $\delta$ Aoxpós.

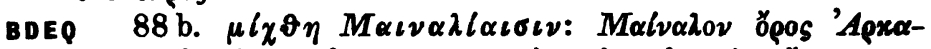

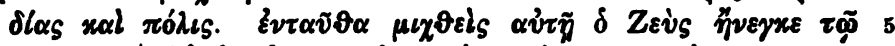

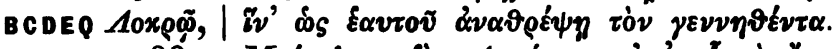

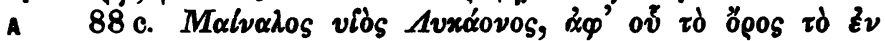

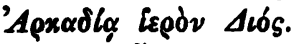

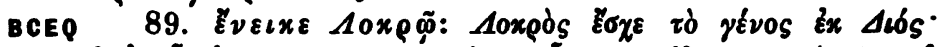

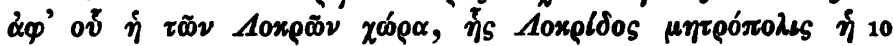
'Oส०ข์s. ${ }^{86}$ B

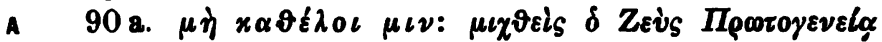

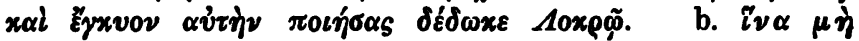

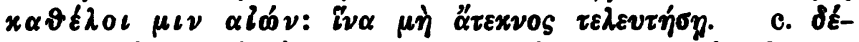

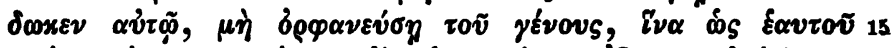

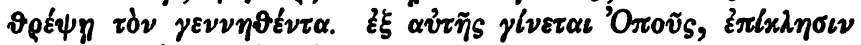

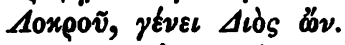

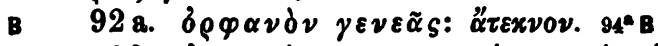

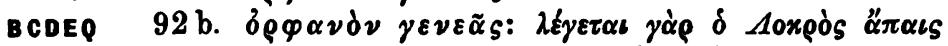

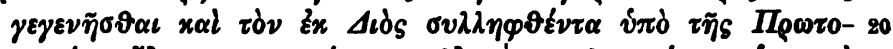

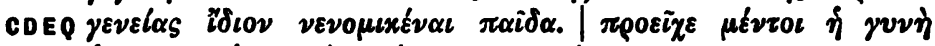

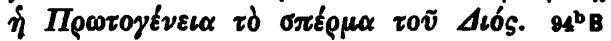

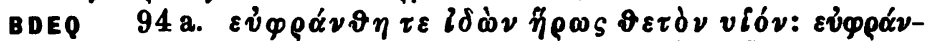

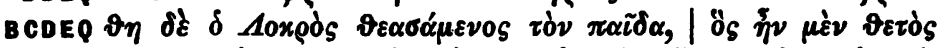

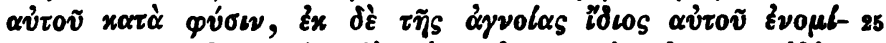

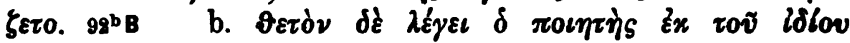

4 sch. Ap. Rh. 1, 1687 sch. Theocr. 1, 124

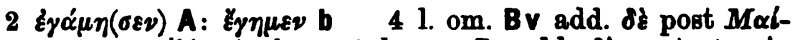

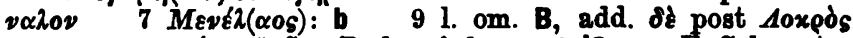

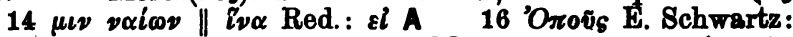

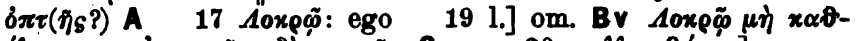

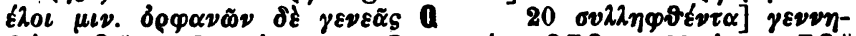

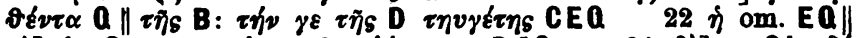

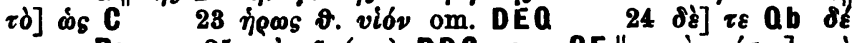

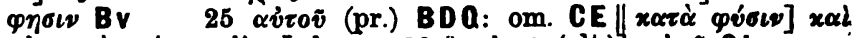

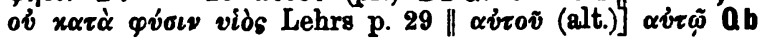




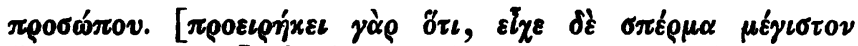

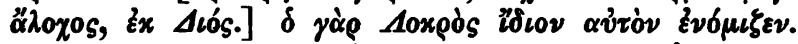

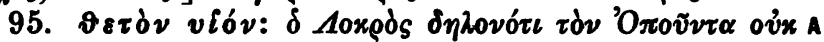

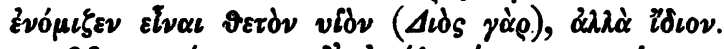

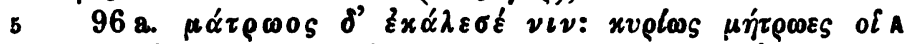

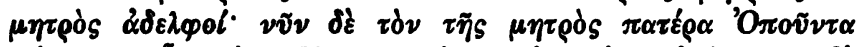
$\mu \eta \dot{v}$ Aoxpov.

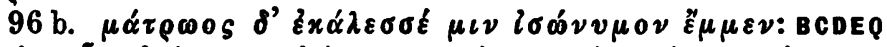

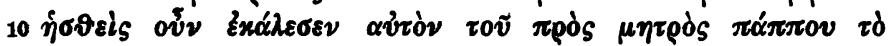

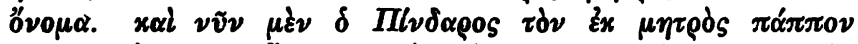

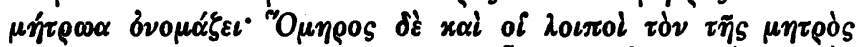

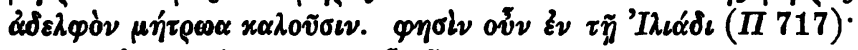

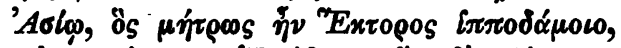

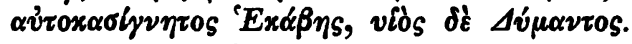

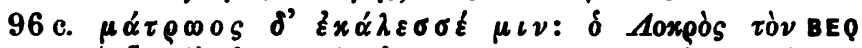

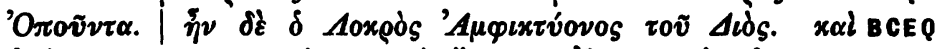

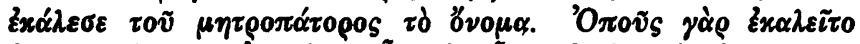

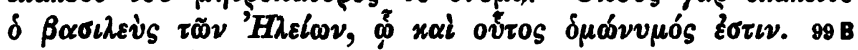

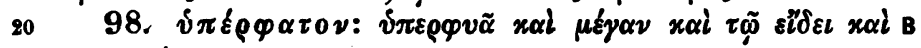

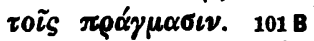

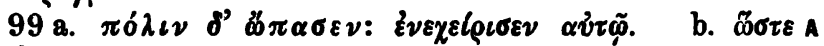

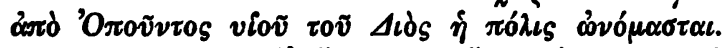

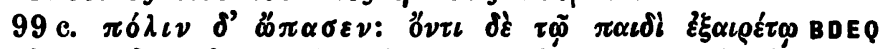

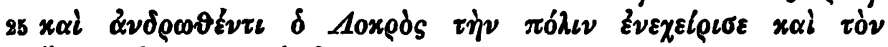

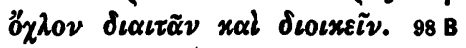
5 cfr. ad sch. 0. VI, 130
11 sch. BT $\Pi 717$

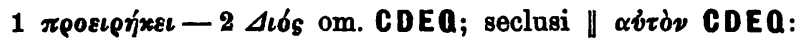

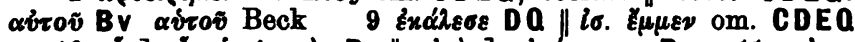

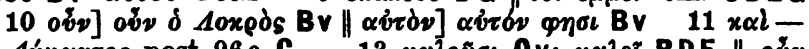

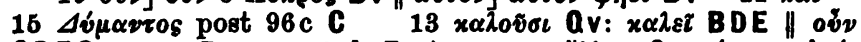

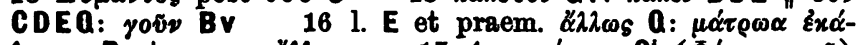

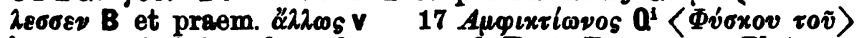

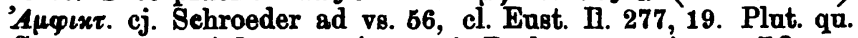
Graec. $15 \| \Delta \iota \dot{s}] \Delta \varepsilon v x \alpha \lambda i \omega v o s$ cj. Beck 18 tò om. EQ

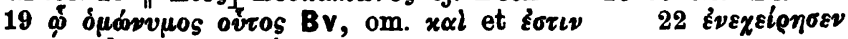

24 1. om. B $\| \pi \delta \imath_{\ell \epsilon}$ D 


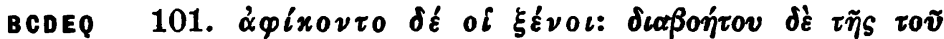

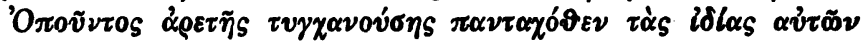

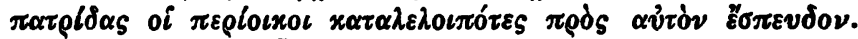

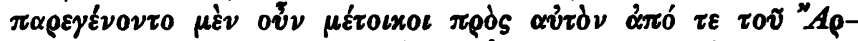

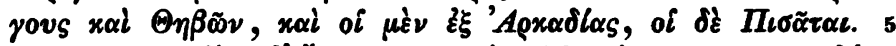

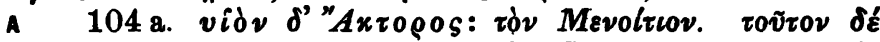

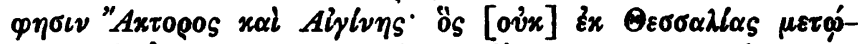

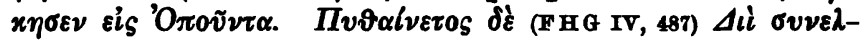

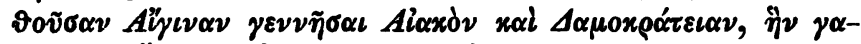

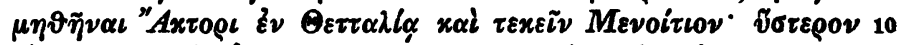

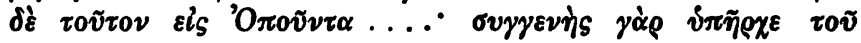

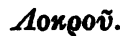

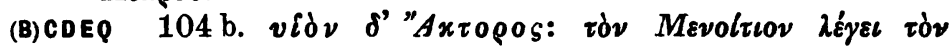

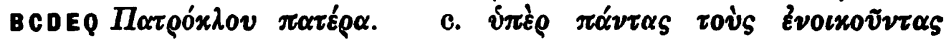

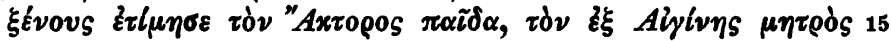

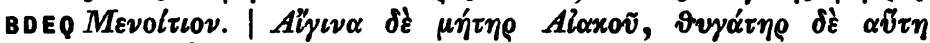

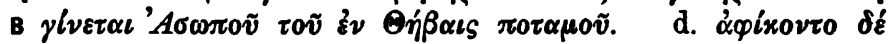

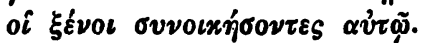

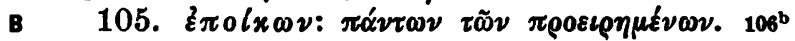

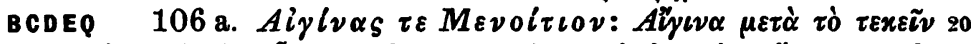

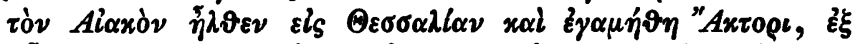

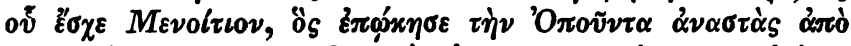

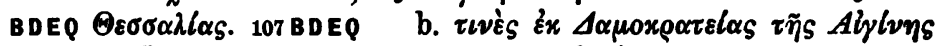

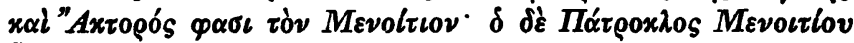

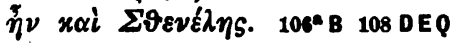

6 sch. D $\Sigma$ 9. sch. A ibid. 10. 1120 Eust. Il. 113, 2

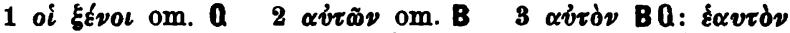

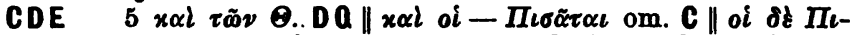

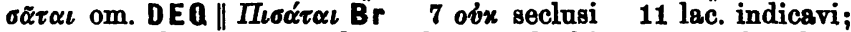
E2 $\sigma_{\varepsilon i \nu}$ suppl. b 13 sch. $104 \mathrm{~b}$ (cum l.) hic om. B; cfr. ad 15

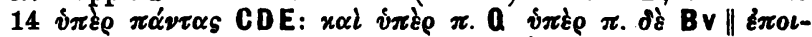

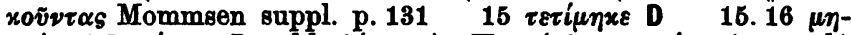

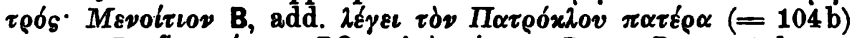

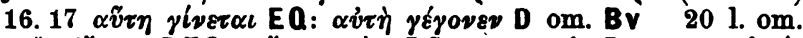

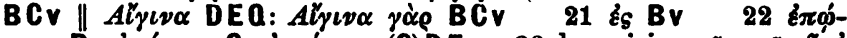

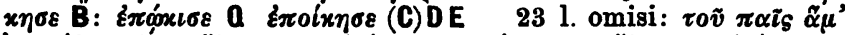

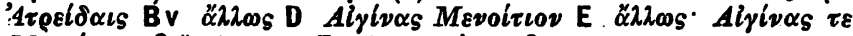

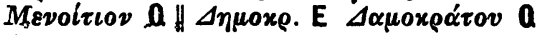




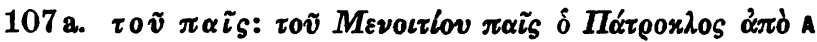

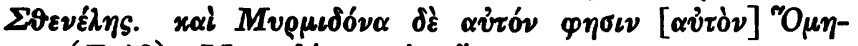

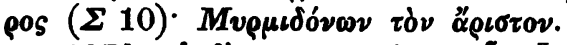

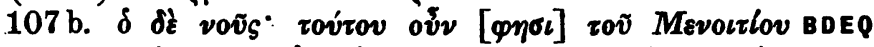

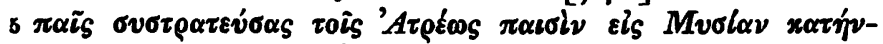

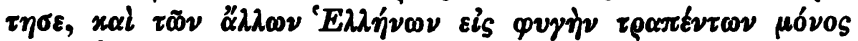

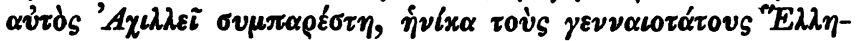

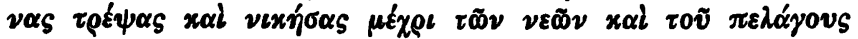

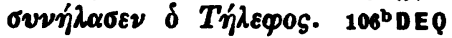

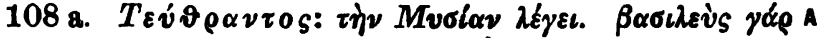

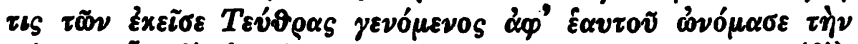

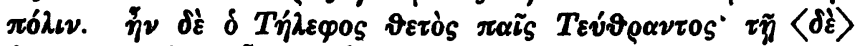

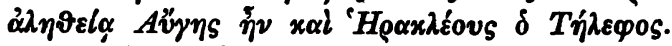

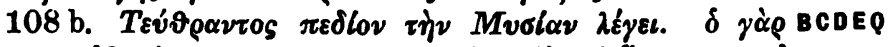

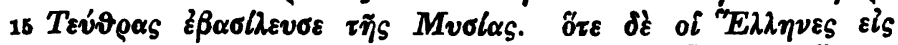

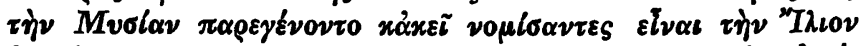

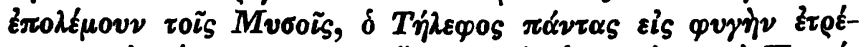

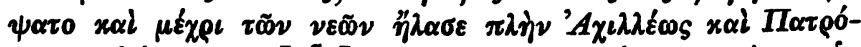

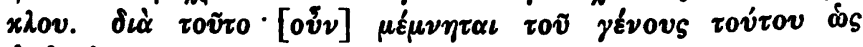

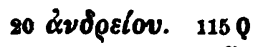

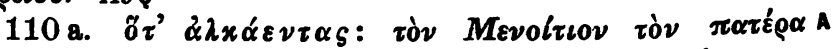

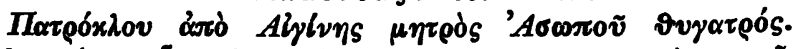

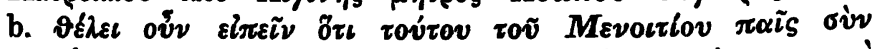

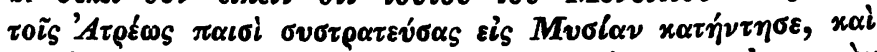

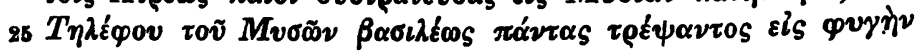

1 Apollod. 3, 13, 8, 4. sch. Ap. Rh. 1, 69. Cram. anecd.

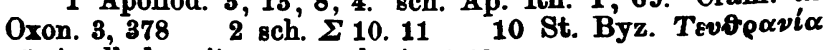
16 Apollod. epit. 3, 17. sch. A $A 69$

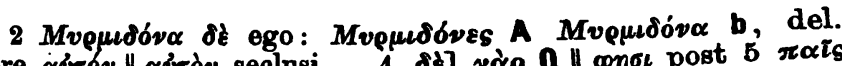

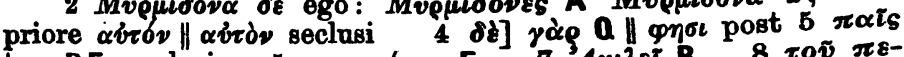

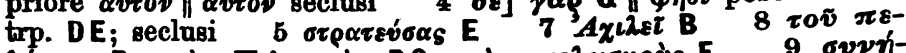

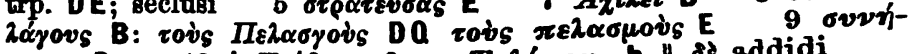

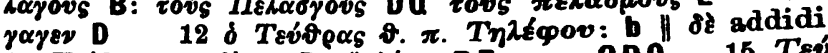

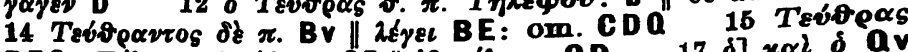

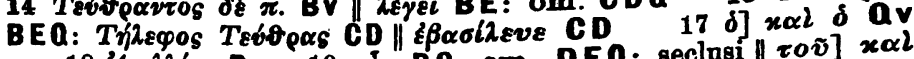

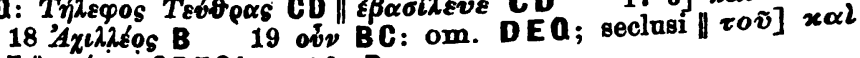

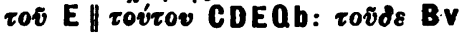




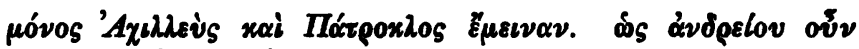

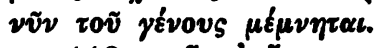

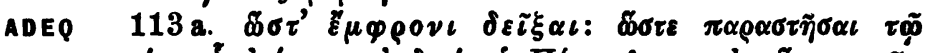

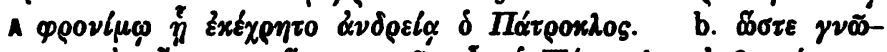

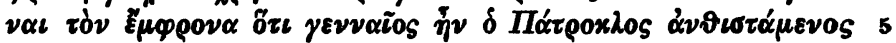
$T \eta \lambda \dot{q} \varphi q$.

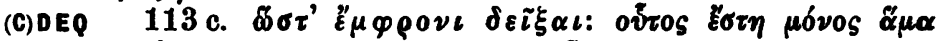

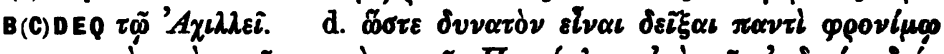

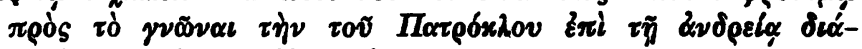

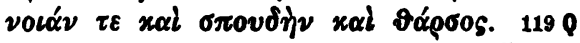

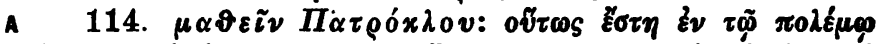

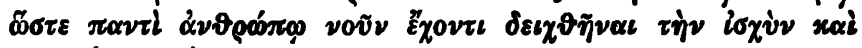

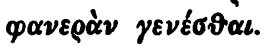

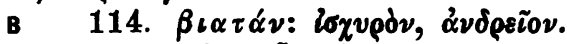

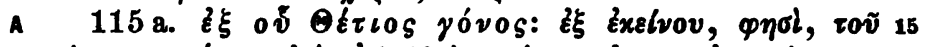

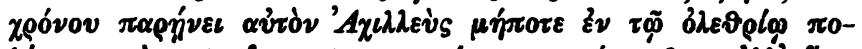

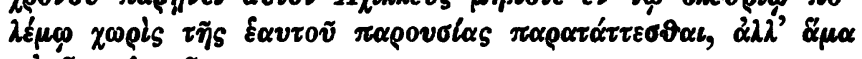

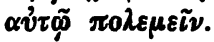

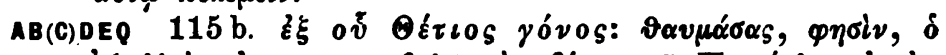

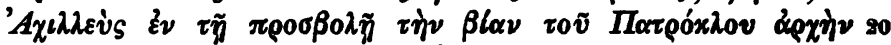

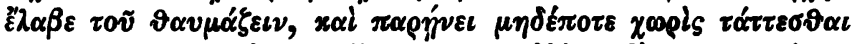

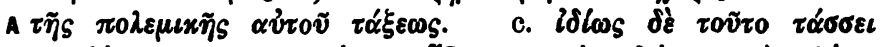

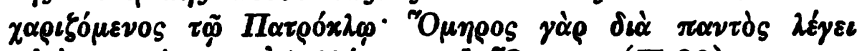

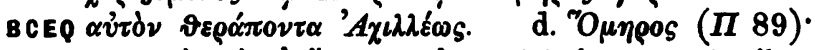

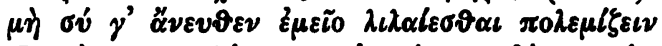

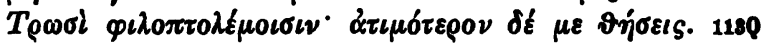

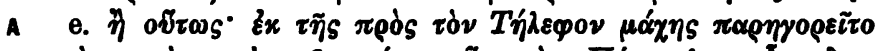

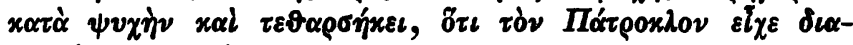
$\pi \alpha \nu \tau \delta s=\pi \alpha \rho \alpha \sigma \tau \alpha \dot{\tau} \tau \eta \nu$.

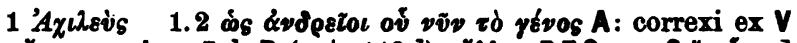

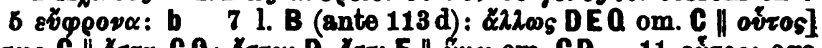

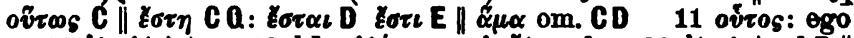

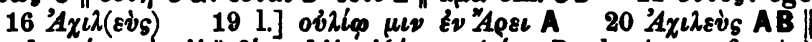

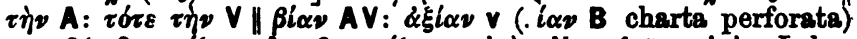

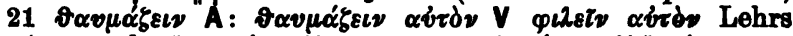

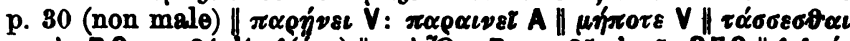

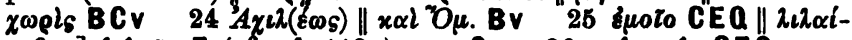

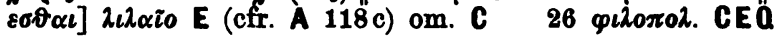


118 a. $\sigma \varphi \varepsilon \tau \hat{\varepsilon} \rho \alpha s: \stackrel{\alpha}{\nu \tau i} \tau 0 \tilde{v} \tau \tilde{\eta} S \varepsilon \tilde{\alpha} s$.

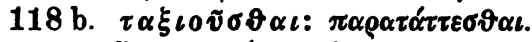

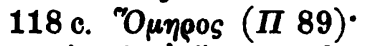

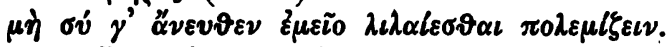

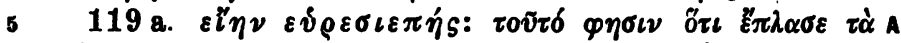

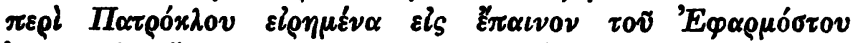

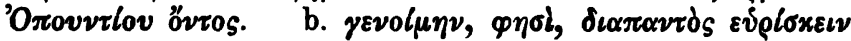

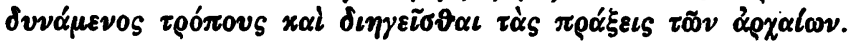

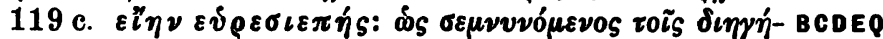

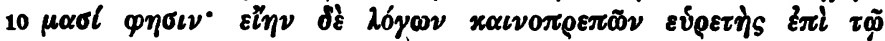

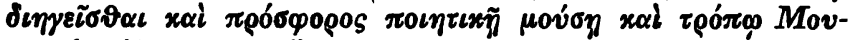

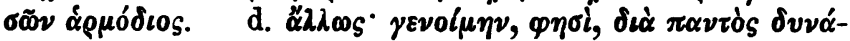

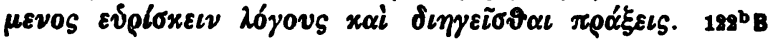

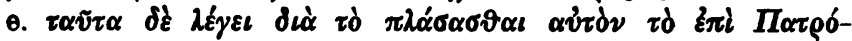

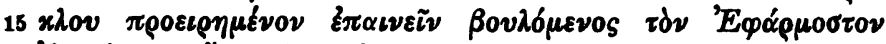

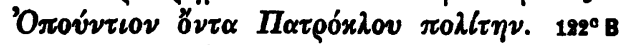

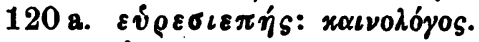
BEQ

120 b. \& \& $\alpha \gamma \varepsilon i \sigma \vartheta \alpha \iota: \delta \iota \eta \gamma \varepsilon i \sigma \vartheta \alpha \iota$. $\mathbf{8}$

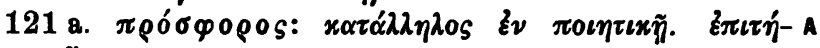

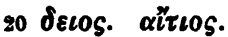

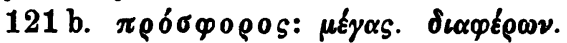

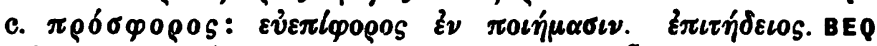

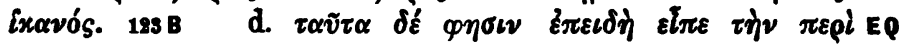

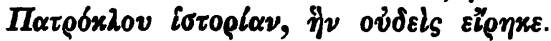

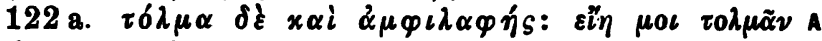

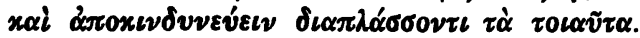

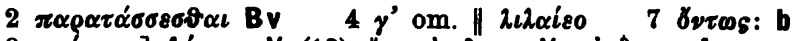

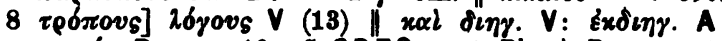

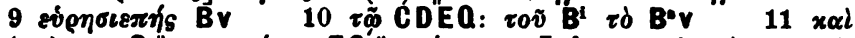

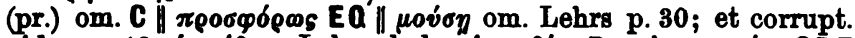

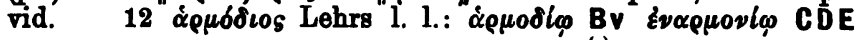

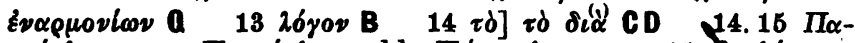

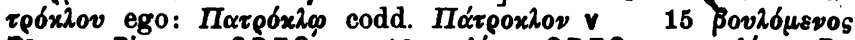

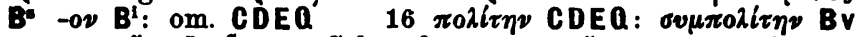

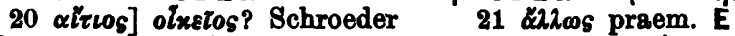

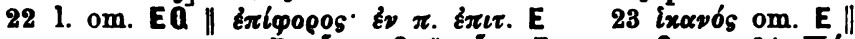

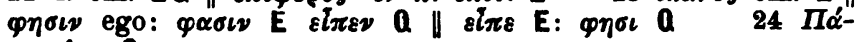
rpoxhov $\mathrm{a}$ 


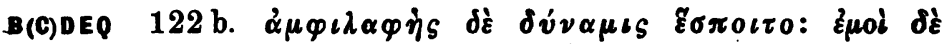

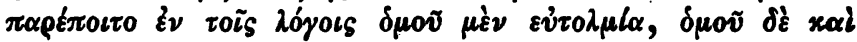

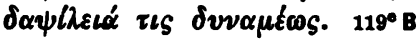

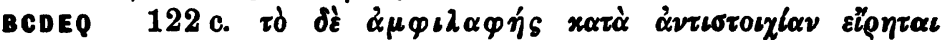

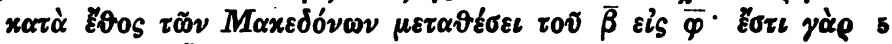

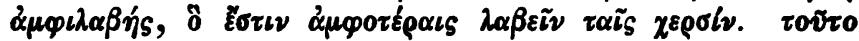

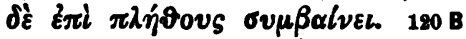

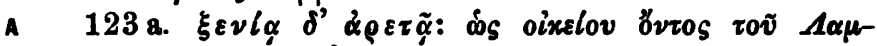

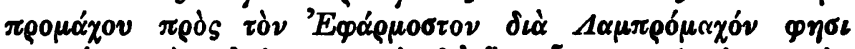

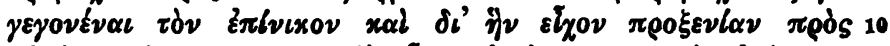

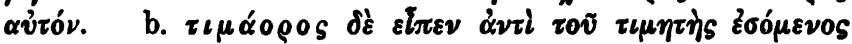

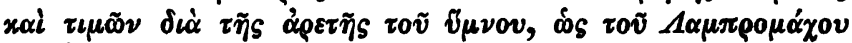

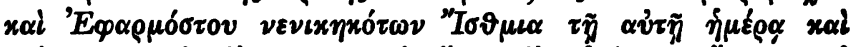

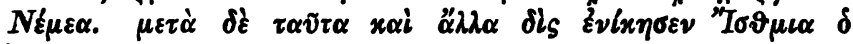

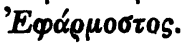

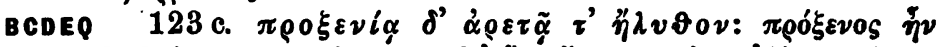

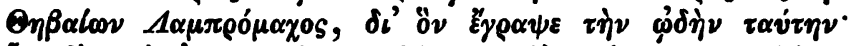

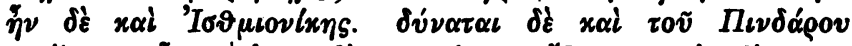

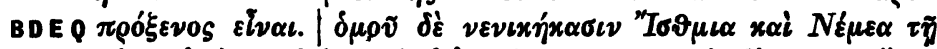

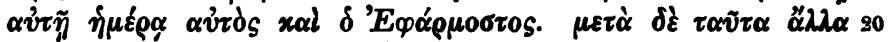

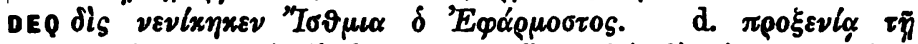

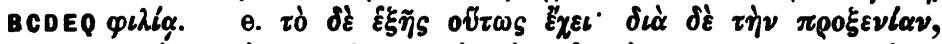

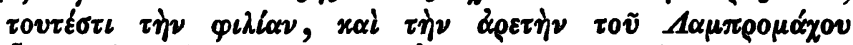

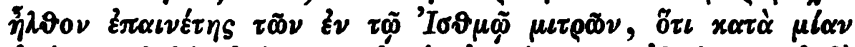

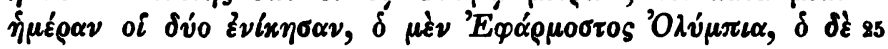

4-7 lex. Bachm. 81, 13. EM 91, 21. EG 48, 47. Suid.

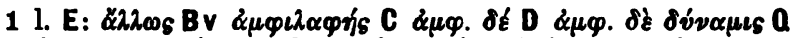

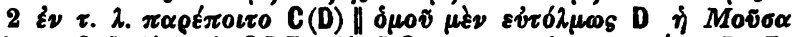

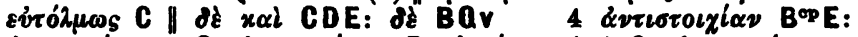

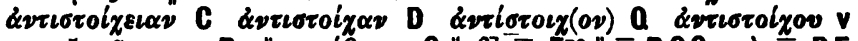

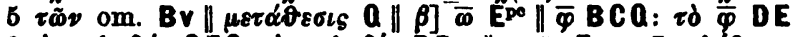

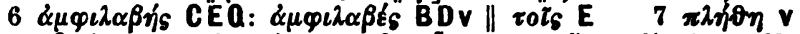

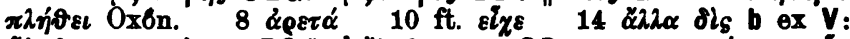

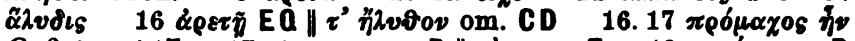

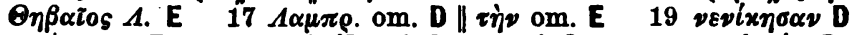

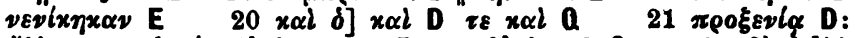

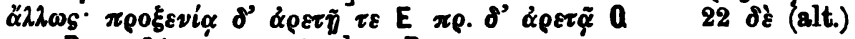
om. D 24 pro $\mu t \tau \rho \tilde{\omega} \nu$ lac. B 


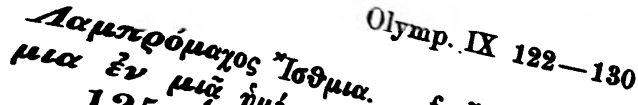

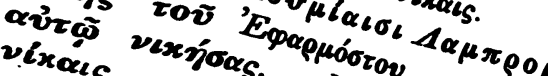

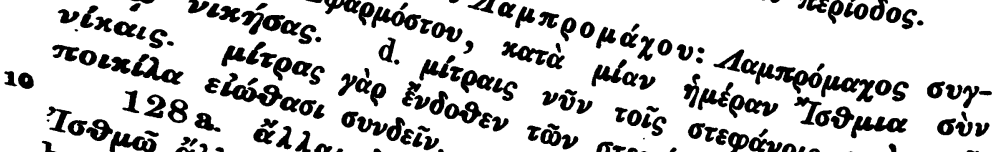

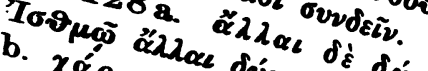

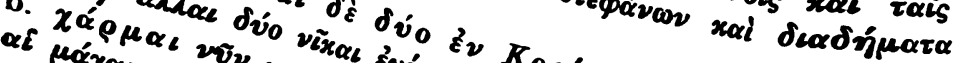

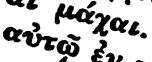

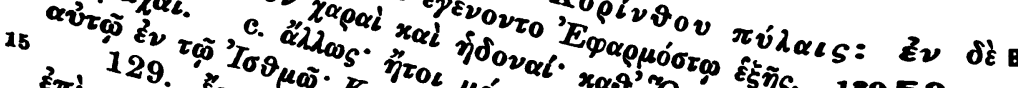

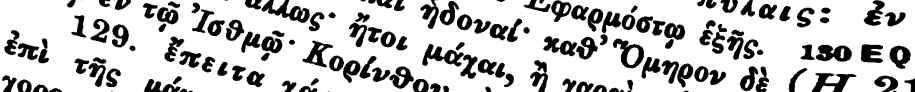

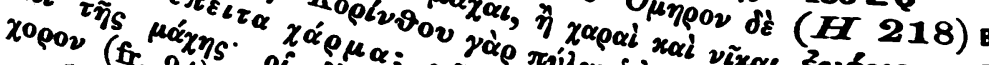

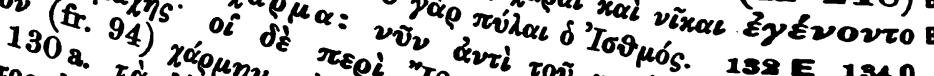

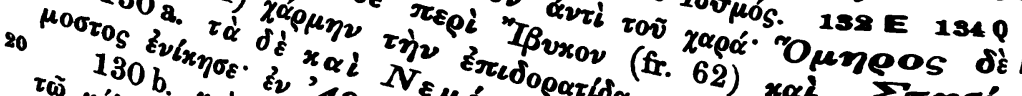

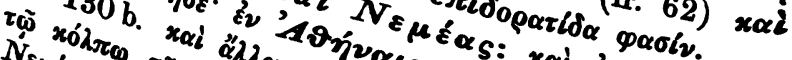

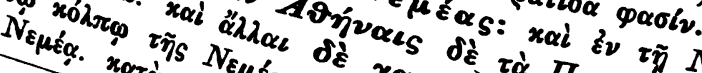

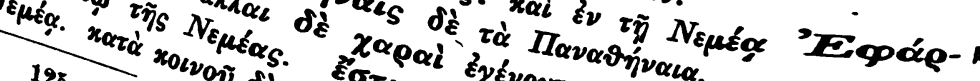

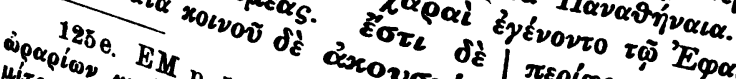

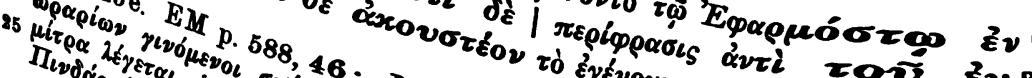

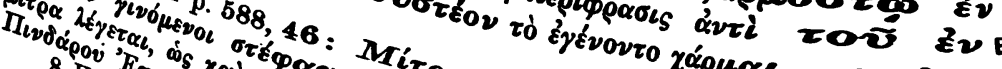

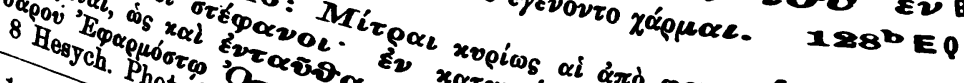

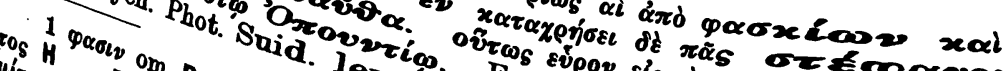

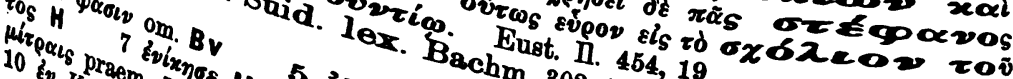

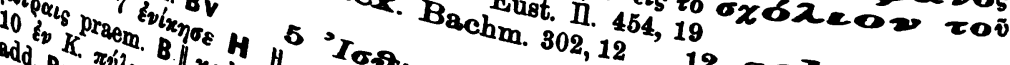

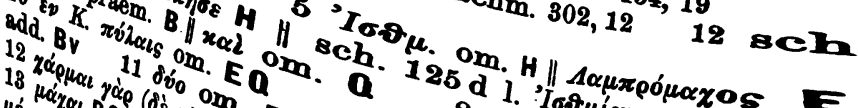

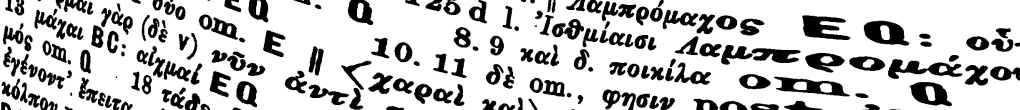

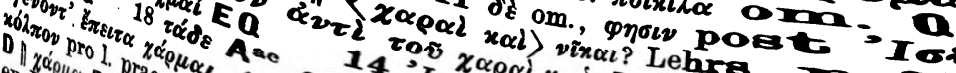

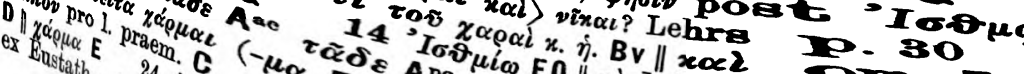

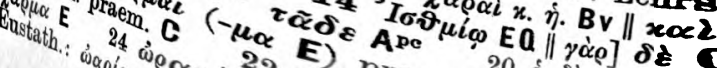

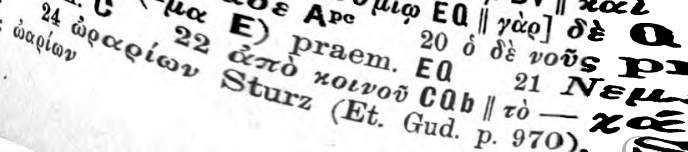
ima $11 \delta$ I os xor 


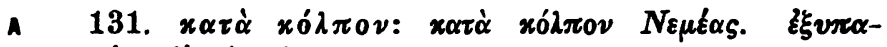

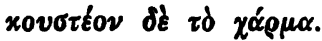

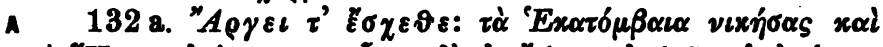

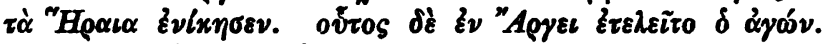

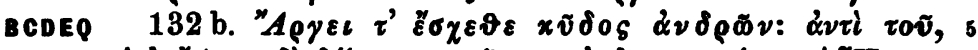

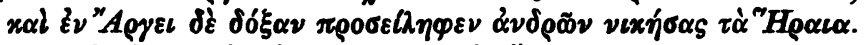

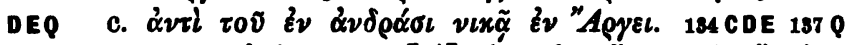

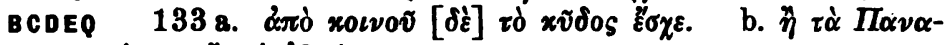

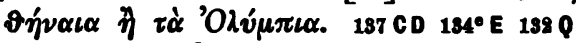

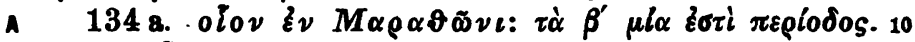

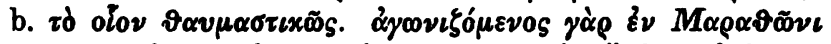

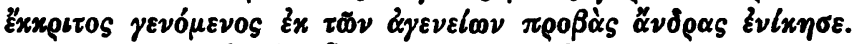

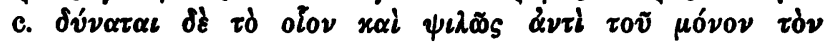
$\dot{\alpha} \gamma \tilde{\omega} \omega \alpha$.

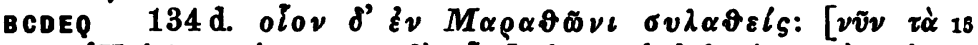

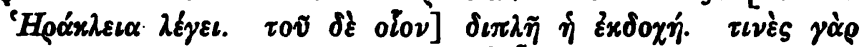

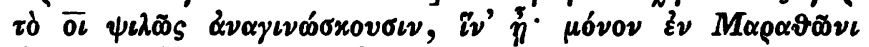

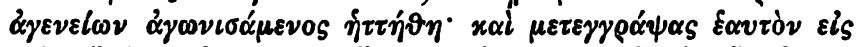

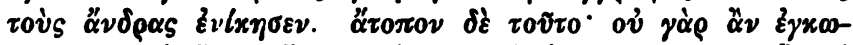

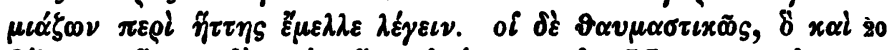

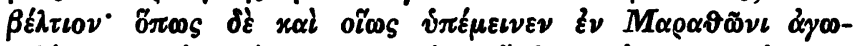

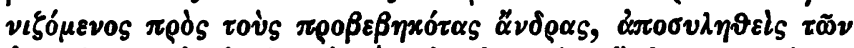

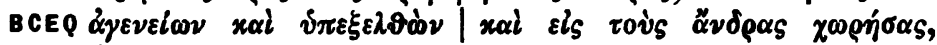

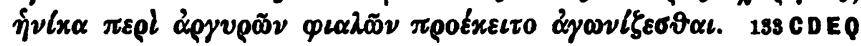

3 Hes. 'Ex ${ }^{\circ} \tau \delta \mu \beta \alpha \iota \alpha$.

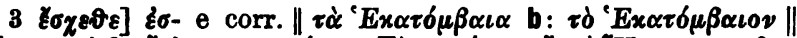

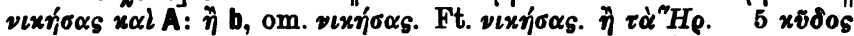

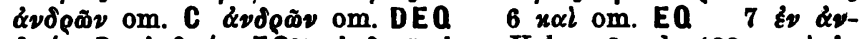

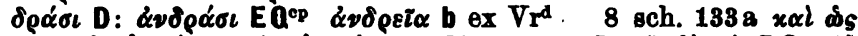

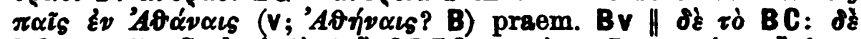
DQ om. E. Seclusi dzे\|

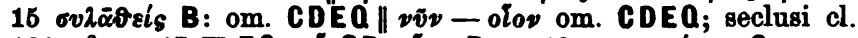

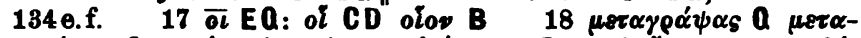

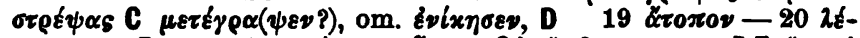

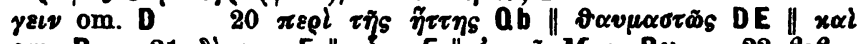

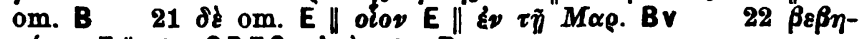
$x \dot{\sigma} \tau \alpha_{S}$ E $\| \tilde{\omega} \nu$ CDEQ: $\alpha \pi \dot{\delta} \tau \tilde{\omega} \nu \mathrm{BV}$ 


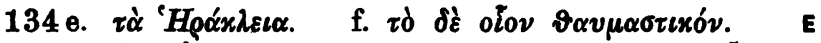

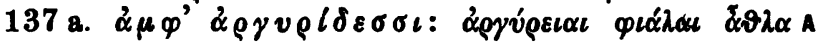

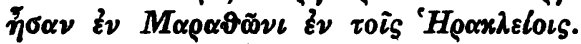

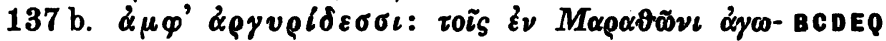

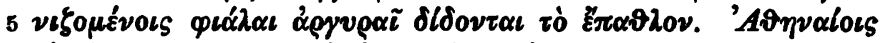

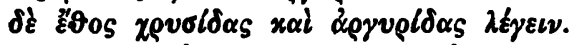

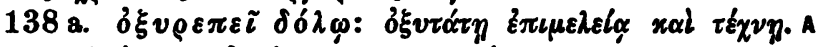

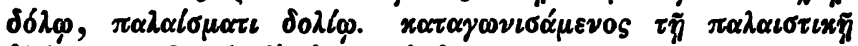

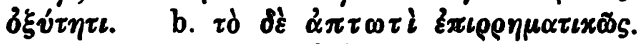

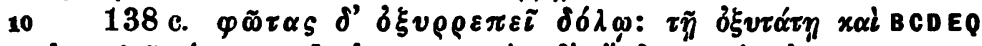

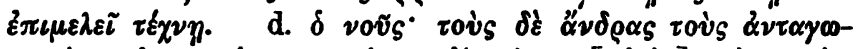

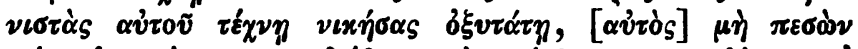

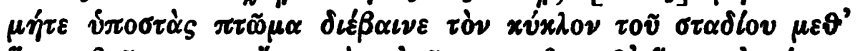

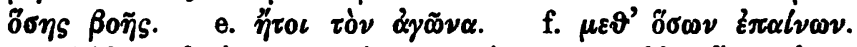

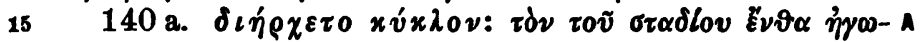

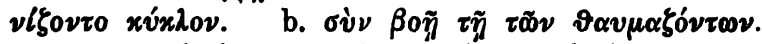

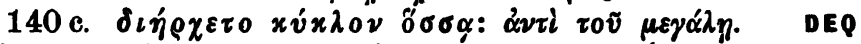

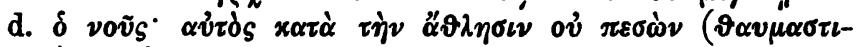

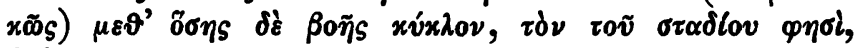

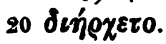

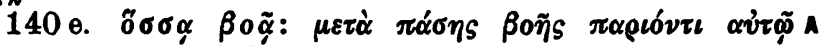

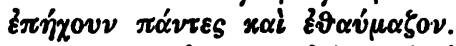

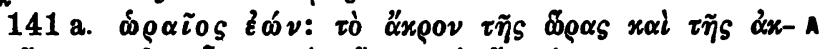

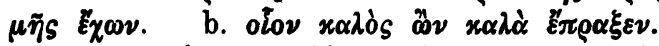

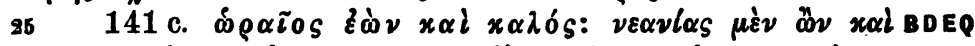

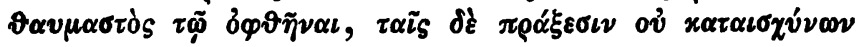

5 sq. Athen. 11, 502 a. lex. Bachm. 141, 22

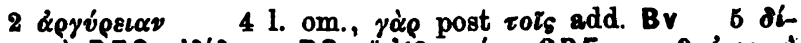

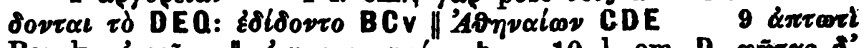

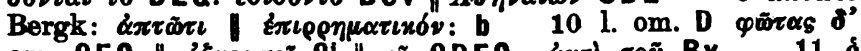

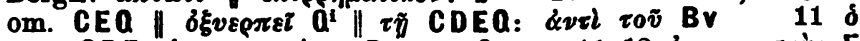

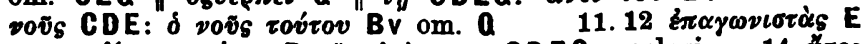

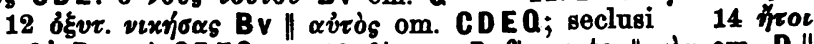

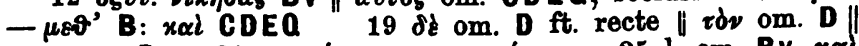

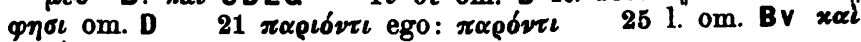
xalós om. D 


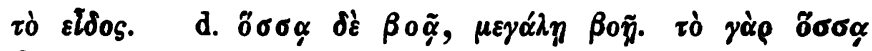
$\vartheta \alpha v \mu \alpha \sigma \tau \iota x \tilde{\omega}$.

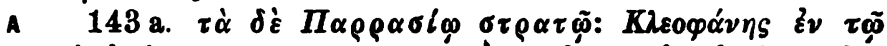

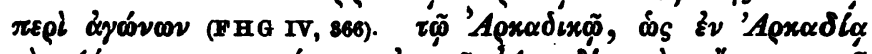

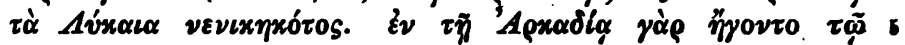

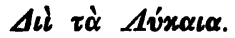

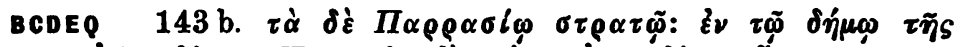

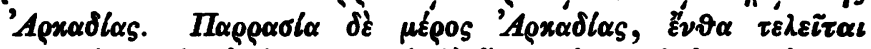

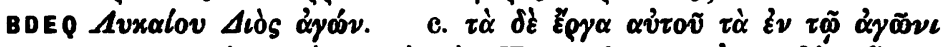

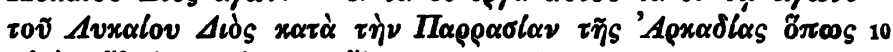

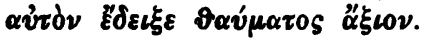

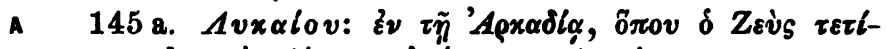

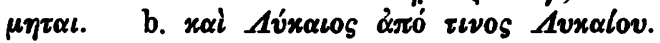

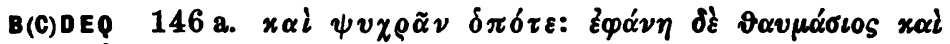

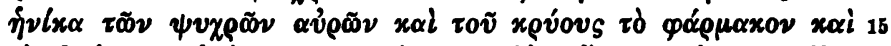

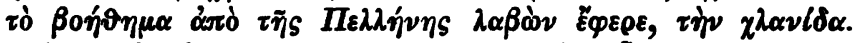

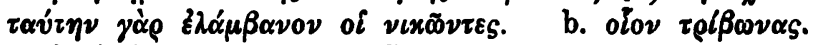

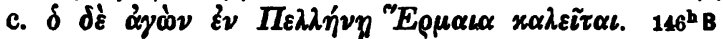

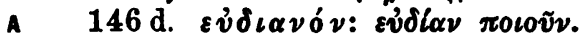

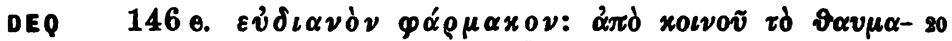
CEH

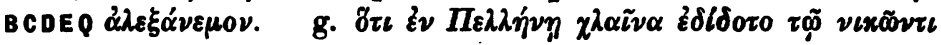

18 sqq. sch. Ar. Av. 1421. Strab. 8, 386. Phot. et Hesych. $\Pi \varepsilon \lambda \lambda \eta v \iota x \alpha l \quad \chi^{\lambda \alpha i \nu \alpha \iota .}$ Poll. 7, 67.

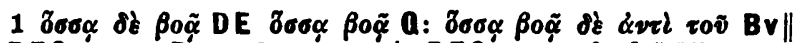

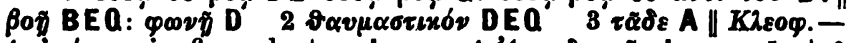

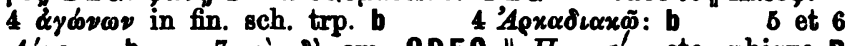

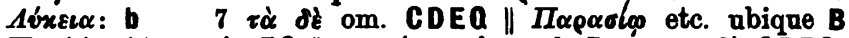

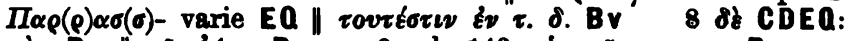

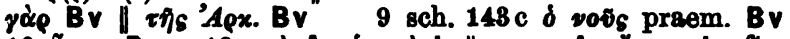

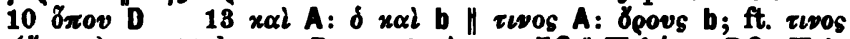

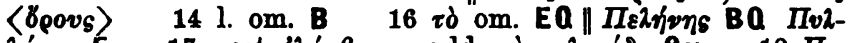

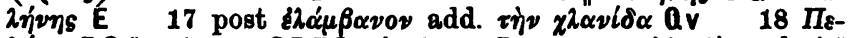

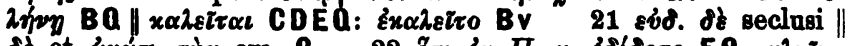

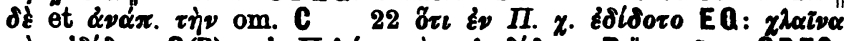

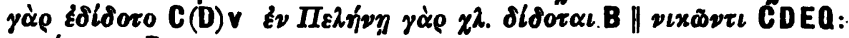
$\nu \iota x \dot{\gamma}^{-\alpha} \alpha \nu \iota$ B 


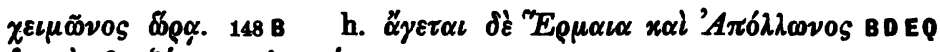

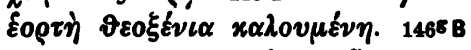

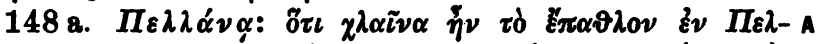

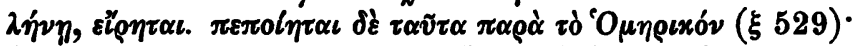

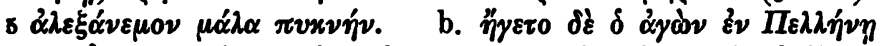

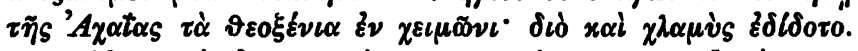

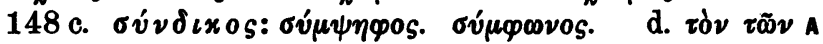

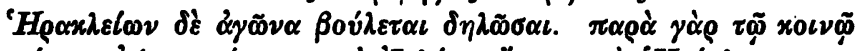

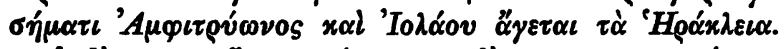

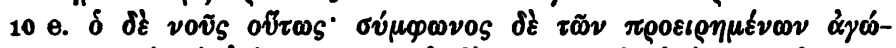

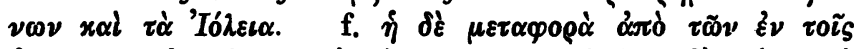

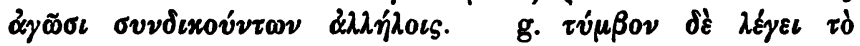

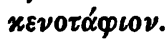

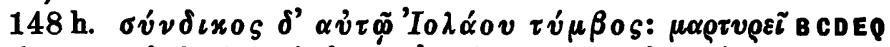

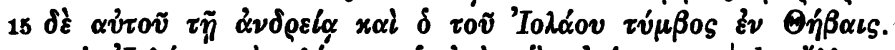

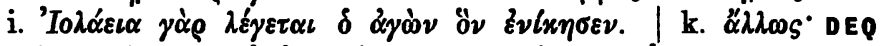

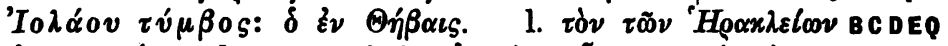

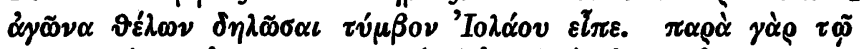

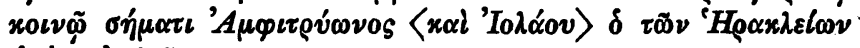

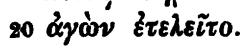

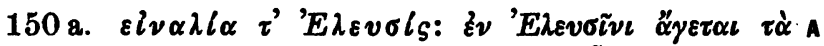

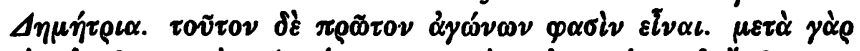

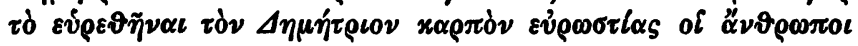

1 Paus. 7, 27,4 22-p. 302, 3 EM 743, 25: ì бँ

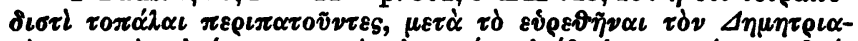

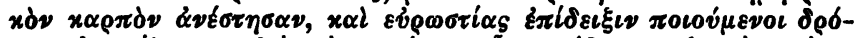

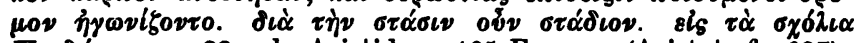

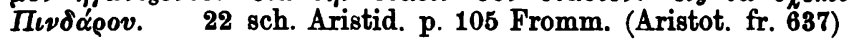

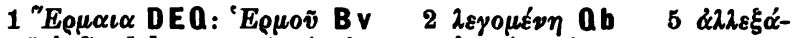

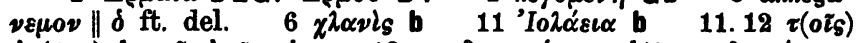

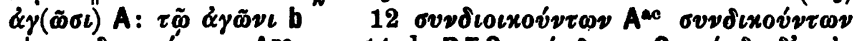

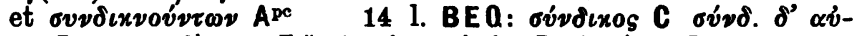

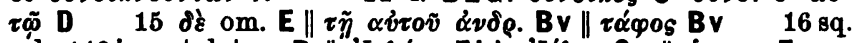

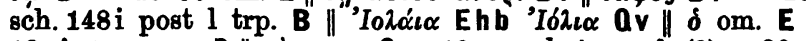

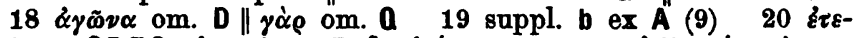

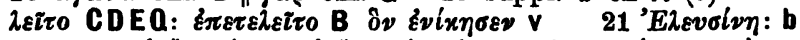

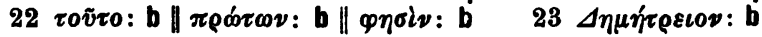




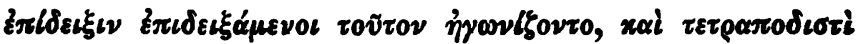

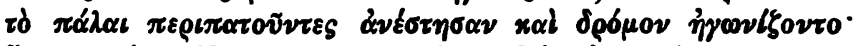

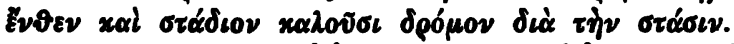

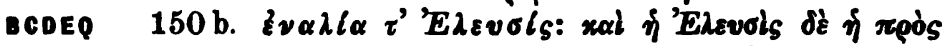

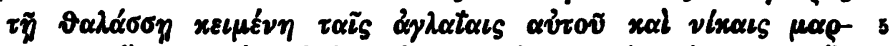

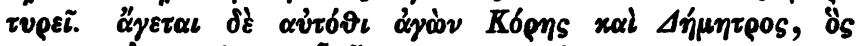

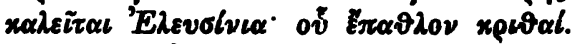

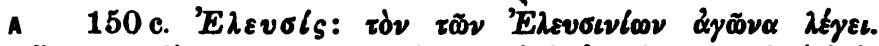

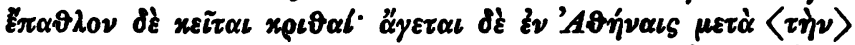

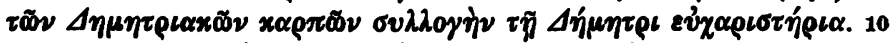

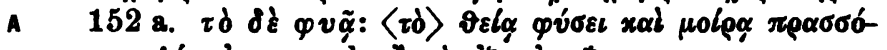

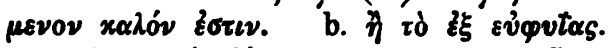

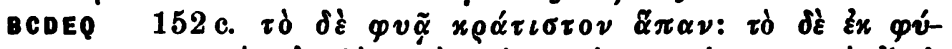

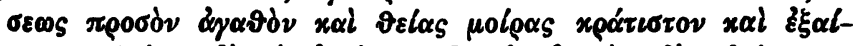

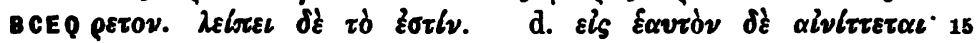

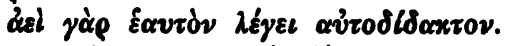

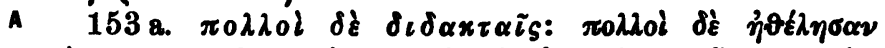

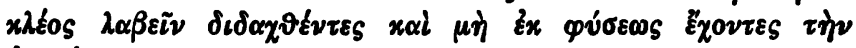

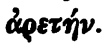

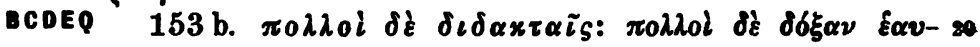

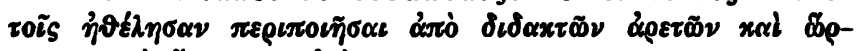

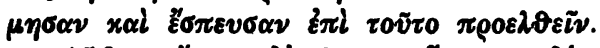

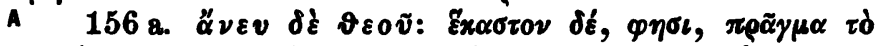

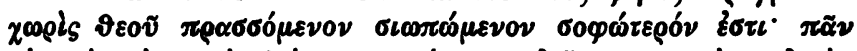

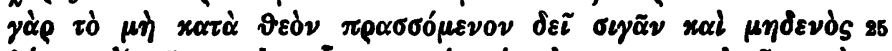

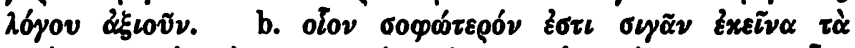

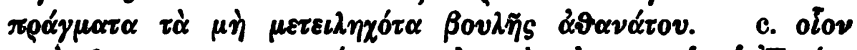

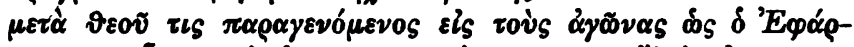

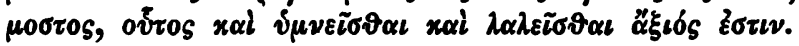

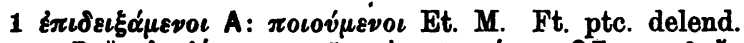

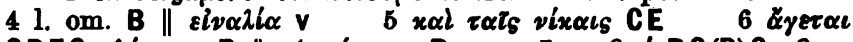

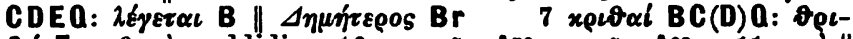

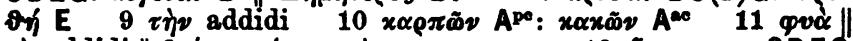

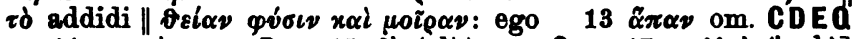
$14 \pi \rho \circ \sigma \partial \nu$ om. D $15 \delta \xi$ (alt.) om. C $17 \pi 0 \lambda \lambda_{0 l}$ (in 1.)] $\pi 0 \lambda \downarrow 201$. om. B $\| \varphi \eta \sigma l$ post $\delta \xi$ add. BV $21 \pi \varepsilon \rho \iota \pi 0 \iota r j \sigma \alpha \sigma \theta \alpha \iota$

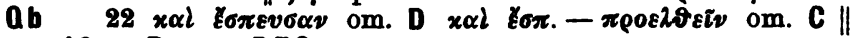

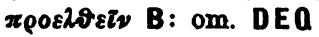




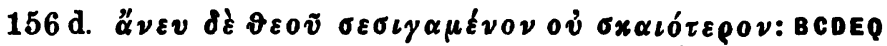

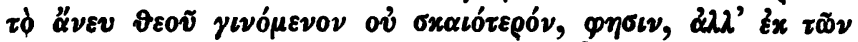

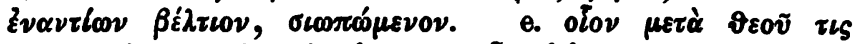

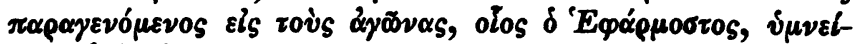

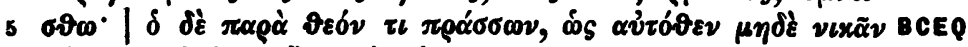

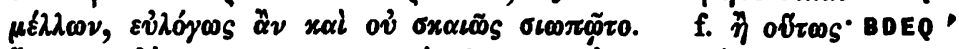

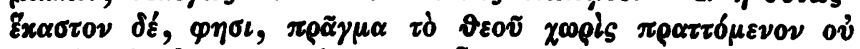

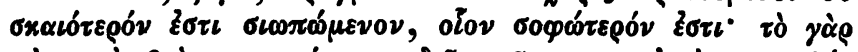

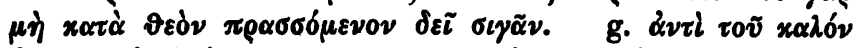

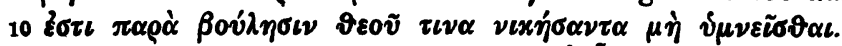

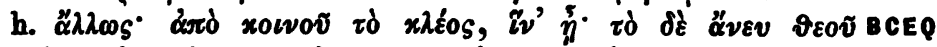

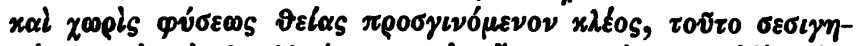

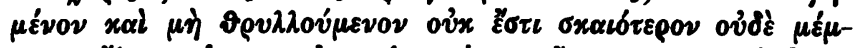

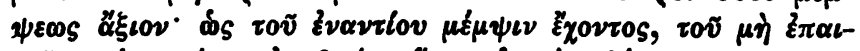

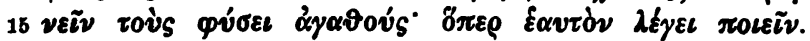

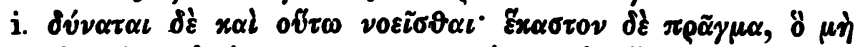

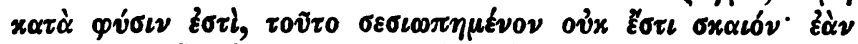

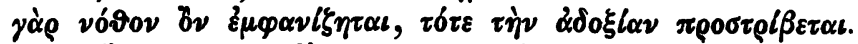

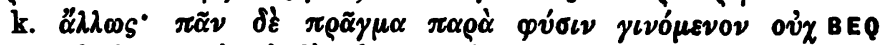

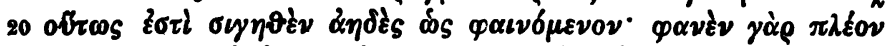

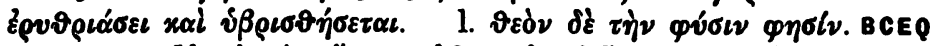

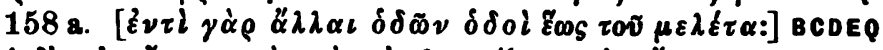

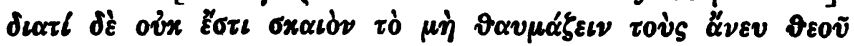

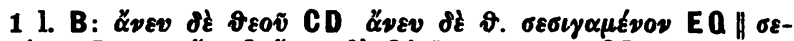

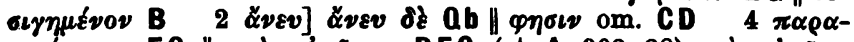

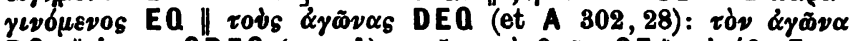

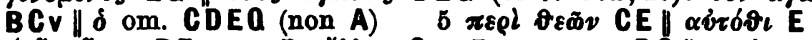

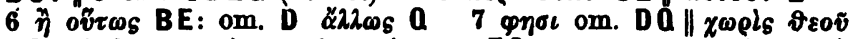
DQ (et A 302, 24) 8 o )

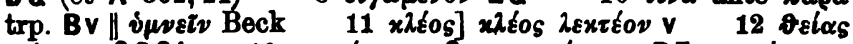

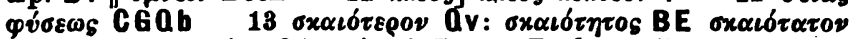

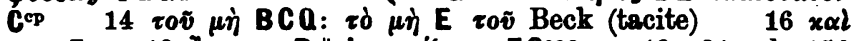

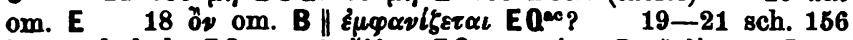

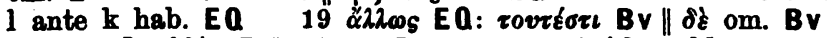

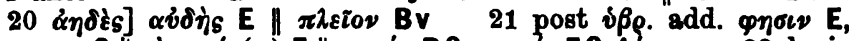

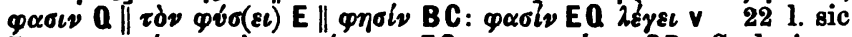

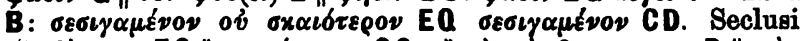

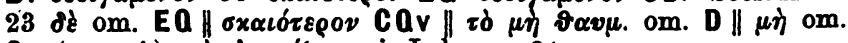

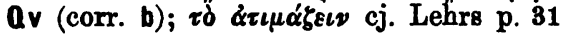




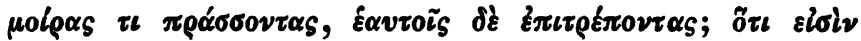

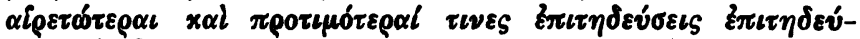

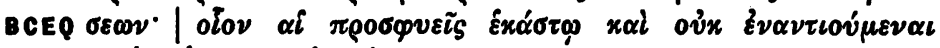

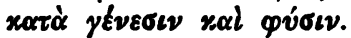

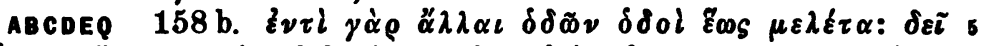

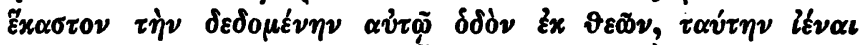

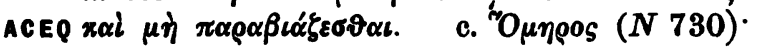

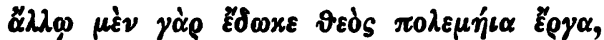

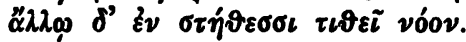

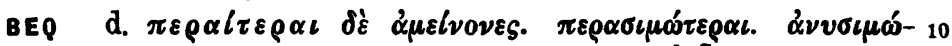

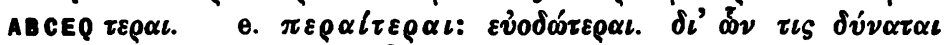

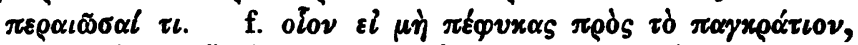

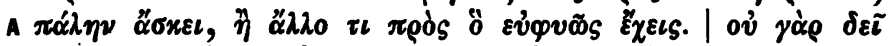

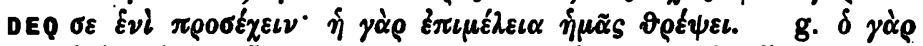

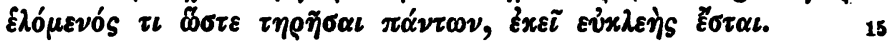

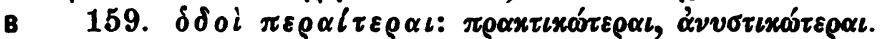

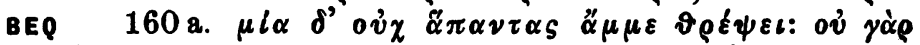

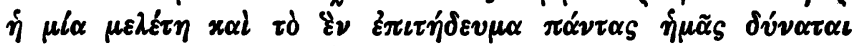

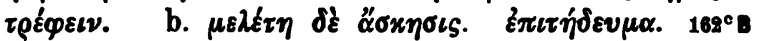

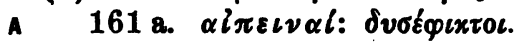

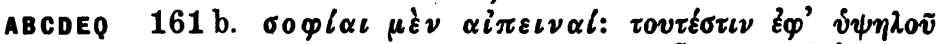

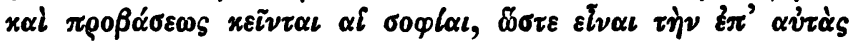

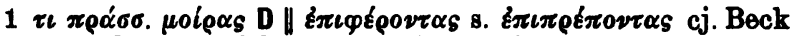

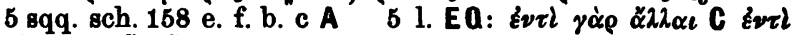

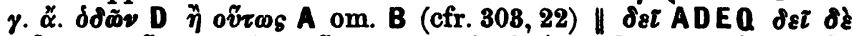

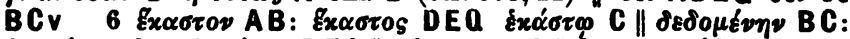

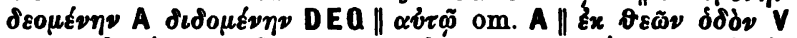

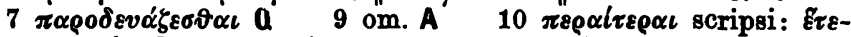

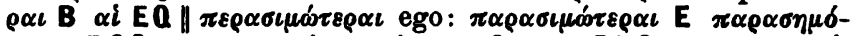

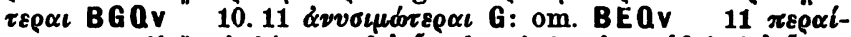

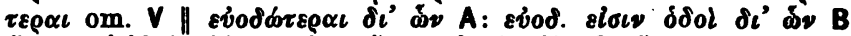

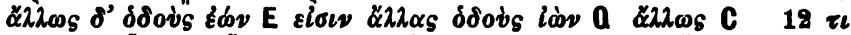

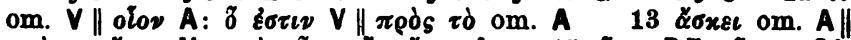

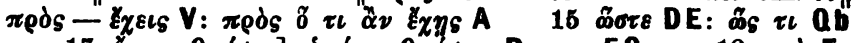

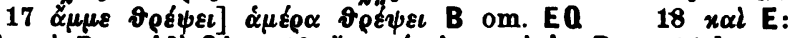

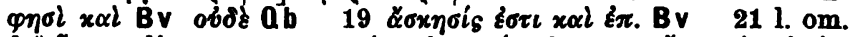

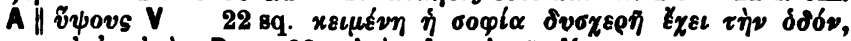

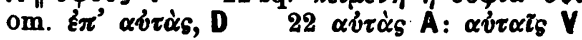




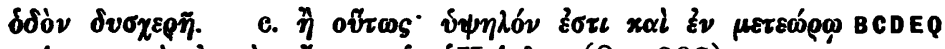

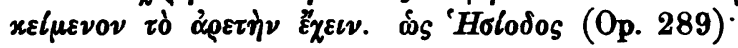

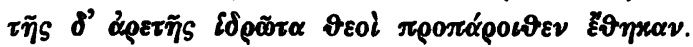

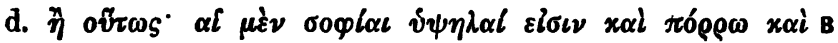
$5 \delta v \sigma \chi \varepsilon \rho \varepsilon \tilde{c}$. $168^{d} \mathrm{~B}$

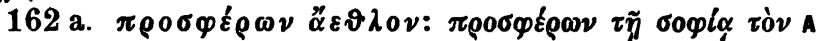

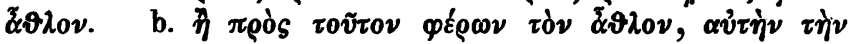

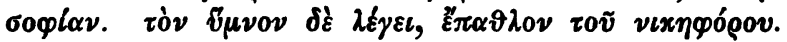

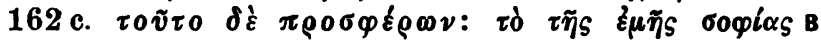
$10 \varepsilon \pi \tau t \tau \dot{\eta} \delta \varepsilon v \mu \alpha$. $161 \mathrm{~B}$

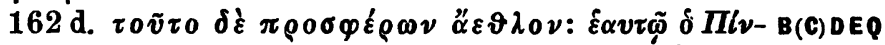

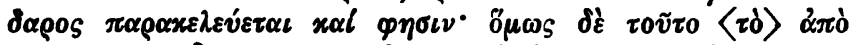

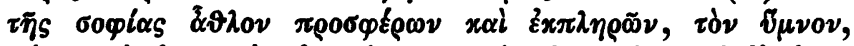

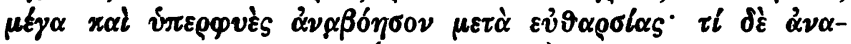

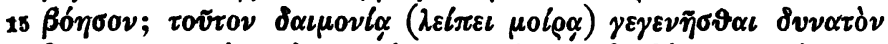

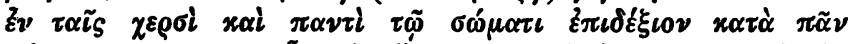

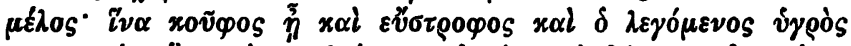

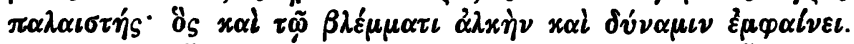

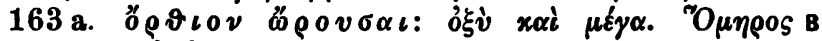

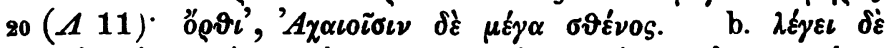

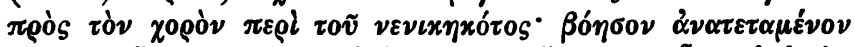

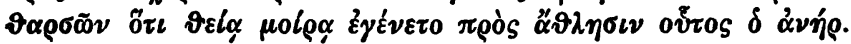

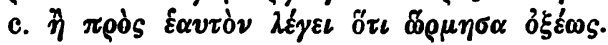

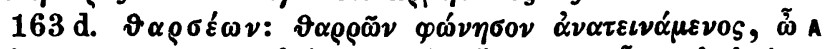

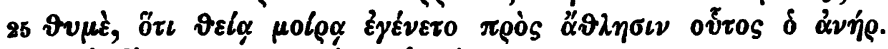

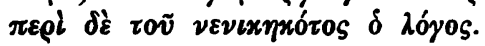

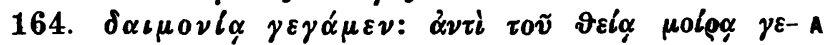

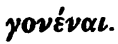

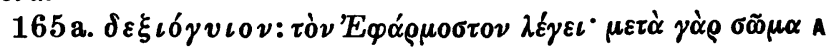

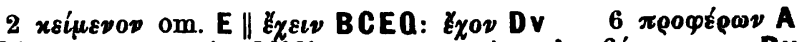

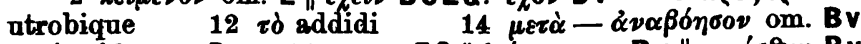

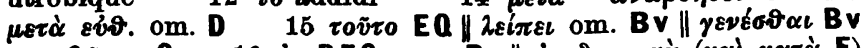

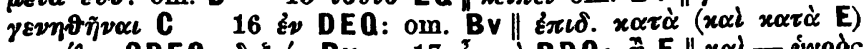

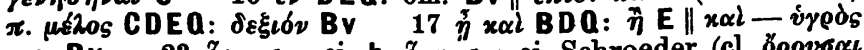

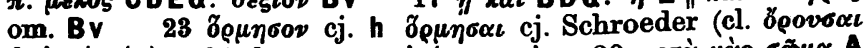

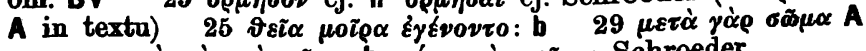

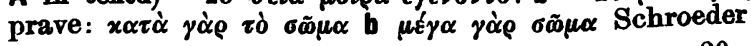

Bghou. m Prodarur ed. Drachmann. 


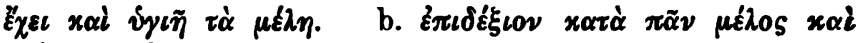

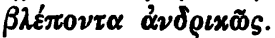

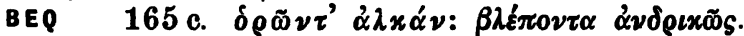

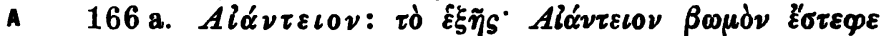

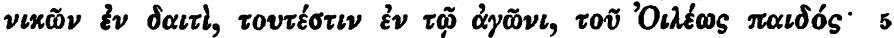

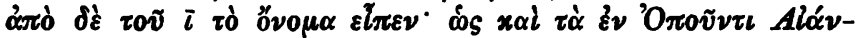

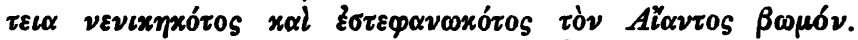

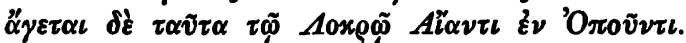

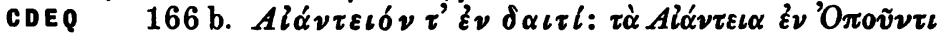

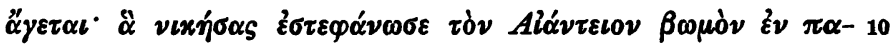

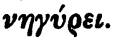

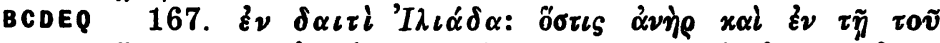

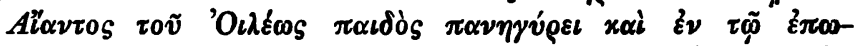

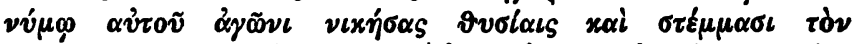

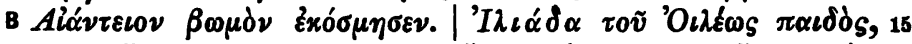

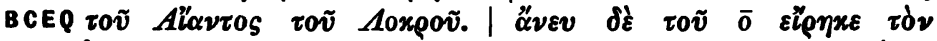

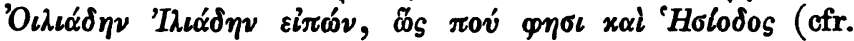
fr. 142).

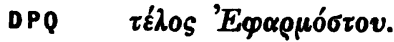

\section{Scholia in Olympionicarum carmen $\mathbf{X}$.}

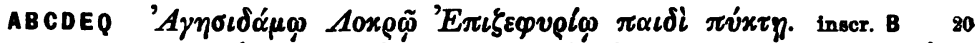

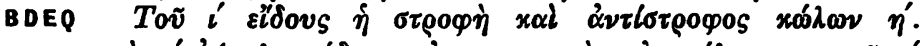

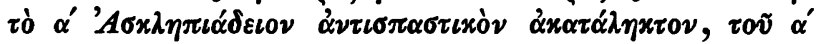

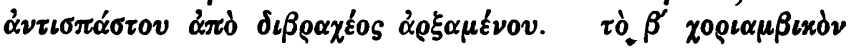

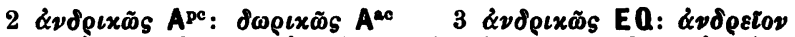

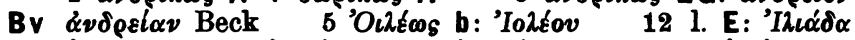

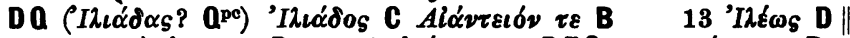

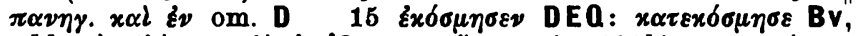

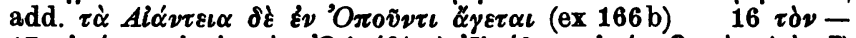

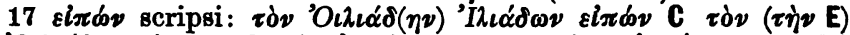

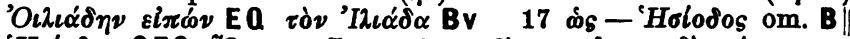

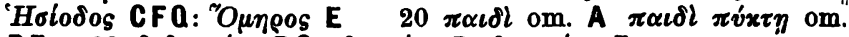

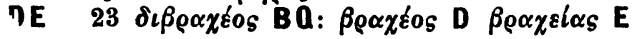




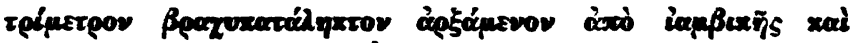

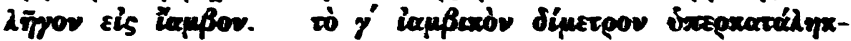

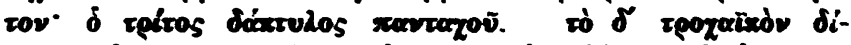

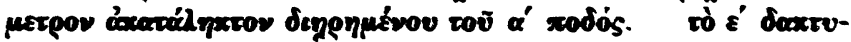

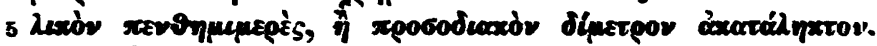

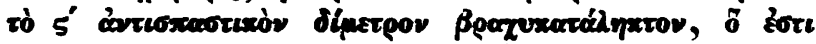

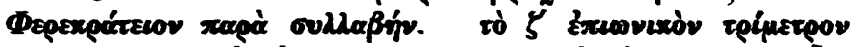

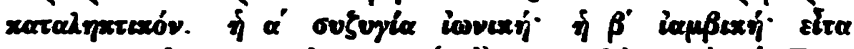

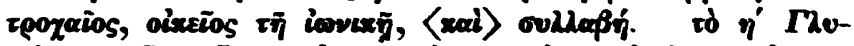

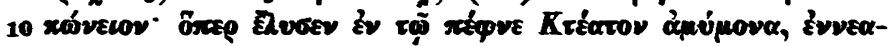
ớllaßiov.

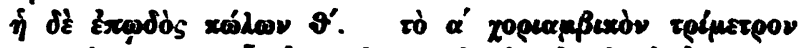

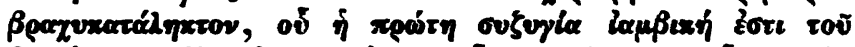

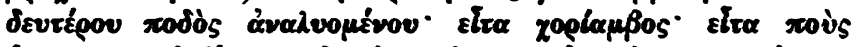

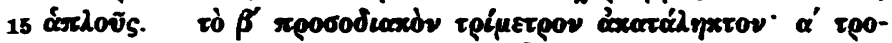

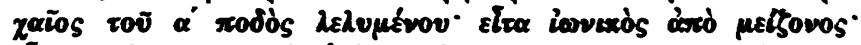

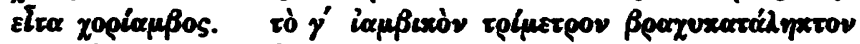

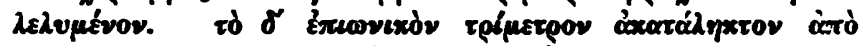

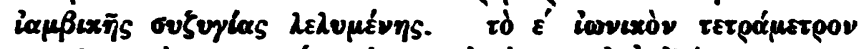

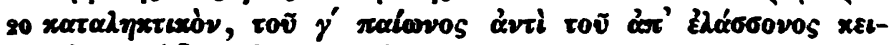

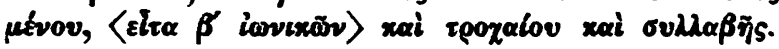

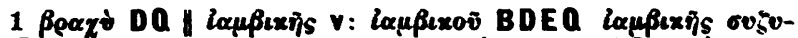

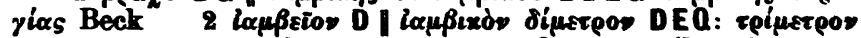

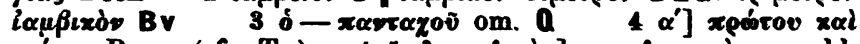

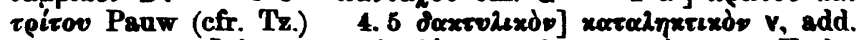

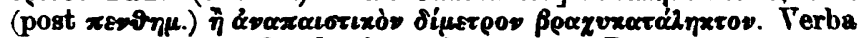

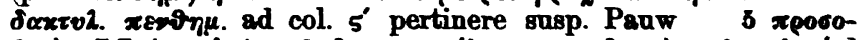

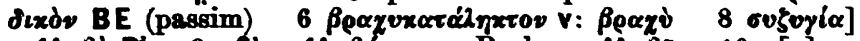

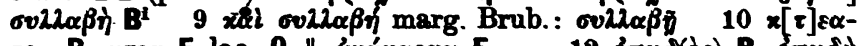

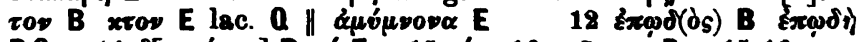

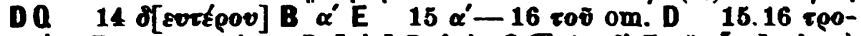

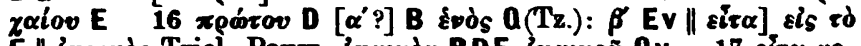

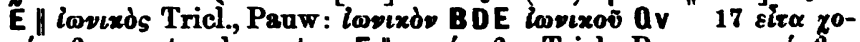

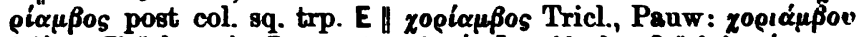

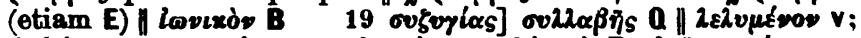

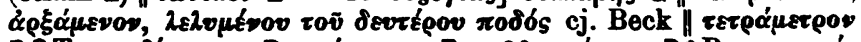

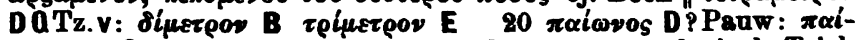
ovos BEQv \&uri roṽ B: ¿vil DEQ 21 supplevi cl. Tricl.

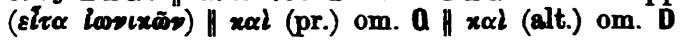




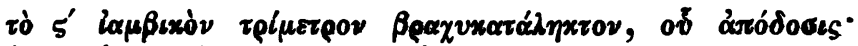

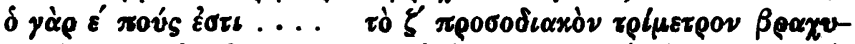

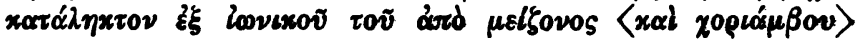

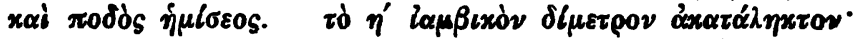

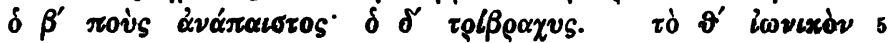

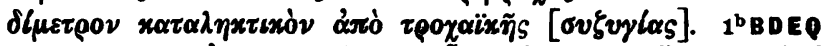

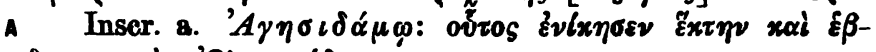

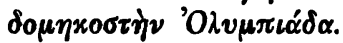

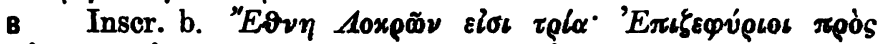

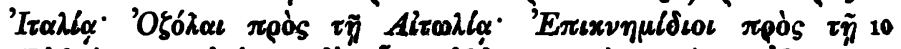

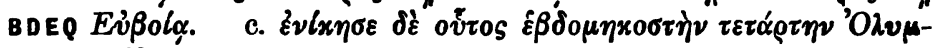
$\pi \iota \alpha \dot{\delta} \alpha$. metr. B $18 D E Q$

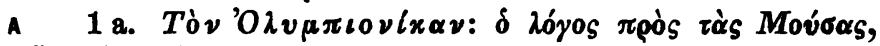

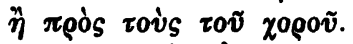

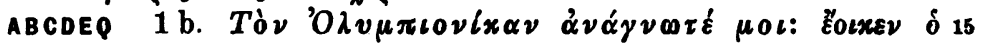

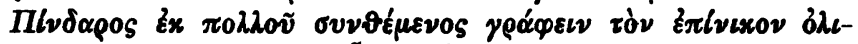

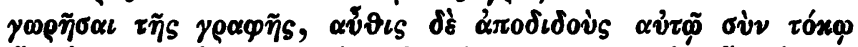

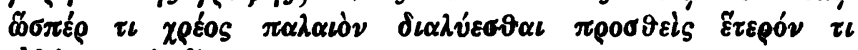

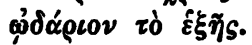

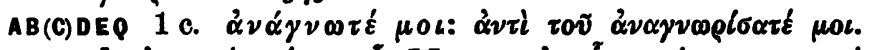

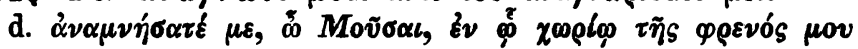

9 sch. A B 527

1 ov $-2 \varepsilon \sigma \tau \iota .$. B (7-9 litt. evan., supremae duae ft. $\tau \iota$;

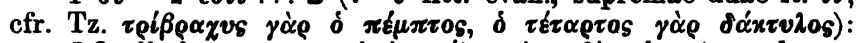

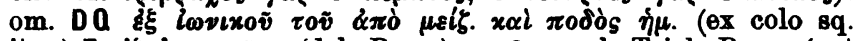

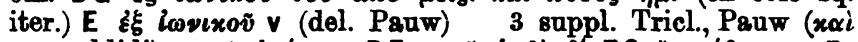

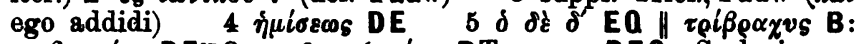

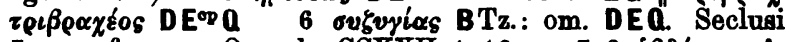
7 sqq. cfr. pap. Oxyrrh. CCXXII, $1,167.8 \xi \beta \delta \delta \mu \eta v:$ b

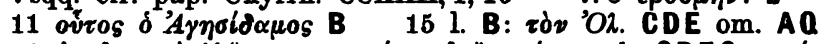

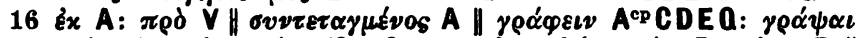

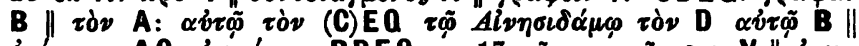

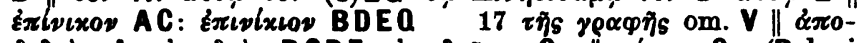

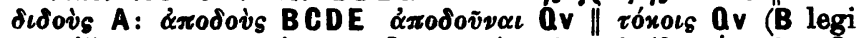

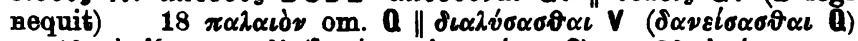

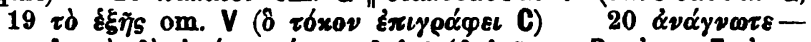

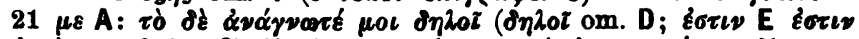

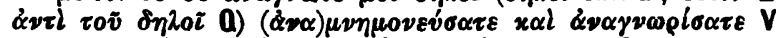

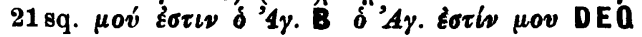




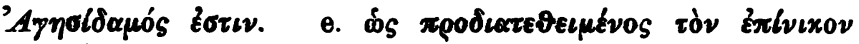

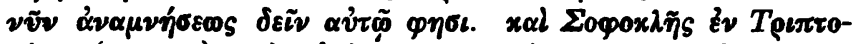

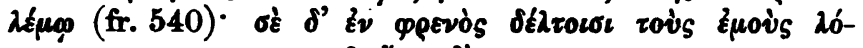

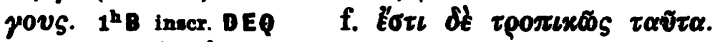

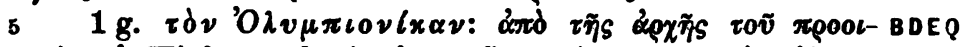

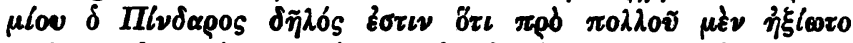

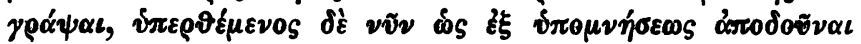

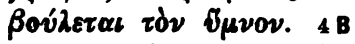

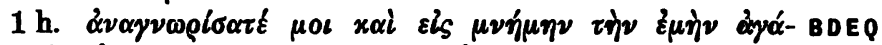

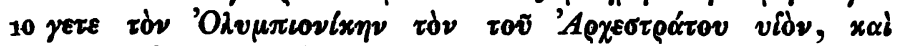

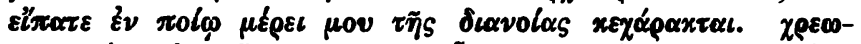

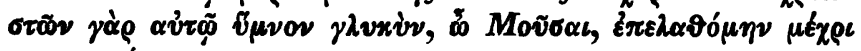

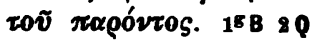

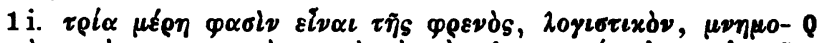

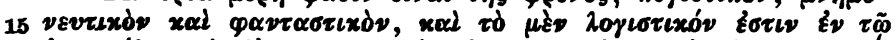

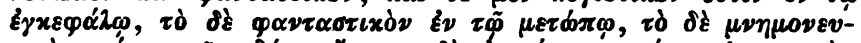

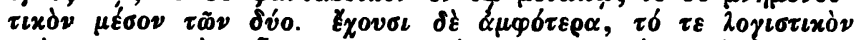

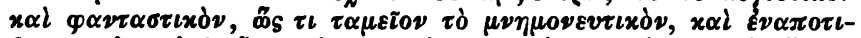

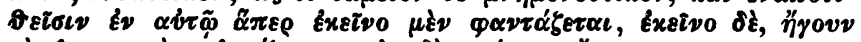

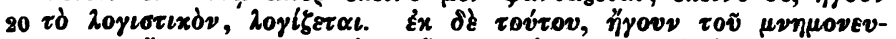

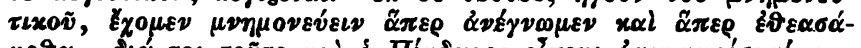

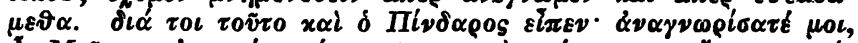

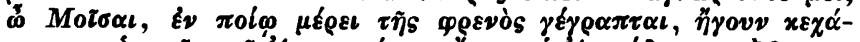

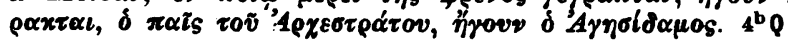

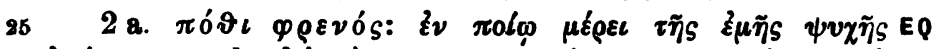

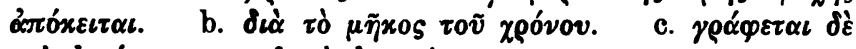

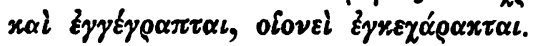

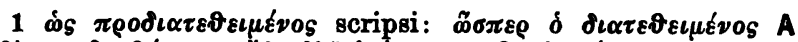

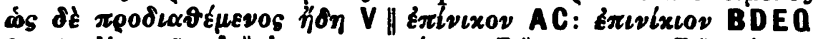

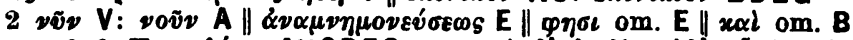

2. 3 TP d' Ev Scaliger $\|$ post $q \rho e v d s$ lac. D 5 l. B: om. D (add. yovv

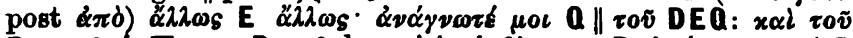

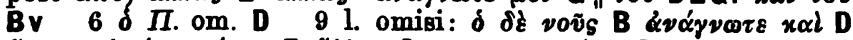

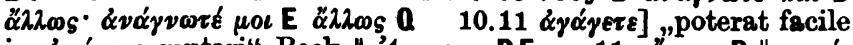

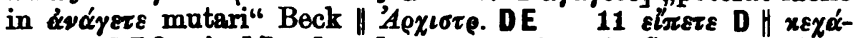

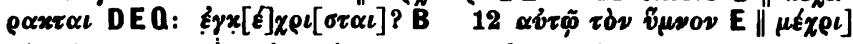

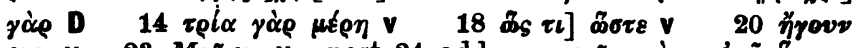

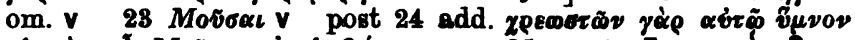

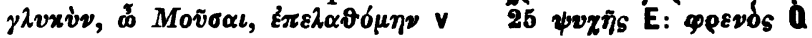




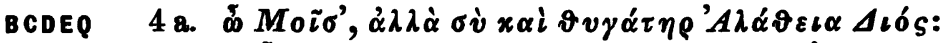

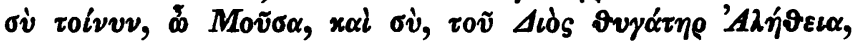

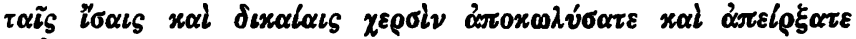

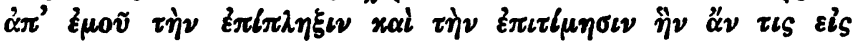

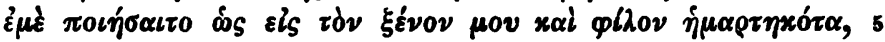

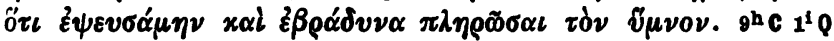

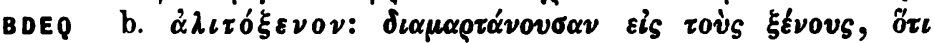

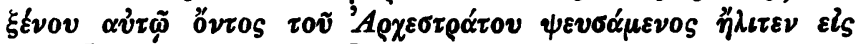

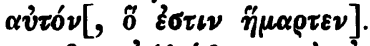

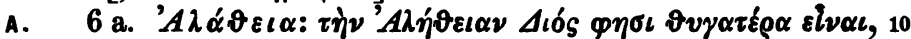

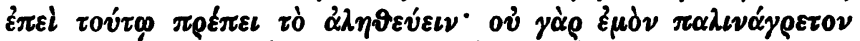

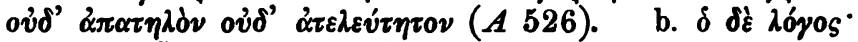

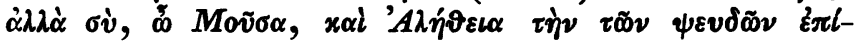

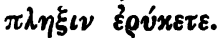

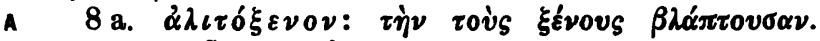

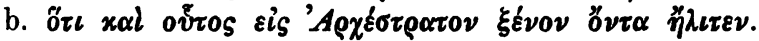

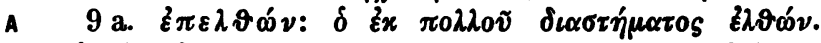

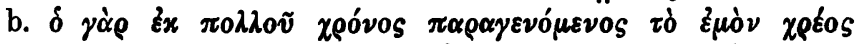

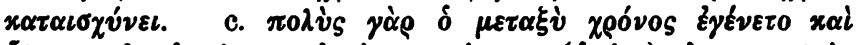

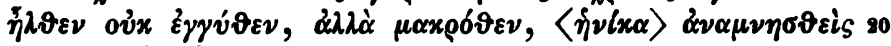

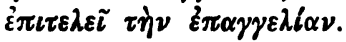

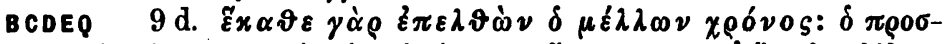

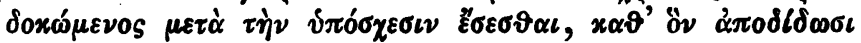

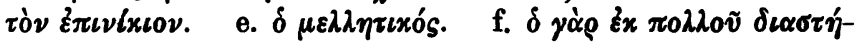

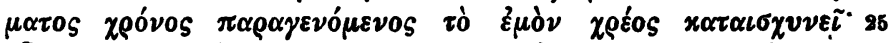

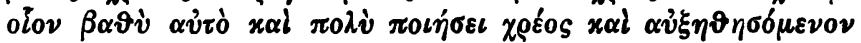

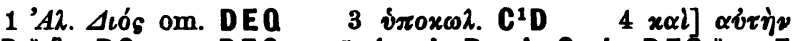
$x \alpha \ell$ D $\| \hat{\nu} \nu$ BC: om. DEQ 5 क́s $\varepsilon i_{S}$ B: $\varepsilon l_{S}$ C $\dot{\omega}$ DEQ $\| \mu \varepsilon$ E

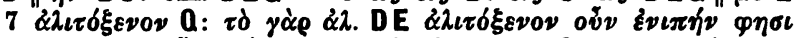

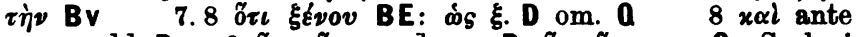

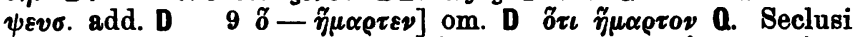

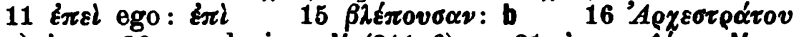

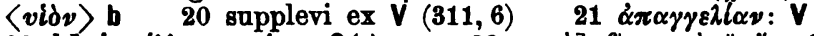

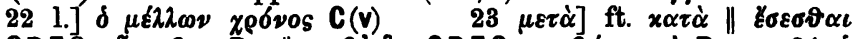

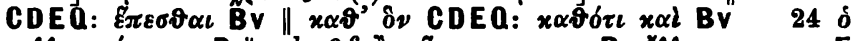

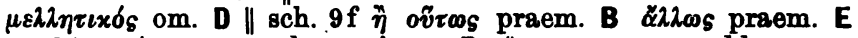

25 post $\pi \alpha \rho \alpha \gamma$. sch. $9 \mathrm{~g}$ inser. Bv $\| x \alpha \tau \alpha \iota \sigma \chi v i v \varepsilon l$ codd., $\mathrm{v}$

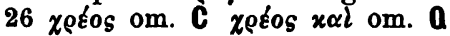




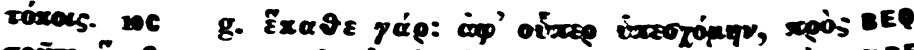

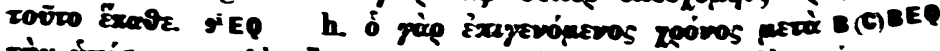

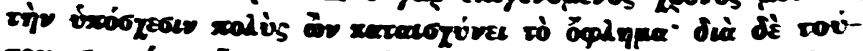

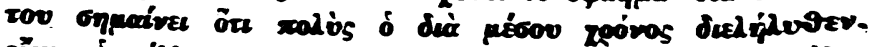

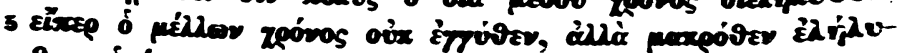

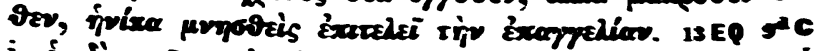

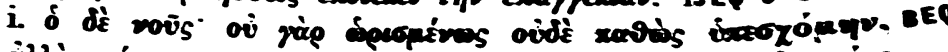

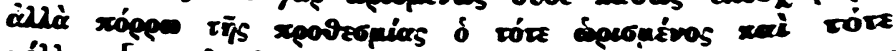

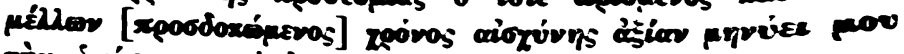

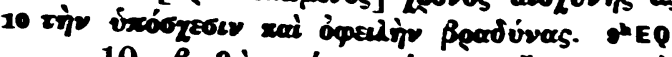

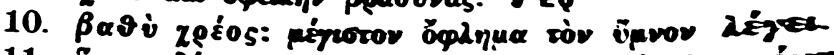

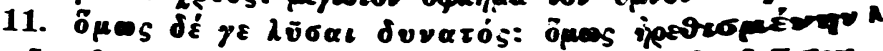

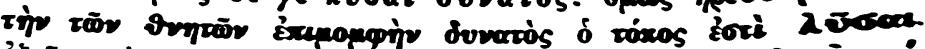

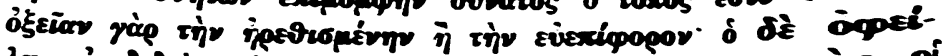

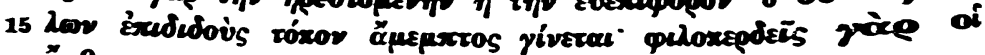
âr powios.

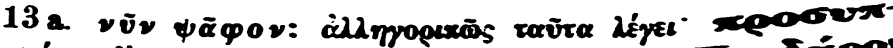

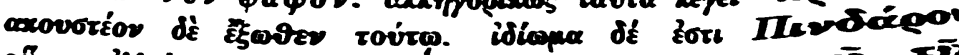

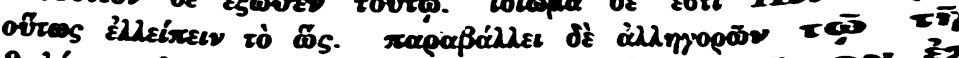

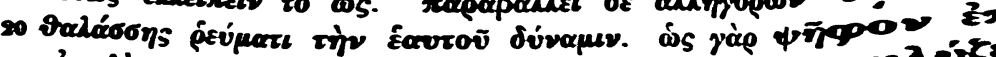

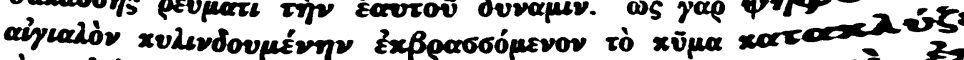

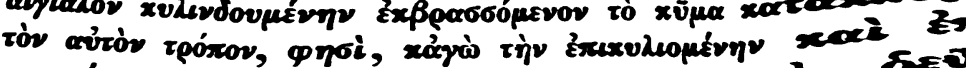

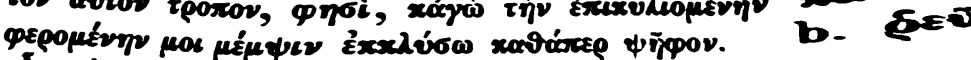

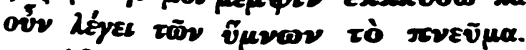

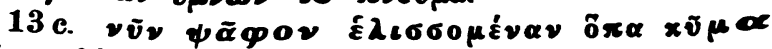

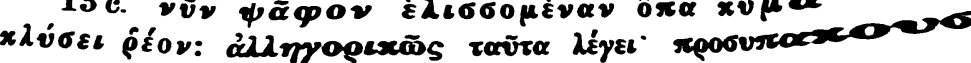

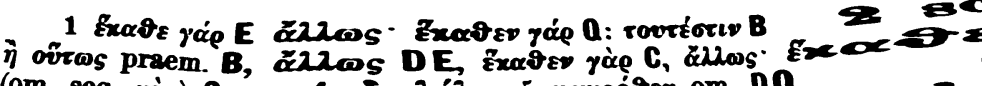

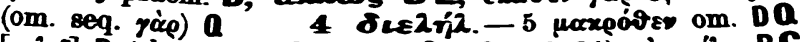
[relei] D (chart. perfor.; Cfr. A 310,21): exerther BCE

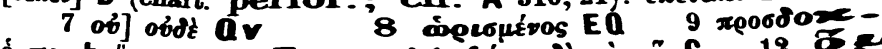
o xe. h $\|$ Hov om. E ego: ôxep A om. :

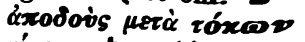

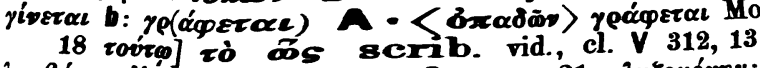

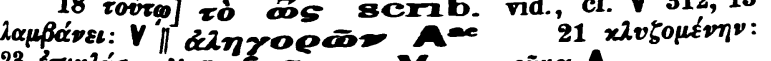

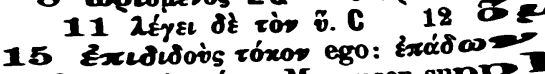

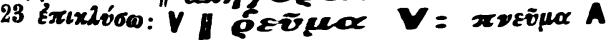




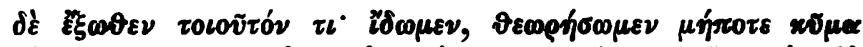

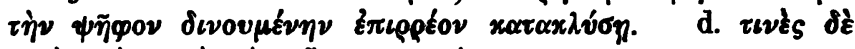

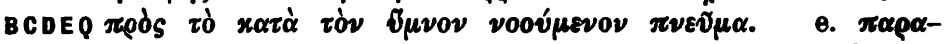

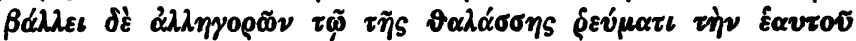

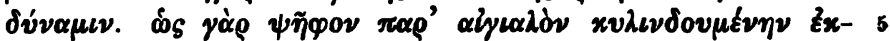

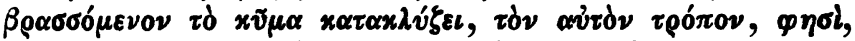

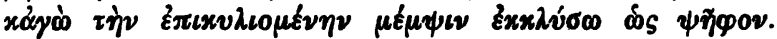

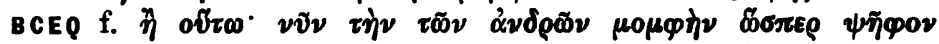

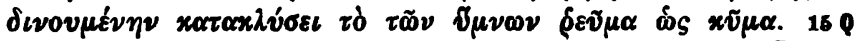

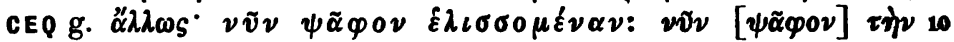

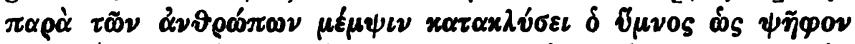

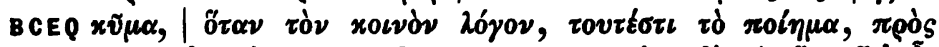

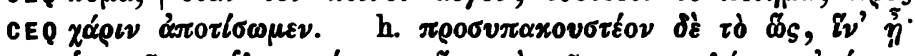

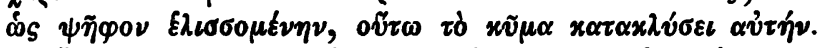

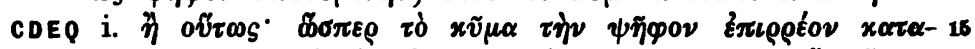

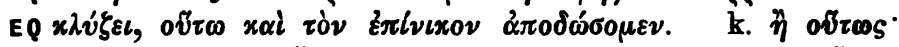

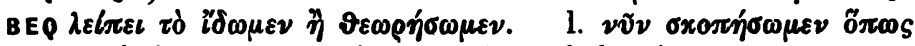

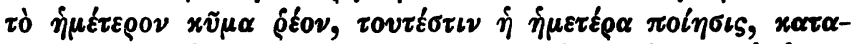

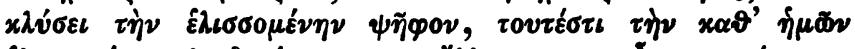

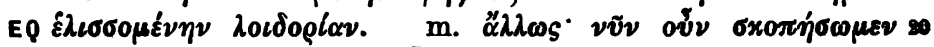

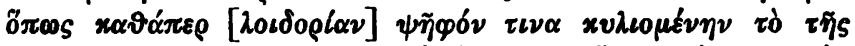

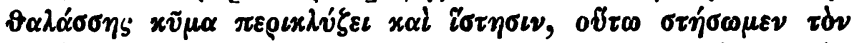

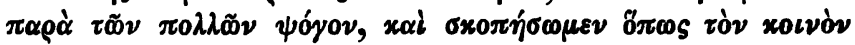

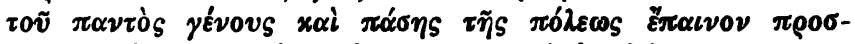

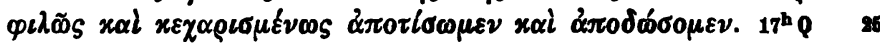

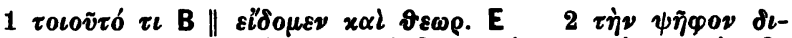

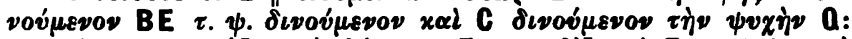

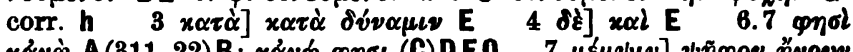

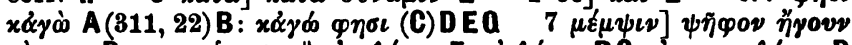

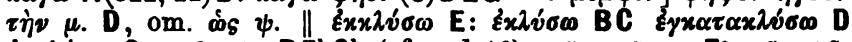

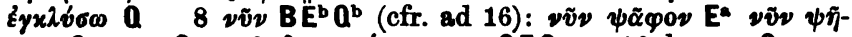

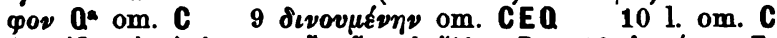

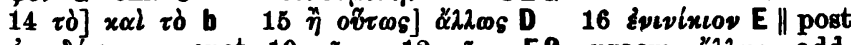

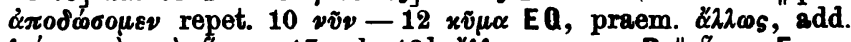

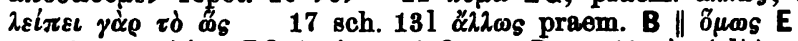

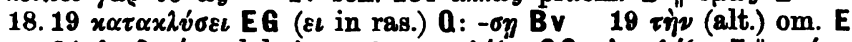

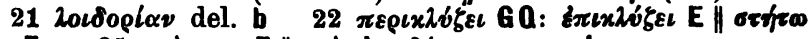

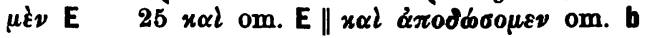




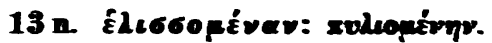

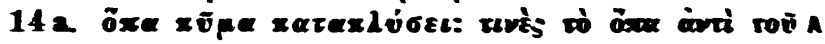

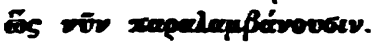

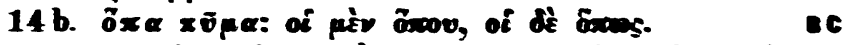

515 a zocvòv lógov: 'Apiorespos rounò lógrov wìn a

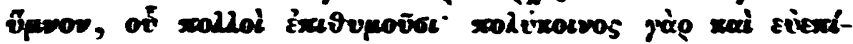

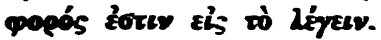

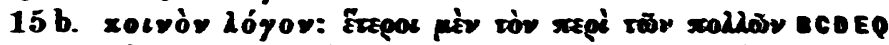

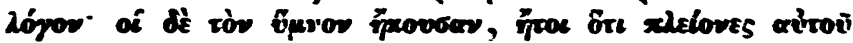

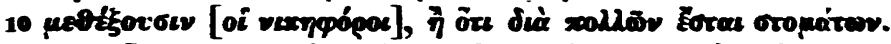

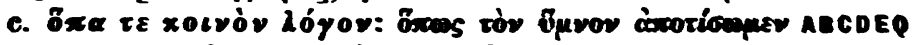

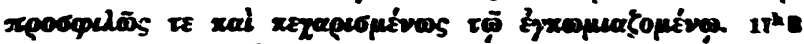

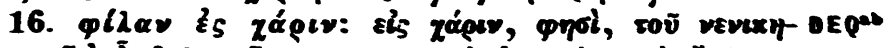

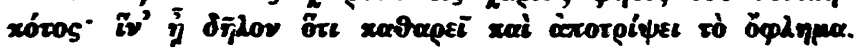

$1520 \varphi^{\circ} 19 \varphi^{b}$

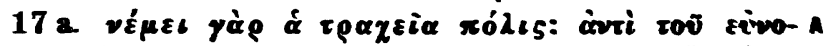

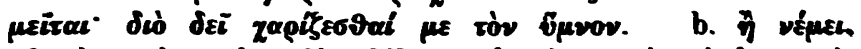

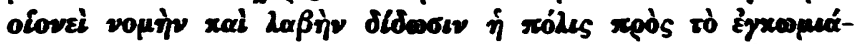

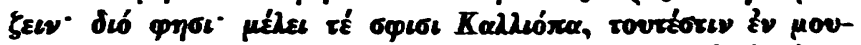

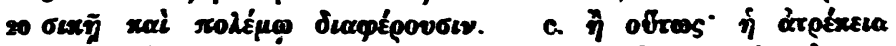

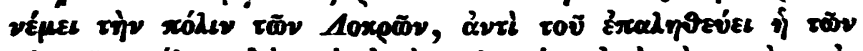

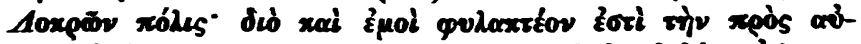

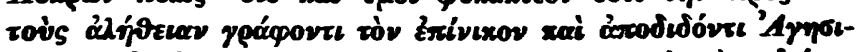

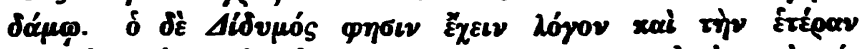

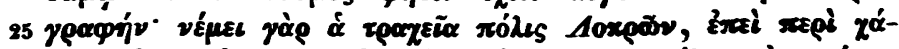

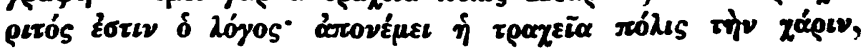

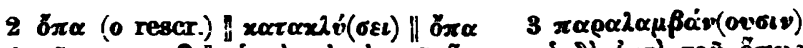

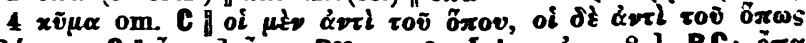

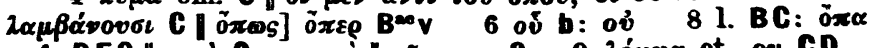

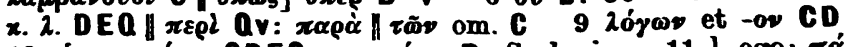

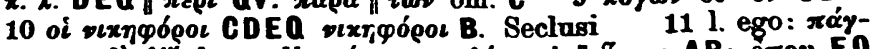

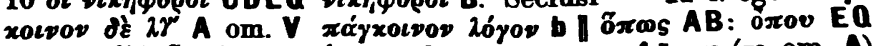

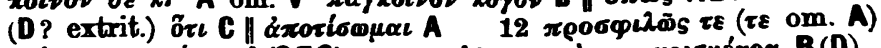

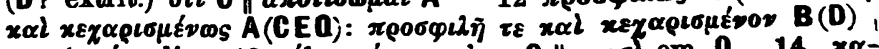

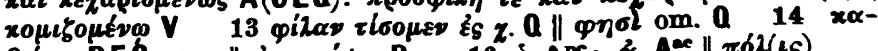

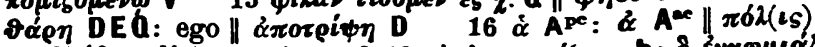

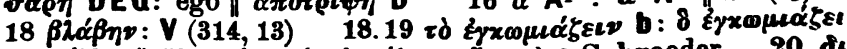

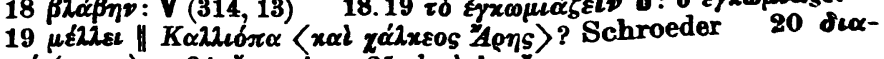

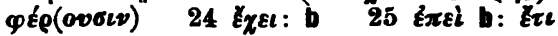




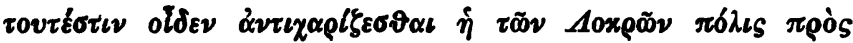

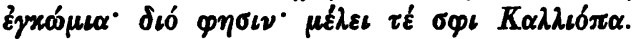

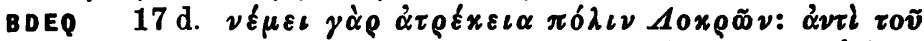

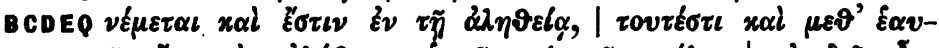

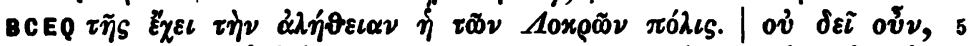

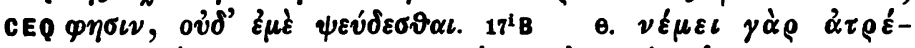

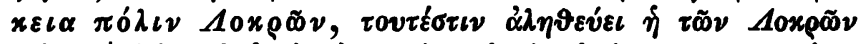

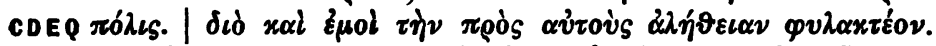
f. छ̇ $\pi \varepsilon i$ yà

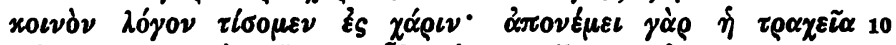

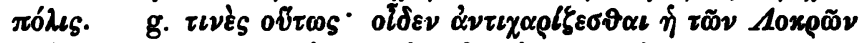

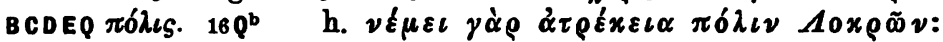

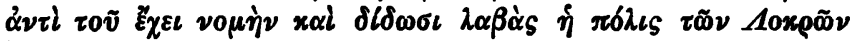

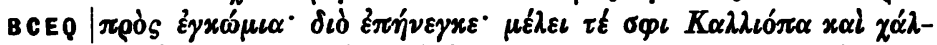

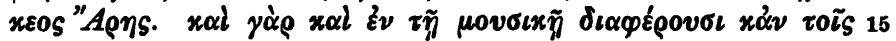

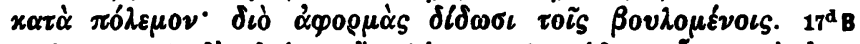

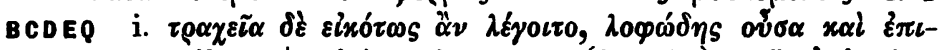

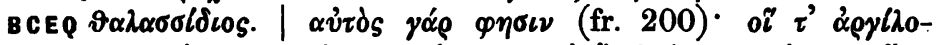

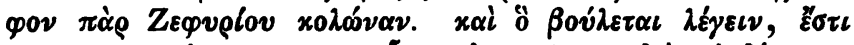

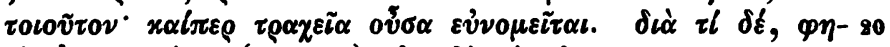

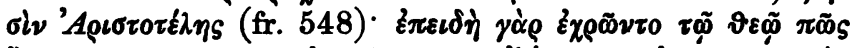

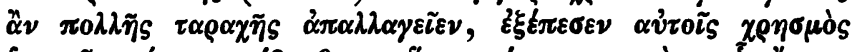

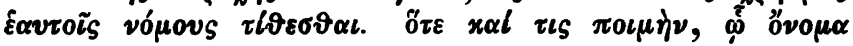

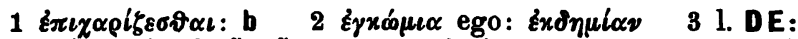

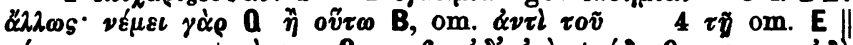
ข

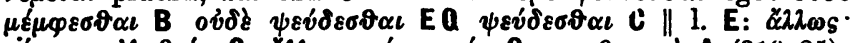

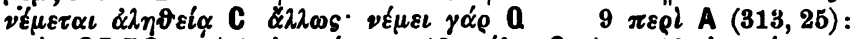

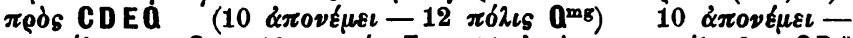

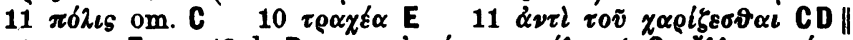

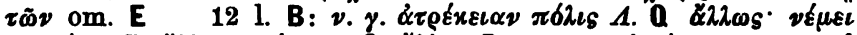

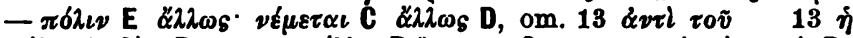

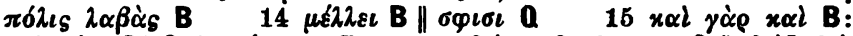

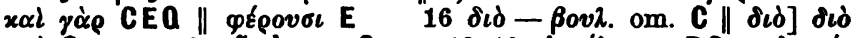

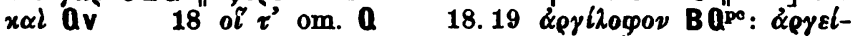

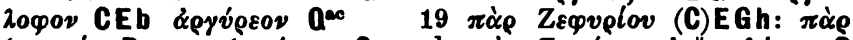

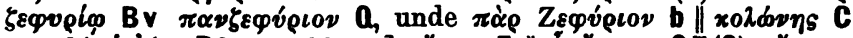

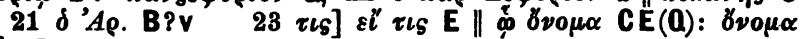
$\delta$ ' $\dot{\eta} \boldsymbol{v} \mathrm{BV}$ 


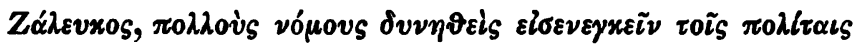

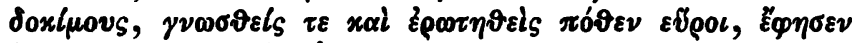

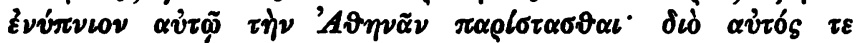

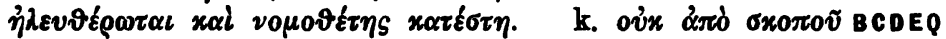

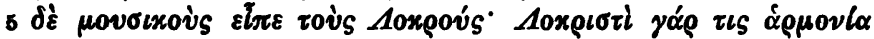

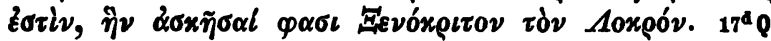

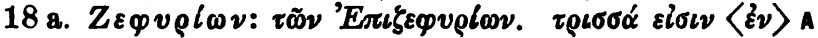

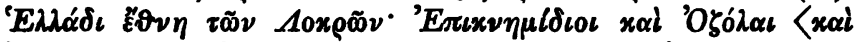

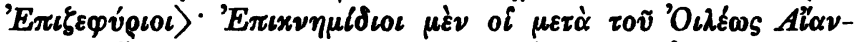

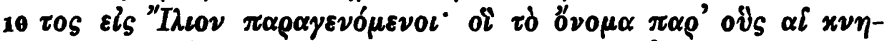

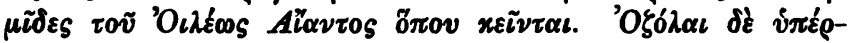

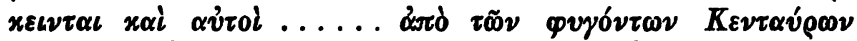

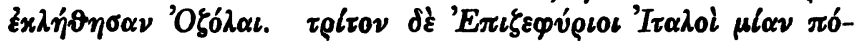

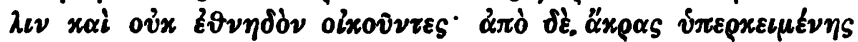

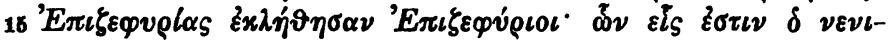
xๆxต́s.

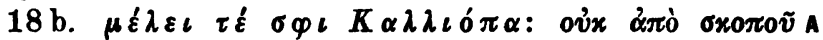

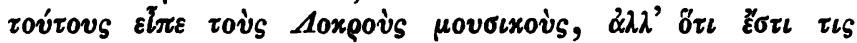

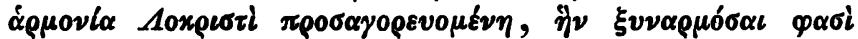

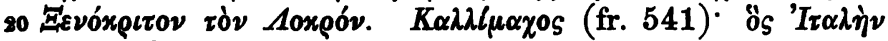

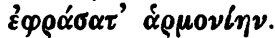

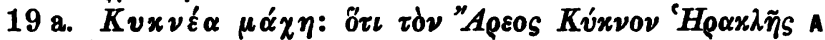

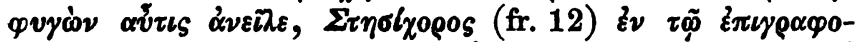

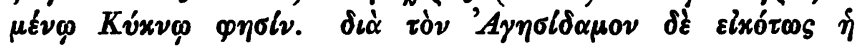

\section{7 sch. A B 527}

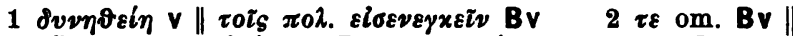

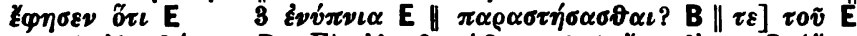

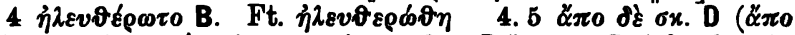

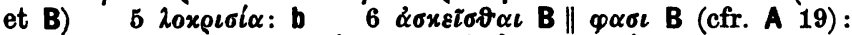

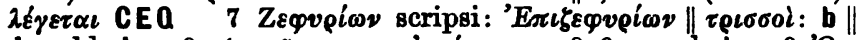

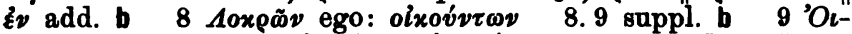

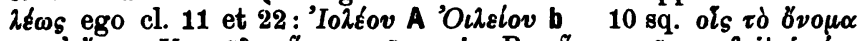

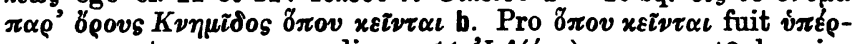
$x \varepsilon \iota \nu \tau \alpha \iota$; cetera non expedio 11 ' $I 0 \lambda\left(\varepsilon \omega_{\omega}\right)$ : ego 12 lac. indicavi H $\varphi v \gamma \delta \dot{v \tau \omega \nu}$ corruptum; an $\pi v \theta 0 \mu \varepsilon v \omega \nu$ ? cfr. Strab. IX 427

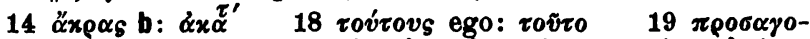

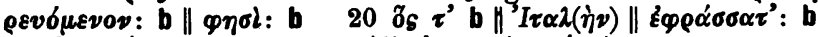

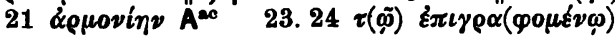




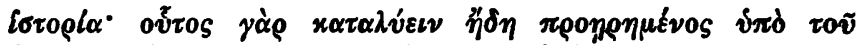

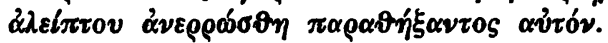

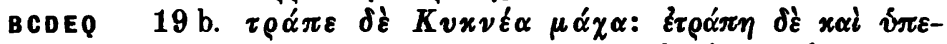

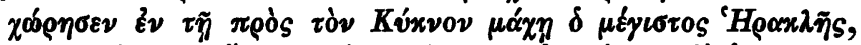

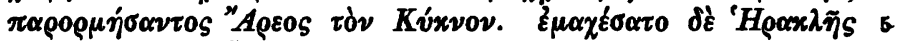

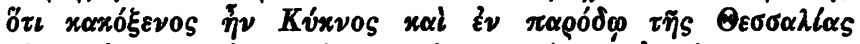

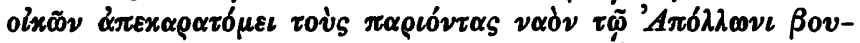

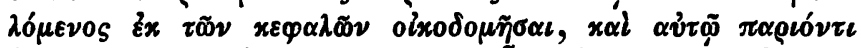

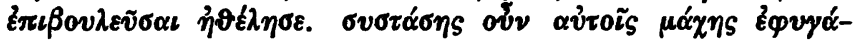

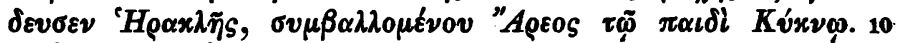

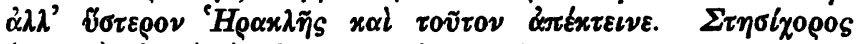

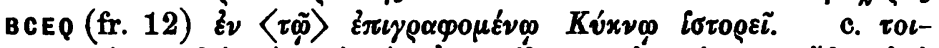

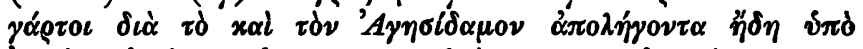

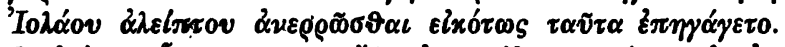

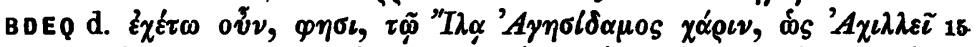

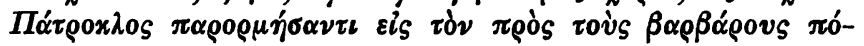
$\lambda \in \mu 0 \nu .210$

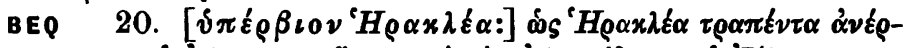

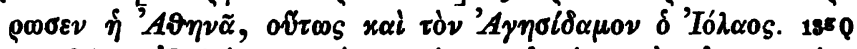

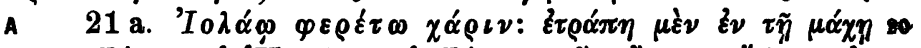

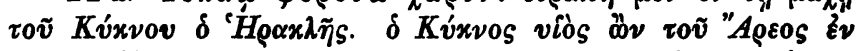

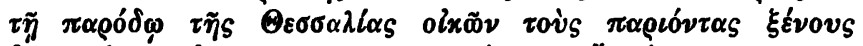

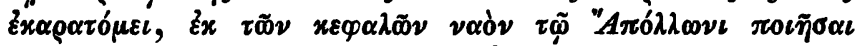

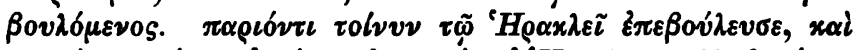

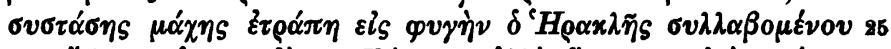

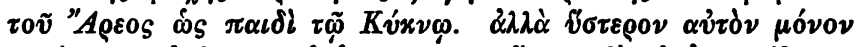

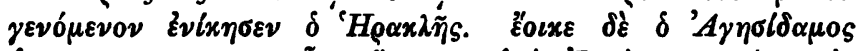

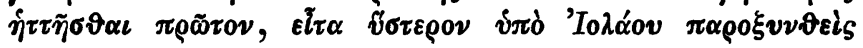

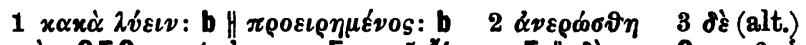

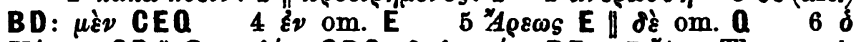

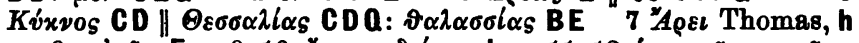

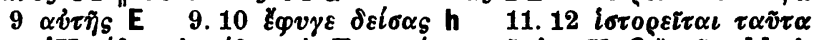

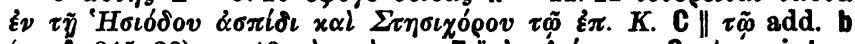

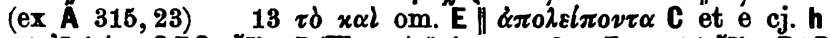

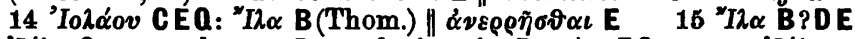

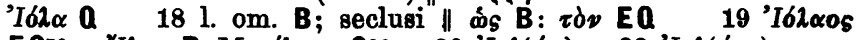

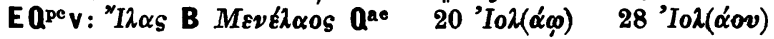




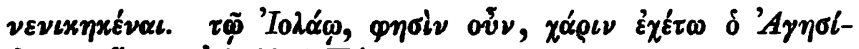

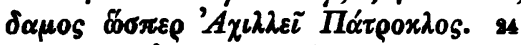

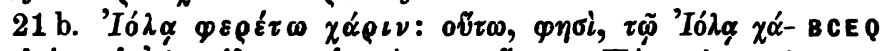

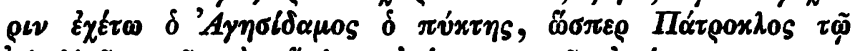

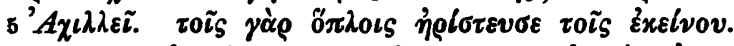

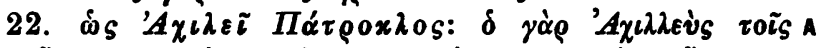

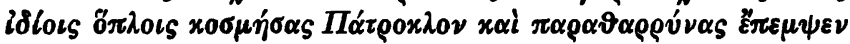

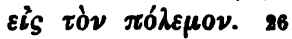

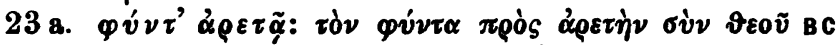

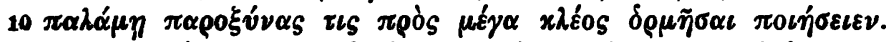

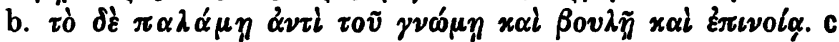

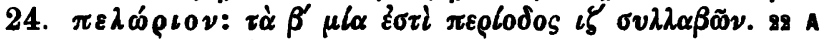

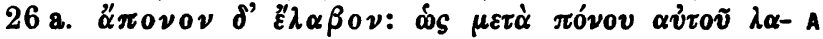

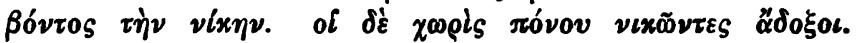

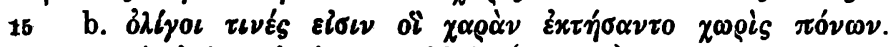

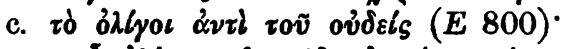

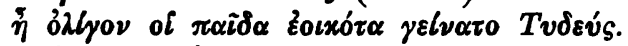

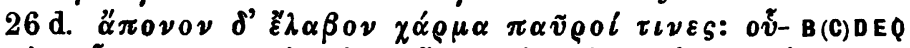

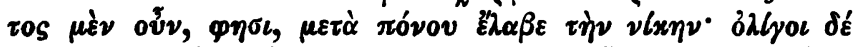

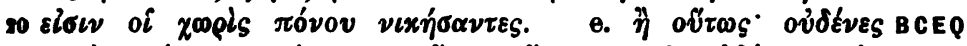

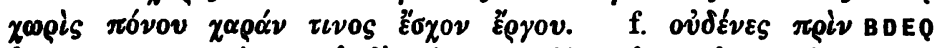

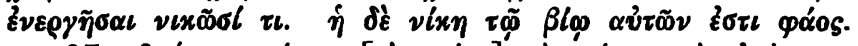

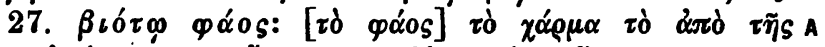

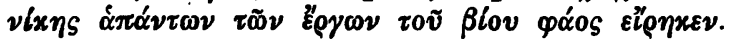

\section{6 sch. A E 800}

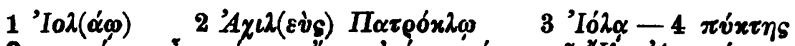
(C) EQ:

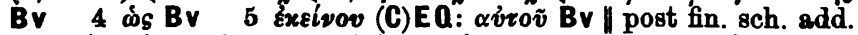

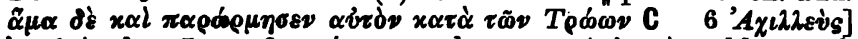

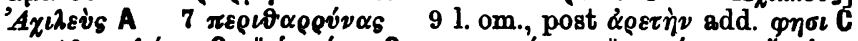

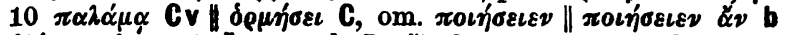
16 bilyov: b $17 \hat{\eta} 18 \mathrm{l}$. B: $\sum_{\alpha \alpha \beta o \nu}-\tau \iota \nu \varepsilon s$ om. C $\pi$. $\tau \iota \nu \varepsilon s$

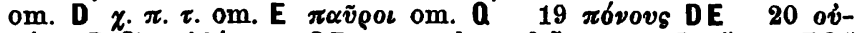

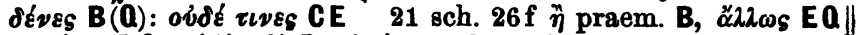

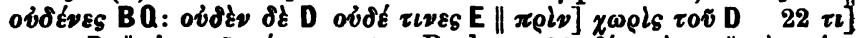

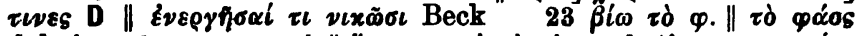

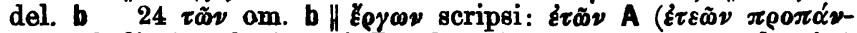

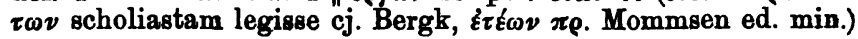




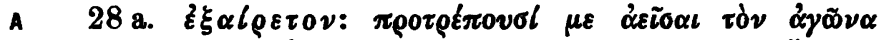

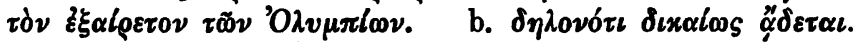

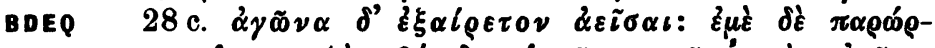

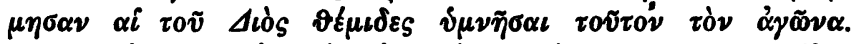

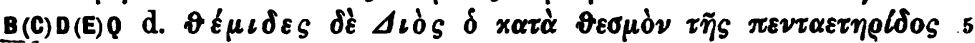

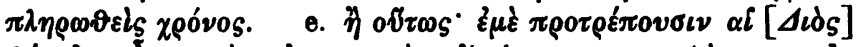

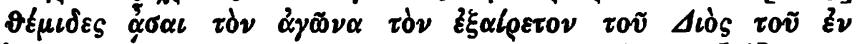

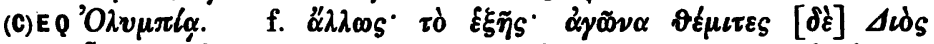

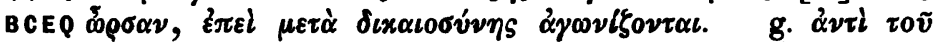

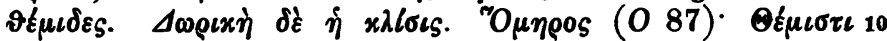
$\delta_{\varepsilon}^{\prime} x \alpha \lambda \lambda \iota \pi \alpha \varrho \eta^{\prime} \omega$.

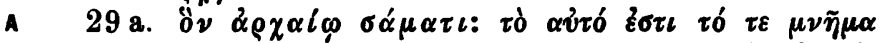

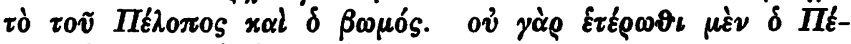

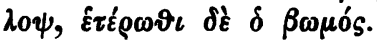

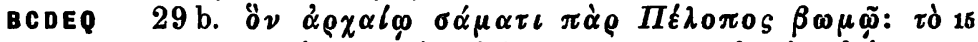

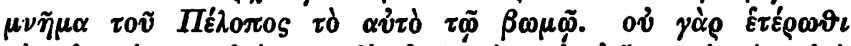

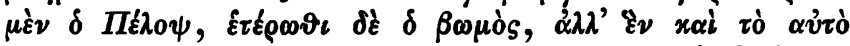

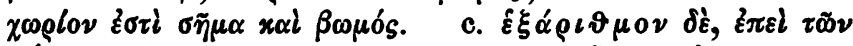

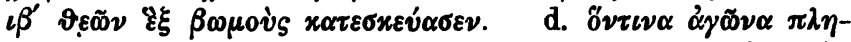

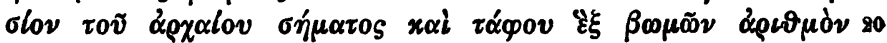

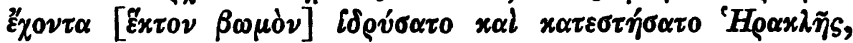

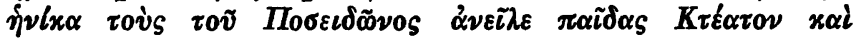

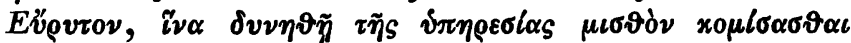

9 Eust. Il. 735, 52. Od. 1618, 29, al. EM 445, 19

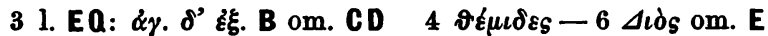

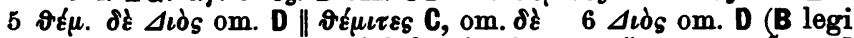

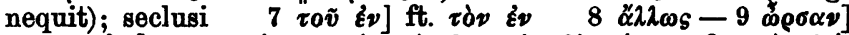

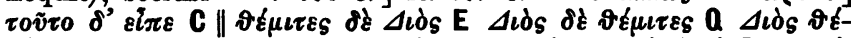

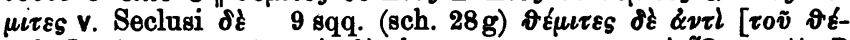

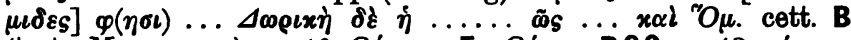

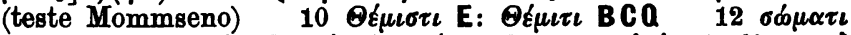

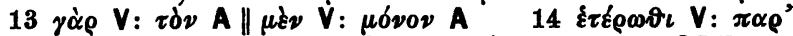

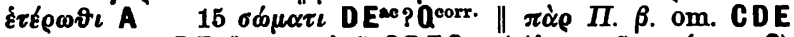

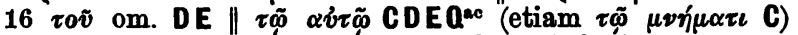

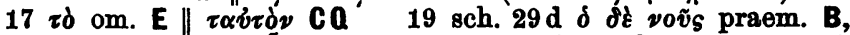

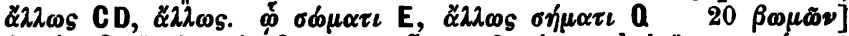

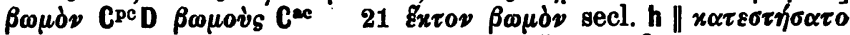

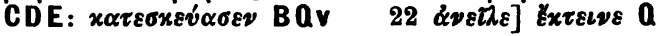




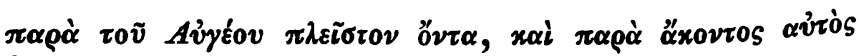

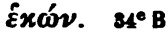

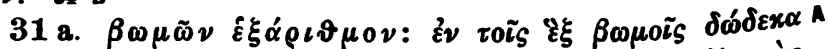

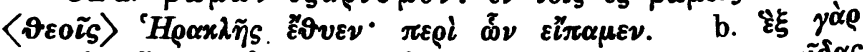

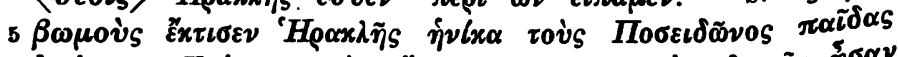

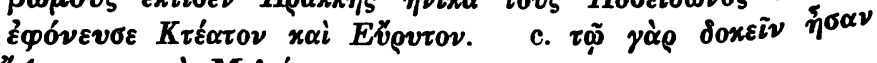

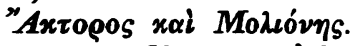

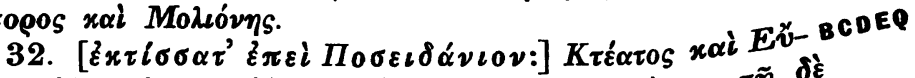

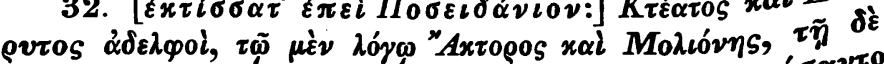

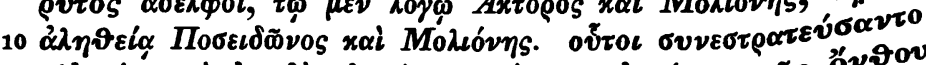

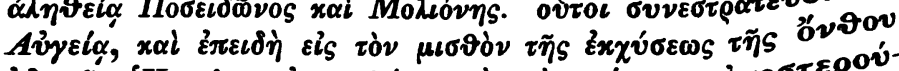

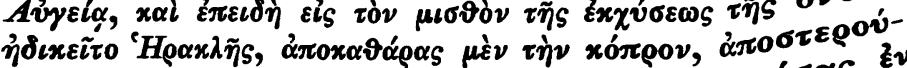

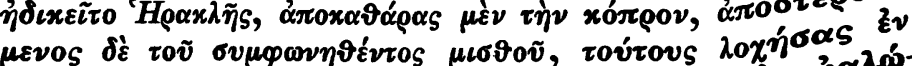

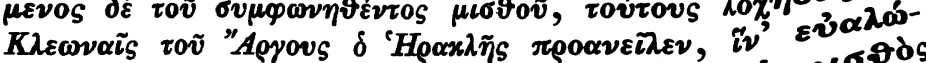

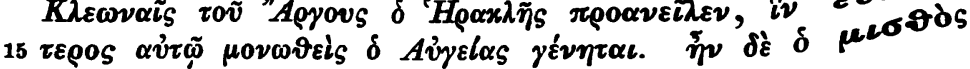

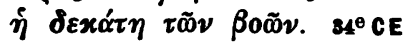

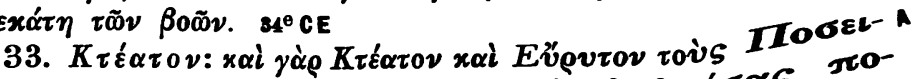

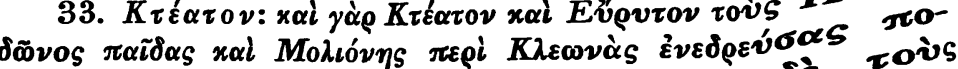

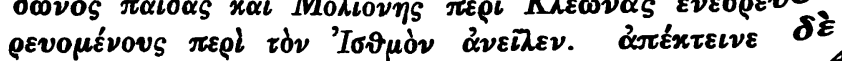

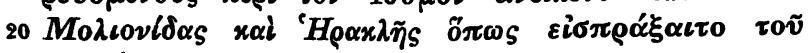

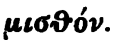

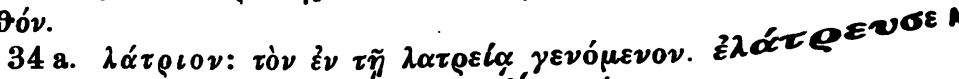

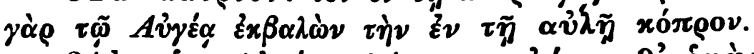

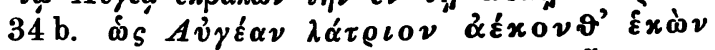

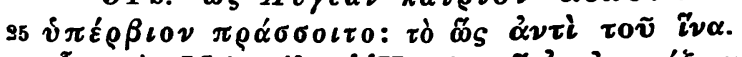

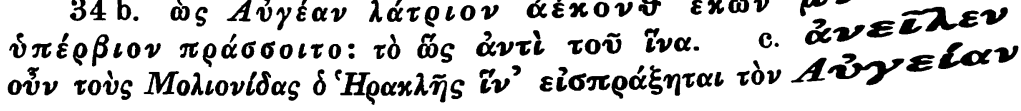

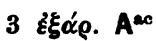
$x \lambda(\tilde{\eta} s)$ $6 \tau \tilde{\omega} A^{*}: \tau \dot{\delta} A^{1}$

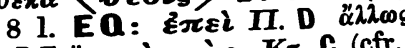

A 11 IEIe

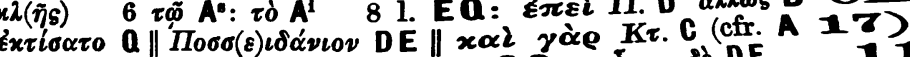

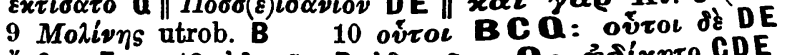

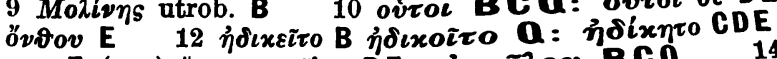

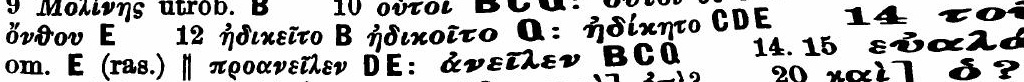

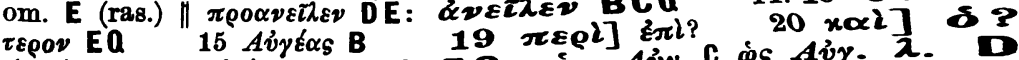

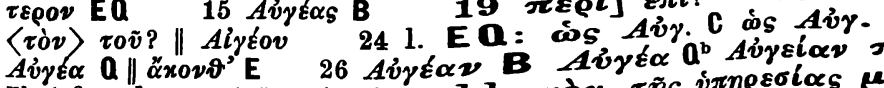

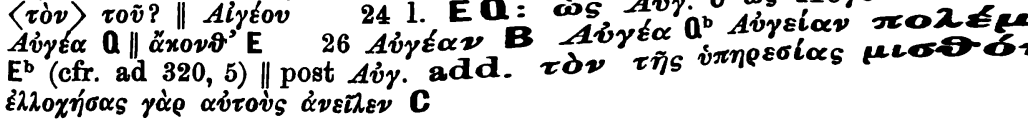




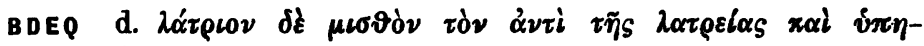

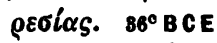

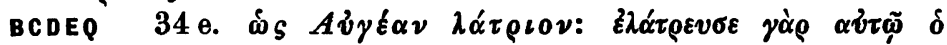

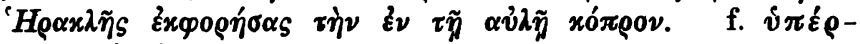

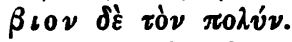

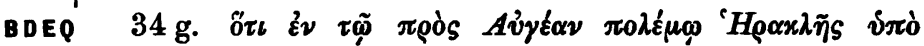

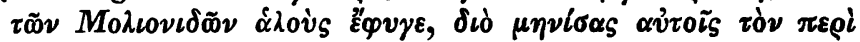

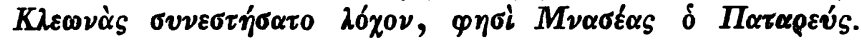
$32 \mathrm{~B}^{\mathrm{a}} 84^{\mathrm{b} C E^{*}} 42 \mathrm{E}^{\mathrm{b}} Q^{\mathrm{b}}$

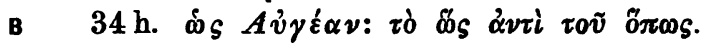

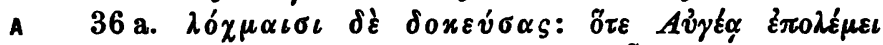

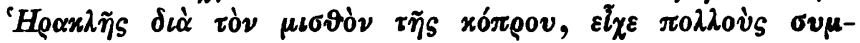

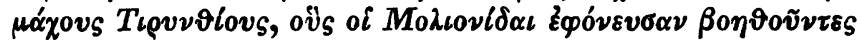
Á̀ý́a.

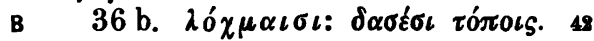

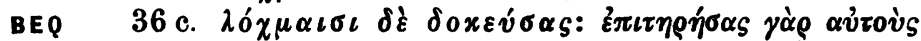

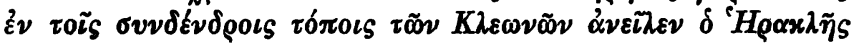

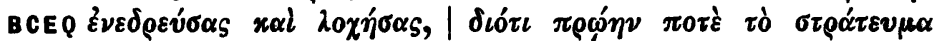

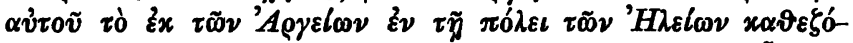

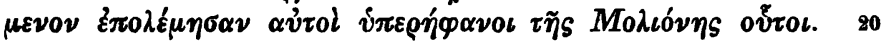

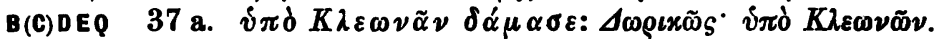

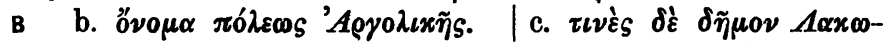

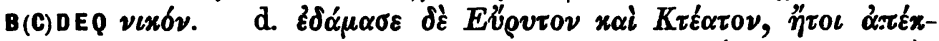

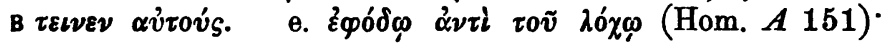

24 sch. BT $A 151$

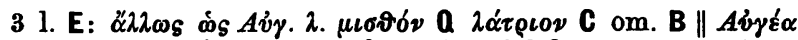
EQ $5 \tau \dot{\eta} \nu \pi 0 \lambda \lambda \dot{\eta} v$ E $\|$ post $f$ repet. C BEQ $6-9$ post 39

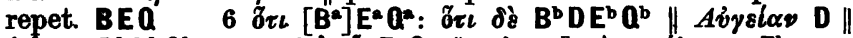

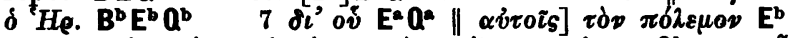

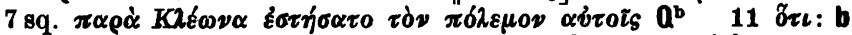

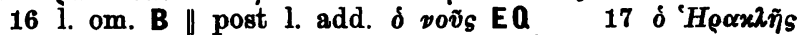

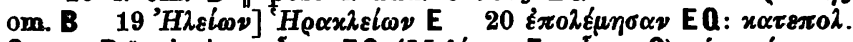

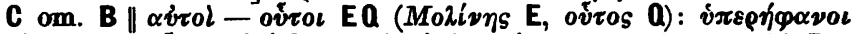

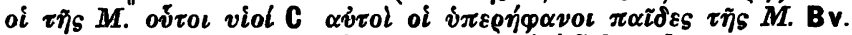
Locus corruptus; $\alpha$ o่ ol oi $\dot{v} \pi$. $\tau \tilde{\eta} s ~ M$. viol Schroeder $21 K \lambda \varepsilon-$

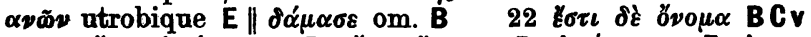

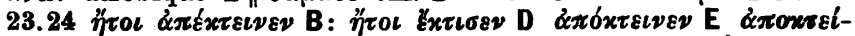

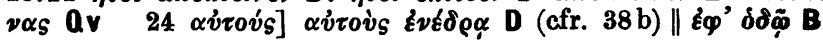




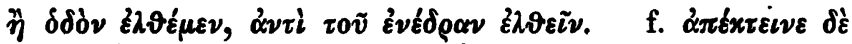

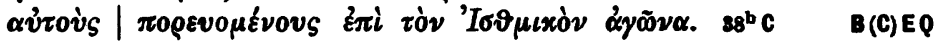

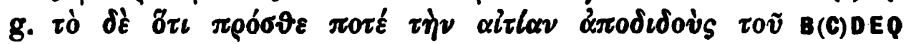

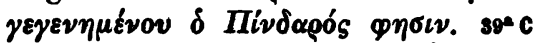

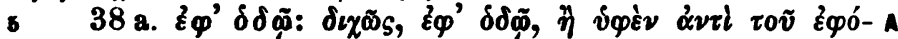
$\delta \omega, \tau \tilde{\eta} \dot{\varepsilon} v \varepsilon \delta \rho \alpha$.

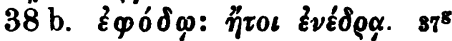

C

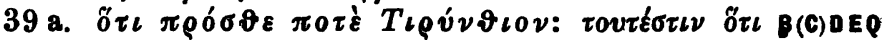

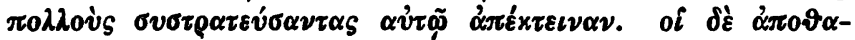

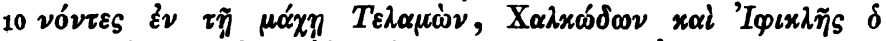

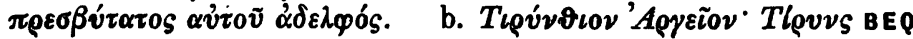

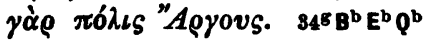

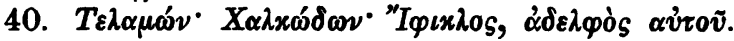

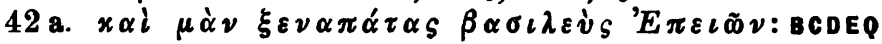

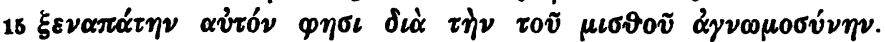

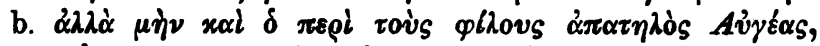

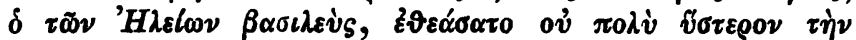

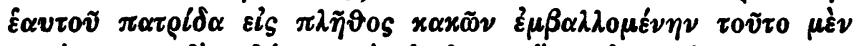

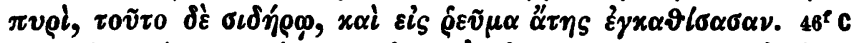

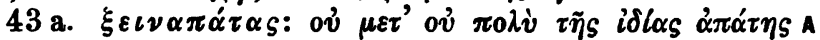

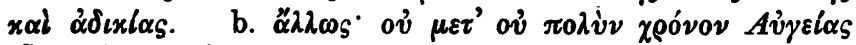

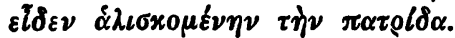

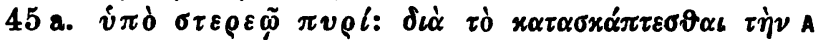

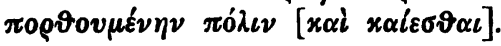

13 Paus. 8, 14, 9; 15, 5-7

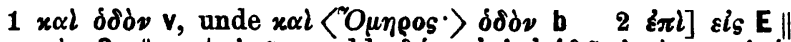

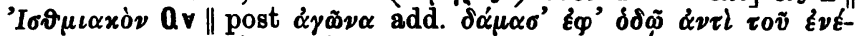

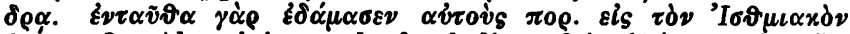

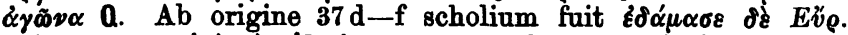

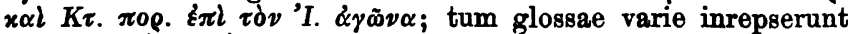

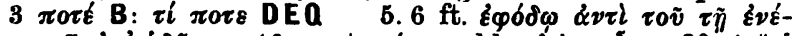

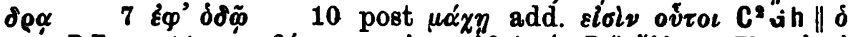

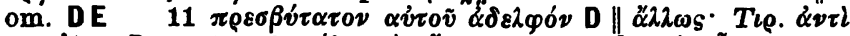

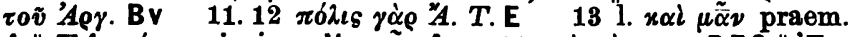

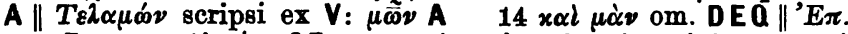

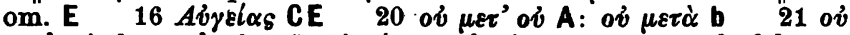

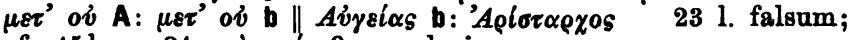

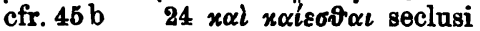

Bohol. ix Pnoarox ed Drachmann. 


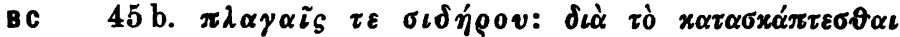

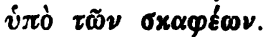

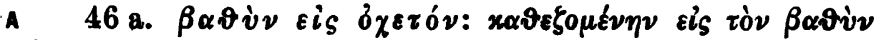

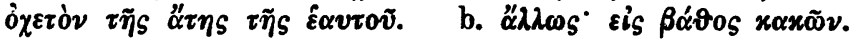

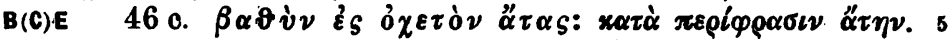

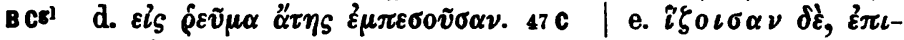
$\varkappa \alpha \vartheta \eta \mu \varepsilon v \eta \nu$.

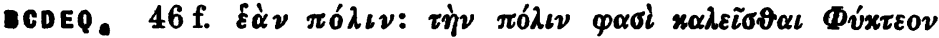

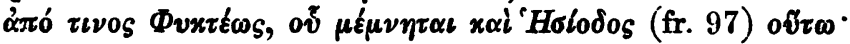

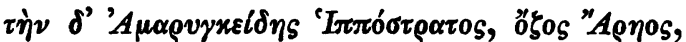

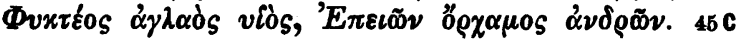

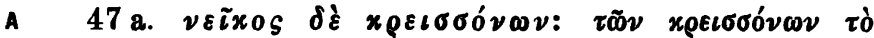

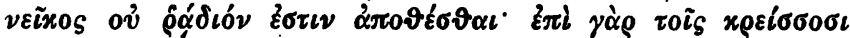

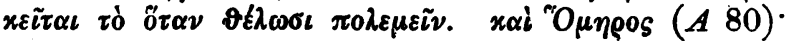

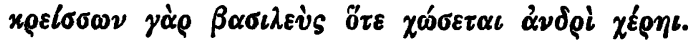

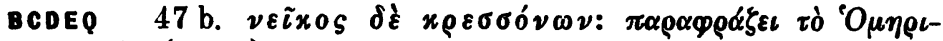
xóv $(A$ 80).

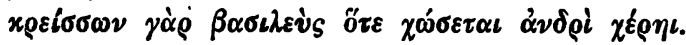

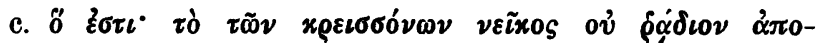

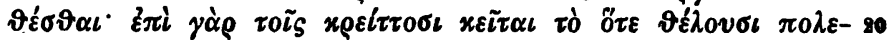

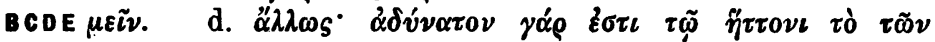

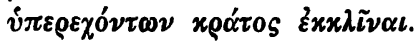

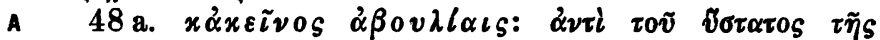

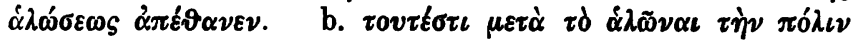

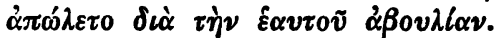

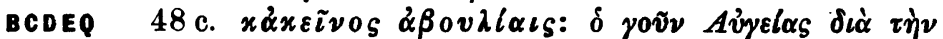

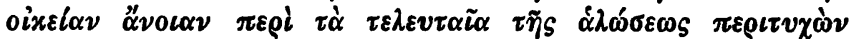

5 $\beta \alpha \vartheta \dot{v} \nu$ \&́s om. E 8 1. Q: om. BC $\tau \dot{\alpha} v \pi$. E $\tau \dot{\alpha} v \pi \delta$

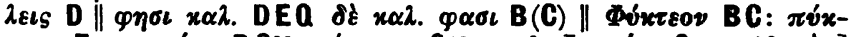

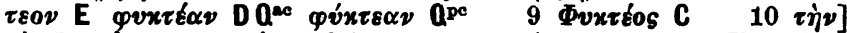

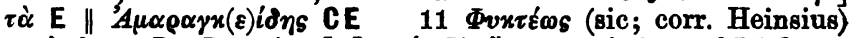

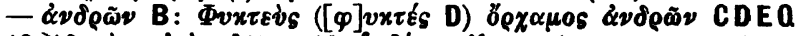

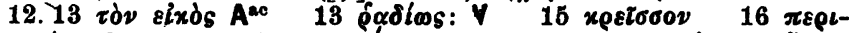

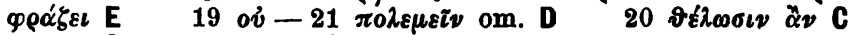

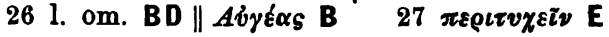




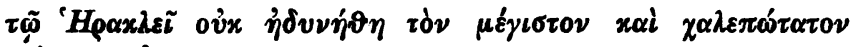

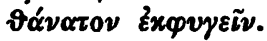

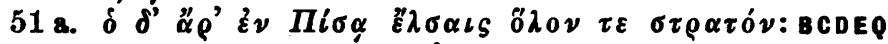

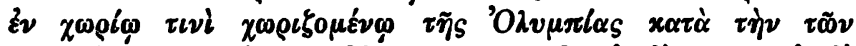

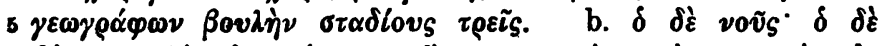

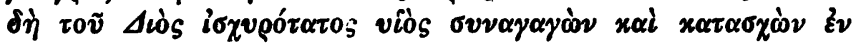

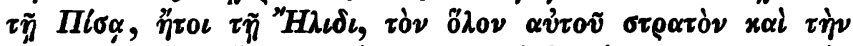

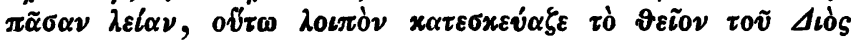

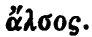

10

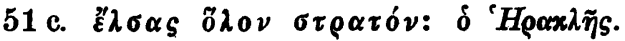

d. $\sigma v v-B D E Q$ $\delta \eta^{\prime} \sigma \alpha \varsigma$. $\quad \sigma v y x \lambda \varepsilon l \sigma \alpha \varsigma . \quad x \alpha \tau \alpha \sigma \chi \omega \dot{\nu} . \quad x \alpha \vartheta \iota \delta \rho v \sigma \alpha \varsigma$.

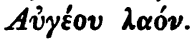

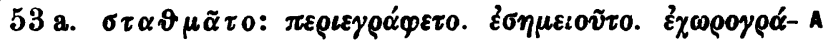
$\varphi \varepsilon \iota . \quad \dot{\pi} \pi \mathrm{i} \varepsilon \iota$.

53 b. $\sigma \tau \alpha \vartheta \mu \tilde{\alpha} \tau 0: \quad \alpha \alpha \tau \varepsilon \mu \varepsilon \tau \rho \varepsilon \tilde{\tau} \tau . \quad \pi \varepsilon \rho\llcorner\omega ́ \rho \iota \xi \varepsilon . \quad \pi \varepsilon \rho \iota \varepsilon \sigma x \dot{\varepsilon} \pi-\operatorname{BCDEQ}$

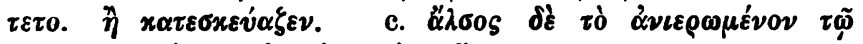

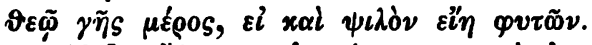

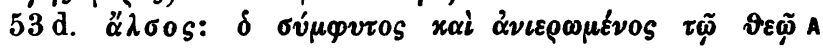
гósos.

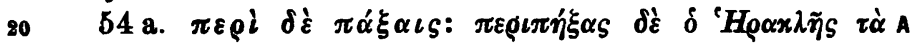

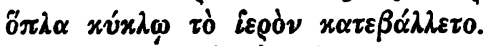

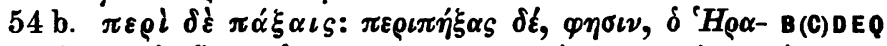

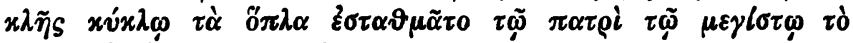

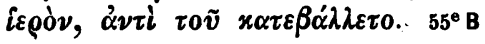

16 cfr. ad sch. 0 . III 31

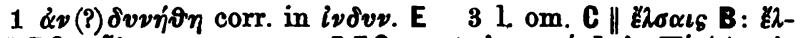

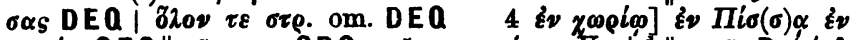

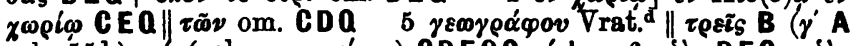
sch. 55b): $\tau$ (vel $\tau \rho \iota \alpha x o \sigma i o v s)$ CDEGQ 5 h 6 viòs DEQ: vios

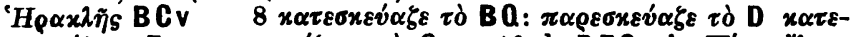

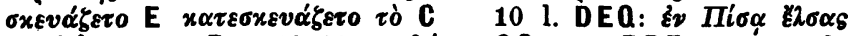

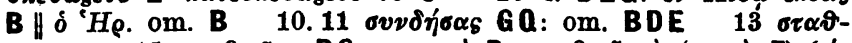

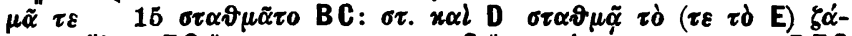

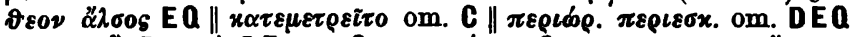

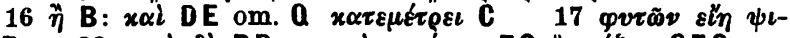
$\lambda \omega^{\prime}$ E $22 \pi \varepsilon \rho l \delta \xi$ B D: $\pi \alpha \tau \rho l \mu \varepsilon \gamma i \sigma \tau \phi$ EQ \| $\pi \alpha^{\prime} \xi \alpha \xi$ CEQ $24 x \alpha \tau \varepsilon \beta \alpha^{\prime} \lambda \varepsilon \tau o$ D $x \alpha \tau \varepsilon \beta \alpha^{\prime} \lambda \varepsilon \tau \varepsilon$ E 


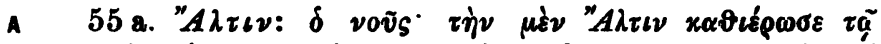

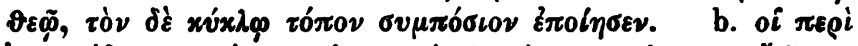

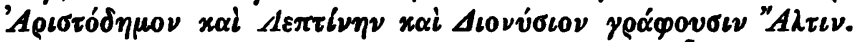

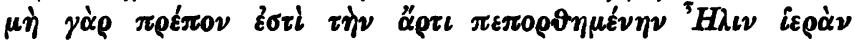

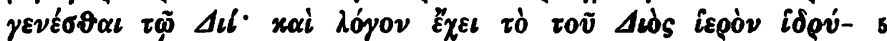

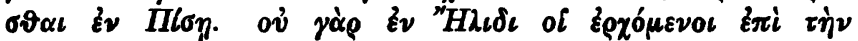

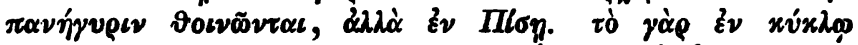

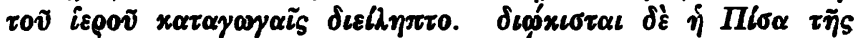

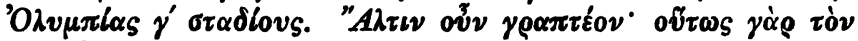

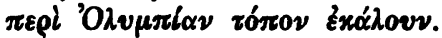

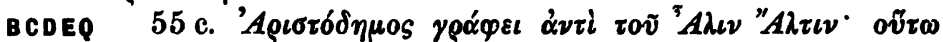

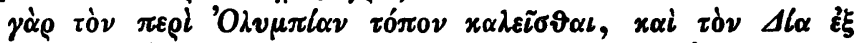

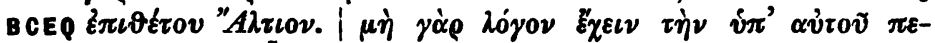

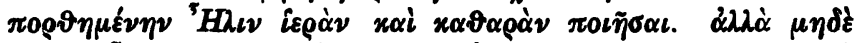

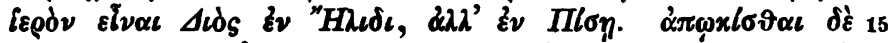

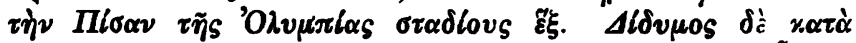

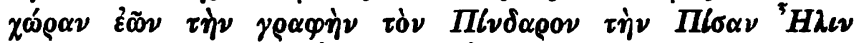

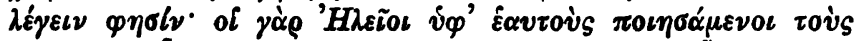

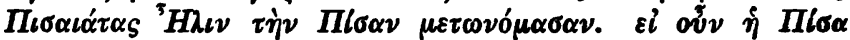

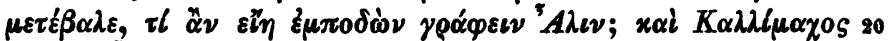

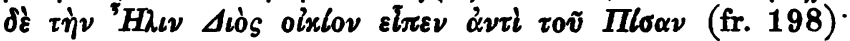
${ }^{3} H$ H

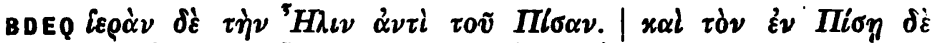
$\Delta l_{\alpha}$ 'H

9 Paus. V 10, 1

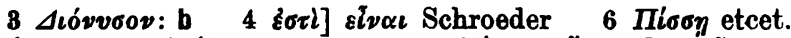

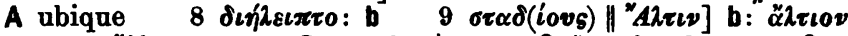

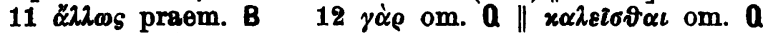

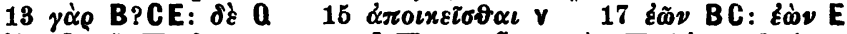

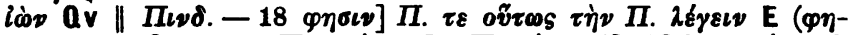

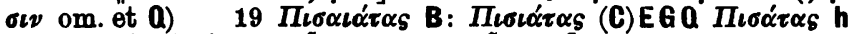

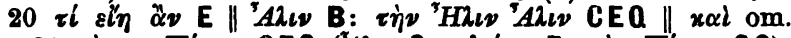
EQ $21 \tau \dot{\eta} \nu-\Pi i \sigma \alpha \nu$ CEQ ( $A \lambda \iota \nu$ Q, olxi $\left.\alpha \nu \mathrm{E}, \tau \dot{\eta} \nu \Pi \Pi^{\prime} \sigma \alpha \nu \mathrm{CQ}\right)$ :

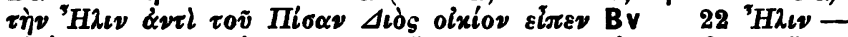

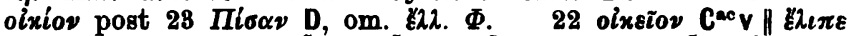
Qv $\left.\| \varphi \imath \lambda_{\varepsilon i} \mathrm{C}^{\alpha v} \mathrm{23}{ }^{5} \mathrm{H} \lambda \iota \nu\right]{ }^{3} H \lambda \iota v \varepsilon i \pi \varepsilon v$ E $24 \varepsilon i \pi \varepsilon v$ 'HR. K. dz Q $\|$ " $A$ lilos D EQ: 'Aletos B 


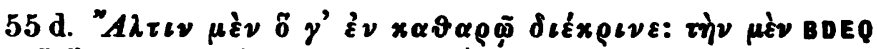

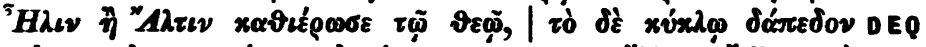

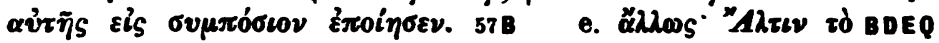

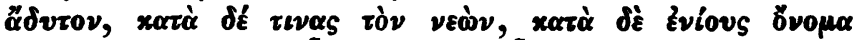

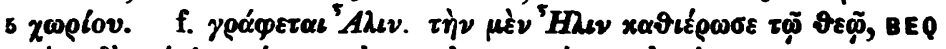

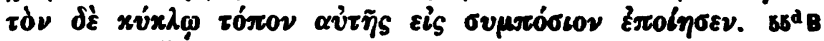

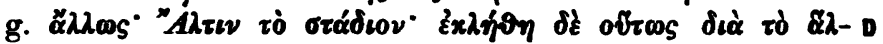

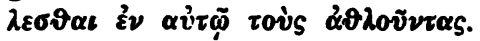

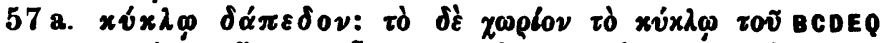

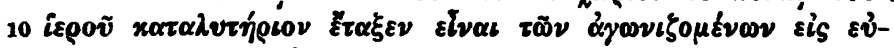

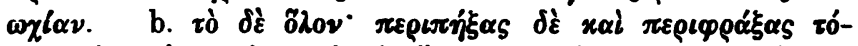

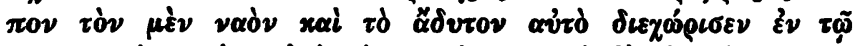

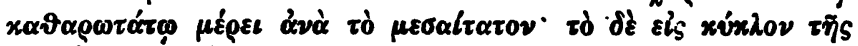

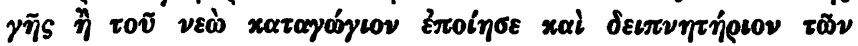
$15 \xi \dot{\varepsilon} v \omega \nu$. $5^{\circ} \mathrm{B}$

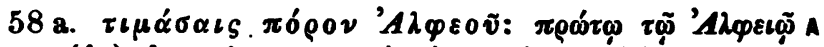

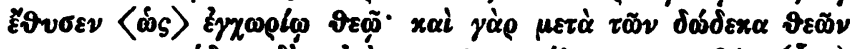

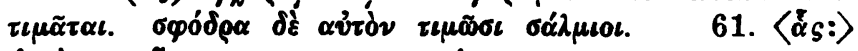

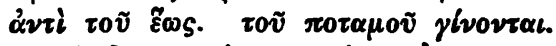

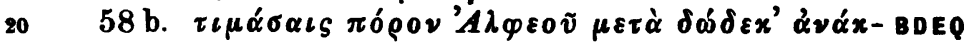

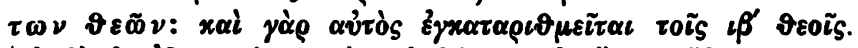

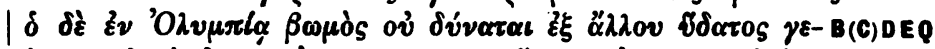

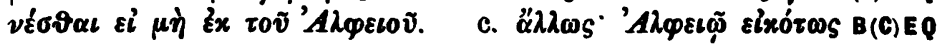

22 cfr. Paus. 5, 13, 11

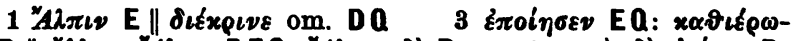

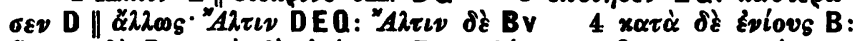

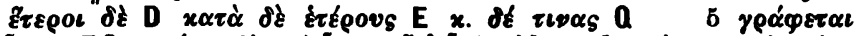

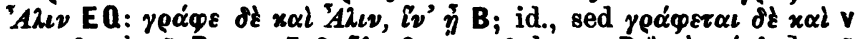

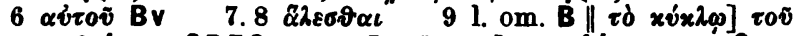

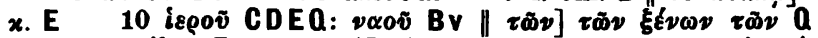

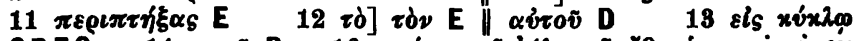

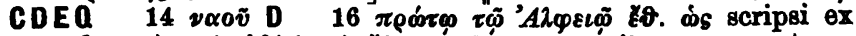

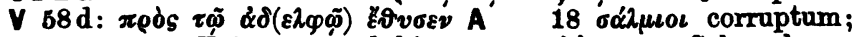

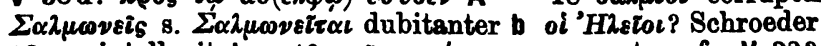

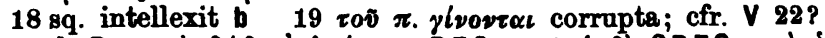

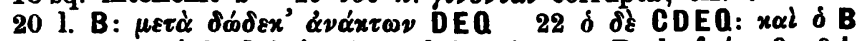

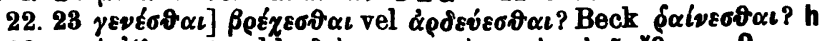

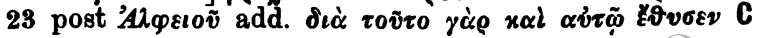




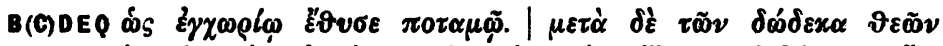

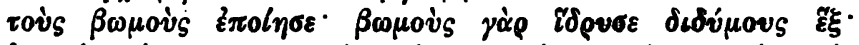

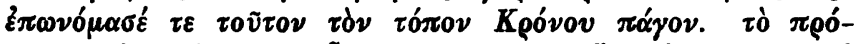

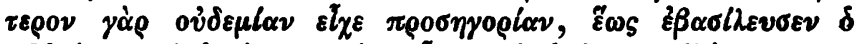

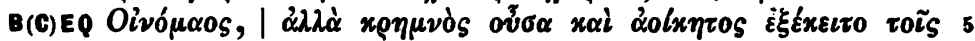

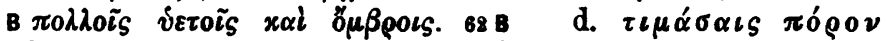

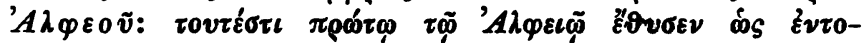
$\pi l \omega \vartheta \varepsilon \tilde{\varphi}$.

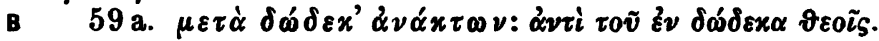

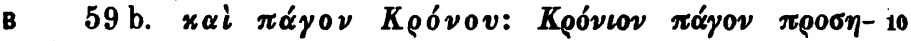

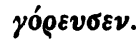

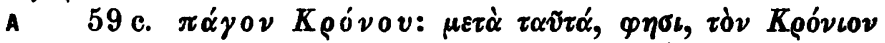

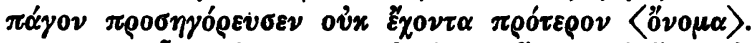

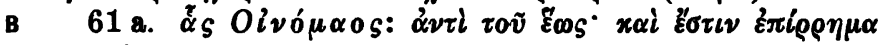

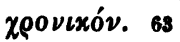

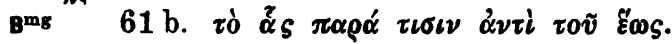

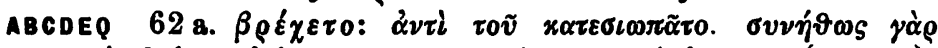

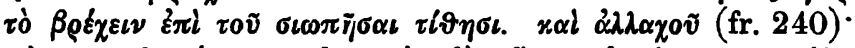

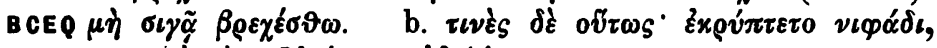

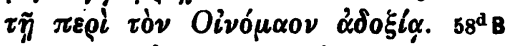

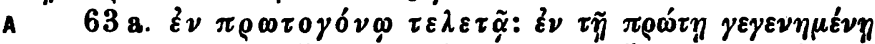

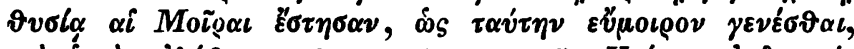

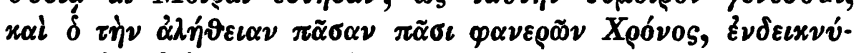

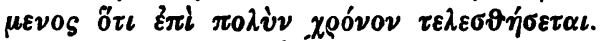

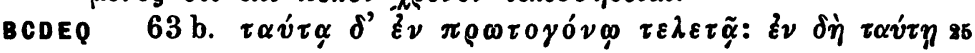

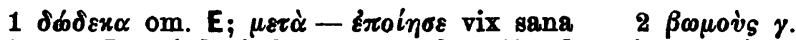

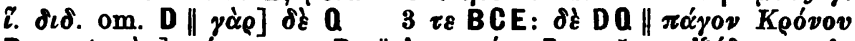

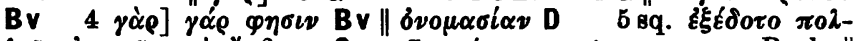

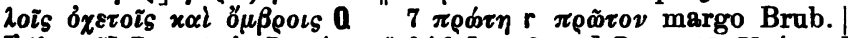

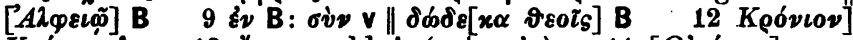

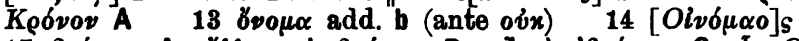

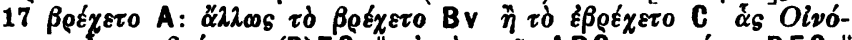

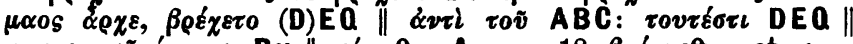

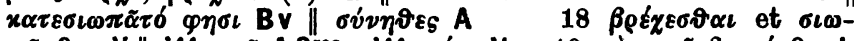

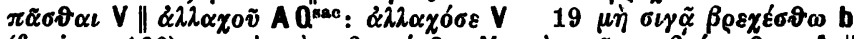

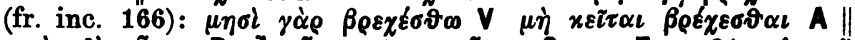

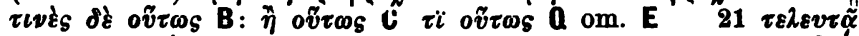
$22 \pi \alpha \rho \varepsilon \sigma \tau \eta \sigma \alpha \nu$ V? (sch. 63c) $25 \tau \varepsilon \lambda \varepsilon \varepsilon \tilde{\alpha} \tilde{a}$ B: $\tau \varepsilon \lambda \varepsilon v \tau \tilde{\alpha}(C) E$ om. DQ $\| \varepsilon \dot{\varepsilon}] \varepsilon i \mathrm{E}$ 


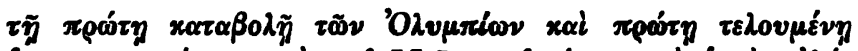

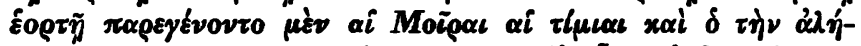

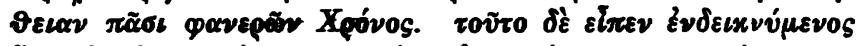

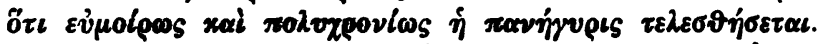

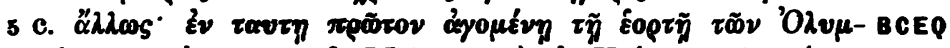

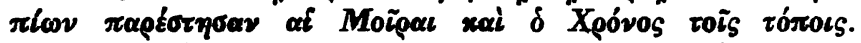

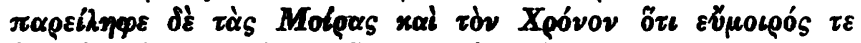

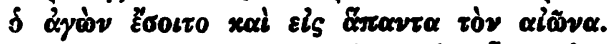

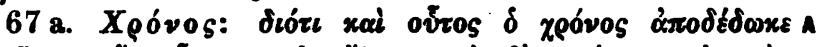

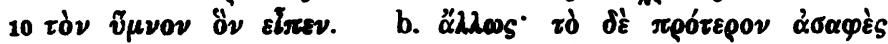

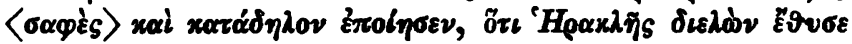

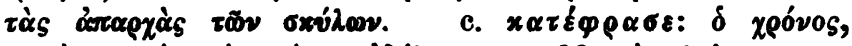

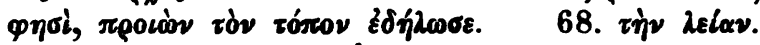

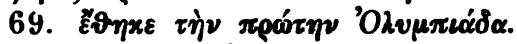

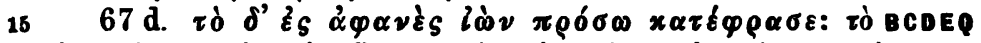

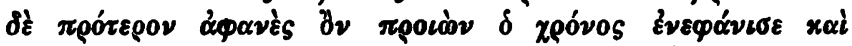

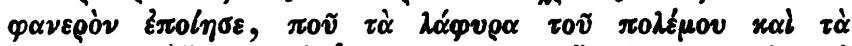

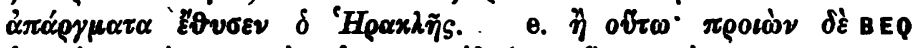

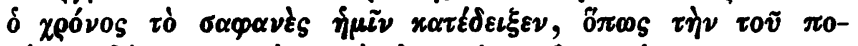

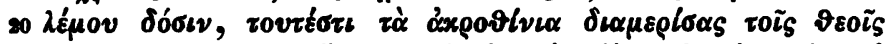

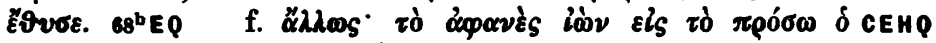

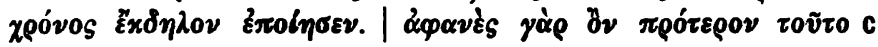

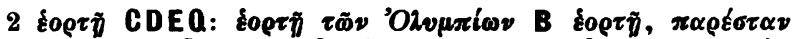
$\mu \varepsilon \dot{\nu} v \quad 3 \pi \tilde{\alpha} \sigma l] \pi \tilde{\alpha} \sigma \alpha \nu$ Qb $\| \varphi \alpha \nu \varepsilon \rho \tilde{\nu} \nu$ B (cfr. A):

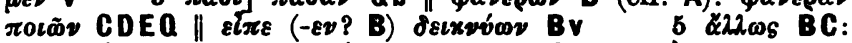

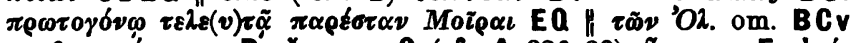

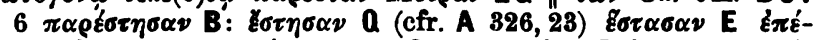

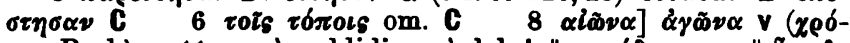

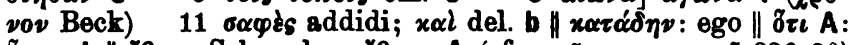

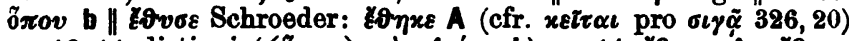

13. 14 distinxi (

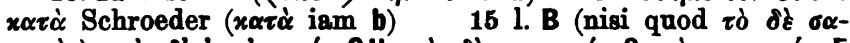

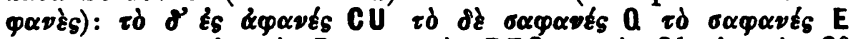

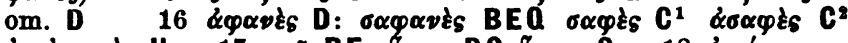

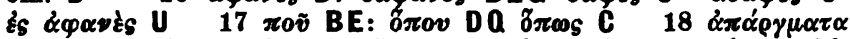

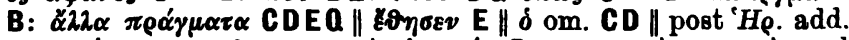

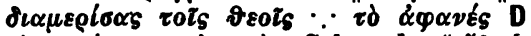

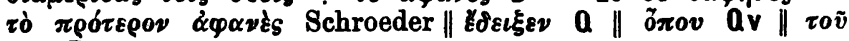
om. B v 


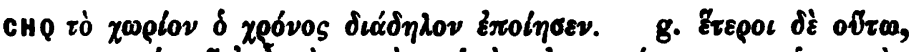

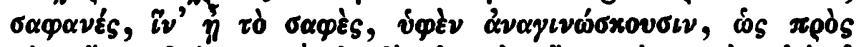

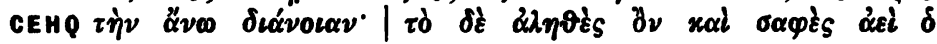

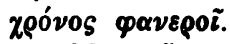

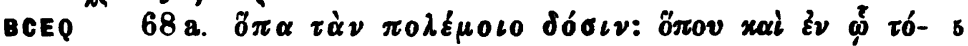

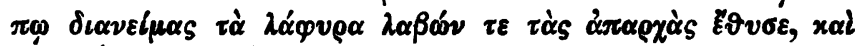

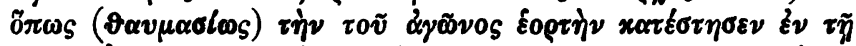

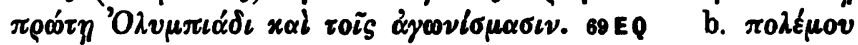

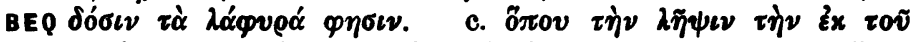

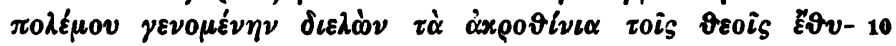
бEv. G7'EQ

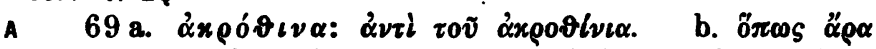

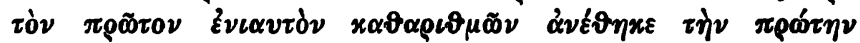

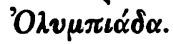

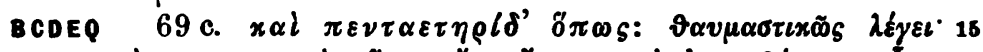

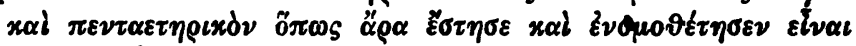

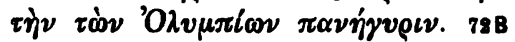

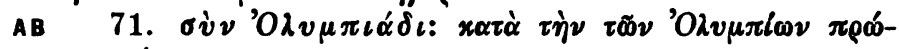

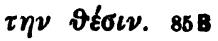

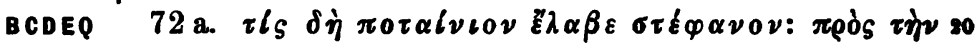

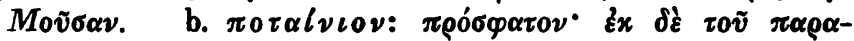

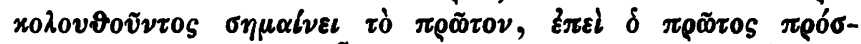

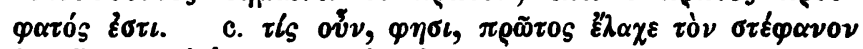

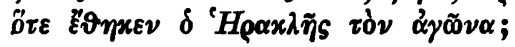

21 sch. Aesch. Prom. 102

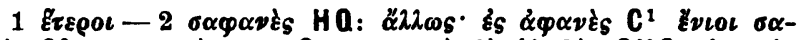

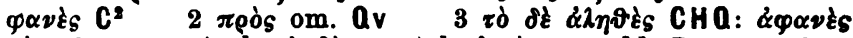

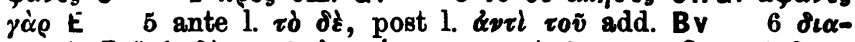

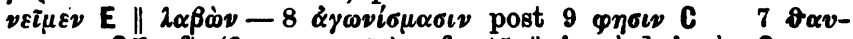

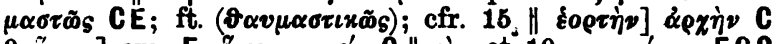

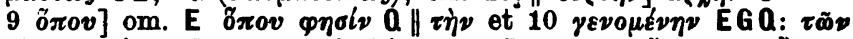

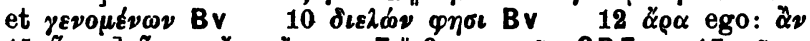

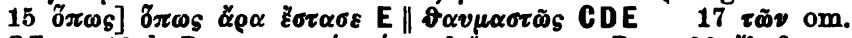
CE 18 l. B: $v i x \alpha \varphi o \rho i \alpha \iota \sigma i \tau_{\varepsilon}$ A $\| \tau \tilde{\omega} \nu$ om. B $20 \varepsilon_{\lambda \alpha \beta \varepsilon} \sigma \tau$. B: om. CDEQ $\| \pi \rho \dot{s}-21 \pi 0 \tau \alpha i v i o v$ om. CD $20.21 \pi \rho \dot{s} s$.

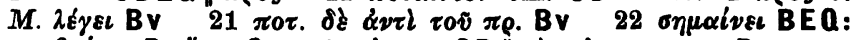

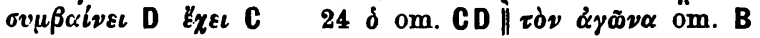




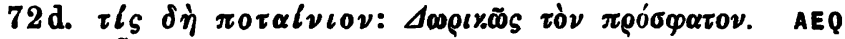

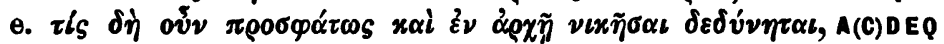

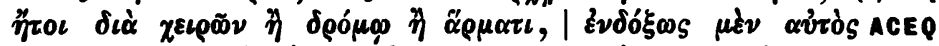

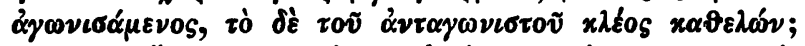

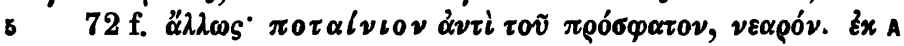

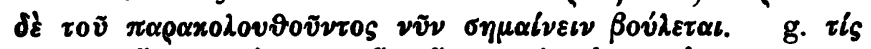

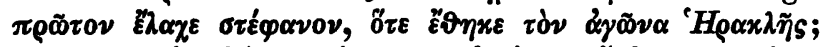

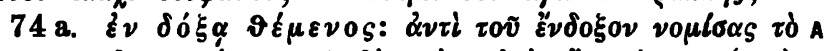

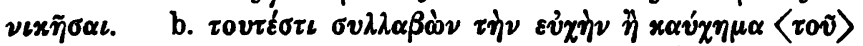

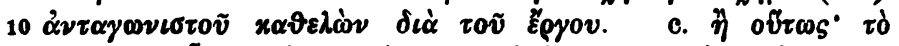

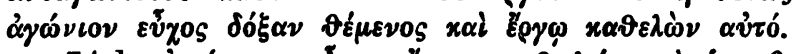

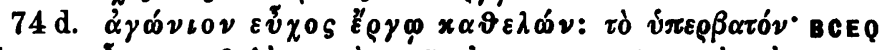

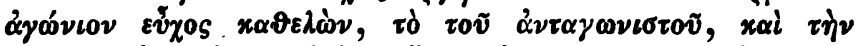

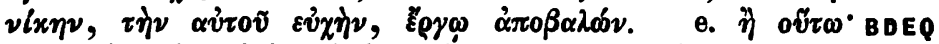

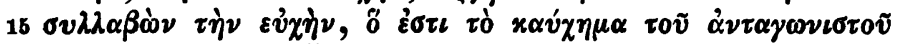

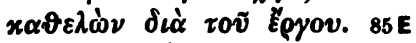

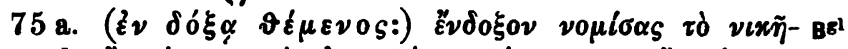

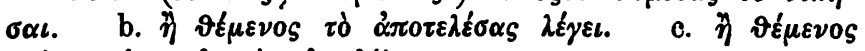

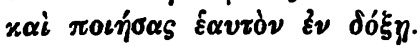

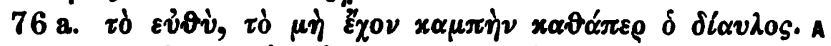

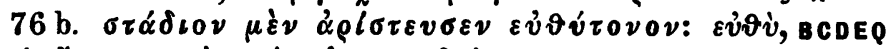

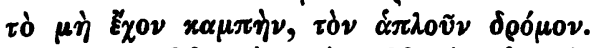

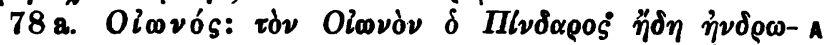

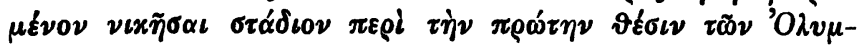

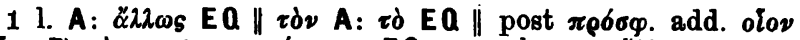

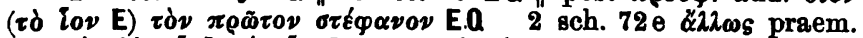

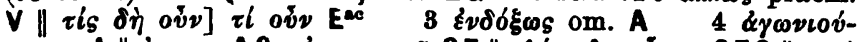

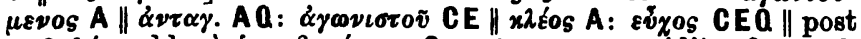

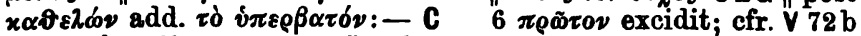

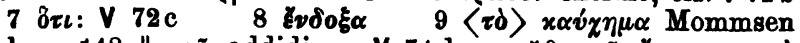

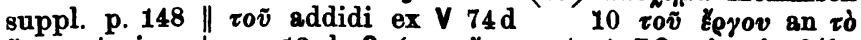

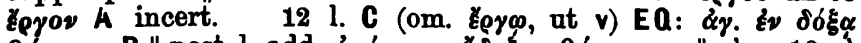

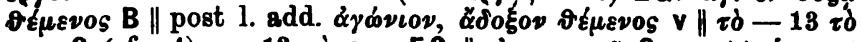

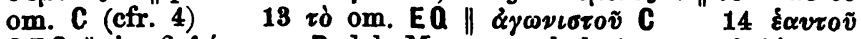
CEQ \| $\alpha \pi \delta \beta \alpha \lambda \dot{c} \nu \nu$ om. B del. Mommsen 1. 1. (ex $x \alpha \tau \alpha \beta \alpha \lambda \dot{\omega} \nu$ or-

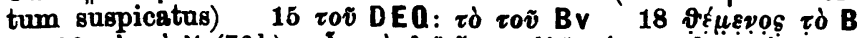

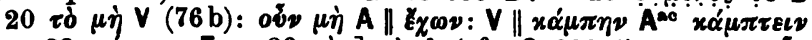

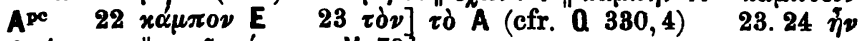

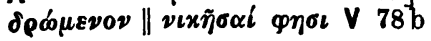




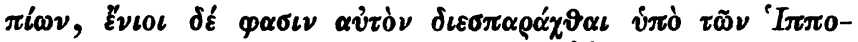

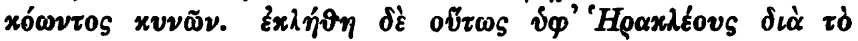

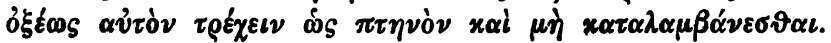

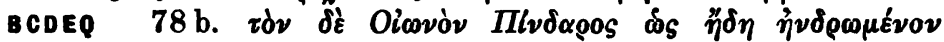

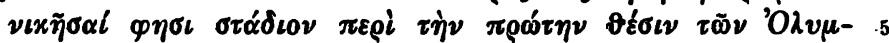

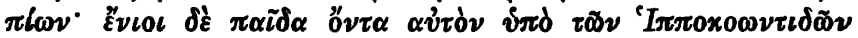

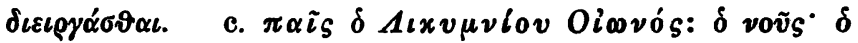

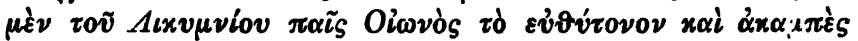

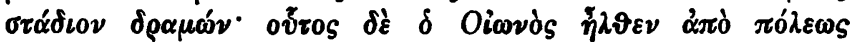

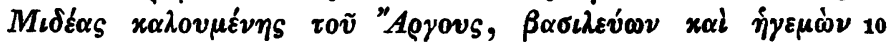

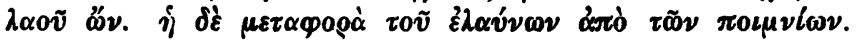

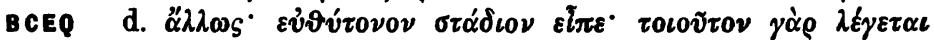

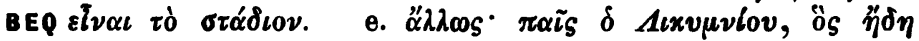

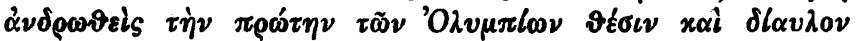

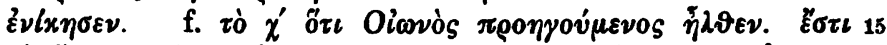

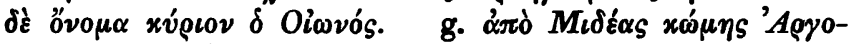

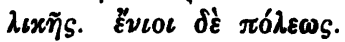

А $\quad 78 \mathrm{~h} . M_{\iota} \delta \varepsilon \alpha \vartheta \varepsilon v:$ $\alpha \pi \delta M_{\iota} \delta \varepsilon \alpha s$.

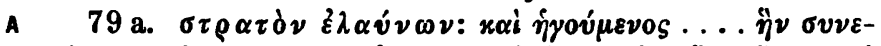

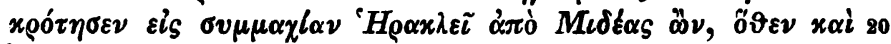

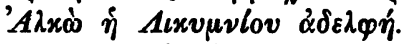

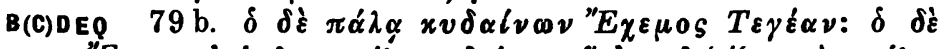

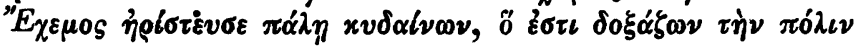

\section{Apollod. 2, 7, 3, 3. Paus. 3, 15, 4}

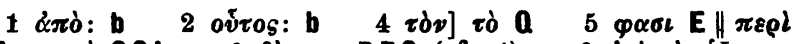

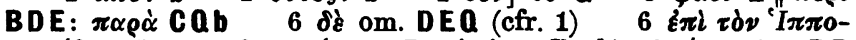

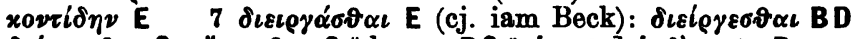

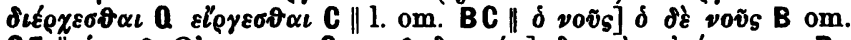

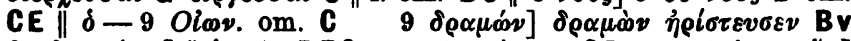

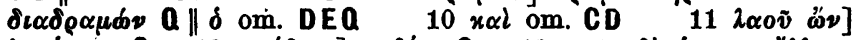

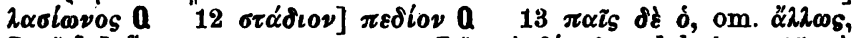

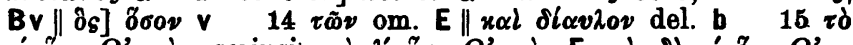

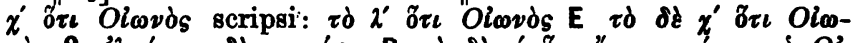

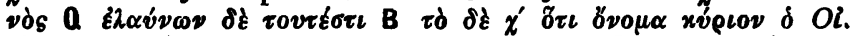
cj. b $16 \delta \mathrm{om}$. EQ || $x \operatorname{co} \mu \eta \mathrm{s}-13 \delta \varepsilon \mathrm{om}$. EQ $18 \mu \eta \delta \varepsilon \alpha \vartheta \varepsilon v$ :

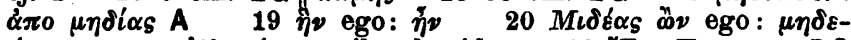

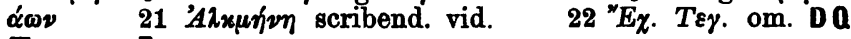
T\&y. om. E 


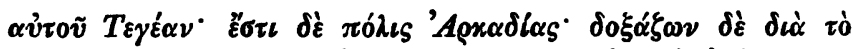

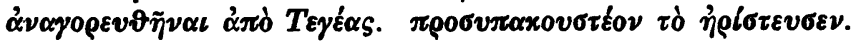

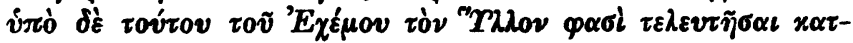

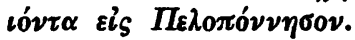

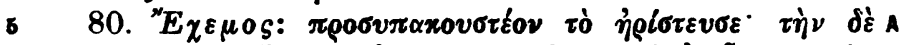

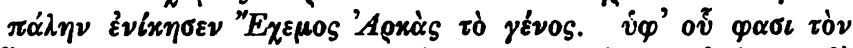

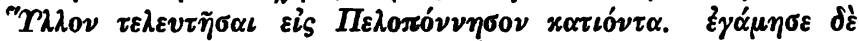

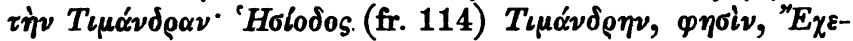

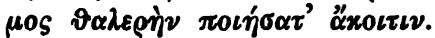

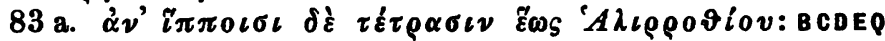

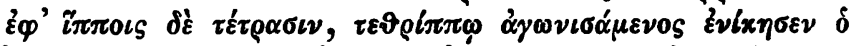

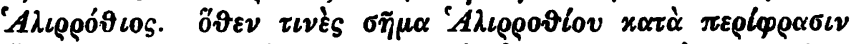

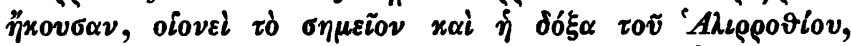

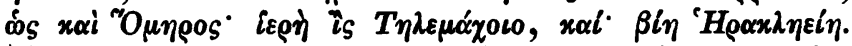

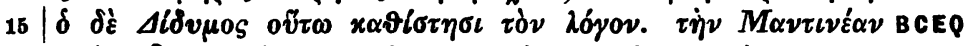

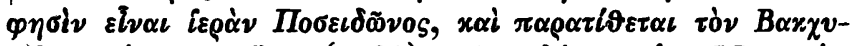

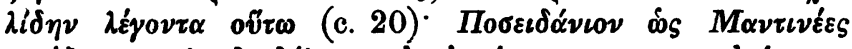

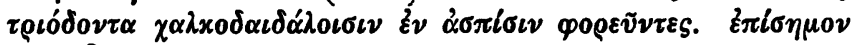

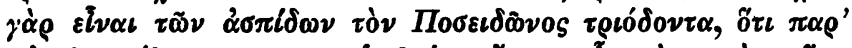

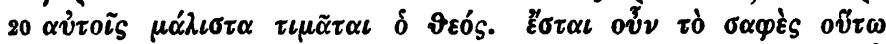

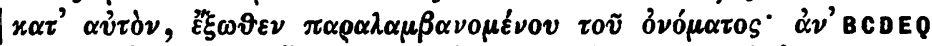

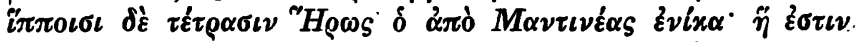

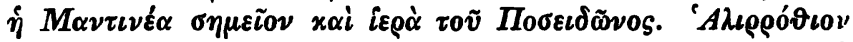

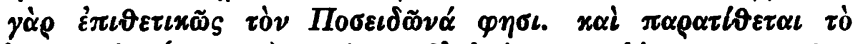

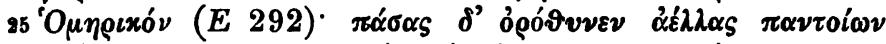

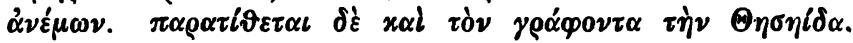

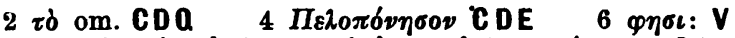

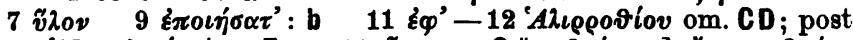

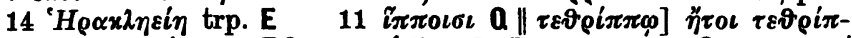

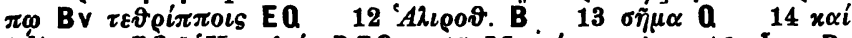

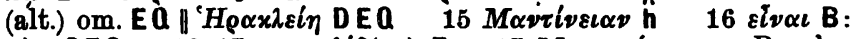

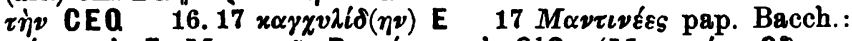

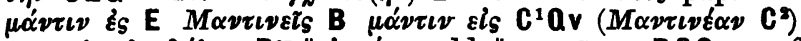

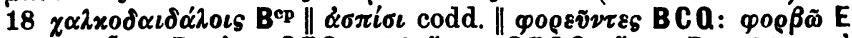

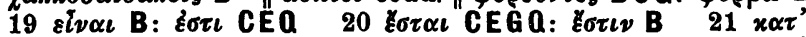

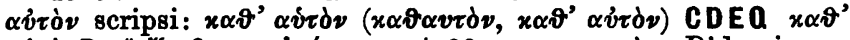

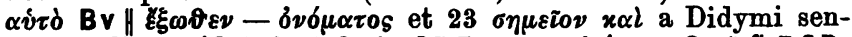

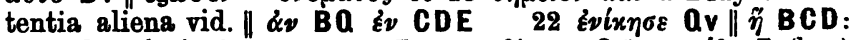

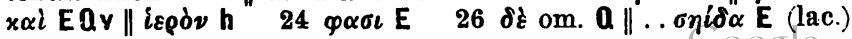




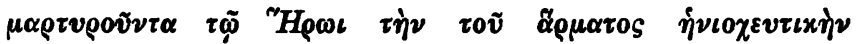

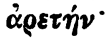

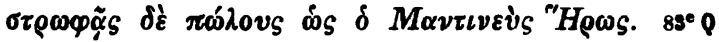

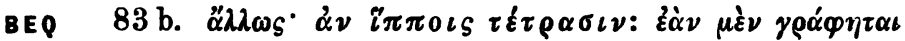

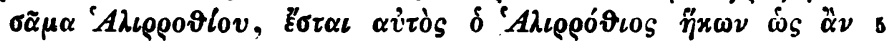

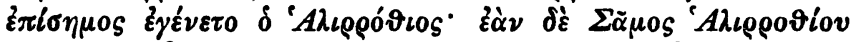

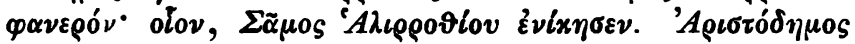

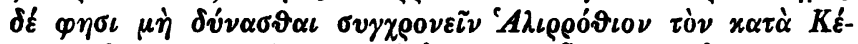

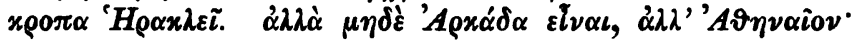

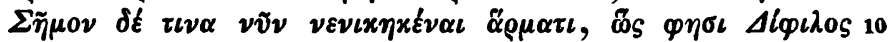

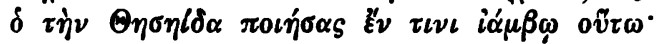

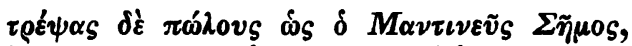

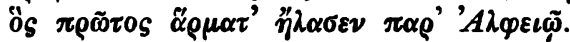

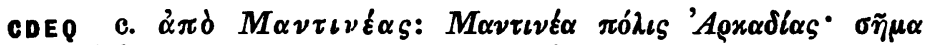

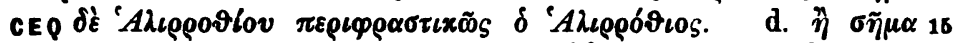

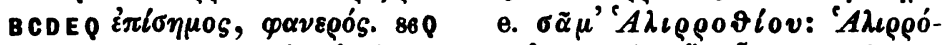

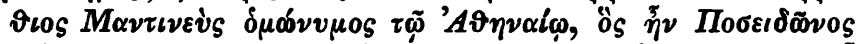

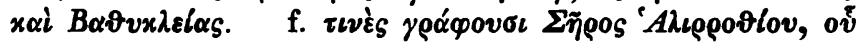
$\mu \varepsilon ́ \mu \nu \eta \tau \alpha \iota$ 'Holodos (fr. 106)'

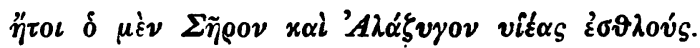

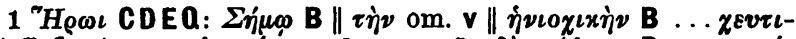

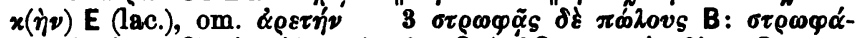

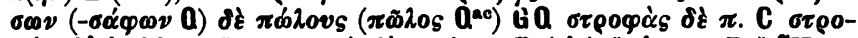

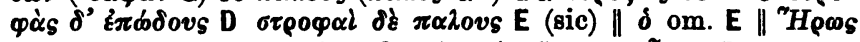

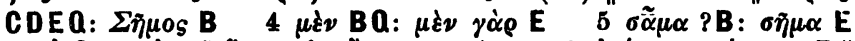

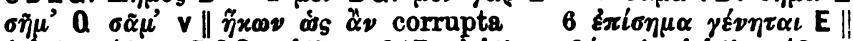

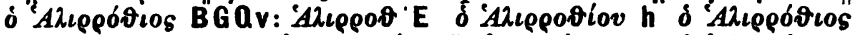

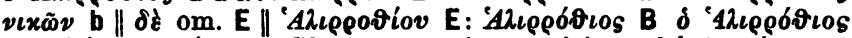

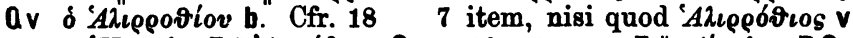

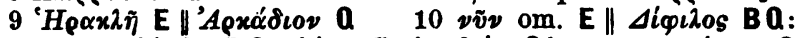

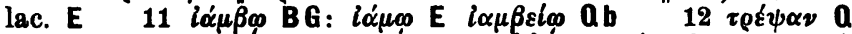

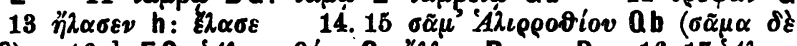

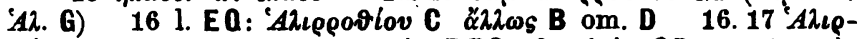

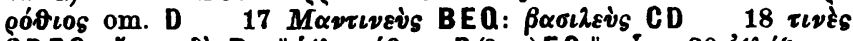

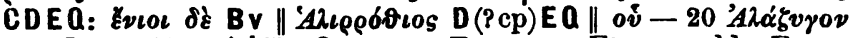

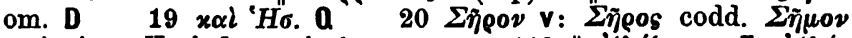

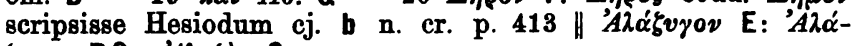

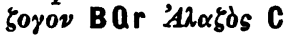




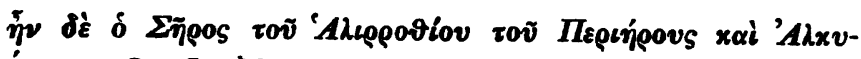
óvฑS. $71 \mathrm{~B} 86 \mathrm{E} 83^{\mathrm{b}} \mathrm{Q}$

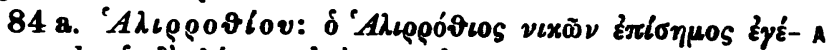

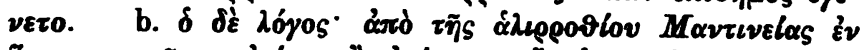

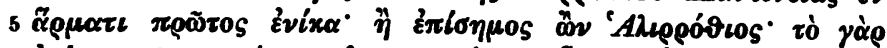

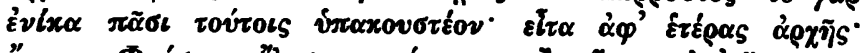

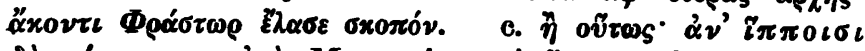

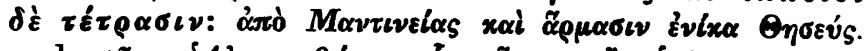

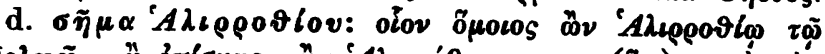

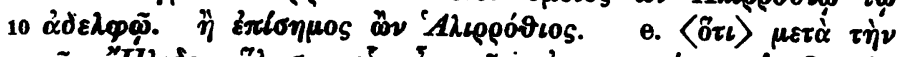

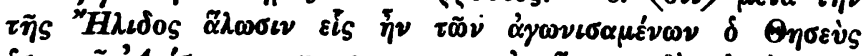

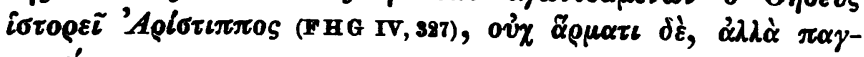
xportio.

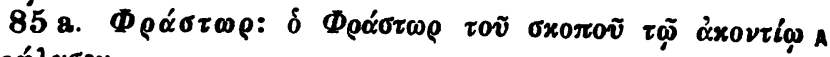

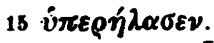

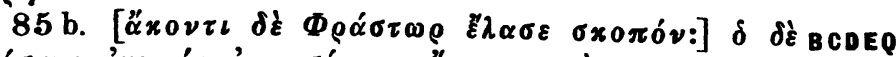

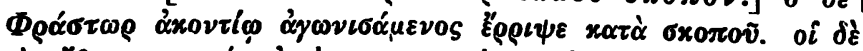

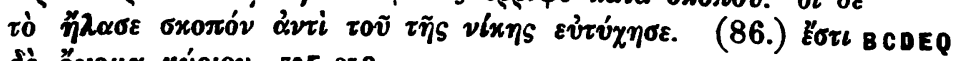

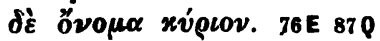

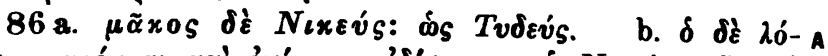

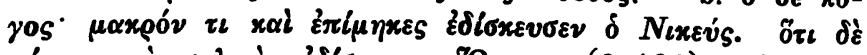

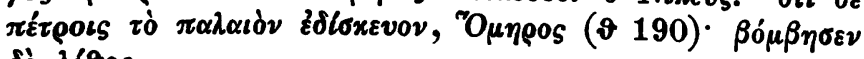
$\delta \dot{\varepsilon} \lambda$ lGos.

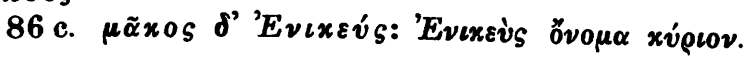
BCDEP

21 sch. $\vartheta 190 . \Psi 826$

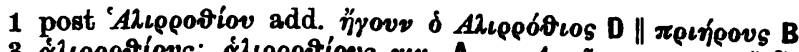

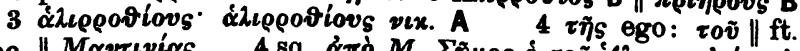

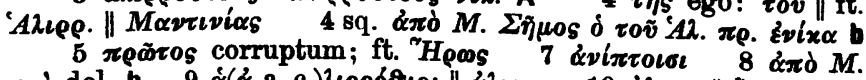

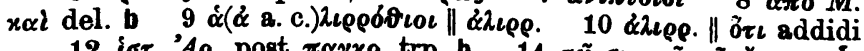

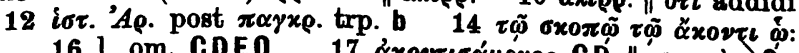

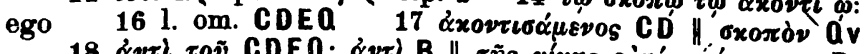

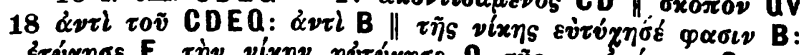

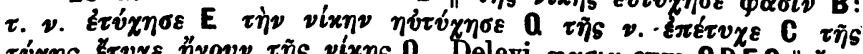

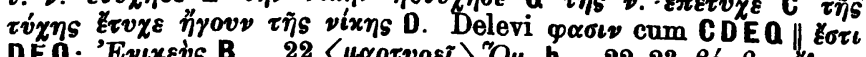

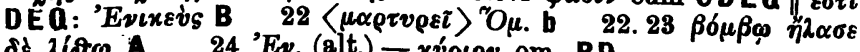
$\delta \xi \lambda i \theta \omega$ A 24 'Ev. (alt.) - xv́ 


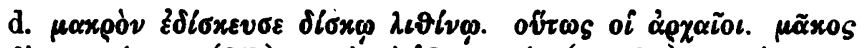

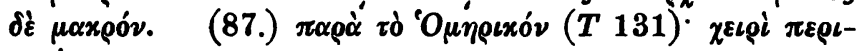

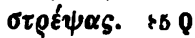

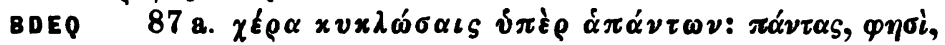

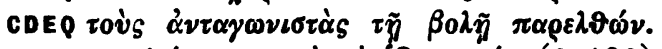

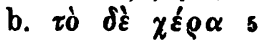

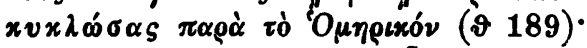

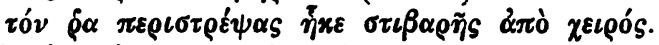

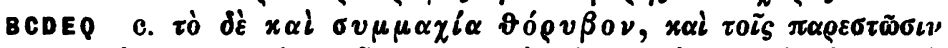

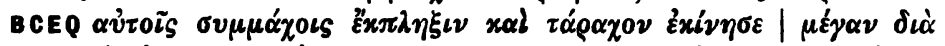

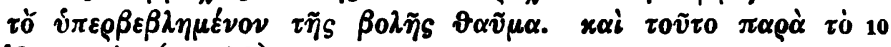

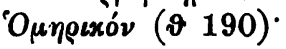

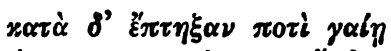

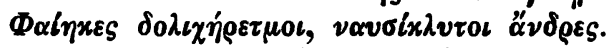

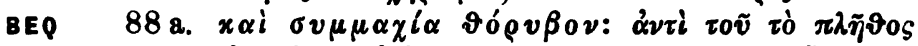

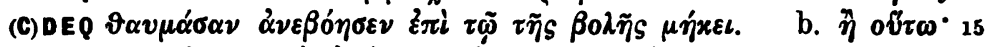

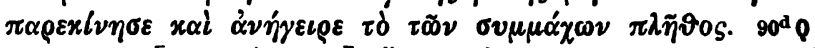

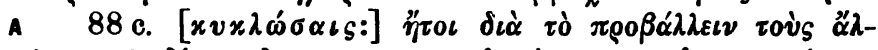

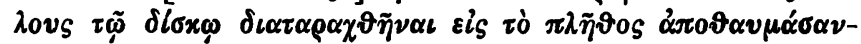

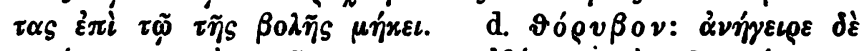

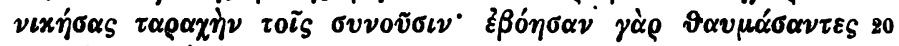

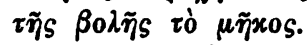

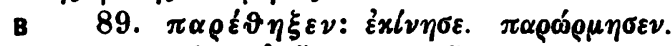

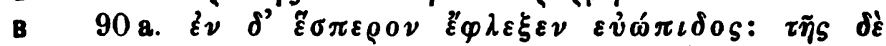

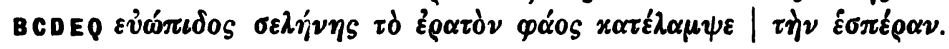

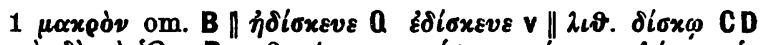

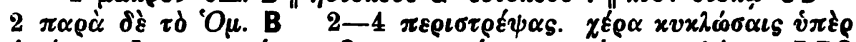

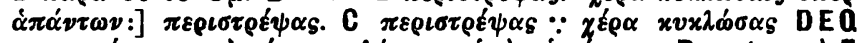

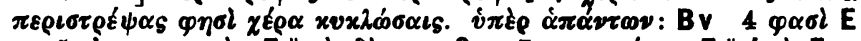

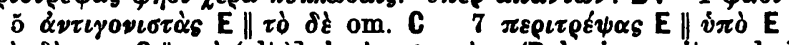

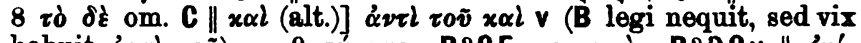

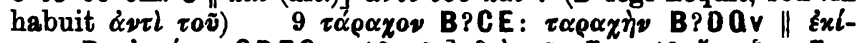

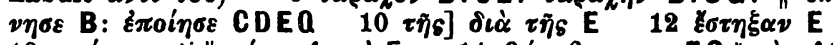

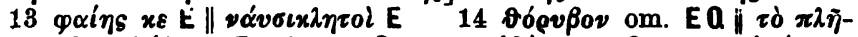

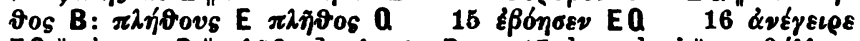

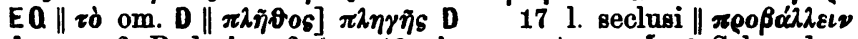
A: $\pi \rho 0 \sigma \beta$. Red. $i \pi \varepsilon \rho \beta$. b 18 eis corruptum; $\varepsilon i \pi \varepsilon ?$ Schroeder;

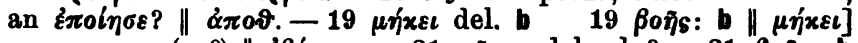

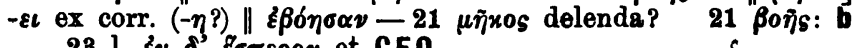

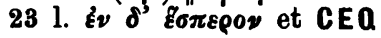




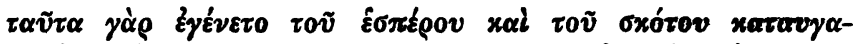

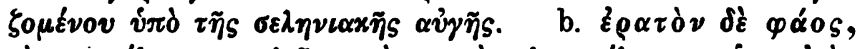

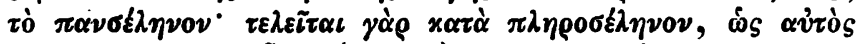

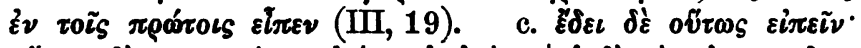

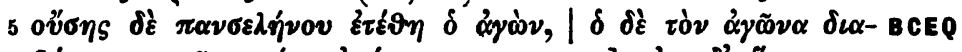

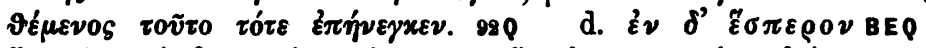

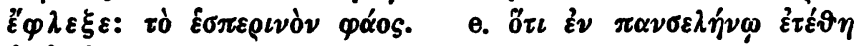
ó áyóv. $90^{*} 0$

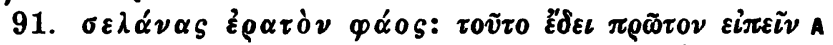

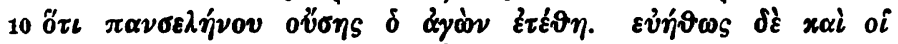

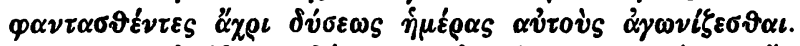

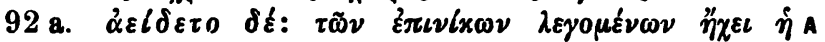
$\Pi l \sigma \alpha$.

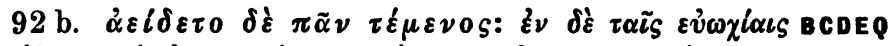

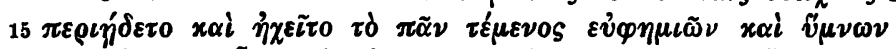

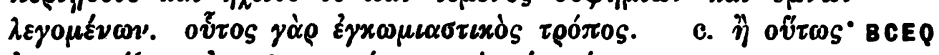

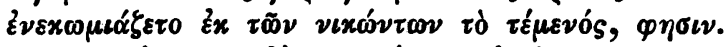

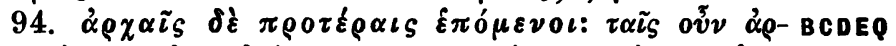

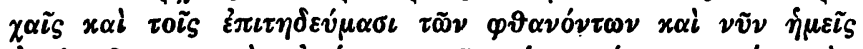

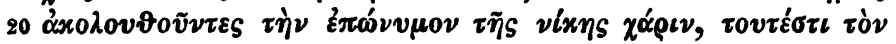

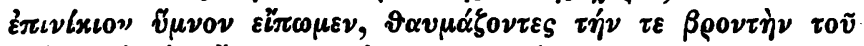

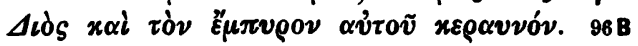

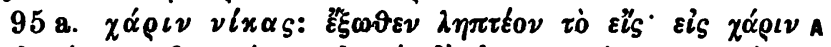

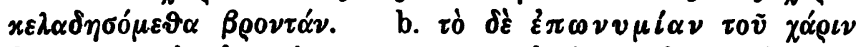

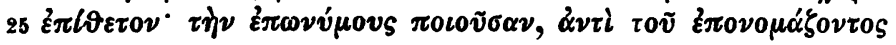

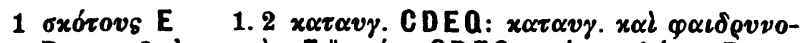

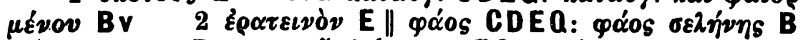

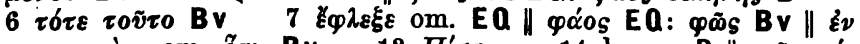
$\pi \alpha \nu \sigma . \gamma \dot{\alpha} \rho$, om. ö $\tau \iota, \mathrm{Bv} 13 \Pi i \sigma \sigma \alpha 14 \mathrm{l}$. om. D $\| \pi \tilde{\alpha} \nu \tau \varepsilon \dot{\varepsilon}-$

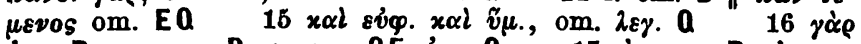

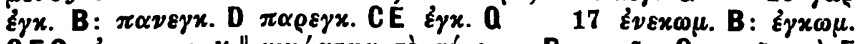

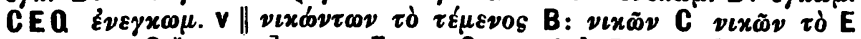

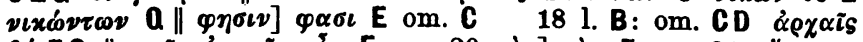

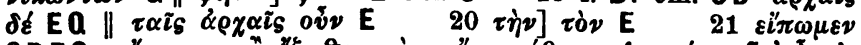

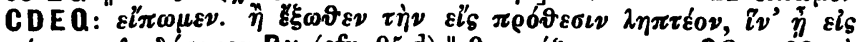

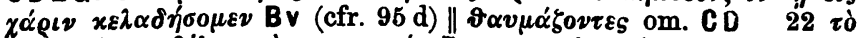

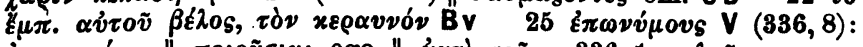

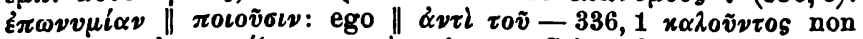

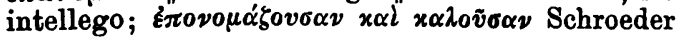




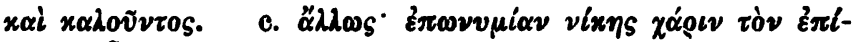
vเxov $\varepsilon l \pi \varepsilon v$.

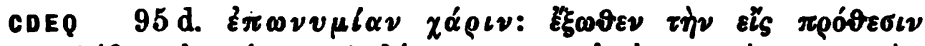

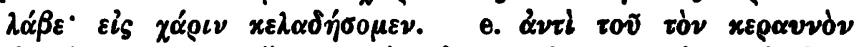

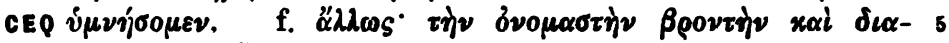

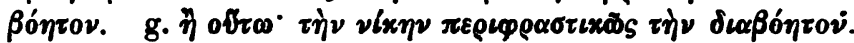

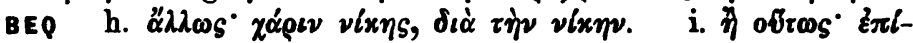

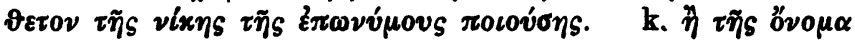

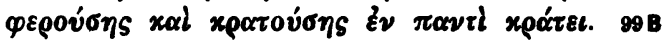

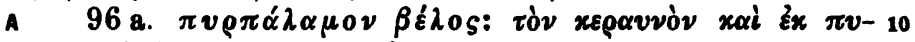

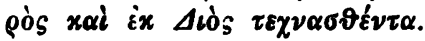

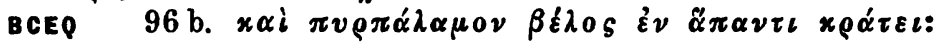

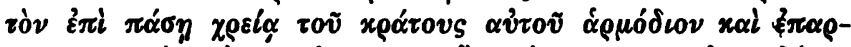

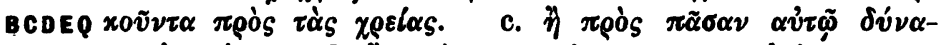

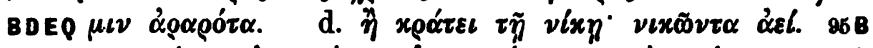

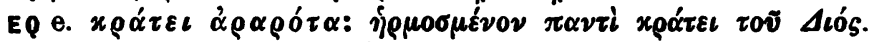

A 99 a. $\chi \lambda \iota \delta \tilde{\omega} \sigma \alpha \delta \dot{\varepsilon} \mu 0 \lambda \pi \alpha \alpha^{\prime}: \tau \rho v \varphi \tilde{\sigma} \sigma \alpha \delta \dot{\varepsilon} \dot{\eta} \mu 0 \lambda \pi \dot{\eta} \tau \tilde{\omega} \nu$

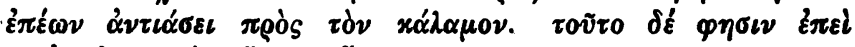

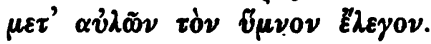

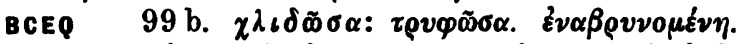

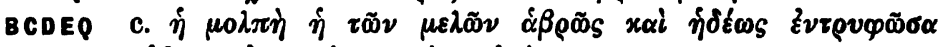

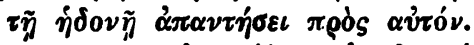

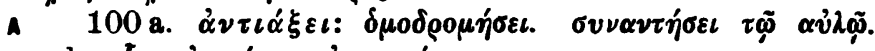

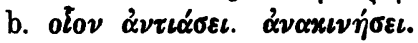

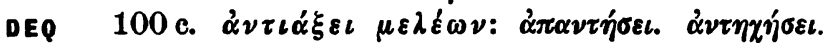

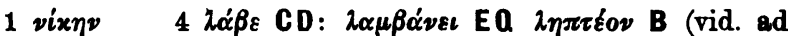

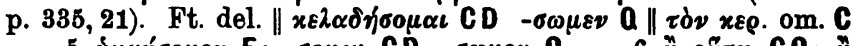

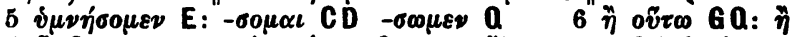

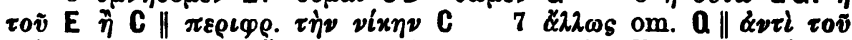

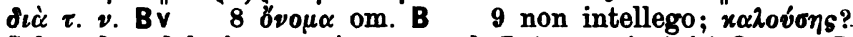

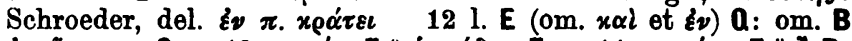

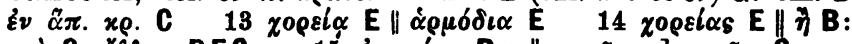

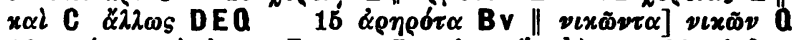

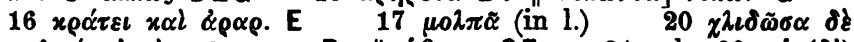

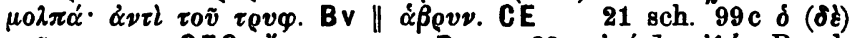

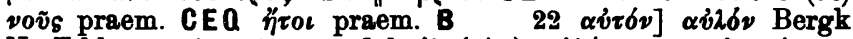

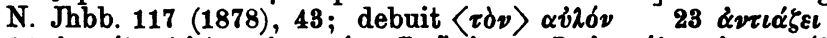

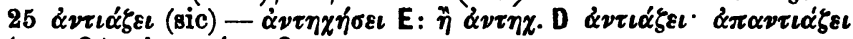

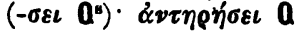




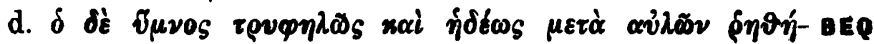
бetres. $100^{\circ} \mathrm{E}$

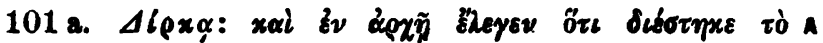

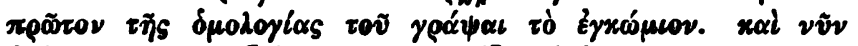

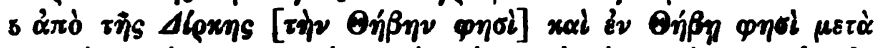

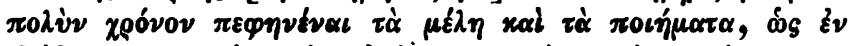

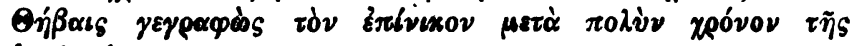

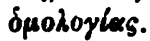

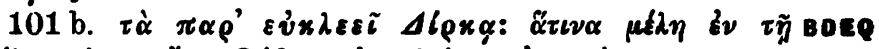

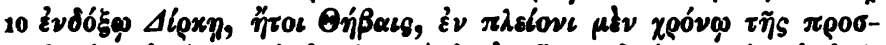

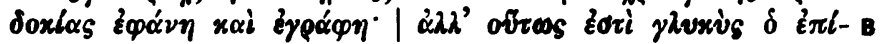

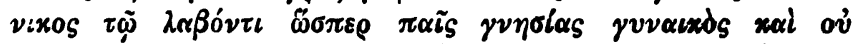

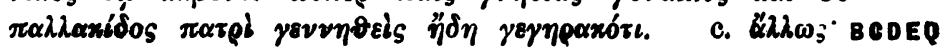

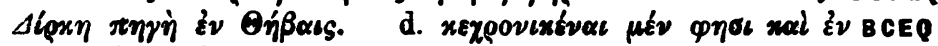

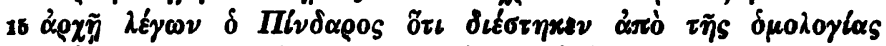

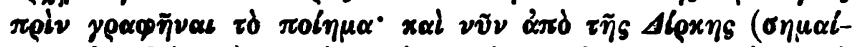

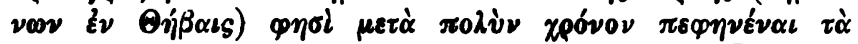

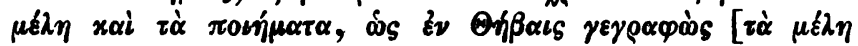

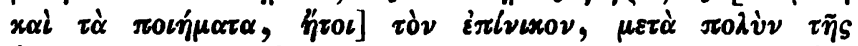

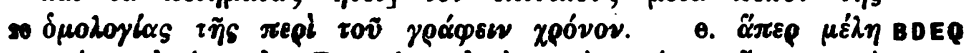

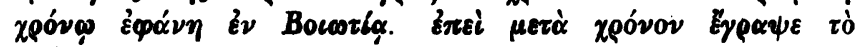
$\pi 0 i \eta \mu \alpha . \quad 108^{\circ} \mathrm{E} 100^{\circ} \mathrm{O}$

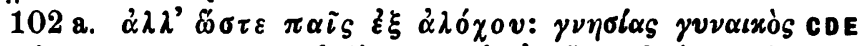

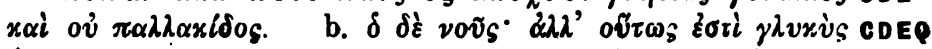

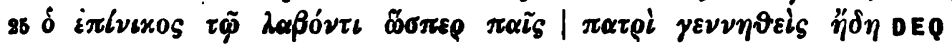

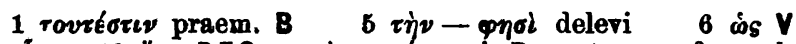

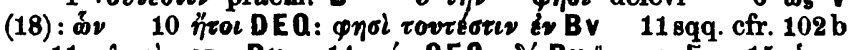

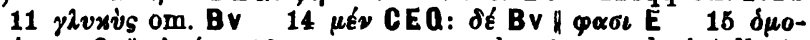

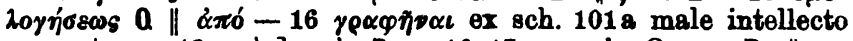
corrupta $16 \pi \rho i v] \pi \rho o g$ E $16.17 \sigma \eta \mu$. है $\theta$. om. BV $\| \sigma \eta-$ $\mu \alpha i v \omega \nu$ Q: $\sigma \eta \mu \alpha i v(o v)$ E $\sigma v \mu \beta \alpha i v(o v)$ C. Cfr. $5 \quad 17 \pi \varepsilon \varphi v x \varepsilon \dot{-}$

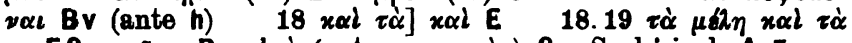

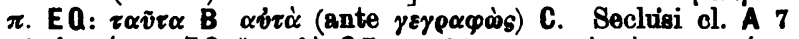

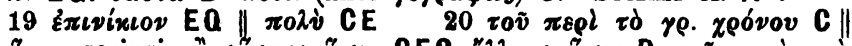

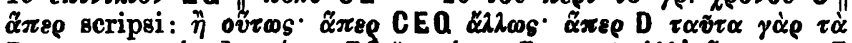

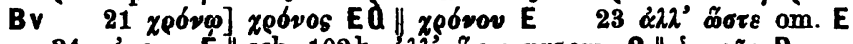

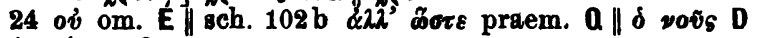
25 Ė $\pi$ เvíxios Q

Sohor. Ix Priparum ed. Drachmann. 


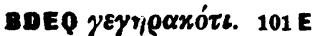

c. $\delta \delta \dot{\varepsilon} \nu 0 \tilde{v} \varsigma^{\circ}$ o

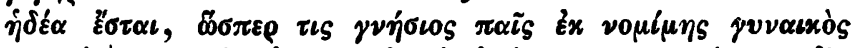

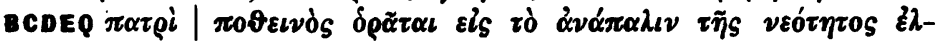

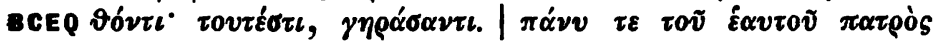

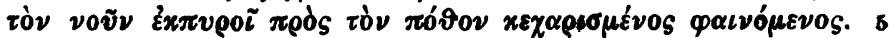

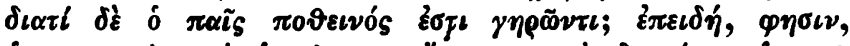

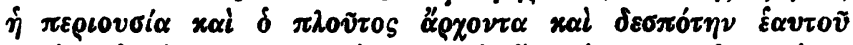

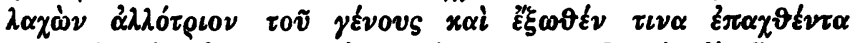

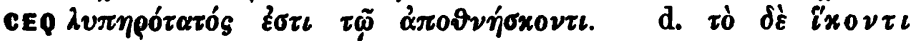

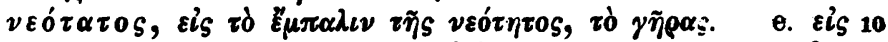

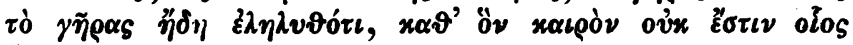
$\ddot{\alpha} \lambda \lambda_{0} \quad \gamma \varepsilon \nu \nu \tilde{\eta} \sigma \alpha \varepsilon$.

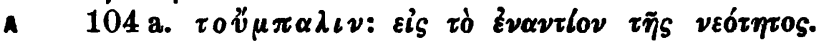

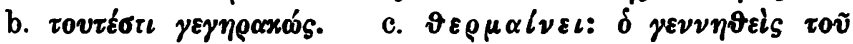

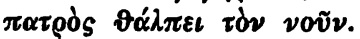

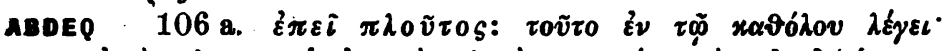

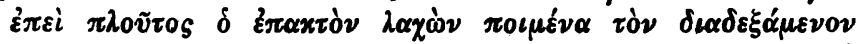

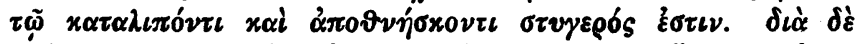

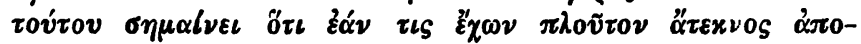

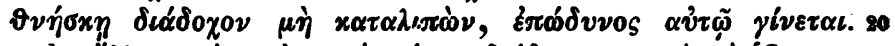

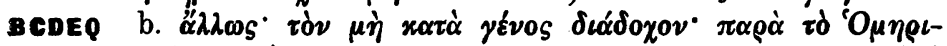

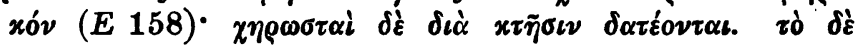

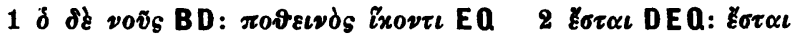

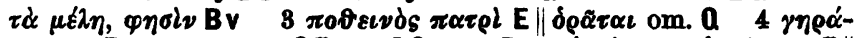

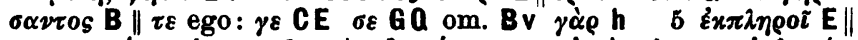

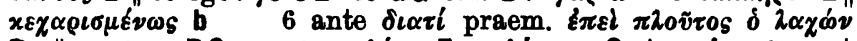

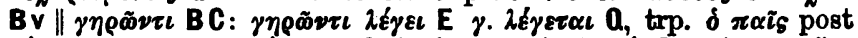

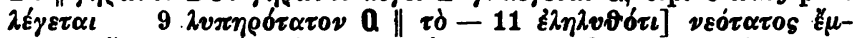
$\pi \alpha \lambda \iota \nu$,

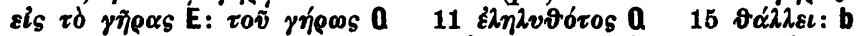

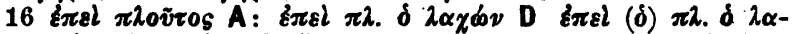

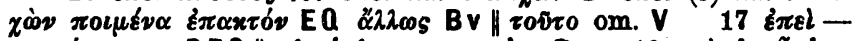

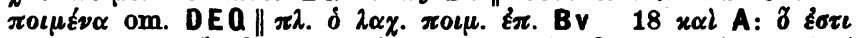

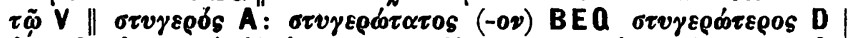

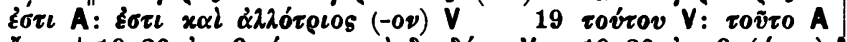

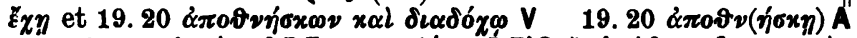

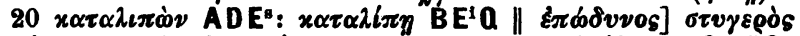

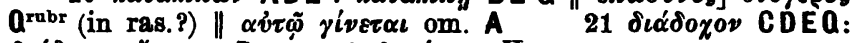

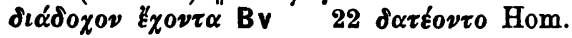




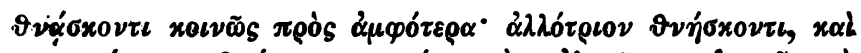

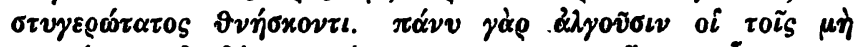

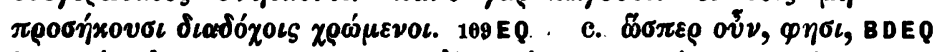

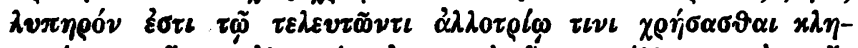

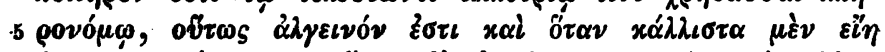

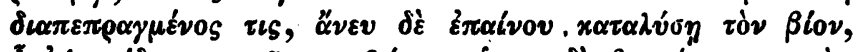

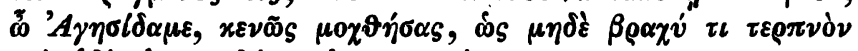

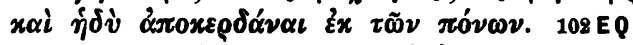

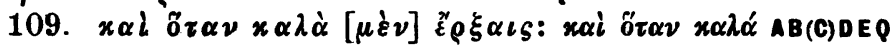

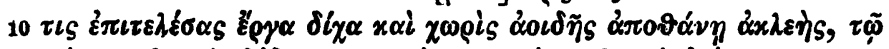

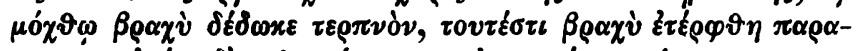

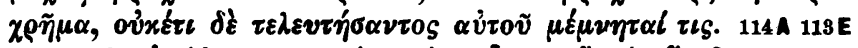

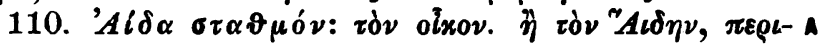

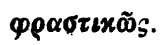

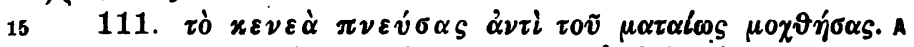

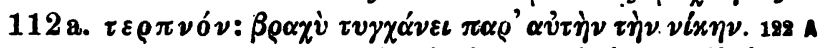

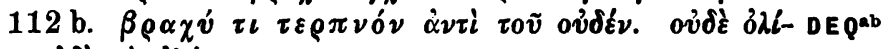

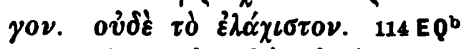

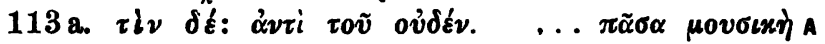

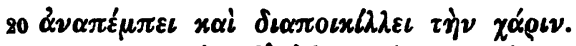

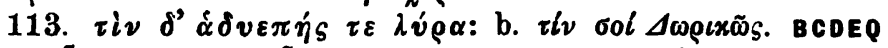
c. $\sigma 0 i$ ov้v,

17 sch. E 800

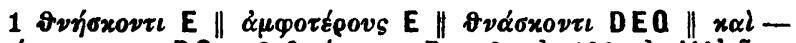

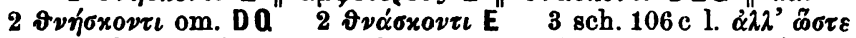

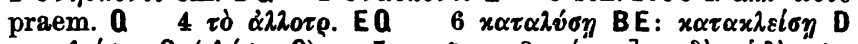

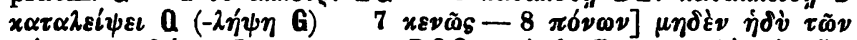

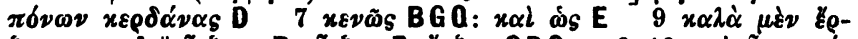

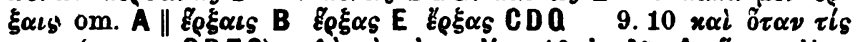

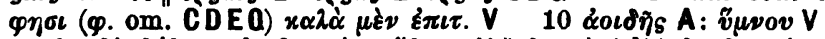

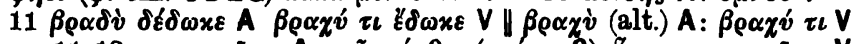

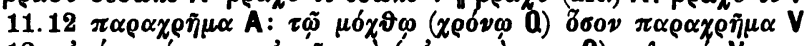

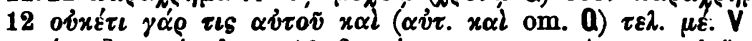

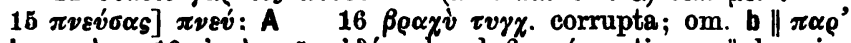

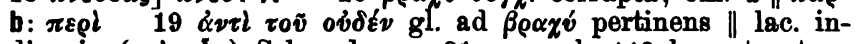
dicavi; 〈 d post $22 \chi_{\alpha}^{\alpha} \rho \iota v$ inser. Bv 21 l. om. E $\| \dot{\eta} \delta v \varepsilon \pi . \mathrm{DQ}$, om. $\tau \varepsilon \lambda \dot{\rho} \rho \alpha \|$

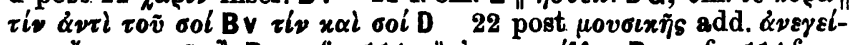

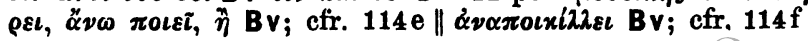




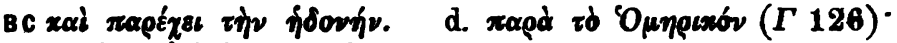

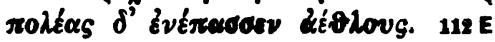

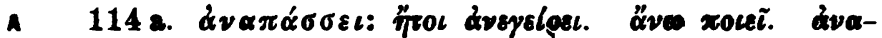

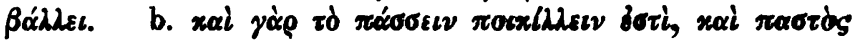

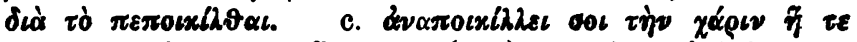

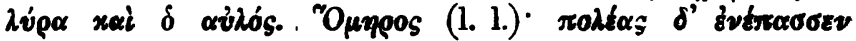
át?lovs.

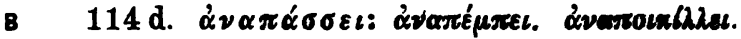

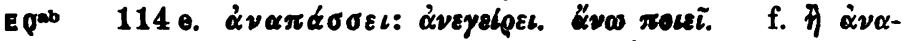

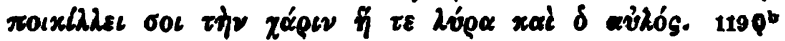

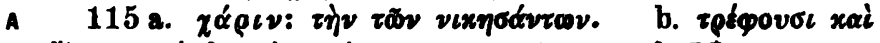

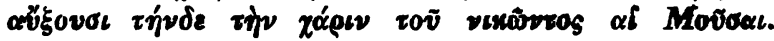

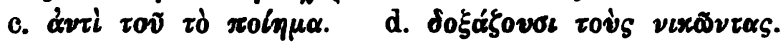

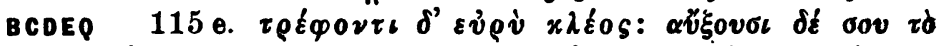

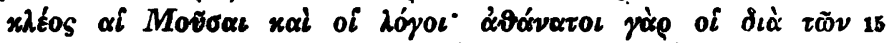

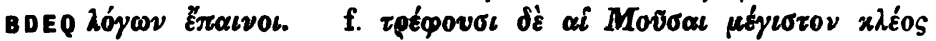

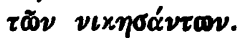

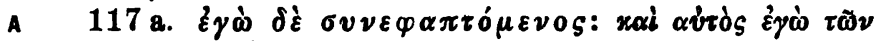

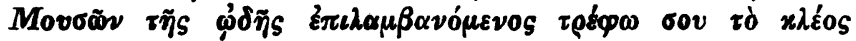

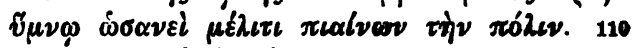

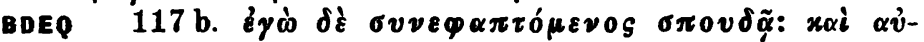

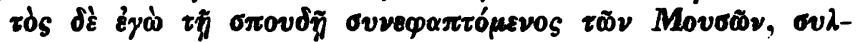

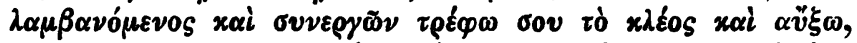

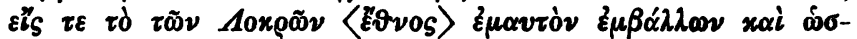

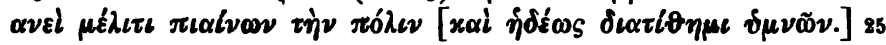

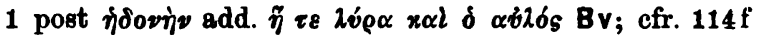

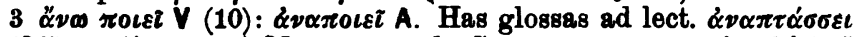
(GU) pertinere put. Mommsen sch. Germ. p. $56 \quad 3.4 \alpha v \alpha \beta \alpha ́ d \lambda \varepsilon i]$

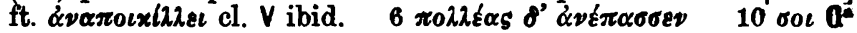

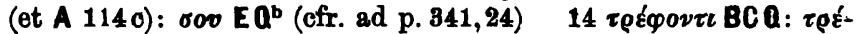

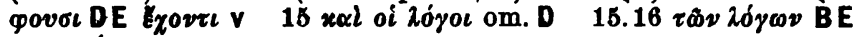

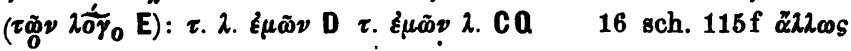

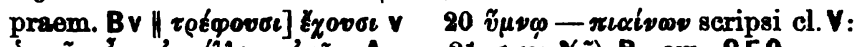

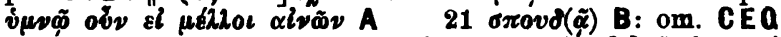

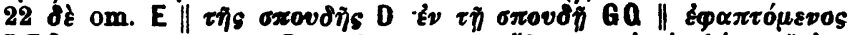

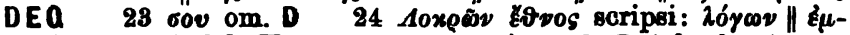

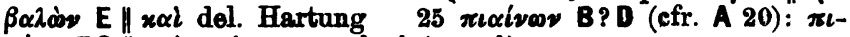
$\alpha i v \omega$ EQ $\| x \alpha i-v \mu v \omega v$ seclusi (om. A) 


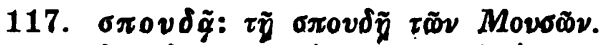

$\mathbf{B}$

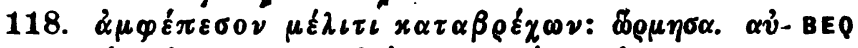

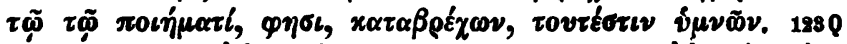

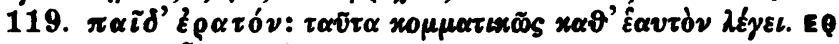

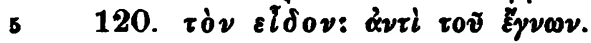

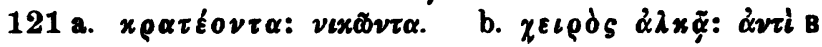

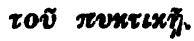

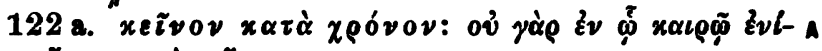

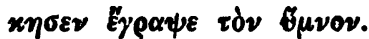

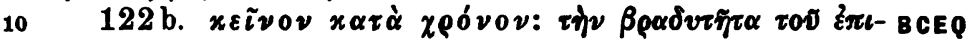

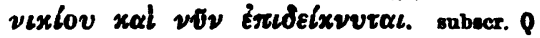

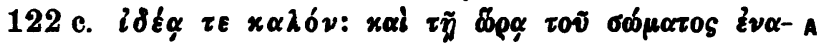

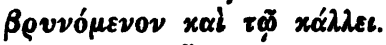

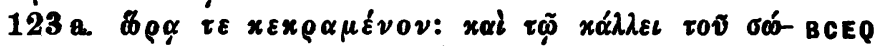

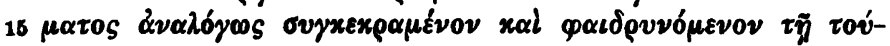

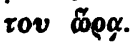

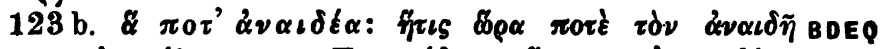

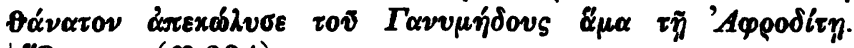

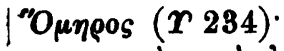

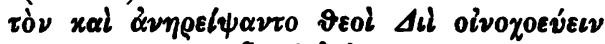

BCDEQ

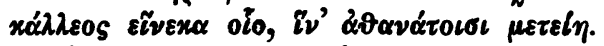

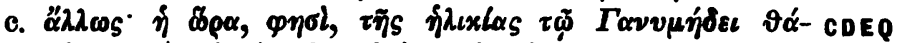

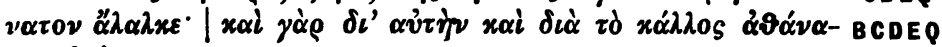

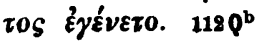

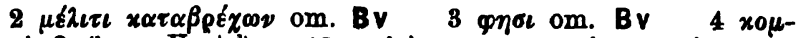

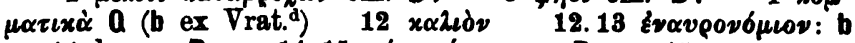

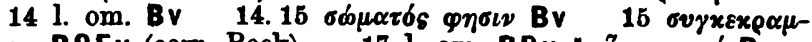

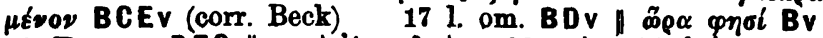

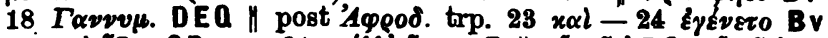

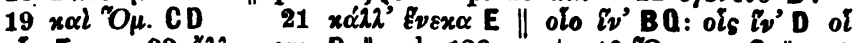

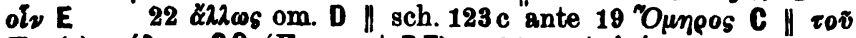

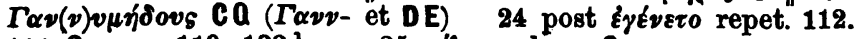
$114 \mathrm{Q}$; seq. 119. $122 \mathrm{~b} \quad 25 \tau \in \mathcal{L}_{0}$ s subser. $\mathrm{O}$ 


\section{Scholia in Olympionicarum carmen XI.}

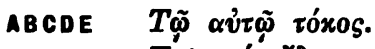

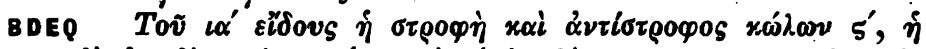

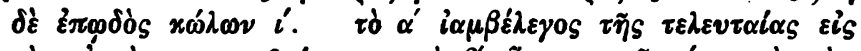

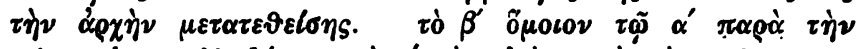

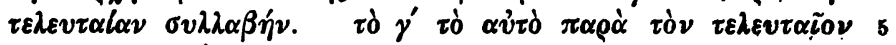

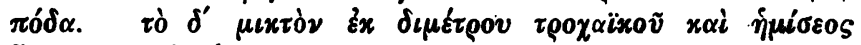

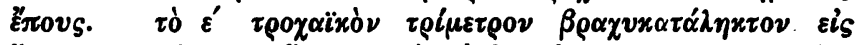

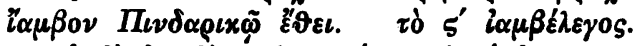

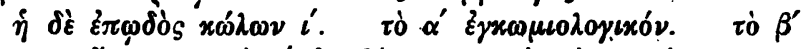

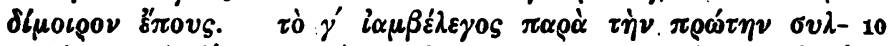

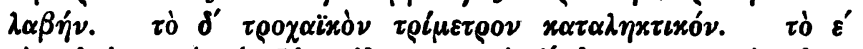

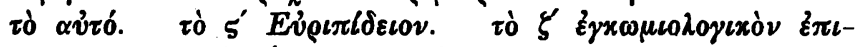

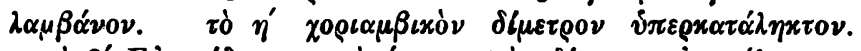

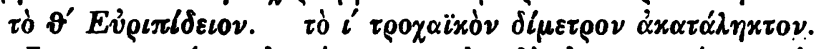

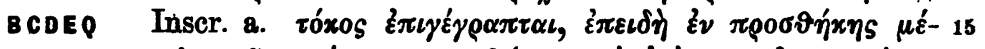

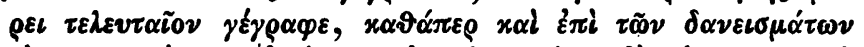

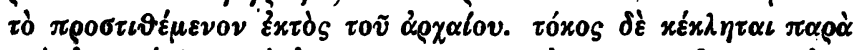

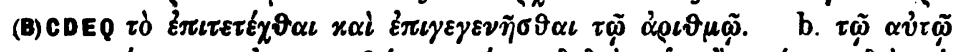

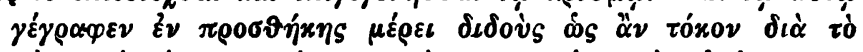

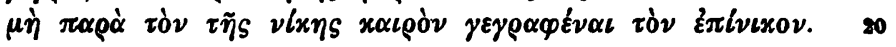

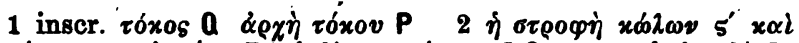

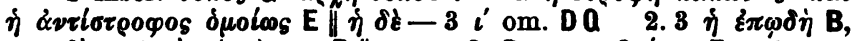

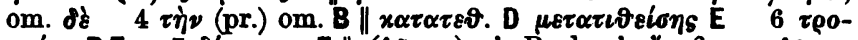

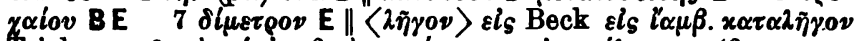

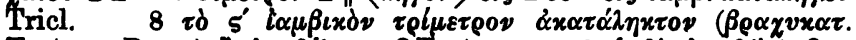

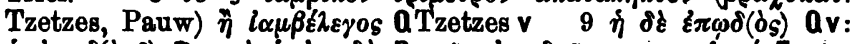

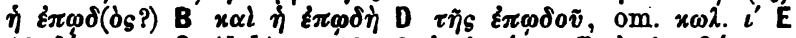

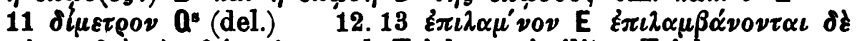

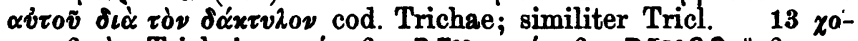

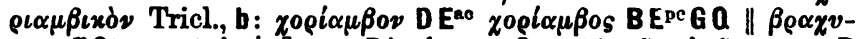

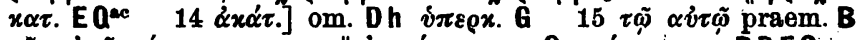

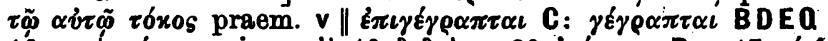

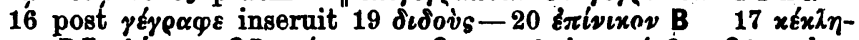

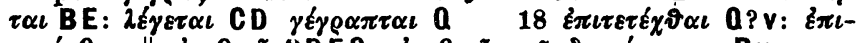

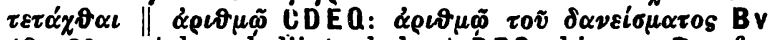
18-20 post l. scholii $1 \mathrm{c}$ habent $D E Q$; hic om. B, cfr. ad 16

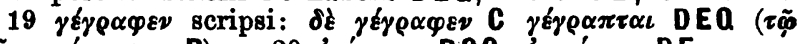

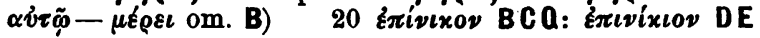




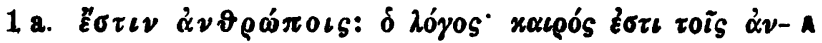

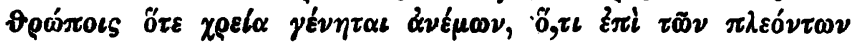

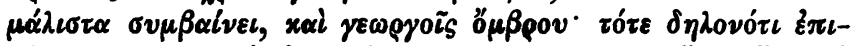

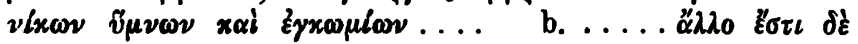

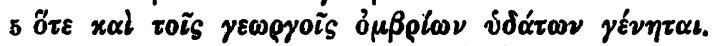

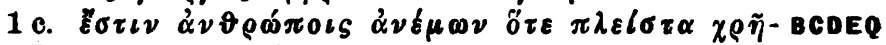

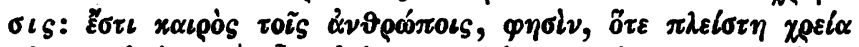

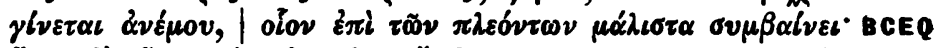

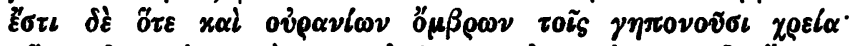

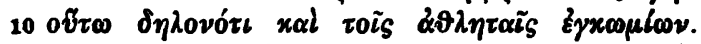
d. $\alpha \lambda \lambda \omega s^{\circ} \in Q$

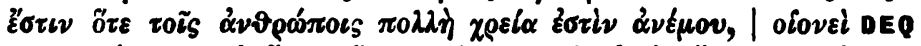

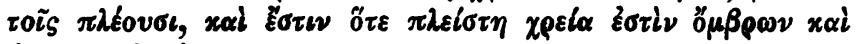

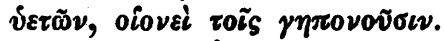

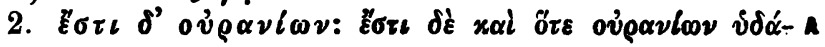

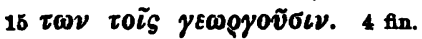

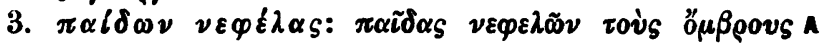

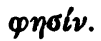

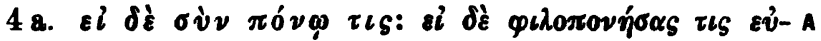

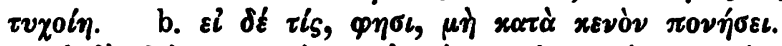

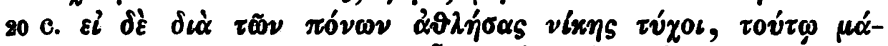

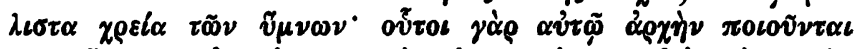

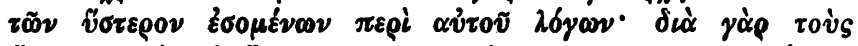

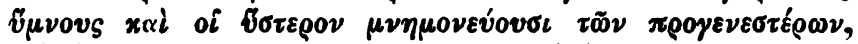

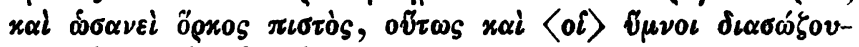

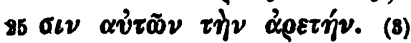

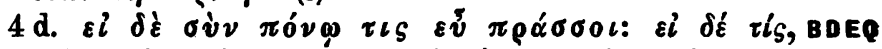

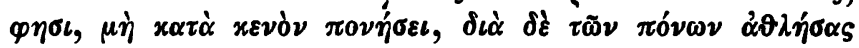

1 sch. $1 \mathrm{a}$ et $\mathrm{b}$ cum sch. $0 . \mathrm{X}$ coniunxit A 3 ö $\mu \beta \rho o v$ : ego $\|$

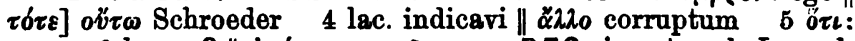
ego 6 1. om. C $\| \dot{\alpha} \nu \xi \mu \omega o \nu-\chi \rho \hat{\sigma} \sigma \iota_{\zeta}$ om. DEQ, inserto sch. Inscr. b

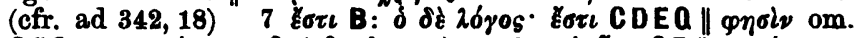

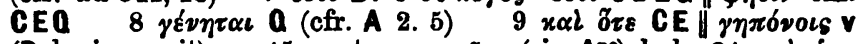

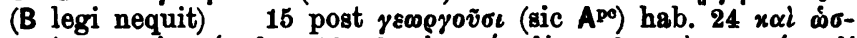

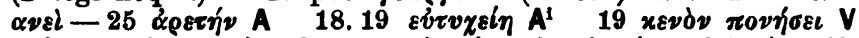

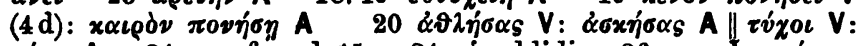

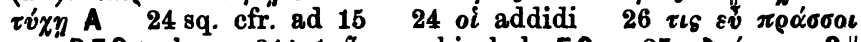

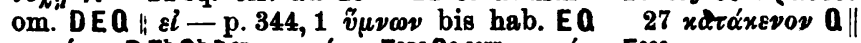
$\pi 0 \nu \eta^{\prime} \sigma \varepsilon \iota \mathrm{BE}^{\mathrm{b}} Q^{\mathrm{b}} \mathrm{D}^{\mathrm{c} p}: \pi 0 \iota \eta^{\prime} \sigma \varepsilon l \mathrm{E}^{\mathrm{apc}} Q^{\mathrm{a}}$ corr $\pi 0 \iota \eta^{\prime} \sigma \eta \mathrm{E}^{\mathrm{aac}}$ 


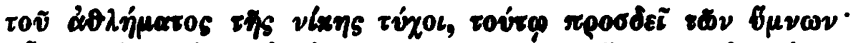

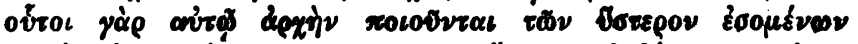

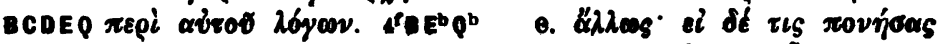

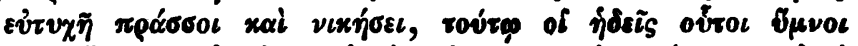

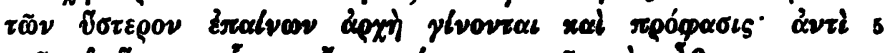

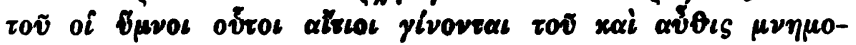

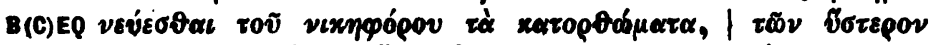

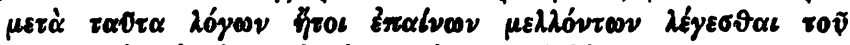

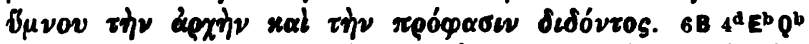

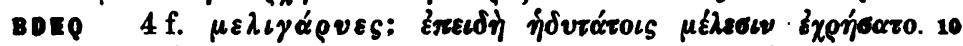

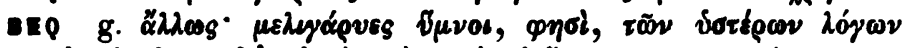

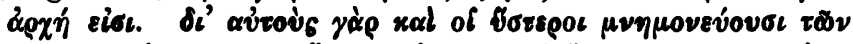

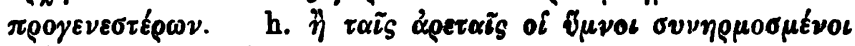

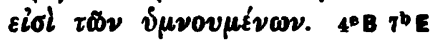

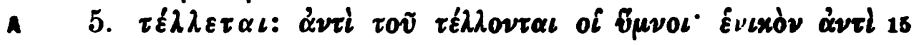
$\pi \lambda \eta \vartheta v v \tau\llcorner x 00$.

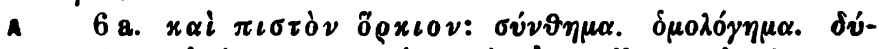

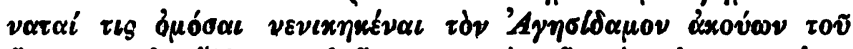

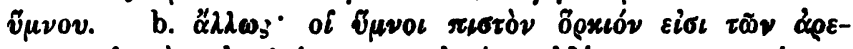

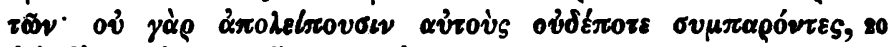

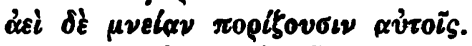

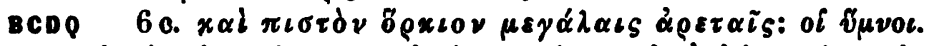

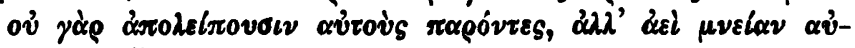

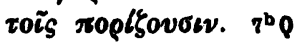

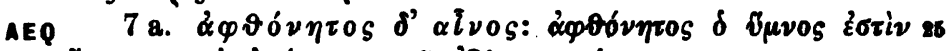

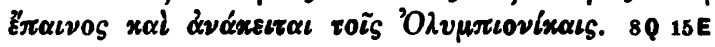

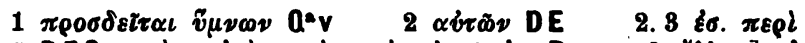

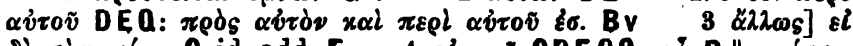

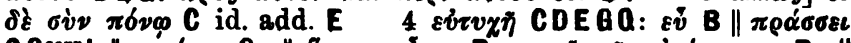

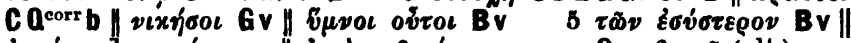

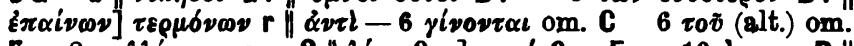

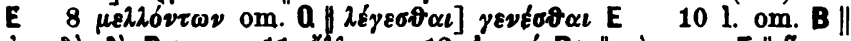

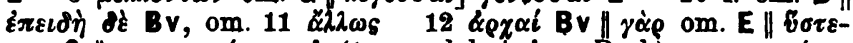

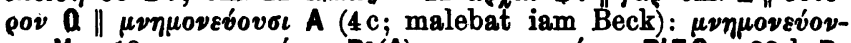

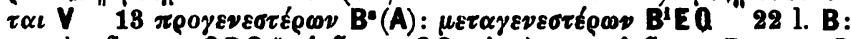

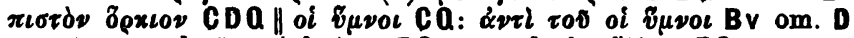

25 sqq. sch. 7 a et b trp. EQ 25 l. A: \&2A20s EQ 25 sq.

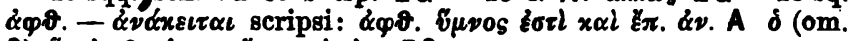

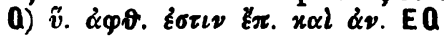




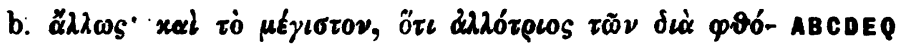

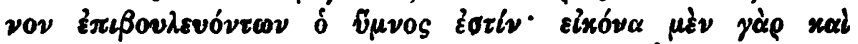

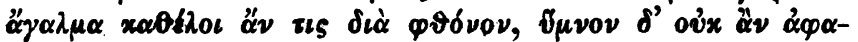

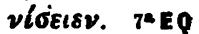

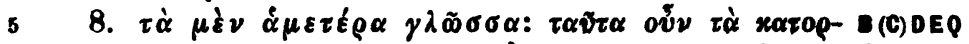

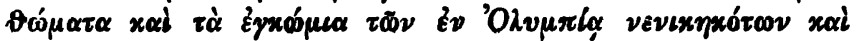

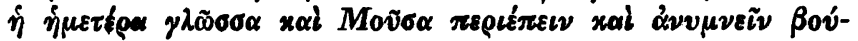
$\lambda i \tau \alpha \iota$.

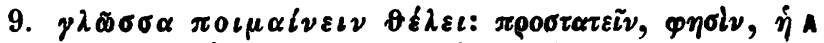

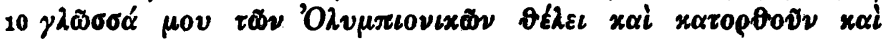

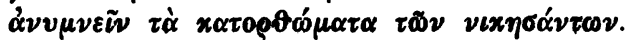

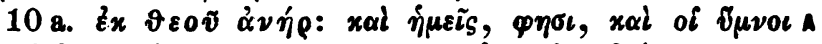

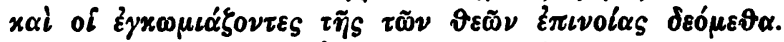

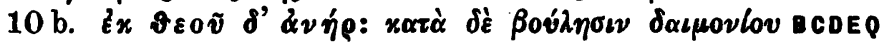

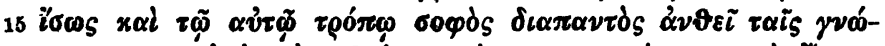

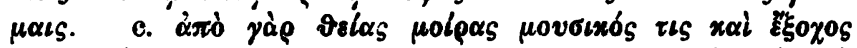

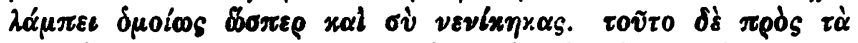

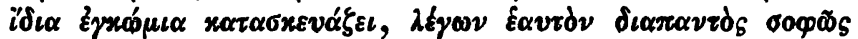
$\varphi \rho \alpha ́\} \varepsilon เ \nu$.

30 11.

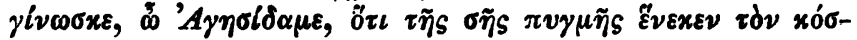

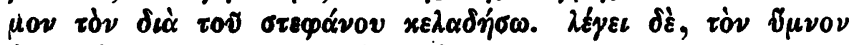

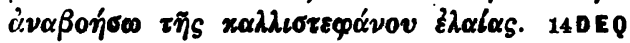

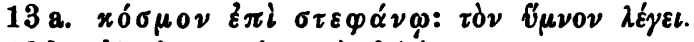

CDEQ

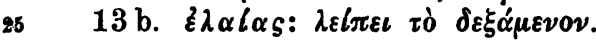

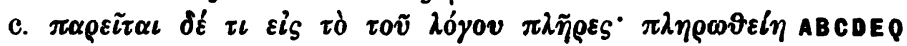

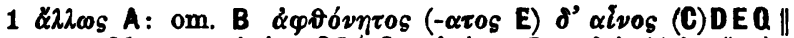

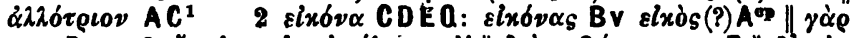

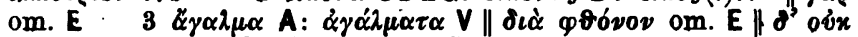

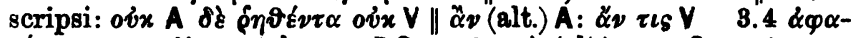
víбol 8. $-\sigma \eta \mathrm{V}$ 5 l. om. DQ $6 x \alpha i$ (alt.) om. C $9 \pi \rho 0-$

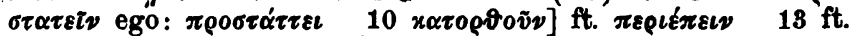

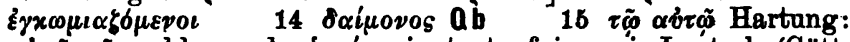
$\alpha b \tau \tilde{\omega} \tau \tilde{\omega}$ codd.; unde $\delta \mu o i \omega s$ in textu fuisse cj. Leutsch (Grott. gel. Anż. 1861, p. 1549), praeeunte Hartungio ( $\left.\delta \mu \omega_{\xi}\right) 17 \delta \mu$ oíos

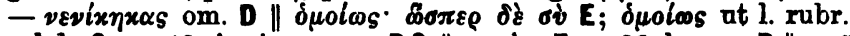

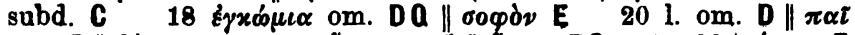

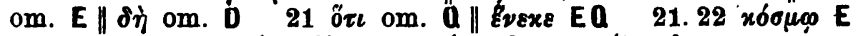
$26 \pi \alpha \rho \varepsilon \tilde{\tau} \alpha \iota$ dé $\tau \iota$ V: $\pi \alpha \rho \alpha \pi \eta^{\prime} \xi \varepsilon \iota A^{\star c}-\pi \alpha i \zeta \varepsilon \iota A^{p c}$ 


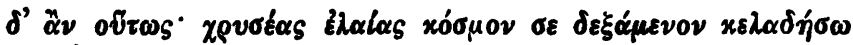

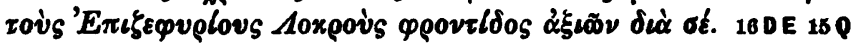

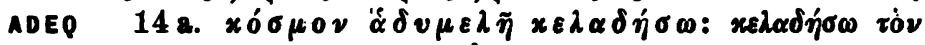

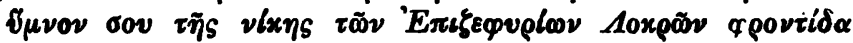

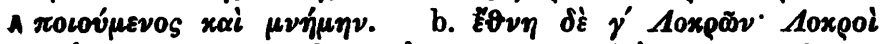

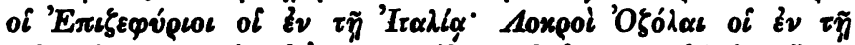

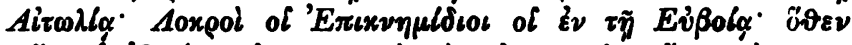

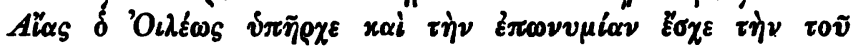

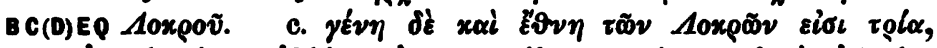

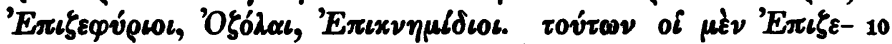

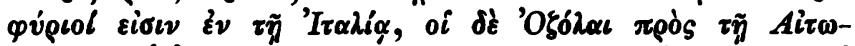

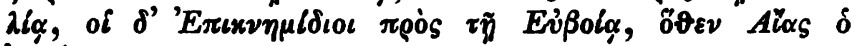
'Oìź⿴囗. 18 DEQ

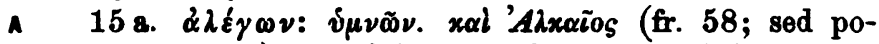

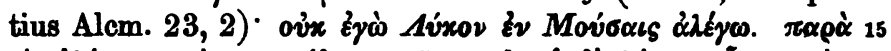

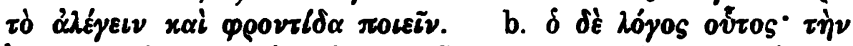

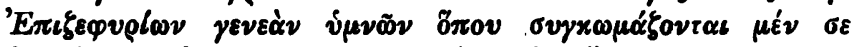

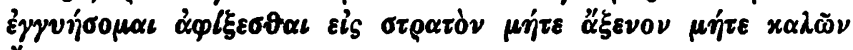

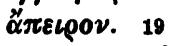

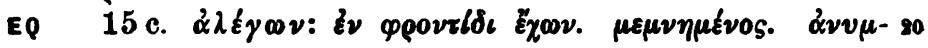

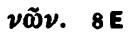

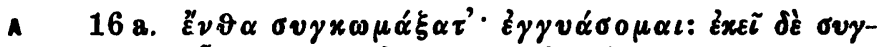

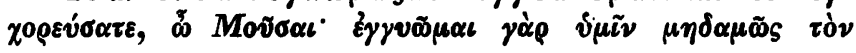

5 sch. A B 527

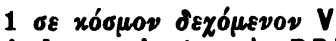

2 Aoxpov̀s qe. \&. C: 2óyovs

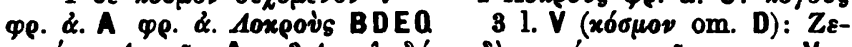

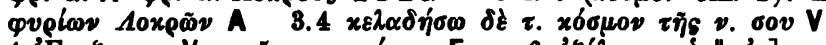

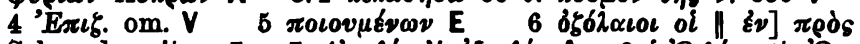

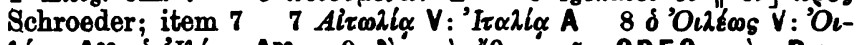

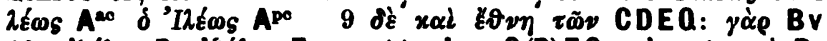

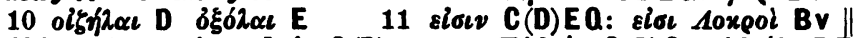

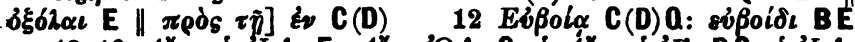

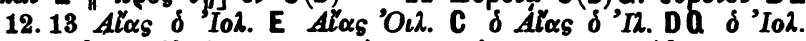

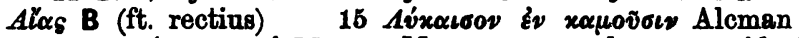

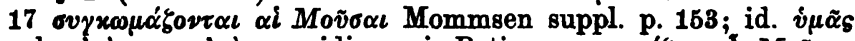

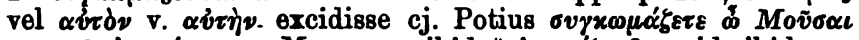

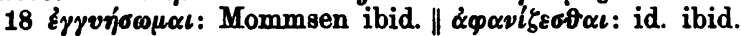
23 ทे $\mu \tau$ : 


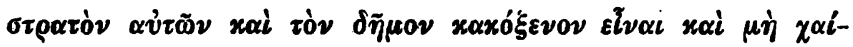

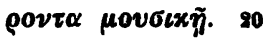

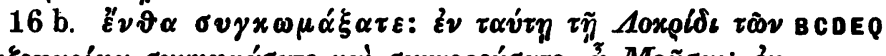

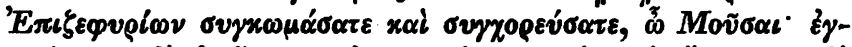

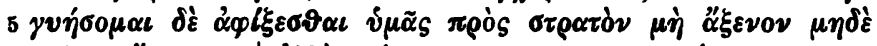

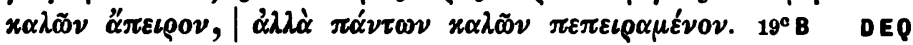

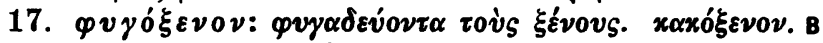

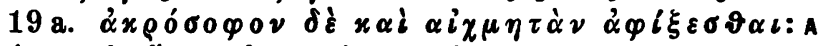

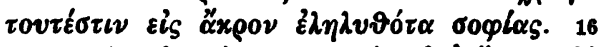

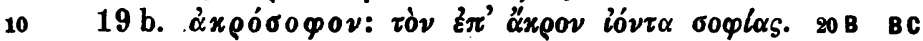

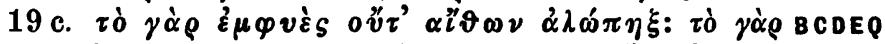

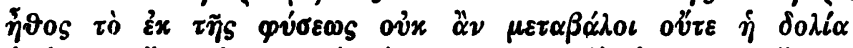

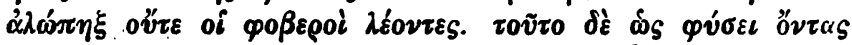

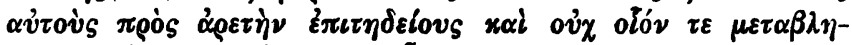

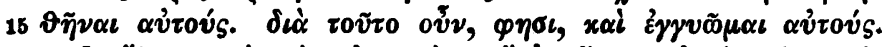

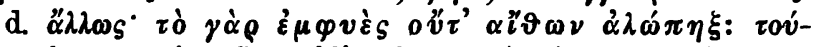

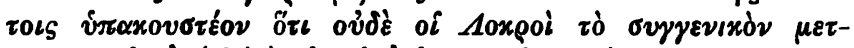

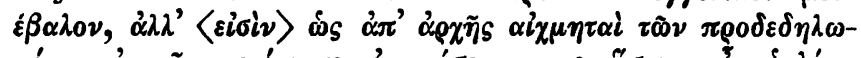

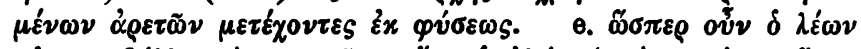

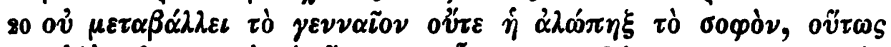

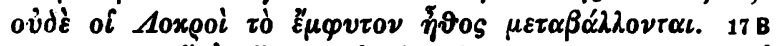

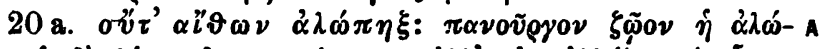

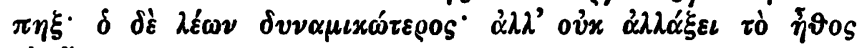

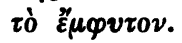

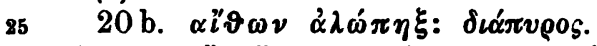

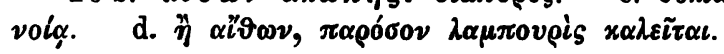

25 Ap. soph. 13, 27. Hesych. EM 32, 33. Zon. 68

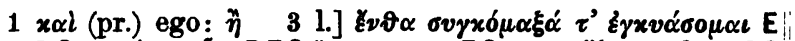

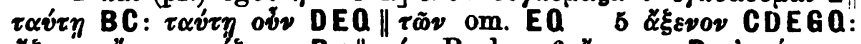

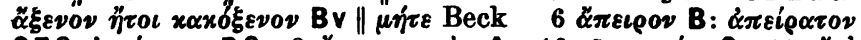

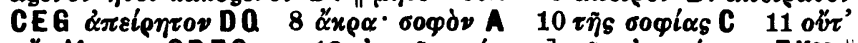

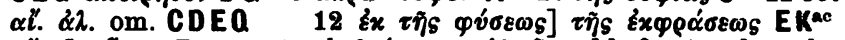

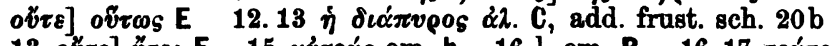

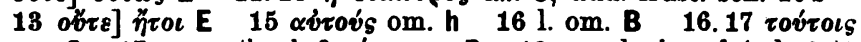
om. D $17 \sigma v \gamma \gamma$ : $i \grave{\eta} \nu \dot{\alpha} \nu \delta \rho \varepsilon i \alpha \nu \mu \varepsilon \tau$. D 18 supplevi ex A (sch. 21):

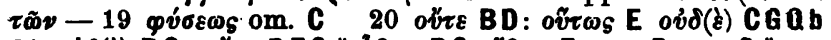

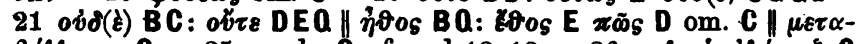

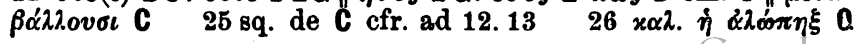




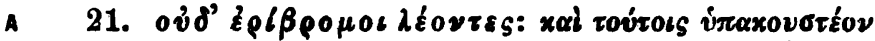

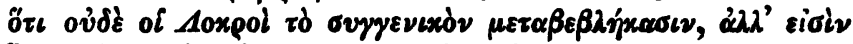

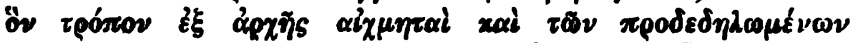

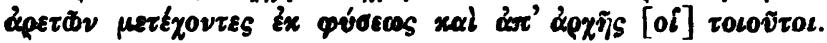

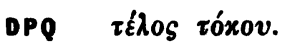

\section{Scholia in Olympionicarum carmen XII.}

Ac 'E

BDEF 'E

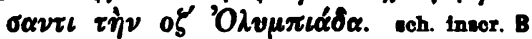

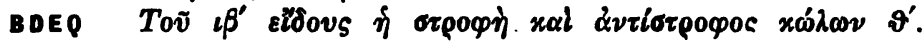

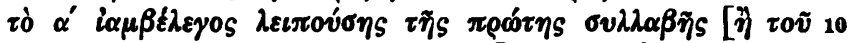

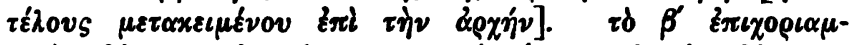

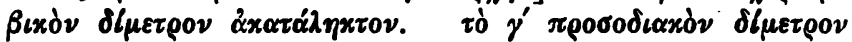

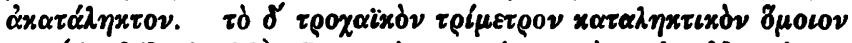

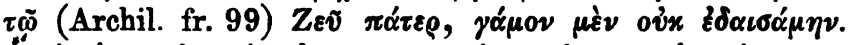

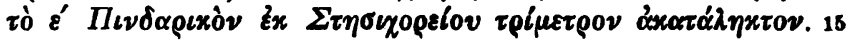

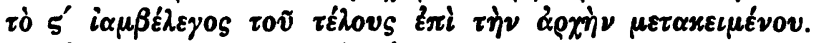

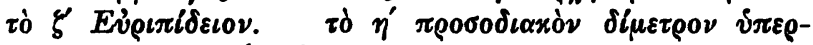

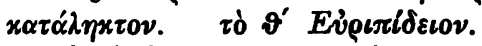

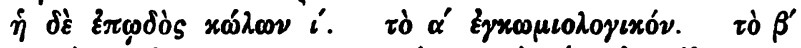

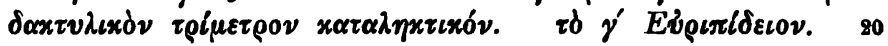

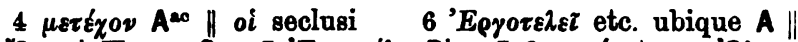

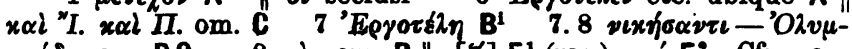
$\pi \iota \alpha \dot{\delta} \alpha$ om. Da $8 \tau \eta \dot{\nu}$ om. B \| o[క] $F^{1}$ (ras.) $\pi \eta^{\prime} F^{2}$. Cfr. pap.

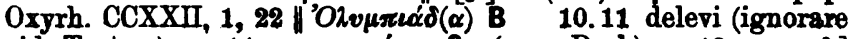

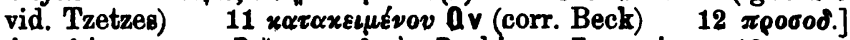

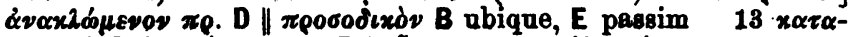

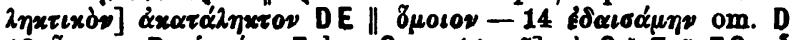

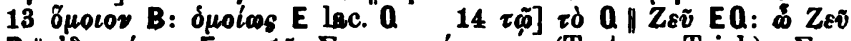

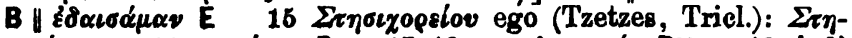

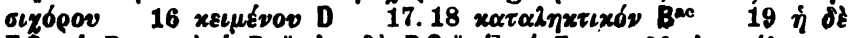

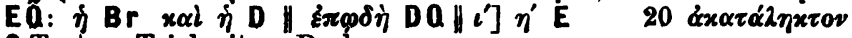
Q Tzetzes Tricl., item Beck 


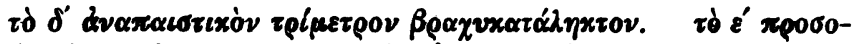

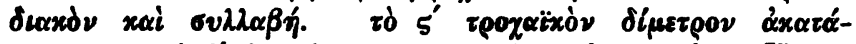

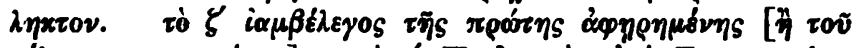

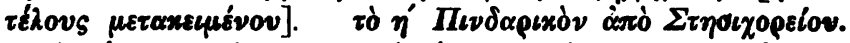

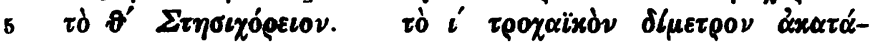

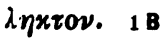

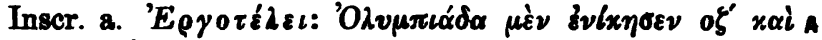

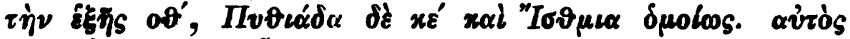

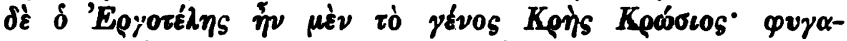

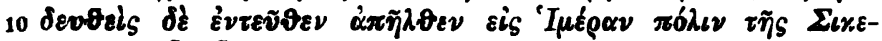

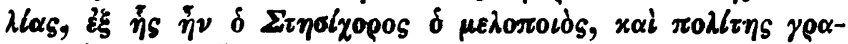

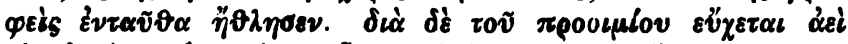

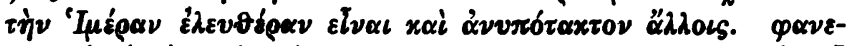

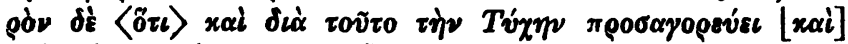

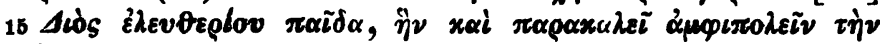
'I $\mu$ épouv.

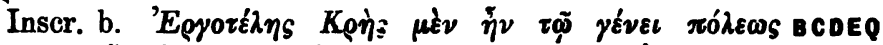
$K \nu \omega \sigma \sigma o \tilde{v}$, ôs $\eta \gamma$

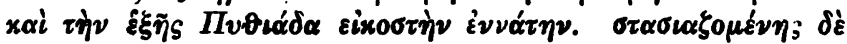

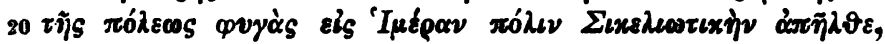

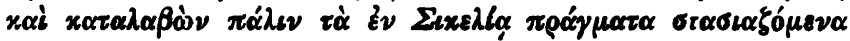

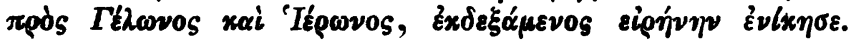

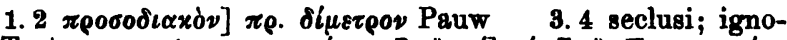

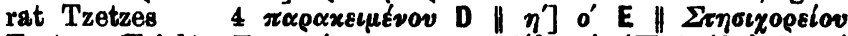

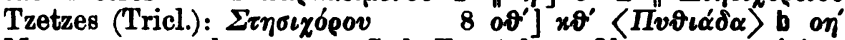
Mommsen suppl. p. 155. Sed Ergotelem 01. 78 non vicisse constat ex Pap. Oxyrh. CCXXII; at de loco emendando desperand. vid. $o \vartheta^{\prime}$ ex $x \vartheta^{\prime}$ ortum vid. $\| x \varepsilon^{\prime} A:\langle x \alpha i\rangle x \varepsilon^{\prime}$ b

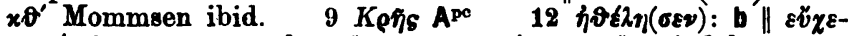

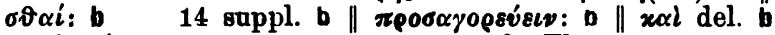
$15 \alpha \mu \varphi i \pi v \lambda_{\imath}$ : $17-$ p. 350,2 cfr. Thom. p. 376, 21

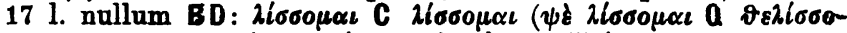

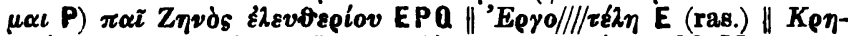

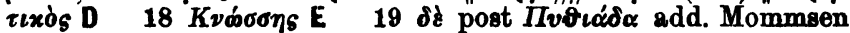

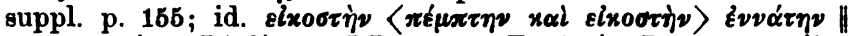

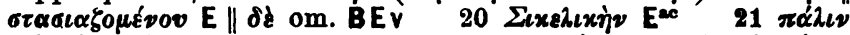

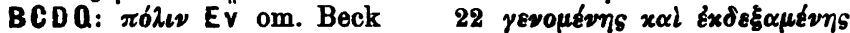

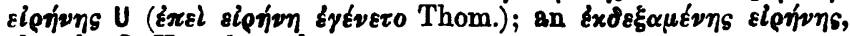
cl. sch. O. II, 34 b. $39 \mathrm{~b}$ ? 


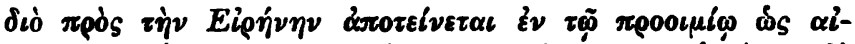

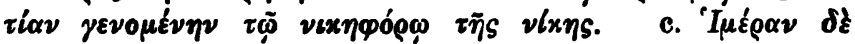

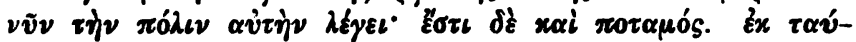

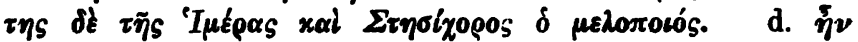

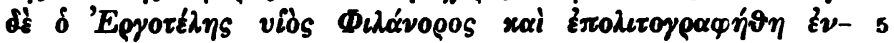
$\tau \alpha \tilde{v} \vartheta \alpha$. metr. B

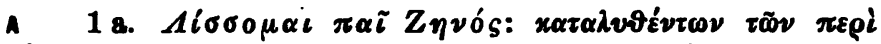

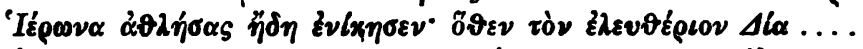

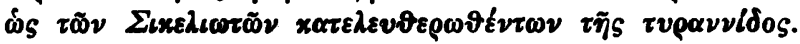

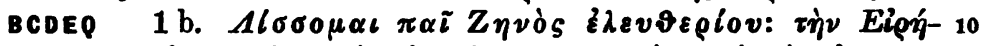

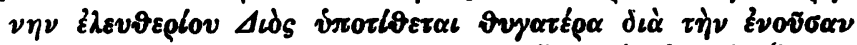

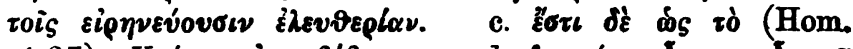

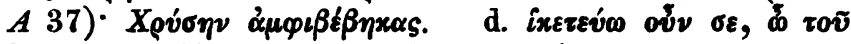

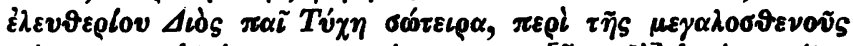

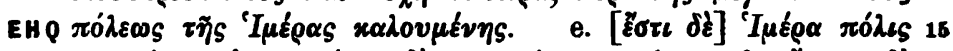

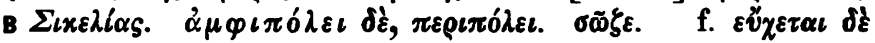

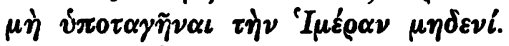

EQ 4 a. $\tau i v:$ ool $\Delta \omega \rho$ Lxõos.

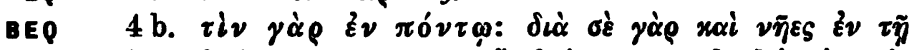

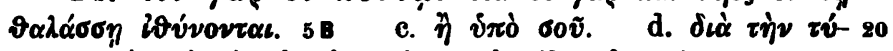

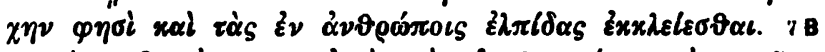

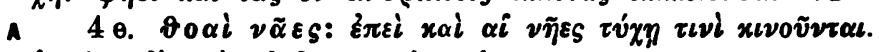

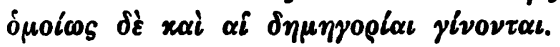

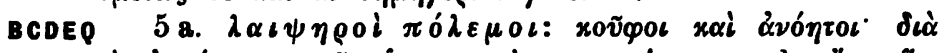

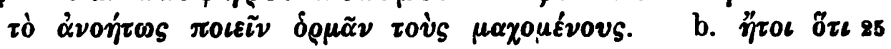

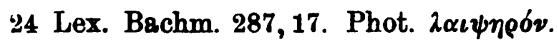

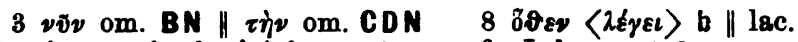
indicavi $9 \cos A$ : $\varepsilon \pi i$ b 10 sqq. cfr. Lehrs p. $118 \mathrm{sqq}$.

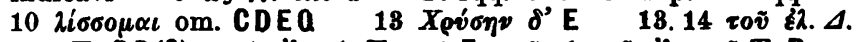
$\pi \alpha \tau$ T. C D (0): $\tau 0 \tilde{v} \varepsilon \lambda$. $\Delta$. T. $\pi \alpha \tilde{\imath} \mathrm{E} \tau 0 \tilde{\Delta} \Delta . \tau 0 \tilde{v} \varepsilon \lambda$. $\pi \alpha \tau T$. BV

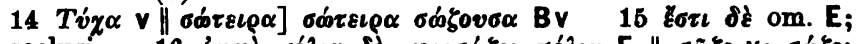

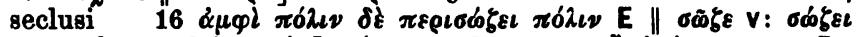

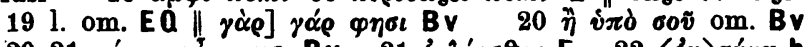

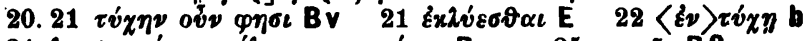

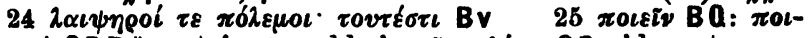

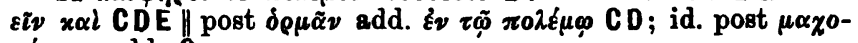
$\mu \varepsilon \dot{\nu} 0 v s$ add. Qv 


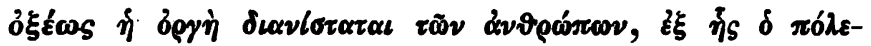
Mos. 8 B

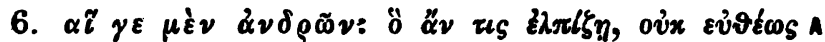

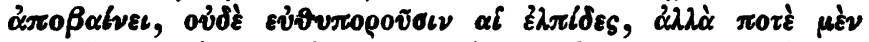

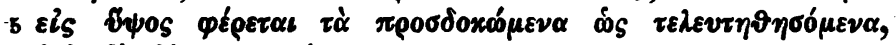

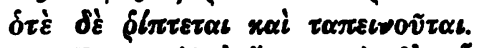

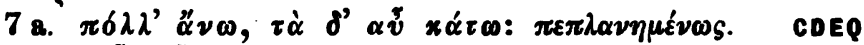

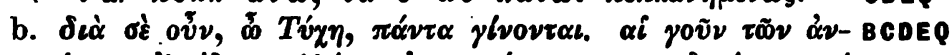

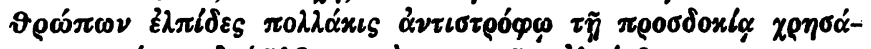

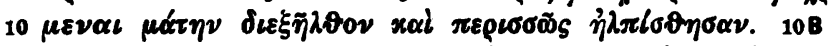

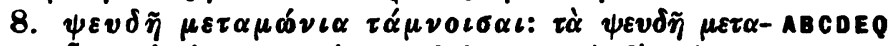

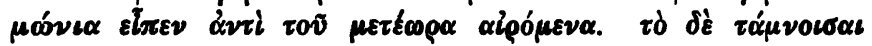

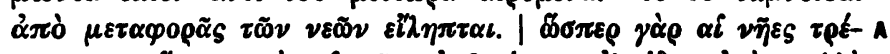

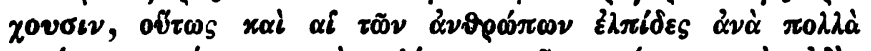

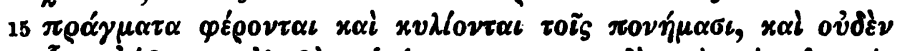

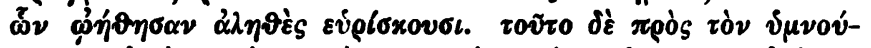

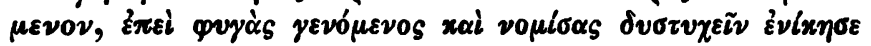
$\pi \alpha \rho \dot{\alpha} \pi \rho 0 \sigma \delta 0 x l \alpha \nu$. AdB $^{\mathrm{d}}$

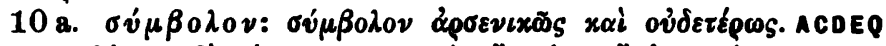

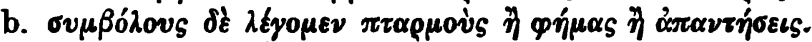

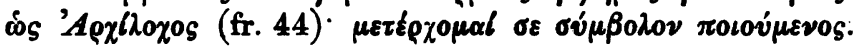

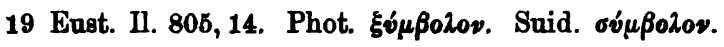

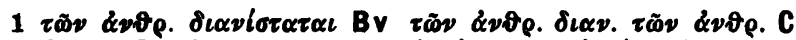

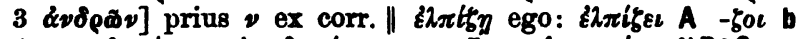

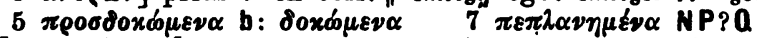

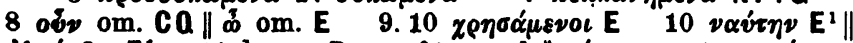

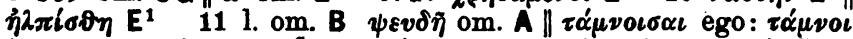

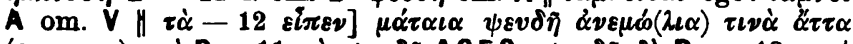

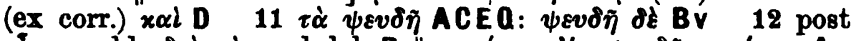

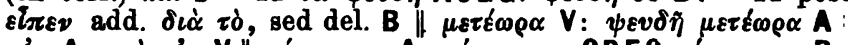

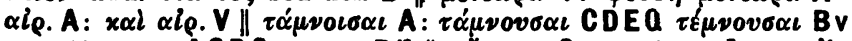

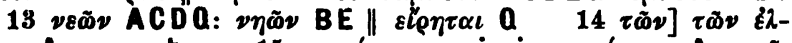

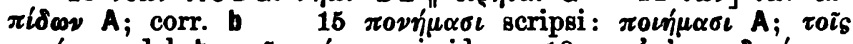

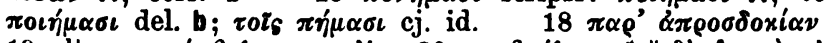

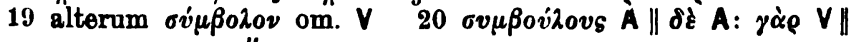

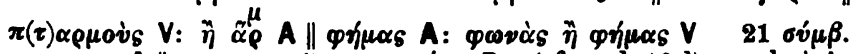

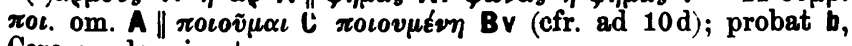
Cererem loqui ratus 


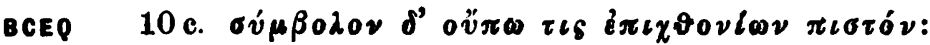

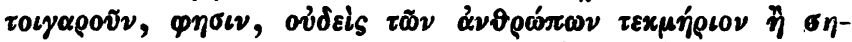

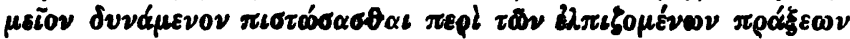

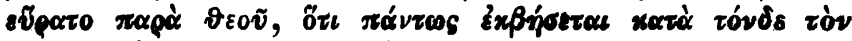

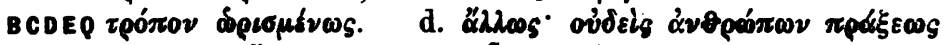

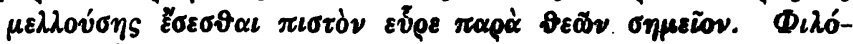

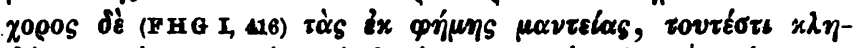

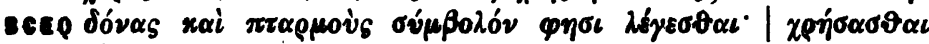

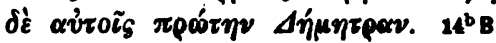

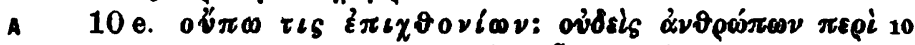

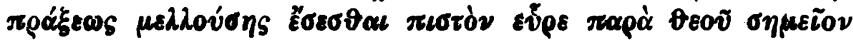

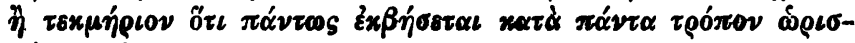

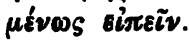

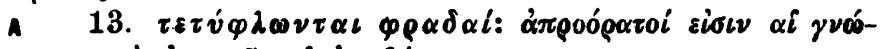

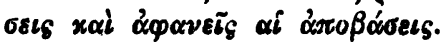

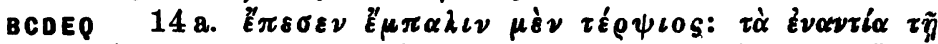

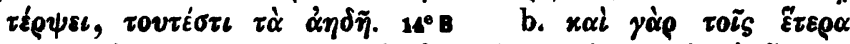

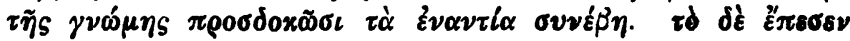

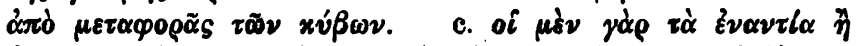

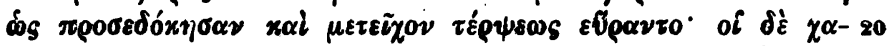

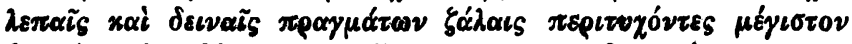

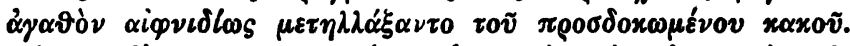

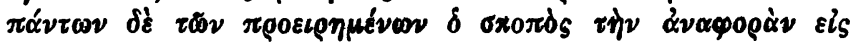

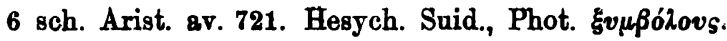

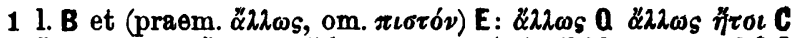

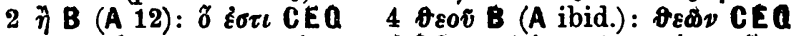

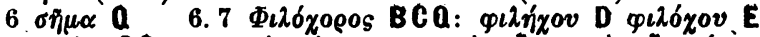

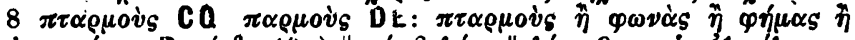

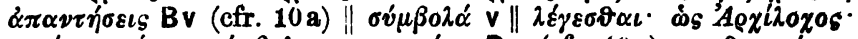

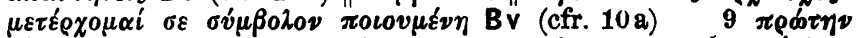

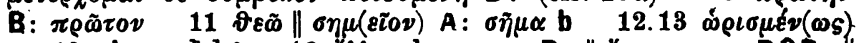

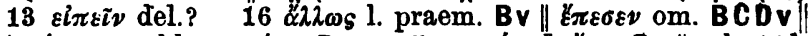

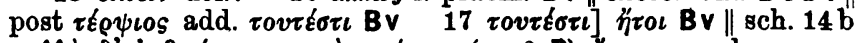

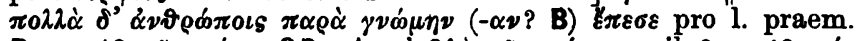

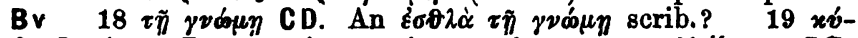

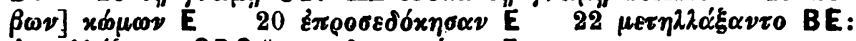

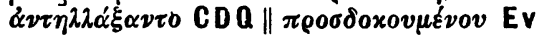


$\tau \dot{\alpha} \nu$ 'E

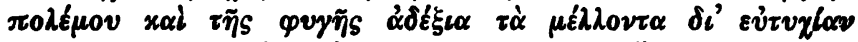

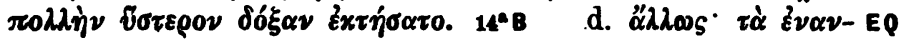

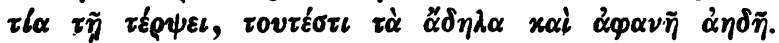

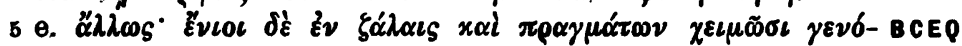

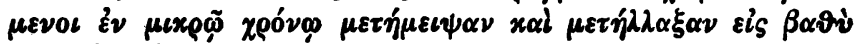

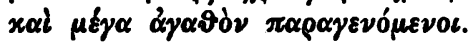

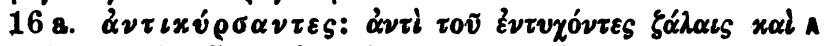

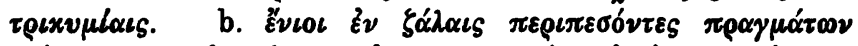

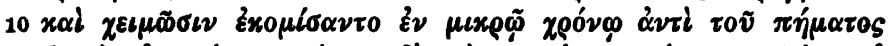

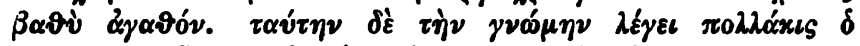

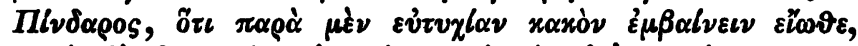

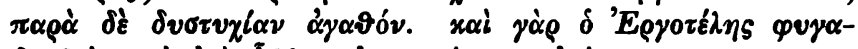

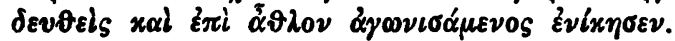

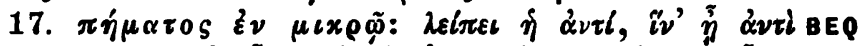

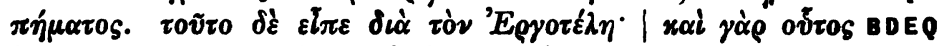

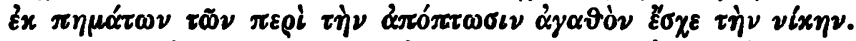

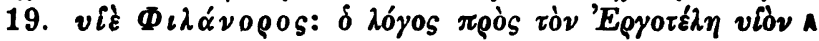

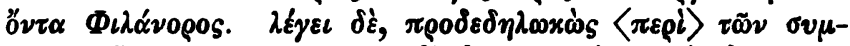

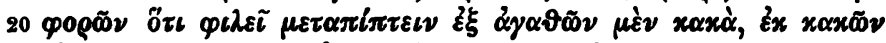

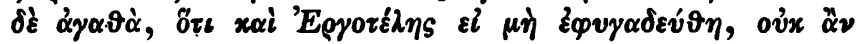
$\dot{\eta} \gamma \omega \nu$ i $\alpha \tau \sigma$.

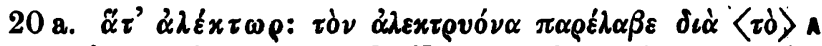

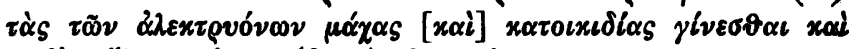

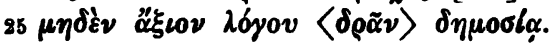

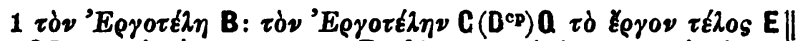

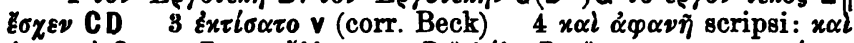

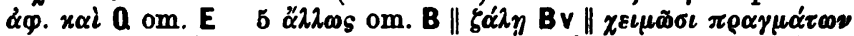
BV 5.6 $6 \varepsilon v \delta \mu \varepsilon v \alpha$ E $6 \varepsilon_{S}$ B $7 x \alpha \downarrow \mu \varepsilon y \alpha \alpha \gamma \alpha \vartheta \delta \nu$ B (cfr. A

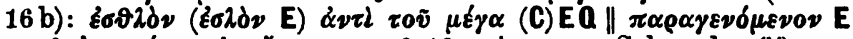

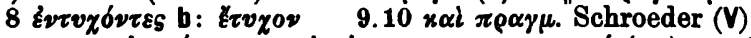

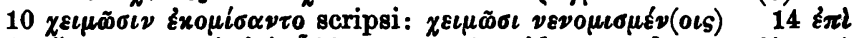

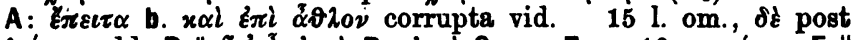

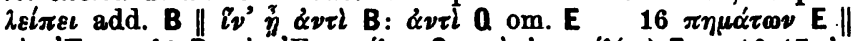
$\tau \dot{~} \nu$ 'E

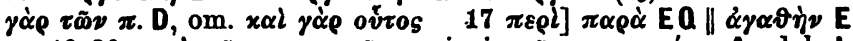

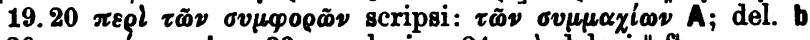

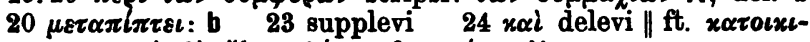

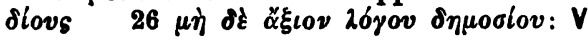

Bchor. m Prwdarux ed. Drachmann. 


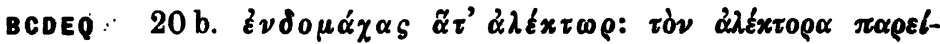

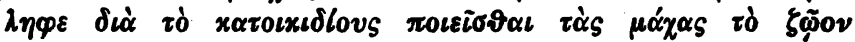

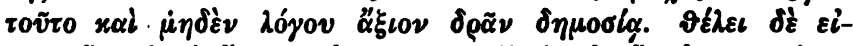

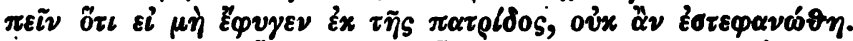

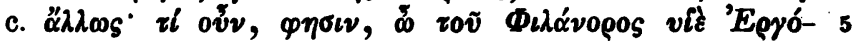

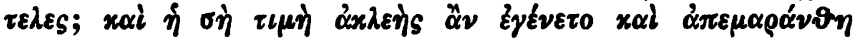

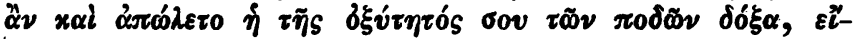

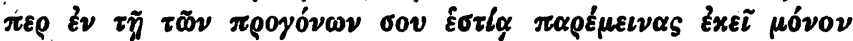

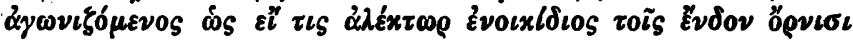

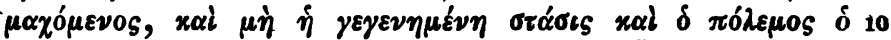

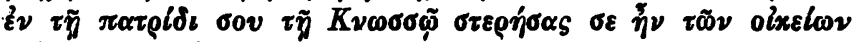

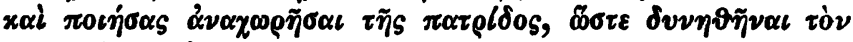

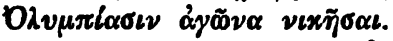

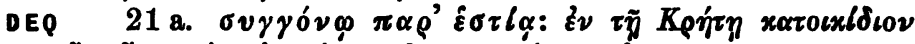

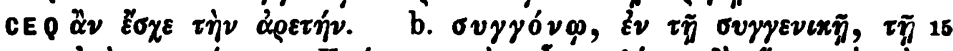

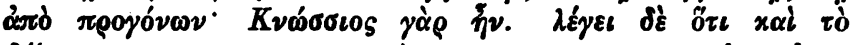

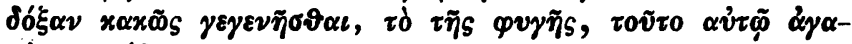
จ่v $\pi \rho \circ \varepsilon \beta \eta$.

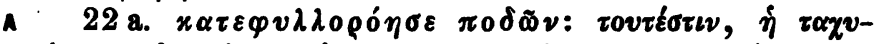

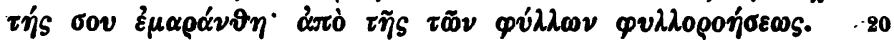

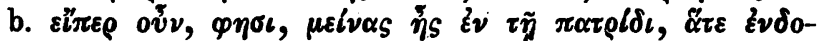

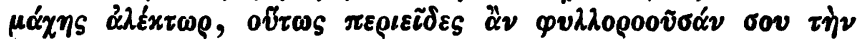

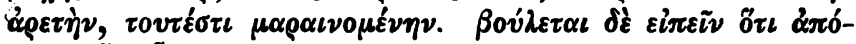

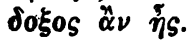

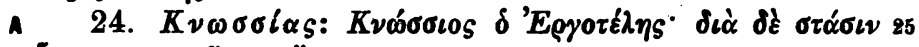

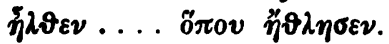

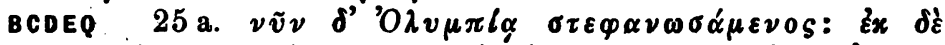

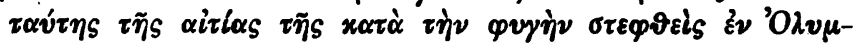

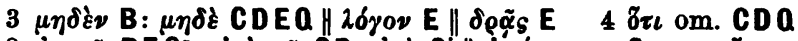

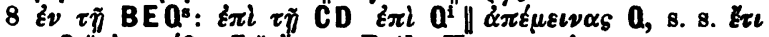

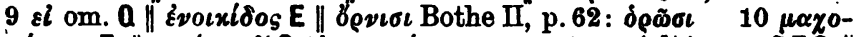

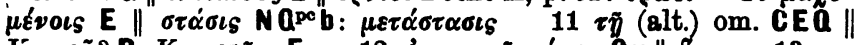

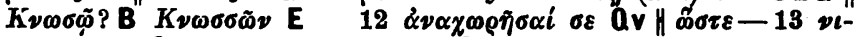

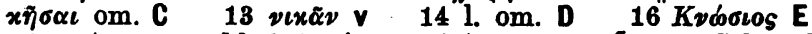

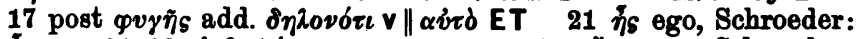

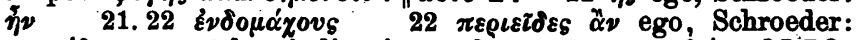
$\pi \varepsilon \rho \imath \delta^{\delta} \varepsilon \sigma \alpha \nu 26$ lac. indicavi 27 l. om. D $28 \alpha i \tau i \alpha$ CDEQ:

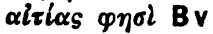




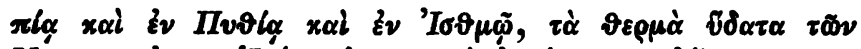

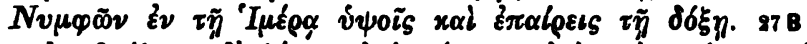

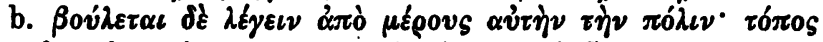

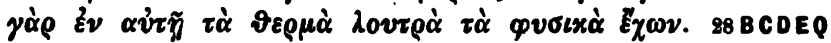

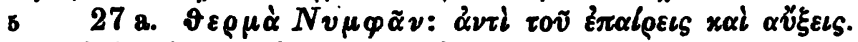

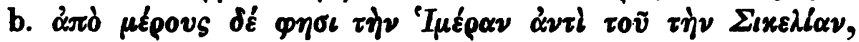

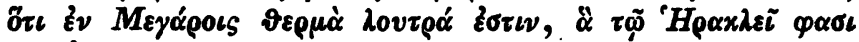

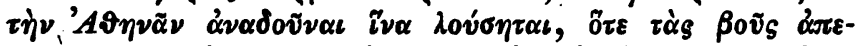

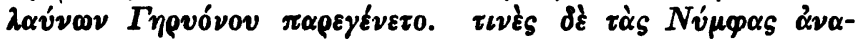

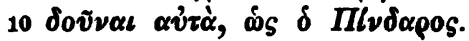

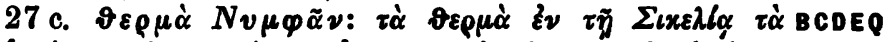

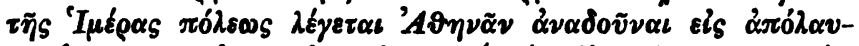

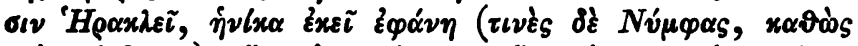

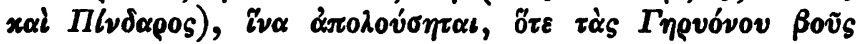

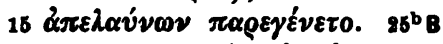

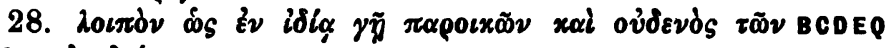

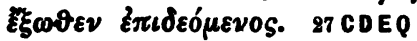

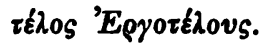

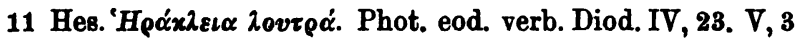

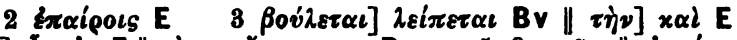

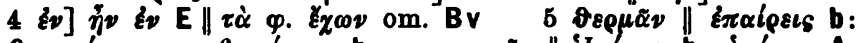

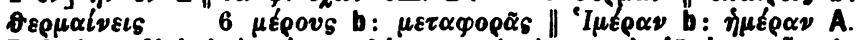

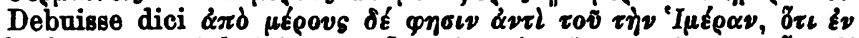

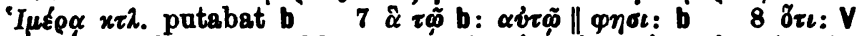

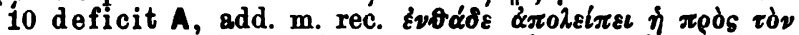

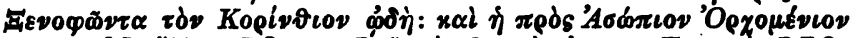

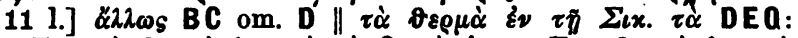

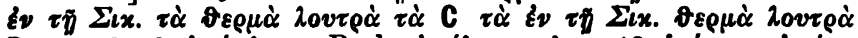

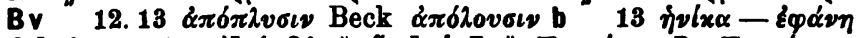

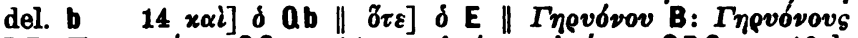

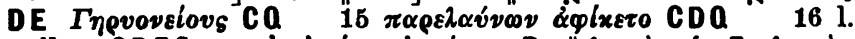

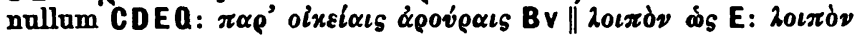
$x \alpha \ell$ is CDQ $\dot{\text { ws }} \mathrm{BV}$ 


\section{Scholia in Olympionicarum carmen XIII.}

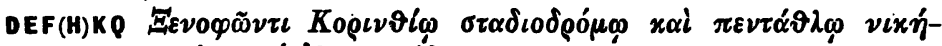

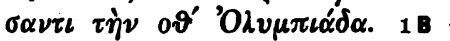

C

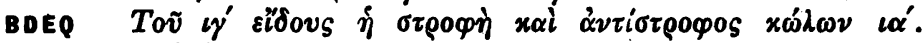

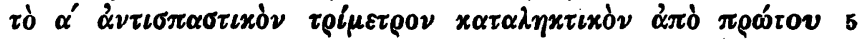

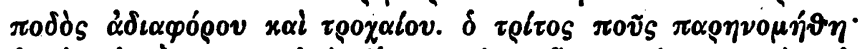

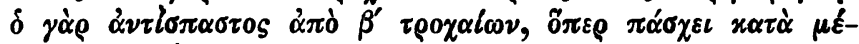

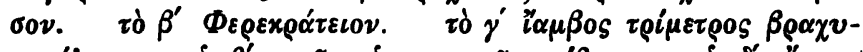

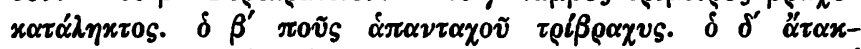

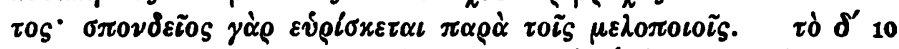

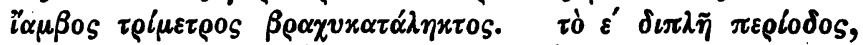

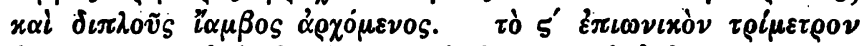

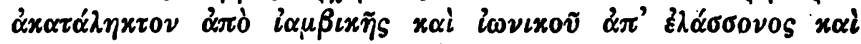

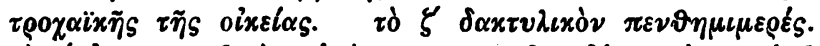

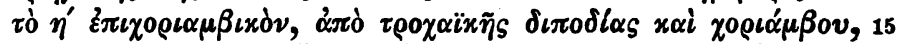

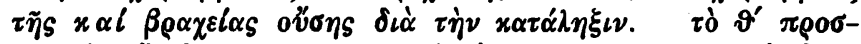

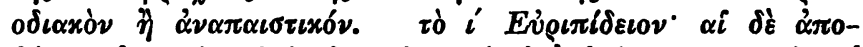

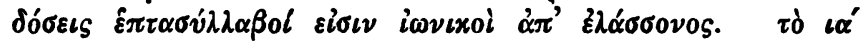

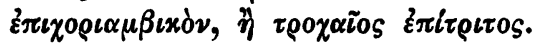

$2 \tau \dot{\eta} \nu$ om. E H $\left.\sigma \vartheta^{\prime}\right] \pi \vartheta^{\prime} F^{2} \quad 5 x \alpha \tau \alpha \lambda \eta x \tau \iota x \dot{\nu} \nu$ Q v : om.

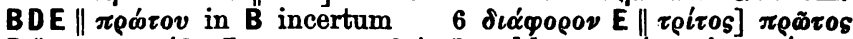

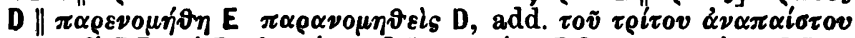

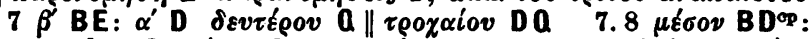

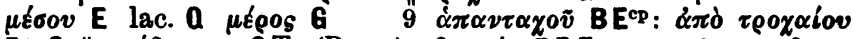

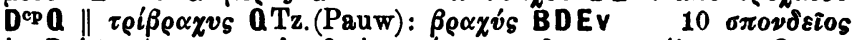

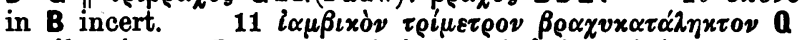

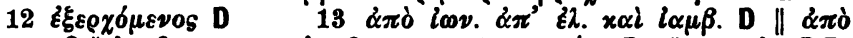

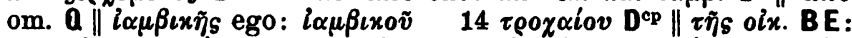

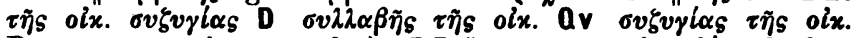

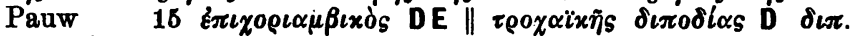

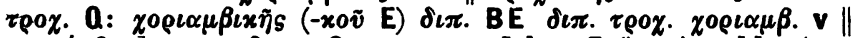

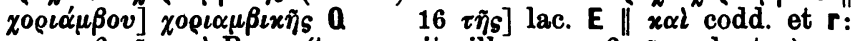

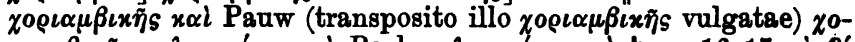

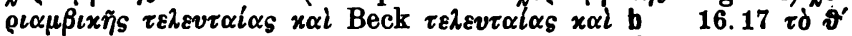

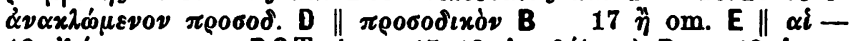

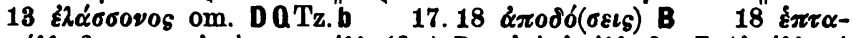

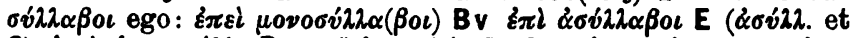

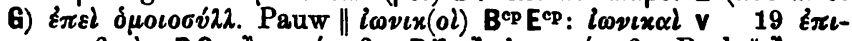

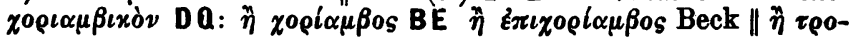




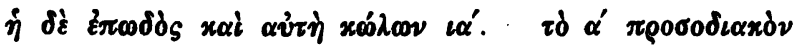

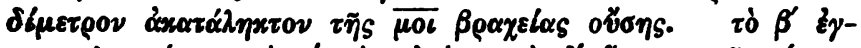

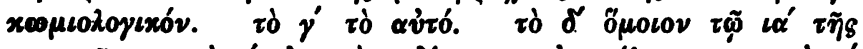

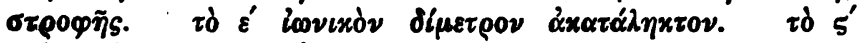

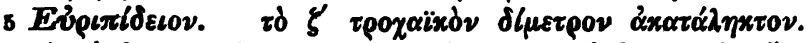

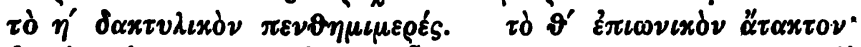

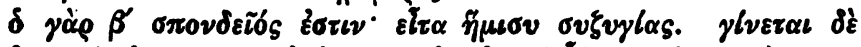

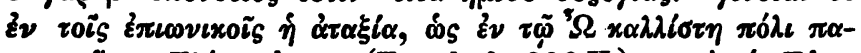

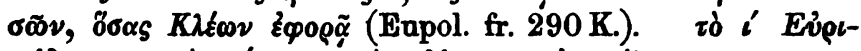

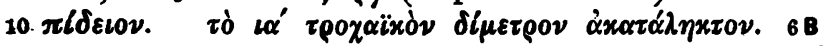

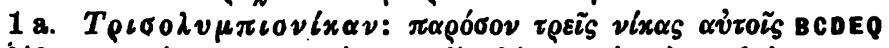

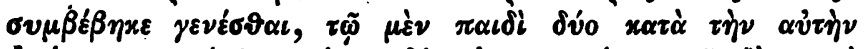

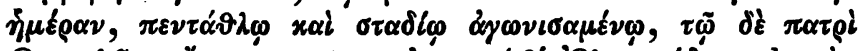

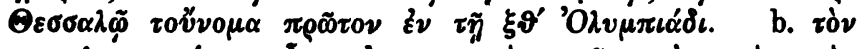

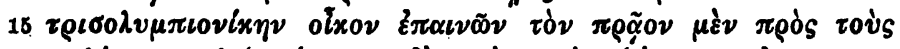

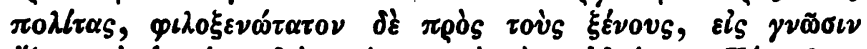

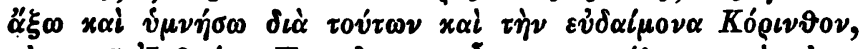

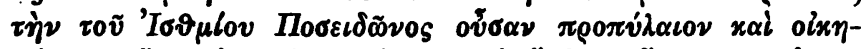

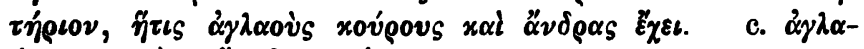

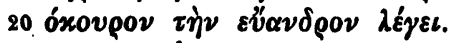

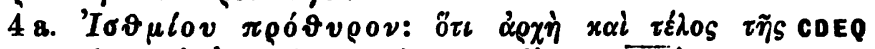

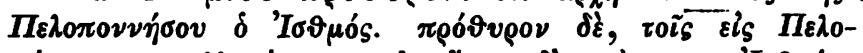

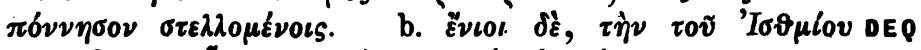

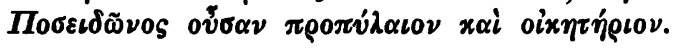

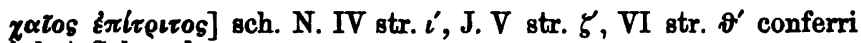
inbet Schroeder

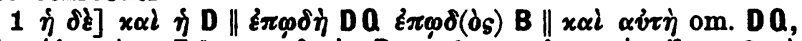

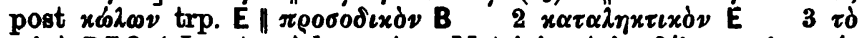

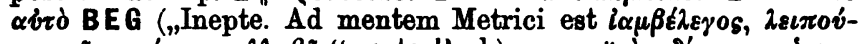

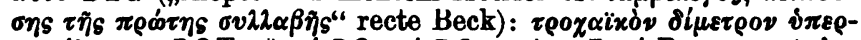

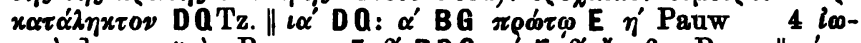

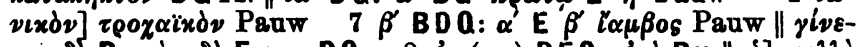

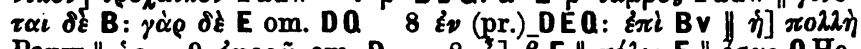

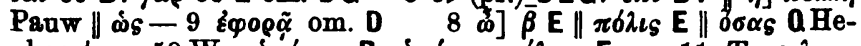

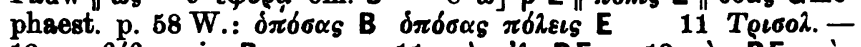

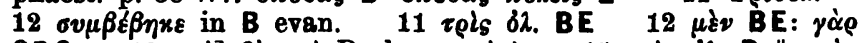

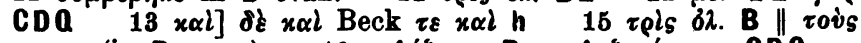

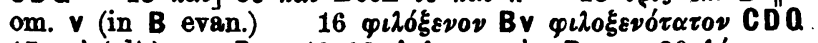

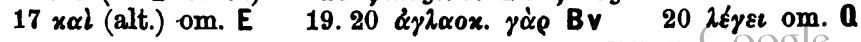




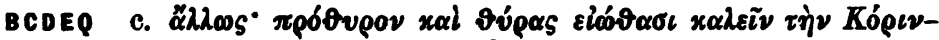

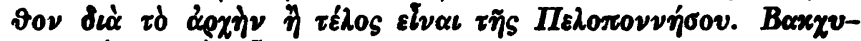

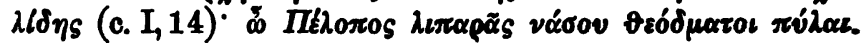

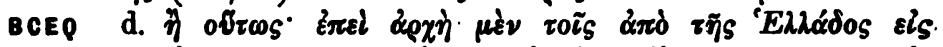

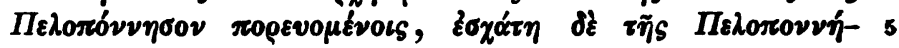
бov. motr. B

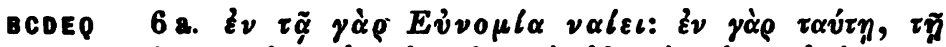

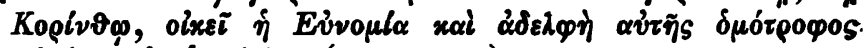

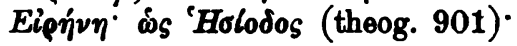

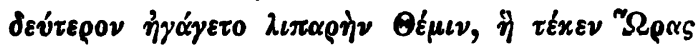

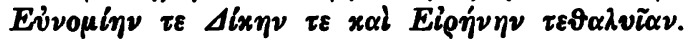

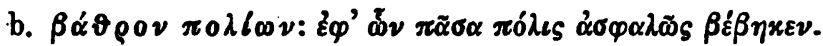

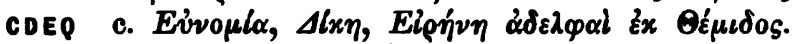

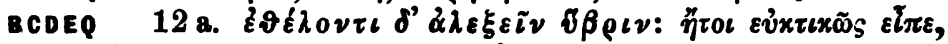

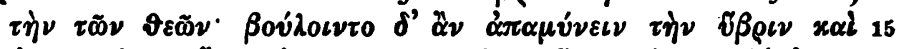

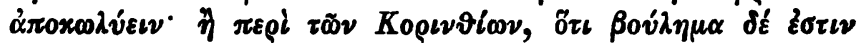

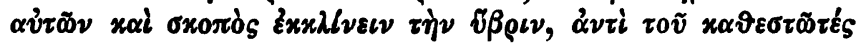

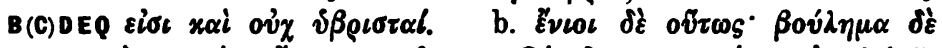

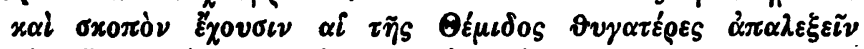

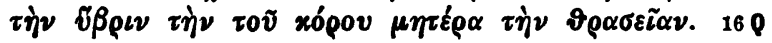

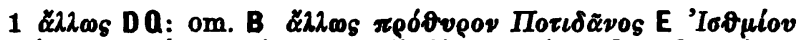

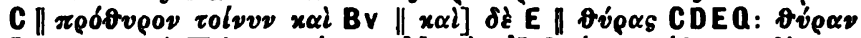

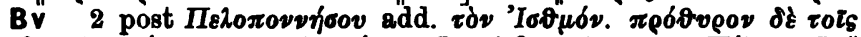

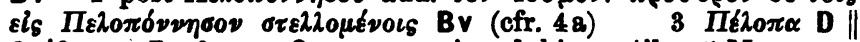
$\vartheta \varepsilon 6 \delta \mu \eta \tau o l$ E $-\delta \mu \eta \tau \alpha \iota$ a 7 sq. „sine dubio mutilum" Mommsen

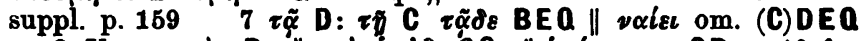

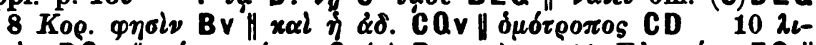

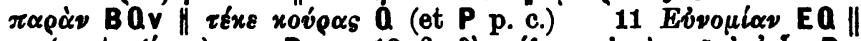

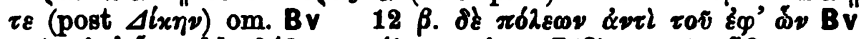

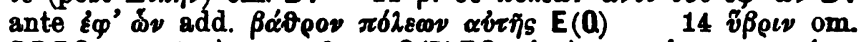

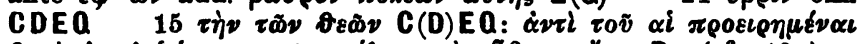

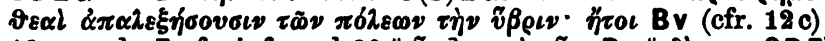
16 sqq. de E cfr. infra ad $20 \| \tilde{\delta} \tau i] \varphi \eta \sigma i \nu \tilde{z} \tau \iota \mathrm{Bv} \| \delta \xi \mathrm{om}$. CDE $\mathrm{E}^{\mathrm{b}} \mathrm{Q}$

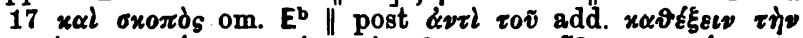

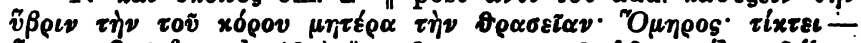

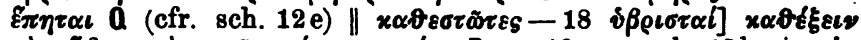

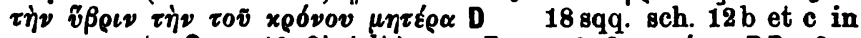

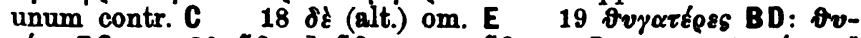

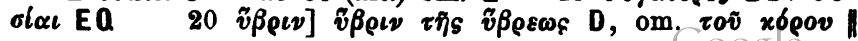




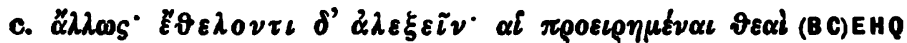

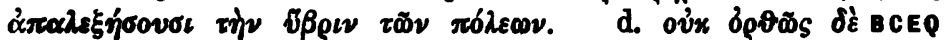

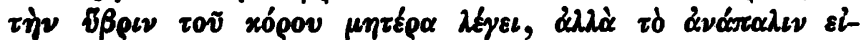

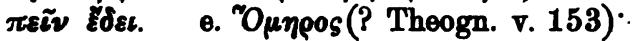

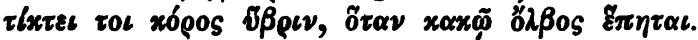

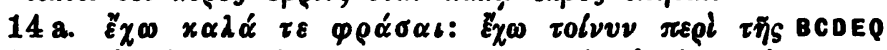

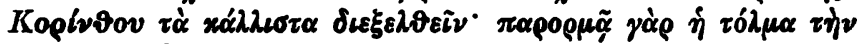

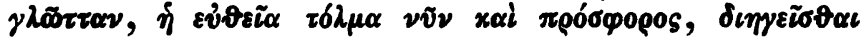

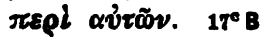

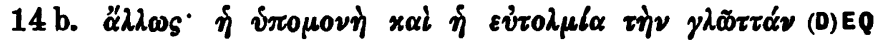

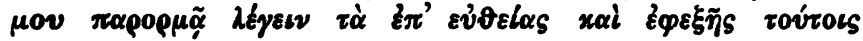

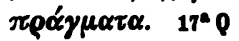

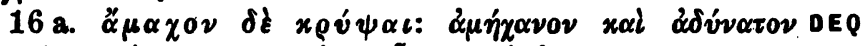

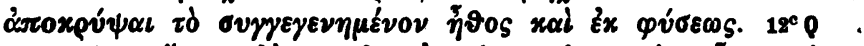

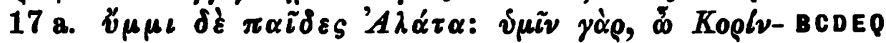

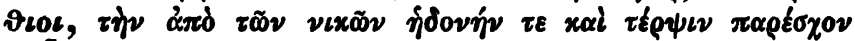

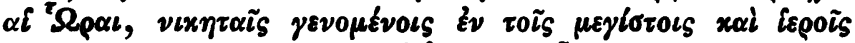

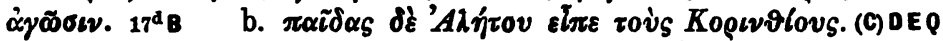

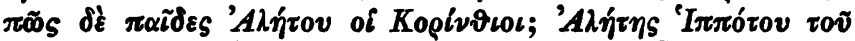

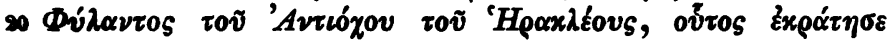

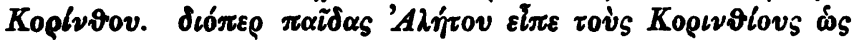

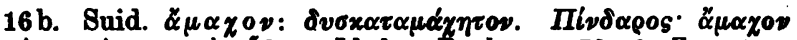

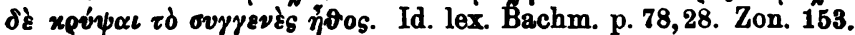
19 et 360, 1 Paus. 2, 4, 3. EM 61, 60

$\tau \grave{\eta} \nu$ (tert.) om. DE $\|$ post $\theta \rho \alpha \sigma \varepsilon t \alpha \nu$ hab. 12e, dein repet. $\pi \varepsilon \rho l$

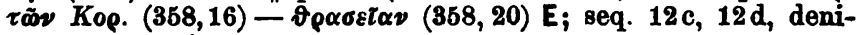
que 12 e repet.

1 sq. de B cfr. ad $358,15 \| 1$. om. E $2 \alpha \pi \alpha \alpha$. BE: $\alpha \lambda$.

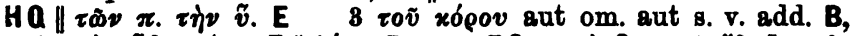
ante $\tau \dot{\eta} \nu \tilde{v} \beta \rho \iota \nu$ trp. E $\| \lambda \xi \xi \varepsilon \varepsilon$ B: om. EQ $\varphi \eta \sigma i$ C $4 \xi \delta \varepsilon \iota] \mu \tilde{\alpha} \lambda-$

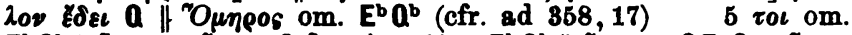

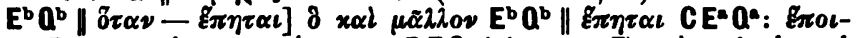

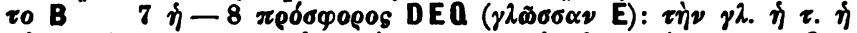

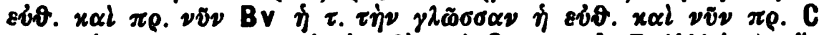

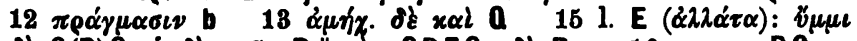

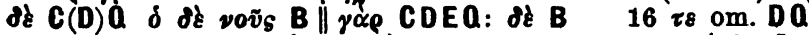

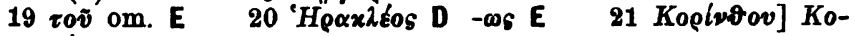
pevolove $E$ 


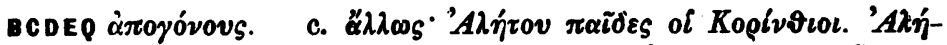

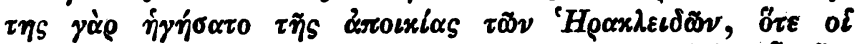

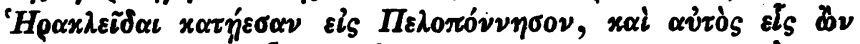
$\tau \tilde{\omega} \nu$ 'H

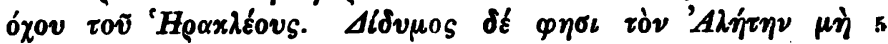

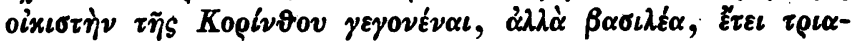

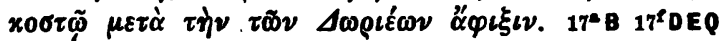

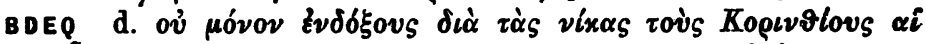

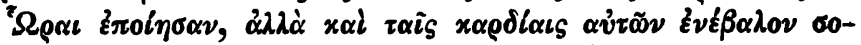

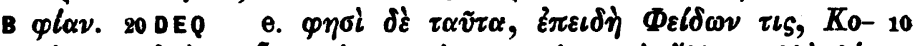

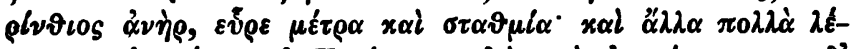

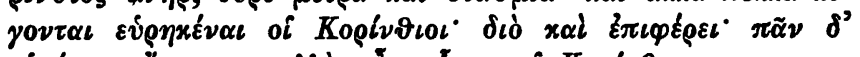

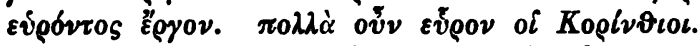

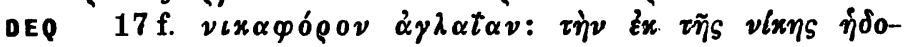
vंभ. $17^{\mathrm{d}} \mathrm{DEO}$

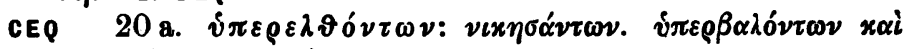

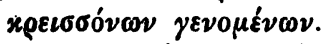

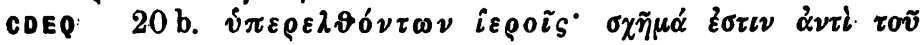

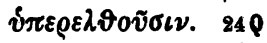

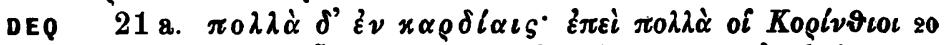

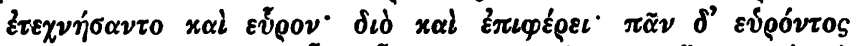

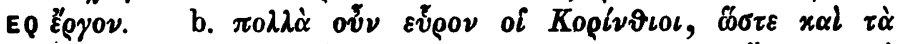

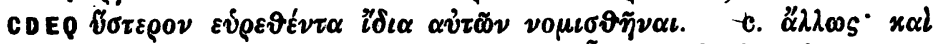

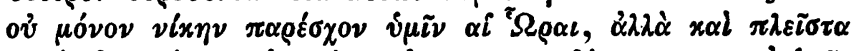

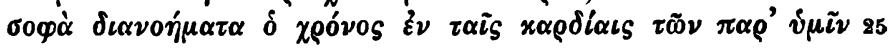

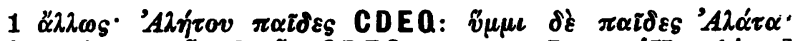

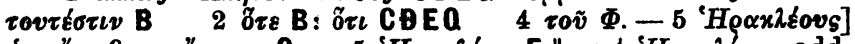

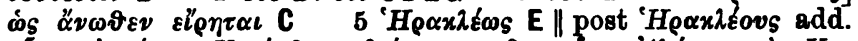

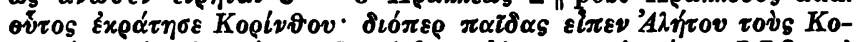

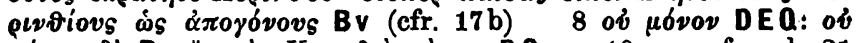

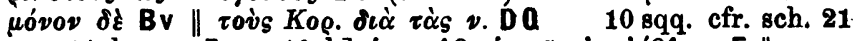

$14 \mathrm{l}$. om. D $16 \mathrm{l}$.]

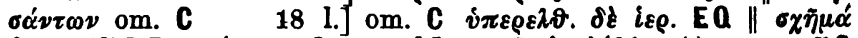

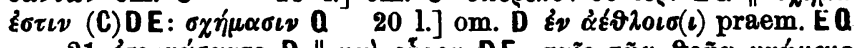

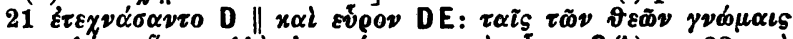

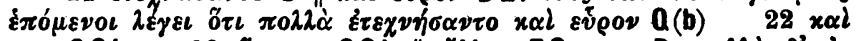

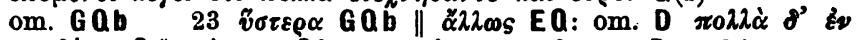

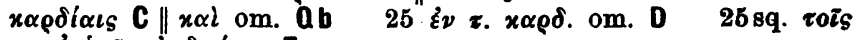
$\pi \alpha \rho^{\prime} \dot{\eta} \mu \tilde{i} \nu$ ¿ $\alpha \delta \rho \alpha^{\prime} \sigma \iota \nu \mathrm{E}$ 


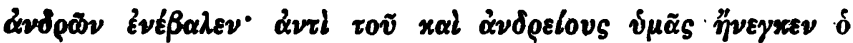

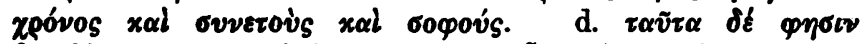

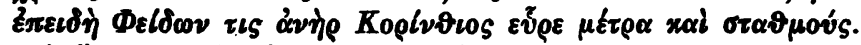

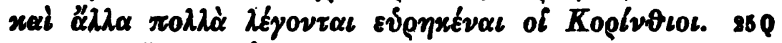

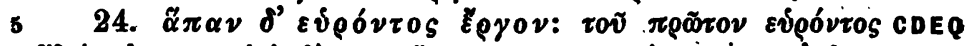

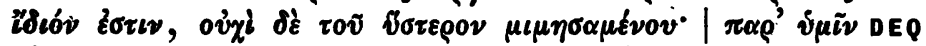
gà

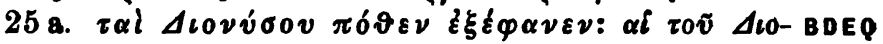

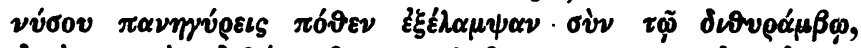

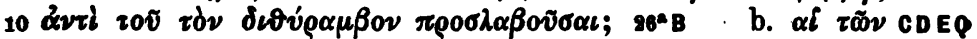

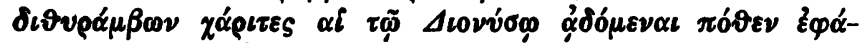

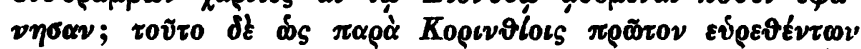

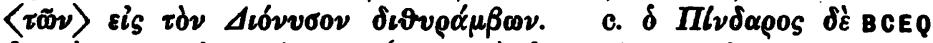

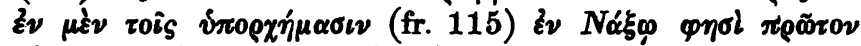

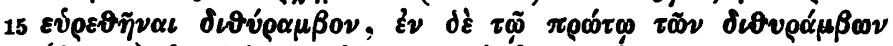

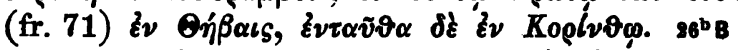

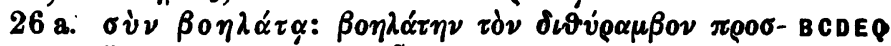

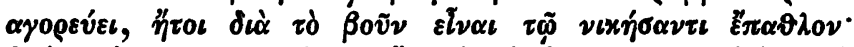

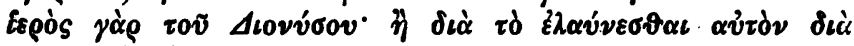

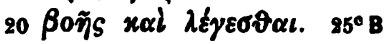

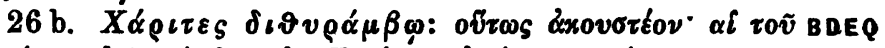

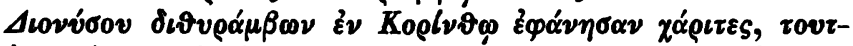

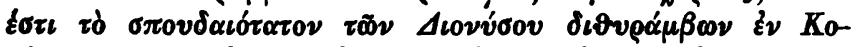

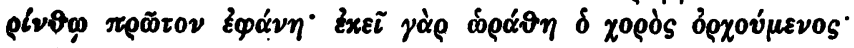

24-p. 362, 5 Tz. ad Lyc. p. 252 M.: $\Delta \omega \theta \dot{v} \rho \alpha \mu \beta o \nu$ dz̀

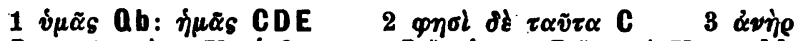
om. D $4 x \alpha i-K o \rho i v \theta \iota \iota l$ om. D \| oi om. E H post Koo. add.

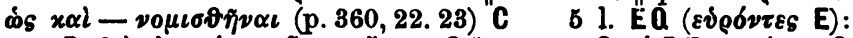

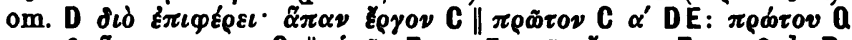

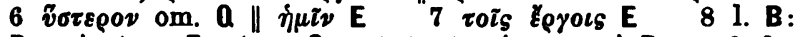

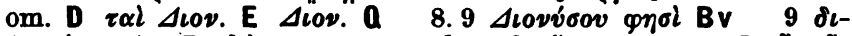

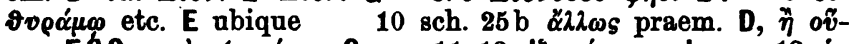

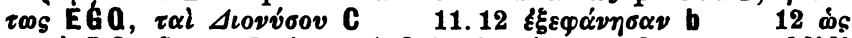

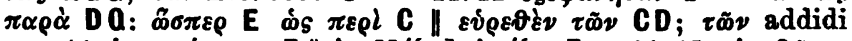

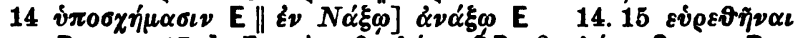

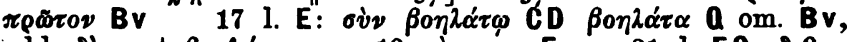

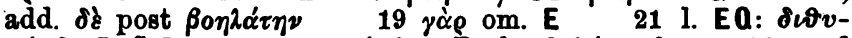

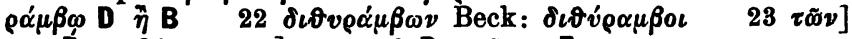

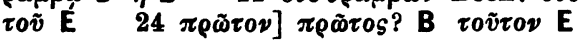




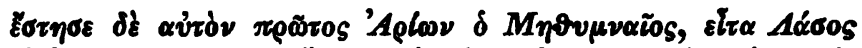

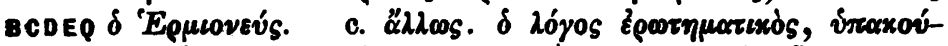

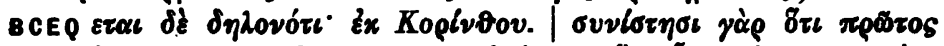

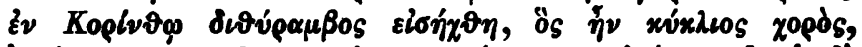

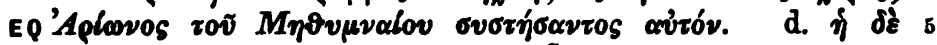

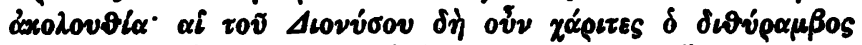

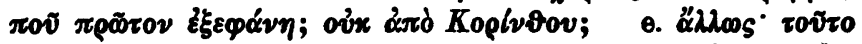

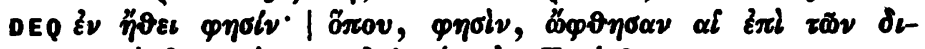

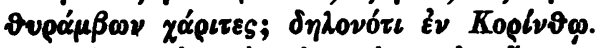

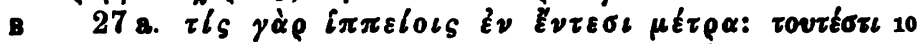

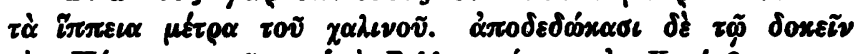

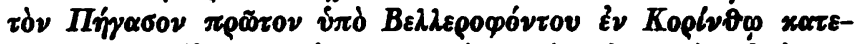

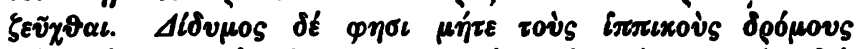

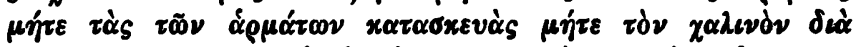

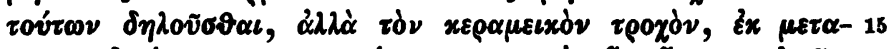

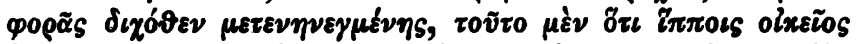

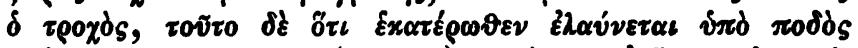

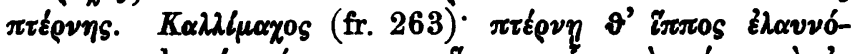
BCDEQ $\mu \varepsilon \nu O S$. b. $\tau l_{S}$ yó

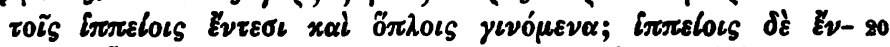

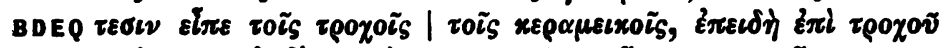

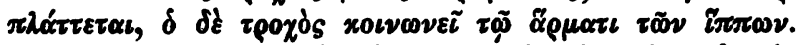

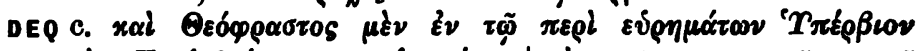

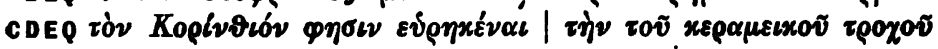

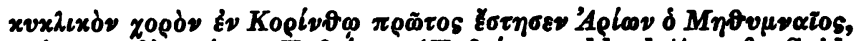

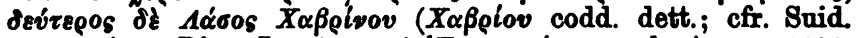

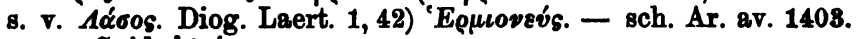
1 Suid. 'Apicv.

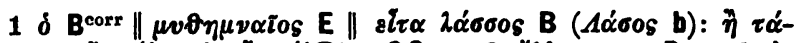

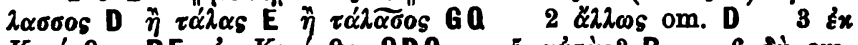

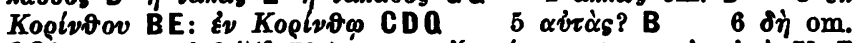

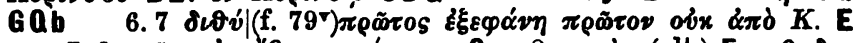

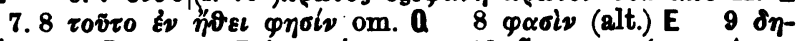

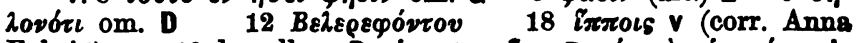

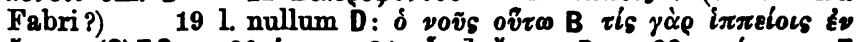

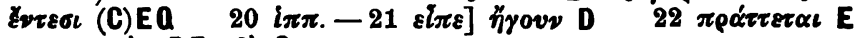
$23 \mu \dot{\varepsilon} \nu$ DE: ḋे Qv 


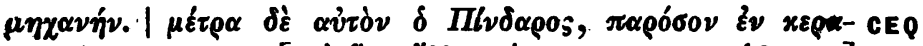

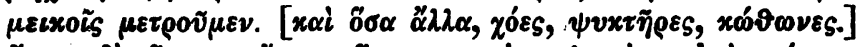

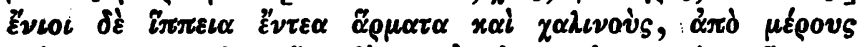

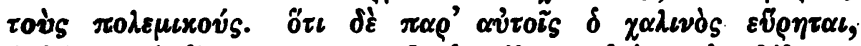

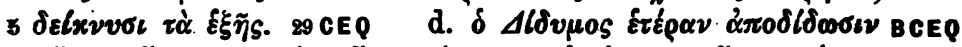

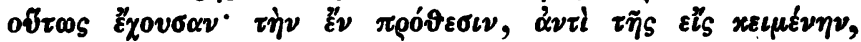

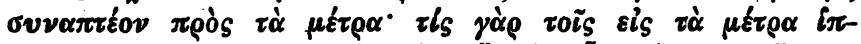

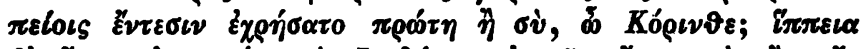

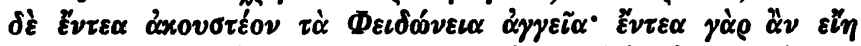

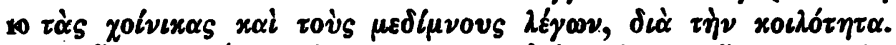

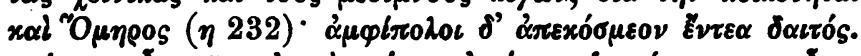

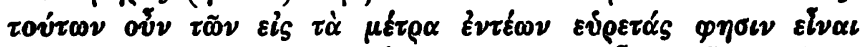

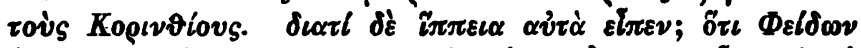

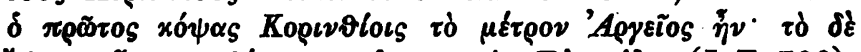

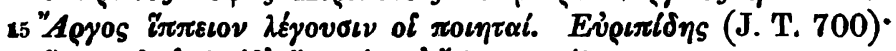

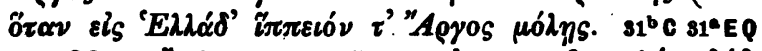

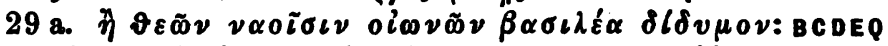

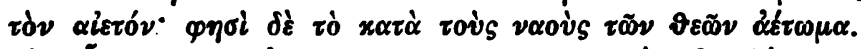
$\tau l_{S}$ ov้v,

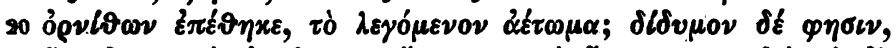

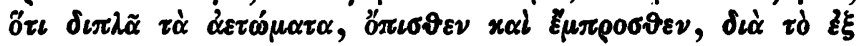

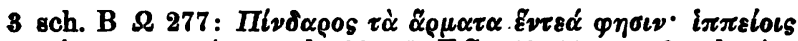

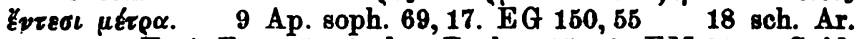
av. 1110. Eust. I. 1352, 38. lex. Bachm. 35, 10. EM 21, 1. Suid.

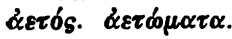

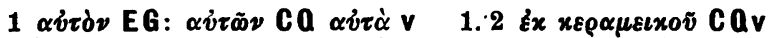

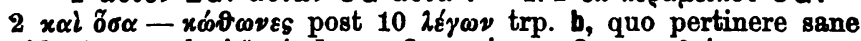

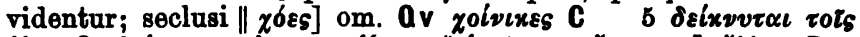

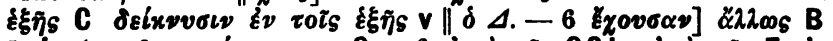

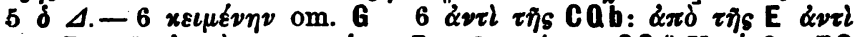

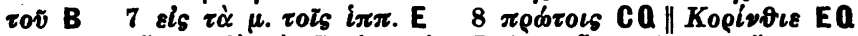

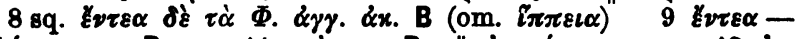

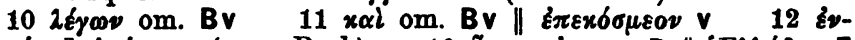

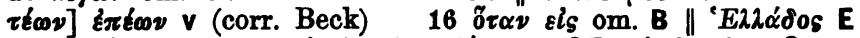

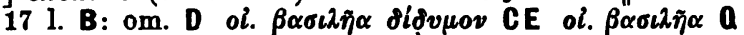

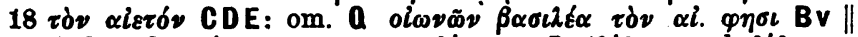

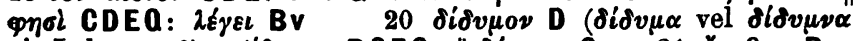

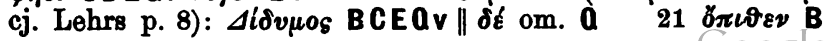




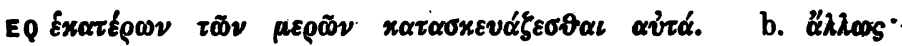

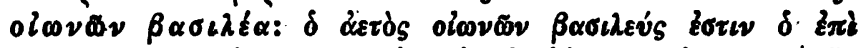

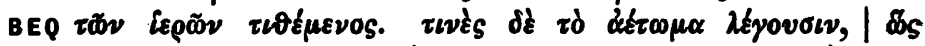

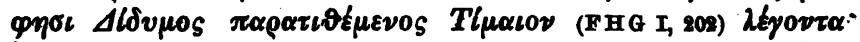

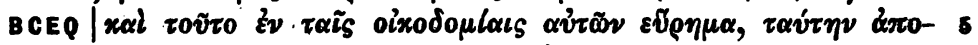

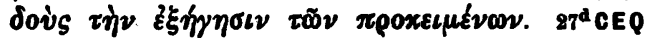

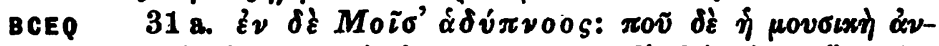

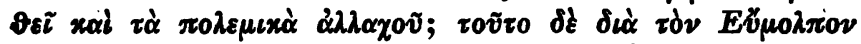

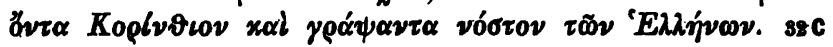

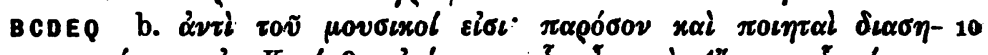

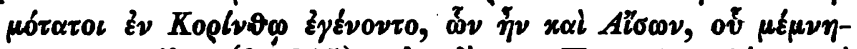

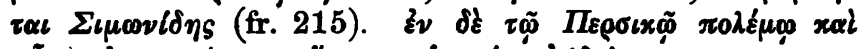

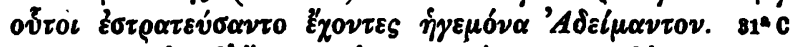

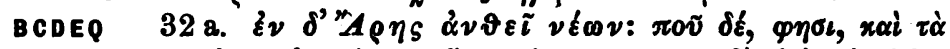

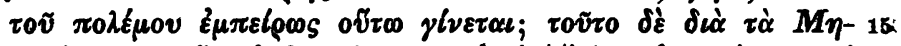

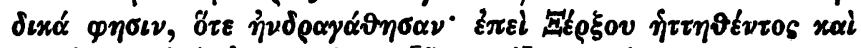

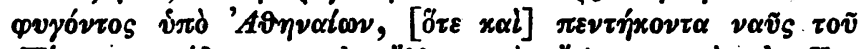

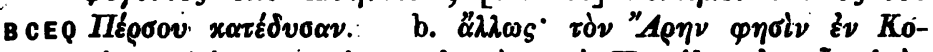

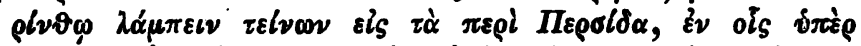

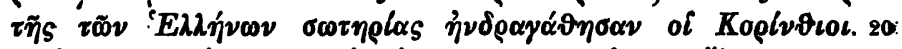

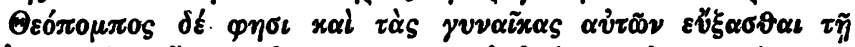

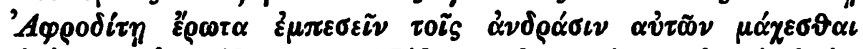

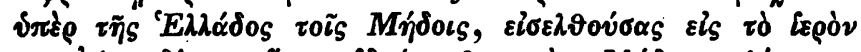

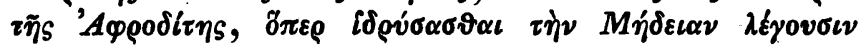

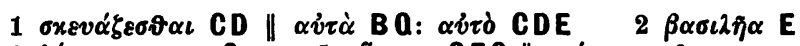

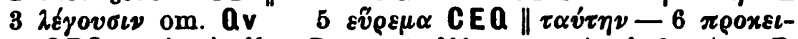

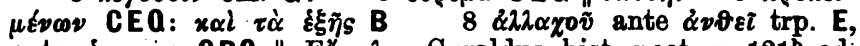

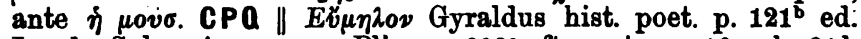
Lugd., Salmasius exerc. Plin. p. 603a, ft. recte 10 sch. 31 b

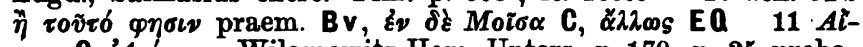

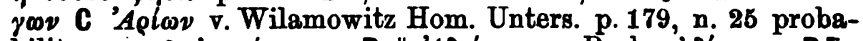

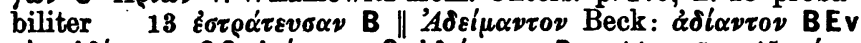

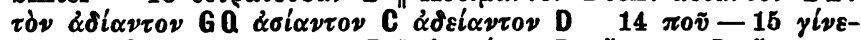

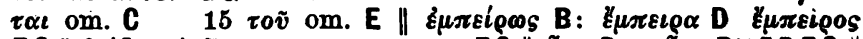

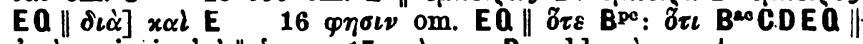

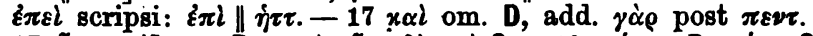

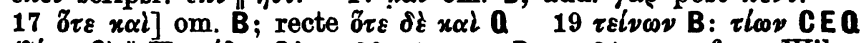

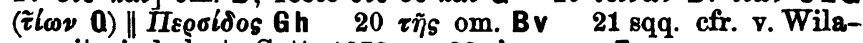

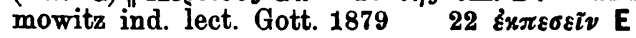




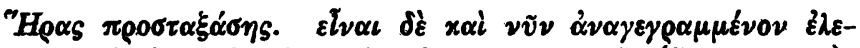

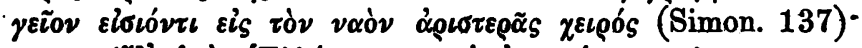

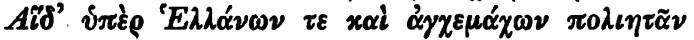

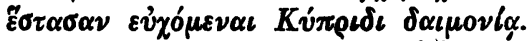

5

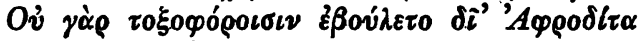

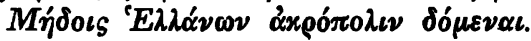

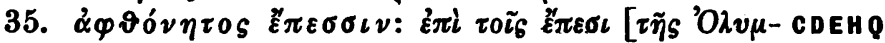

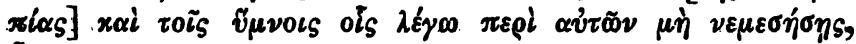
๓ Z Z

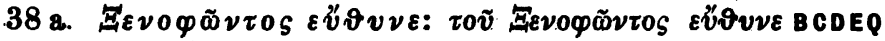

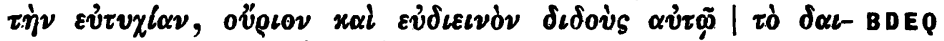

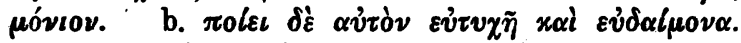

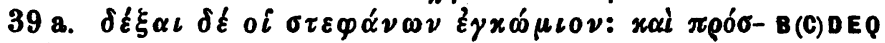

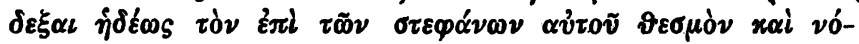

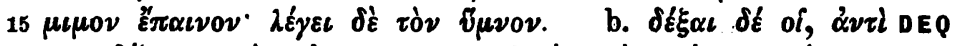

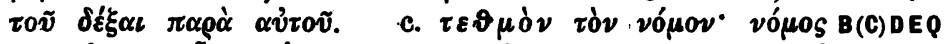
yó

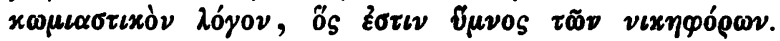

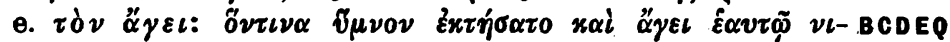

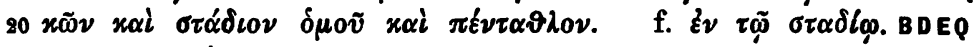

g. $\tau \tilde{\eta} s$ v $l_{x \eta}$.

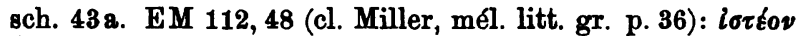

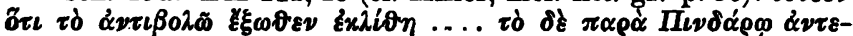

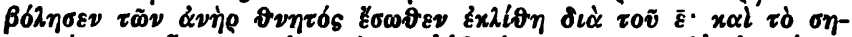

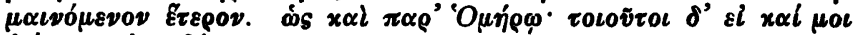

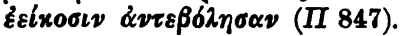

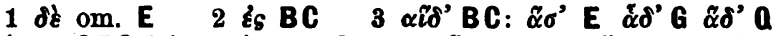

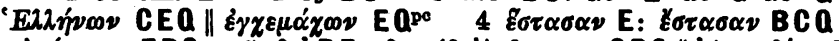

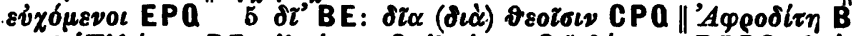

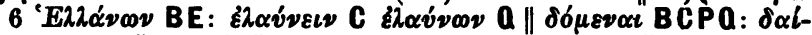

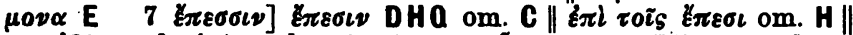

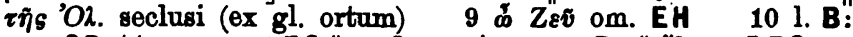

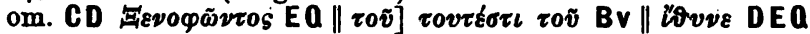

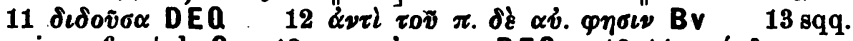

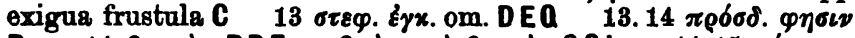

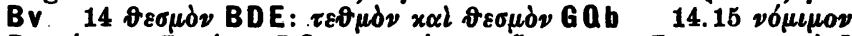

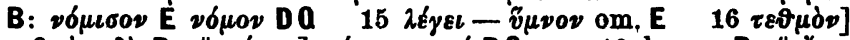

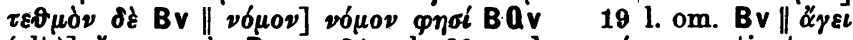

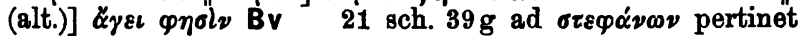




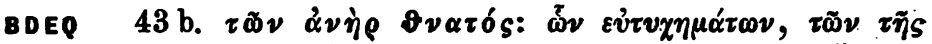

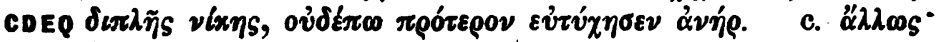

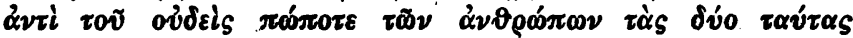

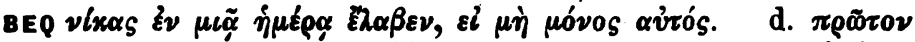

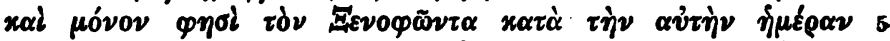

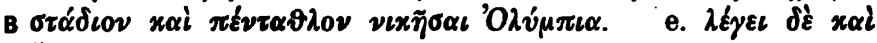

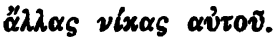

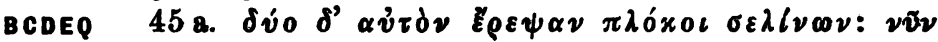

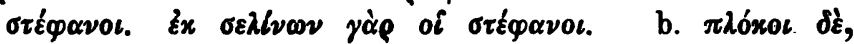

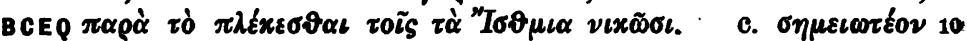
ถ̋

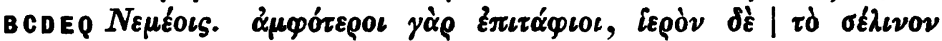

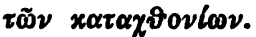

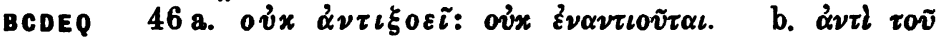

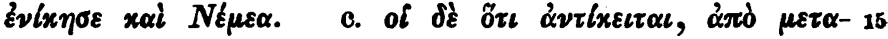

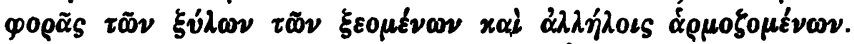

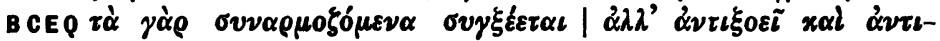

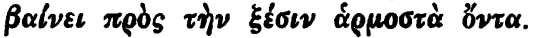

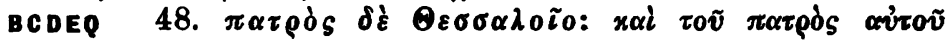

10 Plut. qu. conv. 5, 314 et 15 EM 114, 24. EG 60, 31(?)

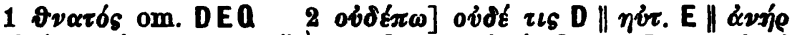

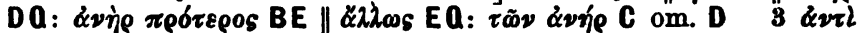

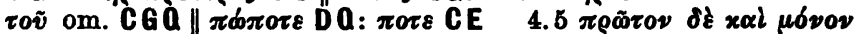

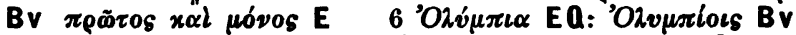

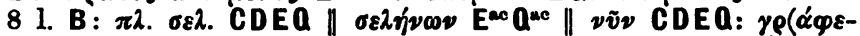

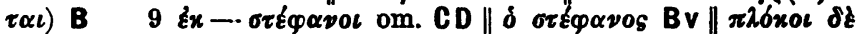

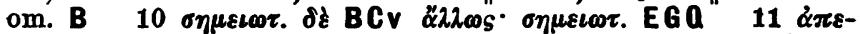

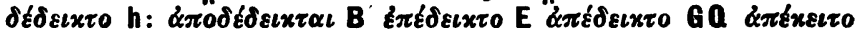

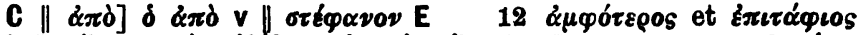

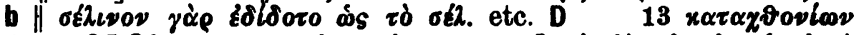

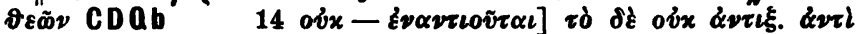

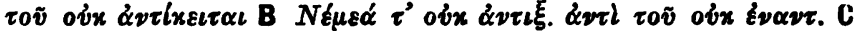

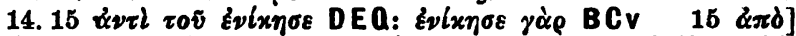
$\varepsilon x \mathrm{Q}{ }^{17} \sigma v \nu \alpha \rho \mu \sigma \zeta \sigma \nu \tau \alpha \mathrm{E} \|[\sigma v \gamma \xi \varepsilon \varepsilon] \tau \alpha \iota \mathrm{B}$, serv. acc. $\langle 0 \mho\rangle \sigma v \gamma \xi \varepsilon-$

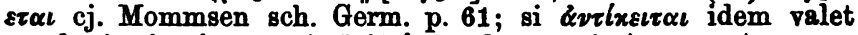

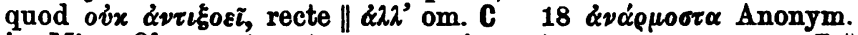
in Misc. Obs. 10 (1739) p. 428, vix recte 19 ๘oขี om. E ||

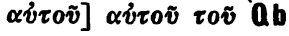




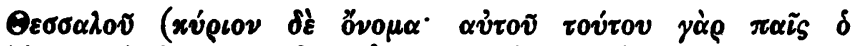

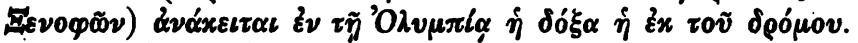
49. $\langle\alpha i \bar{\gamma} \lambda \alpha:\rangle \dot{\alpha} v \tau i$ rov $\dot{\eta} v i x \eta$.

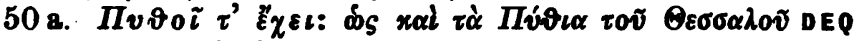

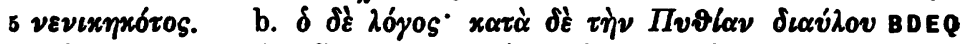

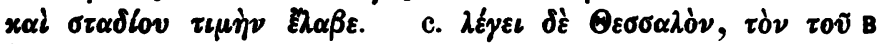

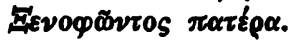

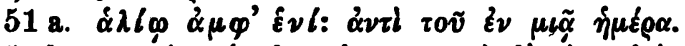

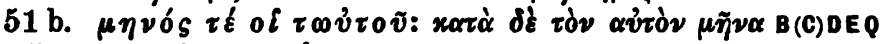

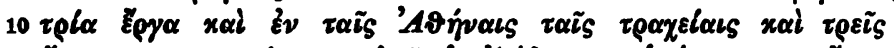

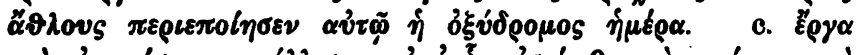

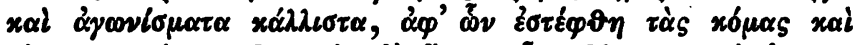

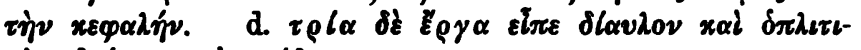

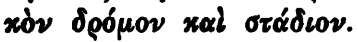

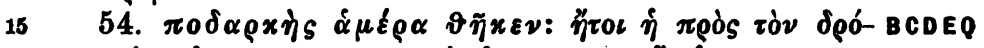

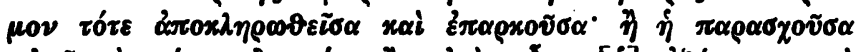

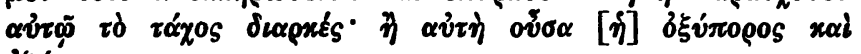

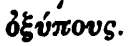

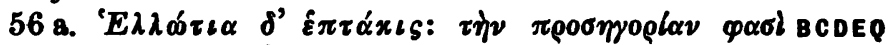

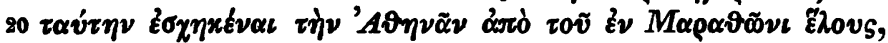

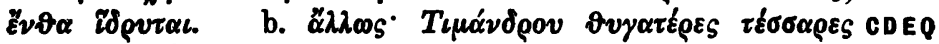

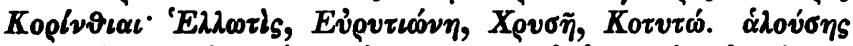

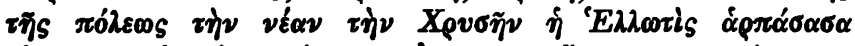

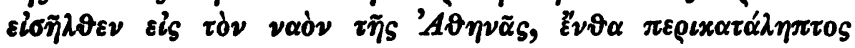

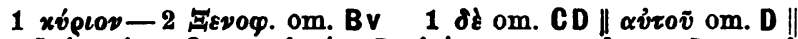

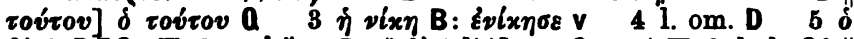

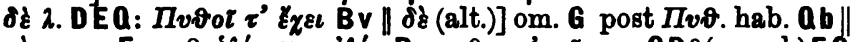

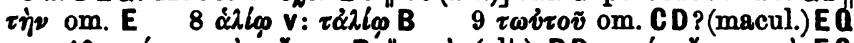

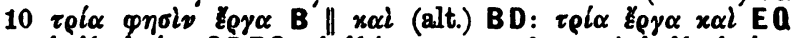

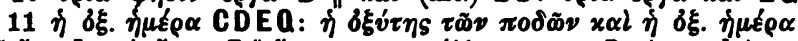

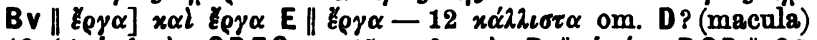

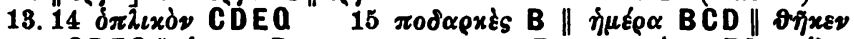

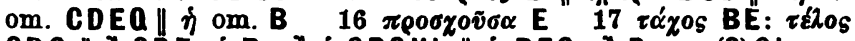
CDQ

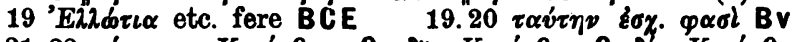

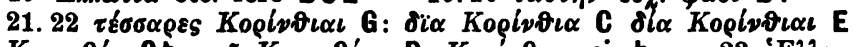

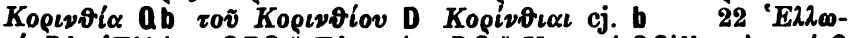

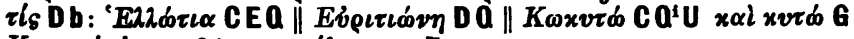

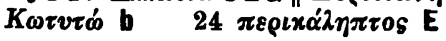




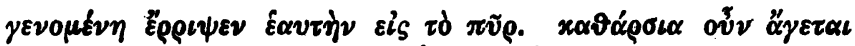

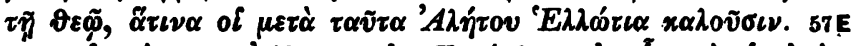

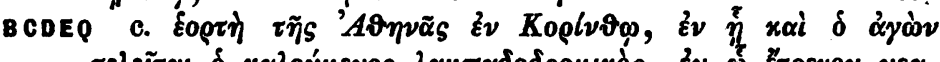

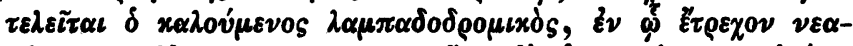

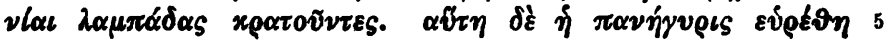

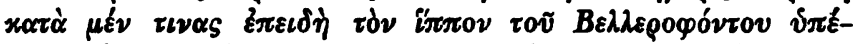
$\tau \alpha \xi \varepsilon \nu$ गे

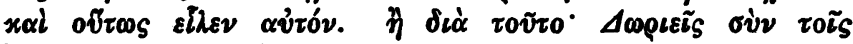

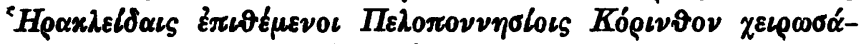

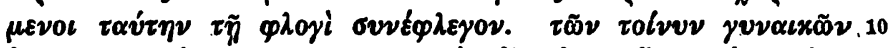

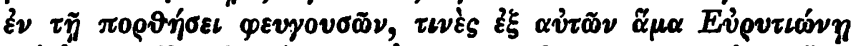

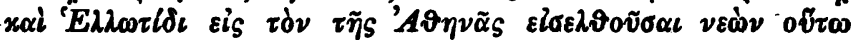

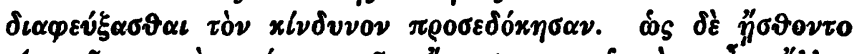

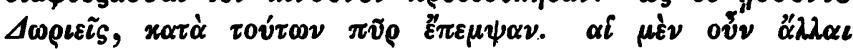

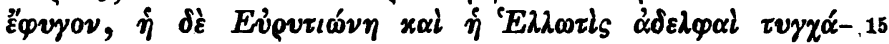

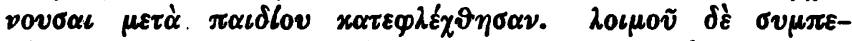

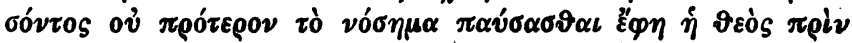

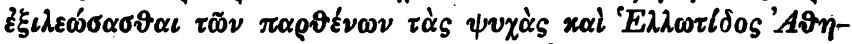

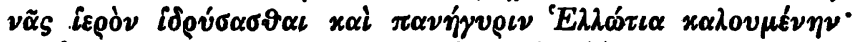

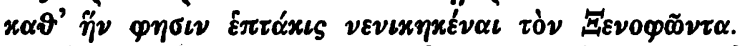

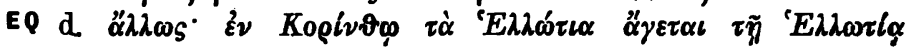

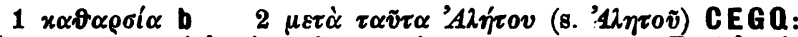

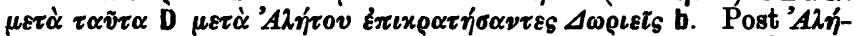

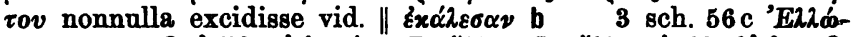

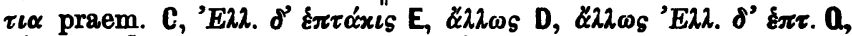

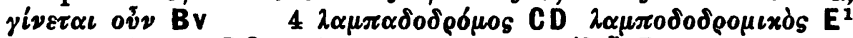

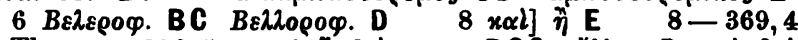

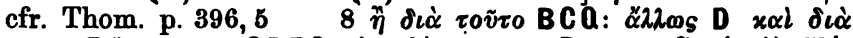

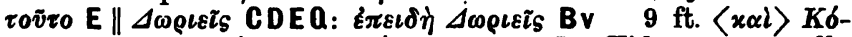

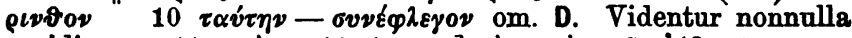

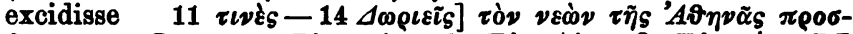

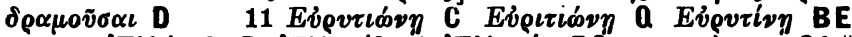

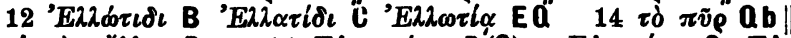

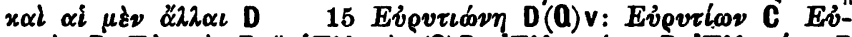

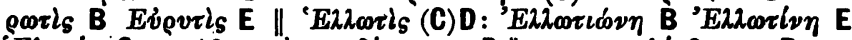

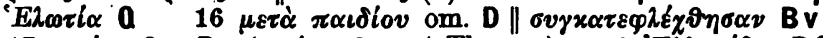

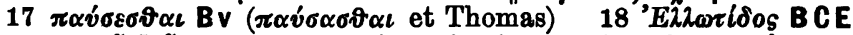

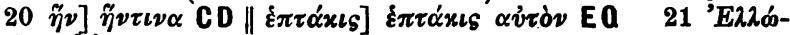
$\tau \iota \alpha$ etc. ubique $E$ 


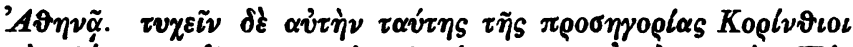

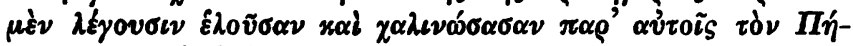

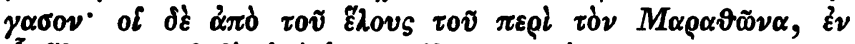

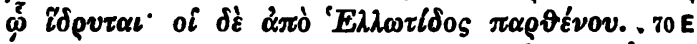

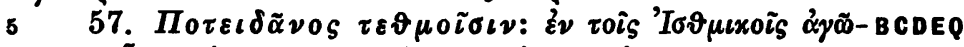

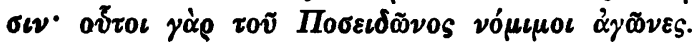

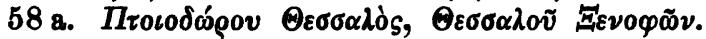

CDEQ

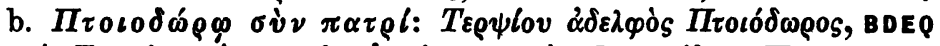

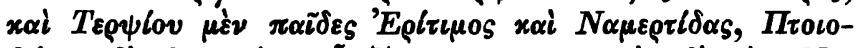

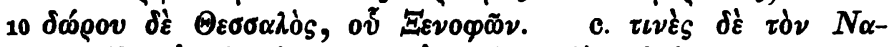

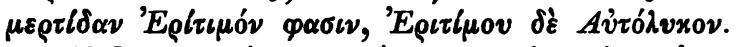

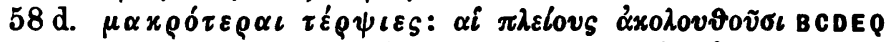

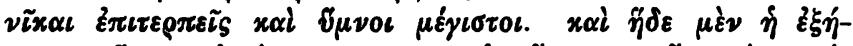

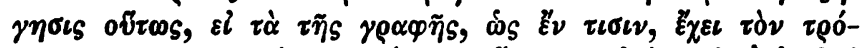

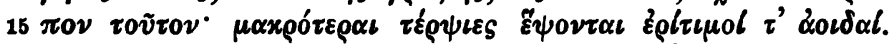

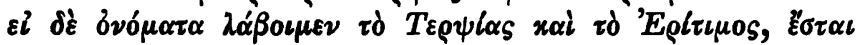

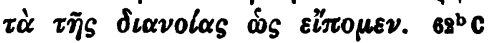

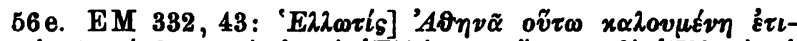

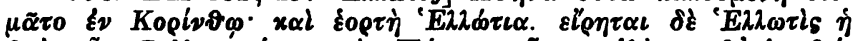

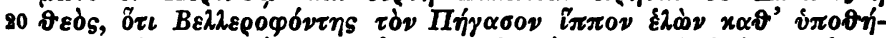

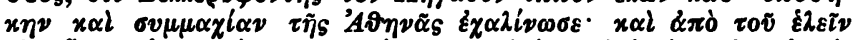

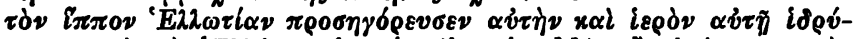

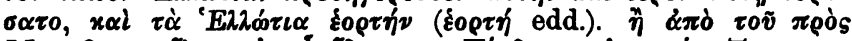

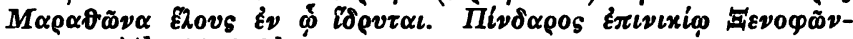
25 ros. - Ath. 15, $678 \mathrm{~b}$.

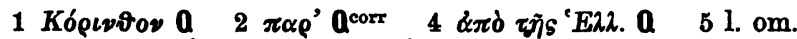

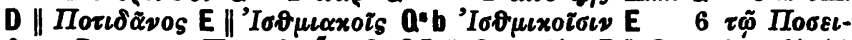

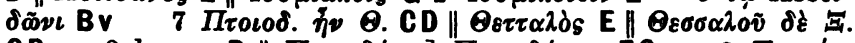

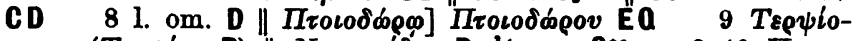

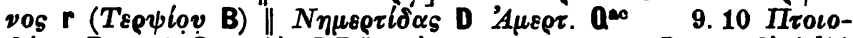

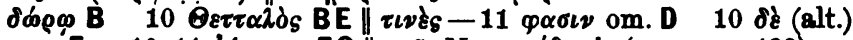

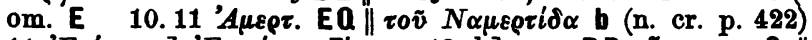

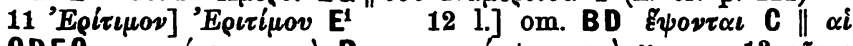

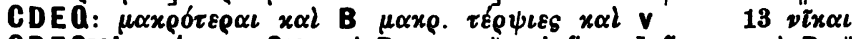

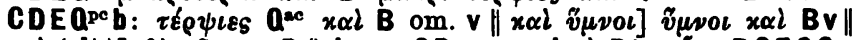

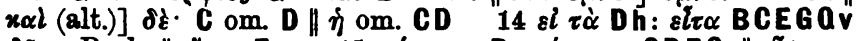

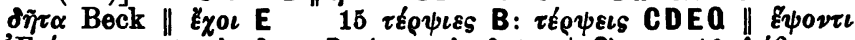
'E

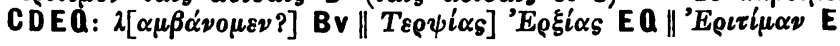

Soror. m Pmbarux ed. Drachmann. 


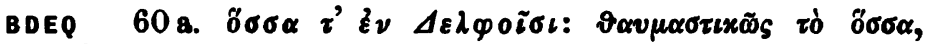

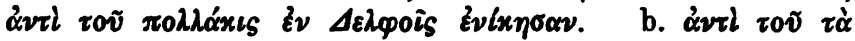

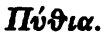

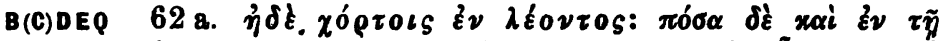

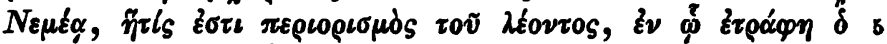

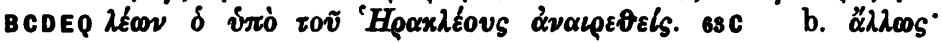

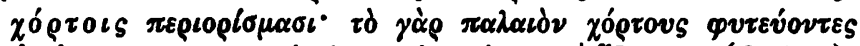

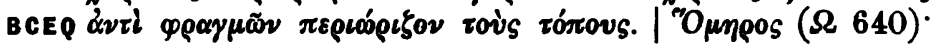

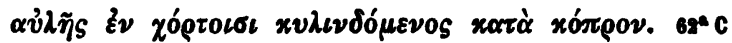

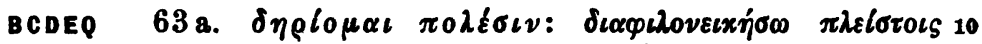

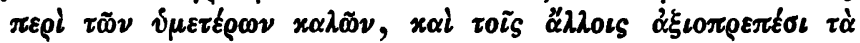

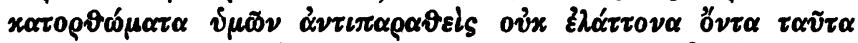

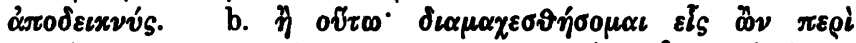

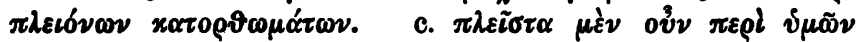

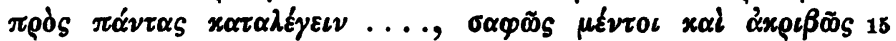

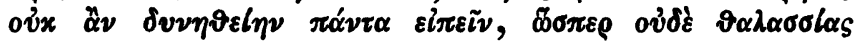

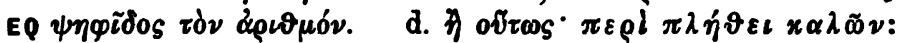

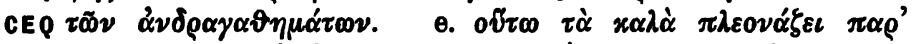

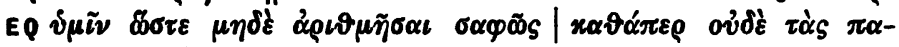

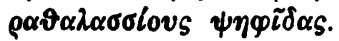

7 sch. DT $\Omega$ 640. Eust. Il. 1369, 16. EM 813, 54. sch. AD A 774. Eust. I1. 883, 45

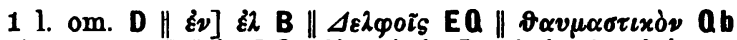

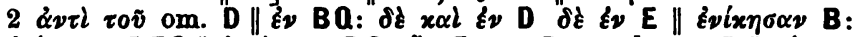

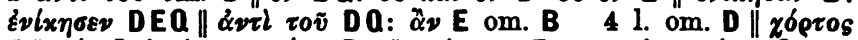

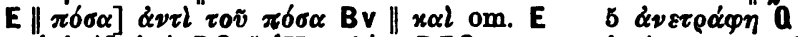

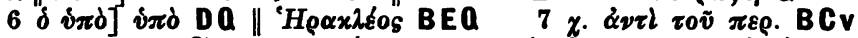

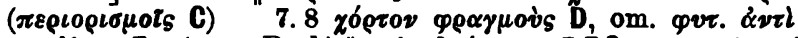

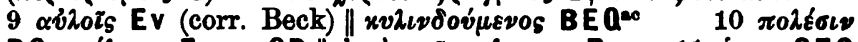

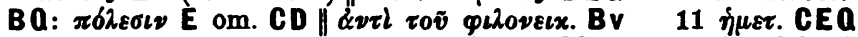

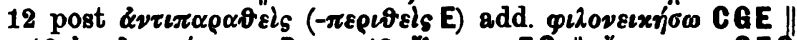

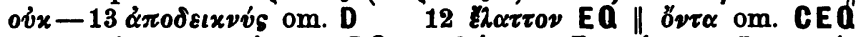

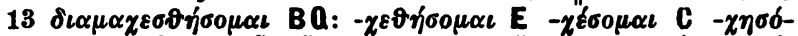
$\mu \varepsilon \theta \alpha$ D 14 sch. $63 \mathrm{c}$ ฑे

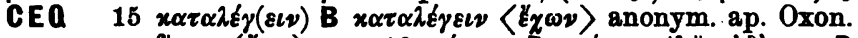

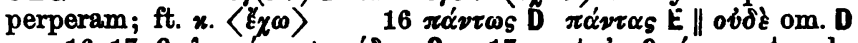
16. $17 \vartheta \alpha \lambda \alpha \sigma \sigma i c \nu \psi \eta \varphi i \delta \omega \nu$ C 17 post $\alpha \rho i \theta \mu \sigma v$ repet. sch.

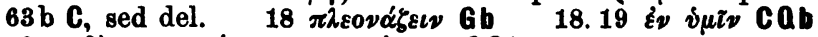

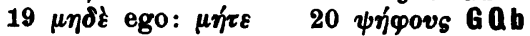




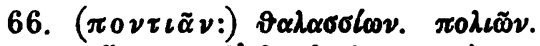

Bel

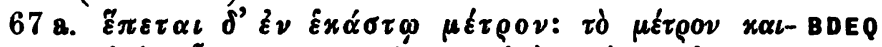

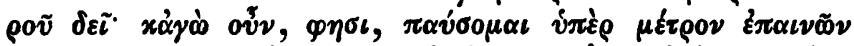

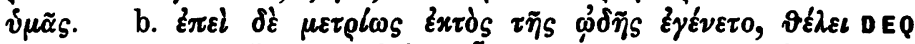

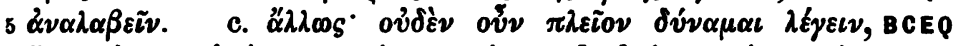

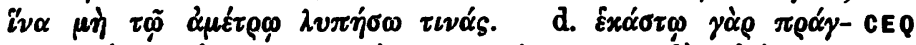

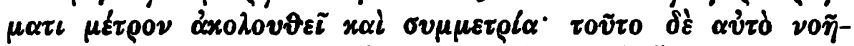

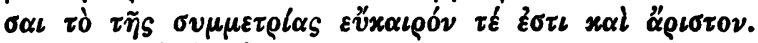

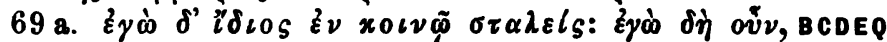

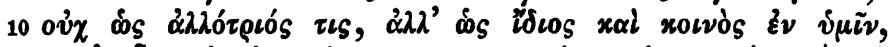

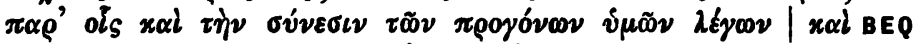

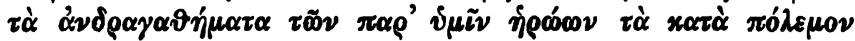

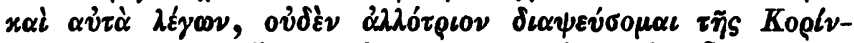

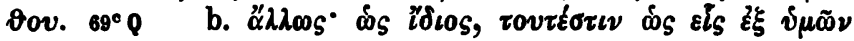

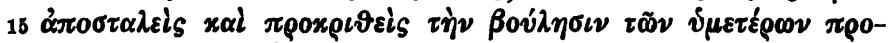

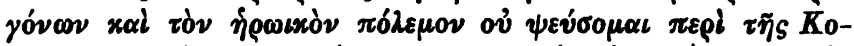

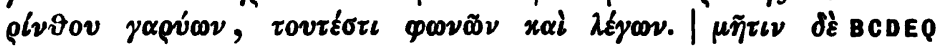

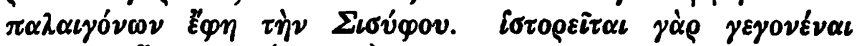

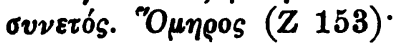

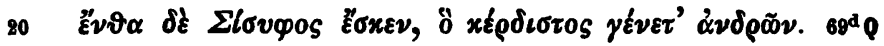

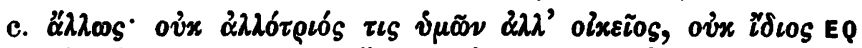

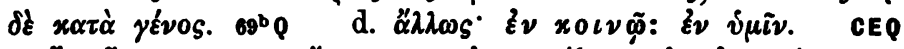

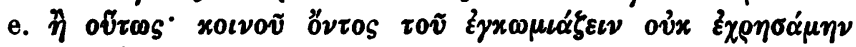

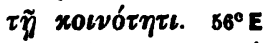

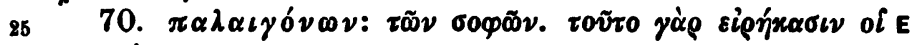

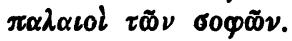

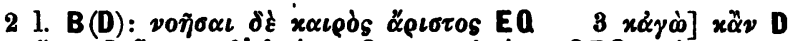

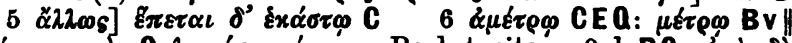

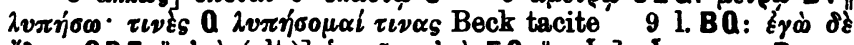

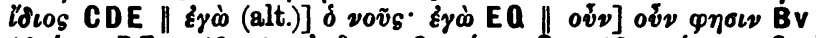

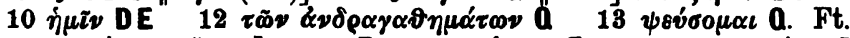

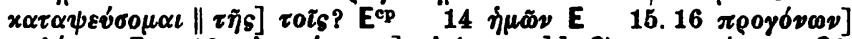
$\pi 0 \lambda \varepsilon \mu \omega \nu$ E 16 ov́ $\psi \varepsilon v ́ \sigma o \mu \alpha \iota]$ ov (8. v. add. G) $x \alpha \tau \alpha \psi \varepsilon v ́ \sigma o \mu \alpha \iota$ G b

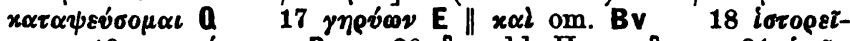

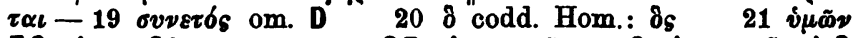

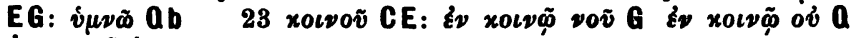

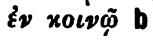




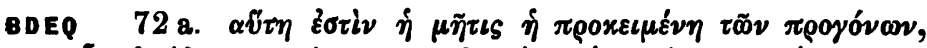

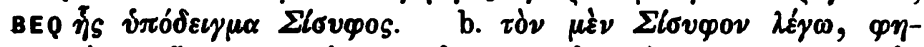

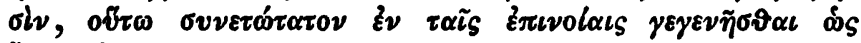

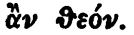

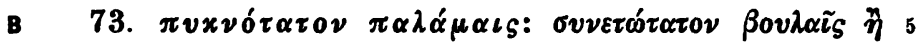
grópuass.

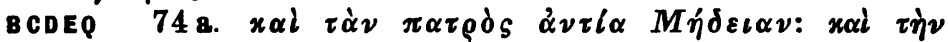

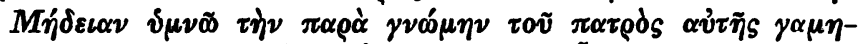

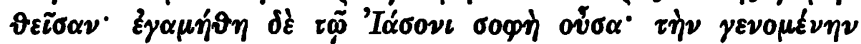

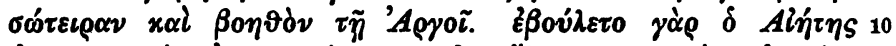

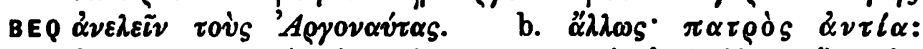

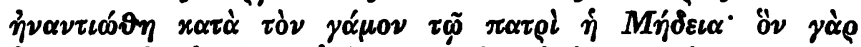

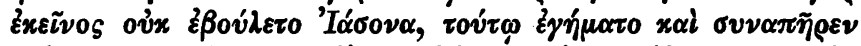

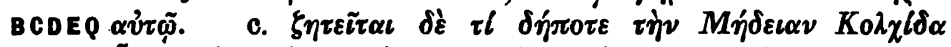

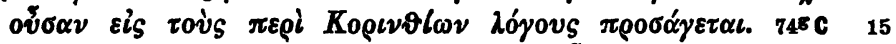

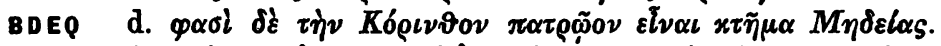

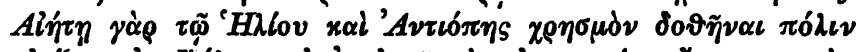

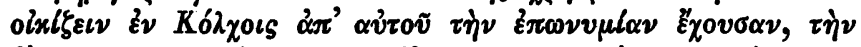

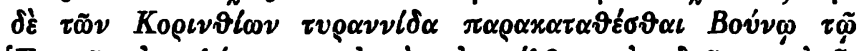

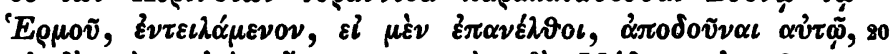

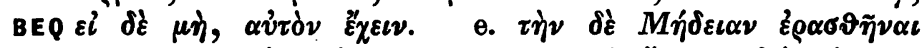

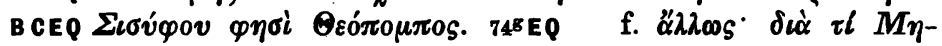

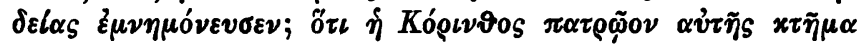

22 soh. Eur. Med. 9. Tzetzes ad Lyc. 174. 1024. Paus. 2, 3, 10

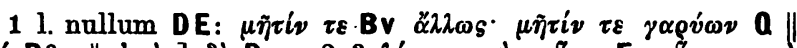

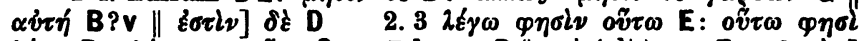

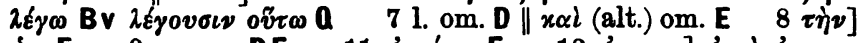

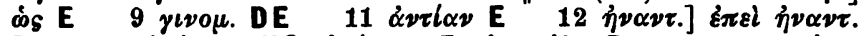

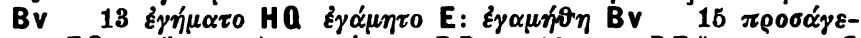

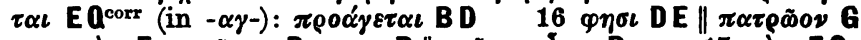

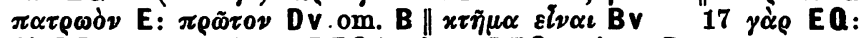

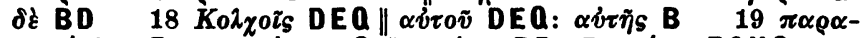

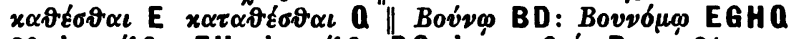

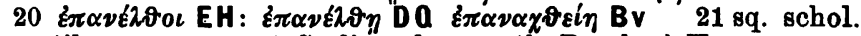
mutilum esse censet Seeliger lex. myth. Roscheri II, 2493 $22-373,7$ cfr. Thom. p. 401,1 22 ờ $\lambda 2 \omega s-23 \dot{\varepsilon} \mu \nu \eta \mu$. om. C $22 \delta \iota \dot{\alpha} \tau i$ EGQb: $\delta \iota \dot{\alpha} \tau o v \tau \tau$ Bv 


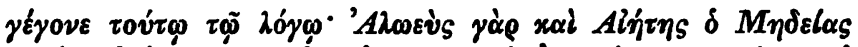

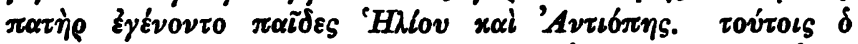

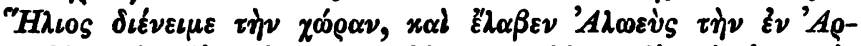

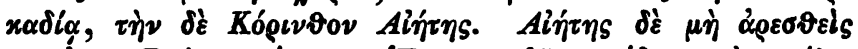

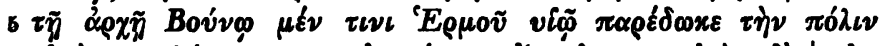

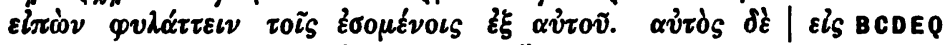

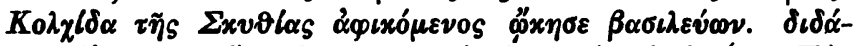

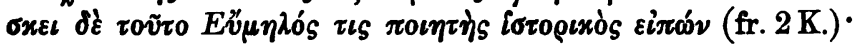

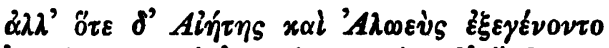

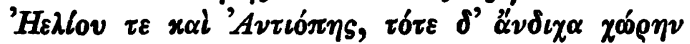

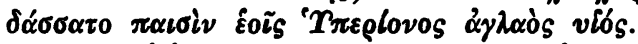

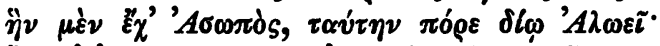

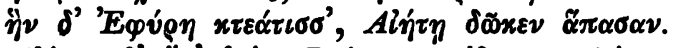

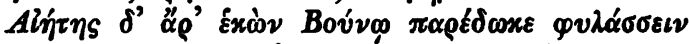

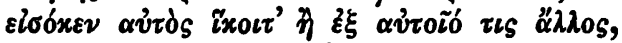

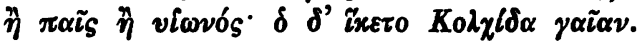

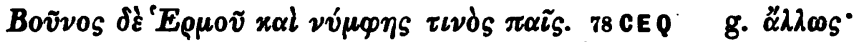

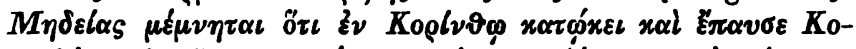

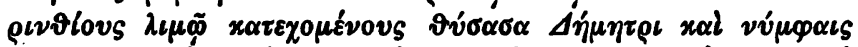

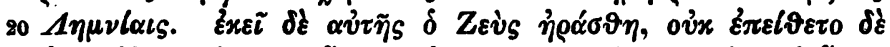

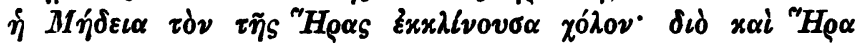

1 jò B (8. v.) EQ: om. C Eur. Thom. $\tau \varepsilon v \| \delta$ om. E

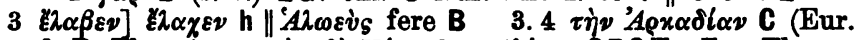

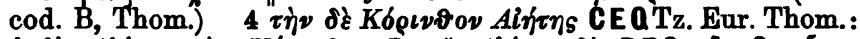

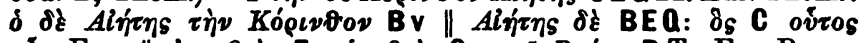

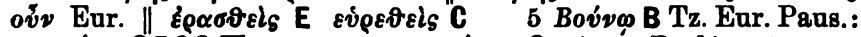

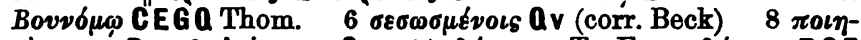

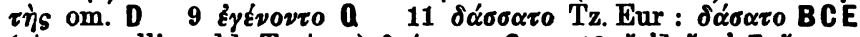

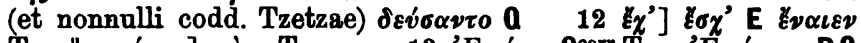

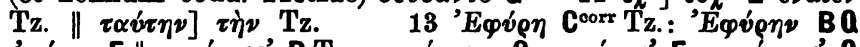

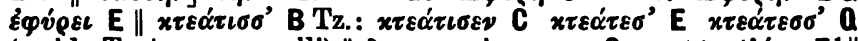
(codd. Tzetzae nonnulli) $\| \delta \tilde{\alpha} x \varepsilon \pi \varepsilon \rho i \tilde{\alpha} \sigma \alpha \nu \mathrm{C} 14$ Alí $\tau \eta \mathrm{E}^{1} \|$

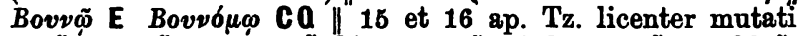

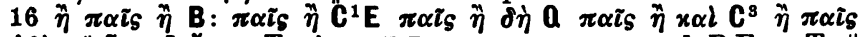

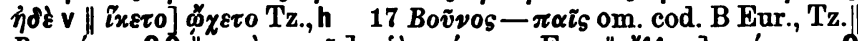

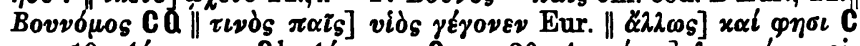

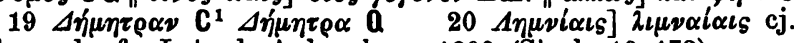
Beck; sed cfr. Lobeck Aglaoph. p. 1209 (Strab. 10, 472)

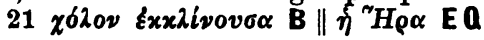




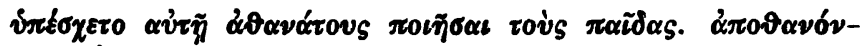

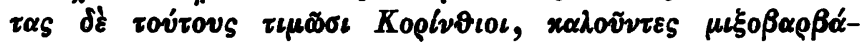
pOUS. TLCEP

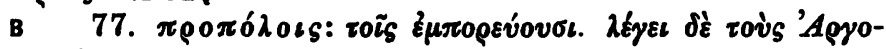

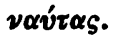

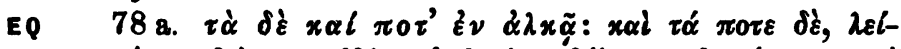

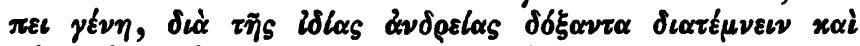

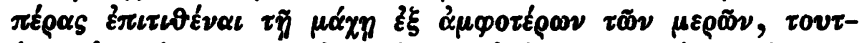

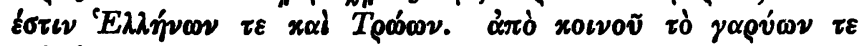

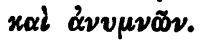

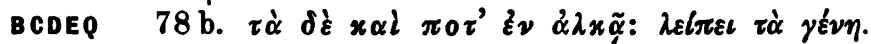

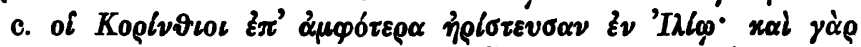

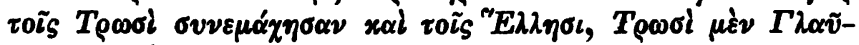

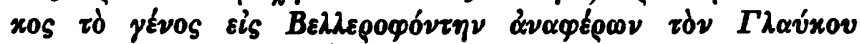

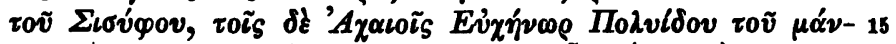

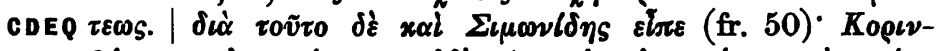

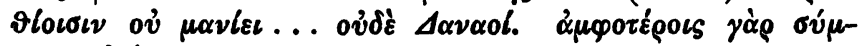

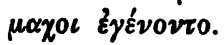

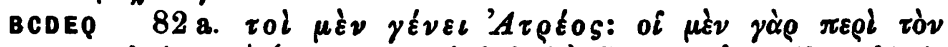

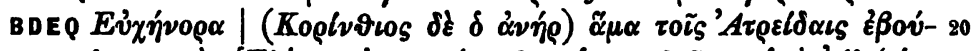

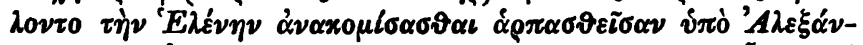

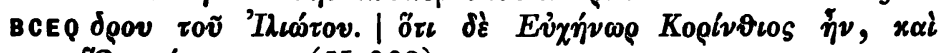

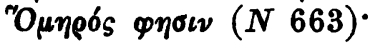

11 (78b-82 a) Cramer anecd. Par. 1, 284, 32. Plut. Dio. 1

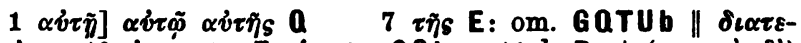
$\mu \varepsilon \tau \nu$ b $10 \dot{\alpha} v \mu \nu \tilde{\omega} \nu \mathrm{E}: \dot{v} \mu \nu \tilde{\omega} \nu \mathrm{GQb} 11 \mathrm{l}$. B et (om. $\tau \dot{\alpha} \delta \dot{\varepsilon})$

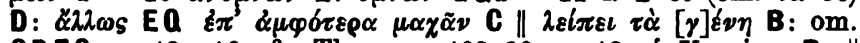
CDEQ 12-16 cfr. Thom. p. 402, 2012 of $K$. qijoiv BV

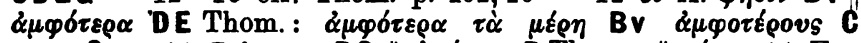

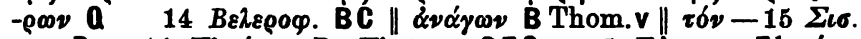

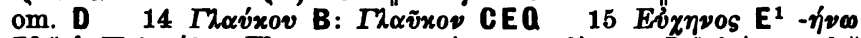

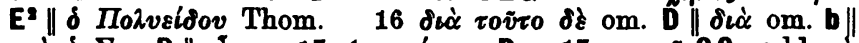

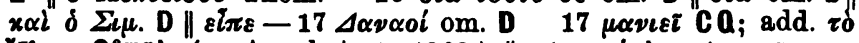

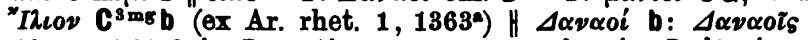
19-p. 275, 2 in B partim. evan. 19 'Atoḱos D 'Atpéwos EQ:

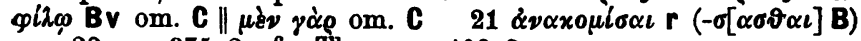
$22-$ p. 375, 2 cfr. Thom. p. 403, 2 


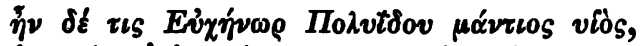

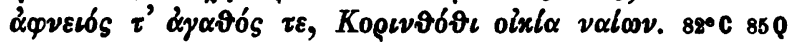

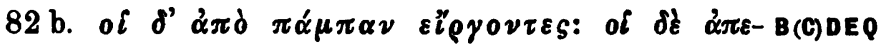

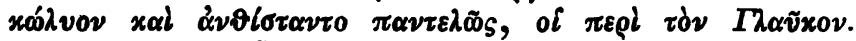

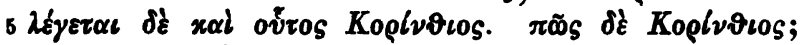

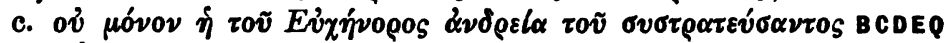

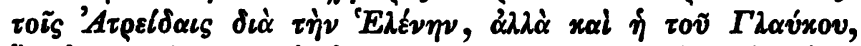

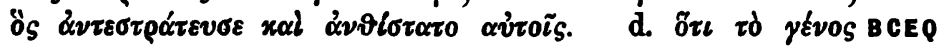

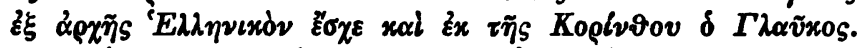

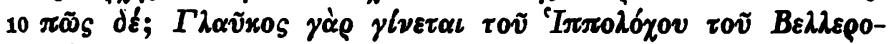

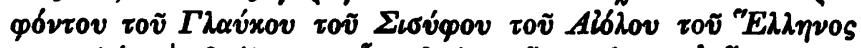

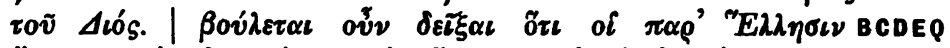

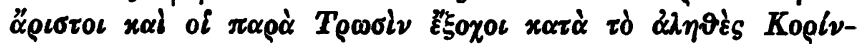

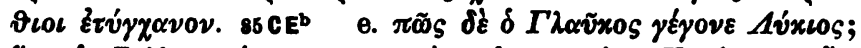

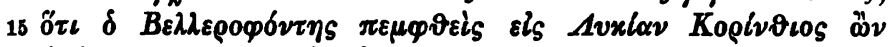

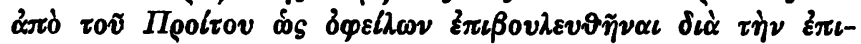

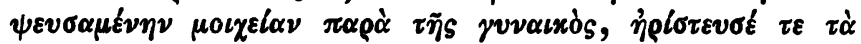

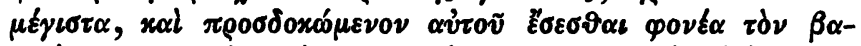

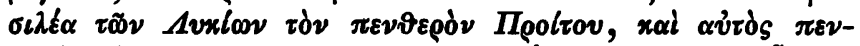

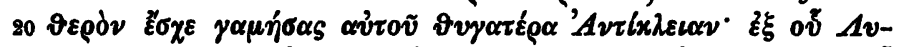

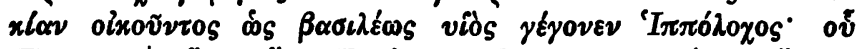

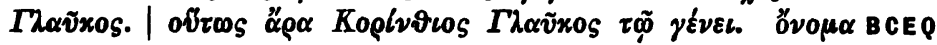

2 oixiay E 3 sqq. $82 \mathrm{bcd}$ post $82 \mathrm{e}$ repet. E. - Ft. $82 \mathrm{c}$ ante $12 \beta 0 v ́ d \varepsilon \tau \alpha \iota$ transponendum, et tres interrogationes (5.10.

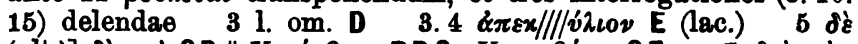

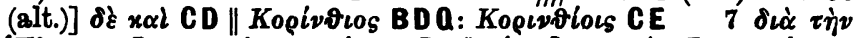

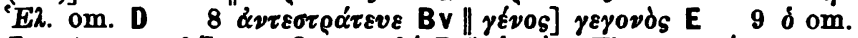

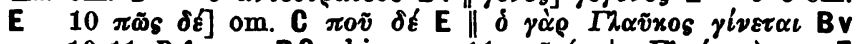

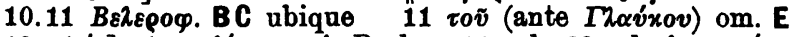
$12 \Delta \iota \delta$ s] $\Delta \varepsilon v x \alpha \lambda i \omega v o s$ cj. Beck 14 sch. $82 \mathrm{e}$ l. Éx Avxíss

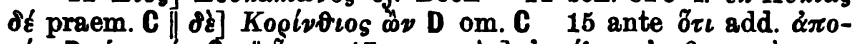

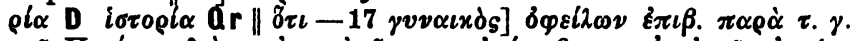

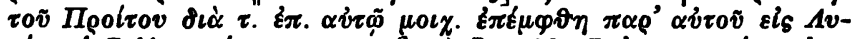

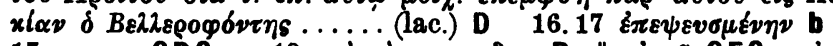

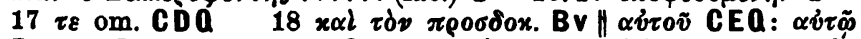

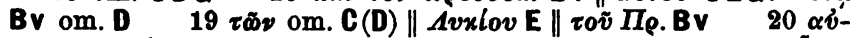

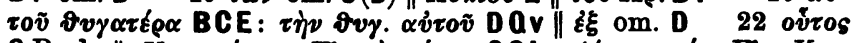

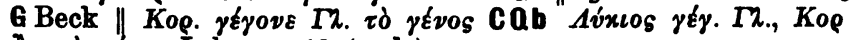

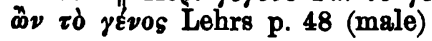




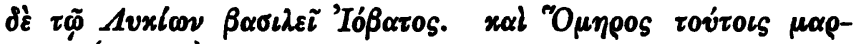

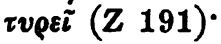

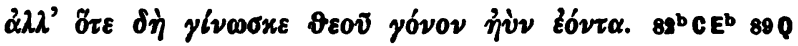

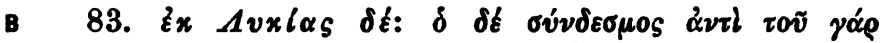

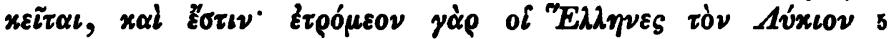
Гגа⿰ี⿻上丨.

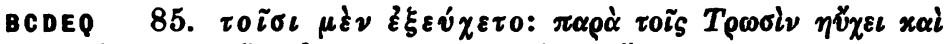

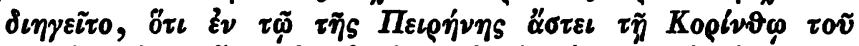

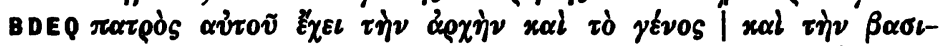

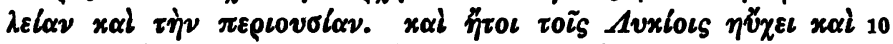

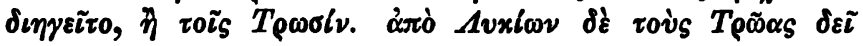

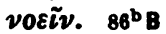

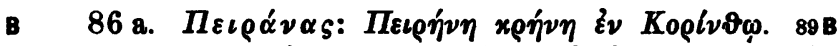

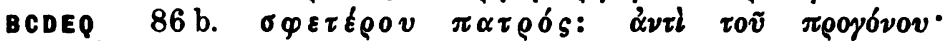

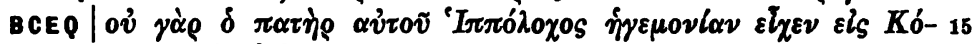

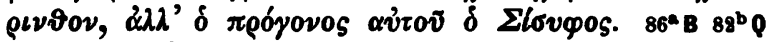

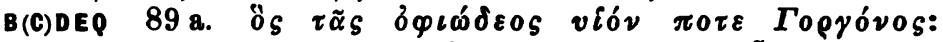

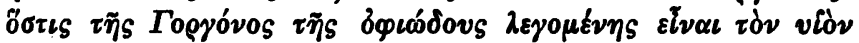

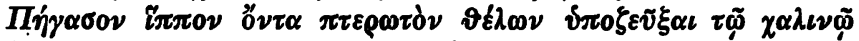

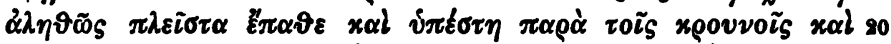

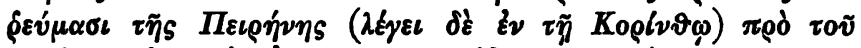

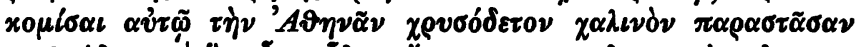

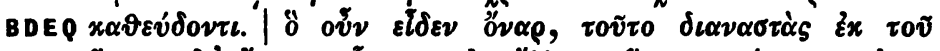
CЕН

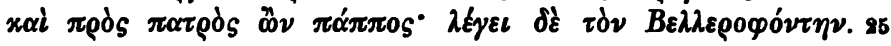

13 EM 668,4 . EG 457,31

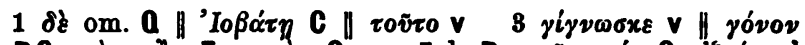

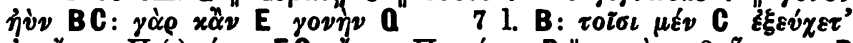

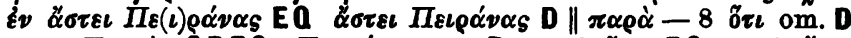

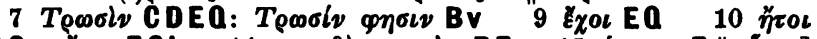
BDQ: $\varepsilon l^{\prime} \tau \iota$ EGh $14 \sigma \varphi$. $\delta \xi \pi \alpha \tau \rho \dot{s} s$ BE $15 \delta$ om. E $\| \varepsilon l_{x \in \nu]}$

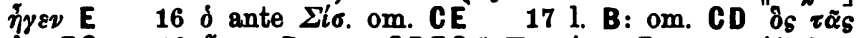

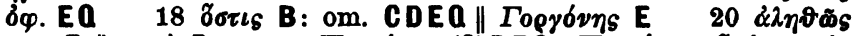

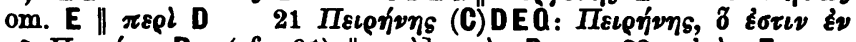

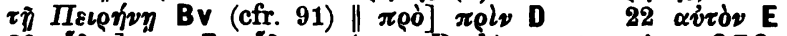

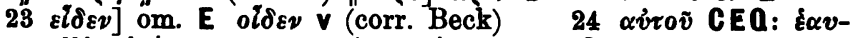
$\tau o \tilde{v} \mathrm{Hh} \dot{\alpha} \sigma i o v$ v $25 x \alpha \imath-\pi \dot{\alpha} \pi \pi 0$ om. C 


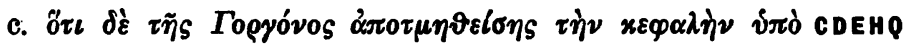

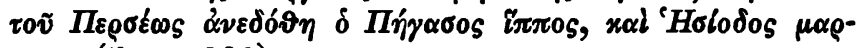

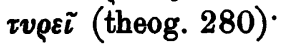

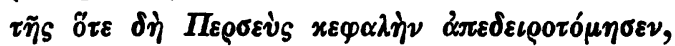

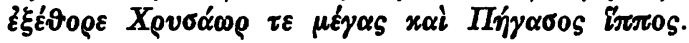

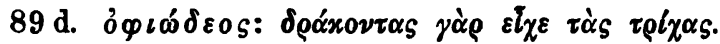

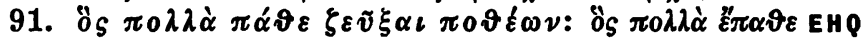

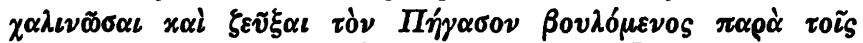

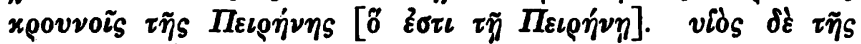
$10 M \varepsilon \delta 0 v \dot{\sigma} \eta s \delta \Pi \Pi_{\eta} \gamma \alpha \sigma o s$.

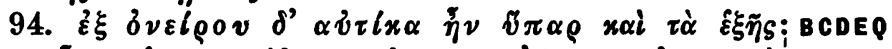

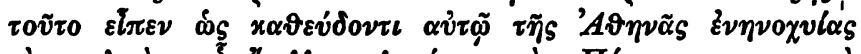

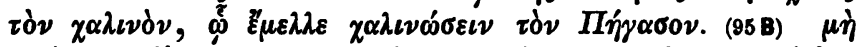

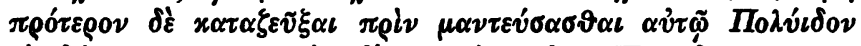

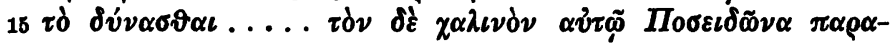
$\sigma \chi \varepsilon \tilde{\nu} \nu .97 \mathrm{~B} 99^{\circ} \mathrm{C}$

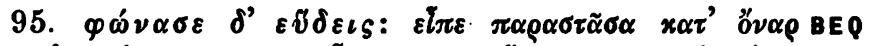

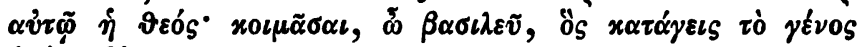

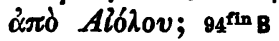

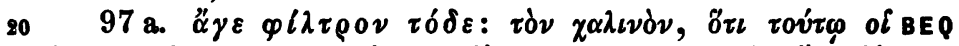

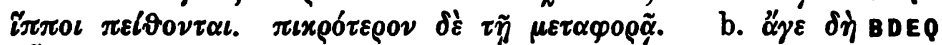

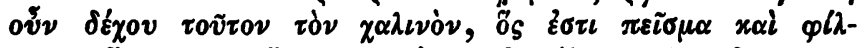

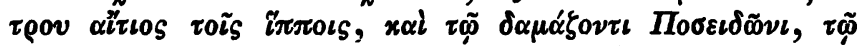

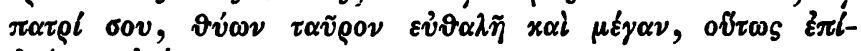
$25 \delta \varepsilon เ \xi o v \alpha u ̛ r o ́ v$.

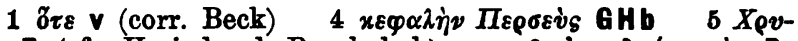

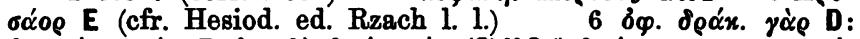

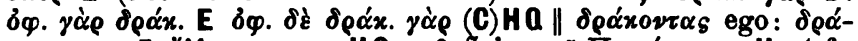

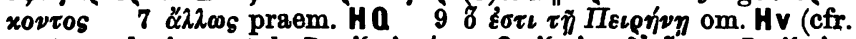

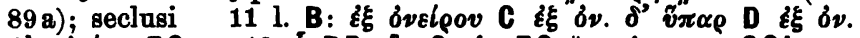

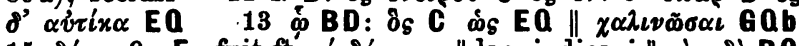

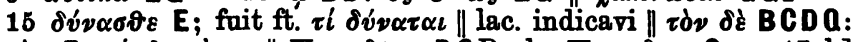

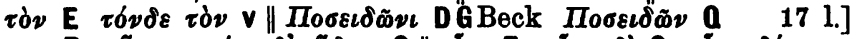

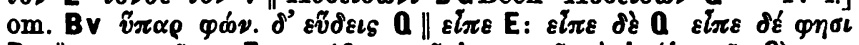

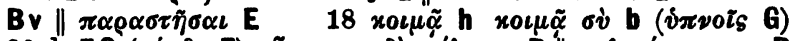

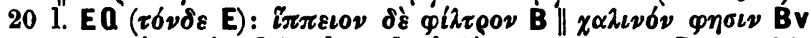

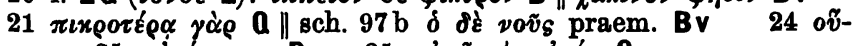

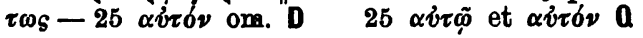




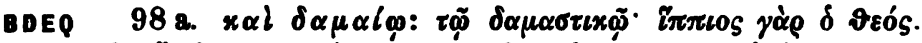

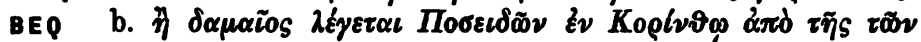

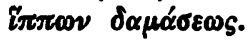

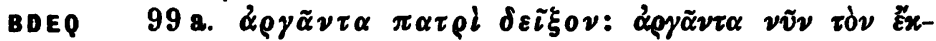

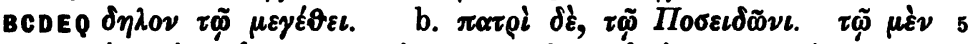

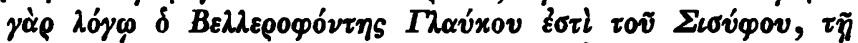

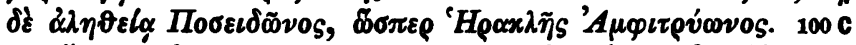

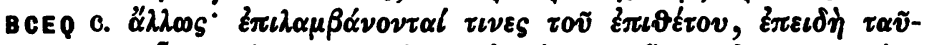

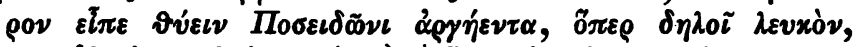

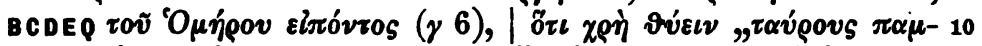

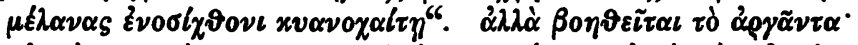

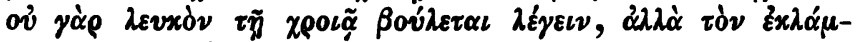

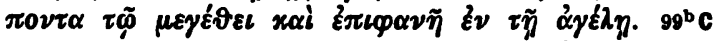

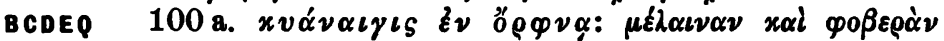

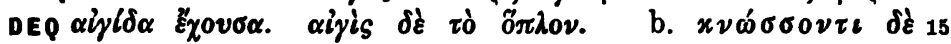
«

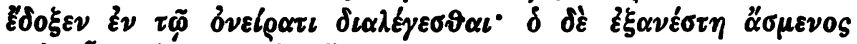

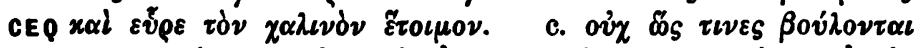

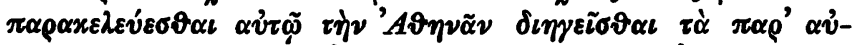

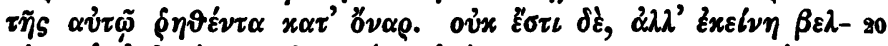

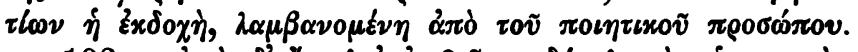

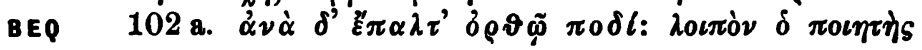

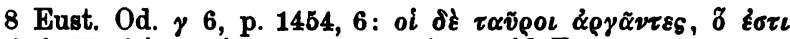

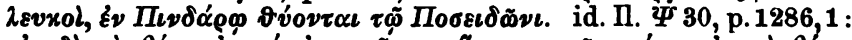

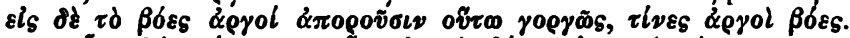

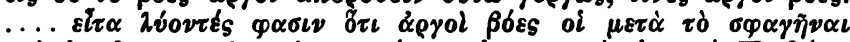

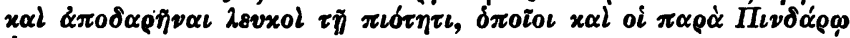

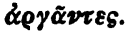

$1 x \alpha l] x \alpha \underline{i} \tau 0 \iota$ vel

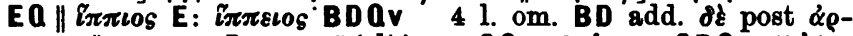
$\gamma \tilde{\alpha} \nu \tau \alpha \| \nu \tilde{\nu} \nu$ om. D $5 \tau \tilde{\omega}$ (alt.) om. CQ $6 \delta$ om $\operatorname{CDQ} 7{ }^{\prime} A \mu$.

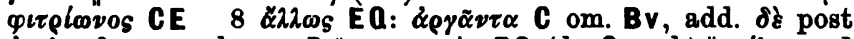

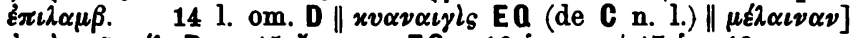

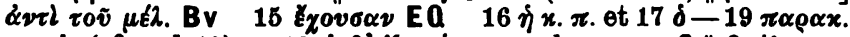

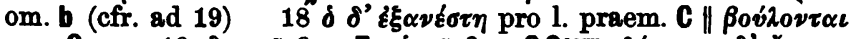

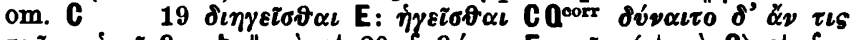

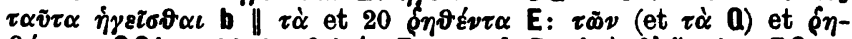

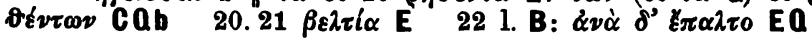




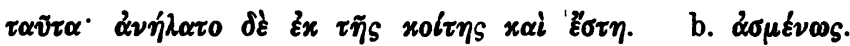
$\pi \varepsilon \rho x \alpha \rho \eta ่ s$ ด̋v.

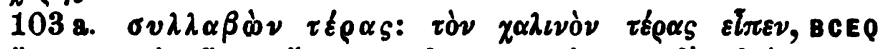

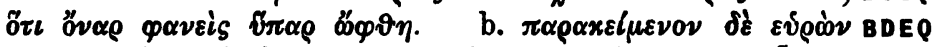

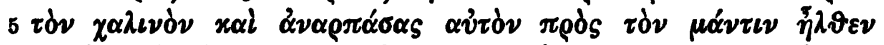

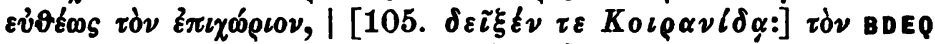

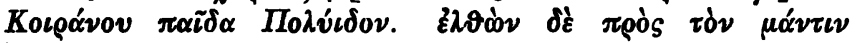

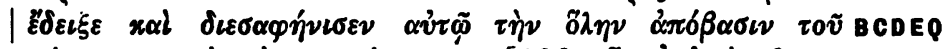

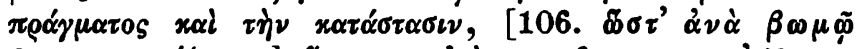

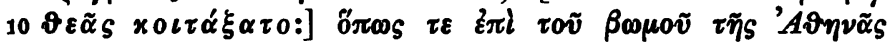

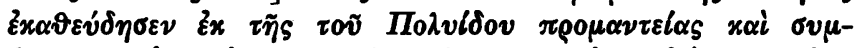

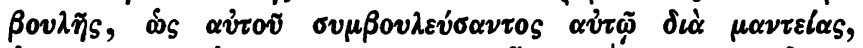

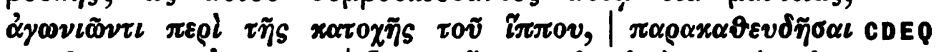

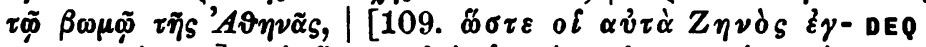

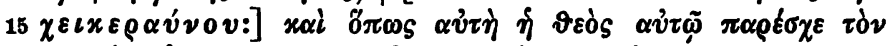

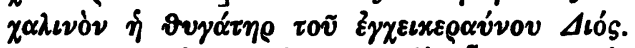

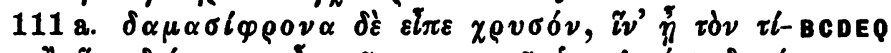

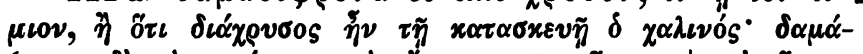

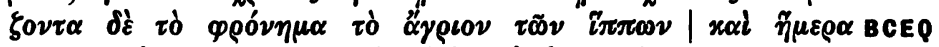

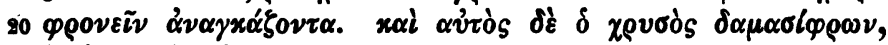

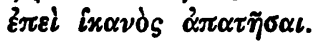

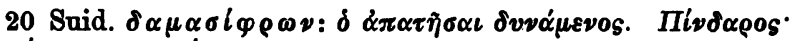
$\delta \alpha \mu \alpha \sigma i \varphi \rho o v \alpha$ $\chi \rho v \sigma \delta \nu$.

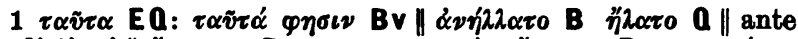

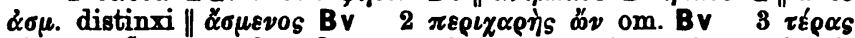

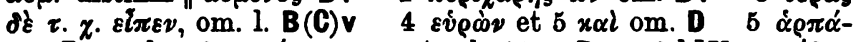

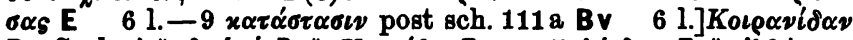

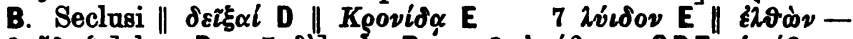

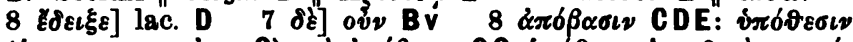

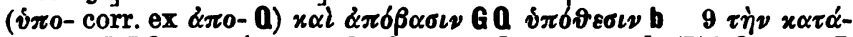
$\sigma \tau \alpha \sigma \iota v$ DE Q: $x \alpha \tau \alpha \dot{\sigma} \sigma \alpha \sigma \iota \nu$ B $\alpha \pi 0 x \alpha \tau$. C 9.10 l. (D)E Q: om. B

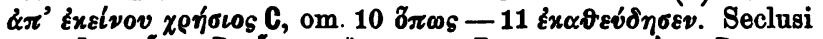

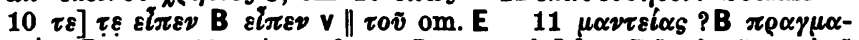

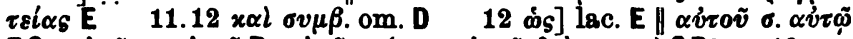

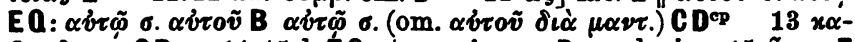

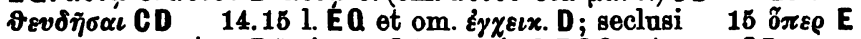

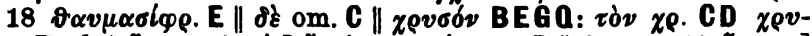

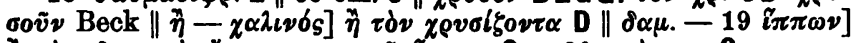

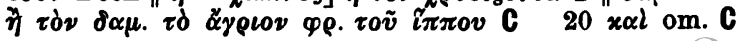


BCDEQ 111 b. $\varepsilon v v \pi v l_{\varphi} \delta^{\prime} \stackrel{\gamma}{\alpha} \tau \alpha \dot{\alpha} \chi \iota \sigma \tau \alpha \pi \varepsilon \ell \vartheta \varepsilon \sigma \vartheta \alpha \iota: \tau 0 \tilde{v} B \varepsilon \lambda d \varepsilon-$

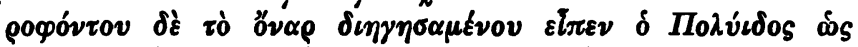

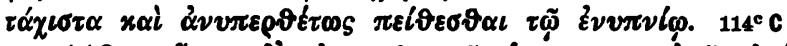

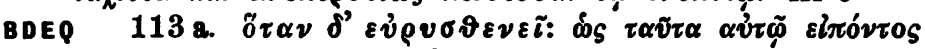

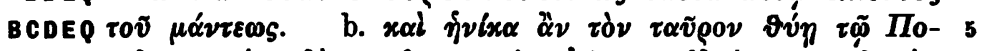

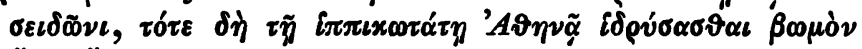

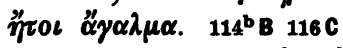

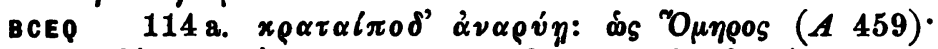

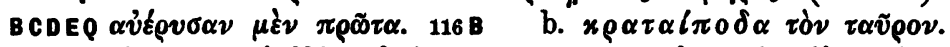

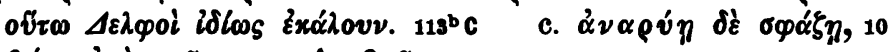

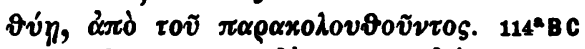

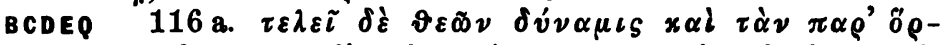

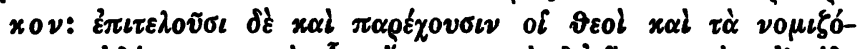

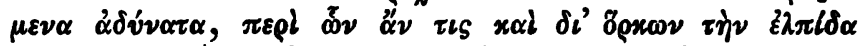

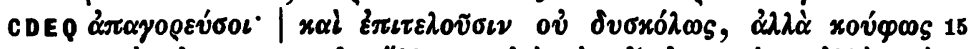

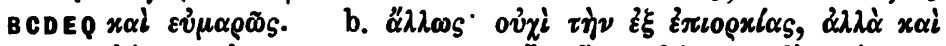

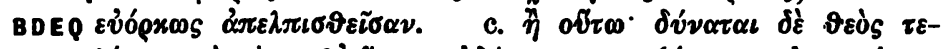

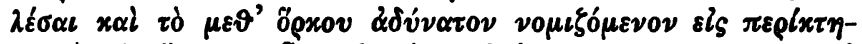

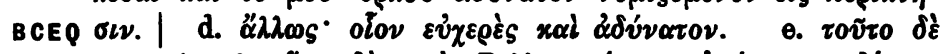

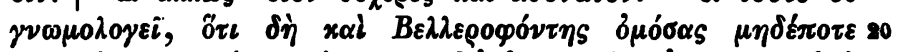

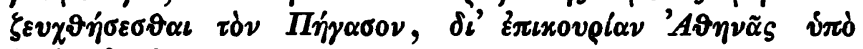

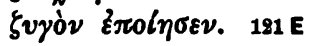

$1 \delta^{\prime} \underset{\alpha}{\alpha}$ E in textu: $\delta \alpha \iota$ D $\delta \xi$ B EHQ $\| \pi \varepsilon i \theta \varepsilon \sigma \theta \alpha \iota$ om. (C) DEQ

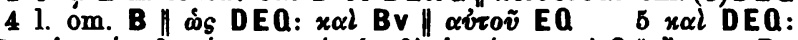

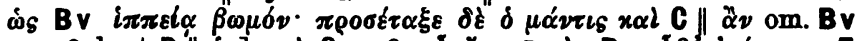

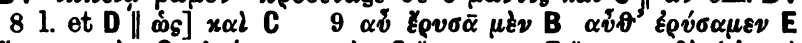

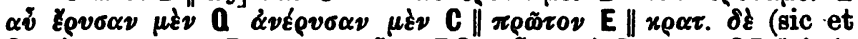

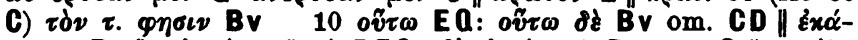

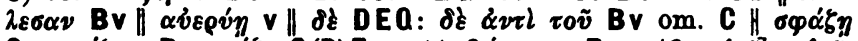

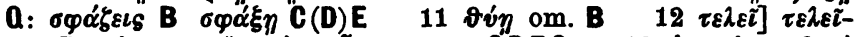

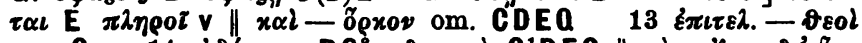

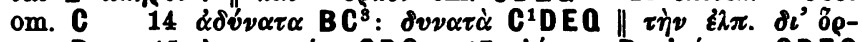

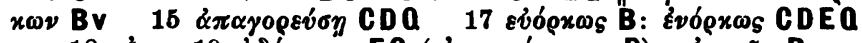

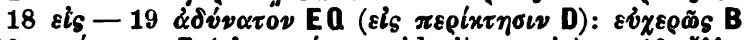
18. $19 \pi \varepsilon \rho i x \tau \iota \sigma \iota \nu ~ E ~\left(\varepsilon i_{s} \pi \varepsilon \rho i x \tau\right.$. quid sit, nescio) $19 \alpha \alpha \lambda \omega s-$

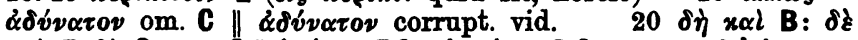

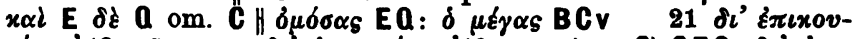

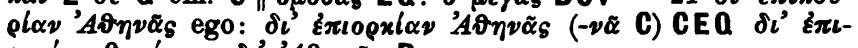

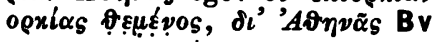




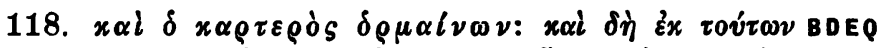

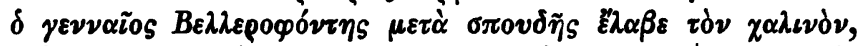

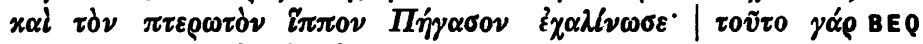

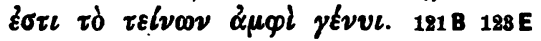

6

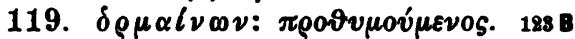

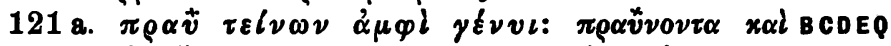

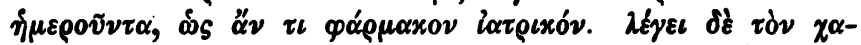

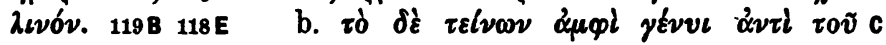
$\chi \alpha \lambda \varepsilon \nu \tilde{\nu} \nu$.

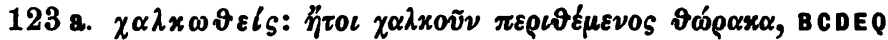

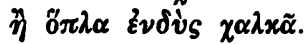

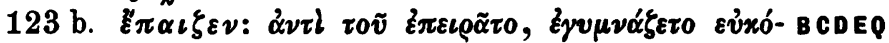

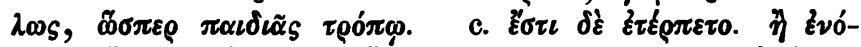

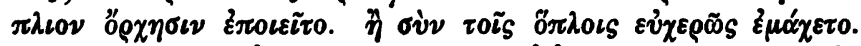

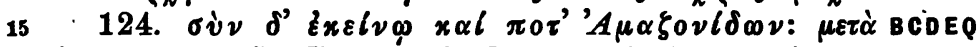

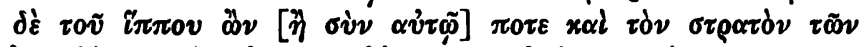

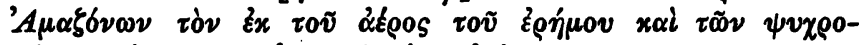

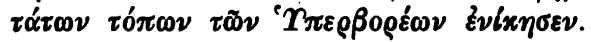

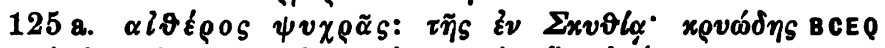

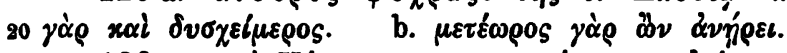

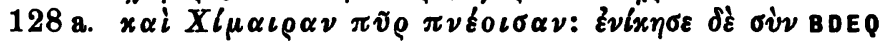

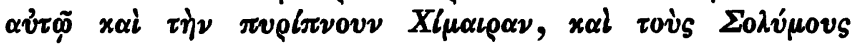

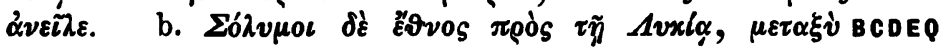

1 1. DEQ: $\delta \delta \xi$ voṽs Bv

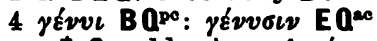
xov $\pi \rho \alpha \dot{v} \mathrm{C}$, add. $\tau \dot{\partial} \nu \chi_{\alpha} \lambda_{\ell} \nu \sigma_{\nu}$

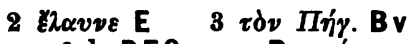

6 1. DEQ: om. B $\varphi \alpha \rho^{\prime} \mu \alpha-$

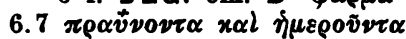

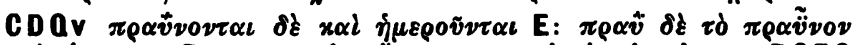

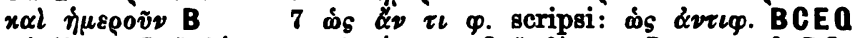

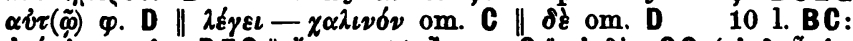

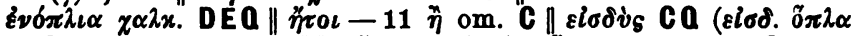

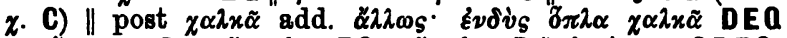

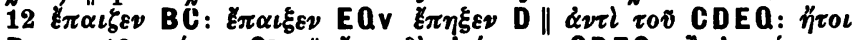

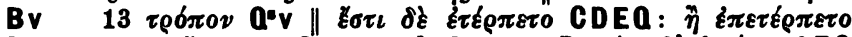

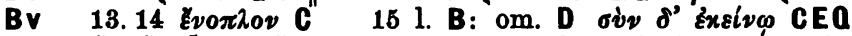

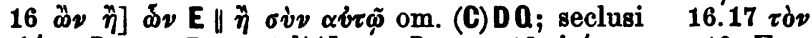

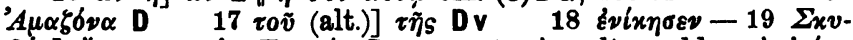

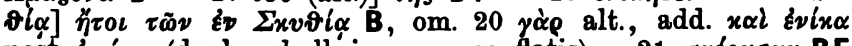

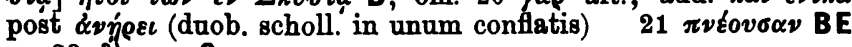
23 de om. C 


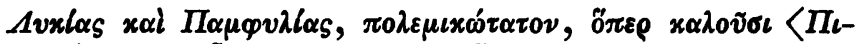

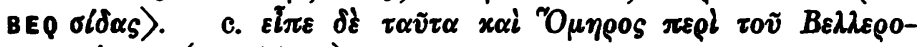
góviov (Z 155 sq.).

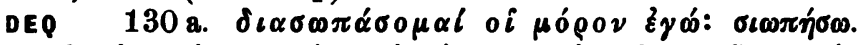

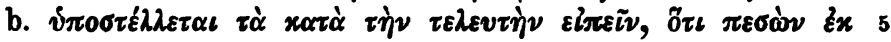

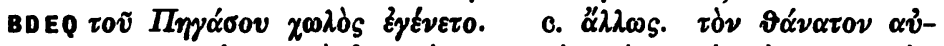

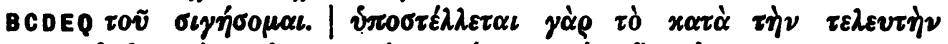

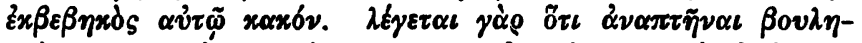

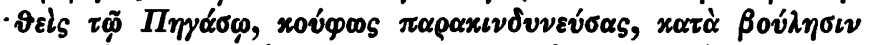

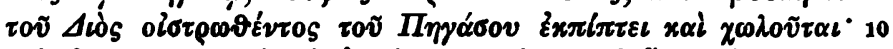

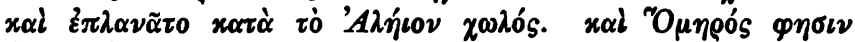
(Z 201)

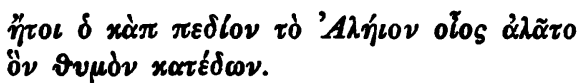

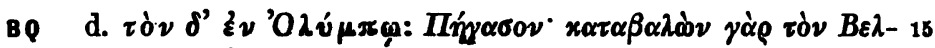

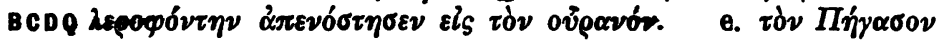

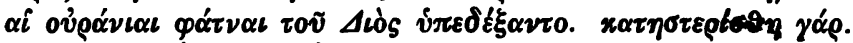

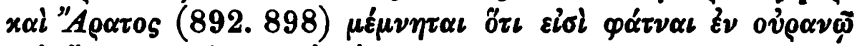

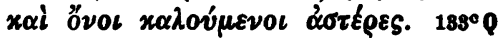

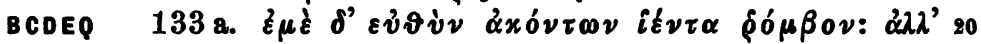

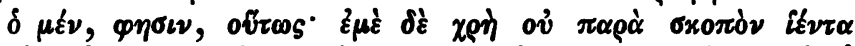

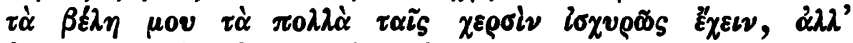

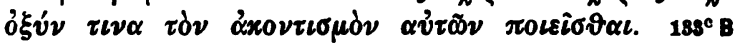

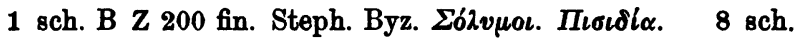
AB Z 155.

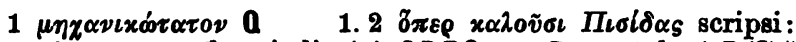

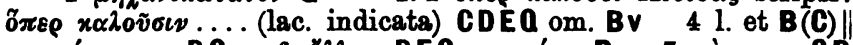

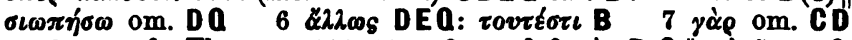

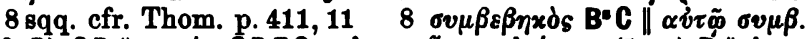

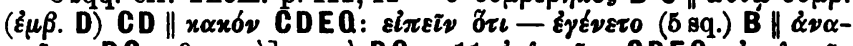

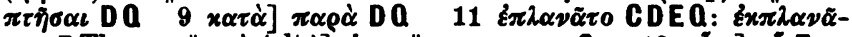
$\tau \alpha \iota$ B Thom. $v \| x \alpha i$ (alt.)] $\dot{\omega} s v \| \varphi \eta \sigma \iota v$ om. C 13 olos] of E

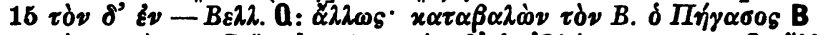

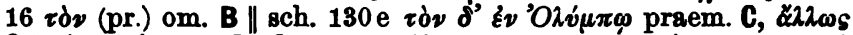

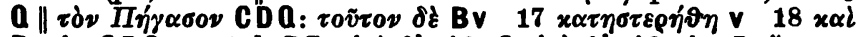

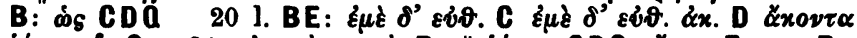

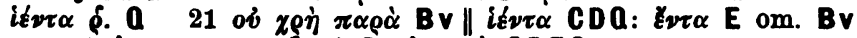

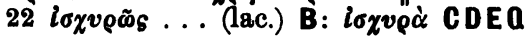




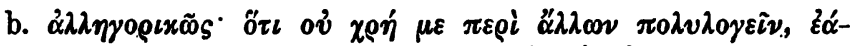

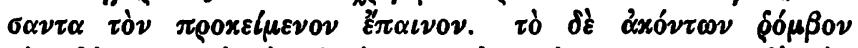

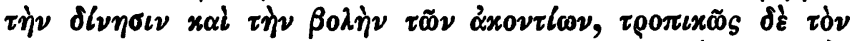

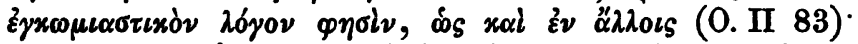

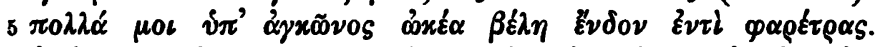

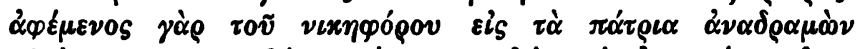

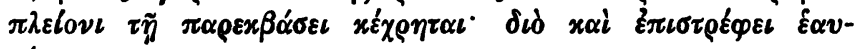
$\tau o ́ v . ~ 136$ BQ

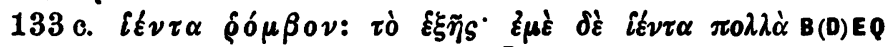

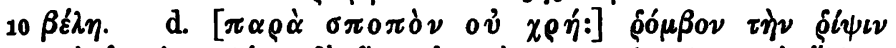

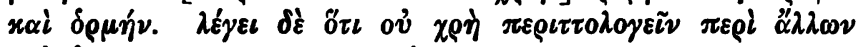

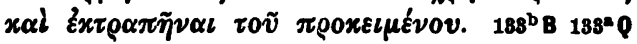

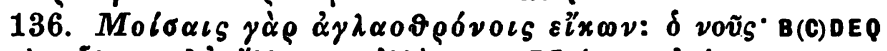
ở yò

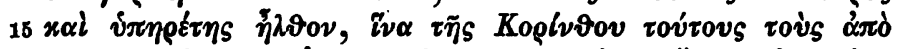

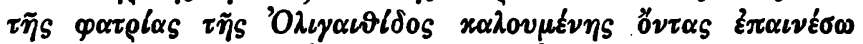

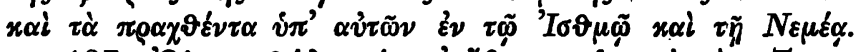

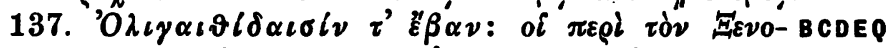

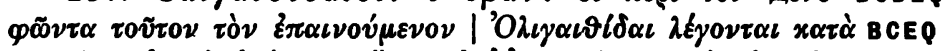

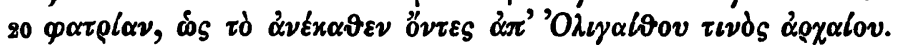

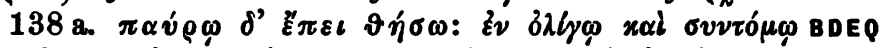

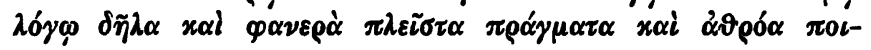

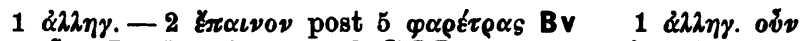

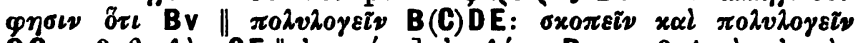

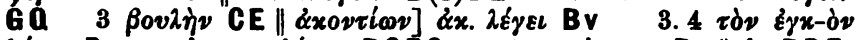

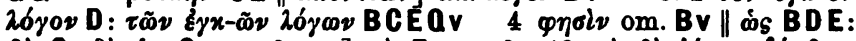

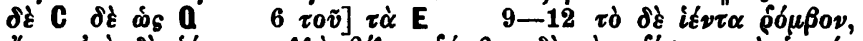

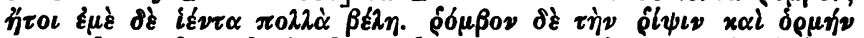

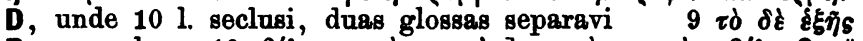

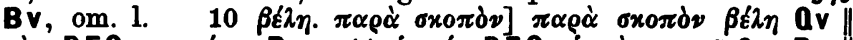

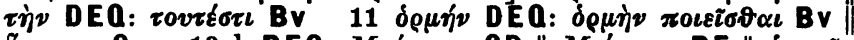

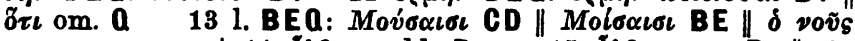

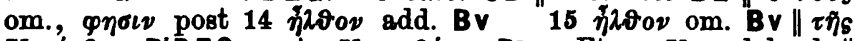

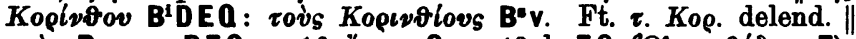

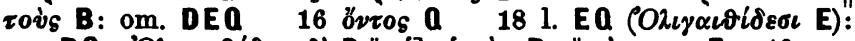

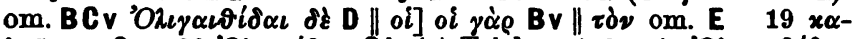

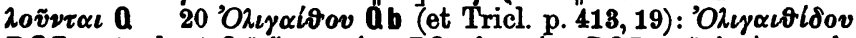

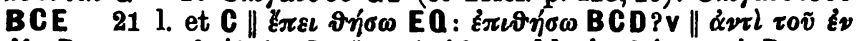

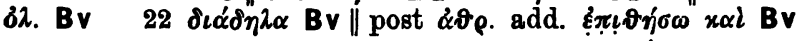




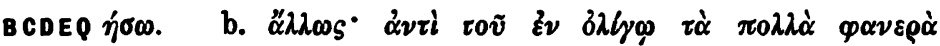

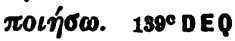

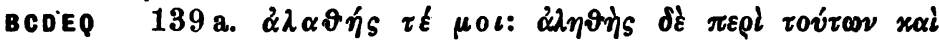

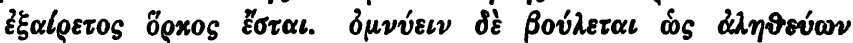

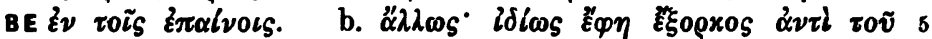

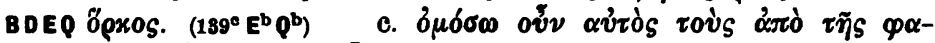

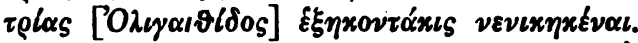

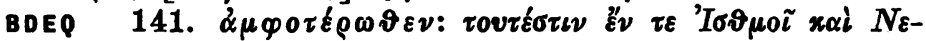

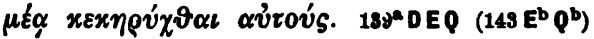

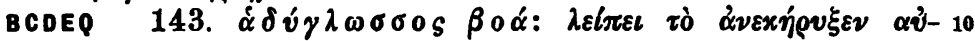

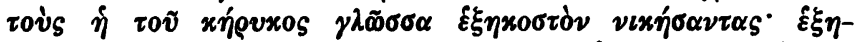

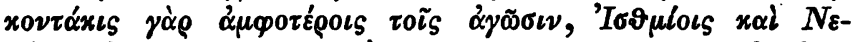

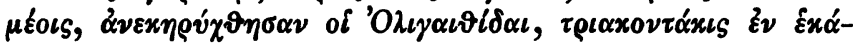

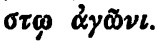

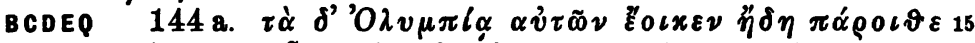

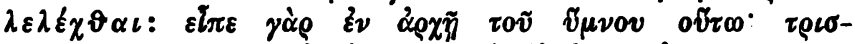

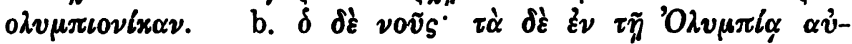

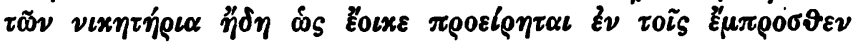

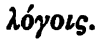

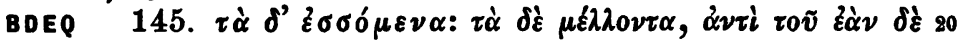

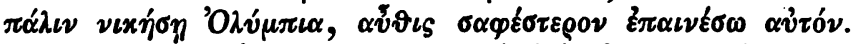

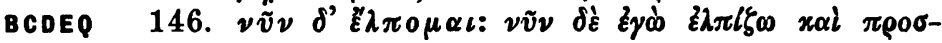

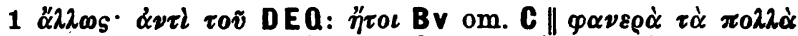

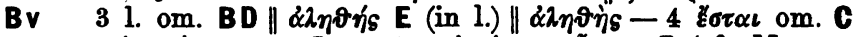

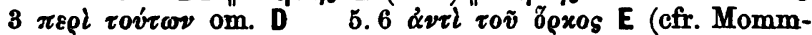

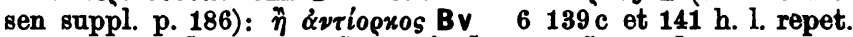

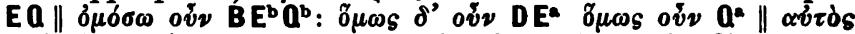

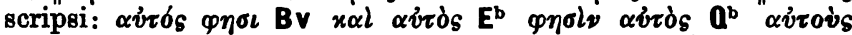

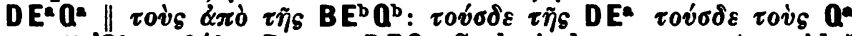

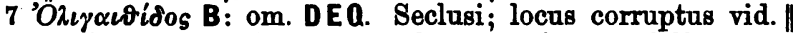

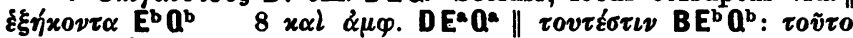

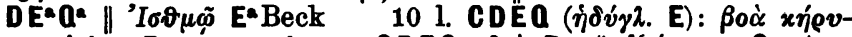

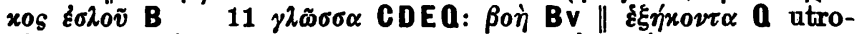

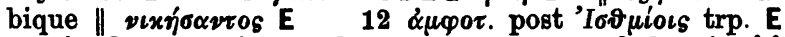
$13 \lambda^{\prime}$ BCDE: $\tau \rho \iota \alpha^{\prime} x o v \tau \alpha$ Q (post $\left.\dot{\alpha} \gamma \tilde{\omega} \nu \imath\right) v$ 15 l. B: $\tau \dot{\alpha} \delta^{\circ}{ }^{\circ} O \lambda v \mu-$

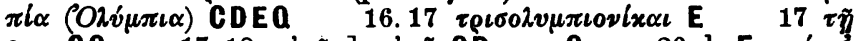
om. CQ 17.18 $\alpha \dot{v} \tau \tilde{\omega} \nu] \alpha \dot{v} \tau \tilde{\omega}$ CD om. Q 20 l. E: $\tau \alpha^{\prime} \tau^{3}$

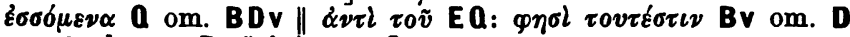
22 l. om. Bv \| Éro om. C 


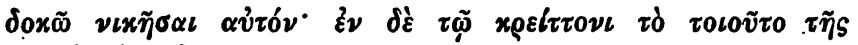

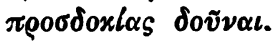

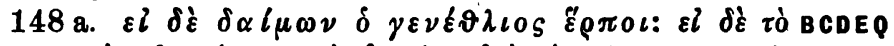

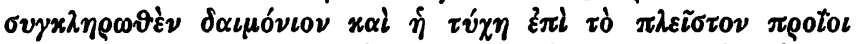

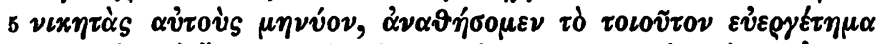

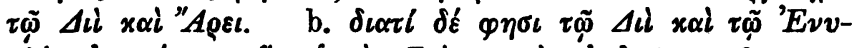

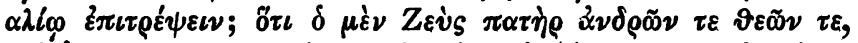

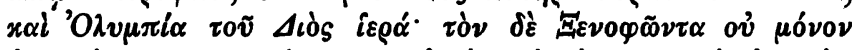

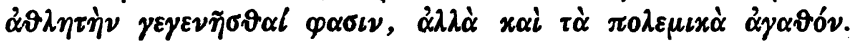

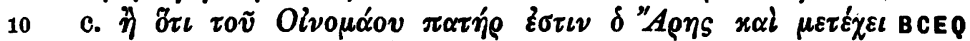

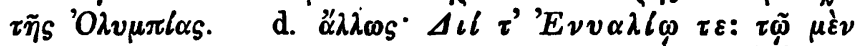

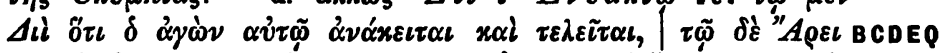

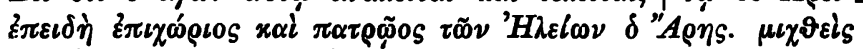

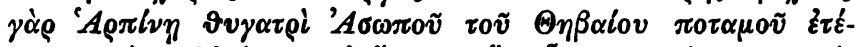

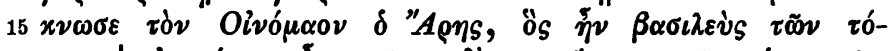

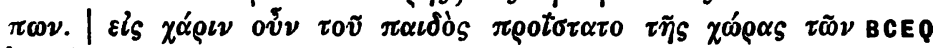
'Hiciov.

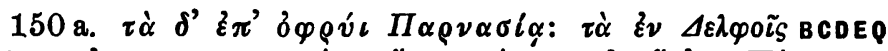

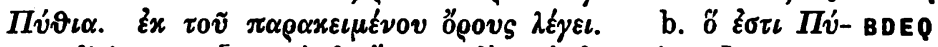

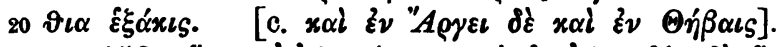

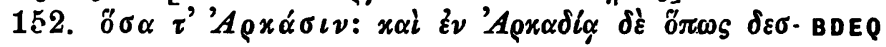

13 St. Byz «ڤ

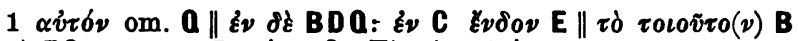

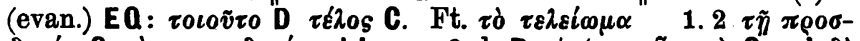

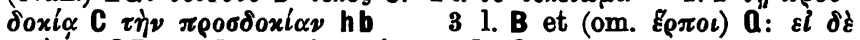

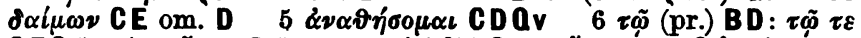

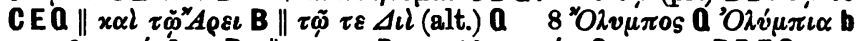

$9 \gamma \varepsilon v \varepsilon \sigma \theta \alpha \iota$ Bv $\| \varphi \eta \sigma \iota \nu$ D $10 \pi \alpha \tau \eta \dot{\rho}$ Cv: om. BDEQ

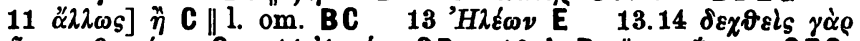

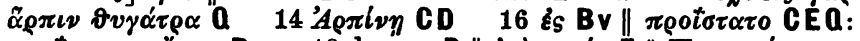

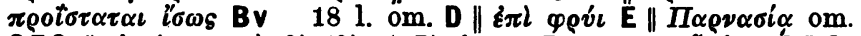

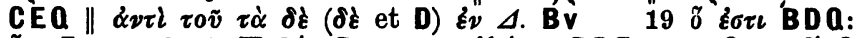

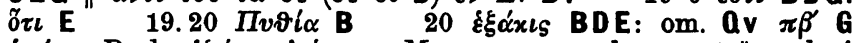

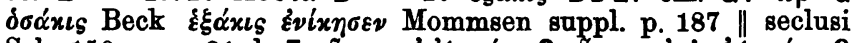

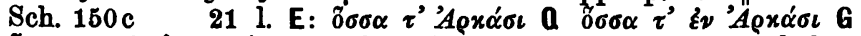

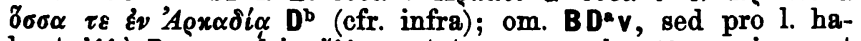
bent $\alpha \lambda \lambda \dot{\alpha} \mathrm{Bv}$, quod in $\alpha \lambda \lambda \alpha$ mutatum cum sch. $150 \mathrm{c}$ coniungunt h b $21 \delta^{2} D^{\mathrm{b}} E G Q$ : $\tau \varepsilon D^{\alpha}$ om. B 


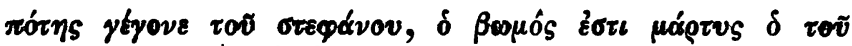

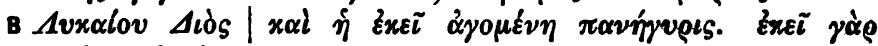

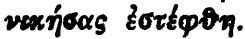

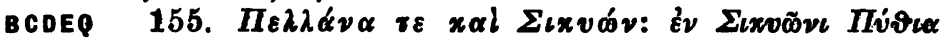

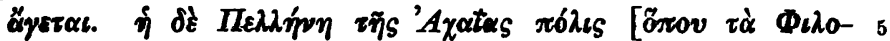

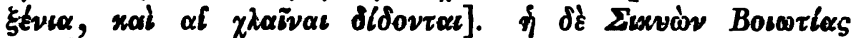

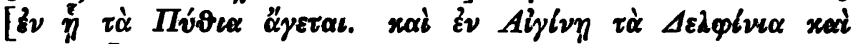
Aláx́čla].

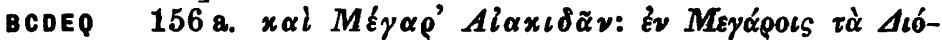

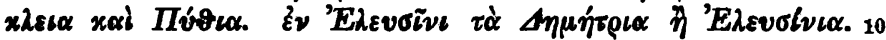

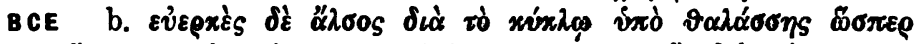

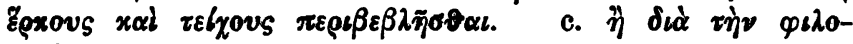
$\xi \varepsilon v l \alpha v$.

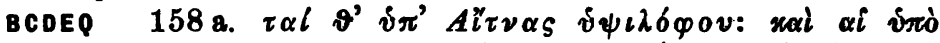

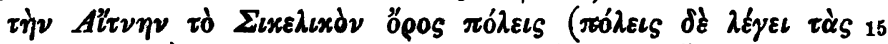

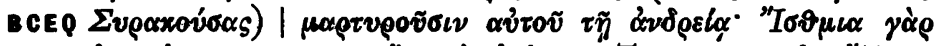

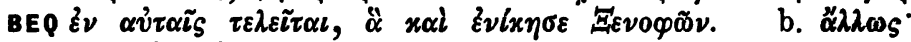

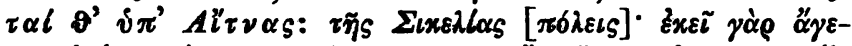

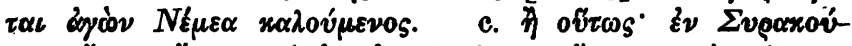

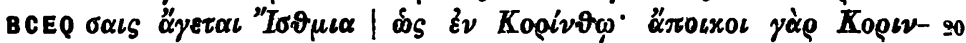

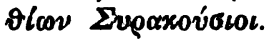

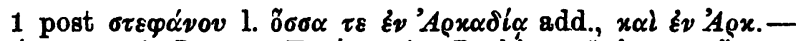

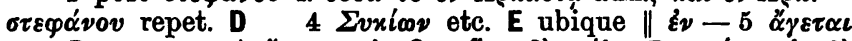

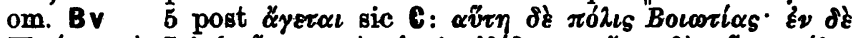

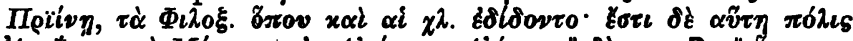

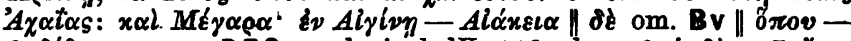

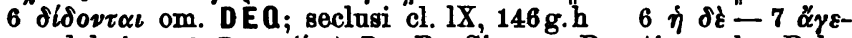

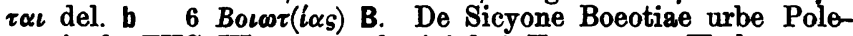
monis fr. FHG III, 135 conferri inbet Unger par. Theb. p. 363

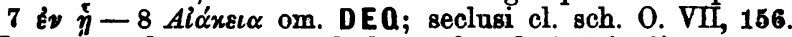

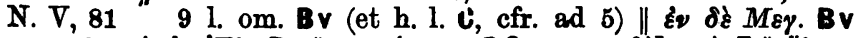

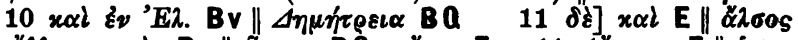

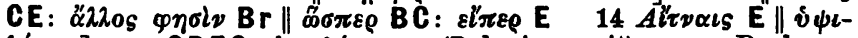

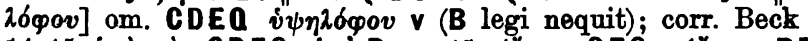

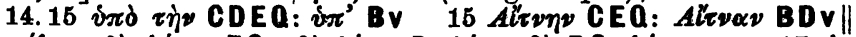

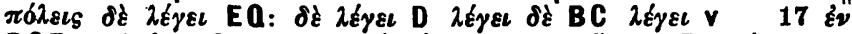

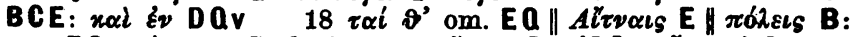

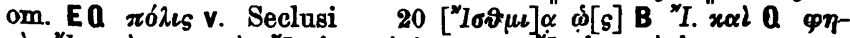

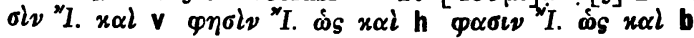




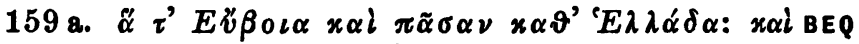

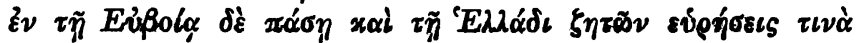

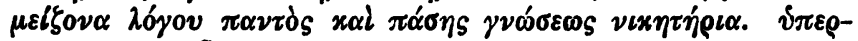

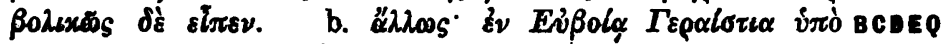

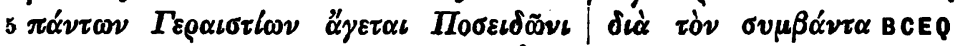

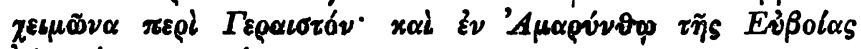

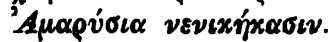

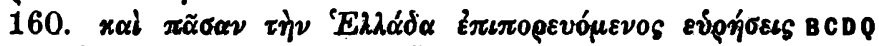
$\mu \varepsilon l \xi o v \alpha$ हैอ

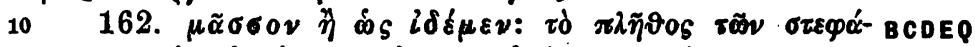

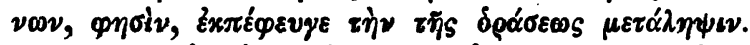

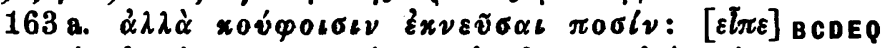

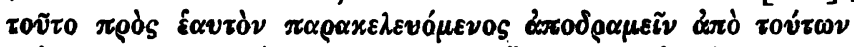

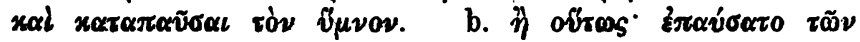

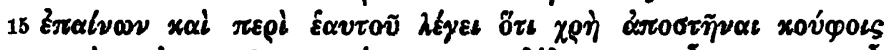

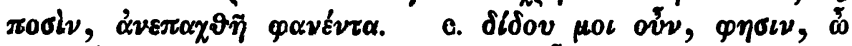

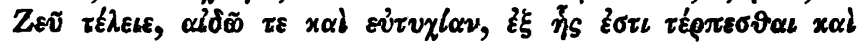

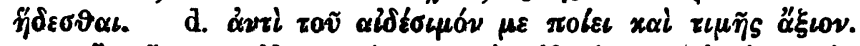

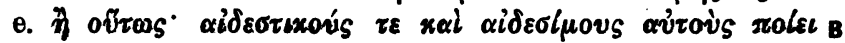

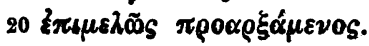

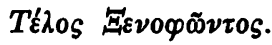

1 1. B: $\ddot{\alpha} \tau^{\prime} E \ddot{z} \beta o \iota \alpha$ D (cfr. ad 4) $\alpha \tilde{\imath} \tau^{\prime} E z \beta o \iota \alpha \iota$ E $\tilde{\alpha} \tau^{\prime} E \ddot{v}-$

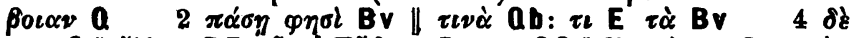

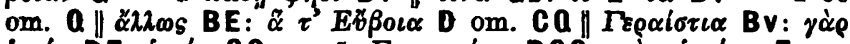

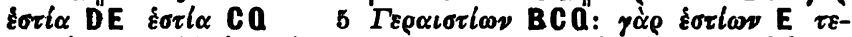

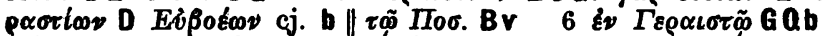

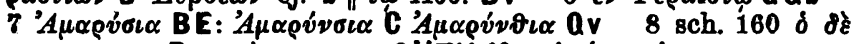

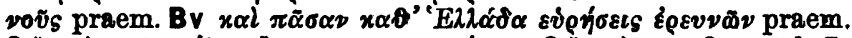

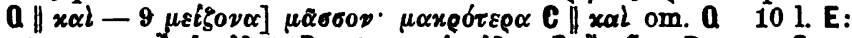

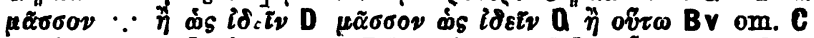

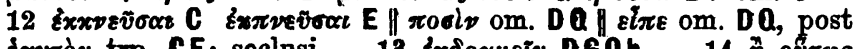

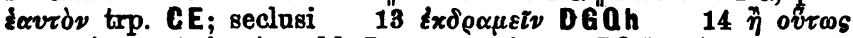

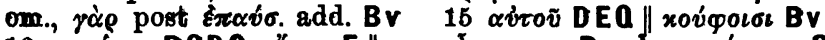

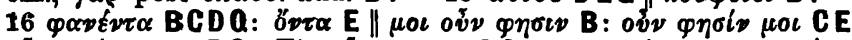

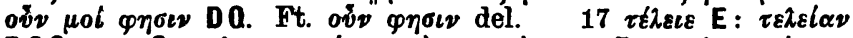

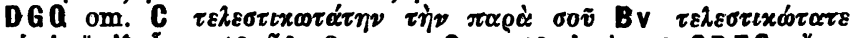

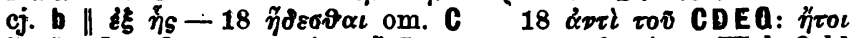

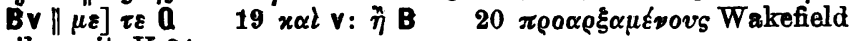
silv. crit. II 94 


\section{Scholia in 0lympionicarum carmen XIV.}

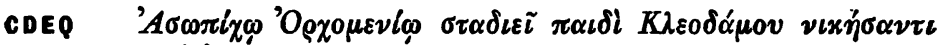

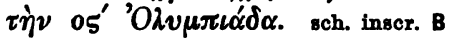

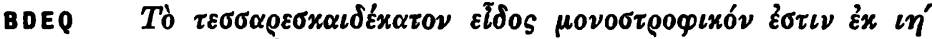

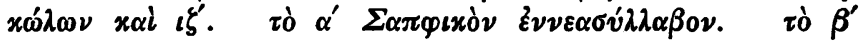

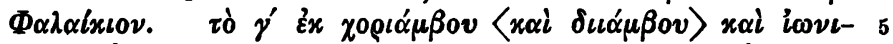

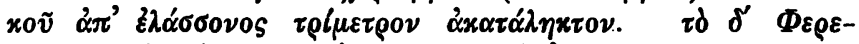

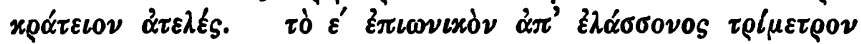

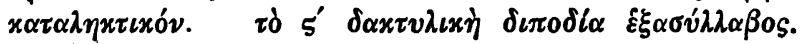

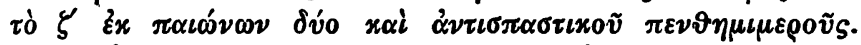

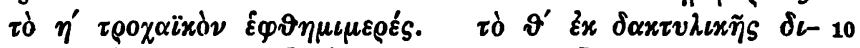

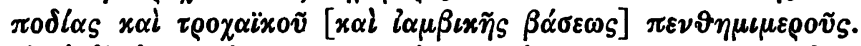

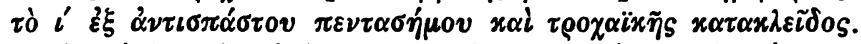

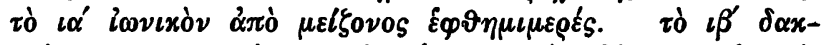

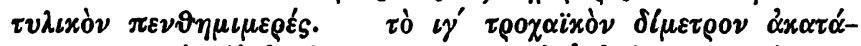

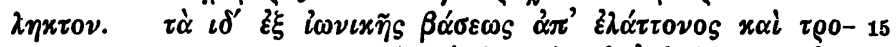

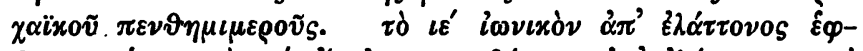

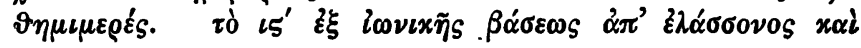

De $\beta$ (i. e. Vat. 1333) cfr. praef.

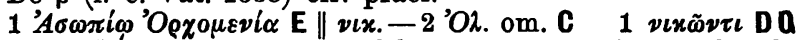

2 o $\mathrm{D}$. Utrumque numerum falsum esse ostendit pap. Oxyrh.

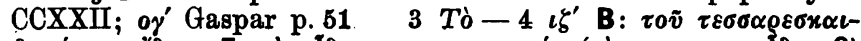

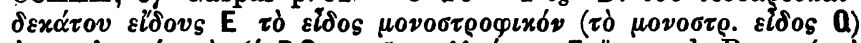

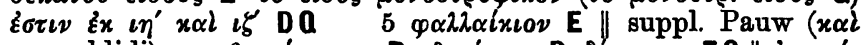

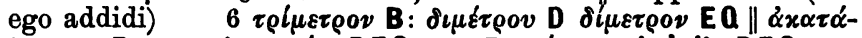
$\lambda \eta x \tau 0 \nu$ B: $x \alpha \tau \alpha \lambda \eta x \tau \iota x \delta \nu$ DEQ $7 \tau$ व

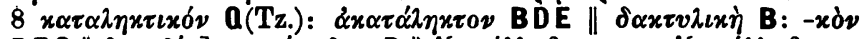

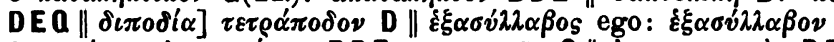

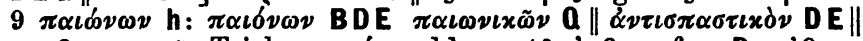

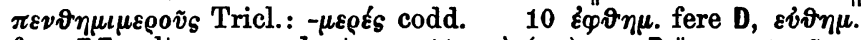

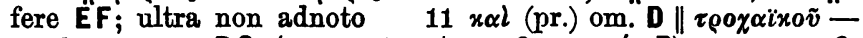

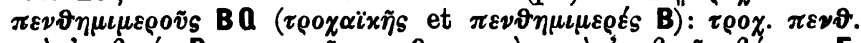

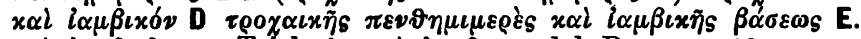
noi $i \alpha \mu \beta$. $\beta$. om. Tricl., b $x \alpha i l \alpha \mu \beta \iota x \tilde{y} s$ del. Pauw $12 \pi \varepsilon v \tau \alpha-$

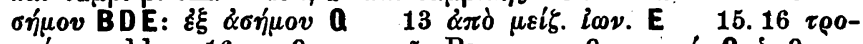

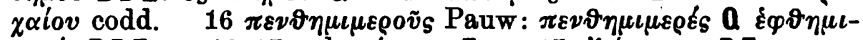

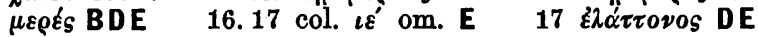




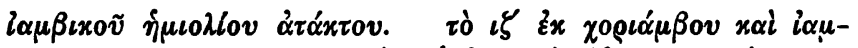

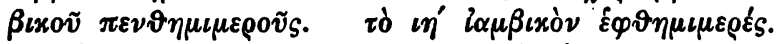

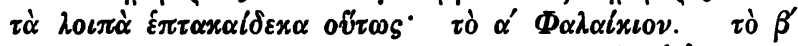

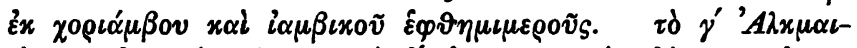

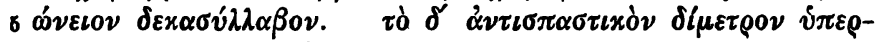

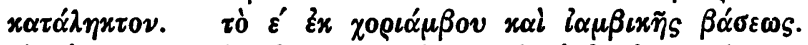

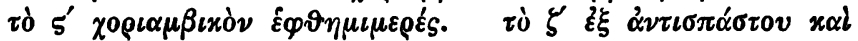

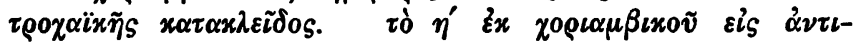

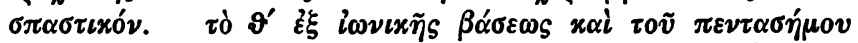

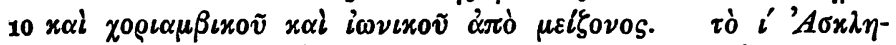

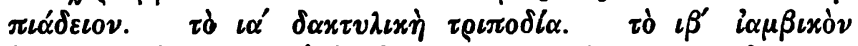

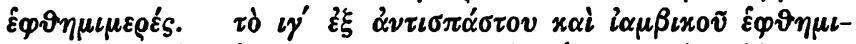

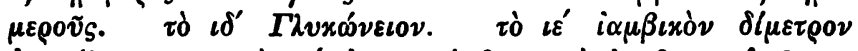

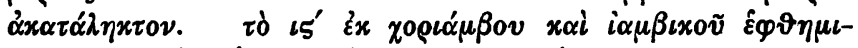

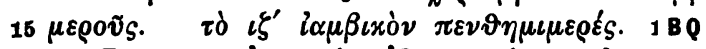

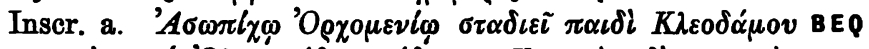

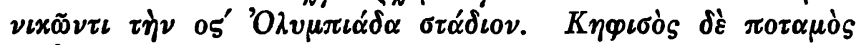

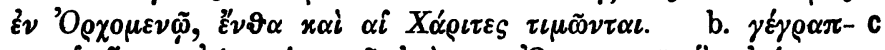

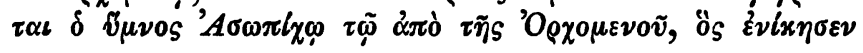

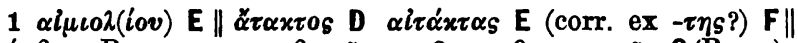

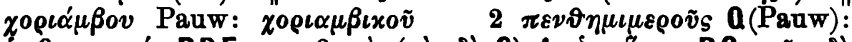

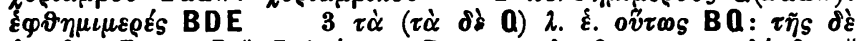

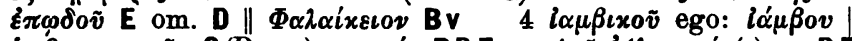

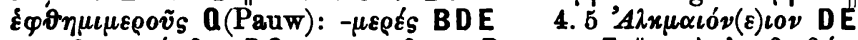

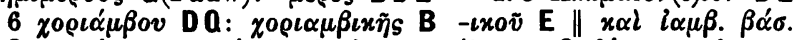

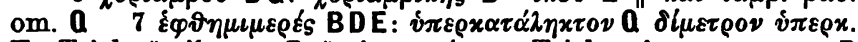
Tz. Tricl. \| $\xi_{\xi}$ om. E $\| \dot{\alpha} \nu \tau \iota \sigma \pi \alpha^{\prime} \sigma \tau 0 v$ Tricl.: $\alpha \nu \tau \iota \sigma \pi \alpha \sigma \tau \iota x o \tilde{v}$ B

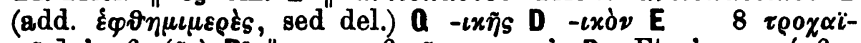

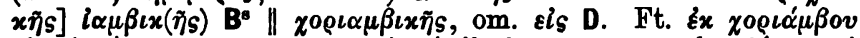

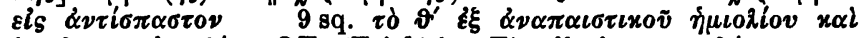

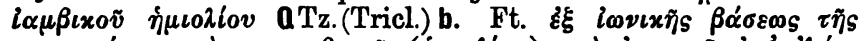

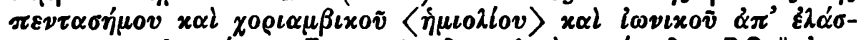

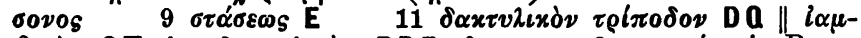
$\beta \iota x \dot{\nu} \nu$ QTz.b : $\delta \alpha x \tau v \lambda \iota x \dot{\nu} \nu$ BDE $\delta \alpha x \tau$. $\pi \varepsilon \nu \vartheta \eta \mu \iota \mu \varepsilon \rho \varepsilon_{s}$ cj. Pauw

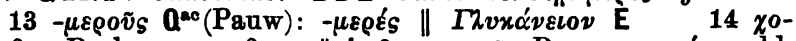

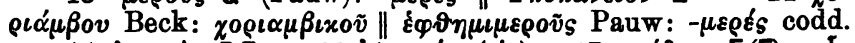

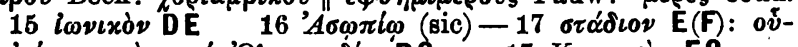

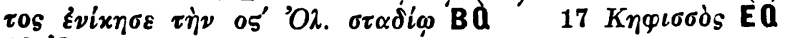

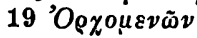




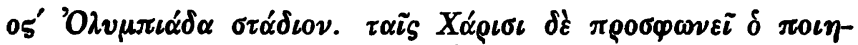

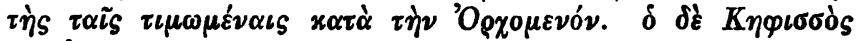

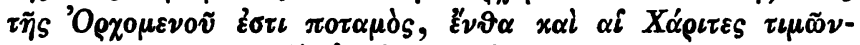

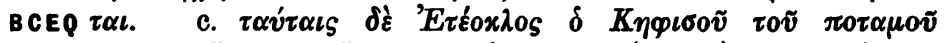

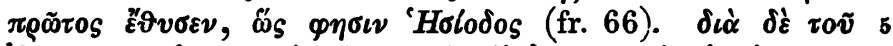

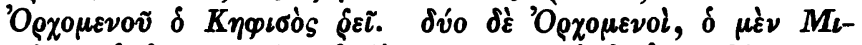

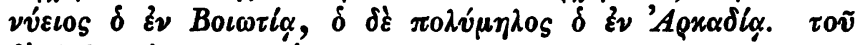

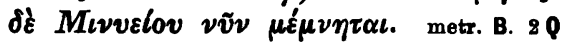

BCDEP 1 a. $K \alpha \varphi \iota \sigma l \omega \nu \delta \delta \dot{v} \tau \omega \nu \lambda \alpha \chi 0 \tilde{i} \sigma \alpha \iota: \varepsilon \pi \varepsilon \iota \delta \eta\rangle$

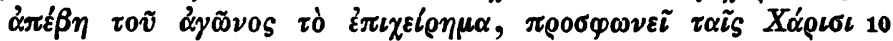

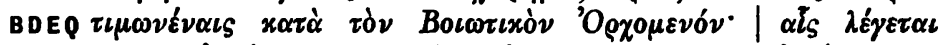

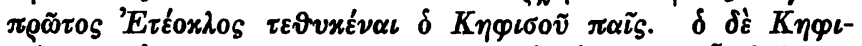

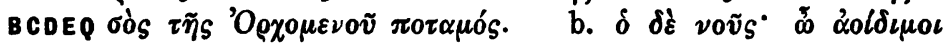
$\beta \alpha \sigma l \lambda \varepsilon l \alpha \iota$ Xó

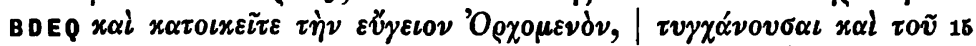

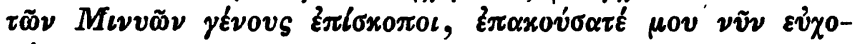

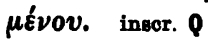

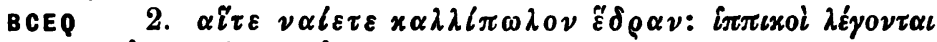
of 'O

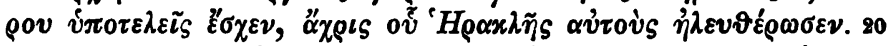

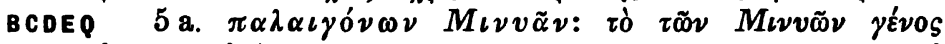

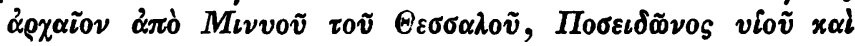

4 Strab. 9, 414. Paus. 9, 35, 1. sch. Theocr. 16, 1046 sch. AD $B$ 511. EM 635, 4. Tzetzes ad Lyc. $874 \quad 19$ Tzetzes ad Lyc. 874

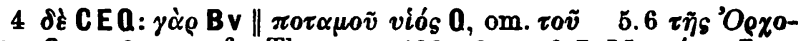
$\mu \varepsilon \nu \tilde{\omega} \nu$ C 6 sqq. cfr. Thom. p. 423, 12 6.7 Mivvंios E. $7 \delta$ (pr.) om. O\| $\delta$ (tert.) om. Q 8 Mivvniov E 9 l. om.

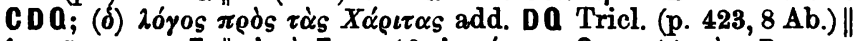

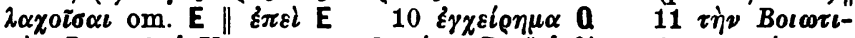

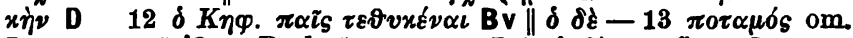

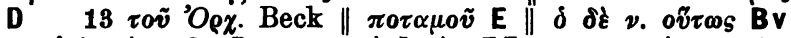

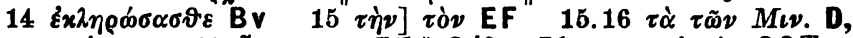

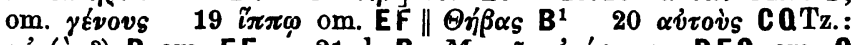

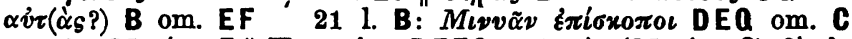

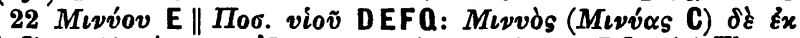

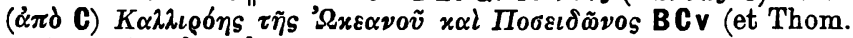
p. 423, 14); cfr. sch. 5 c 


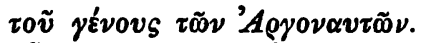

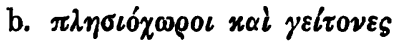

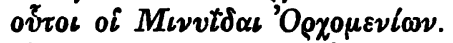

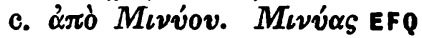

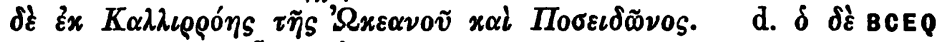

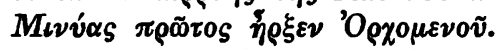

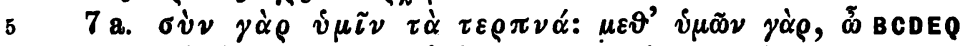

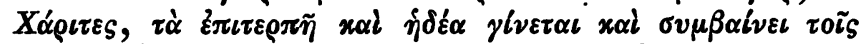

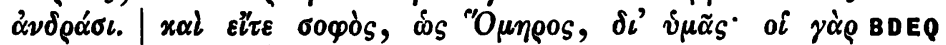

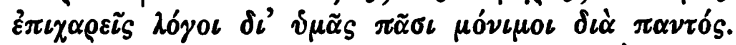

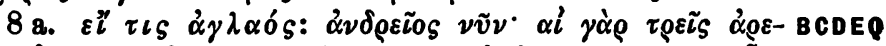

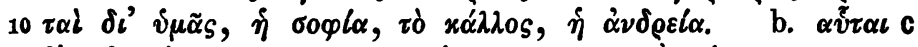

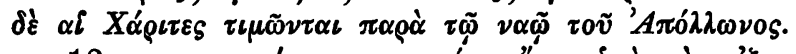

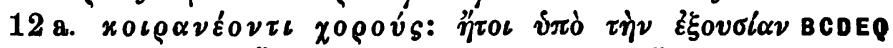

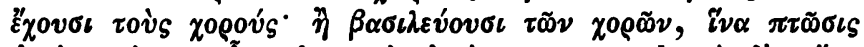

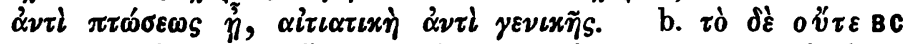

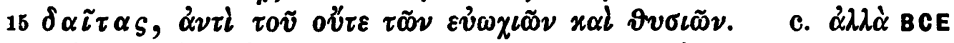

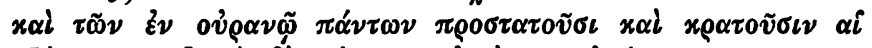

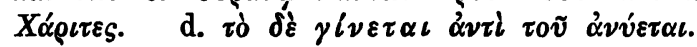

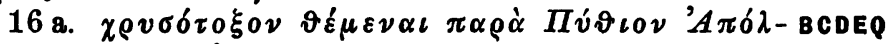

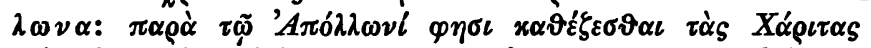

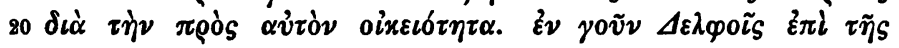

2 Tzetzes ad Lyc. 87420 Macrob. Sat. 1, 17, 13. Plut. de mus. 14. Paus. 9, 35, 3

1 post 'A

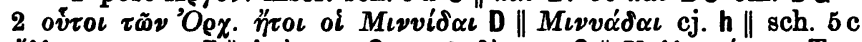

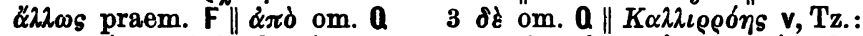

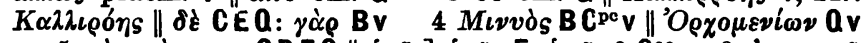

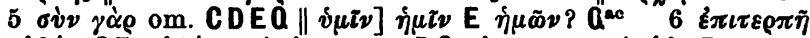

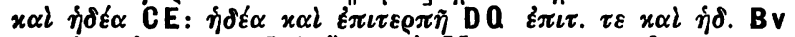
$7 x \alpha i-v \mu \tilde{\alpha} s$ om. D $\| \varepsilon i^{\prime} \tau \iota_{S} \mathrm{cj}$. Mommsen suppl. p. $196 \|$ oi

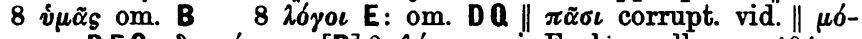

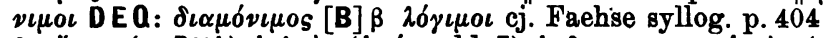

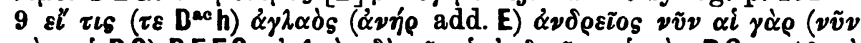

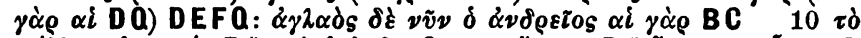

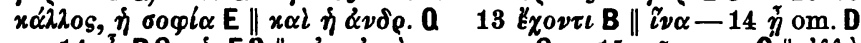

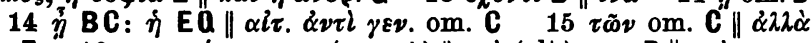

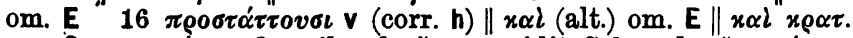

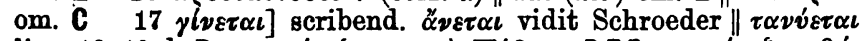

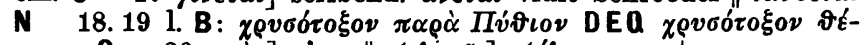

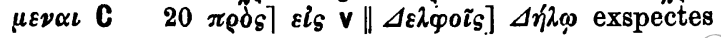




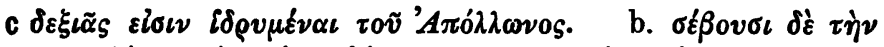

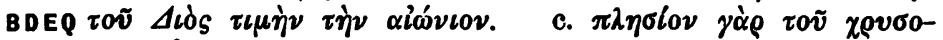

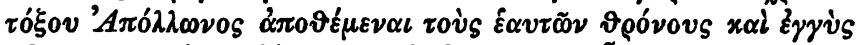

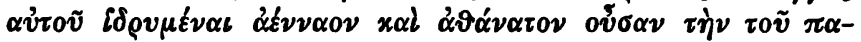

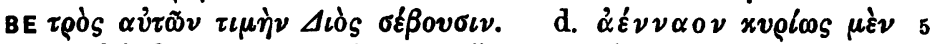

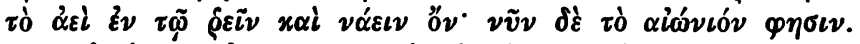

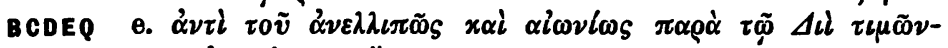

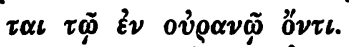

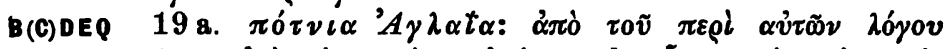

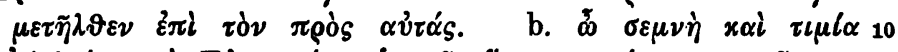

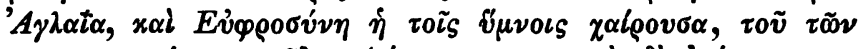

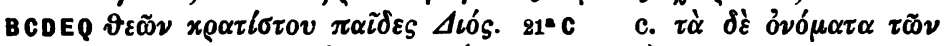

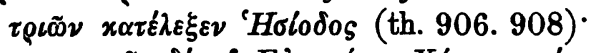

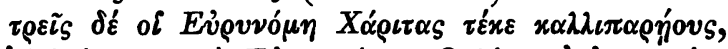

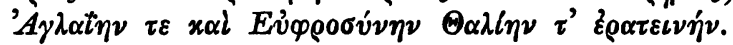

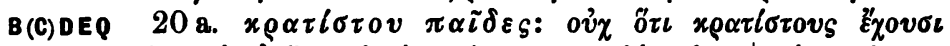

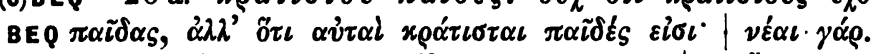
BCE

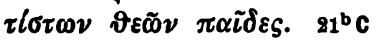

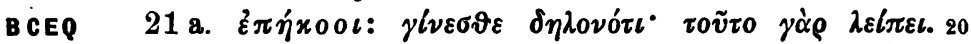

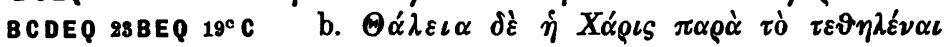

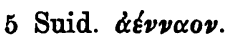

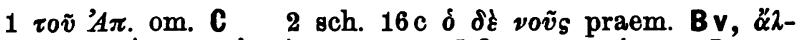

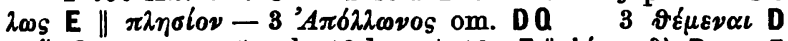

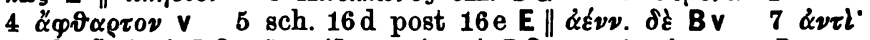

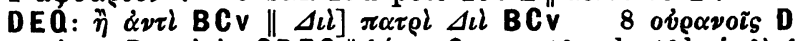

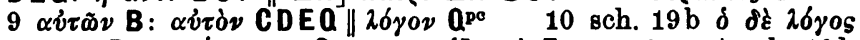

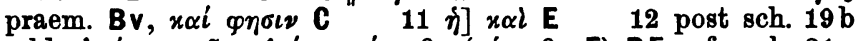

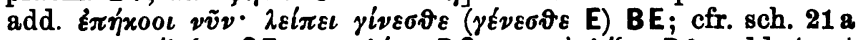

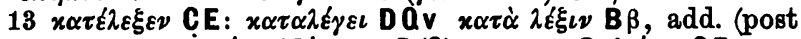

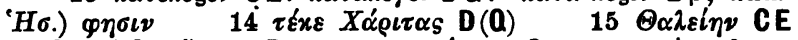

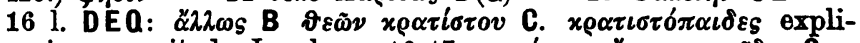

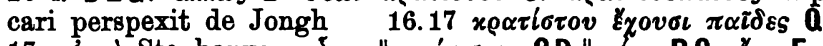

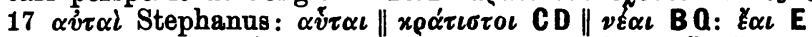

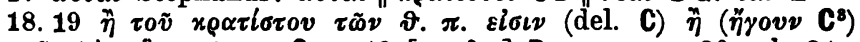

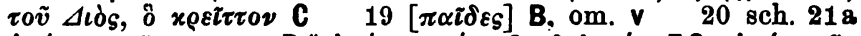

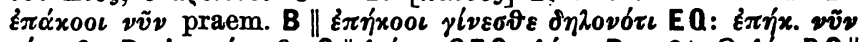

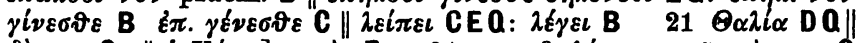

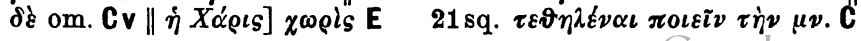




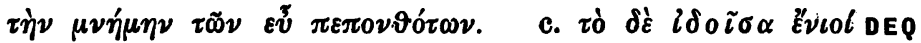

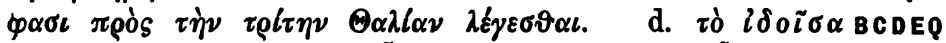

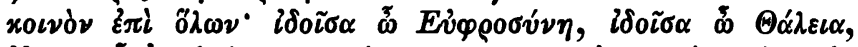

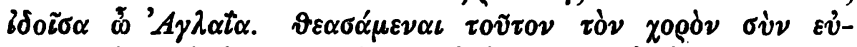

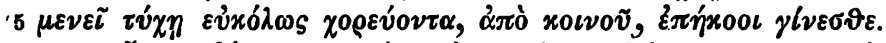

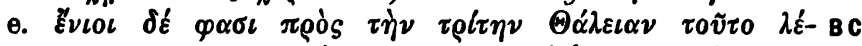

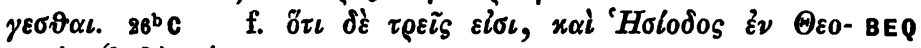

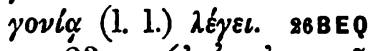

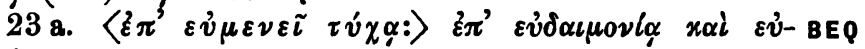
$10 \mu o \iota \rho l \alpha .21^{b}$ BEQ

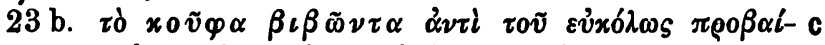

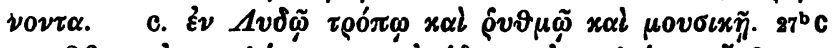

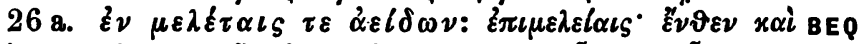

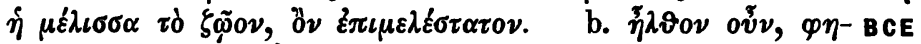

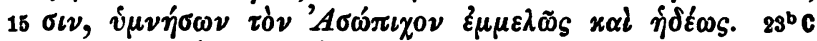

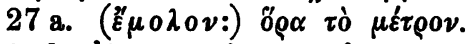

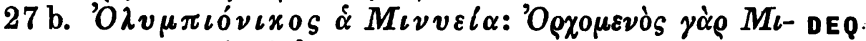

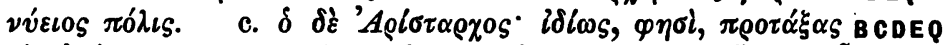

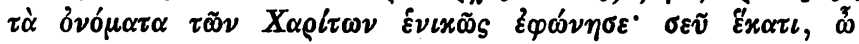
20 Xá

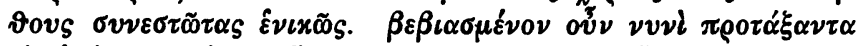

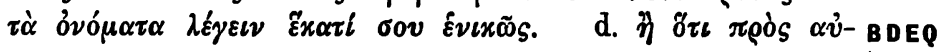

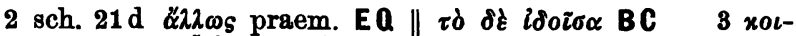

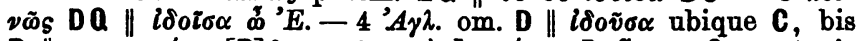

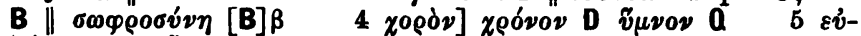

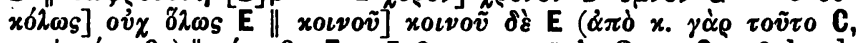

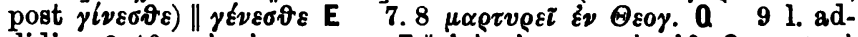

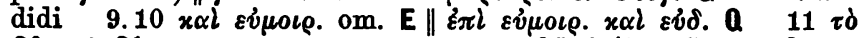

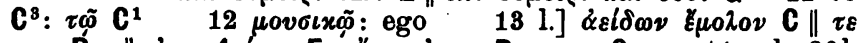

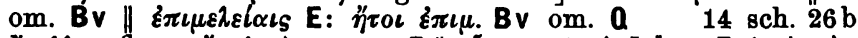

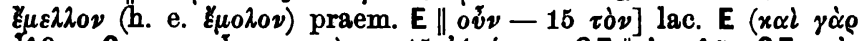

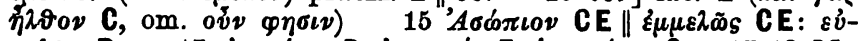

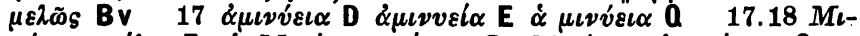

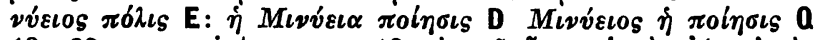

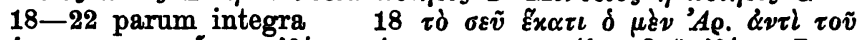

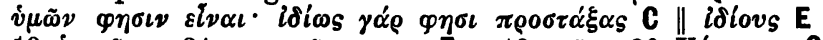

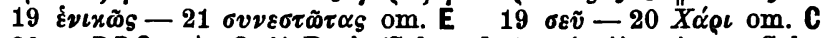

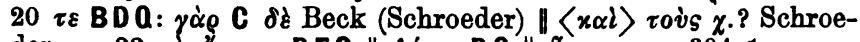

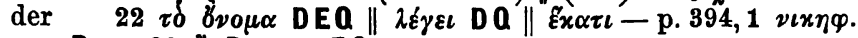
om. D 22 ฑे B: om. EQ 


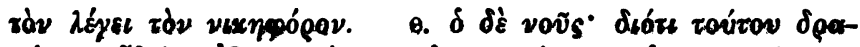

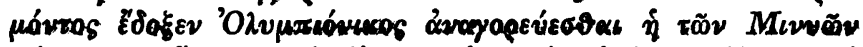

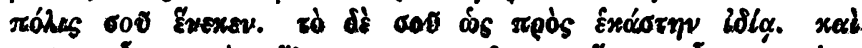

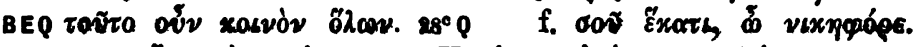

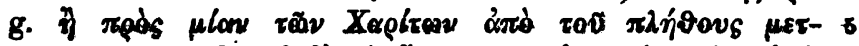

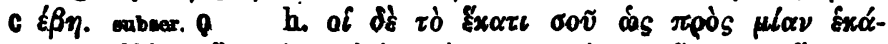

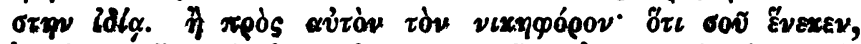

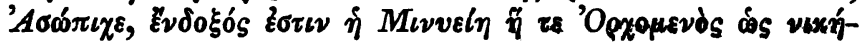
бavios.

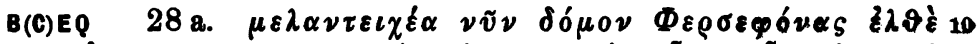

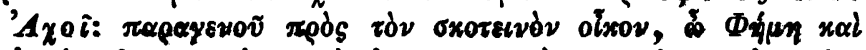

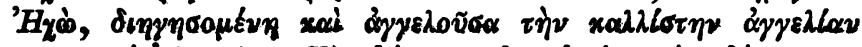

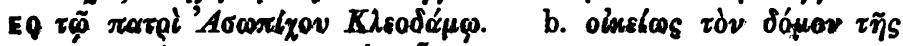

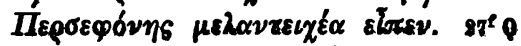

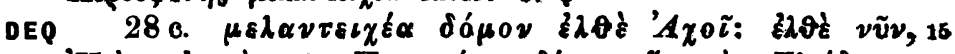

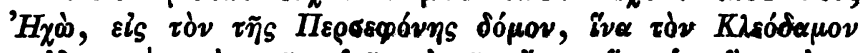

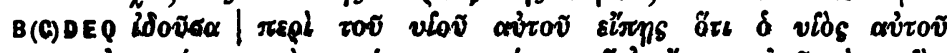

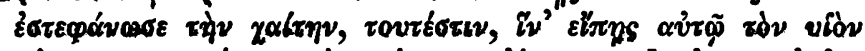

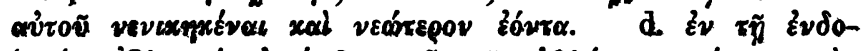

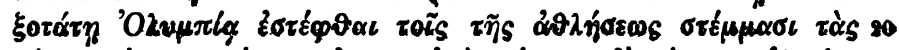

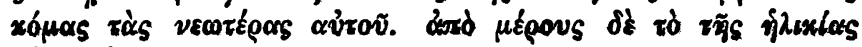
vtev $\lambda \xi \xi \delta i .280$

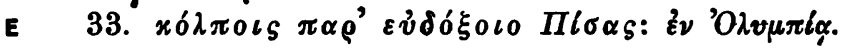

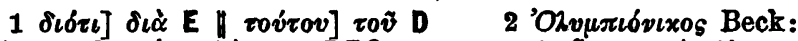

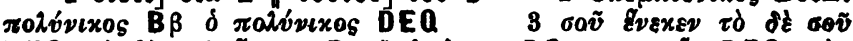

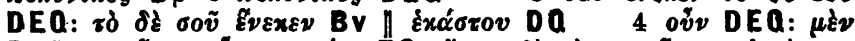

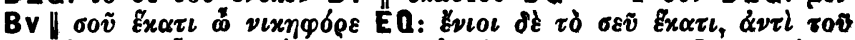

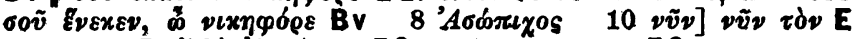

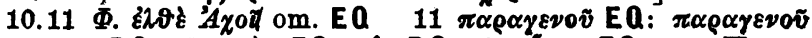

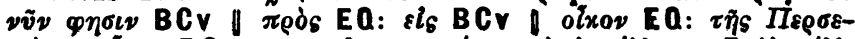

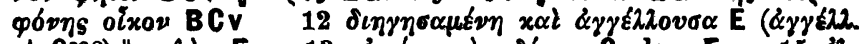

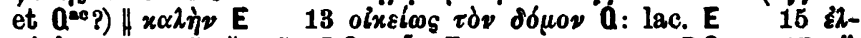

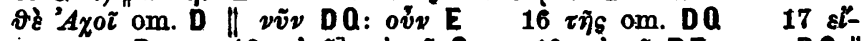

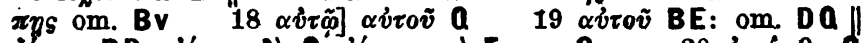

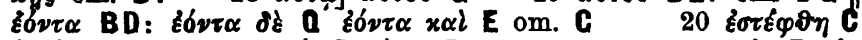

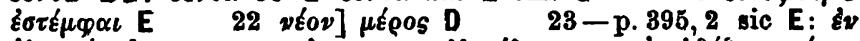

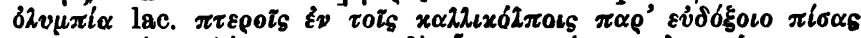

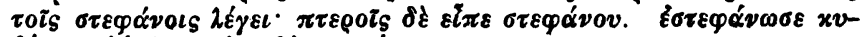
$\delta \imath_{\mu \omega \nu} \dot{\alpha} \varepsilon \hat{\imath} \lambda \omega \nu \cdot \dot{\varepsilon} \pi \varepsilon \iota \delta \dot{\eta}-\sigma \tau \dot{\varepsilon} \varphi \alpha \nu 0 S$ 


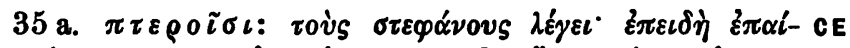

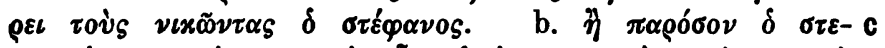

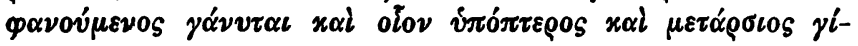

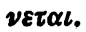

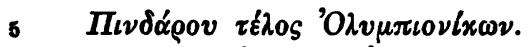

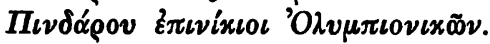

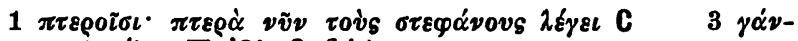

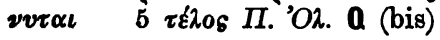


Digitizod by Google 
- i

.

1
1
1
1
1
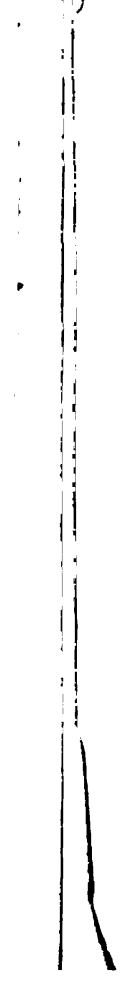

$\because: \because \bullet: \bullet: \vdots:$ 


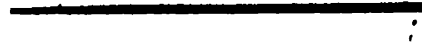

i

t.

Digitized by $\mathrm{GOg} l e$ 


\section{Wissensehaftliche Ausgaben griechischer und lateinischer Schriftsteller in Voriage ron B. G. Teubner 冤 in Leipzig.}

Sammluns wiseoneefinticher Commentere min siechisehen und romisciren Sehriftetolfern. Aetna. Von S. Sudhaus. geh. th 6. - in Leinw. Bob. Hh 7.Inores Buch III. Von R. Heinze. geh. Kh4. - in Leinw. geb. K. 5. Sophokles Flektra. Von G. Kaibol, geh. th 6., in Loinw. geb. Kh 7.-

\section{Iritische und kommontierte Aavesber.}

Nene Eracheinungen 1901-1908.

Acts Apostolorum: B. L ucss.

Demetril Phalerel qui dicitur de elocutione libellus. Praefatus recensuit adnotavitque $\mathrm{L}$. Raderm ach ex. geh. th 5.-

Didascaliae apontolorum fragmenta Veronensia Latins. Accedunt canonum qui diountur apostolorum et Aegyptiorum reliquise. Fr. E dm: Ha uler. Faso. I: Praefatio, fragmenta, imagines. geh. Hh 4.-

Euangelinm sec. Matthaeum cum var. lect. ed. Fr. Bl \& s g. geh. MA 3.60. Puripidis fabulae edd. R. Prinz et N. Wecklein. geh.

Vol. I. P. I: Modes. Ed. II. |Vol. II.P.III: Bacchae. kh 8.th 2.40.

" I. " II: Alcestis. Fd. II. ch 1.80 .

"II. "IV: Hersclidae. M.2.-

" II. "V: Hercules. Ah 2.40.

" II. " VI: Iphig. Aul. th $\mathbf{8 . 8 0}$.

I. „III: Hecubs. $\mathscr{H} 1.60$.

"III. " I: Andromacha. M. 8.40.

I. "IV: Hlectra. M 8.-

I. "V: Ion. th 8.80 .

I. "VI: Helena NA 3.-

I. "VII: Cyclops. $M 1.40$.

"I. "I: Iphlg. Trur. M.8.40.

"I. "II: Supplic. Kh 8.-

" III. " II: Hippolytus.M. 8.80 .

" III. "III: Orestes. k 2.80.

"III " IV: Phoenissee. Ka.80.

"III. "V: Trosdes. M 2.80.

"III. "VI: Bhesus. K3.60.

Grammatioi Graecl. Para III. Scholia in Dionysii Thracis artem grammaticam rec. A. Hil g a rd. geh. Hh 36.-

Hestodi carmina. Accedit Homeri et Hesiodi certamen. Rec. A. Rzaoh. geh. Wh 18. -

Homer1 carmina. Recensuit et selects leotionis varfetate instruxit A. Ludwich. Pars prior: חias. Vol prius. geh. th 16.-

Q. Horati Flacoi opera reco. O. Keller et A Holder. Vol. I. Carminum libri IV, Epodon liber, Carmen saeculare. Iterum reo. O.K eller. geh. Kh12.- - Vol.IT, Fasc. I. Sermonum libri II. geh.Mh4. - Vol.II, Fasc.II. Epistulae, De arte poetica. geh.Kh6.-

[Lueas.] Acta .Apostolorum s. L. ad Theophilum lib. alter. Sec. formam quae vid. Romanam rec. Fr. Blass. geh. th 2.-

[-] Evangelinm sec. Lneam s. I. ad Theoph. lib. prior. Sec. formam quas vid. Romanam rec. Fr. Blass. geh. H4.-

Pindari carmina prolegg. et comm. Instr. ed.W. Christ. geh. Kh 14. P od. I c h roeder. Ed. V. geh. Th 14.-

Platonis Sophista ed. O. A pelt. geh. 位 5.60.

Statius' Silvae, herangg. u. orkl. v. Fr. Vollmer. geh. the 16.Stoicorum veterum fragmente coll. I. abeA r $n$ im. Vol.II. geh. Kh 14.- Theophrasts Charaktere, hrsg., ubers. u. erkl. v. d. Philol. Gesellsch. zu Leipzig. geh. Ah 6.-

Thucydidis historiae rec. C. Hude. Tom. I. geh. Mh 10.- Tom. II. geh. th 12.- 




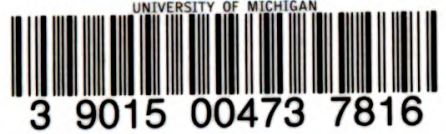

\section{DO NOT REMOVE OR}

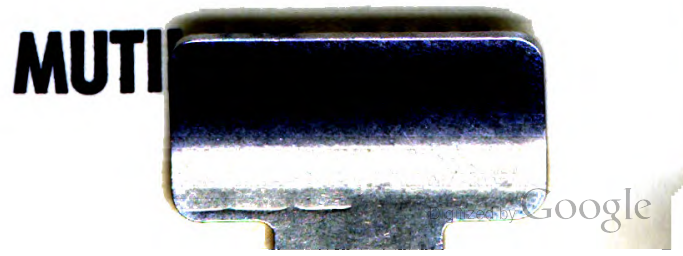


